SANDIA REPORT

SAND98-2252

Unlimited Release

Printed October 1998
1-ISO619-Rev ded 12690

$$
\begin{gathered}
\text { RECFIVED } \\
\text { HOV } 161998 \\
\text { OSTI }
\end{gathered}
$$

\title{
Proceedings of US/Japan Workshop (97FT5-06) on High Heat Flux Components and Plasma Surface Interactions for Next Fusion Devices
}

Richard Nygren and Diana Kureczko

\section{Prepared by}

Sandla National Laboratories

Albuquerque, New Mexico 87185 and Livermore, California $\mathbf{9 4 5 5 0}$

Sandia is a multiprogram laboratory operated by Sandia Corporation, a Lockheed Martin Company, for the United States Department of Energy under Contract DE-AC04-94AL85000.

Approved for public release; further dissemination unlimited. 
Issued by Sandia National Laboratories, operated for the United States Department of Energy by Sandia Corporation.

NOTICE: This report was prepared as an account of work sponsored by an agency of the United States Government. Neither the United States Government nor any agency thereof, nor any of their employees, nor any of their contractors, subcontractors, or their employees, makes any warranty, express or implied, or assumes any legal liability or responsibility for the accuracy, completeness, or usefulness of any information, apparatus, product, or process disclosed, or represents that its use would not infringe privately owned rights. Reference herein to any specific commercial product, process, or service by trade name, trademark, manufacturer, or otherwise, does not necessarily constitute or imply its endorsement, recommendation, or favoring by the United States Government, any agency thereof, or any of their contractors or subcontractors. The views and opinions expressed herein do not necessarily state or reflect those of the United States Government, any agency thereof, or any of their contractors.

Printed in the United States of America. This report has been reproduced directly from the best available copy.

Available to DOE and DOE contractors from

Office of Scientific and Technical Information

P.O. Box 62

Oak Ridge, TN 37831

Prices available from (615) 576-8401, FTS 626-8401

Available to the public from

National Technical Information Service

U.S. Department of Commerce

5285 Port Royal Rd

Springfield, VA 22161

NTIS price codes

Printed copy: A99

Microfiche copy: A0I

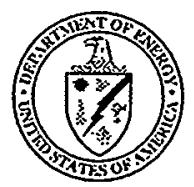




\section{DISCLAIMER}

\section{Portions of this document may be illegible electronic image products. Images are produced from the best available original document.}


SAND98-2252

Unlimited Release

Printed October 1998

\title{
Proceedings of US/Japan Workshop (97FT5-06) \\ On
}

\section{High Heat Flux Components and Plasma Surface Interactions for Next Fusion Devices}

\author{
San Francisco, California \\ December 8 - 11, 1997 \\ Edited by \\ Richard Nygren \\ Diana Kureczko \\ Sandia National Laboratories \\ P.O. Box 5800 \\ Albuquerque, New Mexico 87185-1129
}

\begin{abstract}
The 1997 US-Japan Workshop on High Heat Flux Components and Plasma Surface Interactions for Next Step Fusion Devices was held at the Warwick Regis Hotel in San Francisco, California, on December 8-11, 1997. There were 53 presentations as well as discussions on technical issues and on planning for future collaborations, and 35 researchers from Japan and the US participated in the workshop.
\end{abstract}

Over the last few years, with the strong emphasis in the US on technology for ITER, there has been less work done in the US fusion program on basic plasma-materials interactions and this change in emphasis workshops. The program this year emphasized activities that were not carried out under the ITER program and a new element this year in the US program was planning and some analysis on liquid surface concepts for advanced plasma facing components.

The program included a ceremony to honor Professor Yamashina, who was retiring this year and a special presentation on his career. 
Page Intentionally Left Blank 
Table of Contents

Session I: Activities in present and near term devices

I-1

(O. Motojima \& S. Berk)

Page

Opening Remarks

R. Nygren (Sandia), S. Berk (DOE),

N. Noda (NIFS), K. Wilson (Sandia)

Present Status of LHD

$\mathrm{I}-4$

O. Motojima (NIFS)

Divertor, first wall and PSI issues in LHD I-40

N. Noda (NIFS)

$\begin{array}{lr}\text { Status of NSTX and PSI issues } & \text { I-69 }\end{array}$

M. Peng (PPPL)

Design \& initial operation of W-shaped divertor in JT-60U I-98

K. Masaki (JAERI)

Progress in DIII-D

$\mathrm{I}-126$

C. Wong (GA)

Highlights and plans for C-MOD .............[Paper not available] MIT/Nygren (Sandia)

\section{Session II: PFC Development for near term devices}

II-1 (K. Nakamura \& C. Wong)

Utilization of high $\mathrm{Z}$ materials as PFCs

II-3

T. Tanabe (Nagoya U.)

Development of $\mathrm{W}$ brush armor

G. Wille (Boeing)

Development of high heat flux components at JAERI

II-51

K. Nakamura (JAERI)

Be-Cu Joining

II-76

C. Cadden (Sandia)

Problems and Evaluation of plasma facing materials

II-98

N. Yoshida (Kyushu U.) 


\section{N. Noda \& K. Wilson}

Small personal history on plasma surface interactions

III-3

T. Yamashina (Hokkaido U.)

Session IV: wall conditioning, sputtering, erosion

IV-1

T. Tanabe \& Y. Hirooka

Wall conditioning at the start up phase of LHD

IV-3

A. Sagara (NIFS)

RF wall conditioning

IV-11

D. Cowgill (Sandia)

Erosion/redepostion of high- $\mathrm{Z}$ materials in a linear

Divertor simulator

IV-31

N. Ohno (Nagoya U.)

Erosion and impurity effects on PFC materials in PISCES-B

IV-51

R. Doerner (UCSD)

Recent erosion/redeposition analysis

IV-69

Sze/Brooks (ANL)

Dependence of graphite erosion yield on irradiation flux

Close to actual edge plasma

IV-77

Y. Ueda (Osaka U.)

DiMES experiments

IV-95

D. Whyte (GA)

Reflected neutral particle spectra on MAP

IV-112

S. Ohtsu, K. Kobayashi, S. Tanaka (U. Tokyo)

Session V: Plasma Studies

S. Luckhardt (UCSD)

Effects of turbulent fluctuations on boundary ion

Temperatures in PISCES

$\mathrm{V}-3$

S. Luckhardt (UCSD)

TFTR Experiments with $\mathrm{Li}$

$\mathrm{V}-18$

B. Skinner (PPPL) 
Deposition of $\mathrm{Li}$ on a probe in TFTR

Y. Hirooka (UCSD)

Session VI: Development Issues for Near Term PFCs

VI-1

A. Sagara \& C. Wong

Discussion, development issues for near term PFCs

VI-1

A. Sagara \& C. Wong

Session VII: PFM issues and development

N. Yoshida \& R. Causey

VII-1

W/Cu layers resistant to erosion and tritium permeation

VII-3

M. Shibui (Toshiba)

Review of recent work on removing tritium from PFCs

B. Skinner (PPPL)

VII-20

Chemical compatibility of $\mathrm{C}$ with $\mathrm{Be}$

VII-40

Ashida \& K. Watanabe (Toyama U.)

Tritium retention in $\mathrm{Be}$

VII-62

R. Causey (Sandia)

Modeling of $\mathrm{H}$ isotope retention/release in PFC materials

A. Grossman (UCSD).......... [Paper Not Available]

\section{Session VIII: First Wall Development}

VIII-1

M. Tillack \& N. Noda

HPD approaches, core radiation and He blanket, ST example

VWI-3

B. Wong (GA)

Concept of FliBe blanket in FFHR

VIII-17

A. Sagara (NIFS)

APEX high fusion power density evaluation

VIII-30

N. Morley (UCLA)

Damage in the plasma facing part of the first wall

VIII-50

N. Yoshida (Kyushu U.)

Protective coating at the plasma facing part of first wall

VIII-73

N. Noda (NIFS) 
Plasma spray coating development

VIII-82

Castro/Nygren (LANL)

Recent progress at PPI in plasma spraying

VIII-98

S. Odell (Plasma Processes)

Session IX: PSI/PFM Issues and Collaboration

IX-1

N. Noda \& R. Nygren

Discussion on PSI/PFM issues and collaborations

IX-3

N. Noda \& R. Nygren

Session X: Panel on Future PFC Concepts

M. Tillack \& Y. Ueda

ALPS summary

$\mathrm{X}-3$

C. K. Sze (ANL)

Heat removal issues with liquid metal PFCs

R. Nygren (Sandia)

Helium cooling experiments and prospect

$\mathrm{X}-36$

Baxi (GA)

Comments on liquid/pebble divertor

$X-54$

Y. Ueda (Osaka U.)

Novel concept for a moving belt PFC

Y. Hirooka (UCSD)

He self pumping summary

R. Nygren (Sandia)

Characterization of liquid metal surface

X-99

R. Bastasz (Sandia)

Reflected neutral particle spectra on MAP ... [Paper Not Available]

S. Ohtsu, K. Kobayashi, S. Tanaka (U. Tokyo) 
Discussion of PFC Collaborations

XI-3

R. Nygren, A. Sagara, N. Noda, S. Luckhardt

Session XII: Supplement Session

XII-1

K. Masaki \& D.K. Sze

More activities / results in Japan

XII-3

N. Noda

Simulation Experiments on Screening of

Lithium by boundary plasma

XII-4

H. Sugai, H. Toyoda

Hydrogen Absorption/Desorption by

Oxygen Contaminated Boron film

XII-7

H. Eiki, K. Tsuzuki

Joining of C/C Composite with Oxygen-Free

Copper by Titanium Foil

XII-9

Tatsuo Oku, Yosho Imamura, et. al.

Evaluation of High Z Metals

XII-16

S. Yamazaki

High heat flux testing of neutron irradiated divertor modules

XII-21

R. Duwe, J. Linke, M. Rodig, R. Nygren 
Remarks on the outlook for collaborations

Motojima/Noda, S. Berk

Summary/discussion: Liquid surface PFCs \& collaborations.

R. Nygren \& A. Sagara

Summary/discussion: other PFCs \& collaborations

N. Noda \& S. Luckhardt

Summary/discussion: Development. Issues for near term PFCs

A. Sagara \& C. Wong

Summary/discussion: PSI/PFM issues \& collaborations

N. Noda \& R. Nygren

Closing remarks

N. Noda \& R. Nygren

Appendix A: Workshop Agenda 
Session I: Activities in Present and Near Term Devices

I-1 


\section{I-2}




\section{J-US WORKSHOP \\ ON HHFC/PSI FOR NEXT FUSION DEVICES}

(97 FT5-06)

- WORKSHOP SERIES HAS BEEN PRODUCTIVE AND MUTUALLY BENEFICIAL

$\Rightarrow$ USDOE WILL CONTINUE ITS SUPPORT

- CHANGE IN USDOE RESPONSIBILITY FOR HHFC/PSI PROGRAMS

$\Rightarrow$ MARVIN COHEN RETIRED IN JAN. 1997

$\Rightarrow$ SAM BERK REPLACED MARVIN (ALSO RESPONSIBLE FOR FNT PROGRAMS)

- J-US HHFC/PSI COLLABORATION WILL BE STRENGTHENED IN FUTURE

$\Rightarrow$ US FUSION PROGRAM BUDGET IS STABLE

$\Rightarrow$ FUNDING FOR SUCH COLLABORATIONS WILL INCREASE (US TECHNOLOGY R\&D WILL BE LESS FOCUSED ON ITER AFTER FY1998)

$\Rightarrow$ HHFC/PSI WORK WILL BE LARGEST ELEMENT OF US TECHNOLOGY R\&D

$\Rightarrow$ USDOE VALUES COLLABORATION WITH JAPAN AND LOOKS FORWARD TO BUILDING ON PAST SUCCESSES. 


\author{
US/Japan Workshop on PSI \\ December 8-11, 1997 \\ Warwick Regis Hotel, San Francisco, USA \\ Present Status of LHD
}

$=$ LHD

Osamu Motojima

National Institute for Fusion Science 322-6 Oroshicho, Toki 509-52, Japan.

1, Introduction to LHD Project

Missions in Fusion Physics and Technology,

Specifications of LHD

2, Construction Status/Engineering Achievements

3, Experimental Planning

Commissioning Tests

First Plasma Start up Scenario

Heating System (NBI, Gy rotron, ICRF)

4, Summarizing 


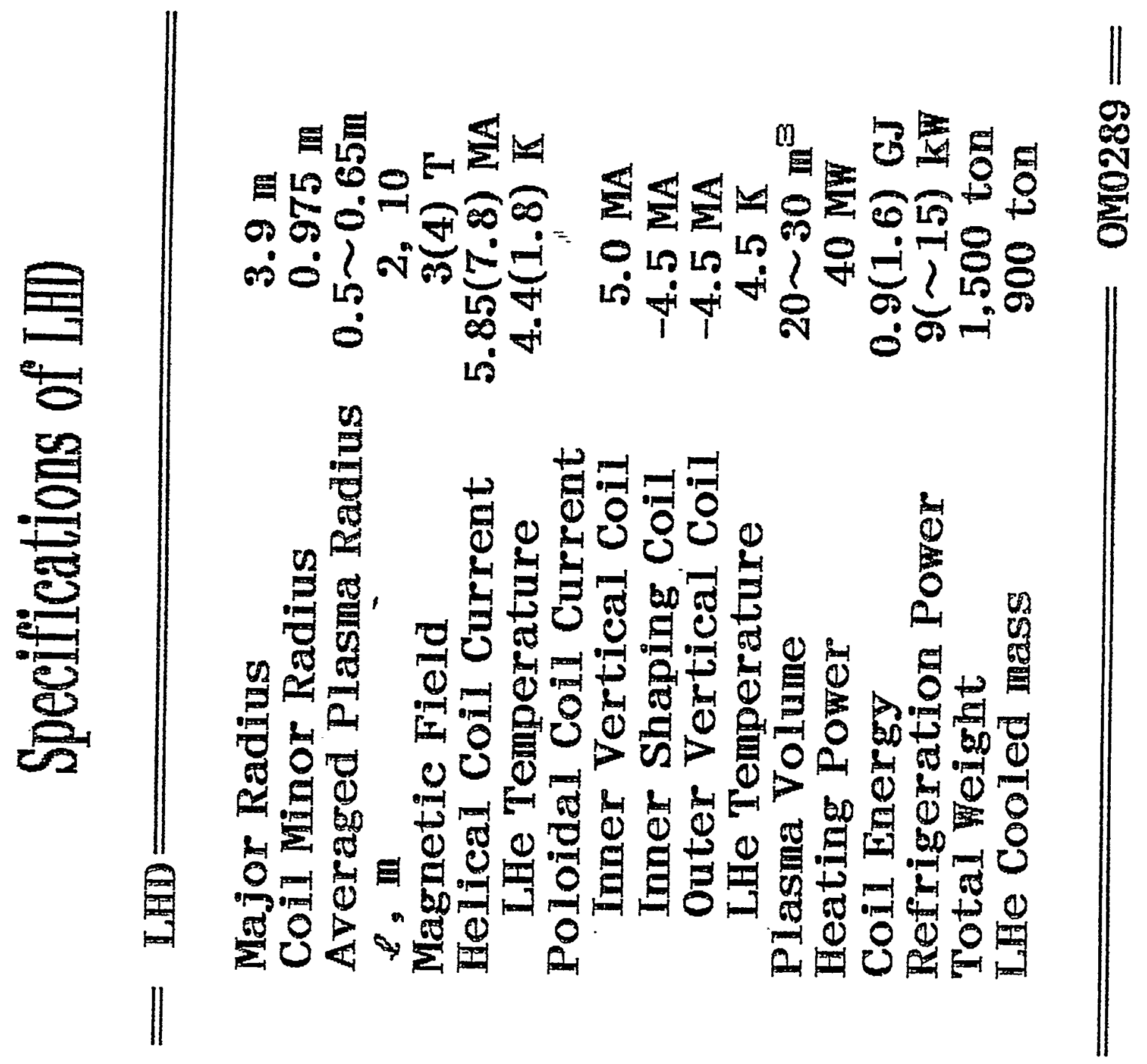




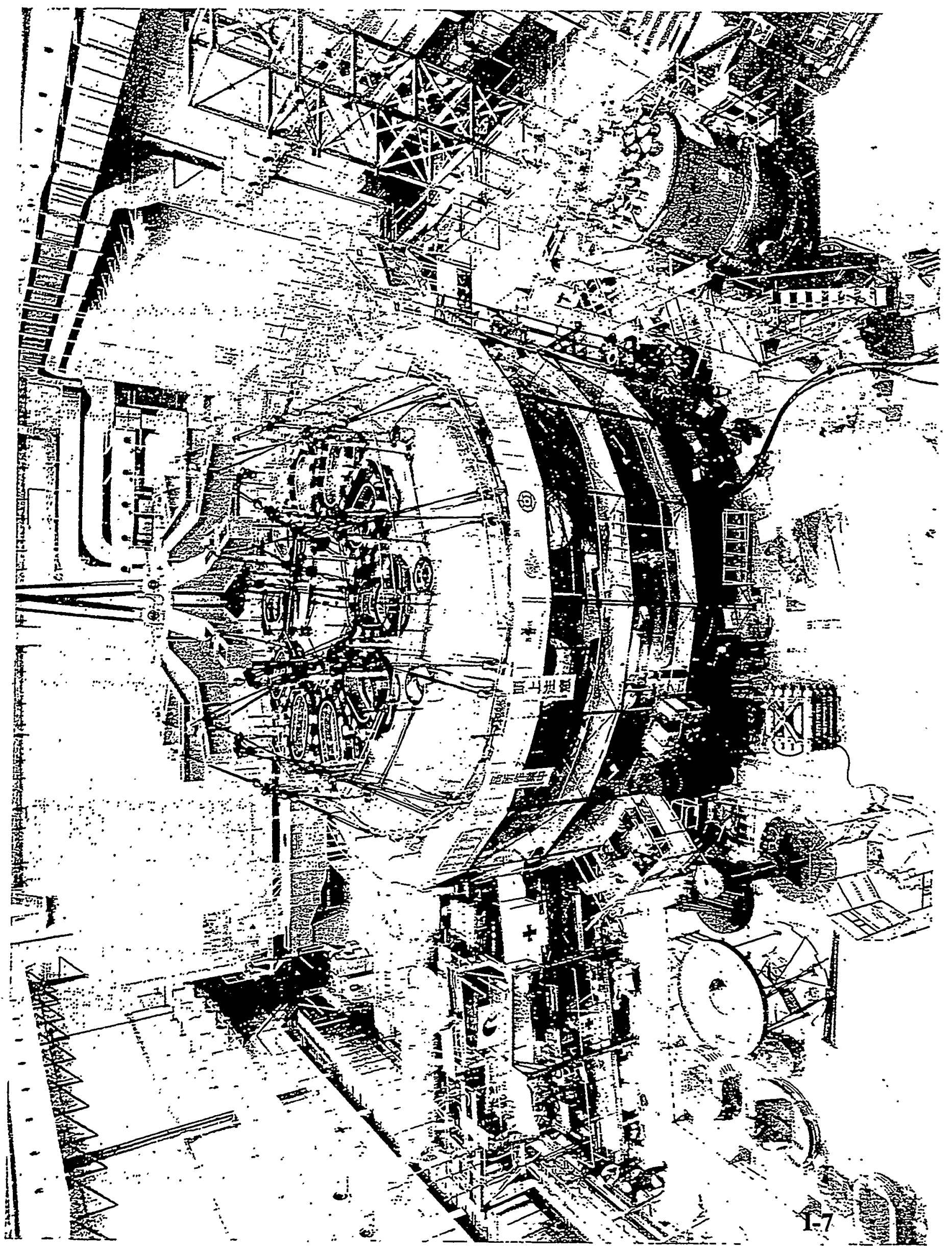




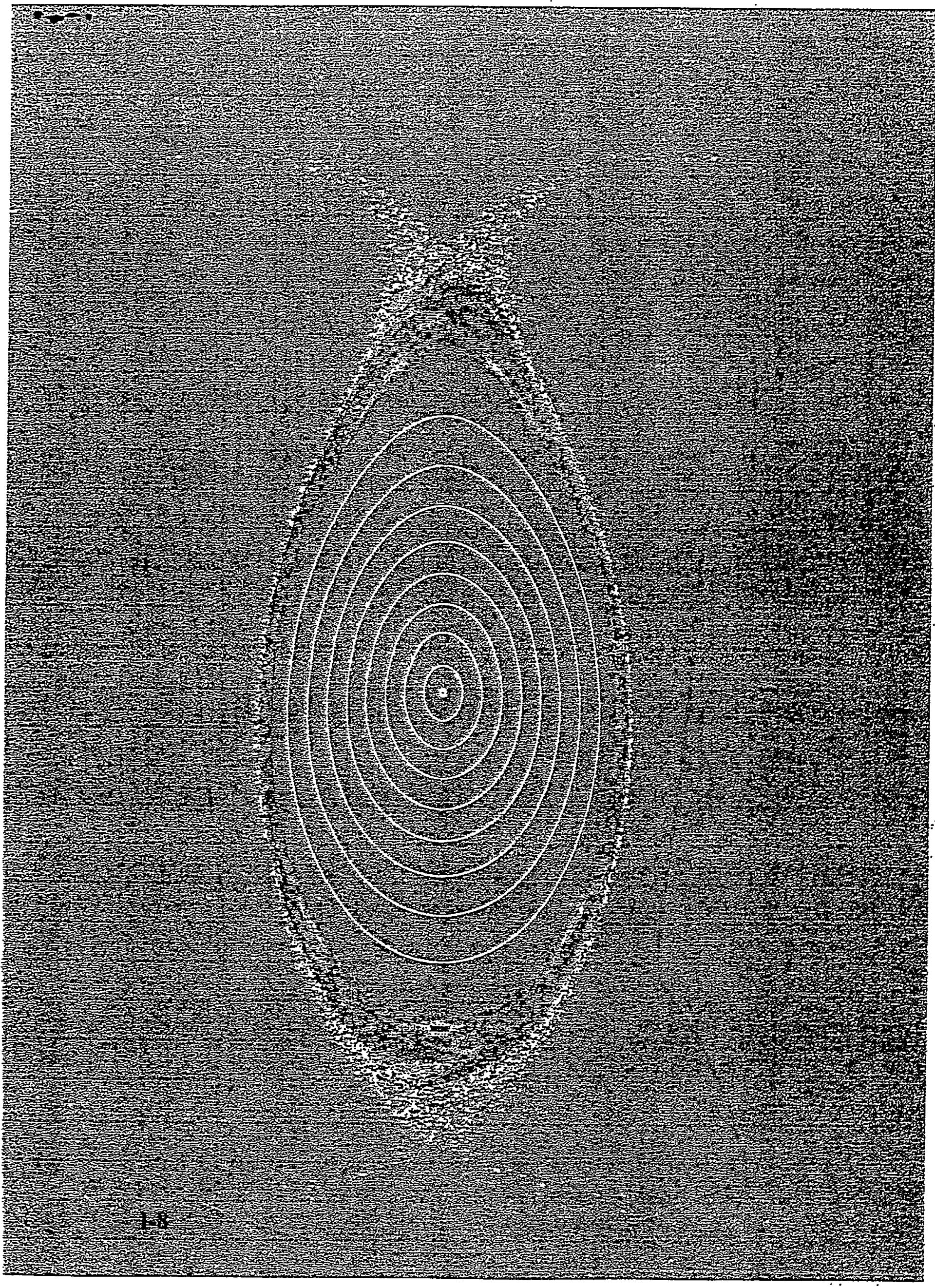




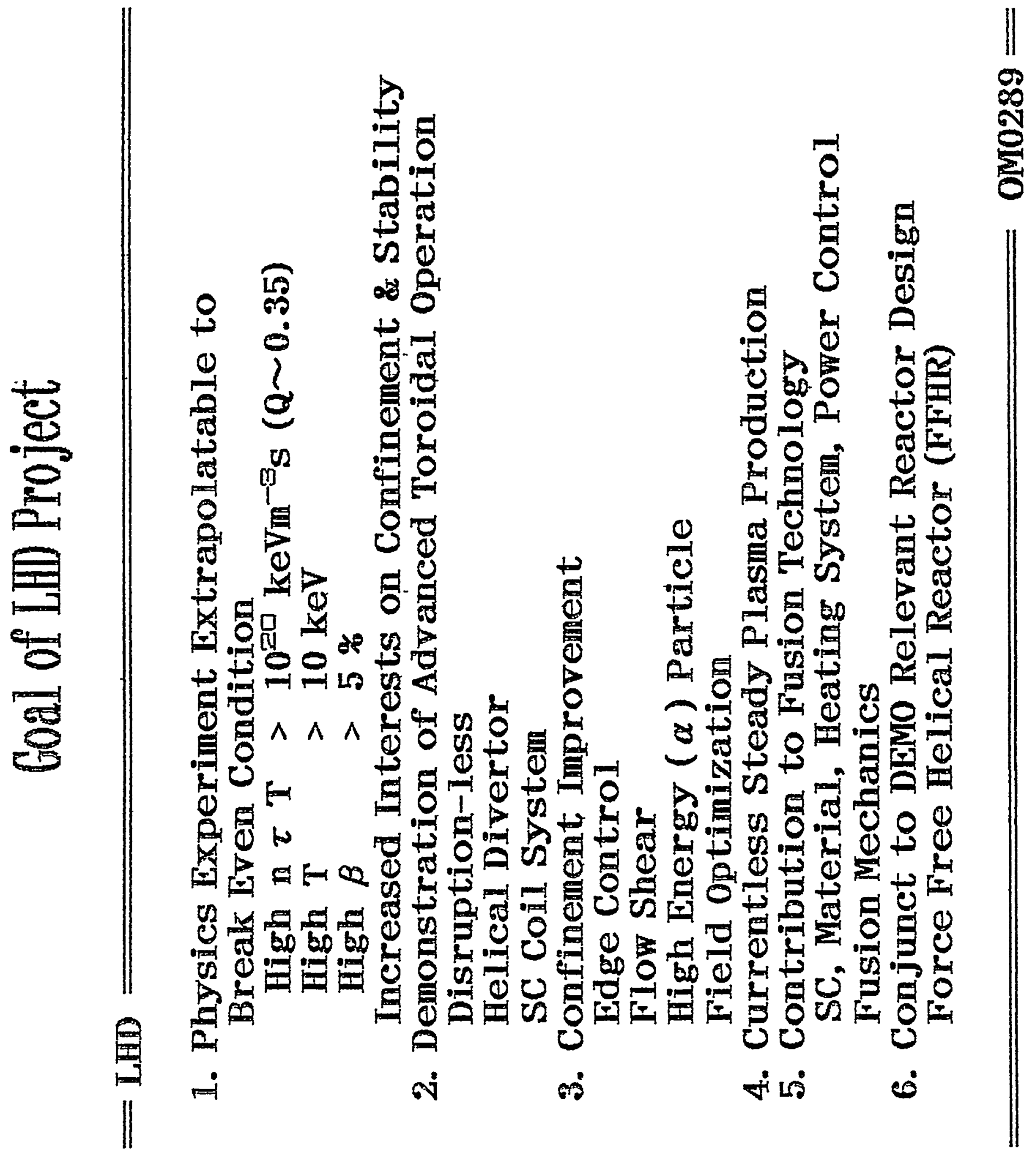




\section{Platform for experimental study}

\begin{tabular}{|c|cccc|}
\hline Device & $\mathrm{R}(\mathrm{m})$ & $\mathrm{a}(\mathrm{m})$ & $x_{0}$ & $\varkappa_{\mathrm{a}}$ \\
\hline ATF (ORNL) & 2.04 & 0.27 & 0.26 & 1.00 \\
CHS (NIFS) & 0.94 & 0.20 & 0.31 & 1.10 \\
Helioton E (Kyoto U.) & 2.17 & 0.21 & 0.51 & 2.75 \\
W7-AS (IPP,MPG) & 2.00 & $0.11-0.18$ & $0.33-0.54$ & $0.33-0.54$ \\
LHD (NIFS) & 3.9 & 0.6 & 0.4 & 1.3 \\
\hline
\end{tabular}

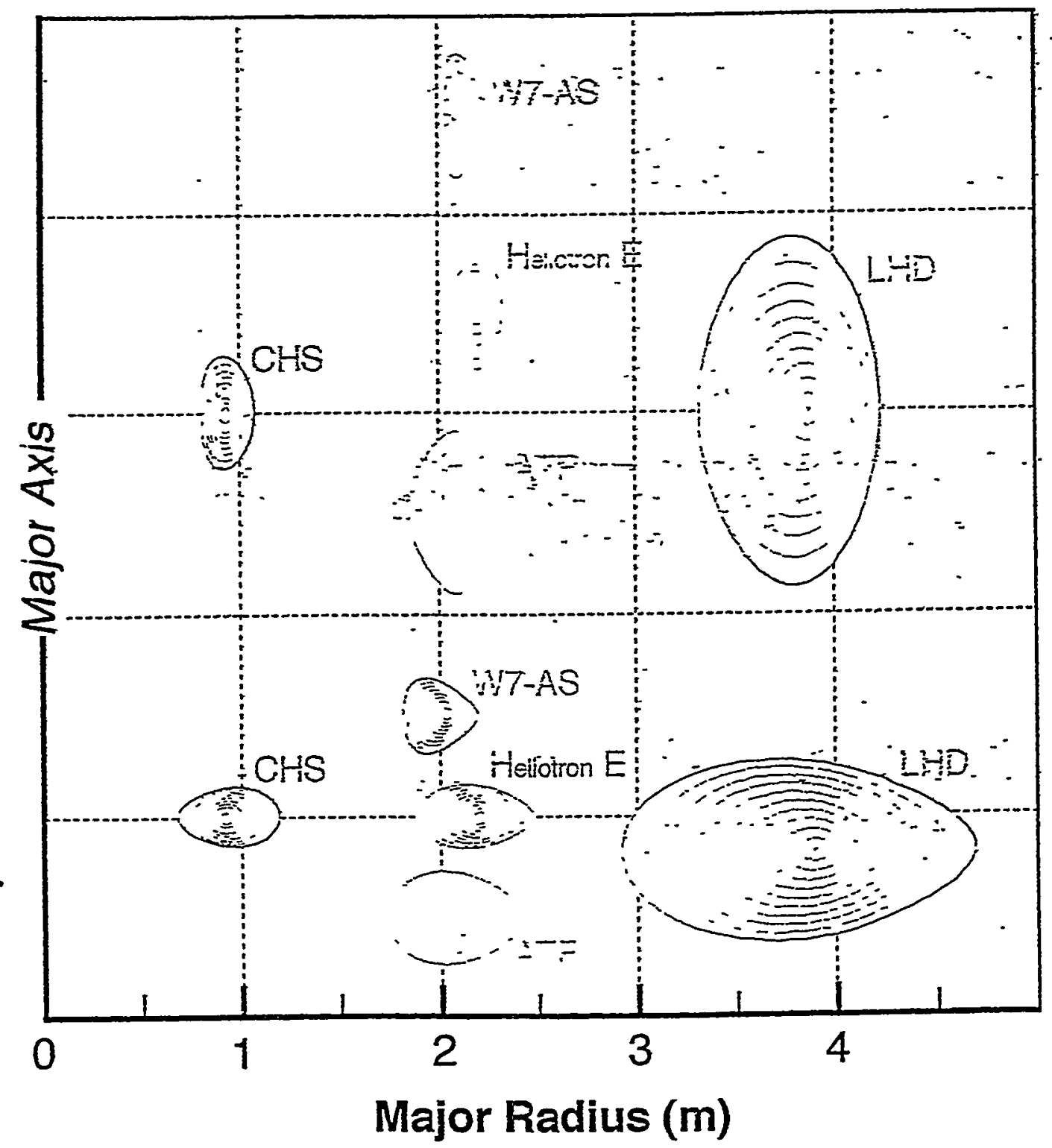




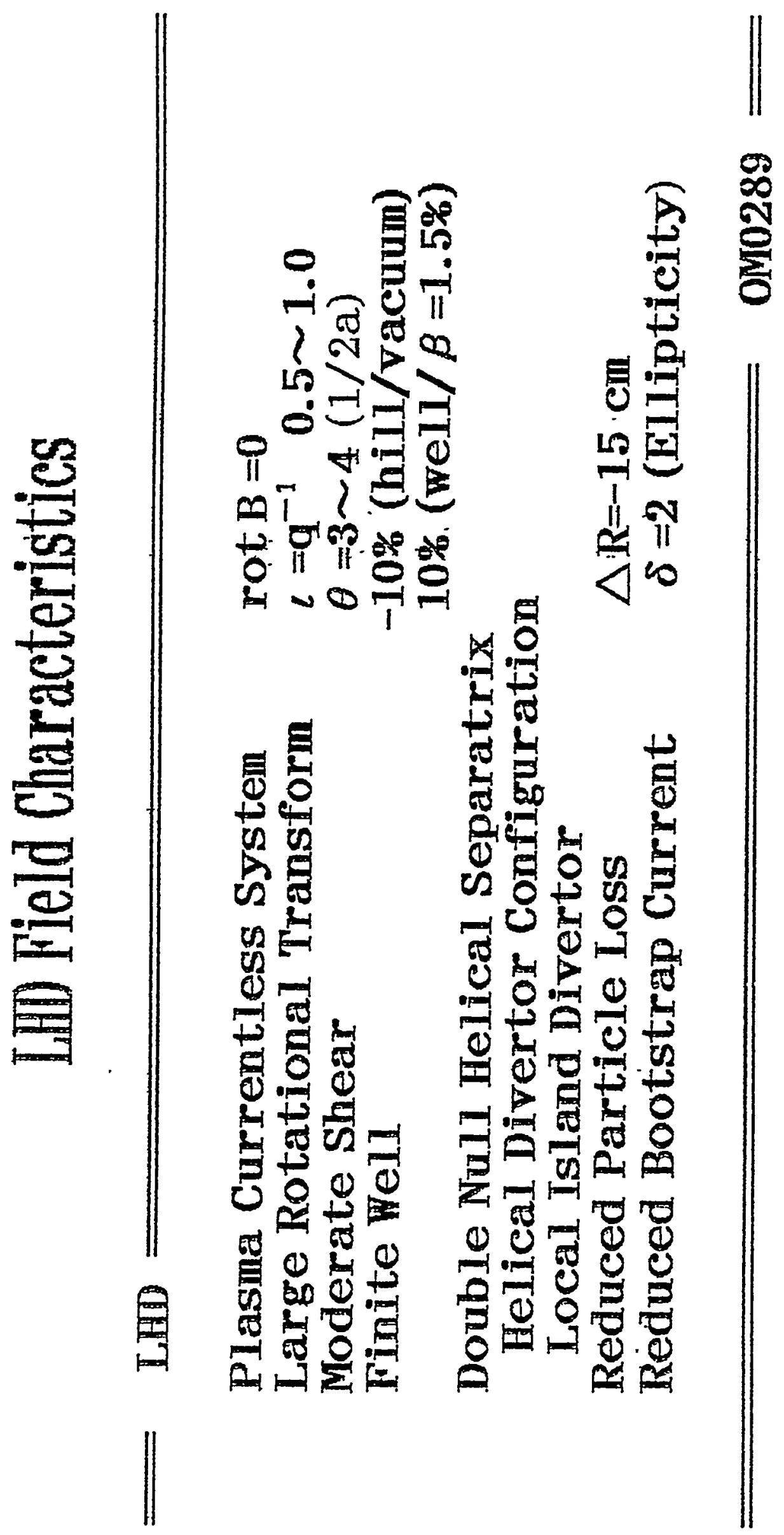


"әseqezep әрош-7 у

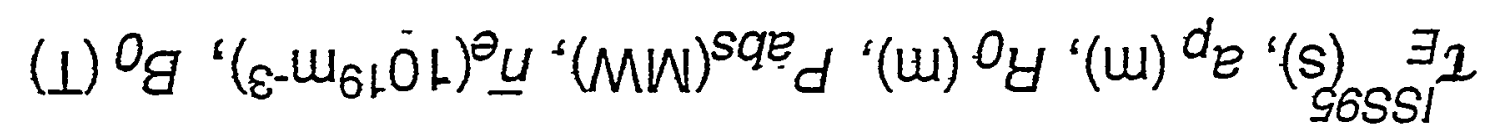

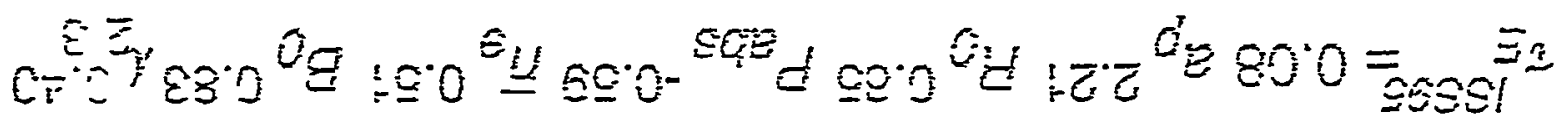
001
$\therefore-0$
$2-01$
$\varepsilon-0 !$

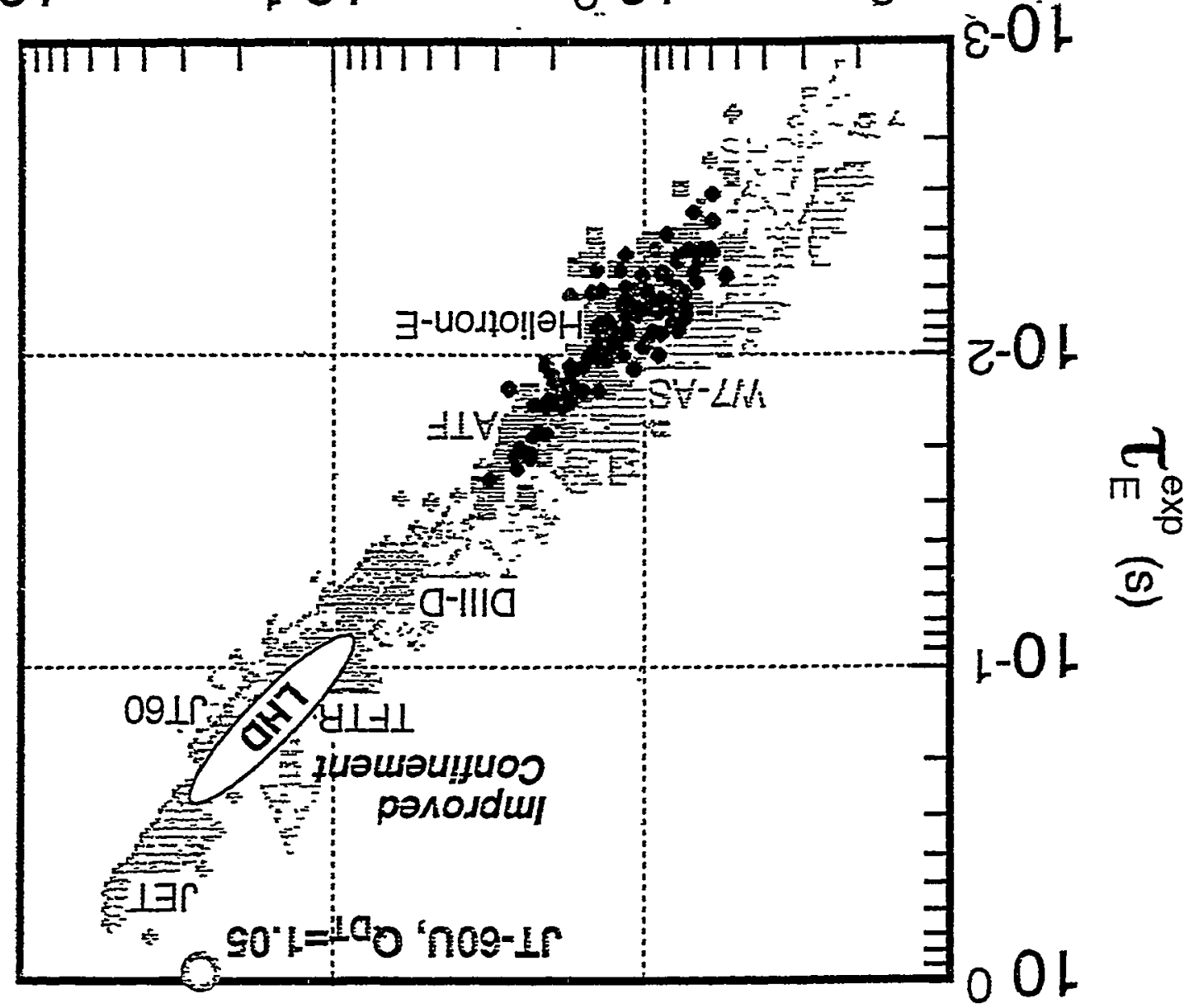

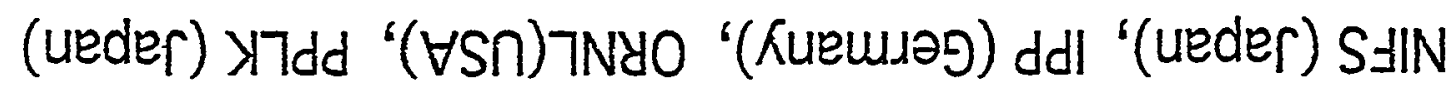

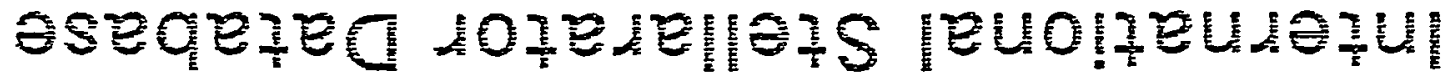




\section{Magnetic Field Optimization Base}

$=$ LHD

0. Intensive Theoretical Study $\rightarrow \quad$ till 1990.

1. Basic Size of Machine $\rightarrow B=3 T, R=3.9 \mathrm{~m}$, and Plasma Volume $30 \mathrm{~m}^{3}$

Close Regime to the Break Evien

2. Field Configuration $\rightarrow \quad m=10 / 1=2$ Continuous Helix

Reducing the Adoption of Unfavorable Field Harmonies

3. Coil System $\rightarrow$ Super Conducting Coils

Two Helical Coils and 3 Sets of Poloidal Coils

4. Edge Control $\rightarrow$ Helical Divertor and Local Island Divertor

5. Heating System $\rightarrow \sim$ 3OMW with NBI, ICRF and ECRH.

Stead State 
1. Large Magnetic Rotational Transform and Medium Shear

2. Established Empirical Global Confinement Scaling Law.

in the Vicinity of Plateau Regime

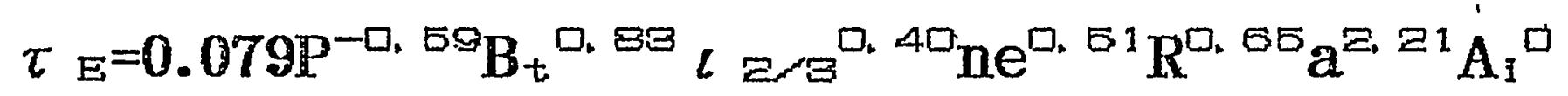

(ISS95-scaling)

- Gyro-Reduce Bohm Type -

3. Neoclassical Type Diffusion Process in Lower-Collision Regime Ion Root Condition $\rightarrow$ Accessible High $\mathbf{n} \tau \mathbf{T}$.Plasma Electron Root Condition $\rightarrow$ Accessible High T Plasma

* Radial electric field plays an important role in the combination of plasma rotation and flow shear.

4. MHD Stability optimized

Satisfying Mercier Criterion and Ballooning Mode Limit

* Expected average $\beta$ value is 5\%. 


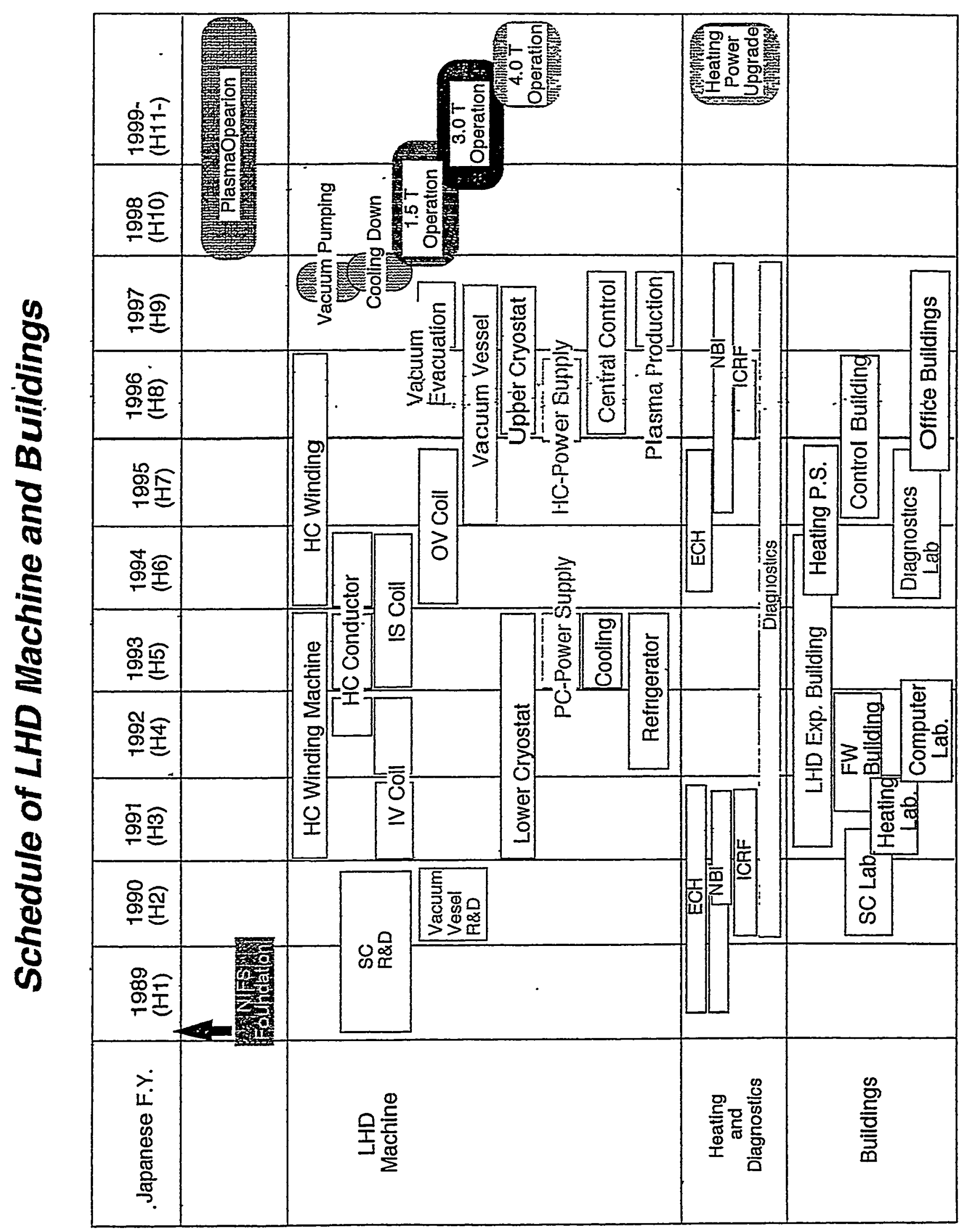

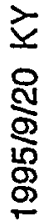




\section{Progress Summary of LWD Construction}

LEDD

Complete: $95 \%$ of Construction Schedule

1. Coil Fabrication Completed Helical Coil

Poloidal Coils(OV)

2. Vacuum Chamber Completed $\rightarrow$ The Biggest Critical Path Diameter: $7.8 \mathrm{~m}, 13 \mathrm{kA}$ Nominal 450 turns $\times 2$ Profile Accuracy $\pm 2 \mathrm{~mm}$ Diameter: $11.1 \mathrm{~m}:-31.3 \mathrm{kA}$ Nominal. 8 Double Pancakes $x 2$ Profile Accuracy $\pm 2 \mathrm{~mm}$ Profile Accuracy $\pm 10 \mathrm{~mm}$ Up/Down Port 10, Inside 6 Horizontal 10, Tangential 4

3. Helical Divertor Panel Under Construction Divertor R \& D Continued Graphite Tile Heat Load Test (ACT)

4. Power Supply Completed

Real Road Test with Fuse, Switch $16 / 23 \mathrm{kA}$ Nominal, 100 shots Reliability Check

5. SC Bus line Completed

6. LHe Liquefier Completed Test Operation $10^{-\infty}$.

9 lines, $32 \mathrm{kA} 、 5 \mathrm{kV}, 5$ Hold T. Tube

$2,700 \mathrm{l} / \mathrm{hr}$ equivalent

7. Control System Under Intensive Construction

Center Contro1 System, Timing System (Time Sequence),

Experimental LAN, LHe Liquefier Control System, Monitoring System $2,000 \mathrm{ch}$, Interlock System for Safety Operation

8. Utilities Completed

Underground Stage, Pressured Air Piping, Wireless System, Safety Sensors、Key Lock System, Gate Valve System, etc. 


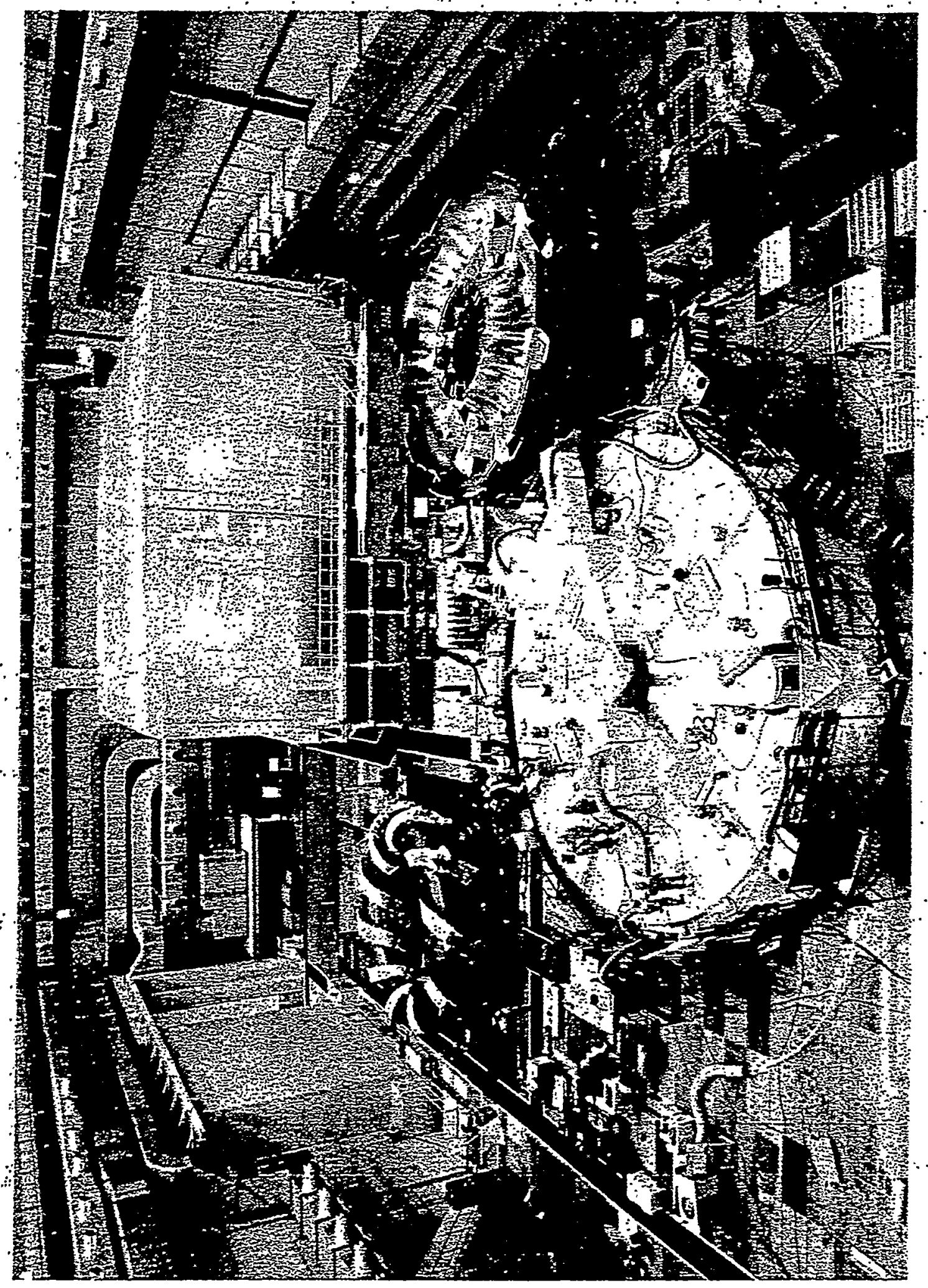




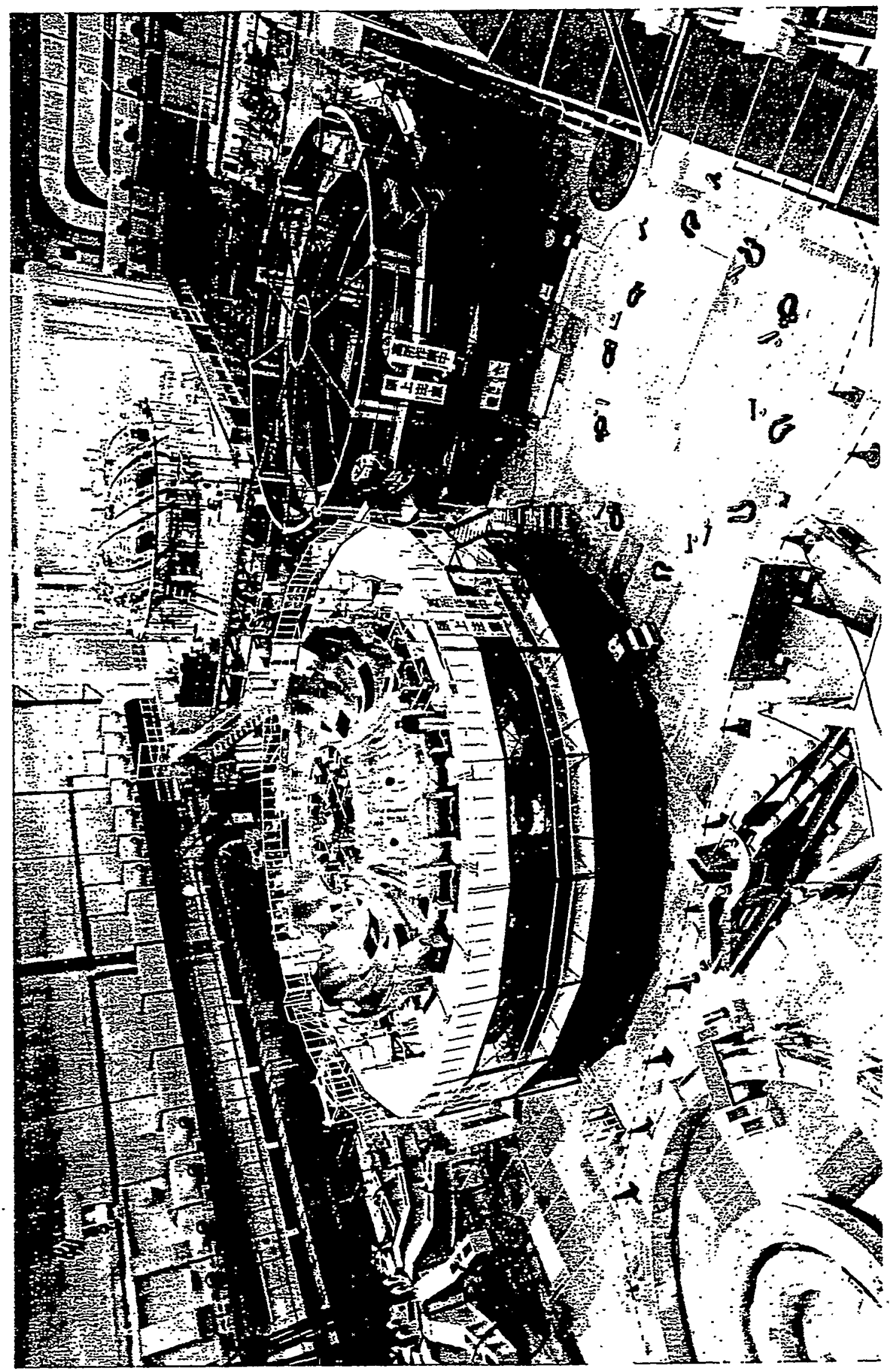




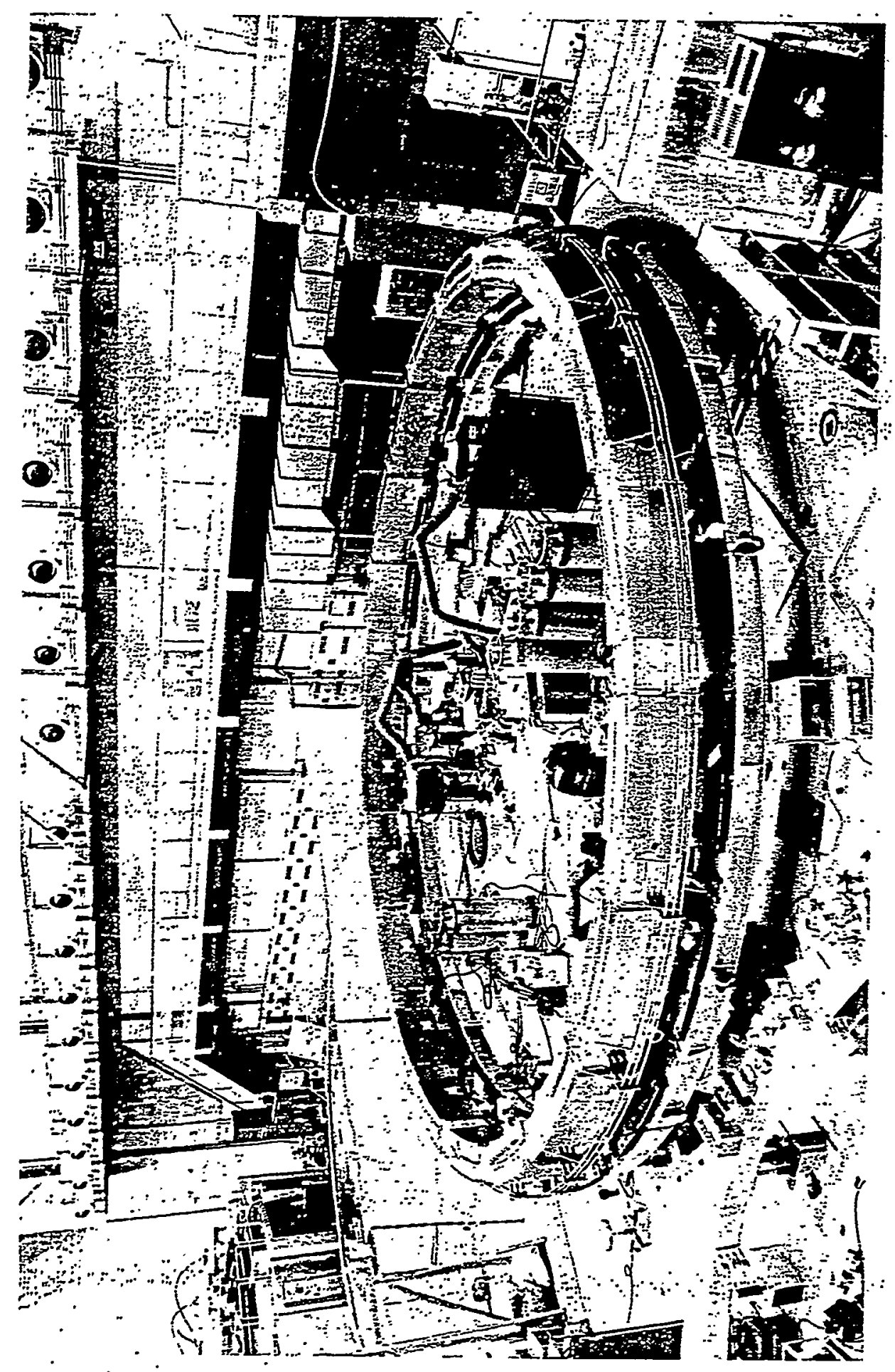




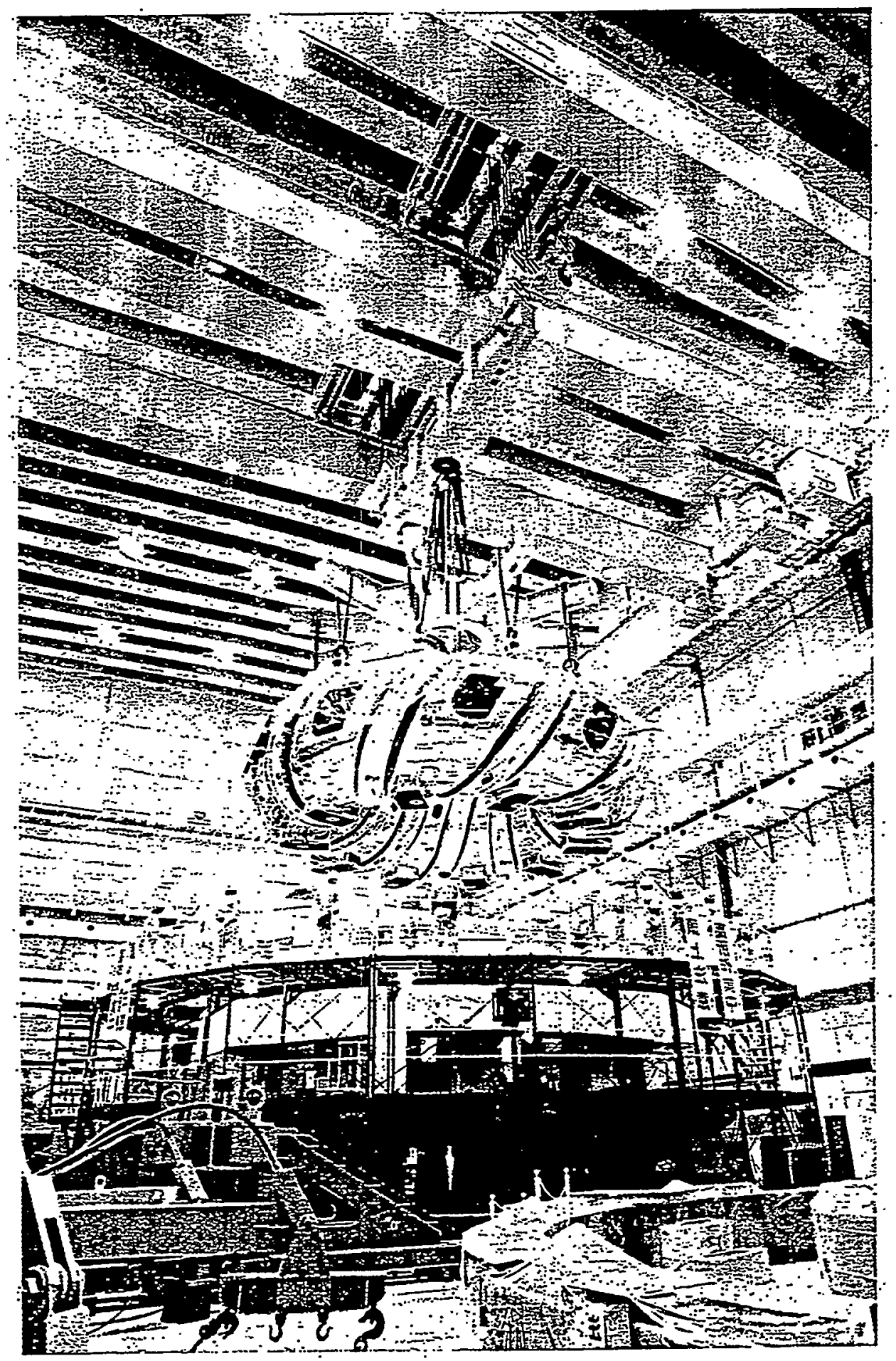


窟

t.

(5)

(1)

(1)

40 (1)

(19

(1)

1)

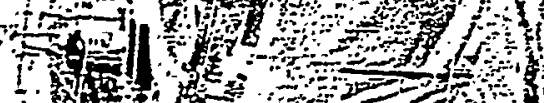

a

(1)

Non

$\because$ O

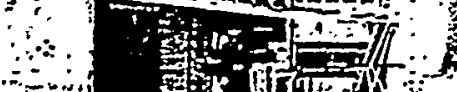

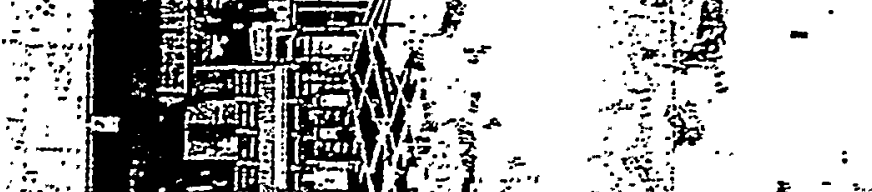

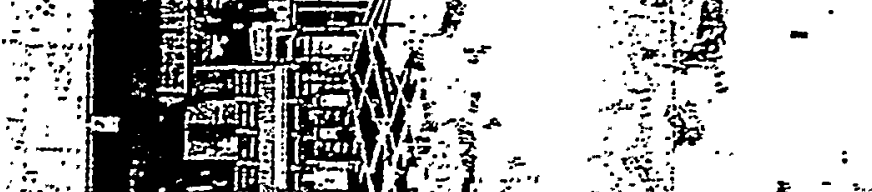

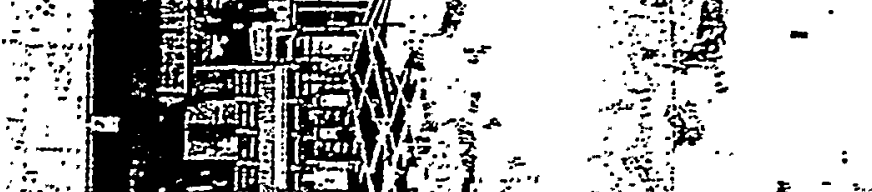

ard

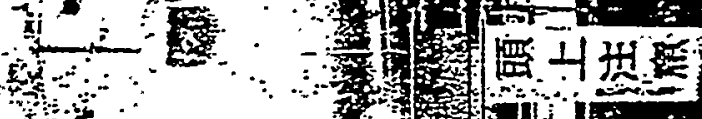

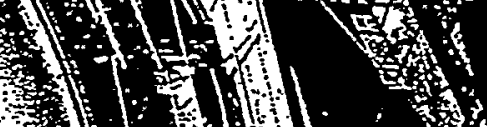

$\therefore \dot{0}=0$

$\therefore$,

on

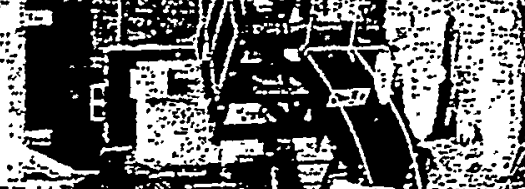

$\rightarrow 00$ ond

IIIIITHA

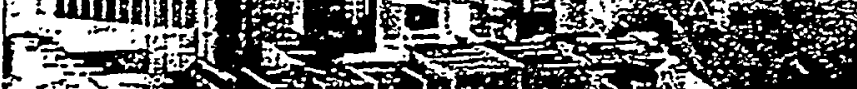

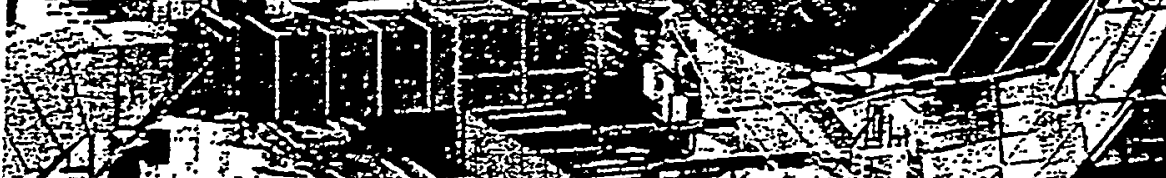

1.

1.

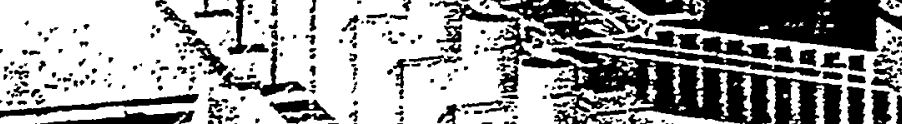

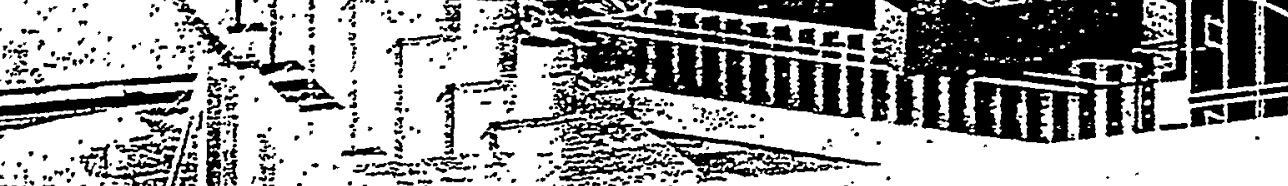
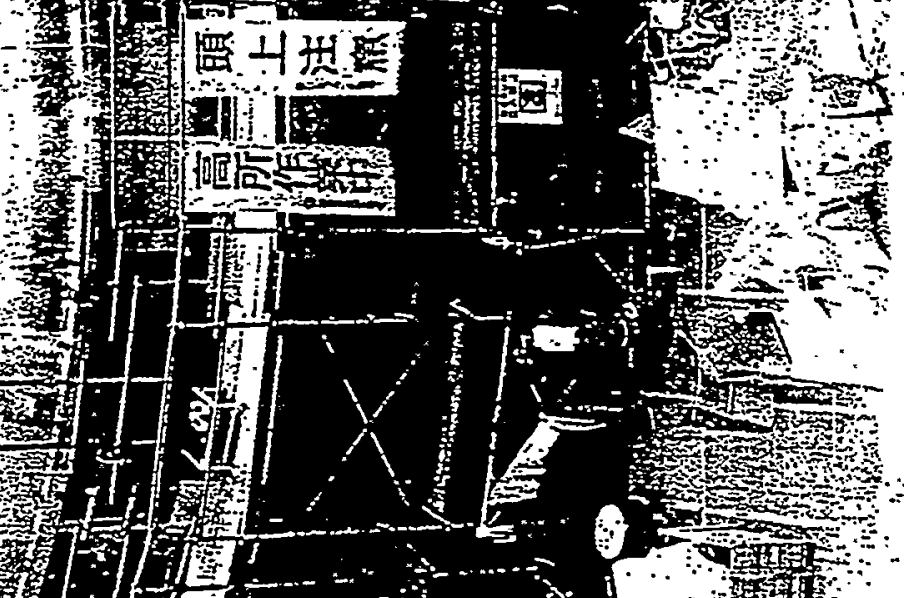

iili 
minges

1.

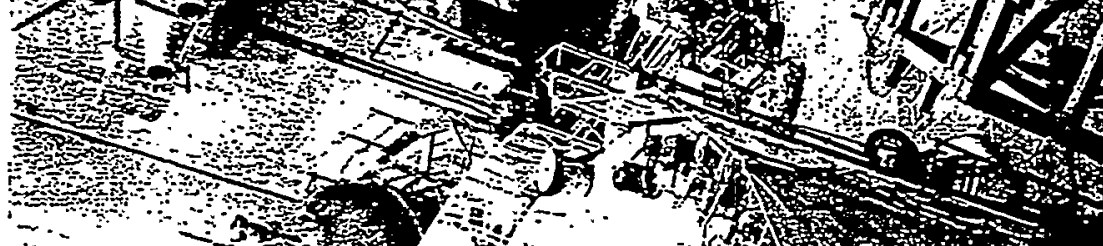

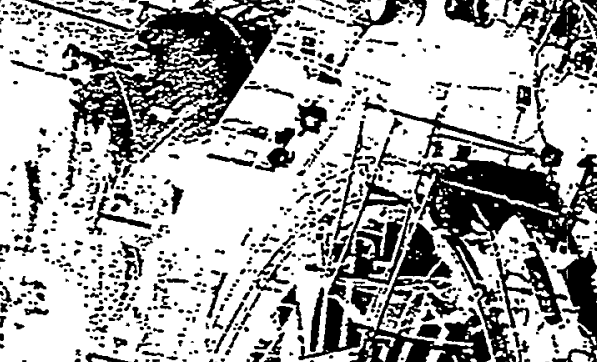
1)

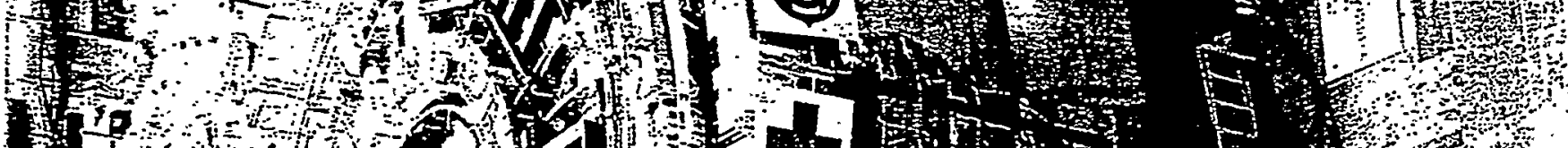
(1)

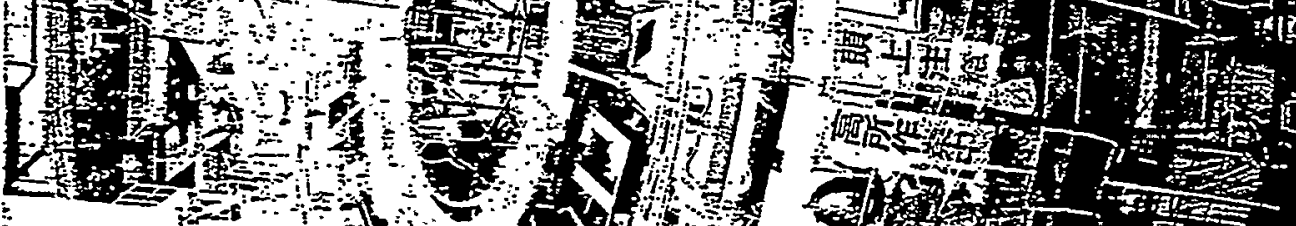
(1) (n) $\frac{1}{3}$

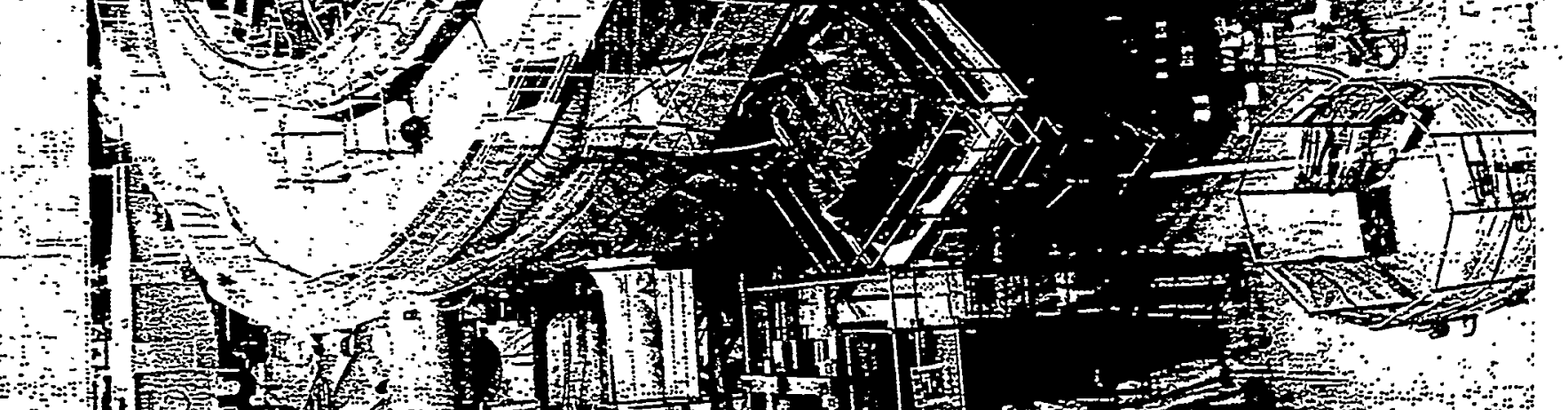

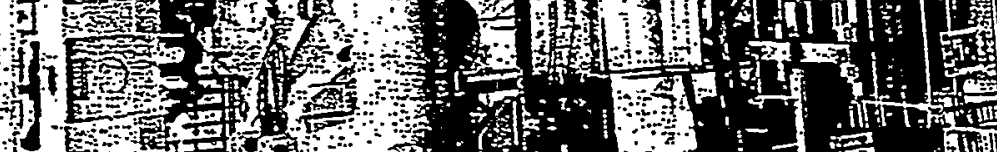

- 5 ( )

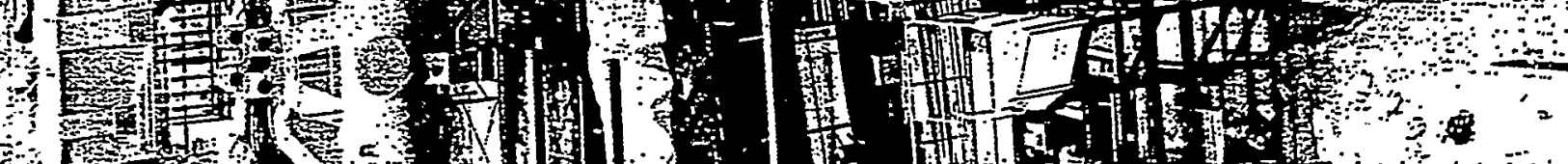

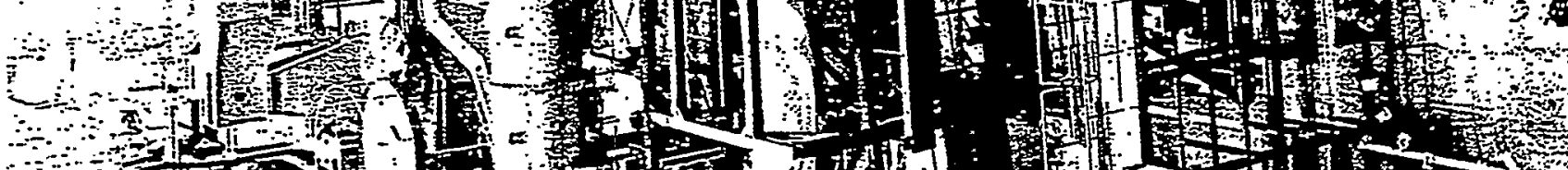

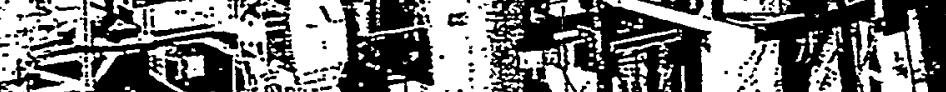

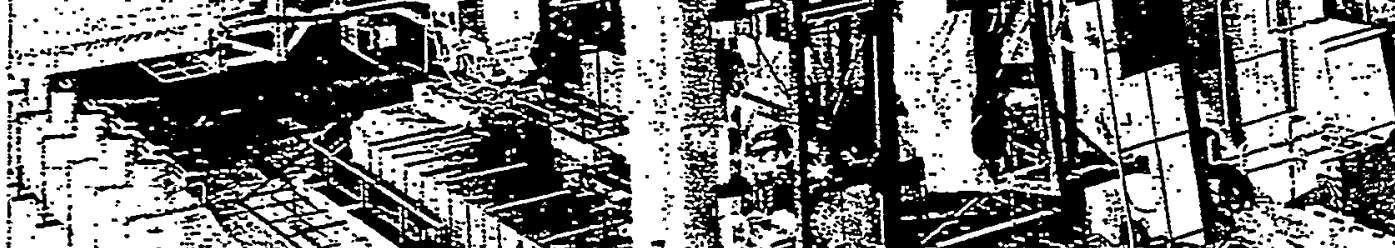

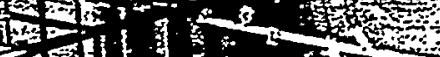

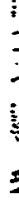
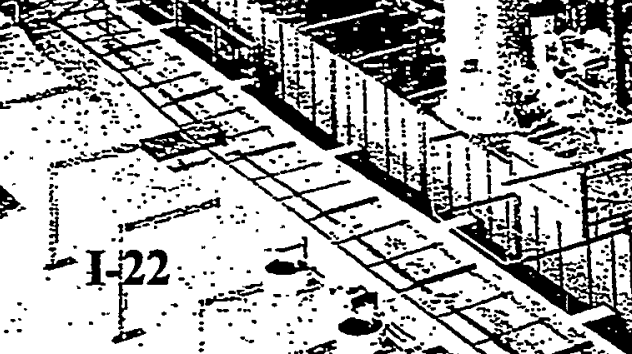
留 (2)

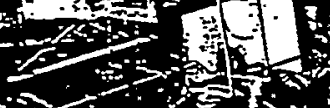

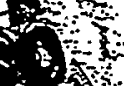

政

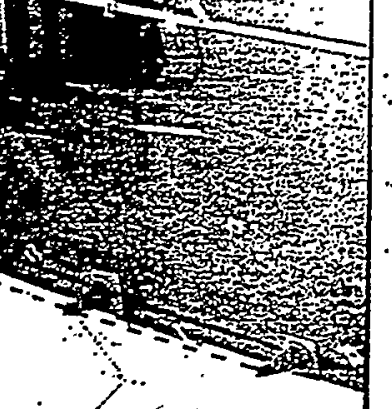




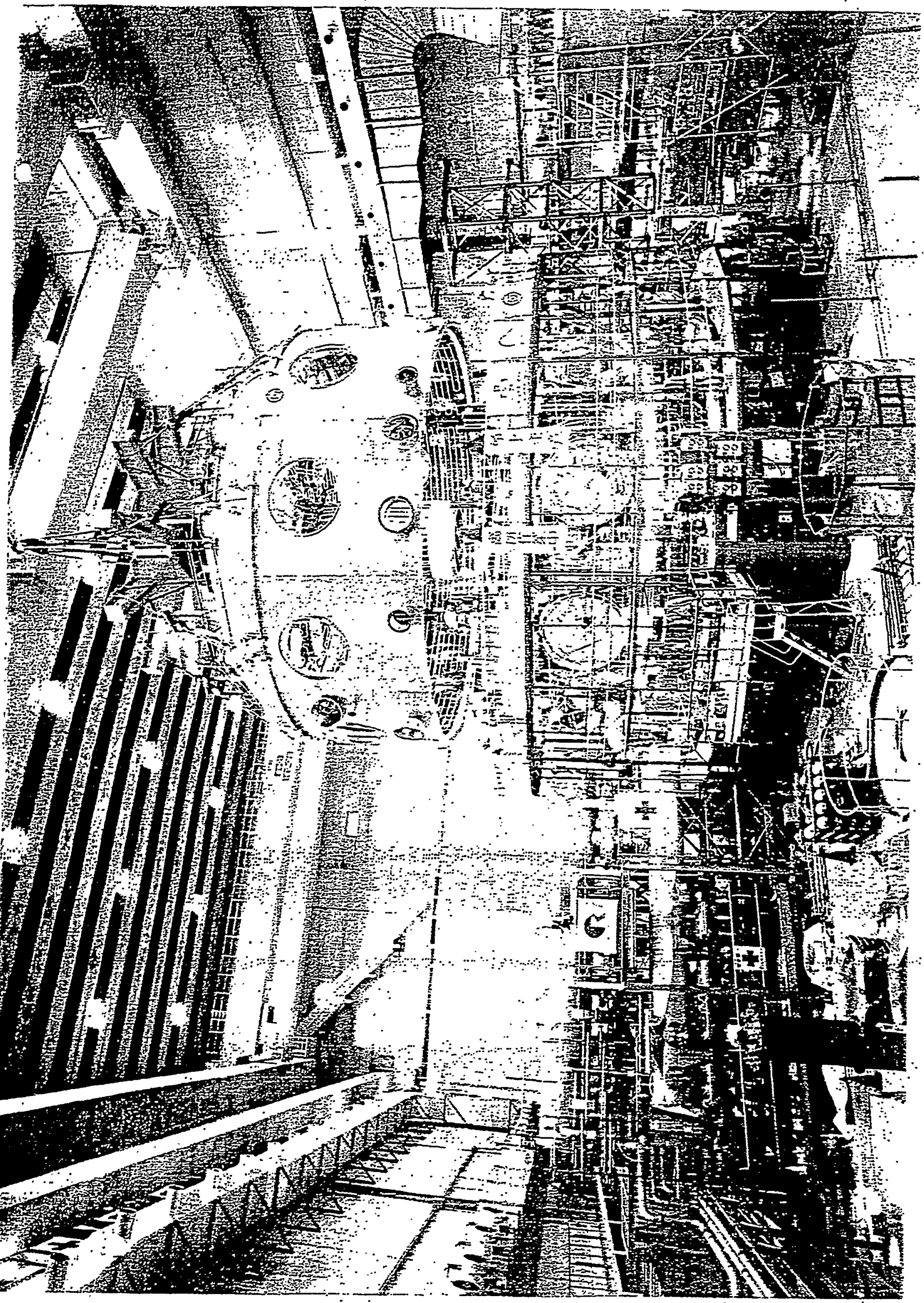




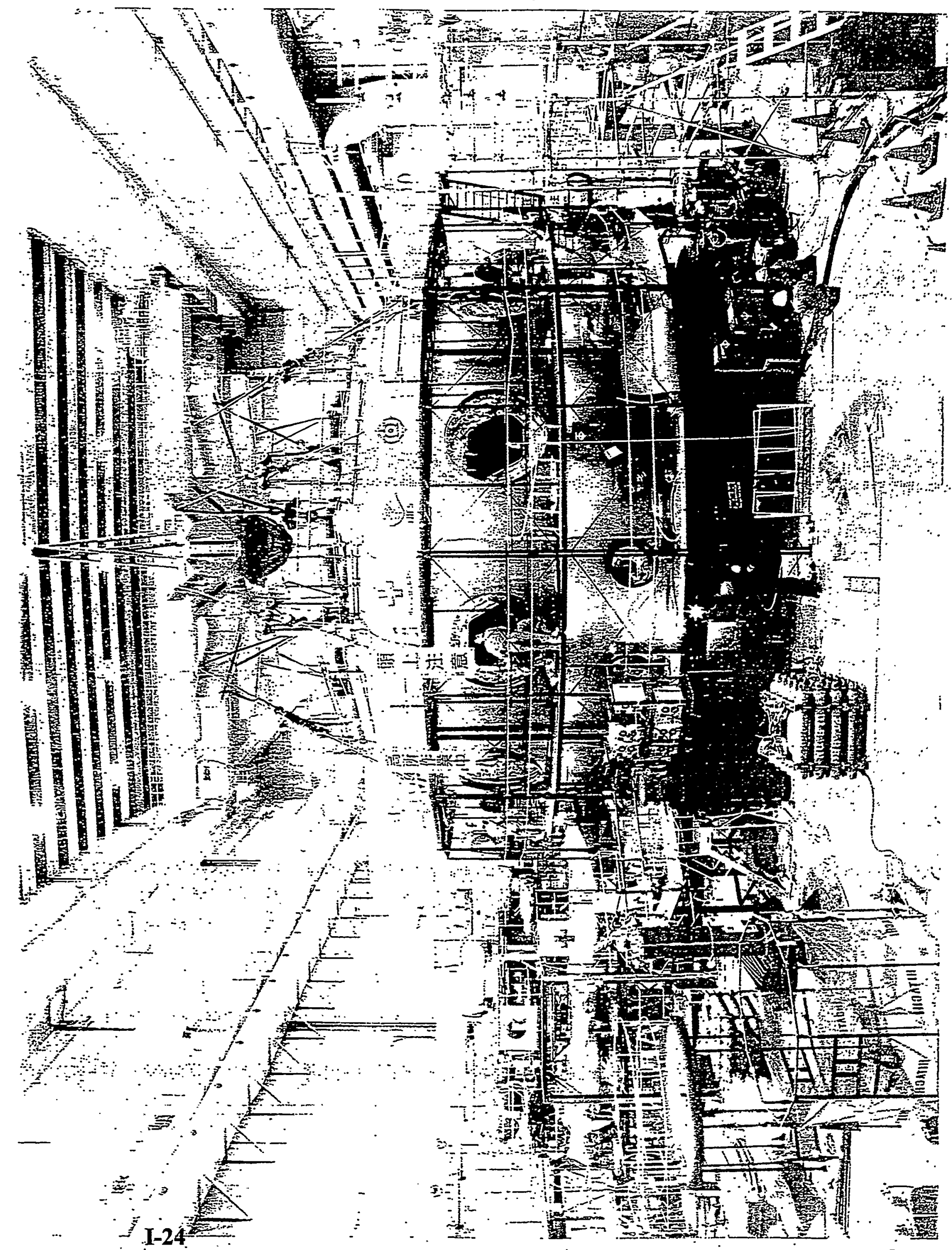




\section{Near-Future Plan of LHD Experiments}

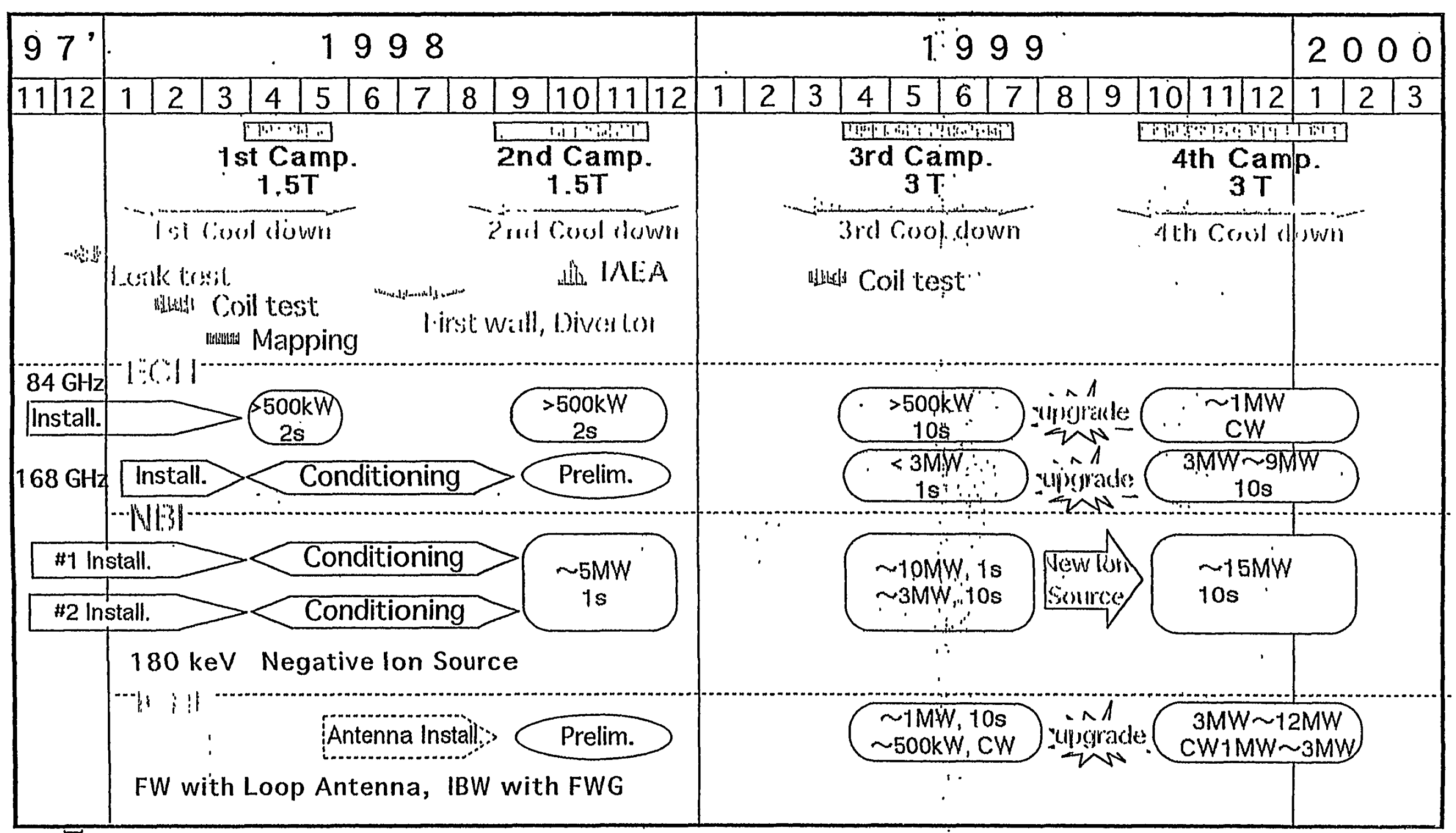


r

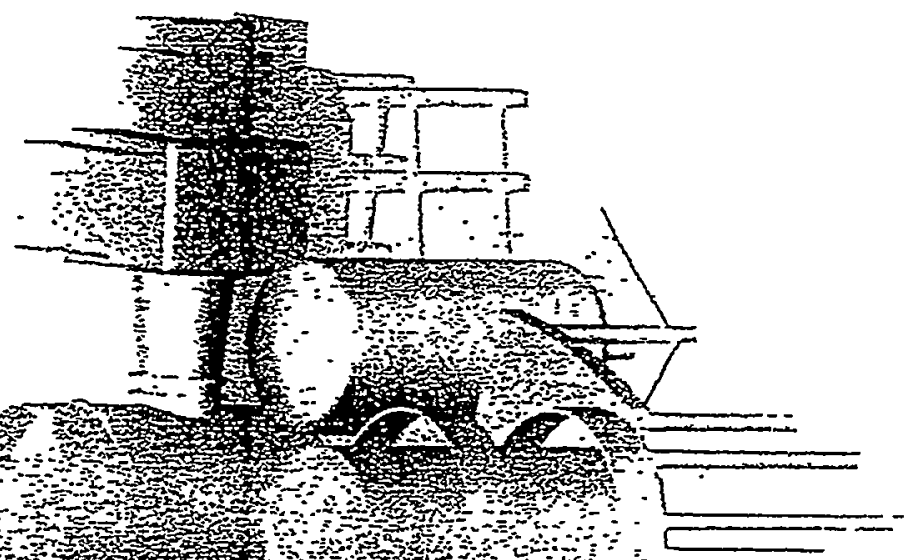

(n)

Now
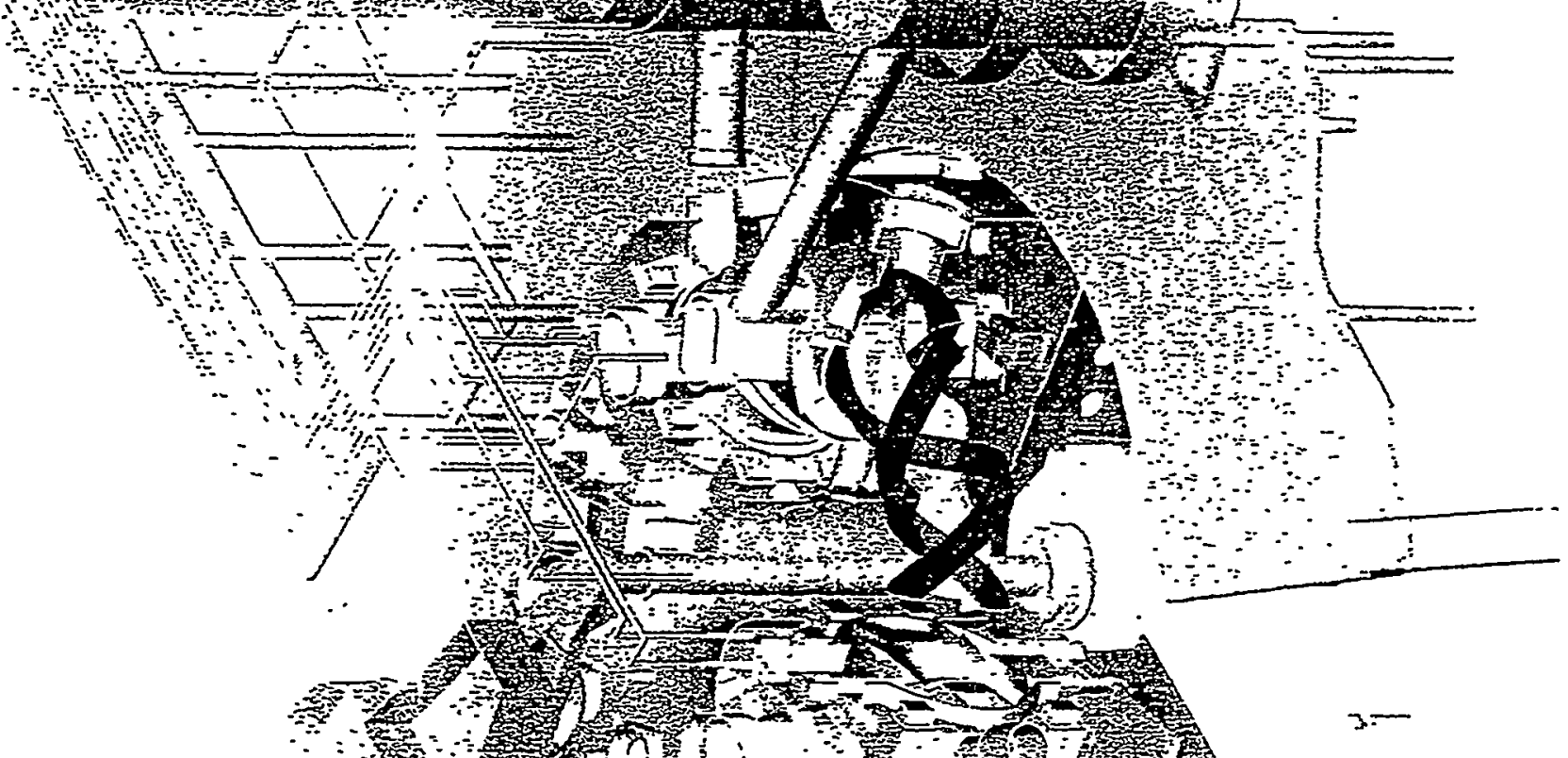

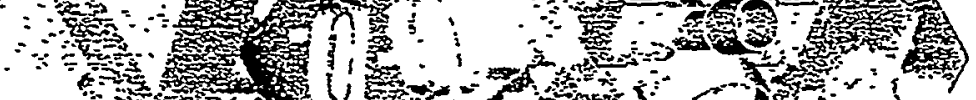

$\therefore$, on

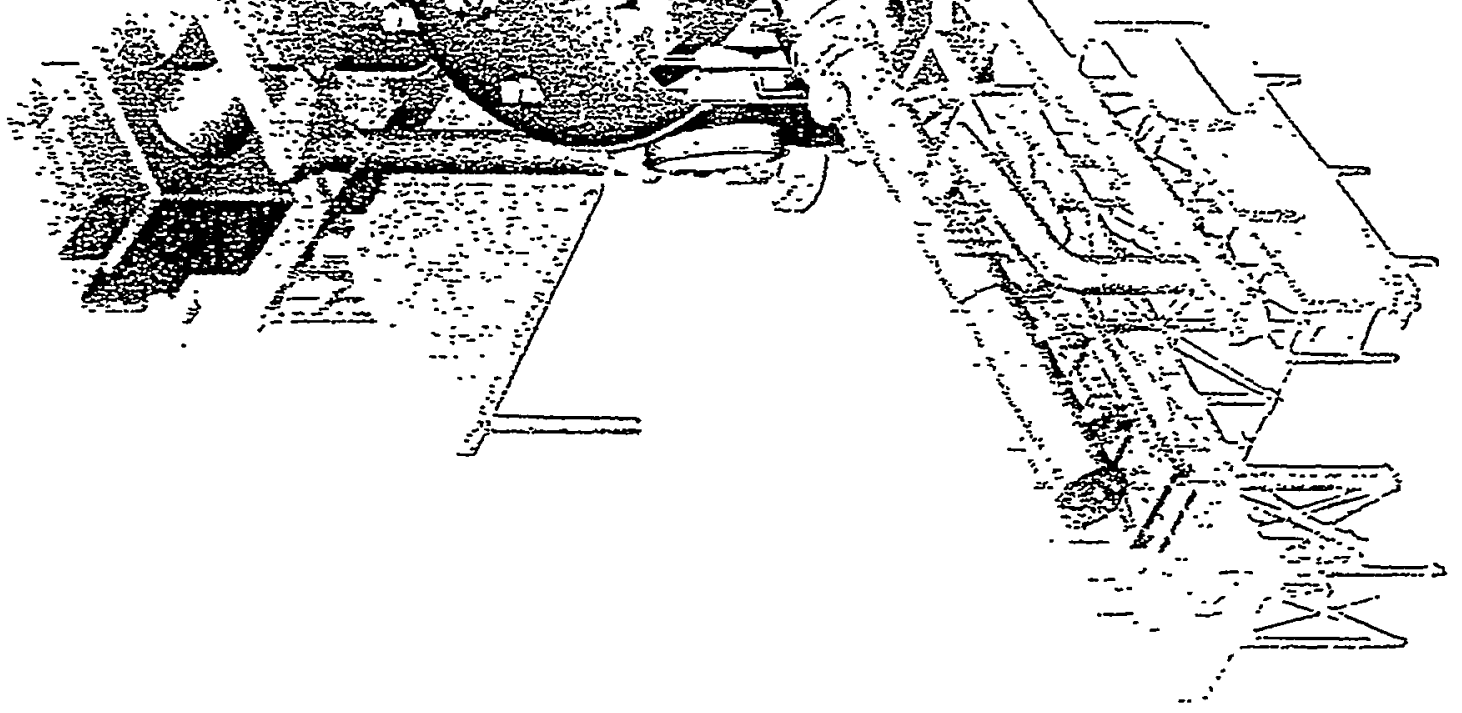




\section{大電力中性粒子入射加熱裝置}

Neutral Beam Injection Heating System.

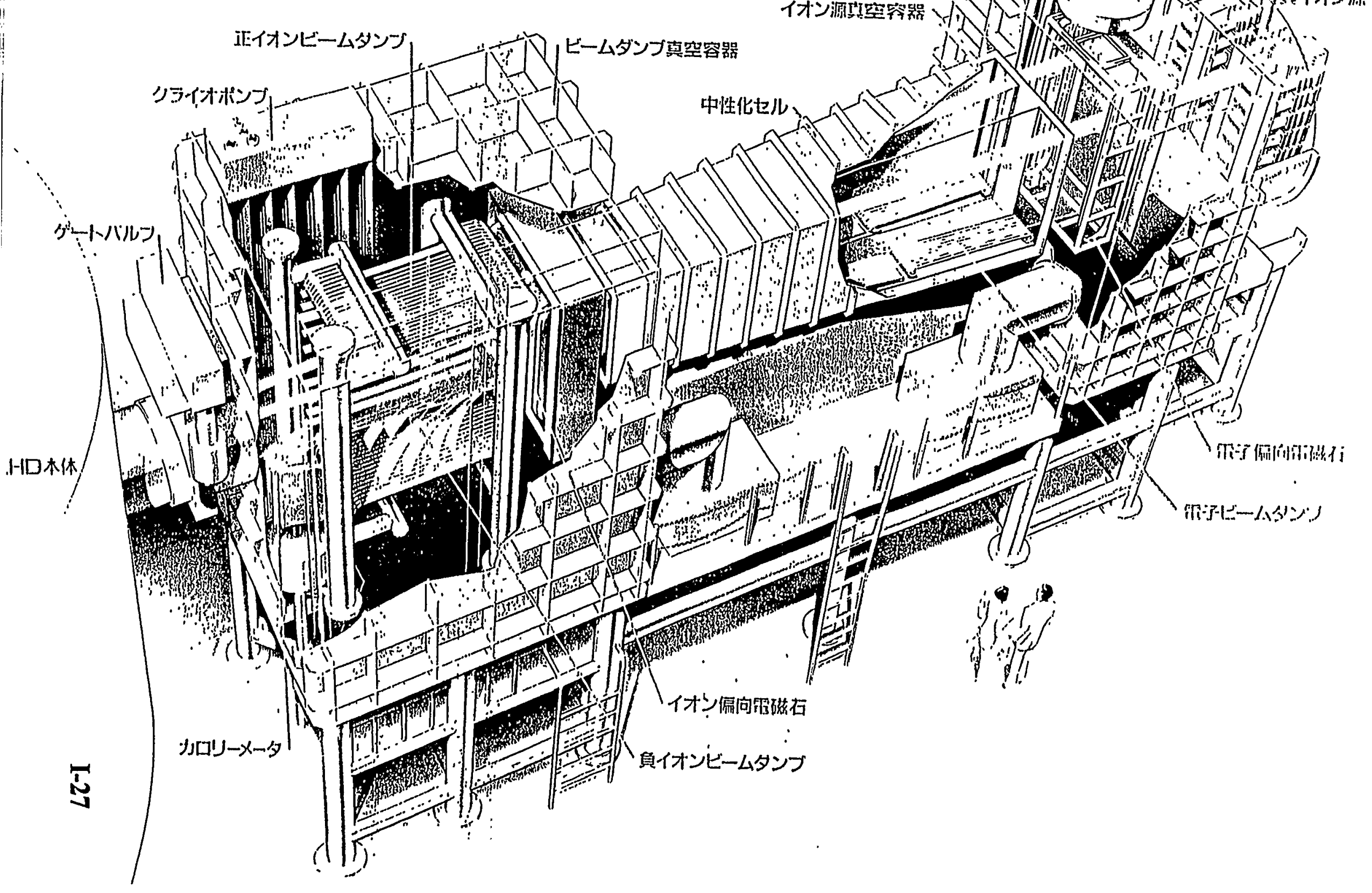




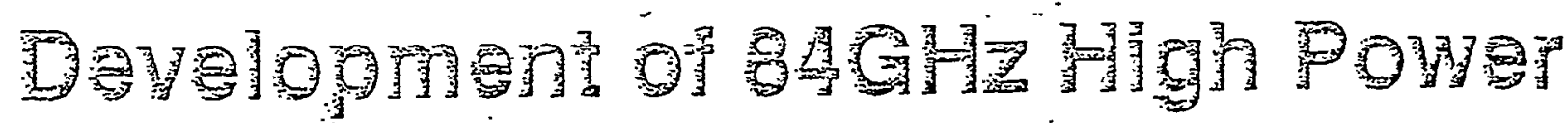

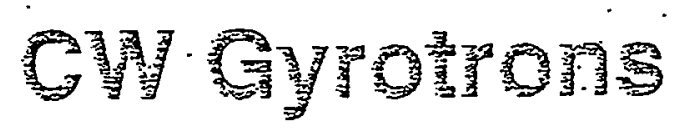

- $400 \mathrm{~kW} 10,5 \mathrm{sec}, 500 \mathrm{~kW} 2 \mathrm{sec}_{2}, 100 \mathrm{~kW} 30 \mathrm{~min}$. oscllation

- The maximim power is limited by window temperature rise.

- The gas pressure increment prevented from obtaining higher duty and CW operations.

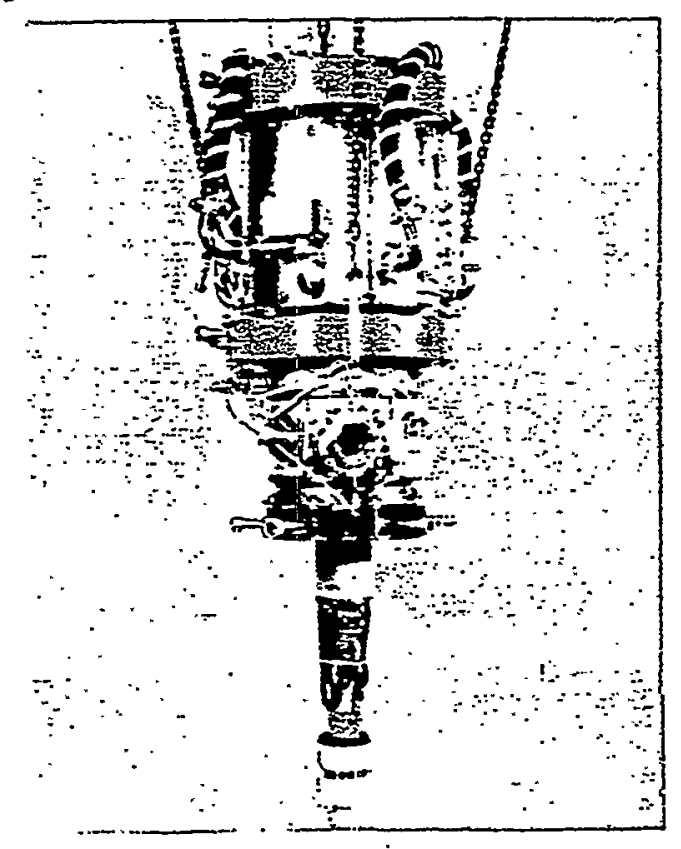

84GHz CW gyrotron

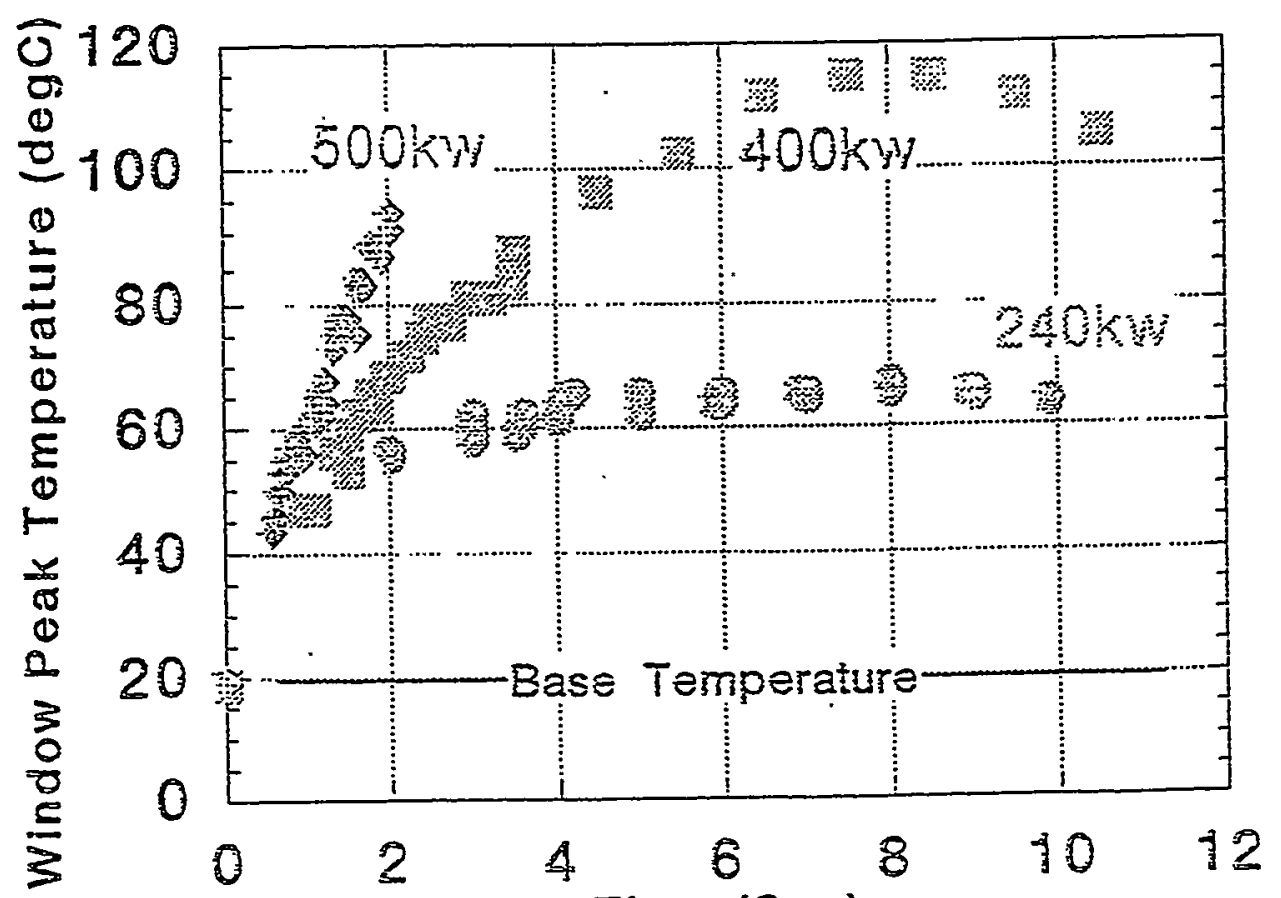

Time (Ses)

Variation of peak window temperature during RF pulse 


\section{Physics Axis}

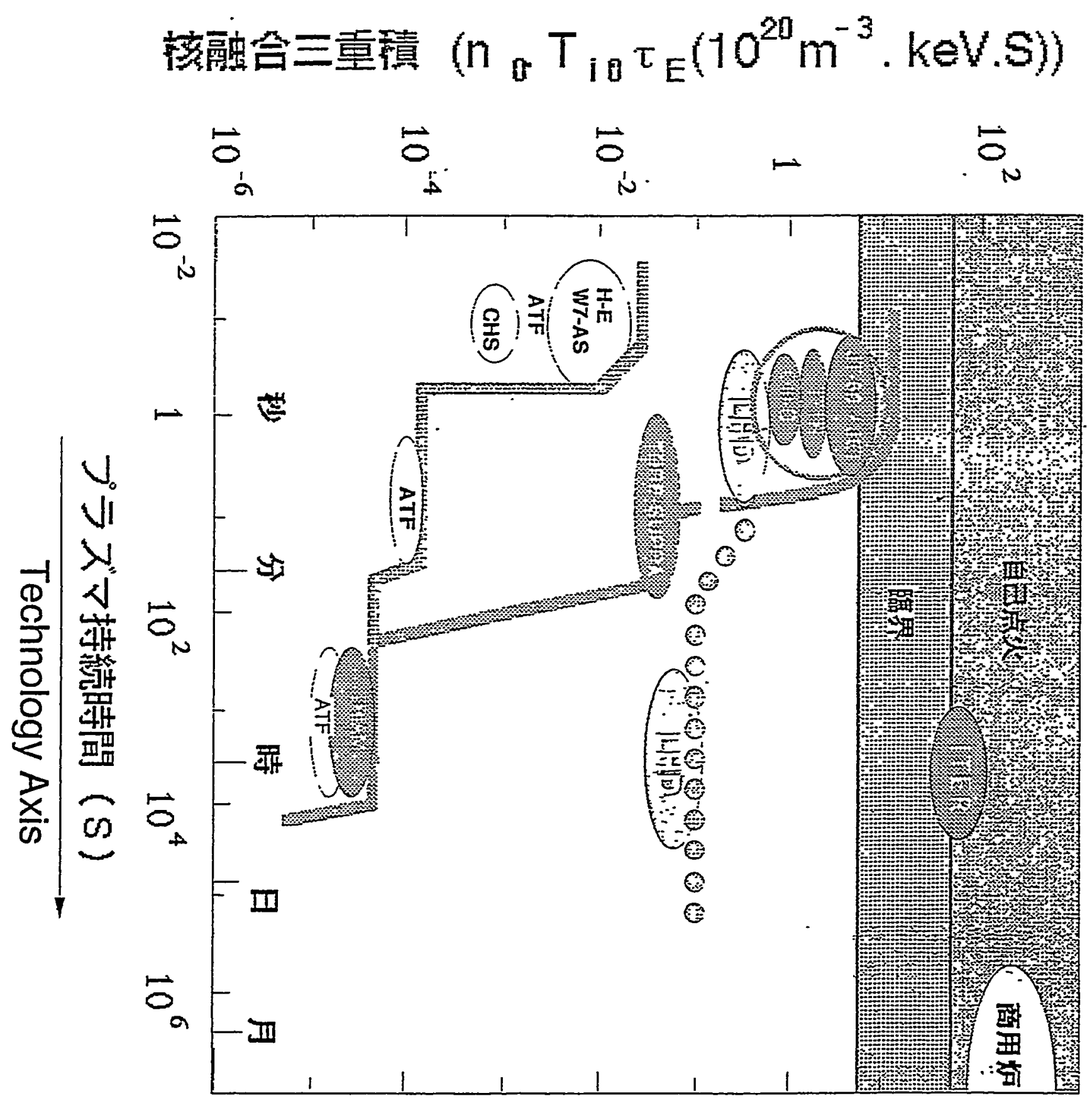




\section{Heating Power and Wall Load}

$=$ LHD

$\begin{array}{lllll}\text { Heating Power } & \text { ECRH } & 10 & \text { MW } & (10 s) \\ & & 3 & \text { MW } & (\text { cW }) \\ & \text { NBI } & 20 & \text { MW } & (10 s) \\ & \text { ICRF } & 9 & \text { MW } & (10 s) \\ & & 3 & \text { MW } & (c W)\end{array}$

Max. Heat load on Divertor Plate

$5 \mathrm{MW} / \mathrm{m}^{2} \quad(10 \mathrm{~s}, 20 \mathrm{MW})$

$10 \mathrm{MW} / \mathrm{m}^{2} \quad(5 \mathrm{~s},>30 \mathrm{MW})$

$0.75 \mathrm{MW} / \mathrm{m}^{2}$ ( $\mathrm{cW}, 3 \mathrm{MW}$ )

$2.5 \mathrm{MW} / \mathrm{m}^{2}$ ( $\left.\mathrm{cW}, 10 \mathrm{MW}\right)$

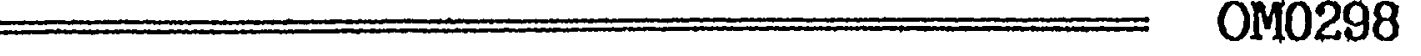




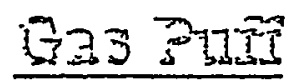

- $\mathrm{H}_{2}, \mathrm{D}_{2}, \mathrm{He}$ : up to $300 \mathrm{~Pa} \cdot \mathrm{m}^{3} / \mathrm{s}$ from 9 inlets $\left(100 \mathrm{~Pa} \cdot \mathrm{m}^{3} / \mathrm{s}\right.$ from single inlet for the initial operation)

- $\operatorname{Ar}\left(>50 \mathrm{~Pa} \cdot \mathrm{m}^{3}\right)$ : Plasma shutdown

- High purification control $\sim 10 \mathrm{ppb}$

Pellet Injegtors

Fuieler

- 10-barrel single-stage pipe gun

- $\mathrm{H}_{2}, \mathrm{D}_{2}: 1.0 \sim 3.8 \mathrm{~mm} \phi$

- $500 \sim 1500 \mathrm{~m} / \mathrm{s}$

Tracer-encapsulated pellet

- $\mathrm{Li}$ and $\mathrm{C}$ encapsulated in $\mathrm{H}_{2}$

- 800m/s

Impurity pellet

- Hydrocarbon, Al, Li, C, Ti etc. : $0.3 \sim 1 \mathrm{~mm} \phi$

- $300 \sim 500 \mathrm{~m} / \mathrm{s}$

IIBI

- Negative ion source

- Balanced injection with 2 beam lines

- $180 \mathrm{keV}$ and $90 \mathrm{~A}$ in total

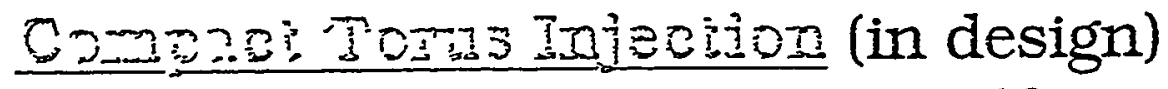

- Contained particle $1 \times 10^{19}, \mathrm{~V}_{\mathrm{CT}^{\sim}} 300 \mathrm{~km} / \mathrm{s}$

- Collaboration with Himeji Institute of Technology 


\section{Fueling Scenario in LHD Experiment}

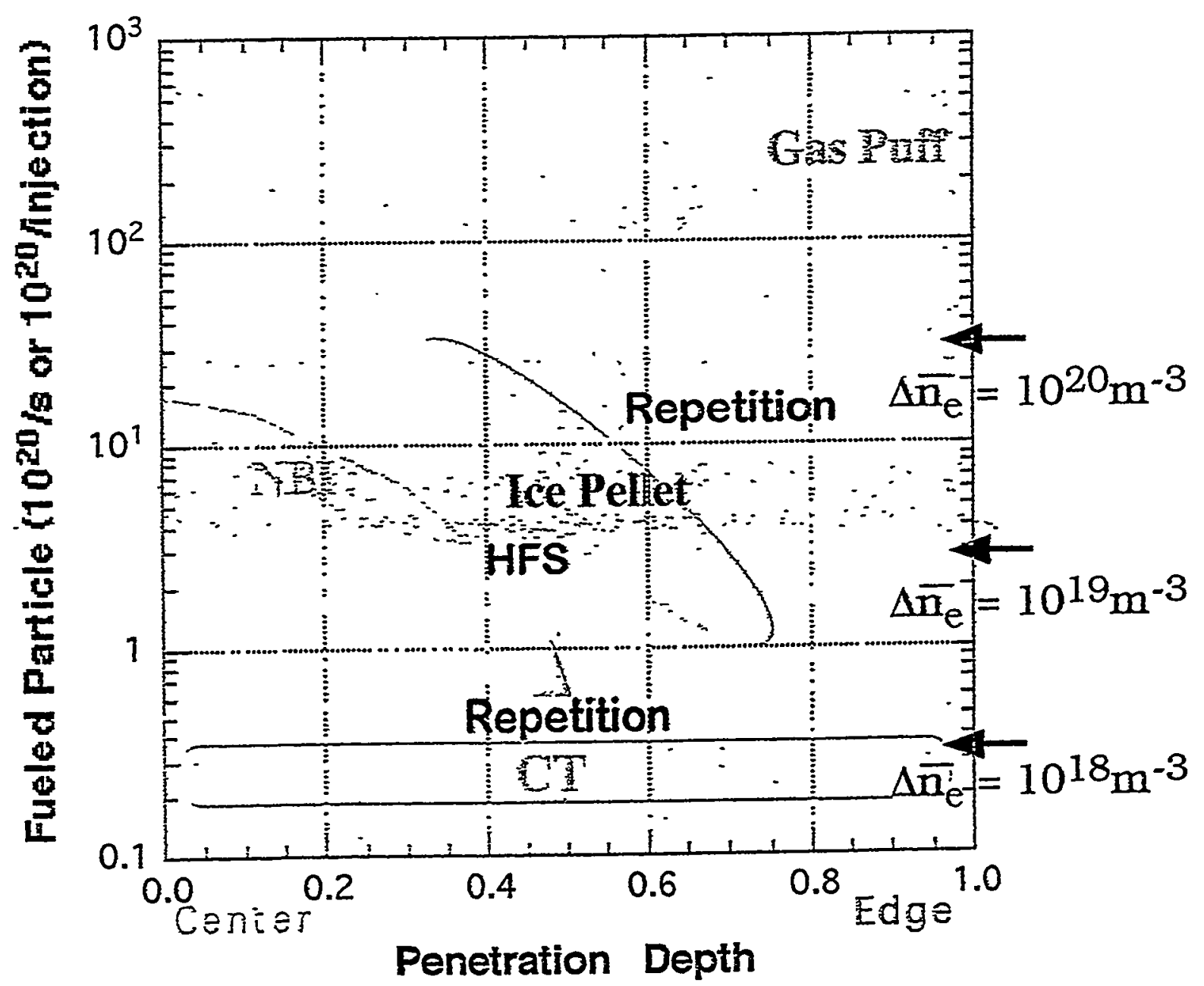

iote:

Fuelang efficiency

NBI 100\%, Pellet 20 100\%,

CT 15 30\%, GasPuff 2 20\%

Consistency with capability of pumping systems

Main pumps, LID, Divertor

Specific pellet injectors

Tracer-encapsulated pellet and

I-32 Impurity pellets ( $\mathrm{Li}, \mathrm{Al}, \mathrm{C}$, etc)

for transport studies 
Helical Divertor (HD) $\quad \sim$ Helical divertor geometry

*:High recycling operation

- high density, low temperature $\left(n_{\mathrm{div}}>10^{20} \mathrm{~m}^{-3}\right)$

- edge radiative cooling for safe heat removal

* Low recycling operation by efficient pumping

- low density, high temperatưure $\left(n_{\text {div }} 10^{17} \mathrm{~m}^{-3}\right)$

- significant improvement of $\tau_{\mathrm{E}}$ i.e., H-mode.

SHC boundary $\sim$ Helical + Island divertor geometries $~$

* Low density, high temperature, steep gradient at LCFS

- favorable for H-mode

* High density, low temperature in ergodic boundary

- favorable for radiative cooling

$m / n=1 / 1$ island:

Simultaneous achievement of - -mode and radiative Cooling

Local Island Divertor (LID) $\because \sim \sim$ Island divertor geometry $~$ * Closed divertor with high efficient pumping system

- low recycling operation for confinement improvement

* No leading edge problem

* Efficient discharge cleaning.

$m / n=1 / 1$ island 

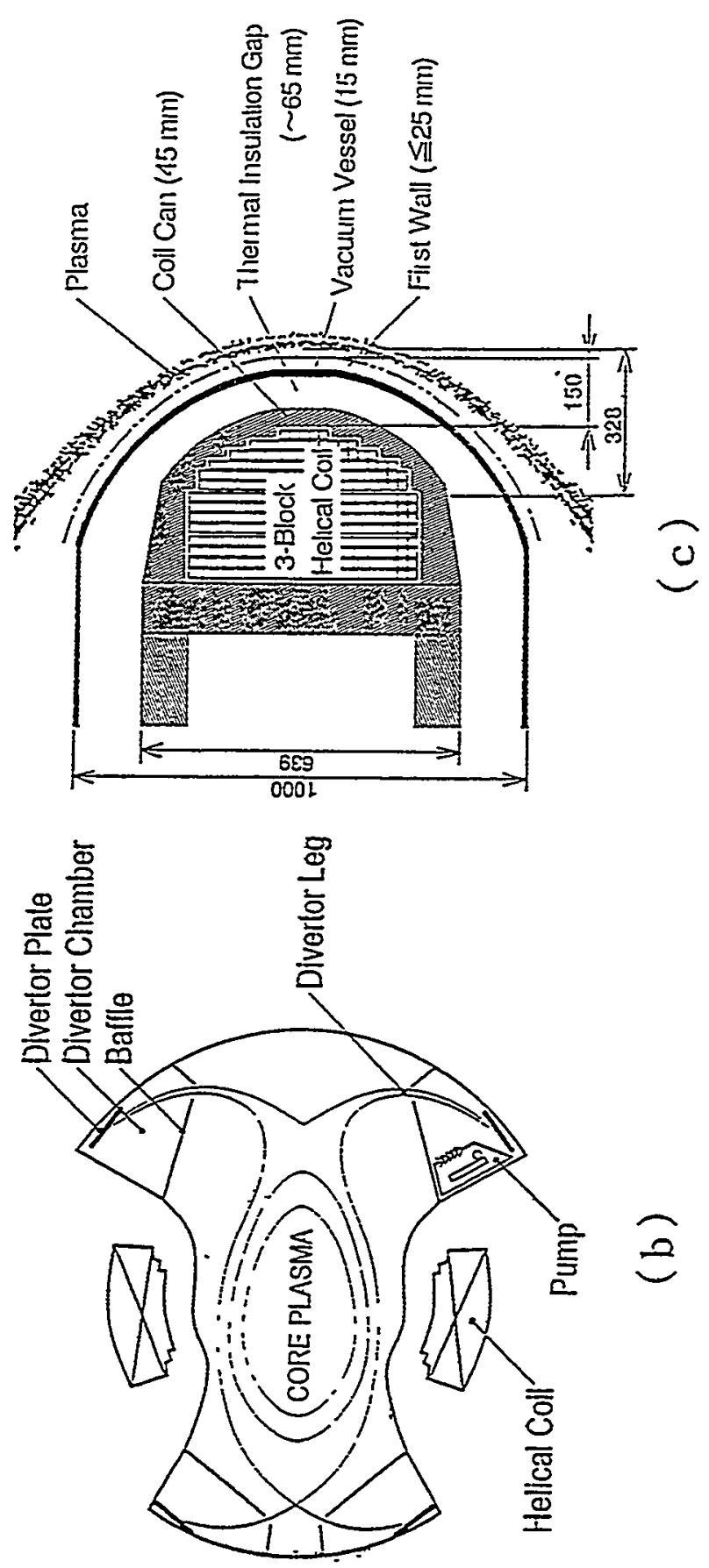

I-34

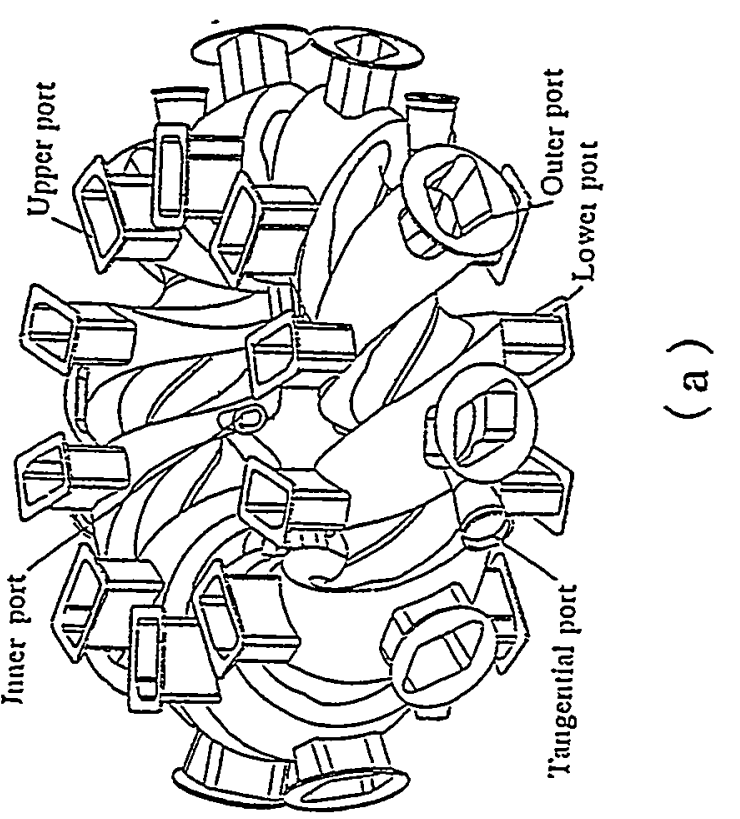




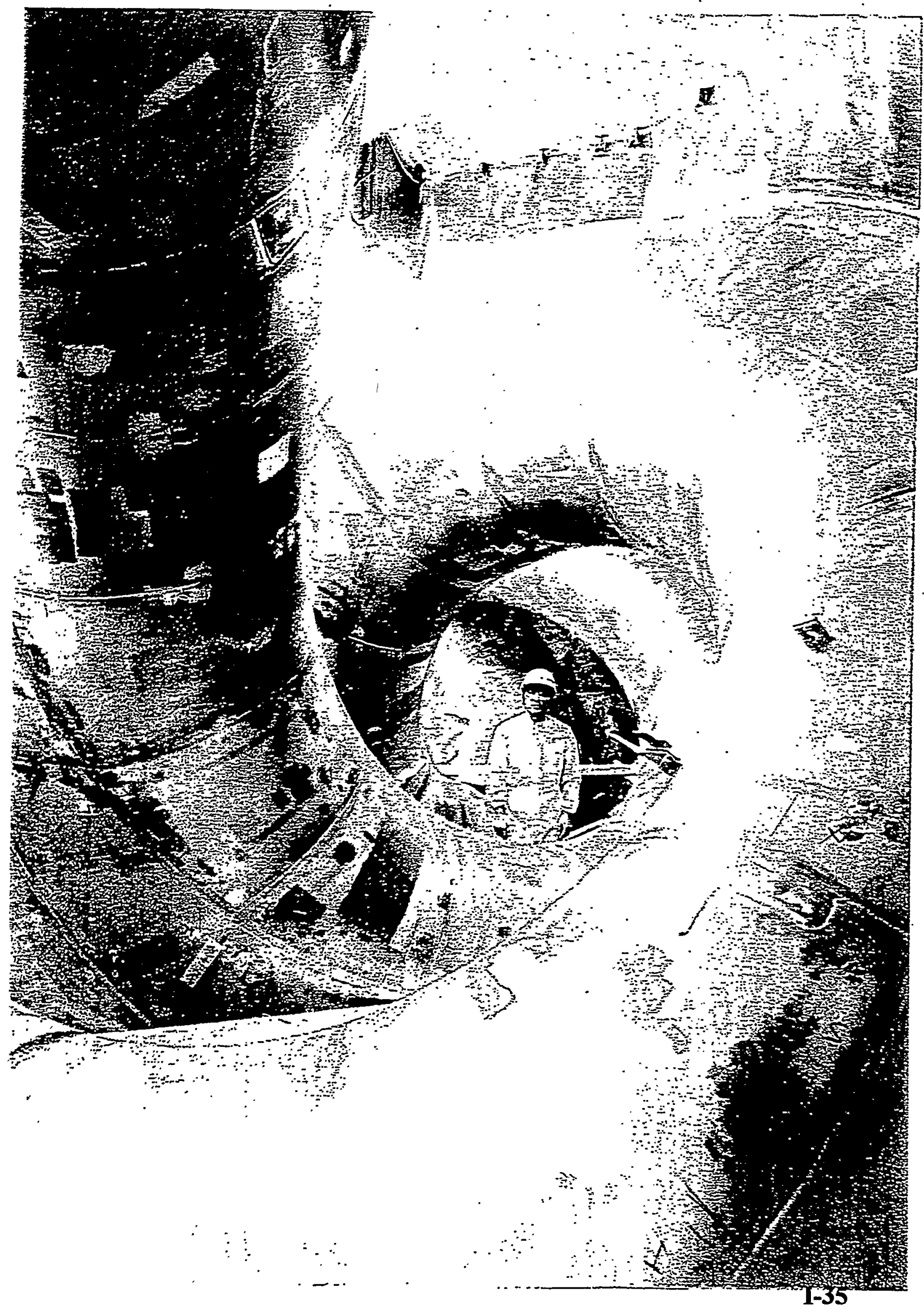




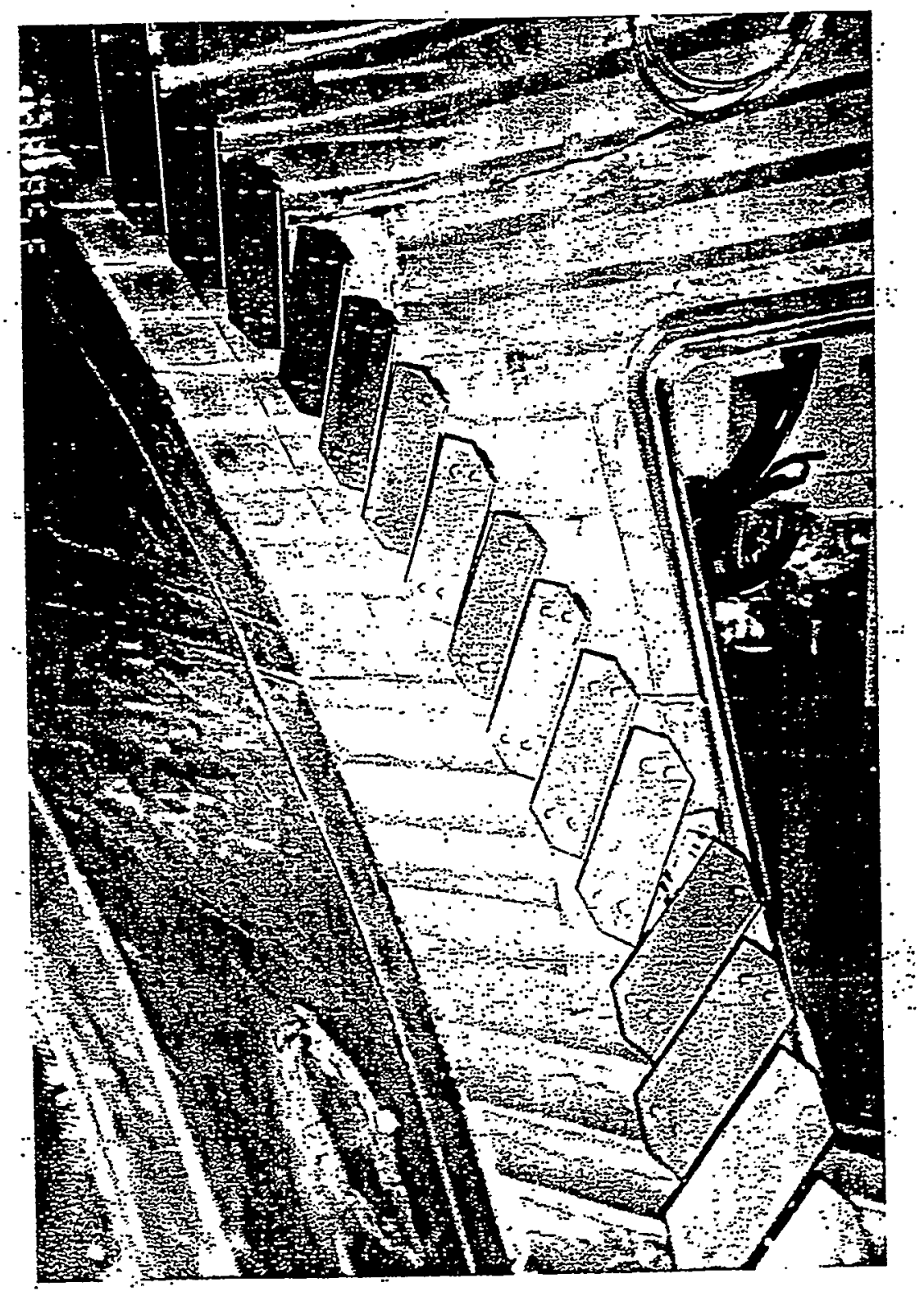




\section{SHC boundary configuration}

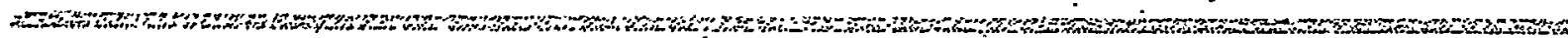

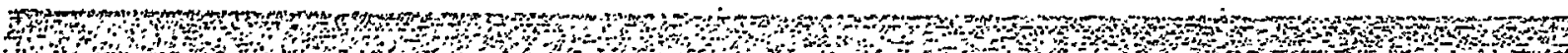

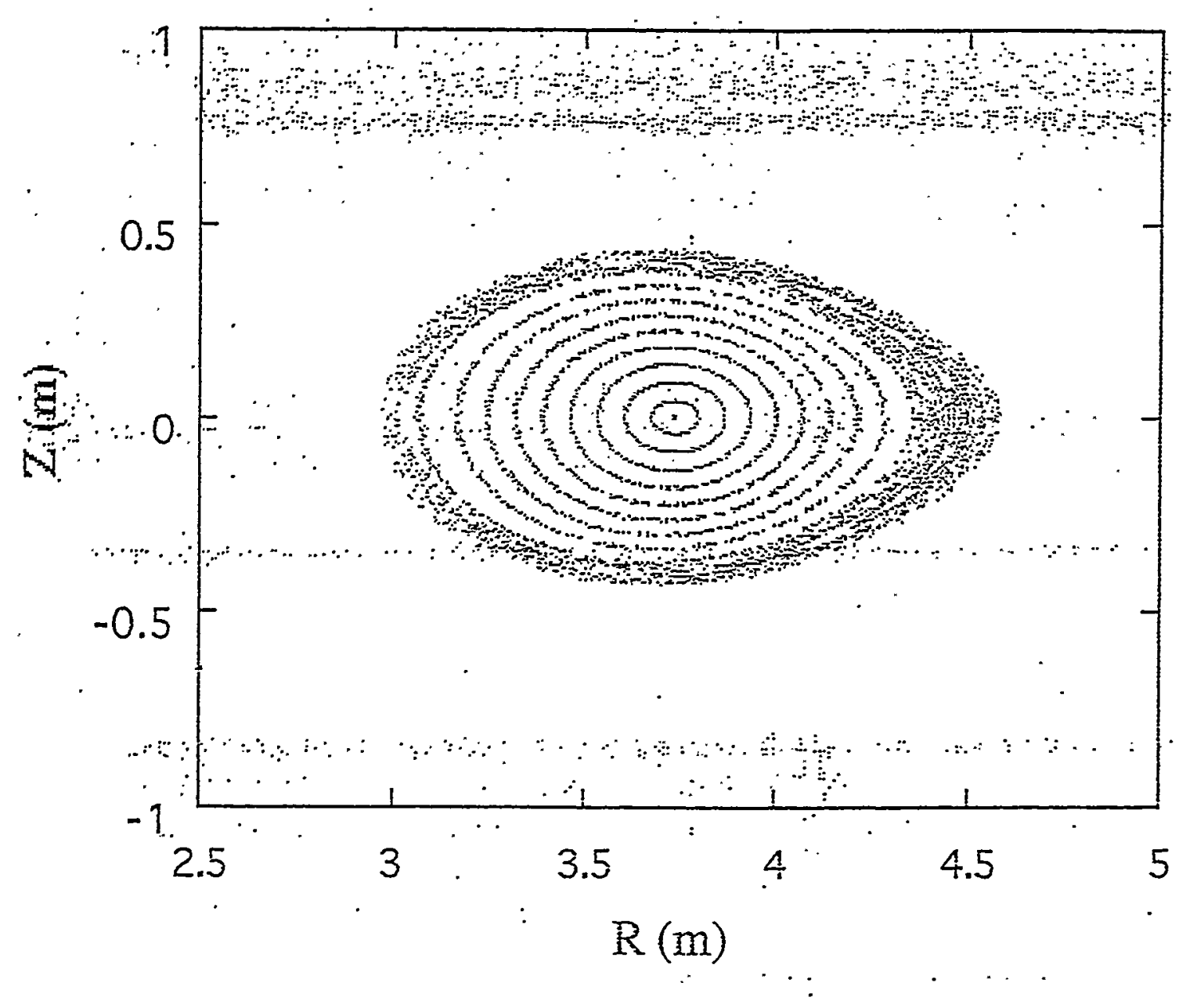
T) 


\section{RESEARCH PROGRESS BY HELIOTROON CONCEPT}

$\Xi$

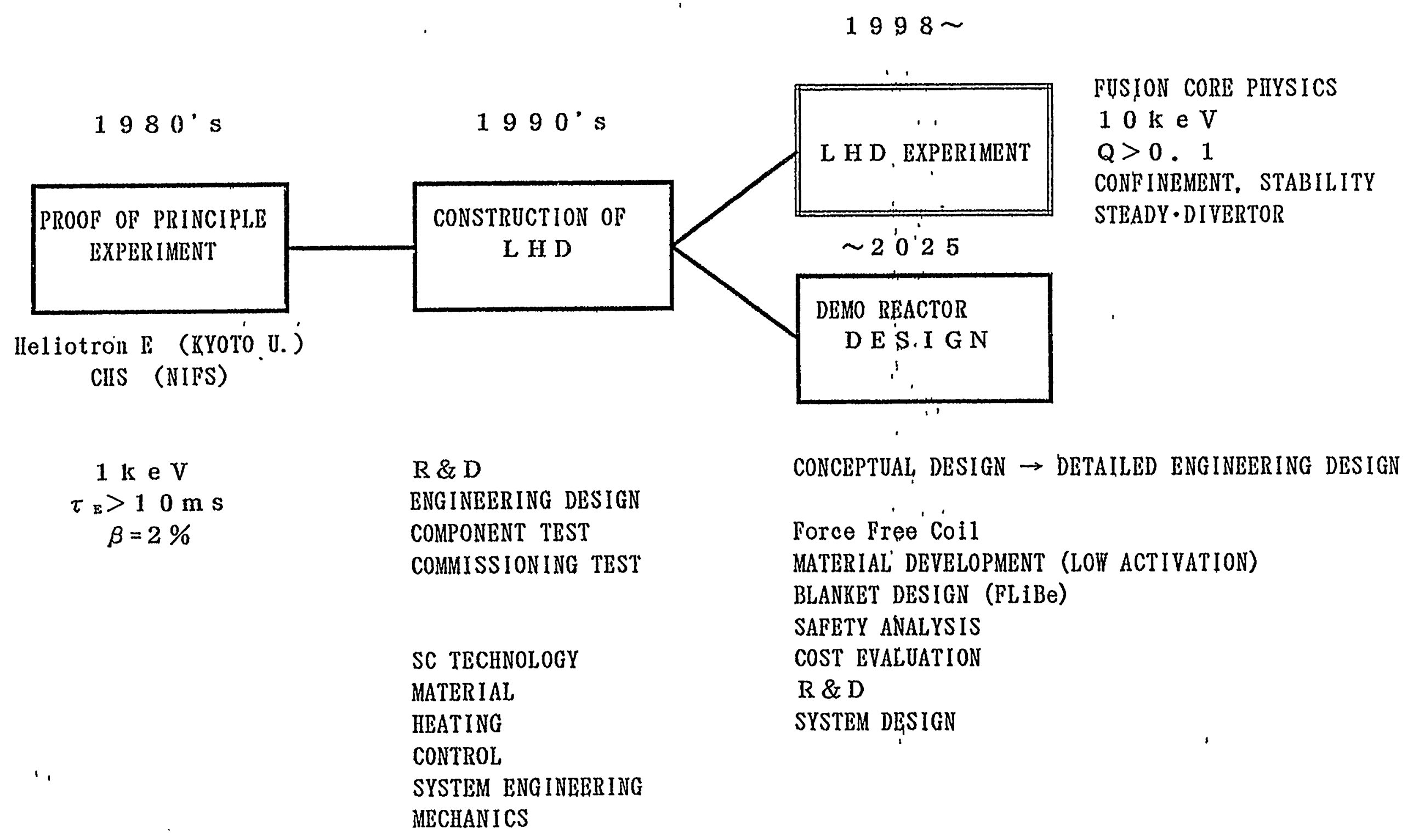




\section{US/Japan Workshop on Advanced Fueling December 2-3, 1997 \\ Lawrence Livermore National Laboratory, USA \\ Status of LHD and Fueling Plans}

$\Longrightarrow$ LHD

(

\section{Osamu Motojima}

National Institute for Fusion Science

322-6 Oroshicho, Toki 509-52, Japan

1, Introduction to LHD Project

Missions in Fusion Physics and Technology

Specifications of LHD

2, Construction Status

3, Experimental Planning

Commissioning Tests

First Plasma Start up Scenario

Heating System (NBI, Gy rotron, ICRF)

4, Fueling Plans

Pellet Injection, CT Injection, Gas Puff System

w

5, Summarizing 


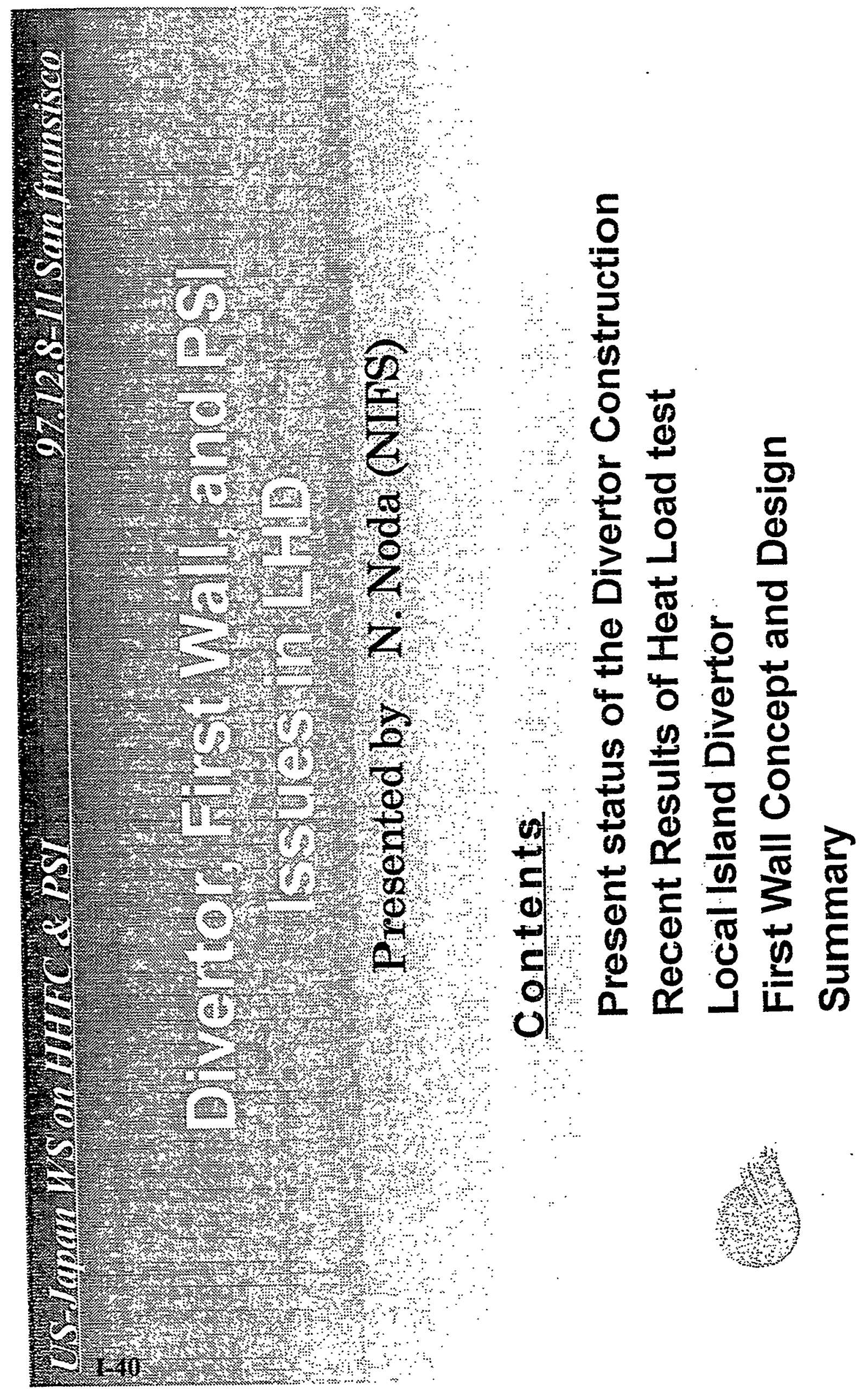




\section{Present Status of the Divertor Construction}

Concept is

Helically Running Discrete Bar Array

Final Goal is

Steady State Removal of $>10 \mathrm{MWW} / \mathrm{m}^{2}$

Stepwise Approach

Mechanically joined $\mathrm{C}$-armor to $\mathrm{Cu}$ Heat sink and SS cooling tube

Fabrication of the Divertor is going on 


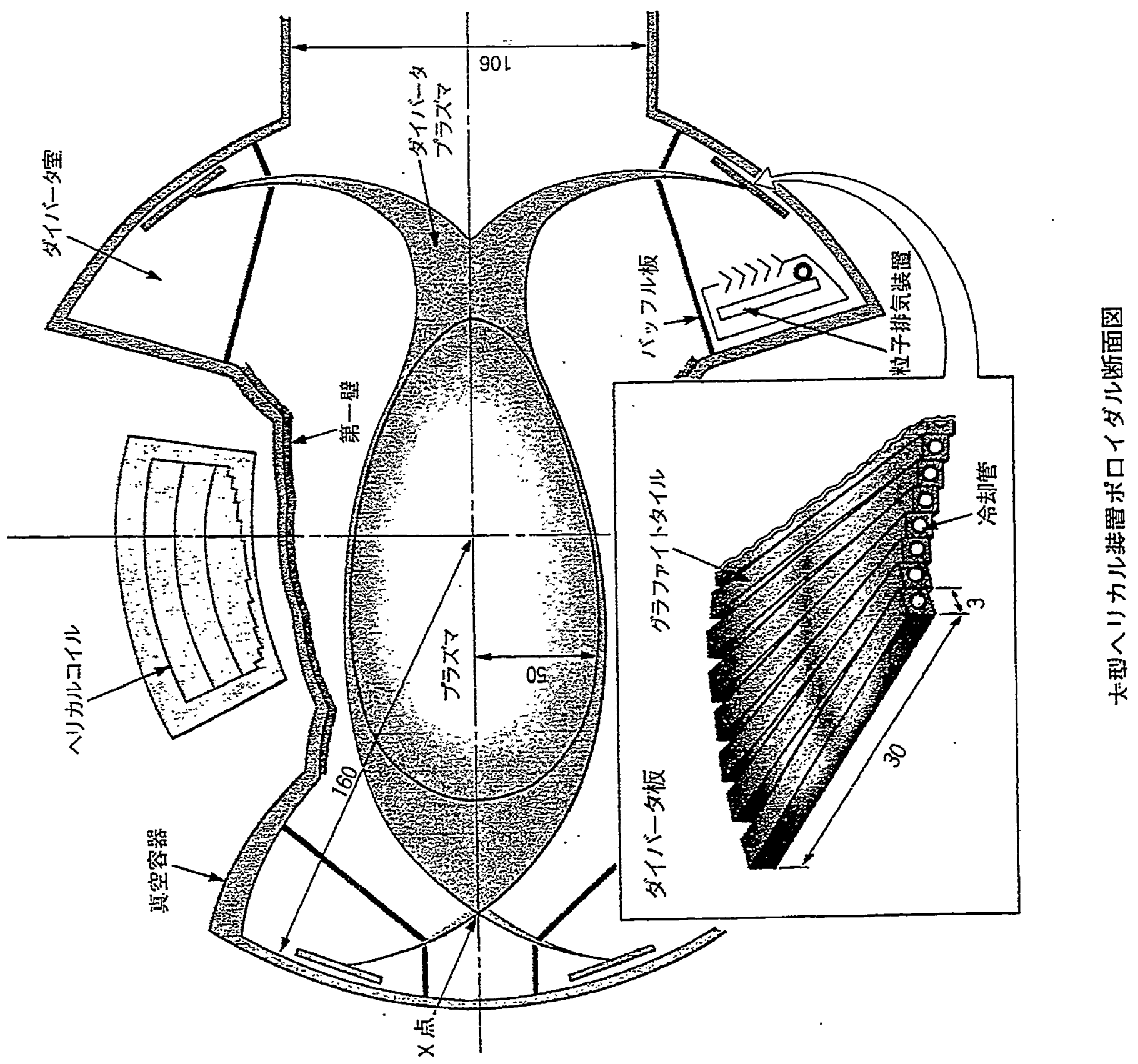




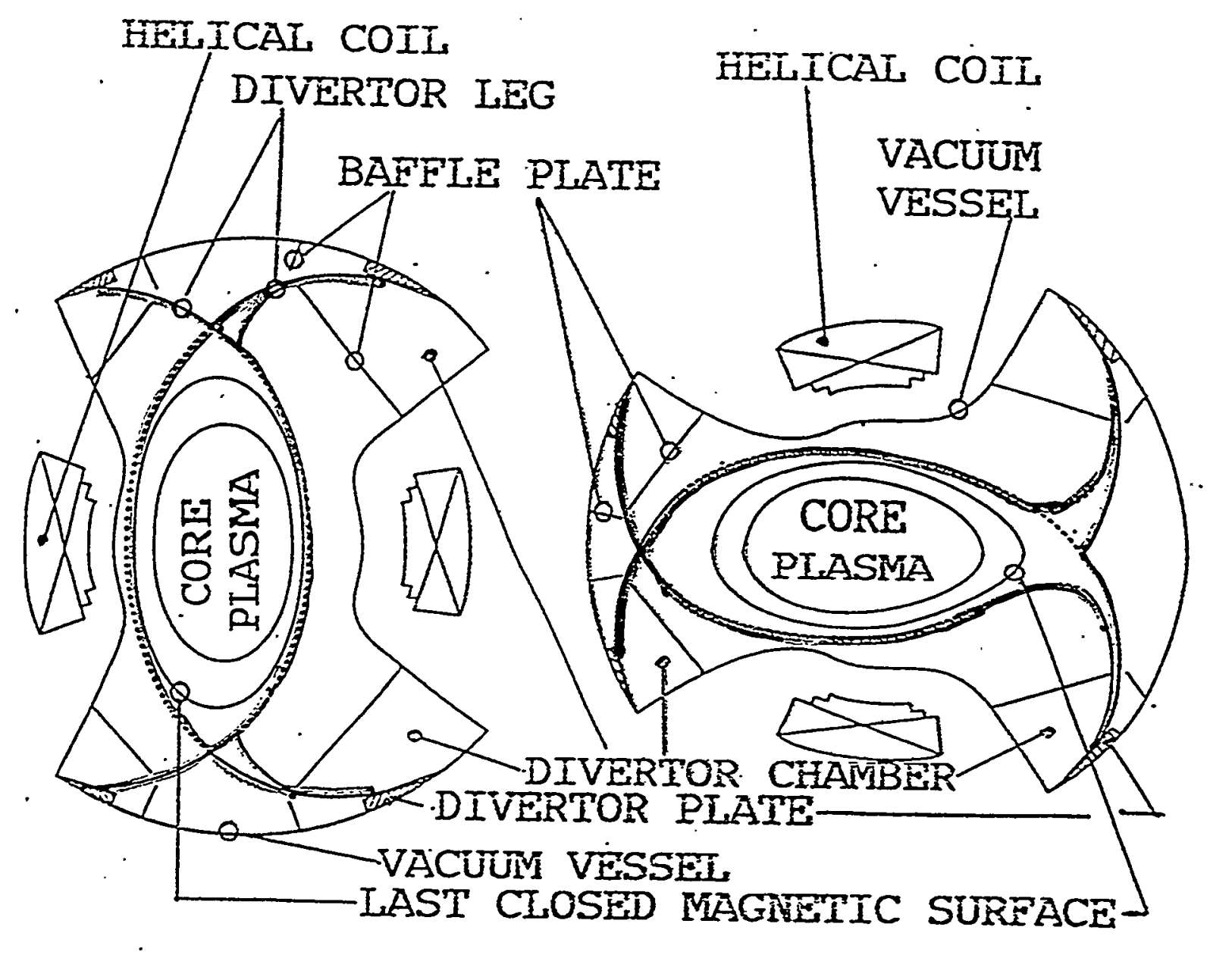

Fig. 1 Cross sectional view of the LHD device Torus axis is on the left hand side. 


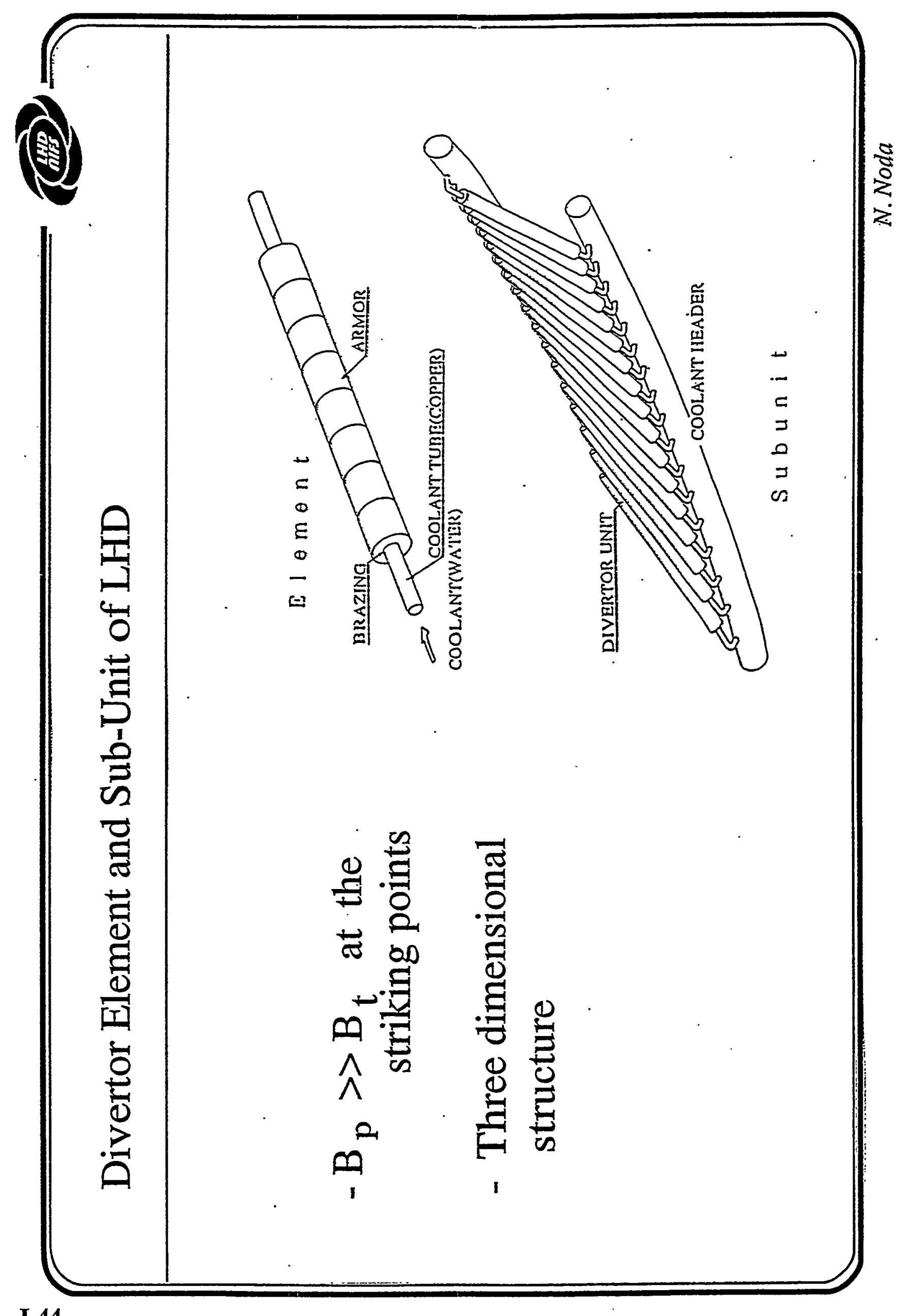




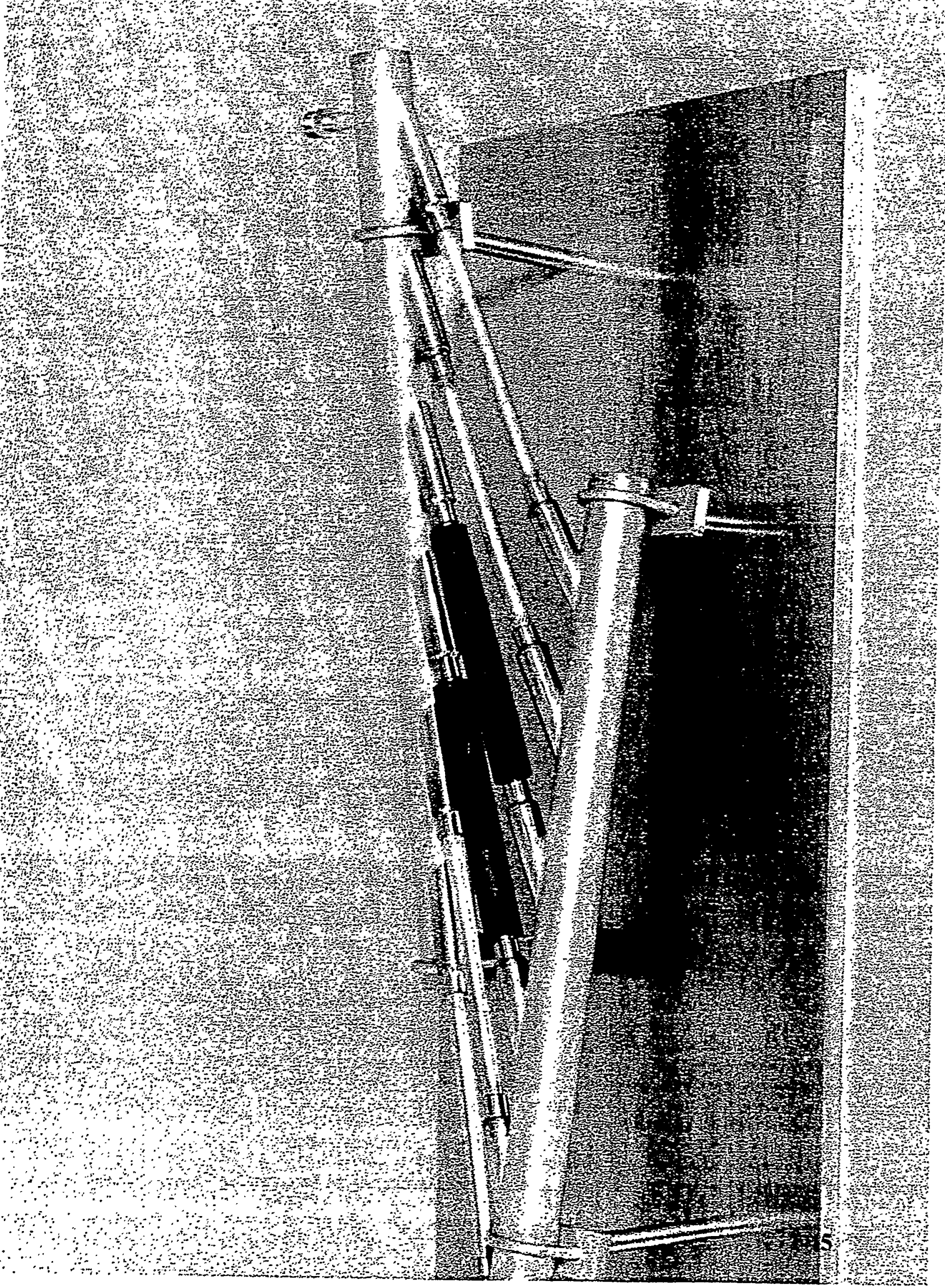




\section{Stepwise approach of Divertor design}

- To start with graphite armors mechanically joined to OFHC because of limited knowledgeabout the heat distribution flexibility of the MJ type configuration limitation of budget in the initial phase

- To learn the heat-load distribution during the initial phase experiments

- To replace them to brazed type elements from the highest. heat load sectiorts 

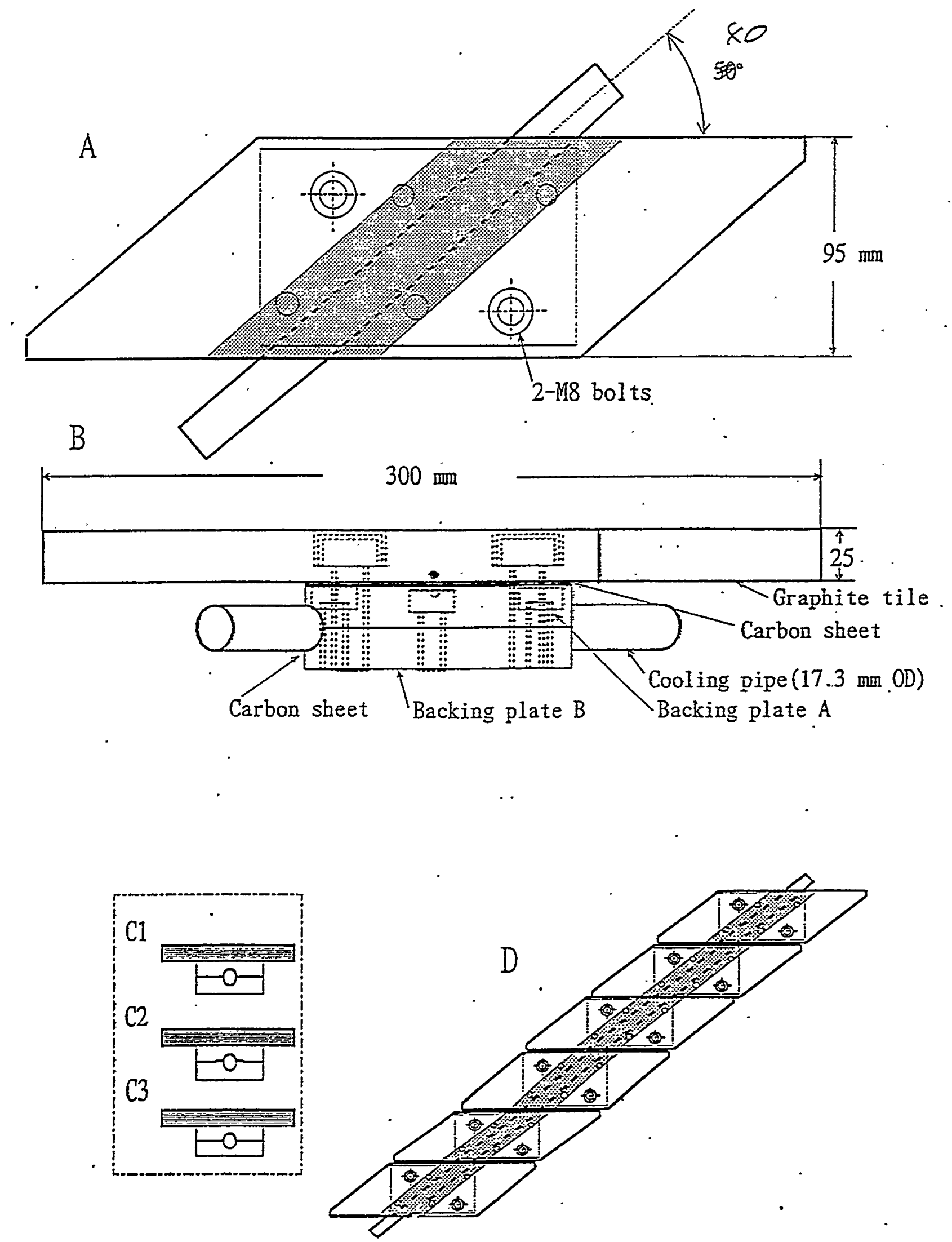

図 1 特性評価用容サイズ機瀻的接合材（タイプ9) 


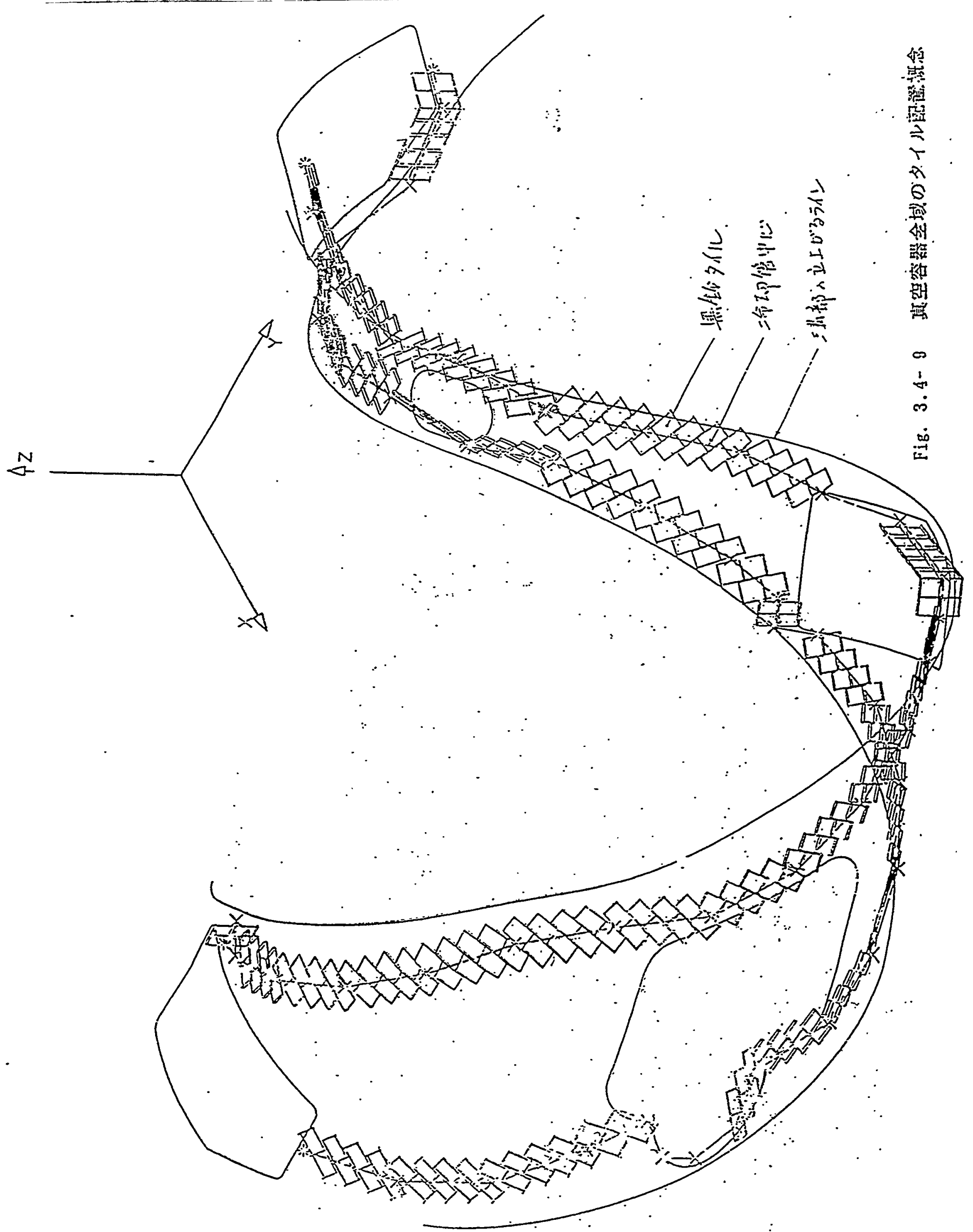




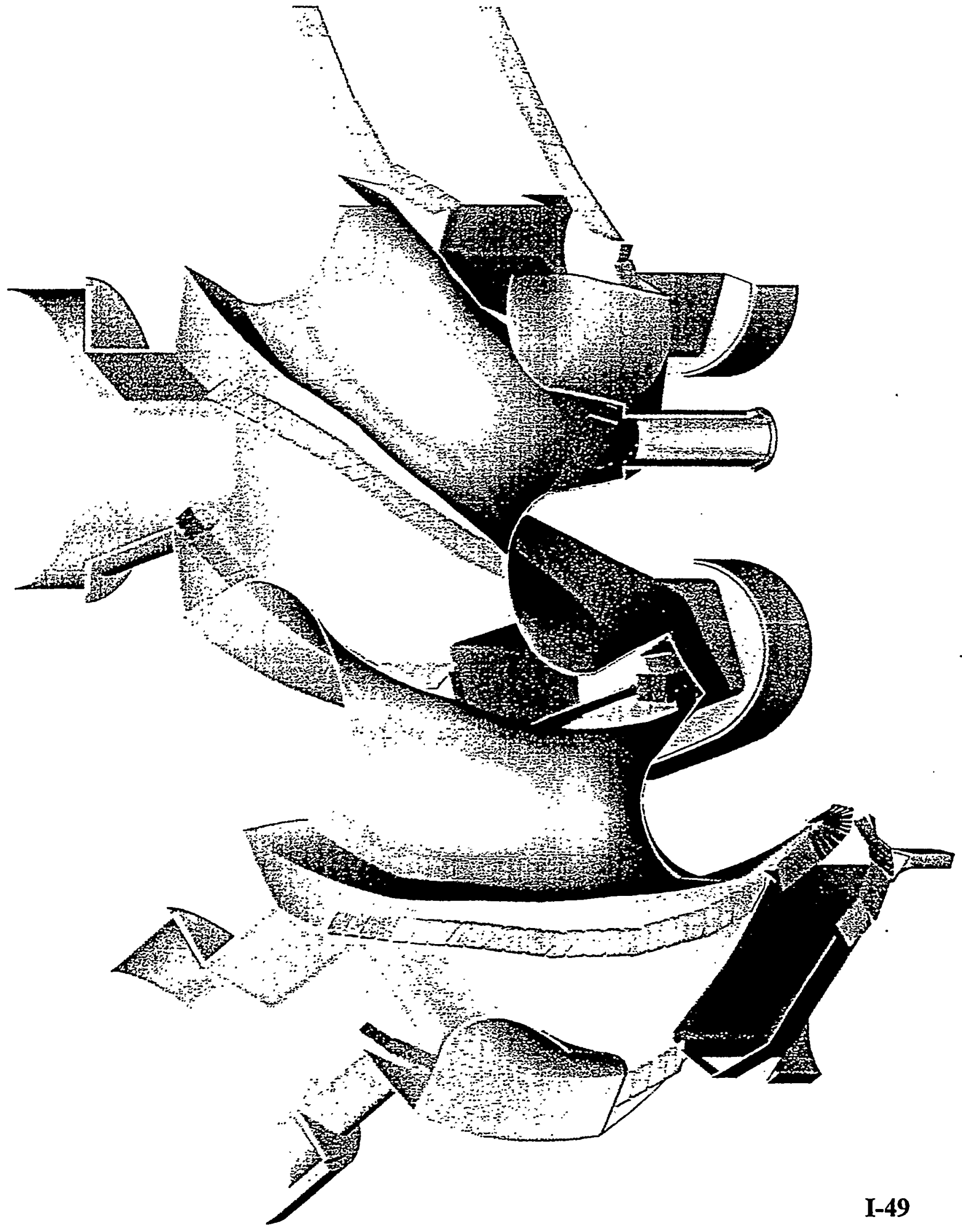




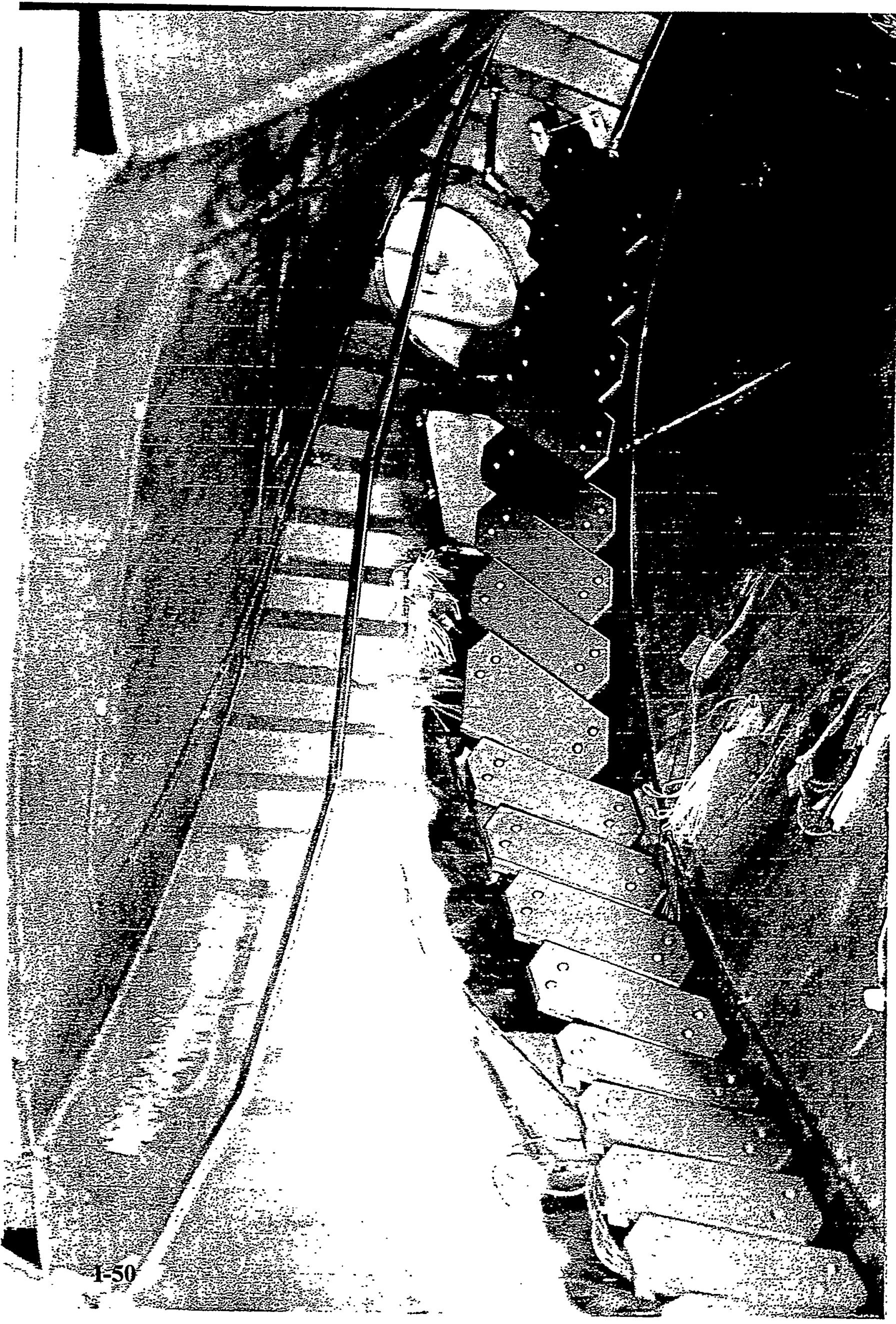




\section{Thermocouple Measurement Hole}

to -

\section{Isotropic Graphite Tile: \\ Hole: $1.1 \mathrm{~mm}$ \\ Thermocouple: $1.0 \mathrm{~mm}$}

\section{Cu Heat Sink: \\ Hole: $1.7 \mathrm{~mm}$ \\ Thermocouple: $1.6 \mathrm{~mm}$}

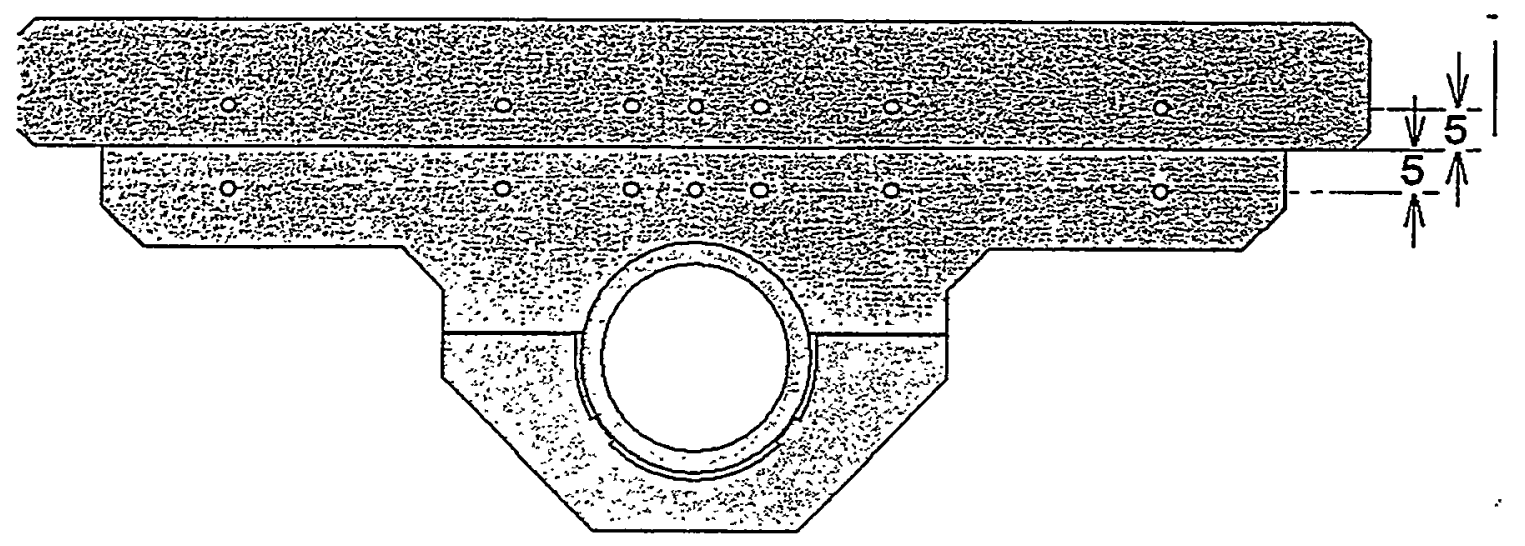




\section{Temperature Profile of Divertor}

\section{$0.3 \mathrm{MW} / \mathrm{m}^{2}$, at $1000 \mathrm{~s}$}

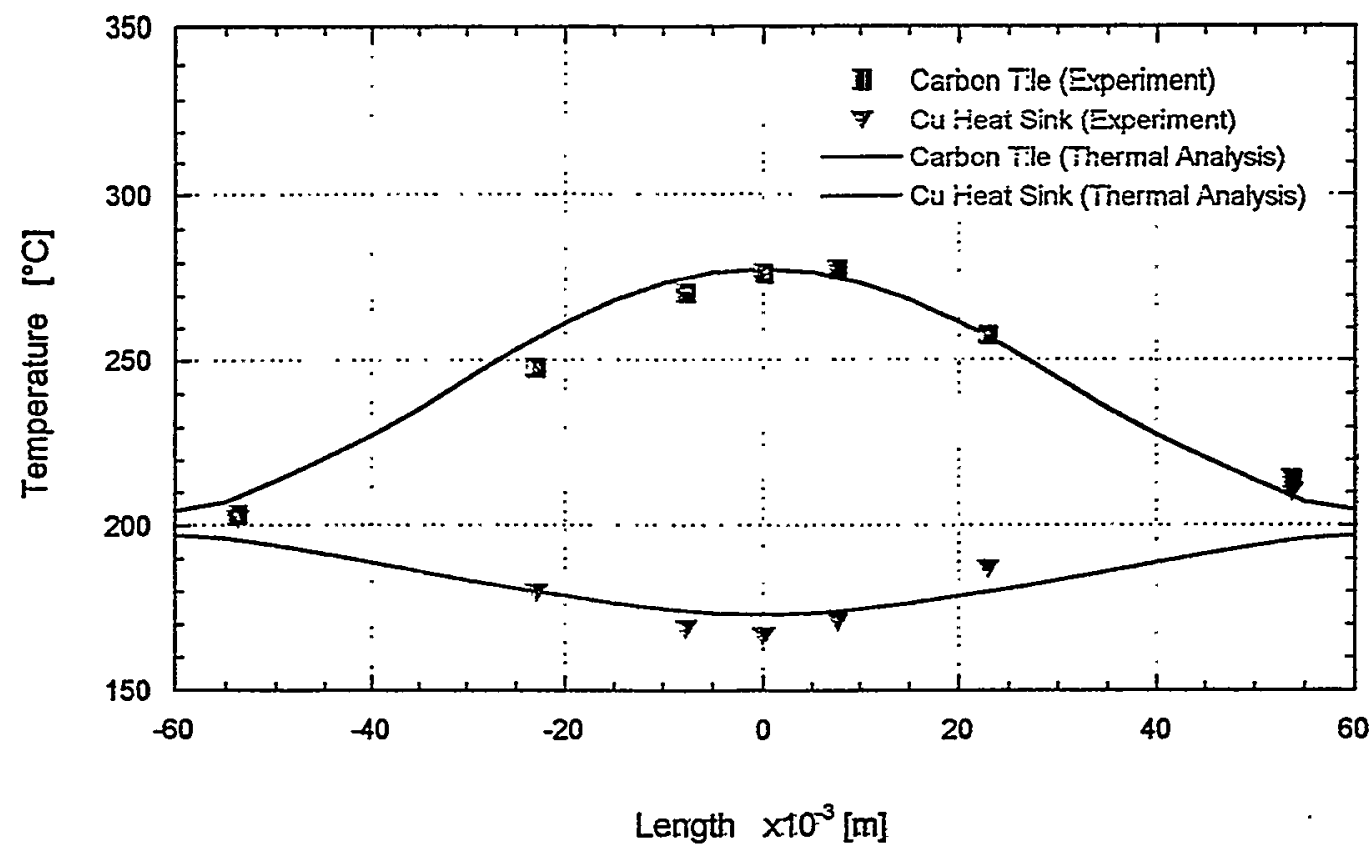

Temperature of Isotropic Graphite Tile

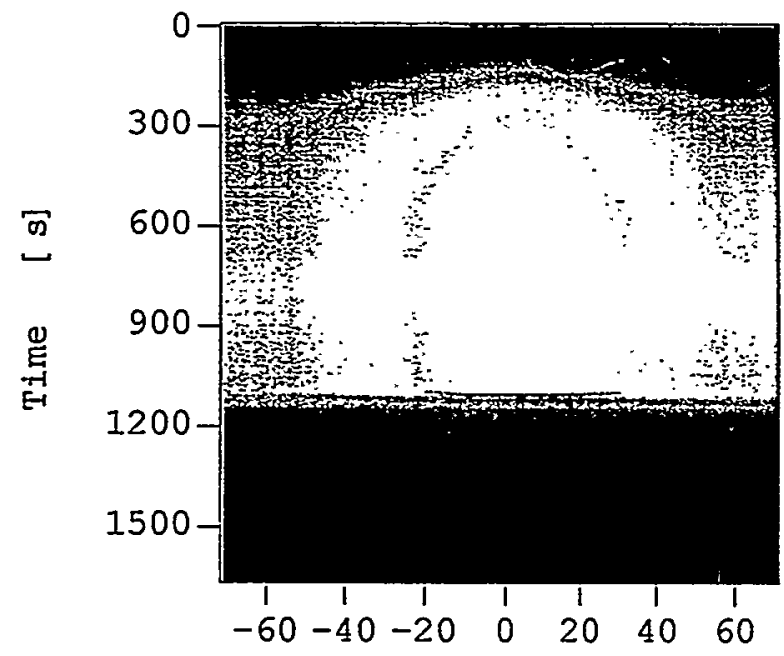

Distance from Center [mm]

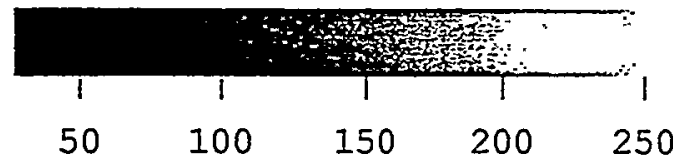

I-52

Temperature $\quad\left[{ }^{\circ} \mathrm{C}\right]$
Temperature of

$\mathrm{Cu}$ Heat Sink

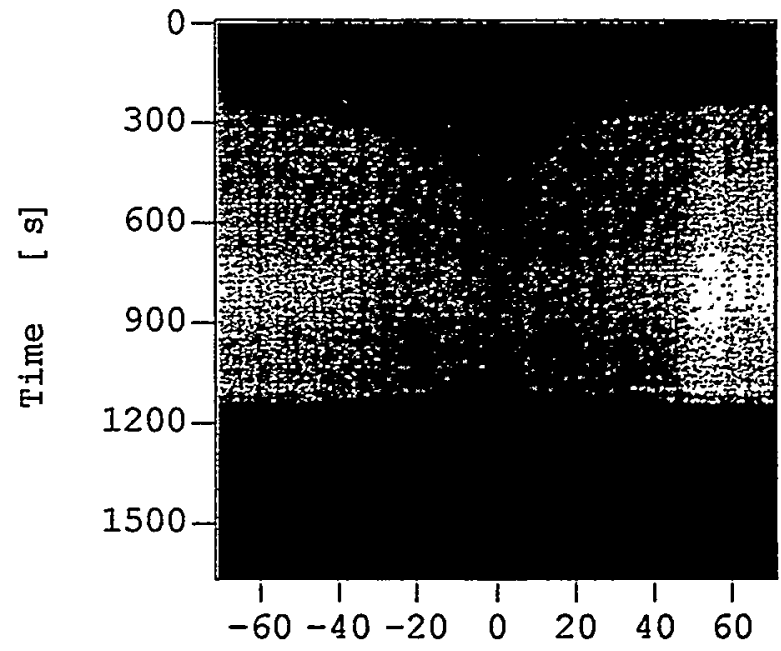

Distance from Center [mm]

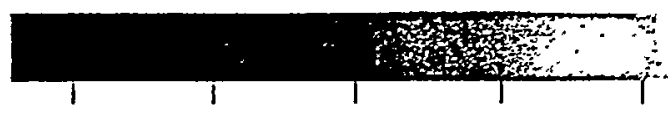

50

150

200

Temperature $\quad\left[{ }^{\circ} \mathrm{C}\right]$ 


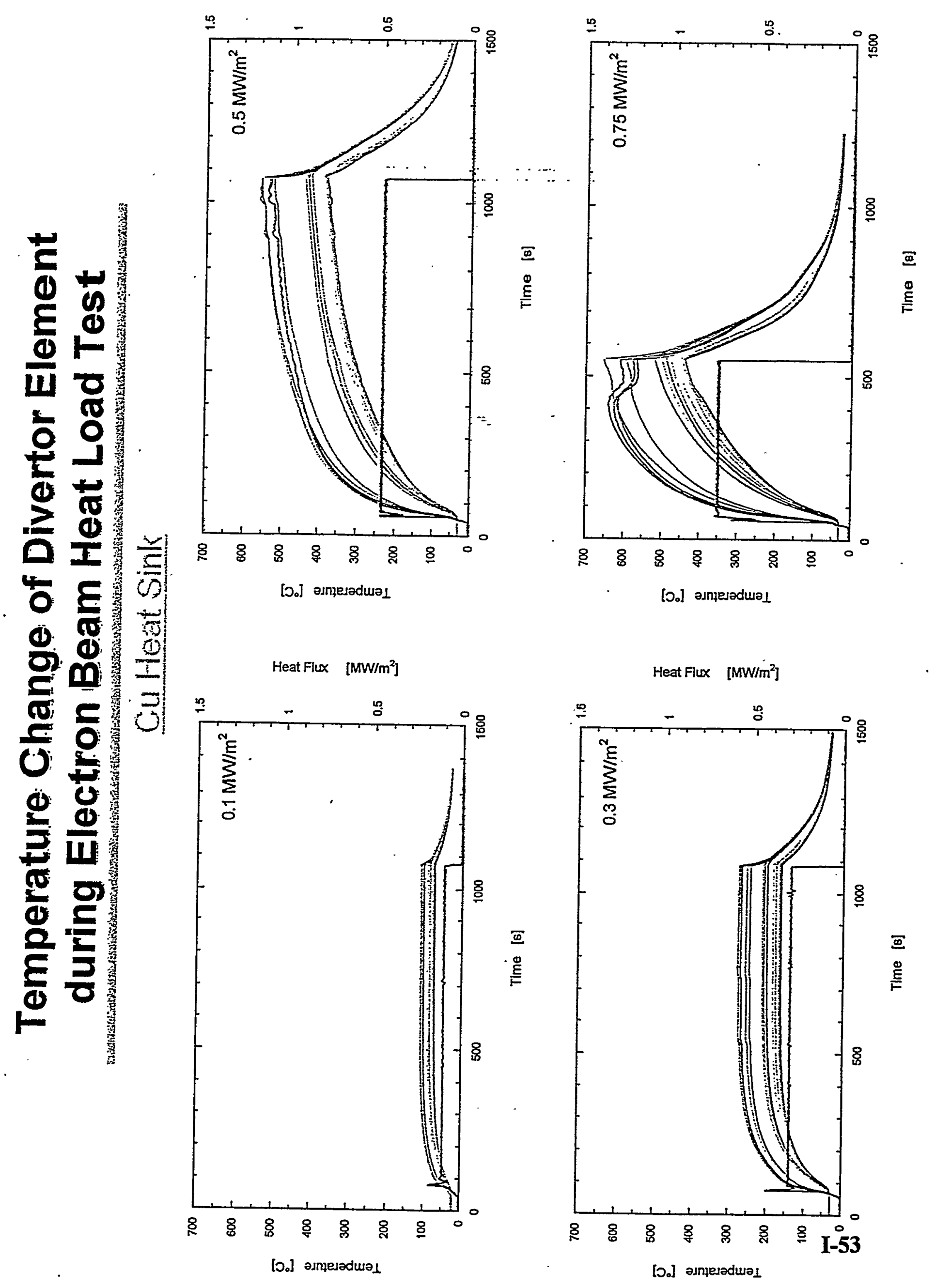


Heat Flux [MW/m]

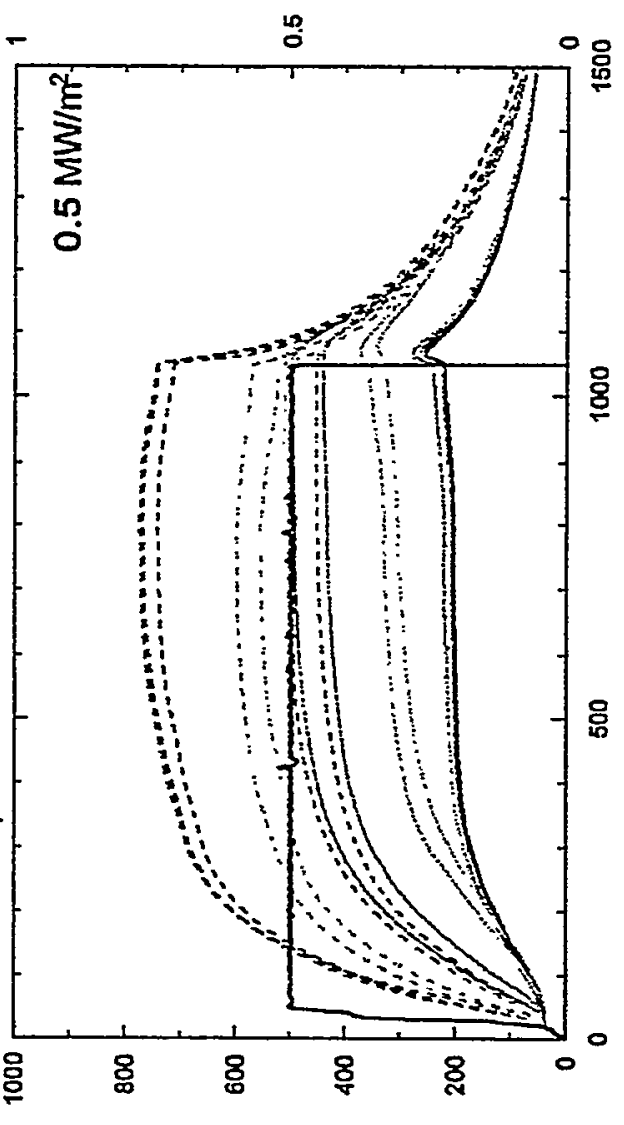

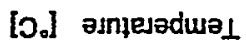

Heat Flux [MW/min]

8
Heat Flux [MW/m]

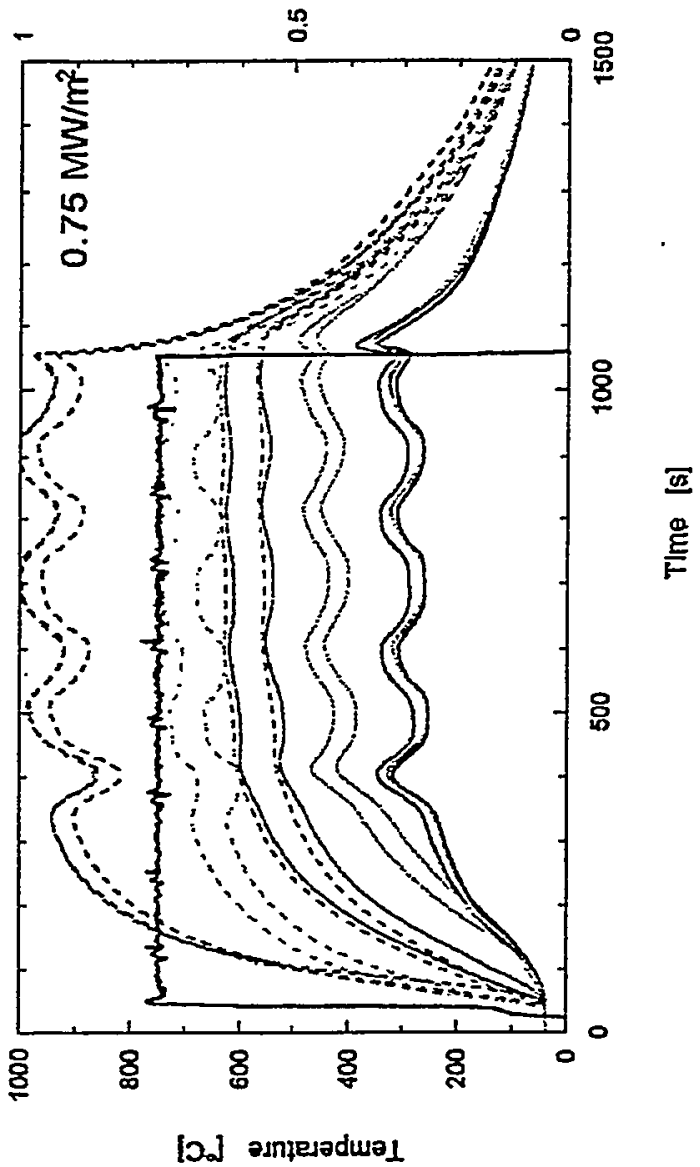

Heat Flux [MW/mi]

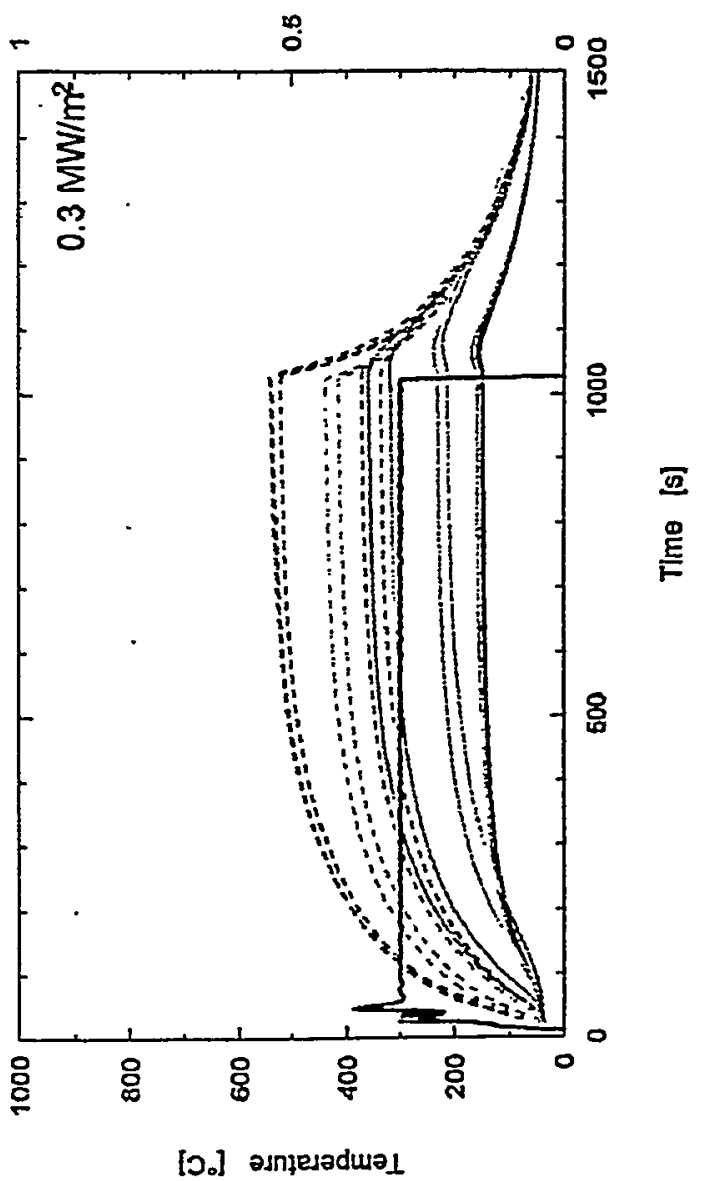



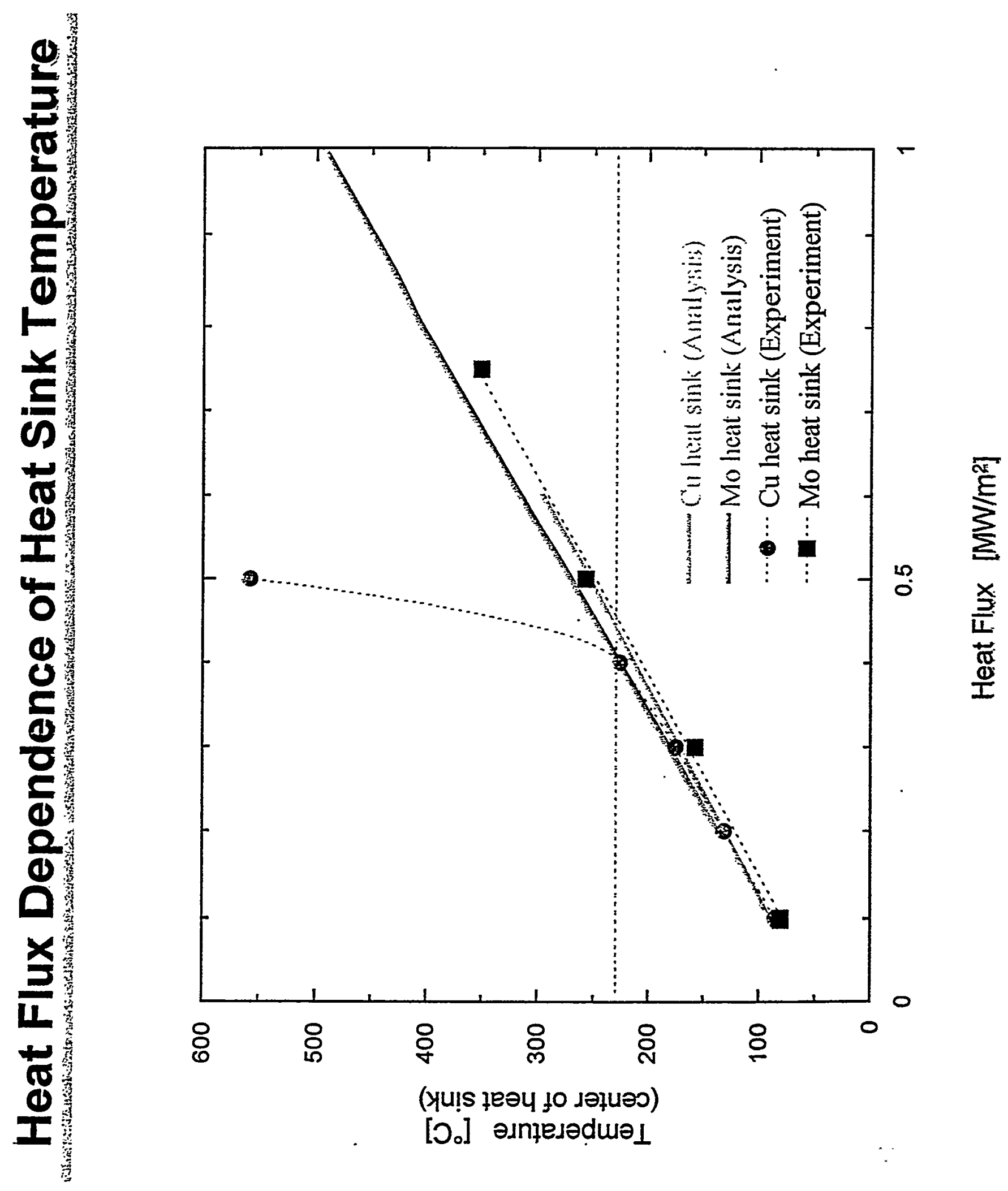
$\mathrm{m} / \mathrm{n}=1 / 1$ のコンポーネント

を加えた磁気面

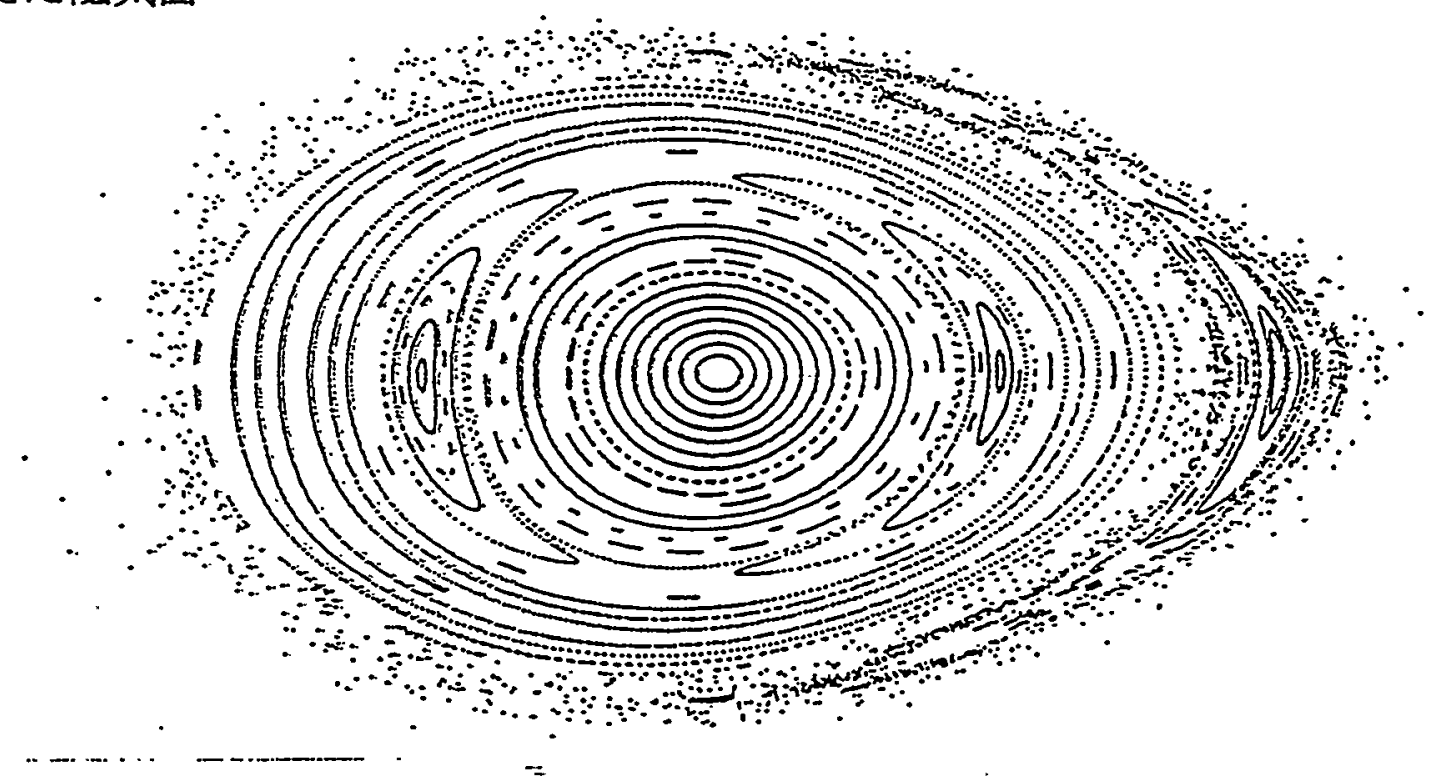

$\mathrm{m} / \mathrm{n} \equiv 2 / 1$ 学打方消すコンポ

一ネンにを加えた磁気面

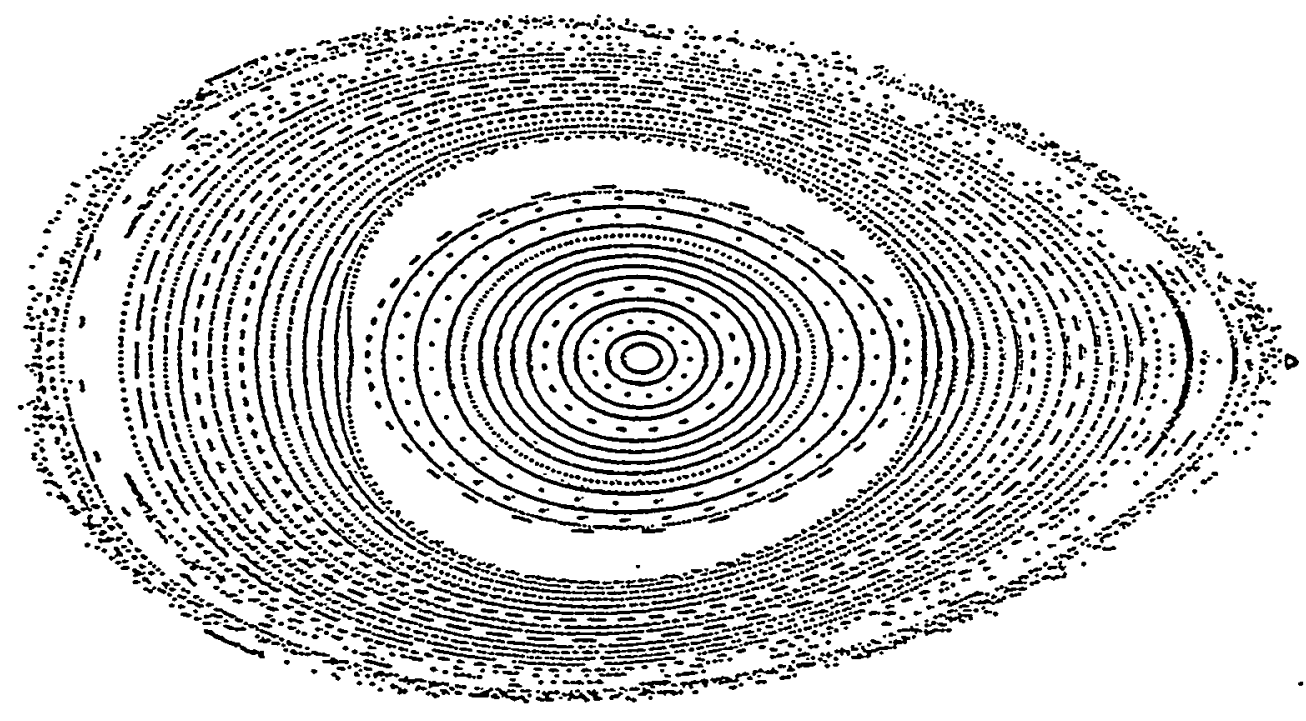

$\mathrm{m} / \mathrm{n}=1 / 1$ の广イランドだけ

が形成された磁気面

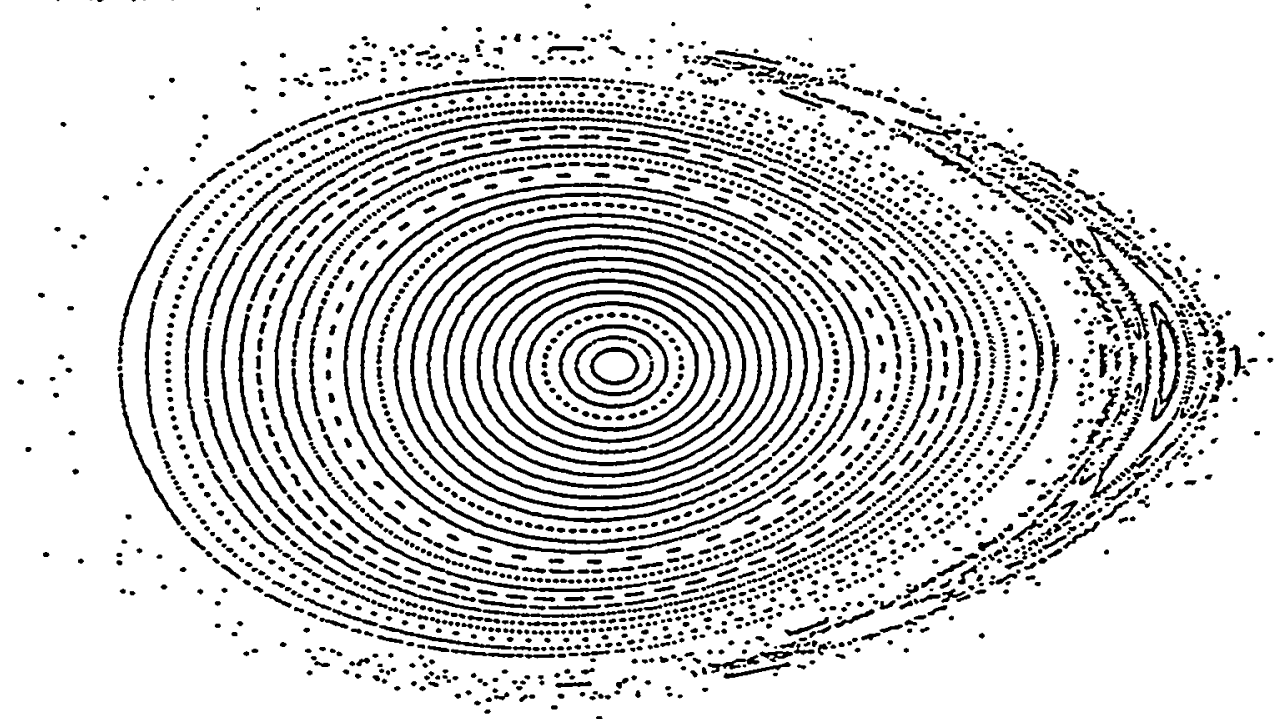




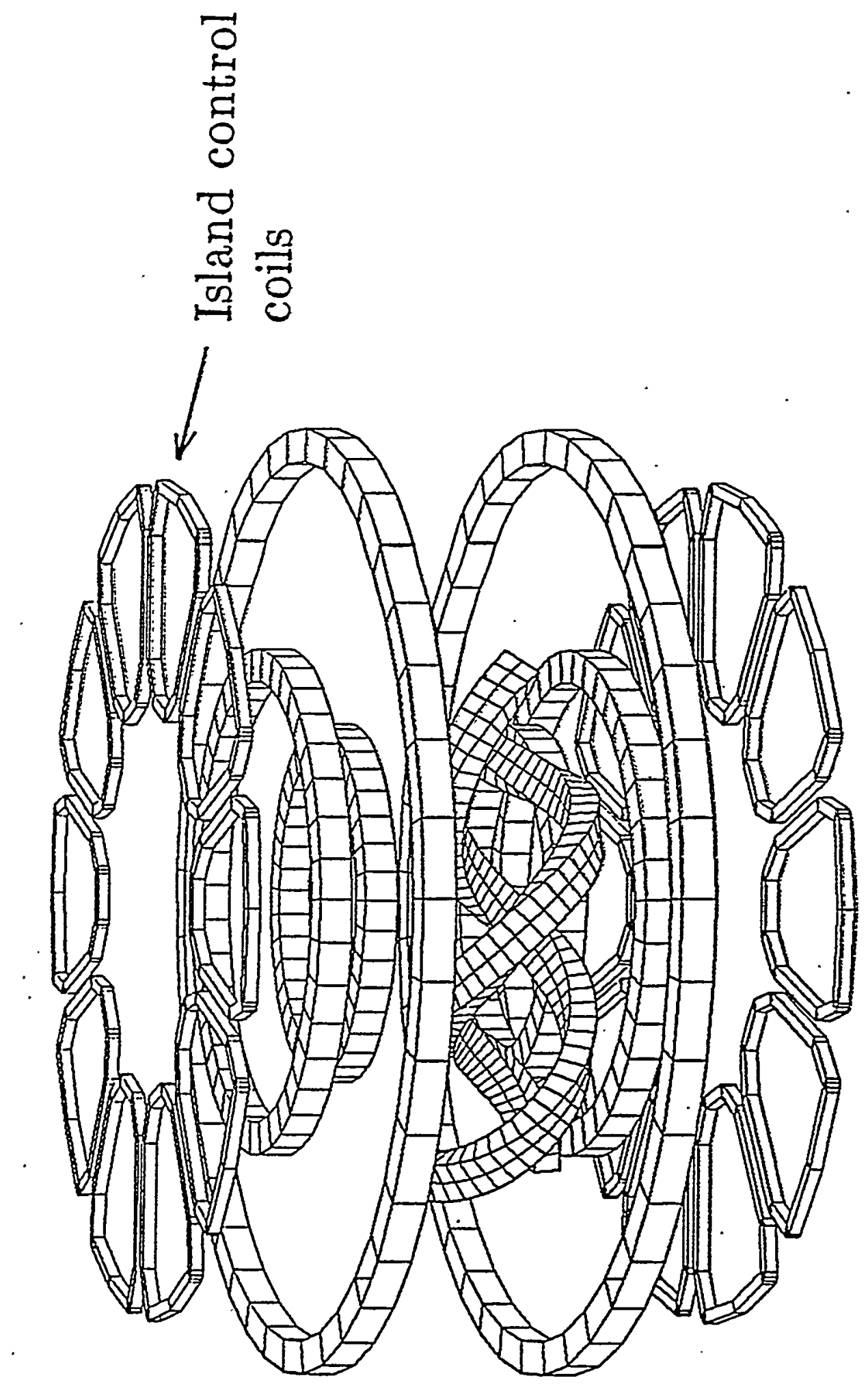




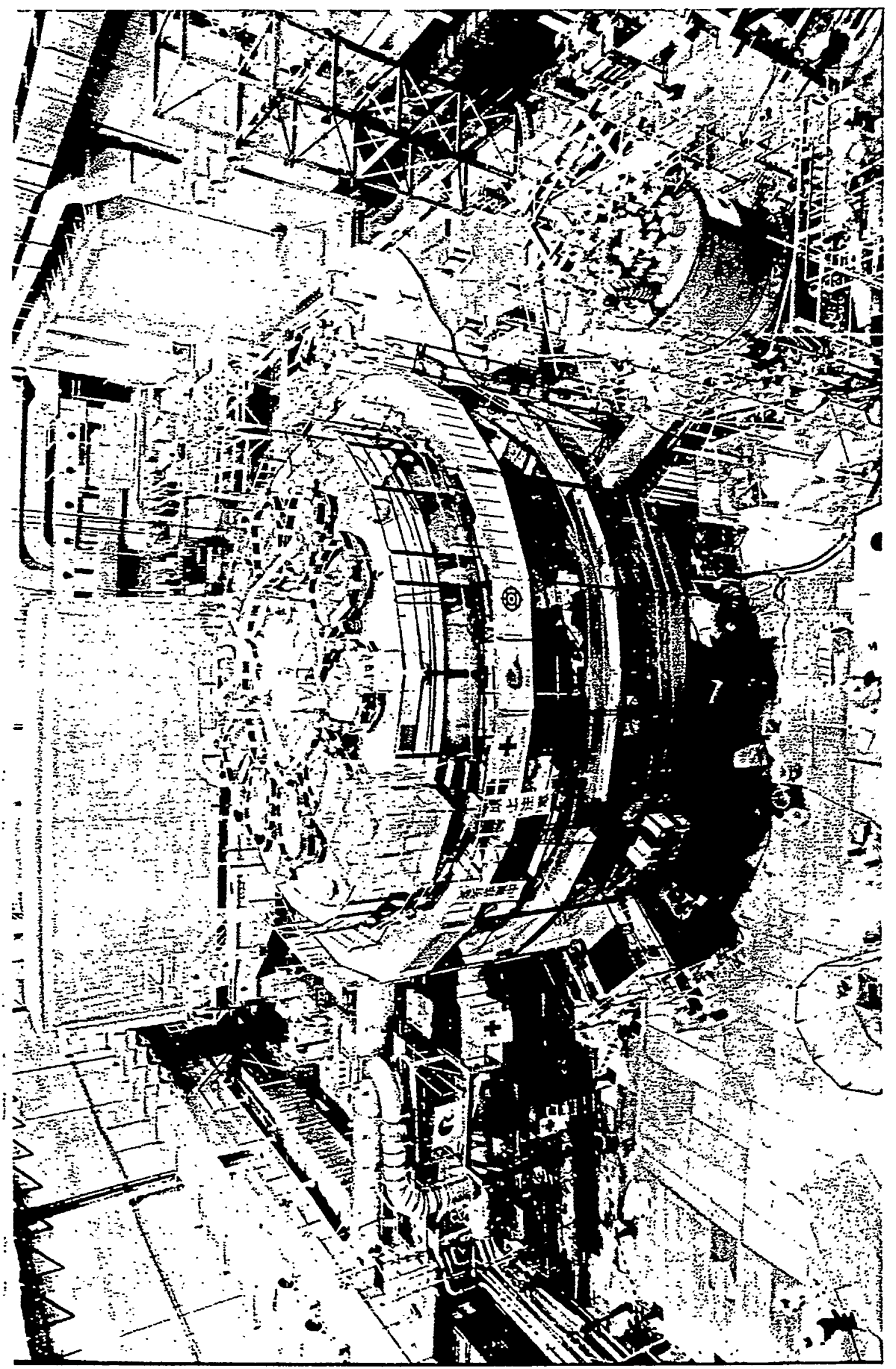



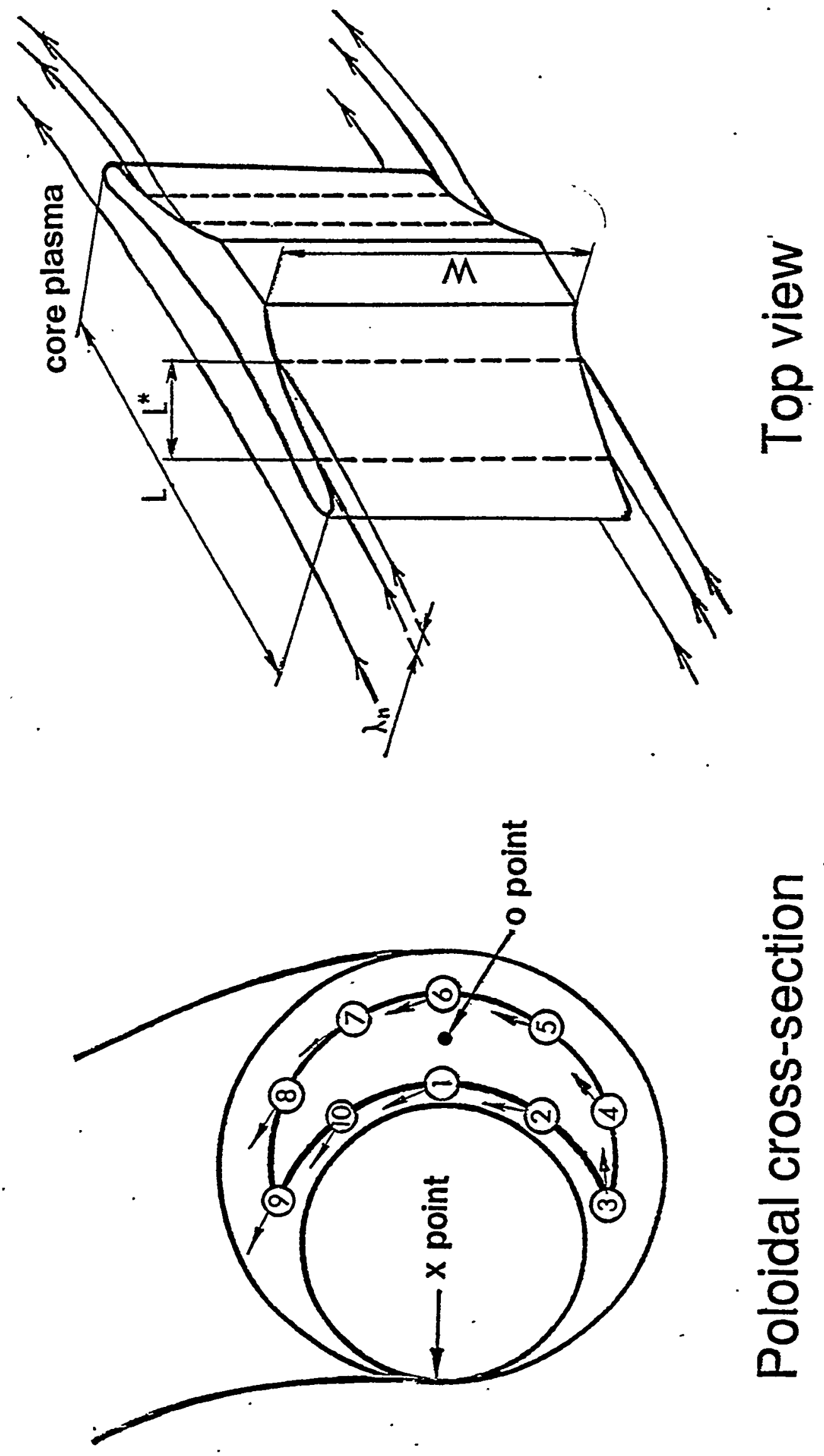


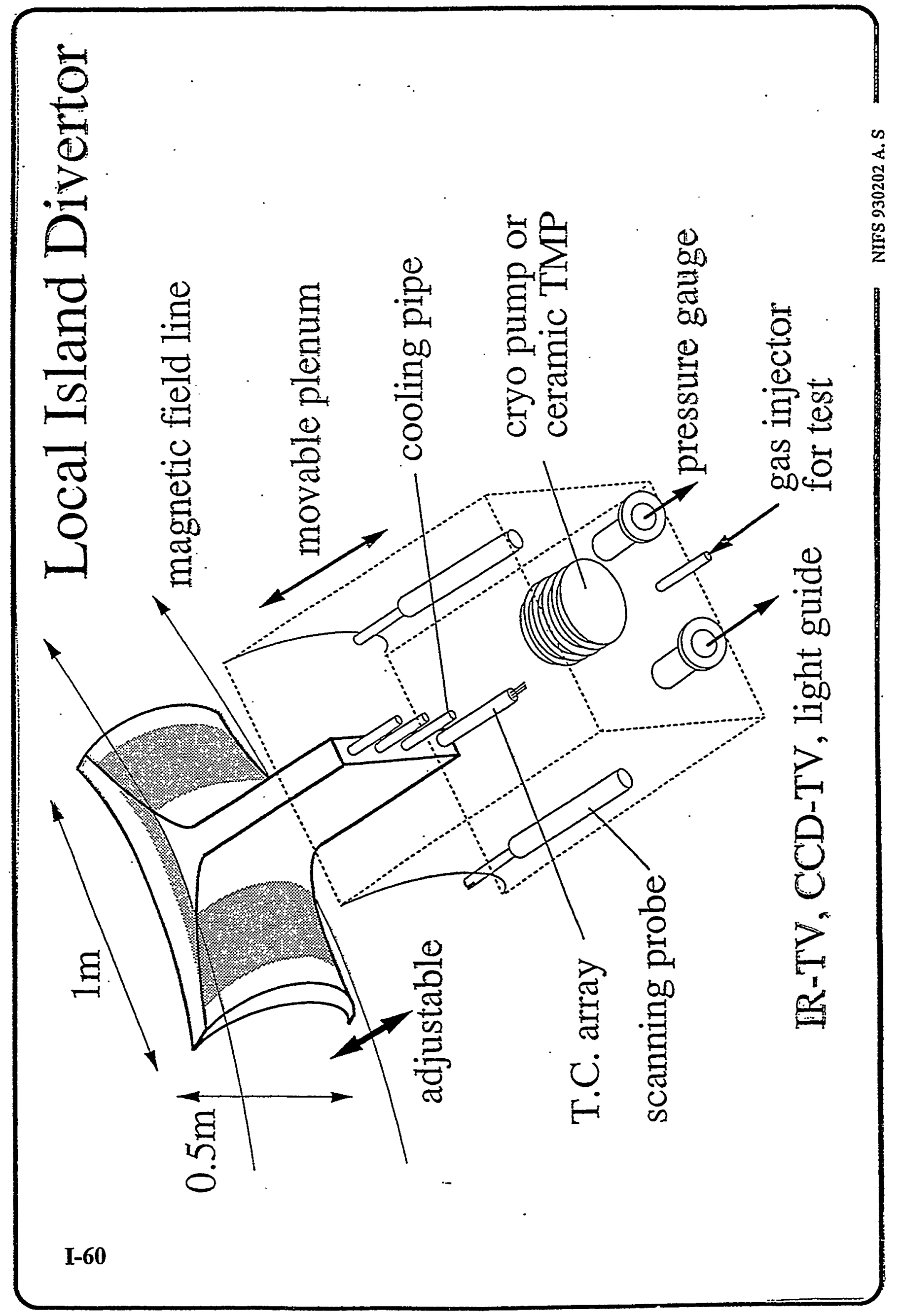




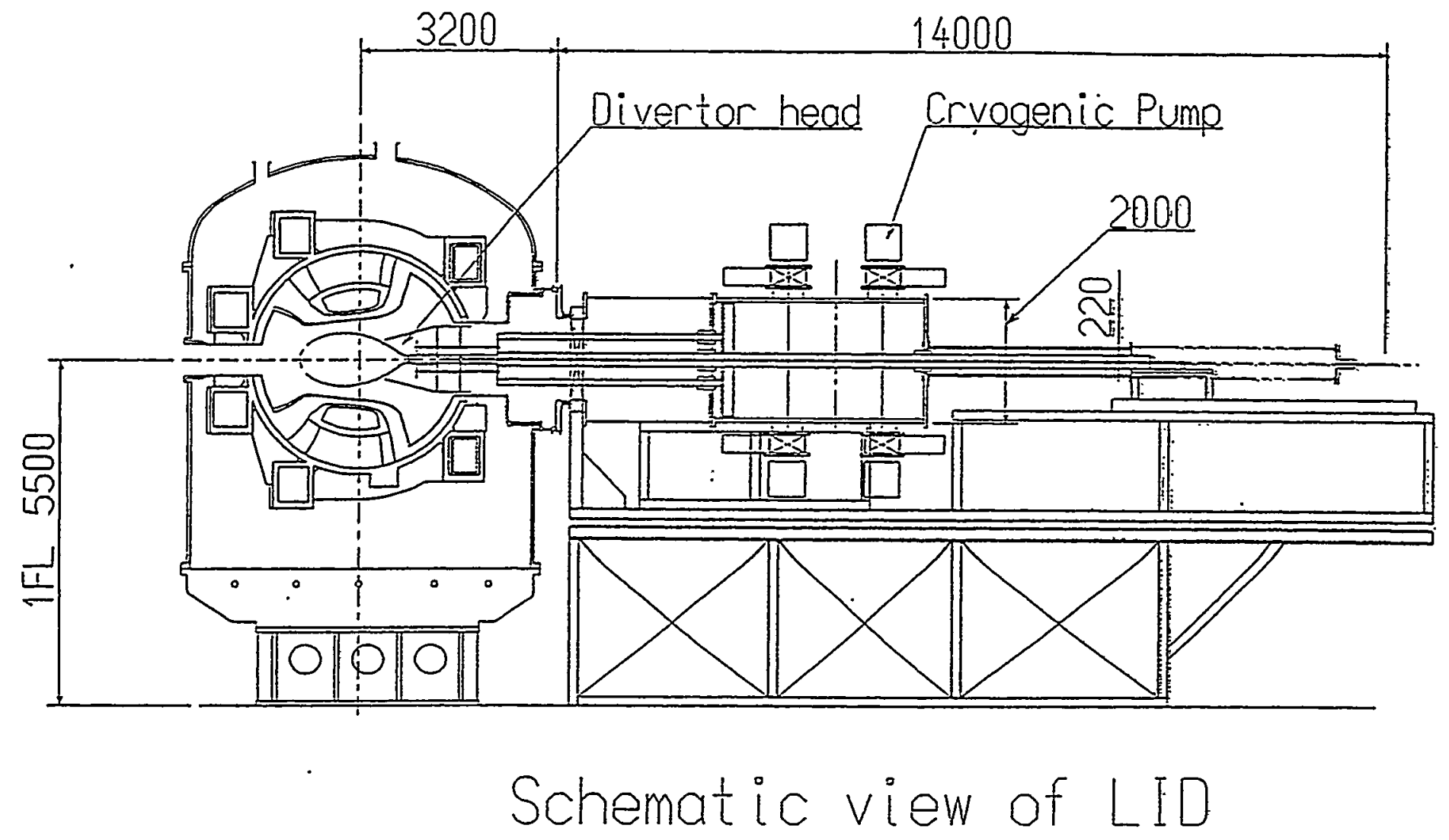

Pumping system : Cryogenic pump

. Pumping speed . $\quad \sim 100,000 \mathrm{Tit} / \mathrm{s}$

Pumping capacity . $\quad-300,000$ torr.lit.

Maximum pumping flux $\sim 75$ torr.lit./s

Steady-state heat load :

$\begin{array}{ll}\text { Heat.load } & \sim 1.5 \mathrm{MW} \\ \text { Averaged Heat flux } & \sim 5 \mathrm{MW} / \mathrm{m}^{2} \\ \text { Maximum Heat flux } & \sim 10 \mathrm{MW} / \mathrm{m}^{2}\end{array}$

Cooling system : Plasma facing material C/C composite brazed to copper plate cooled by water 


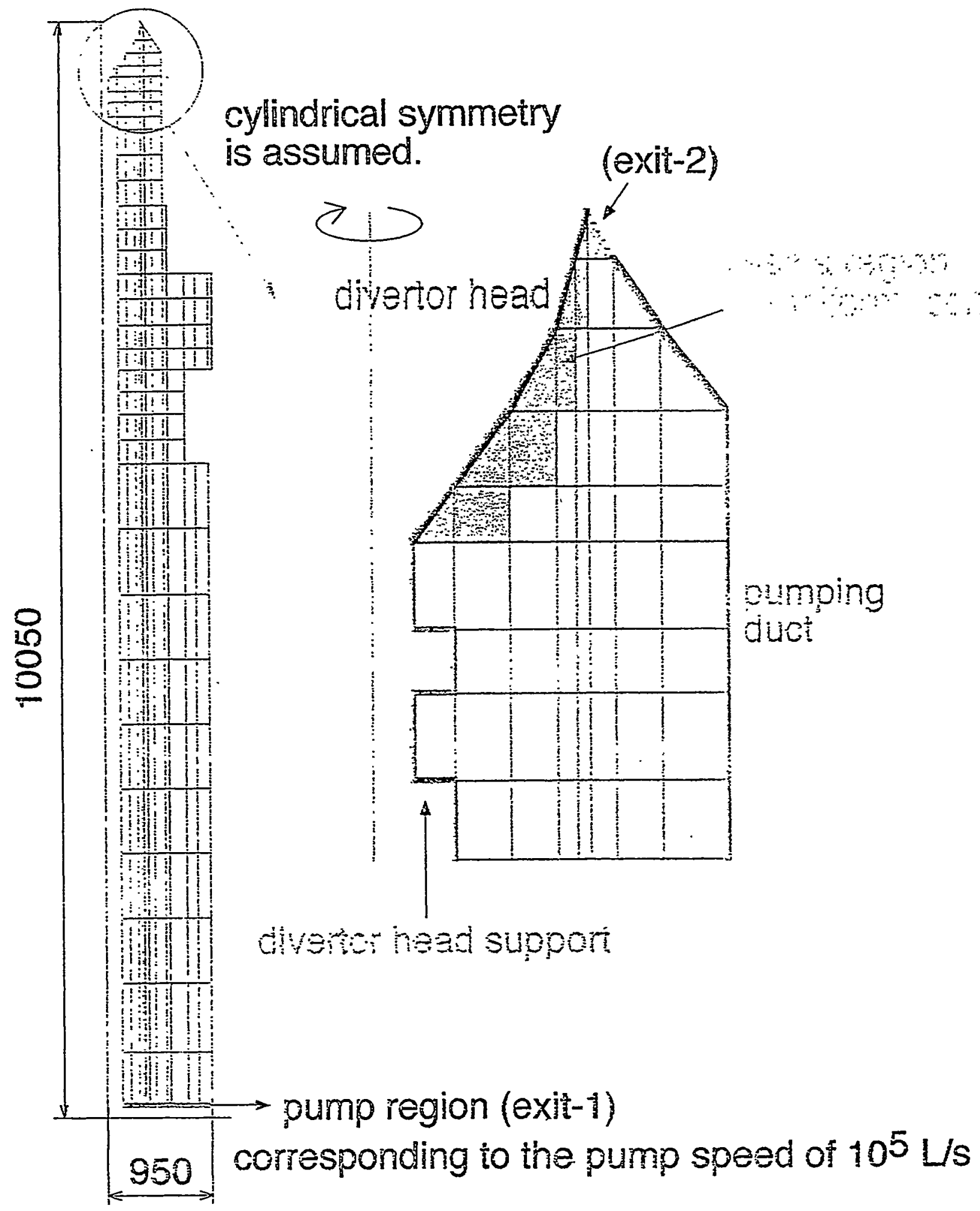

This system has two particle exit. 


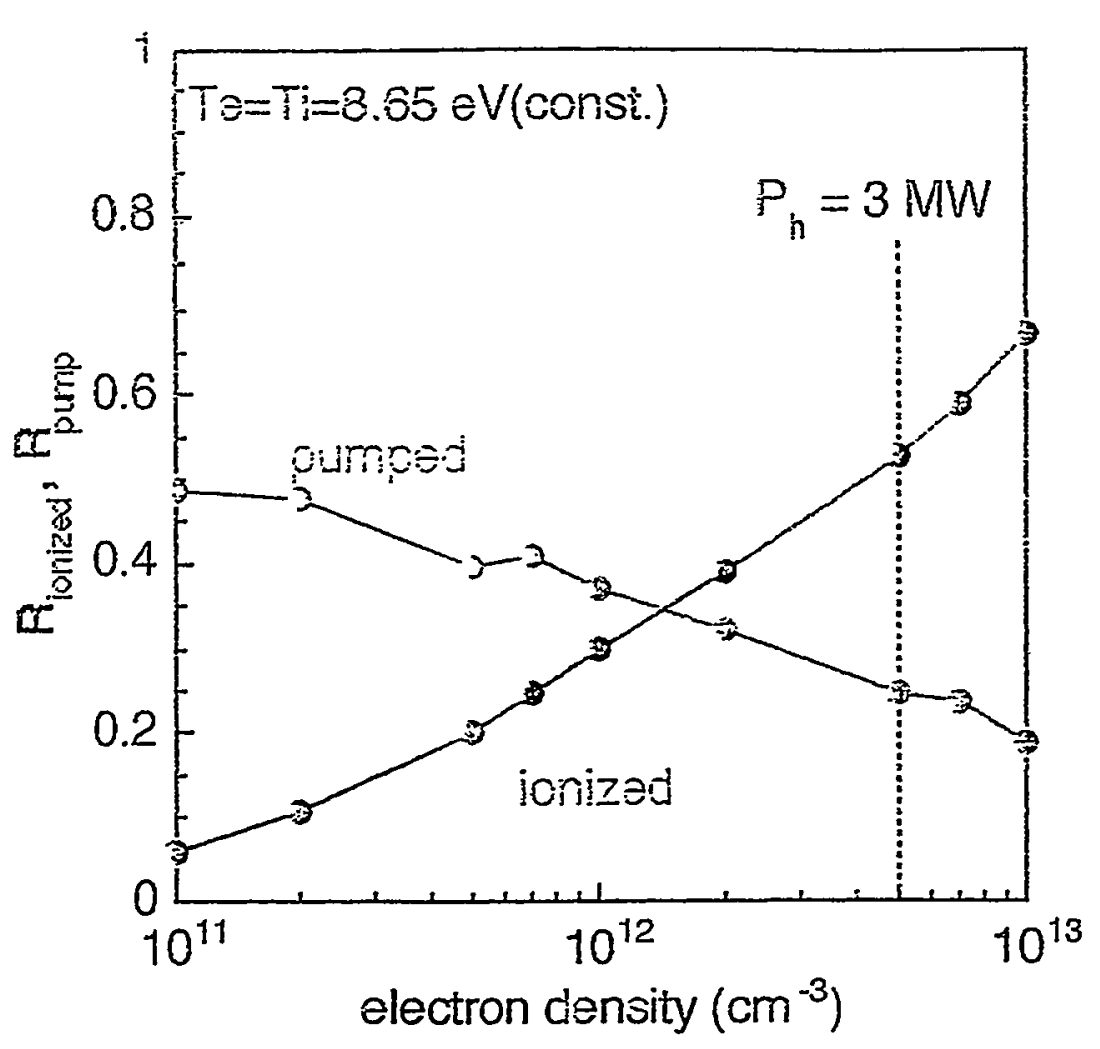

Pumping efficiency as the function of plasma density

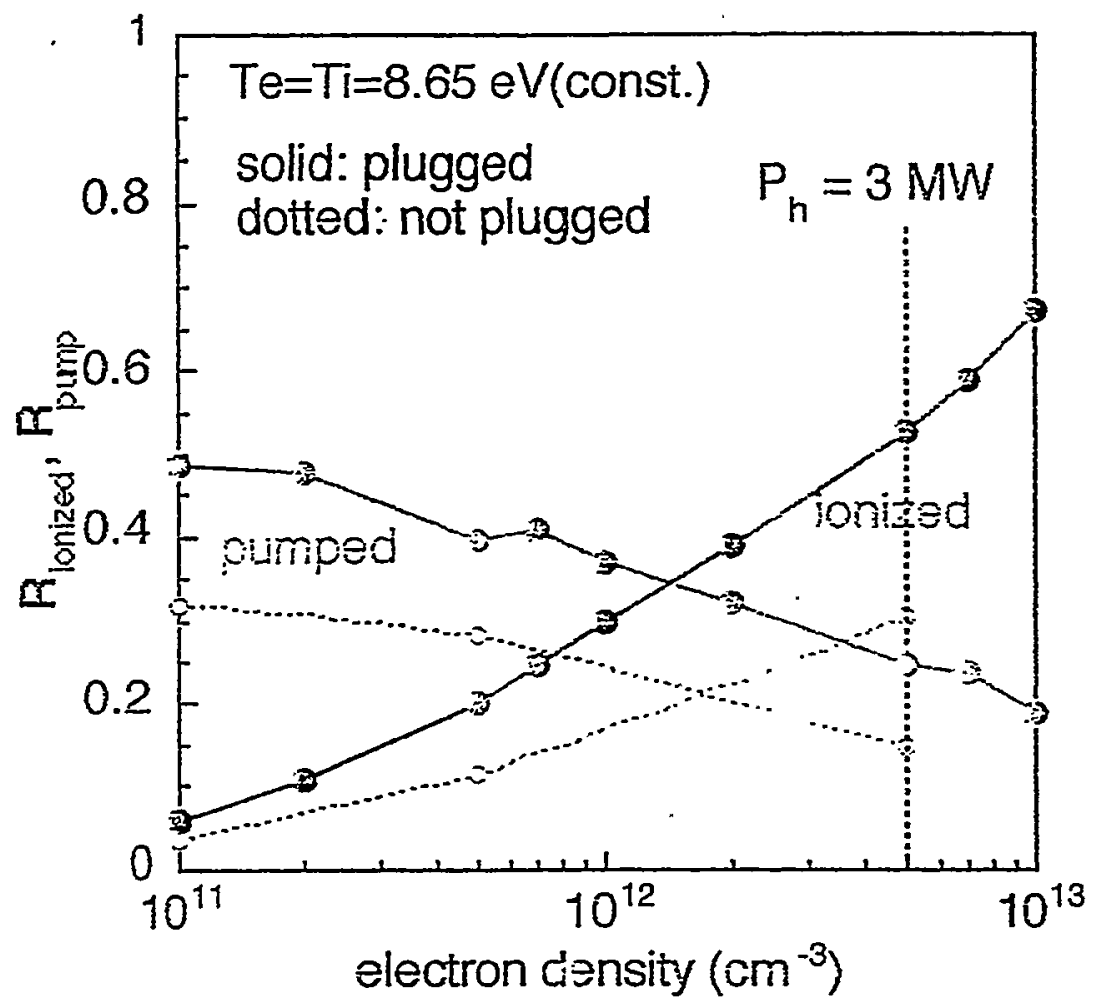

The effect of the plasma plaugging

$R_{\text {pump }}=\frac{N_{\text {pump }}}{N_{\text {total }}}=\frac{N_{\text {pump }}}{\mathbb{N}_{\text {ionized }}+\mathbb{N}_{\text {duct }}+N_{\text {pump }}}, \mathbb{P}_{\text {ionized }}=\frac{N_{\text {ionized }}}{N_{\text {ionized }}+N_{\text {duct }}+N_{\text {pump }}}$ 


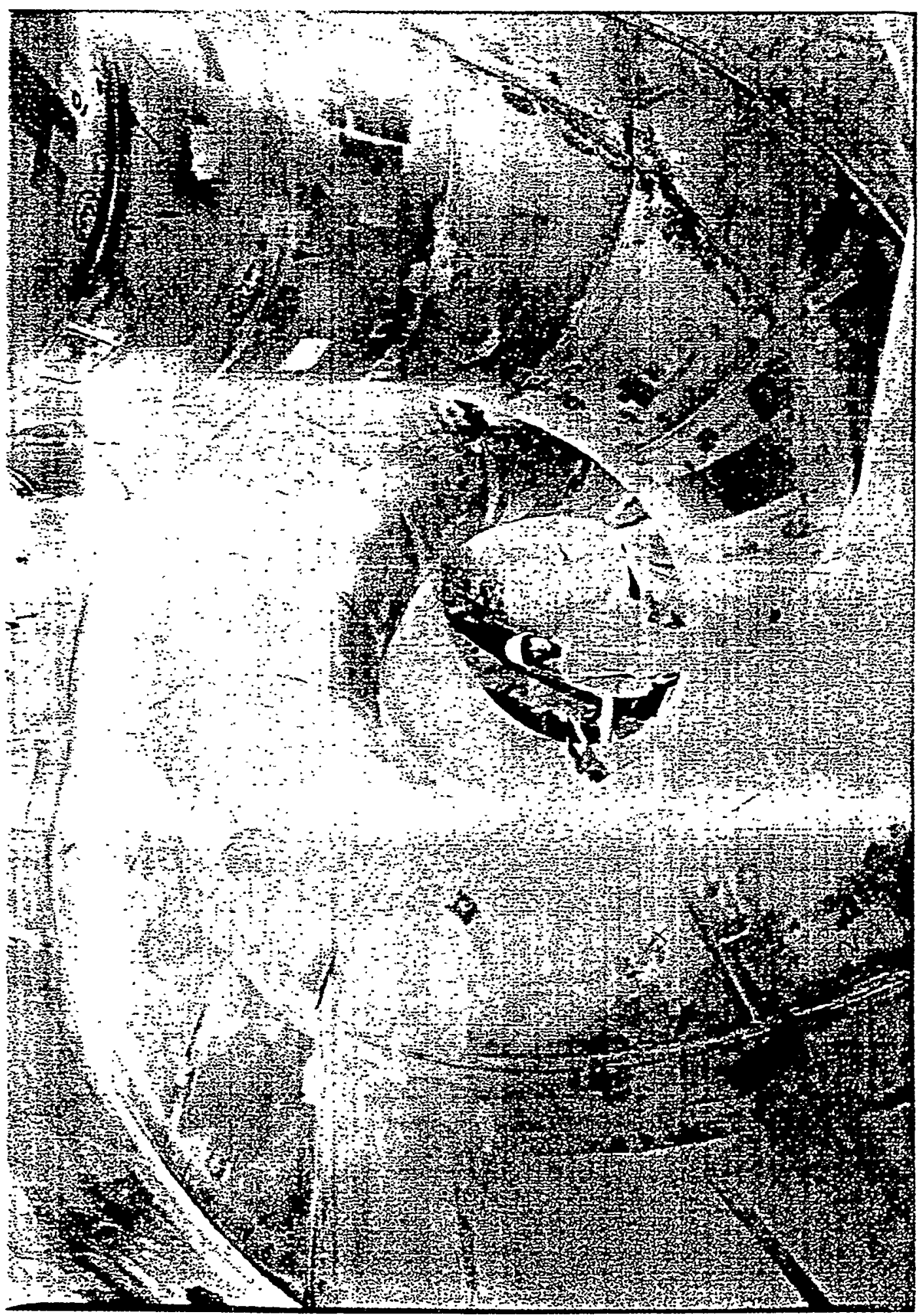




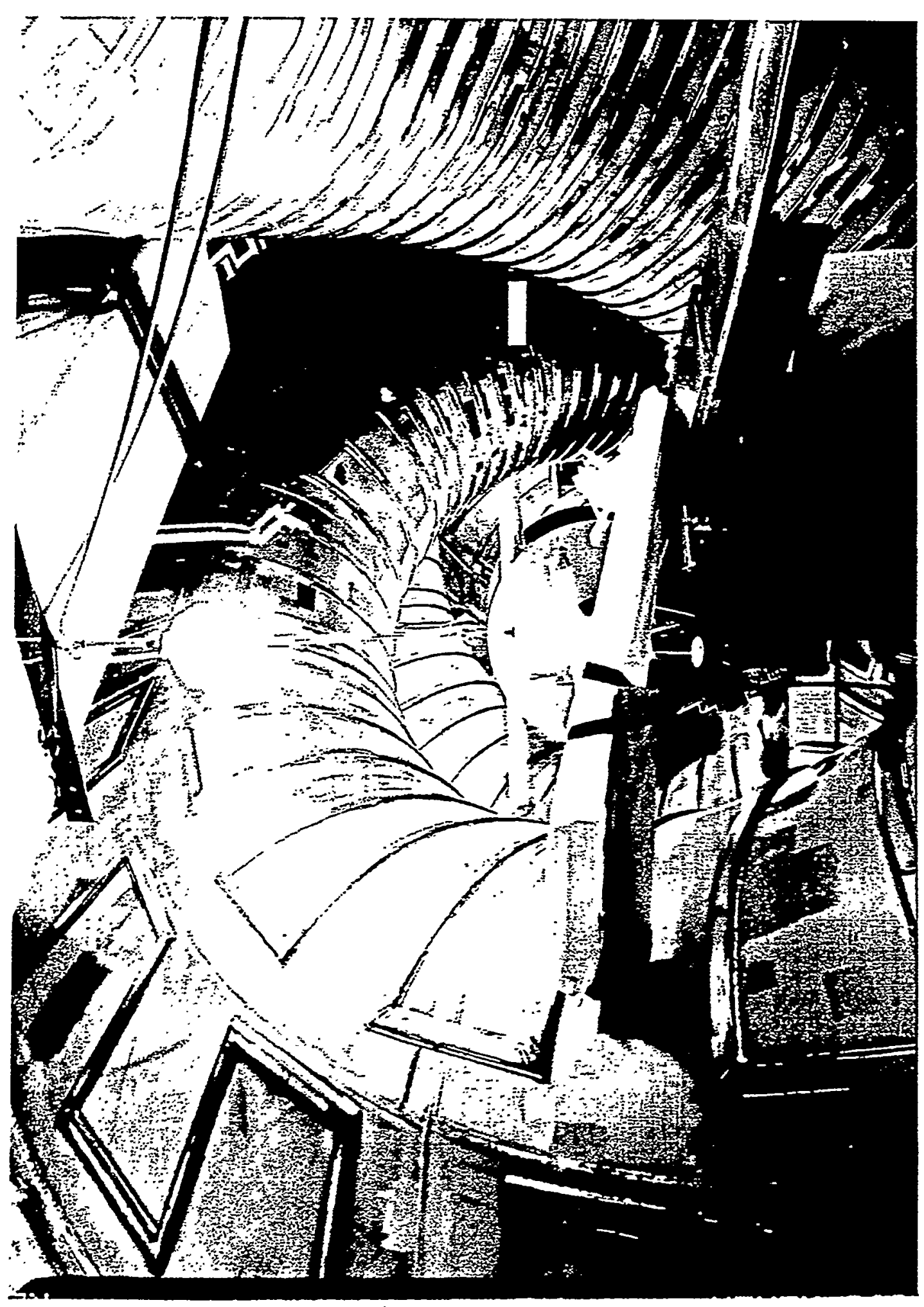




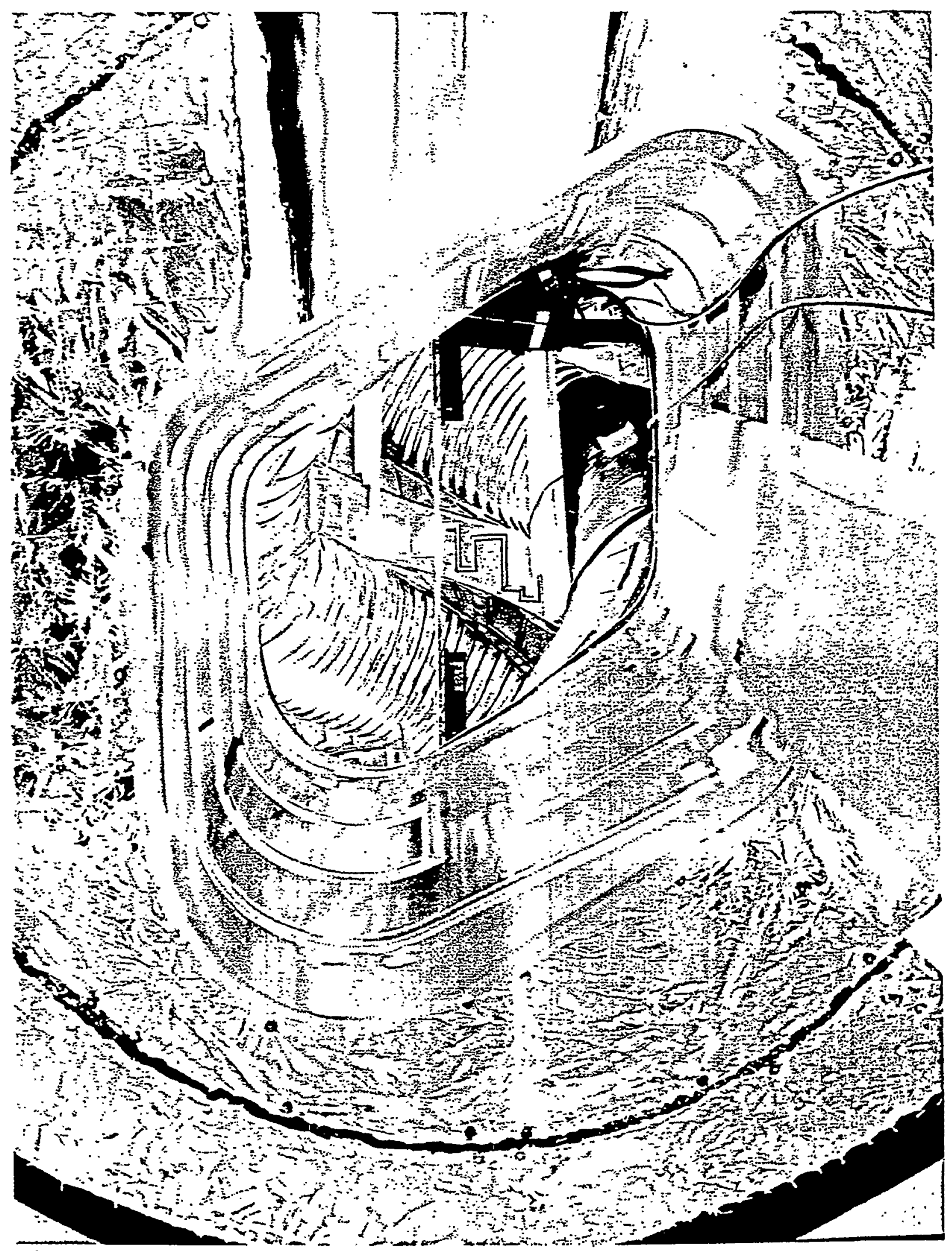




\section{First Wall Concepts}

First Wall

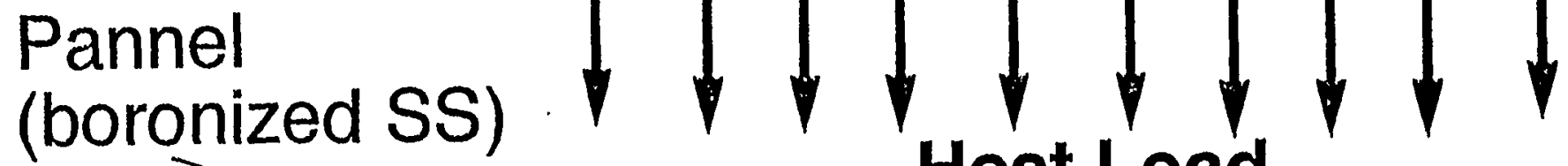

Plasma

Cooling Channel

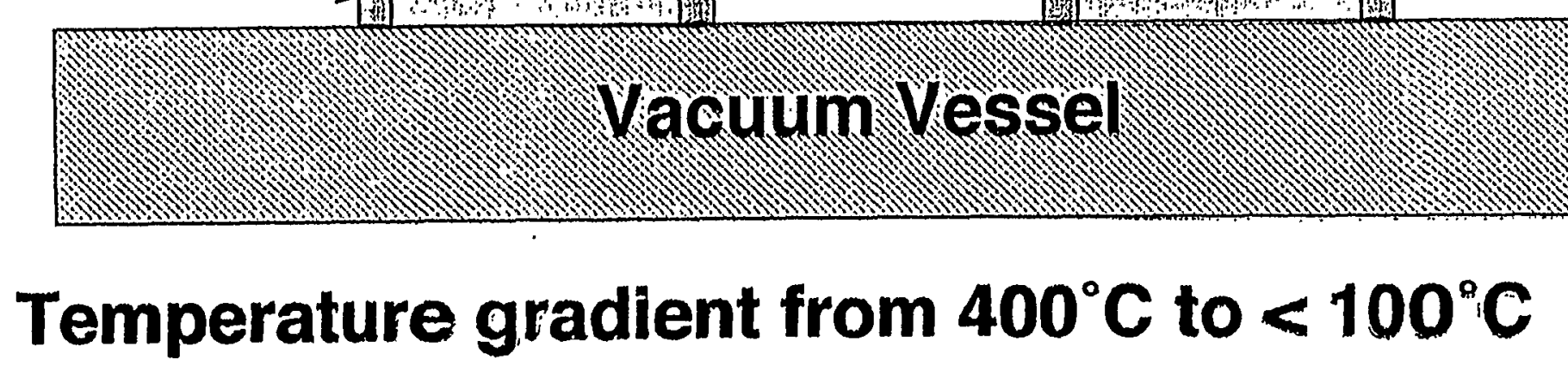




\section{Summary}

- Fabrication of the Divertor is going on

- Heat load limitation is determined by thermal deformation of $\mathrm{Cu}$ heat sink

- Heat load of $0.75 \mathrm{MW} / \mathrm{m}^{2}$ cleared by Mo

- Aux. coils for LID installed

- LID head design completed

- Vacuum vessel completed, cooling channels under welding process 


\title{
(1D) NS
}

\section{NSTX Status and PSI Issues}

\author{
Martin Peng \\ ORNL@PPPL
}

High Heat Flux Components \& Plasma Surface Interactions for Next Fusion Devices

$\tilde{8}$

December 8-11, 1997

San Francisco, California, USA 


\section{Spherical Torus Plasma Has Exciting Possibilities of High Performance in Fusion Science}
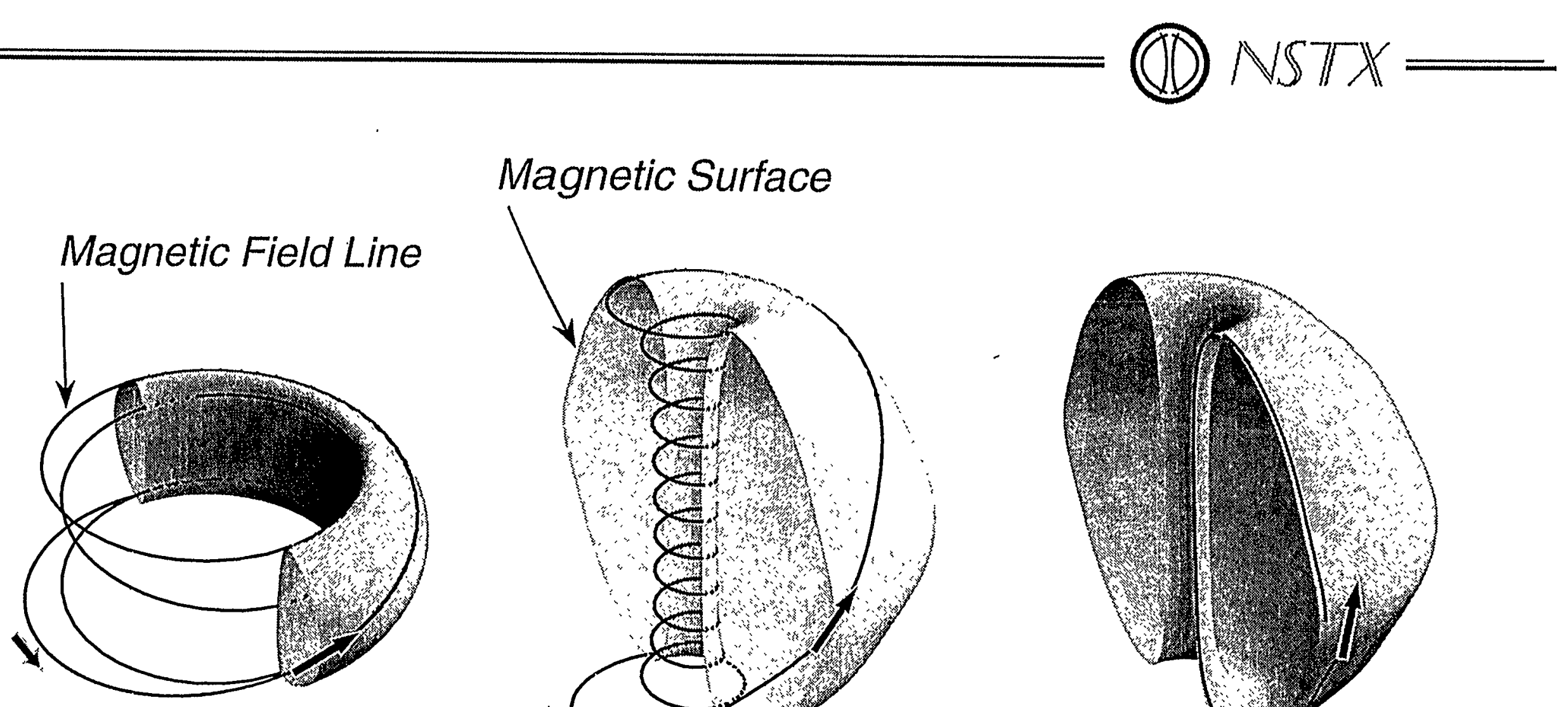

Magnetic Surface
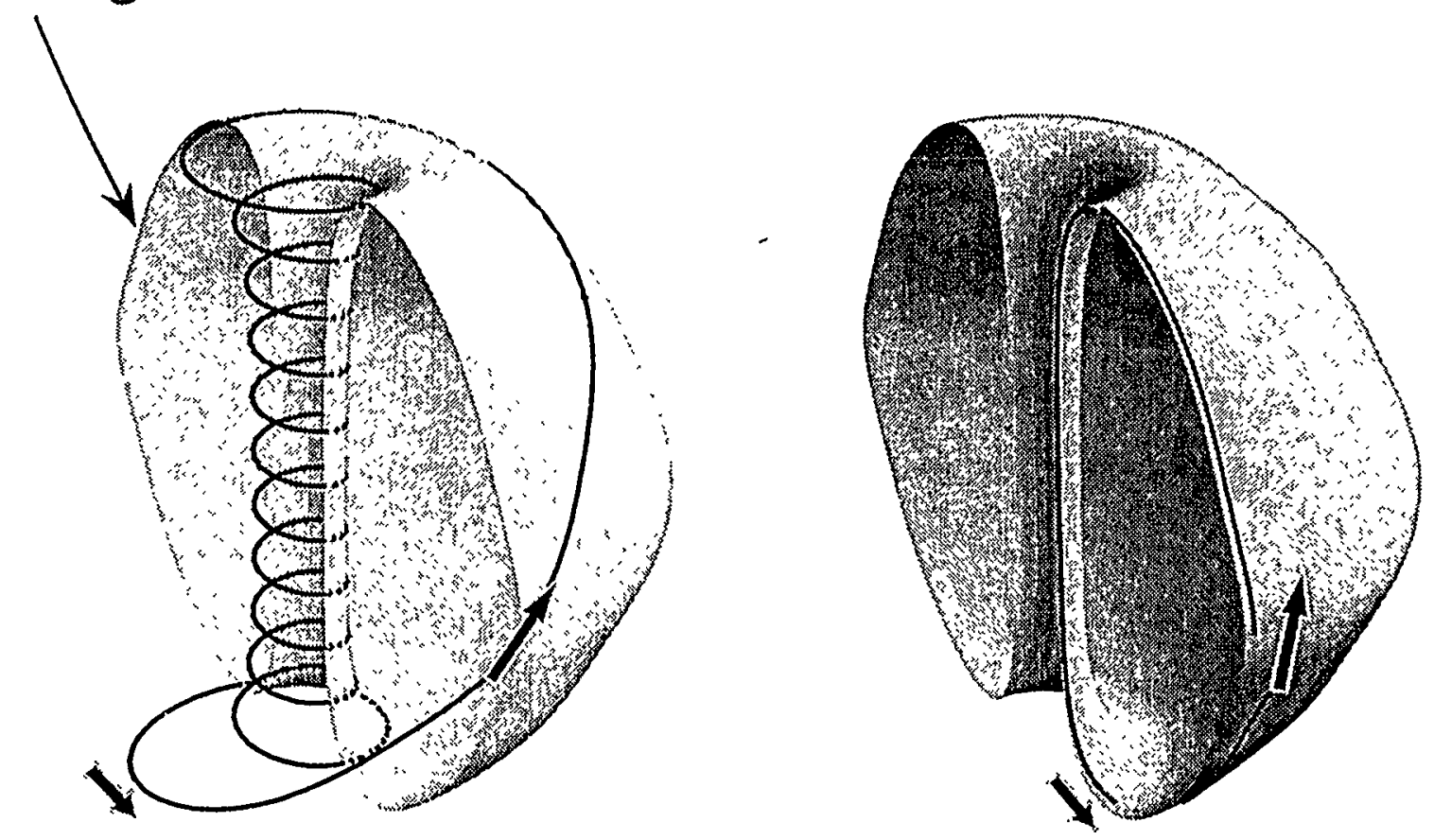

Tokamak Plasma

Spherical Torus Plasma (safety factor $q=12$ ) (safety factor $q=4$ ) 


\section{START Reached $\sim \mathbf{3 0} \%$ in Average Toroidal $\beta$ in Well-Confined NBI Plasma (Gates, Sykes et al.)}
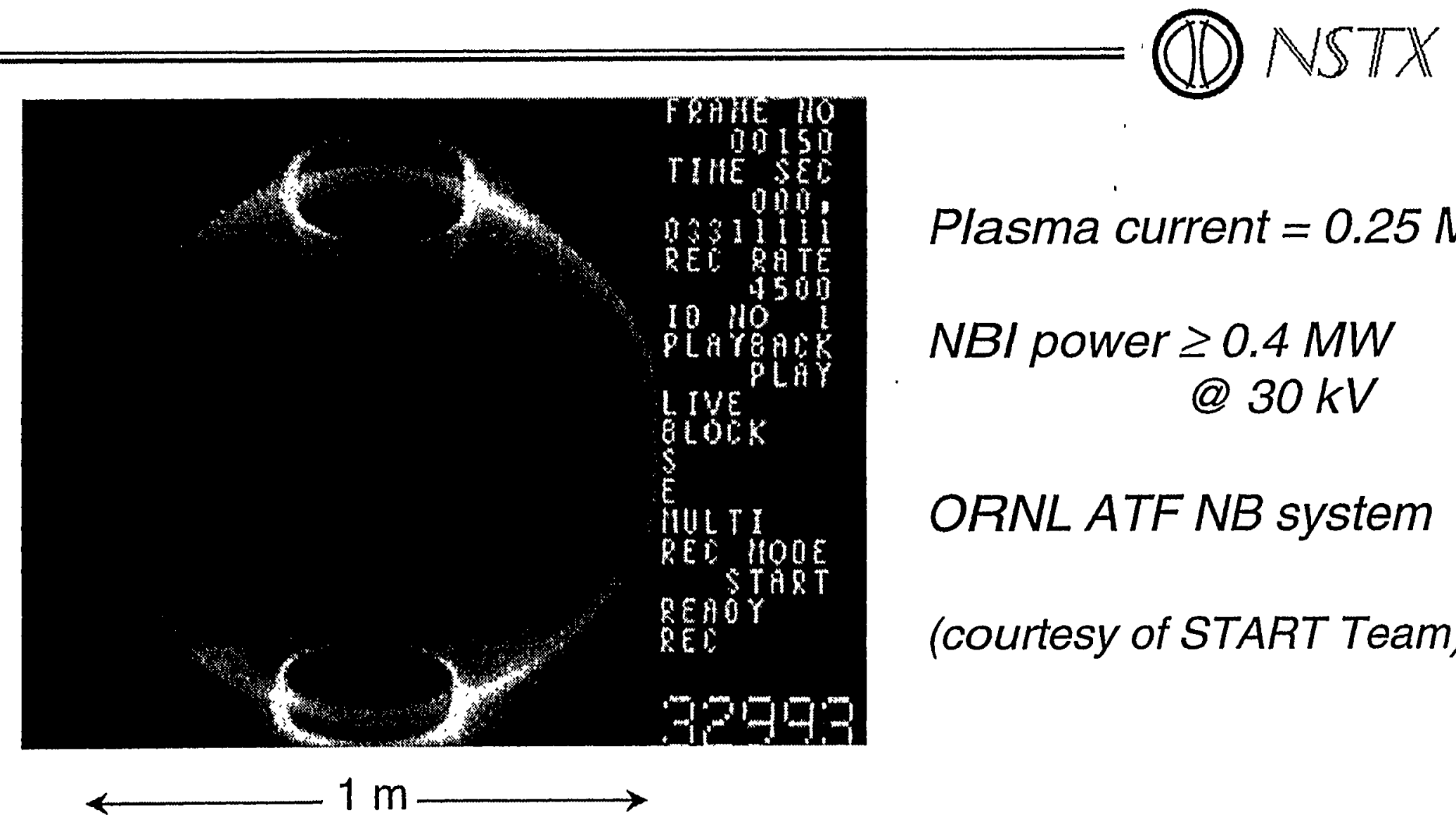

Plasma current $=0.25 \mathrm{MA}$

NBI power $\geq 0.4 \mathrm{MW}$

$@ 30 k V$

ORNL ATF NB system

(courtesy of START Team)

- Central $\beta>60 \%$ and average toroidal $\beta \sim 30 \%$

- Increased operating space and improved plasma

- Recent Upgrades: NBI power $1 \mathrm{MW}$; field duration $\sim 30 \mathrm{~ms}$

- MAST will upgrade the NBI systems from ORNL 


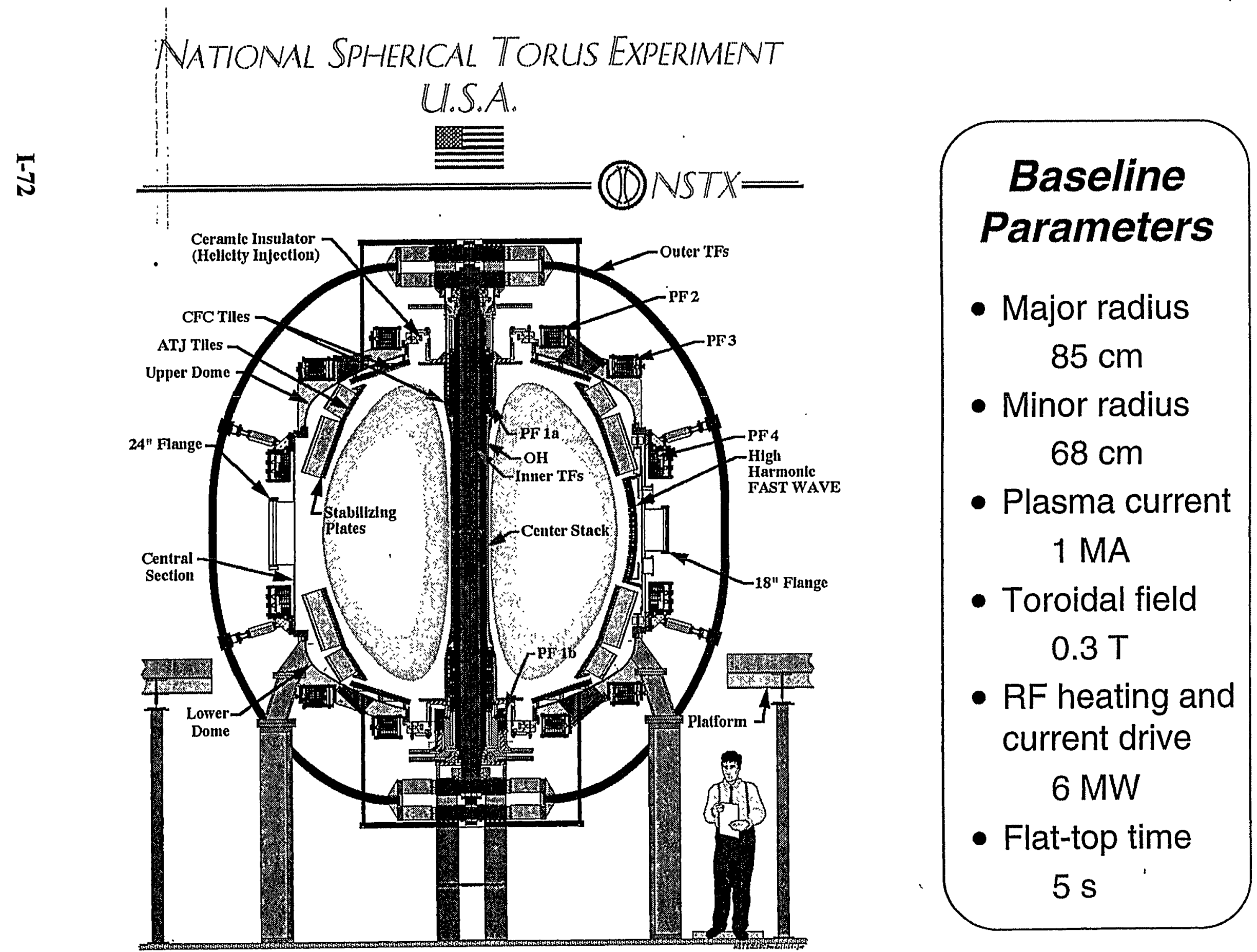




\section{The Main Mission of NSTX is to Prove the Physics Principles for Attractive VNS and Power Plant}
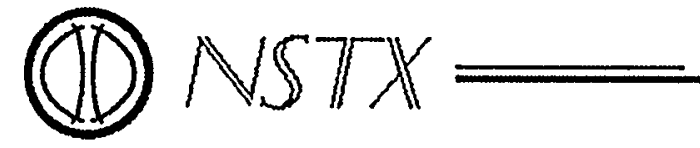

- High beta, high confinement, high bootstrap current fraction simultaneously and near steady state

- Noninductive current startup and maintenance to eliminate Ohmic solenoid and minimize size for next steps

- Feasible power and particle handling to permit practical plasma facing components

\section{Related Research Opportunities}

- Further improvements in configuration: R/a $\rightarrow 1$, bridging to CTs (spheromak, FRC, spherical RFP, etc.)

- Excellence in plasma and fusion science 
NATIONAIL SPHIERICAIL TORUIS EXPERIMIENT U.S.A.

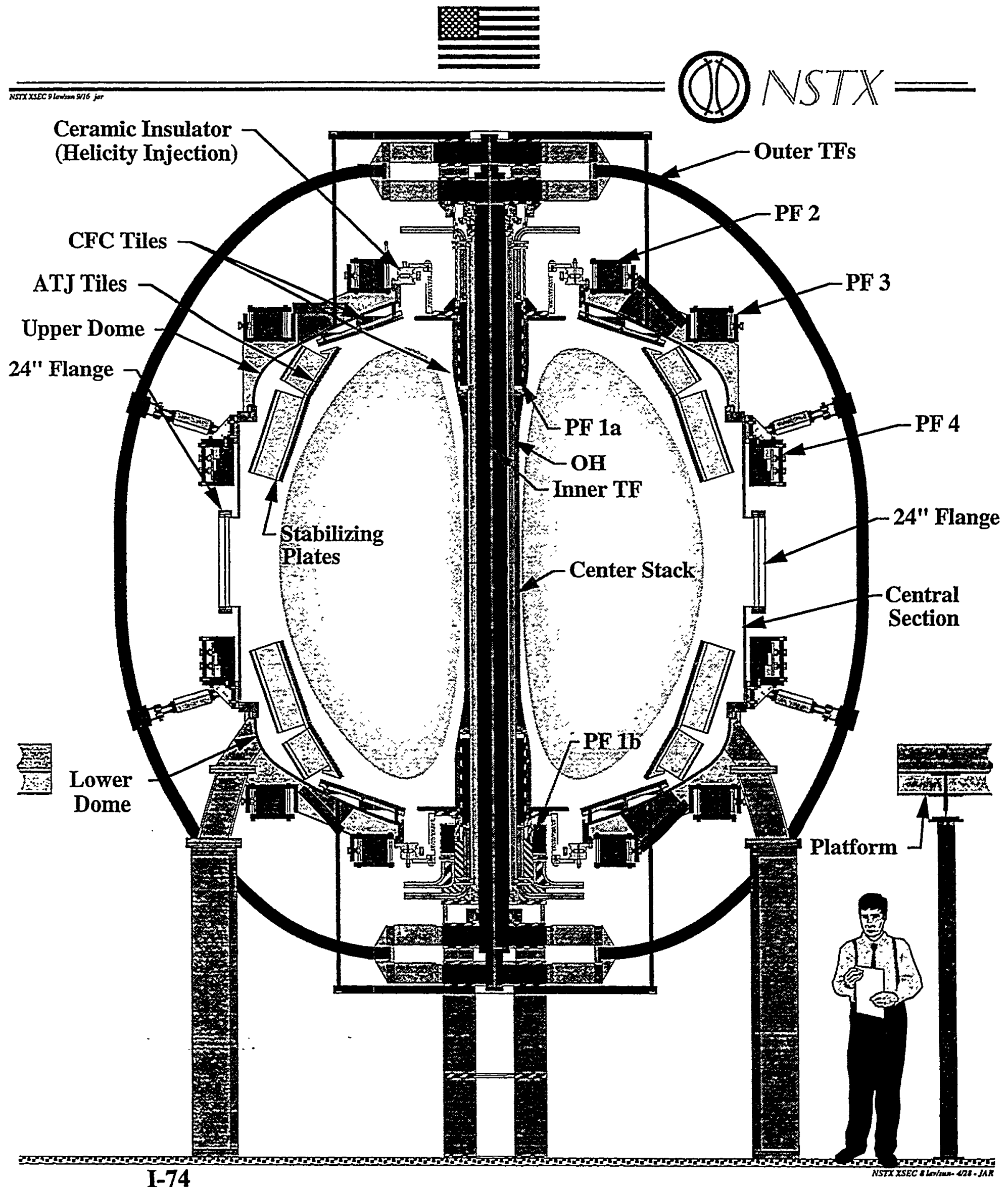


$\mathbb{N} \mathbb{S} \mathbb{X} \mathbb{X} \mathbb{E} \mathbb{S} T \mathbb{C E L L}$

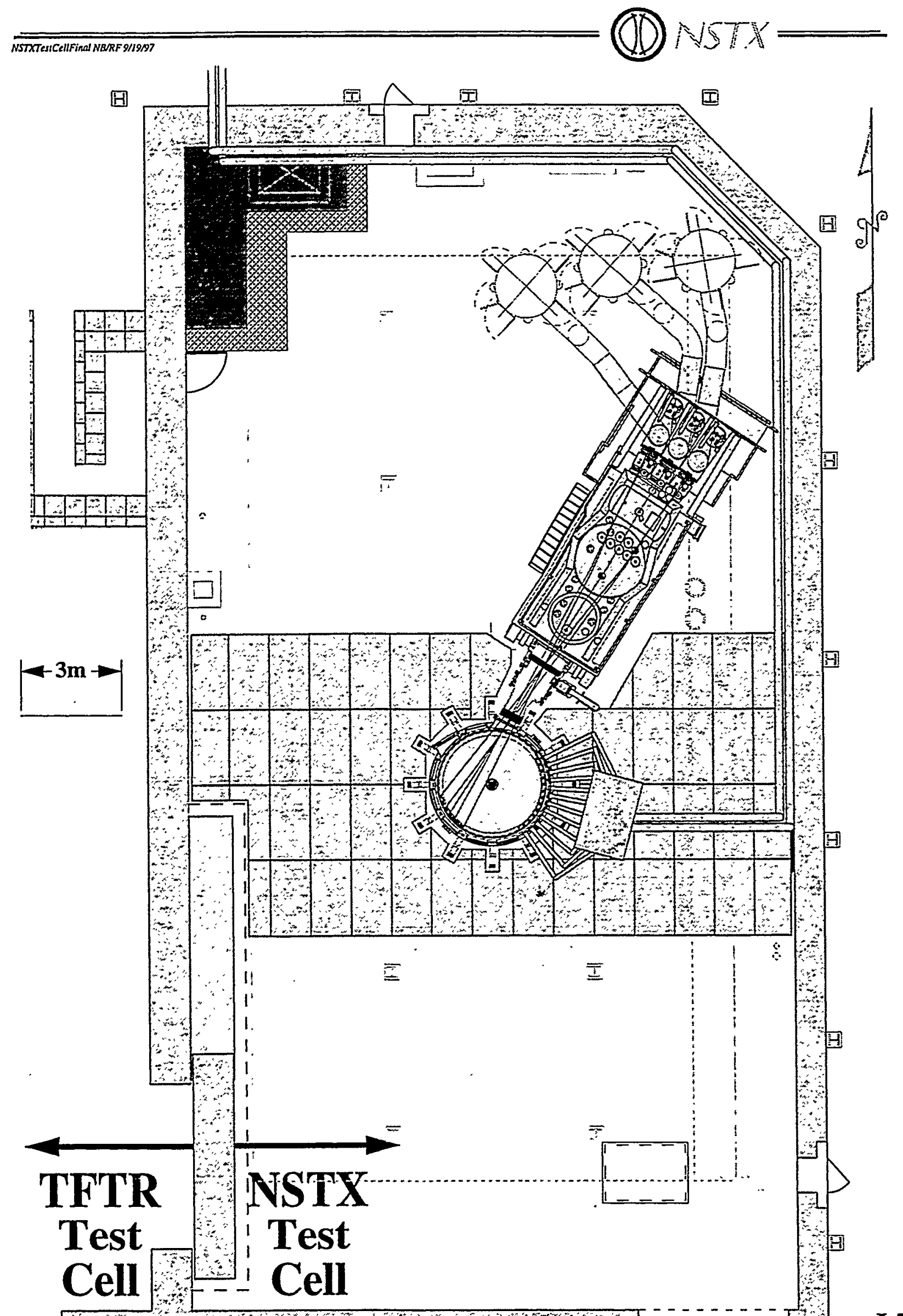



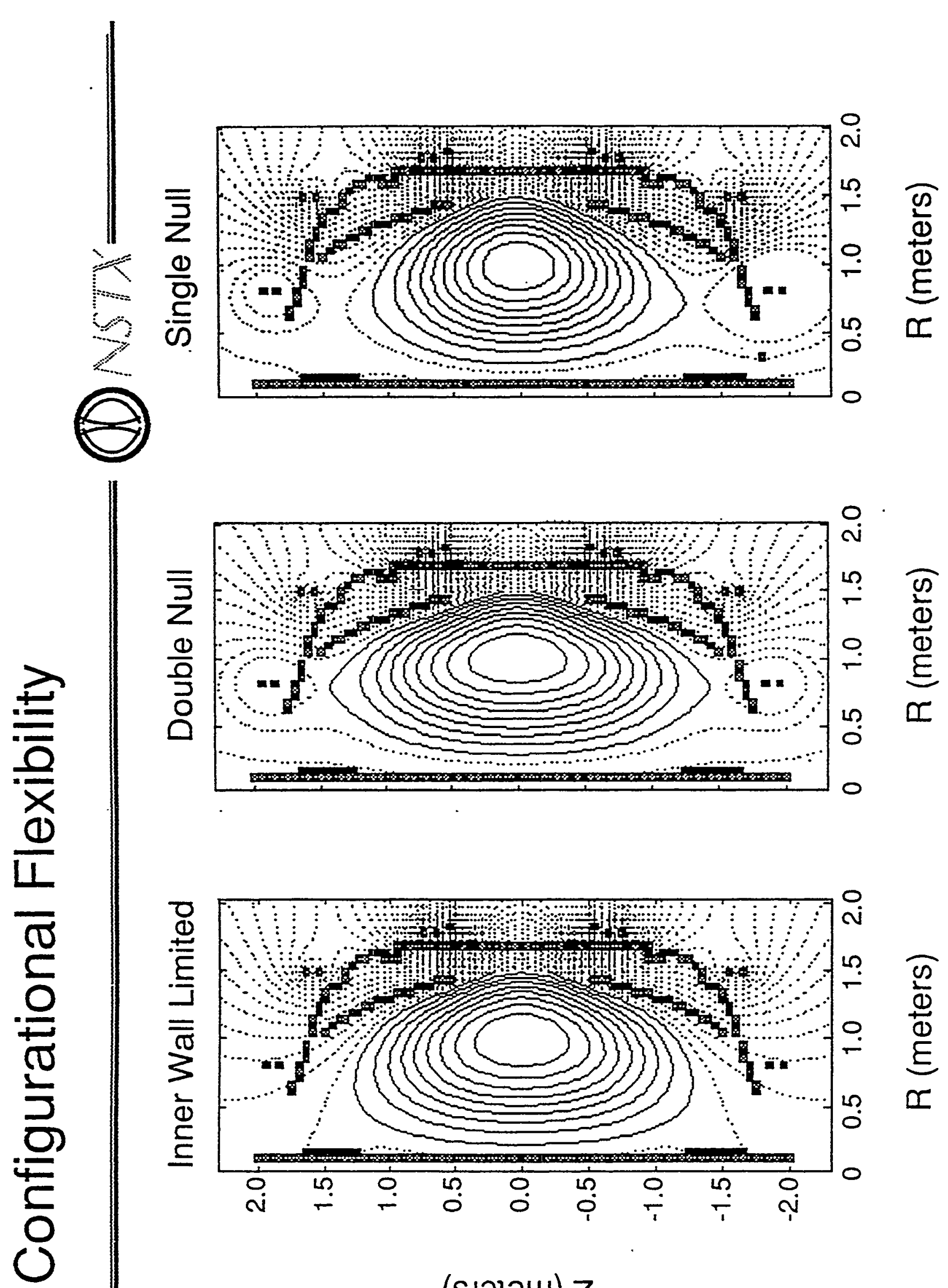


\section{$\kappa=3$ Equilibrium \\ $(\mathrm{R} / \mathrm{a}=0.7 / 0.5 \mathrm{~m})$}

$$
\begin{aligned}
& \text { PF1a }=0.75 \mathrm{kA} / \text { turn } \\
& \text { PF2 }=8.2 \\
& \text { PF3 }=-11.9 \\
& \text { PF4 }=-6.5
\end{aligned}
$$
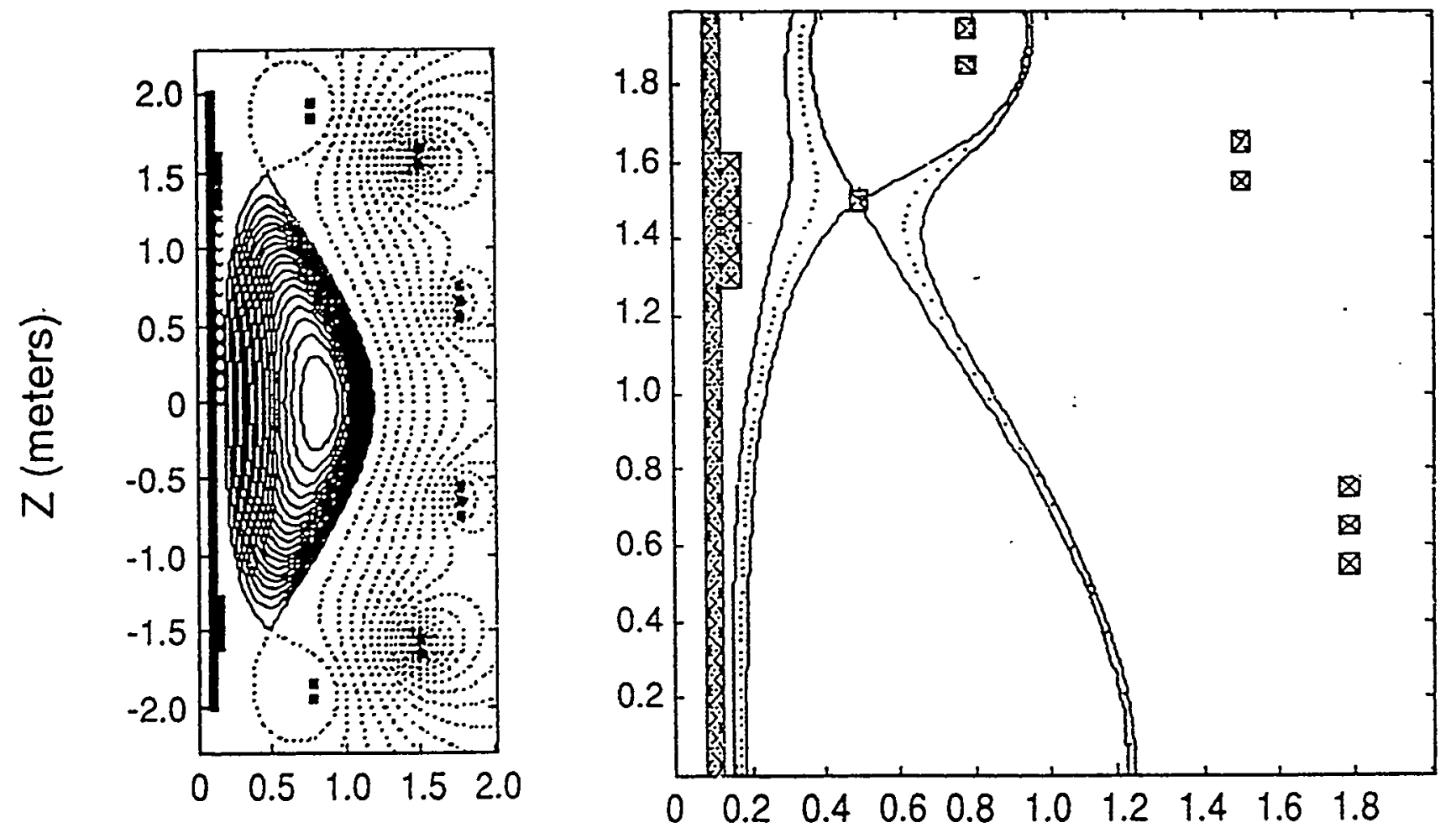

$R$ (meters)

- Ideally unstable to $n=0$ axisymmetric mode with plates in original position

- Relocation of passive plates farther inboard required for maximizing MHD stability 


\section{2-D CALCULATONS SHOW \\ DIV

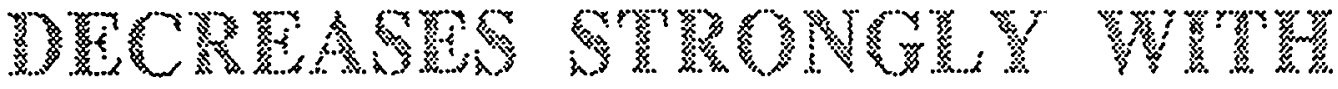 \\ DIVTRTOR PADIATON}

- Peak heat flux decreases linearly with divertor radiation until plasma begins to detach from divertor (radiation $60 \%$ )

- Peak heat flux dominated by convection for radiation fraction > $65 \%$

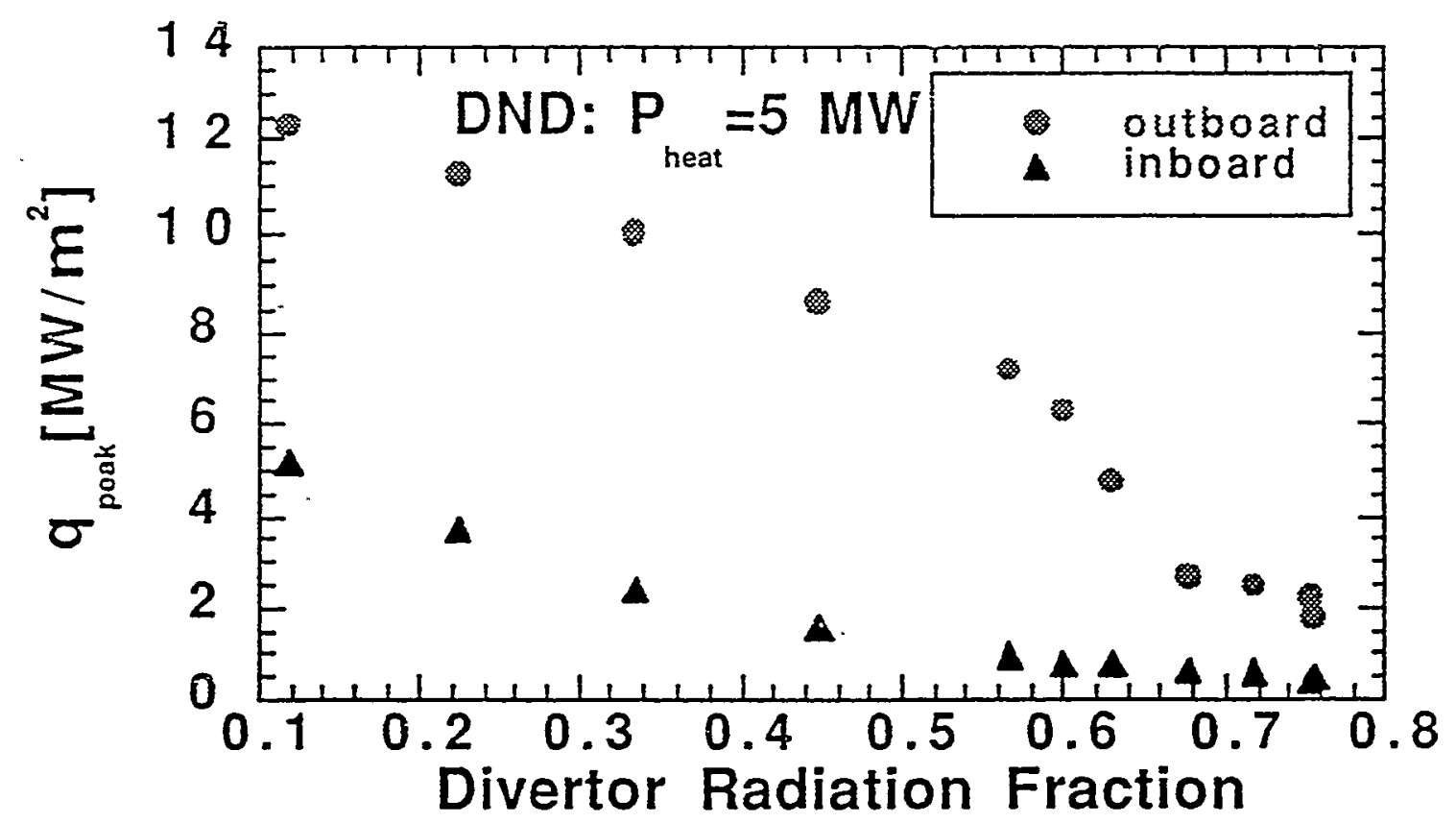

- Divertor radiation increased with multiplier to hydrogenic radiation to simulate impurities 


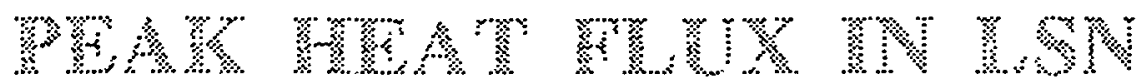

COMWMIM

W

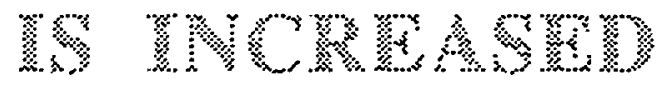

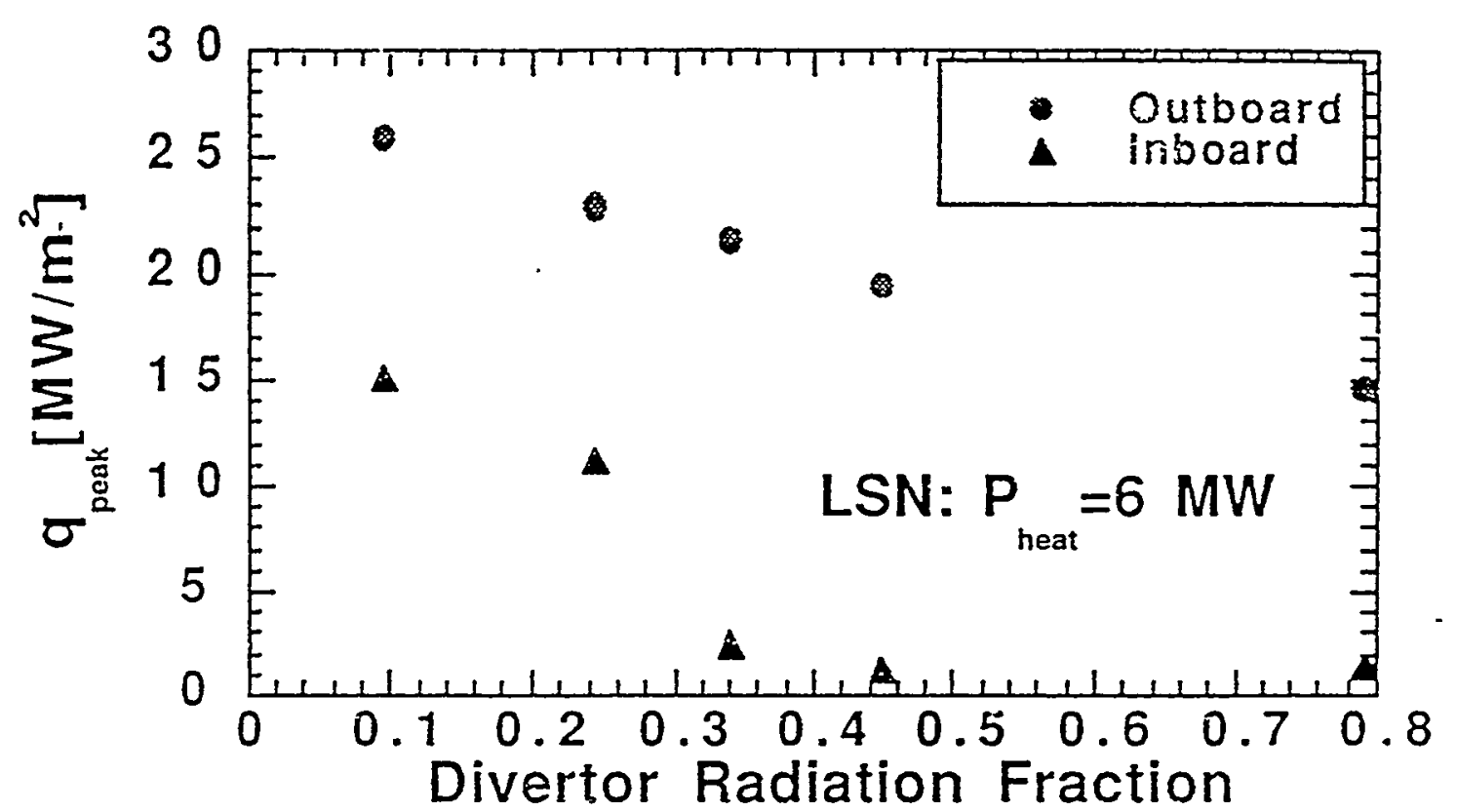

- Most of the increase in divertor radiated power fraction occurs on the inboard leg, driving the inboard heat flux down quickly

- This phenomenon is caused by the in/out geometric size difference in ST's: the inner SOL cross-sectional area and is $1 / 2-1 / 3$ of the outer SOL area, leading to a similar in/out power split 


\section{SOL for Naturally Diverted (ND) Plasma in NSTX Exhibits Unique Magnetic Geometry}

(1) $\mathbb{N} \mathbb{S T X}=$
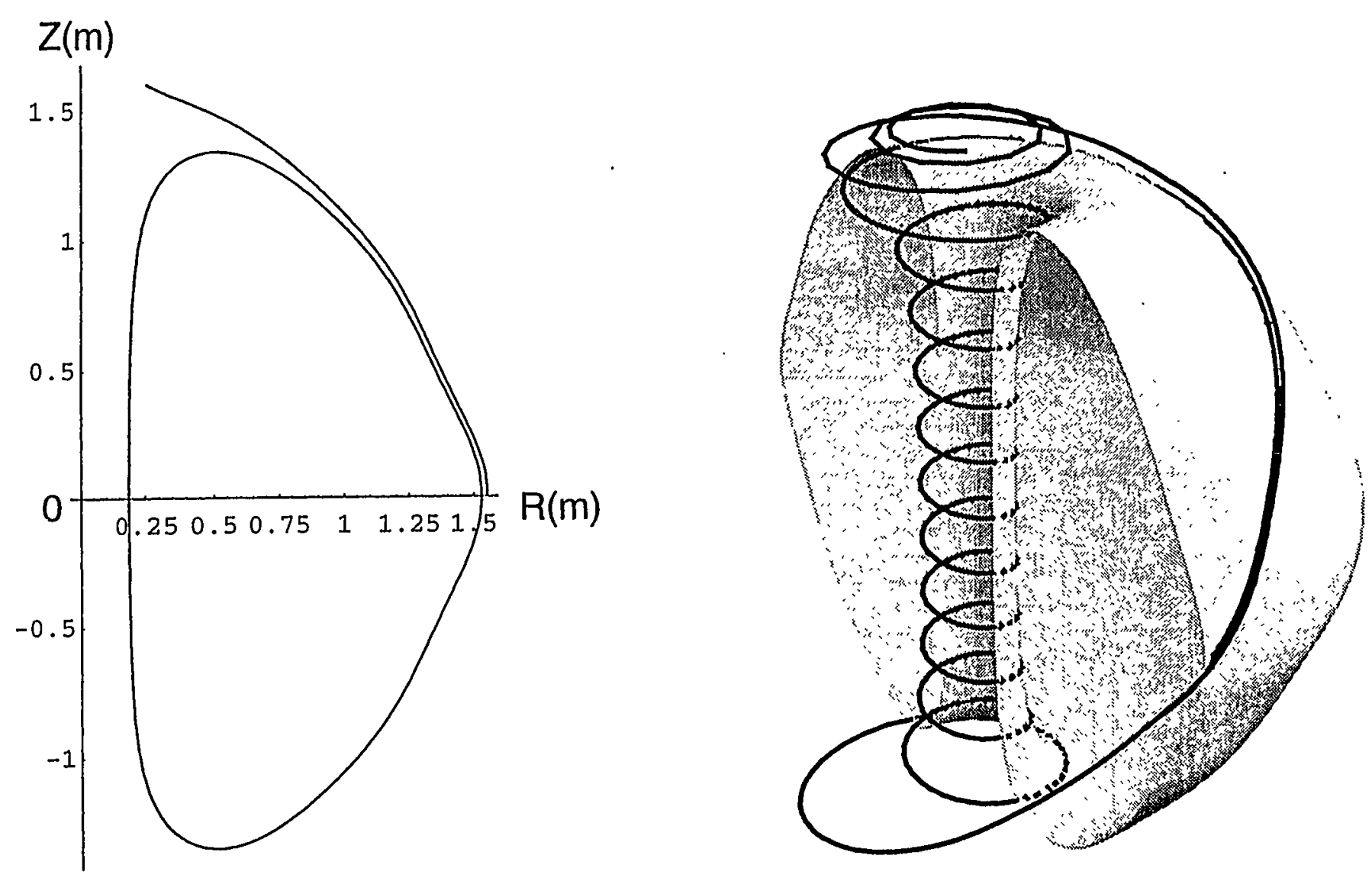

ND Plasma, $A=1.26, \kappa=2.0, \delta=0.52, q_{a}=13+, \beta_{t}=25 \%$ 


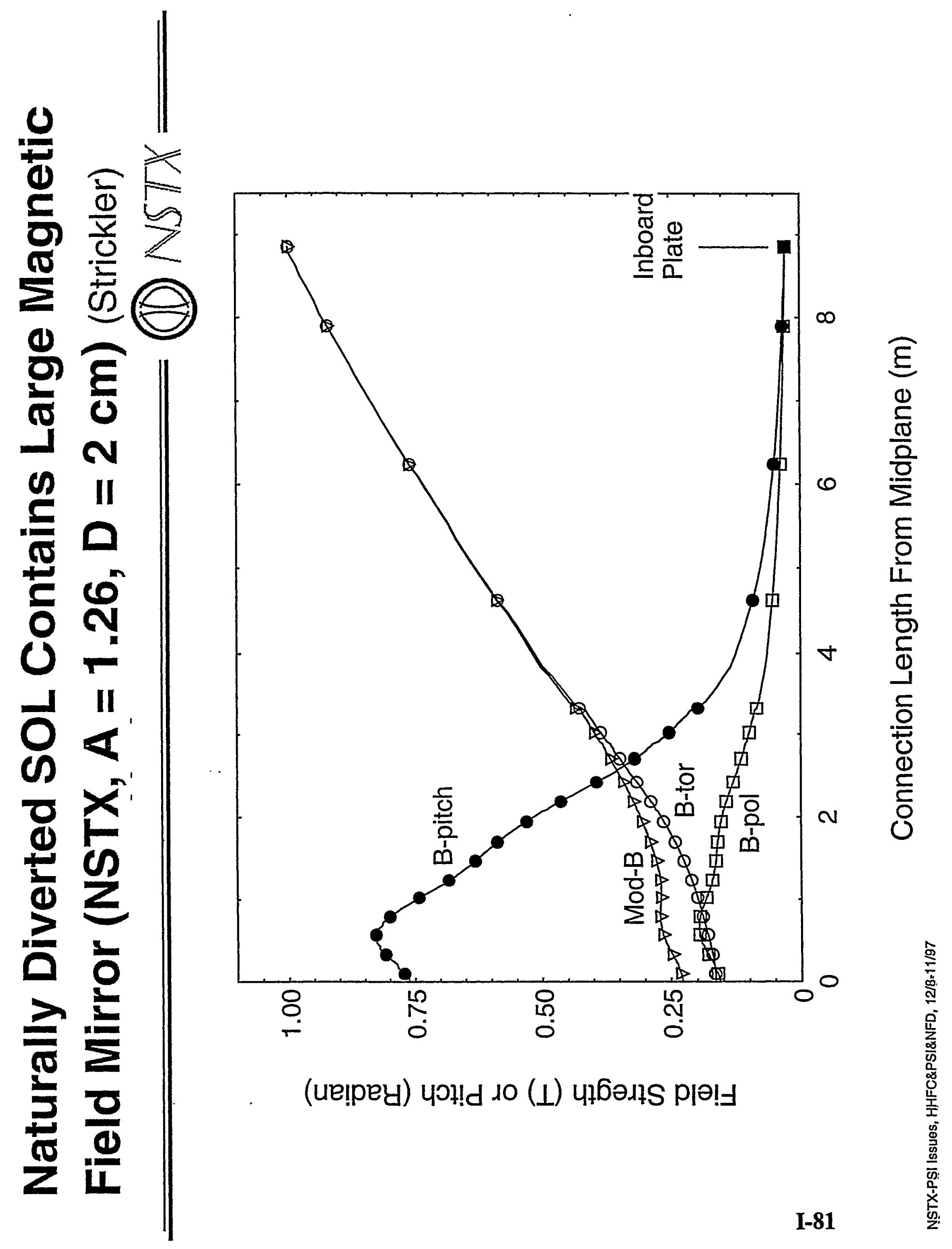




\section{Natural Divertor Increases SOL Flux Tube} Expansion and Connection Length (Strickler)

$$
(A=1.26, \kappa=2.0, \delta=0.52, q=13+)
$$

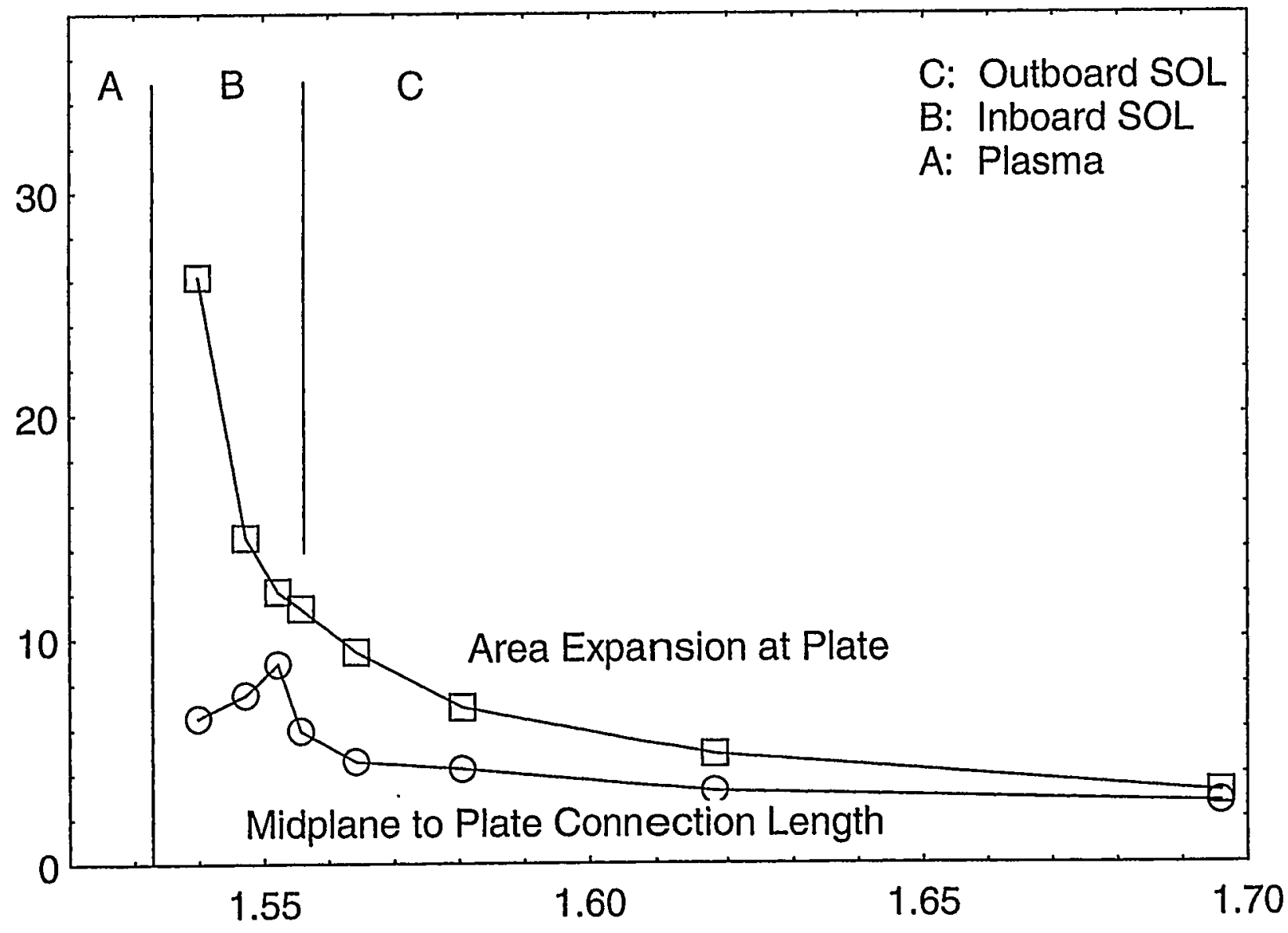

Major Radius at Midplane (m) 


\section{Requirements/Design Criteria - Thermal loads}

- Normal Operation, peak surface flux

- Center stack $200 \mathrm{~W} / \mathrm{cm}^{\wedge} 2$ peak

- Inboard divertor

$\sim 700 \mathrm{~W} / \mathrm{cm}^{\wedge} 2$ for single null

- OB divertor

$\sim 1100 \mathrm{~W} / \mathrm{cm}^{\wedge} 2$ for $\lambda=3 \mathrm{~cm}$

$\sim 1700 \mathrm{~W} / \mathrm{cm}^{\wedge} 2$ for $\lambda=1.5 \mathrm{~cm}$

(peak from calc $=1400 \mathrm{~W} / \mathrm{cm}^{\wedge} 2$ )

- Passive plates $\sim 10 \mathrm{~W} / \mathrm{cm}^{\wedge} 2$

- Disruption

- Peak surface flux: TBD

- NBI shine (upgrade only)

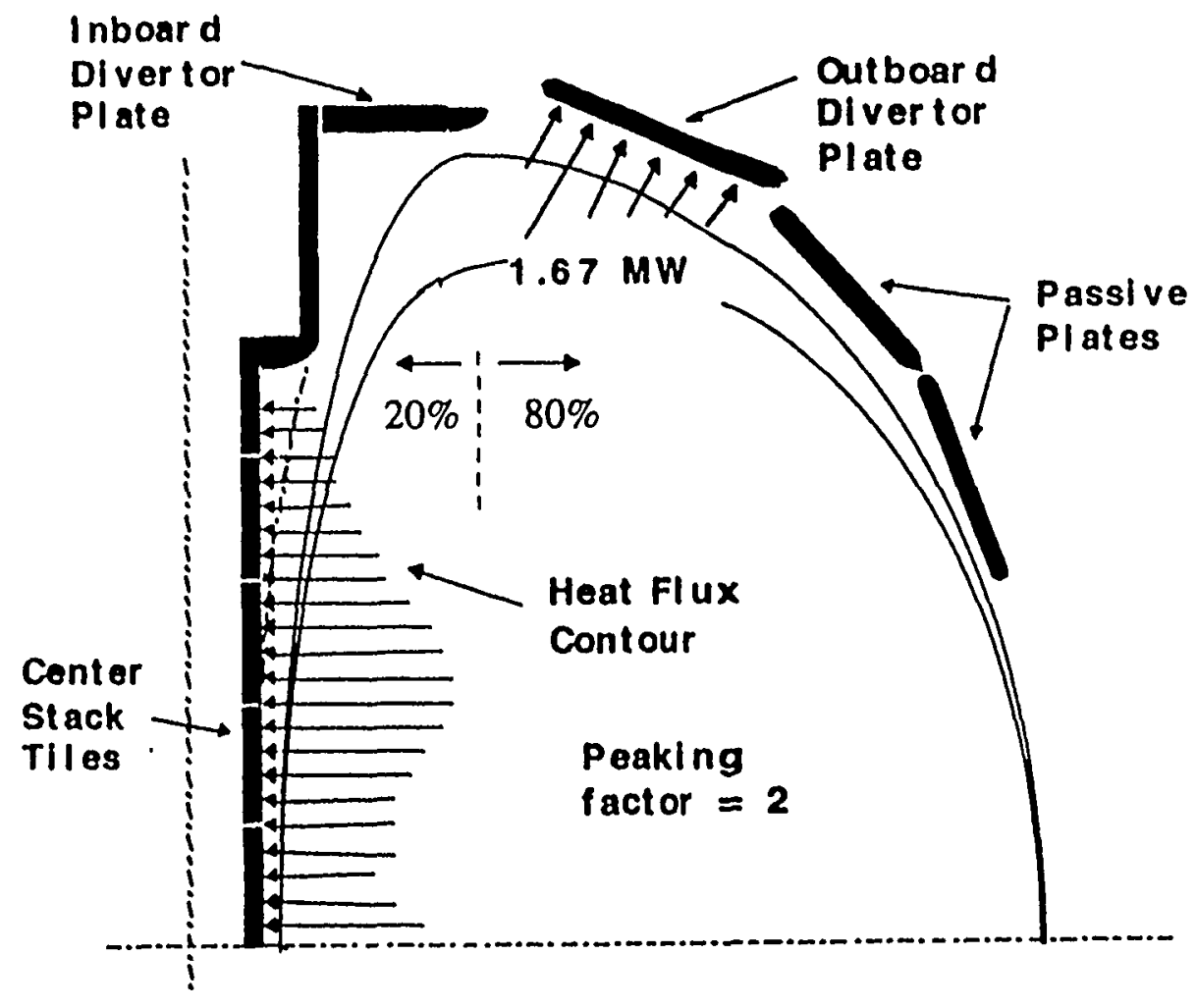

do

- Peak surface flux: TBD 


\section{¿outboard Divertor Plate Cooling/thermal analysis}

- Copper plate is cooled at 50 to $100 \mathrm{C}$ and baked out to $350 \mathrm{C}$ by Dowtherm

- Analysis shows feasibility for divertor tile operating at about $1500 \mathrm{~W} / \mathrm{cm}^{\wedge} 2(\lambda-2 \mathrm{~cm})$

- Divertor tile can be returned to $150 \mathrm{C}$ after pulse in 3 minutes

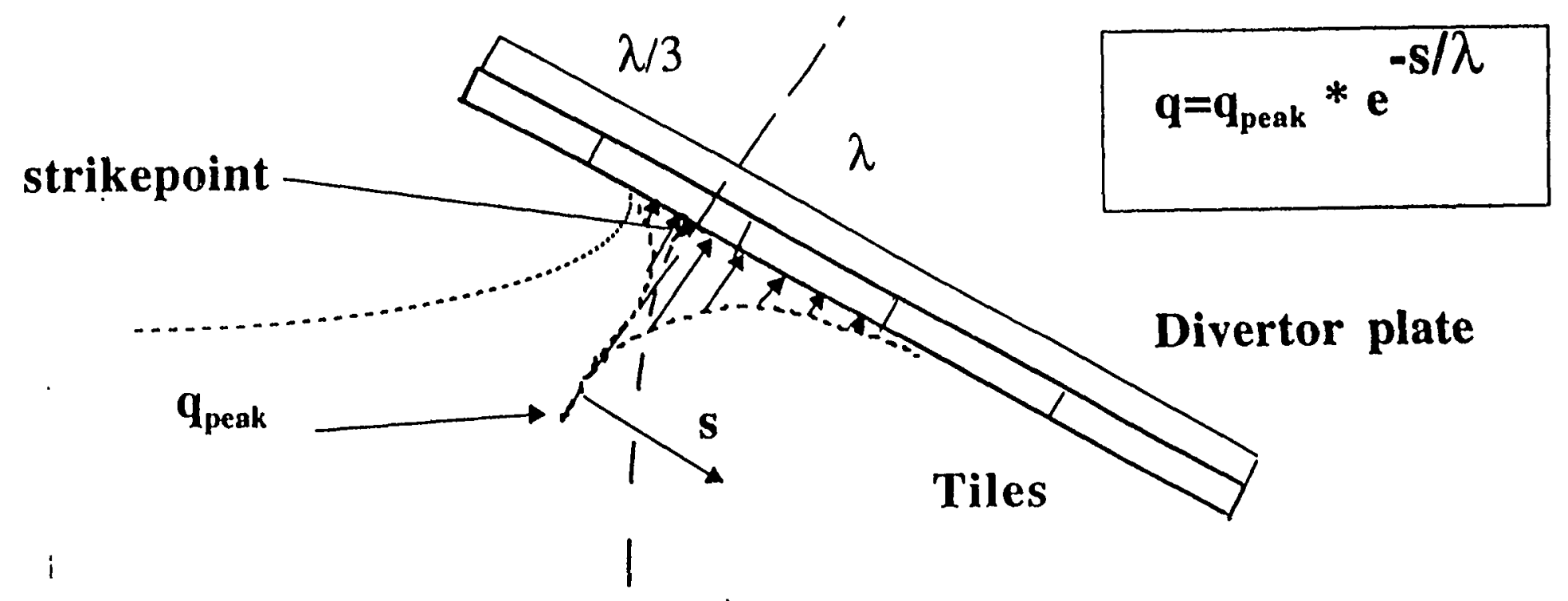




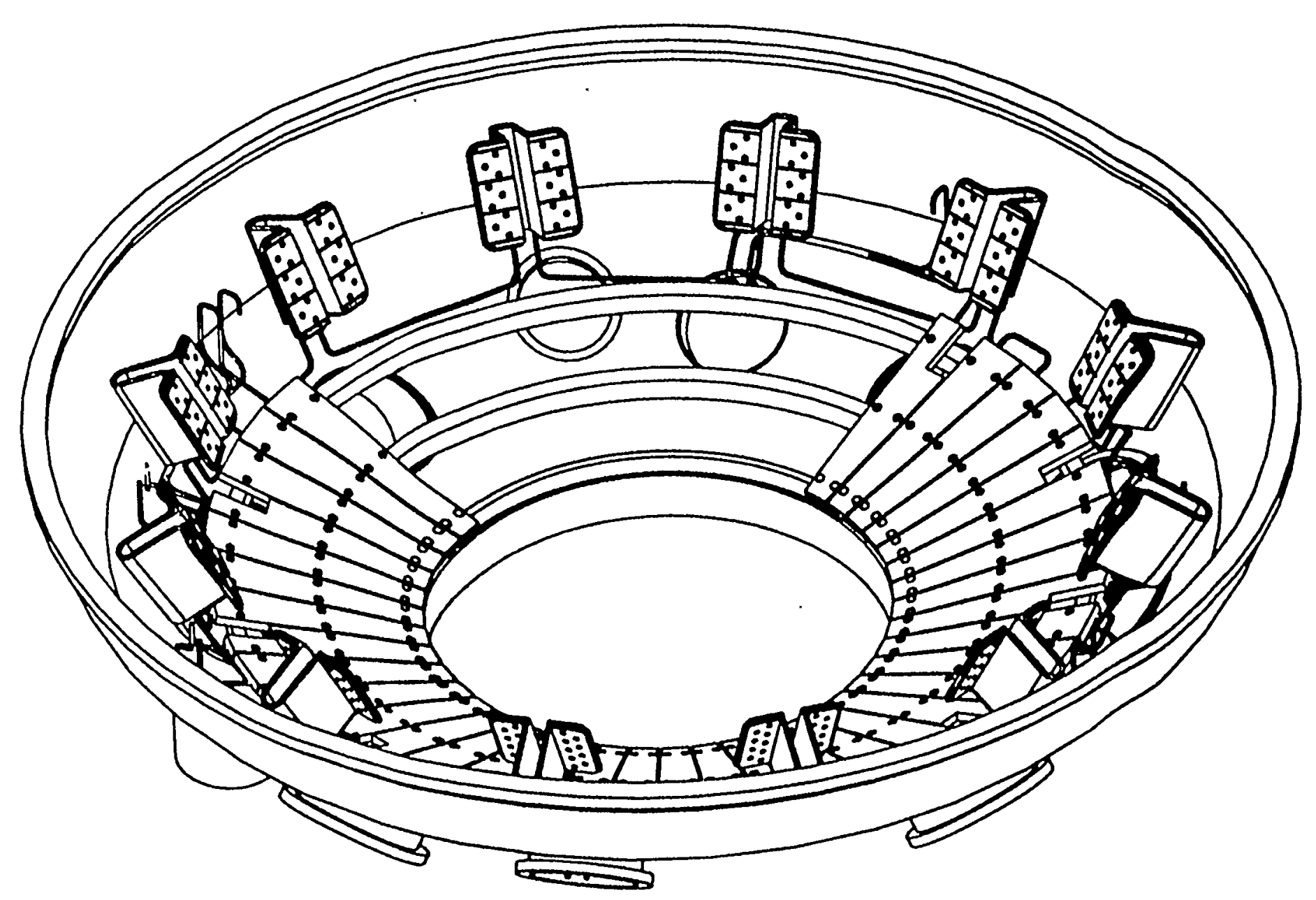

SIMPLFD REP: SHELL sid 


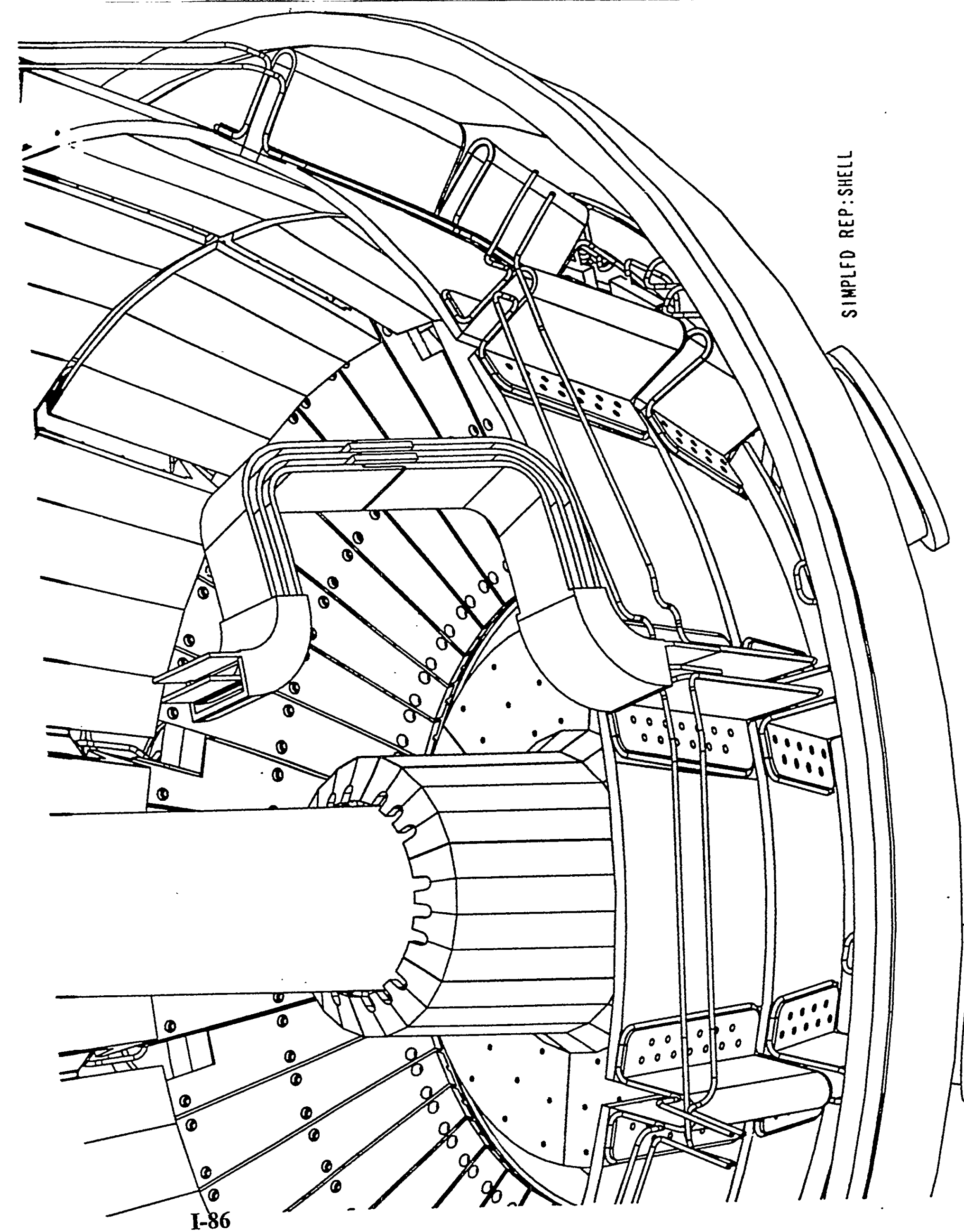




\section{(10) $N S T X=$}

\section{Center Stack Tile Design - Vertical Bolted Rail}

TZM rails with carbon covers clamp vertical rows of tiles

- Robust, all metal retainers

- Graphite tiles, FMI-4D carbon-carbon covers

- Good thermal expansion capability

- Fair, controlled thermal isolation

- Multiple fasteners per tile

- Relatively simple parts

$\stackrel{1}{1}$

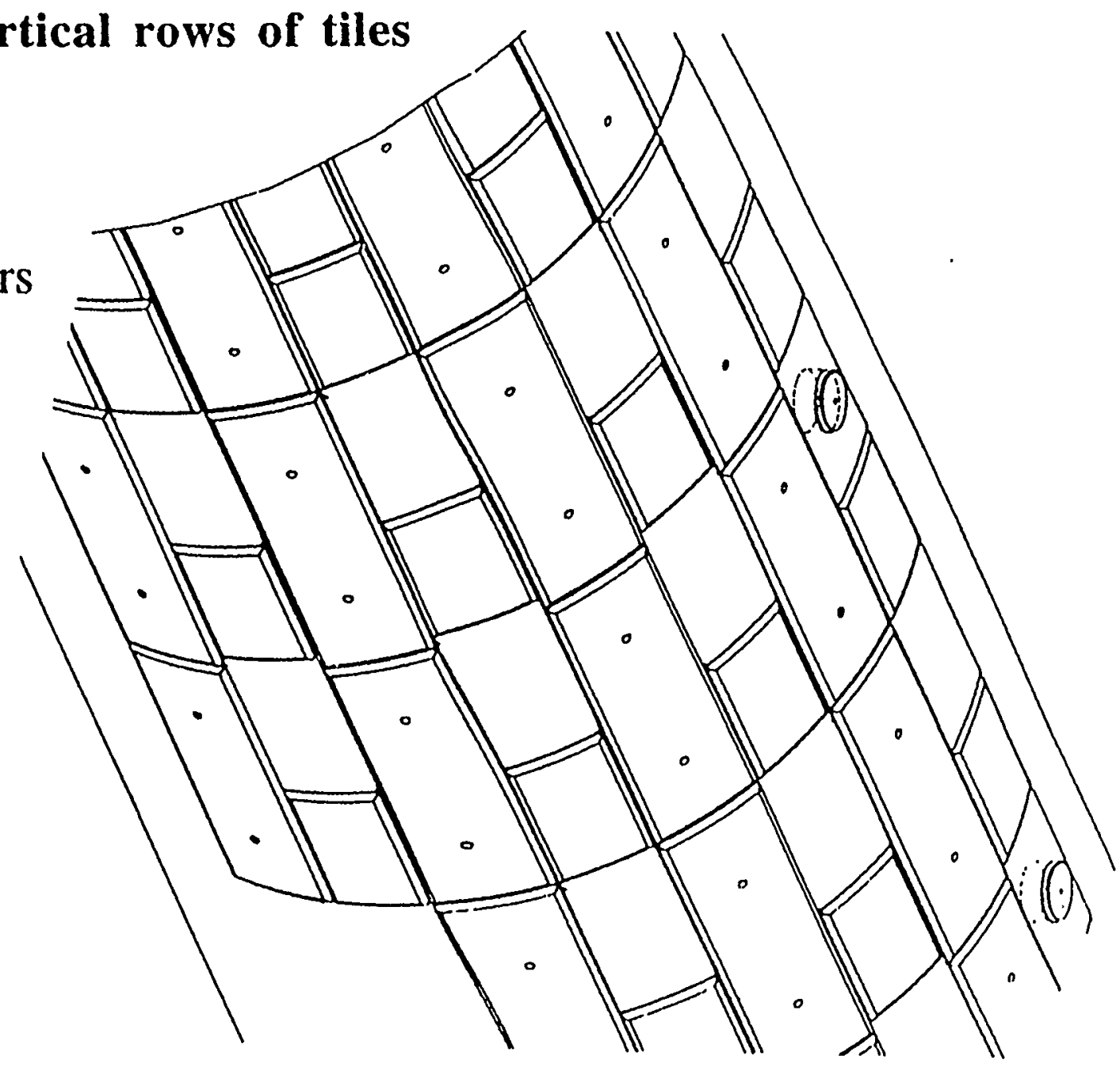




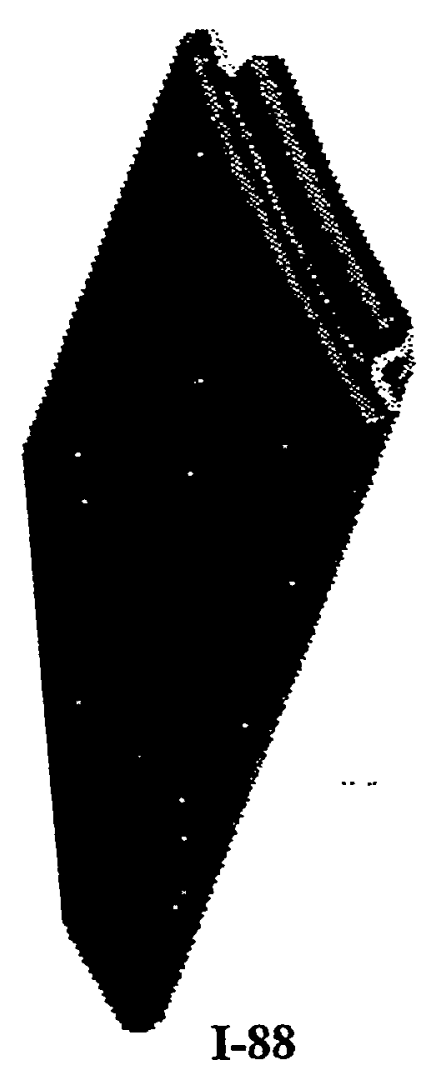




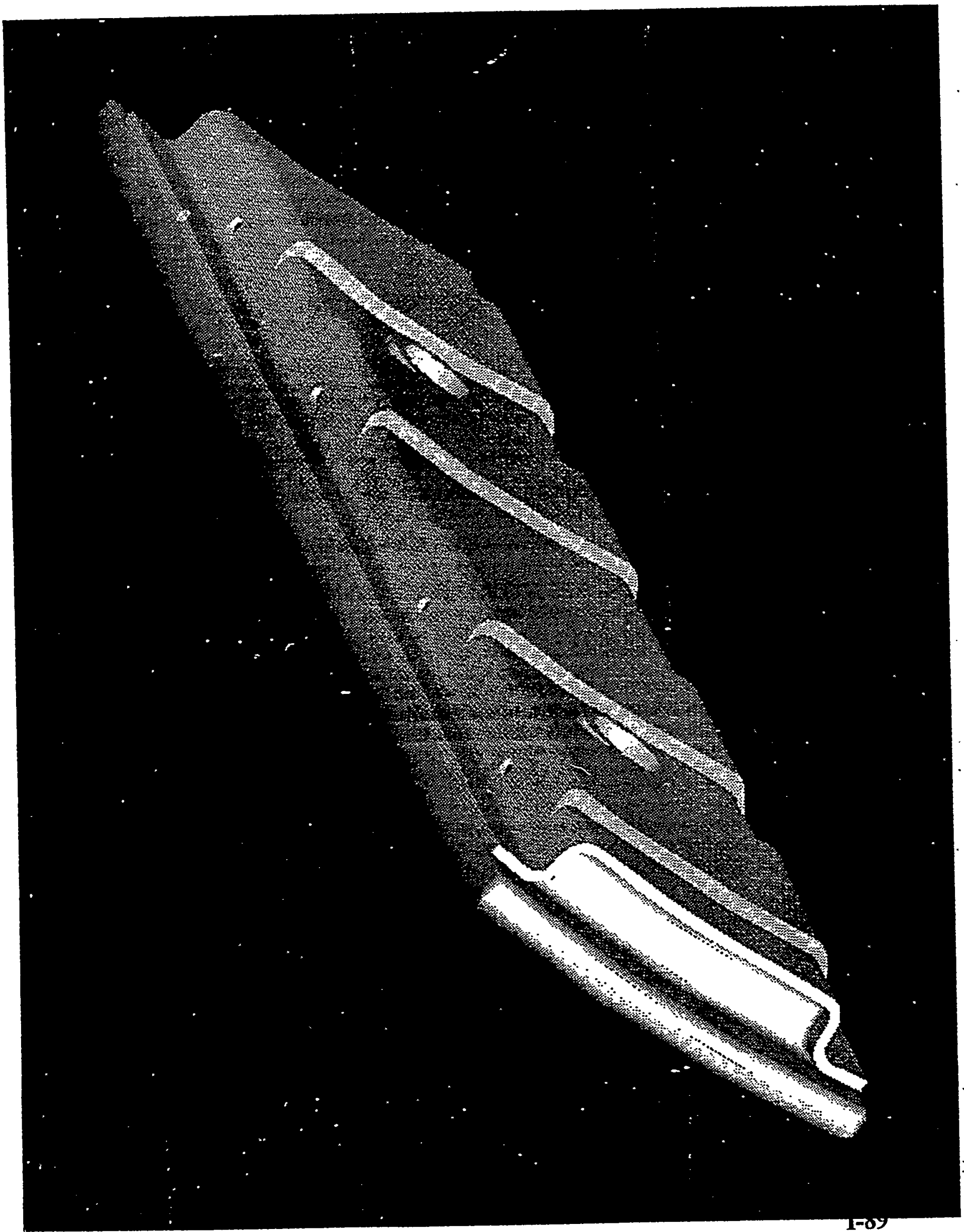




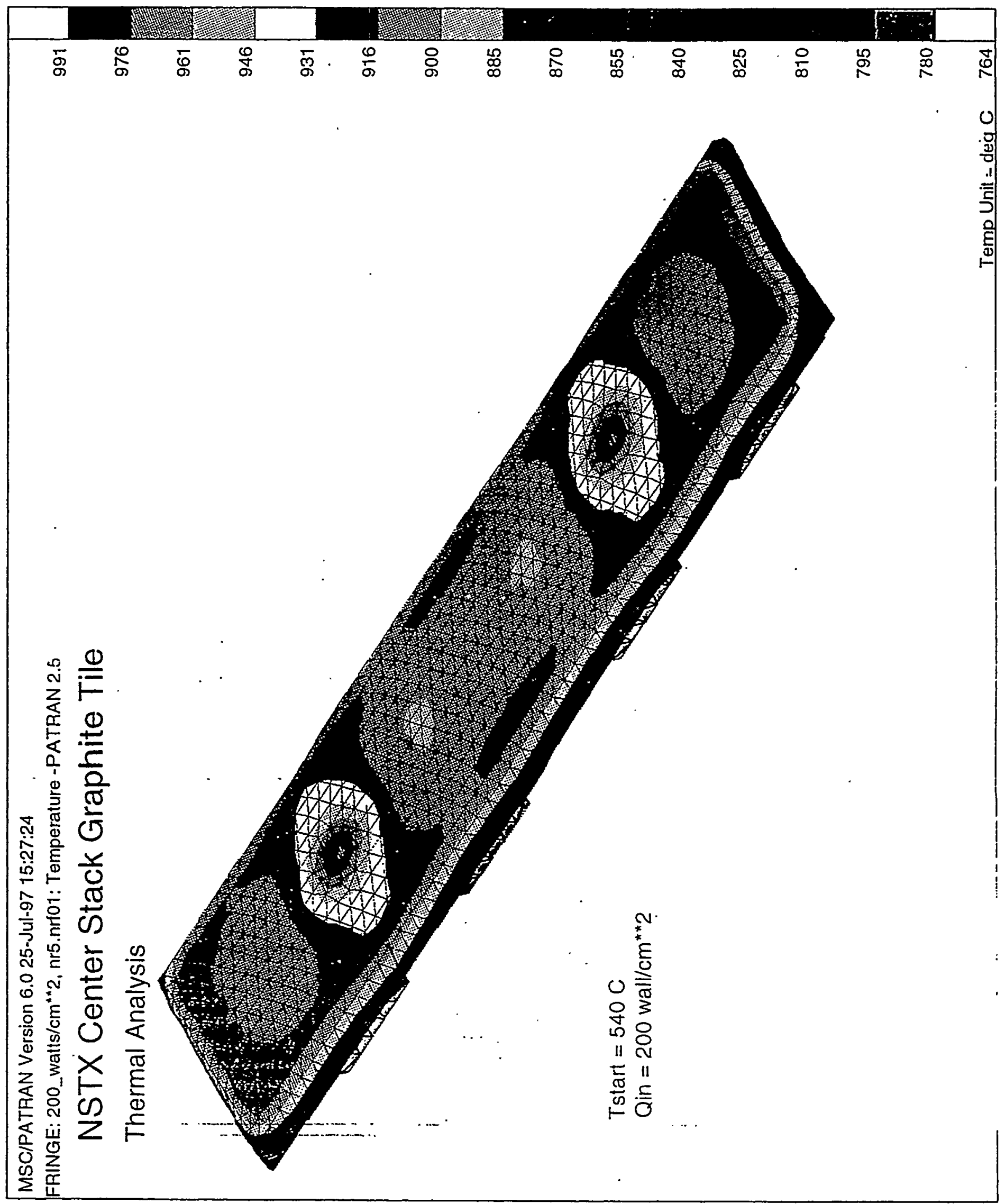




\section{NSTX Research Will Cover a Wide Range of Topics and Provide Ample Opportunities for Collaboration}

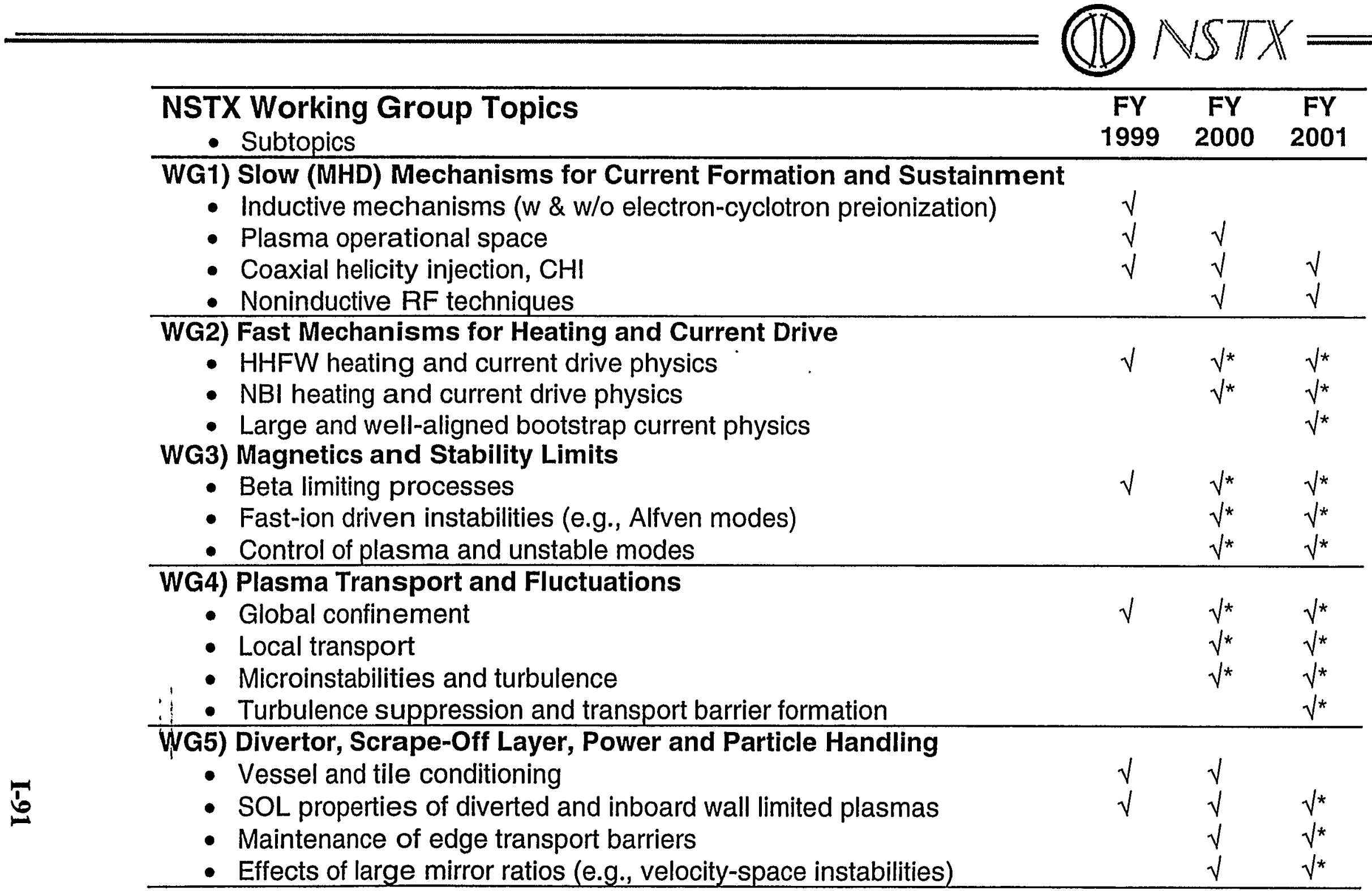




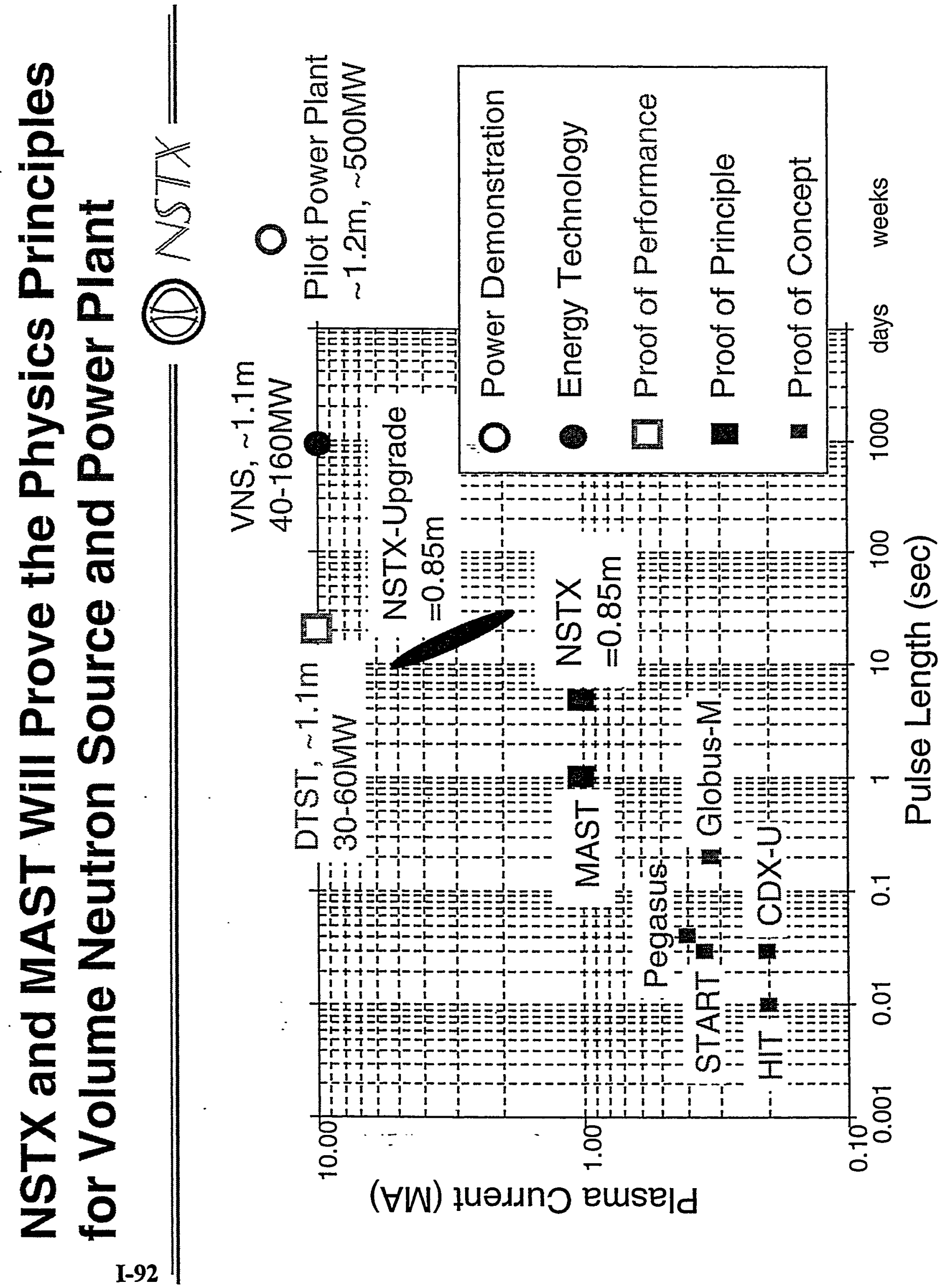




\section{ST Development Pathway Provides a Good Example of Potential Benefits from Innovation}

(1)Pathway

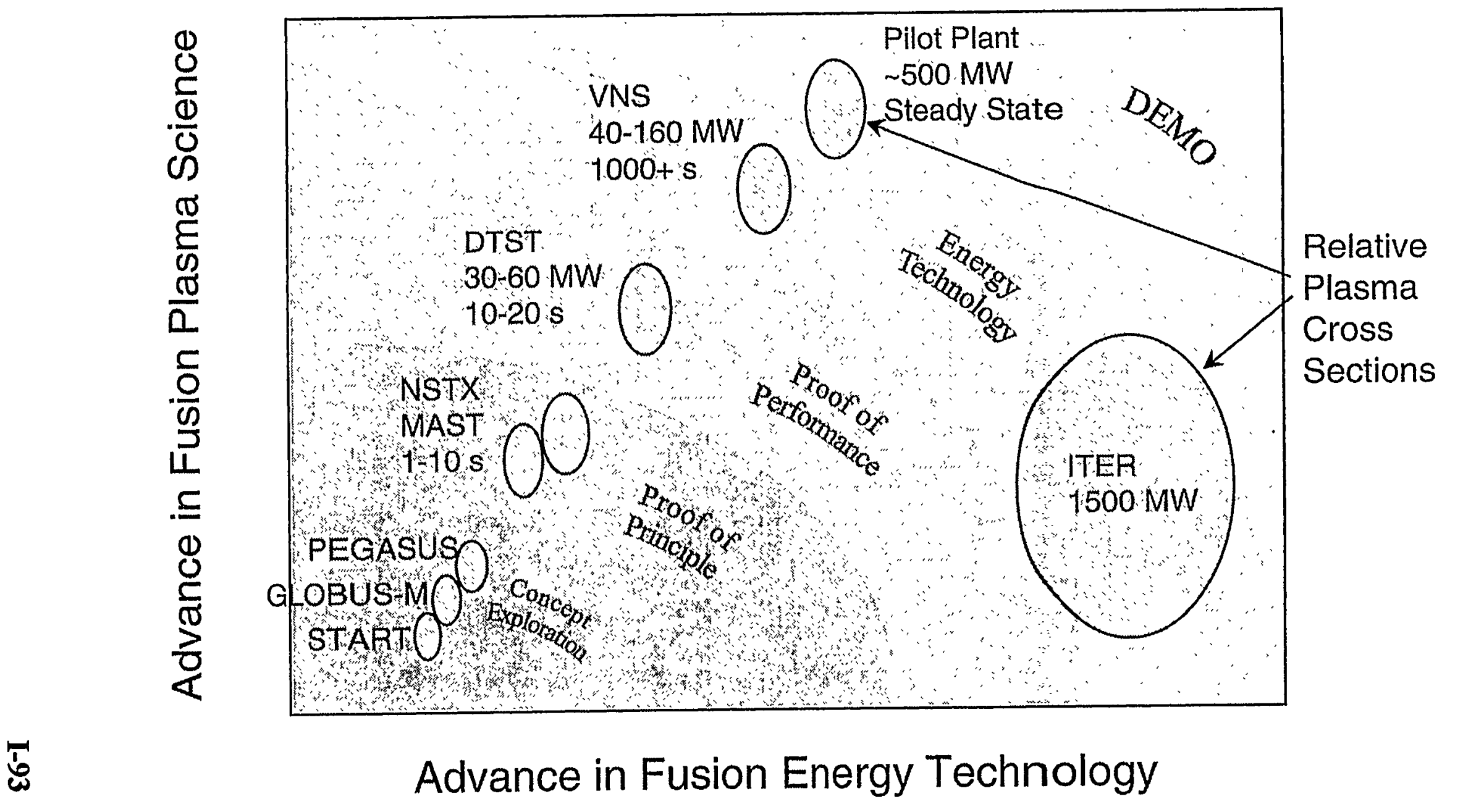




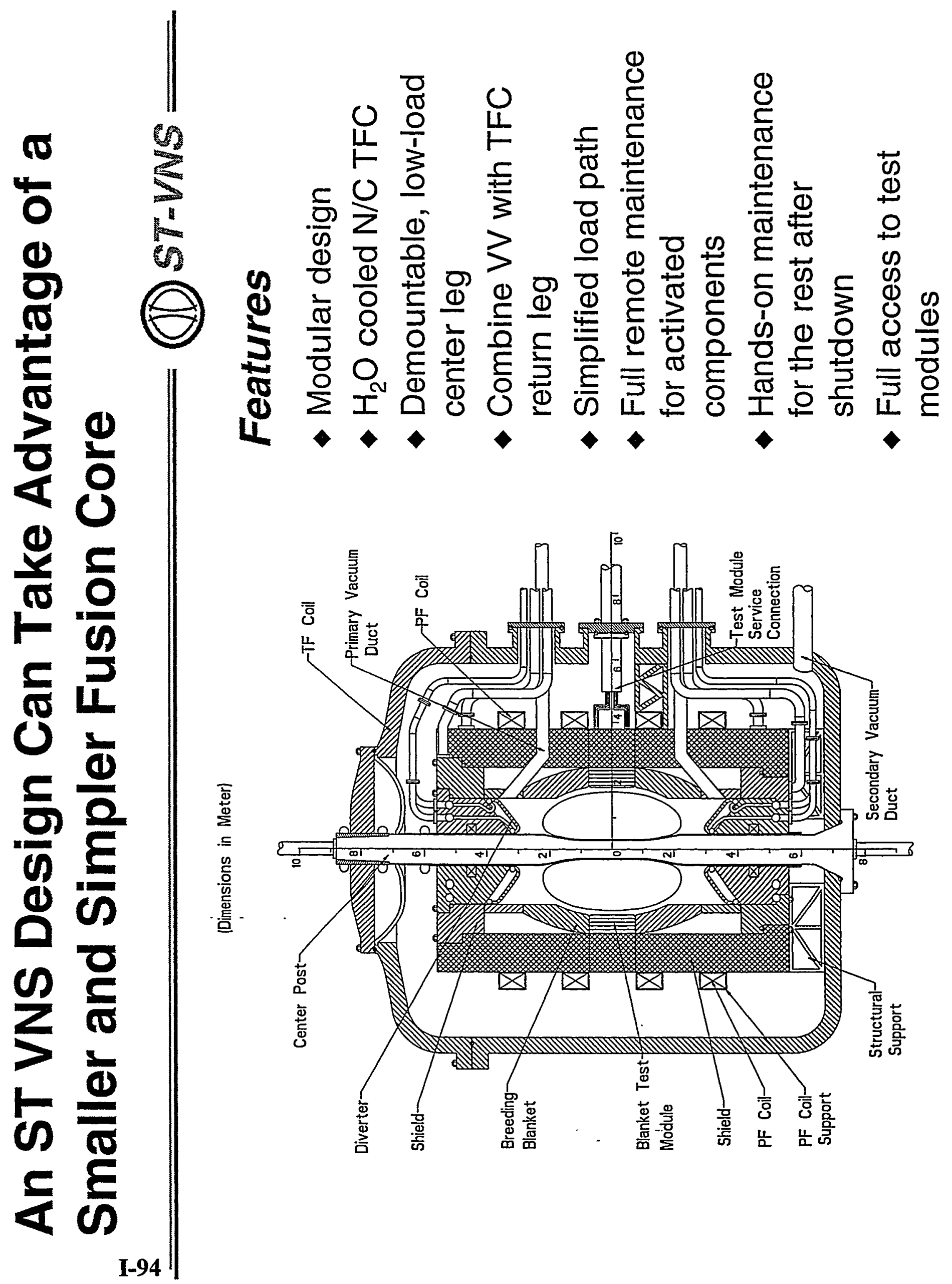




\section{Naturally Diverted Plasma in ST-VNS Show Mostly Diverted SOL}

(11) ST:VNS

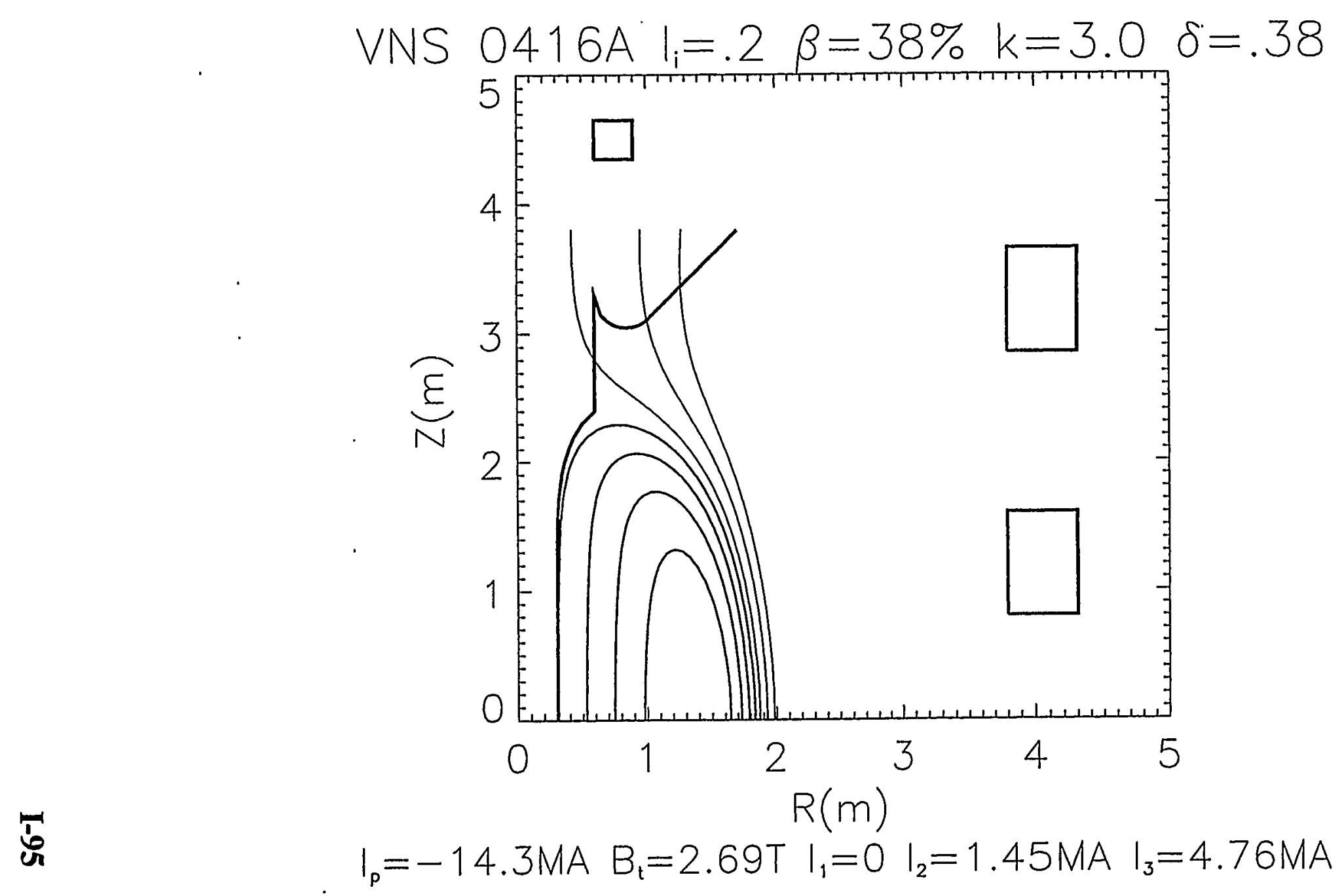




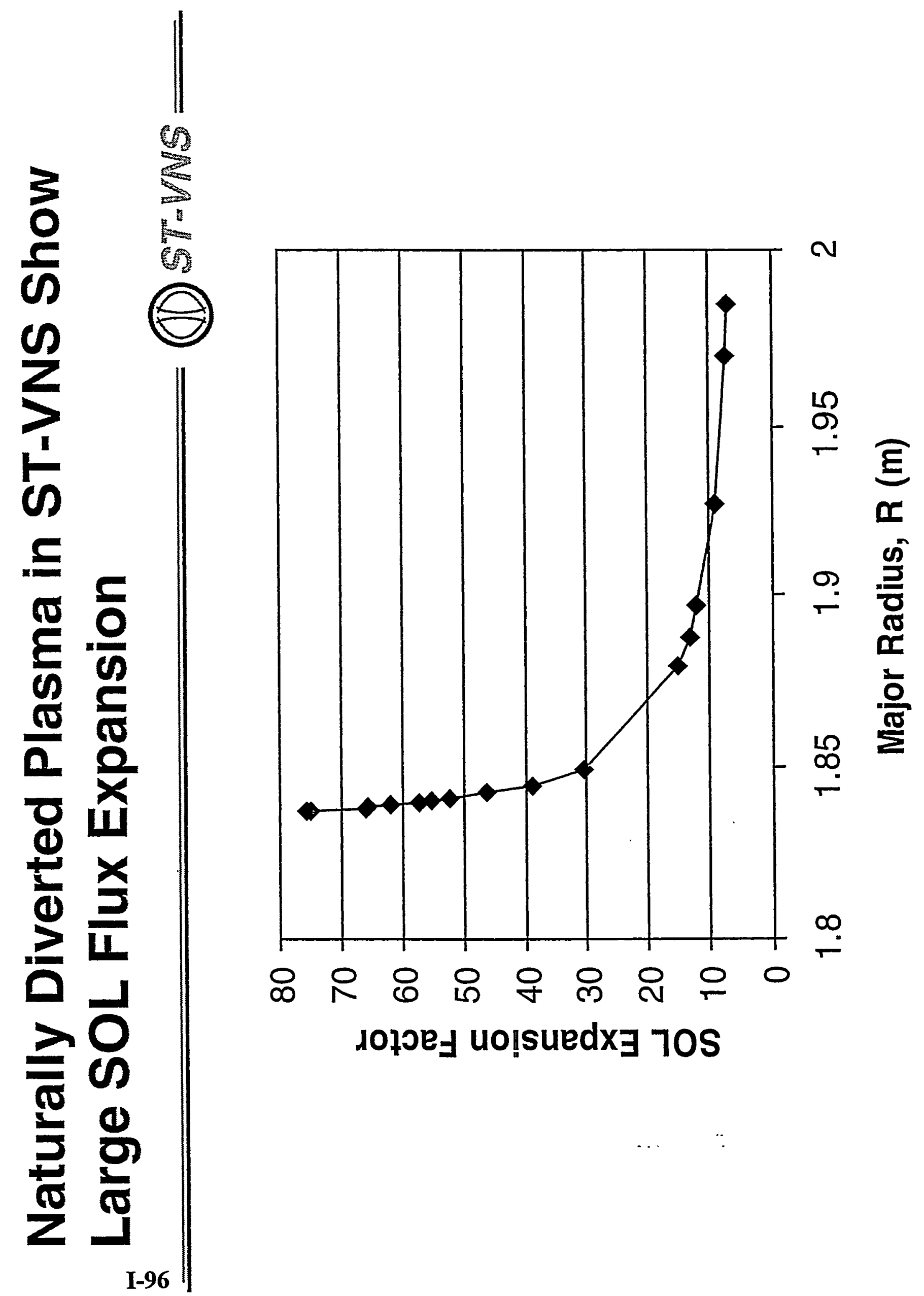




\section{Summary}

- Spherical Torus plasma has exciting possibilities for high performance in fusion plasma science

- NSTX will prove the physics principles for attractive VNS and power plants, which may have high demands on HHFC \& PSI performances

- NSTX Research will cover a wide range of topics and provide ample opportunities for collaboration, including HHFC \& PSI enabling technology

- NSTX Research Program is being prepared for start of operation in May 1999.

- HHFC \& PSI researchers are encouraged to collaborate and utilize the NSTX device and facility 

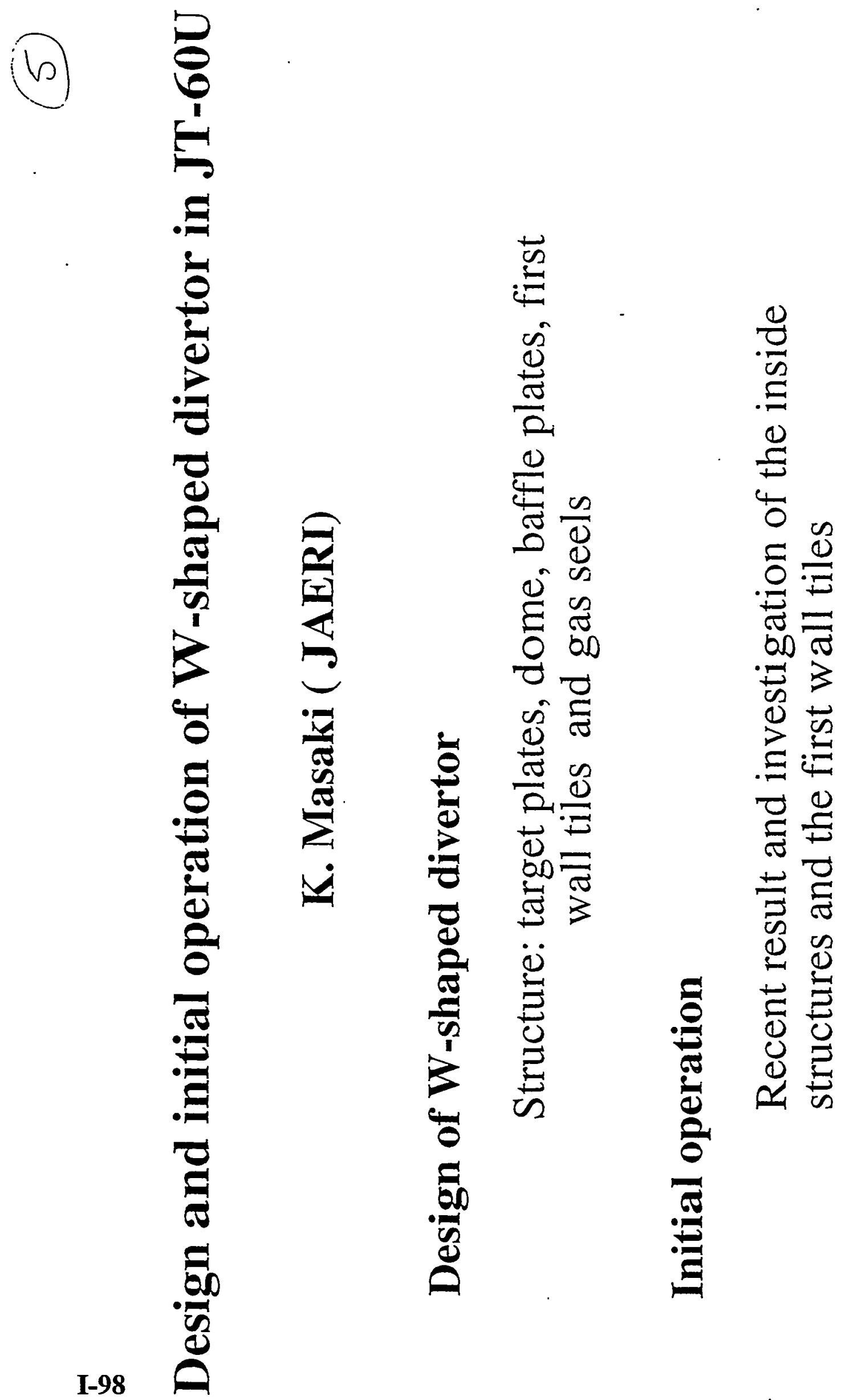


\section{Modification from open divertor to $\mathrm{W}$-shaped divertor}

8

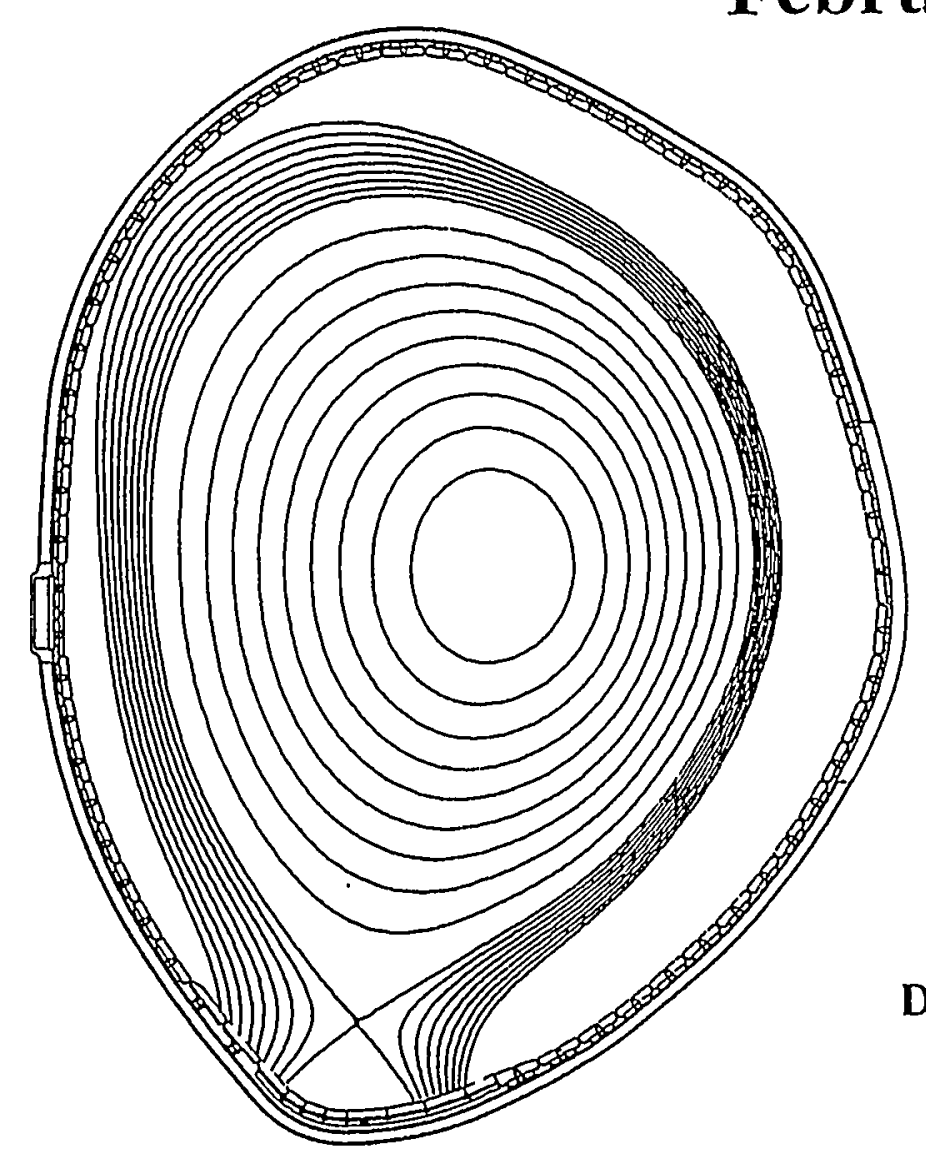

Open divertor
The work of this modification started end of February in 1997, and was completed in May.

\section{Objective}

to realize radiative divertor plasma and good H-mode confinement simultaneously.

Designed value 3MA, 4T $30 \mathrm{MW}$ (net heaing power)

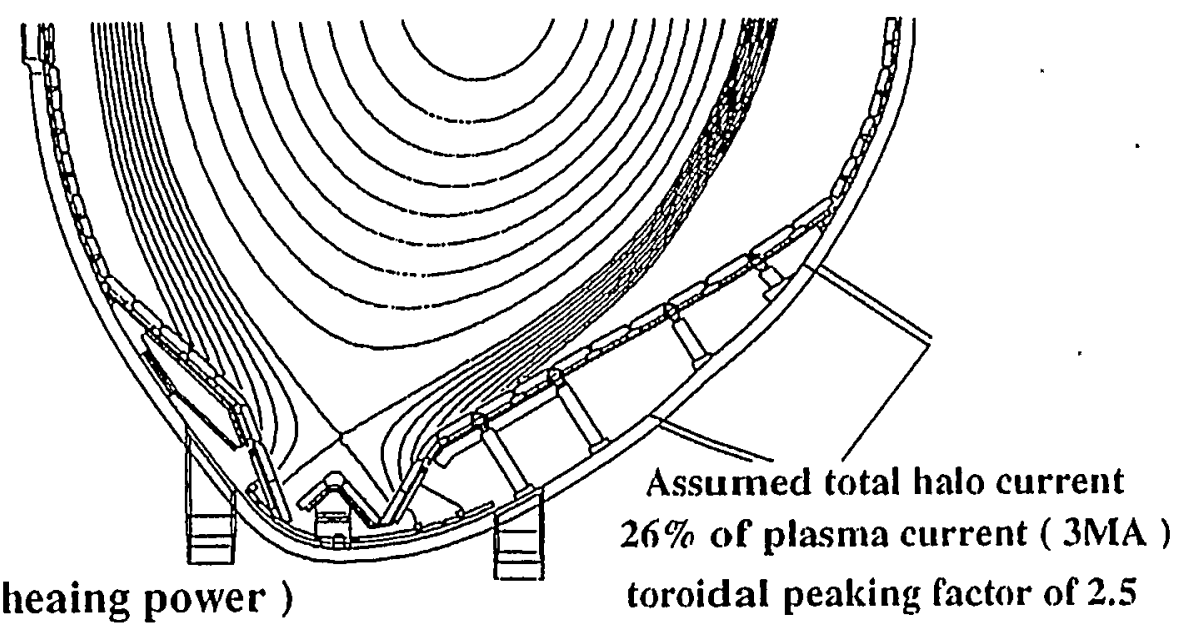

W-shaped divertor 


\section{Structure of W-shaped divertor \\ s}

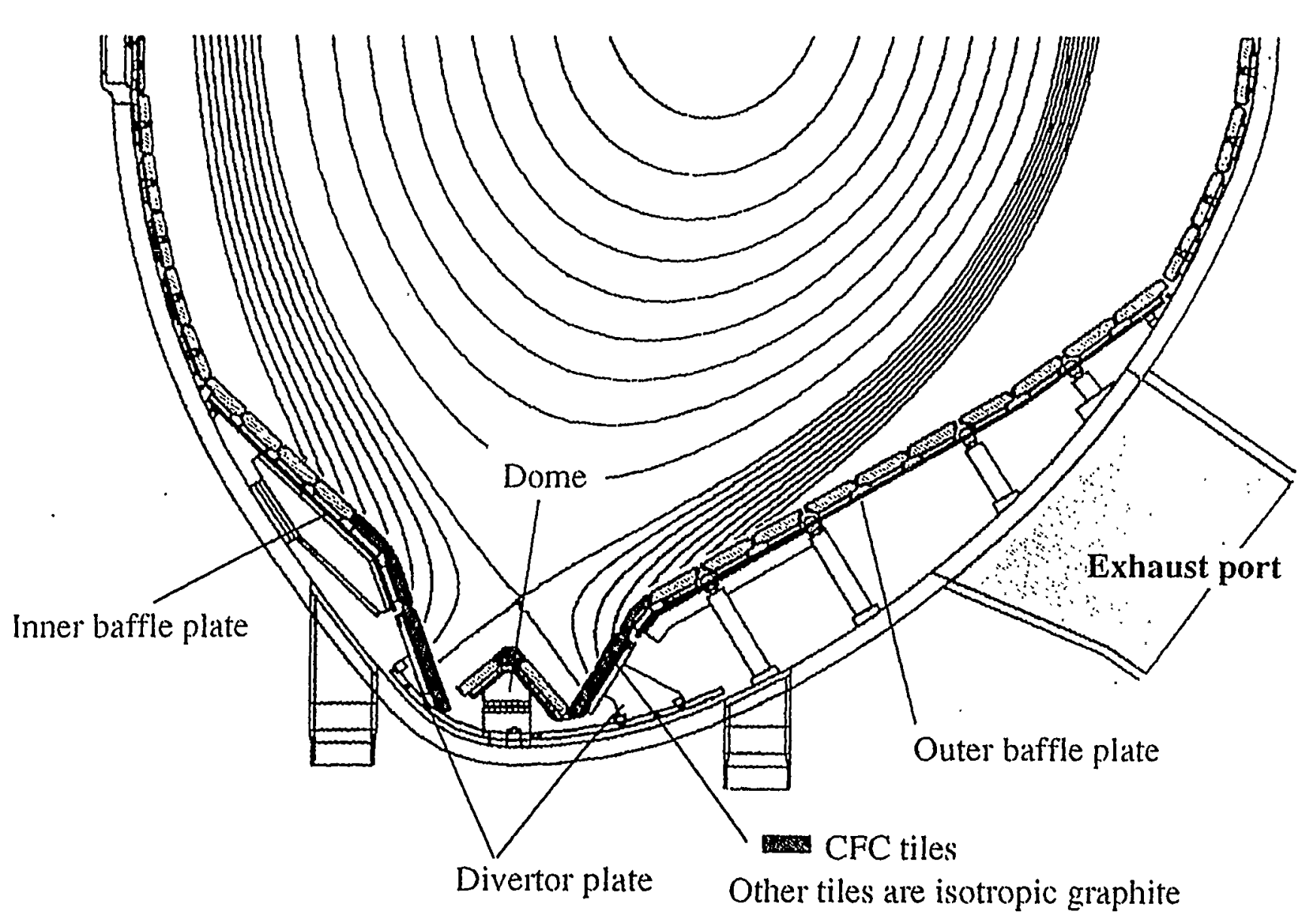

$<$ Inclined target $>$

High recycling, dense and cold divertor

$<$ Baffle $>$

to suppress back flow

of neutral particles

$<$ Dome $>$

to reduce generation of carbon impurity

$<$ Pumping $>$ (only inner divertor)

Particle control 


\section{Location of gas injection port, exhaust port and NBI port}

Divertor exhaust port

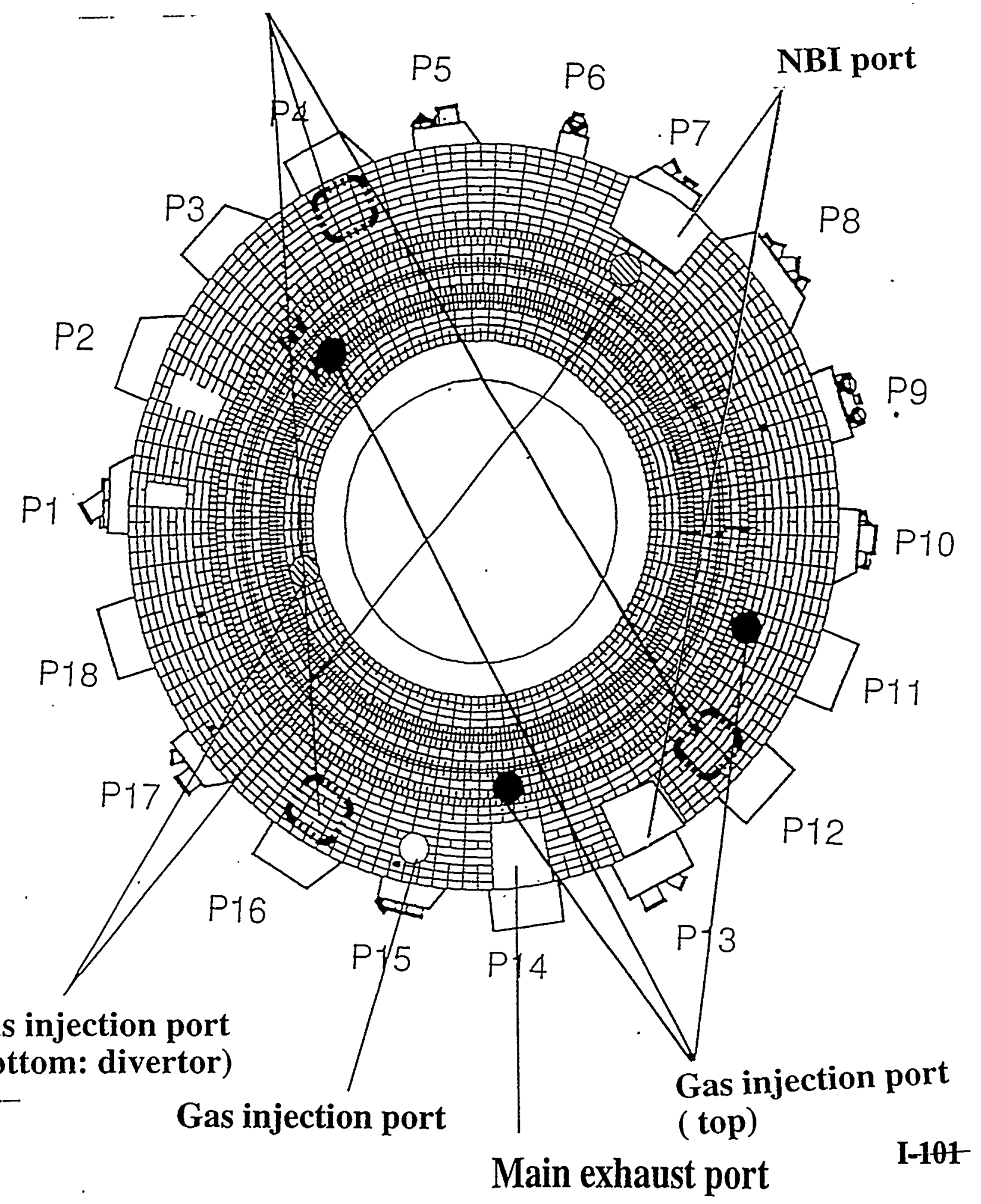




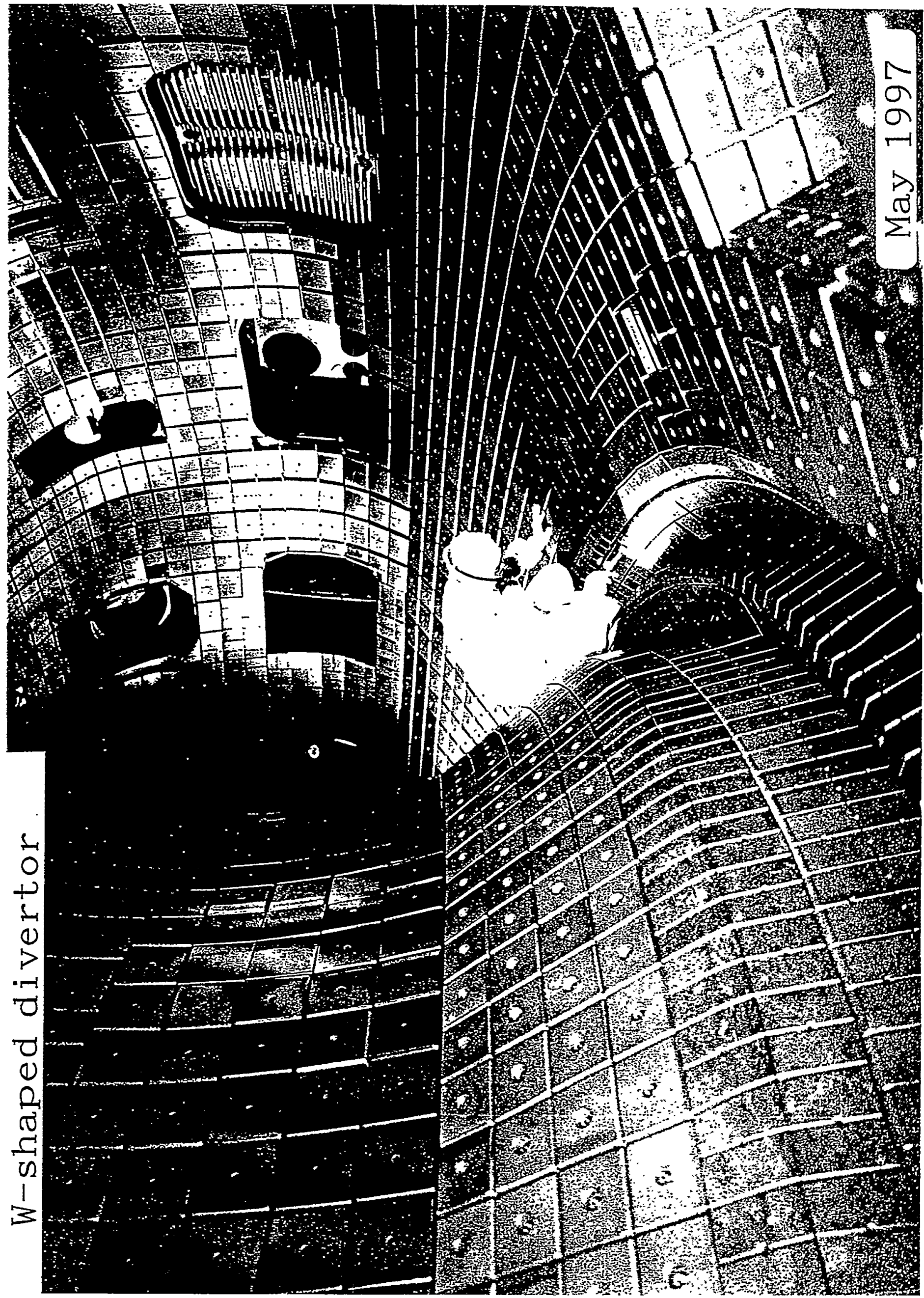




\section{Inner divertor ( 1 unit )}

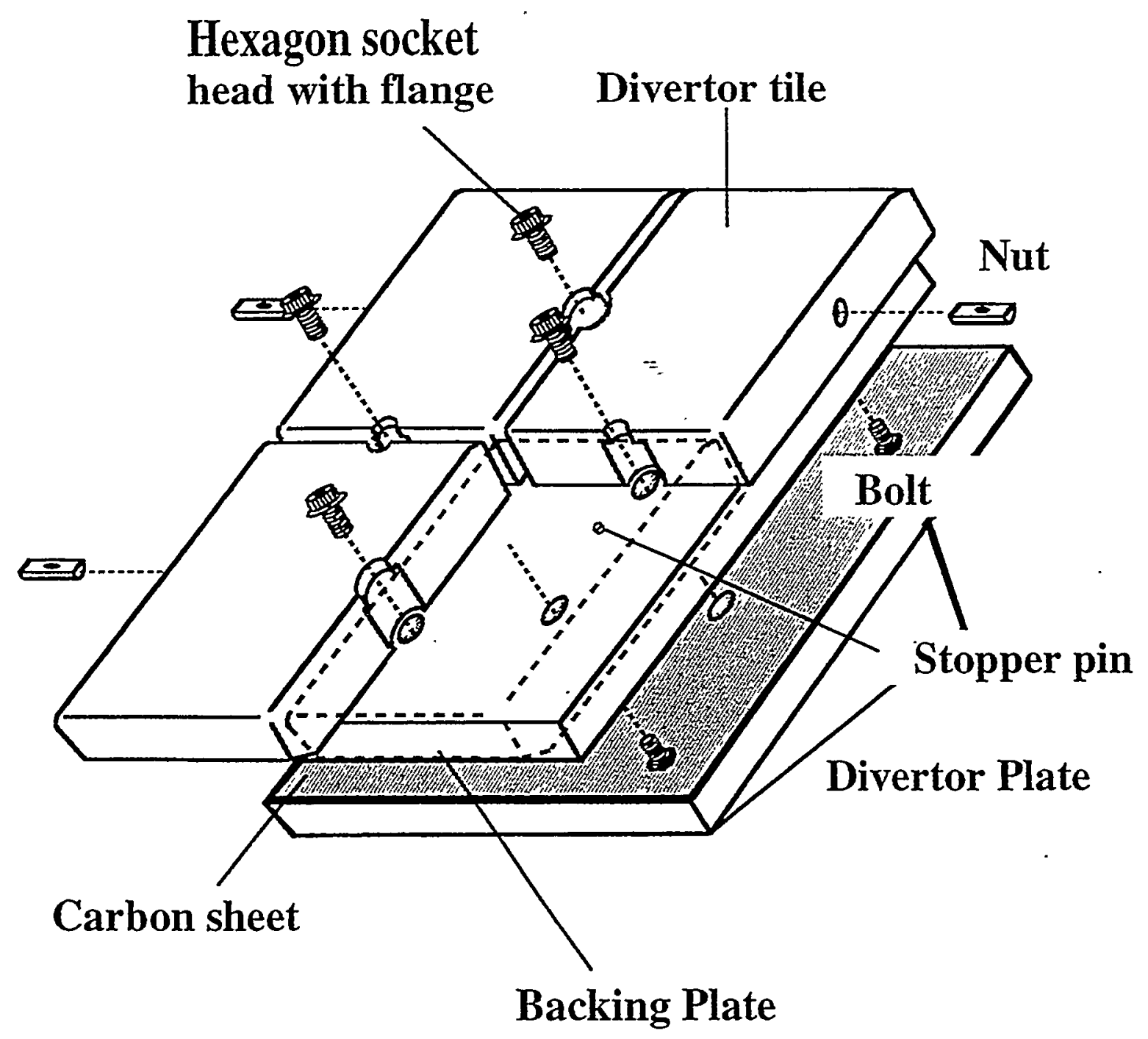

Inner divertor : 125 units outer divertor: 125 units Total 250 units

These divertor tiles were designed as the surface is circumscribed (inner divertor) and inscribed (outer divertor ) in each circles of inner and outer divertor plates.

These tiles were tapered to avoid the heat concentration to the tile edge. 


\section{Schematic view of tile alignment}

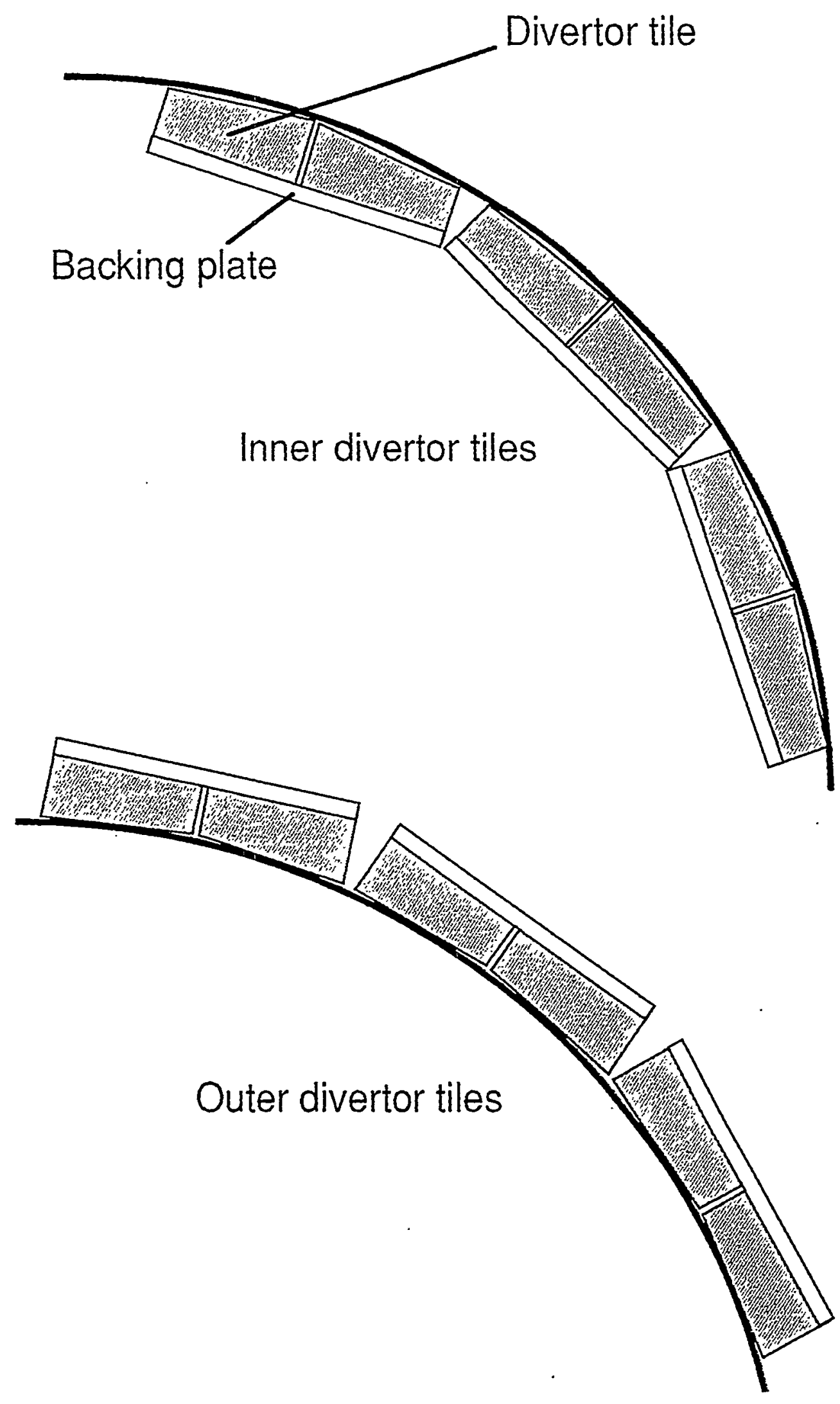




\section{Taper of divertor tile \\ (Inner divertor)}

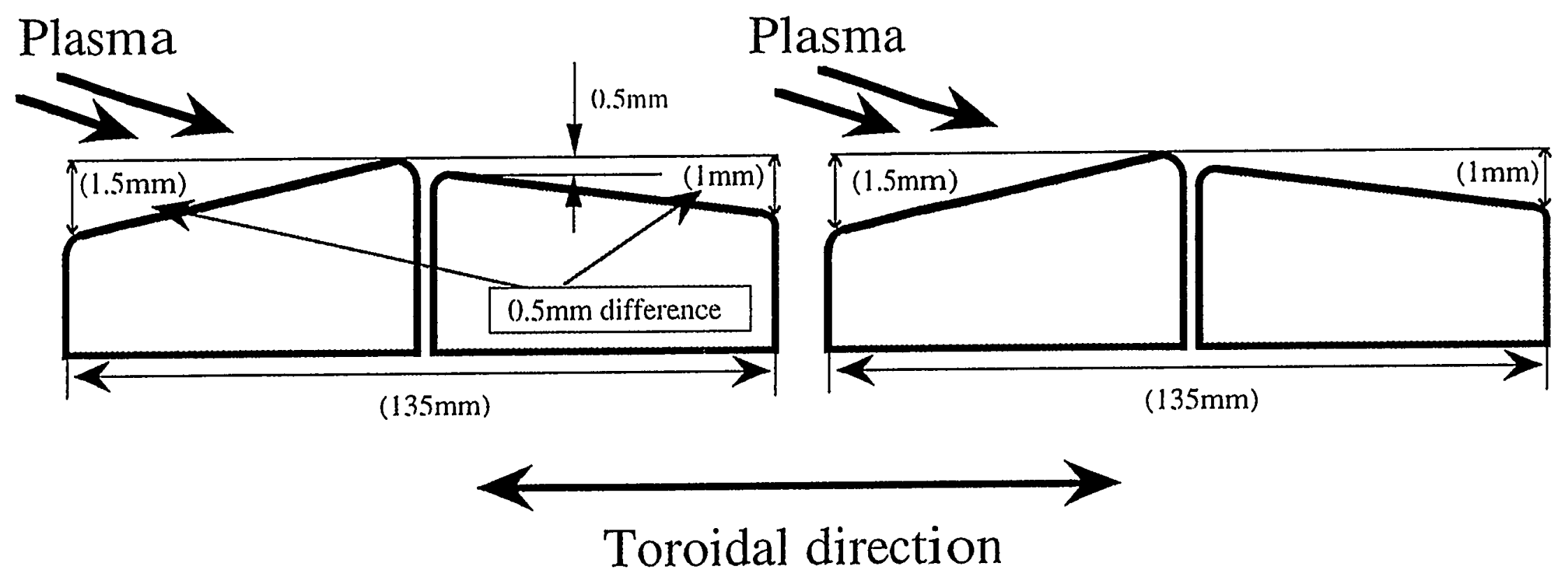

無 
홍

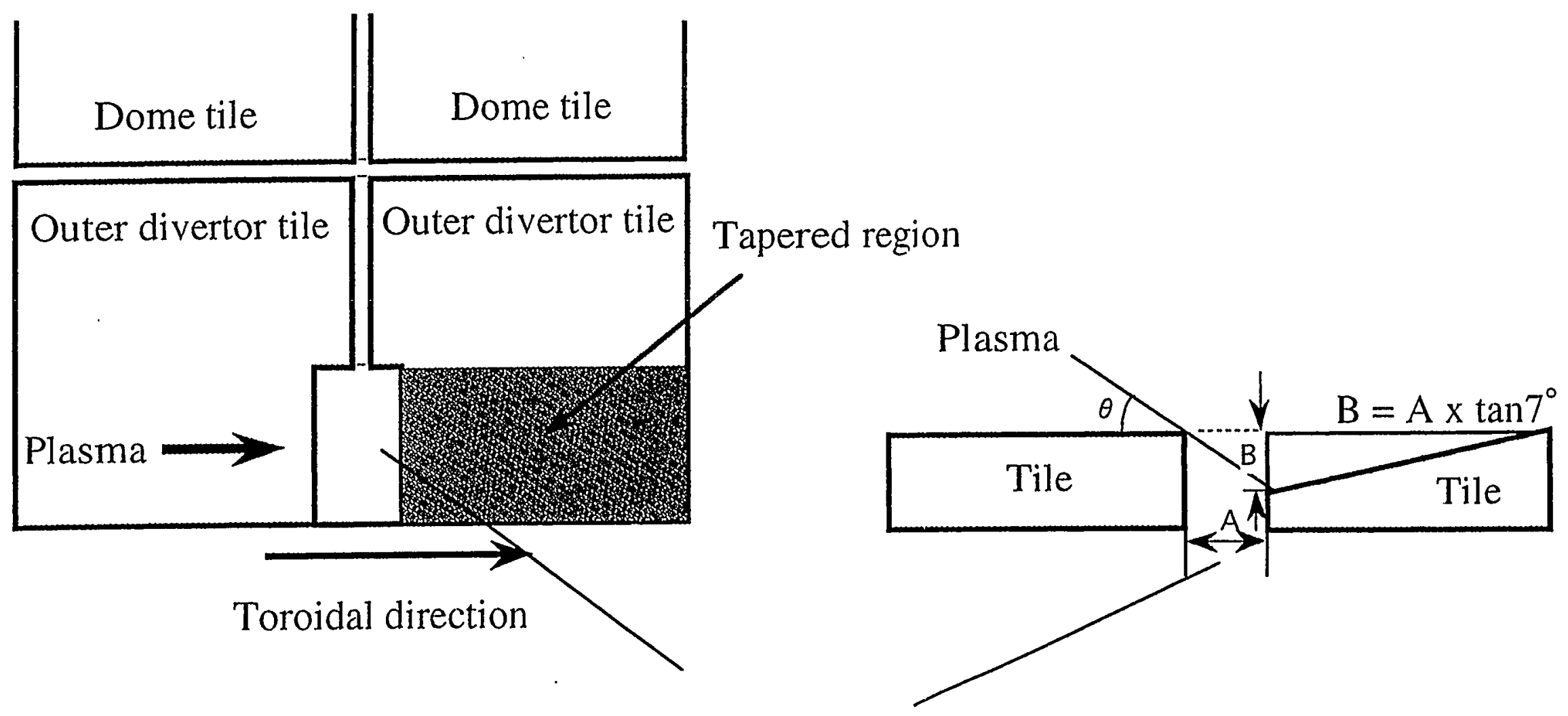

Opening space for measurement 


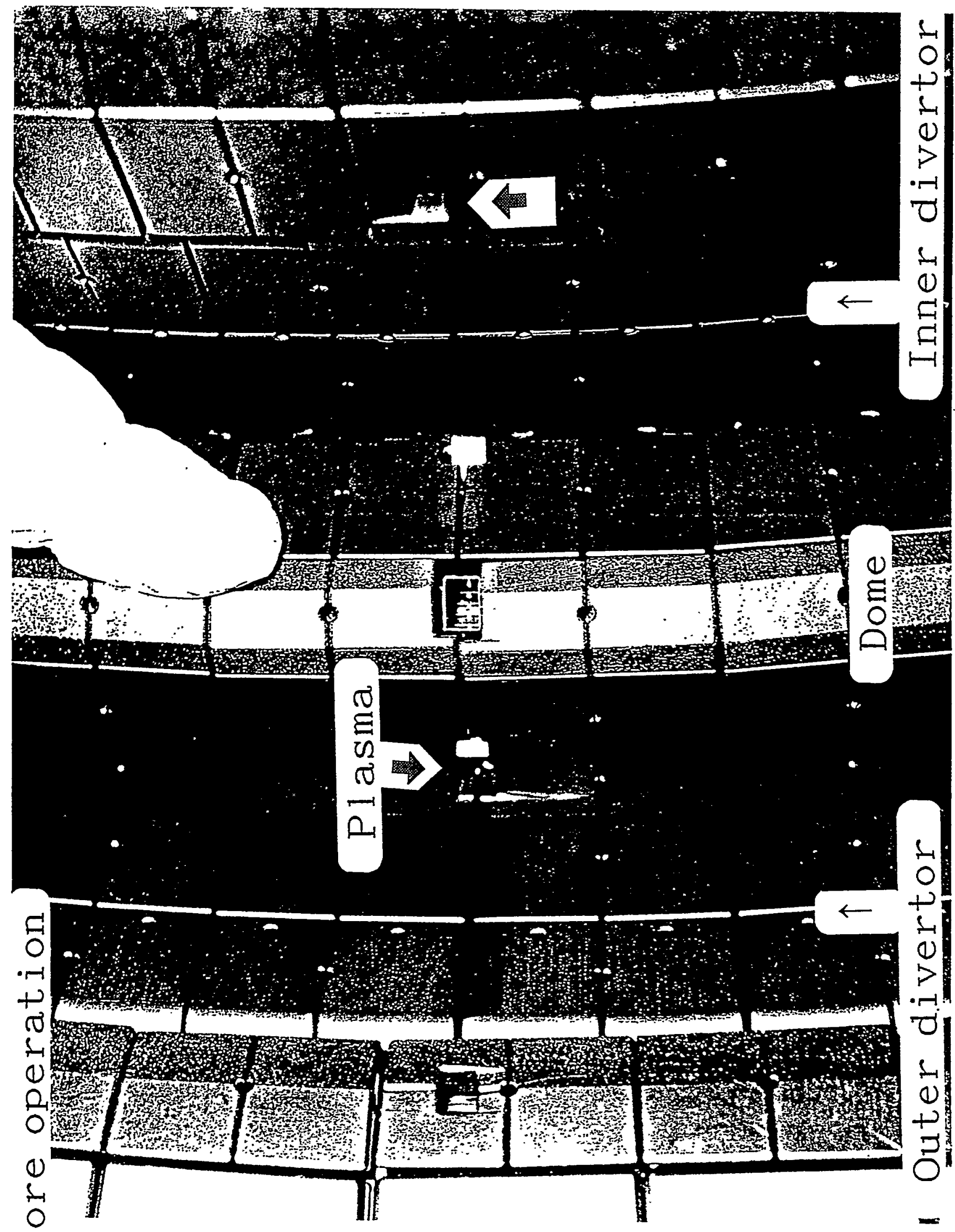

4

(1) 


\section{Dome (1 unit )}

Hexagon socket head with flange

Holes for stopper pin

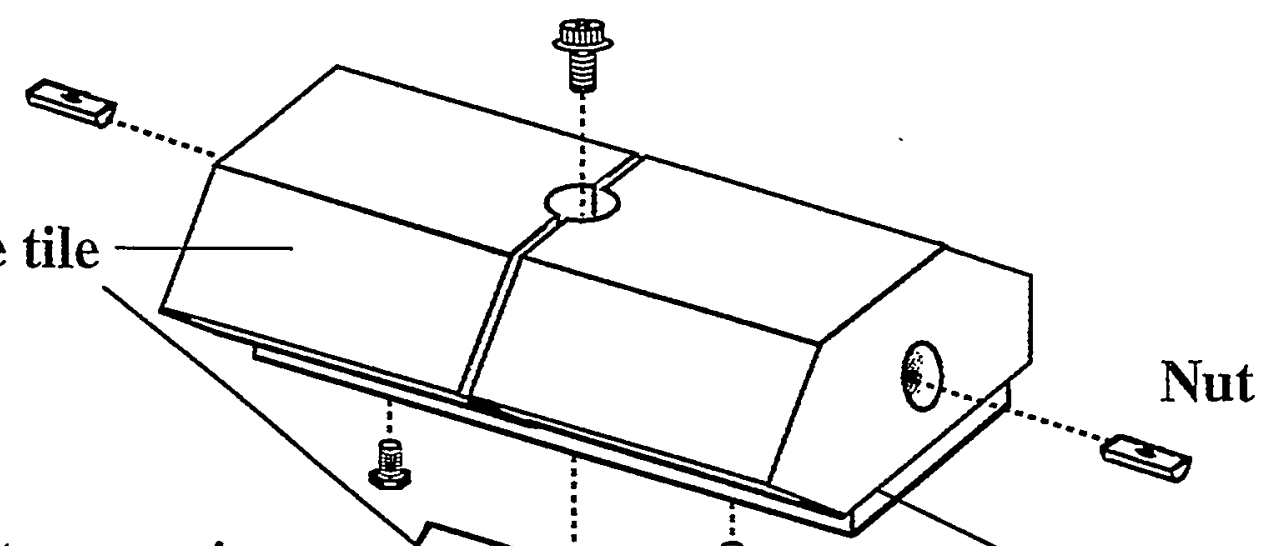

Dome tile

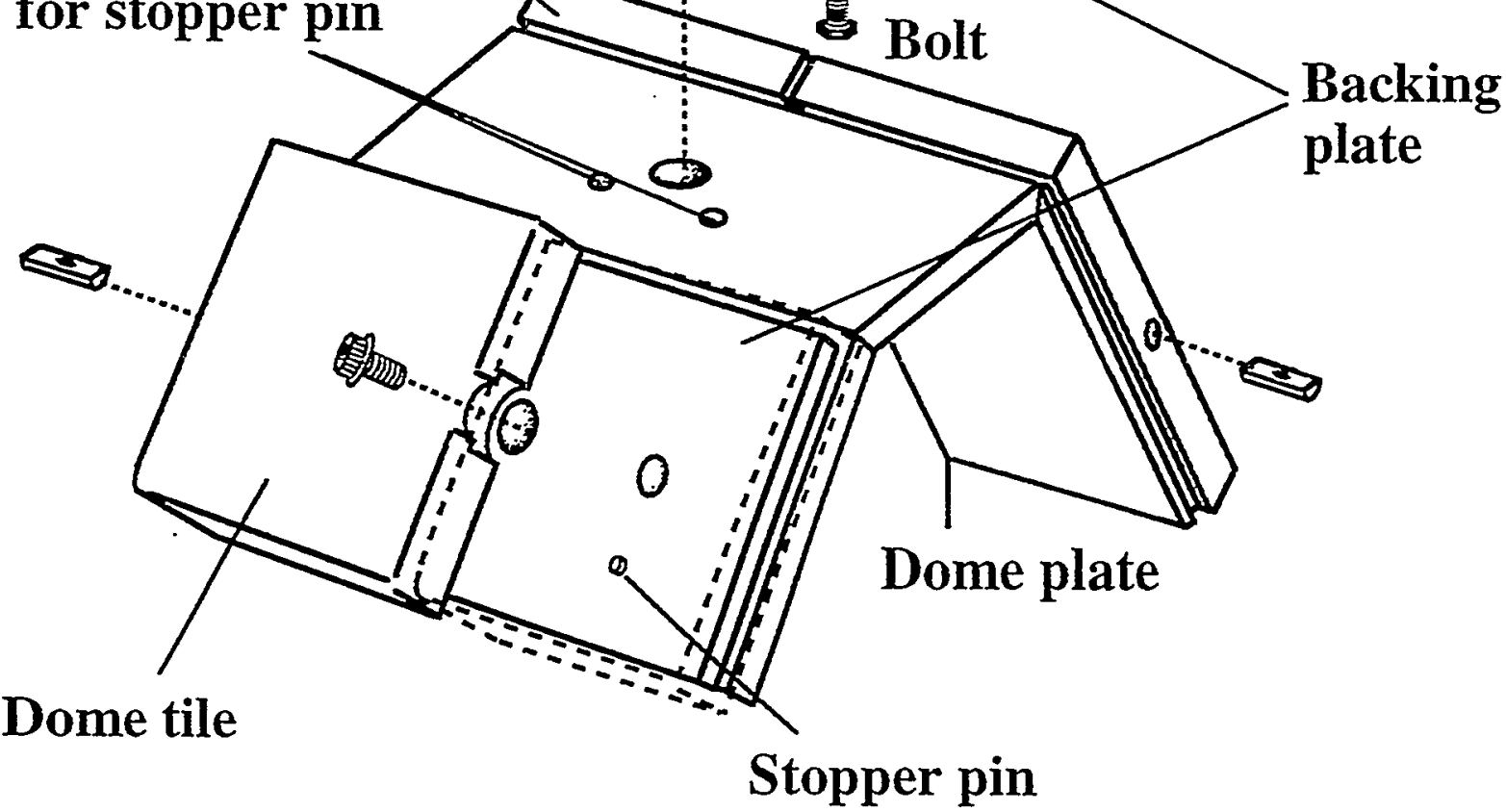

Total : 125 units 


\section{Inner and outer baffle plates}

Inner baffle plate

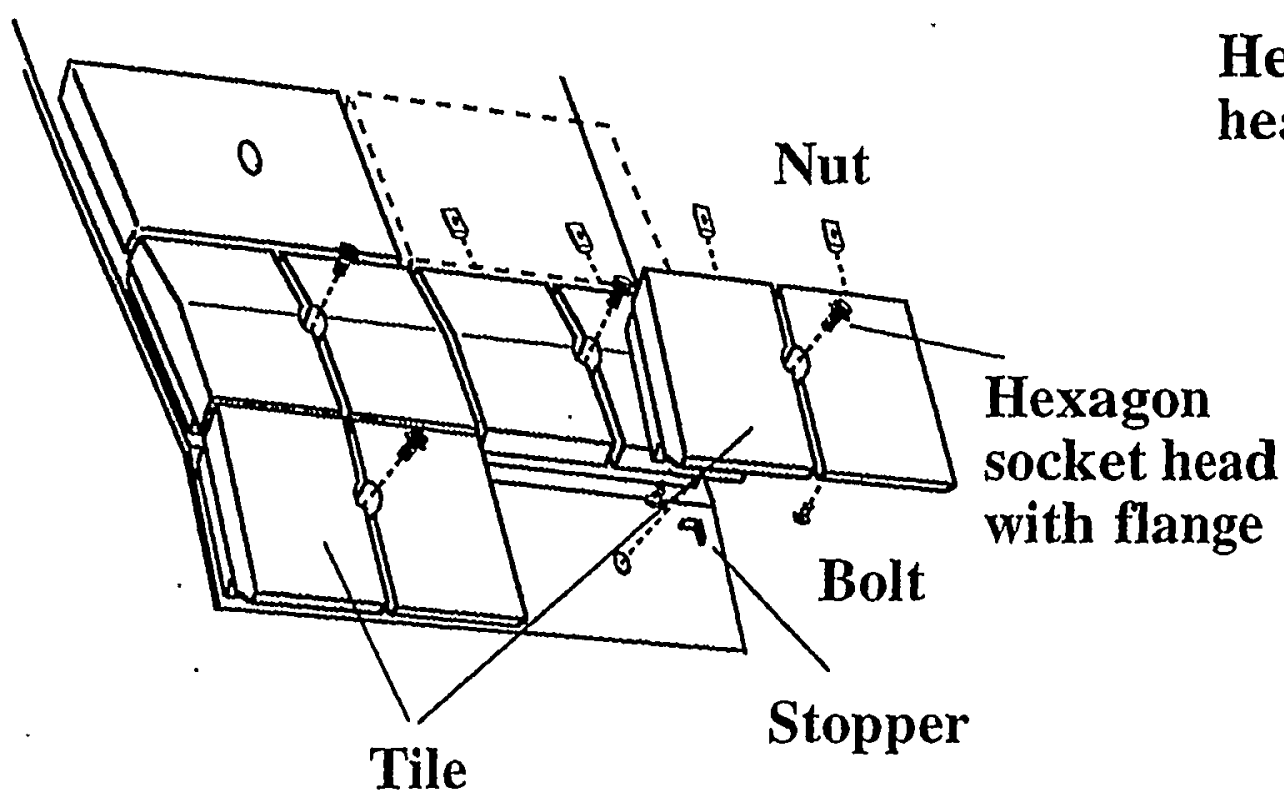

Inner baffle plate : 72 plates
Outer baffle plate

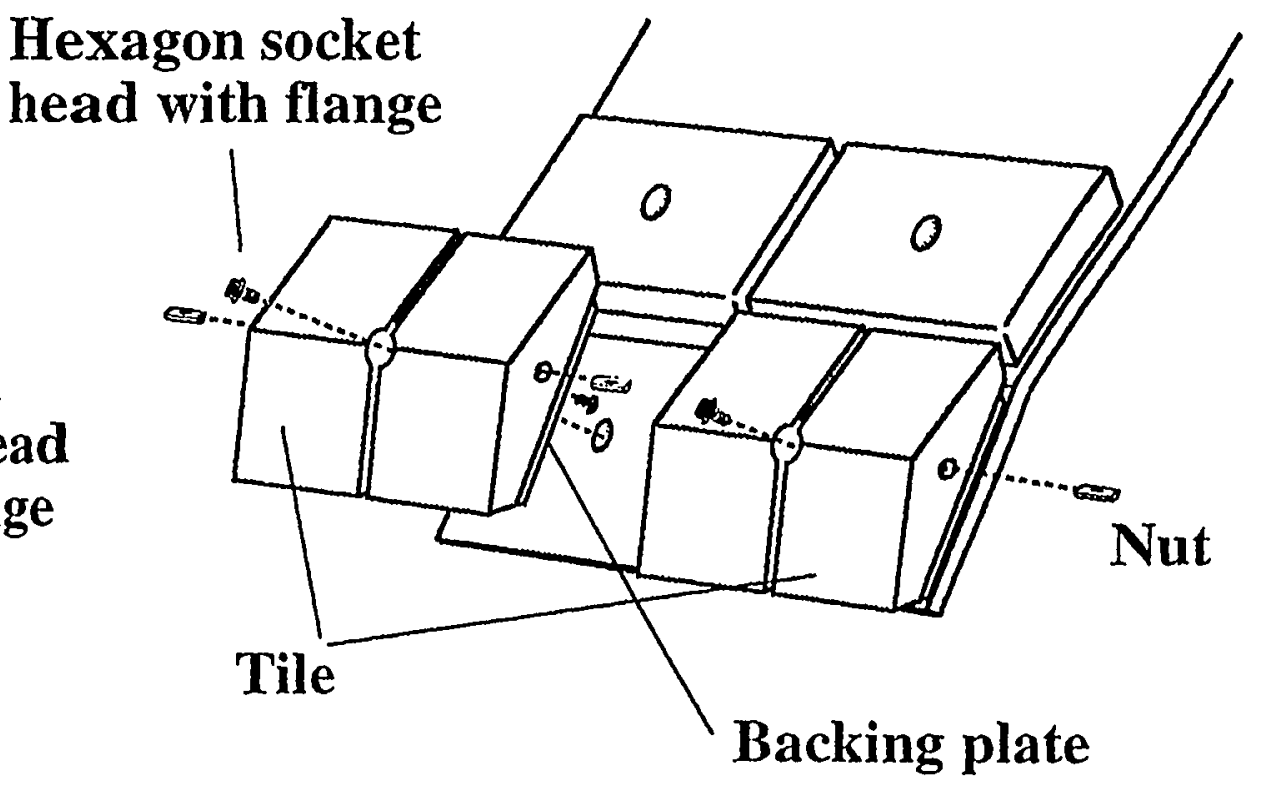

Outer baffle plate : 72 plates

These baffle tiles were designed as the surface is circumscribed (inner baffle ) and

$\frac{1}{6}$ inscribed ( outer baffle) in each circles of inner and outer divertor plates.

These tiles were tapered to avoid the heat concentration to the tile edge. 


\section{Baffle plate tile}

Hexagon socket head with flange

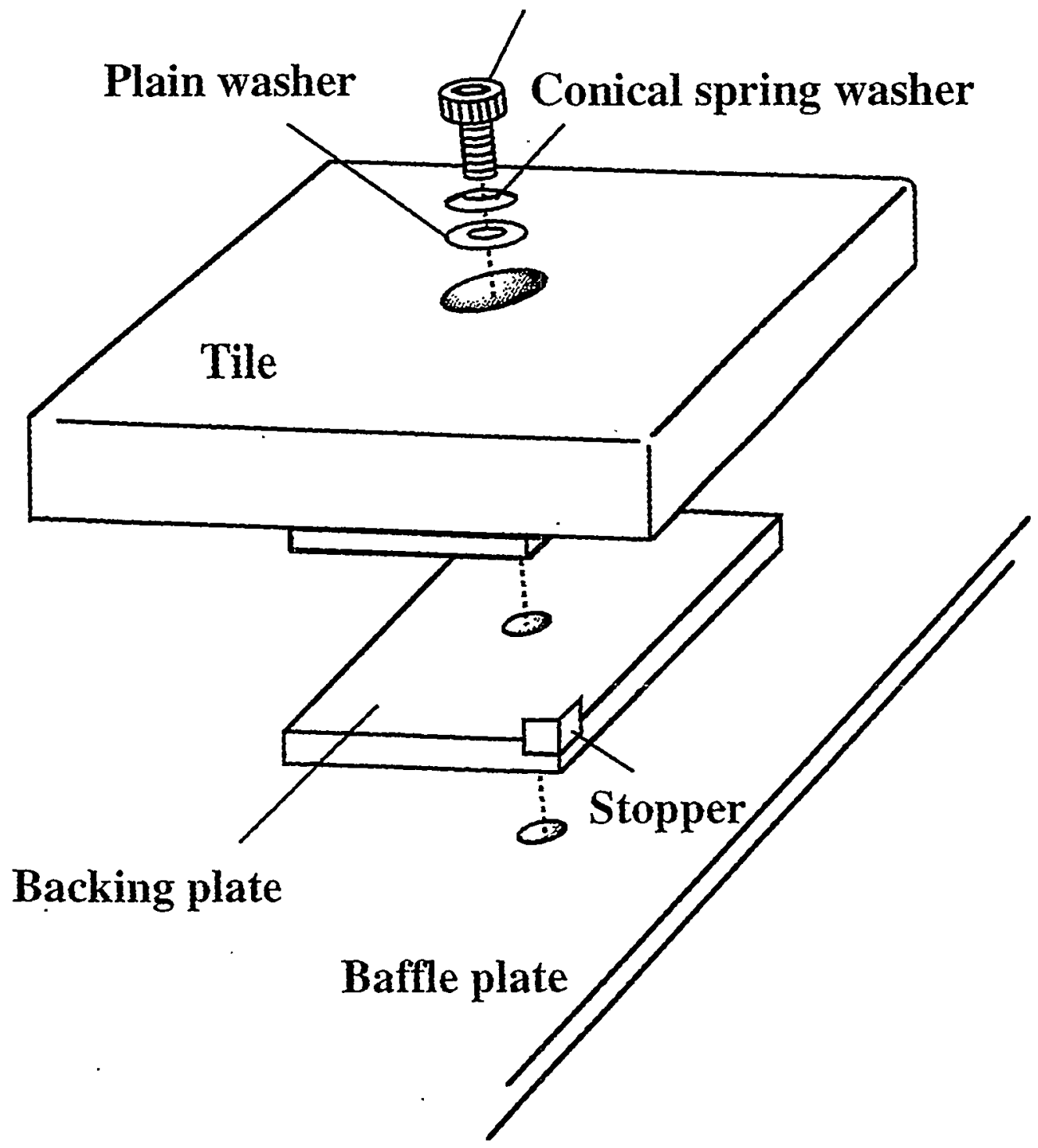


Gap and level difference between each tile

\begin{tabular}{|l|c|c|}
\hline \multicolumn{1}{|c|}{ section } & gap & level diff. \\
\hline inner baffle (T) & $>1.5$ & $-1 \sim 0^{*}, 2 \sim 0$ \\
\hline in. baffle/div. (P) & $3.5 \sim 4.5$ & \\
\hline inner divertor (T) & $>1.0$ & $-1.5 \sim 0$ \\
\hline dome (T) & $0.5 \sim 2.0$ & $-2 \sim 0$ \\
\hline outer divertor (T) & $>1.5$ & $-1.5 \sim 0$ \\
\hline out. baffle/div. (P) & $3.5 \sim 4.5$ & \\
\hline outer baffle (T) & $1.5 \sim 2$ & $-1 \sim 0^{*}, \pm 2$ \\
\hline
\end{tabular}

(T) : Toroidal direction

level diff.

(P) : Poloidal direction

$\underline{m}$

$-1 \sim 0 *$ : Near the Divertor region

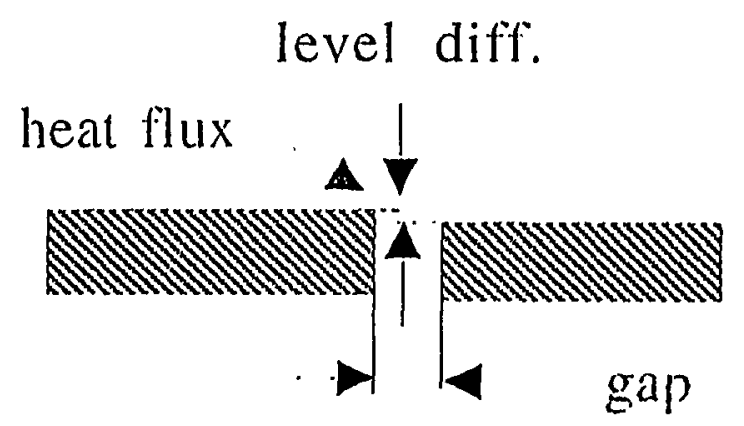




\section{Thermal analysis}

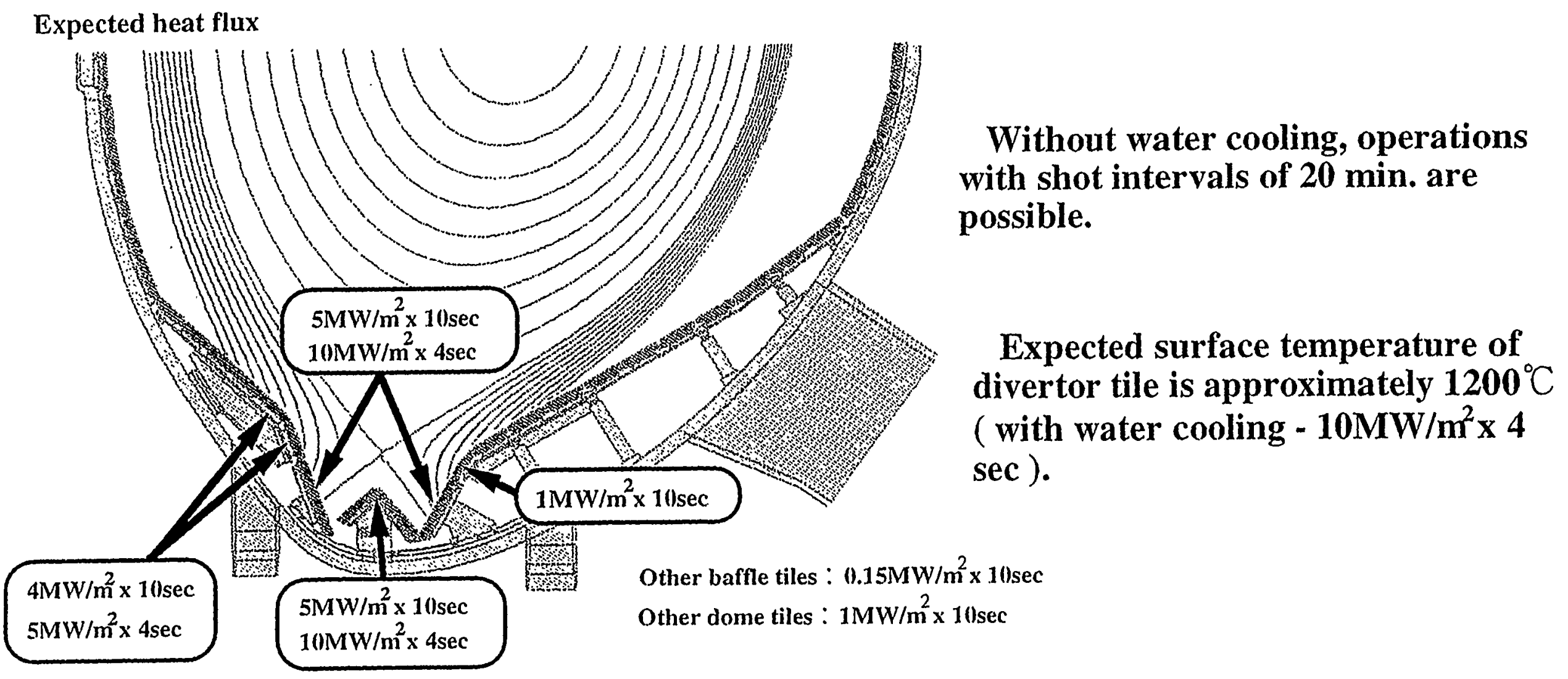




\section{Structure of gas seal ( baffle palte )}

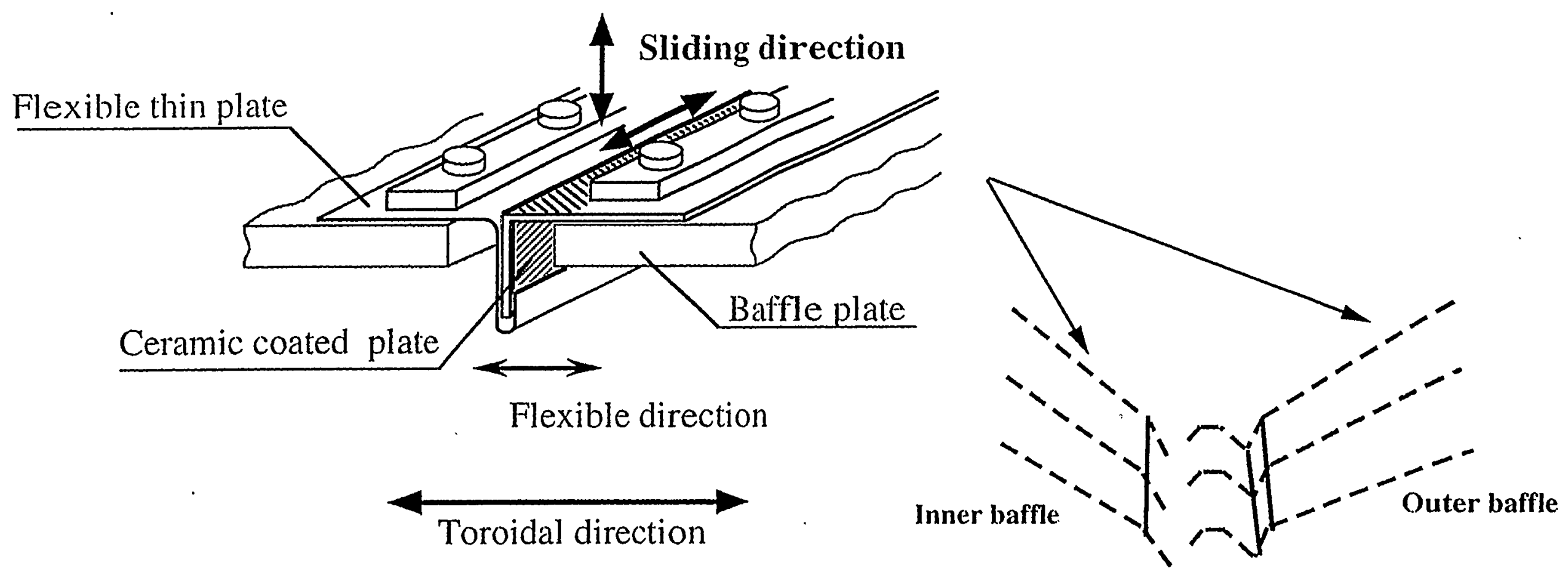

Poloidal gaps between the segmented baffle sturctures are sealed by inserted-sliding mechanizm.

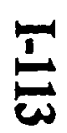

Sliding parts are insulated by sprayed ceramic coating to avoid arcing across the gaps. 


\section{Structure of gas seal (divertor palte and dome plate )}

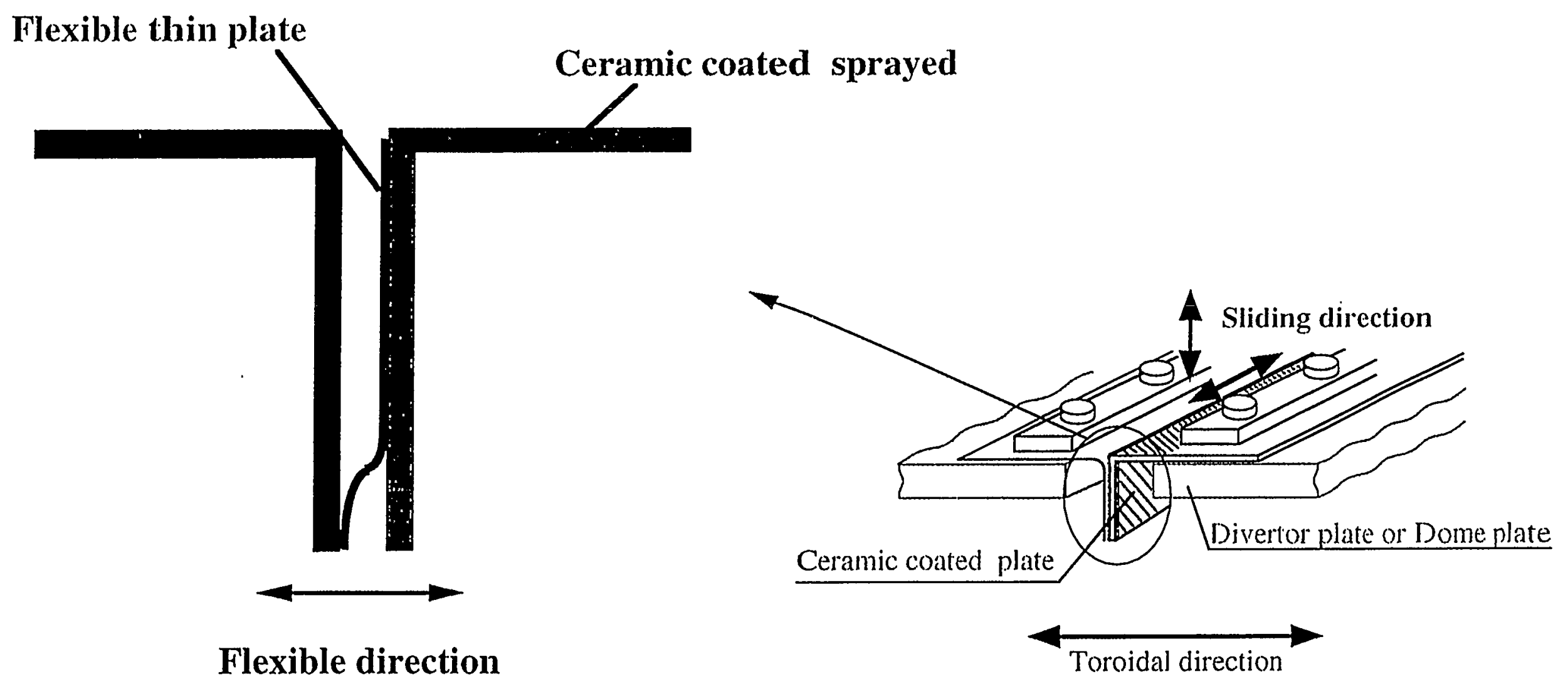




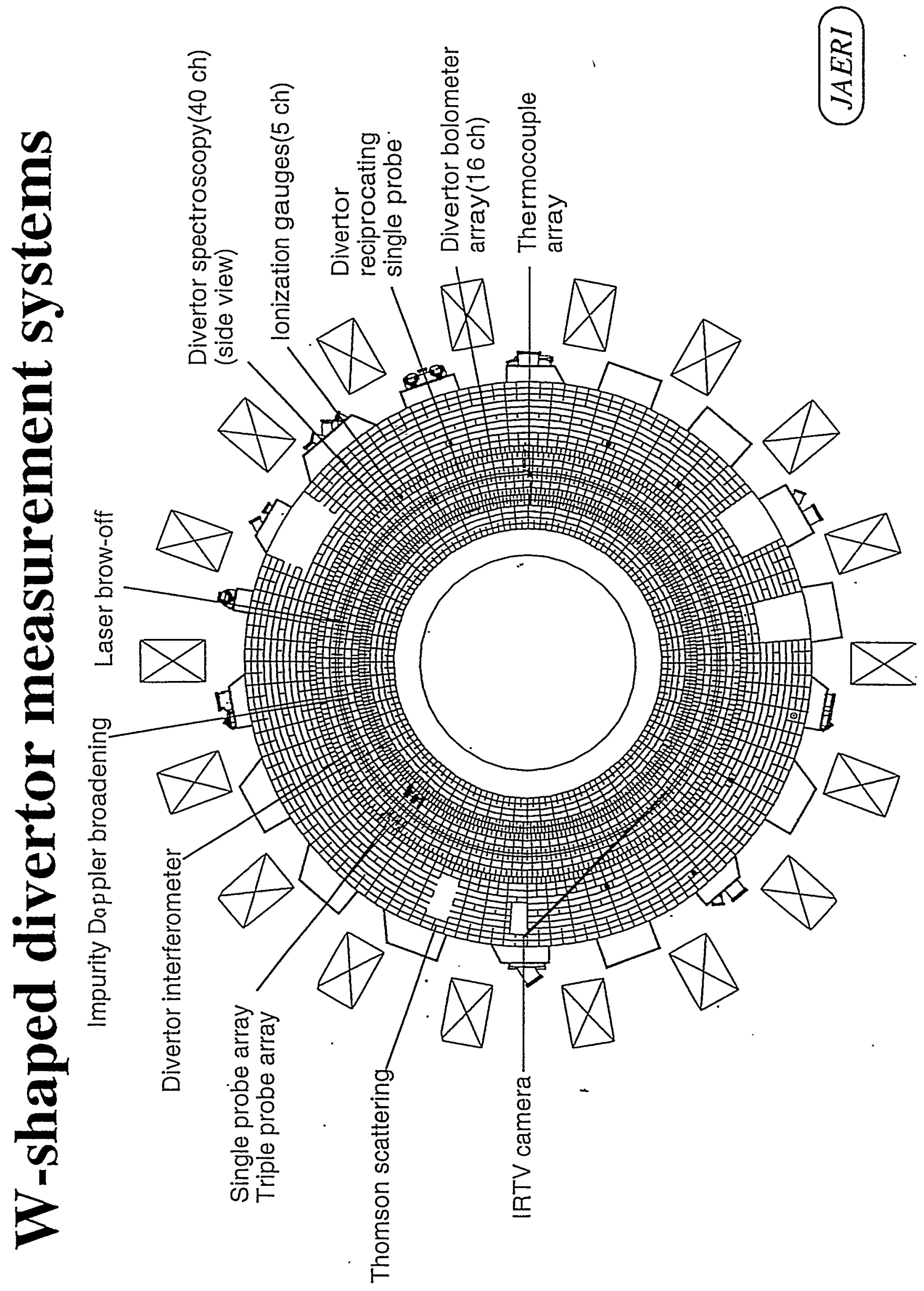




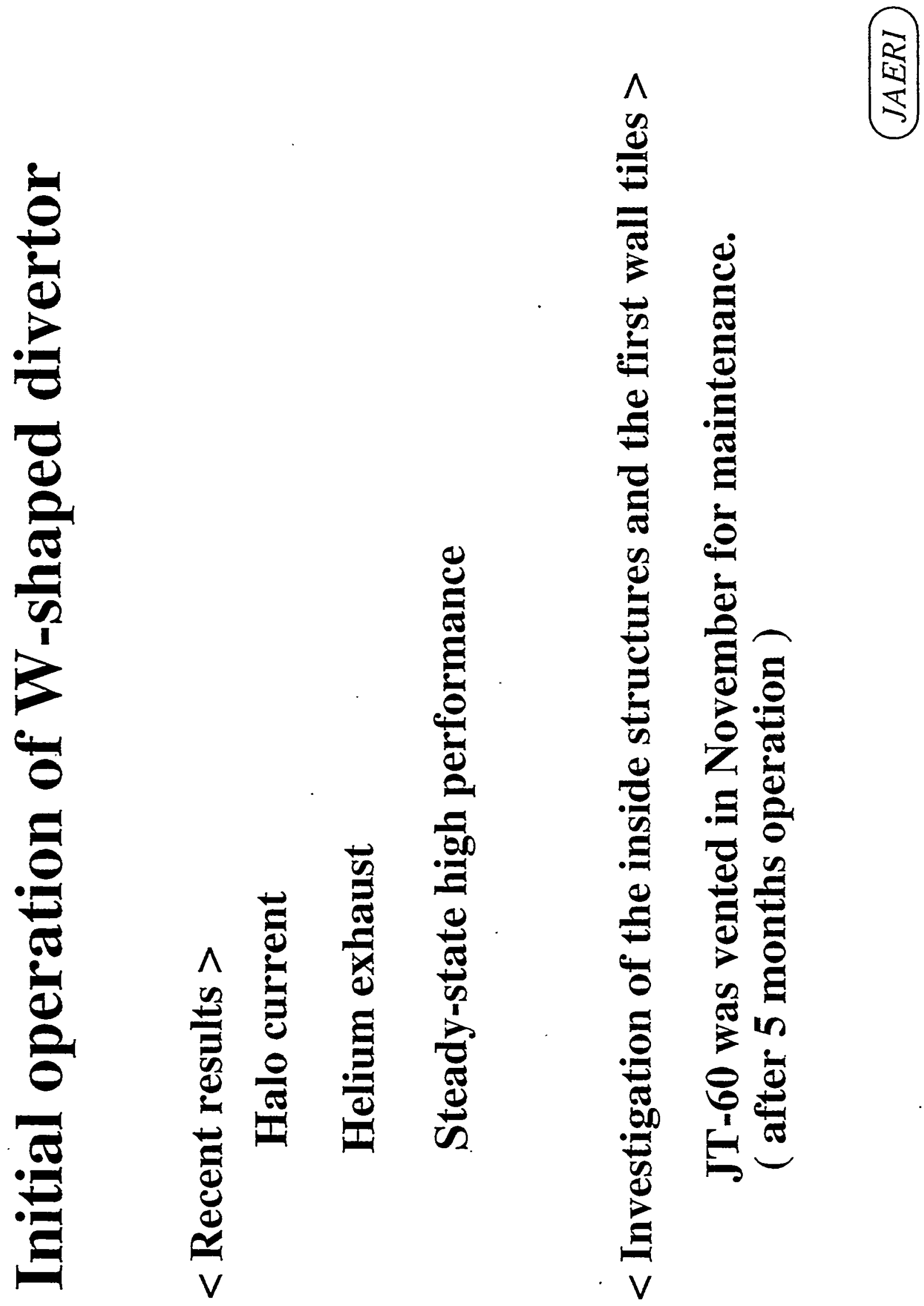




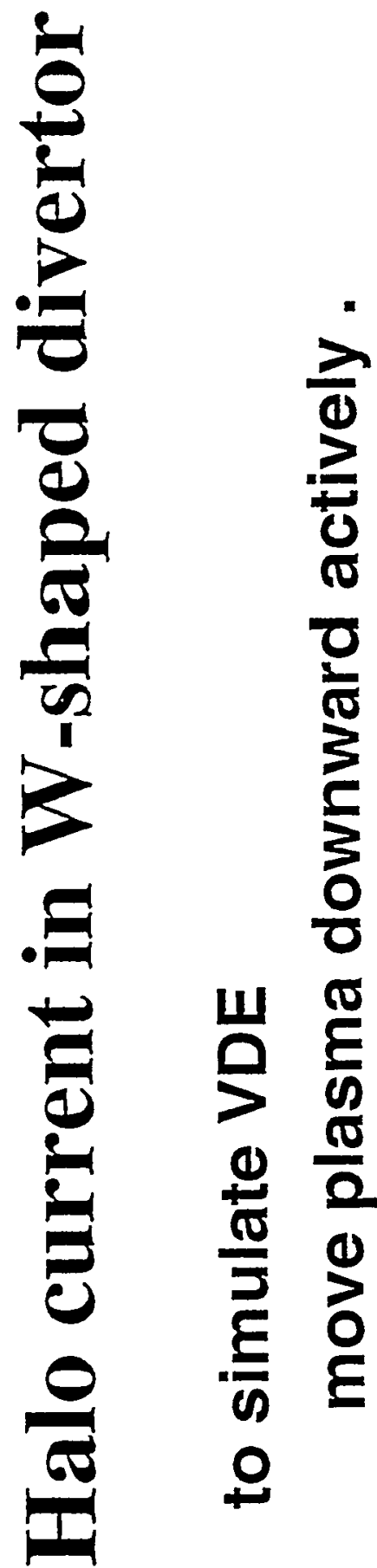

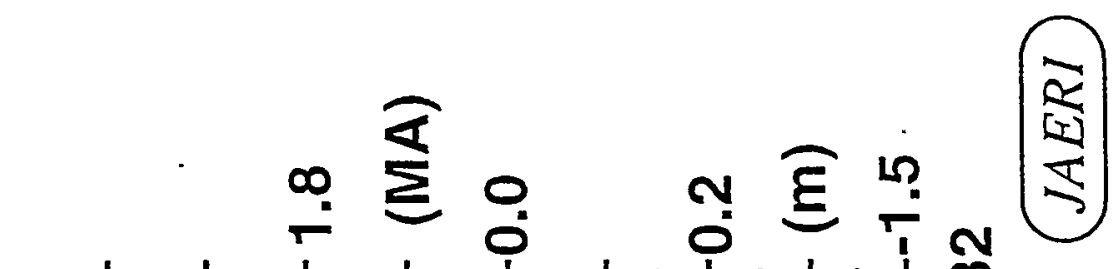
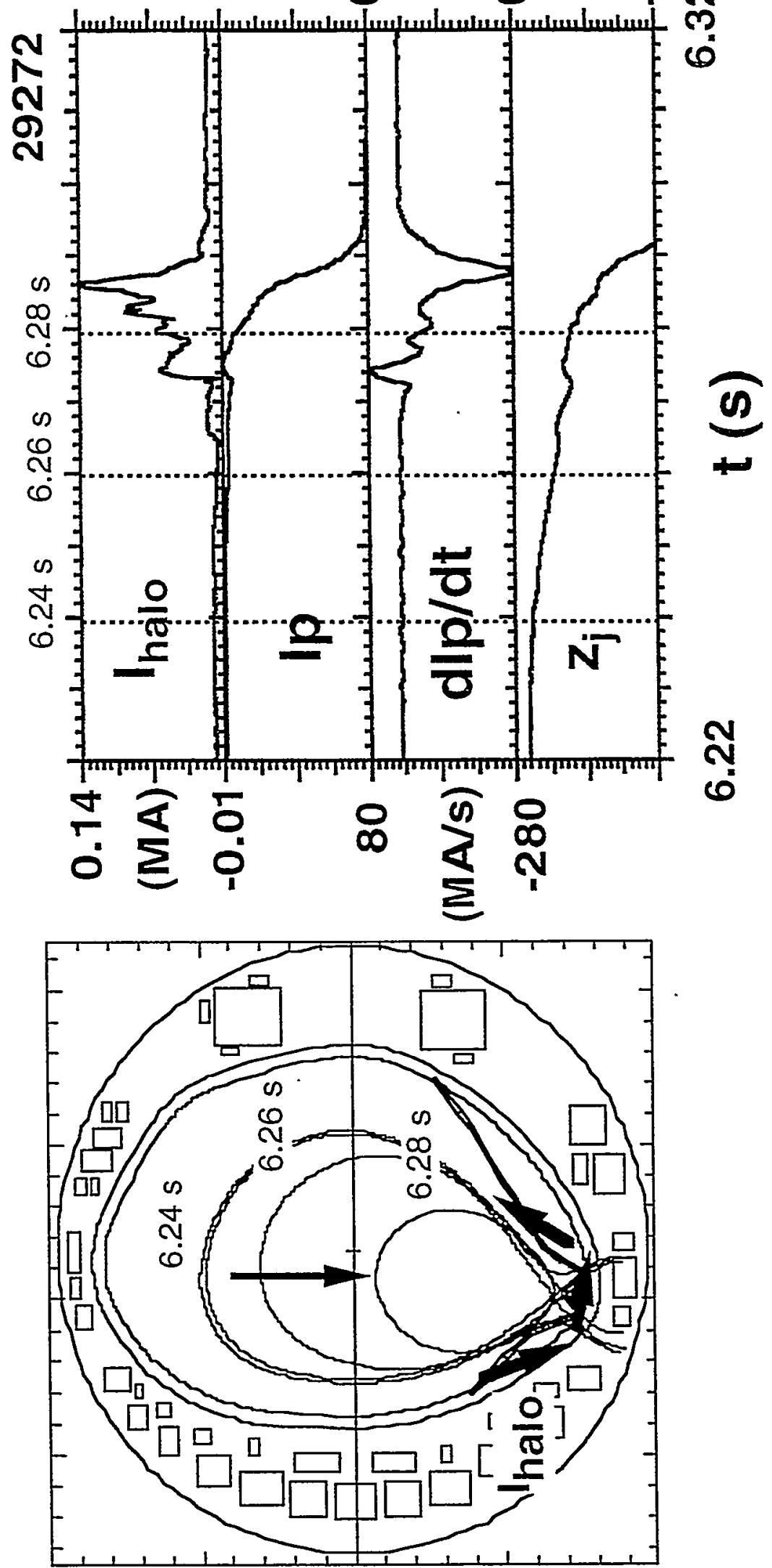
[Specification]

- Material of coiling wire : MI (Mineral Insulation)cable (1 $\mathrm{mm} \Phi)$

- Sheath : Inconel

- Working temperature : $<500^{\circ} \mathrm{C}$

- Cross section : $0.6 \mathrm{~m}^{2} / \mathrm{m}$

- Frequency Responce : $<5 \mathrm{kHz}$

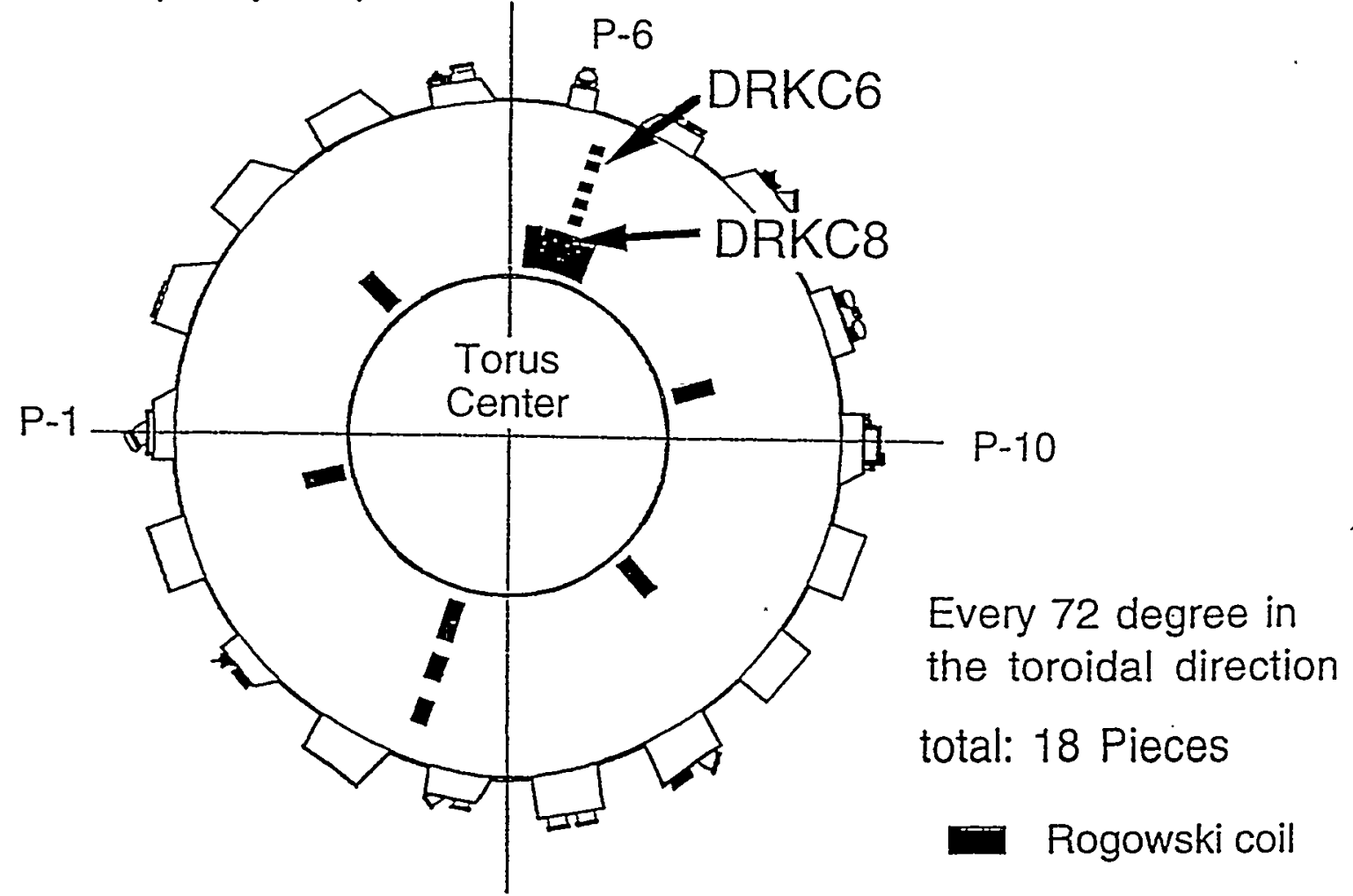

Top View of Vacuum Vessel

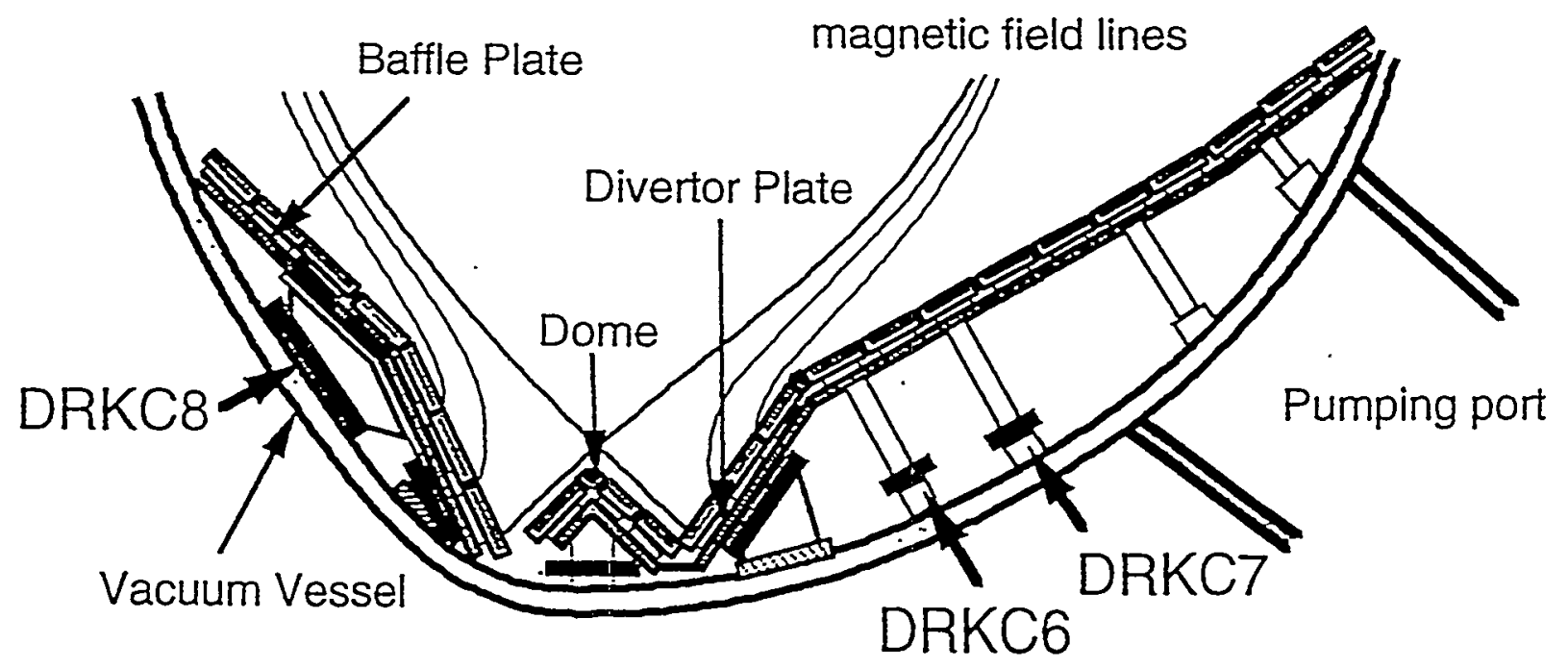

Side View of Vacuum Vessel

(P-6 section)

Rogowski coil 

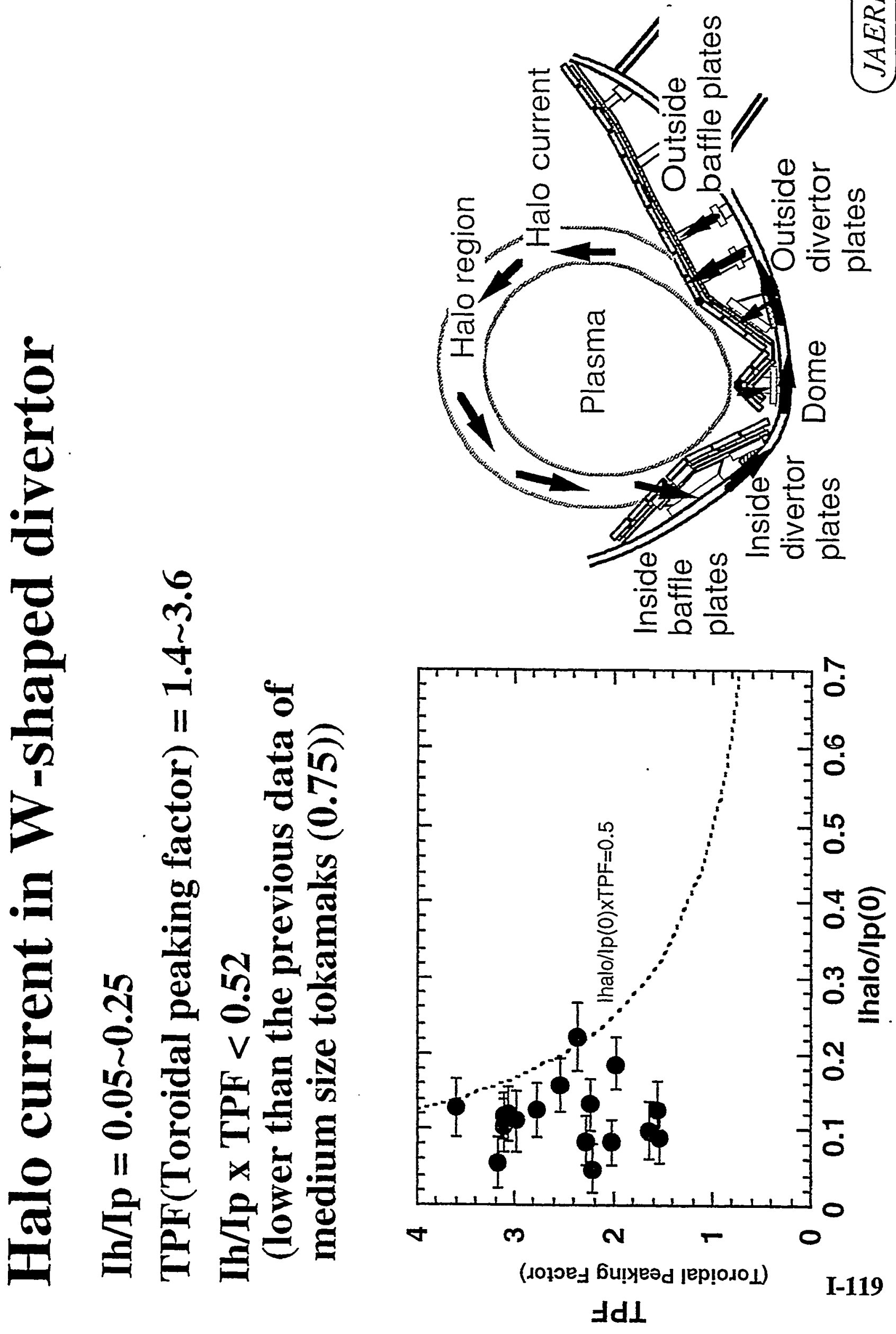


\section{Steady-state high performance with $\mathrm{W}$-shaped divertor}

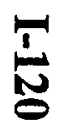

Steady-state ELMy H-mode for 9sec

No serious increase in recycling and carbon impurity

$\sim 22 \mathrm{MW}$ (NBI) x 9sec : 200MJ

Surface temperature of divertor tile exceeded $1000^{\circ} \mathrm{C}$

$1.5 \mathrm{MA} / 3.5 \mathrm{~T}$

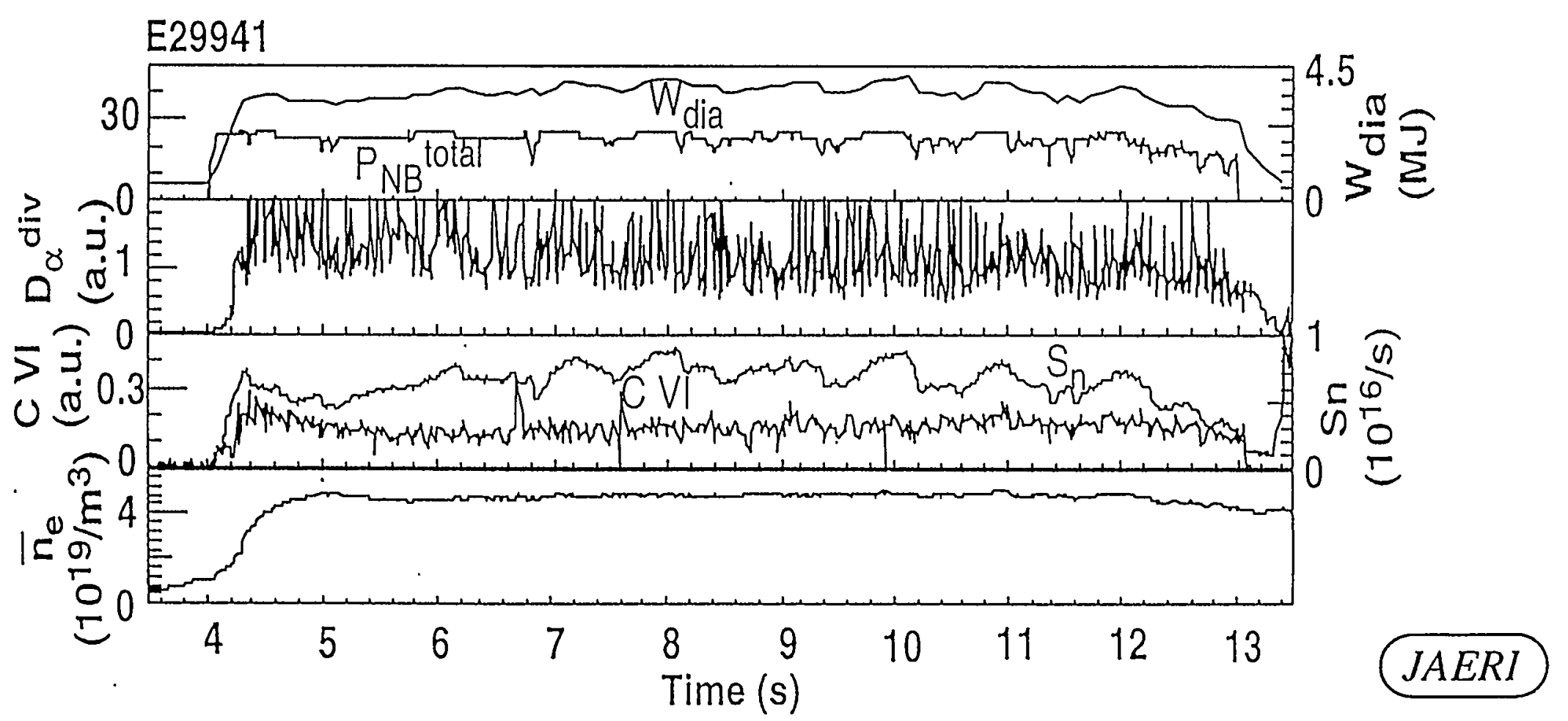




\section{Helium exhaust}

OHelium beam injection into ELMy H-mode.

$01.4 \mathrm{MA} / 3.5 \mathrm{~T}$

Helium exhaust was demonstrated with divertor pump ( argon frost cryopumps for He exhaust ).

$\tau_{\mathrm{He}}^{*} / \tau_{\mathrm{E}}=4$

( In ITER ELMy H-mode, $<8 \sim 15$ )

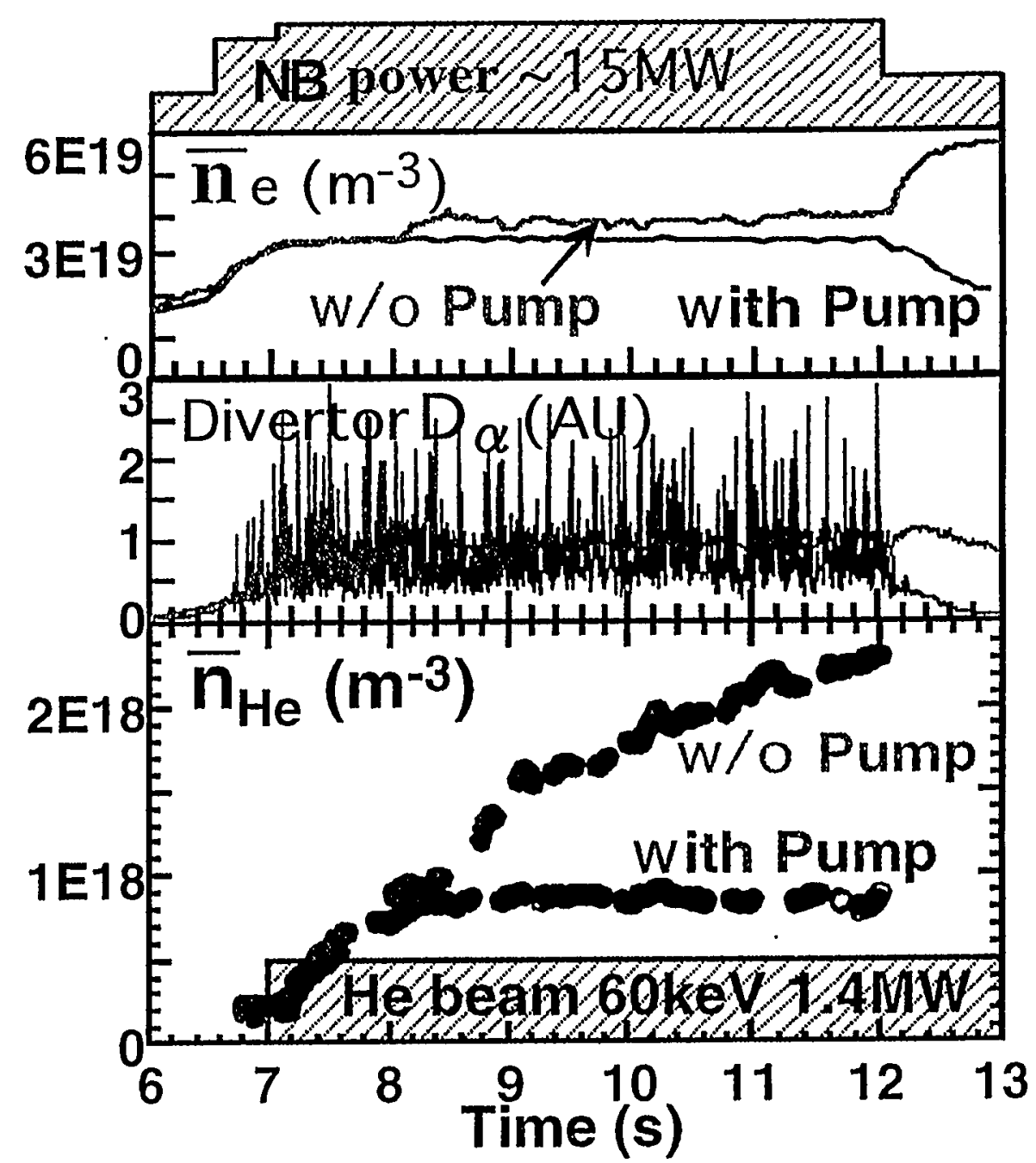

$\stackrel{T}{N}$ 


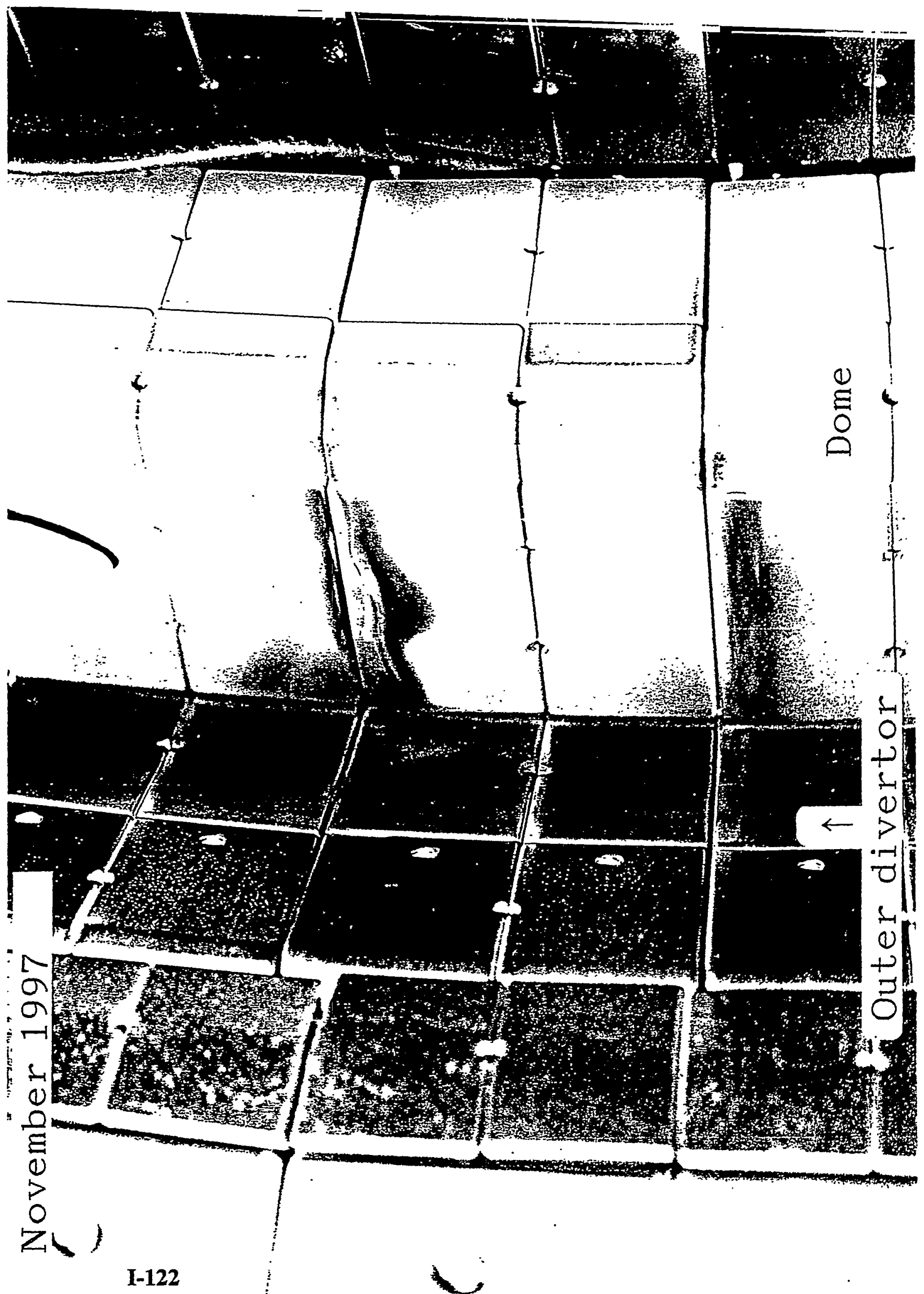




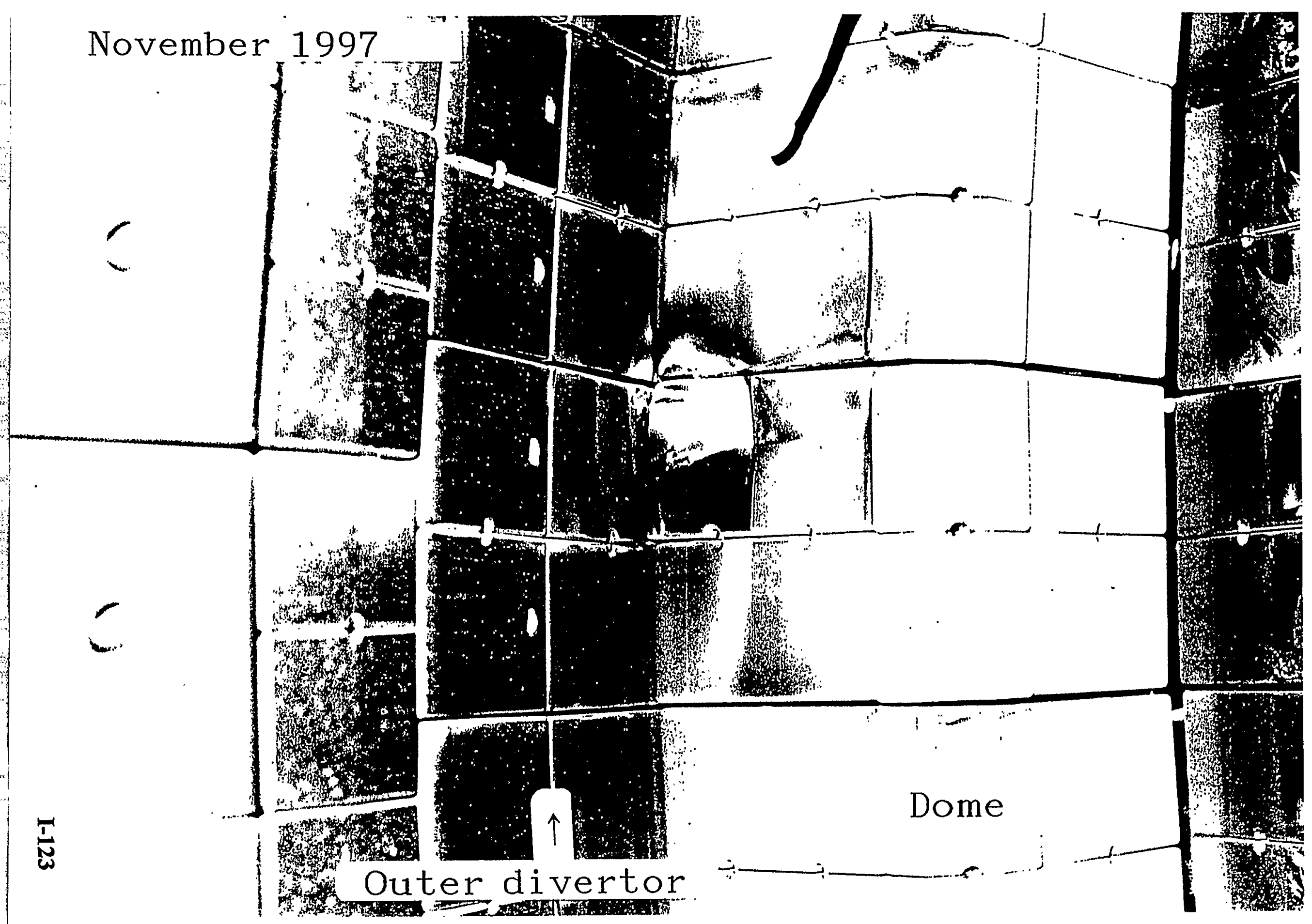




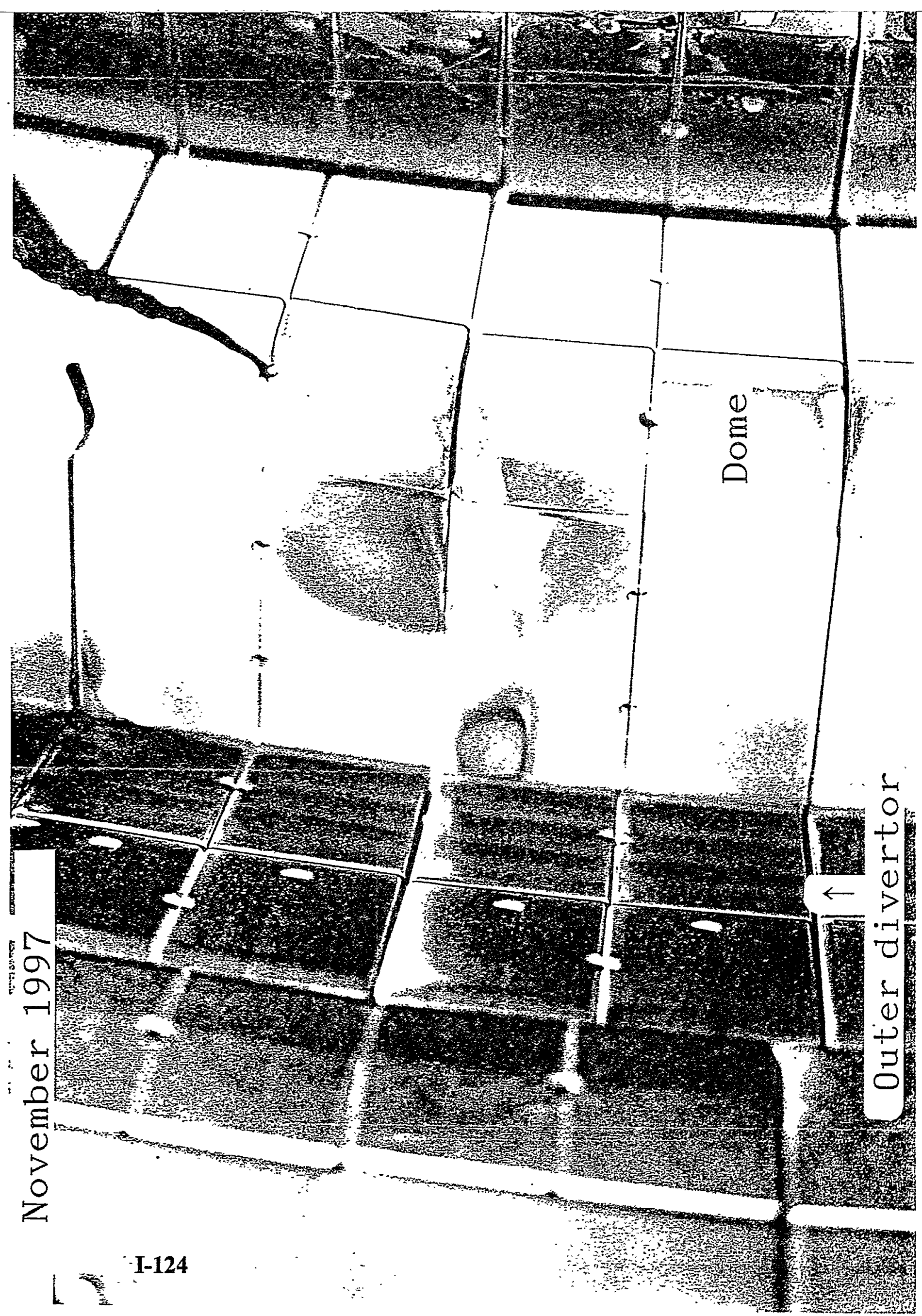




\section{Summary}

JT-60U divertor was modified to $\mathrm{W}$-shaped divertor CFC tiles were used for divertor target tile. Operations with intervals of 20 minutes are possible. Insulated structure was adopted for gas seal.

$\bigcirc$ Halo current $\quad \mathrm{Ih} / \mathrm{Ip}=0.05 \sim 0.25, \mathrm{TPF}=1.4 \sim 3.6, \mathrm{Ih} / \mathrm{Ip} \times \mathrm{TPF}<0.52$

Helium exhaust was successfully demonstrated with divertor pump.

Steady-state ELMy H-mode for 9s was observed.

Dome tile severely eroded.

Two tiles were broken due to thermal shock.

Thick deposition layer was observed on the inner divertor tiles

空 


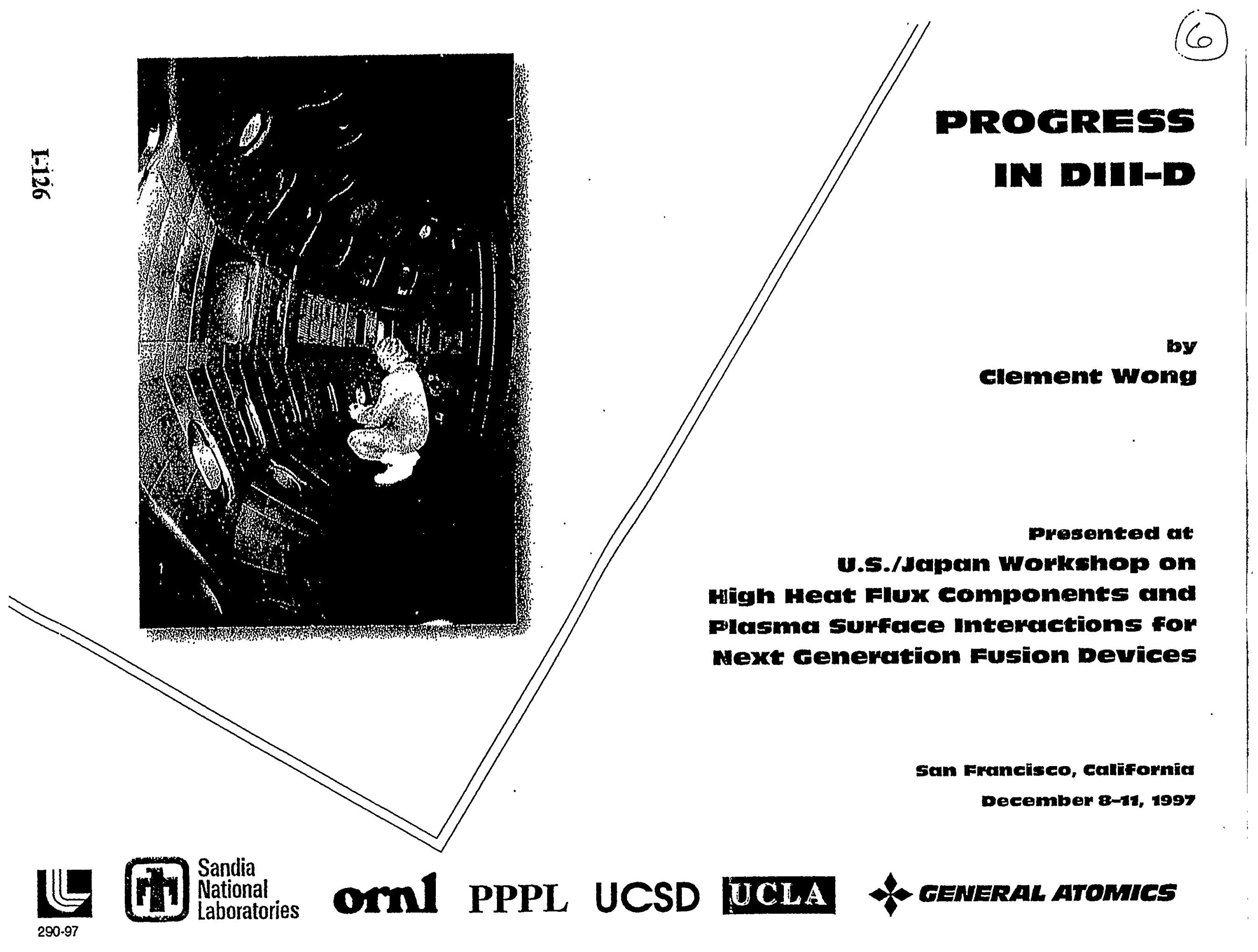




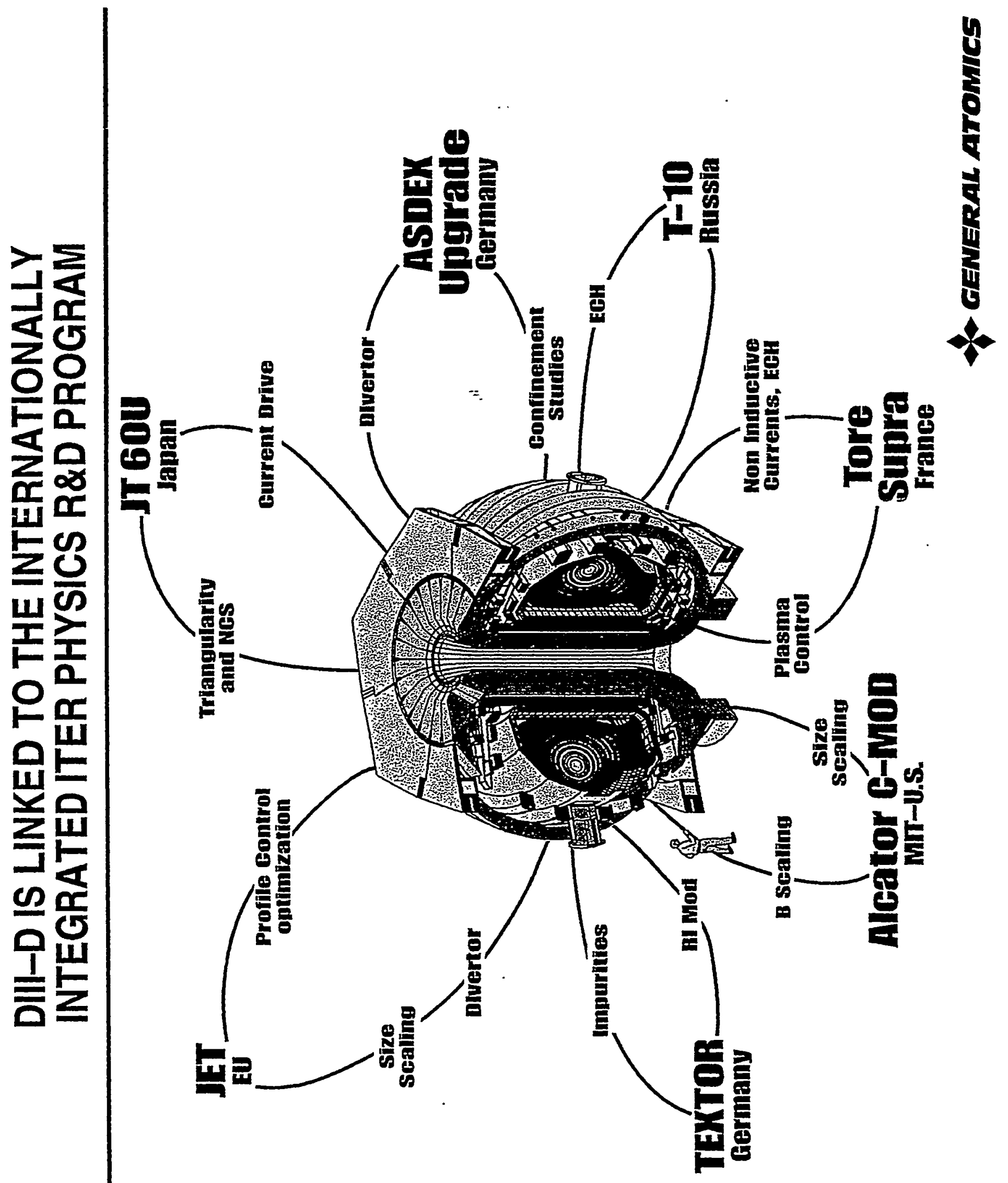



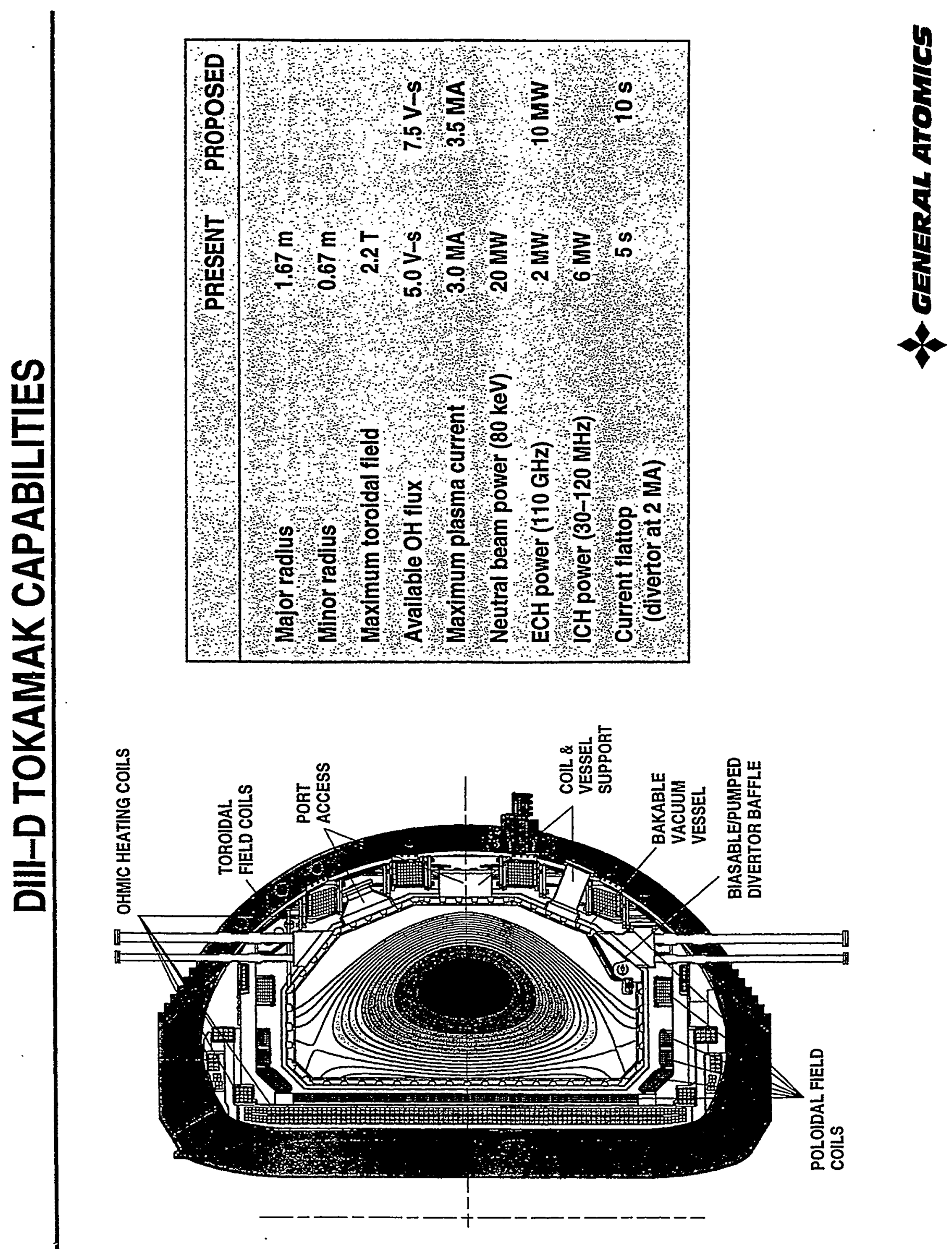


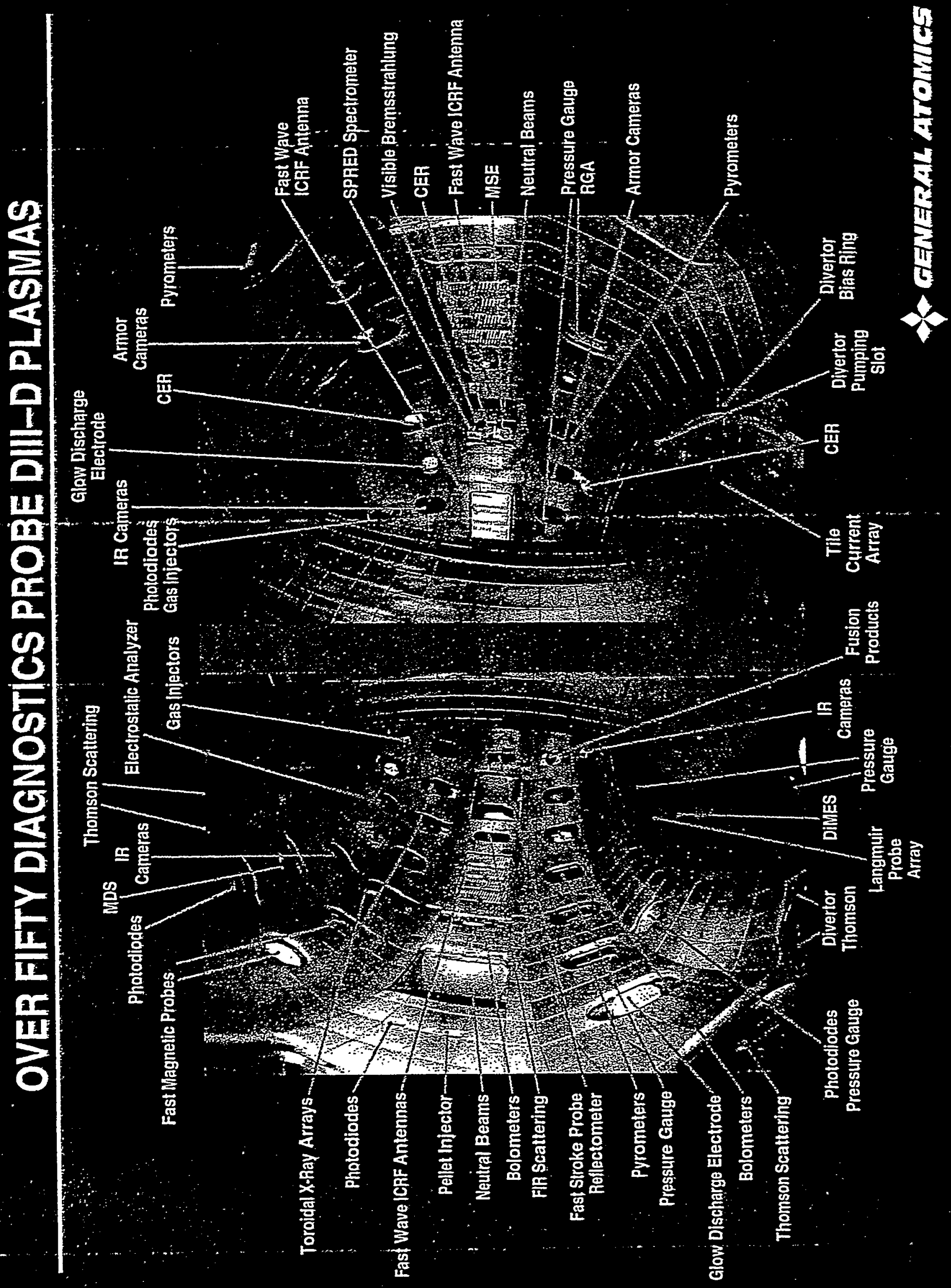


- Transport

- Developed H-mode edge pedestal scaling

- Triggered core transport barrier expansion and contraction

- MHD stability and descriptions

- Demonstrated disruption halo current dependence on vertical instability growth rate and mitigation with killer pellet injection

- Increased neoclassical tearing mode beta limit with $q(r)$ and $\delta$

- Studied wall stabilization and resistive wall modes

- Divertor and boundary

- Developed understanding of parallel energy transport and dissipation (convection and recombination)

- Achieved density control with pumped closed divertor

- Wave-particle

- Utilizing steerable ECH to test transport theories

- Demonstrated on-axis current drive 


\section{H-mode Pedestal and Plasma Performance}

- In stiff ITG-mode turbulent transport models, the core transport coefficients depend strongly on the plasma edge parameters which enter as a boundary condition.

o ITER $H$ and $Q$ increase with $T_{i}^{\text {PED }}$

(Results at right from IFS/PPPL model, taken from "Memorandum on Confinement Projections," FESAC ITER Confinement Reviews, M.Kotschenreuther and W. Dorland.)
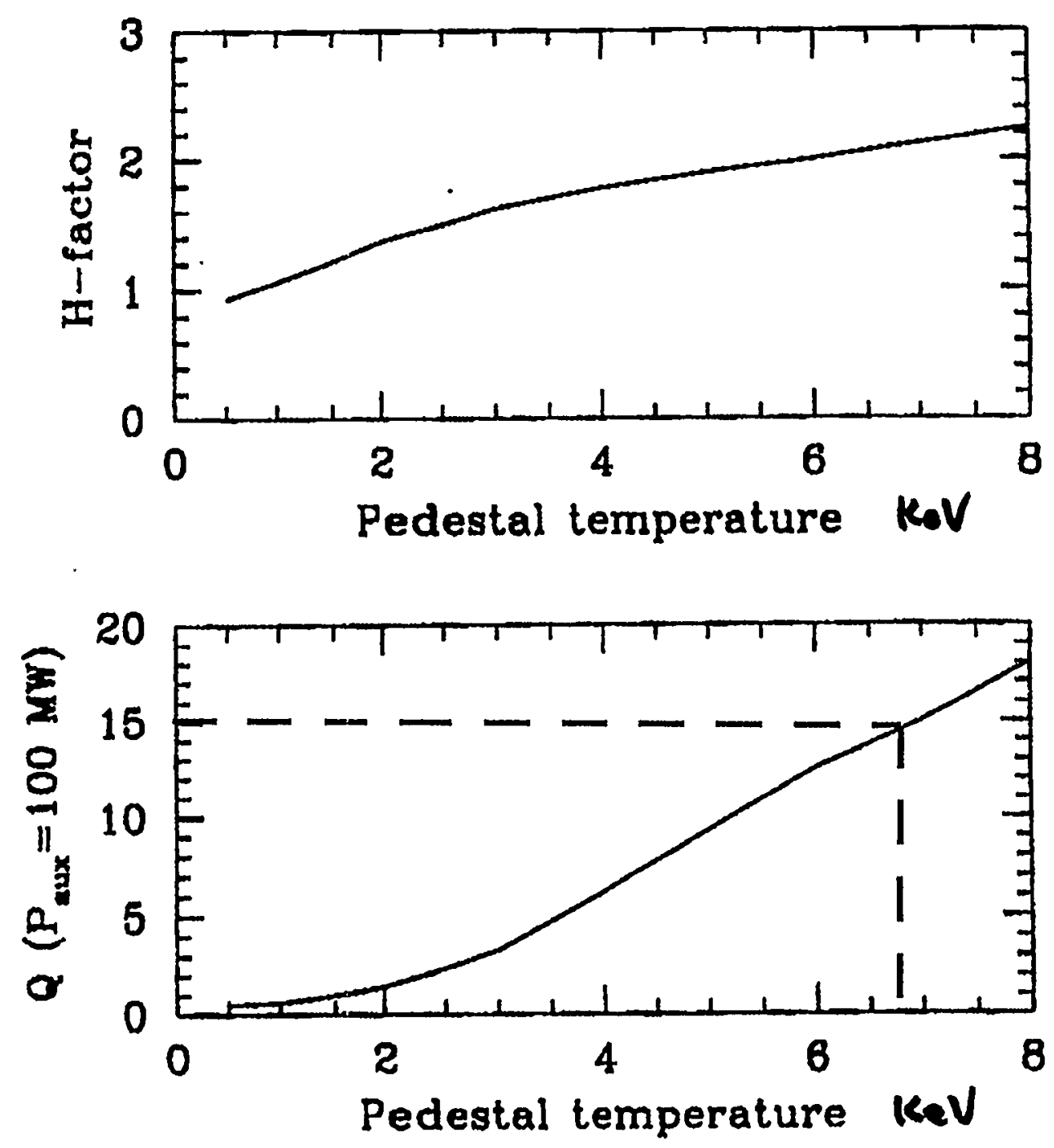

GENERAL ATOMICS 

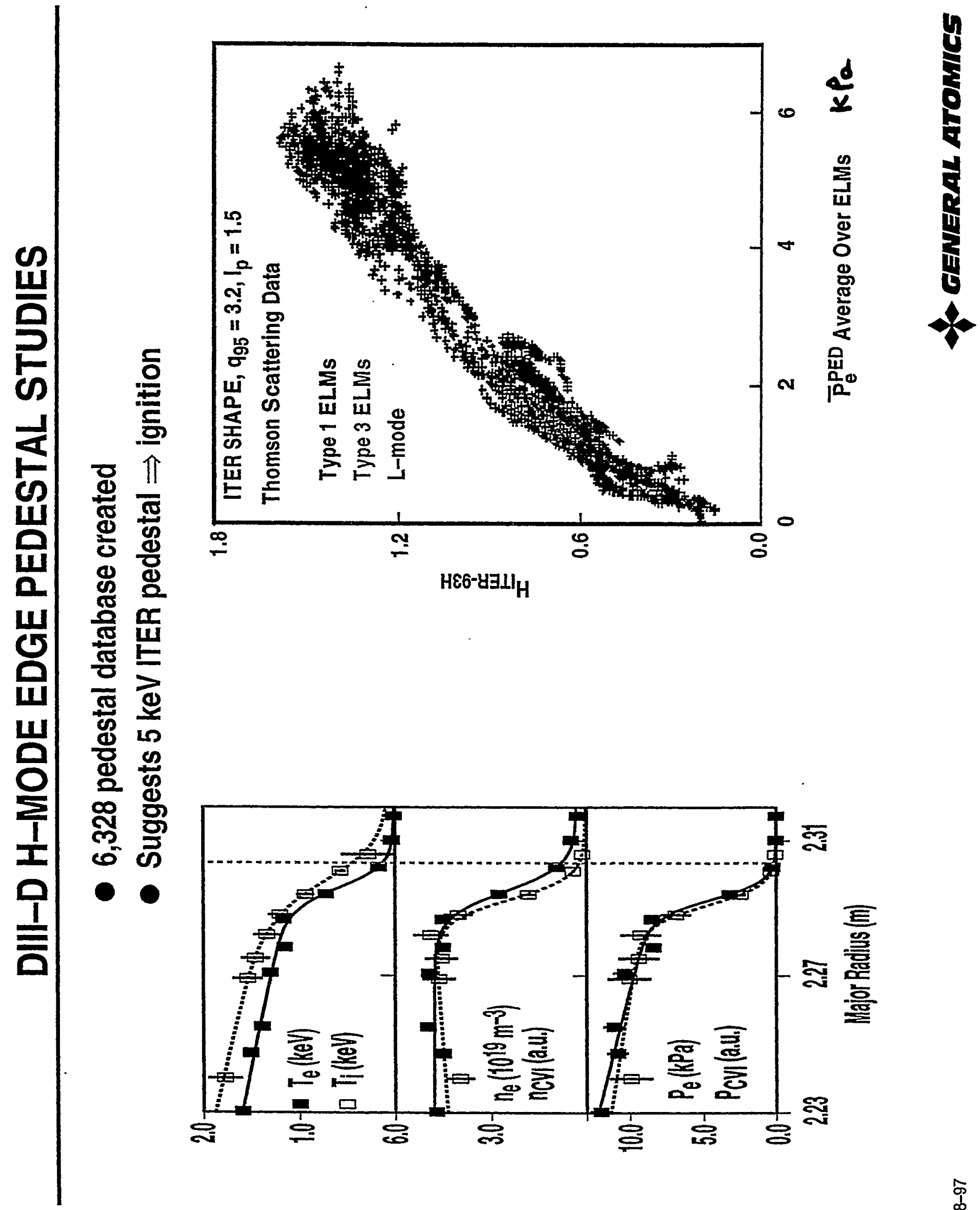


\section{Halo Current and Toroidal Peaking Factor Reduced by \\ Neon and Argon "Killer" Pellet Injection into VDE Disruption}

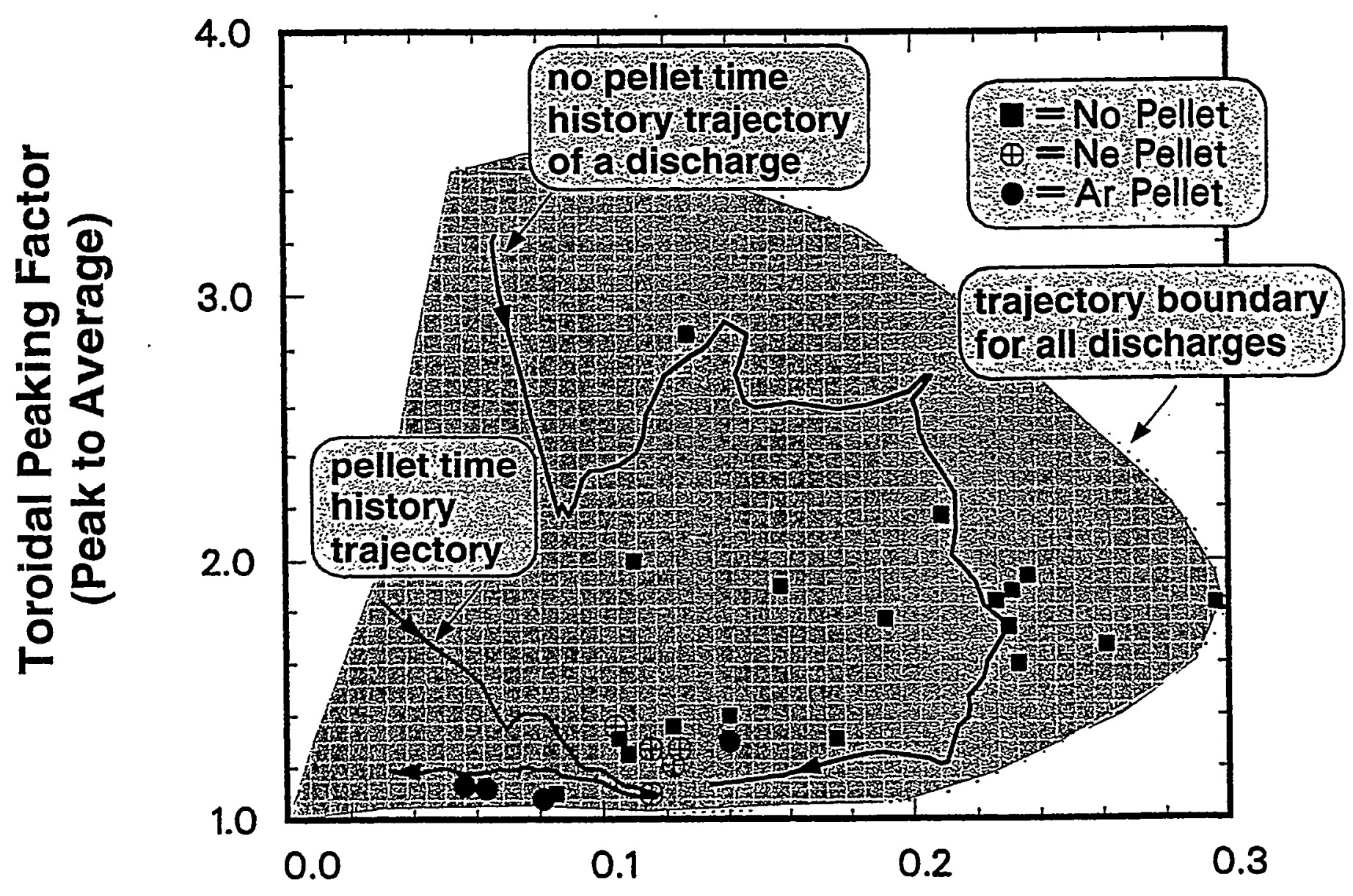

\section{Halo Current / Plasma Current}

Pure neon pellets reduce the vessel loading by a factor of $4-5$

Pure argon pellets reduce the vessel loading by a factor of 8-10 


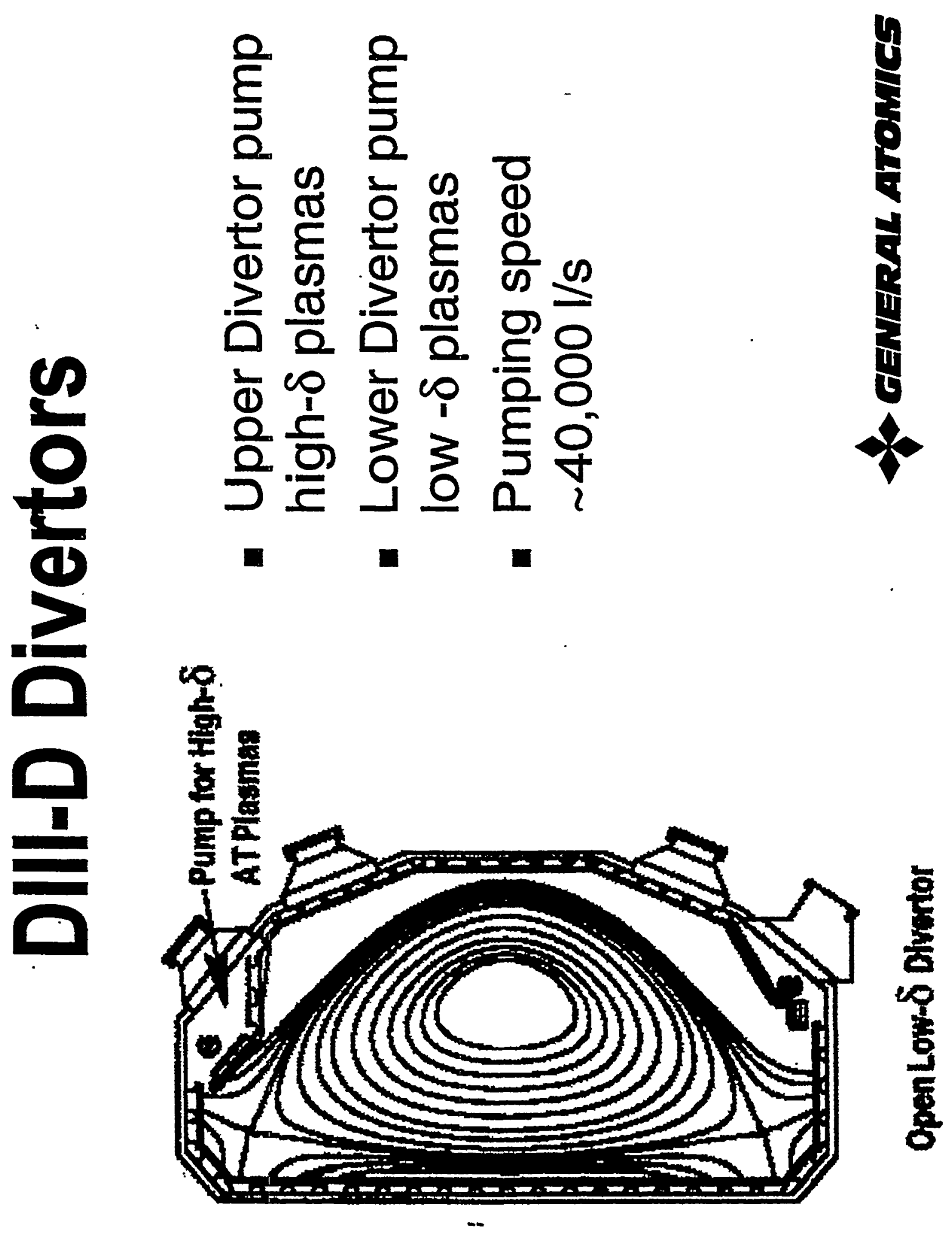




\section{DIII-D DIVERTOR PLAN FOR PARTICLE CONTROL}

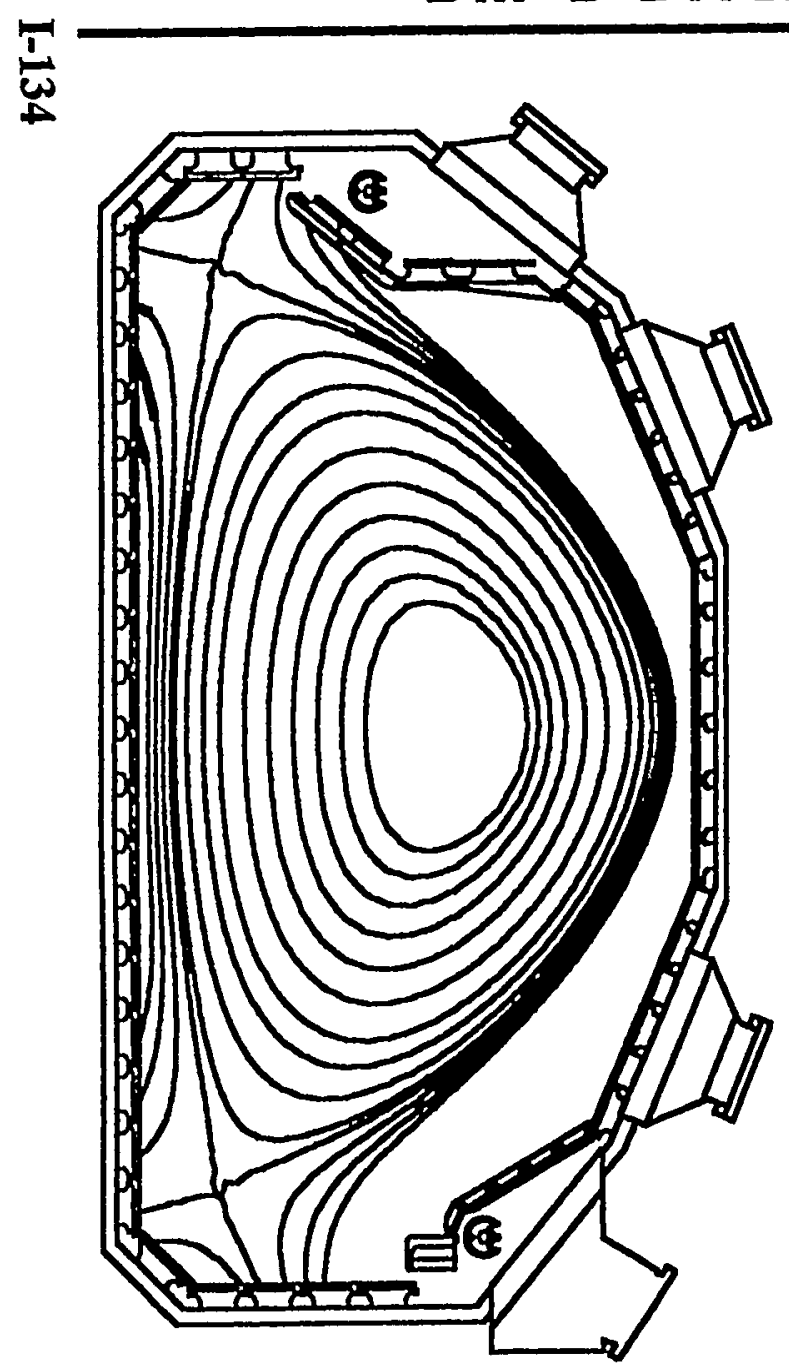

1997

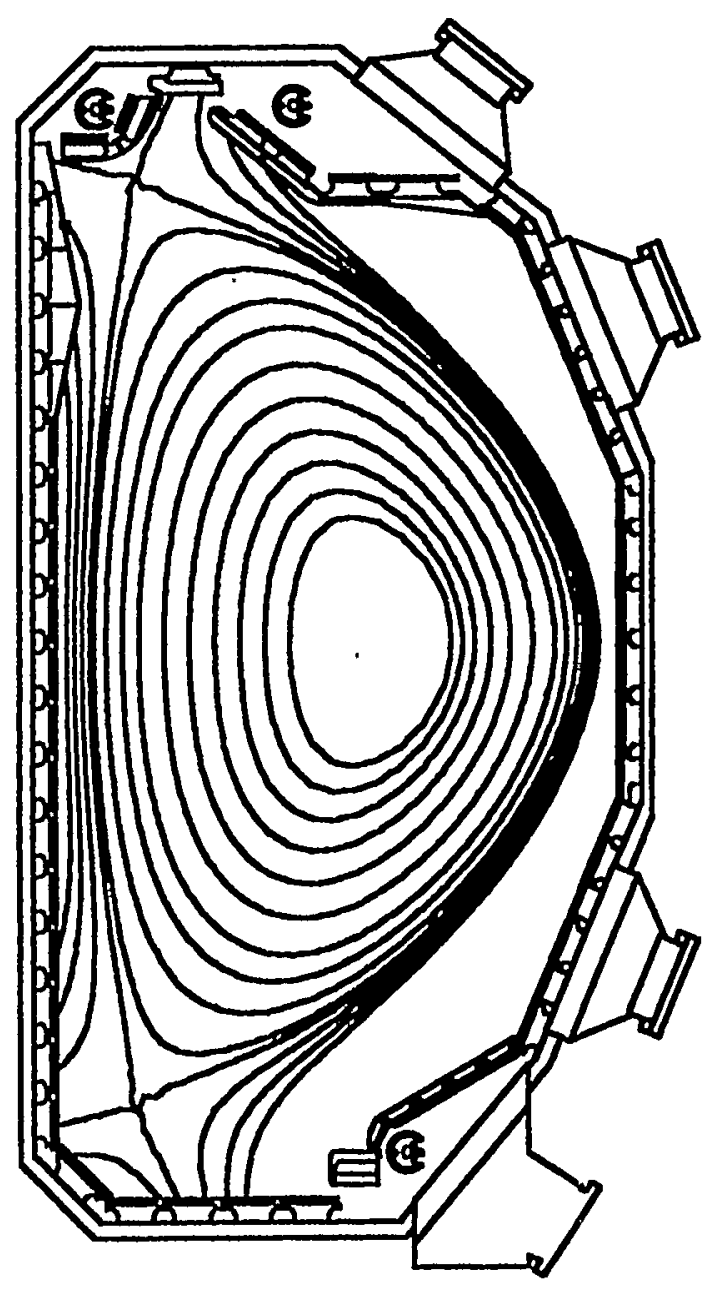

1999

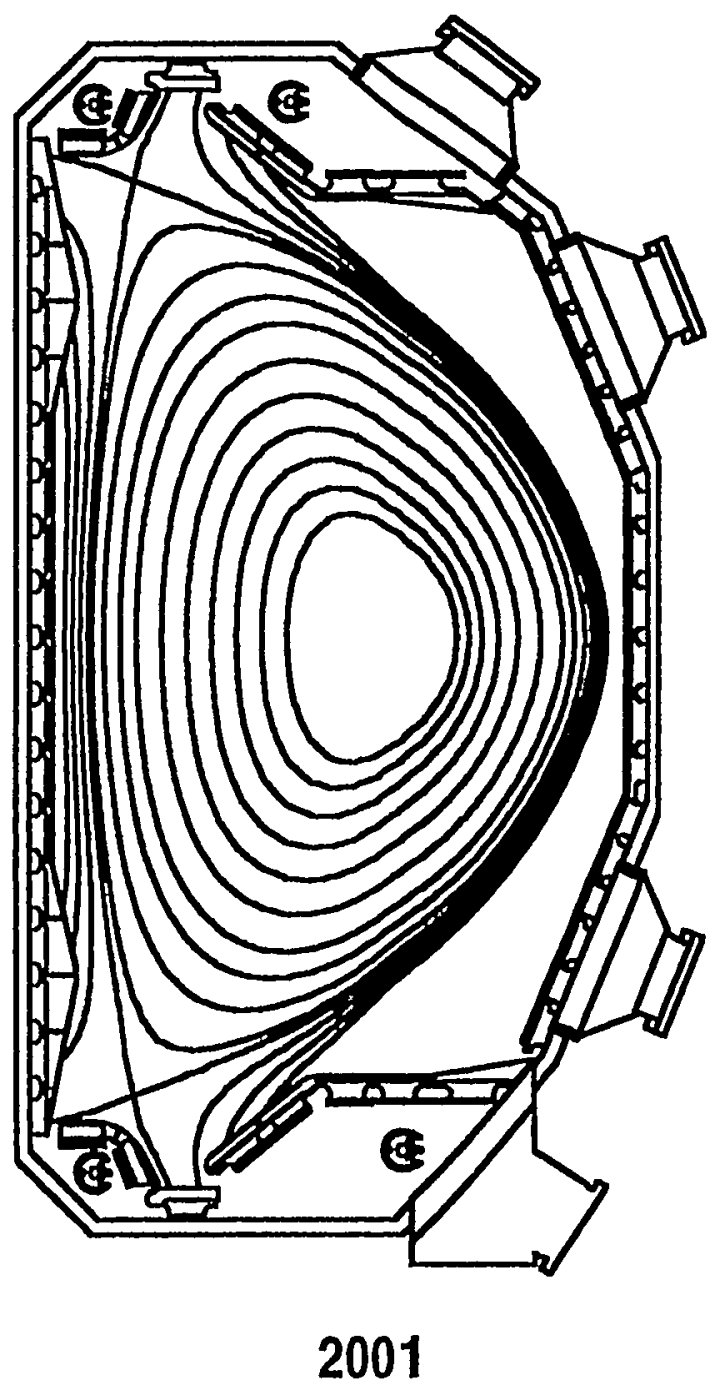

* general atomics 


\section{Results from the New High- $\delta$ Upper Pump and Baffle on DIII-D}

Presented for the AT+D Campaign on DIII-D by S. L. Allen Lawrence Livermore National Laboratory

- $\mathrm{n}_{\mathrm{e}}$ control achieved in high- $\delta$ Plasmas

$\Rightarrow n_{\mathrm{e}} / I_{\mathrm{p}} \sim 2.5$ (ELMing H-mode), similar to low- $\delta$

$\Rightarrow$ Impurity density similar in low- $\delta$, high- $\delta$, and pumped

- Open vs. Closed divertor comparisons have shown:

$\Rightarrow$ Reduction in core ionization and midplane $\mathrm{H} \alpha$

$\Rightarrow$ Exp. results are similar to UEDGE+DEGAS predictions

$\Rightarrow \tau_{\mathrm{e}}$ similar

- Experiments with high- $\delta$ DN plasmas in progress

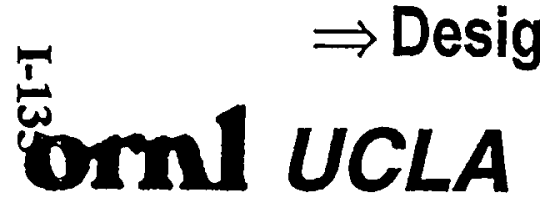




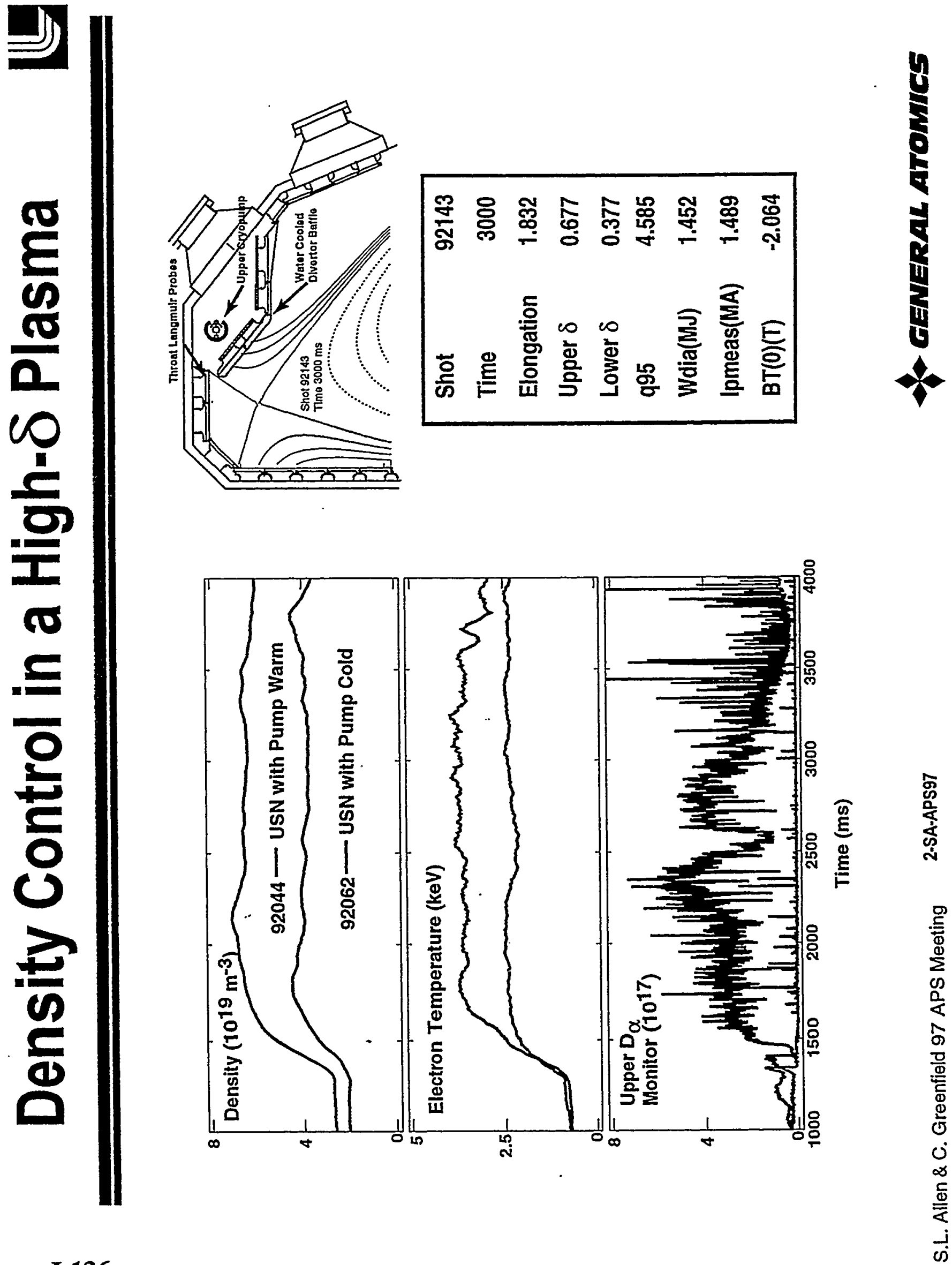




\section{Low $\mathrm{T}_{\mathrm{e}}$, High $\mathrm{n}_{\mathrm{e}}$ in Radiative Divertor}

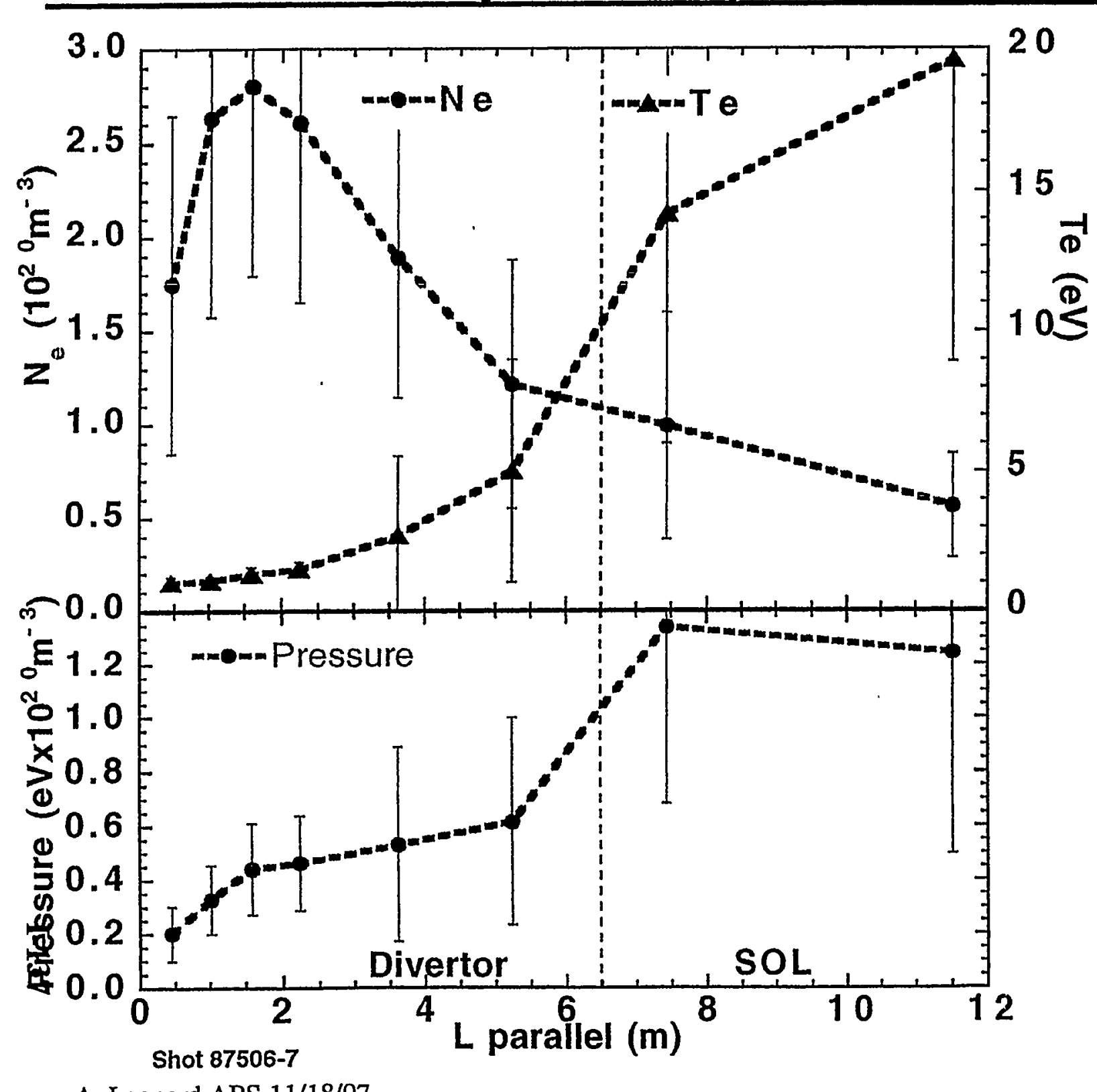

- Very low $T_{e}, 1-3 \mathrm{eV}$ through much of divertor.

- Very high density, $\sim 3 \times 10^{20} \mathrm{~m}^{-3}$.

- Large pressure drop near $X$-point, gradual decline to divertor plate. 


\section{Summary of Results}

$\underset{\infty}{\stackrel{T}{\omega}}$
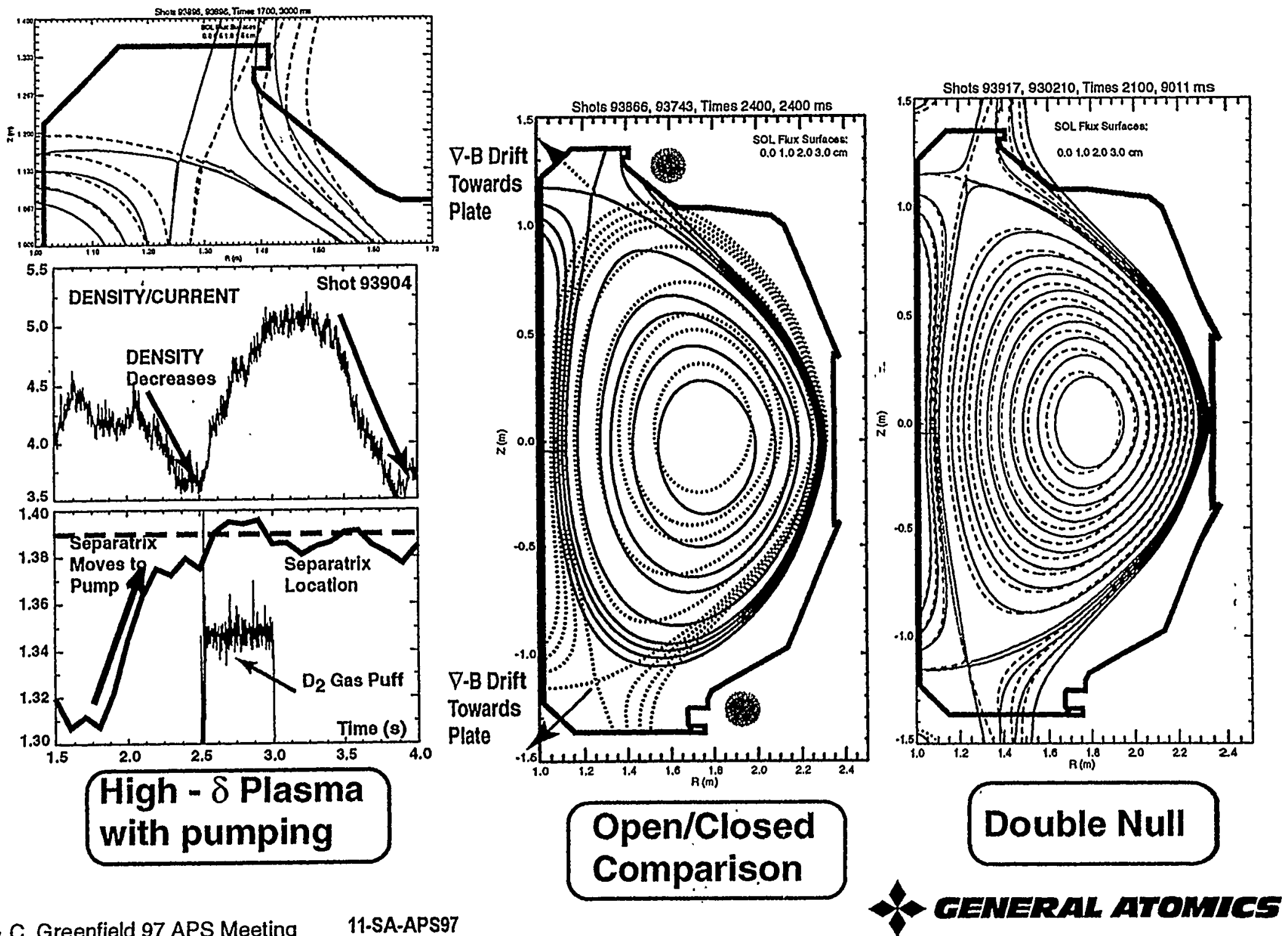

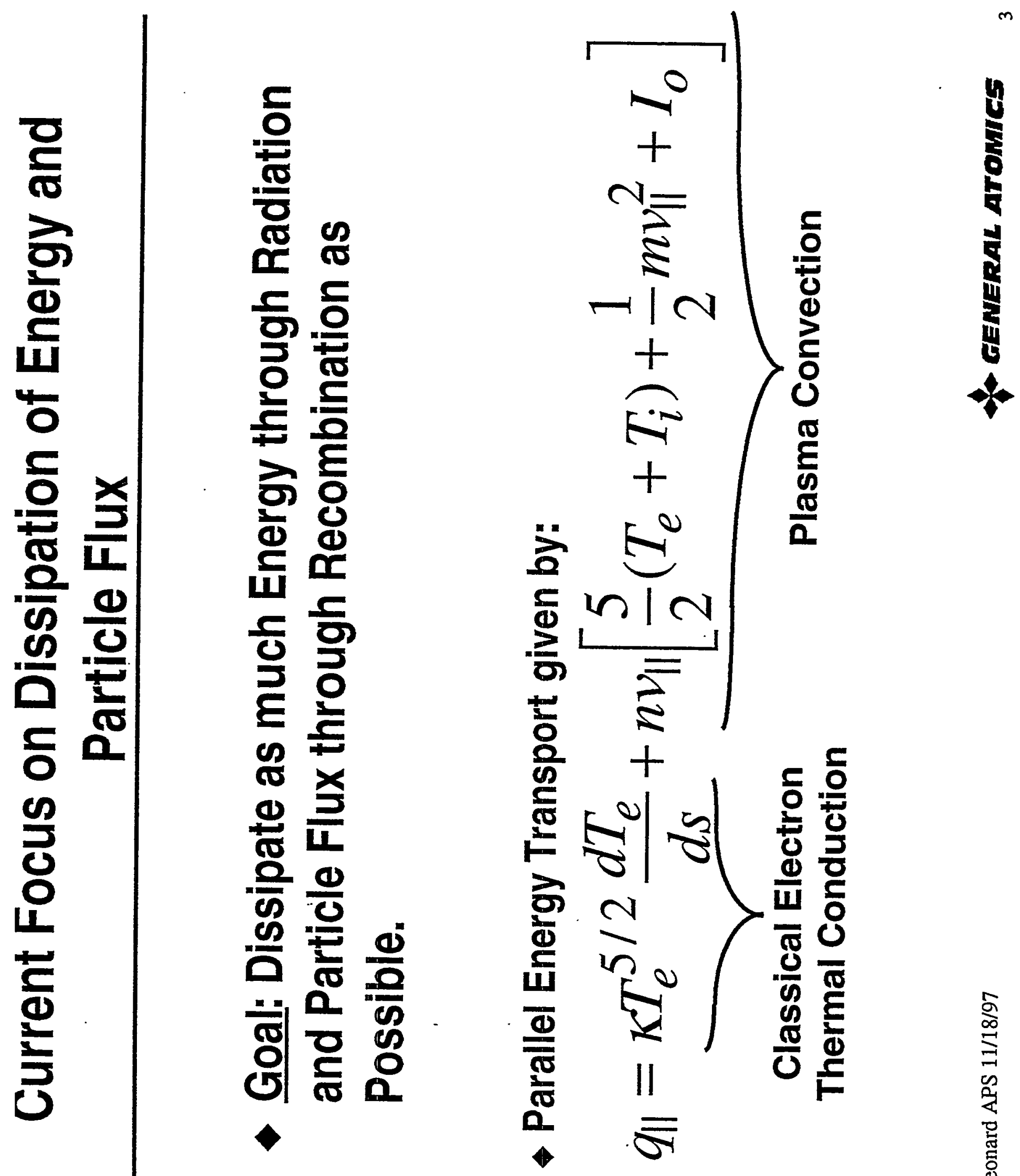

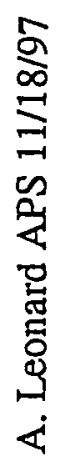




\section{DIII-D Achieves Heat Flux Reduction without Highly}

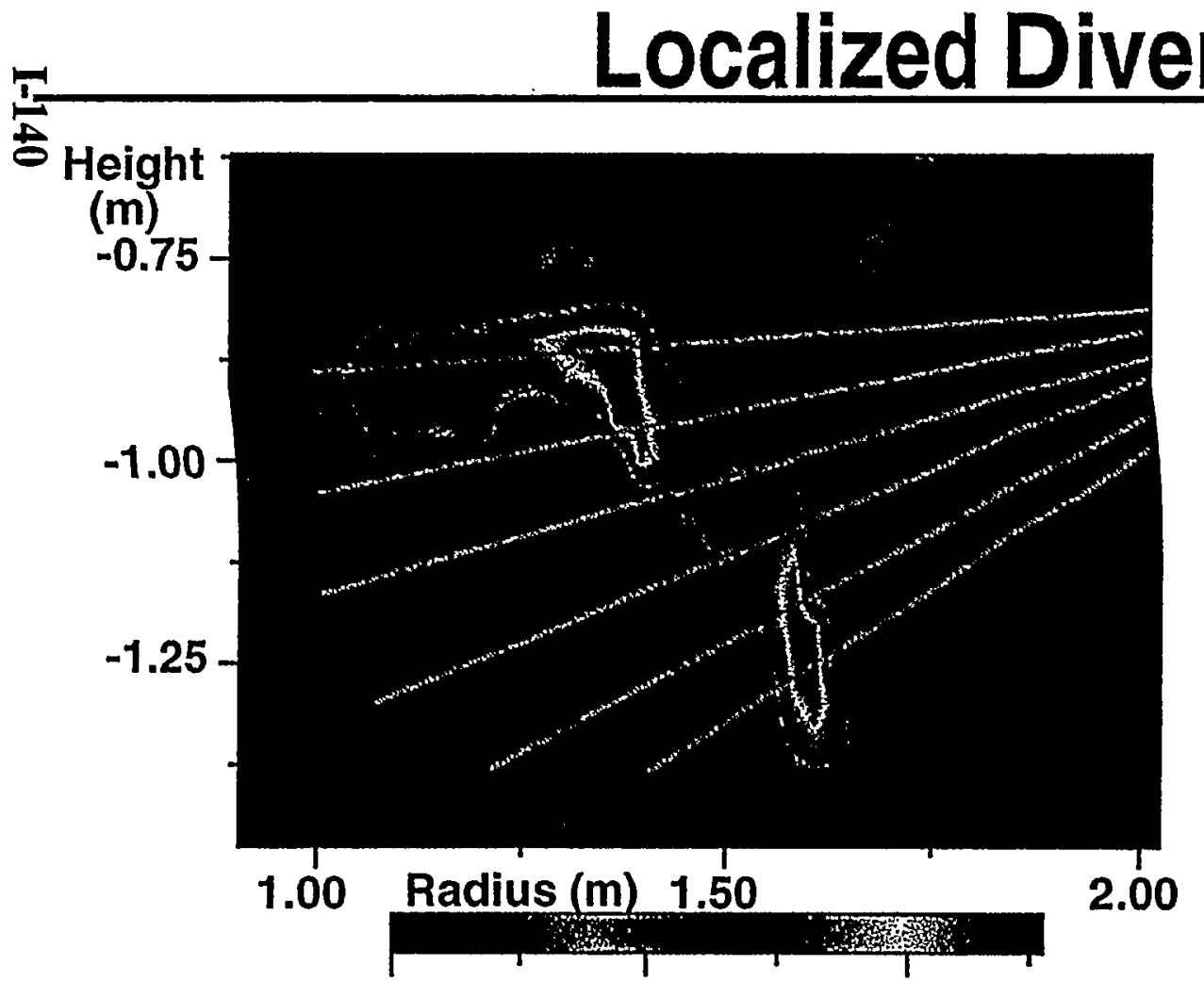

- 6 bolometer chords from X-point to divertor target; less than 2:1 variation in divertor radiation.

- Factor of 3 peak heat flux reduction.

- Carbon the dominant impurity.

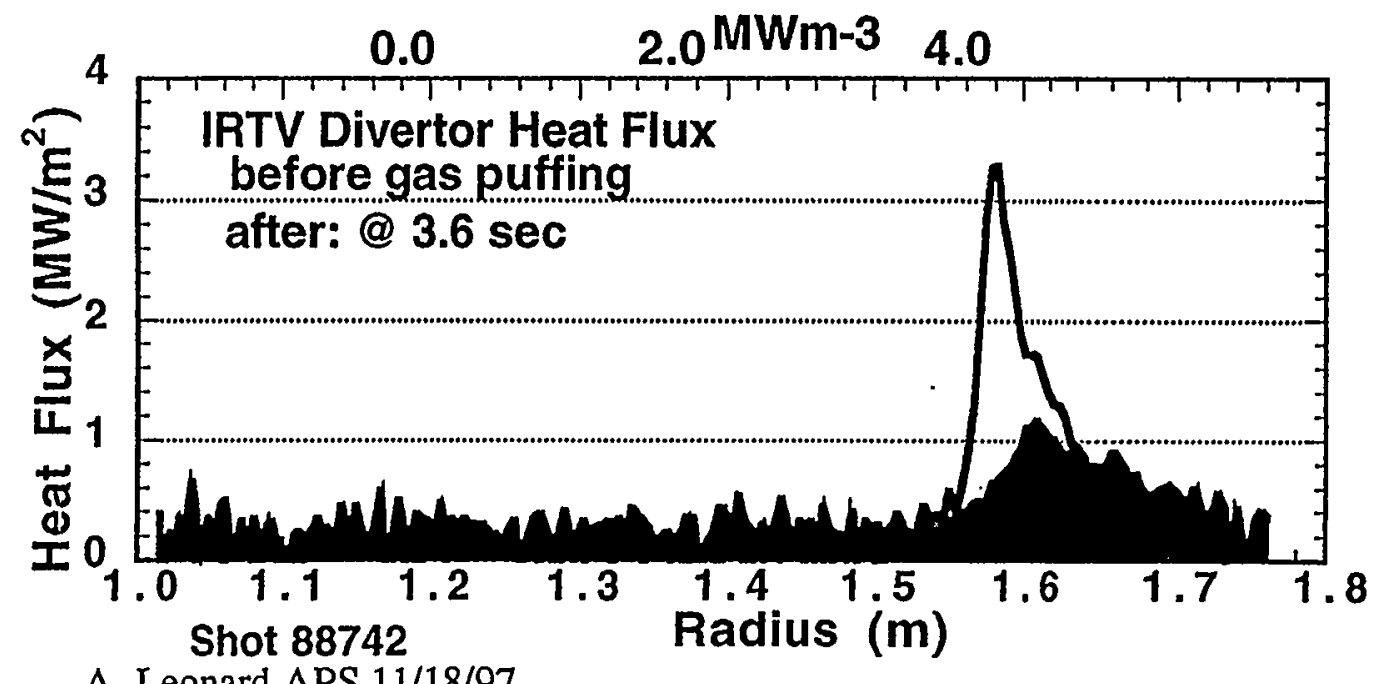

A. Leonard APS 11/18/97 


\section{Carbon and Deuterium Radiation Spread through Divertor}
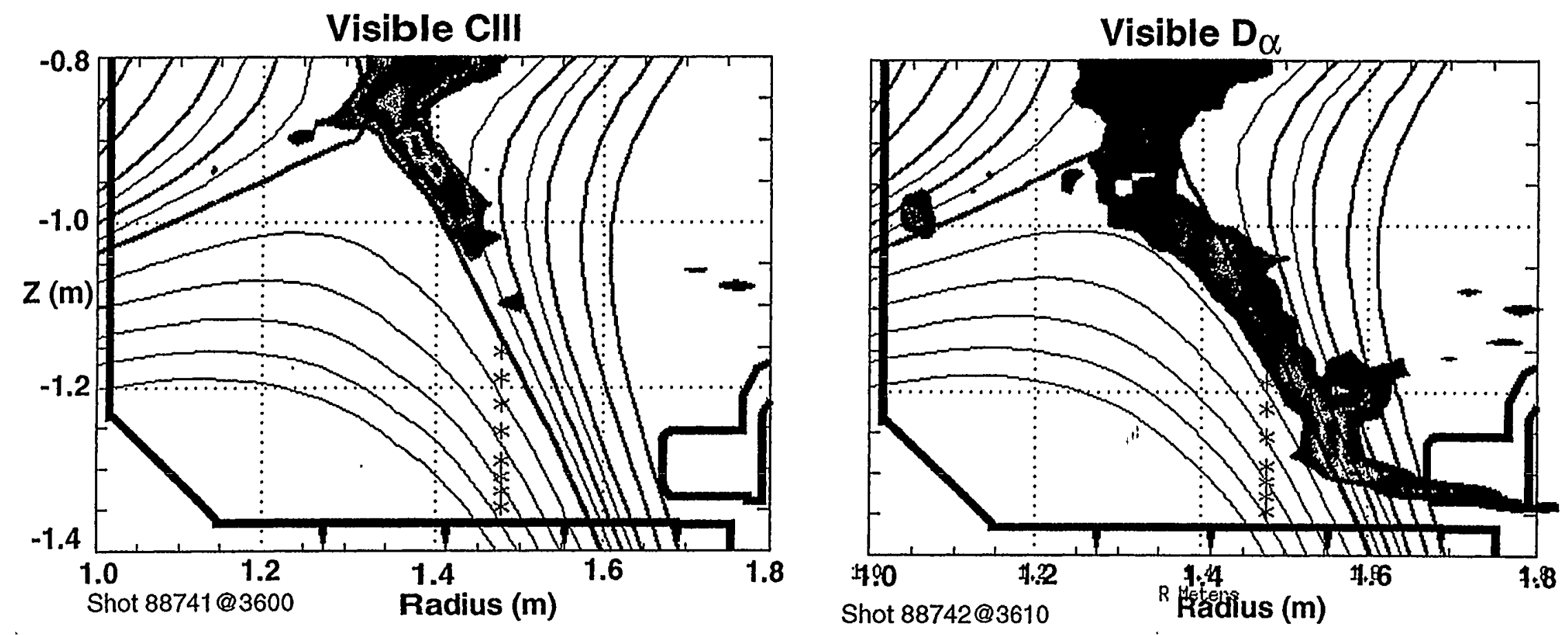

- VUV spectroscopy indicates CIII peaks where $\mathrm{T}_{\mathrm{e}} \sim 10 \mathrm{eV}$.

- Both Carbon and Deuterium radiation spread over larger region than

$\underline{\underline{z}}$ conduction model would indicate. 


\section{$T_{e}$ in Radiative Divertor cannot Support Observed Energy Flux through Conduction}

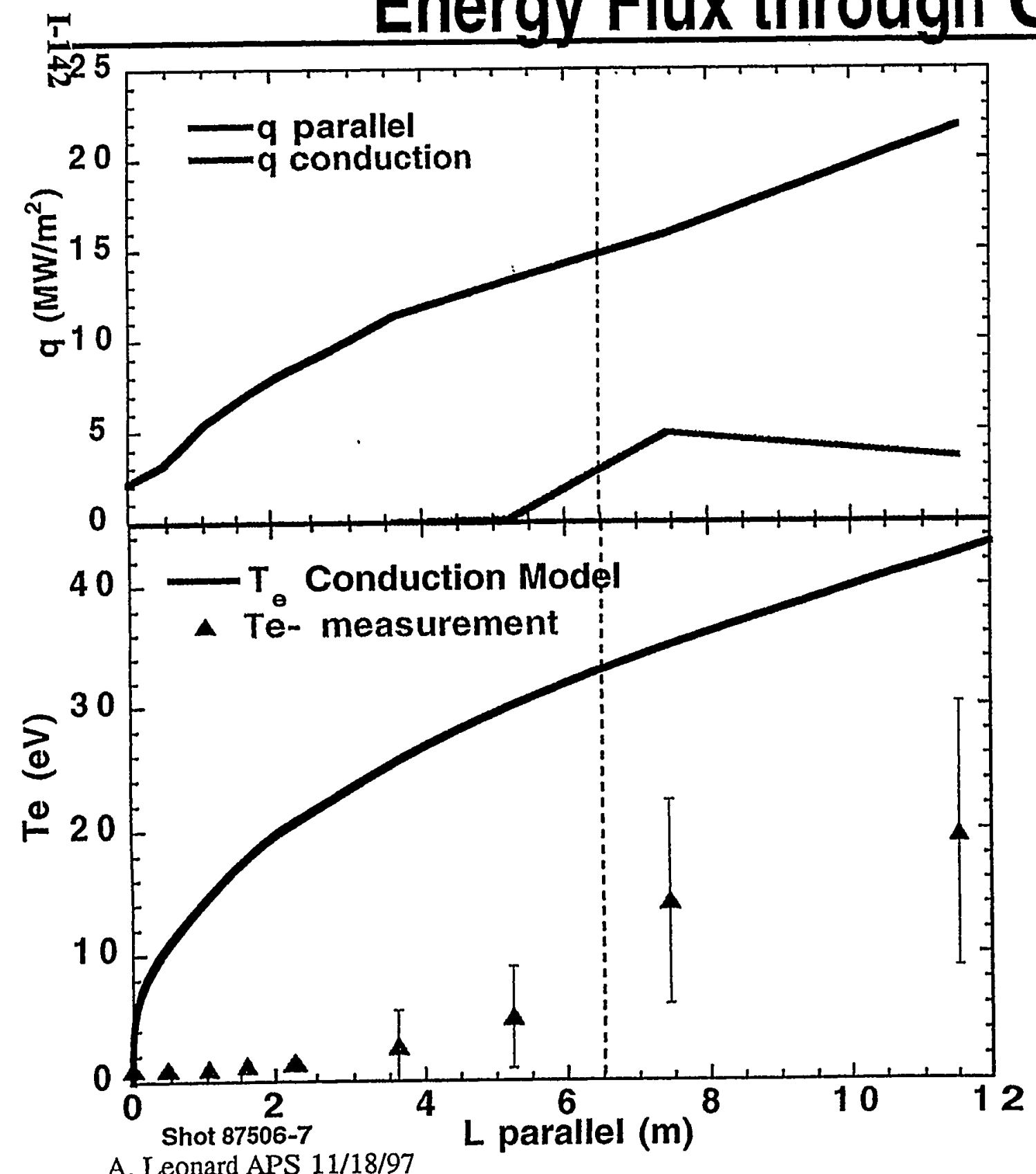

- About $90 \%$ of power flowing into divertor is radiated.

- Measured $T_{e}$ can support very little energy flux.

- Region of 8-12 eV, $\Delta \mathrm{L} \sim 1-2 \mathrm{~m}$, larger than implied by conduction. Volume consistent with total Carbon Radiation.

- Large volume of cold plasma, $\mathrm{T}_{\mathrm{e}}<2 \mathrm{eV}$. 


\section{Conclusions}

- Power balance coupled with $n_{e}$ and $T_{e}$ profile measurements allow study of parallel energy transport processes in the divertor and SOL.

- Plasma filow can greatly expand the plasma volume at high density and low temperature for efficient low $\mathbf{Z}$ impurity radiation and plasma recombination.

- It is possible to exceed limits on radiation and recombination predicted by conductive transport models.

- New 2D diagnostics are begining to measure the plasma flow and recombination profiles in the divertor.

$\frac{5}{\omega}$ 


\section{Divertor Detachment in DIII-D Helium Plasmas}

D.N. Hill, Lawrence Livermore National Laboratory

and the DIII-D Divertor Group

- Motivation

- Partially Detached Divertor Operation (PDD) is the reference scenario for power handling in ITER

- All divertor experiments show peak heat flux reduction with gas puffing.

- Scaling to ITER or other future high power density tokamaks requires understanding the underlying physics 


\section{Helium Operation Helps Identify Relevant Atomic Processes Associated with Divertor Detachment}

Likely steps to detachment

1. Gas puffing drives radiation $\Uparrow$

2. Increased radiation drives $T_{\text {ediv }} \Downarrow$

3. Ionization moves upstream

4. Convection carries remaining energy in divertor

5. High $n_{e}$ low $\mathrm{T}_{\mathrm{e}}$ allow volume recombination

6. Recombination reduces plate ion current

\section{How helium should be different}

No chemical sputtering: carbon radiation $\Downarrow$

Higher density to produce same radiation

Longer $\lambda_{\text {mip }}$ yields detachment at higher $T_{\theta}$

No molecules

Less recombination $\Rightarrow$ less drop in $I_{\text {pate }}$ 

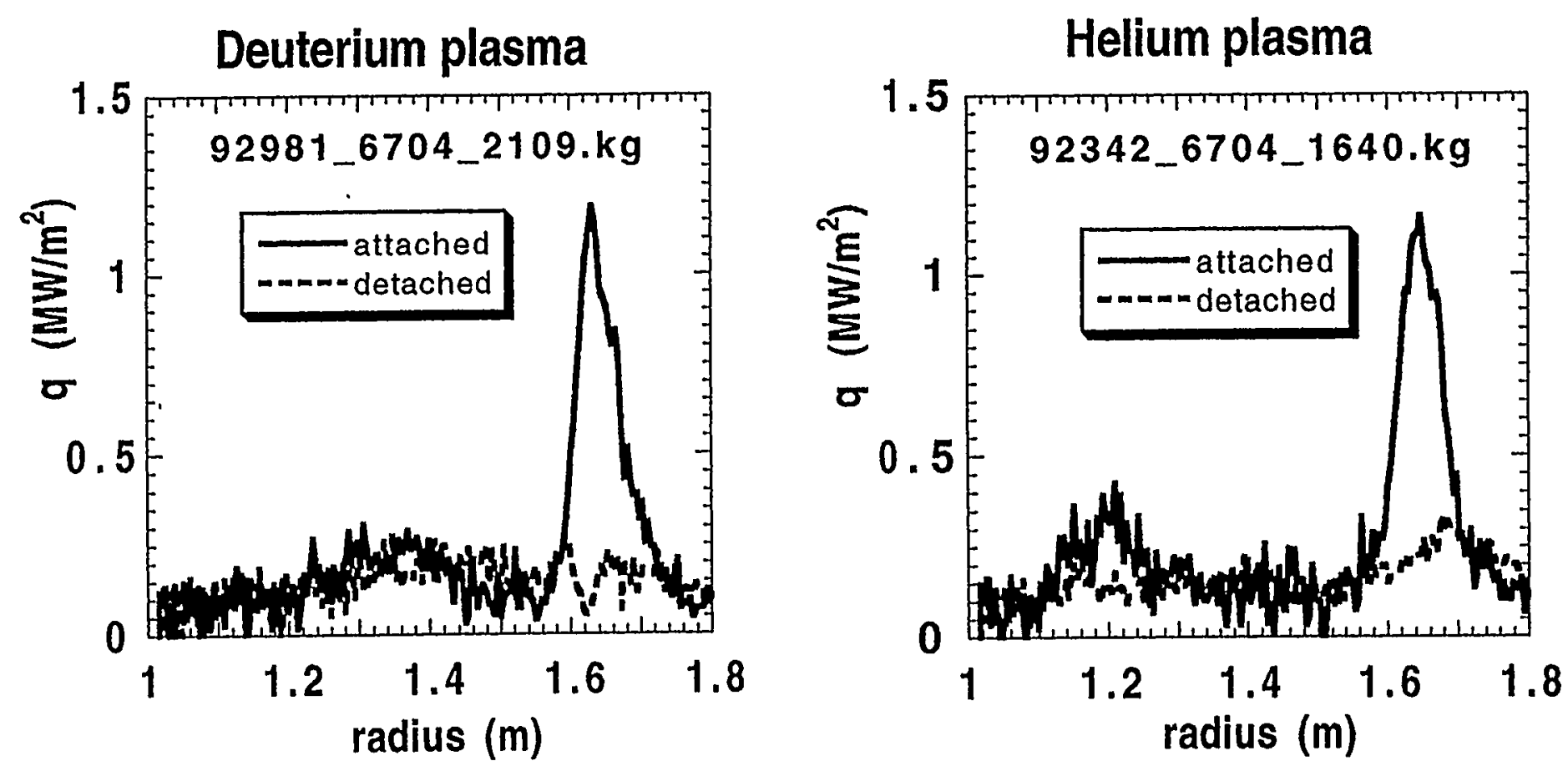

- Lower single-null, $P_{\text {beam }}=2 M W, H$-mode in deuterium, L-mode in helium

- Peak heat flux reduced by a factor of four

- ELMs and radiation produce most of residual. 
Radiation Profiles in Helium Show A Maximum Near X-point, as in Detached Deuterium Plasmas
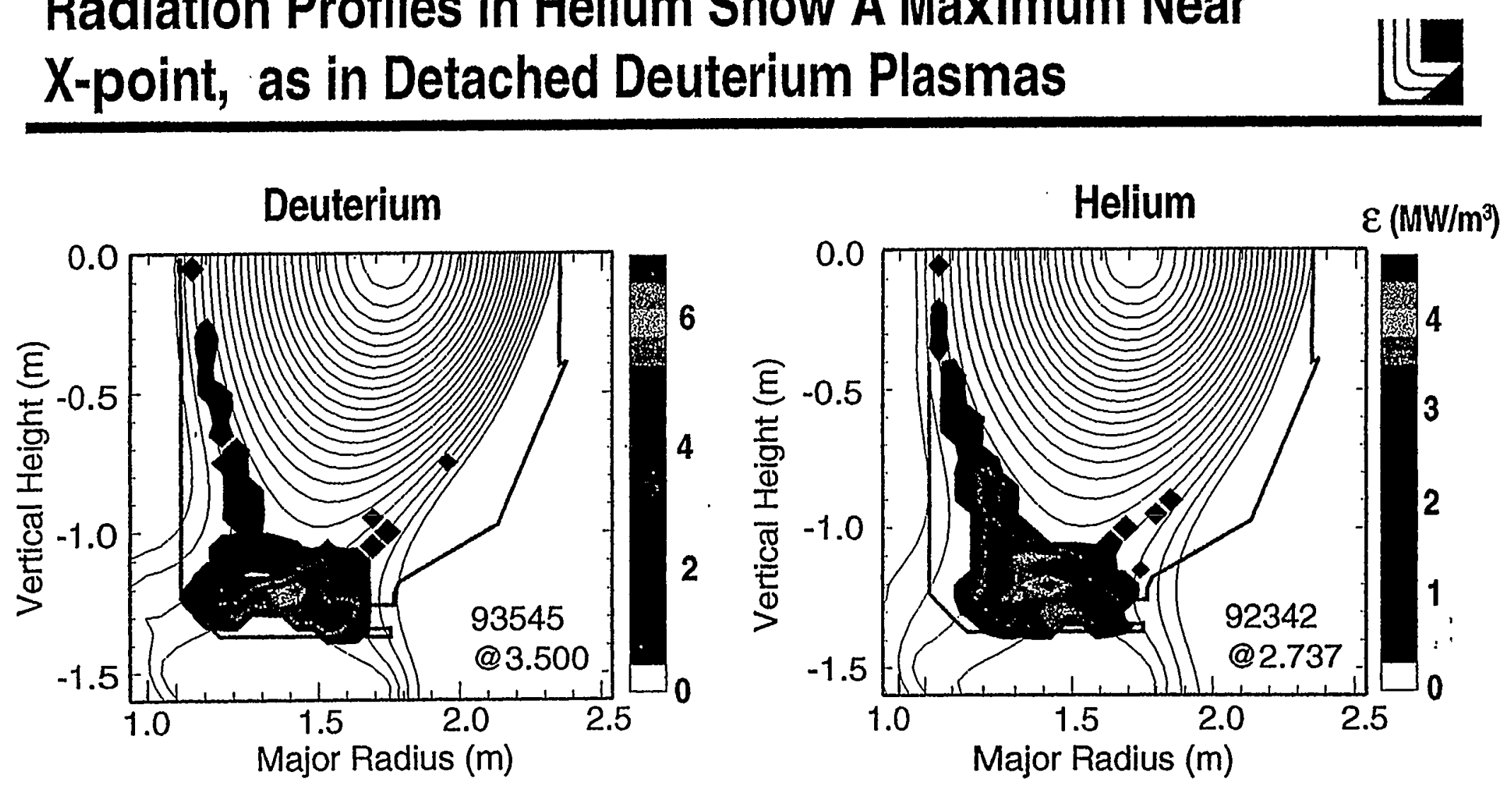

- Both plasmas at similar density, but $D^{+}$plasma has $2 x$ more input power.

- Both plasmas have emissivity peaked near x-point after detachment.

$\underset{5}{5}$

- Helium plasma has more distributed radiation. 


\section{CIII emission is reduced by a factor of at least 5 in helium detached plasmas.}

- Distribution of $\mathrm{C} \mathrm{III} \mathrm{is} \mathrm{different} \mathrm{in} \mathrm{helium} \mathrm{plasma.}$

- C III 465nm filter FWHM = 3nm may pick up He Il emission at $468.6 \mathrm{~nm}$

- Reduction in carbon emission is larger than infered from these images.
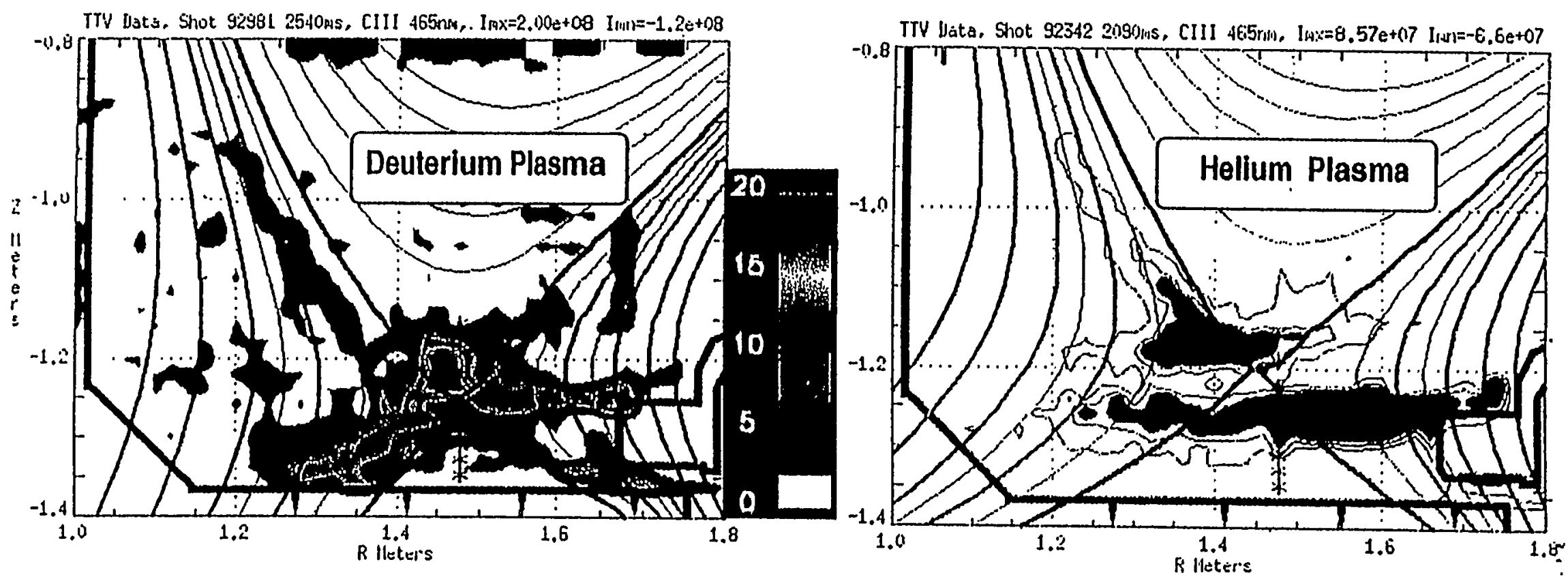

C III images reconstructed from a tangentially viewing filtered TV system. 


\section{Summary}

- Helium operation lowers carbon concentration by a factor of four or more

- core and divertor content both reduced, especially during helium puffing

- suggestive of chemical sputtering effect, but may be due to different confinement times in the core or different screening effects

- A radiative divertor with significant heat flux reduction can be obtained in helium plasmas: helium replaces carbon as the main radiator

- The behavior of the divertor plasma is similar to that of deuterium plasmas

- similar threshold density

- similar radiation distribution

- similar heat flux reduction and profiles, but no drop in ion flux

- similar divertor density and temperature in the detached state. 


\section{PROGRESS IN DIII-D KEY RESULTS PRESENTED}

- H-mode edge pedestal determined and results suggested the ignition of ITER

- Killer pellet injection can reduce VDE disruption vessel loading by a factor 4-10

- $\mathrm{n}_{e}$ control achieved in the new high- $\delta$ upper pump and baffled divertor

- Radiative divertor experiments indicate the importance of plasma convection including the effects of recombination

- Comparison of $D$ and He gas puffing shows helium can replace carbon as main radiator and chemical sputtering as the main source for carbon 

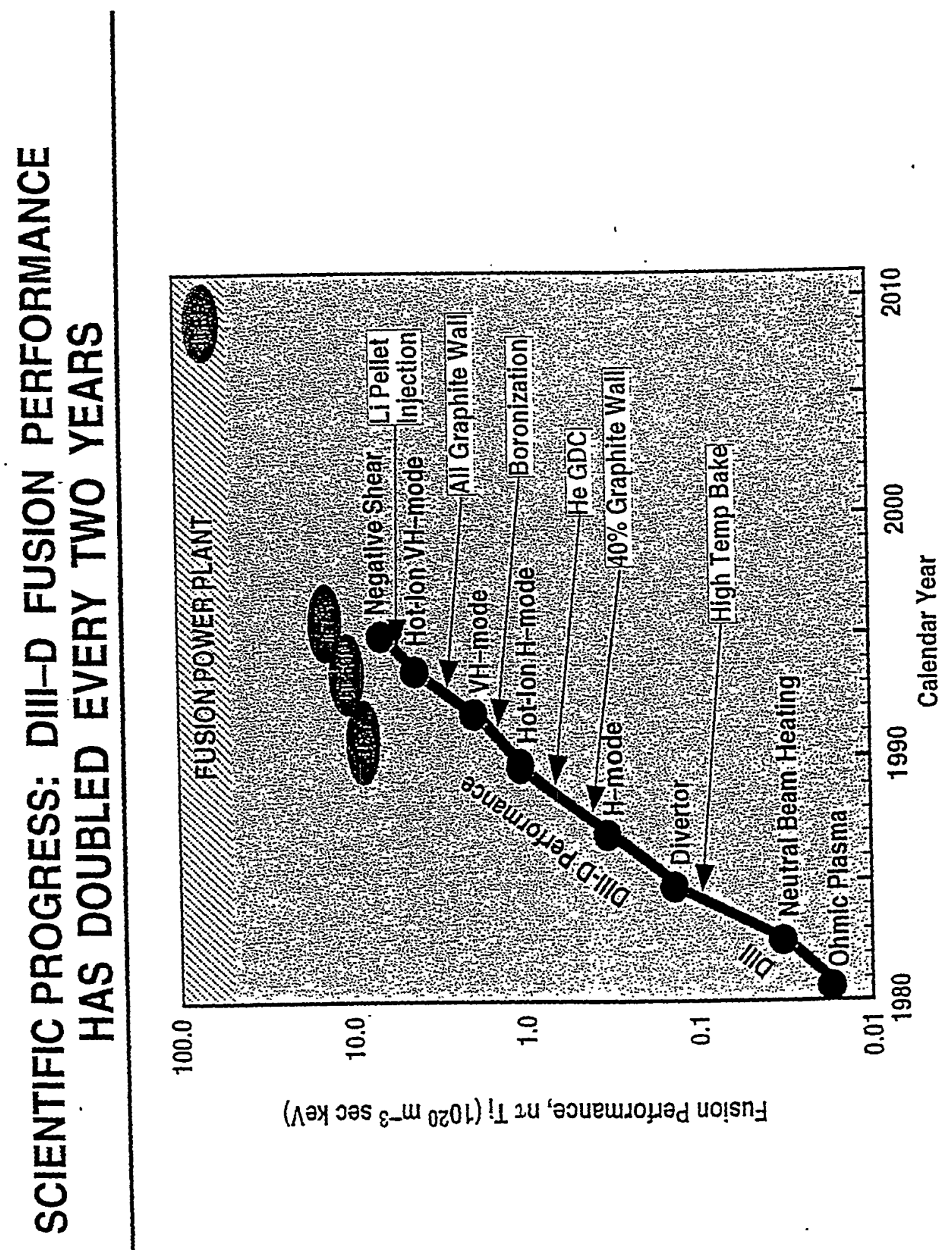

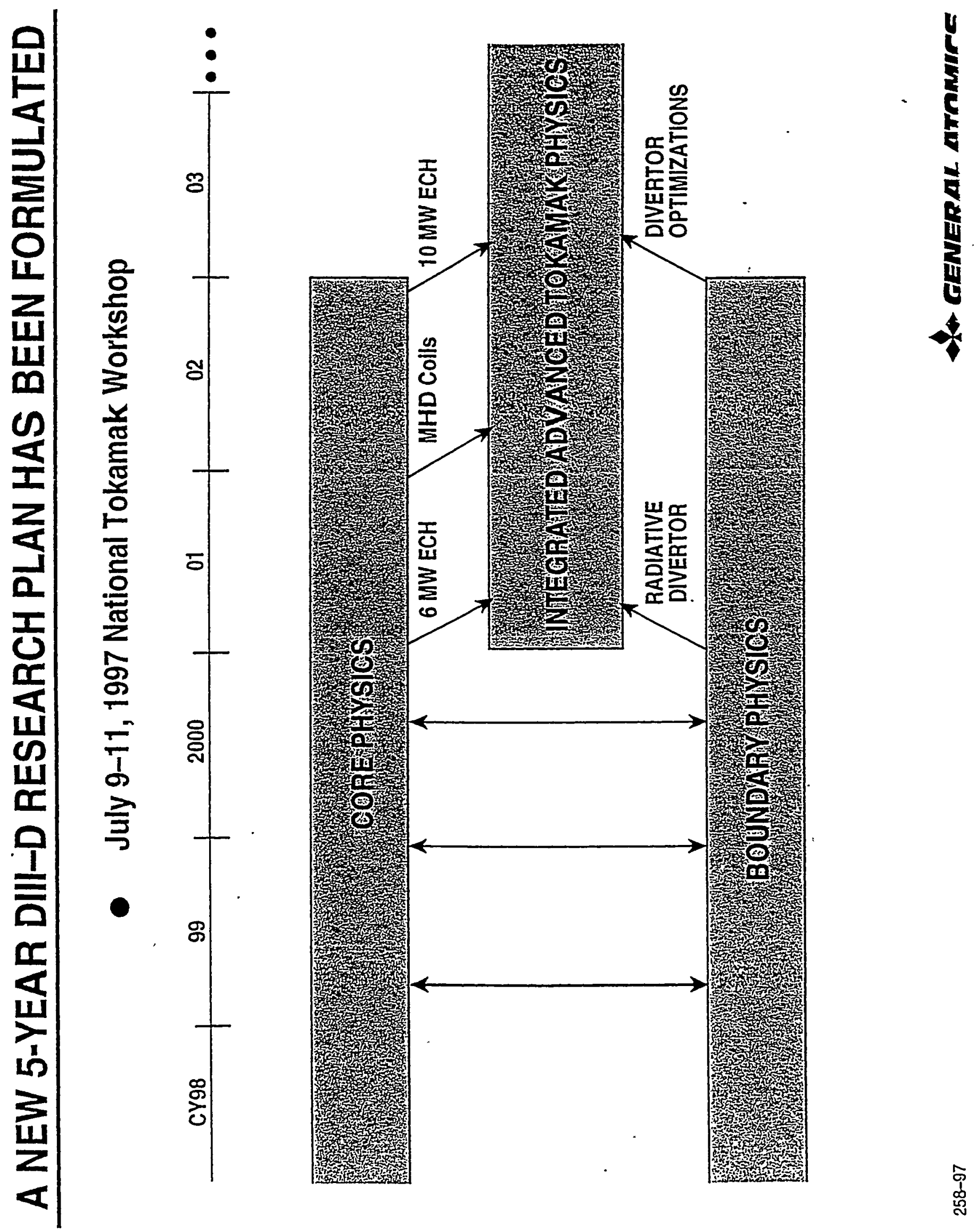
$=$ 
Session II: PFC Development for Near Term Devices

\section{II-1}




\section{CHRSE}

Nagoya University

Center for Integrated Research in Science and Engineering

\section{On the Utilization of High Z Materials as Plasma Facing Component}

T. Tanabe, CIRSE, Nagoya University

M. Akiba, JAERI

Y. Ueda, . Osaka University

K. Ohya, Tokusima University

M. Wada, Doshisha University

V. Philipps, Julich Research Center

Contents

1. Introduction

2. Influence on plasma performance

3. Erosion and redeposition

4. Energy deposition and reflection

5. Material responce to high heat load

6. Hydrogen effect

7. Summary and conclusions 


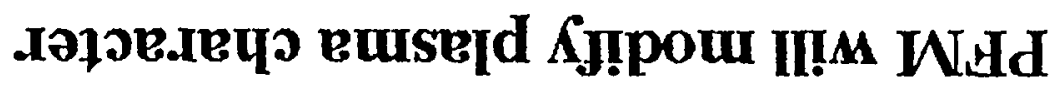
$\mathrm{Z}$ पo̊!บ $01 \mathrm{Z}$ MOI ய0.J

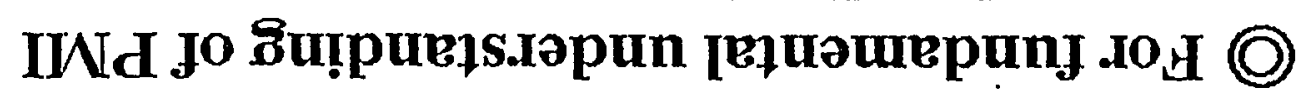

euseId uody əouənLIUI euse[d uo әəuənโJuI

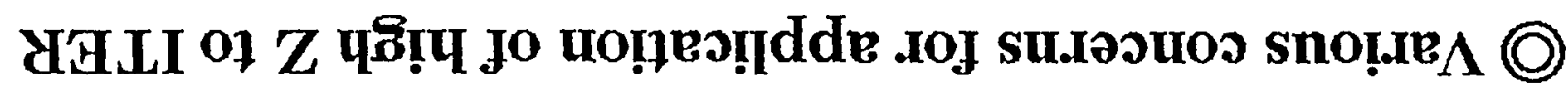

\section{uo!ponpodpuI 'I}



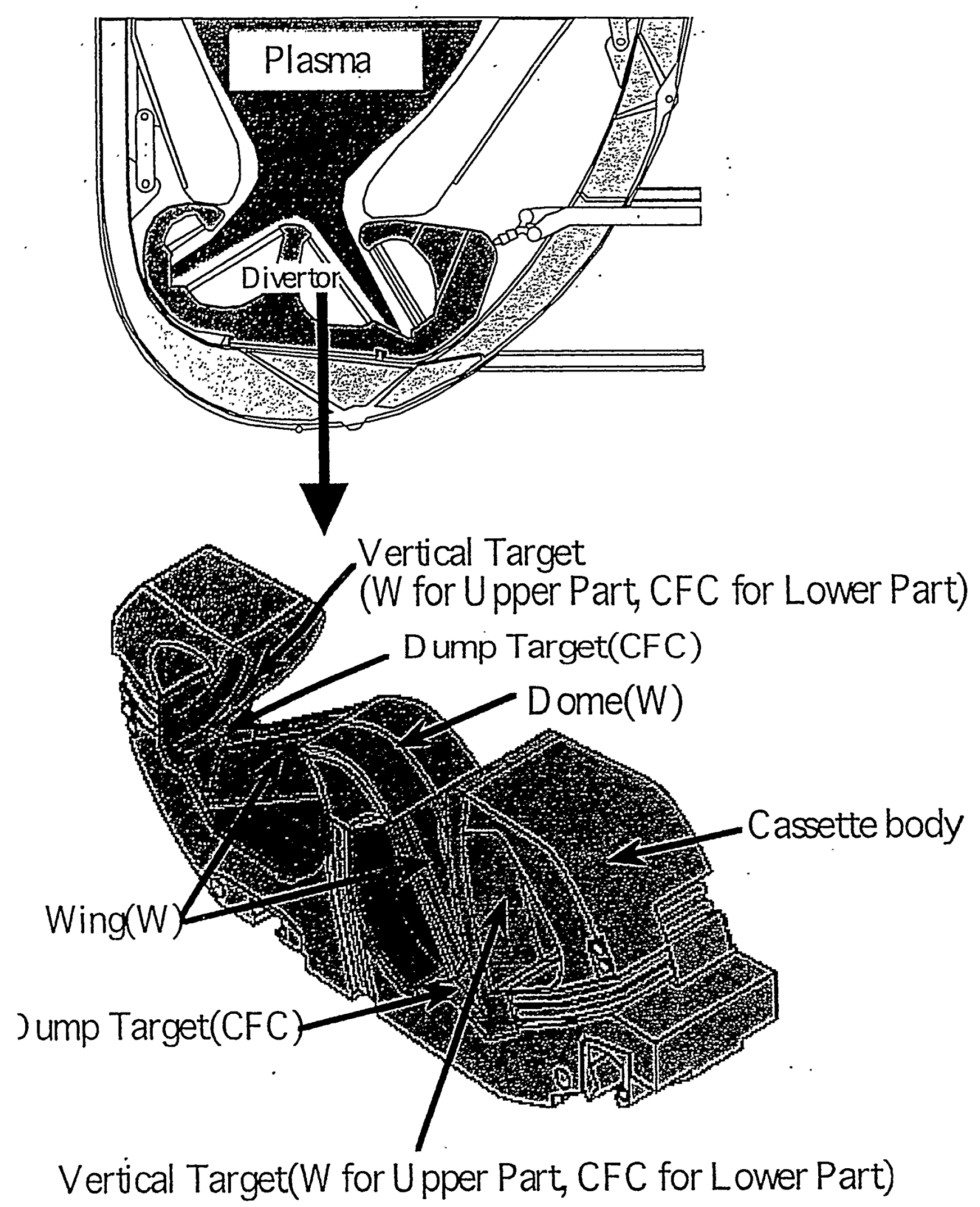

\section{Divertor Cassette of ITER (Vertical Target Option)}




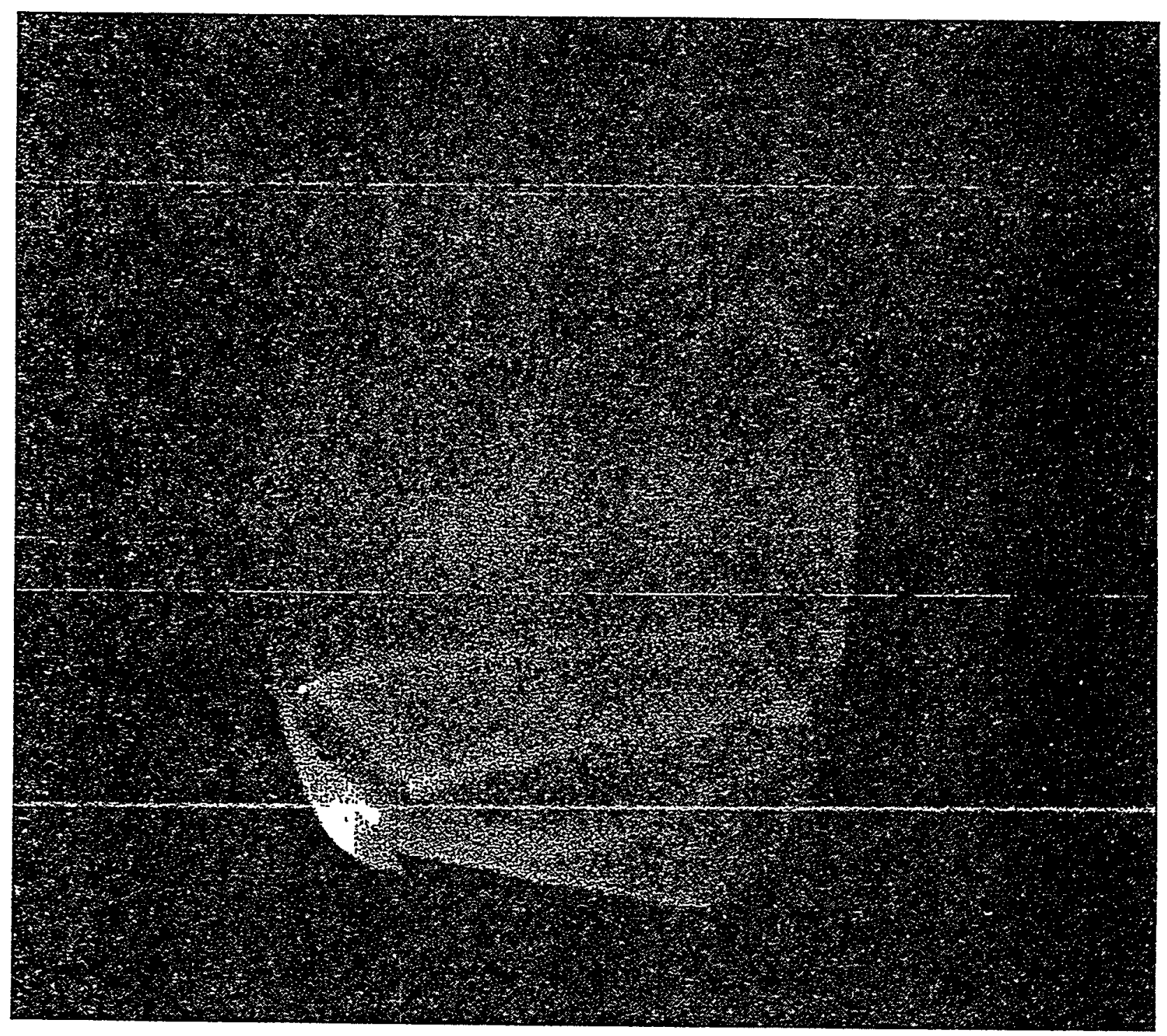

TEXTORプラズマとALT-Iトロイダル

ポンプリミター（接線方向の㥶より撮影）

詳細はP 1 参照 


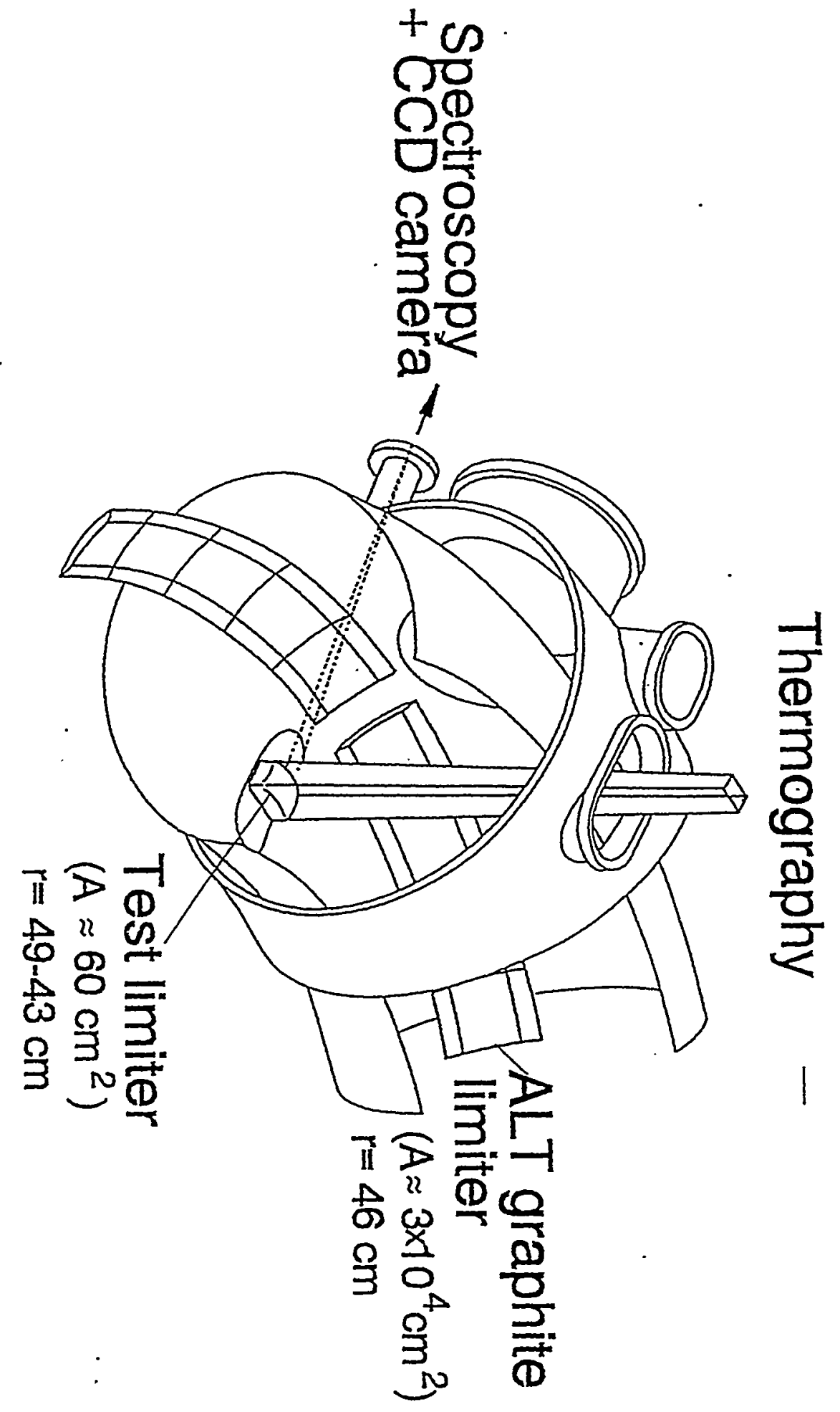

$1.8 .-1$ 


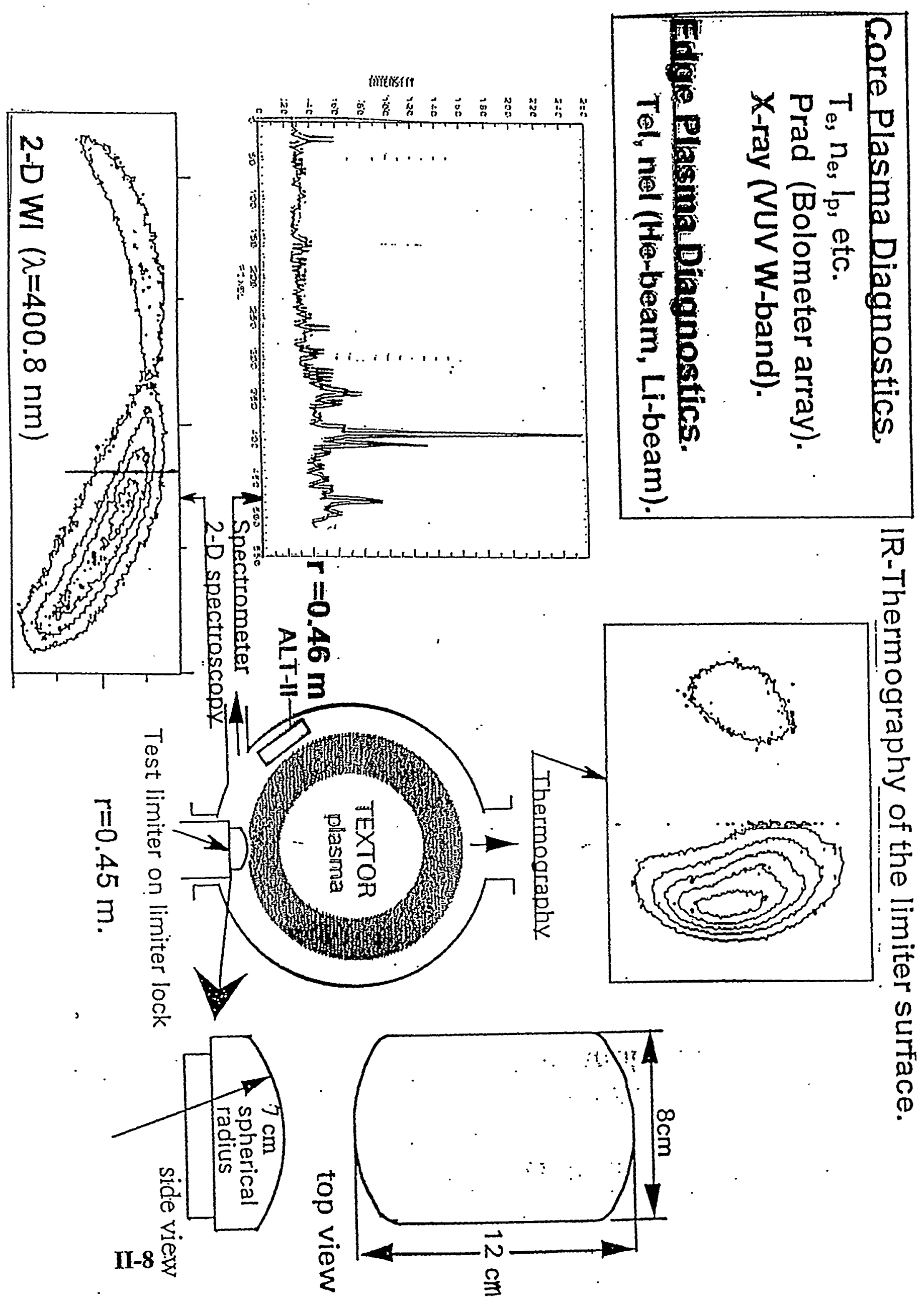




\section{Experimental setup in TEXTOR}

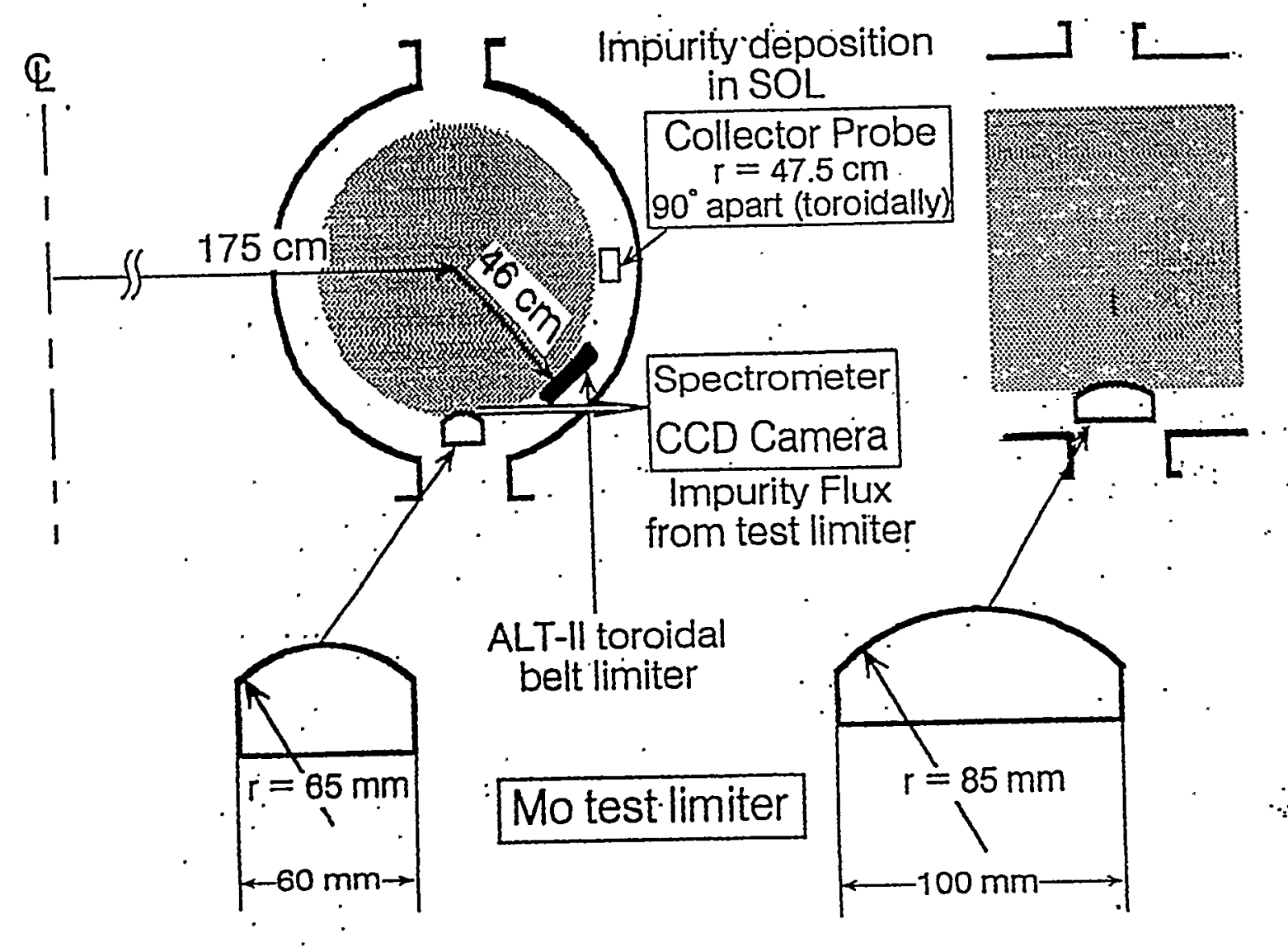

$\frac{E_{\text {lim }}}{E_{\text {conv }}} \lesssim 5 \%$ (in this presentation)

$\longrightarrow$ Most of $\mathrm{E}_{\text {cony }}$ flows into ALT-II

$E_{l l m}$ : Deposition.energy to the test limiter

$E_{\text {conv: }}$ Total convective energy

- $E_{\text {conv }}=\int\left(P_{\text {neat }}-P_{\text {rad }}\right) d t$ 
-SYEUEYO ว8.IEI

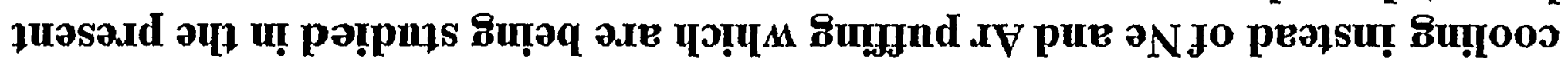

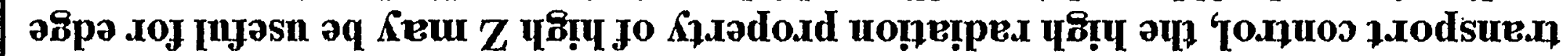

วqеฺ!ns $\Lambda q$ papgo

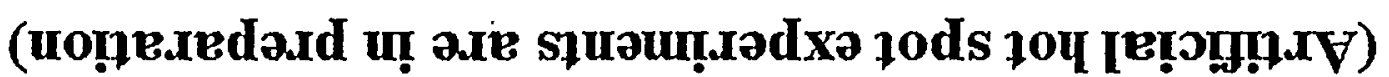

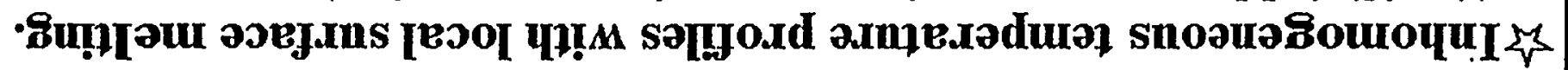

- YOLX'AL

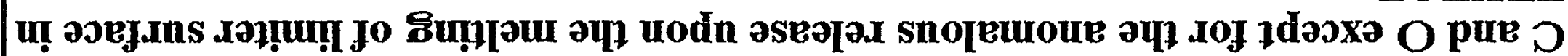

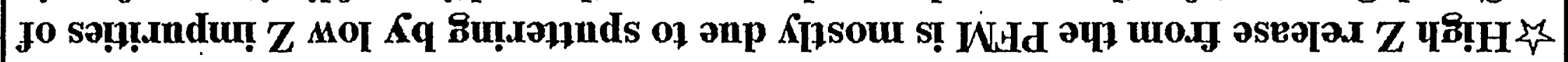

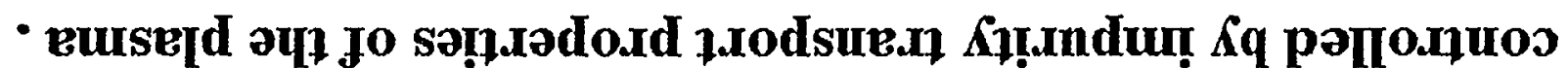

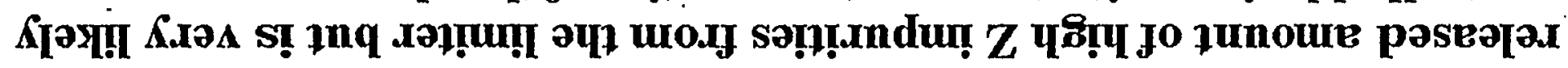

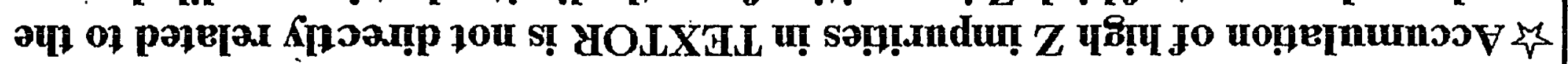

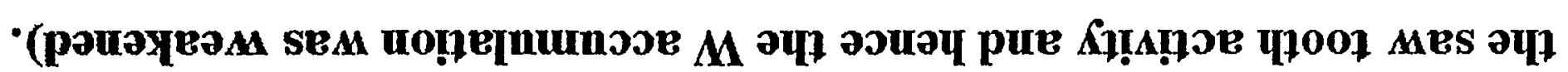

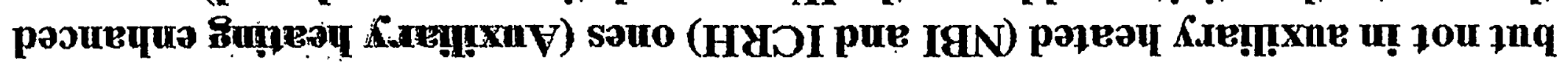

MOd ${ }^{2}$

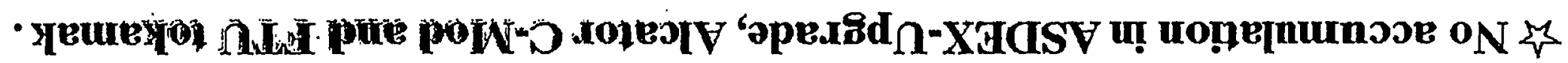

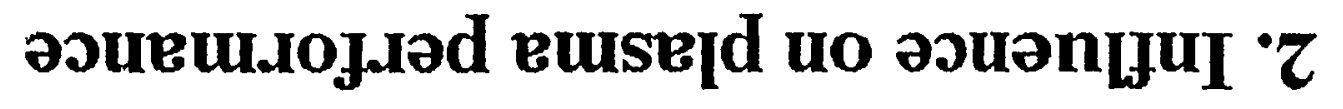


Evolution of $T_{e}, \bar{n}_{\theta}$ and $P_{\text {rad }}$

for $\mathrm{OH}$ and $\mathrm{NBI}$ plasmas
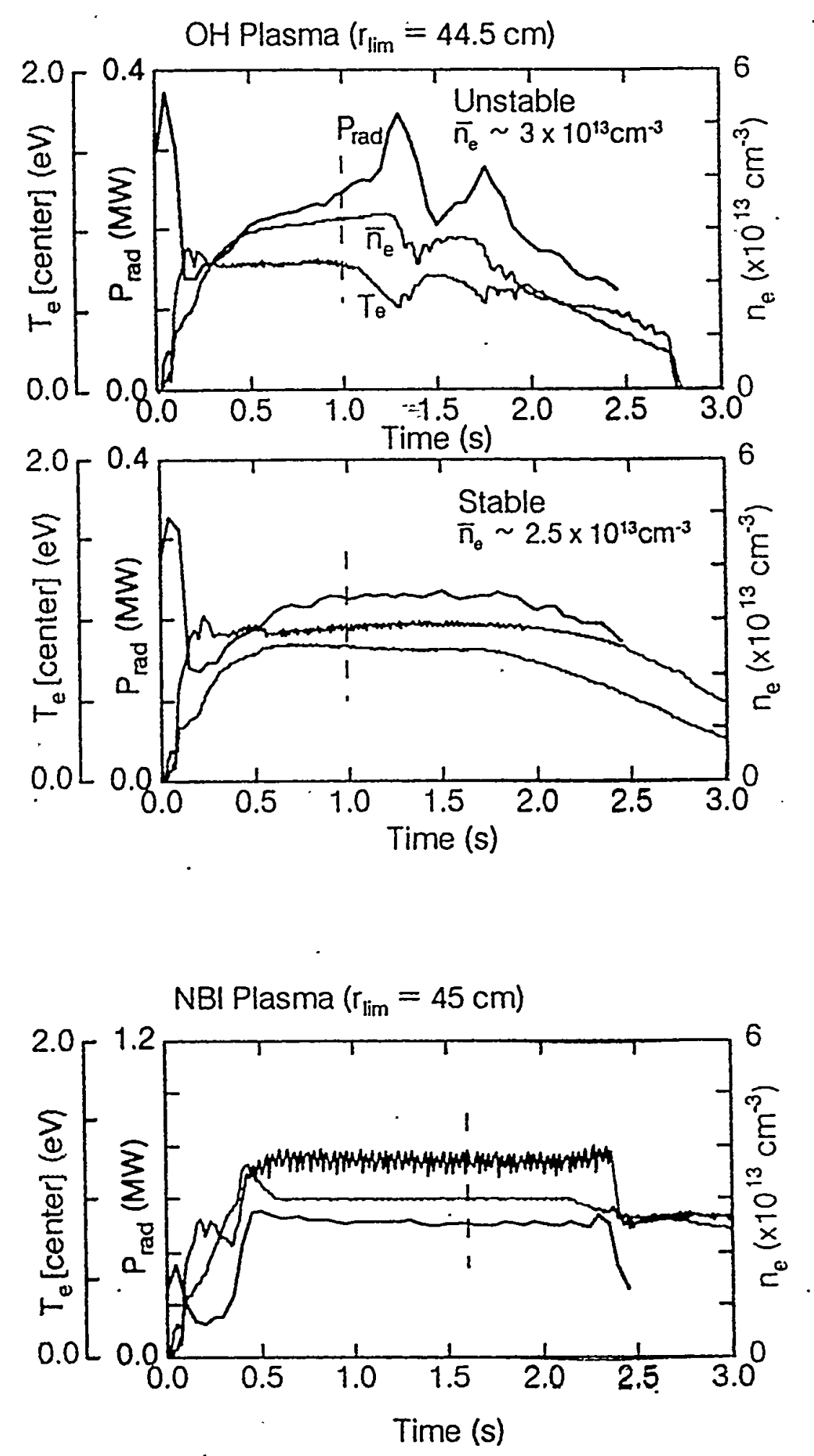


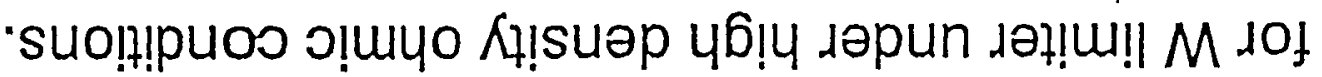

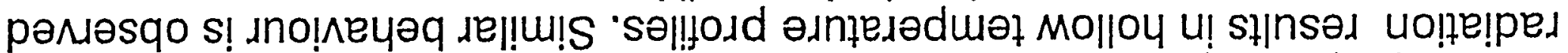

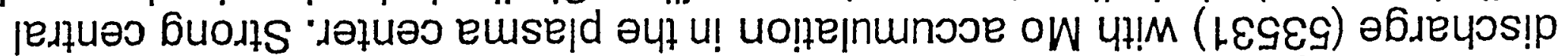

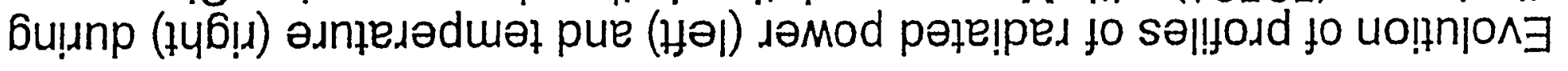
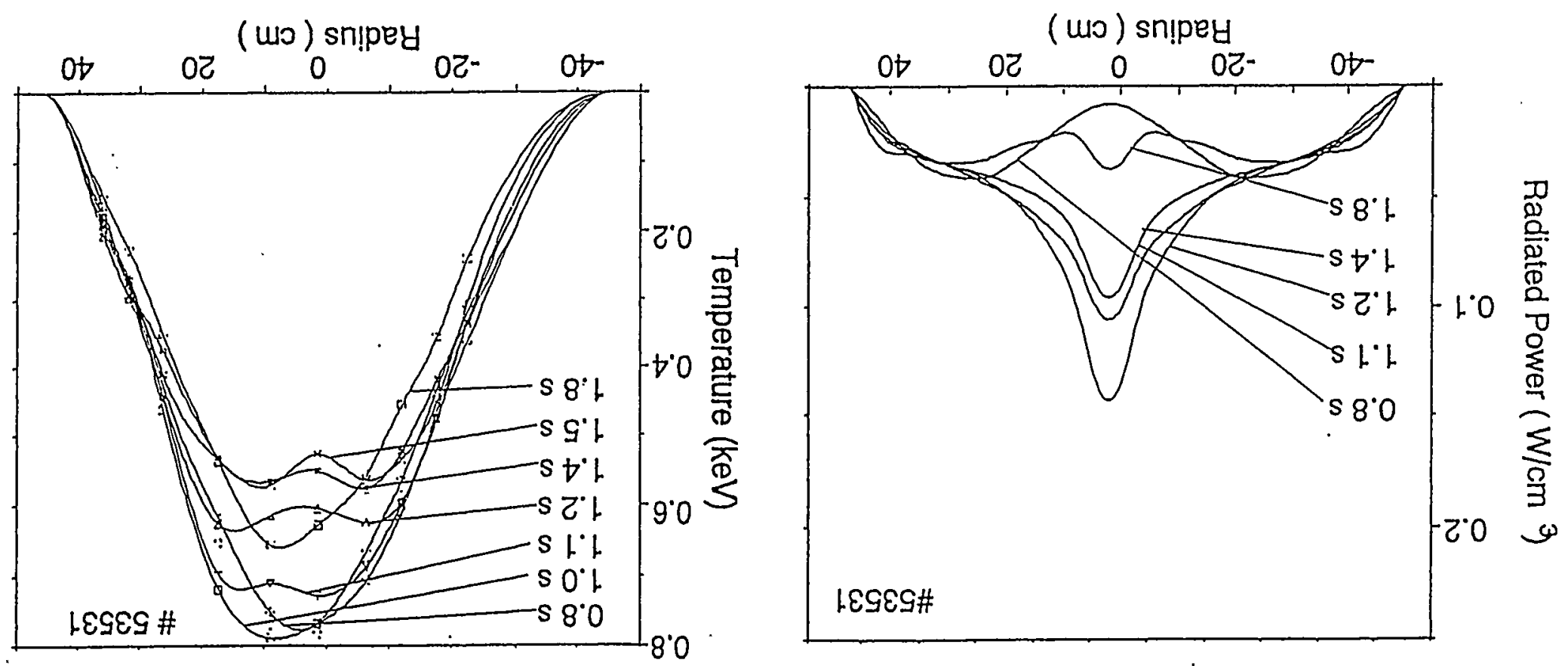
Deposited Energy (kWs)

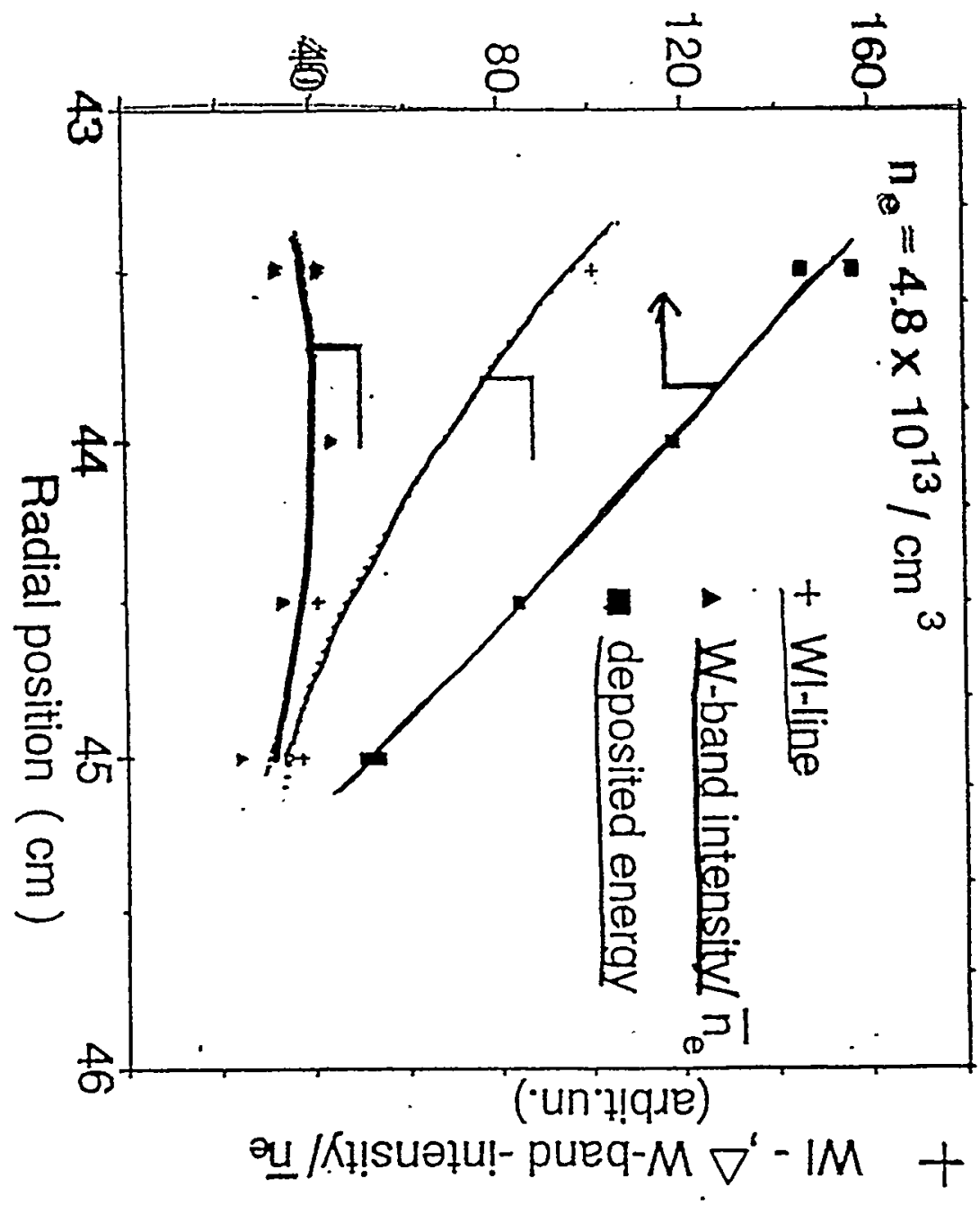

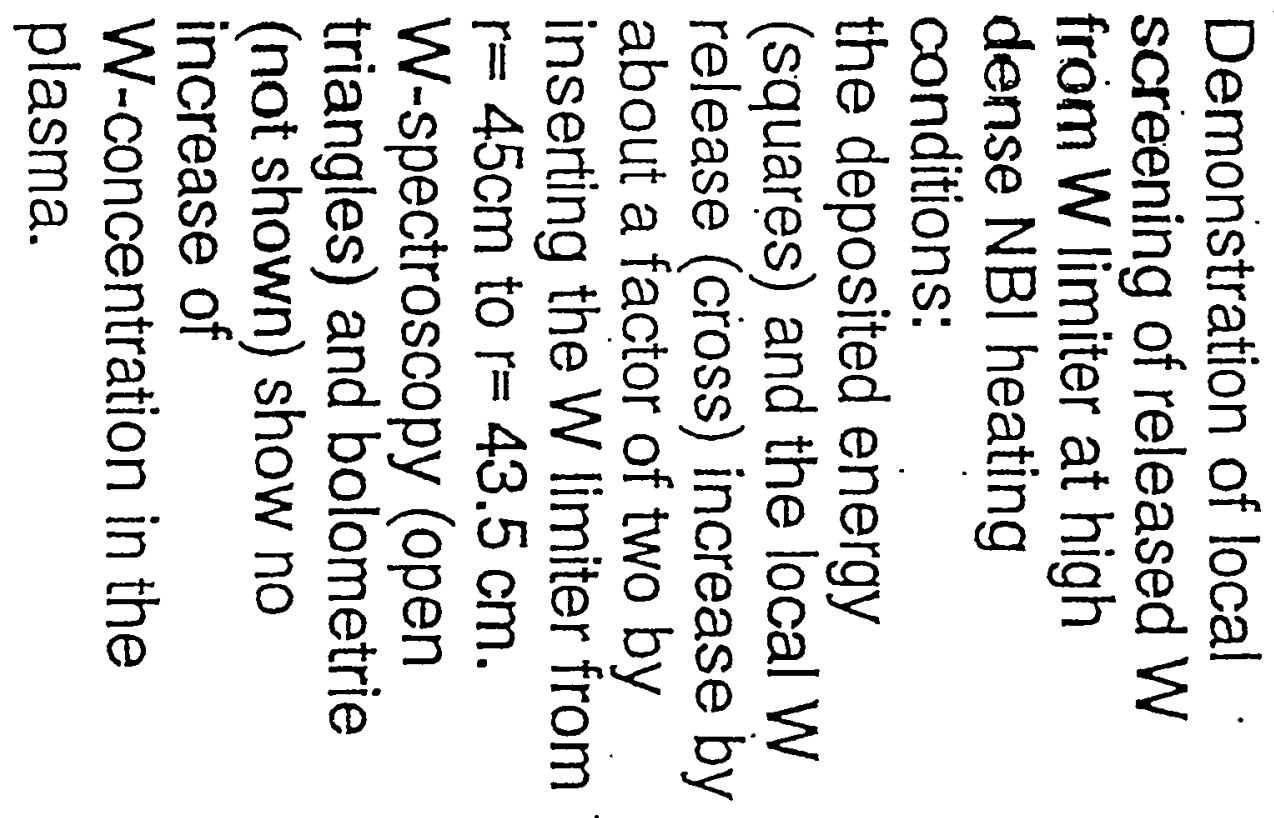


Accumulation of high $\mathrm{Z}$ impurities is not directly related to the released amount of high $\mathrm{Z}$ impurities from the limiter

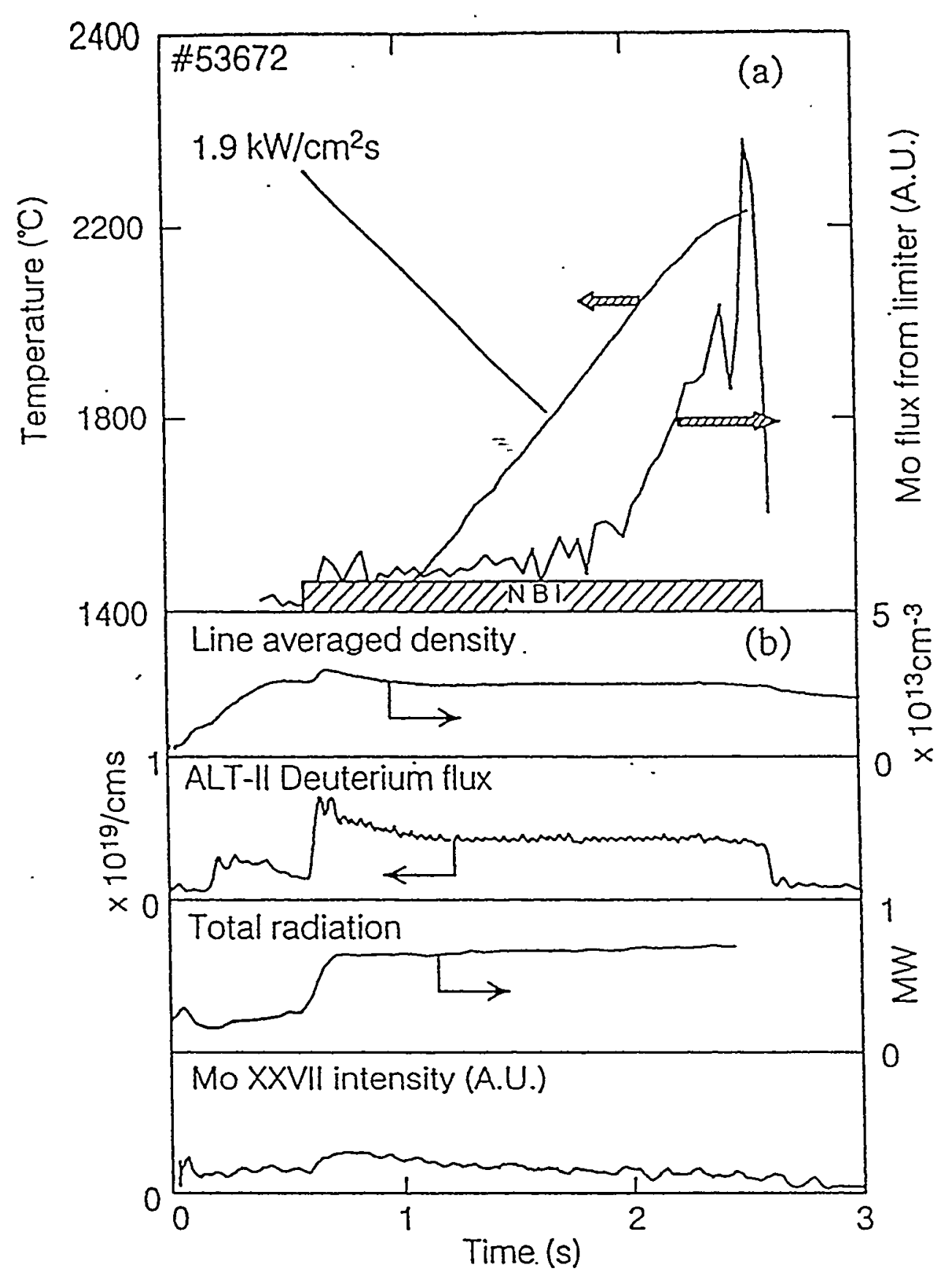



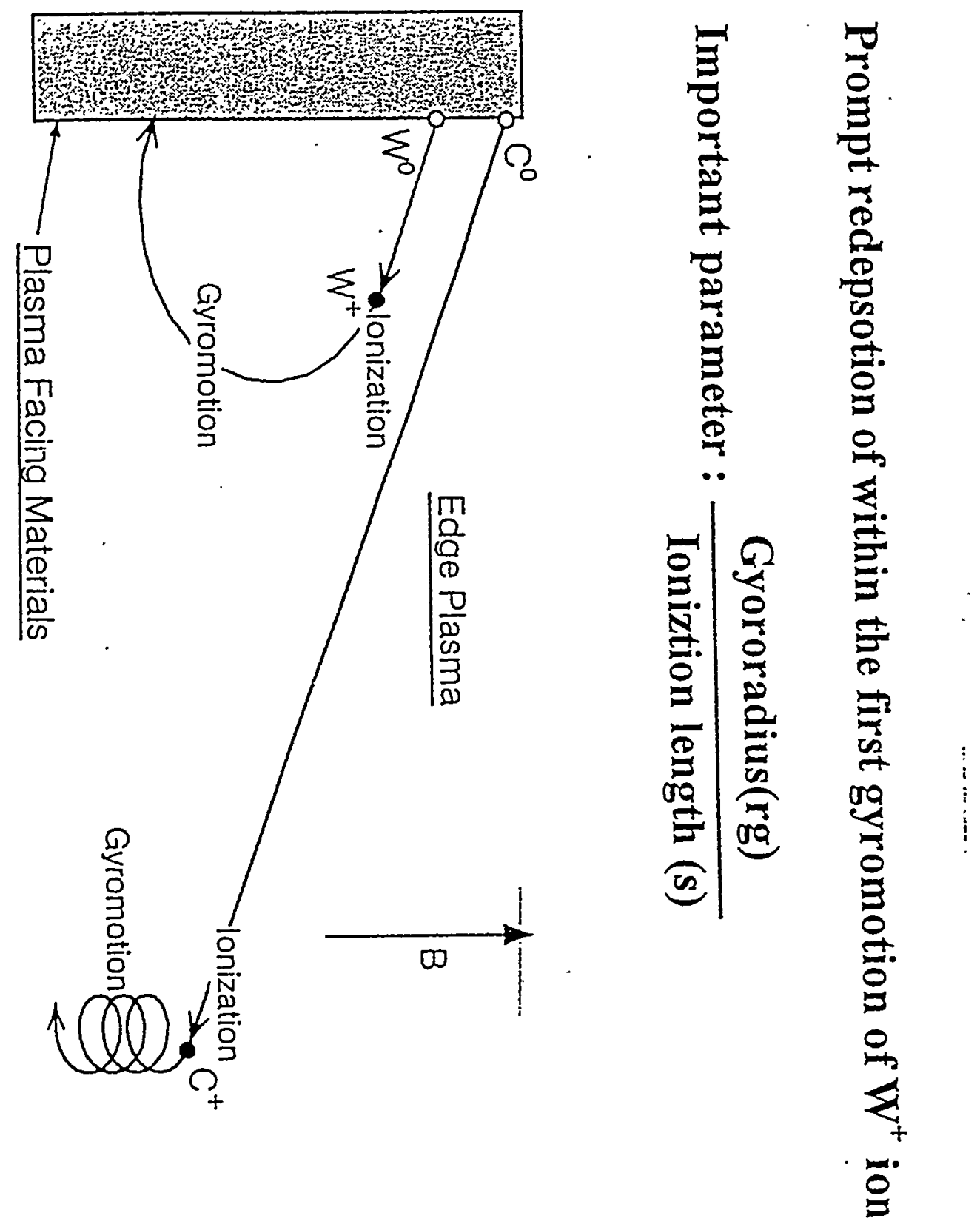
Ionization:Length of Mo Neutral

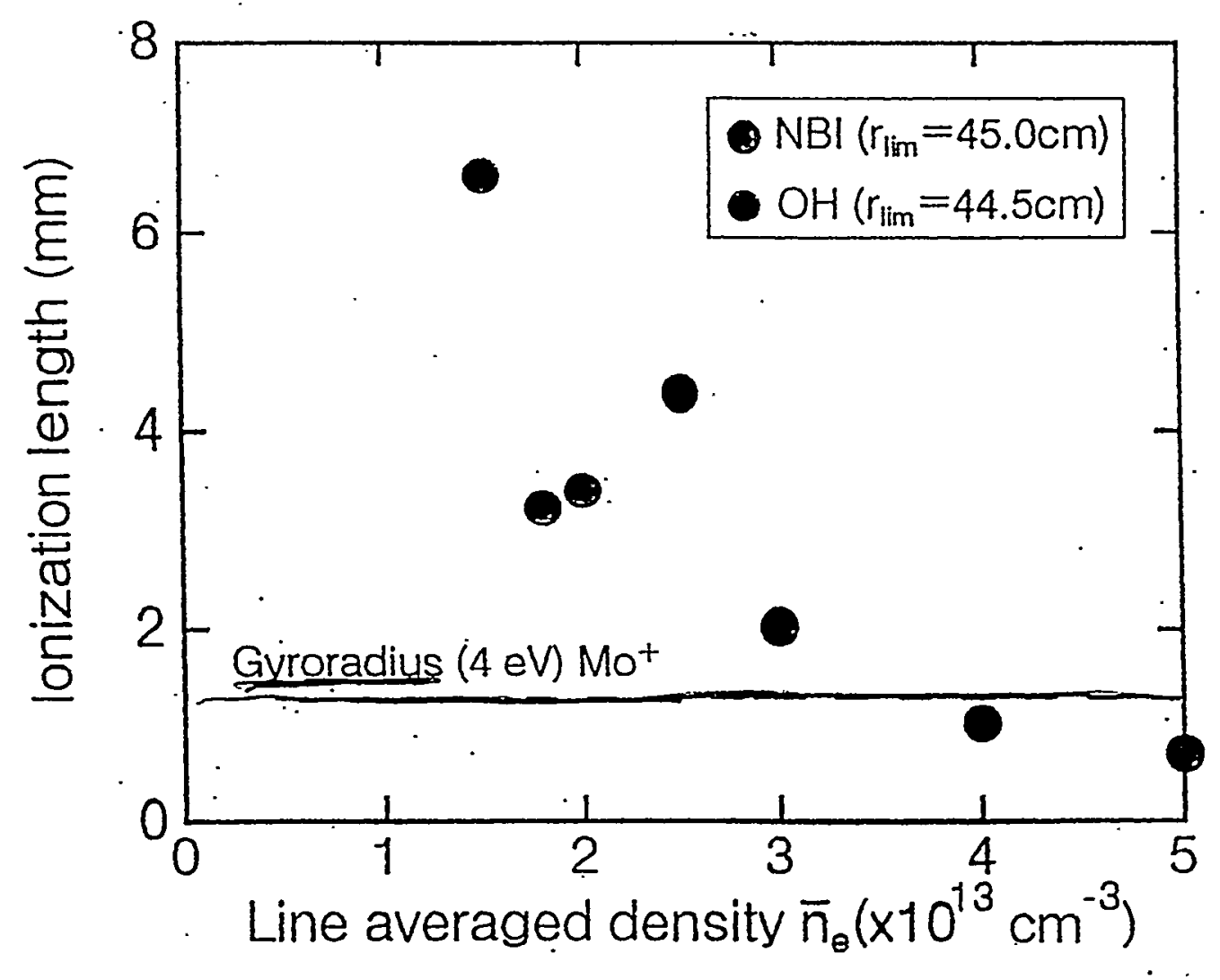

In high density NBI plasma $\left(\bar{n}_{\mathrm{e}} \geq 4 \times 10^{13} \mathrm{~cm}^{-3}\right.$, $n_{\theta}$ [limiter surface] $\left.\gtrsim 1 \times 10^{13} \mathrm{~cm}^{-3}\right)$, lonization length of $\mathrm{Mo}$ is less than gyroradius of $\mathrm{Mo}^{+}$ and probability of prompt deposition is much high.

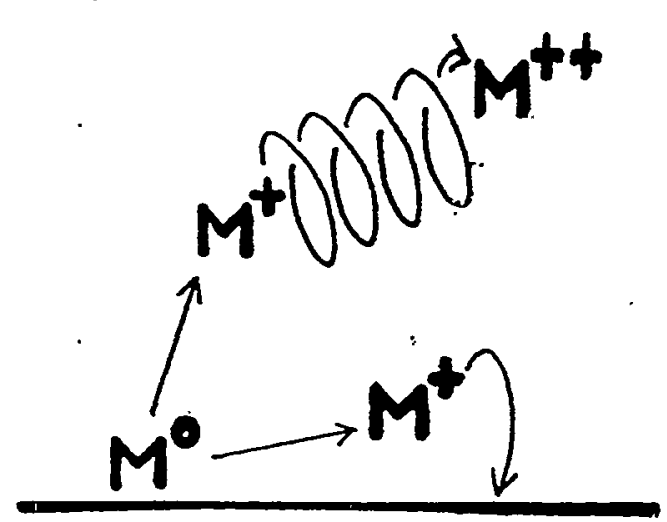




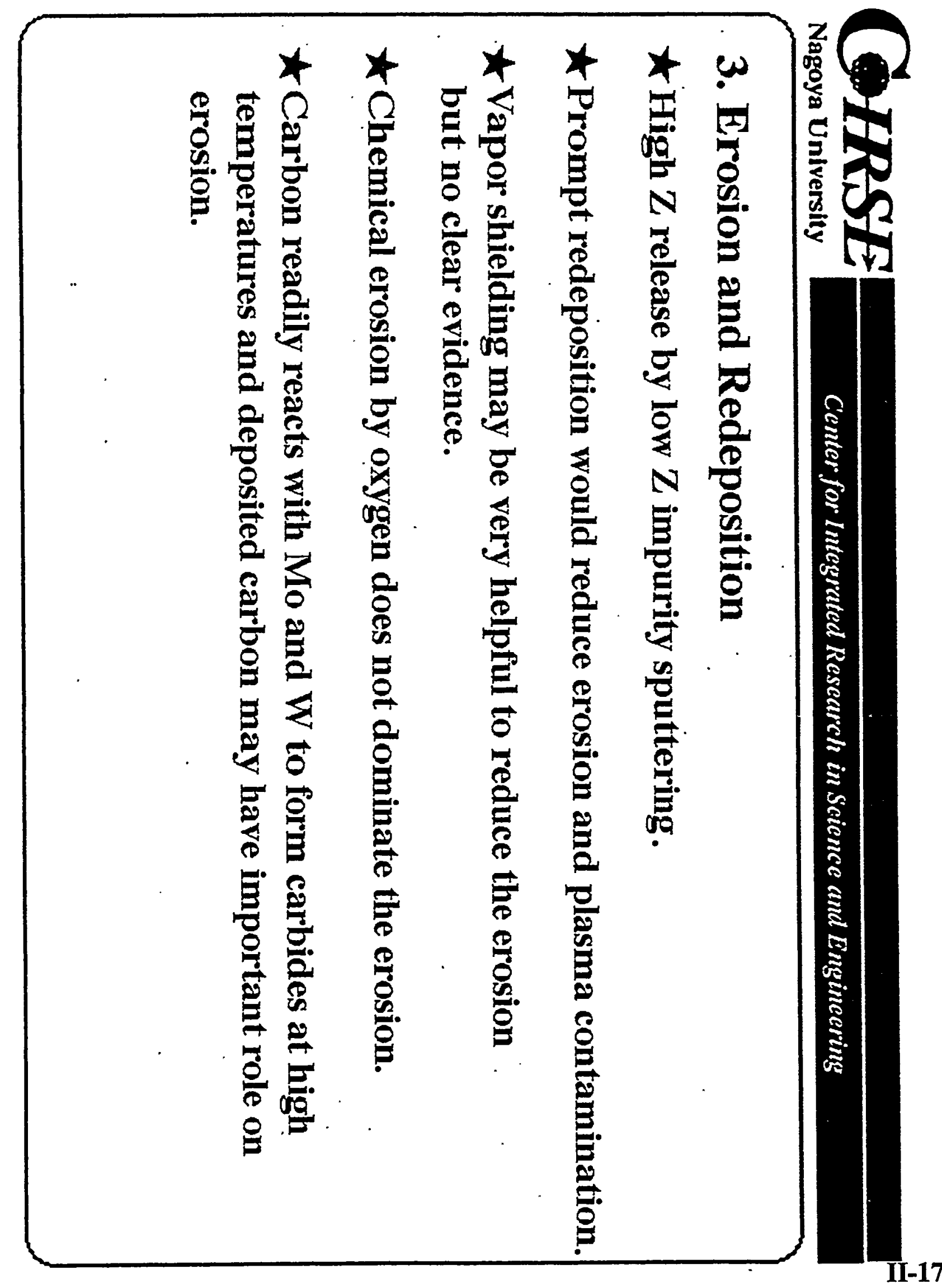


Number of atoms

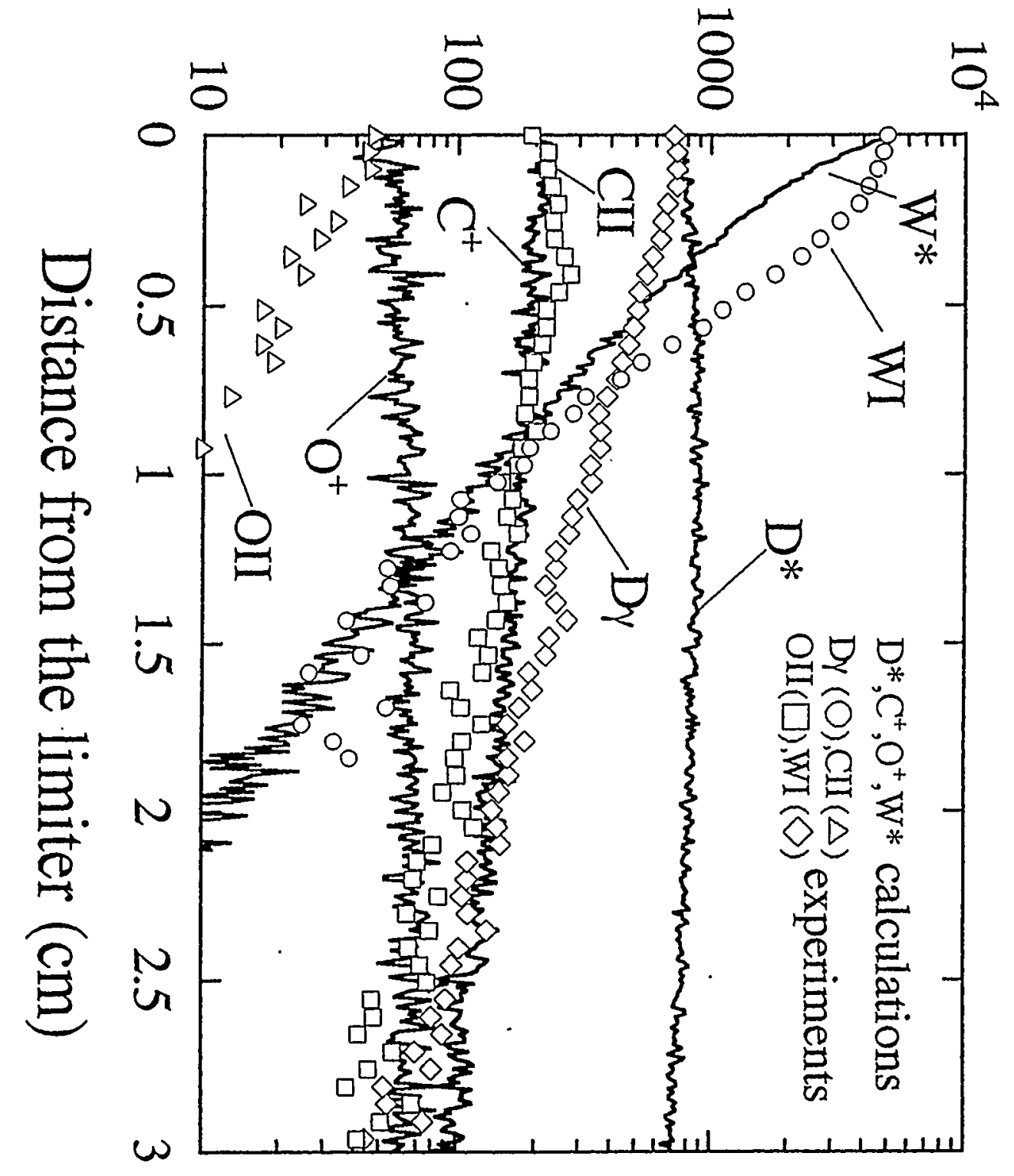


DEPOSITION RATES (FLUXES) OF HIGH - Z METALS ON THE COLLECTOR PROBES EXPOSED TO NBI HEATED PULSES

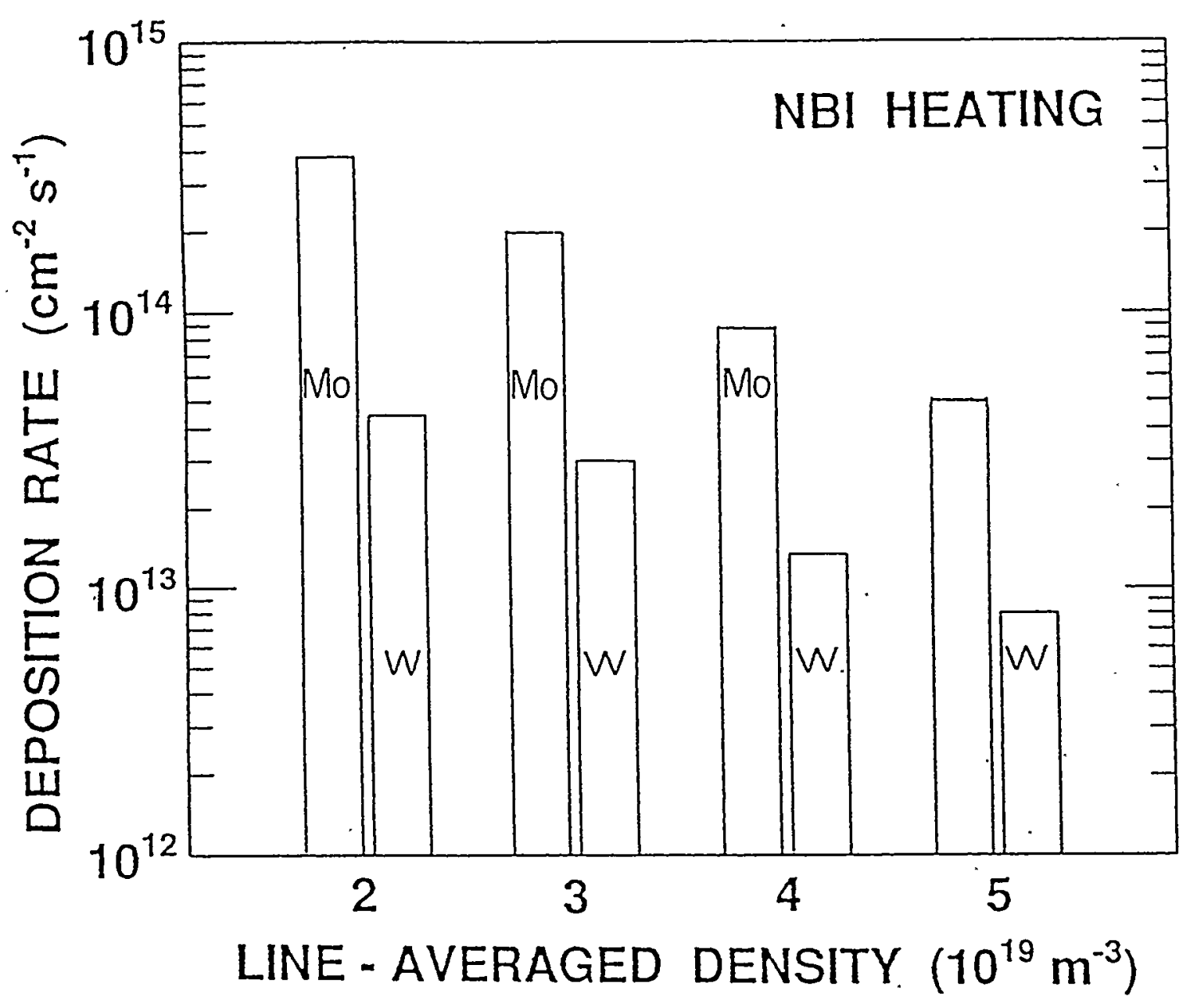

RESULTS:

1. $\Phi_{\mathrm{Mo}}: \Phi_{\mathrm{W}}=7: 1$

2. THE DECREASE OF HIGH - $Z$ FLUXES (EROSION) WITH THE INCREASE OF ELECTRON DENSITY. 


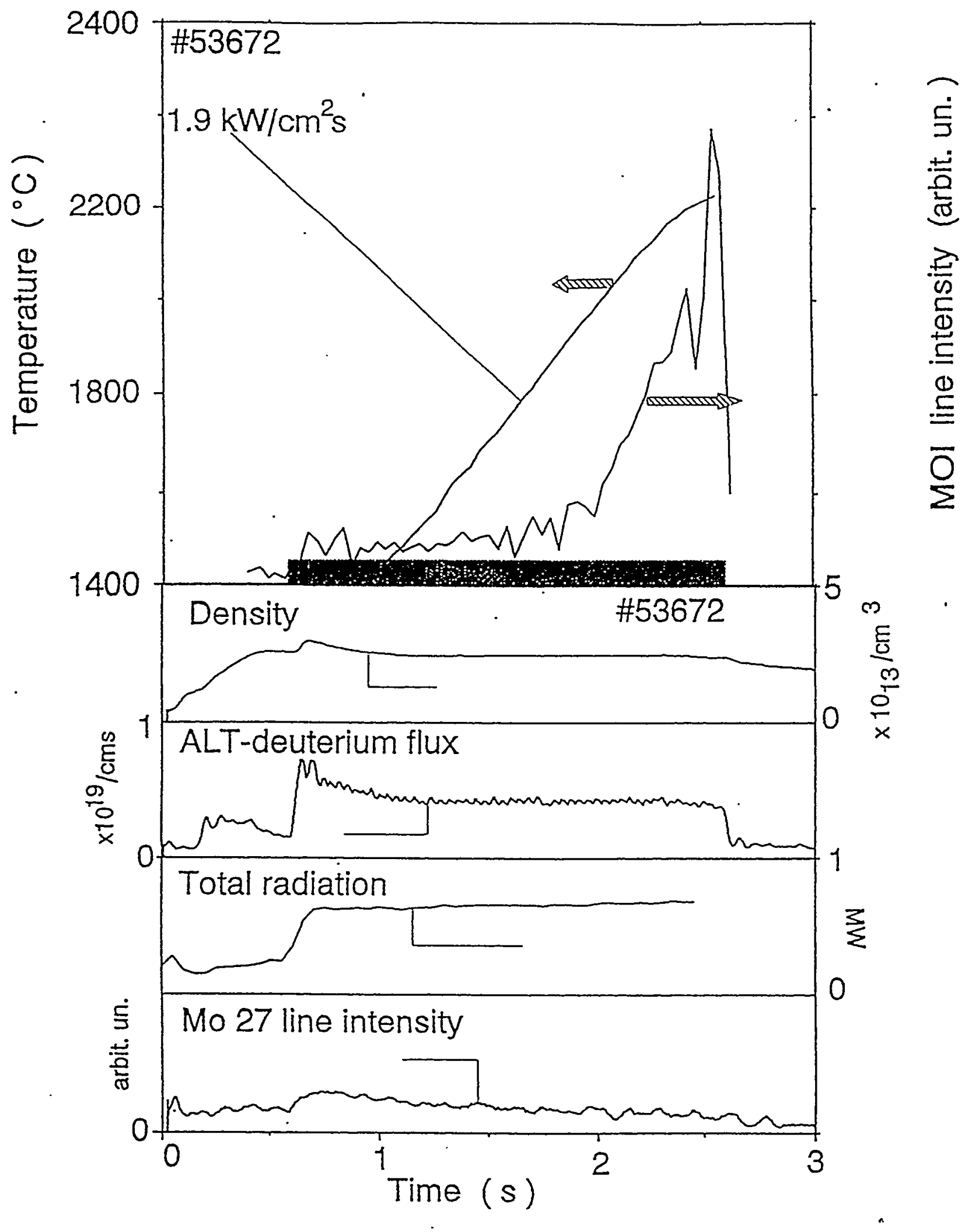

Fig.5. Time sequences of various plasma parameters for the particular shot where the Mo-limiter subjected to surface melting as seen in the increase of the MoI line in the figure. II-20 


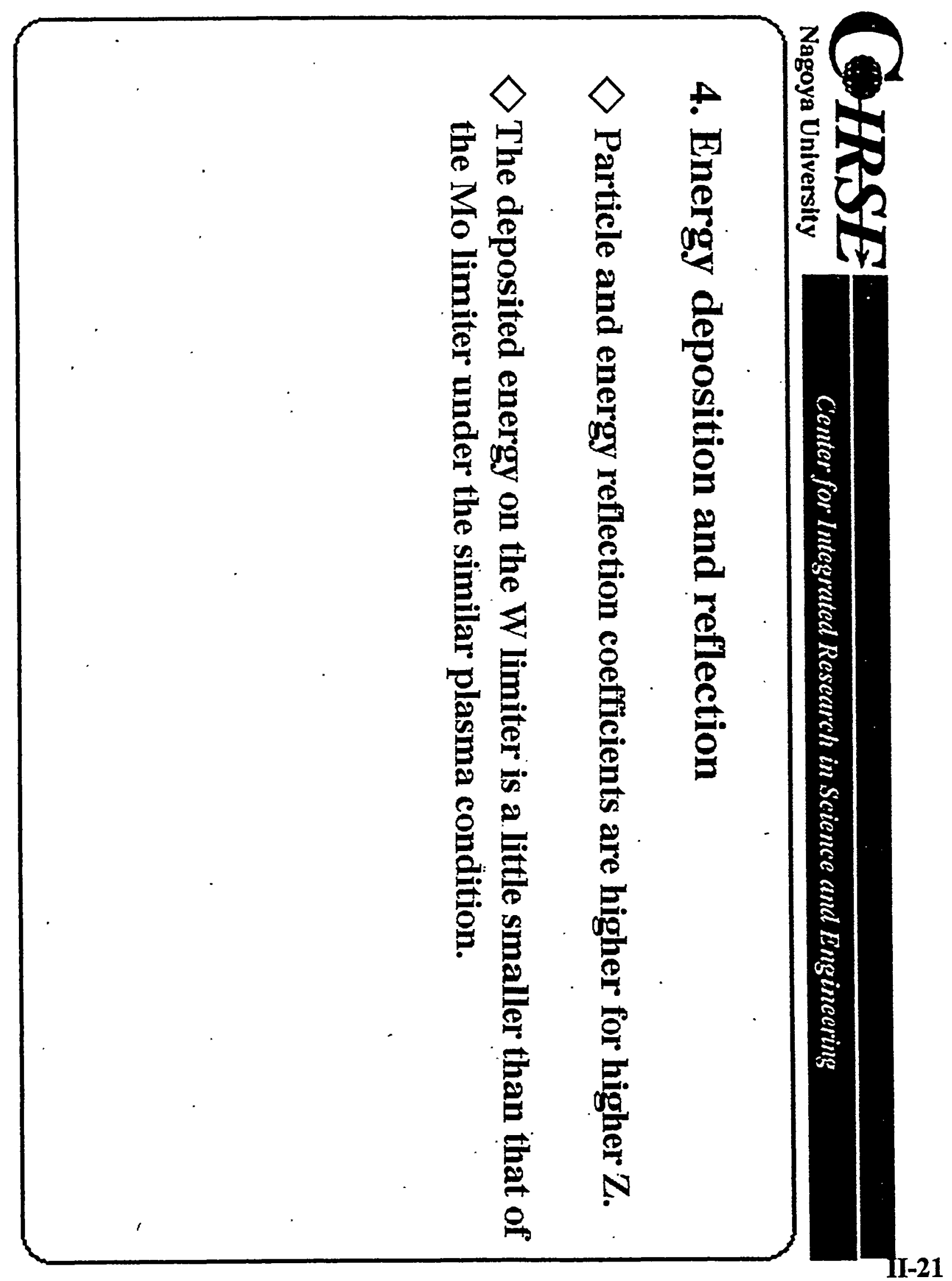



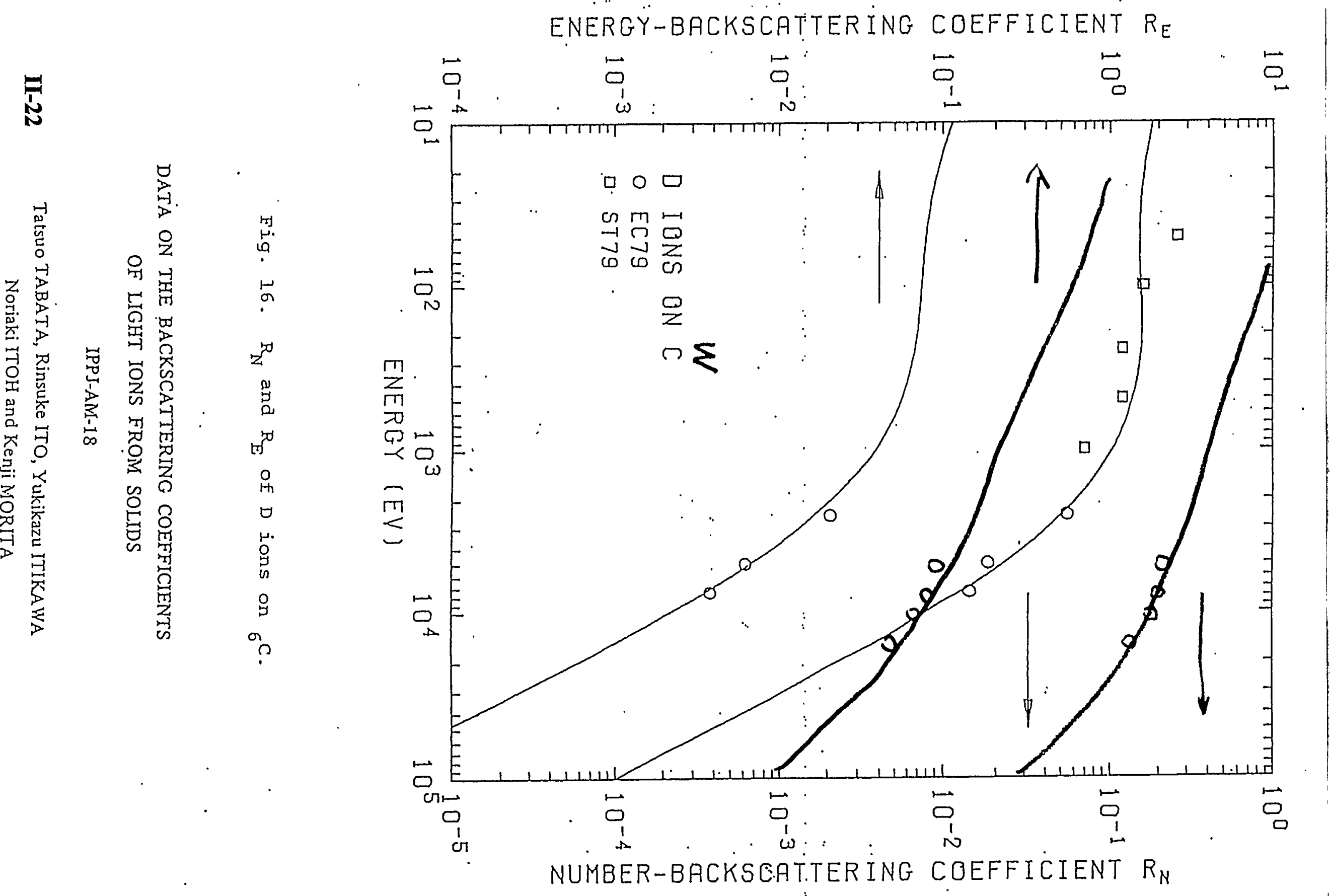


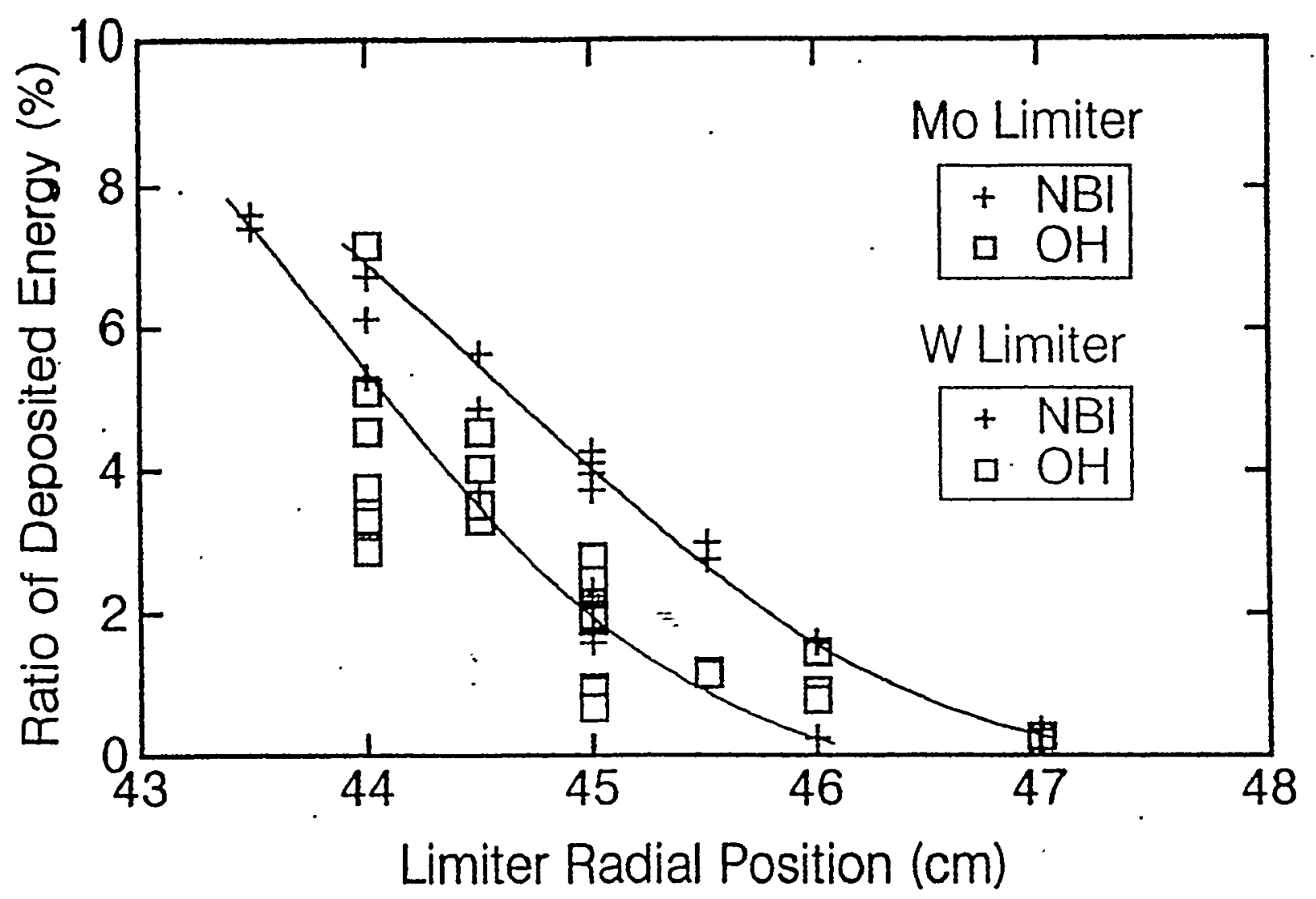

Ratio of Deposited Energy : $E_{L i m} / E_{c o n}$

Convective loss Energy : $E_{\text {con }}$

$E_{\text {con }}=\int\left(P_{\mathrm{OH}}+P_{\mathrm{NBI}}-\mathrm{P}_{\mathrm{rad}}\right) d t$

Ratio of Deposited Energy to Limiter vs Limiter Radial Position 


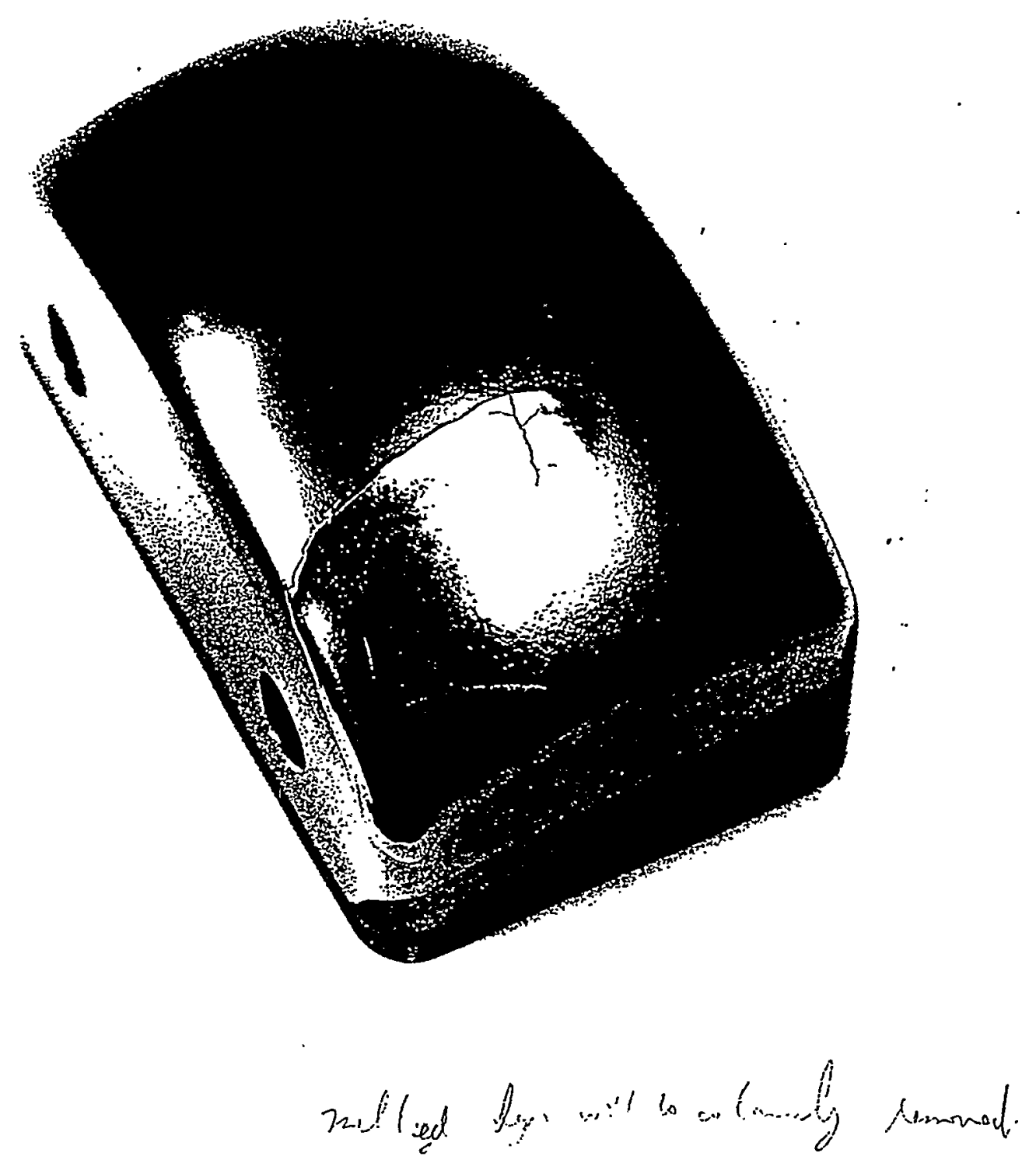




$$
8
$$


T921t360_asc
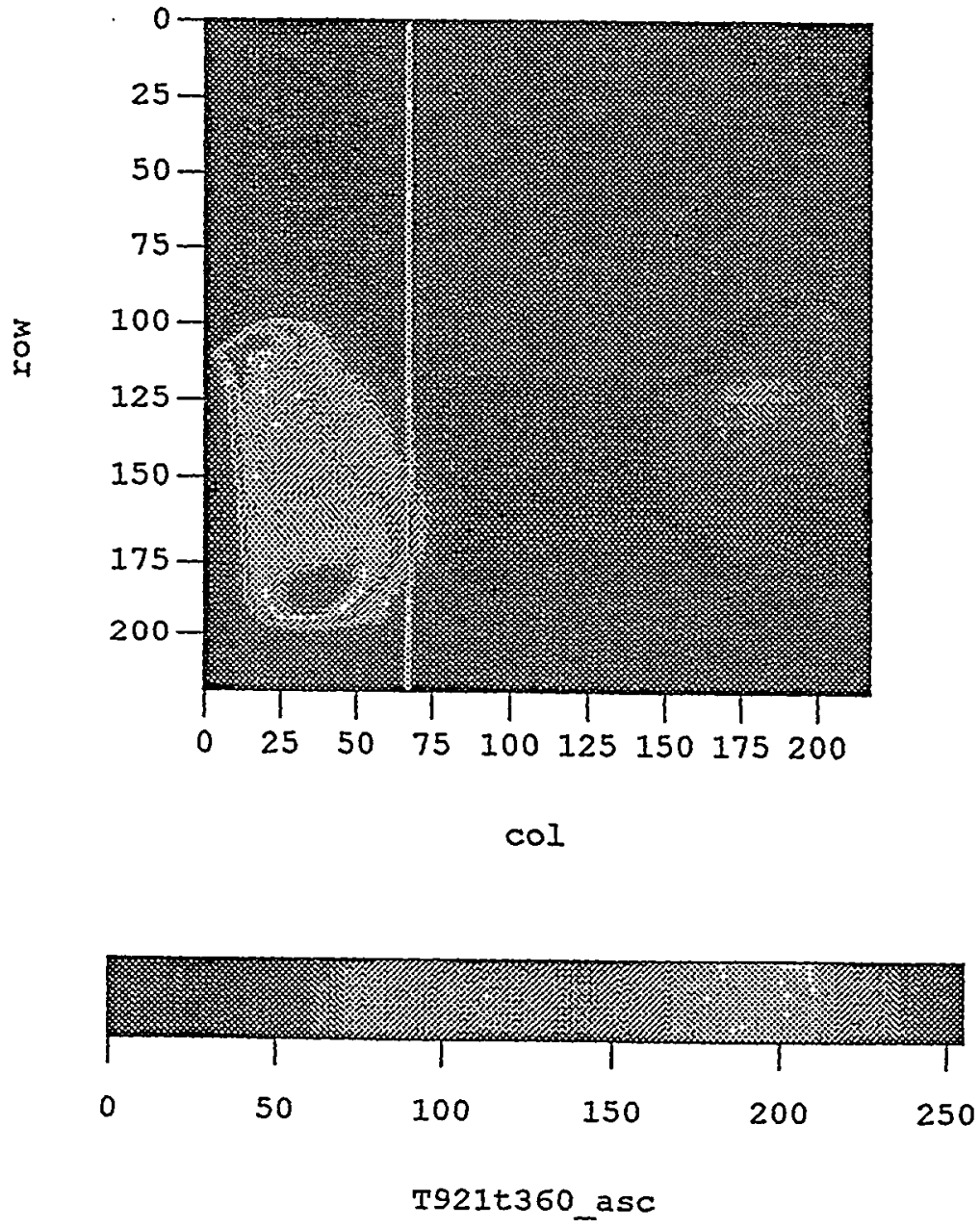

$7 \ln 20, y) \quad 0 \because \therefore-$ 


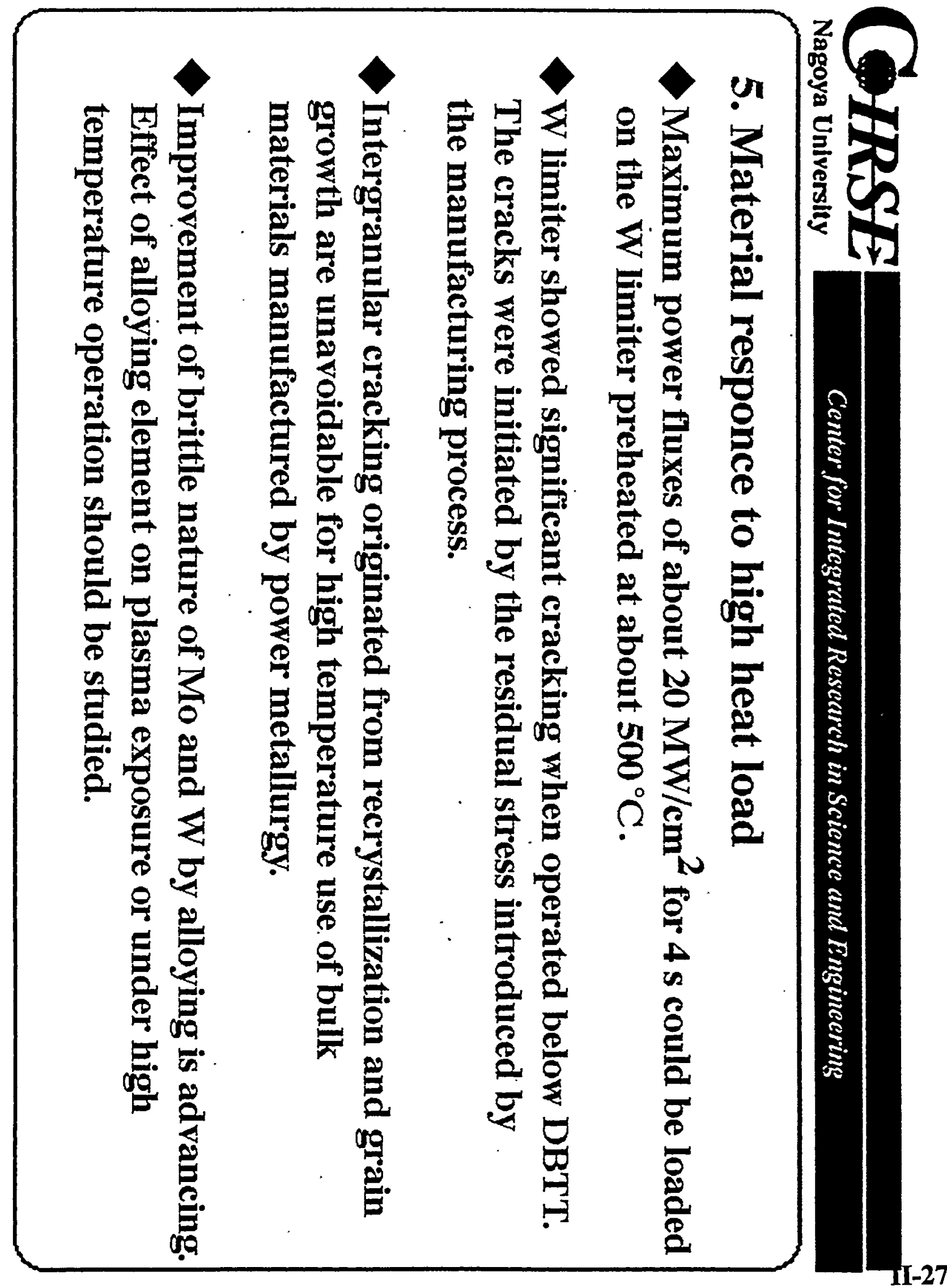



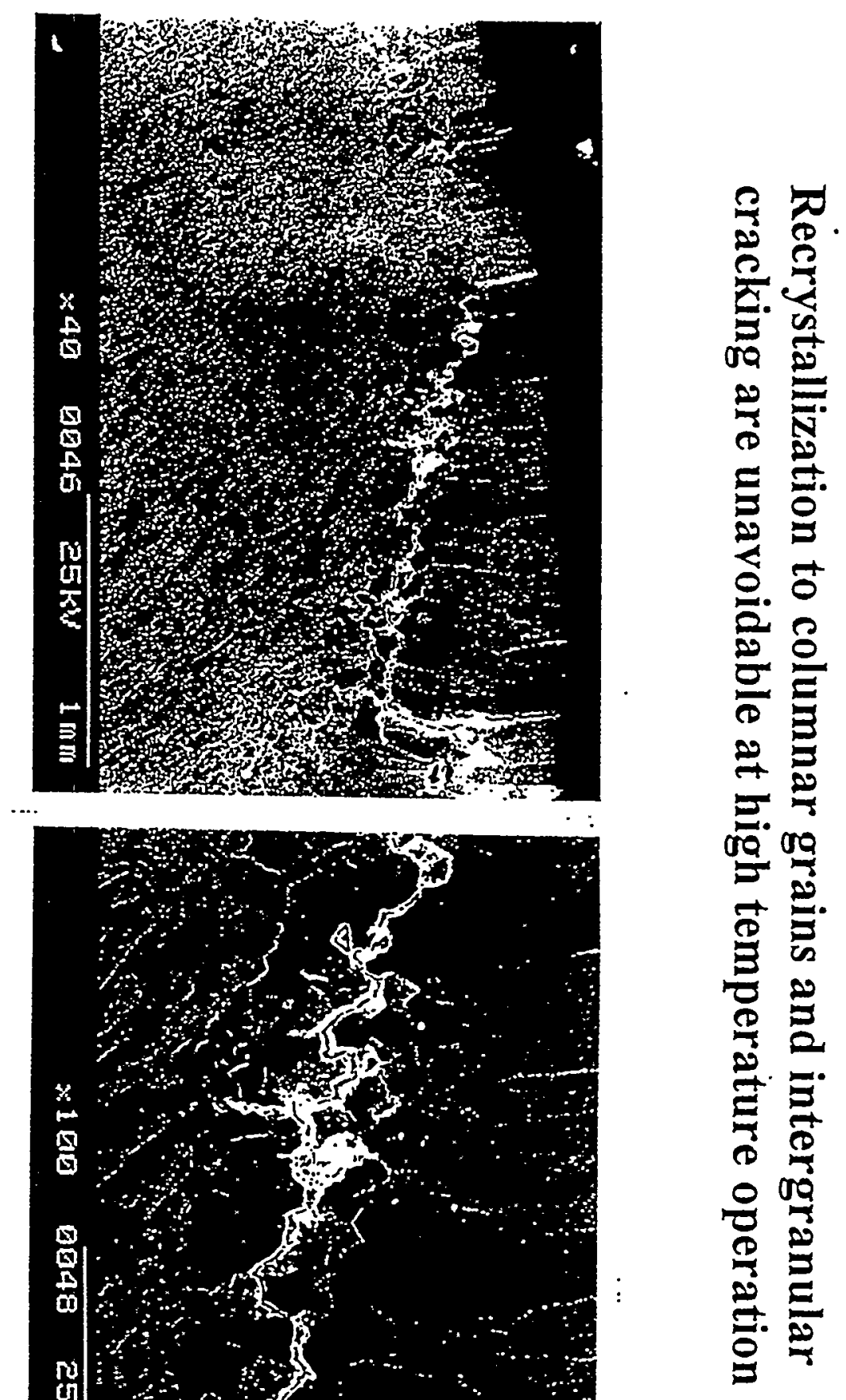


\section{Manufacturing and high heat flux loading of tungsten coatings on fine grain graphite for the ASDEX-Upgrade divertor}

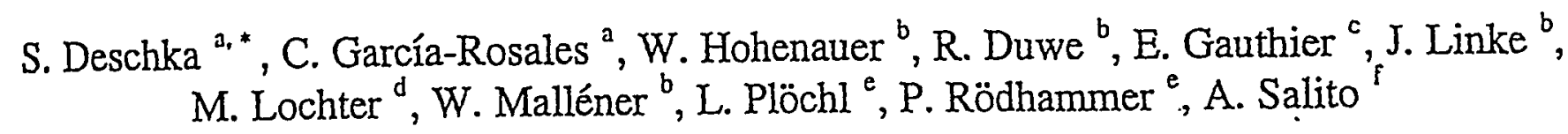
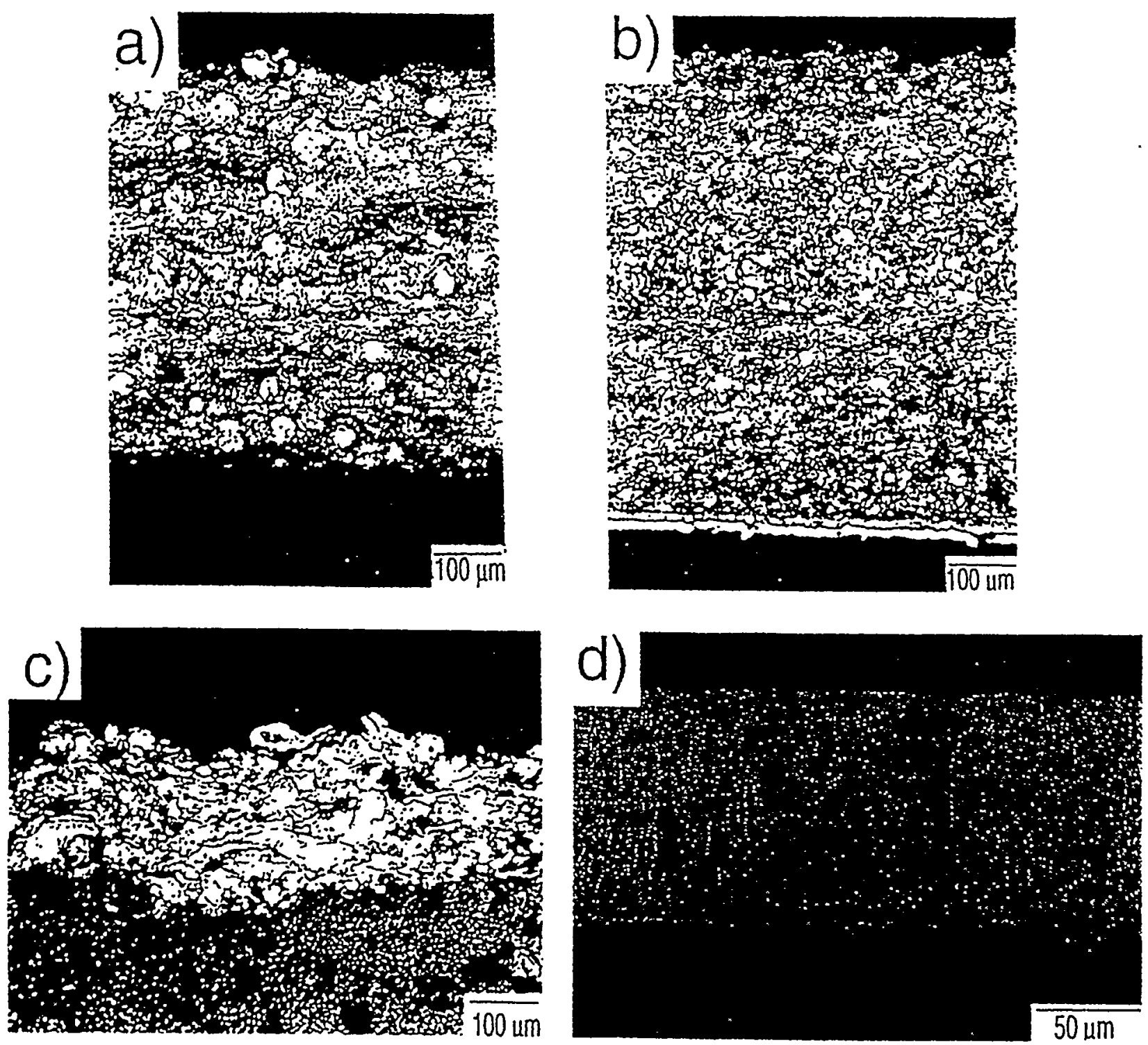

Fig. 1. Metallographic sections of the four different types of coatings: (a) VPS-coating, KFA Jülich, (b) VPS-coating with Re-containing intermediate layer, P/SM AG, (c) IPS-coating, CEN Cadarache, (d) PVD-coating from Plansee with Re-containing interht 8 iat layer 


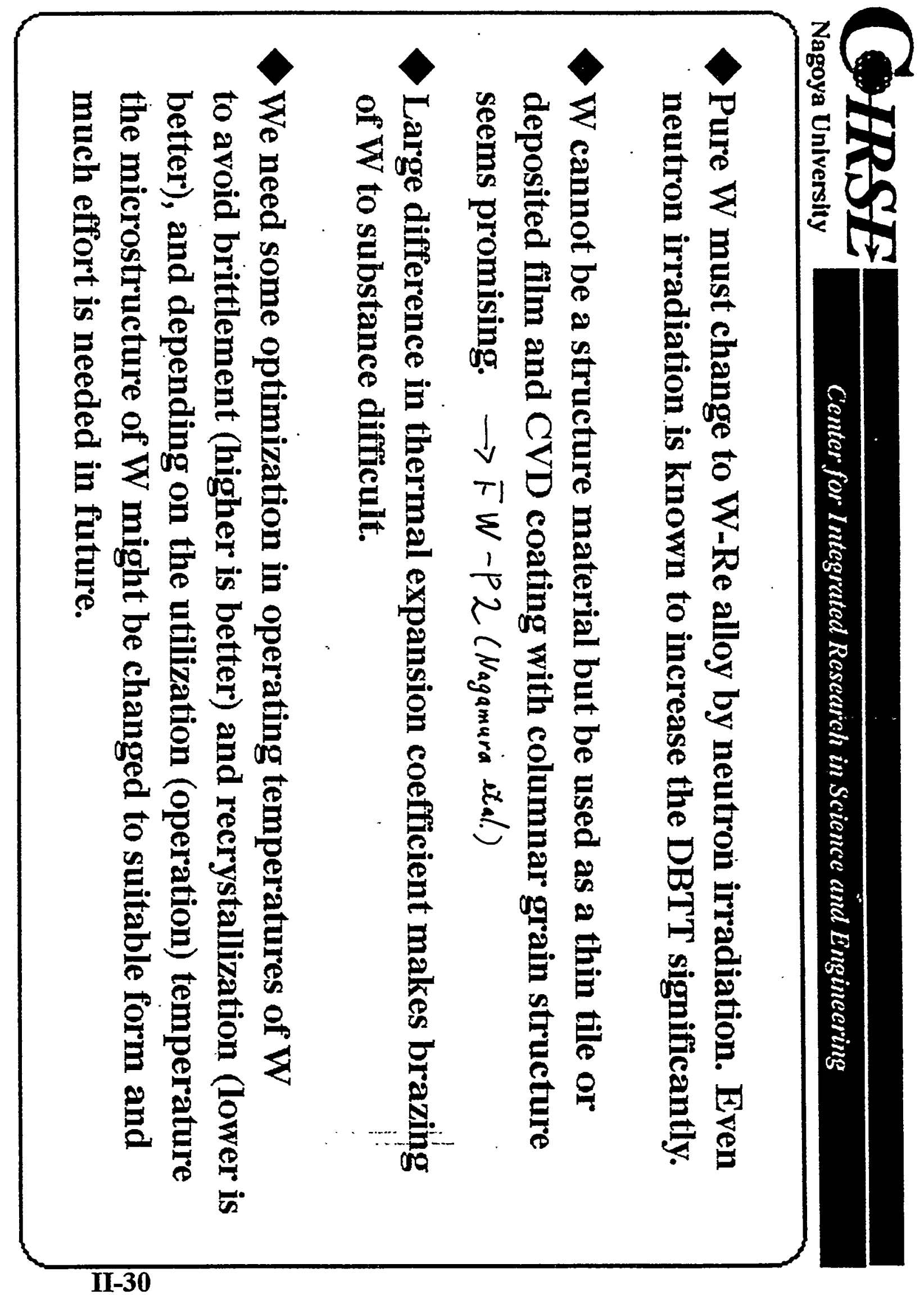




\section{Weight Loss(mg)}

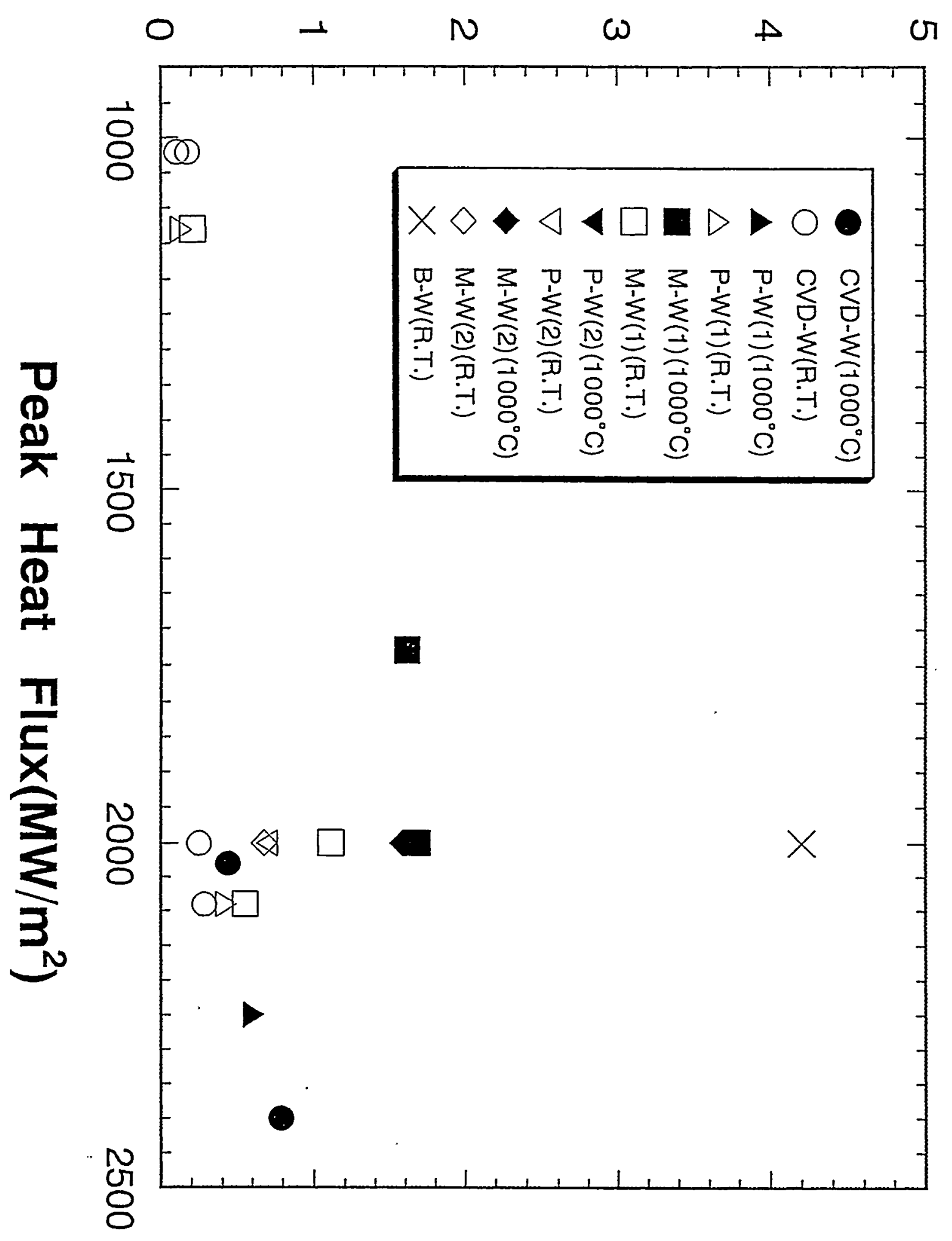



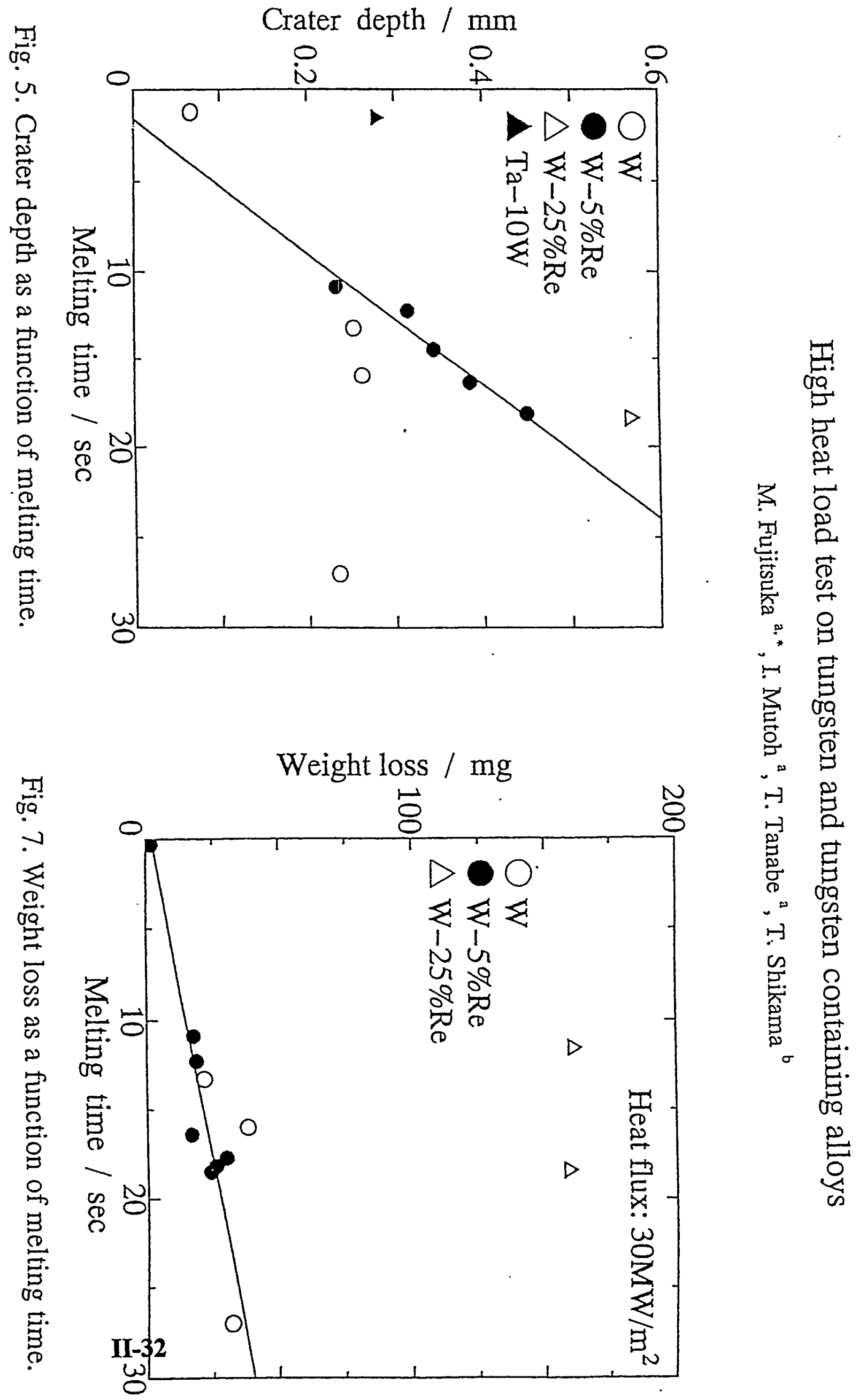


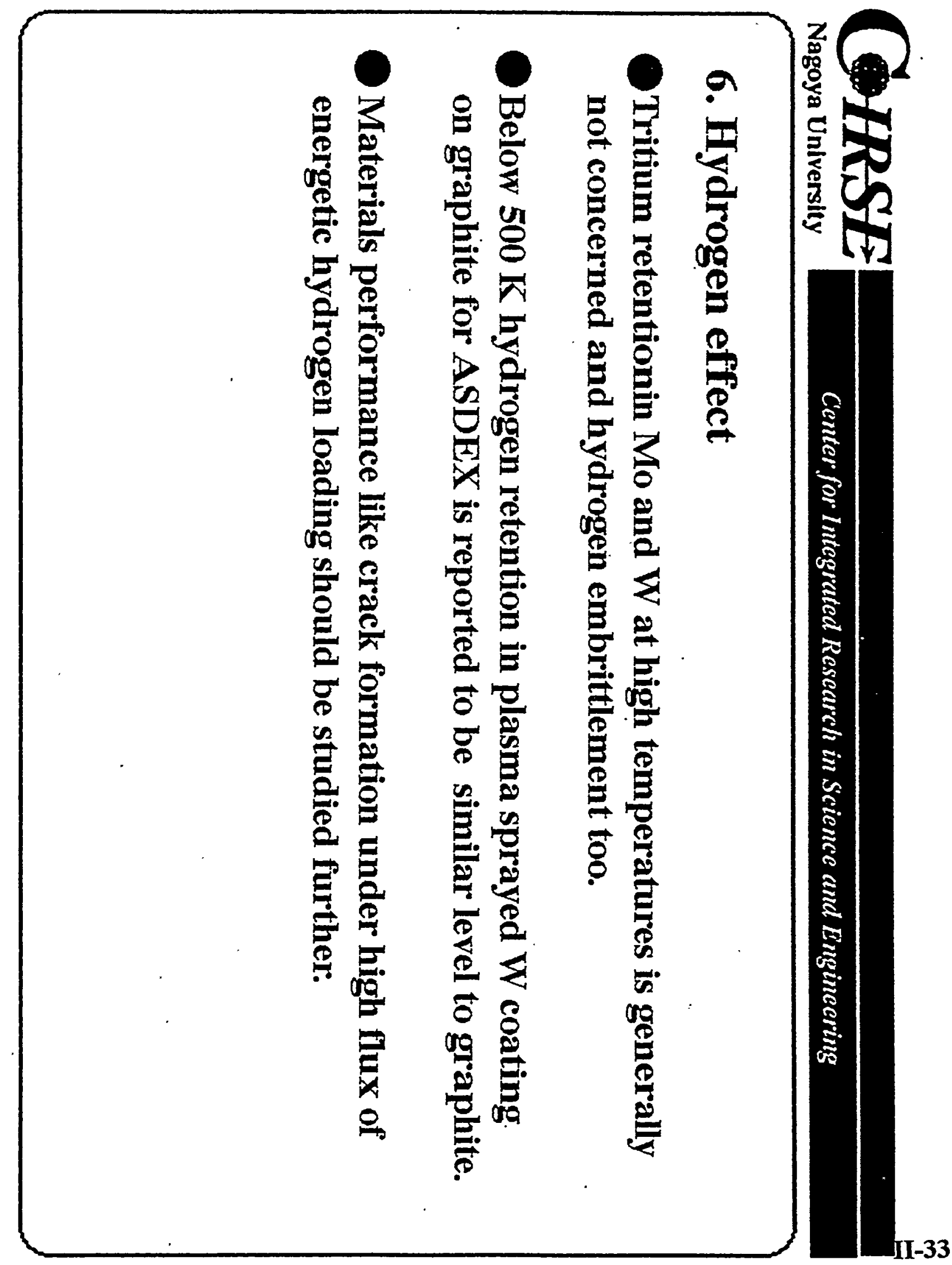




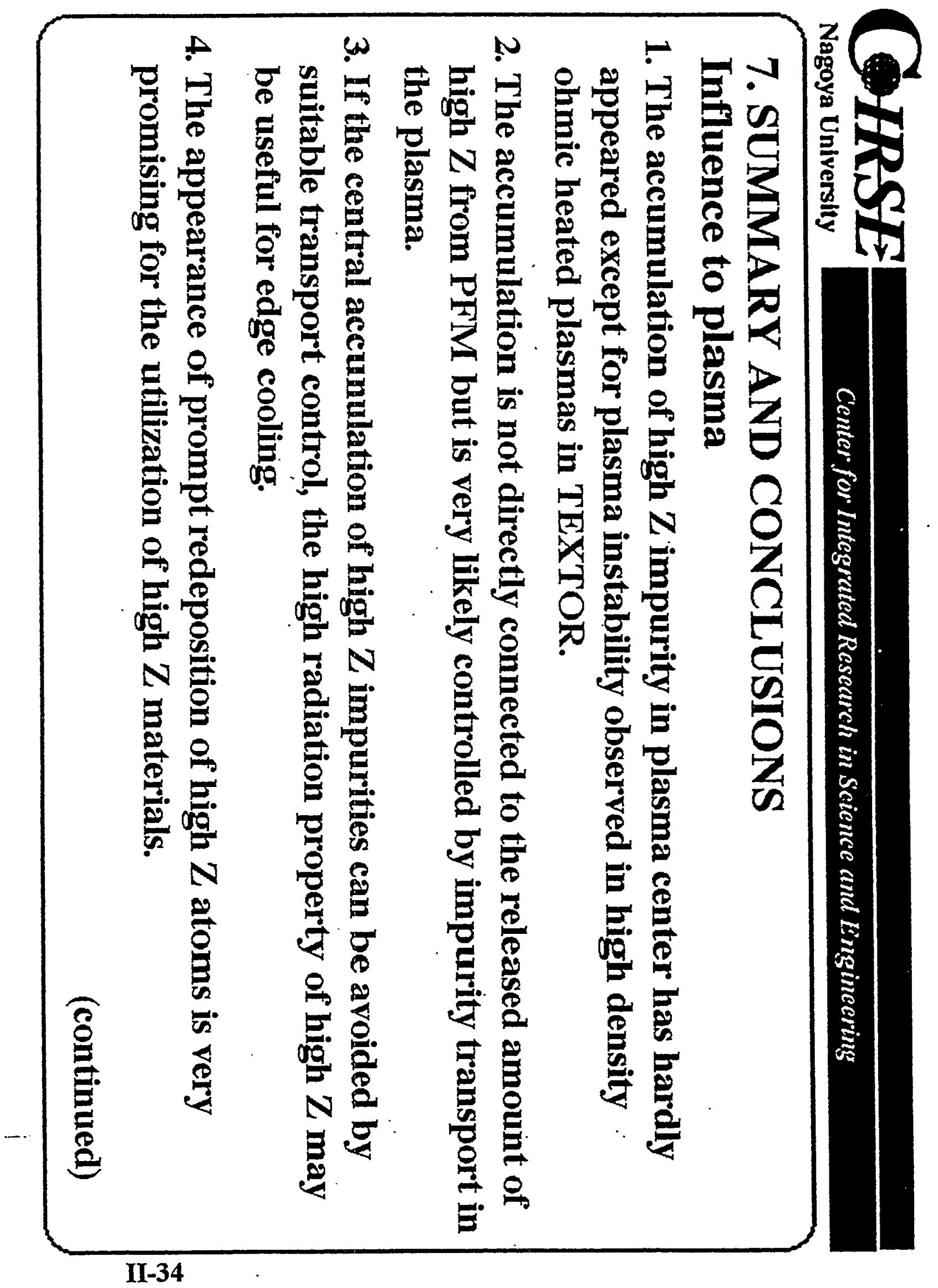




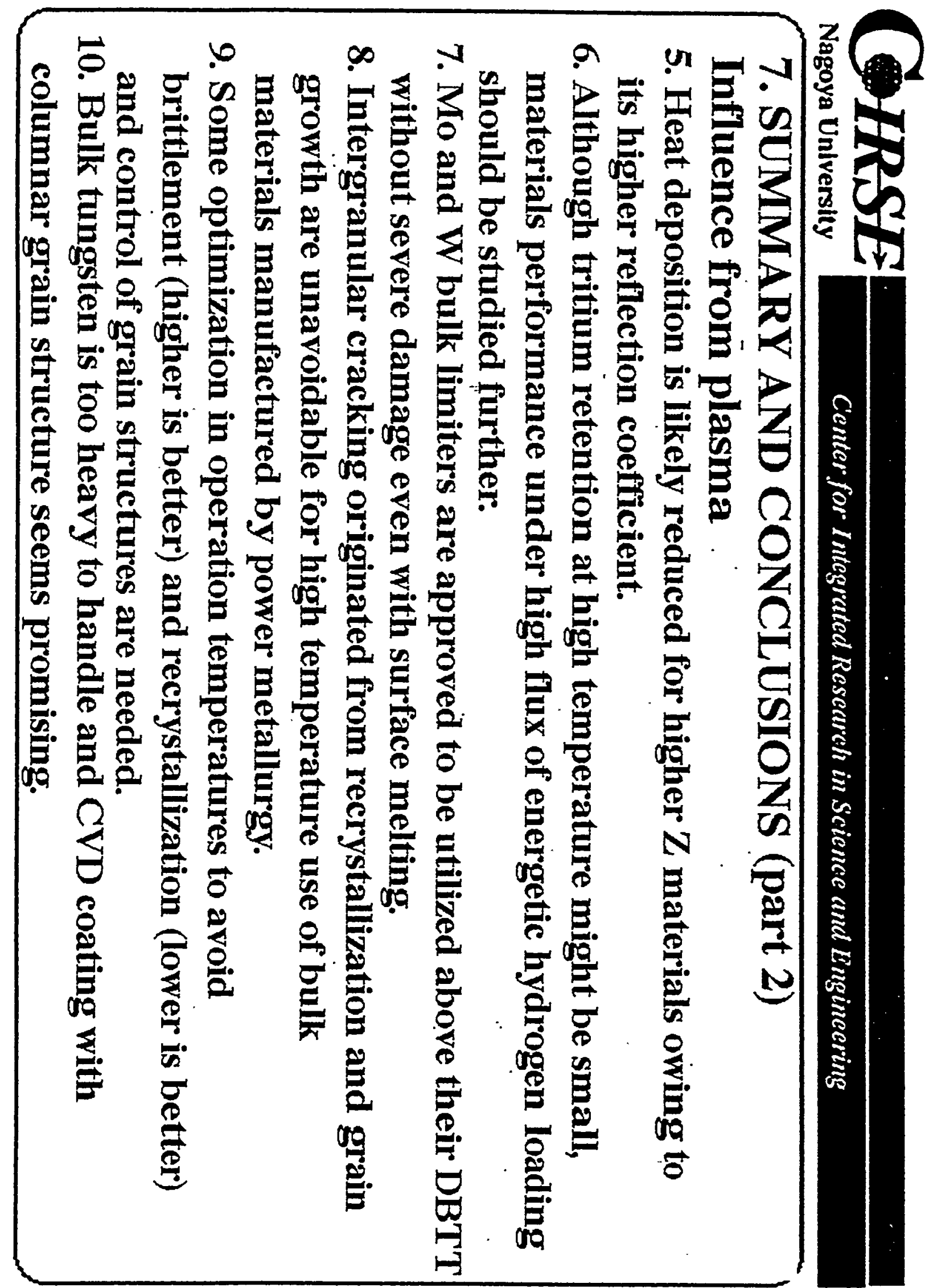




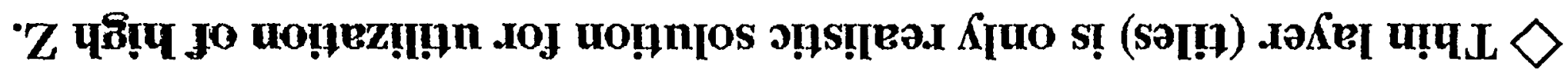

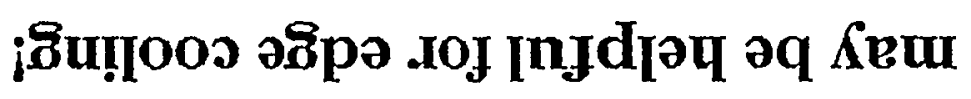

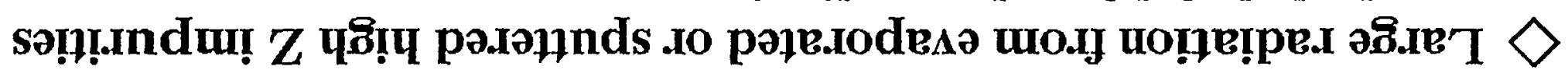

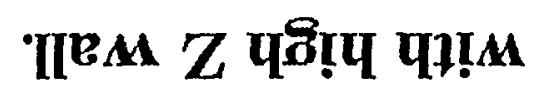

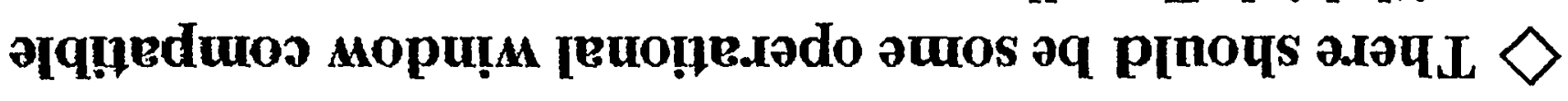

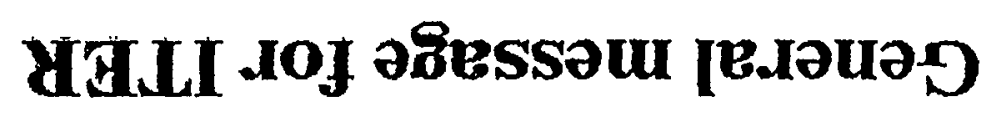




\title{
Tungsten Brush Development ITER Divertor Task T221
}

US-Japan Workshop on Fusion Technology

\author{
San Francisco, CA \\ 8 - 9 December 1997
}




\section{CONTRIBUTORS}

U.S. Home Team

v SANDIA NATIONAL LABS - NEW MEXICO

- R. Watson, M. Ulrickson

$v$ SANDIA NATIONAL LABS - LIVERMORE

- C. Cadden, B. Odegard

$v$ BOEING - ST. LOUIS

- J. Davis, D. Deuser, D. Driemeyer, R. Lederich, K. Slattery, J. Wille

v PLASMA PROCESSES

- T. McKechnie, S. O'Dell

v SURMET CORPORATION

- R. Cooke, N. Gunda, S. Sastri 


\section{Tungsten Brush Fabrication - 3 Methods}

U.S. Home Team

PLASMA SPRAY METHOD

1) Fixture Pointed W Rods in Honeycomb

2) Plasma Spray $\mathrm{Cu}$ to Tips of Rods

3) HIP Diffusion Bond to $\mathrm{CuCrZr}$ Heat Sink at $450^{\circ} \mathrm{C}-550^{\circ} \mathrm{C} / 200 \mathrm{MPa} / 180 \mathrm{~min}$
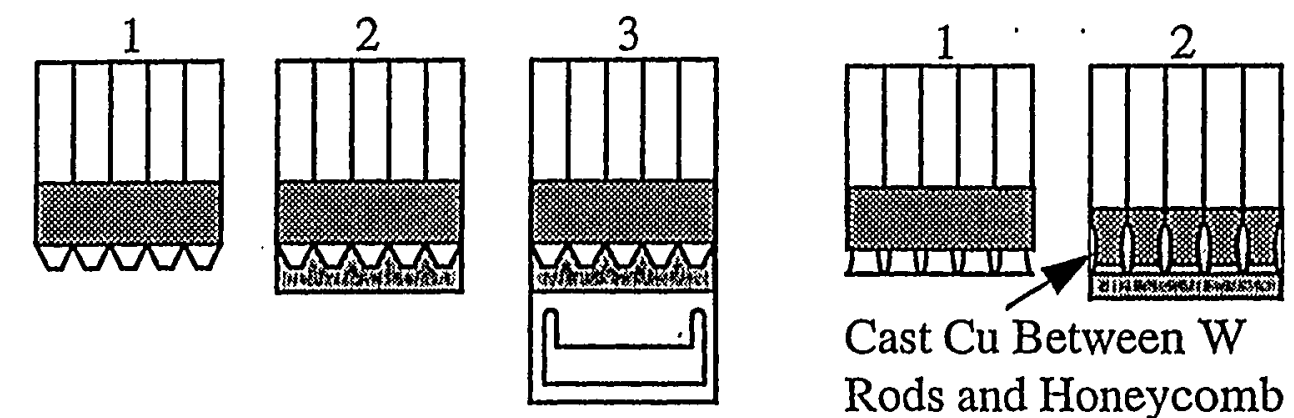

Cast Cu Between W Rods and Honeycomb

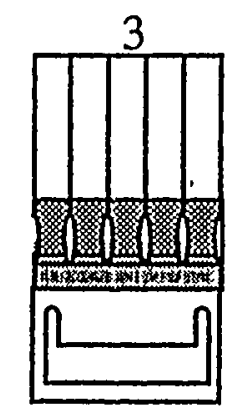

1) Fixture Tapered W Rods in Honeycomb

2) Cast $\mathrm{Cu}$ to Tips of Rods

3) HIP Diffusion Bond to $\mathrm{CuCrZr}$ Heat Sink at

\section{DIRECT DIFFUSION BOND METHOD} $450^{\circ} \mathrm{C} / 200 \mathrm{MPa} / 180 \mathrm{~min}$

1) Fixture Pointed W Rods in Honeycomb \& PVD Coat Rod Tips with Diffusion Aid

2) HIP Diffusion Bond to CuCrZr Heat Sink (a) at $450^{\circ} \mathrm{C} / 200 \mathrm{MPa} / 180 \mathrm{~min}$, Driving Rods into OFHC Cladding (b)
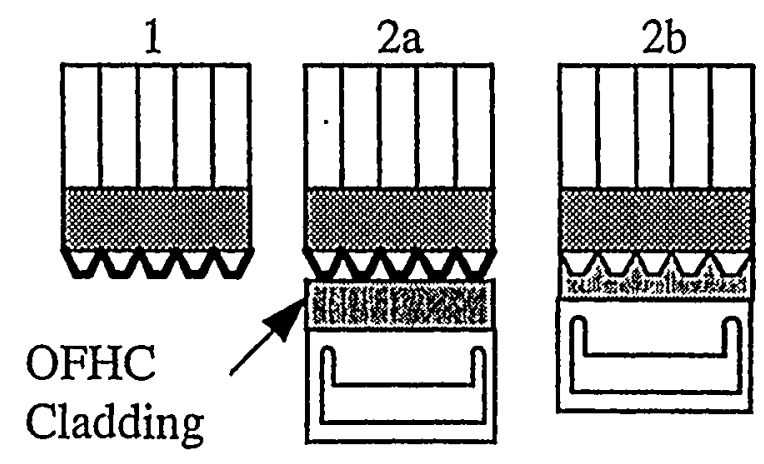


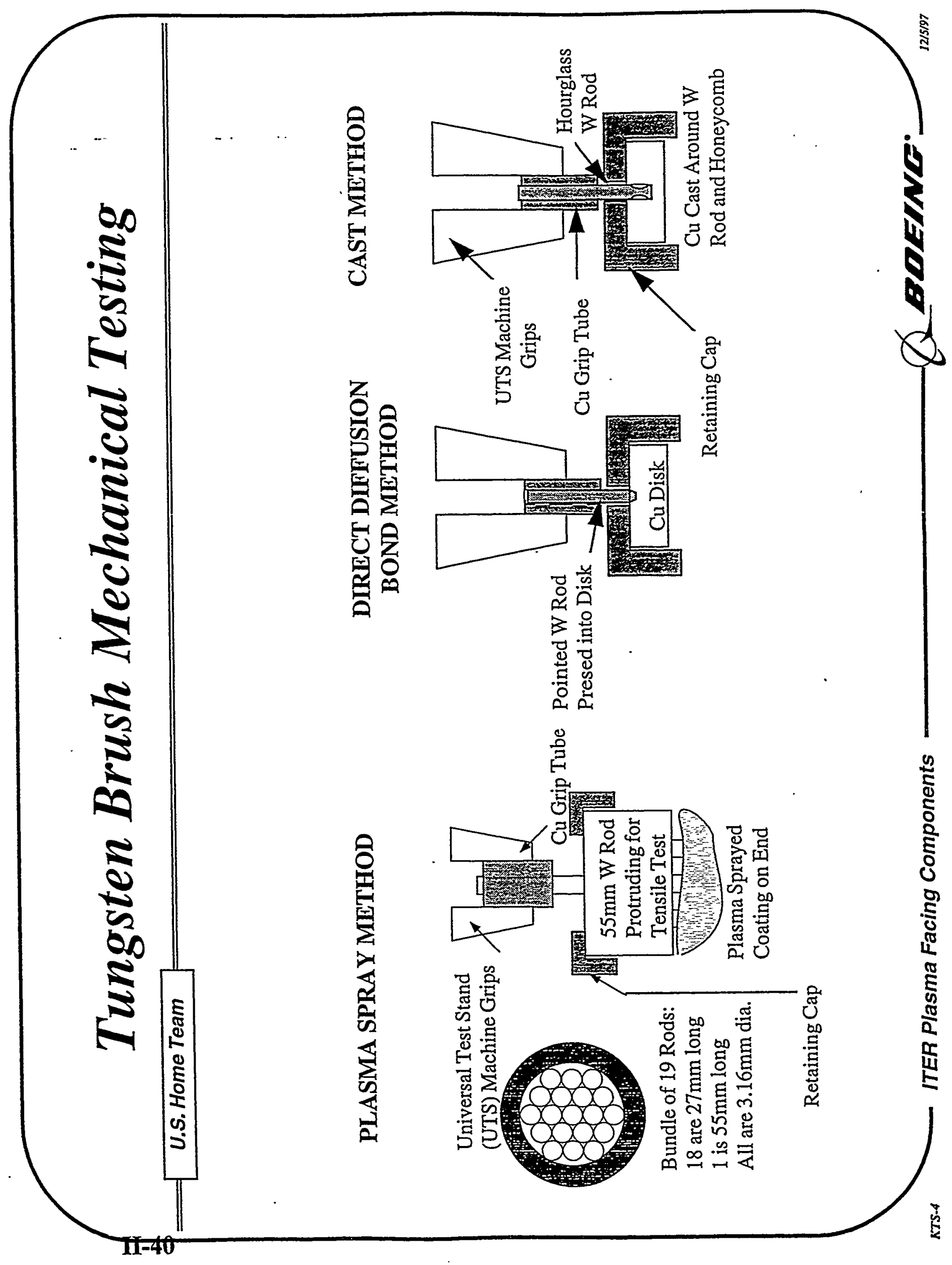




\section{Mechanical Testing Results}

PLASMA SPRAY METHOD - ALL TESTS @ $280^{\circ} \mathrm{C}$

\begin{tabular}{|c|c|c|}
\hline $\begin{array}{c}\text { PLASMA SPRAYED } \\
\text { COATING }\end{array}$ & $\begin{array}{c}\text { THERMAL TREATMENT } \\
\text { CYCLES }\end{array}$ & $\begin{array}{c}\text { FAILURE STRESS } \\
\text { (MPa) }\end{array}$ \\
\hline $\mathrm{Cu}$ & Vacuum Anneal $900^{\circ} \mathrm{C} \&$ HIP & 139 \\
\hline $\mathrm{Cu}$ & HIP & 136 \\
\hline Fine Ni $\cdot$ & Vacuum Anneal $900^{\circ} \mathrm{C}$ & 110 \\
\hline Fine Ni. & Vacuum Anneal $600^{\circ} \mathrm{C} \&$ HIP & 118 \\
\hline Fine Ni & Vacuum Anneal $900^{\circ} \mathrm{C} \&$ HIP & 108 \\
\hline Fine Ni & HIP & 101 \\
\hline Coarse Ni & Vacuum Anneal $600^{\circ} \mathrm{C}$ & 119 \\
\hline Coarse Ni & Vacuum Anneal $900^{\circ} \mathrm{C} \&$ HIP & 109 \\
\hline Coarse Ni & HIP & 107 \\
\hline PPI-1 & Vacuum Anneal $600^{\circ} \mathrm{C}$ & 141 \\
\hline PPI-1 & HIP & 97 \\
\hline
\end{tabular}

CAST METHOD - ALL TESTS @ $280^{\circ} \mathrm{C}$ Grip Tube Slipped and Test Discontinued $370 \mathrm{MPa}$ for Cu cast on $5 \mathrm{~mm}$ of rod $425 \mathrm{MPa}$ for $\mathrm{Cu}$ cast on $6 \mathrm{~mm}$ rod

Failure Stress Refers to Axial Stress in W Rod When Bond Failed 


\section{Mechanical Testing Results}

\section{DIRECT DIFFUSION BOND METHOD}

\begin{tabular}{|c|c|c|c|c|c|}
\hline $\begin{array}{l}\text { SPECIMEN IDENTIFICATION } \\
\text { \& CU ALLOY BASE IF NOT OFHC }\end{array}$ & COATING & TIP & $\begin{array}{c}\text { THERMAL } \\
\text { TREATMENTS }\end{array}$ & $\begin{array}{l}\text { TEST TEMP } \\
\left({ }^{\circ} \mathrm{C}\right) \\
\end{array}$ & $\begin{array}{l}\text { FAILURE STRESS } \\
\text { (MPa) }\end{array}$ \\
\hline 316 & PVD Nb & $45^{\circ} 1 / 2$ taper & none & 300 & 112 \\
\hline 317 & PVD Cu & $45^{\circ} 1 / 2$ taper & none & 300 & 67 \\
\hline $319 R$ & PVD Ni & $45^{\circ} 1 / 2$ taper & none & 280 & 94 \\
\hline $320,323,340,346,349$ & PVD Nb \& Ni & $45^{\circ} 1 / 2$ taper & none & 300 & $133,106,101,120,110$ \\
\hline 341 & PVD Nb \& Ni & $45^{\circ} 1 / 2$ taper & none & RT & 310 \\
\hline 353 & PVD Nb \& Ni & $45^{\circ} 1 / 2$ taper $2 \mathrm{~mm}$ deep & none & 280 & 179 \\
\hline 354 & PVD Nb \& Ni & $45^{\circ} 1 / 2$ taper $3 \mathrm{~mm}$ deep & none & 280 & 156 \\
\hline 355 & PVD Nb \& Ni & $45^{\circ}$ full taper $4 \mathrm{~mm}$ deep & none & 280 & 123 \\
\hline 356 & PVD Nb \& Ni & flat & none & 280 & 0 \\
\hline 359 (CuCrZr Cn A) & PVD Nb \& Ni & $45^{\circ} 1 / 2$ taper $2 \mathrm{~mm}$ deep & none & 280 & 2 \\
\hline 360 (CuCrZr Cn HT) & PVD Nb \& Ni & $45^{\circ} 1 / 2$ taper $2 \mathrm{~mm}$ deep & none & 280 & 5 \\
\hline $361(\mathrm{CuCrZr} \operatorname{Cn} \mathrm{A})$ & PVD Nb \& Ni & $45^{\circ}$ full taper $4 \mathrm{~mm}$ deep & none & 280 & 0 \\
\hline 357 & PVD Nb, Nb \& Ni & $45^{\circ} \quad 1 / 2$ taper & $1800^{\circ} \mathrm{C}, 30 \mathrm{~min}$ & 280 & 47 \\
\hline 321 & PVD Cu \& Ni & $45^{\circ} 1 / 2$ taper & none & 300 & 75 \\
\hline 324 & PVD Cu \& Ni & $45^{\circ} 1 / 2$ taper & none & 307 & 66 \\
\hline 326 & PVD Ni \& Ni & $45^{\circ} 1 / 2$ taper & $800^{\circ} \mathrm{C}, 60 \mathrm{~min}$ & 300 & 64 \\
\hline 327 & PVD Cu, Ni \& Ni & $45^{\circ} 1 / 2$ taper & $1000^{\circ} \mathrm{C}, 60 \mathrm{~min}$ & 320 & 32 \\
\hline
\end{tabular}

*353, 354, 355, 361 bent during pressing

Failure Stress Refers to Axial Stress in W Rod When Bond Failed 


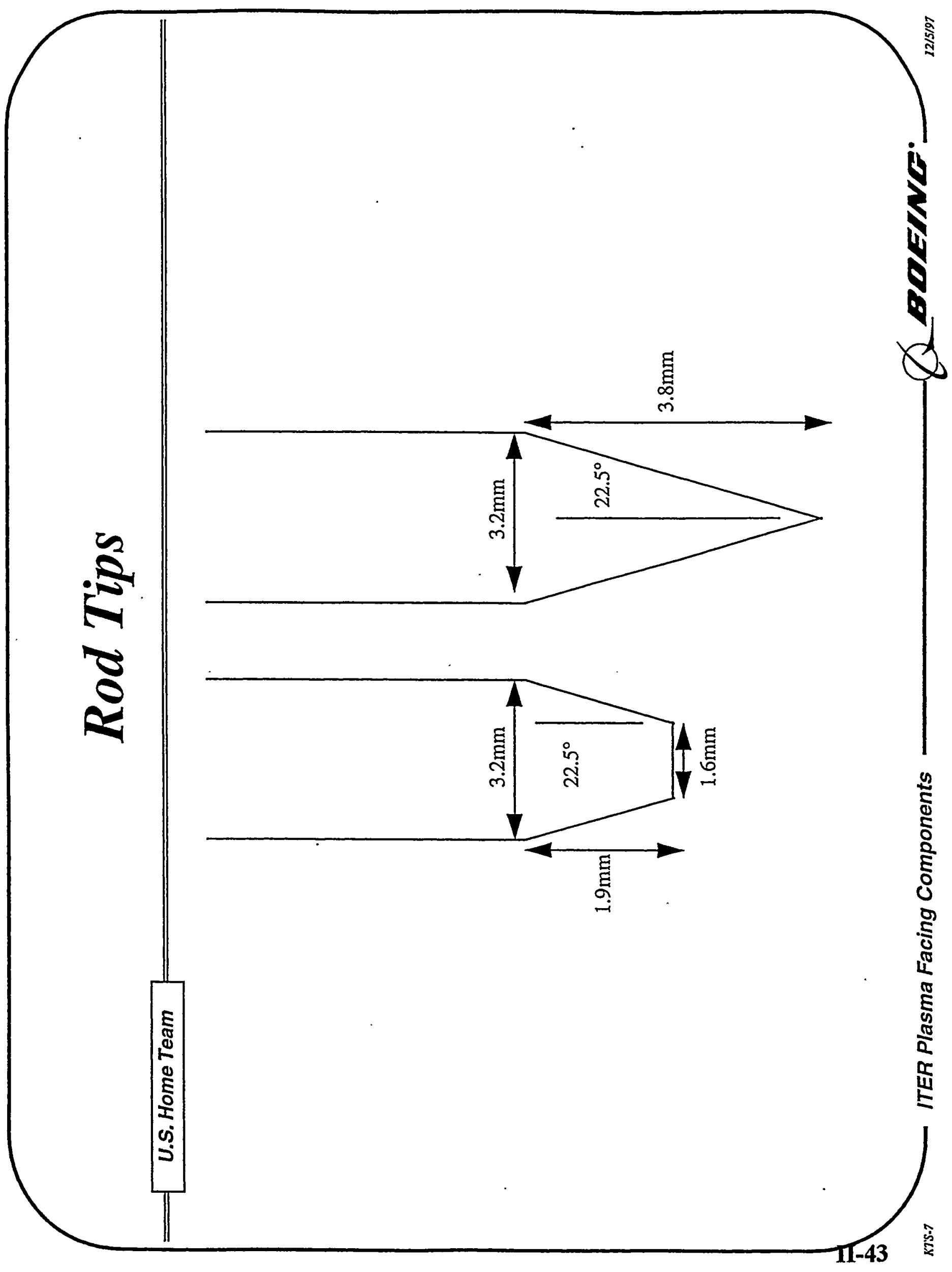




\section{Mechanical Testing Results}

DIRECT DIFFUSION BOND METHOD

\begin{tabular}{|c|c|c|c|c|c|}
\hline $\begin{array}{c}\text { SPECIMEN IDENTIFICATION } \\
\text { \& CU ALLOY BASE IF NOT OFHC }\end{array}$ & COATING & TIP & $\begin{array}{c}\text { THERMAL } \\
\text { TREATMENTS }\end{array}$ & $\begin{array}{c}\text { TEST TEMP } \\
\left({ }^{\circ} \mathrm{C}\right) \\
\end{array}$ & $\begin{array}{c}\text { FAILURE STRESS } \\
\text { (MPa) }\end{array}$ \\
\hline 328 & PS PPI-1 PVD Ni \& Ni & $45^{\circ} 1 / 2$ taper & $1000^{\circ} \mathrm{C}, 60 \mathrm{~min}$ & 280 & 129 \\
\hline 329 & PS PPI-2 PVD Ni \& Ni & $45^{\circ} 1 / 2$ taper & $1000^{\circ} \mathrm{C}, 60 \mathrm{~min}$ & 280 & 44 \\
\hline 330,358 & PS fn Ni PVD Ni \& Ni & $45^{\circ} 1 / 2$ taper & $1000^{\circ} \mathrm{C}, 60 \mathrm{~min}$ & 280 & 175,131 \\
\hline 331 & PS cs Ni PVD Ni \& Ni & $45^{\circ} 1 / 2$ taper & $1000^{\circ} \mathrm{C}, 60 \mathrm{~min}$ & 280 & 73 \\
\hline 332 & PS Cu PVD Ni \& Ni & $45^{\circ} 1 / 2$ taper & $1000^{\circ} \mathrm{C}, 60 \mathrm{~min}$ & 280 & 20 \\
\hline 334 & SUR-1 & $45^{\circ} 1 / 2$ taper & none & 280 & 130 \\
\hline 335 & SUR-2 & $45^{\circ} 1 / 2$ taper & none & 280 & 66. \\
\hline 337 & SUR-4 & $45^{\circ} 1 / 2$ taper & none & 280 & 33 \\
\hline 338 & SUR-5 & $45^{\circ} 1 / 2$ taper & none & 280 & 73 \\
\hline 339 & SUR-6 & $45^{\circ} 1 / 2$ taper & none & 280 & 106 \\
\hline 347 & SUR-7 & $45^{\circ} 1 / 2$ taper & none & 280 & 21 \\
\hline 348 & SUR-8 & $45^{\circ} 1 / 2$ taper & none & 280 & 121 \\
\hline 350 & Cast Cu \& PVD Ni & $45^{\circ} 1 / 2$ taper & none & 280 & 12 \\
\hline 351 & Cast Cu \& PVD Ni & flat & none & 280 & 151 \\
\hline
\end{tabular}

Failure Stress Refers to Axial Stress in W Rod When Bond Failed ITER Plasma Facing Components 


\section{W/Cu Interfaces - Plasma Sprayed}

U.S. Home Team

Interface Between W and Plasma Sprayed Cu with Ni Bond Coat
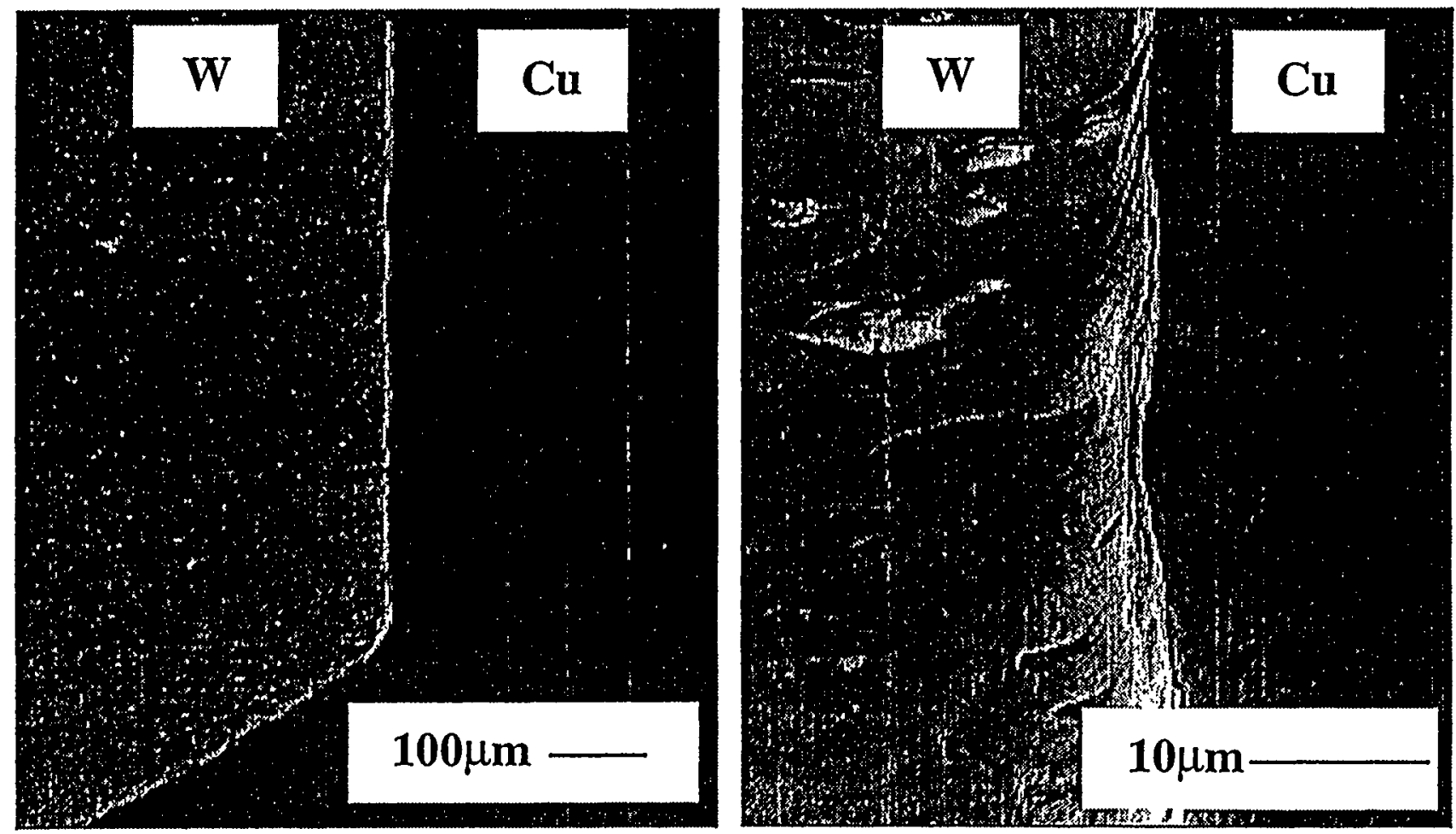


\section{W/Cu Interfaces - Cast Method}

U.S. Home Team

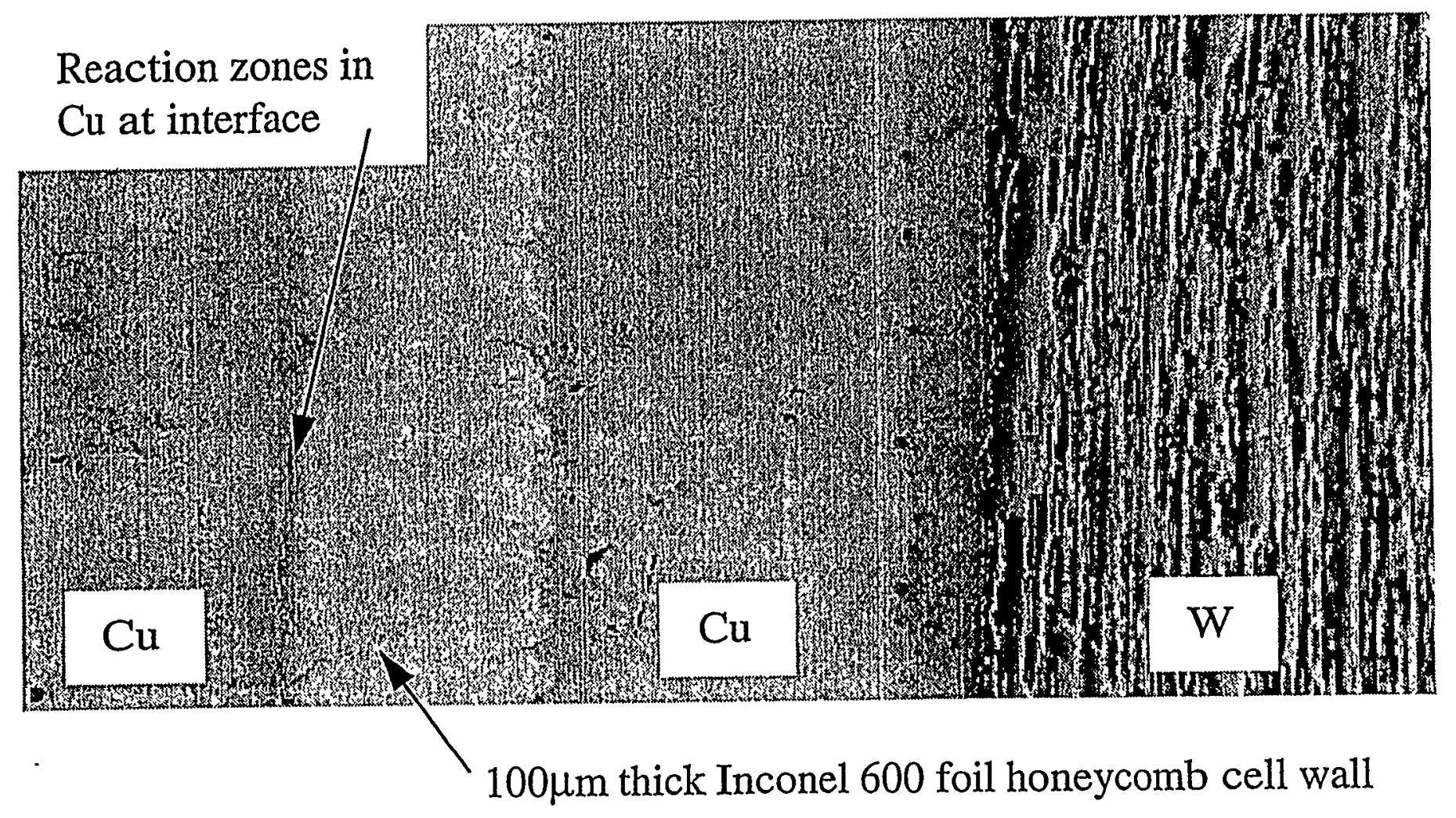

ITER Plasma Facing Components

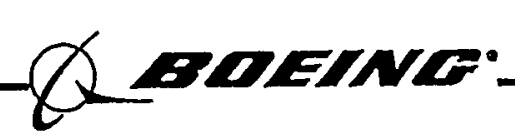




\section{Cast Cu Around W Rod}

U.S. Home Team

Specimen 393 - Grip Tube Slipped at 370MPa
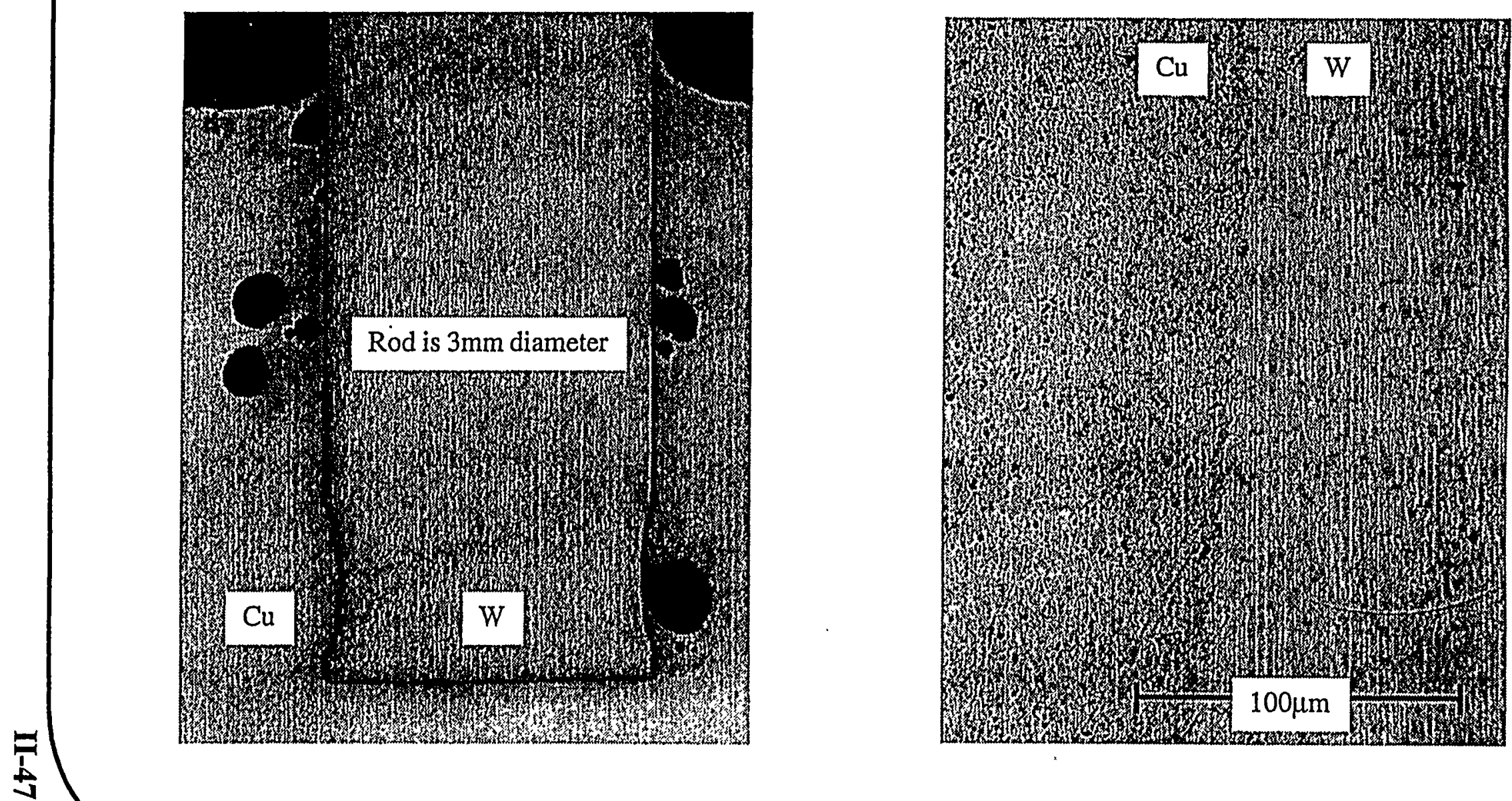


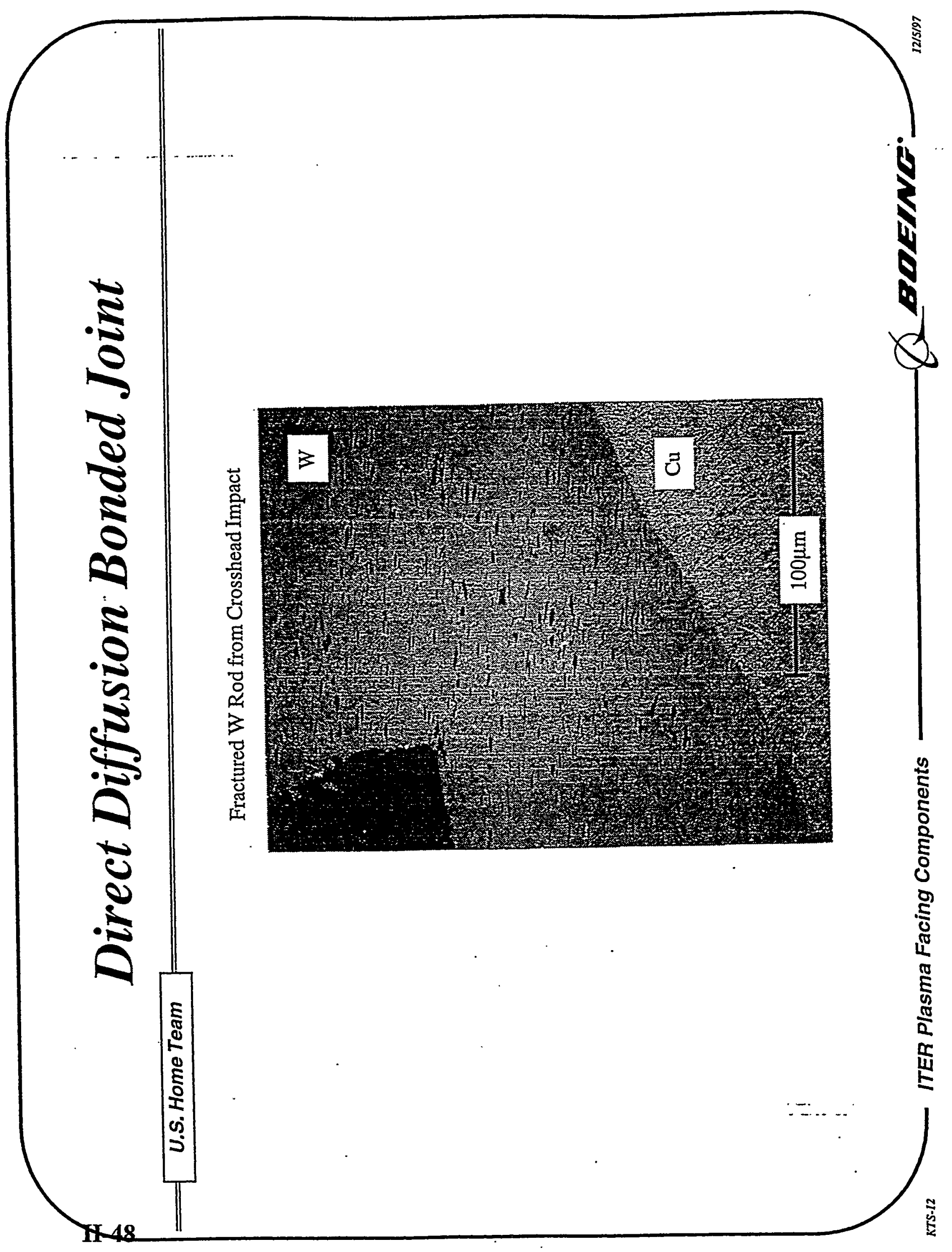




\section{Tungsten Brush Mock-ups PW-9 and PW-4}

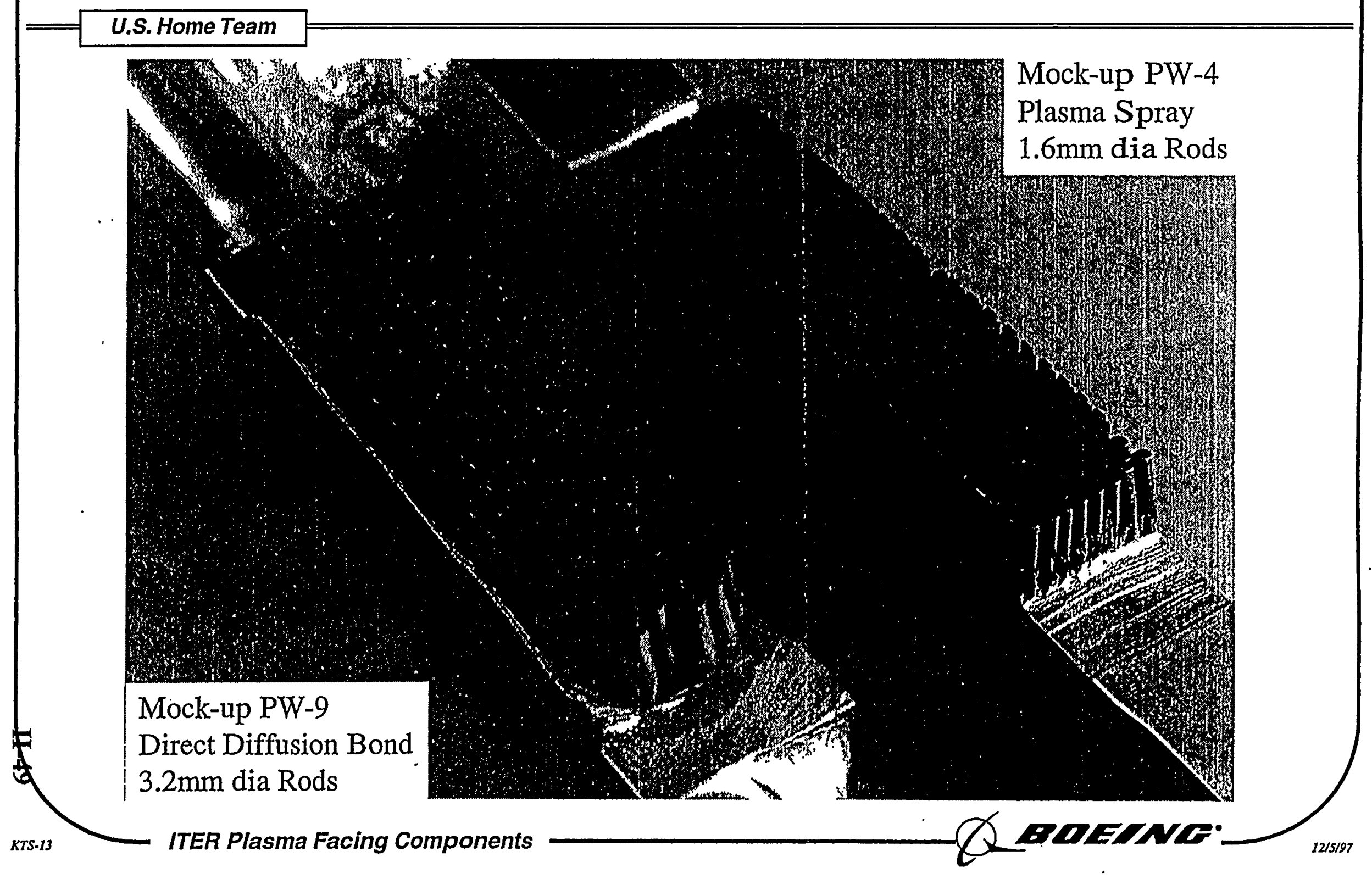




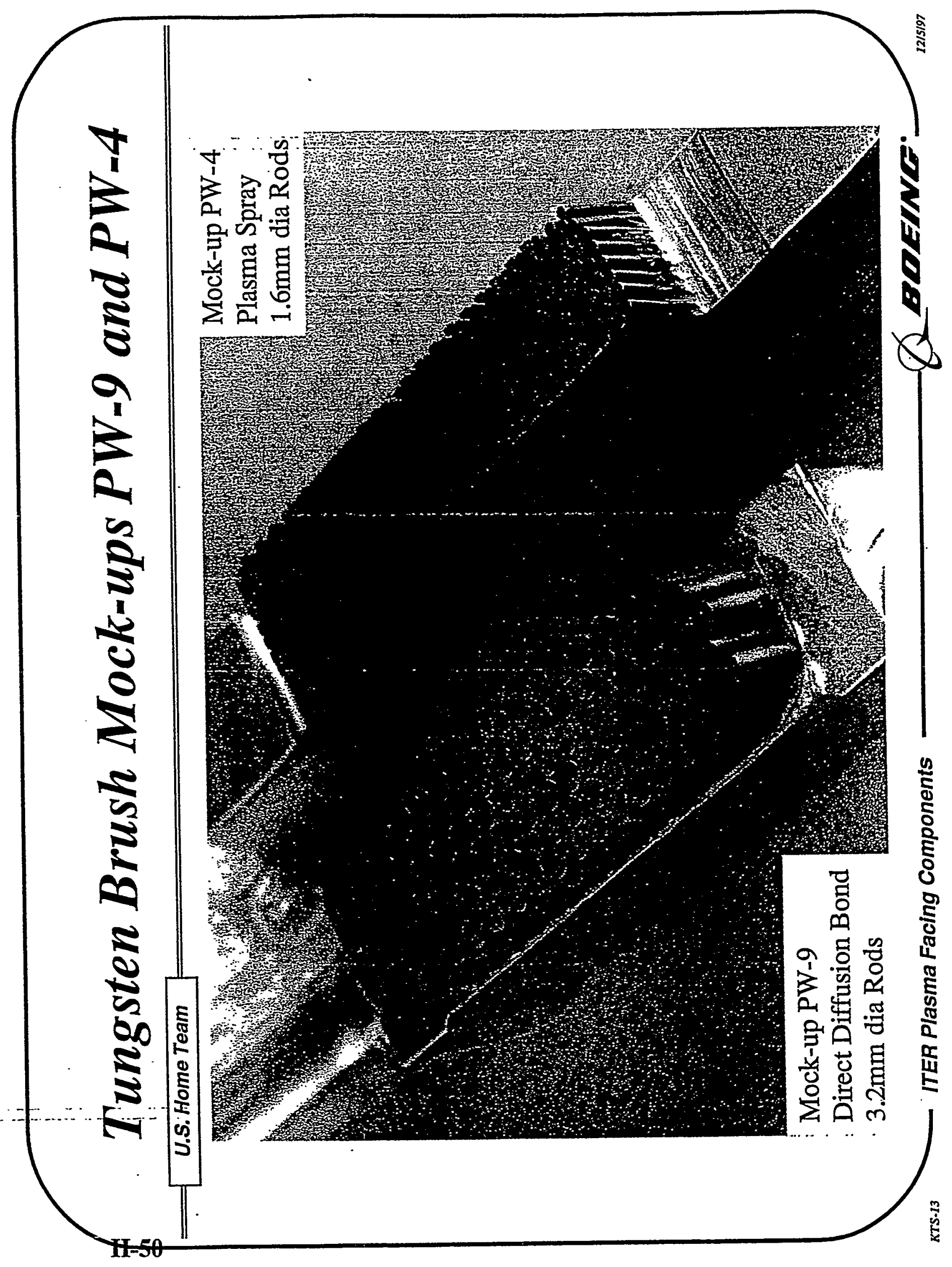


US-Japan Workshop on Plasma Material

Interaction and High Heat Flux Components,

December 8-11, 1997,

Sandia National Lab., San Francisco, US

\title{
Development of High Heat Flux Components at JAERI
}

\author{
K. Nakamura \\ JAERI
}

1. Overview

2. R\&Ds on Divertor Component Development

空

3. Summary 


\section{Progress in 1997}

1D/3D hybrid CFC was newly developed, and withstood up to a heat load of $20 \mathrm{MW} / \mathrm{m}^{2}, 15 \mathrm{~s}$.

口 $5 \mathrm{~mm}$ thick CVD-W was successfully coated on both OFHC Cu and W/Cu heat sink. Small divertor mock-ups meet the ITER steady-state heat load condition; $5 \mathrm{MW} / \mathrm{m}^{2}, 15 \mathrm{~s}$ for 1000 cycles.

$\square$ Full-scale length Vertical Target with W, CFC armors were successfully fabricated.

$\square \mathrm{SiC}$ doped 1D CFC with high thermal conductivity and high thermal shock resistance has been developed.

$\square$ Neutron irradiation of Be, CFCs and CVD-W was finished at JMTR, and post-irradiated tests will be started soon. 


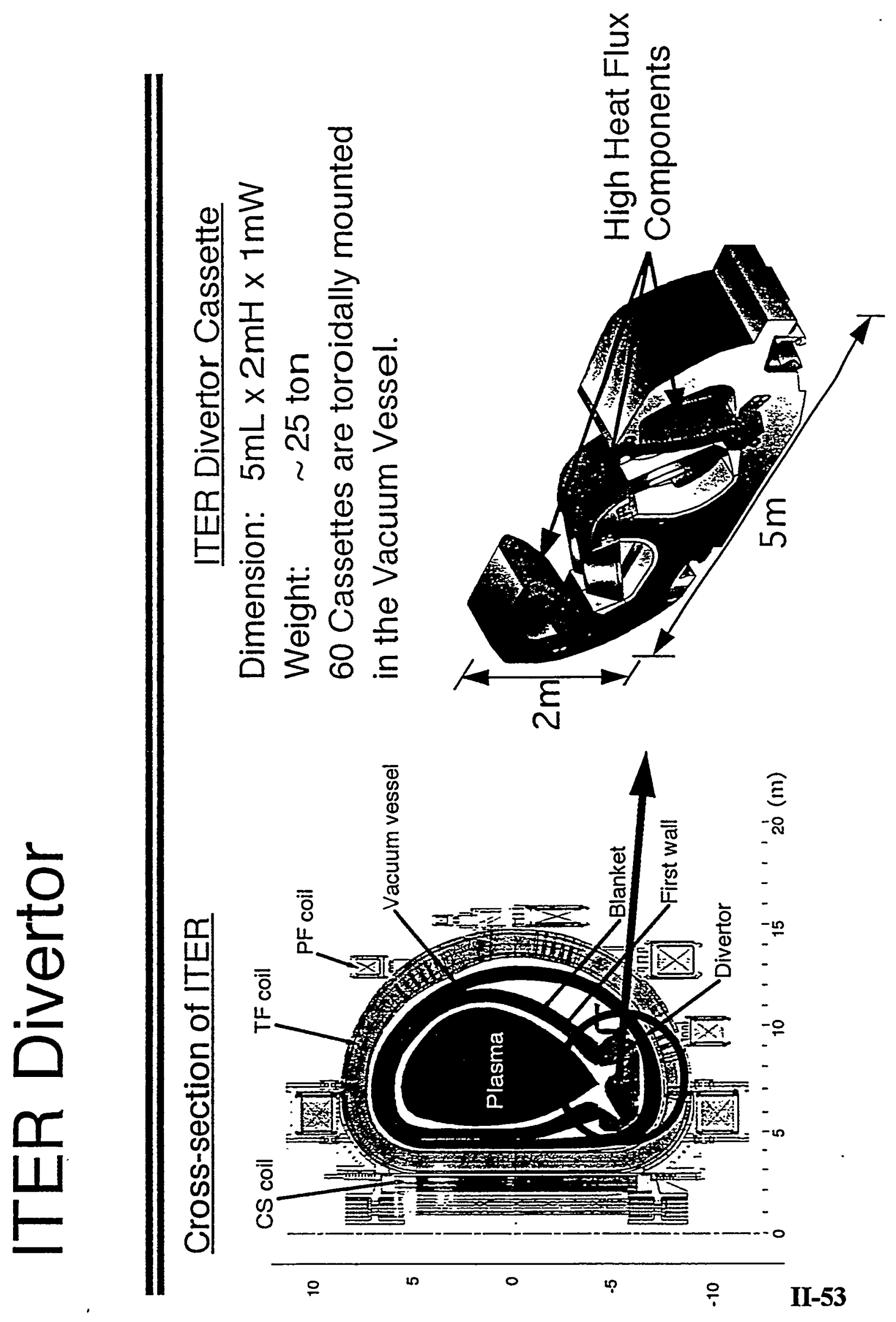


Divertor Cassette

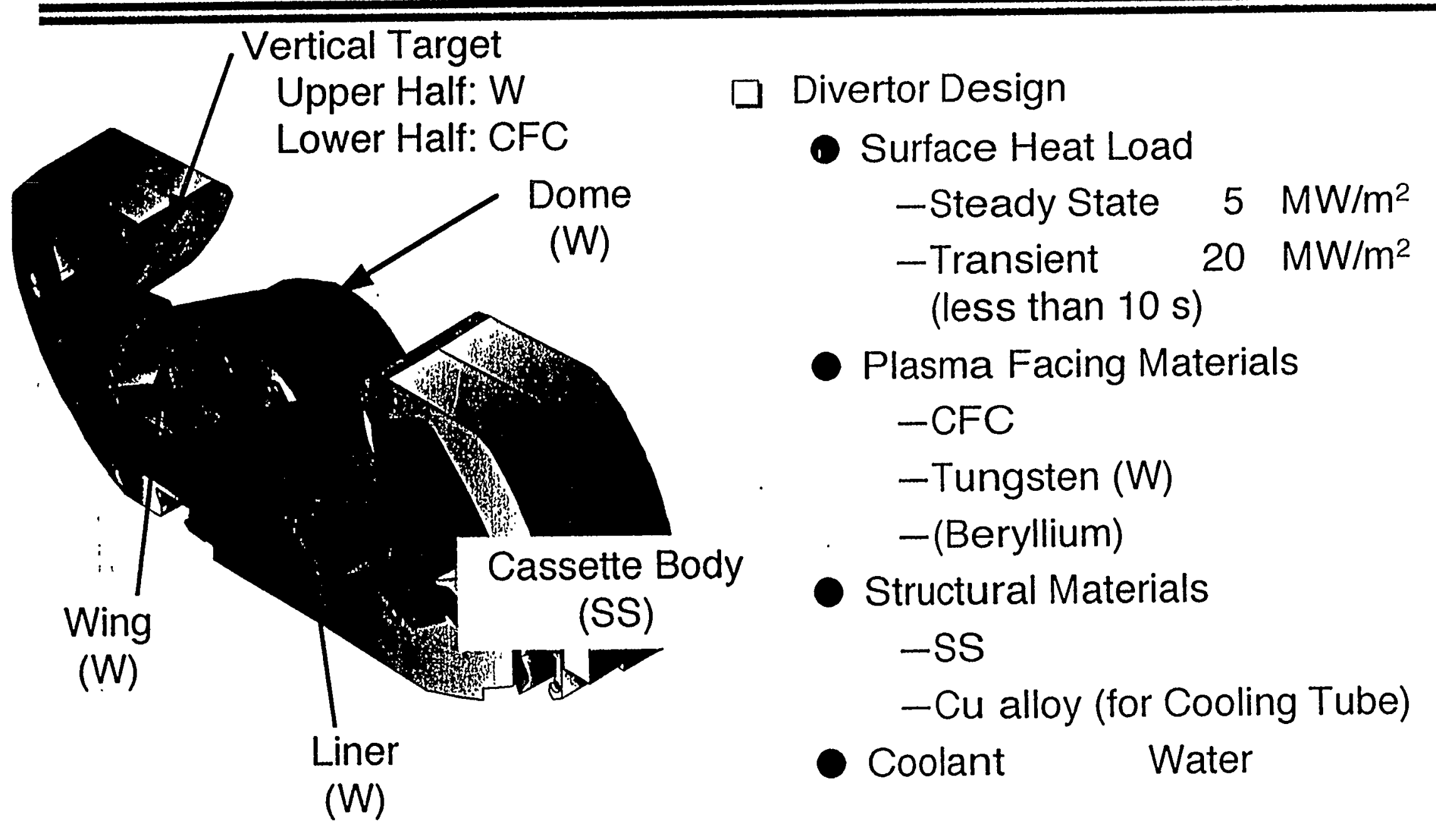




\section{Major Design Parameters of ITER Divertor}

\begin{tabular}{|c|c|c|c|}
\hline Comnonent & & & Wall \\
\hline componemí & & Nominal & Limiter/Baffle \\
\hline Normal Operation & & & \\
\hline Steady-State Heat Load $\left(\mathrm{MW} / \mathrm{m}^{2}\right)$ & $0.2-\underline{5}(10)$ & $0.25-0.5$ & $3-5$ \\
\hline Transient Heat Load $\left(\mathrm{MW} / \mathrm{m}^{2}\right)$ & & & 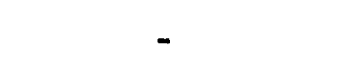 \\
\hline Incident Ion Flux(ions $/ \mathrm{m}^{2} / \mathrm{s}$ ) & $<10^{24}$ & $<10^{20}$ & $<10^{20}$ \\
\hline Incident Ion Energy(eV) & $<100$ & $100-500$ & $100-500$ \\
\hline Neutron Load $\left(\mathrm{MW} / \mathrm{m}^{2}\right)$ & $0.1-1$ & 1 & 1 \\
\hline Plasma Disruption (Thermal Quench) & & & \\
\hline Disruption Heat Load $\left(\mathrm{MJ} / \mathrm{m}^{2}\right)$ & $<100$ & TBD & TBD \\
\hline Duration (ms) & $0.1-3$ & $0.1-3$ & $0.1-3$ \\
\hline Cooling Conditions & & & \\
\hline Coolant & Water & Water & Water \\
\hline Inlet Pressure (MPa) & 4 & $<4$ & $<4$ \\
\hline Inlet Temperature $\left({ }^{\circ} \mathrm{C}\right)$ & 140 & TBD & TBD \\
\hline Materials & & & \\
\hline Plasma Facing Material & CFC, W & CFC, W, Be & $\mathrm{CFC}, \mathrm{W}, \mathrm{Be}$ \\
\hline Structural Material & Cu alloy, ss & Cu alloy, SS & Cu alloy, SS \\
\hline
\end{tabular}




\section{-1996 R\&D-}

\section{D-CFC Armored Divertor Mock-ups}

\section{with Silver-free Braze}
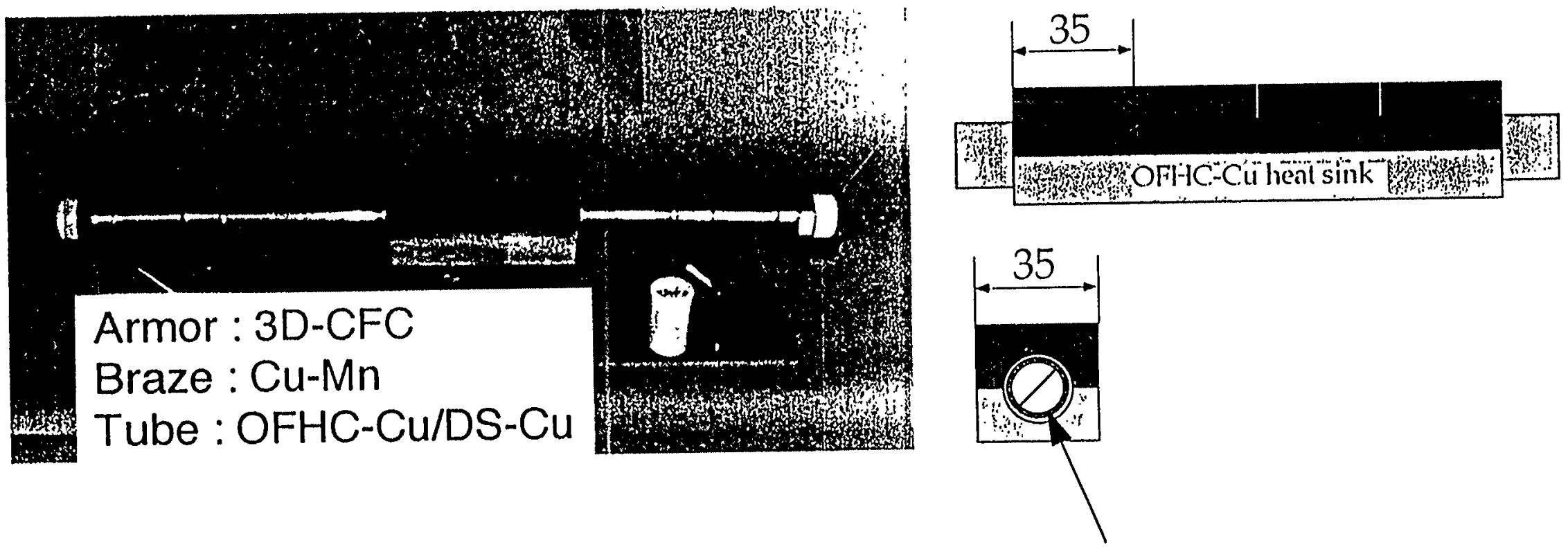

OFHC-Cu cladded DS-Cu tube

- outer skin : OFHC-Cu

- inner core : DS-Cu

- O.D./I.D. = 20/15 


\section{High Heat Flux Experiment on 3D-CFC Divertor Mock-up}
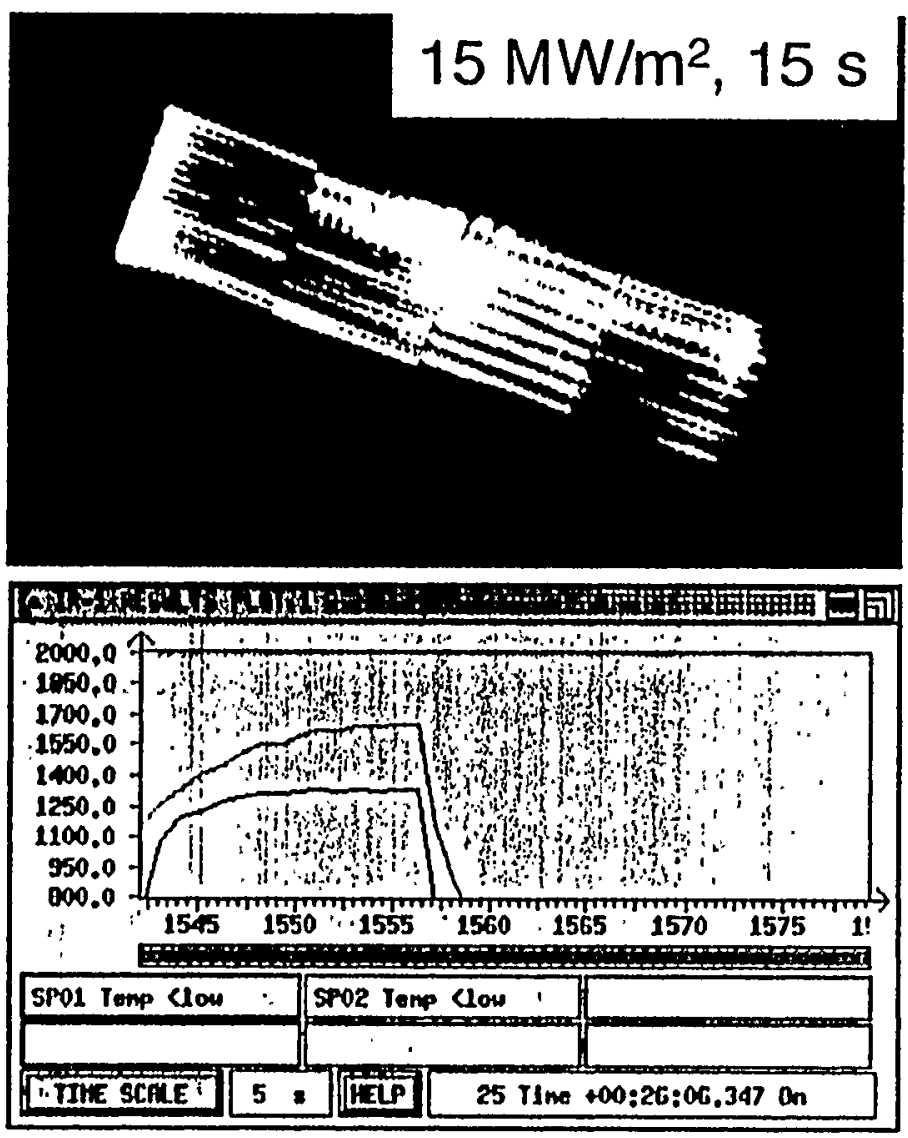

$\square$ 3D-CFC armor tiles silver-free-brazed on DSCu cooling tube endured a heat load of up to $15 \mathrm{MW} / \mathrm{m}^{2}, 15 \mathrm{~s}$ without failure.

$\square$ Fibers brazed onto the cooling tube and the heat sink were well cooled, while thermal conduction was not sufficient for fibers in other directions.

M To achieve higher thermal conduction between fibers, modification of fabrication method of 3D-CFC is on going.

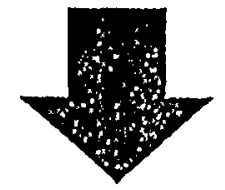

1D/3D Hybrid CFC 


\section{D/3D Hybrid CFC}

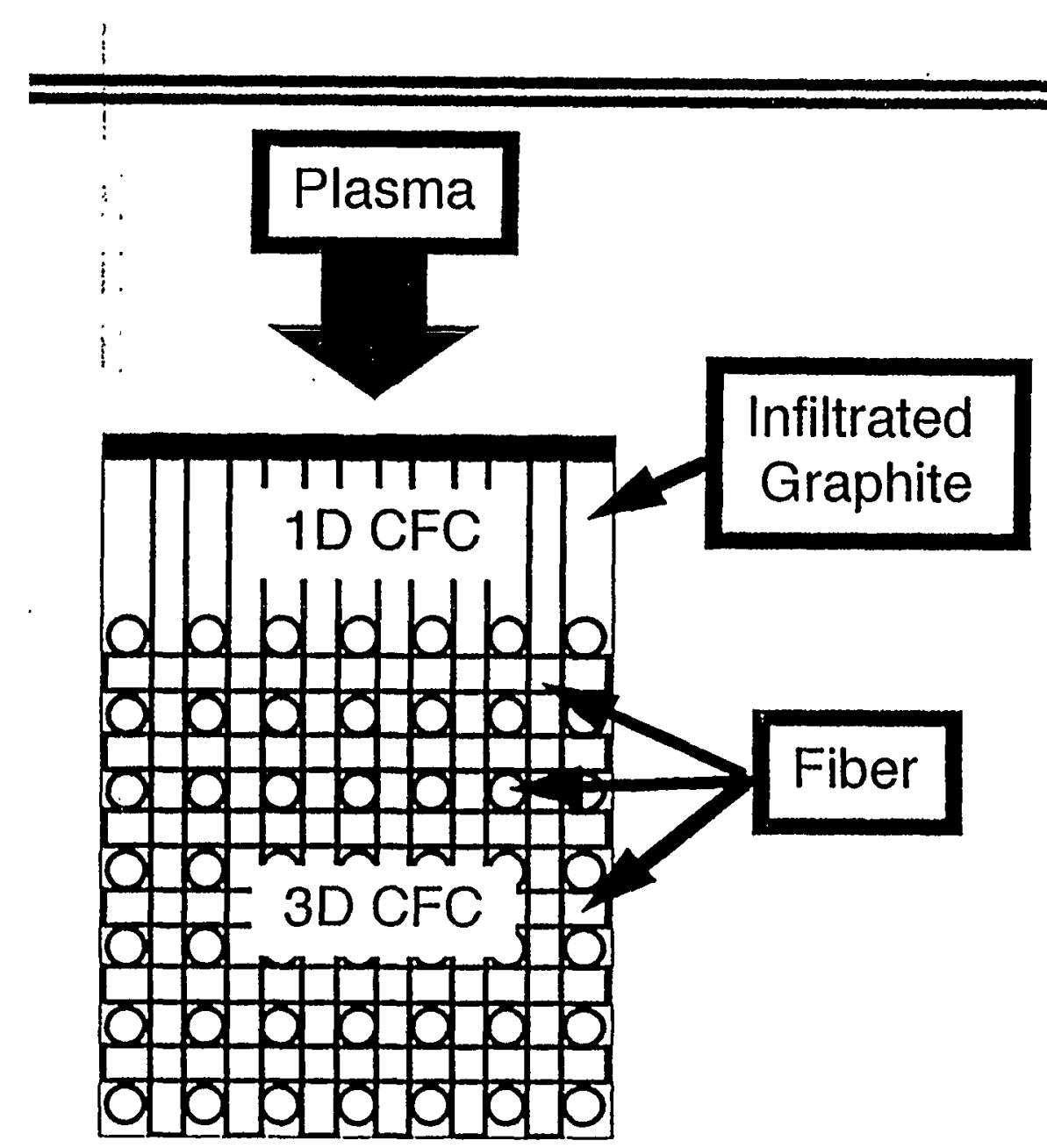

$\square$ Full weaved CFC

$\square$ Graphite powder is infiltrated in the 1D part.

$\square$ Infiltrated graphite is highly graphitized to achieve high thermal conductivity.

$\square$ Thermal Conductivity;

- in 1D $=\sim 550 \mathrm{~W} / \mathrm{m} / \mathrm{K}$

- in $3 D=\sim 450 \mathrm{~W} / \mathrm{m} / \mathrm{K}$ (at room temperature)

Cross-section of Hybrid CFC 


\section{D/3D Hybrid CFC withstands the ITER heat load requirement.}
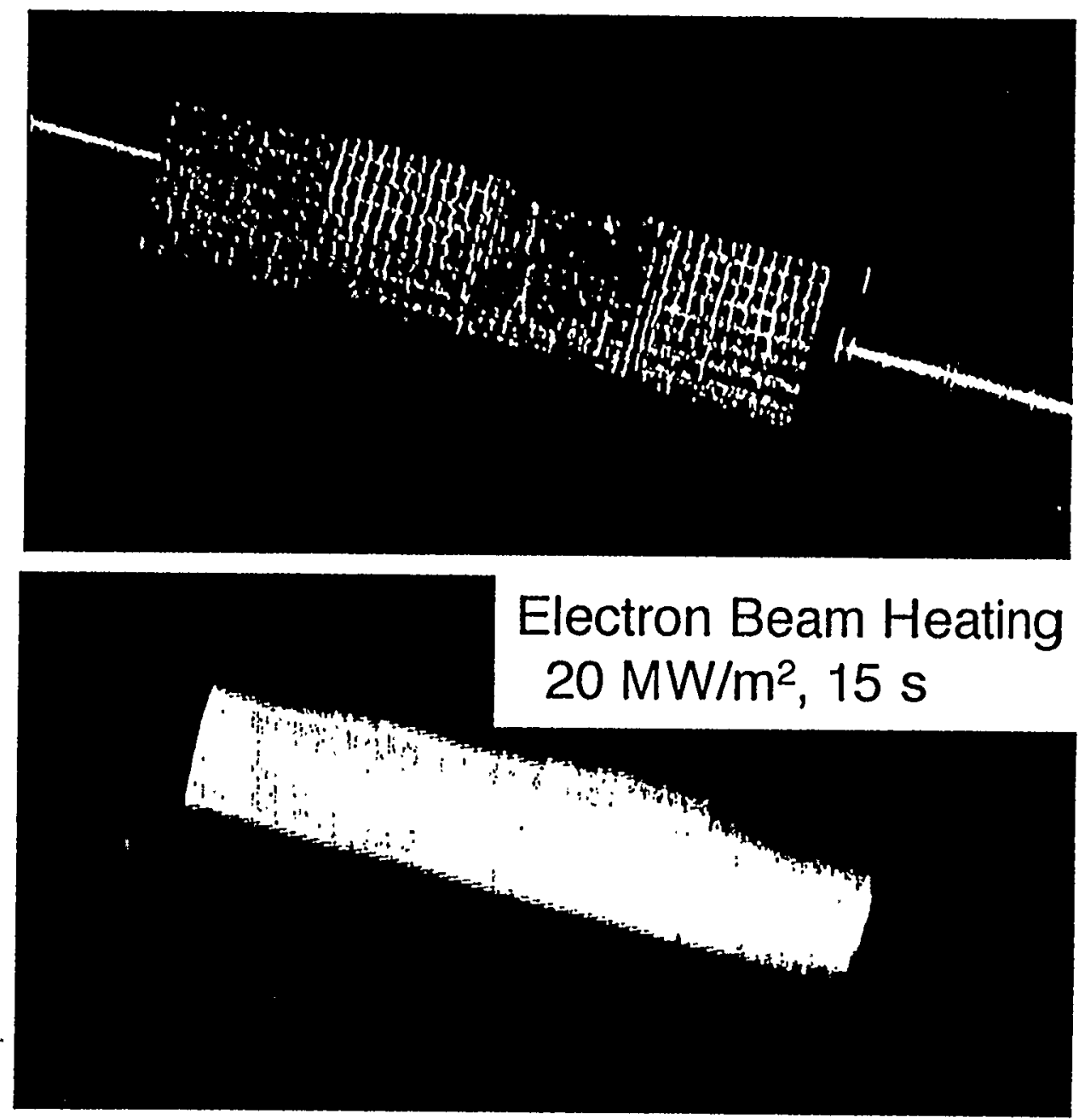

$\square$ The mock-up endured a heat load of $20 \mathrm{MW} /$ $\mathrm{m}^{2}, 15 \mathrm{~s}$.

$\square$ The armor surface is uniformly heated.

$\square$ The surface temperature is reduced, and local erosion of fibers are also reduced. 


\section{$5 \mathrm{~mm}$ thick CVD-W layer was successfully coated on OFHC Cu and on W/Cu heat sinks.}
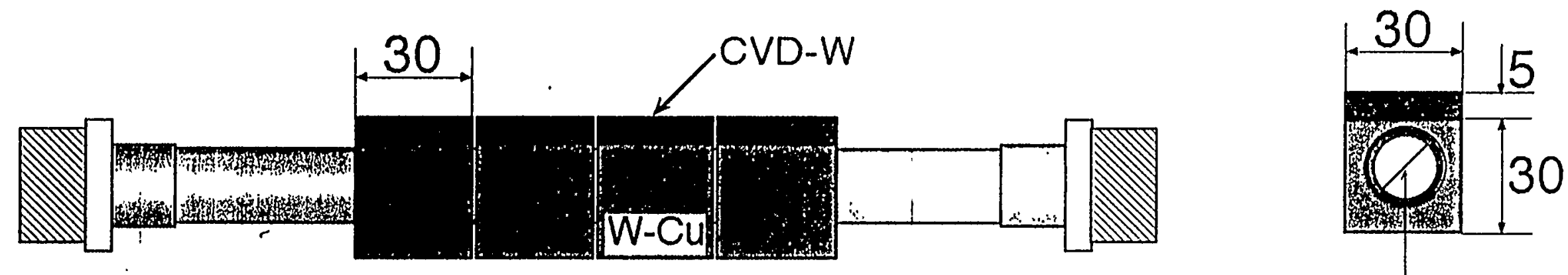

Inner core:DSCu

Outer layer:OFHC-Cu

Tape twist ratio:3

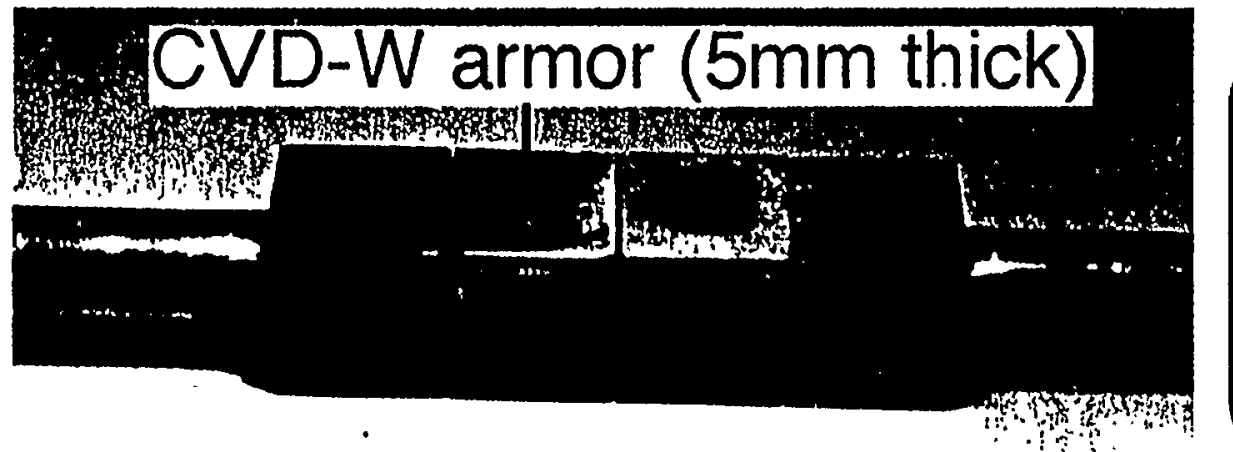

CVD process Working gas : WF 6

Tenv. : $700-750^{\circ} \mathrm{C}$

Coating rate : $0.2 \mathrm{~mm} / \mathrm{h}$ 


\section{Heating Tests on 5mm thick CVD-W coated on the W/Cu heat sink}

Thermal cycling experiment at $5 \mathrm{MW} / \mathrm{m}^{2}, 15 \mathrm{~s}$

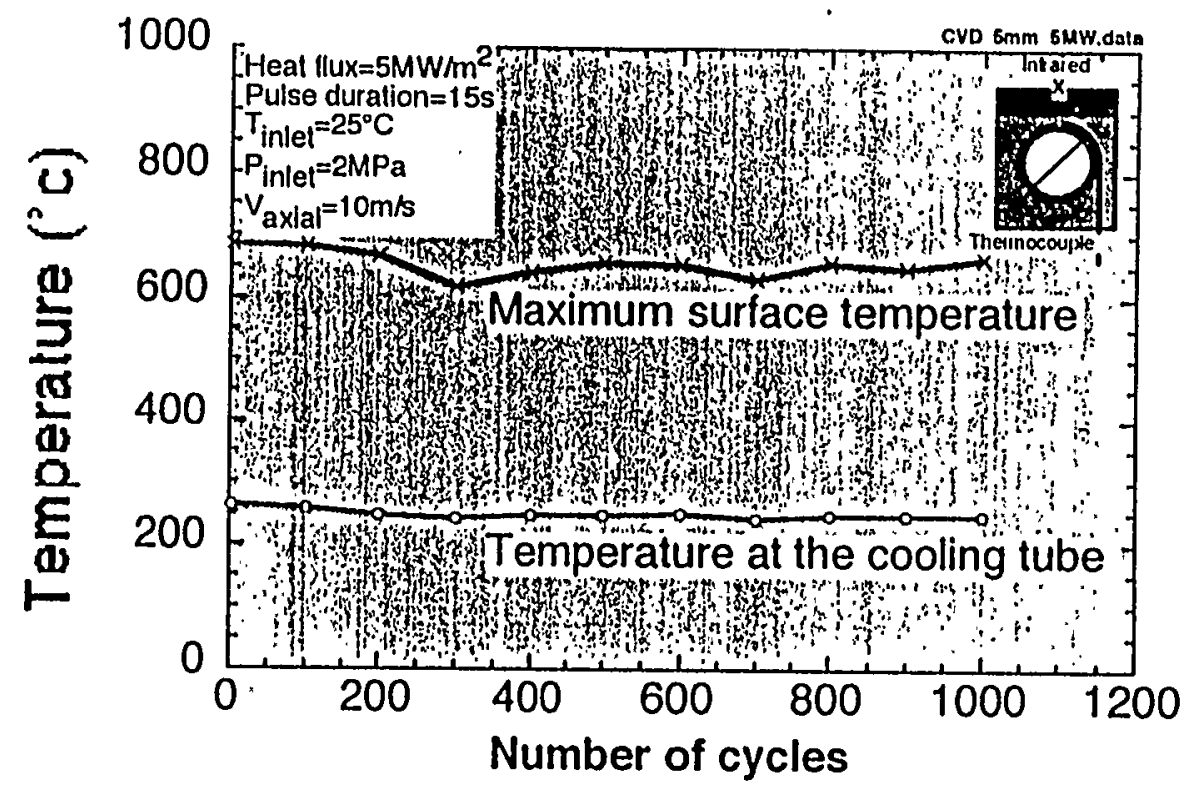

톰
Screening experiment at $20 \mathrm{MW} / \mathrm{m}^{2}, 15 \mathrm{~s}$
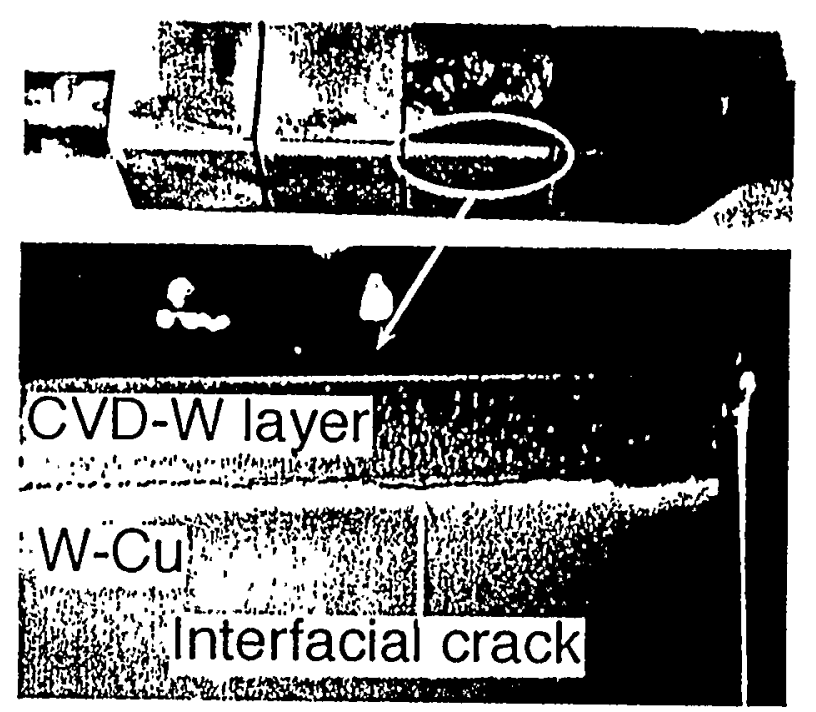

The mock-up survived up to 18 $\mathrm{MW} / \mathrm{m}^{2}$, though the surface was melted at $20 \mathrm{MW} / \mathrm{m}^{2}$. 


\section{Further R\&D's are necessary for coating on the OFHC-Cu heat sink.}

- $5 \mathrm{~mm}$ thick CVD-W was coated on the OFHC-Cu heat sink. :
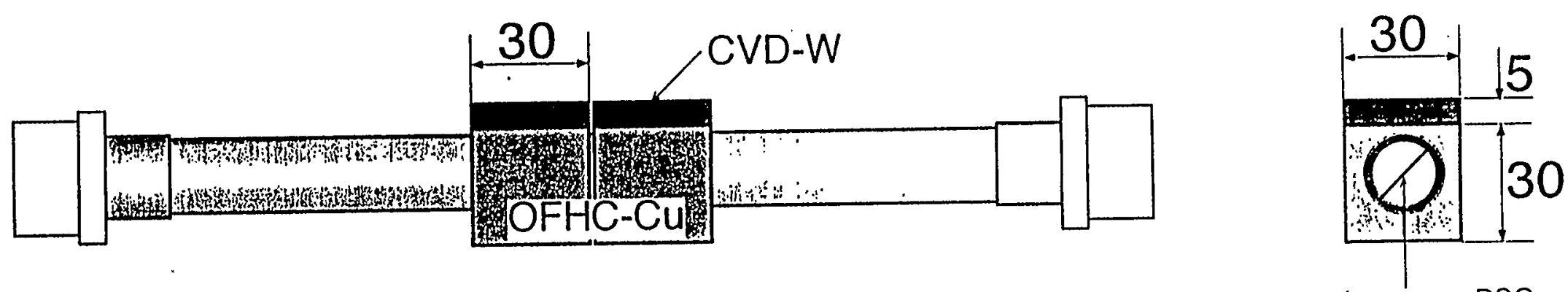

Inner core:DSCu

Tape twist ratio:3

- Thermal cycling experiment at $5 \mathrm{MW} / \mathrm{m}^{2}, 15 \mathrm{~s}$
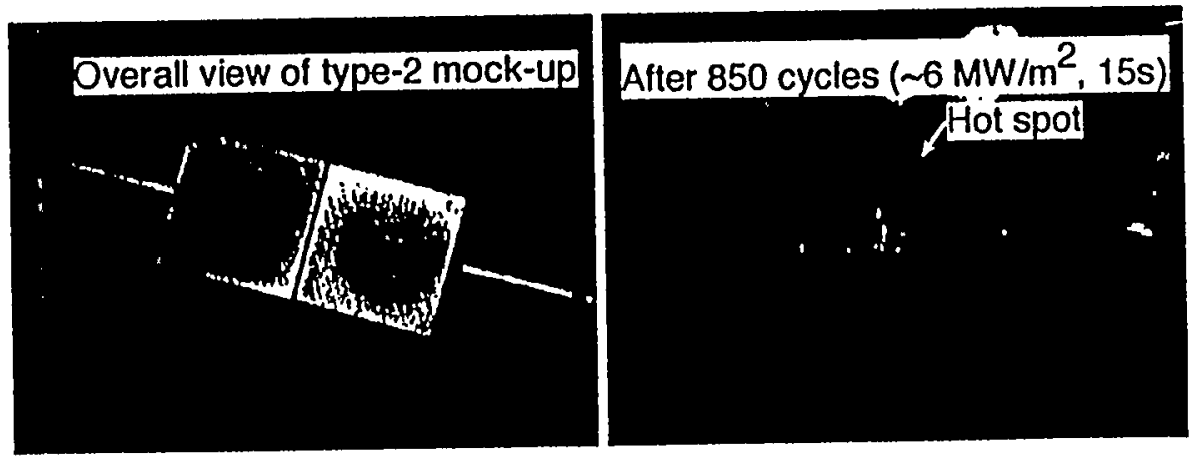

One tile survived more than 2,000 thermal cycles, but the other tile was detached after 850 cycles. 


\section{$3 \mathrm{~mm}$ thick CVD-W was successfully coated on the cylindrical W/Cu heat sink.}
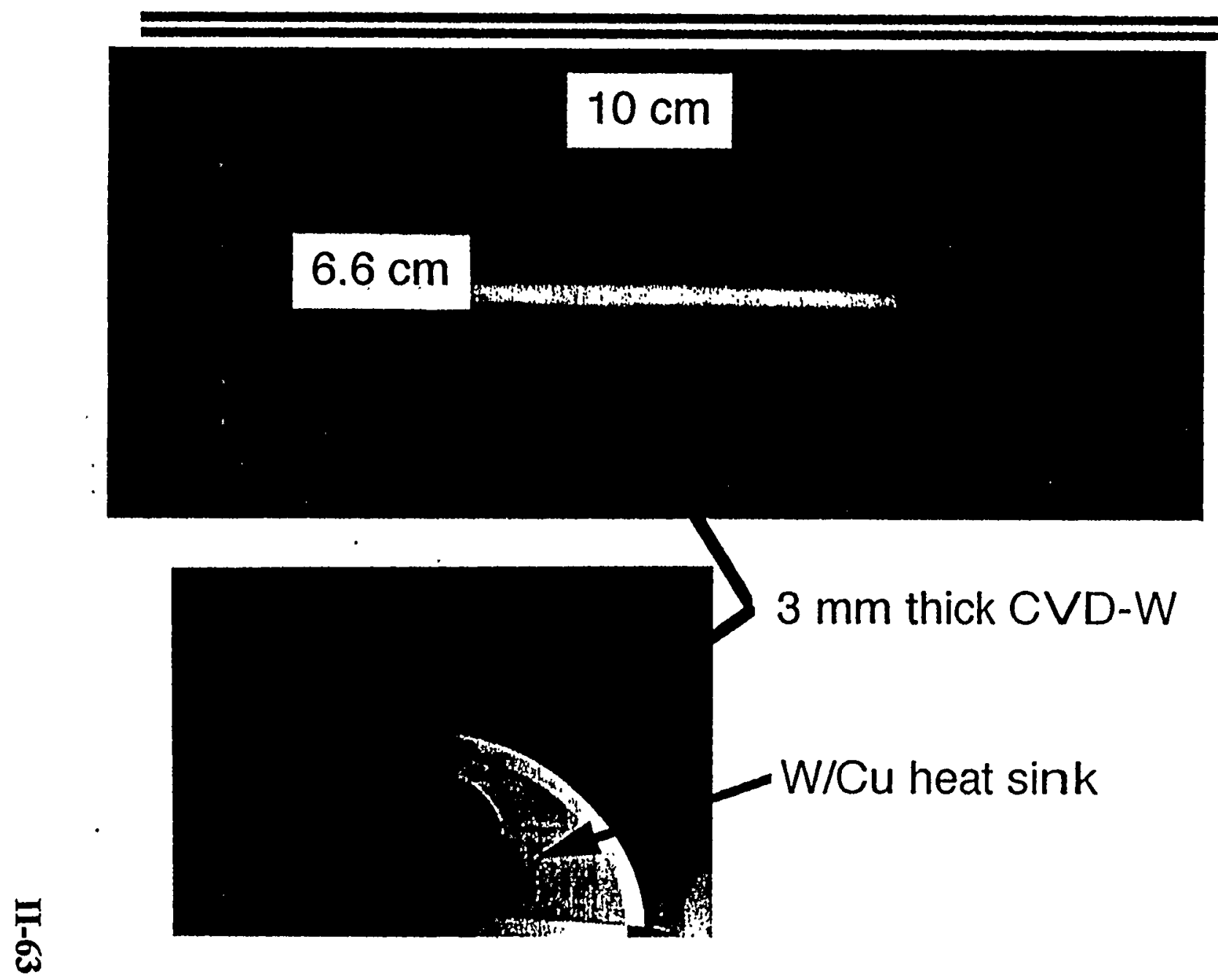

$3 \mathrm{~mm}$ thick CVD-W

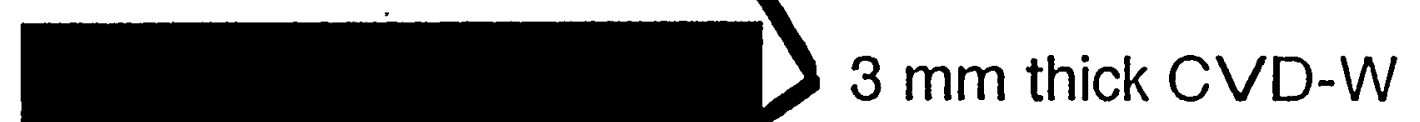

వ్
- The wing edge needs coating technique on a rounded surface.

- $3 \mathrm{~mm}$ thick CVDW was coated on the cylindrical surface of W/Cu. 


\section{- 1996 R\&D - \\ Full-scale Length Vertical Target Mock-ups}

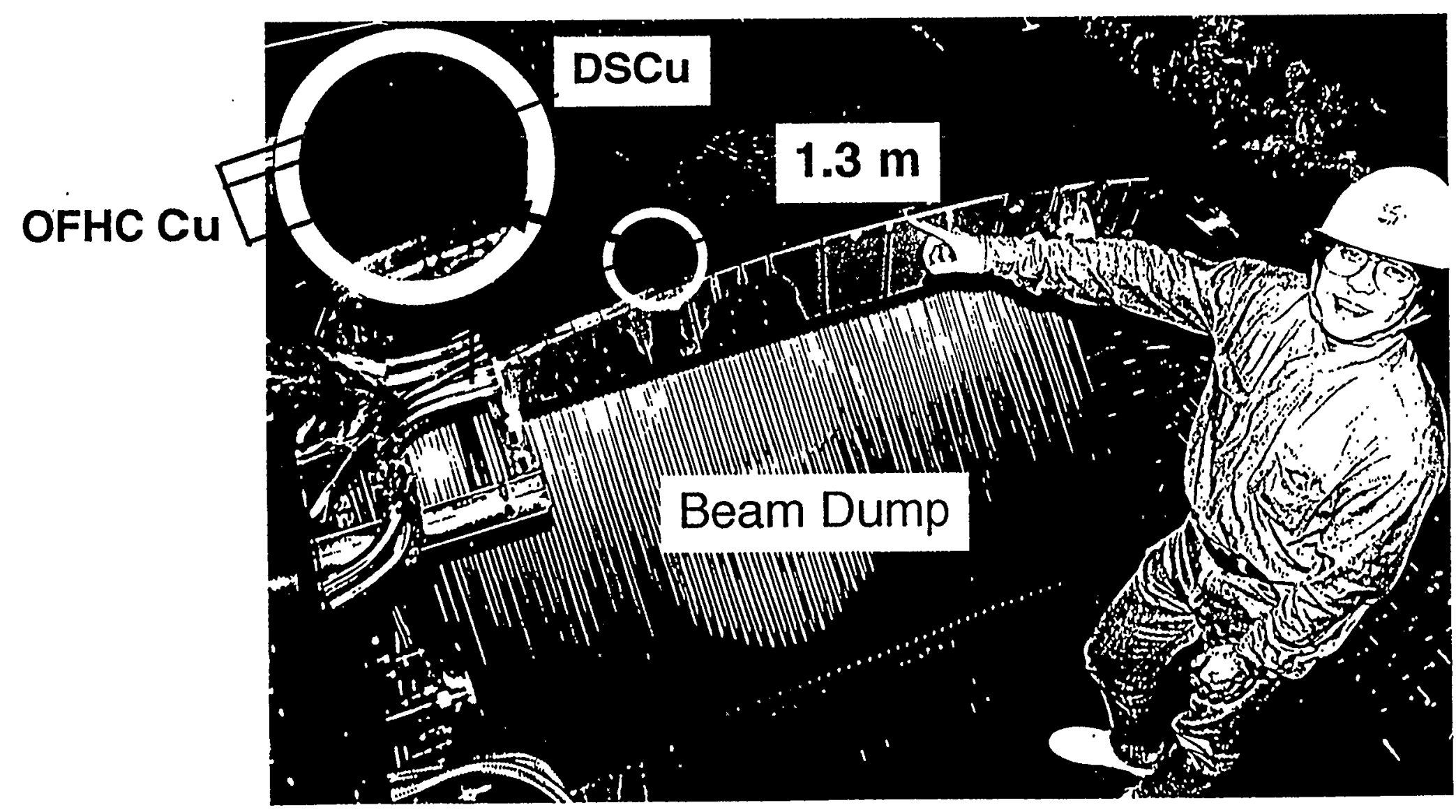




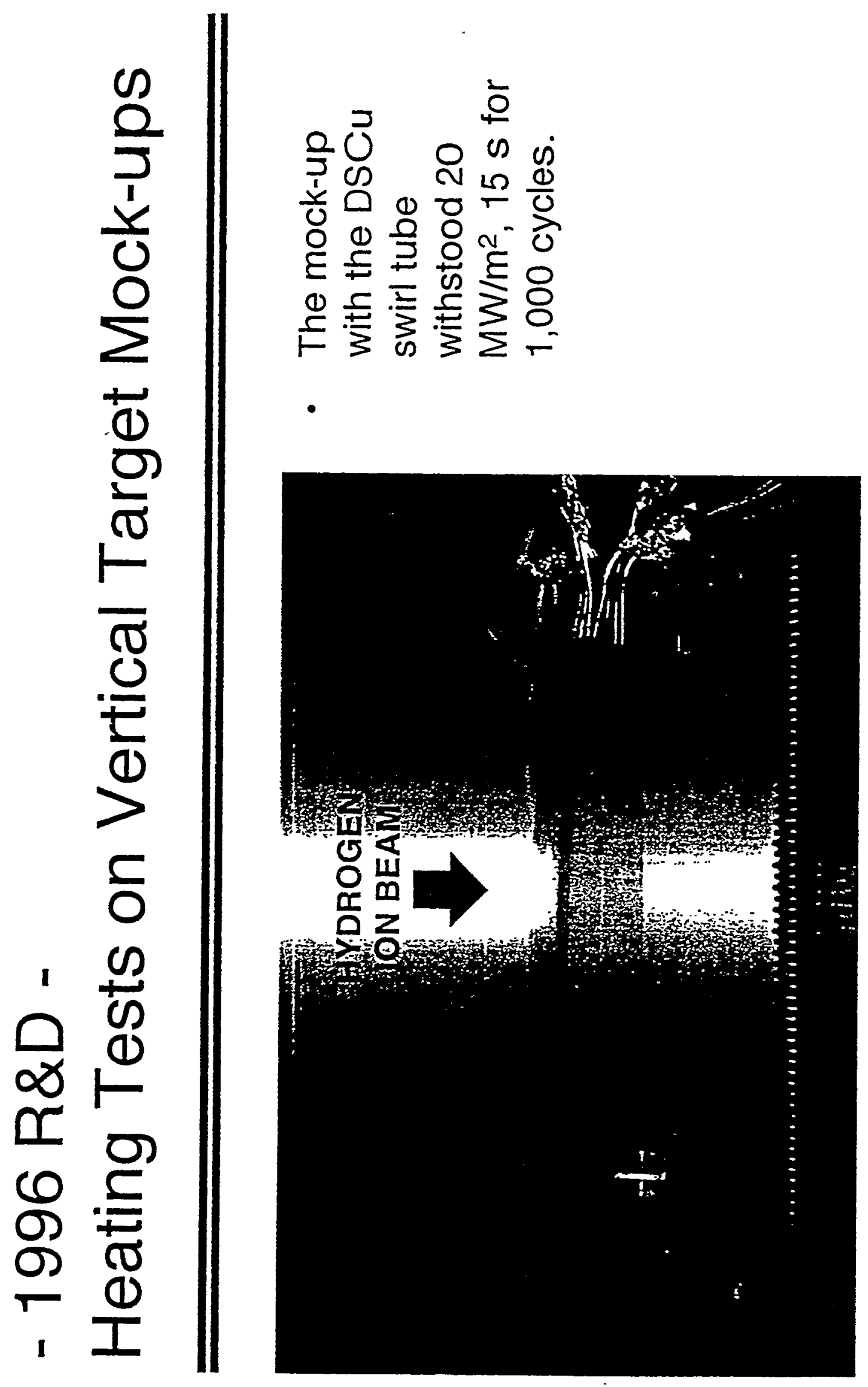




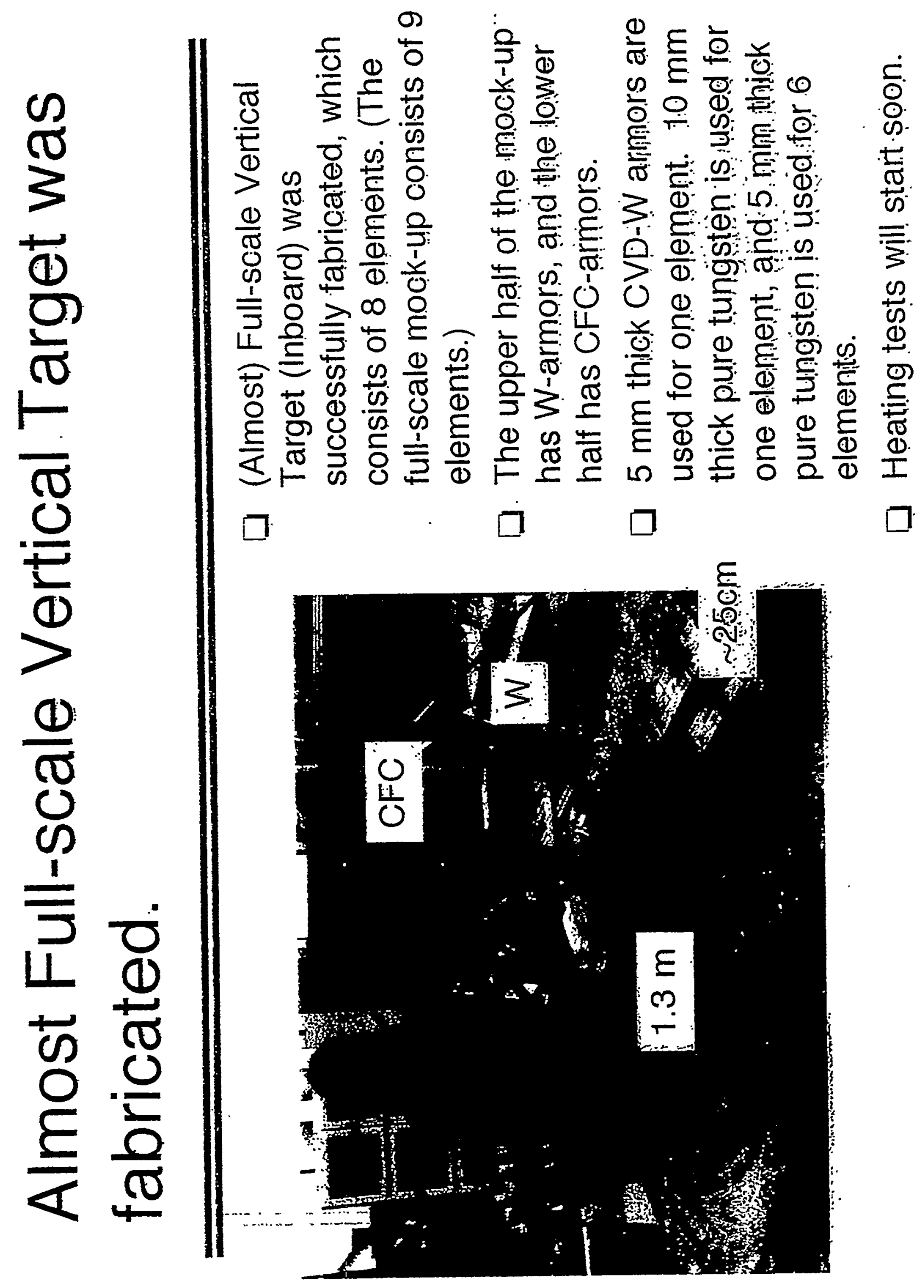


Weight Loss of Doped CFCs and Non-doped CFC with Electron Beam Irradiation

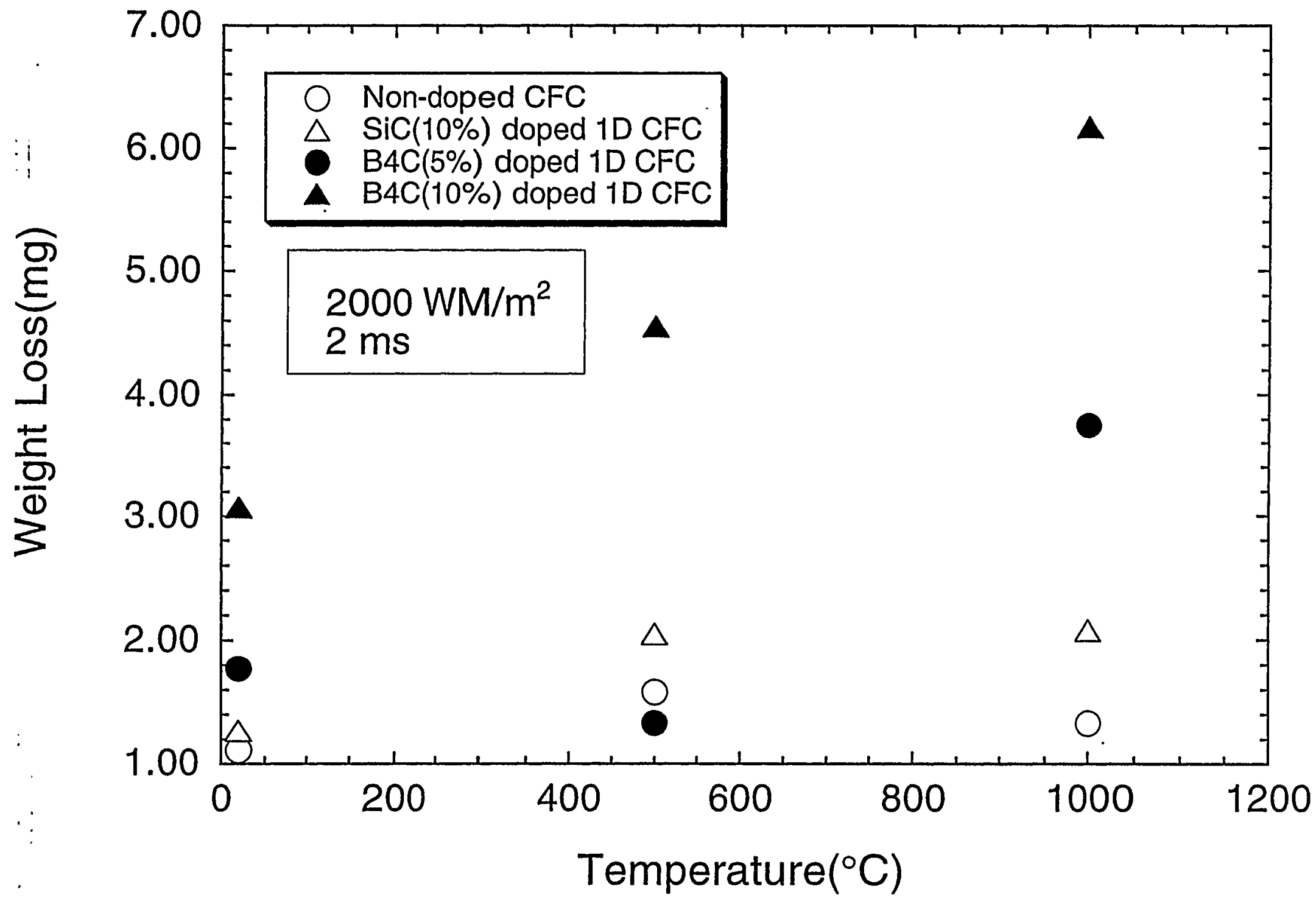




\section{Non-doped CFC}

\section{$\mathrm{SiC}(10 \%)$ doped CFC}
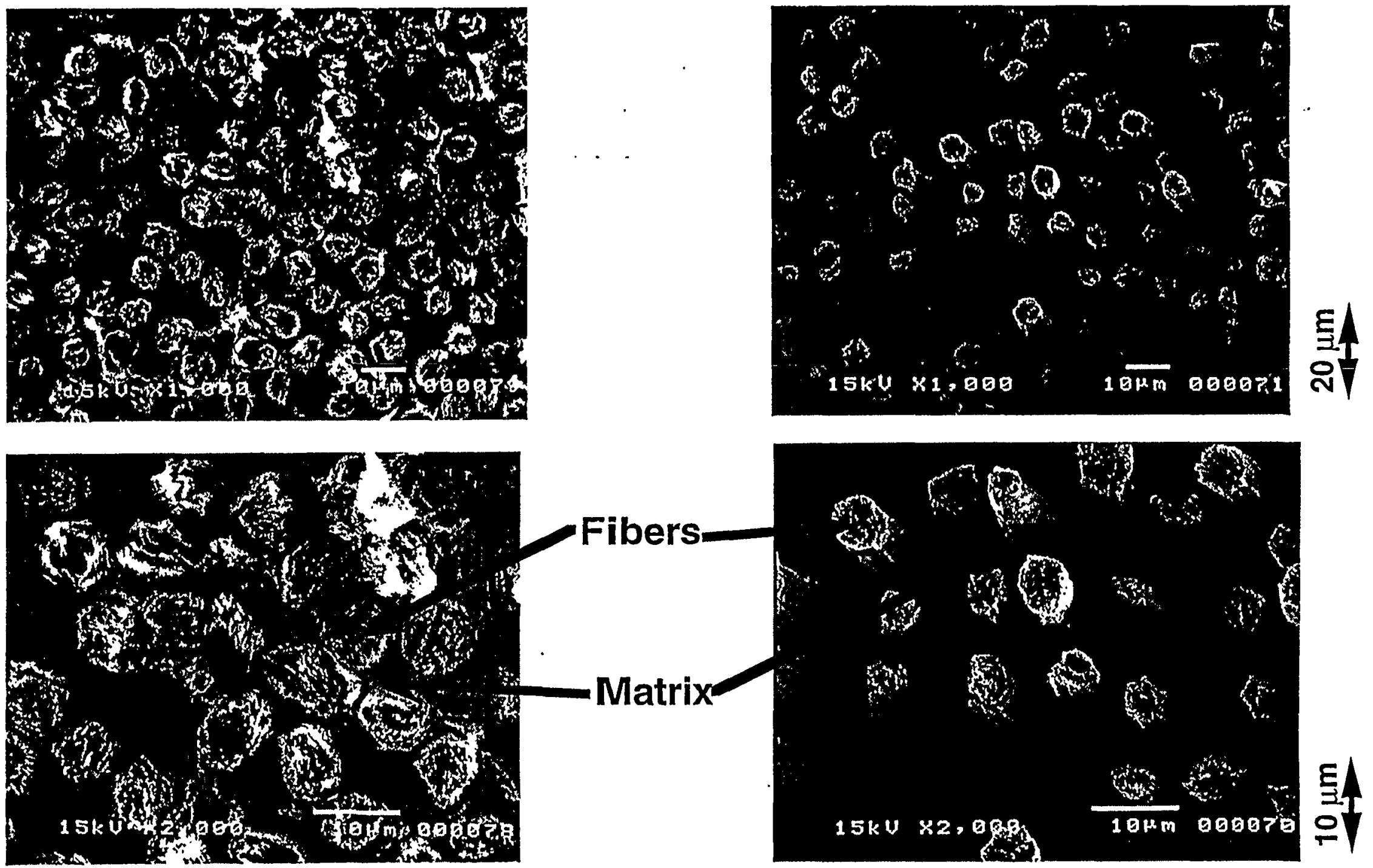

Photographs of electron irradiated $\mathrm{SiC}(10 \%)$ doped CFC and non-doped CFC with a heat flux of $1000 \mathrm{MW} / \mathrm{m}^{2}$ for $4 \mathrm{~ms}$ at RT. The matrix part of SiC doped CFC was largely eroded comparing with that of non-doped CFC. 


\section{Non-doped CFC}

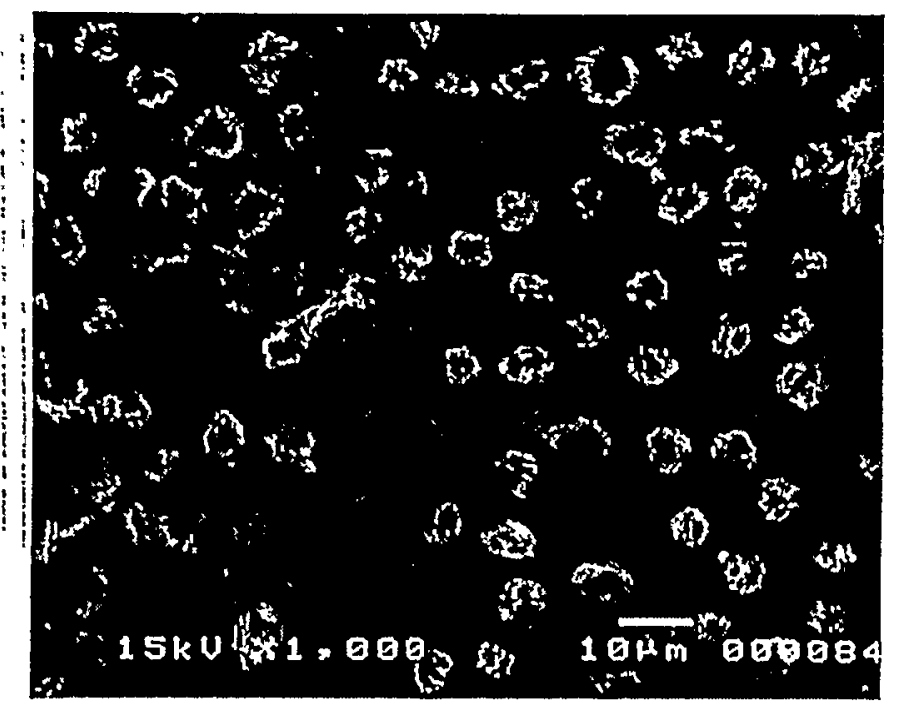

\section{SiC $(10 \%)$ doped CFC}

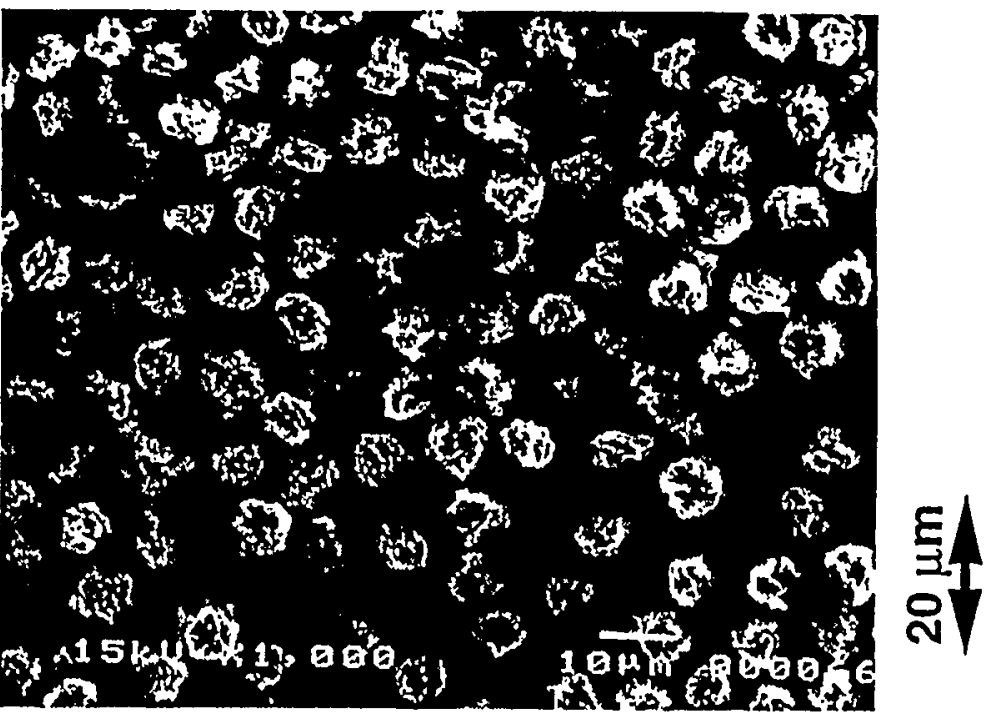

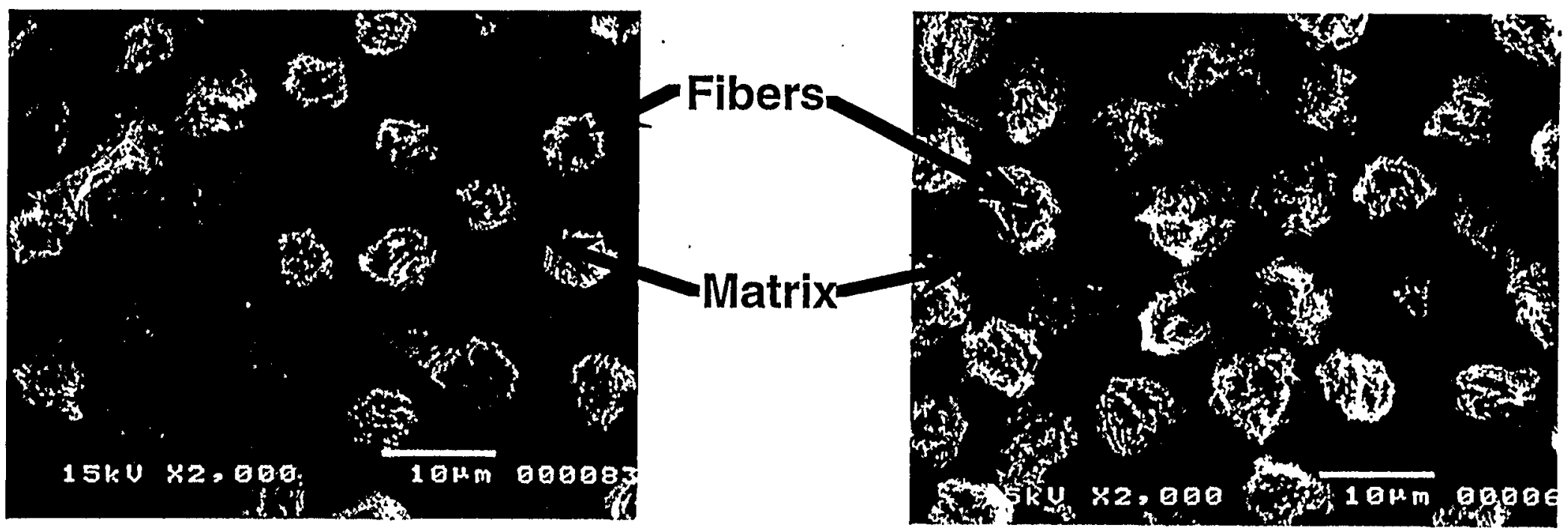
CFC with a heat flux of $1000 \mathrm{MW} / \mathrm{m}^{2}=$ for $4 \mathrm{~ms}$ at $500^{\circ} \mathrm{C}$. The both matrix part of SiC doped and non-doped CFC were not so much eroded. 


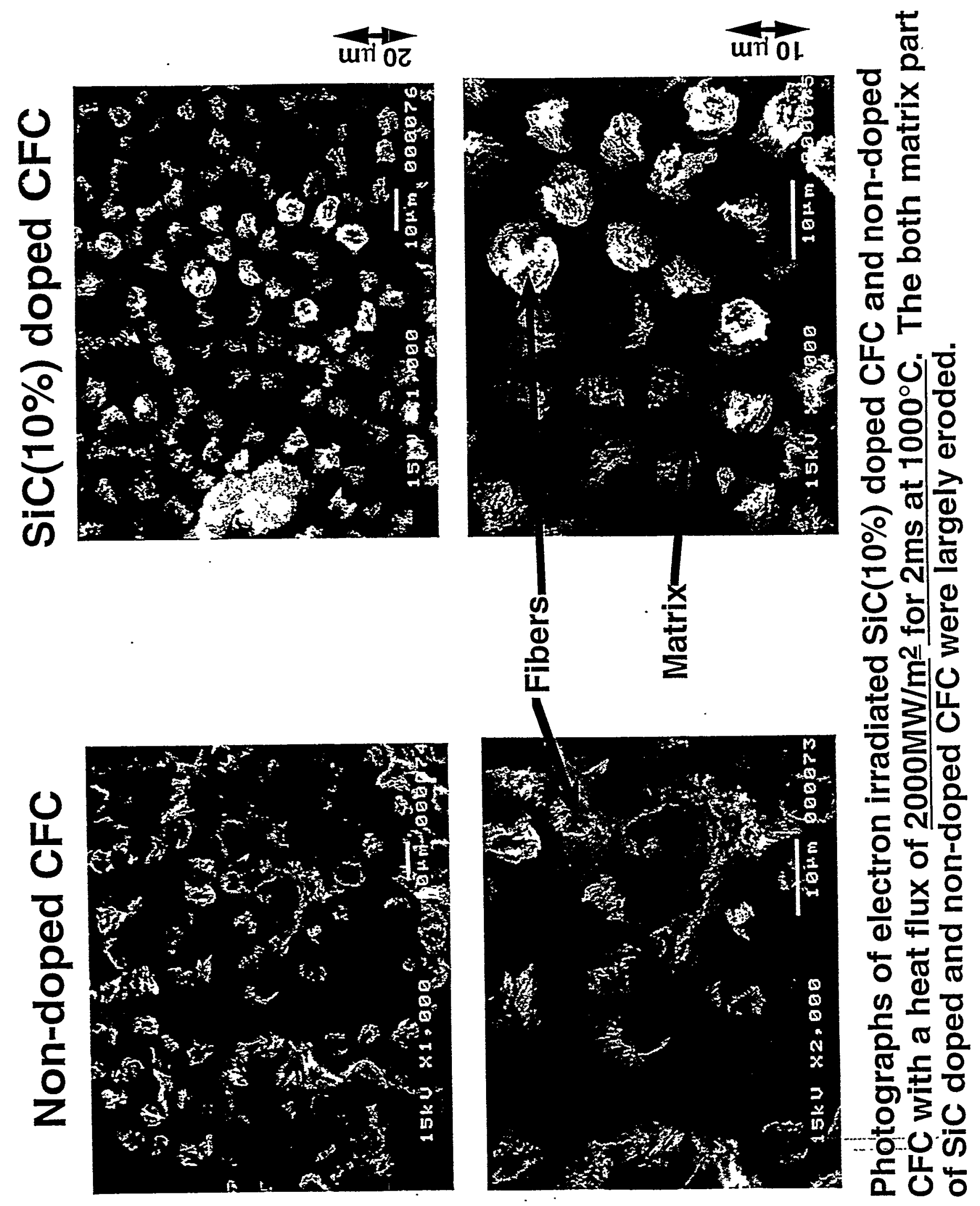




\section{Schedules of Neutron Irradiation and Post-irradiated Tests}

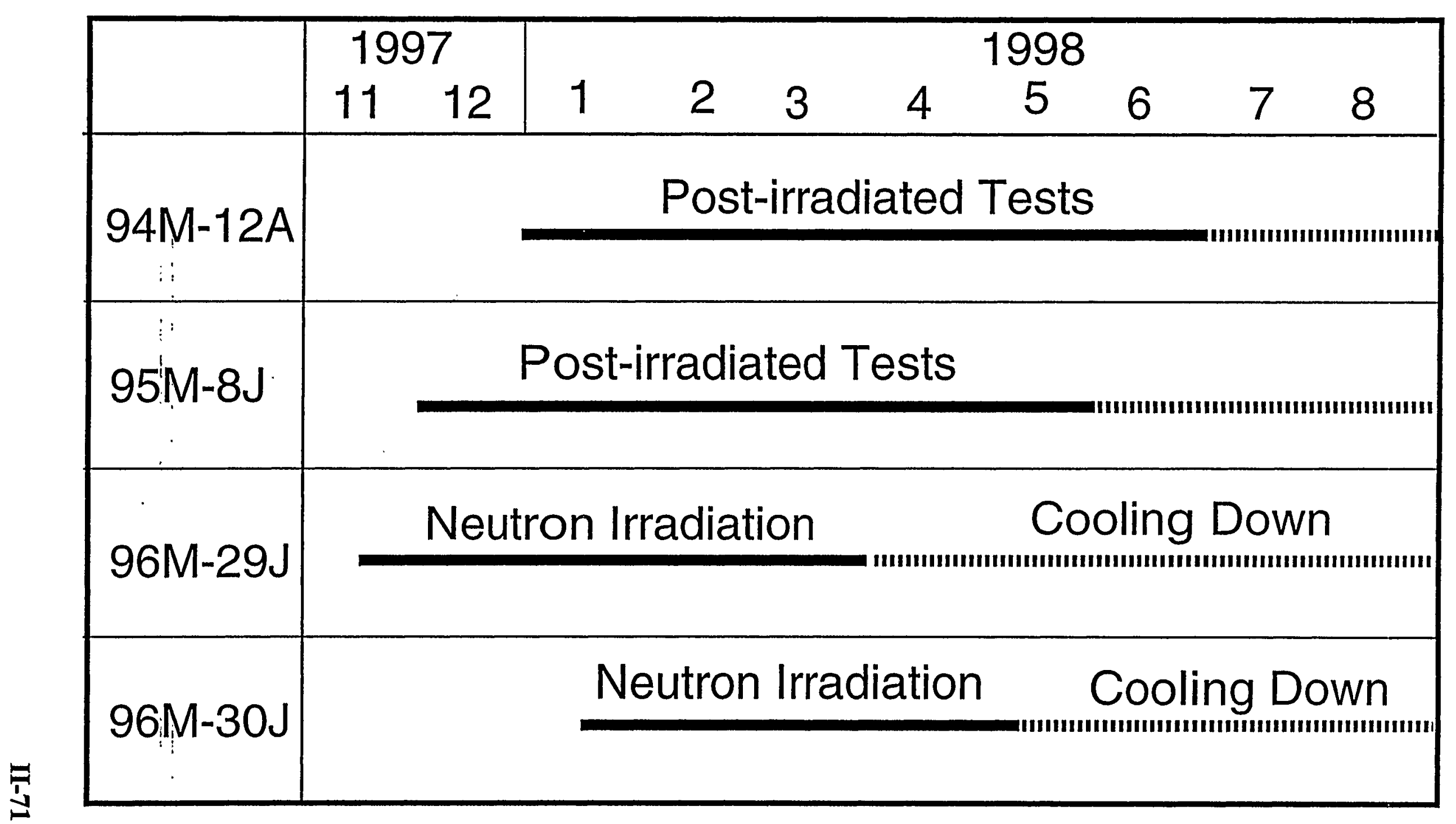




\section{Neutron Irradiated Samples in JMTR}

$94 \mathrm{M}-12 \mathrm{~A}\left(0.925 \mathrm{dpa}, 400 \sim 500^{\circ} \mathrm{C}\right.$, already irradiated):

: Thermal conductivity test

MFC-1(2), CX-2002U(2), PCC-2S(2), Be(2), OFCu(2), DSCu(2),

MFC-1/OFCu(2), MFC-1/DSCu(2)

- Tensile strength test

MFC-1/OFCu(4), Be/OFCu(4), MFC-1/DSCu(4), Be/DSCu(4)

- Bending test

CX-2002U(1), Be(1), OFCu(1), DSCu(1), MFC-1/OFCu(3), Be/OFCu(3), MFC-1/DSCu(3), Be/DSCu(3)

95M-8J(0.3 0.7 dpa, 300 400 ${ }^{\circ} \mathrm{C}$, already irradiated):

- Thermal fatigue test MFC-1/OHCu(2), CVD-W/OHCu(4), $\mathrm{Be} / \mathrm{OHCu}(6)$

- Disruption erosion test

$$
\text { MFC-1(4), CX-2002U(4), NIC-01(4), CVD-W(10), Be(15) }
$$




\section{$96 \mathrm{M}-29 \mathrm{~J}\left(8.3 \times 10^{19} \mathrm{n} / \mathrm{cm}^{2}, \sim 200^{\circ} \mathrm{C}\right.$, under irradiation):}

- Thermal conductivity test

DSCu(3), CuCrZr(2)

- Tensile strength test

OFCu(2), DSCu(2), CuCrZr(2)

- Bending test

OFCu(1), DSCu(2), CuCrZr(1),

- Thermal fatigue test

CVD-W/OFCu(2), MFC-1/OFCu(2), CX-2002U/OFCu(2), NIC-01/OFCu(2)

- Disruption erosion test

MFC-1(2), CX-2002U(2), NIC-01(2), CVD-W(4), P-W(4), Be(4) 
$96 \mathrm{M}-30 \mathrm{~J}\left(6.5 \times 10^{19} \mathrm{n} / \mathrm{cm}^{2}, 300 \sim 500^{\circ} \mathrm{C}\right.$, to be irradiated soon):

- Thermal conductivity test

CVD-W(2), P-W(2), 1D CFC(MFC-1 grade)(2), NIC-01(2)

- Tensile strength test

P-W(4), MFC-1(1), 1D CFC(MFC-1 grade)(2), CX-2002U(1), NIC-01(2),

PCC-2S(1)

- Bending test

P-W(4), MFC-1(1), 1D CFC(MFC-1 grade)(2), NIC-01(2), PCC-2S(1)

- Disruption erosion test

P-W(12), 1D CFC(MFC-1 grade)(3), NIC-01(3) 


\section{SUMMARY}

1D/3D hybrid CFCs are promising as an armor material.

$\square$ A divertor with $5 \mathrm{~mm}$ thick CVD-W armors can meet ITER requirement.

$\square$ Full-scale Vertical Target with W, CFC armors were successfully fabricated. Heating tests will start soon. Full-scale length Wing with CFC armors are also going to be ready soon.

$\square$ SiC doped 1D CFC is also promising as an armor material.

$\square$ Post-irradiation tests will give us very useful information for evaluation of armor material. 


\title{
Be-Cu Joining Technologies for Plasma Facing Components in the ITER Fusion Reactor
}

\author{
B.C. Odegard Jr, C.H. Cadden, R.D. Watson
}

Sandia National Laboratories

\author{
and \\ K.T. Slattery \\ The Boeing Co.
}




\section{Topics}

- Introduction

- Beryllium-Copper Joining Technology

I. Plasma Spraying

II. Brazing

III. Diffusion Bonding

- Summary

- Future Studies

I

US-Japan Workshop, Dec 8-11, 1997, San Francisco

Sandia National Laboratories 


\section{Challenges to Beryllium-Copper Joining}

- Be is chemically reactive with most elements in forming brittle intermetallics. Exceptions are:

$\mathrm{Ge}, \mathrm{Si}, \mathrm{Al}, \mathrm{Ag}$

- Be has limited room temperature tensile ductility $(\sim 5 \%)$

- Coefficient of Thermal Expansion $(\mu \mathrm{m} / \mathrm{m}-\mathrm{K})$

$$
\begin{array}{r}
\mathrm{Be}-11.6 \\
\mathrm{Cu}-16.8 \\
\mathrm{Al}-23.6 \\
\text { AlBeMet-150 - } 17.6
\end{array}
$$

Aluminum seemed the most promising both as a filler metal and a compliant layer. 


\section{Beryllium Reacts with the Copper Alloy at Bonding Temperatures}

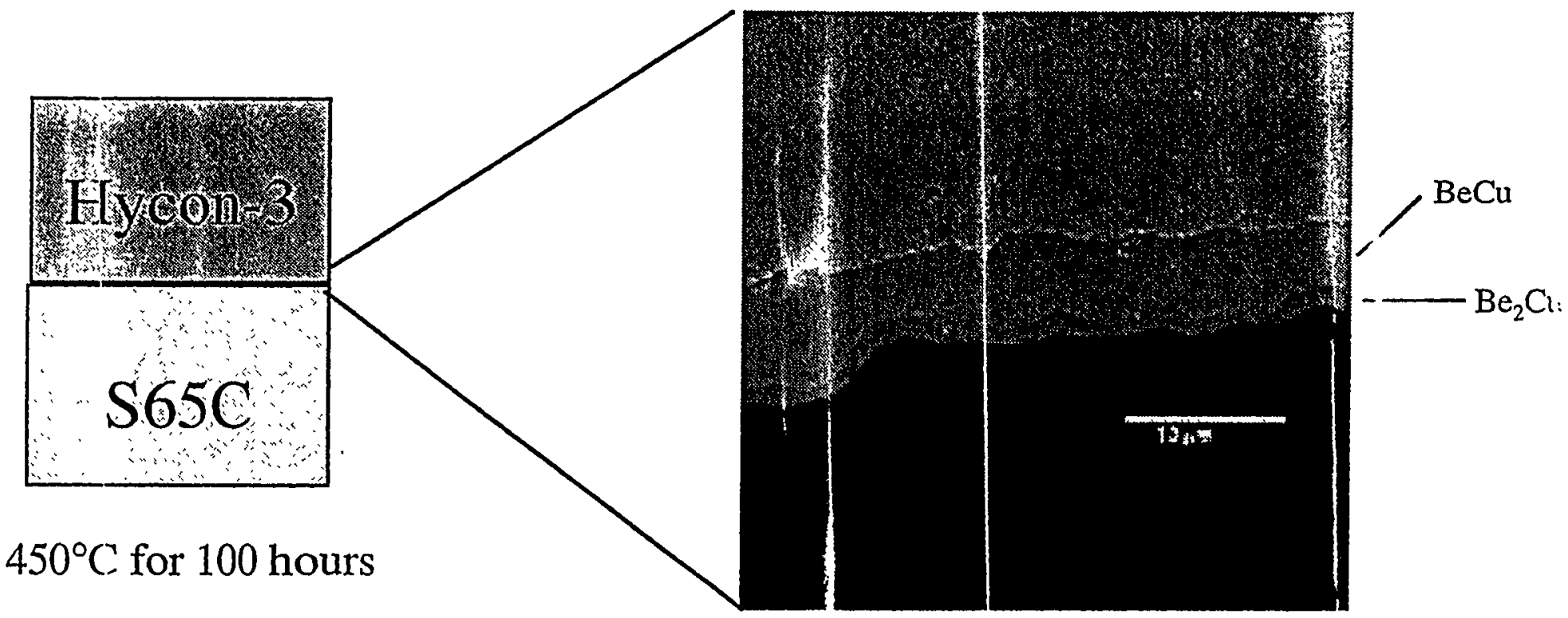

Beryllium reacts with copper to form two intermetalic phases BeCu and $B \epsilon_{2}$ C $\mathrm{A}$ better bonding solution would be to use a diffusion barrier to eliminate this itjong, brittle phase

( Kawamura and Kato, US-Japan Workshop, Jackson Lake, 1995)

\# (Odegard et al, Proc. Nuclear Fusion Tech., Tokyo, 1997) 


\section{Beryllium-Aluminum Phase Diagram Predicts ro Intermetallics and Low Solubility}

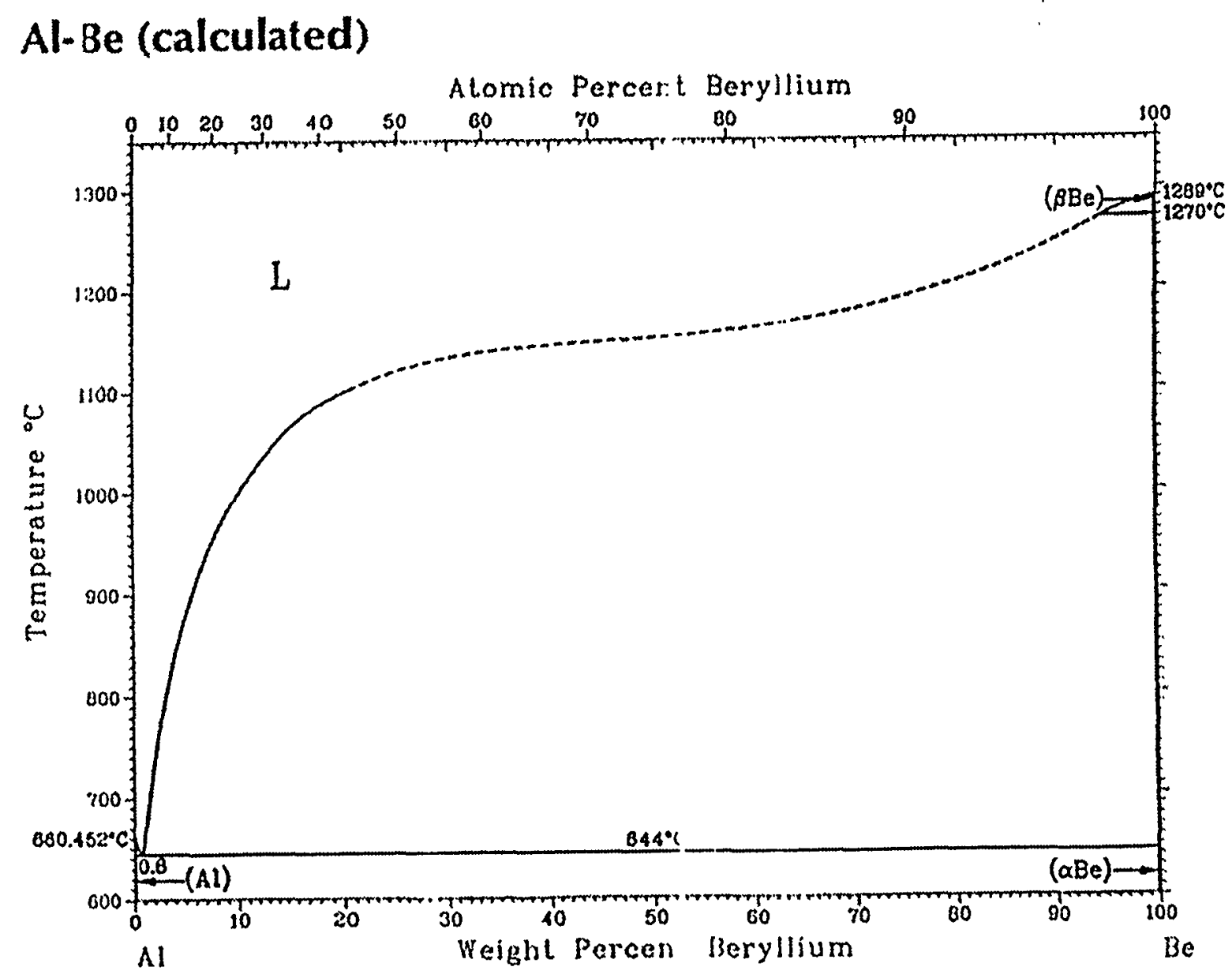




\section{Aluminum-Copper Phase Diagram}

Phase diagram predicts several intermetallics, $10 \% \mathrm{Cu}$ solubility in Al with no intermetallics.

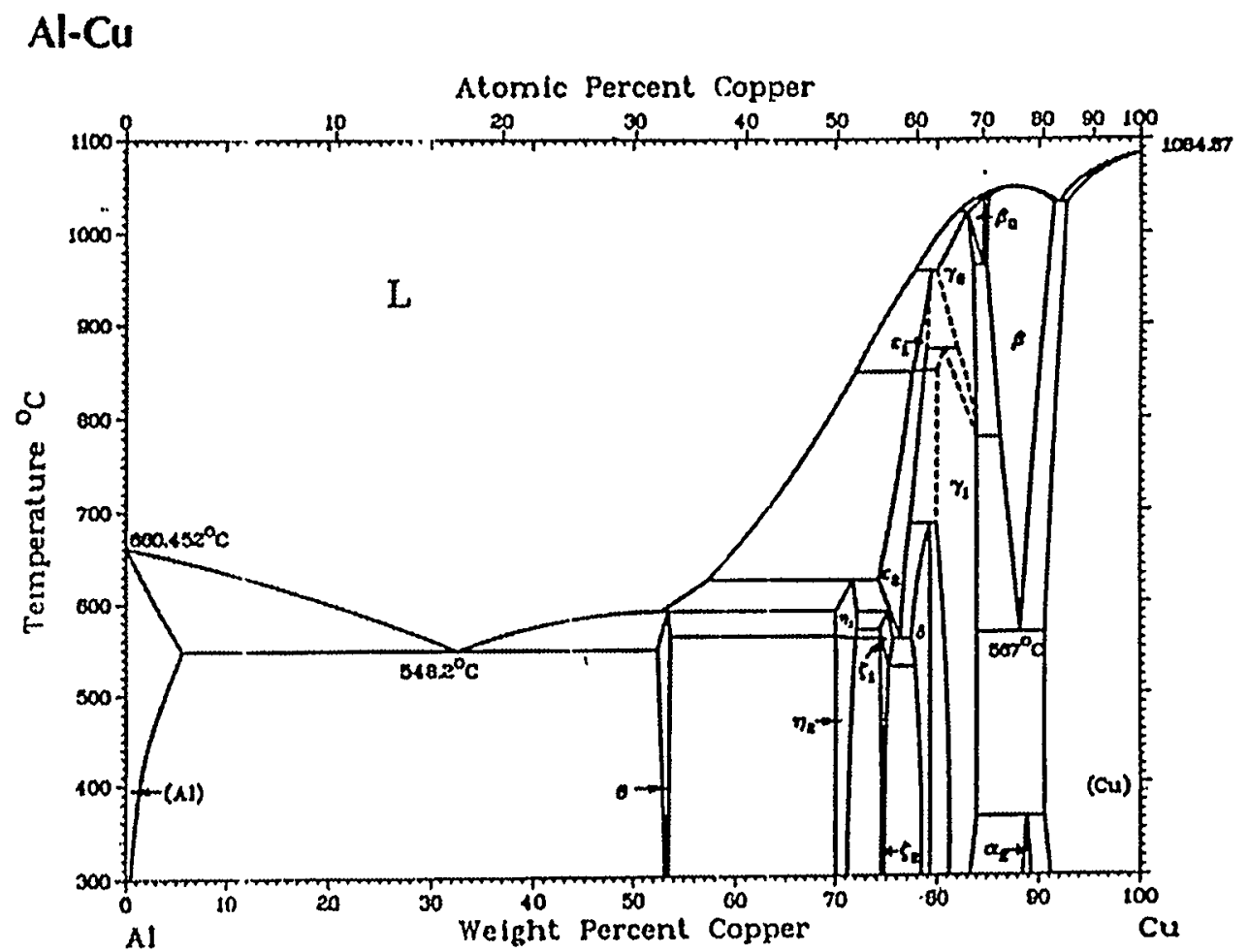

F Joining aluminum to copper without a diffusion barrier was a problem. 


\section{Explosive bonding is an effective method of bonding $1100-\mathrm{Al}$ or AlBeMet-150 to copper alloys.}
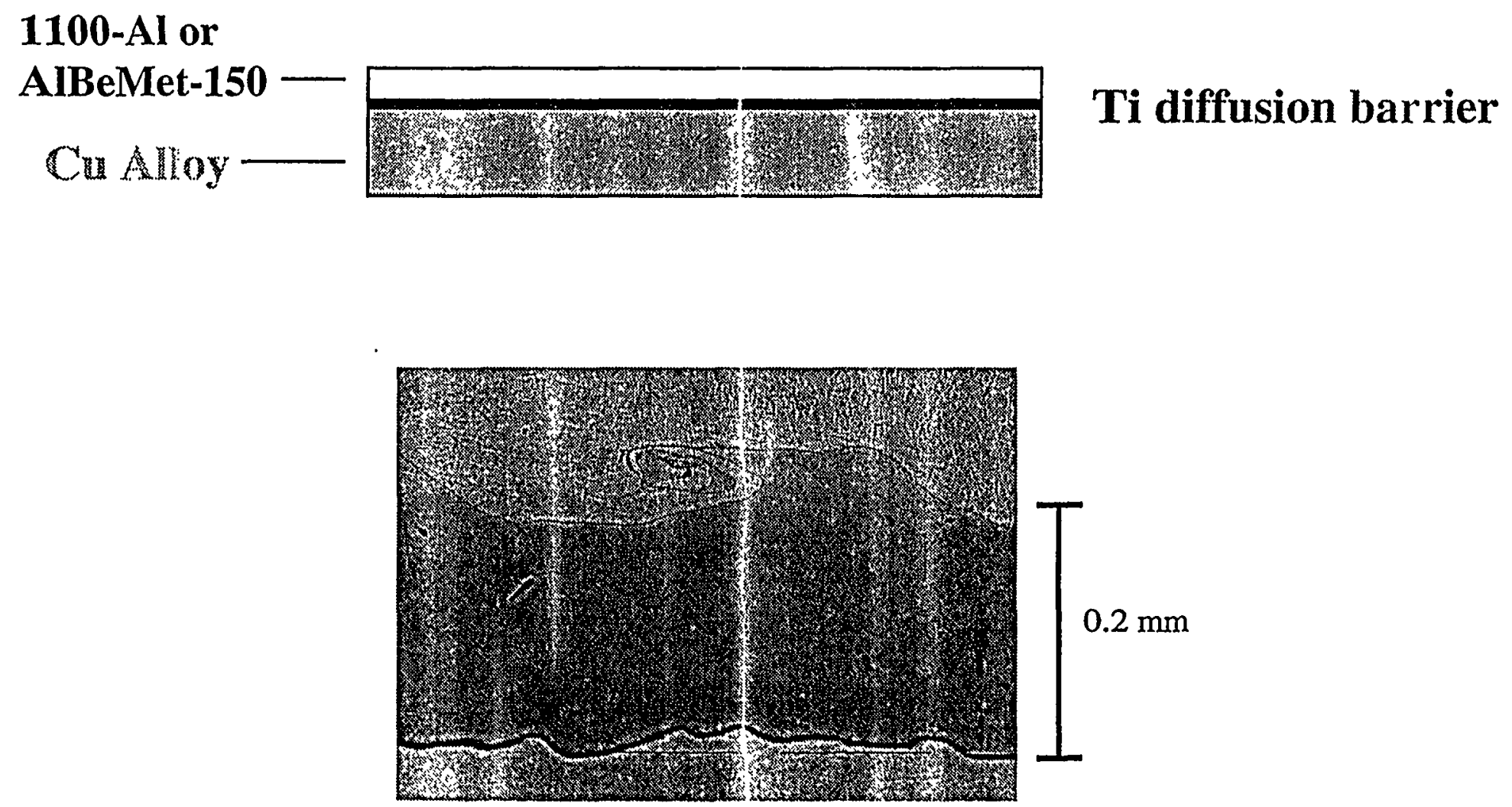


\section{Beryllium to Copper Joining Technology}

- I. Beryllium Plasma Spraying:

1. directly on copper heat sink

2. directly on an aluminum compliant layer

3. as an in situ repair of beryllium tiles

- I. Brazing:

1. directly onto an alüminum compliant layer

2 directly onto an aluminum composite (AlBeMet-150)

- III. Diffusion Bonding:

1. directly onto an aluminum compliant layer

2. directly onto an aluminum composite (AlBeMet-150) 


\section{Plasma Sprayed Beryllium on Copper}

蒂

- LANL Vacuum Plasma Spray Facility developed process which produces high density Be deposits.

- Negative transferred arc (cathodic) cleaning of substrate removes surface oxides prior to spray deposition

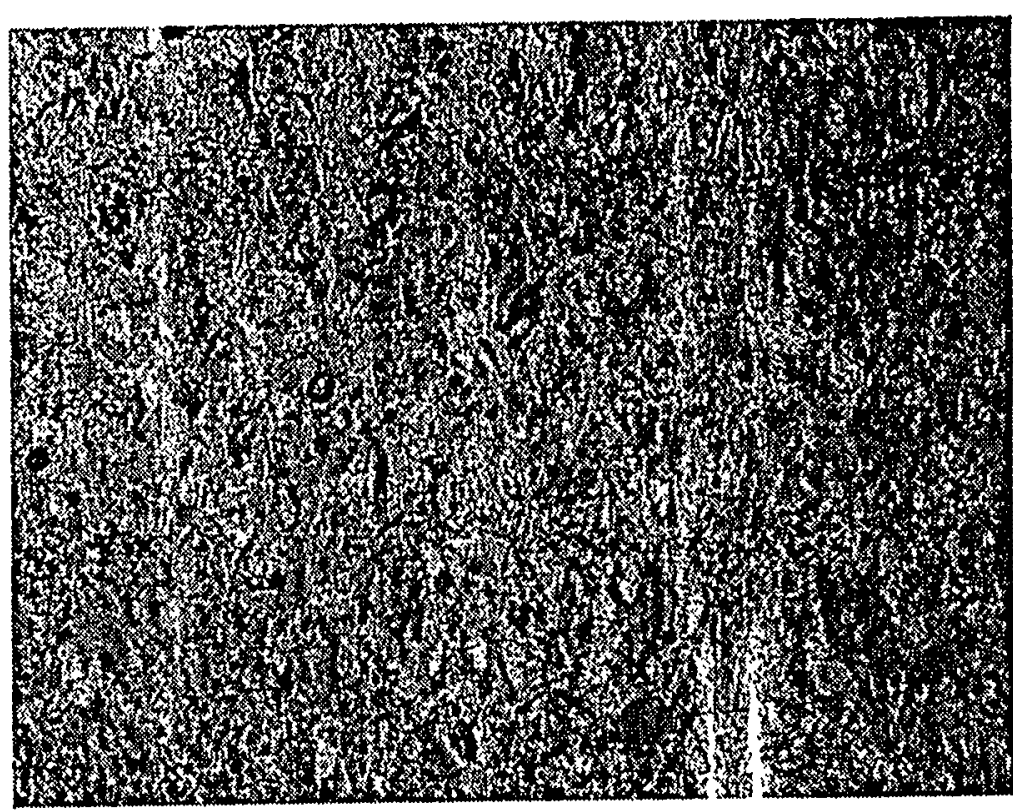

Micrograph of as-plasma spı ayed Be

- Process could be utilized for both initial fabrication and in-situ repair 


\section{Vacuum plasma sprayed Be can produce $\mathrm{Be} / \mathrm{Be}$, $\mathrm{Be} / \mathrm{Cu}$ and $\mathrm{Be} / \mathrm{Al}$ specimens possessing good strength.}

\section{Bond Strength}

VPS Be / HP Be: 110 - 220 MPa

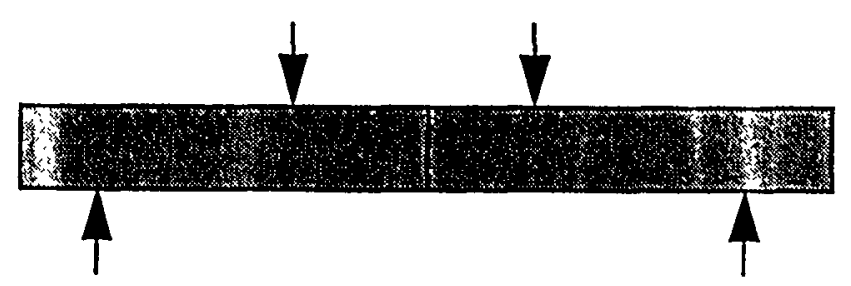

VPS Be / Cu: $\quad \sim 70$ MPa

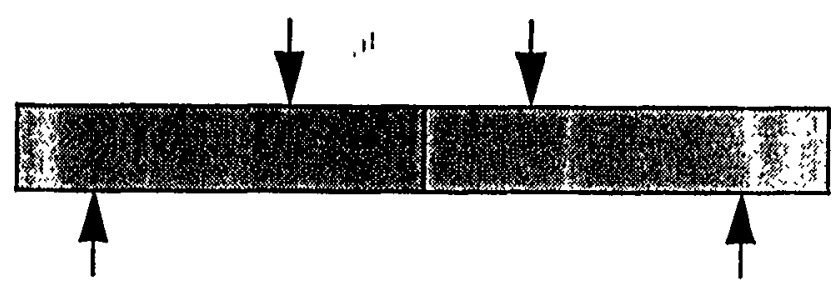

VPS Be / Al: 120 MPa

疍

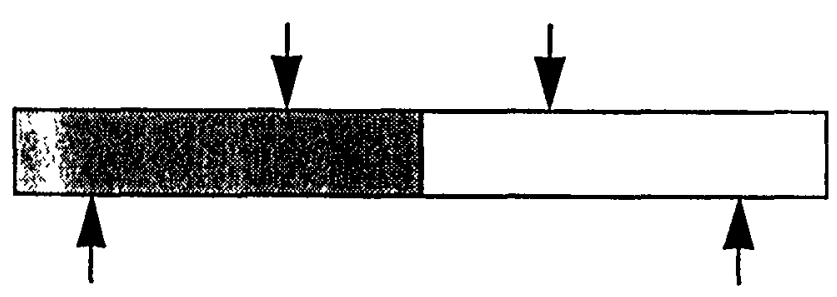




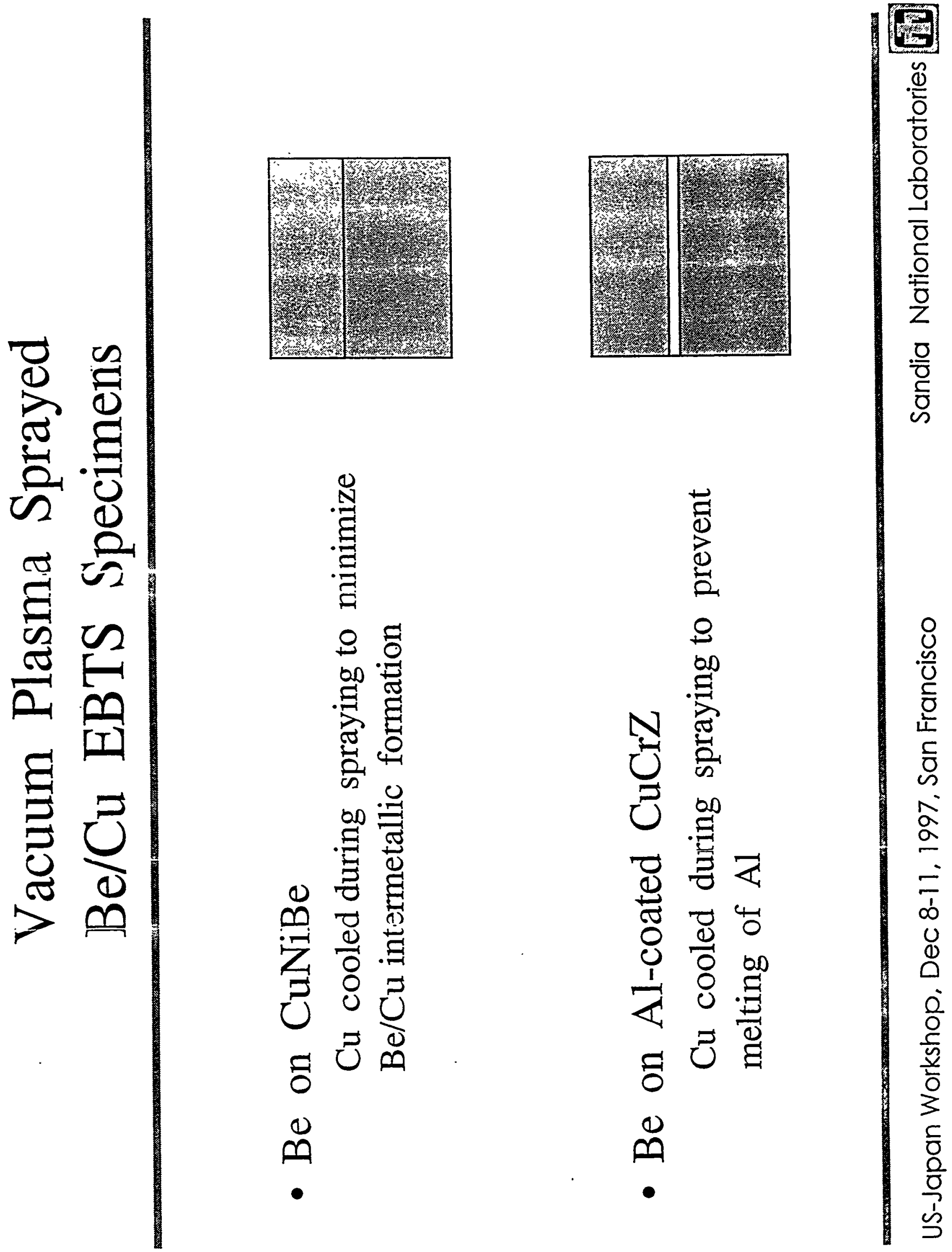




\section{High Heat Flux Test (EBTS) Specimen}

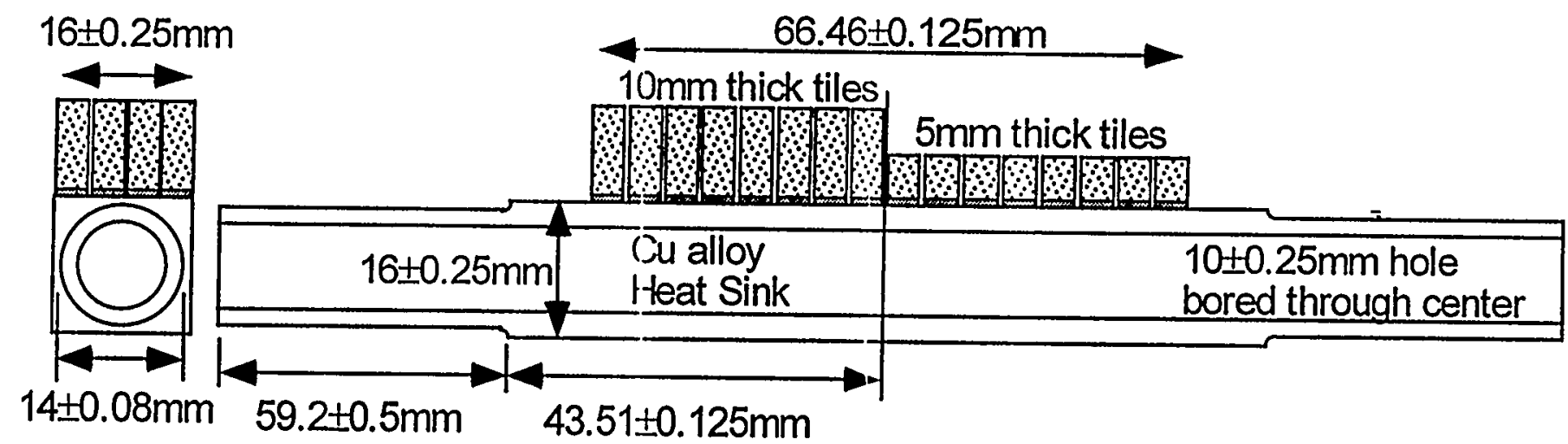

Actively cooled high heat flux sample geometry used at the EBTS facility

봉 at Sandia National Laboratories-New Mexico 


\section{Vacuum Plasma Sprayed

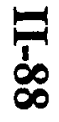 Beryllium-Copper EBTS Specimens}

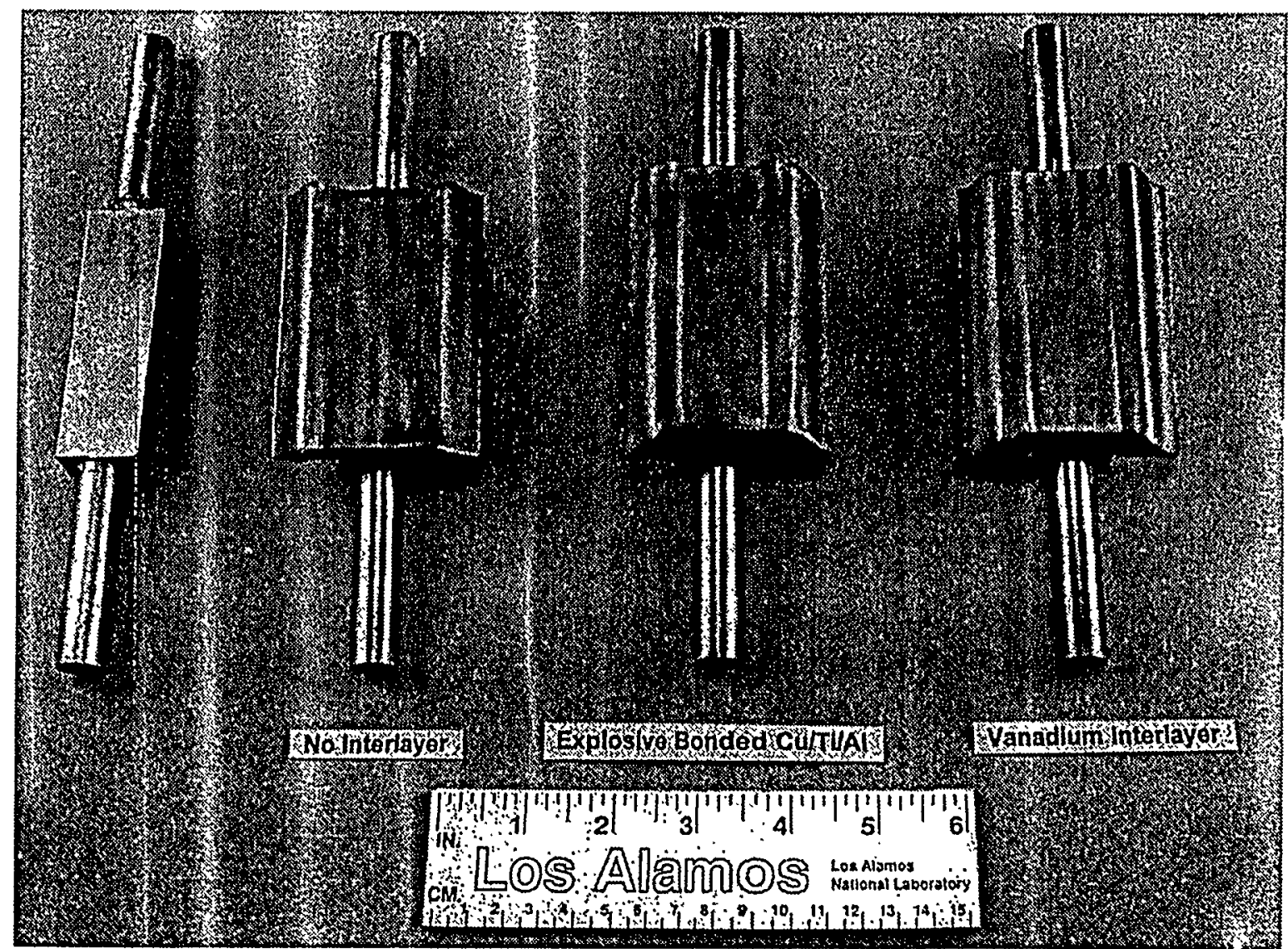

US-Japan Workshop, Dec 8-11, 1997, San Francisco

Sandia Nat'onal Laboratories [ 


\section{EBTS Results for VPS Beryllium on Copper}

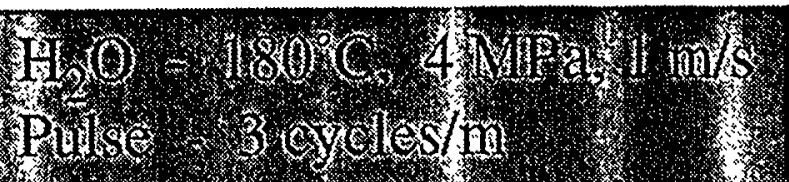

VPS Be on CuNiBe (knurled surface)
$1 \mathrm{MW} / \mathrm{m}^{2}$

3000 cycles

(No damage)

$3 \mathrm{MW} / \mathrm{m}^{2}$

10 cycles

(lateral cracking of $\mathrm{Be}$ no damage at $\mathrm{Be} / \mathrm{Cu}$ bond)

VPS Be on CuCrZr w/aluminum cladding. (knurled surface)
$1 \mathrm{MW} / \mathrm{m}^{2}$ 1400 cycles

(No damage)

$\frac{3 \mathrm{MW} / \mathrm{m}^{2}}{40 \text { cycles }}$

(lateral cracking of $\mathrm{Be}$ no damage at $\mathrm{Be} / \mathrm{Cu}$ bond)

范

US-Japan Workshop, Dec 8-11, 1997, San Francisco

Sandia National Laboratories 盯 


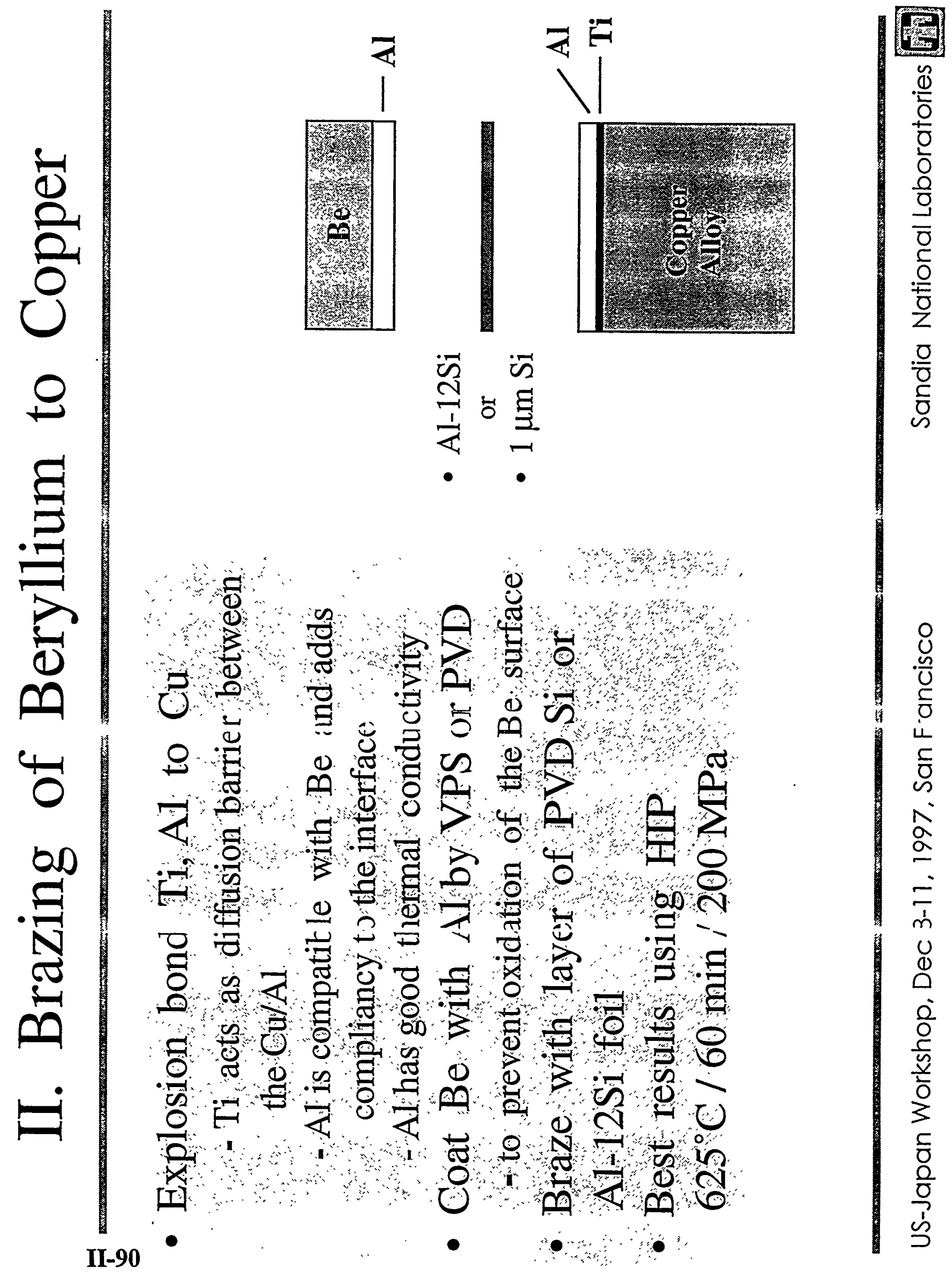




\section{Brazing techniques can produce $\mathrm{Be} / \mathrm{Al} / \mathrm{Cu}$ specimens possessing good strength and ductility.}

\begin{tabular}{|c|c|c|}
\hline & $\begin{array}{c}\text { Fracture Strength } \\
\text { MPa }\end{array}$ & $\begin{array}{c}\text { Elongation } \\
\%\end{array}$ \\
\hline $\begin{array}{r}\mathrm{Be} / \mathrm{Al} / \mathrm{Cu} \\
\mathrm{Al}-12 \mathrm{Si}\end{array}$ & + & $\sim 15$ \\
\hline $\begin{array}{c}\mathrm{Be} / \mathrm{Al} / \mathrm{Cu} \\
1 \mu \mathrm{m} \mathrm{Si}\end{array}$ & + & -3 \\
\hline $1100 \mathrm{Al}$ & 90 & $\sim 35$ \\
\hline
\end{tabular}

Strain measurement was not accurate.

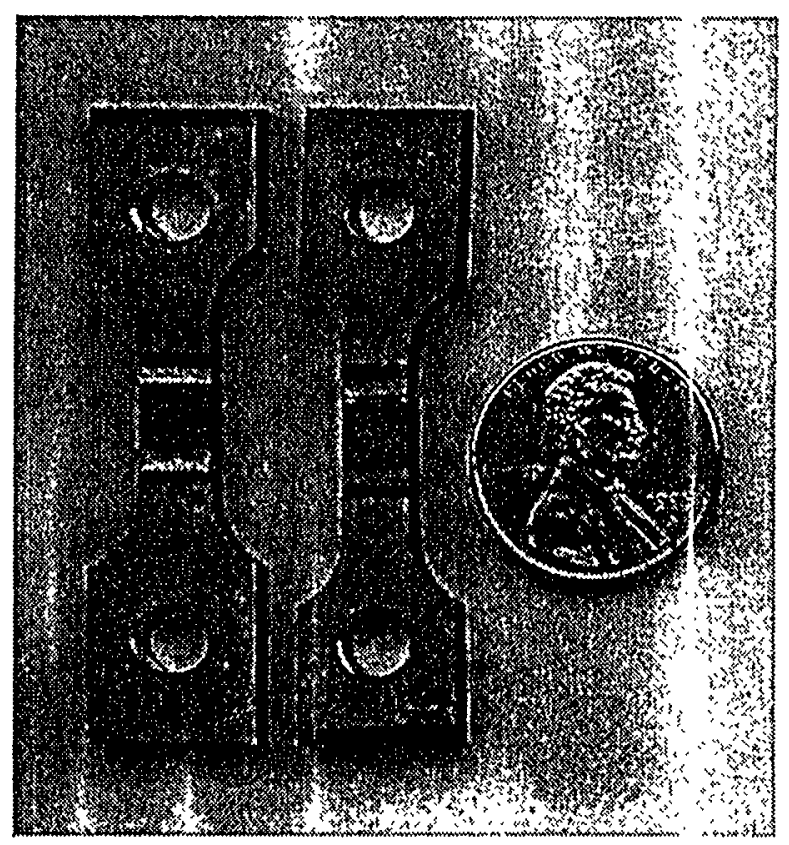

Failure occurred in the aluminum compliant layer. 


\section{Diffusion Bonding of Beryllium to Copper}

- Explosion bond:

- Ti, Al

$-\mathrm{Ti}_{1}, \mathrm{Al}-50 \% \mathrm{Bz}$

- Ti diffusion bar rier $(250 \mu \mathrm{m})$

- Al compatible with Be and adds compliancy

- AlBeMet-150 is stronger than $1100 \mathrm{Al}$ (350 MPa vs $80 \mathrm{MPa}$ - ultimate tensile strength)

- AIBeMet-150 has abetter CTE match with beryllium and copper

Be -11.6, Cu-16.8, Al -23.6. AlBeMet-150-17.6(um/m-K)

- Be etched or Al-coated

- HW Parameters

$600-650^{\circ} \mathrm{C} / 60 \mathrm{~min} / 100 \mathrm{MPa}$

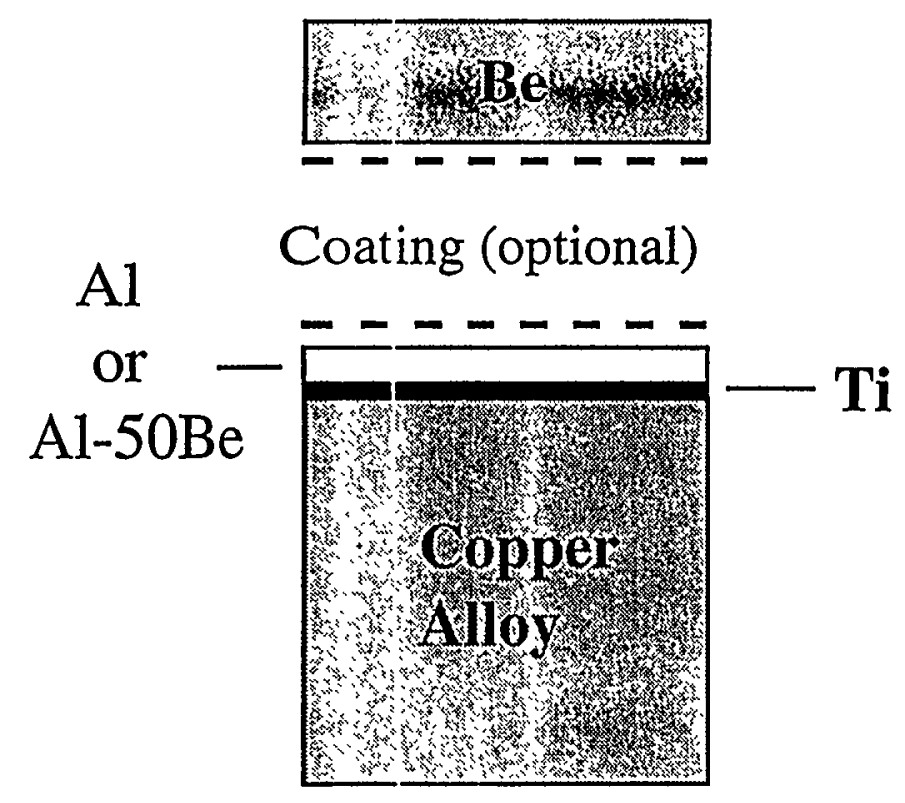




\section{Diffusion bonding techniques can produce $\mathrm{Be} / \mathrm{Al} / \mathrm{Cu}$}

specimens which exhibit good strength and ductility.

1. Cú /Ti/AlBeMet-150 (Cu film) $\mathrm{Be}+\mathrm{PVD} \mathrm{Al}(\mathrm{Cu}$ film$)$

2. $\mathrm{Cu} / \mathrm{Ti} / 1100-\mathrm{Al}(\mathrm{Cu}$ film) $\mathrm{Be}+\operatorname{VPS} \mathrm{Al}(\mathrm{Cu}$ film)

Fracture Strength (MPa)

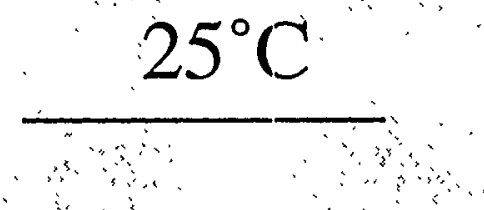

195 $300^{\circ} \mathrm{C}$

115

not tested

3. $\mathrm{Cu} / \mathrm{Ti} / \mathrm{AlB}$ eMet-150 (NaOH)

92

not $\mathrm{Be}\left(\mathrm{HNO}_{3}+\mathrm{HF}\right)$ tested

桌 


\section{EBTS Specimen}

\section{Braze}

$\mathrm{Be}+$ VPS Al

Al-12Si (filler metal)

$\mathrm{Cu} / \mathrm{Ti} / 1100-\mathrm{Al}$

(HIP Parameters - $625^{\circ} \mathrm{C} / 60 \mathrm{~m} / 105 \mathrm{MPa}$ )

Diffusion Bond

$\mathrm{Be}+\mathrm{PVD} \mathrm{Al}$ (Cu film)

$\mathrm{Cu} / \mathrm{Ti} / \mathrm{AlBeMet}-150$ (Cu film)

(HIP Parameters - $625^{\circ} \mathrm{C} / 60 \mathrm{~m} / 105 \mathrm{MPa}$ )
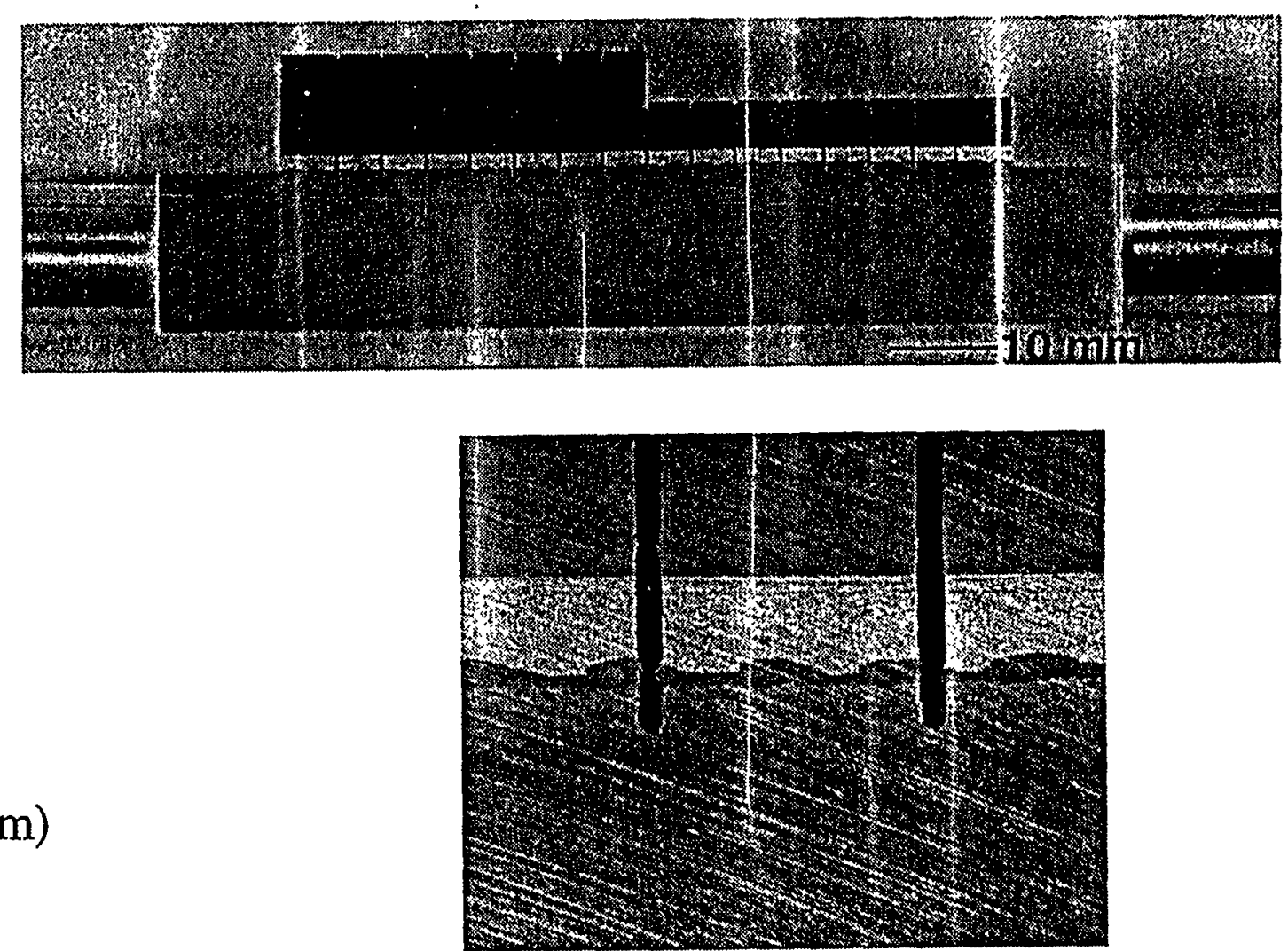


\section{EBTS Results for Brazed and Diffusion Bonded $\mathrm{Be}$ on $\mathrm{Cu}$}

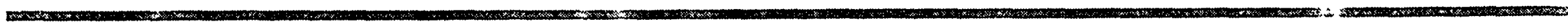

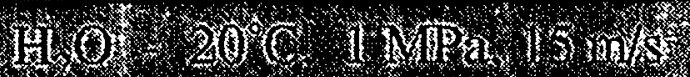

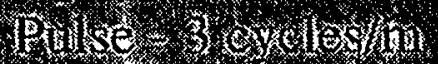

\begin{tabular}{|c|c|c|c|}
\hline & $1 \mathrm{MW} / \mathrm{m}^{2}$ & $3 \mathrm{MW} / \mathrm{m}^{2}$ & $10 \mathrm{MW} / \mathrm{m}^{2}$ \\
\hline raze & 1000 cycles & 1000 cycles & \\
\hline & No damage & No damage & No damag \\
\hline
\end{tabular}

Diffusion Bond

$\frac{1 \mathrm{MW} / \mathrm{m}^{2}}{\begin{array}{l}1000 \text { cycles } \\ \text { No damage }\end{array}} \frac{3 \mathrm{MW} / \mathrm{m}^{2}}{\begin{array}{l}1000 \text { cycles } \\ \text { No damage }\end{array}}$

The mock ups were subjected to several heat loads to $250 \mathrm{MJ} / \mathrm{m}^{2}(0.5 \mathrm{~s})$ in which the beryllium tiles melted. No beryllium tile de-bonding was noted. 


\section{Summary}

ํㅜㅇ

Several joining techniques have been studied as a method Jlogy to join beryllium to a copper alloy heat sink:

1. Aluminum brazing of beryllium to copper with an aluminum or AlBeMet-150 compliant layer;

2. Diffusion bonding of beryllium to copper with an alumin ım or AlBeMet-150 compliant layer;

3. Plasma spraying beryllium directly on copper or an alumi num compliant layer

The results of high heat flux testing suggest that these bonding technologies can be used successfully for PFC applications. 


\section{Future Work - Diffusion Bonding}

- Replace explosive bond with sputtered coatings

- enalbles thinner coatings

- compatible with curved geometries

- Reduce: bonding temperature

- prevent degradation of $\mathrm{CuCrZr}$ mechanical properties

- focus on $\mathrm{Al}$ - $\mathrm{Al}$ bond using diffusion enhancing coatings - $\mathrm{Ge}, \mathrm{Si}, \mathrm{Cu}$ 


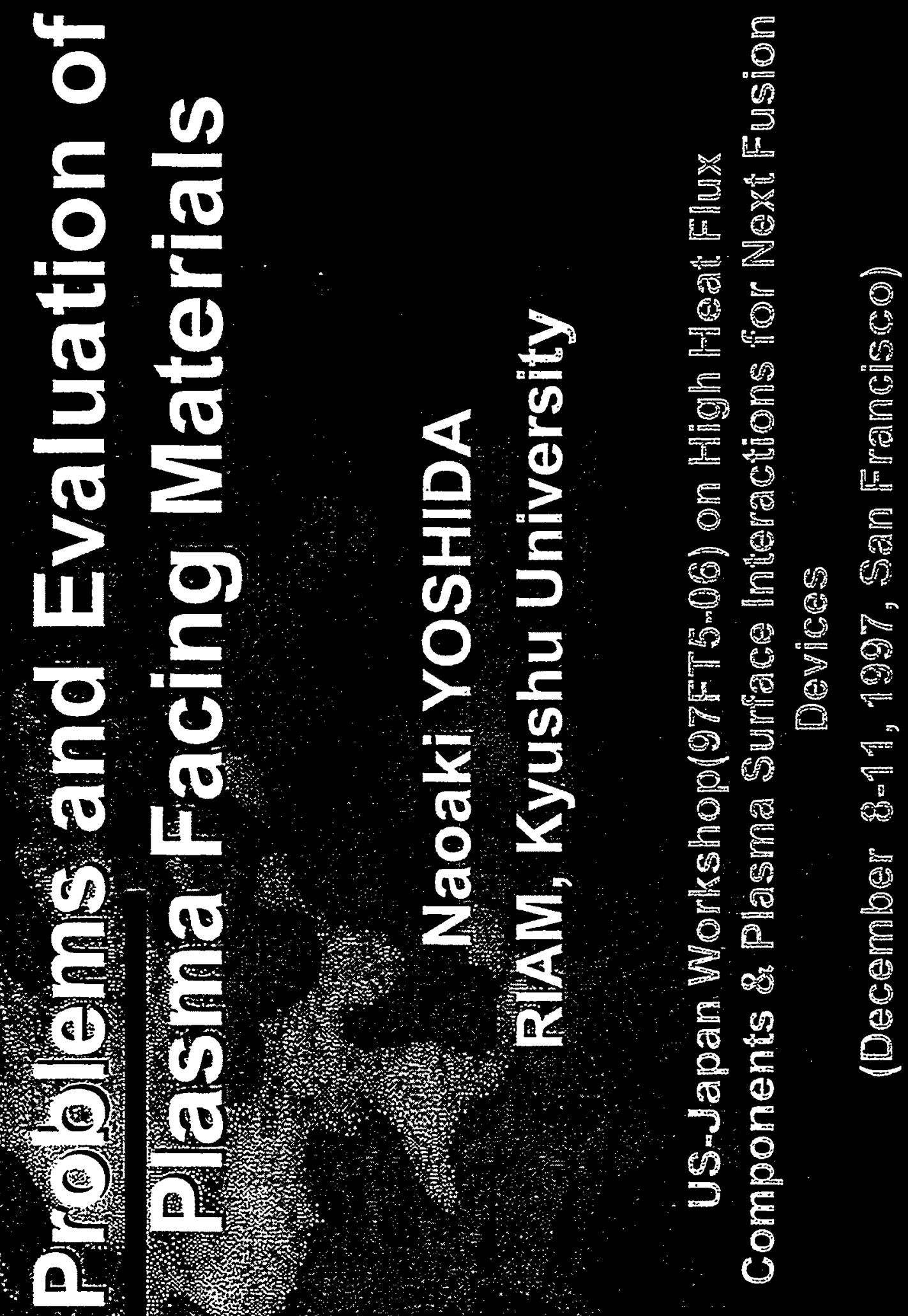




\section{High Heat Load Properties of Tungsten Coated Carbon Materials}

K. Tokunaga, N. Yoshida (RIAM / Kyushu University)

N. Noda (NIFS)

T. Sogabe (Toyo Tanso Co., LTD)

T. Kato (Nippon Plansee K.K) 


\section{Objective}

Was Armor Plate of PFC

$W$ and $W$ alloys seem promising candidate materials for plasma facing components in next fusion experimental devices.

Advantages

low sputtering yield, good thermal properties

Disadvantages

difficulty of machining and welding, very heavy

For technical realization of a $\mathrm{W}$ material:

W coatings on light $\mathrm{CFC}$ by plasma spray or physical vapor deposition (PVD)

$\rightarrow$ good thermal conductivity \& mechanical strength, light weight

PRESENT WORK

- Thick W coatings on CFC as well as isotropic fine graphite was successfully produced.

- High heat flux experiments were performed on the coated samples in order to prove the suitability and load limit of such coating. 


\section{Samples}

- W Coating:

Vacuum plasma spraying technique (VPS) <Plansee> thickness $\cdots 0.5 \mathrm{~mm} \& 1.0 \mathrm{~mm}$. density $\cdots . .92 .5 \%$ of theoretical density

- Substrate Materials:

C/C composite CX-2002U \& Isotropic fine graphite IG-430U <Toyo Tanso Co.> size $\cdots \cdots 20 \mathrm{~mm} \times 20 \mathrm{~mm} \times 10 \mathrm{~mm}$

- Diffusion barrier of Re between W and substrate to suppress the formation of brittle carbide.

- Heat treatment was performed to stabilize microstructure of the sample. 


\section{Thermal Conductivity of Carbon and Tungsten}

旬

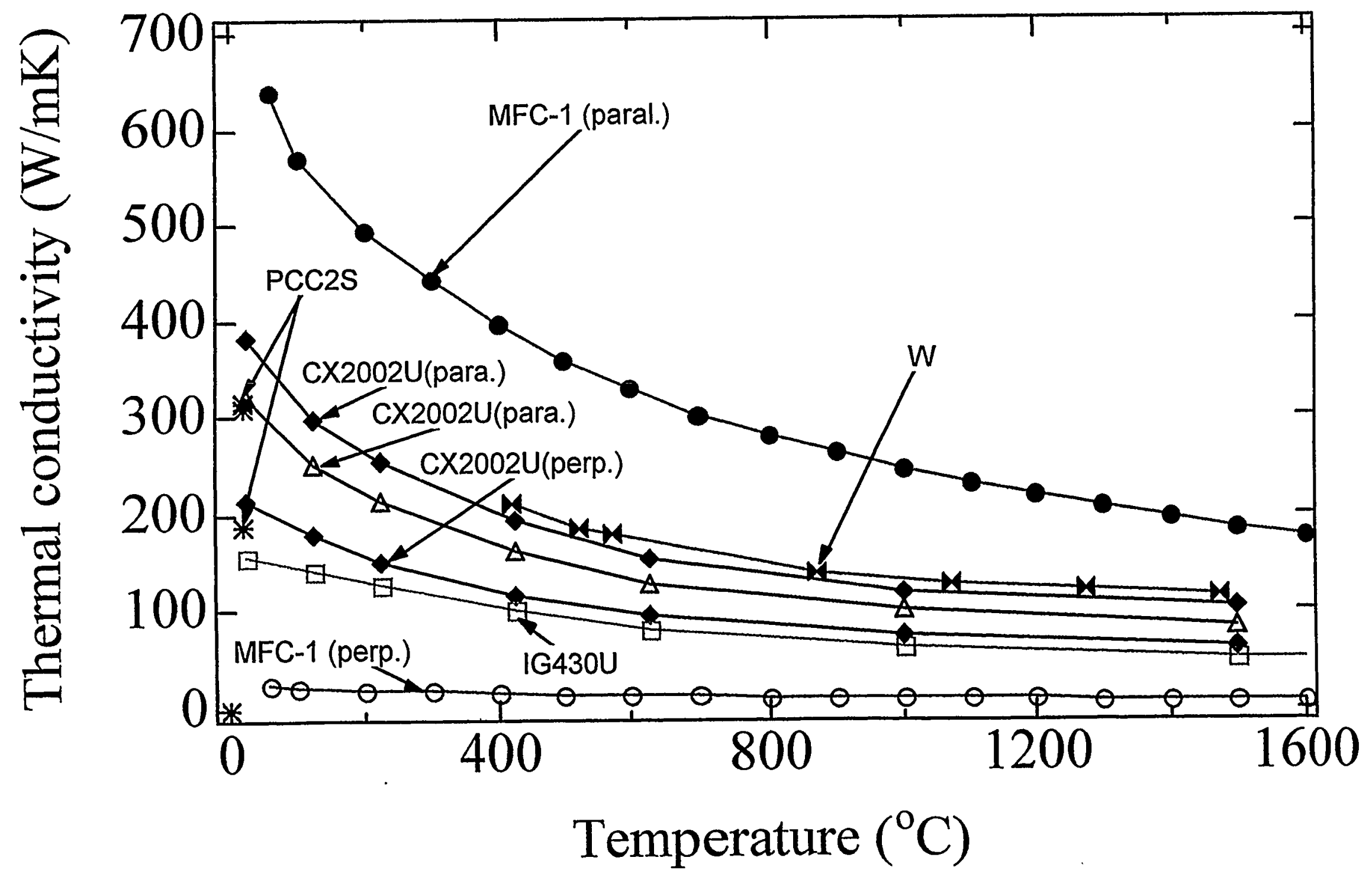




\section{SEM Image of Cross Section}

\section{VPS-W(1 mm thick) coated CX-2002U(\#6)}

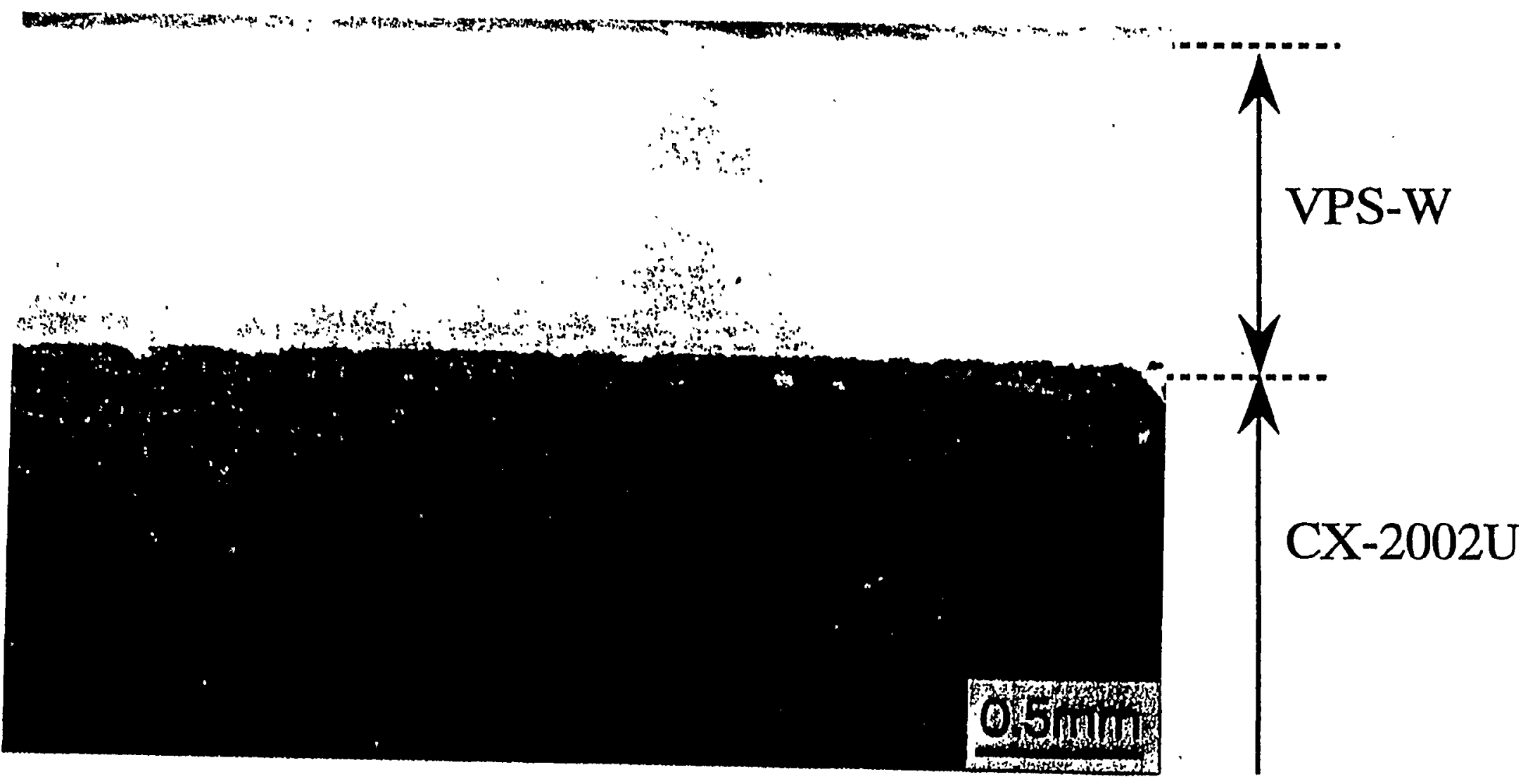

突 


\section{Heat Load Test (1)}

Electron Beam Heat Load Simulator (HLS) at RIAM:

$>$ Electron beam energy : $20 \mathrm{keV}$

$>$ Beam diameter : $8 \mathrm{~mm} \phi$

$>$ Duration of heat load: 10 sec.

Fixing of samples:

$>$ Mechanical fixing on a copper block actively cooled with water.

$>$ Carbon sheet $(0.38 \mathrm{~mm}$ thick) between sample and copper block

Surface temperature:

$>$ two-color optical pyrometers $\left(400-1100{ }^{\circ} \mathrm{C}, 1000-3100{ }^{\circ} \mathrm{C}\right)$.

$>$ scanning optical pyrometer (two-dimensional distribution)

Emitted gases:

$>$ quadrupole mass spectrometer(QMS) 


\section{Heat Load Test (2)}

Estimation of heat flux:

$>$ Heat flux was estimated from the beam diameter and net electron beam current, which was measured by applying a bias voltage to the sample to suppress the secondary electrons.

Estimation of heat removable capavility:

$>$ Temperature difference between inlet and outlet water of cooled copper block was measured by $\Delta T$ system to evaluate heat removable capability of the sample. Water flow rate was also measured.

Observation of Surface Morphology:

$>$ Before and after the irradiation, the sample surface was observed with SEM(scanning electron microscope) 


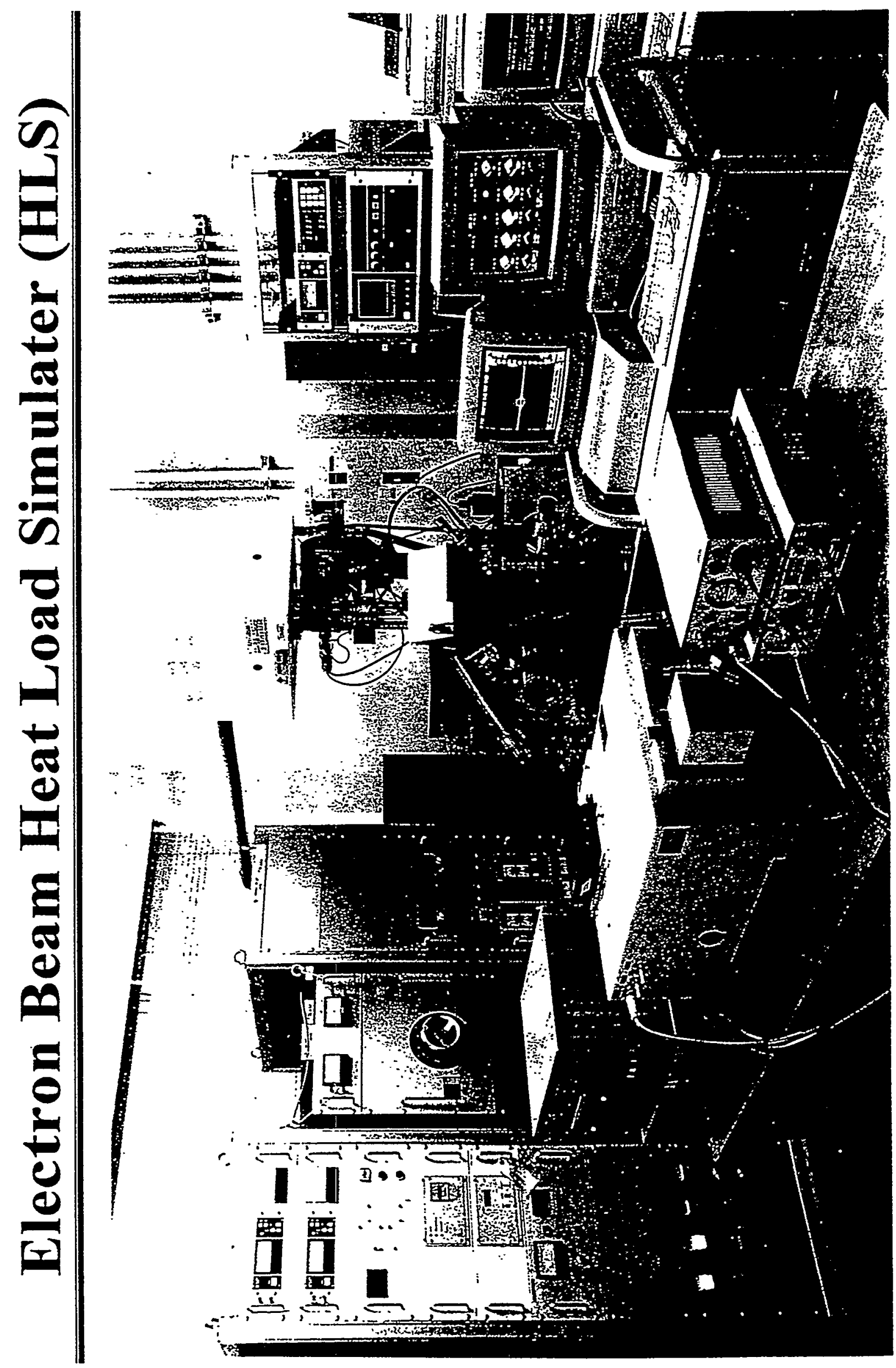




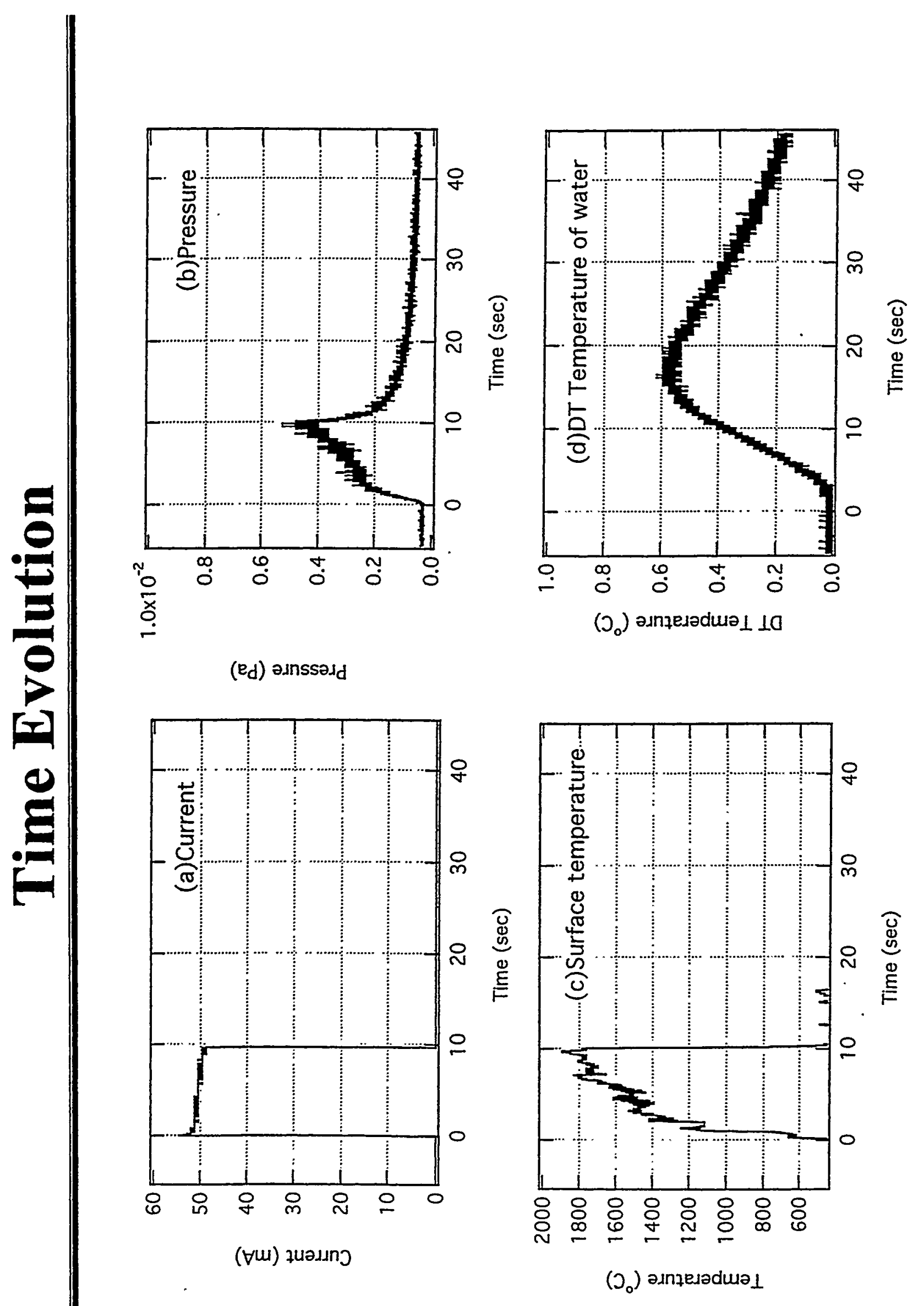




\section{Increasing of Surface Temperature}

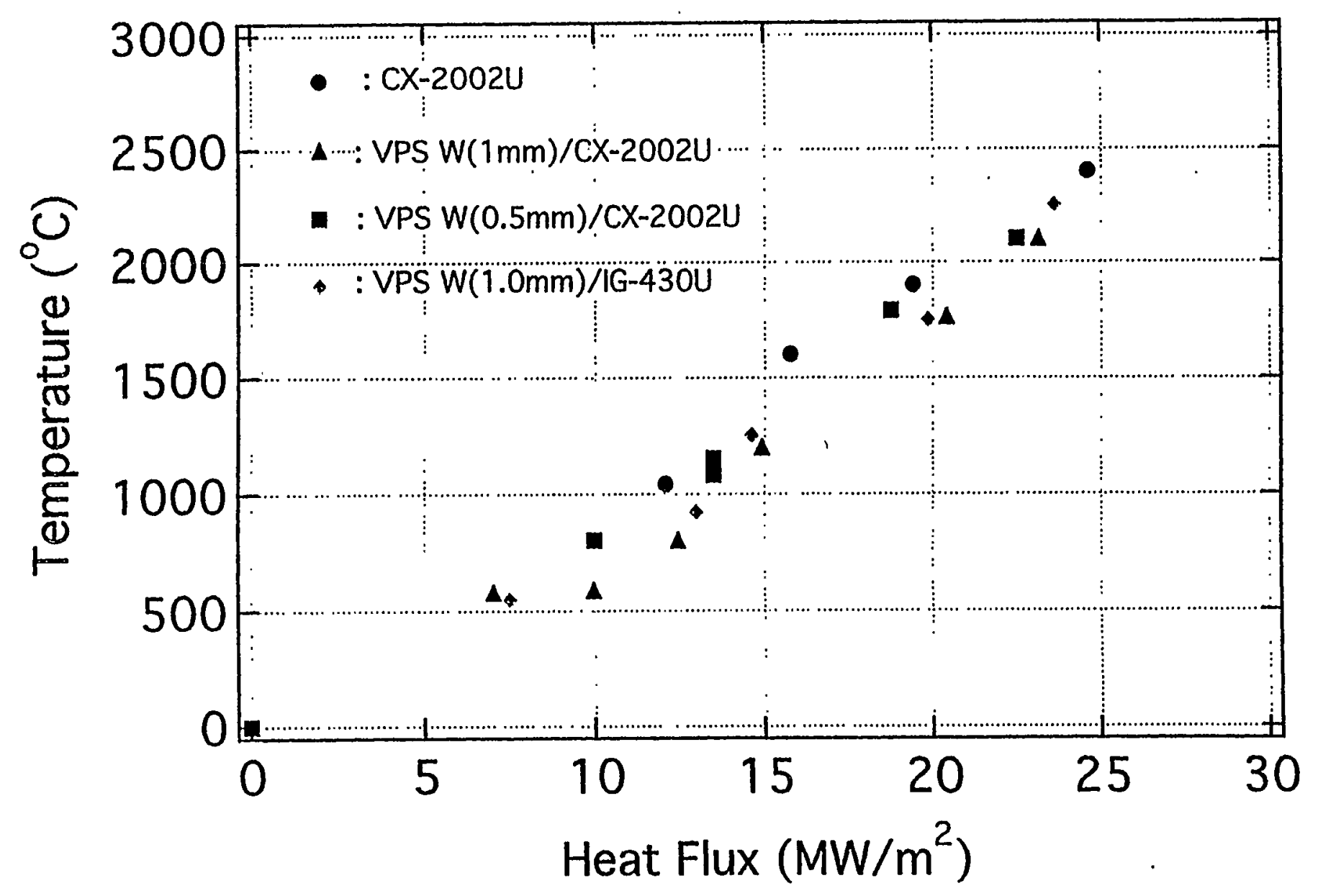




\section{QMS Signal before and during Heat Load Test}

\section{QMS VPS-W(0.5mm)/CX-200U, 6s/10s, 18.6MW/m²}

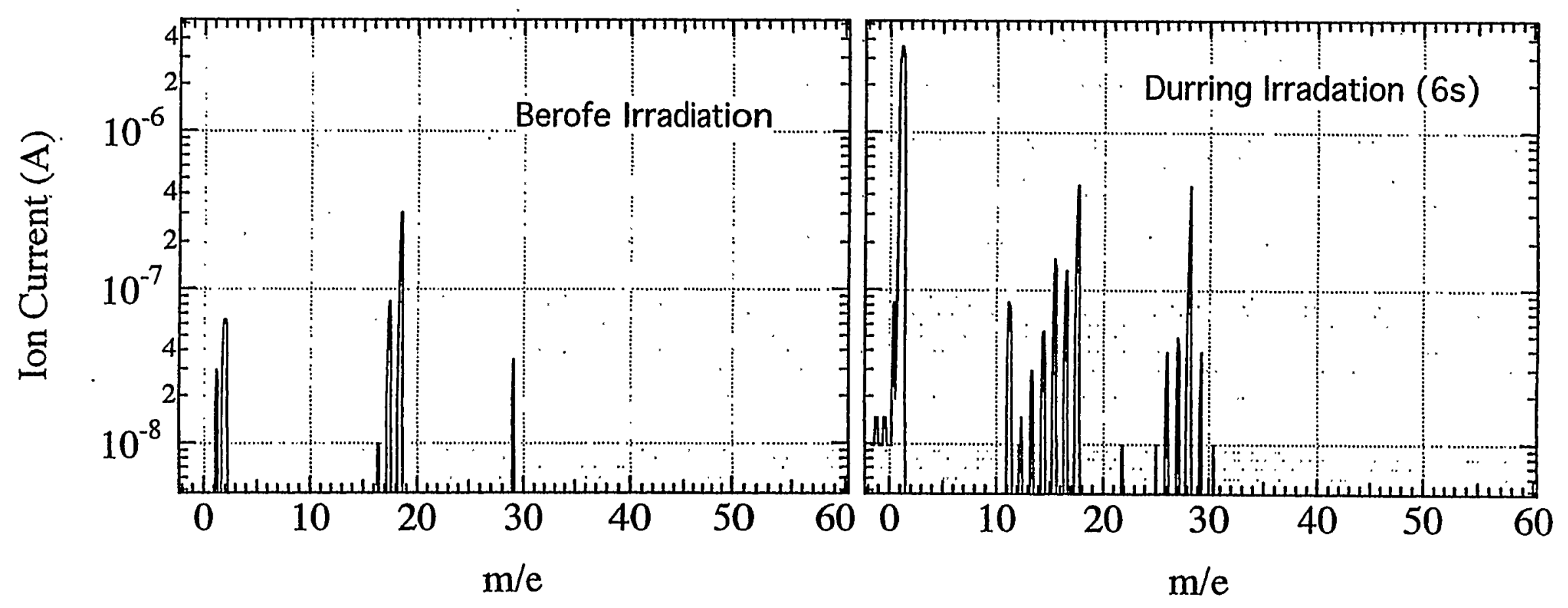

$\frac{6}{6}$ 


\section{SEM Images of Heat Loaded Surface}

(a) VPS-W(1.0mm)/CX-2002U(\#14)

(b) VPS-W(0.5mm)/CX-2002U(\#10)

(c) VPS-W(1.0mm)/IG-430U(\#17)
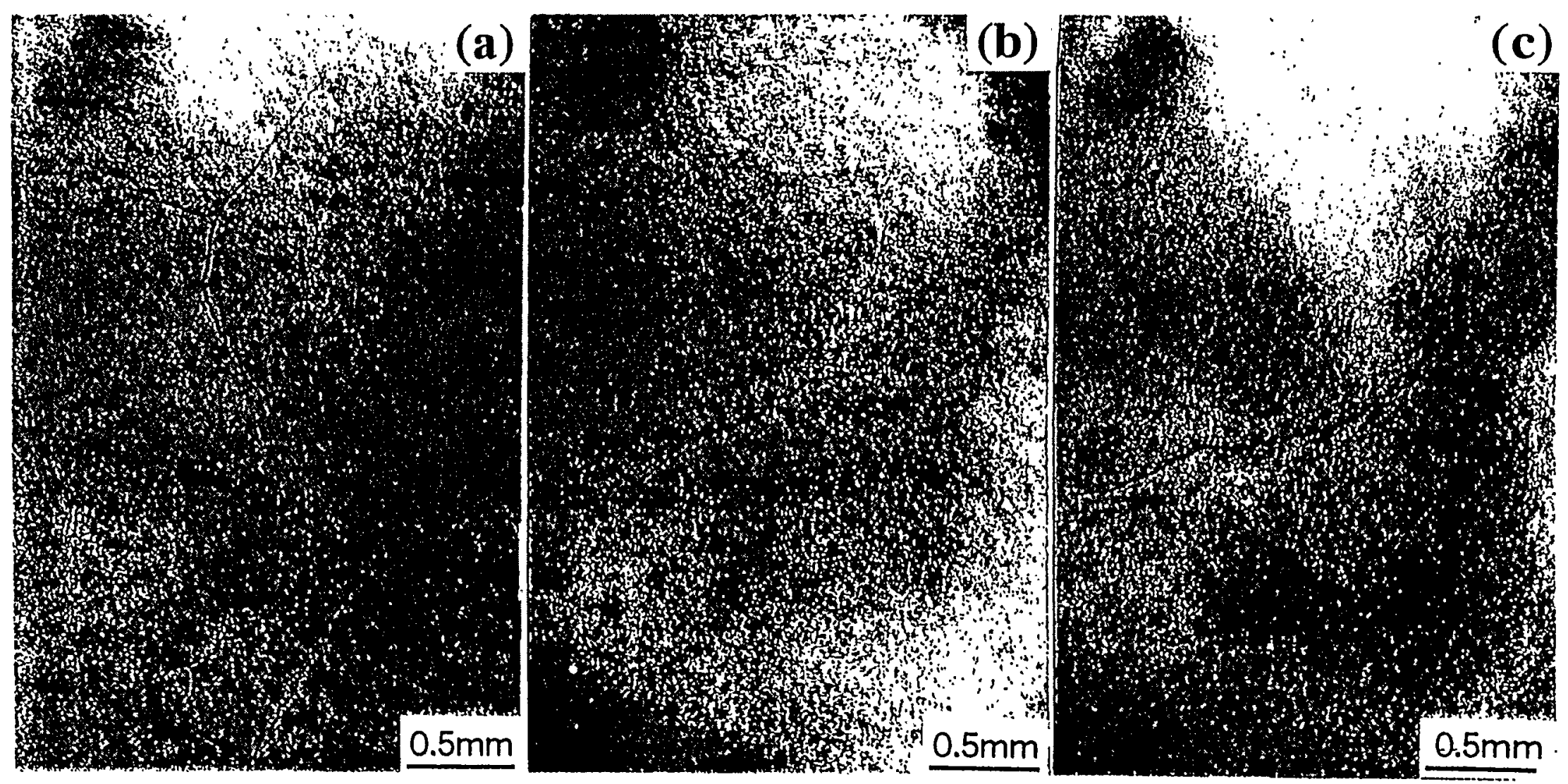


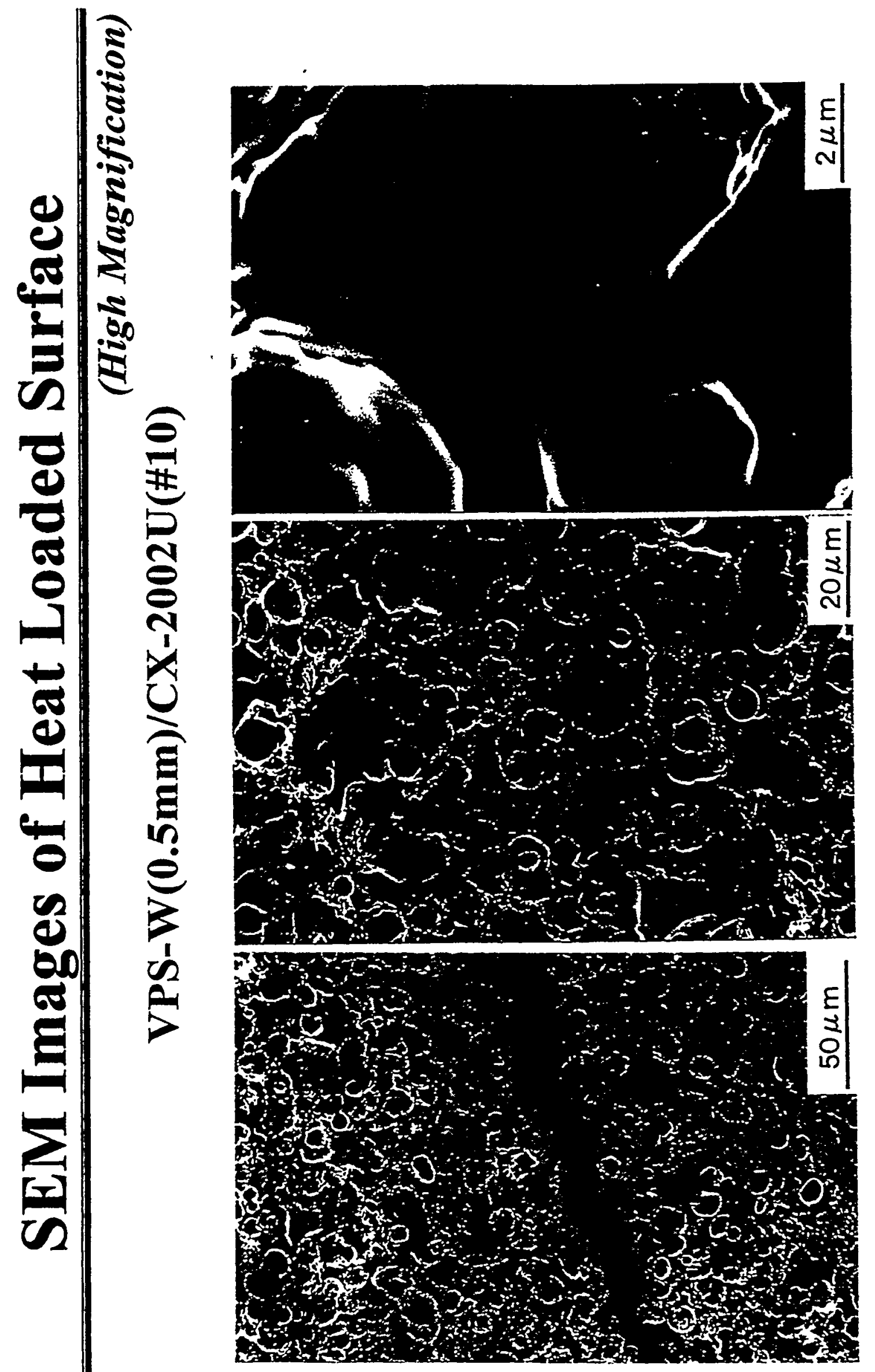




\section{SEM Image of W Surface (before heat load)}

\section{VPS-W(0.5mm thick) coated CX-2002U(\#10)}

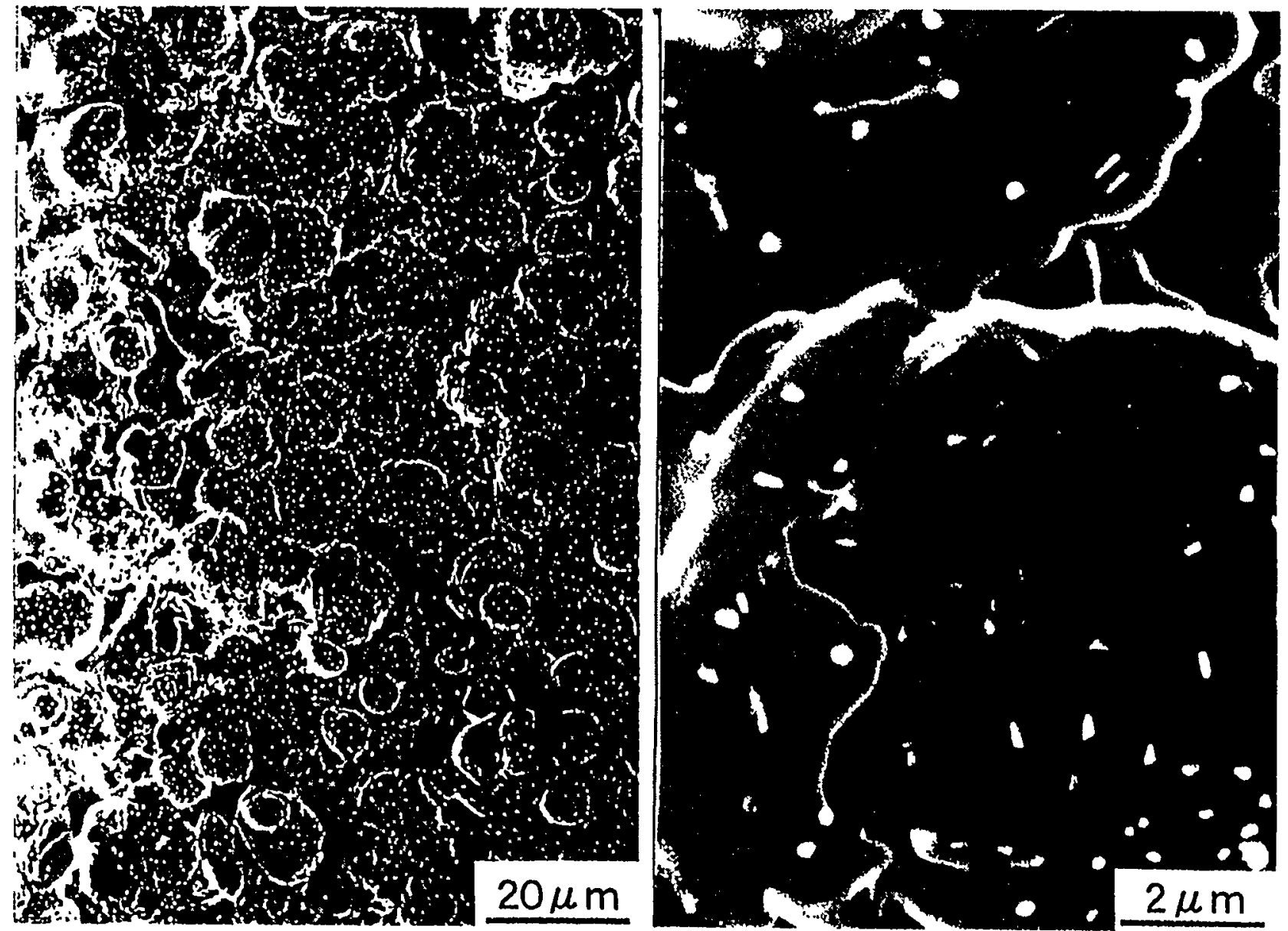




\section{Summary}

- W coatings of $0.5 \mathrm{~mm}$ and $1.0 \mathrm{~mm}$ thick were successfully deposited by Vacuum Plasma Spraying Technique on carbon-carbon fiber composite, $\mathrm{CX}-2002 \mathrm{U}$, and isotropic fine grain graphite, IG-430U.

- High heat flux experiments were performed on the coated and noncoated samples in order to prove the stability and load limit of such coating materials.

- There was little difference in temperature increase among CX-2002U and the coated materials up to $2200^{\circ} \mathrm{C}$. This result indicated that thermal and adhesion properties of the $W$ coated materials were good under high heat flux $\left(\sim 25 \mathrm{MW} / \mathrm{m}^{2}\right)$

- A few large cracks were formed in W coating, but plastic F deformation and micro-cracks due to grain growth by recrystallization were not observed below $2200^{\circ} \mathrm{C}$. The cracks may be formed by local thermal stress due to spot-like electron beam. 


\section{Future Planes}

- Investigation of microscopic change of VPS-W/CFC interface phase change (WC formation), compositional change, Re-crystallization, mechanical properties, etc.

- Thermal fatigue test $\cdots$ estimation of life time

- Heat loading test of actively wáter-cooled mockups

( Jan. 1998) 


\title{
Thermal Response of CFC/OFHC Cooling Pipe Mock-up for LHD/LID
}

\author{
T. Tokunaga, N. Yoshida \\ RIAM / Kyushu University
}

Y. Kubota, S. Inagaki, R. Sakamoto, A. Sagra, A. Komori, A. Noda, N. Ohyabu, O. Motojima National Institute for Fusion Science

Y. Soman Mitsubishi Heavy Industry Co. 


\section{Objective}

LूHD / NIFS

- Helical Divertor:

$10 \mathrm{MW} / \mathrm{m}^{2}$ for $10 \mathrm{~s} \& 0.75 \mathrm{~W} / \mathrm{m}^{2}$ in steady state

$\Longrightarrow \mathrm{C}$ tiles bolted to $\mathrm{SS}$ cooling tube

- Local Island Divitor(LID):

$6-8 \mathrm{MW} / \mathrm{m}^{2}$ in steady state $\Longrightarrow \mathrm{C}$ tiles brazed to Copper

R\&D Issues of LID divertor plate

- Armor material and brazing layer with high thermal conductivity and strong mechanical properties

- High heat transfer of cooling pipe/water

(optimum conditions for pressure, flow rate and temperature)

PRESENT WORK

Evaluation of thermal response and thermal fatigue for the newly developed two $\mathrm{CFC} / \mathrm{Cu}$ armor mock-ups 

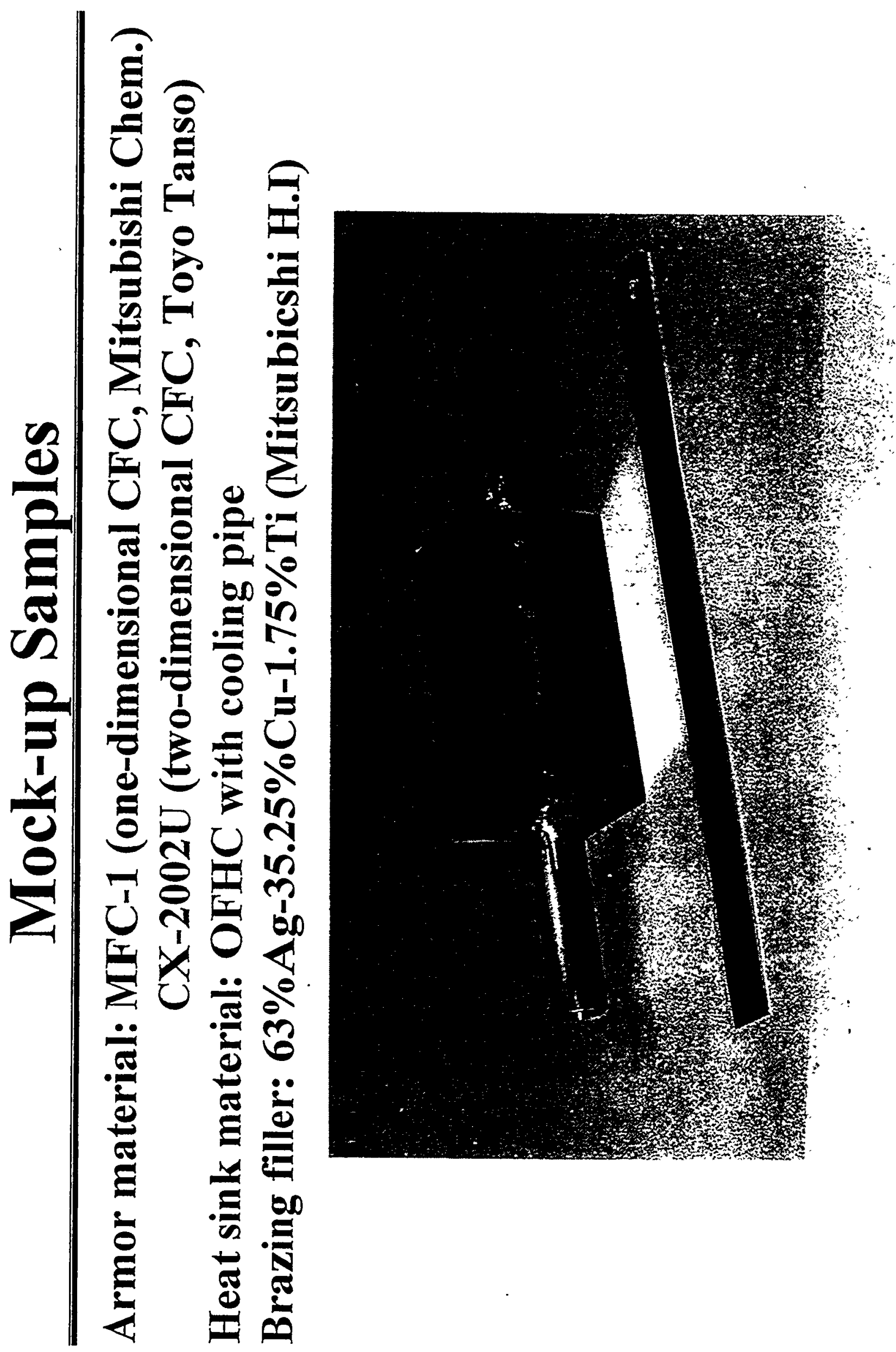


\section{Thermal Conductivity of Carbon Materials}

$\stackrel{⿱ 乛}{\infty}$

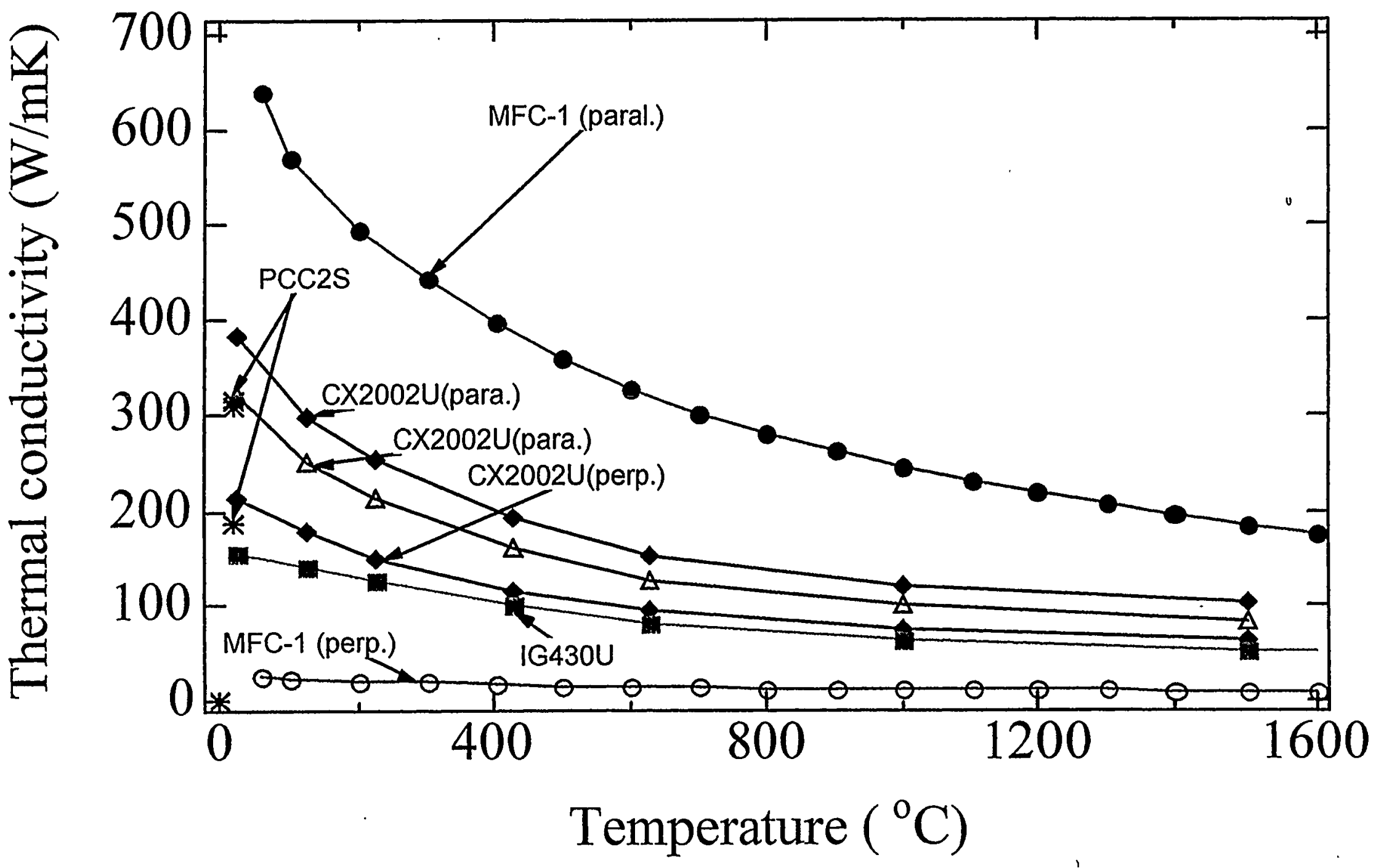




\section{Experimental Procedure}

- Heat Load Testing Device: ACT at NIFS

- Operation Conditions

electron beam: $30 \mathrm{keV}, 60 \mathrm{~s}$, beam size $30 \mathrm{~mm} \times 30 \mathrm{~mm}$

heat flux: $1-16 \mathrm{MW} / \mathrm{m}^{2}$

water cooling : pressure

$0.5 \mathrm{MPa}$

temperature $20-30^{\circ} \mathrm{C}$ (inlet)

flow rate $\quad 7.5 \mathrm{~m} / \mathrm{s}$

- Diagnostic

\begin{tabular}{|l|l|}
\hline surface temperature & pyrometers \\
\hline bulk temperatures & thermocouples (top and bottom of I.F.) \\
\hline heat removal capability & water calorimetry \\
\hline gas analysis & QMA \\
\hline
\end{tabular}




\section{CFC/Armor Mock-up}
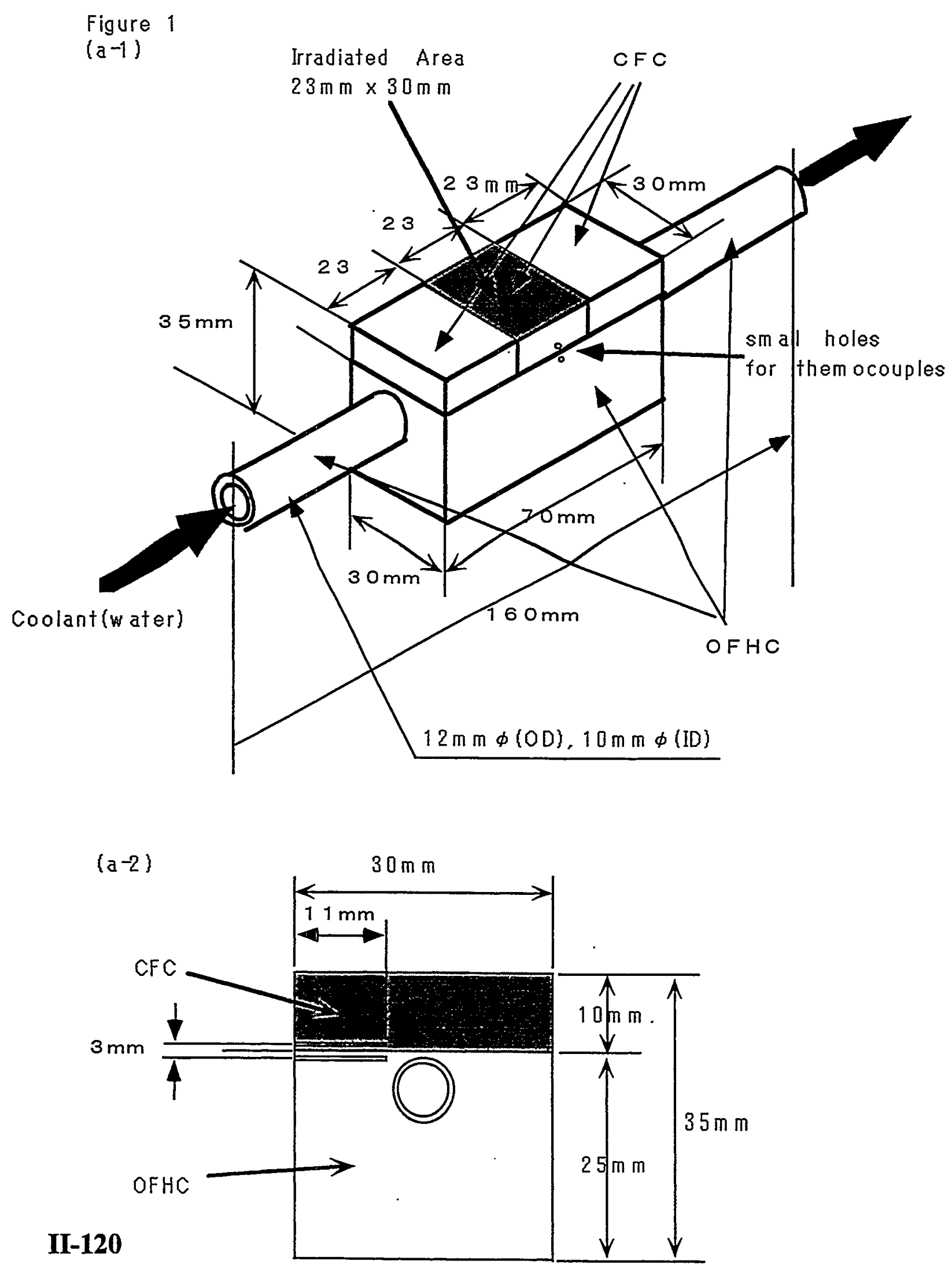


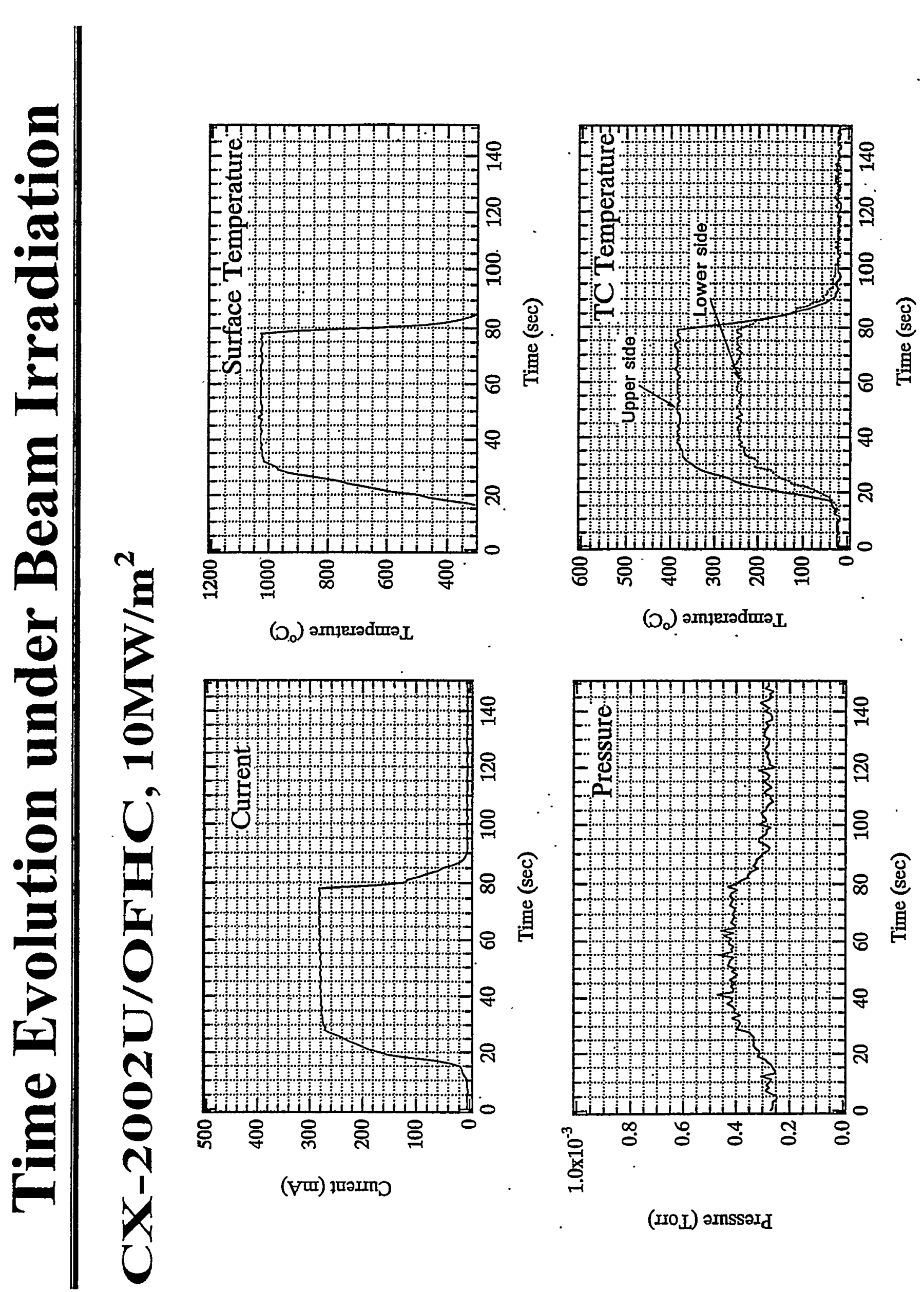



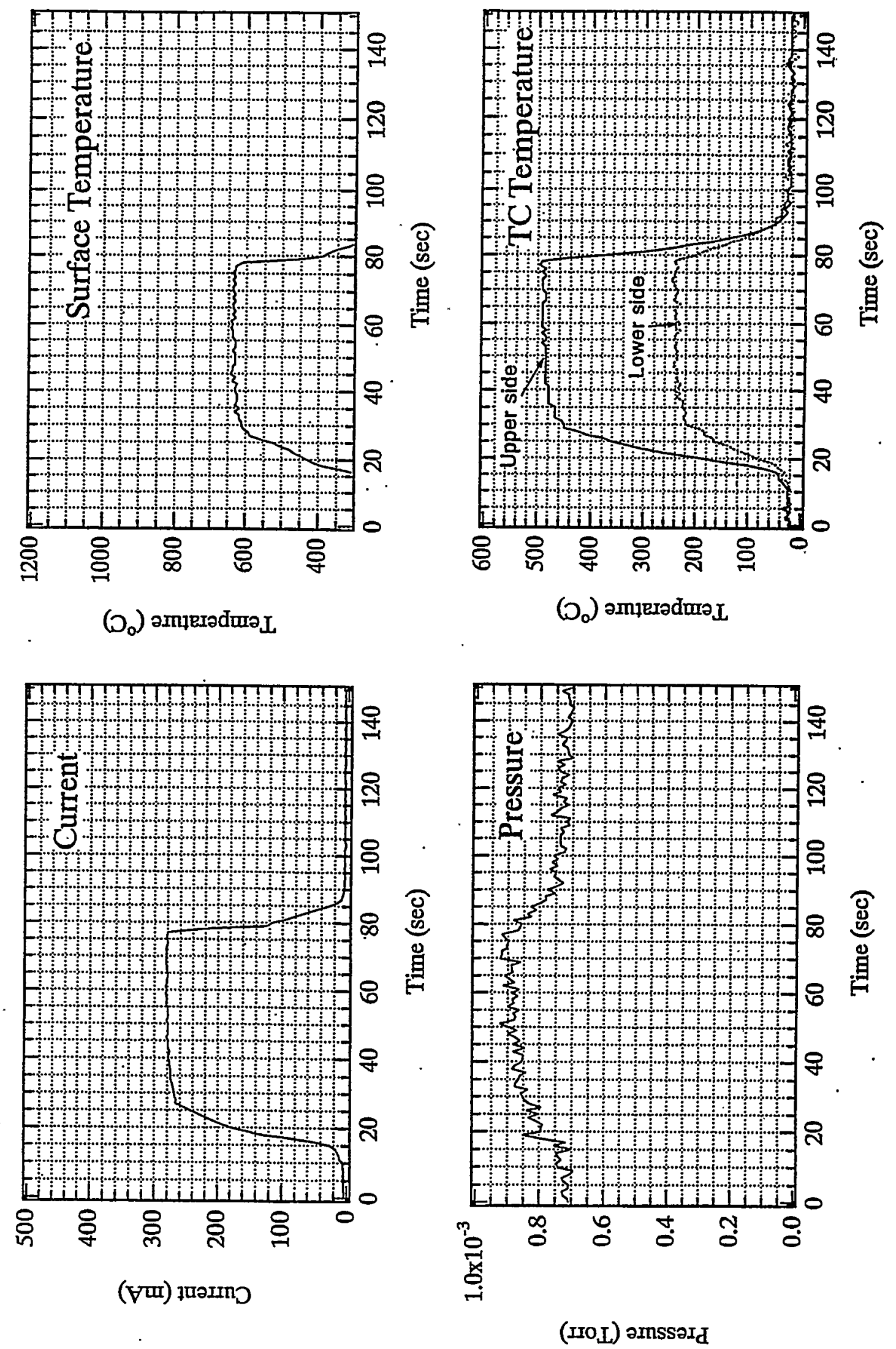

II-122 


\section{Surface Temp. / Heat Flux}
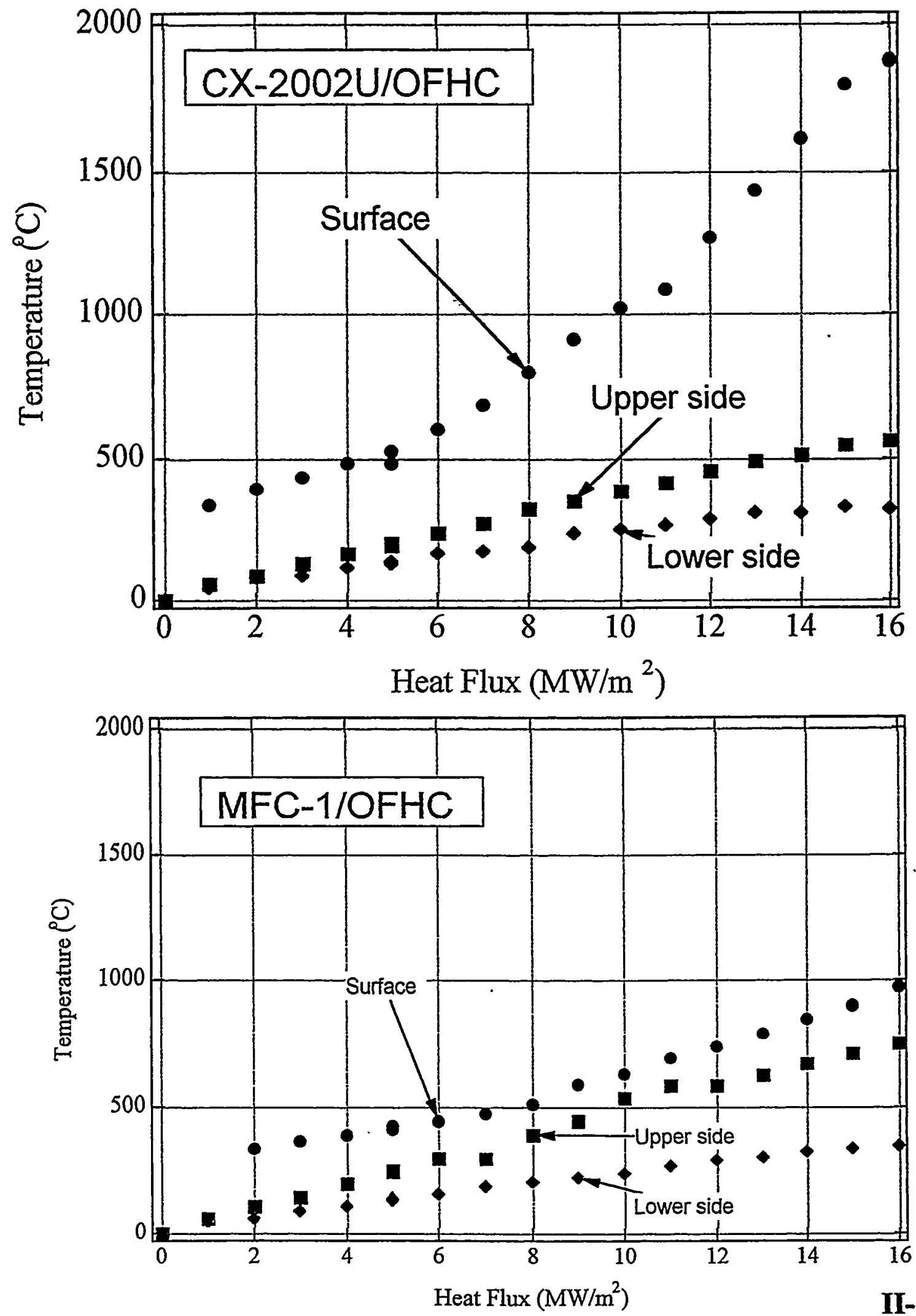

II-123 


\section{Gas Emission}

\section{CX2002U/OFHC, $15 \mathrm{MW} / \mathrm{m}^{2}$}
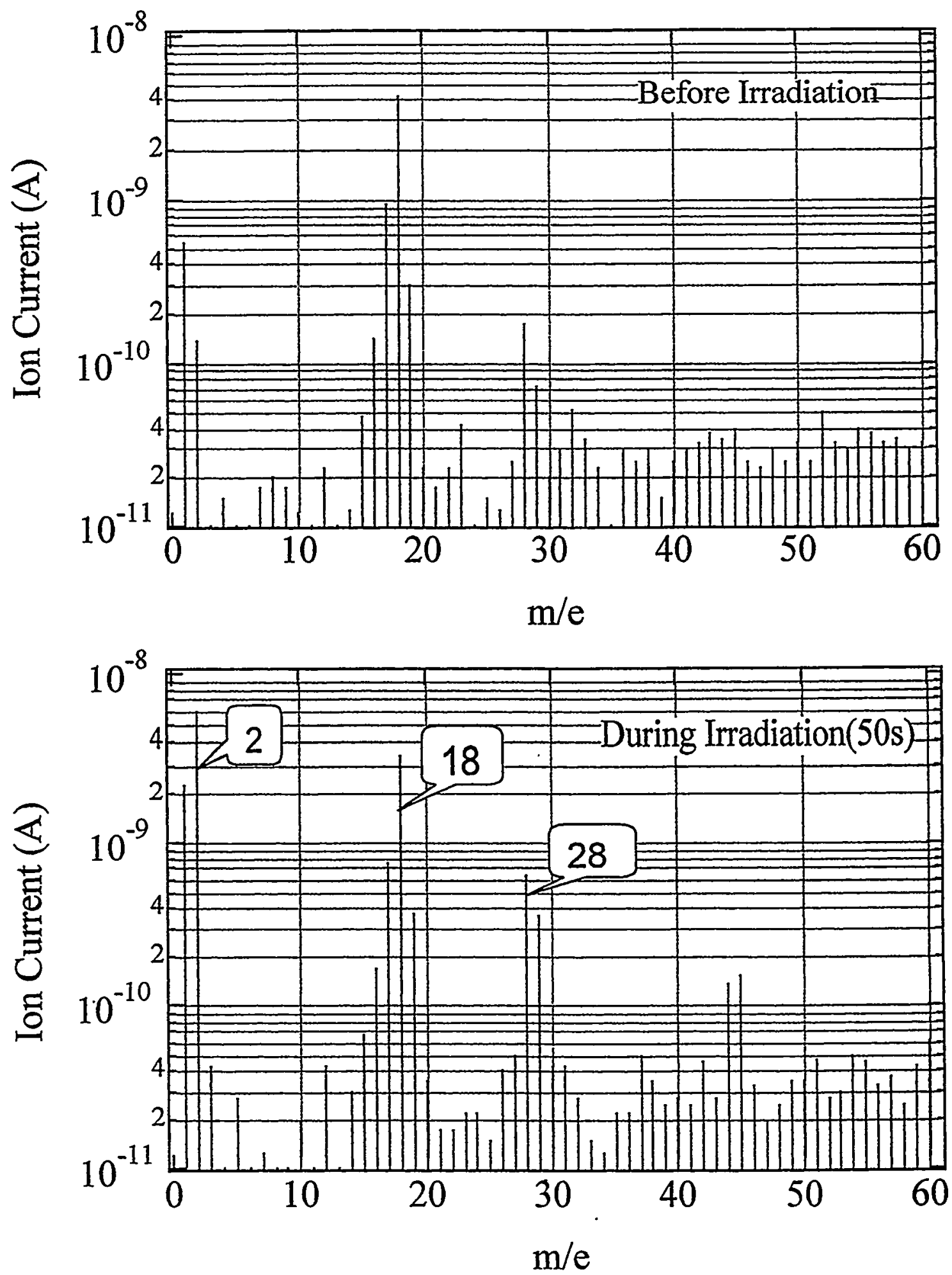


\section{QMS Signal during Heat Loading}

\section{CX-2002U/OFHC, 15MW/m}

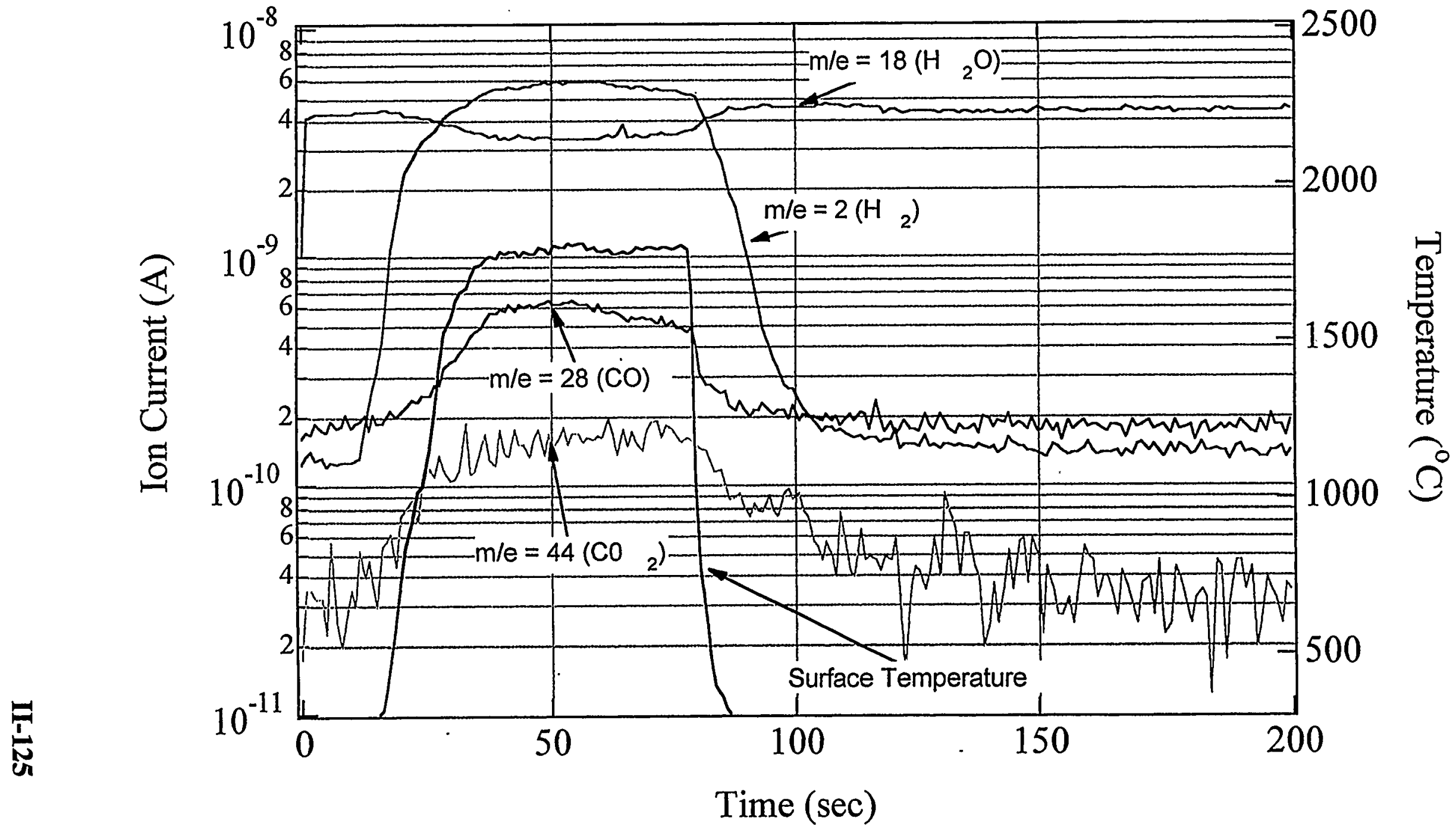




\section{Result of Thermal Fatigue Test}

MFC-1, 10MW $/ \mathrm{m}^{2}, 20{ }^{\circ} \mathrm{C}, 1.0 \mathrm{MPa}, 10 \mathrm{~m} / \mathrm{s}, 20 \mathrm{sec}$

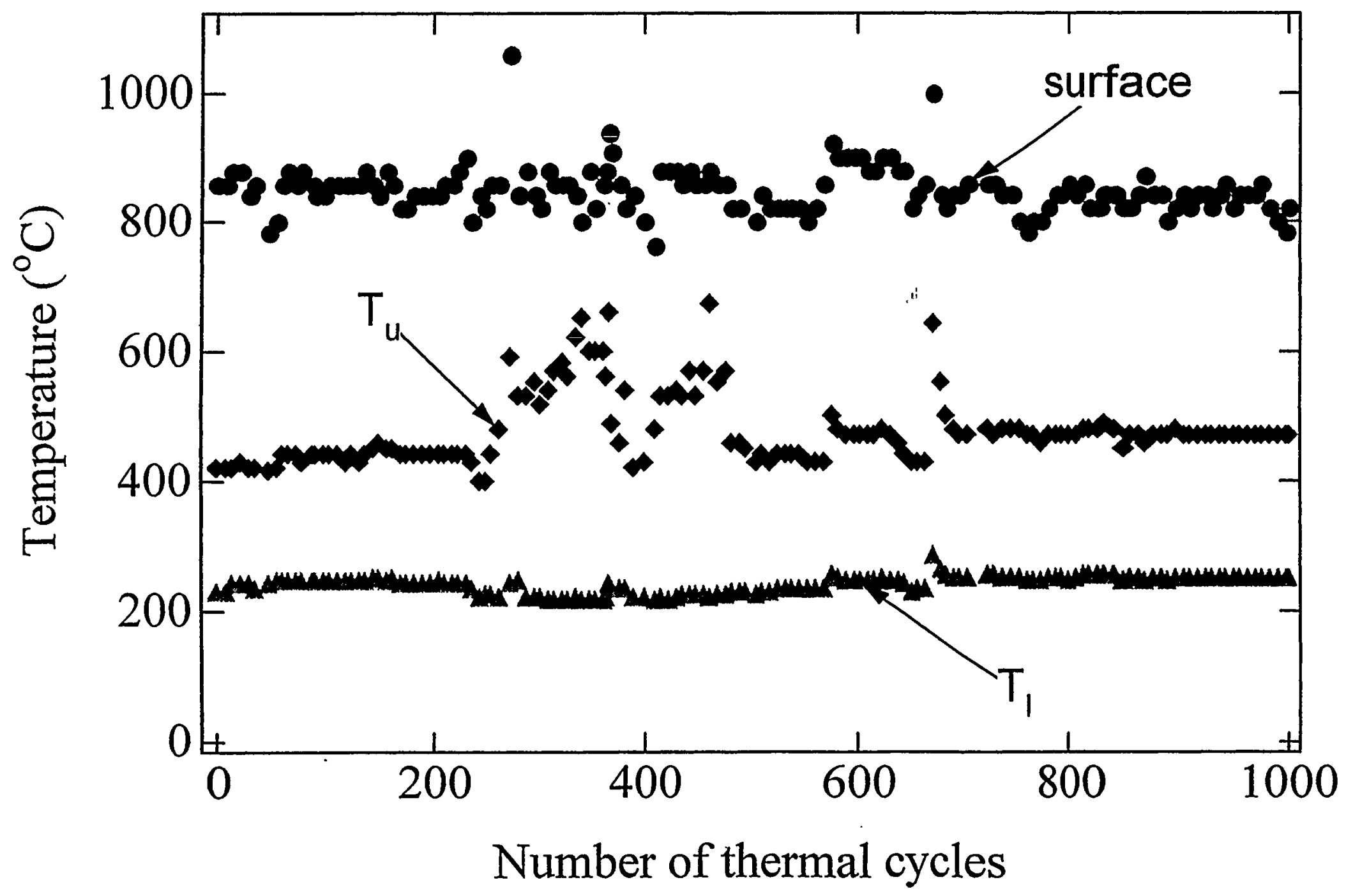




\section{Summary (1)}

- Two mockups of CFC brazed to OFHC for the LID divertor plate were fabricated.

CX2002U/OFHC (two-dimensional CFC)

MFC-1/OFHC(one-dimensional CFC)

- Their thermal response, material damage (and thermal fatigue lifetime tests) by high flux heat loading were examined by using electron beam facilities ACT and HHF.

- The MFC-1 mockup showed excellent heat removal performance due to its high thermal conductivity.

- In the case of $\mathrm{CX}-2002 \mathrm{U}$ mockup, surface temperature is about 胥 $1000^{\circ} \mathrm{C}$ at $10 \mathrm{MW} / \mathrm{m}^{2}$. This satisfies requirement for armor materials of LID/LHD. 


\section{Summary (2)}

- Thermal fatigue test of the MFC-1 mockup was performed (HHF). Though anomalous increase of temperature was not observed until 1000 cycles, strange fluctuation of temperature indicates the possibility of bonding degradation.

- Analysis of residual gas indicates that $\mathrm{H}_{2} \mathrm{O}$ gas reacts with $\mathrm{C}$ at the high temperature CFC surface. Influence to erosion?

\section{In Future:}

- Improvement of brazing for MFC-1/OFHC mockup.

- Thermal fatigue tests for CX2002U/OFHC and MFC-1/OFHC mockups 
Special Session III: Historical Progress in PSI Studies

III-1 


\section{III-2}




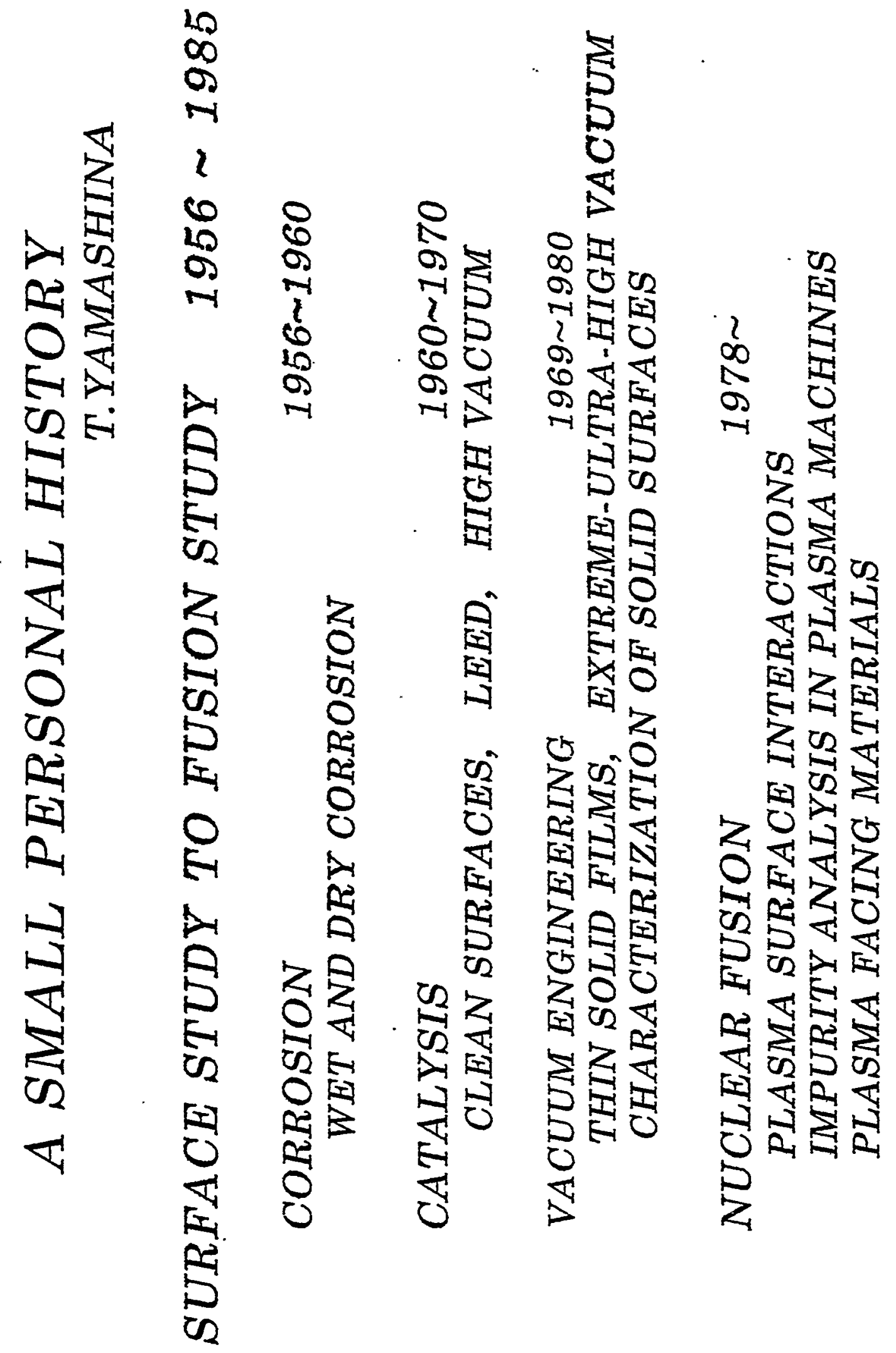


SURFACE-CHARACTERIZATION-TECHNIQUES 1958 1983

1958 QUARZ SPIRAL MICROBALANCE X-RAY DIFFRACTION

1959 HIGH ENERGY ELECTRON DIFFRACTION

1960 ULTRA-HIGH VACUUM WITH OMEGATRON MASS SPECTROMETER (FIRST JAPANESE MADE)

1963 TORTION TYPE MICROBALANCE

1965 QUADRUPOLE MASS SPECTROMETER (FIRST JAPANESE MADE)

1967 ULTRA-HIGH VACUUM SYSTEM SPUTTERING APPARATUS
DRY CORROSION

SURFACE OF METAL OXIDES

CATALYSIS BY CLEAN SURACES LOW PRESSURE CHEMICAL REACTIONS

SURFACE OXIDATION AND REDDUCTION

LOW PRESSURE GAS REACTIONS

THIN SOLID FILMS

REACTIVE SPUTTERING PROCESS 
1970 SURFACE ROUGHNESS FACTOR

1970 (JOINED TO NUCLEAR FUSION STUDY)

1971 AUGER EELECTRON SPECTROSCOPY (FIRST AES IN JAPANESE UNIVERSITY)

1973 AES-SIMS COMBINED SYSTEM

1978 ION-MASS-MICRO-ANALYSIS(IMMA)

1981 XPS-AES COMBINED SYSTEM PROBE-MEASUREMENTS IN JIPPT-II (FIRST EXPERIMENTS WITH REAL PLASMA MACHINE)
SURFACE AREA WITH ATOMICAL $S C A L E$

\author{
PLASMA SURFACE INTERACTIONS \\ IMPURITY DISTRIBUTION OF WALL \\ SURFACES \\ SURFACE SEGREGATION OF \\ BINARY ALLOYS \\ SURFACE CHARACTERIZATION \\ OF FIRST WALL \\ ENERGY DISTRIBUTION OF \\ SPUTTERED IONS
}

1983 PROBE-MEASUREMENTS IN HELIOTRON E 


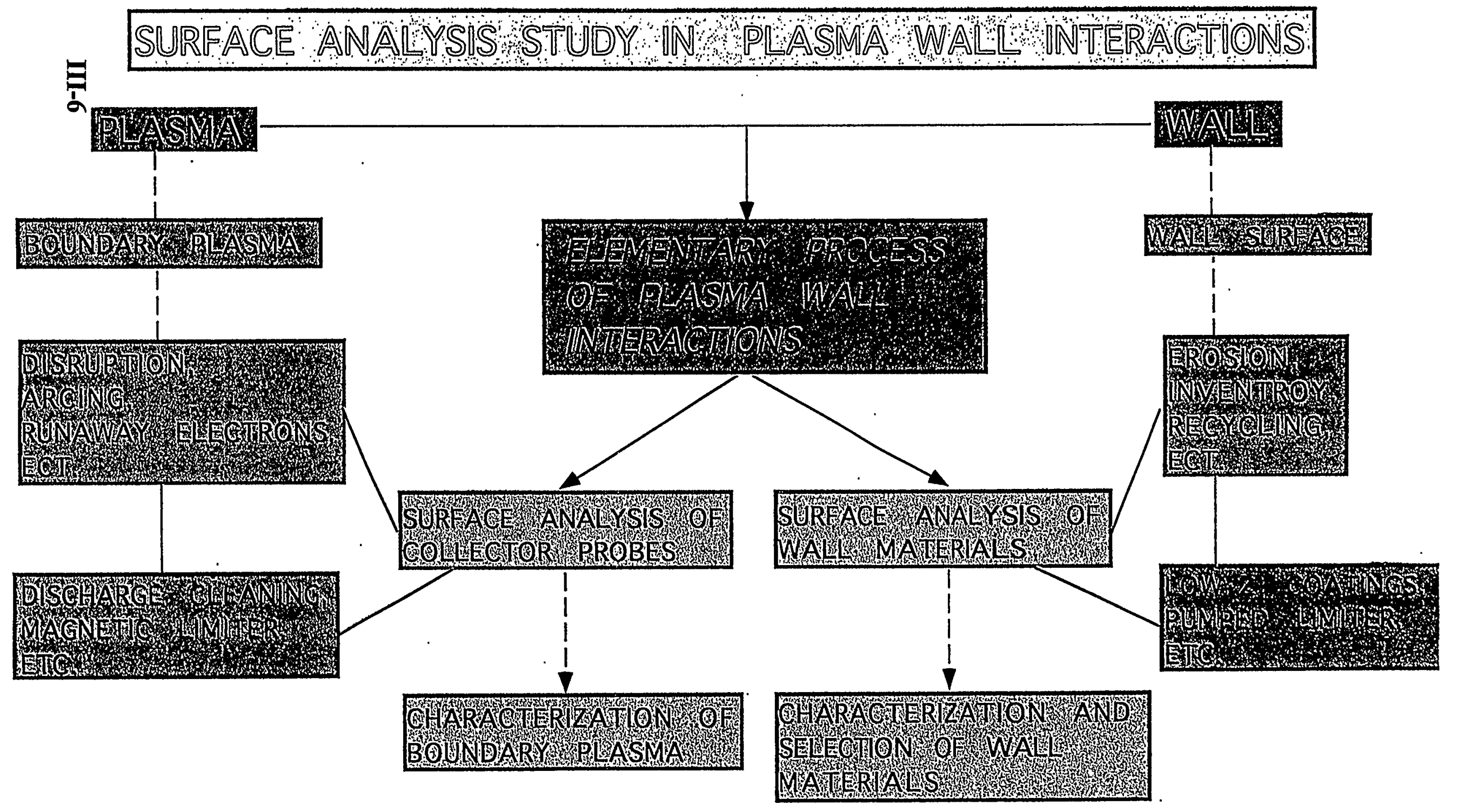




\section{OUR VIEW}

1. Plasma WalZ Interactions $\longleftrightarrow$ Surface Analysis Techniques

2. Torus Machine Experiments $\longleftrightarrow$ Simulation.Experiments

3. Surface Collector Probes $\longrightarrow$ Long Term Exposed Materials

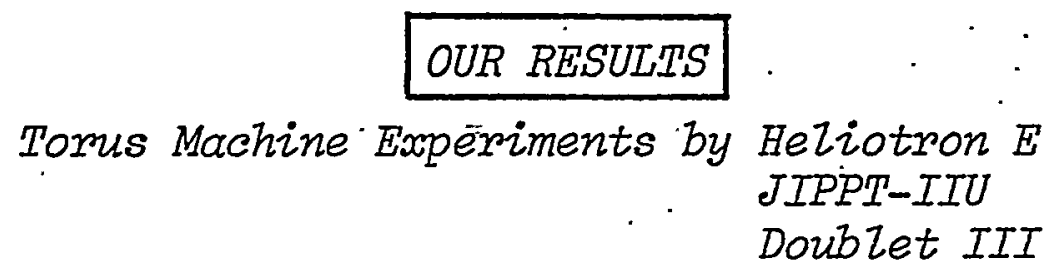

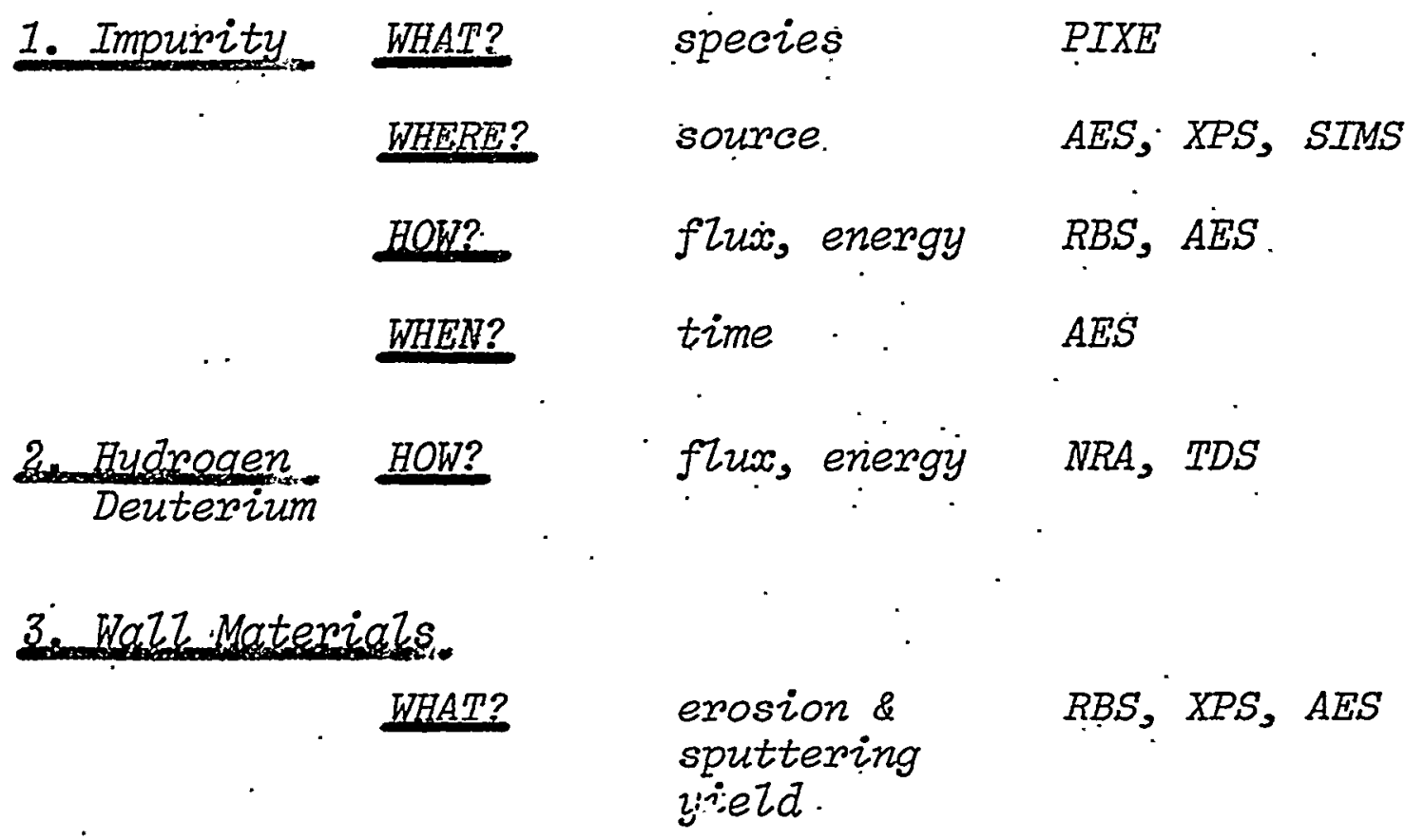




\section{OUR FIRST CONTRIBUTION TO PLASMA.SURFACE.INERACTIONS CONFERENCE}

Journal of Nuclear Materials 75 (1978) 7-13

(c) North-Holland Publishing Company

SPUTTERING PROCESS OF A SILICON CARBIDE SURFACE WITH ENERGETIC IONS BY MEANS OF AN AES-SIMS-FDS COMBINED SYSTEM

Mamoru MOHRI, Kuniaki WATANABE and Toshiro YAMASHINA

Department of Nuclear Engineering, Faculty of Engineering, Hokkaido University, Sapporo, Japan 060

Received 20 February 1978

Surface phenomena on silicon carbide following interaction with energetic hydrogen ions and argon ions have been studied by means of simultaneous, in situ measurements with a combined system of AES-SIMS-FDS (Flash Desorption Spectroscopy). Bombardment by 0.7 and $1.5 \mathrm{keV}$ argon ions was observed to sputter the surface atoms, both silicon and carbon, with the same sputtering yields. In the case of bombardment by hydrogen ions, on the other hand, silicon atoms were sputtered out preferentially through chemical sputtering to form silicon hydrides at room temperature. In-depth composition profiles of silicon carbide irradiated by $100-\mathrm{keV} \mathrm{D}^{+}$ions were also examined by the combined system. 
IMA (Ion Microprobe-Mass Analysis)

Ion beam (Duoplasmatron) $0 \sim 30 \mathrm{KeV}, 0.1 \mathrm{~mm} \phi($ scannihg $), T$ Planking several tens $\mu A\left(H^{+}, D^{+\ldots}\right)$

Mass analyzer (Stigmatic second order $\left\{\begin{array}{l}\text { analyzer (Stigmatic second order } \\ \text { double focussing mass spectrometer) } \\ \text { Microcomputer }\end{array}\right.$

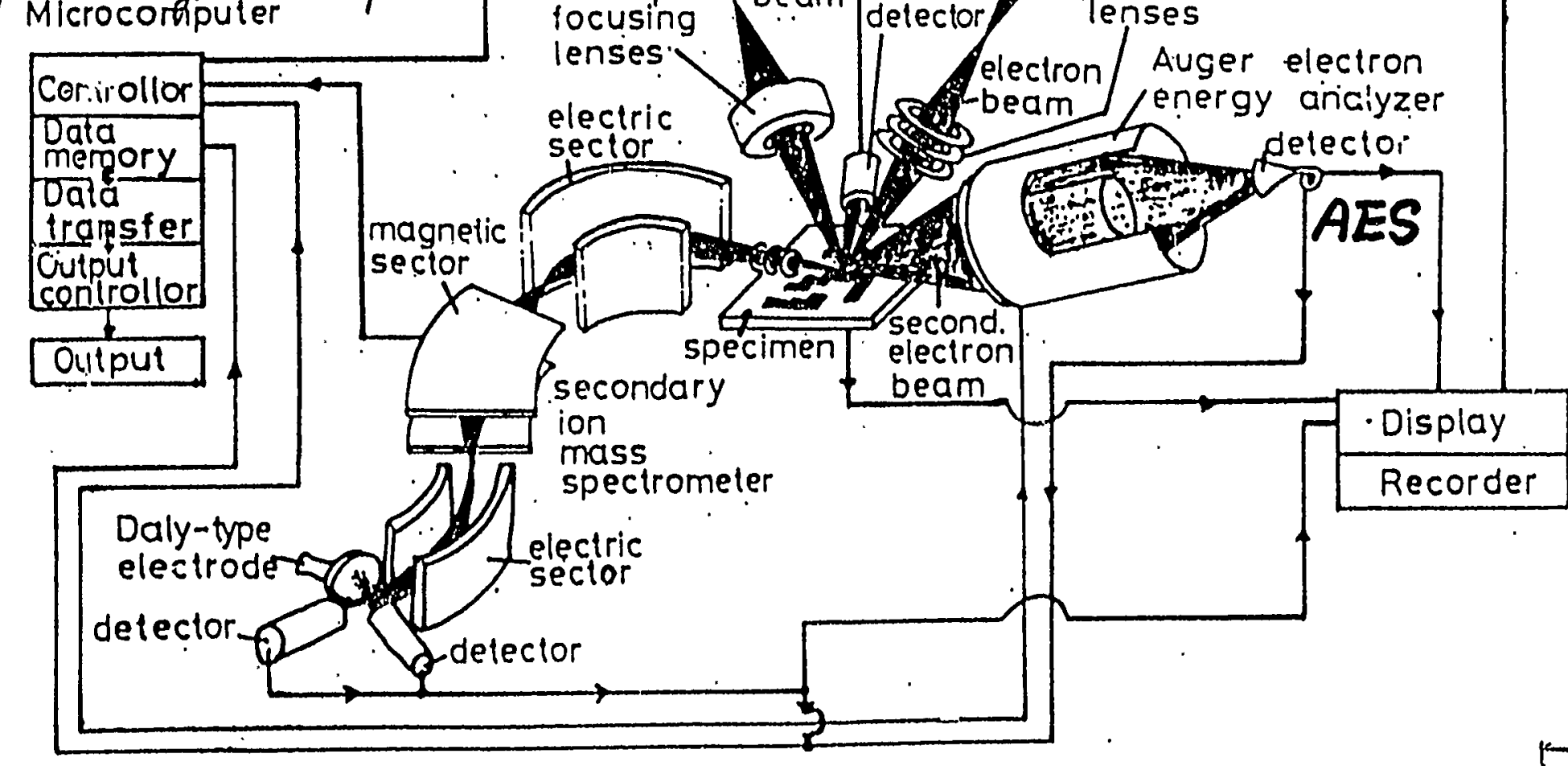

寻

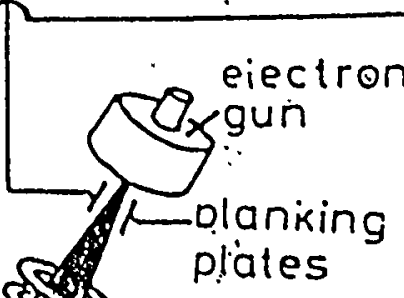

IFig. 1. Schematic construction of the AES-IMA combined system.

1. Sputtering yield measurement by volumetic method

2. Chemical and physical sputtering processes

2 Fnow dictribution analusis ot sputtered species 


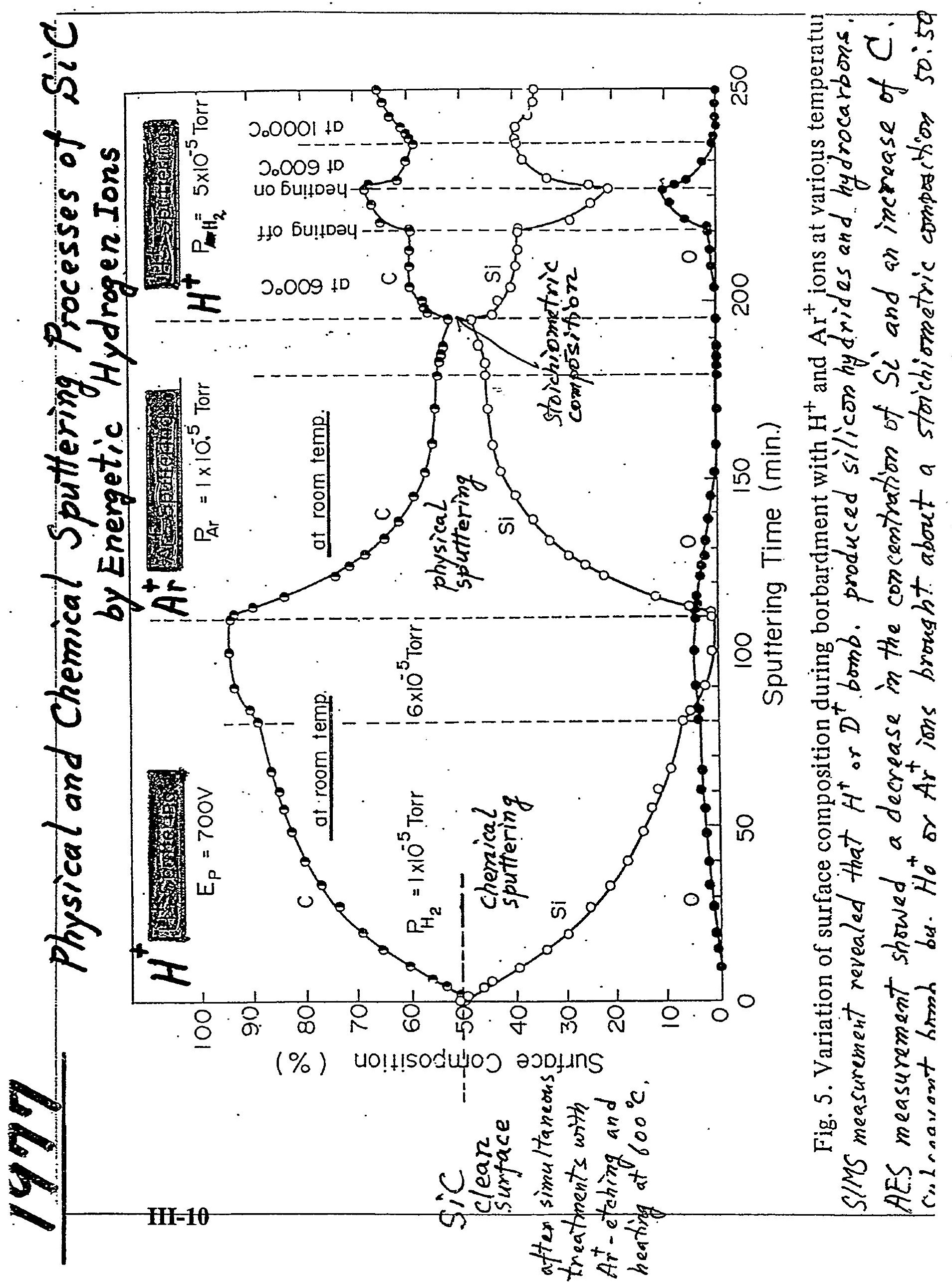




\section{COLLABORATION WITH VARIOUS MACHINES}

\section{$1980 \sim$}

JIPPT-II (NAGOYA UNIV.)

HELIOTORN-E (KYOTO UNIV.)

STORAGE RINGS (CERN)

TEXTOR (KFA)

DOUBLET III (GA)

JT-60, JT-60U (JAERI)

LHD (NIFS)

ARGONNE NL

SANDIA NL (LIVERMORE AND ALBUQUERQUE) OAKRIDGE NL 


\title{
SURFACE PROBE STUDIES IN TORUS DEVICES
}

\author{
JIPP T-IIU 1981 \\ HELIOTRON E 1983
}

1. EFFECTIVE UTILIZATION OF SURFACE ANALYSIS TECHNIQUES

2. TRANSPORT PROCESSES OF IMPURITIES

a) DETECTION OF IMPURITIY SPECIES

b) AREAL DISTRIBUTION OF IMPURITIES

c) ENERGY ESTIMATION OF IMPURITIES

3. TRANSPORT PROCESSES OF PLASMA PARTICLES

a) FLUX OF HYDROGEN IN SCRAPED-OFF LAYER

b) TIME RESOLVED ANALYSIS

c) ENERGY ESTIMATION OF PLASMA PARTICLES 


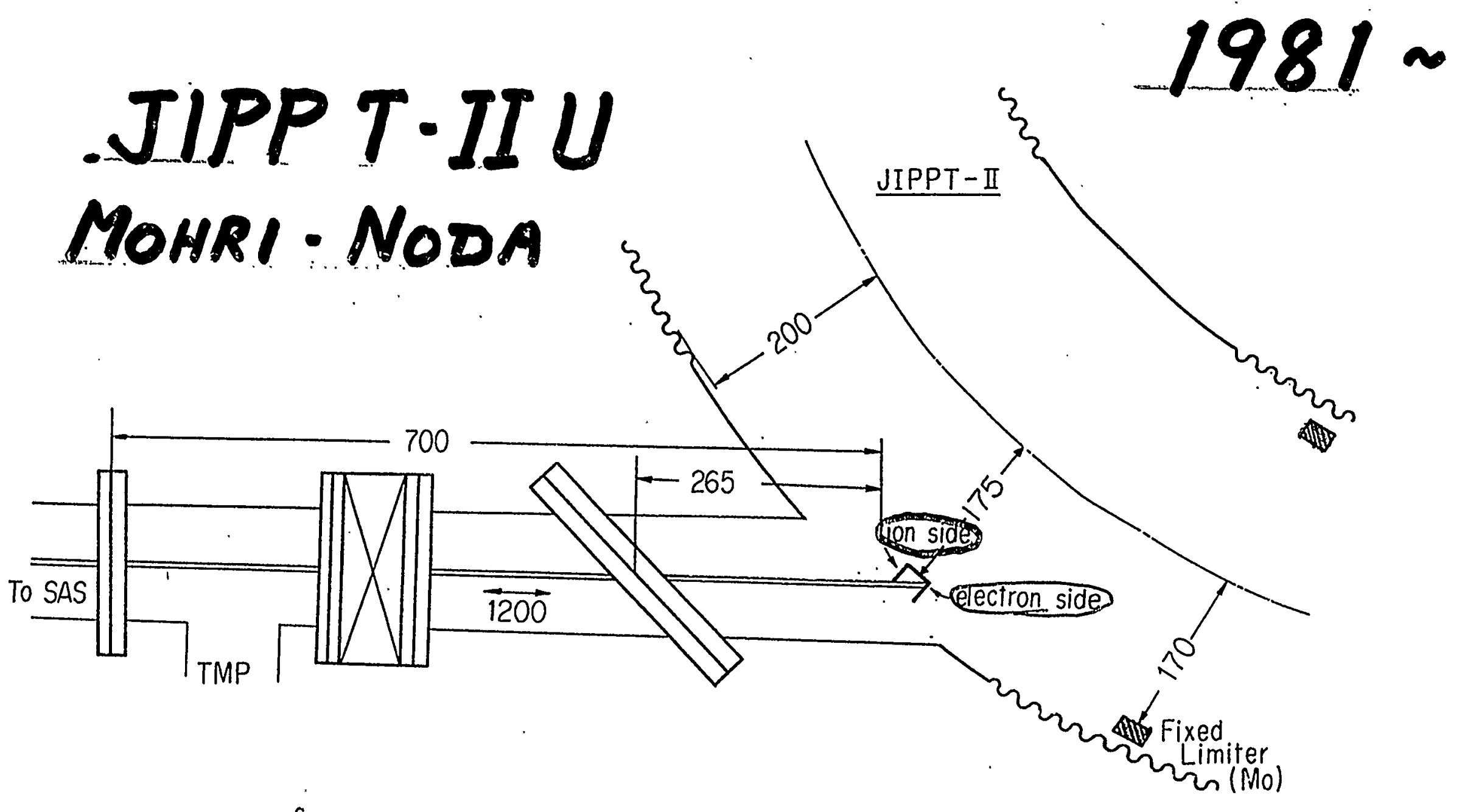

Geometry of the Sample Probe introduced into JipP T-II

思 

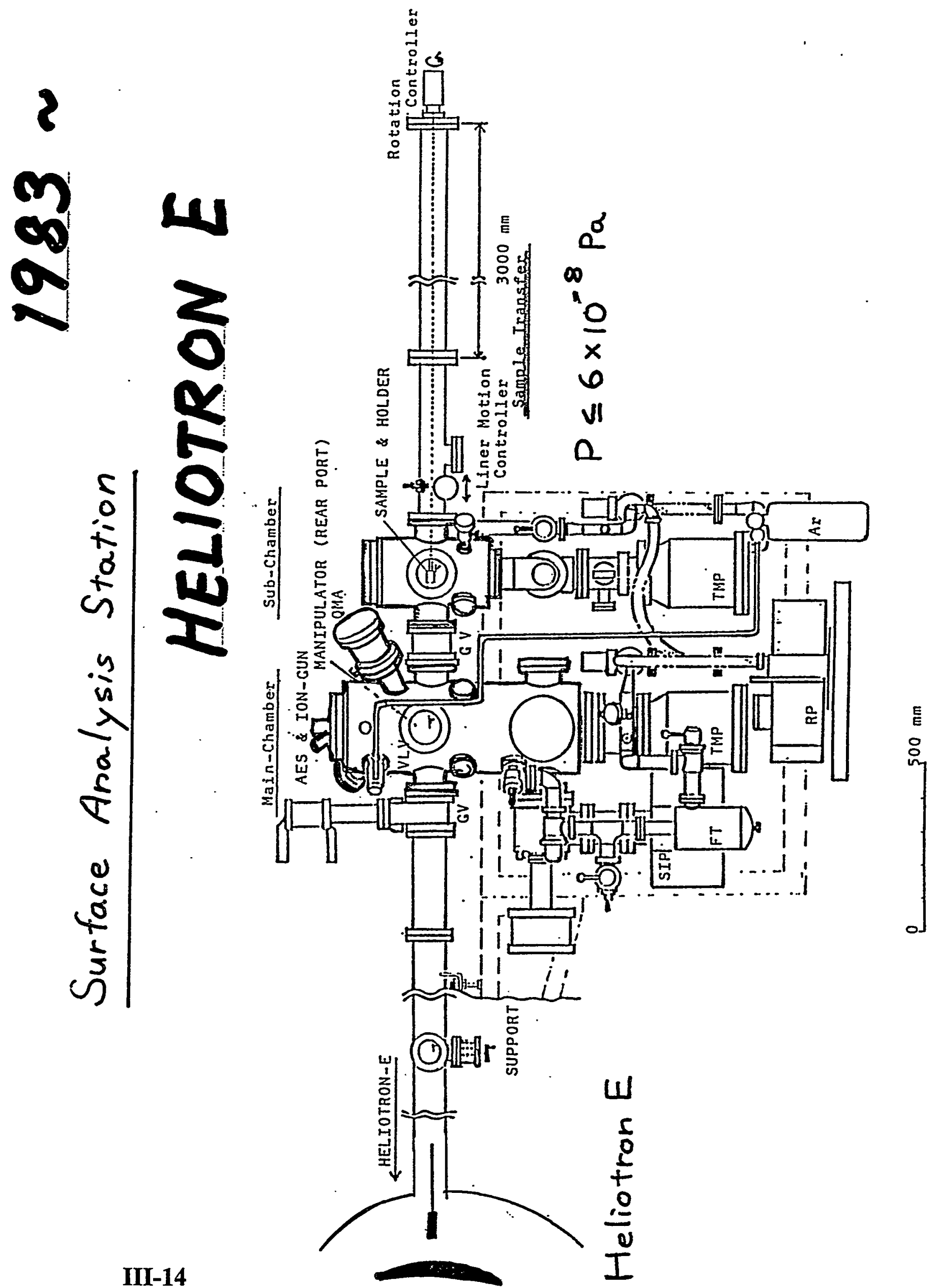


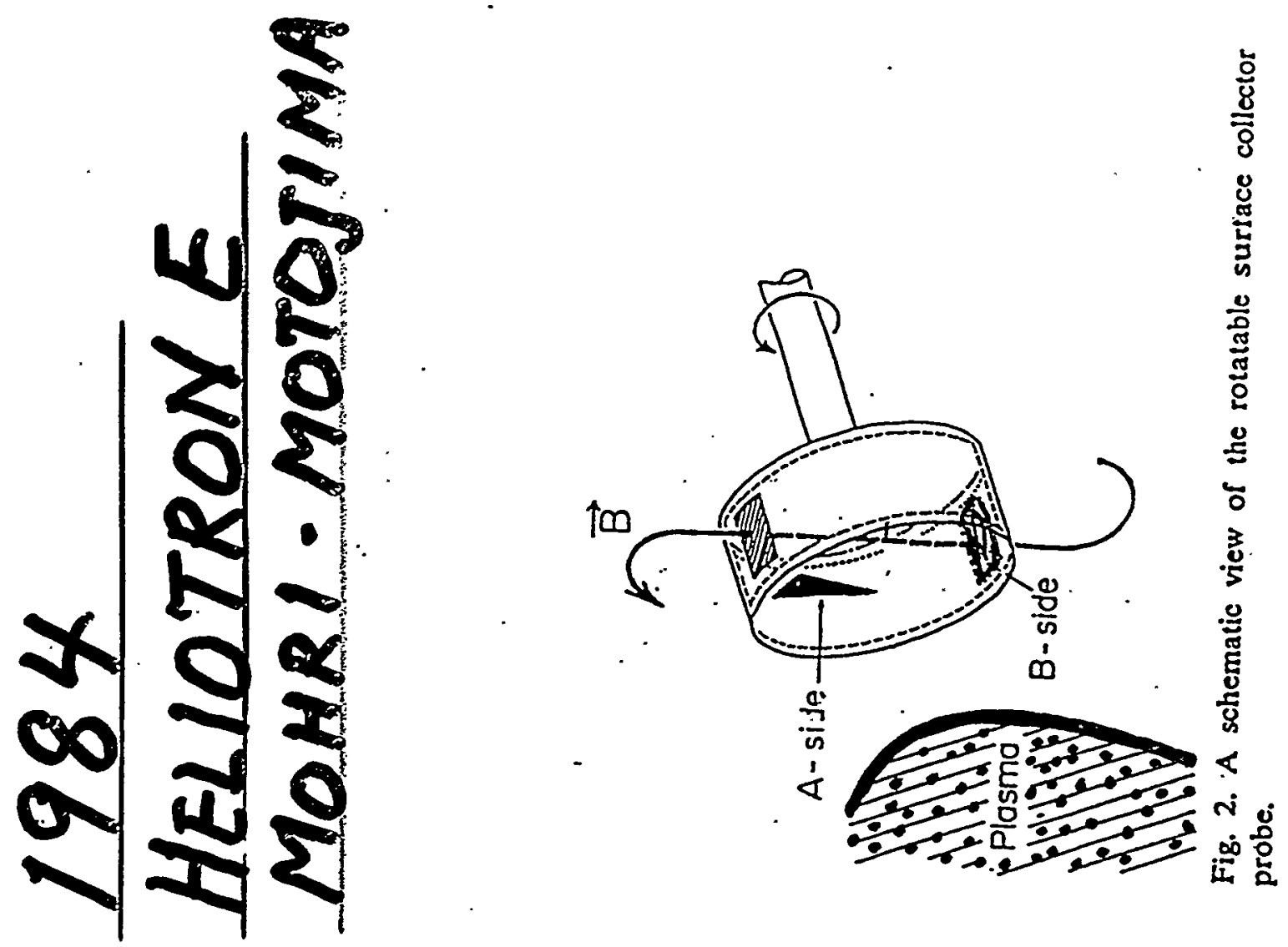

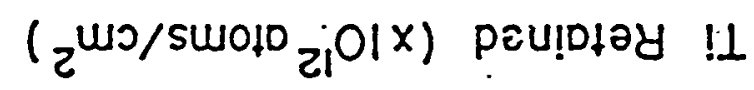

돌

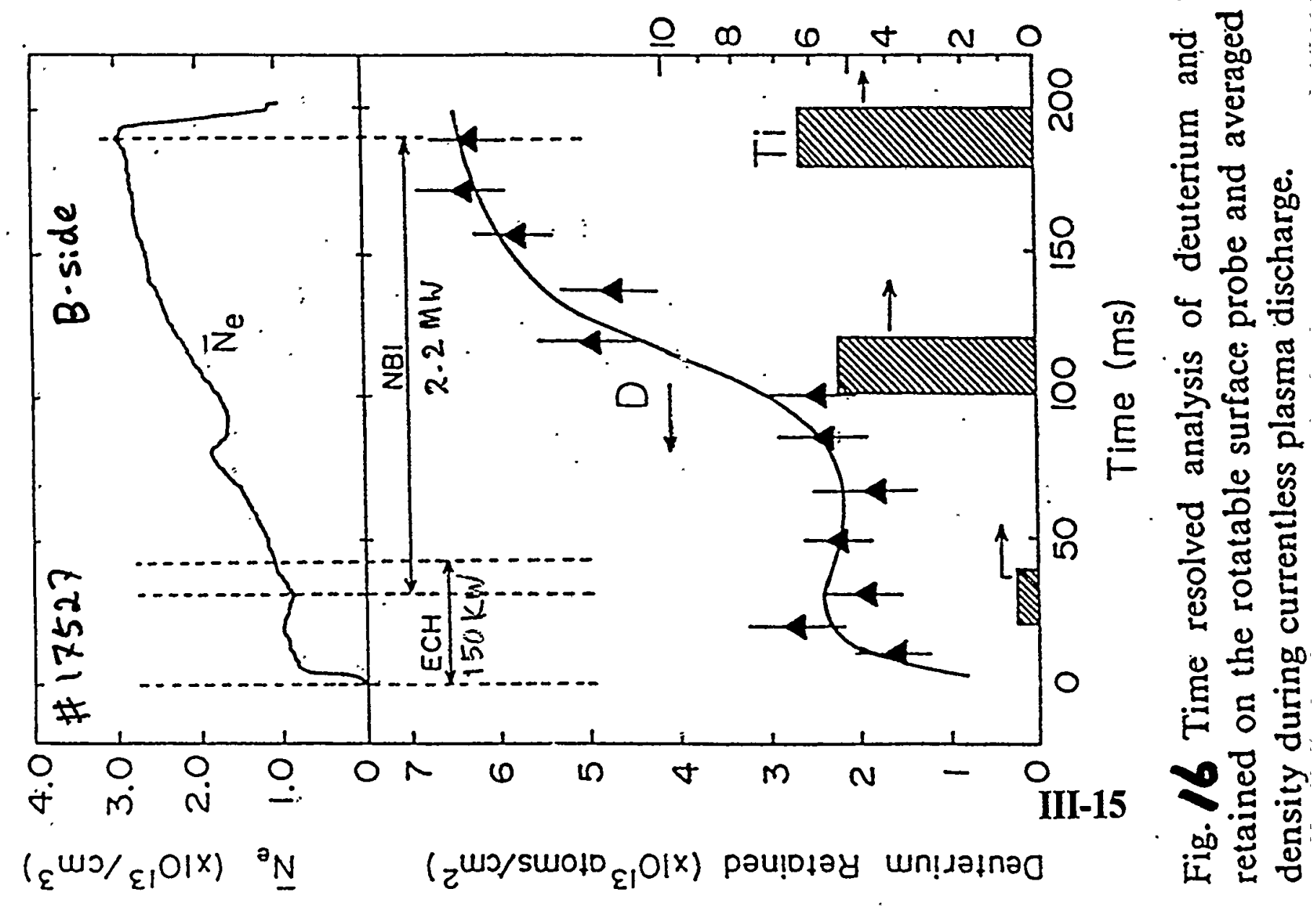




\section{DAMAGE ANALYSIS OF ARMOR / LIMITER TILE $O F$ \\ PLSMA MACHINES}

JIPP-TII (TiC LIMITER)

1982

D-III (SiC LIMITER)

1982

TEXTOR (GRAPHITE ALT-II)

1984

JT-60 (GRAPHITE ARMOR)

1990 


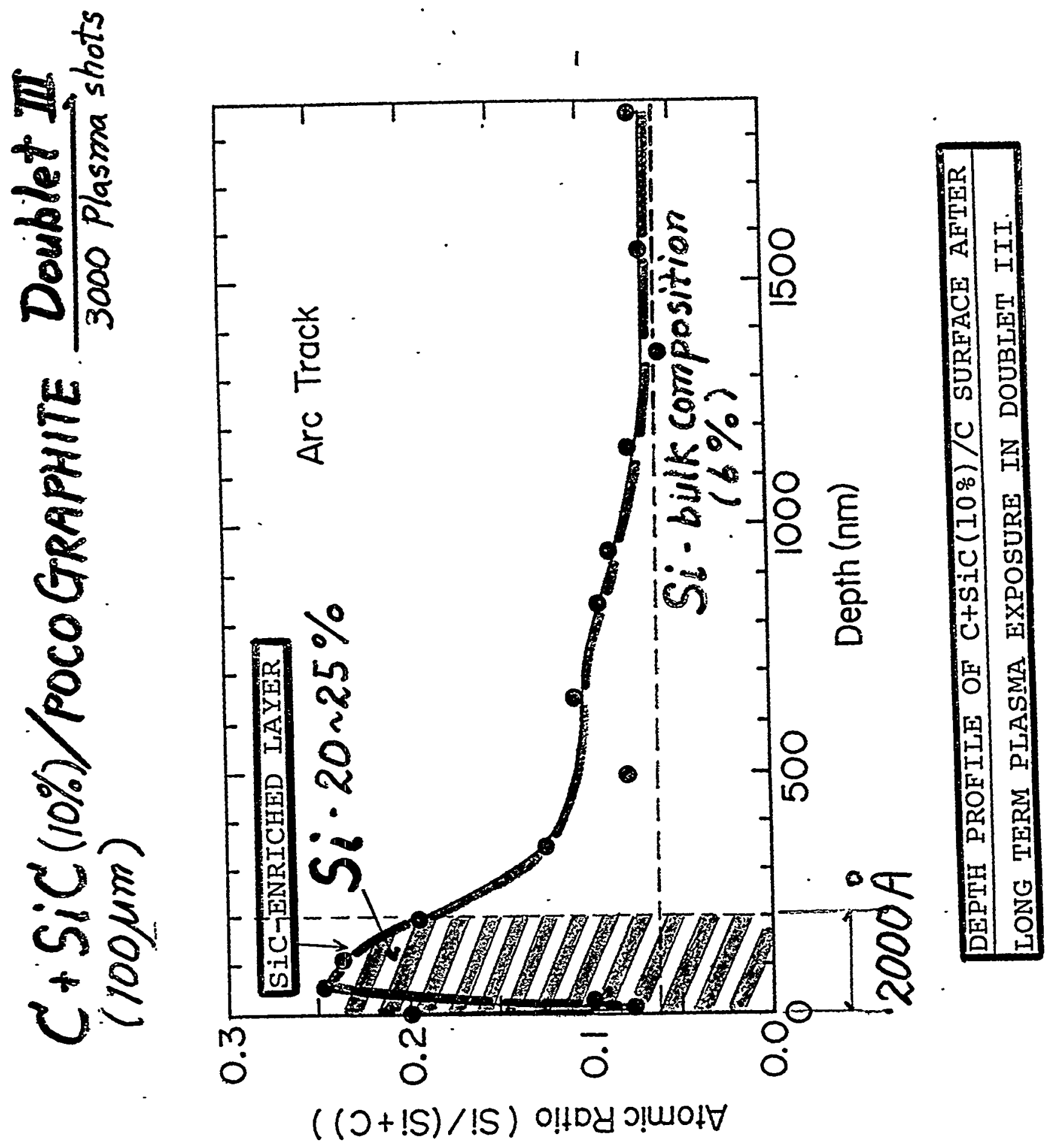


JT.60 1990 AKIBA 
OVERALL CHARACTERIZATIONS OF GRAPHITES AS FUSION FIRST WALL MATERIAL AND EVALUATION OF THE STABILITY AGAINST PLASMAS

INTERIM REPORT

by

Fusion First Wall Material Research Group, Nuclear Fusion Research Project,

The Ministry of Education,

Science and Culture, Japan

January 1989.

Edited by Toshiro Yamashina, Project Leader Hokkaido University, Sapporo, Japan 


\section{JAPANESE COMPANIES}

Table I Product names of graphite materials and the companies. ISOTROPIC GRAPHITES Low Derisity.

\begin{tabular}{|l|l|}
\hline \multicolumn{1}{|c|}{ Company name } & Product name of graphite \\
\hline Ibiden Co. & $\begin{array}{l}\text { T-6P, T-4MP, } \\
\text { ETP-10 }\end{array}$ \\
\hline Nippon Steel Chemical Co. & $\begin{array}{l}\text { 880, \#781 } \\
\text { Tokai Carbon Co. }\end{array}$ \\
\hline Toyo Carbon Co. & $\begin{array}{l}\text { AX650, KMT200K, } \\
\text { AX280K, YPD-K }\end{array}$ \\
\hline Toyo Tanso Co. & $\begin{array}{l}\text { ISO-880U, ISO-630U, } \\
\text { IG-110U }\end{array}$ \\
\hline Hitachi Chemical Co. & PDX-80S, PDX-60S \\
\hline Nippon Carbon Co. & EFG262, EFG301 \\
\hline
\end{tabular}




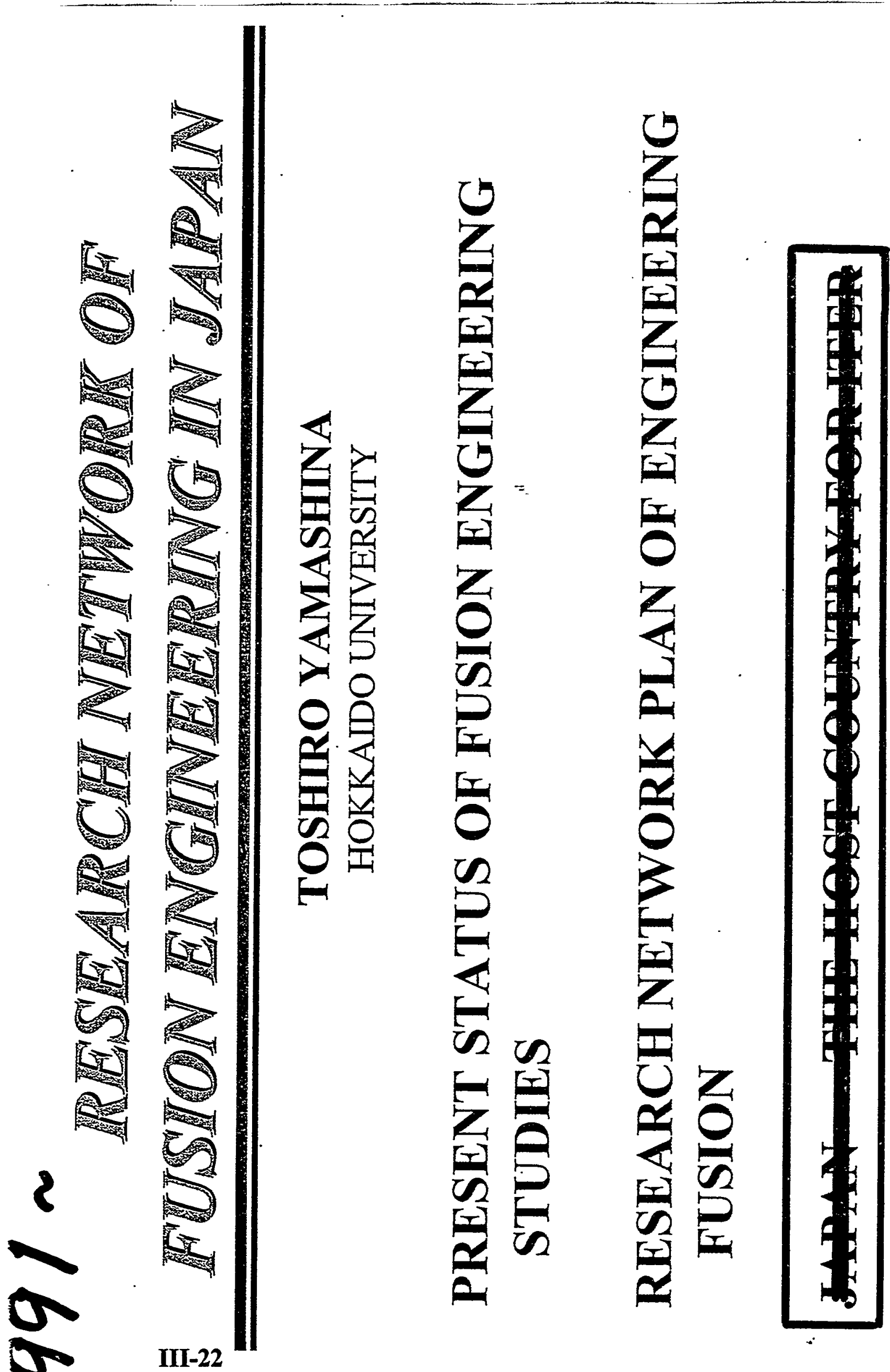




\section{NETWORK PLAN FUSION REACTO ENGINEERING}

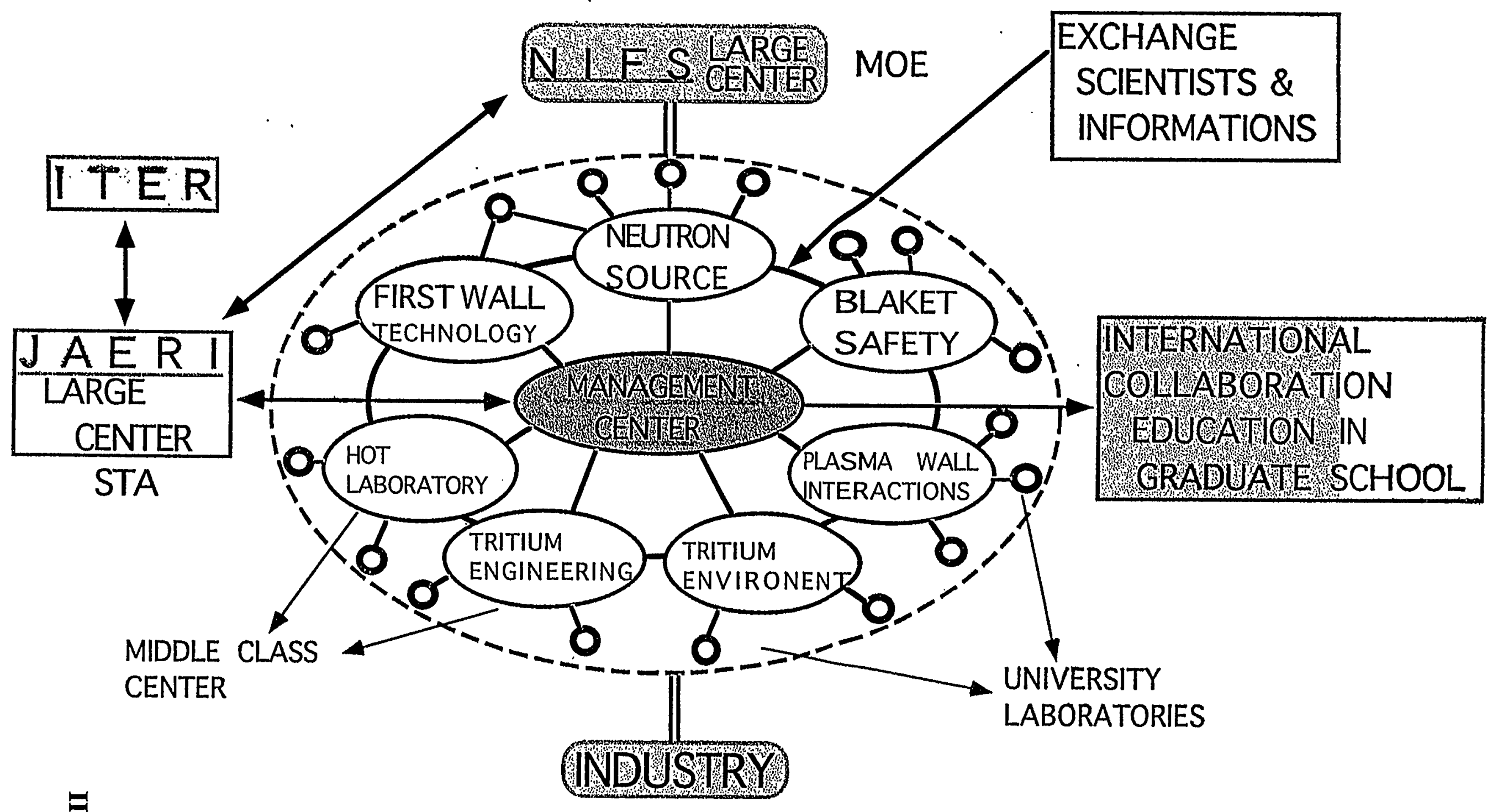




\section{$1981 \sim 1990$}

\section{EFFECTIVE UTILIZATION OF SURFACE ANALYSIS TECHNIQUES TO ENVIRONMENTS}

OUR SIDELINES

\section{MICROANALYTICAL STUDIES OF ENVIRONMENTS AND AIR POLLUTION CAUSED BY STUDDED TIRES OF AUTOMOBILES}

MAMORU MOHRI SUSUMU AMEMIYA SHIGERU MAEDA

SHIN FUKUDA SHIGEKI KATO

$M A S A O$ HASHIBA TOSHIRO YAMASHINA 

(昭和 57 年 12 月 27 日受理)

\title{
Microanalysis of Dust Particles from Road Surface Scraped off by Studded Tires of Automobiles —- Part 2
}

\section{Mamoru MoHri, Susumu Amemiya, Shigeru MaEdA, Shin FUKUDA, Shigeki Kato, Tohru SATAKE, Masao HASHIBA and Toshiro YaMASHINA \\ (Received December. 27, 1982)}

\begin{abstract}
Investigations of particulate substances originating from the use of studded tires of automobiles were performed. The amount of floating dust particles was measured by particle induced X-ray emission spectroscopy (PIXE) as a function of the horizontal distance from a road-edge and the vertical distance from the ground in the city of Sapporo. The results were compared with those of the city of Nagoya. It was found that the amount of floating dust particles in Sapporo was four to five times larger in November and April, while it was much less in February than that in Nagoya. The chemical composition of studs of studded tires and paint of road marking were analyzed by Auger electron spectroscopy (AES) and atomic absorption spectroscopy, respectively. Based upon these measurements the particulate substances collected from a road surface were examined and identified by use of scanning electron microscopy (SEM) and X-ráy microanalyzer (XMA). They could be attributed to mainly pieces of studs, paint from road markings and paving materials. Alveoli of the lungs of dogs and mice which inhaled such dust particles were also examined by SEM and XMA. Ferruginous components were found to segregate on the wall surface of the alveoli.
\end{abstract}


車粉じん分析に用いた：各社分析棫器

SEM (Scanning Electron Microscopy)

走查型電子显夏微叙竟

AES (Auger Electron Spectroscopy)

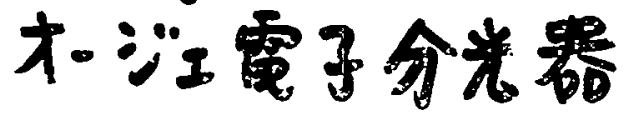

$X M A$ (X-ray Microprobe Analyzer)

X線マイタロアナうイザ-

TEM (Transmission Electron Microscopy)

透遏型電子影微镜

IMA (Ion Micro Probe Mass Analyjer)

イオンマイプアナライザー

PIXE (Particle Induced X-ray Emission) 䊉予励起 $x$ 楾玟出今光

IR (Infrared Spectroscopy)

赤外線合光

XPS (X-ray Photoelectron Spectroscopy) III-26 $x$ 楾光電子分光 


\section{Comparison of Dust Particle Amount}

(Sapporo and Nagoya)

1981 11月
0
500
1000
1500

1982 Feb.

$$
\text { 2月 }
$$

1982 Apr.

$$
\text { 4月 }
$$

1982 June

Amount of dust particles in air of Sapporo and Nagoya measured $\checkmark$ in defferent seasons. (by PIXE) 


\section{$1989 \sim$}

\section{INVITING PROJECT FOR ITER SITE \\ OF. CONSTRUCTION}

EAST AREA OF TOMAKOMAI

HOKKAIDO

(BIG) TOMATOH

- HUGE FLAT AREA (1000 ha)

- EASY ACCESS TO INTERNATIONAL

AIRPORT (SAPPORO) $15 \mathrm{~km}$

- REQUIED COOLANT AVAILABLE FACING TO SEASIDE

- ELECTRIC POWER ABAILABLE $635 \mathrm{MWe}$ :

-MILD WEATHER AND BEAUTIFUL RESORTS 
Session IV: Wall Conditioning, Sputter, Erosion

IV-1 


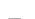




\title{
Wall Conditioning at the Start up Phase of LHD
}

\author{
Akio Sagara \\ National Institute for Fusion Science, Toki 509-52, Japan
}

The first cycle of the LHD plasma operation is scheduled to set off from the end of March, 1998. At this first stage the magnetic flux density at the plasma axis $\mathrm{B}_{0}$ is $1.5 \mathrm{~T}$, and the plasma heating with $84 \mathrm{GHz}$ ECH of the total input power over $0.5 \mathrm{MW}$ is arranged, which is operated using the second harmonics with the cut-off plasma density of $8.8 \times 10^{19} \mathrm{~m}^{-3}$.

According to the estimate of radiation loss using the 1-D time-dependent transport code under the first operation condition [1], it is required for the oxygen concentration in the hydrogen plasma to be less than $1.8 \%$, that is, the Zeff lower than 2 in order to obtain the line averaged plasma density $\left\langle n_{\mathrm{e}}\right\rangle$ of ${ }^{-}$about $2 \times 10^{1} 9_{\mathrm{m}^{-3}}$ with the plasma temperature $\mathrm{Te}(0)$ higher than $1.5 \mathrm{keV}$.

On the other hand, according to the results observed in the wall conditioning procedure performed in the start up phase of CHS [2], ECR discharge cleaning using hydrogen was effective to reduce down partial pressures of $\mathrm{H}_{2} \mathrm{O}, \mathrm{CO}$ and $\mathrm{CH}_{4}$, resulting in suppression of uncontrollable density rise under ECH discharges by mainly reducing down oxygen impurities.

Reduction of oxygen impurities is therefore the main purpose of the wall conditioning at the start up phase of LHD. Suppression of hydrogen recycling is also necessary after conditioning with $\mathrm{H}_{2}$. Standing on this guide line, the main scenario of wall conditioning in LHD has been decided including arrangement of hardware required.

The $300 \mathrm{~kW}$ hot water utility is arranged for baking the vacuum vessel which is made of 316 stainless steel with the total surface area of $777 \mathrm{~m}^{2}$ and the total mass of $77.7 \mathrm{ton}$ including ports and bellows. However, this baking procedure is not sufficient, because the temperature is limited at the max. $100^{\circ} \mathrm{C}$ and there is only one week for baking scheduled before starting the SC coils cooling down.

The main wall conditioning method is the $20 \mathrm{~kW} \mathrm{ECR}(2.45 \mathrm{GHz})-\mathrm{DC}$ with $\mathrm{H}_{2}$. It is expected to take about a half day to evacuate adsorbed $\mathrm{H} 2 \mathrm{O}$ molecules, and to take at least . a few days to reduce oxide layers $[2,3]$. After this procedure, the main discharge is set off with $84 \mathrm{GHz} \mathrm{ECH}$, which is also considered as an effective conditioning of the wall surfaces, especially with repeated short pulse and high power operations of $10-20 \mathrm{~Hz}$ and $10-20 \%$ duty.

Titanium-gettering is arranged as one of backup methods, which covers the $30 \%$ area of $V / V$ and suppresses both of oxygen impurities and hydrogen recycling [4]. The film thickness of only 30 monolayers is sufficient.[5], and the total operation time is limited to avoid peeling off of Ti films thicker than $10 \mu \mathrm{m}$.

Glow discharge with $\mathrm{He}$ is also arranged as the other backup method to reduce oxide layers and to suppress hydrogen recycling after ECR-DC with $\mathrm{H}_{2}$. Based on intensive $R \& D$ results, boronization using glow discharge is scheduled to be put into operation from the 2'nd cycle in 1998.

References :

[1] H. Yamada, 13th meeting of J. Plasma and Fusion Research (1996) 22aD2, p187.

[2] N. Noda, Annual Review of IPP Nagoya University (April, 1988 - May, 1989) p9.

[3] E. Jotaki and S. Itoh, Fusion Eng. Design, 36 (1997) 447.

[4] A. Sagara, J. Nucl. Mater., 93\&94(1980) 847.

[5] S. Besshou and O. Motojima, Annual Review of PPL Kyoto Univ., (1981) p64. 


\section{Wall Conditioning at the Start up Phase of LHD}

Akio Sagara

National Institute for Fusion Science, Japan

\section{The first cycle of the LHD plasma starts from the end of March, 1998}

At the operation condition with

$\checkmark$ the magnetic flux density $B_{0}<1.5 \mathrm{~T}$

$\checkmark$ the plasma heating with $84 \mathrm{GHz}$ ECH

- the total input power $>0.5 \mathrm{MW}$

This condition_requires the_oxygen_concentration less than $1.8 \%$, that is, Zeff $<2$

$\checkmark$ to obtain $\left\langle\mathrm{n}_{\mathrm{e}}>\sim 2 \times 1019 \mathrm{~m}-3\right.$

$\downarrow$ with $\mathrm{Te}(0)>1.5 \mathrm{keV}$ 
Wall Conditioning at the Start up Phase of LHD

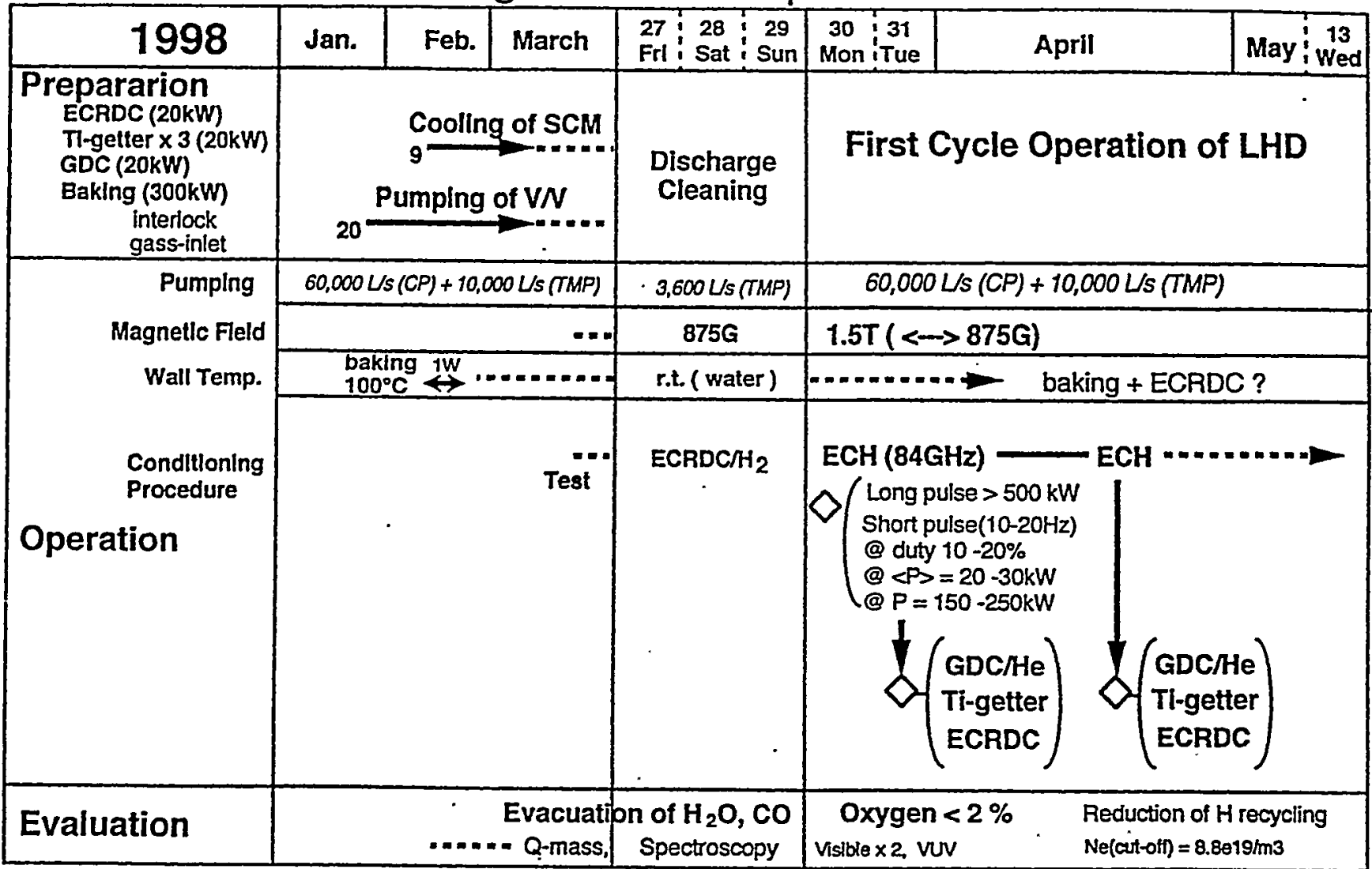

1997.12.3 A.S
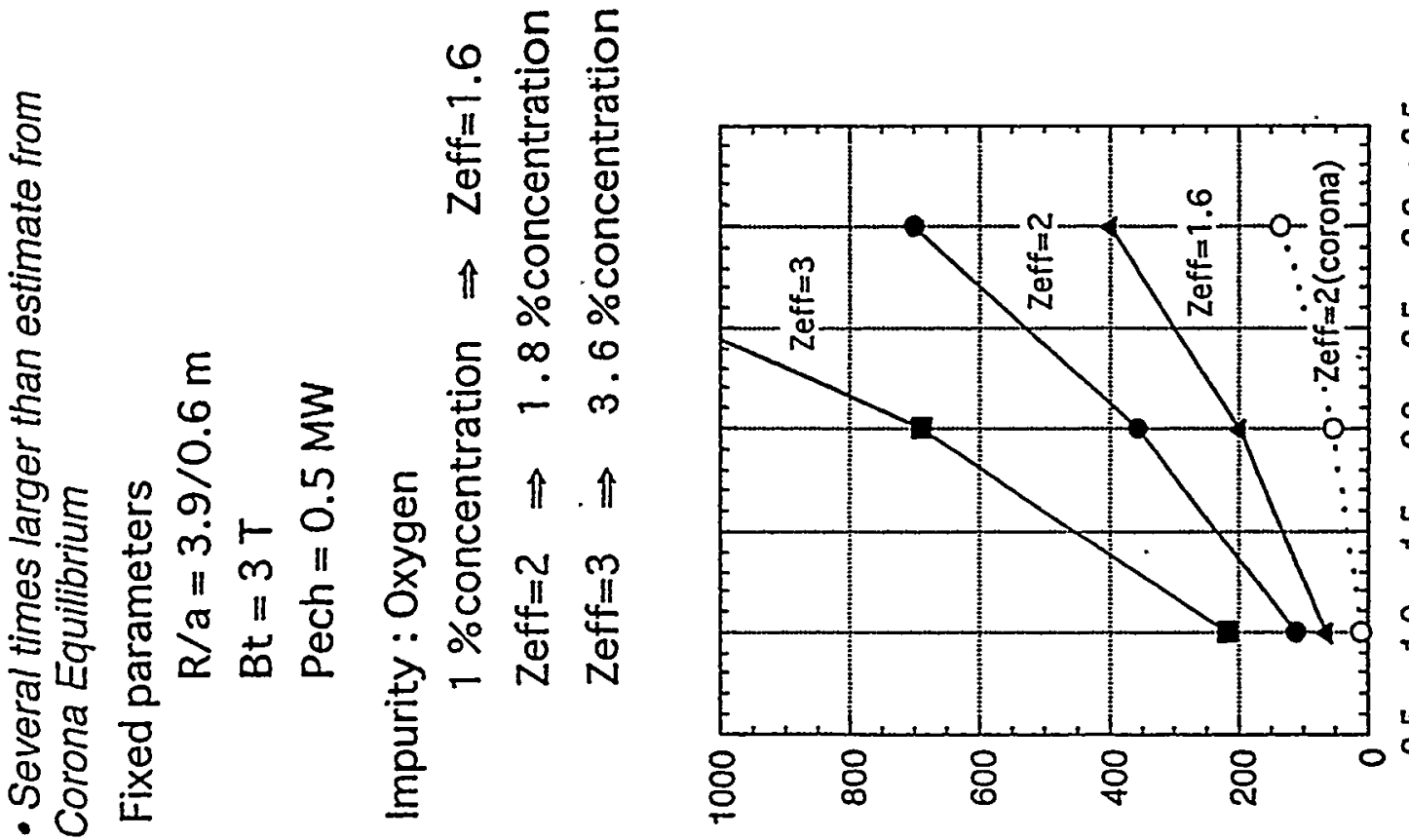

$\stackrel{L}{\circ}$

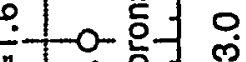

웅

นึ

क

우은

흔

눈

울

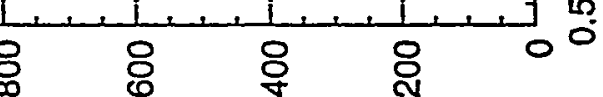

(MY) دәMOd uoṇe!̣pey 


\section{Reduction of oxygen impurities}

\section{is the main purpose \\ of the wall conditioning \\ at the start up phase of LHD}

After pumped down to the range of 10-8 Torr,

the $300 \mathrm{~kW}$ hot water is used for baking the V/N

- made of 316 stainless steel

- the total surface area of $777 \mathrm{~m}^{2}$

the total mass of 77.7 ton

However, this baking procedure is not sufficient, because the temperature is limited at the max. $100^{\circ} \mathrm{C}$ and there is not enough time before the first cycle.

\section{The main wall conditioning method is ECR-DC with $\mathrm{H}_{2}$.}

\subsection{GHz ECR}

$\rightarrow$ input power of $20 \mathrm{~kW}$ $(\sim 50 \mathrm{~W} / \mathrm{m} 2)$

- Remote control

- Water-cooled window

It takes about 10 20h to evacuate adsorbed $\mathrm{H}_{2} \mathrm{O}$. It takes at least a few days to reduce oxide layers. (cf. CHS, JIPP T-IIU, TRIAM-IM)
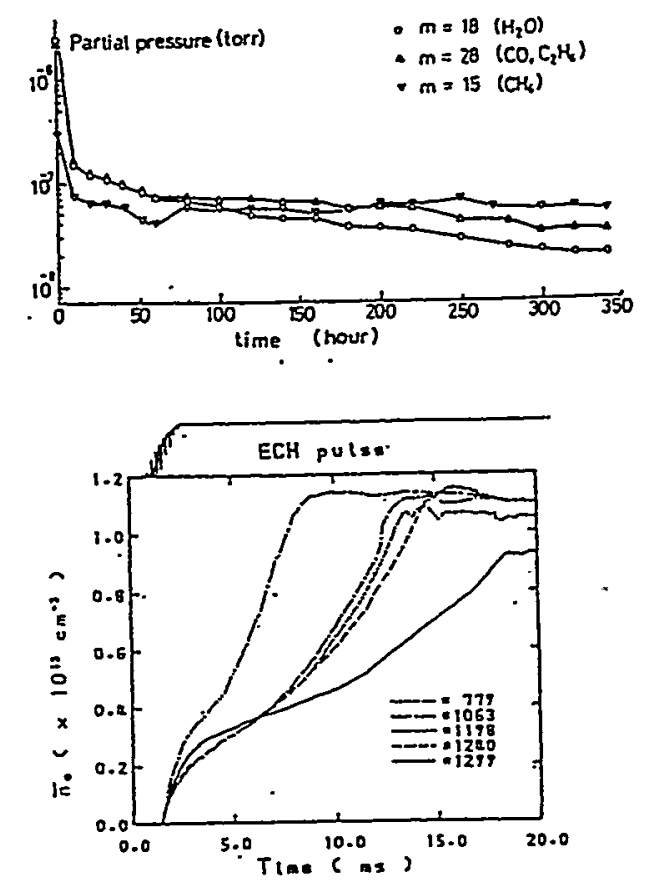

ECR-DC in CHS, 1988, N.Noda 

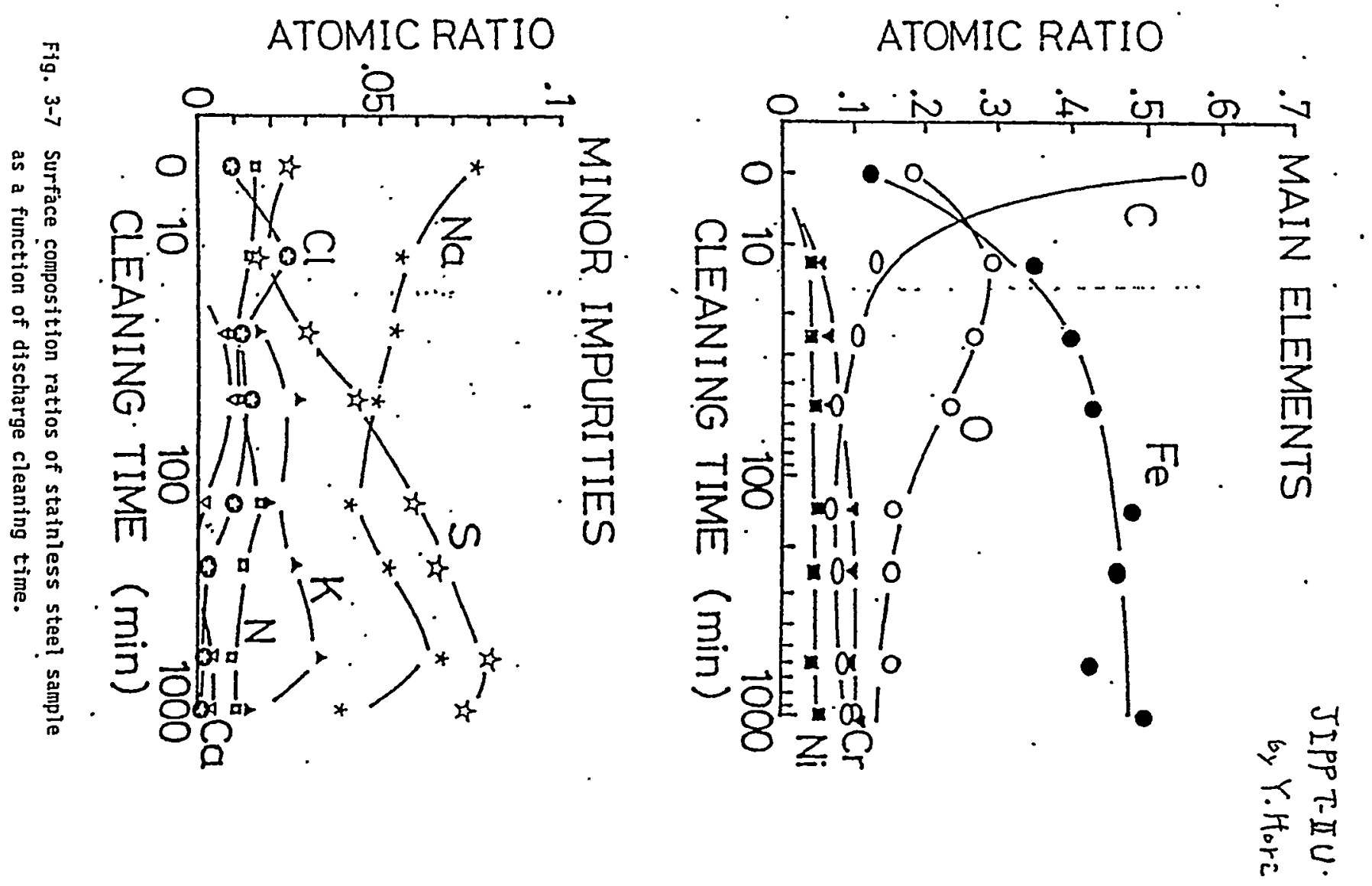

TRIAM-1M E. Jotaki, S. Iloh / Fusion Engineering and Design 36 (1997) 447-450

449
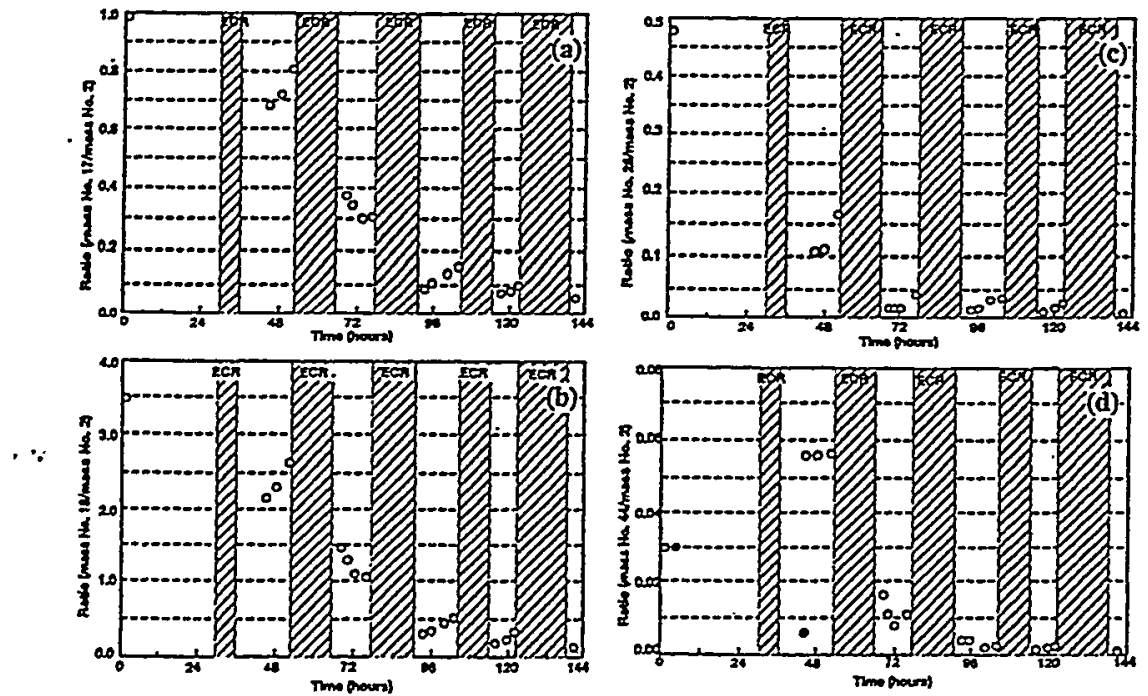

Fig. 2. Dependencies of impurity components on duration of ECR-DC. (a) $\mathrm{OH}$; (b) $\mathrm{H}_{2} \mathrm{O}$; (c) $\mathrm{CO}$ and (d) $\mathrm{CO}_{2}$. Shaded intervals indicate the periods of ECR-DC. The three impurities $a, b$ and $c$ exhibit a similar trend in reduction. However, the tendency for d $\left(\mathrm{CO}_{2}\right)$ differed slightly in that the quantity increased with initial ECR-DC, and thereafter declined in a manner similar to the other impurities. In these graphs, the open circles indicate data after rebuilding, and the closed circles show data from ordinary experimental terms before rebuilding. 


\section{The main $84 \mathrm{GHz} \mathrm{ECH}$ discharges will be effective for reducing oxide layers.}

Especially with repeated short pulse and high power operations

$\checkmark 10-20 \mathrm{~Hz}$

- duty $10-20 \%$

$\checkmark P>=20-30 \mathrm{~kW}$

$\rightarrow P=150-250 \mathrm{~kW}$

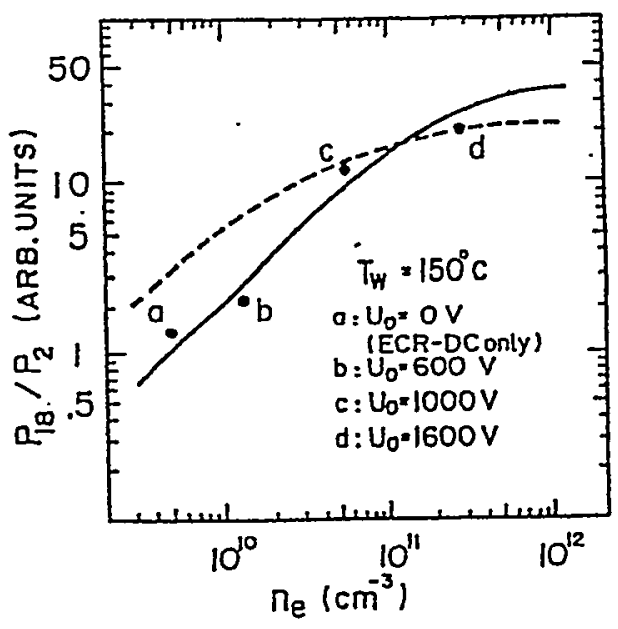

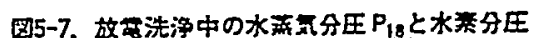

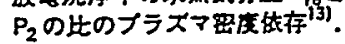

$P_{18} / P_{2}$ vs. $n_{e}$ under DC, '90, N. Noda

\section{Titanium-gettering is arranged as backup to suppress $O$ impurities}

with 3 sets in every $120^{\circ}$, and movable in $50 \mathrm{~cm}$

$-\max .1 \mathrm{~h}$ flashing at $\mathrm{B}=0$

$-\sim 30 \%$ coverage of $\mathrm{V} N$

- Ti films > 30 monolayers

In order to avoid peeling off of Ti films thicker than $10 \mu \mathrm{m}$, - Total operation $<30 \mathrm{hrs}$

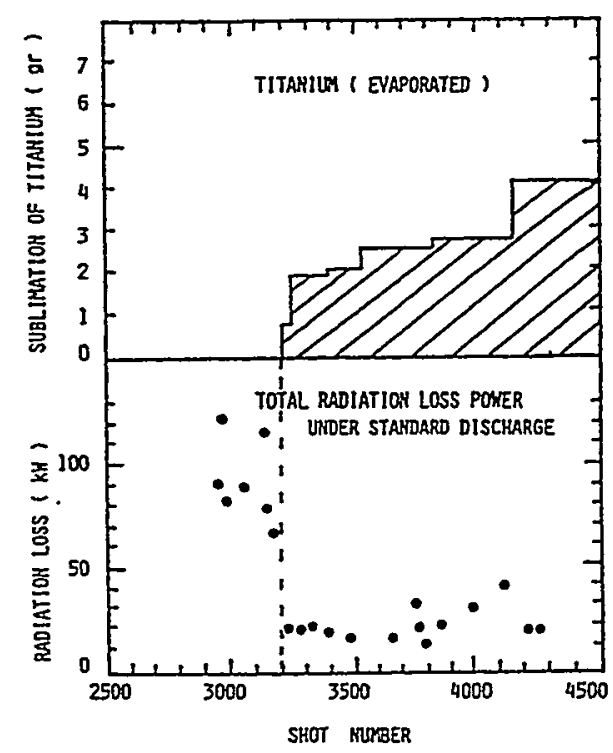

(Heliotron-E, '81) 19, 30\%, 30layers 

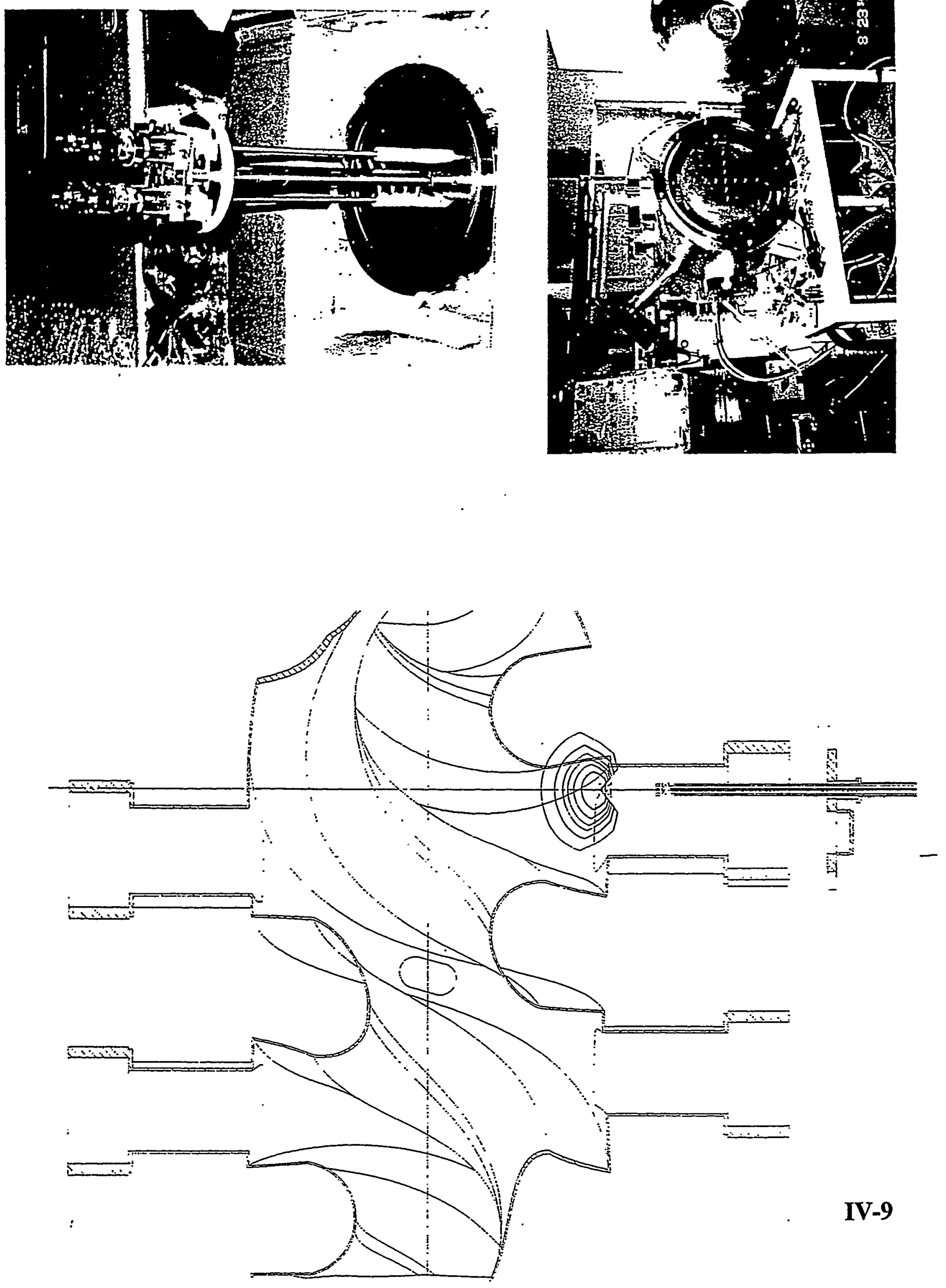


\section{Glow discharge with $\mathrm{He}$ is arranged as the other backup method \\ to reduce $\mathrm{H}$ recycling after ECR-DC}

- One electrode for the 1'st cycle

( 3 electrodes from the 2 'nd cycle)

- Graphite head under inertia cooling

- Boronization using glow discharge is scheduled to be put into operation after the 2'nd cycle in 1998.

\section{Conclusion}

(1) Reduction of $O$ impurities is the main purpose.

(2) Baking at $100^{\circ} \mathrm{C}$ with $300 \mathrm{~kW}$ hot water is arranged.

(3) ECR-DC with $\mathrm{H}_{2}$ is mainly used to evacuate $\mathrm{H}_{2} \mathrm{O}$ in a half day, and to reduce oxide in a few days.

(4) The main $84 \mathrm{GHz} \mathrm{ECH}$ is also effective.

(5) Ti-gettering and G-DC/He are arranged as backup.

(6) Boronization is scheduled after the 2 'nd cycle. 


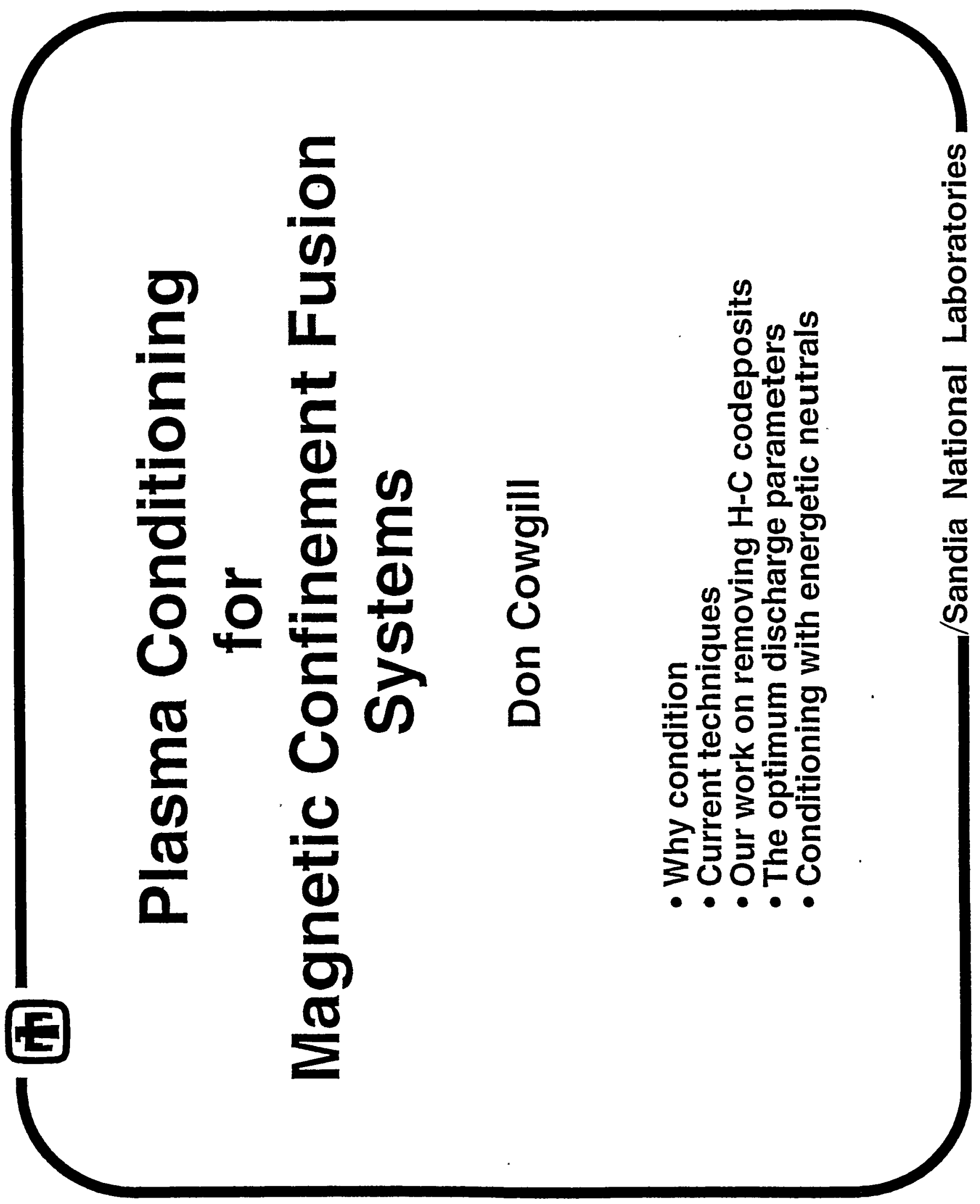

IV-11 


\section{Why is Conditioning Needed?}

- To reduce plasma contamination by minimizing impurity influx from walls and plasma-facing components.

- Surfaces contain hundreds of monolayers of volatile gases (Graphite tiles have huge surface areas: $1 \mathrm{~m}^{2} / \mathrm{gm}$ )

- Particularly important: O-bearing contaminants (water)

- To reduce $\mathrm{H}$-recycling during plasma startup, needed for reliable density control.

- Particularly important for systems with graphite tiles (Hydrogen codeposits with sputtered carbon at $0.4 \mathrm{H} / \mathrm{C}$ )

- To control in-vessel tritium inventory.

- Particularly important for systems with graphite tiles 


\section{Co-Deposition is Expected to be a Major Source of In-Vessel Tritium Inventory}

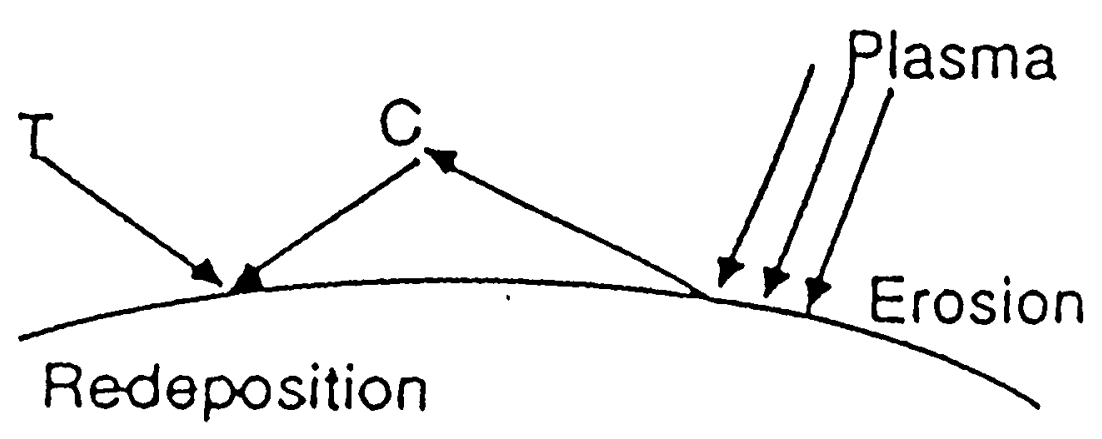

- Tritium concentration $\sim 0.4 \mathrm{~T} / \mathrm{C}$ expected in a DT device

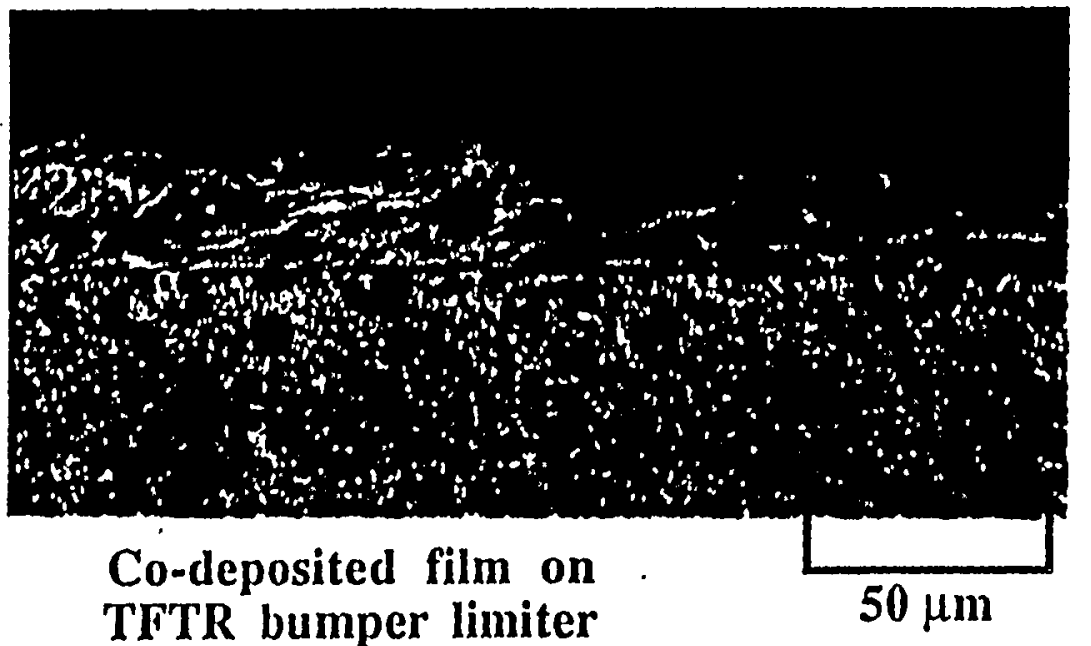

- The thickness of the co-deposited layer increases monotonically with discharge time

- Carbon erosion from high flux areas results in redeposition of carbon along with tritium

TFTR bumper limiter

$50 \mu \mathrm{m}$ 
- Prebaking of invessel materials

- Vacuum bakeout (Outgassing of graphite requires $\mathrm{T}>1000^{\circ} \mathrm{C}$ )

- Operation at elevated temperatures

- Active contaminant gettering with $\mathrm{B}, \mathrm{Be}, \mathrm{Li}, \mathrm{Si}$, etc.

- Discharge conditioning: GDC, TDC, PDC, DDC (H, He)

\begin{tabular}{|l|l|l|}
\hline Machine & Op. Temp. & Conditioning Discharges \\
\hline JET & $300^{\circ} \mathrm{C}$ & GDC, He PDC \\
Tore-Supra & $190^{\circ} \mathrm{C}$ & \\
TFTR & RT & GDC, TDC, He PDC, DDC \\
DIII-D & RT & GDC \\
JT-60 & $250-300^{\circ} \mathrm{C}$ & GDC, He PDC \\
ASDEX-U & RT & GDC, He TDC \\
\hline
\end{tabular}




\section{Needs for Future / Current Machines}

-Tore-Supra: Long pulse, steady-state B-field

- Improved conditioning for impurity control

- New field-on techniques

- LHD: Not bakeable, steady-state B-field

- New field-on techniques

- International Cooperation

- NSTX: Graphite, low bakeout temperature, inertial cooling

- Improved conditioning for impurity and density control

- Have started discussions with PPPL

- JET: High near-surface D and T inventories

- Long conditioning treatments required to keep neutron radiation at acceptible levels

- More rapid/frequent conditioning methods needed 


\section{Glow discharges are used to remove surface impurities}

- $\mathrm{H}(\mathrm{D})$ volatilizes hydrocarbons to methane $\left(\mathrm{C}_{\mathrm{x}} \mathrm{H}_{\mathrm{y}}+\mathrm{H} \Rightarrow \mathrm{CH}_{4}\right)$ and weakly-bound oxides to water $\left(\mathrm{M}_{\mathrm{x}} \mathrm{O}_{\mathrm{y}}+\mathrm{H} \Rightarrow \mathrm{M}+\mathrm{H}_{2} \mathrm{O}\right)$

- He desorbs surface and near-surface $\mathrm{H}$

$(300 \mathrm{eV}$ He range $\approx 3.6 \mathrm{~nm}$ in $\mathrm{C}, 4.5 \mathrm{~nm}$ in $\mathrm{Be}$ )

- $\mathrm{O}$ agressively removes hydrocarbons, volatilizing $\mathrm{C}$ to $\mathrm{CO}$

- He-O rapidly removes thick a-C:H codeposits

\section{We studied the He-O erosion process}

- To optimize discharge parameters for rapid, efficient conditioning,

while minimizing resultant $\mathrm{O}$-contamination

- To identify the best method for use in ITER (steady-state, high B-field) 


\section{Our investigations showed He-O GDC removes $\mathrm{C}$-H codeposits}

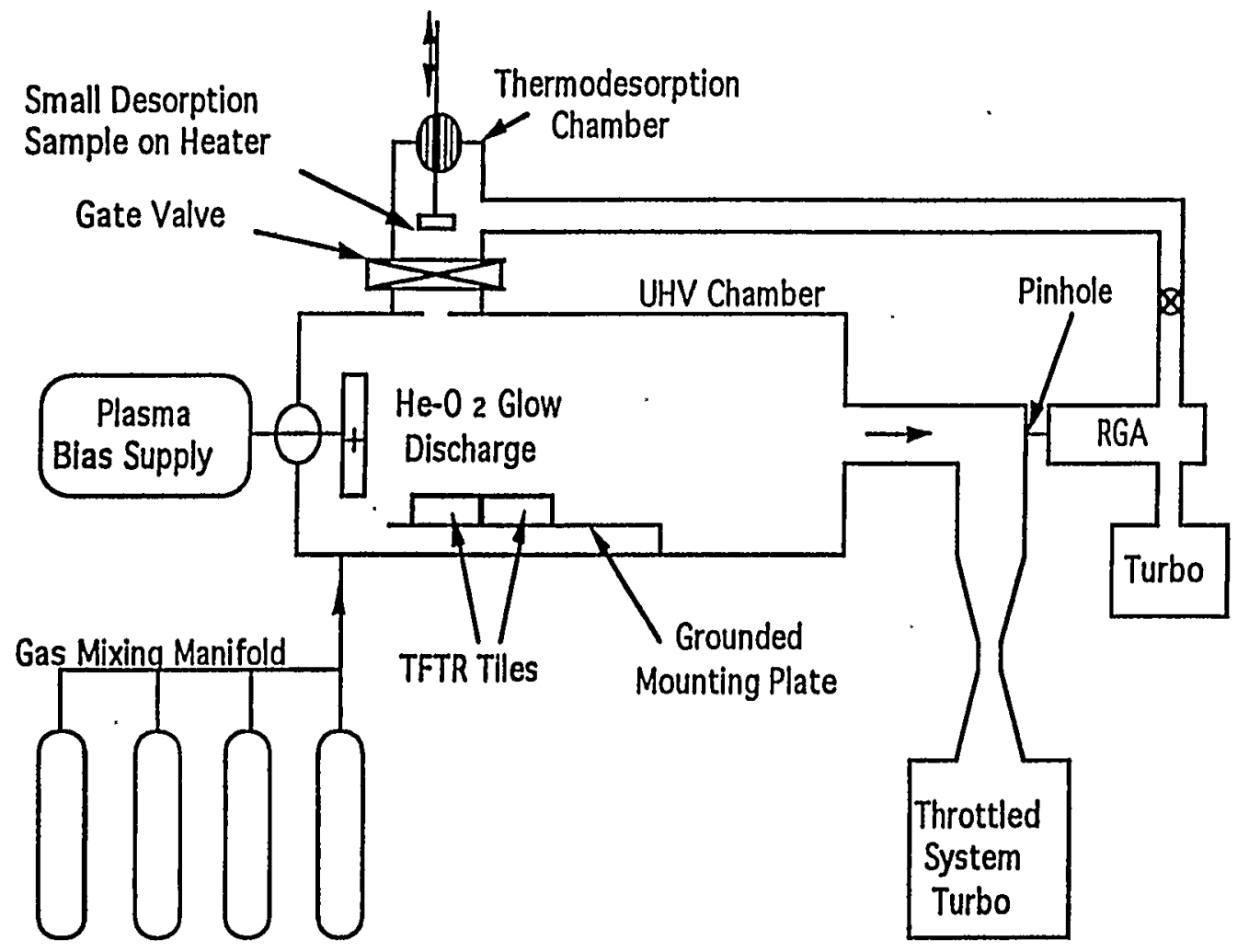

- Codeposit erosion rate $\sim 0.3 \mu / \mathrm{hr}$

- Efficient removal from gap between tiles

- Surfaces roughened

- O-contamination of tiles 


\section{He-Oxygen Glow Discharges Removed Codeposits from TFTR Tiles}

\section{Cross-section of Codeposit}

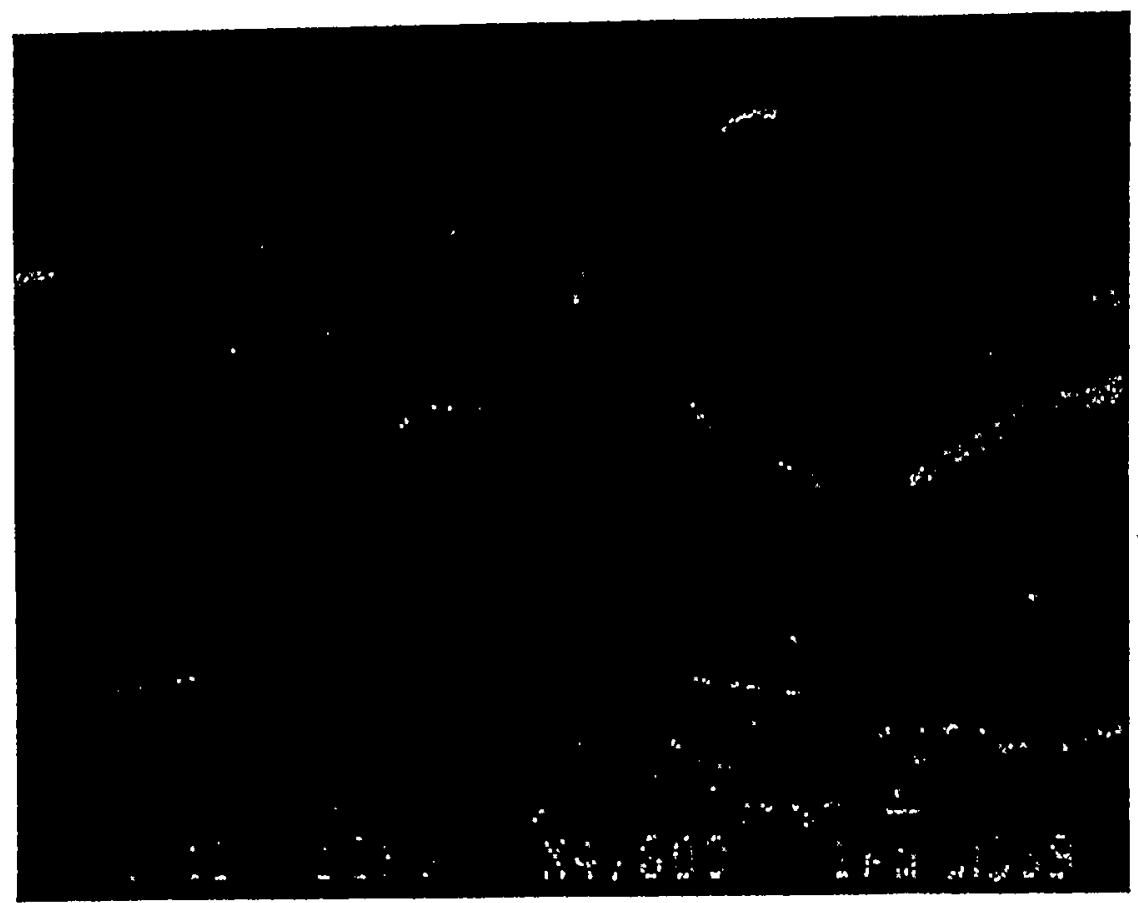

- Erosion is ion-induced:

- Occurs normal to plasma sheath

- Surfaces become highly textured

- Average codeposit erosion rate:

$\approx .07 \mu \mathrm{m} / \mathrm{hr}$ from tiles

$\approx .25 \mu \mathrm{m} / \mathrm{hr}$ from lab. codeposits 


\section{We investigated the mechanisms of codeposit erosion by He-O GDC}
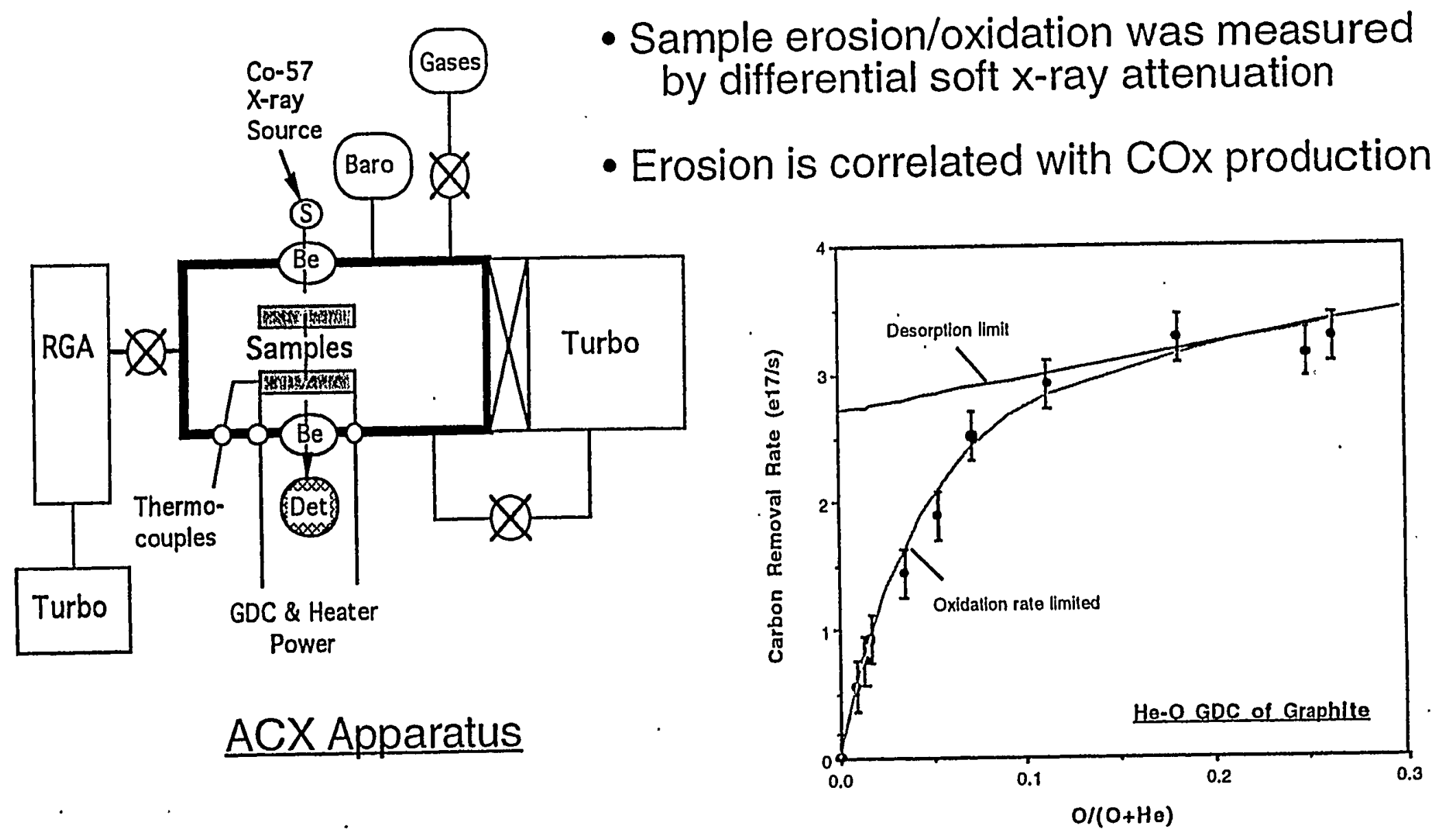


\section{The experiments also showed how bulk O-Contamination can be reduced}

He-O GDC Exhaust Gases

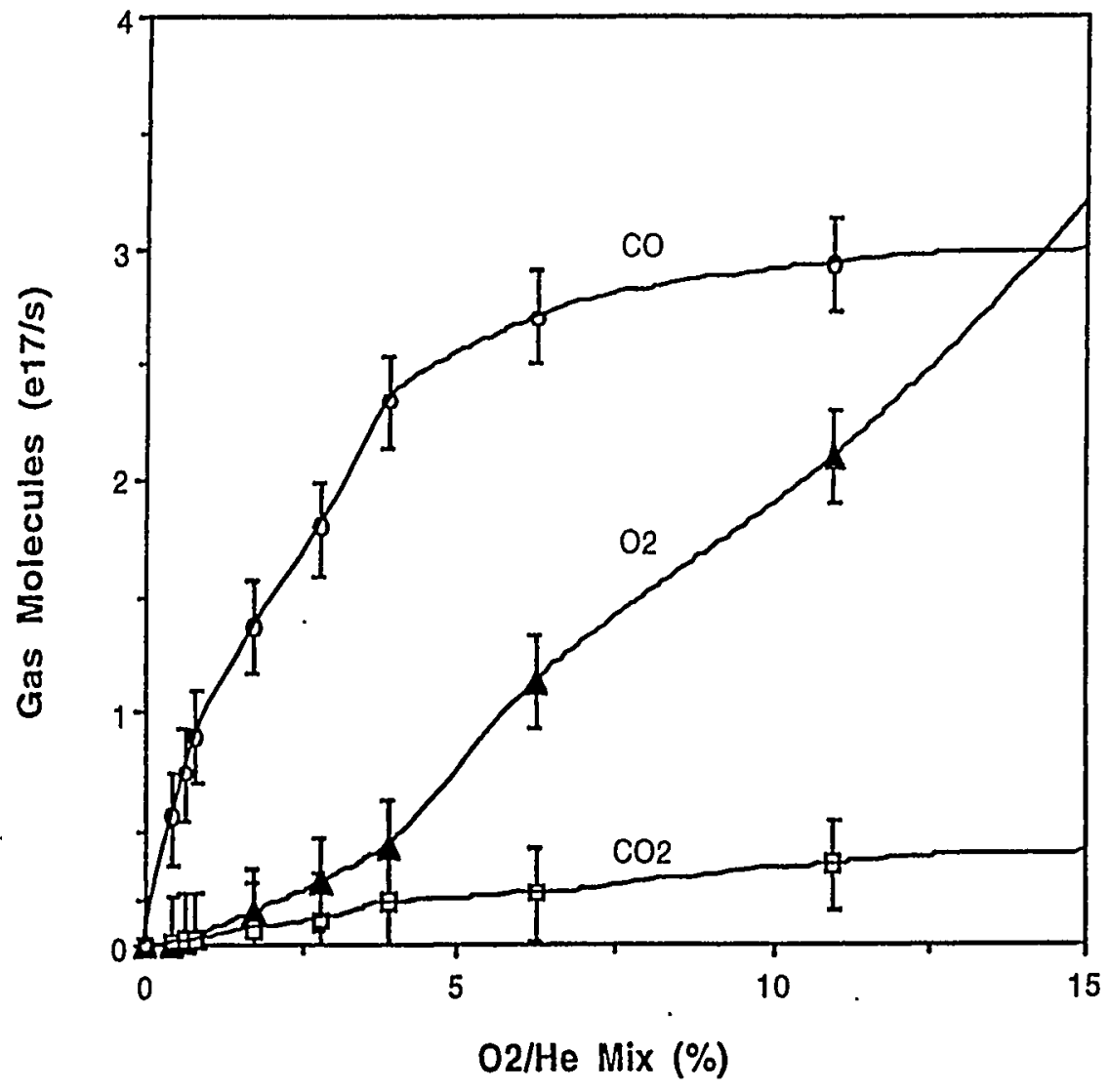

- Input oxygen is rapidly converted to $\mathrm{CO}$ and $\mathrm{CO}_{2}$

- Conversion is even more efficient for Ar-O GDC

- For low oxygen concentration, little molecular $\mathrm{O}_{2}$ remains to permeate into graphite pores. 


\section{Summary of RG-O GDC observations:}

- Slow pumping (long CO residence time) causes $\mathrm{C}$ redeposition and $\mathrm{O}$ reuse.

- In desorption limit, a-C:H codeposits are eroded $\sim 3 \mathrm{x}$ more rapidly than $\mathrm{C}$.

(HCO can be used for discrimination)

- Desorption of $\mathrm{CO}$ increases with impact energy up to few $\mathrm{kV}$.

At $400 \mathrm{eV} \mathrm{He}$, desorption yield $\approx 20 \mathrm{CO} / \mathrm{He}$.

- Erosion is normal to plasma sheath: Shielded surfaces are not conditioned.

Graphite surface becomes textured.

- Observed erosion rates at $2 \mathrm{~mA} / \mathrm{cm}^{2}$, and $>90 \%$ oxygen-use efficiency:

Total carbon etch rate Graphite surface erosion - $\frac{4 \% \mathrm{O} / \mathrm{He} \mathrm{GDC}}{5.2 \times 10^{15} \mathrm{C}^{-\mathrm{cm}^{2}-\mathrm{s}}}$

$1.4 \AA / \mathrm{s}$ $\frac{12 \% \mathrm{O} / \mathrm{Ar} \mathrm{GDC}}{9.5 \times 10^{15} \mathrm{C} / \mathrm{cm}^{2}-\mathrm{s}}$

$2.6 \AA / s$

If texturing is eliminated, overnight conditioning at these rates will remove 6-10 microns of codeposit. 
- Particle impact energies of few hundred eV

- for large desorption yield.

- Particle impact at random angles

- for large yield, good depth, and no texturing.

- Low electron energies (<10 eV)

- for minimal ionization or dissociation of desorbed impurity gases.

- Low background gas pressure $\left(<10^{-5}\right.$ torr),

- for rapid evacuation and low gas throughput. 


\section{He discharges can produce large energetic $\mathrm{He}^{\circ}$ fluxes by charge exchange}

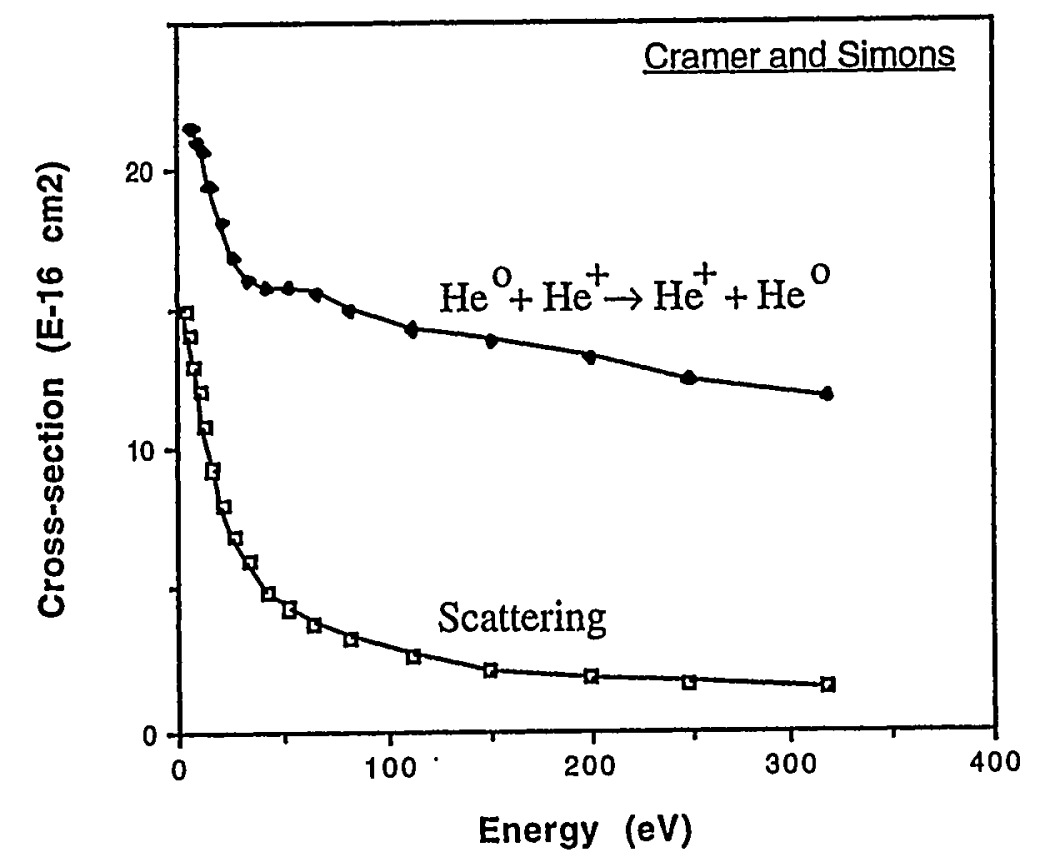

Only He has high charge-exchange, but low scattering, cross-sections $\mathrm{He}$ can be directly energized by a transverse $\mathrm{RF}_{\mathrm{ICR}}$ field Electrons are slowly heated by ion scattering $\mathrm{He}^{\circ}$ energy is determined by the mean-free path to charge exchange 


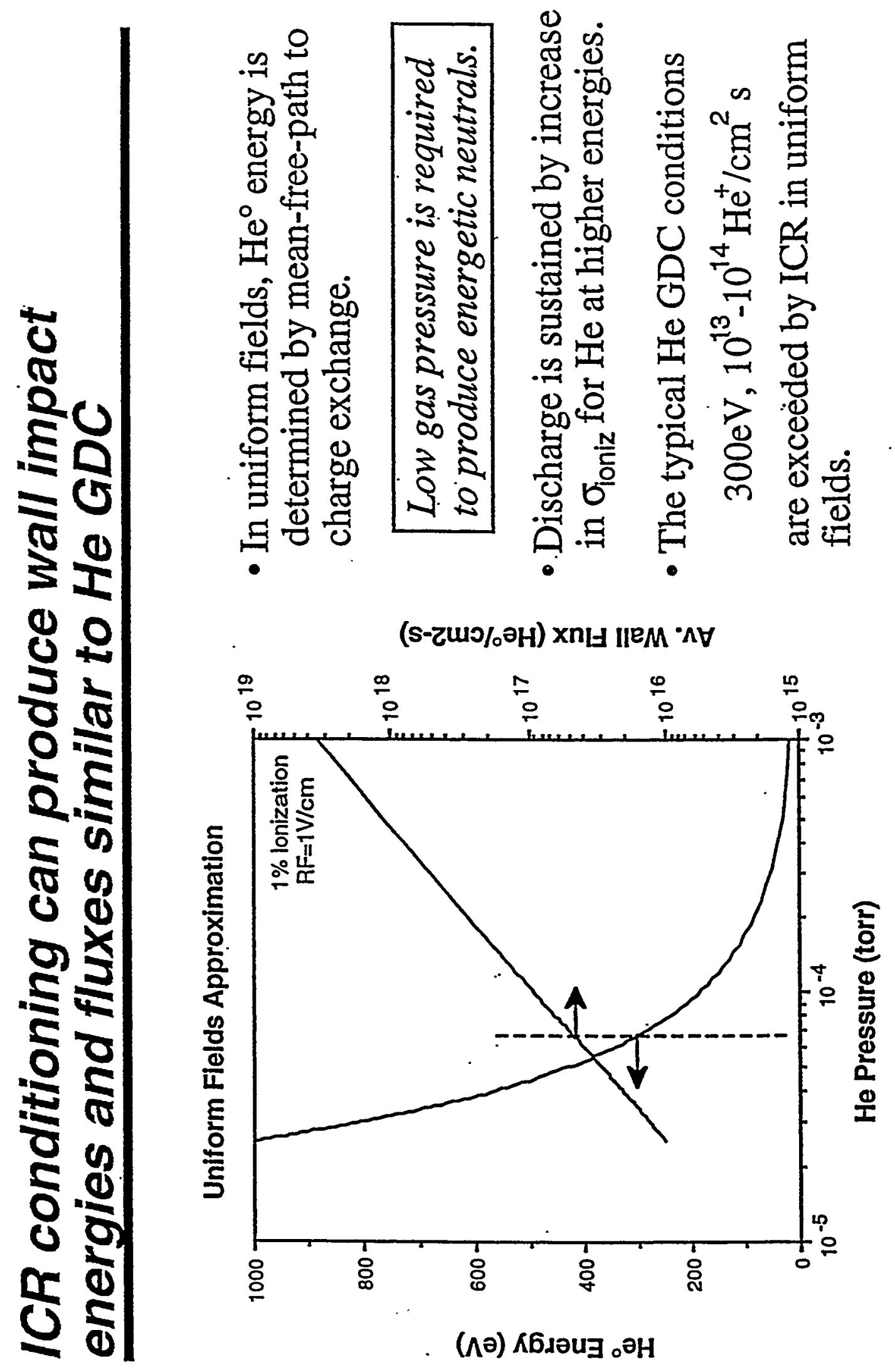




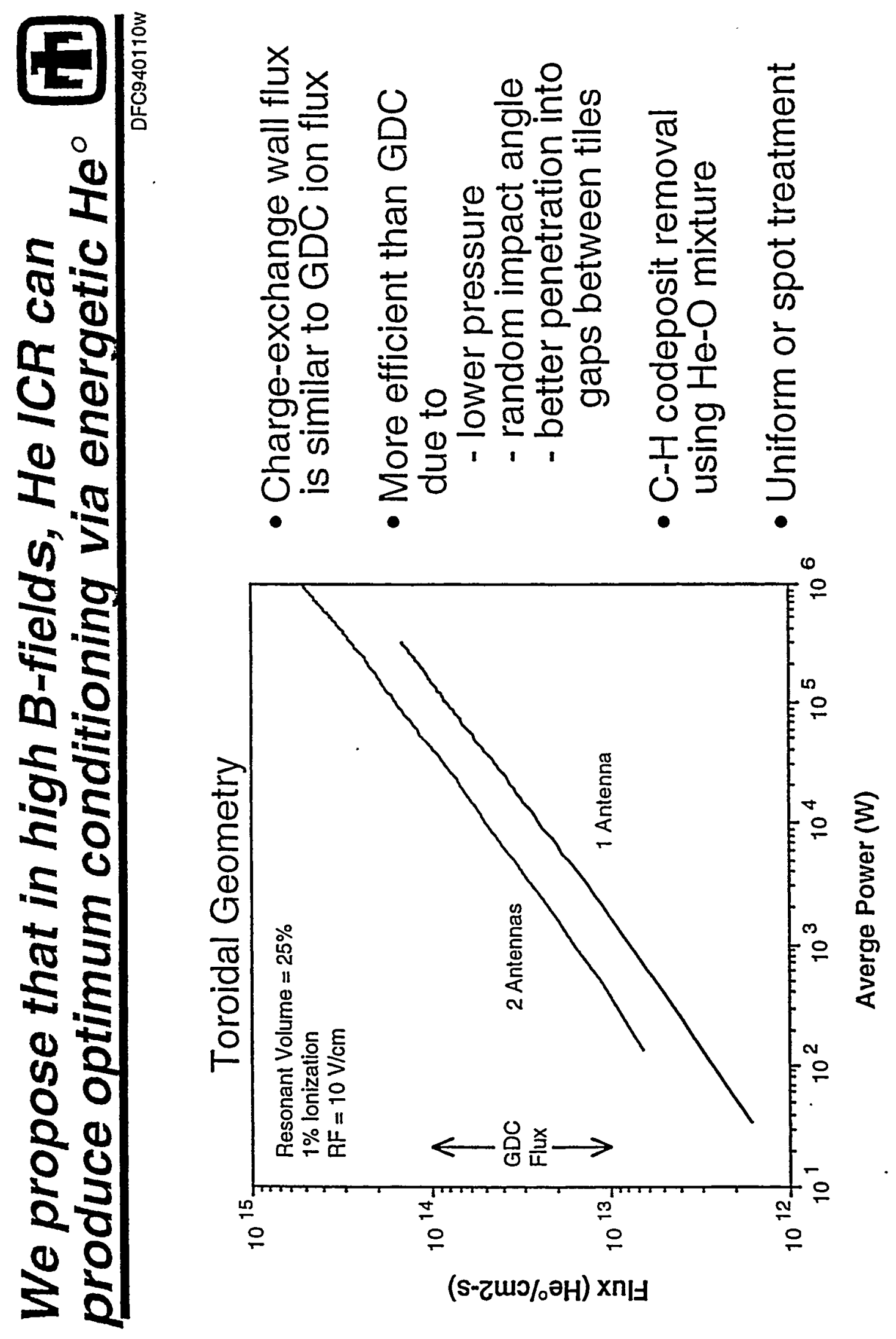




\section{We are investigating $\mathrm{C}-\mathrm{H}$ codeposit removal by plasma discharges in high B-fields}

- Using a 3 Tesla Penning-style trap configuration

- DC and RF excitation

- Determining removal rates for O-containing discharges (He-O) from rate of carbon oxidation

- Separating erosion rates due to ionized and neutral species

- Exploring penetration into shadowed/confined regions

- Varying plasma power, gas flow/mix

- maximize removal rate

- minimize residual O-contamination

We have preliminary data on erosion due to neutrals: atomic-O and $\mathrm{He}^{\circ}$. 


\section{High-field Probe Configuration}

\section{$11 / 6 / 97$}

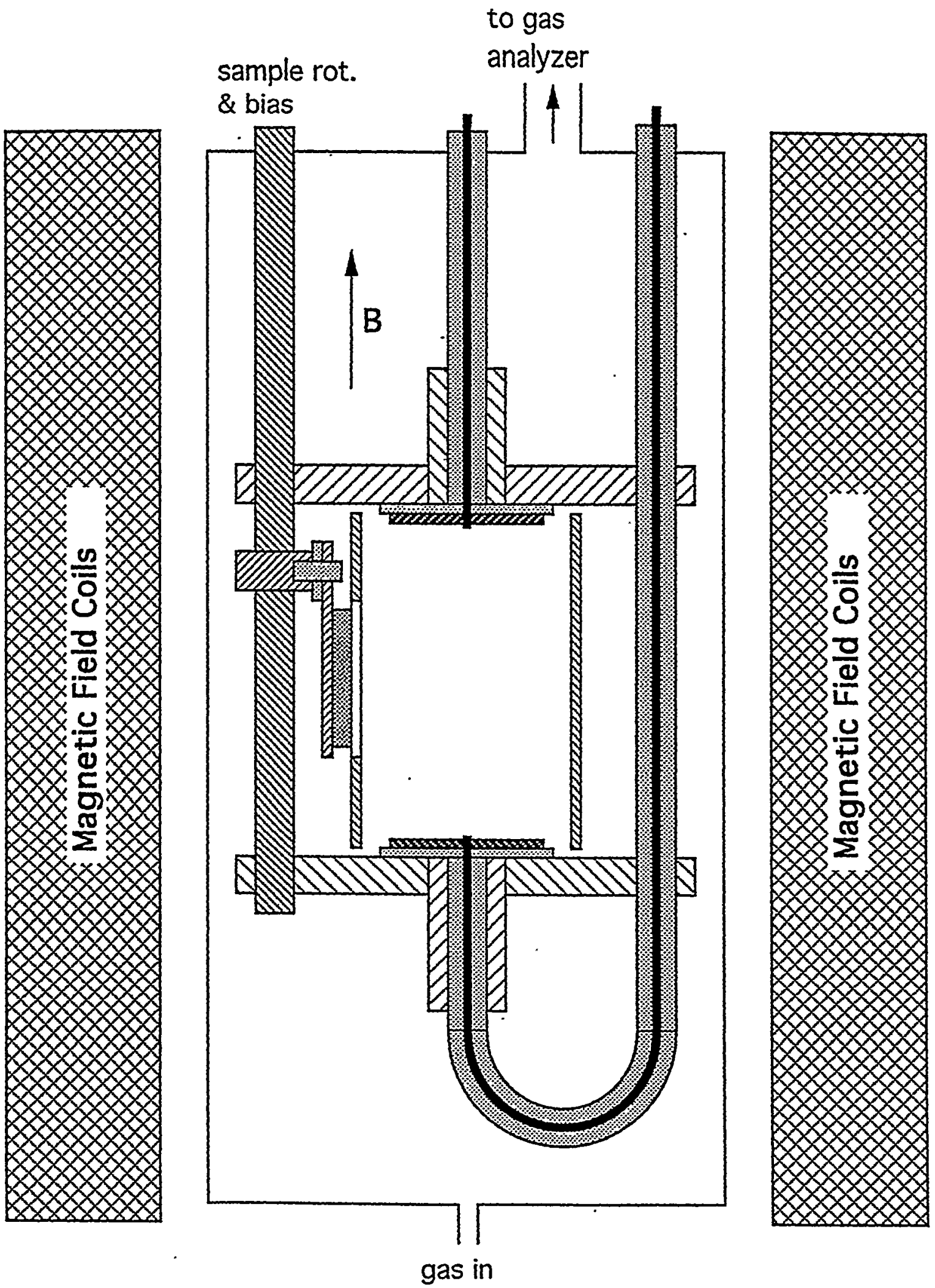




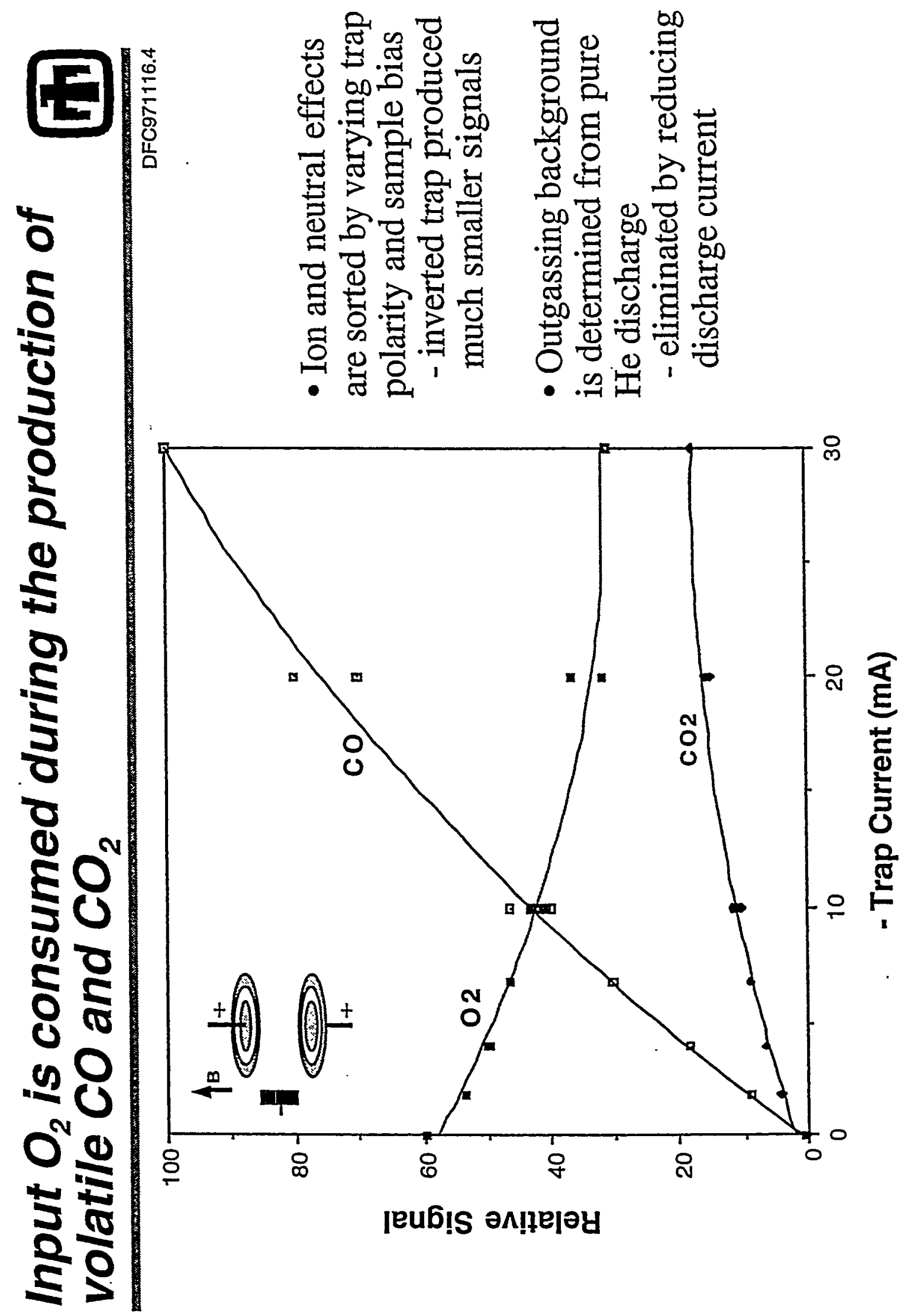

IV-28 


\section{Erosion by neutral species was observed using low power discharges}

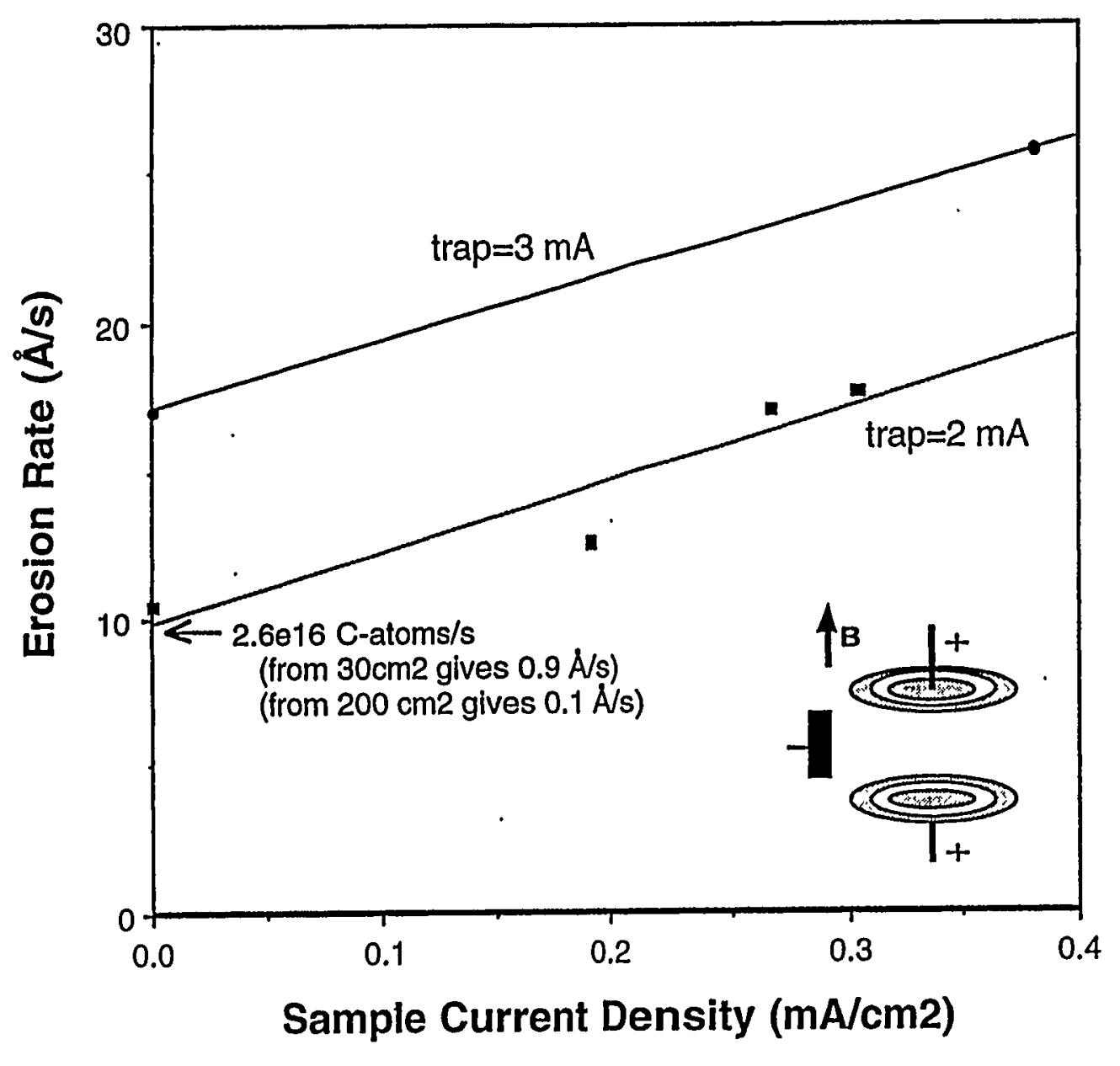

- Erosion by ions increases with sample bias $(-)$ and current

- Erosion by ions is eliminated by zero (or + ) sample bias

- Erosion by neutral increases with trap (plasma) current

- Area eroded by neutrals is larger than sample area

- Carbon is also removed from deposits on walls

Sample Current Density (mA/cm2) 


\section{Summary:}

- We have a simple, versatile test probe for studying codeposit erosion in high magnetic fields.

- Initial studies using Penning discharges show erosion due to neutral species can be separated from erosion due to ions.

- Preliminary measurements indicate atomic-O from a weak $\mathrm{He}-\mathrm{O}$ plasma will cross magnetic field lines and erode carbon materials at $0.1-.3 \mu \mathrm{m} / \mathrm{hr}$.

- Larger erosion rates are expected for $\mathrm{C}-\mathrm{H}$ codeposits $(\sim 3 \mathrm{x})$ using optimized discharges, and elevated sample temperatures.

- The initial studies also indicate atomic-O can reflect off surfaces, allowing it to reach around corners and penetrate into confined regions. 
US-Japan PMI/HHF Workshop in San Fràncisco December 8-11th, 1997

\title{
Erosion and Redeposition
}

\section{of High-Z Materials}

\section{in Linear Divertor Simulator}

\author{
N. Ohno, M. Kojima, Y. Ido, N. Ezumi, \\ and S. Takamura
}

Department of Energy Engineering and Science, Graduate School of Engineering, Nagoya University.

Furo-cho, Chikusa-ku, Nagoya 464-01, Japan 


\section{Outline}

- Introduction

- Erosion and Redepositon Process of Mo with Oblique Incidence of Magnetic Field

- Modification of W Surface by the Low Energy and High Flux Plasma Irradiation

- Introduction of New Divertor Plasma Simulator, NAGDIS-II

- Conclusions 


\section{Introduction}

High Z materials are recently focused for the material of future divertor target plate, because of

(1) good thermal properties,

(2)high threshold value of

physical sputtering;

(3) low hydrogen retention.

Systematic investigation on high Z materials is required including the atomic and transport processes.

-- Linear plasma device with high particle /heat flux plasma and a simple magnetic structure is a suitable one for investigating underlying individual physics in a series of atomic and transport processes.

\section{In this presentation,}

(1) Erosion and redeposition processes of a high

$\mathbf{Z}$ material (Mo) with oblique Incidence of Magnetic Field :

(2) Modification of W surface by the low energy

and high flux $\mathrm{He}$ and $\mathrm{H}$ plasma irradiation (Enhancementof heat load due to thermoelectron emission) 


\section{Experimental Set-up in TPD-I}
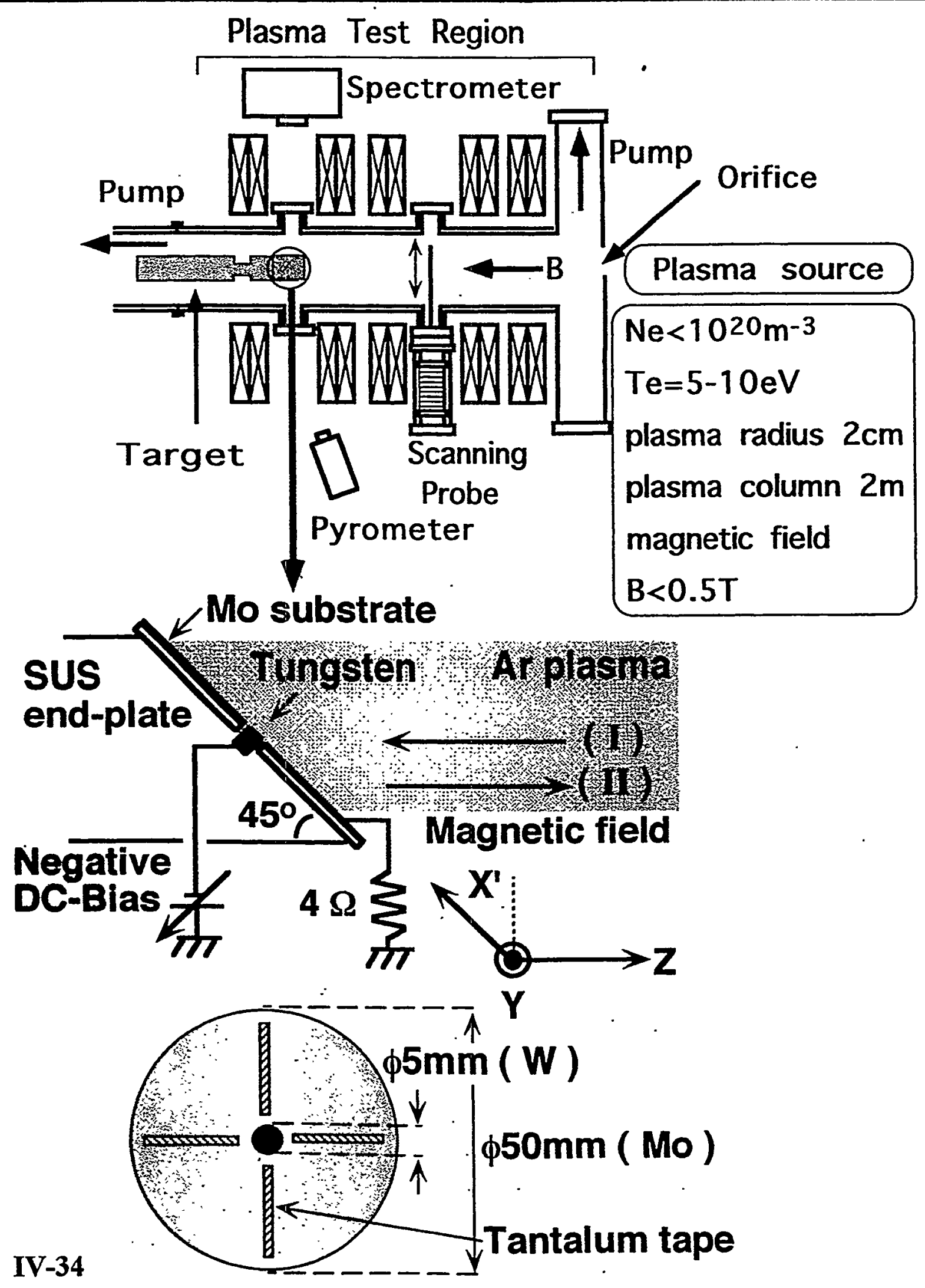

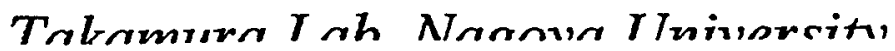




\section{Contour plot of a Mo substrate after Ar plasma exposure}

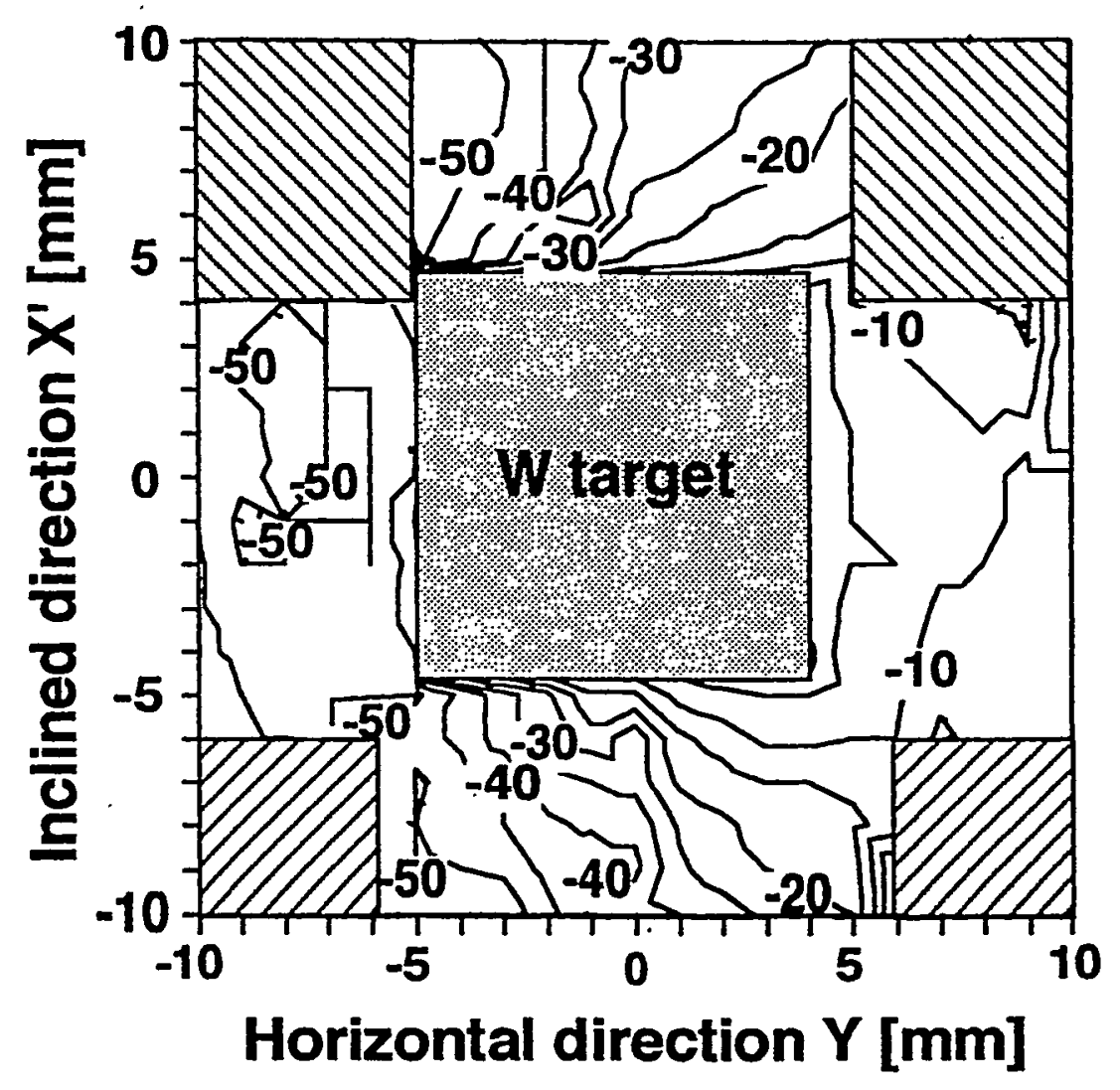

- Exposure time: 90 minutes

- Surface temperature: $1100^{\circ} \mathrm{C}$

- The levels correspond to the erosion depth in $\mu \mathrm{m}$ units.

Mo surface was characterized by Electron Probe Micro Analysis ( EPMA ).

---- Mo, Ta and carbon are only obsered $W$ is not found ( $<100$ p.p.m. ) 


\section{Asymmetric erosion profiles for the \\ different directions of magnetic field}
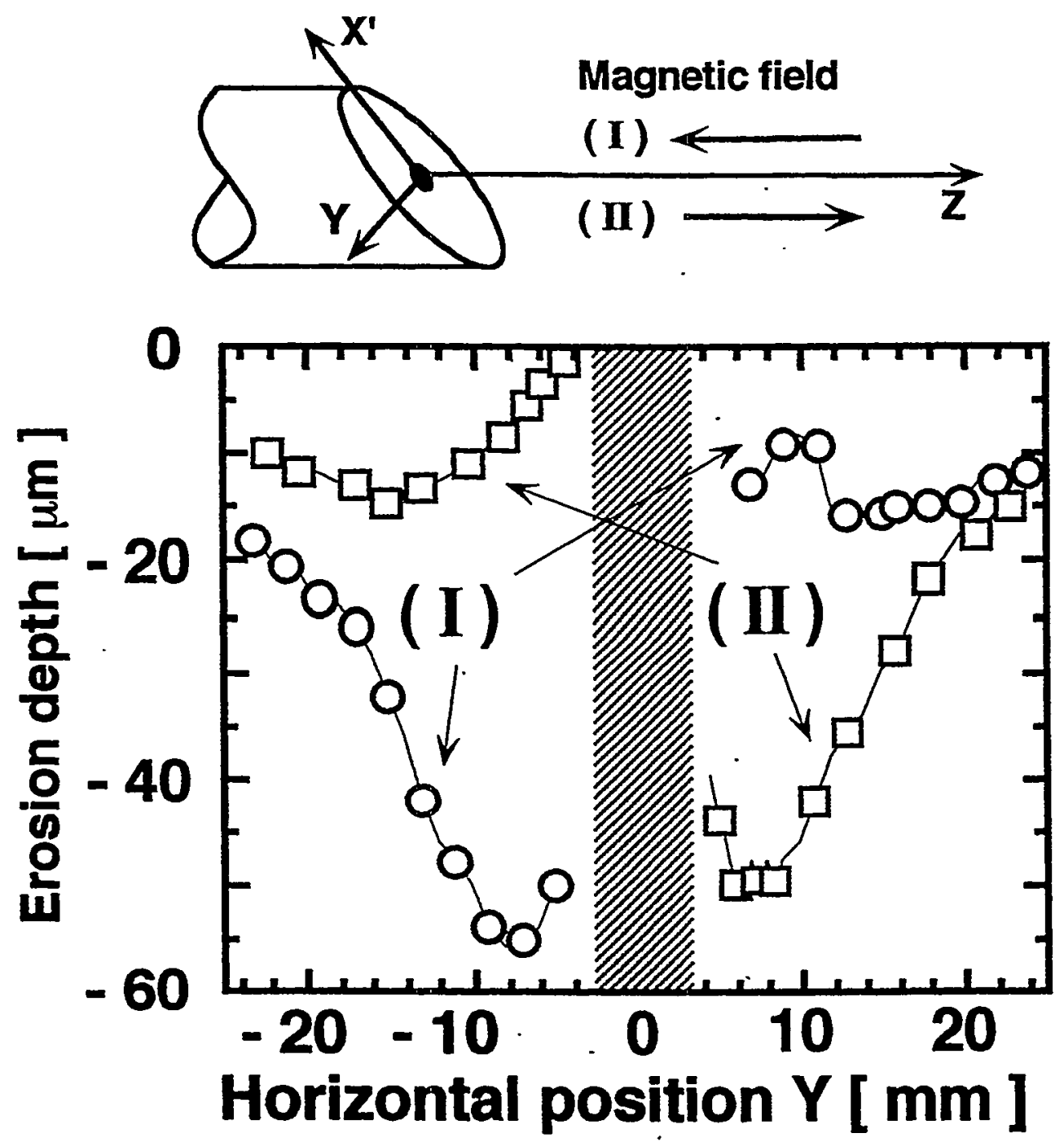

Experimentally observed asymmetric erosion profiles along horizontal direction $Y$ with the different directions of magnetic field. The hatched area shows the position of $\mathrm{W}$ target. 
Radial profiles of plasma paramters for Ar plasma in TPD-I

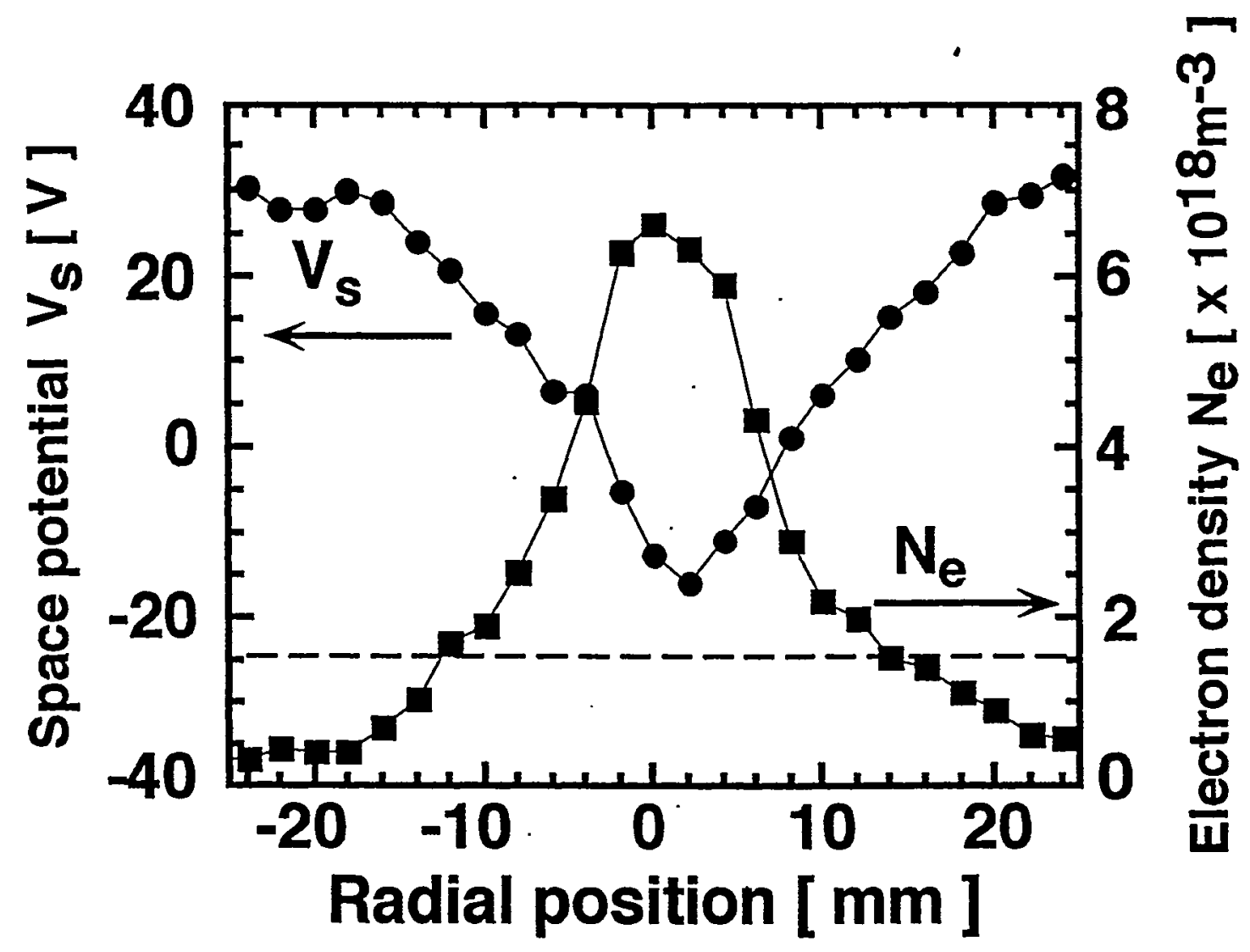

Radial profile of space potential and electron density for argon plasma. The dotted line shows the potential of Mo substrate.

-These parameters are measured using a fastscanning Langmuir probe located at $30 \mathrm{~cm}$ away from the substrate.

- Electron temperature is almost uniform around $7 \mathrm{eV}$. 


\section{3-D Monte Carlo simulation code}

\section{Magnetic field}

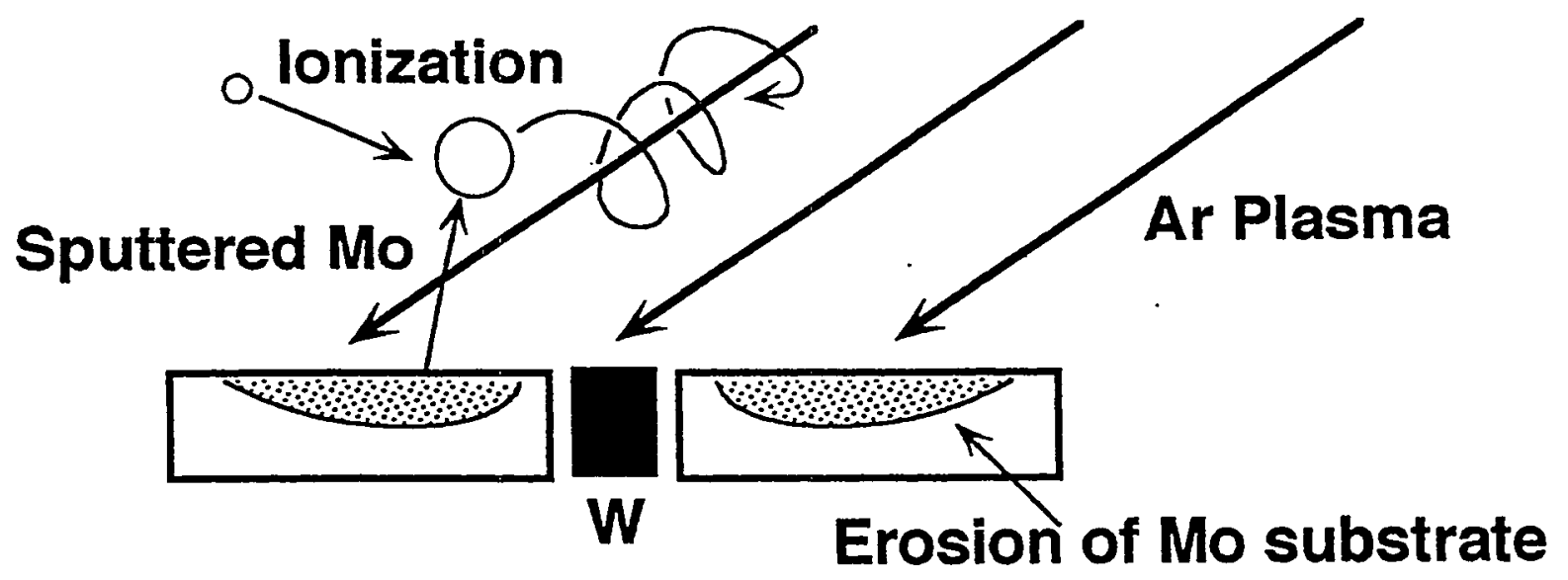

Collision processes in a plasma

- Elastic collisions (rigid sphere collision)

- Coulomb collisions

o Electron impact ionization

o Charge exchange

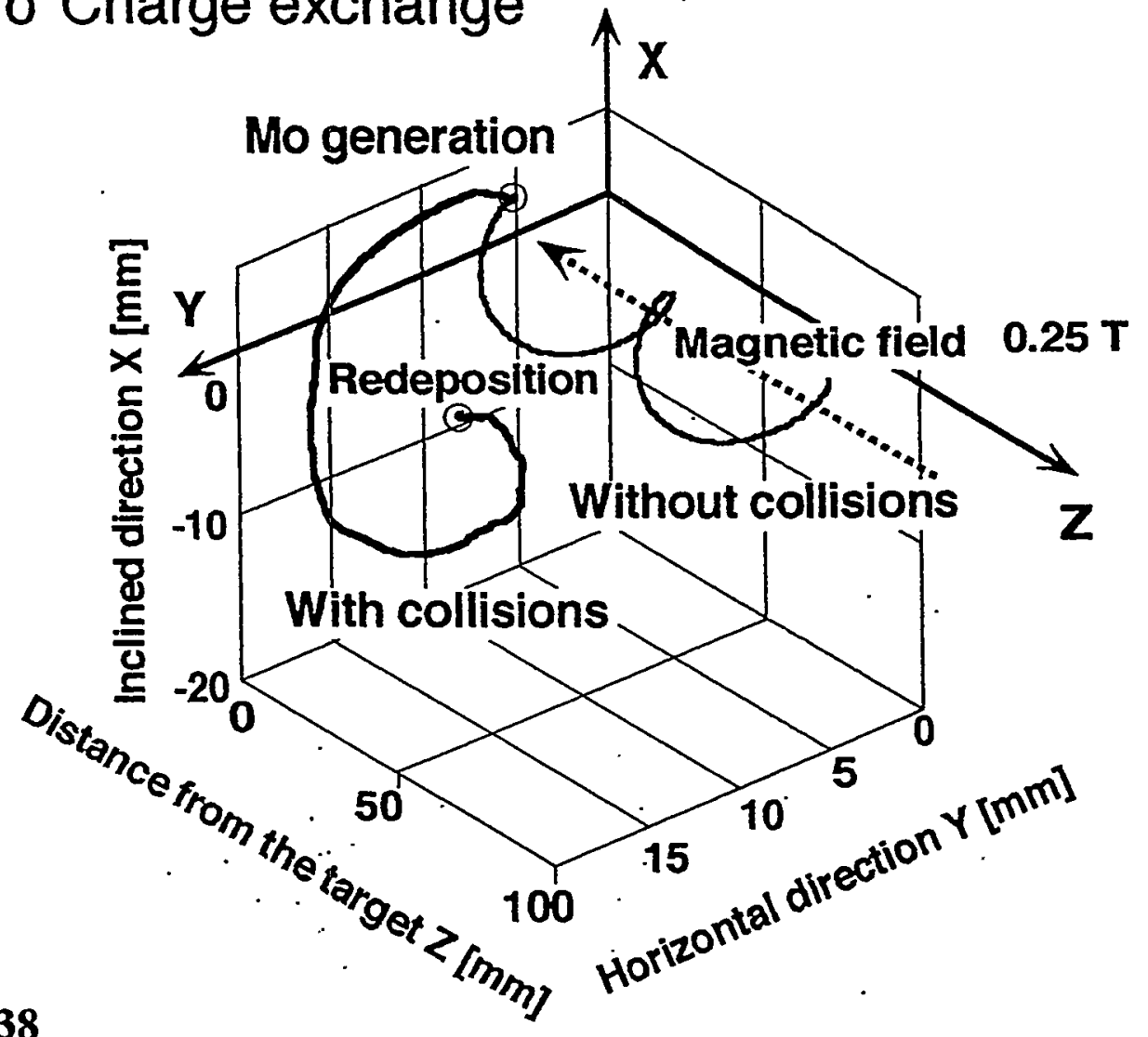




\section{Erosion-redeposition model \\ on a plasma-facing material}

$\Gamma_{i} \cdot Y \cdot Y_{s}:$ Self-sputtered flux

$\Gamma_{i} \cdot Y:$ Sputtered flux ニニニート

$\Gamma_{i}:$ Ion flux

(a) Erosion process

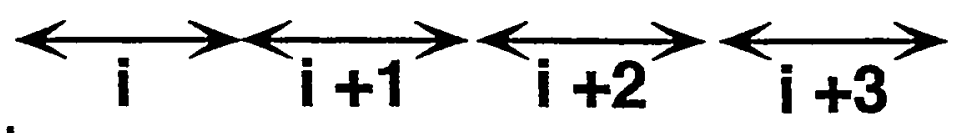

Local erosion depth $d(i, j)[m]$ is given by

$$
\begin{array}{cl}
d(i, j)=\Gamma_{i}(i, j) \times Y(i, j) \times M \times T / \rho . \\
\Gamma_{\mathrm{i}}(\mathrm{i}, \mathrm{j})\left[\mathrm{m}^{-2} . \mathrm{s}^{-1}\right] & : \text { the average flux of plasma ions } \\
Y(\mathrm{i}, \mathrm{j})[\text { atoms } / \text { ion }] & : \text { sputtering yield at that zone } \\
\mathrm{M}[\mathrm{kg}] & : \text { atomic mass of the substrate } \\
\rho\left[\mathrm{kg} \cdot \mathrm{m}^{-3}\right] & : \text { mass density of the bulk } \\
& \text { (Mo is } 1.02 \times 10^{4} \mathrm{~kg} . \mathrm{m}^{-3} \text { ) } \\
\mathrm{T}[\mathrm{s}] & : \text { plasma exposure time }
\end{array}
$$

(b) Redeposition process process

Redeposition depth at $(i+3, j)$

$$
\begin{aligned}
d(i+3, j)=\dot{\Gamma}_{i} & \times Y \times\left\{1-Y_{s}^{1}(i+3, j)\right\} \times M \times T / \rho \\
Y_{s}{ }^{1}(i+3, j) & \text { :the self sputtering yield at the zone }(i+3, j)
\end{aligned}
$$




\section{2-D erosion distributions caluculated by Monter Carlo code}
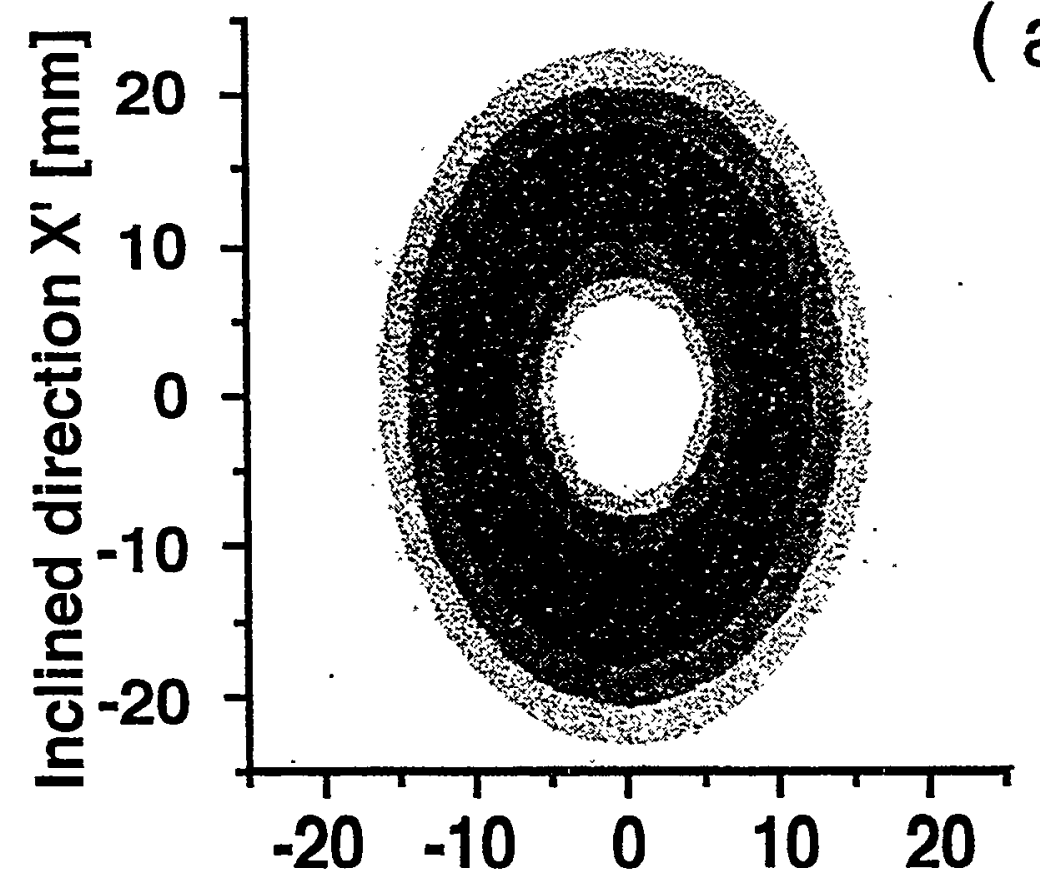

$\begin{array}{ccccc}-20 & -10 & 0 & 10 & 20 \\ \text { Horizontal direction } Y & {[\mathrm{~mm}]}\end{array}$

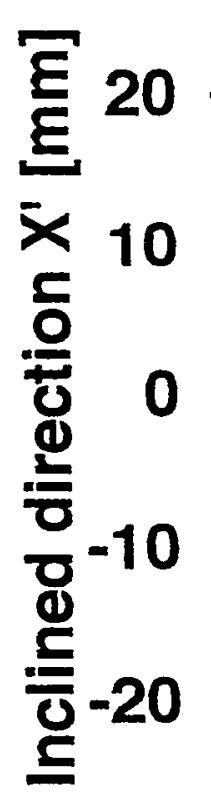
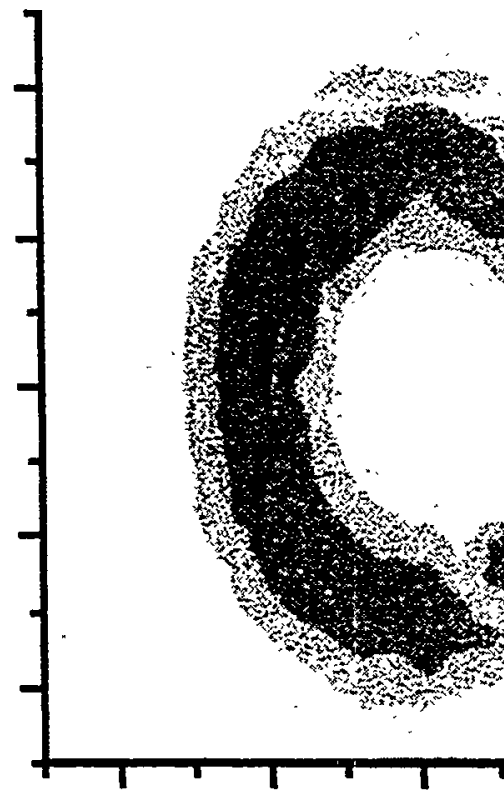

intos 


\section{Sliced erosion profiles \\ in horizontal direction $\left(X^{\prime}=0\right.$ )}

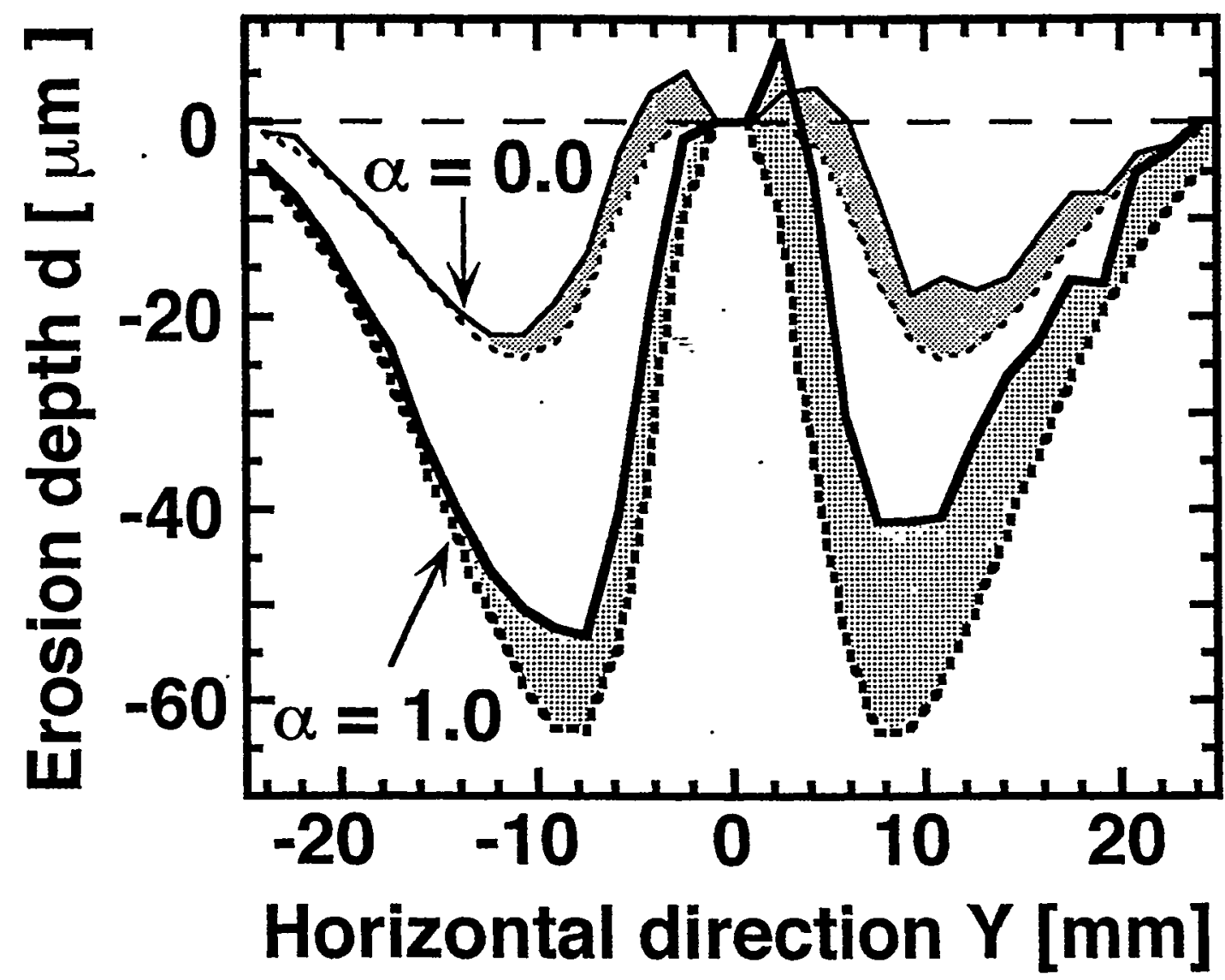

$-\alpha$ : the ratio of $\mathrm{Ar}^{2+}$ density to $\mathrm{Ar}^{1+}$

-Solid and dotted lines show the profiles

with and without redeposition process.

-Shadow regions correspond to the

redeposited depth of sputtered Mo.

-> Asymmetric redeposition profile 


\section{Redeposition of Mo}

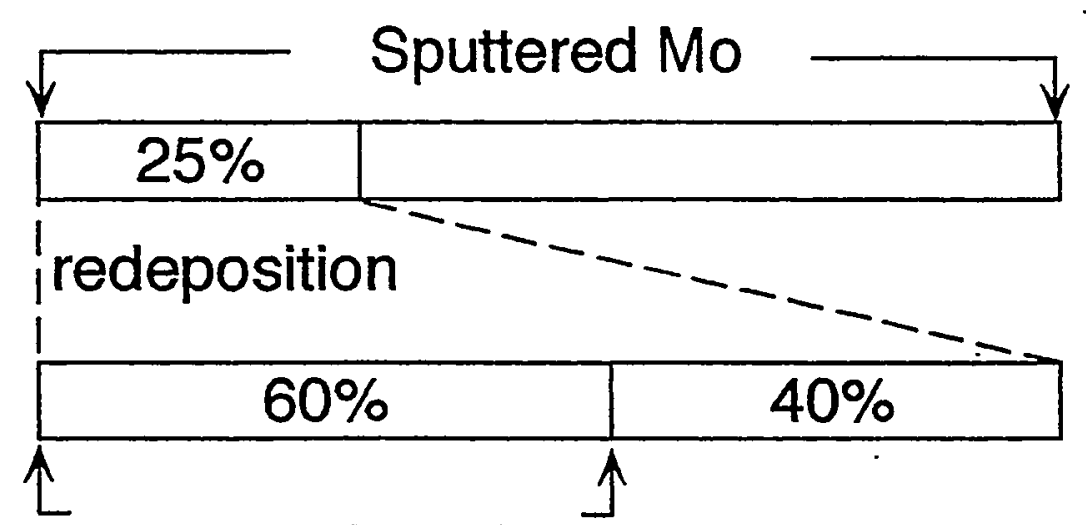

prompt redeposition

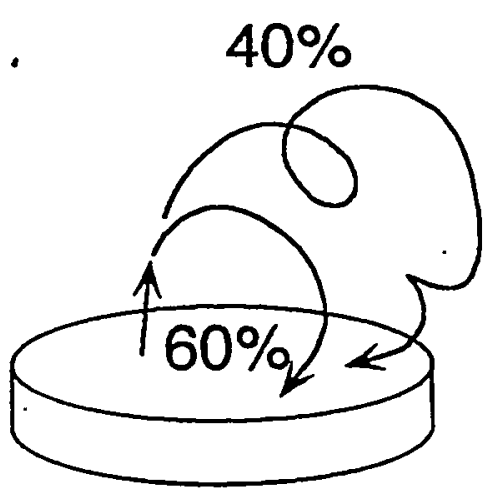

Mo substrate About $25 \%$ of sputtered Mo return back to the substrate as mainly an ionized Mo, and redeposited. About $60 \%$ of their returned particles redeposit within their first gyrations Mo substrate Magnetic field

(a) $\lambda_{i}<r_{t}$

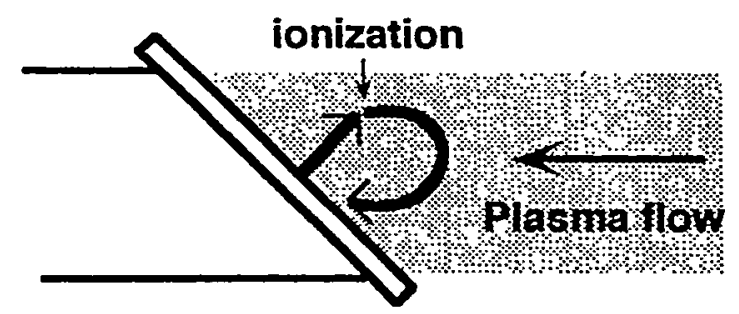
(b) $\lambda_{i}>r_{t}$ with collisions of sputtered $\mathrm{Mo}$ is $2 \mathrm{eV}$ )
- Ionization mean free path $\sim 16 \mathrm{~mm}$

$\left(\mathrm{n}_{\mathrm{e}} \sim 2.0 \times 10^{18} \mathrm{~m}^{-3}, \mathrm{Te} \sim 7 \mathrm{eV}\right)$ - Gyro radius $8 \mathrm{~mm}$ ( $\mathrm{B} \sim 0.25 \mathrm{~T}$, average energy

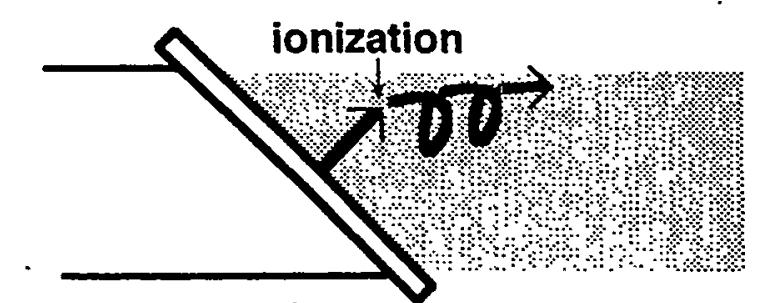

(c) $\mathrm{IV}-42_{i}>r_{\mathrm{t}}$ without collisions 


\section{Erosion of Mo}

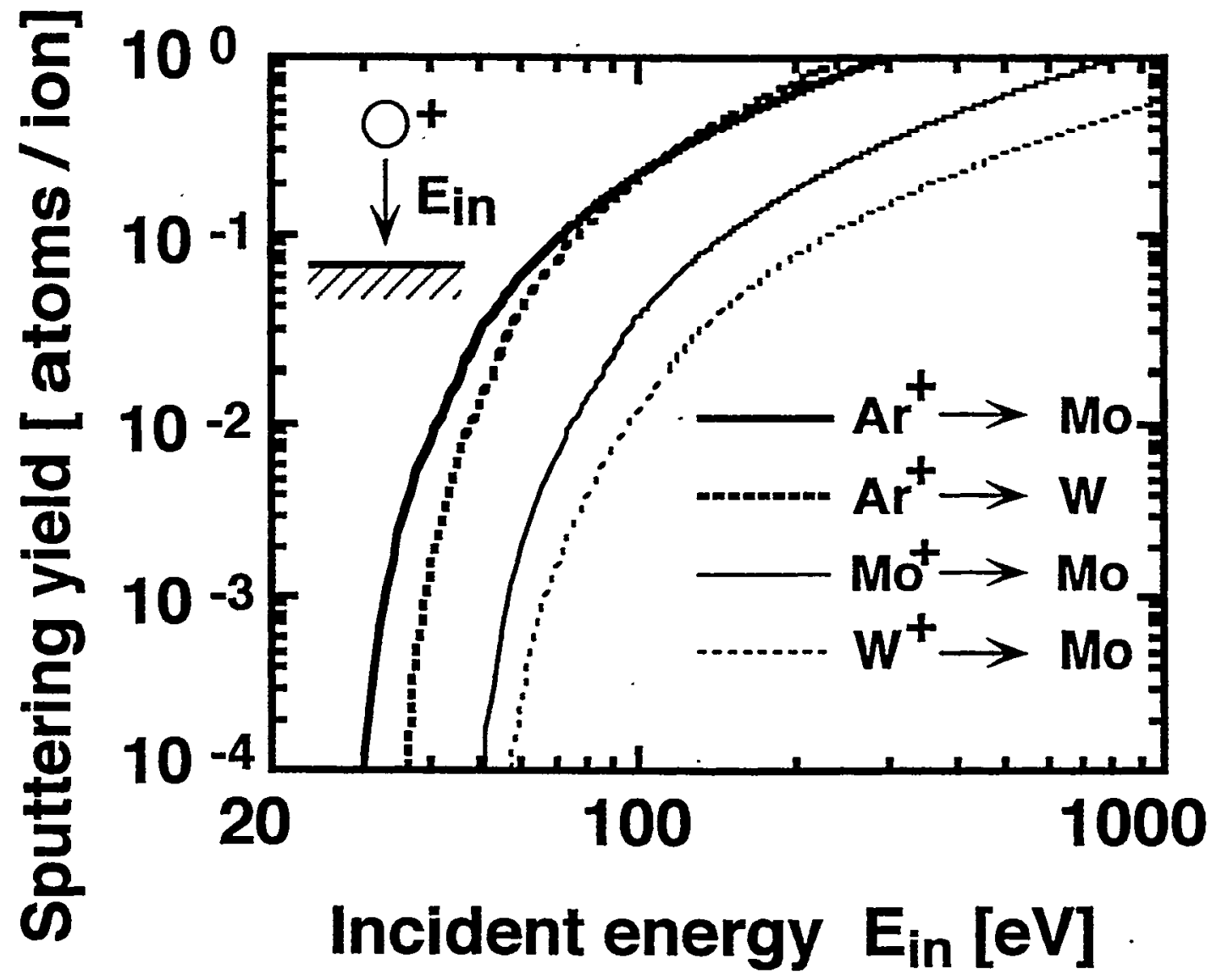

Sputtering yields of various ion-material components at normal incidence, calculated from an empirical formula [ $\left.{ }^{\star}\right]$.

* Y. Yamamura and H. Tawara, Atomic Data and Nucl. Data Tables 62, 149 (1996). 


\section{Summary (1)}

\section{1) Experiments:}

The effect of prompt redeposition of Mo is clearly demonstrated in a linear plasma device. Mo ions which are returned back to the substrate locally redeposit on it depending on the direction of magnetic field due to the effects of prompt redeposition.

\section{2) Numerical analysis:}

To analyze the transport of sputtered particles in a plasma and the subsequent redeposition on a plasmafacing material, a particle simulation (Monte Calro ) code has been developed. We have a qualitative agreement for the erosion profiles with the results of numerical code predictions with the effect of prompt redeposition for high $\mathrm{Z}$ material. However, we have quantitative differences for their erosion rates. 


\section{Experimental set-up for plasma irradiation to W in NAGDIS-I}
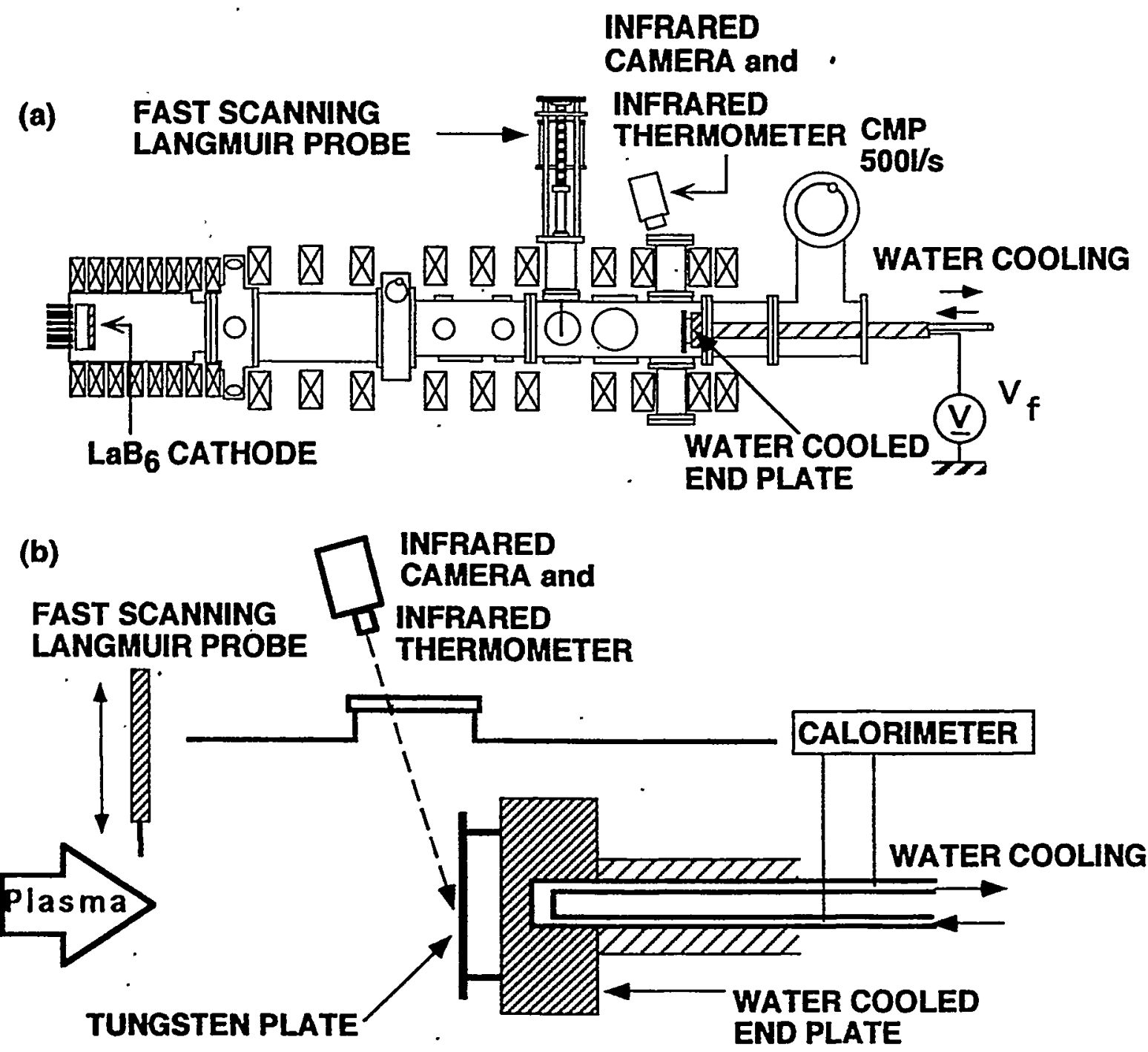

Tungsten can be irradiated by the helium and hydrogen plasmas with the diameter of about $6 \mathrm{~cm}$ and $\mathrm{Te}=5-$ $10 \mathrm{eV}, \mathrm{ne}_{\mathrm{e}}=0.5-4.0 \times 10^{18} \mathrm{~m}^{-3}$, corresponding to the flux of (0.27-3.1) $\times 10^{2.2} \mathrm{He}^{+}$ions $\mathrm{m}^{-2} \mathrm{~s}^{-1}$ and a tungsten plate temperature of up to about $3200 \mathrm{~K}$. 


\section{Experimental results}

A. Tungsten irradiated by the helium plasma

SEM micrographs of tungsten surface show clearly that the microstructure of the tungsten surface is changed due to the helium plasma irradiation.

1) No obvious the change of microstructure of tungsten surface irradiated by helium plasma with the flux of $4.4 \times 10^{21} \mathrm{He}^{+}$ions $\mathrm{m}^{-2} \mathrm{~s}^{-1}$ corresponding to a tungsten plate temperature of $1144 \mathrm{~K}$.
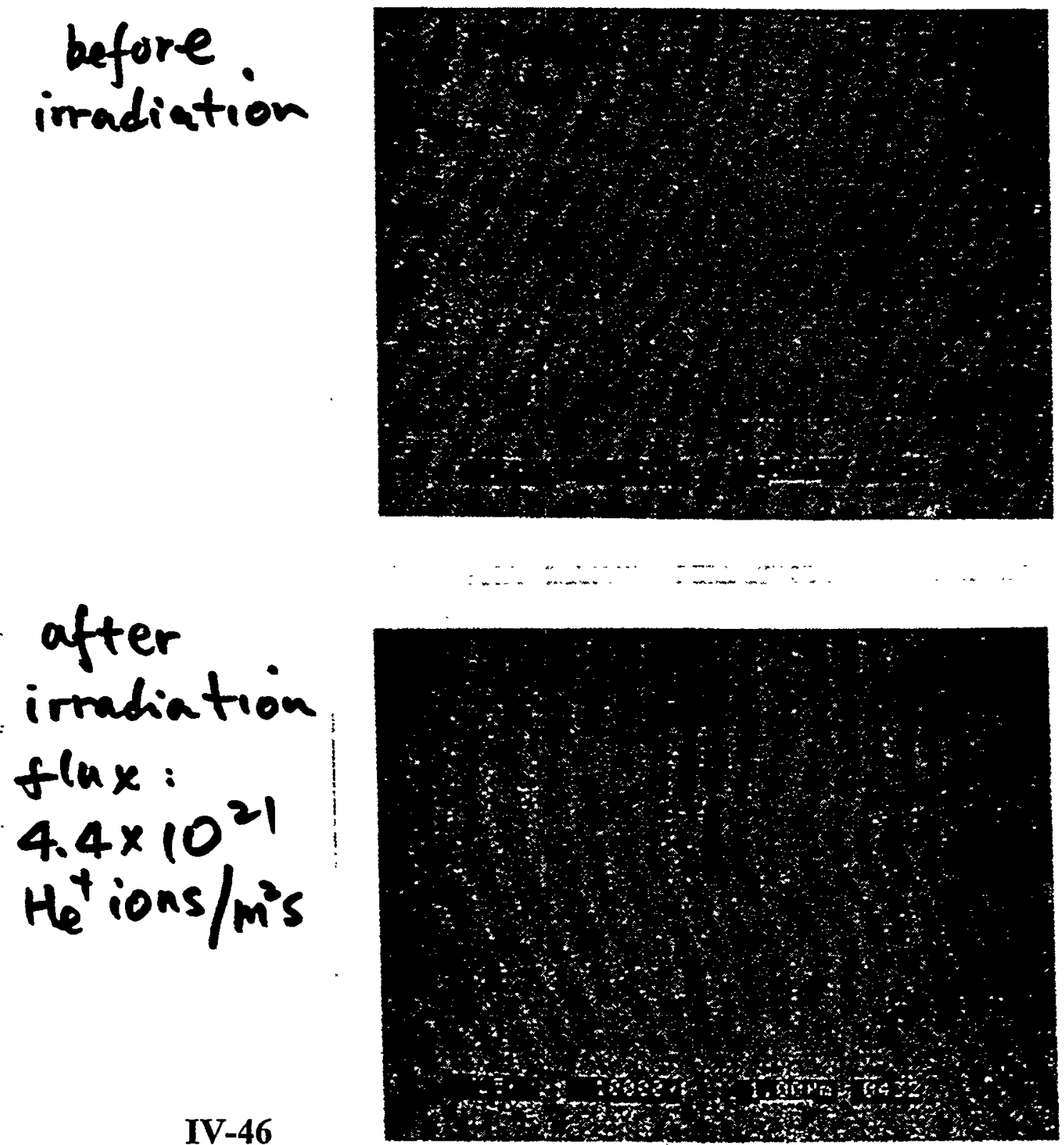
2) Many holes of $0.1-0.5 \mu \mathrm{m}$ in diameter which appear in the tungsten surface irradiated by helium plasma with the higher flux corresponding to a higher tungsten plate temperature

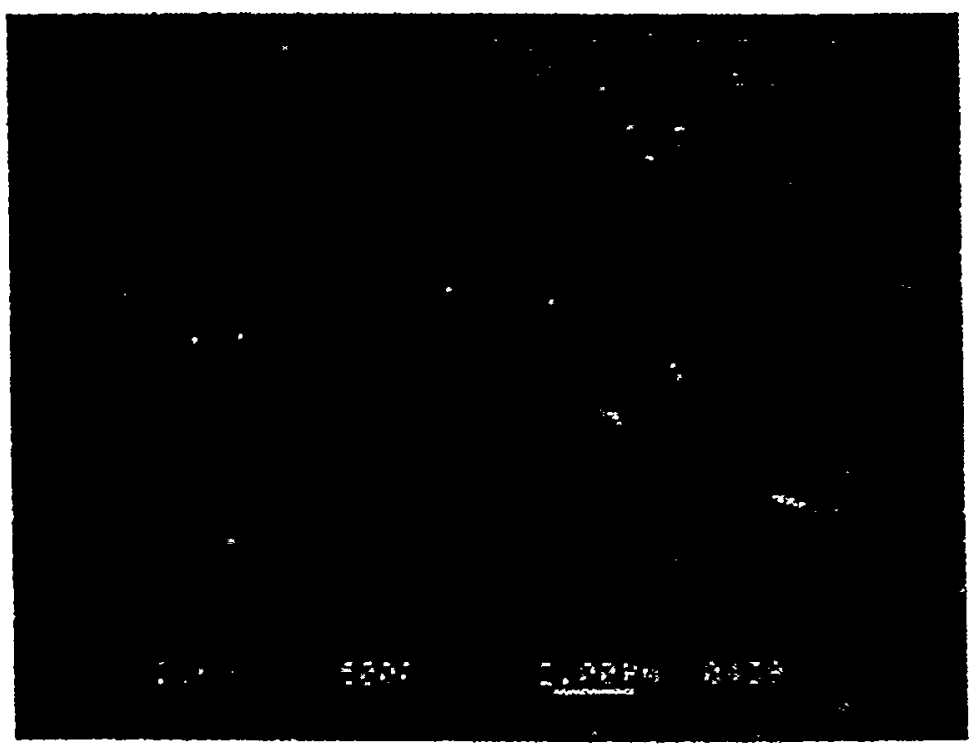

$$
\begin{gathered}
T=1496 \mathrm{~K} \\
\text { Flux } \sim 4.5 \times 10^{21} \mathrm{He}^{+} \\
\text {cons } / \mathrm{m}^{2} \mathrm{~s}
\end{gathered}
$$

Energy $~ 31 \mathrm{eV}$

$T=1580 \mathrm{~K}$

Flux $~ 5.2 \times 10^{24} \mathrm{He}$ ions $/ \mathrm{m}^{2} \mathrm{~s}$

Energy $\sim 30 \mathrm{eV}$ 
3) When tungsten was irradiated by the helium plasma with the more higher flux of $2.4 \times 10^{22} \mathrm{He}^{+}$ions $\mathrm{m}^{-2} \mathrm{~s}^{-1}$ corresponding to a tungsten plate temperature of up to near $3200 \mathrm{~K}$.

i) The size of holes becomes large with the diameter of about $1-4 \mu \mathrm{m}$
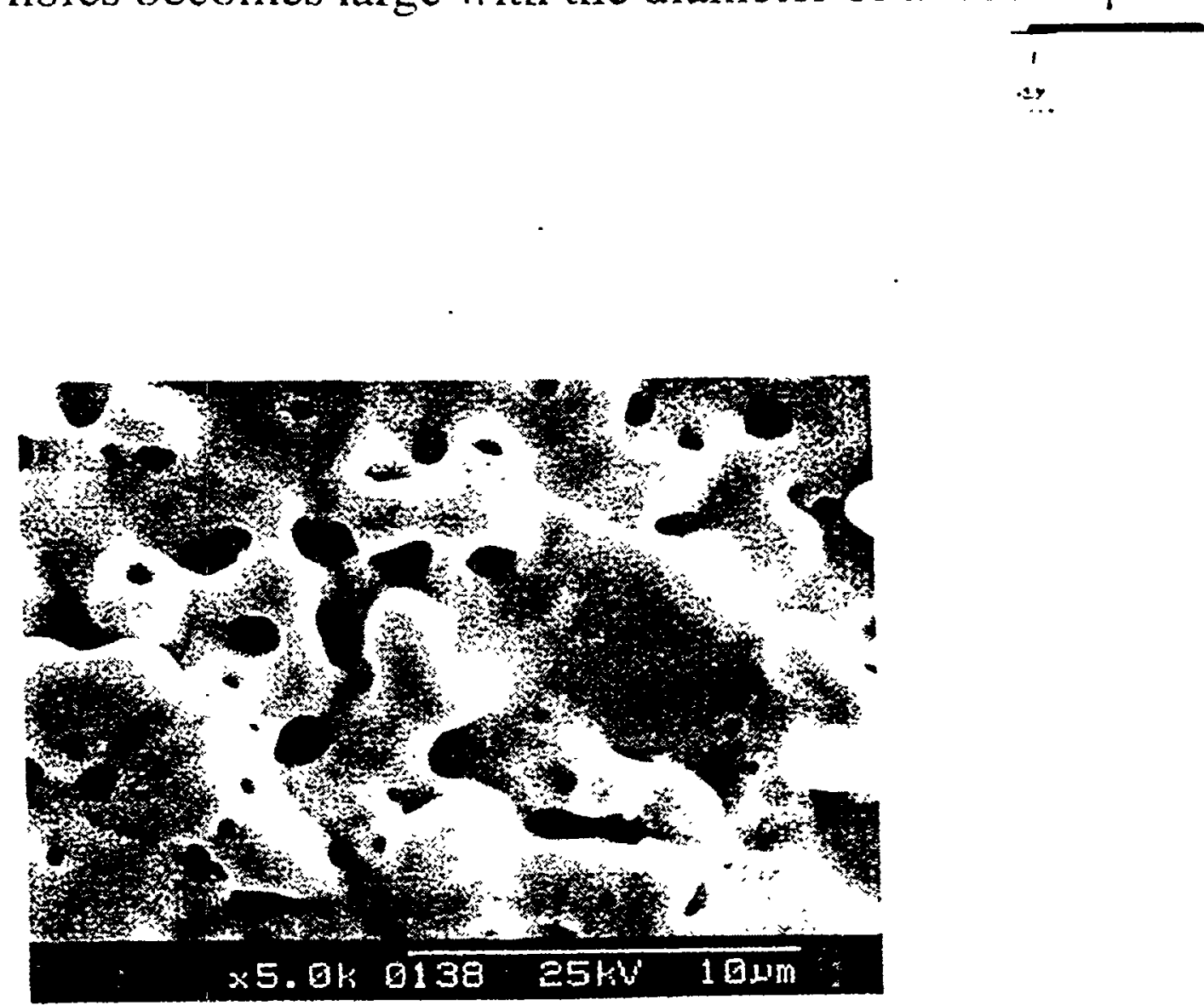

$3200 \mathrm{~K}, \quad 2.4 \times 10^{22} \mathrm{He}^{+}$ions $/ \mathrm{m}^{2} \mathrm{~s}$

ii) A large undulation appear in tungsten surface

iii) The redeposited layers of $W$ are formed at the periphery of the irradiation region on tungsten surface. 


\section{B. Tungsten irradiated by the hydrogen plasma}

To obtain a comparison with different ion-species, the tungsten plate was irradiated by the hydrogen plasma with $3.3 \times 10^{22} \mathrm{H}^{+}$ions $\mathrm{m}^{-2} \mathrm{~s}^{-1}$, corresponding to a tungsten plate temperature of near $2800 \mathrm{~K}$. Surface morphology of tungsten irradiated by the hydrogen plasma is found to be quite different from the helium case.

A large crystal structure appears in the tungsten surface without any holes appearing.

There is no redeposition of $W$ observation in the case of the hydrogen plasma irradiation.

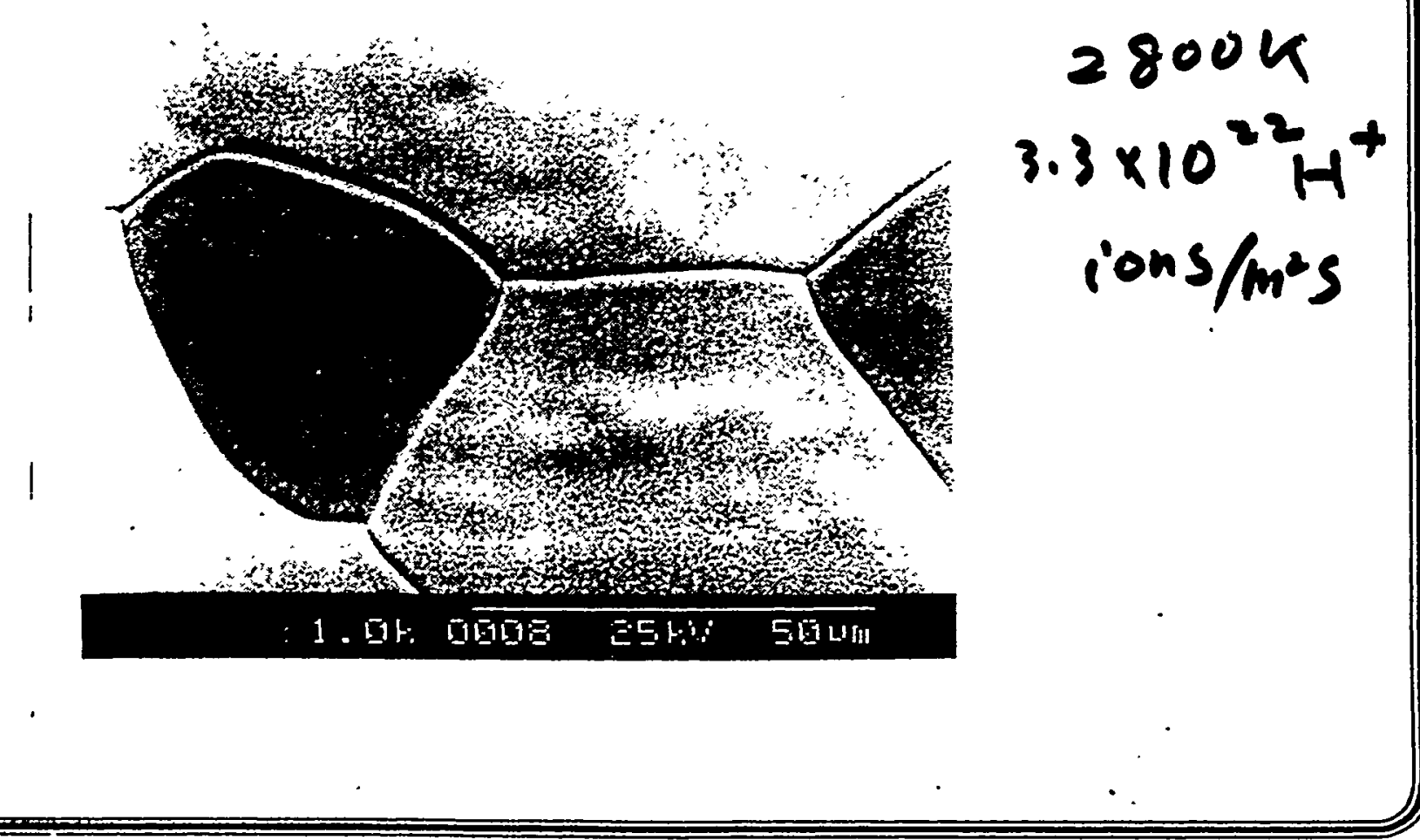




\section{$\operatorname{Summary}(2)$}

For a high temperature tungsten plate irradiated by a low energy and high flux plasma, surface modification is observed in experiments.

A surface undulation are formed and many holes of 0.1- 5 $\mu \mathrm{m}$ in diameter appear in the tungsten surface after the helium plasma irradiation.

The redeposition of $\mathrm{W}$ are observed due to the helium plasma irradiation at high plate temperature.

Microsructure changes of the tungsten irradiated by the hydrogen plasma is found to be quite different from the helium plasma case.

The size and the density of holes has an obvious relation to the incident ion energy, flux as well as the surface temperature distribution on the tungsten surface. But we can't get a quantitative relation in our experiments.

In the experiments we can't controll the ions flux, energy and target plate temperature, separately because tungsten plate is only heated by plasma.

Now a further experiments are considered for getting quantitative relation through some improvement of experimental setup.

1. A controll of plate temperature by external heating power

2. A controll of incident ions energy by bias voltage on plate

3. A controll of incident ions flux by plasma density 


\title{
Erosion and Impurity Effects on PFC Materials in PISCES-B
}

\author{
presented by \\ R. Doerner
}

A summary of work performed by the PISCES-B materials research team. R. Doerner, A. Grossman, S. Luckhardt, R. Seraydarian, F.C. Sze and D. Whyte

Fusion Energy Research Program,

University of California - San Diego, La Jolla, CA. 92093-0417

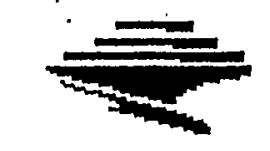

UCSanDiego

work supported under DOE grant \# DE-FG03-95ER-54301.

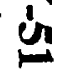


住 Outline:

- Interaction of beryllium with a 'clean' plasma

- erosion

- surface modifications

- deuterium retention

- PISCES-B mixed-material experiments

- Beryllium \& Carbon

- Tungsten \& Carbon

- Conclusions and future directions 
(2)

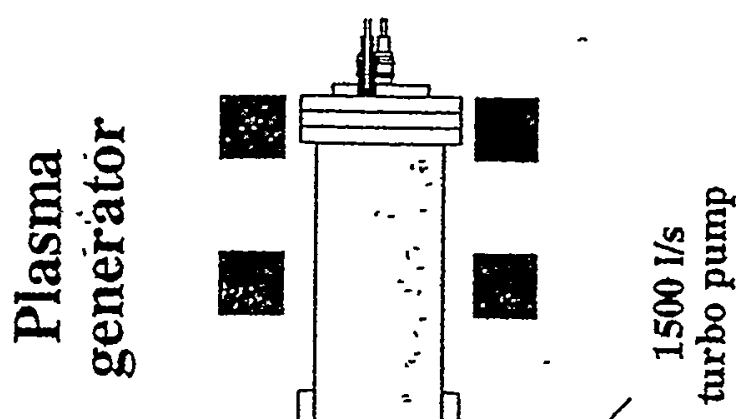




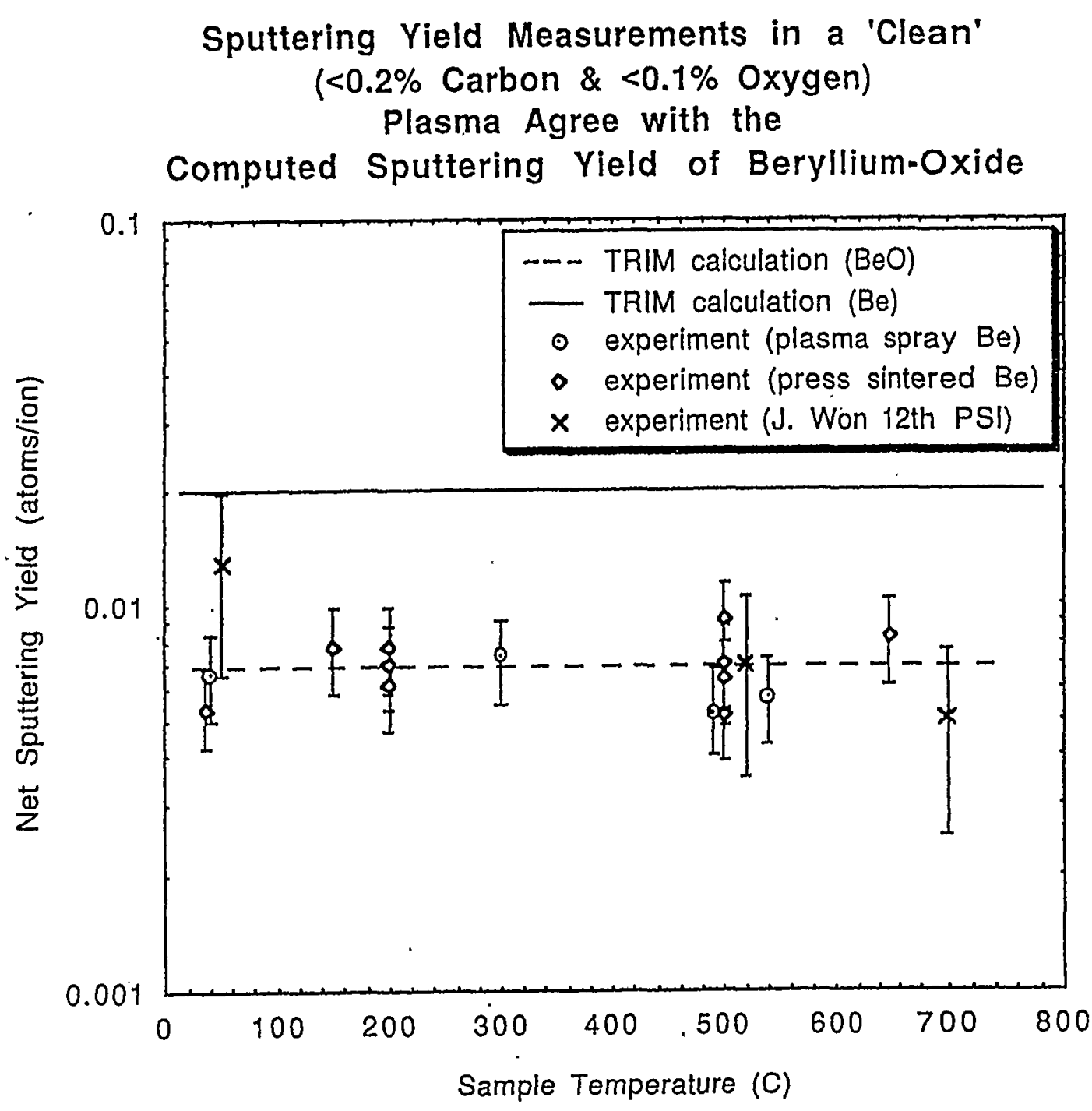


Beryllium samples exposed to plasma bombardment at high temperature exhibit surface damage

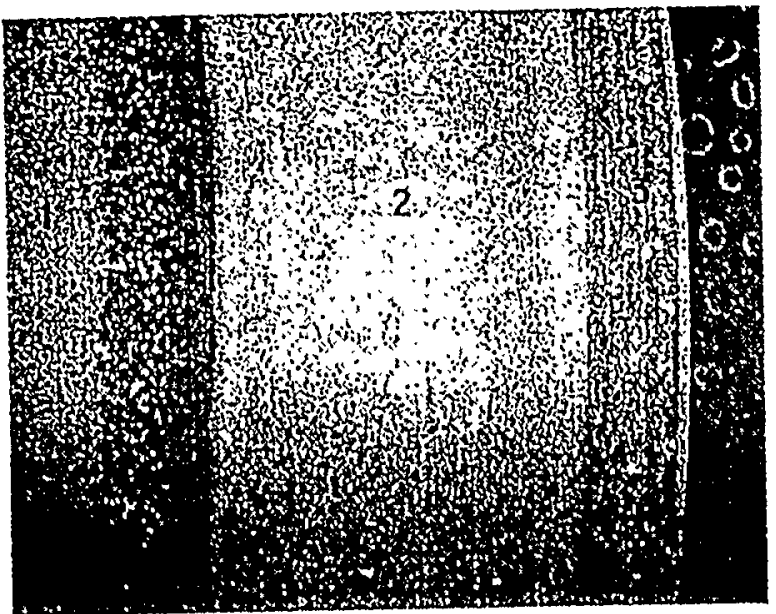

\section{Area 1 - High-temperature exposure $\left(580^{\circ} \mathrm{C}\right)$}

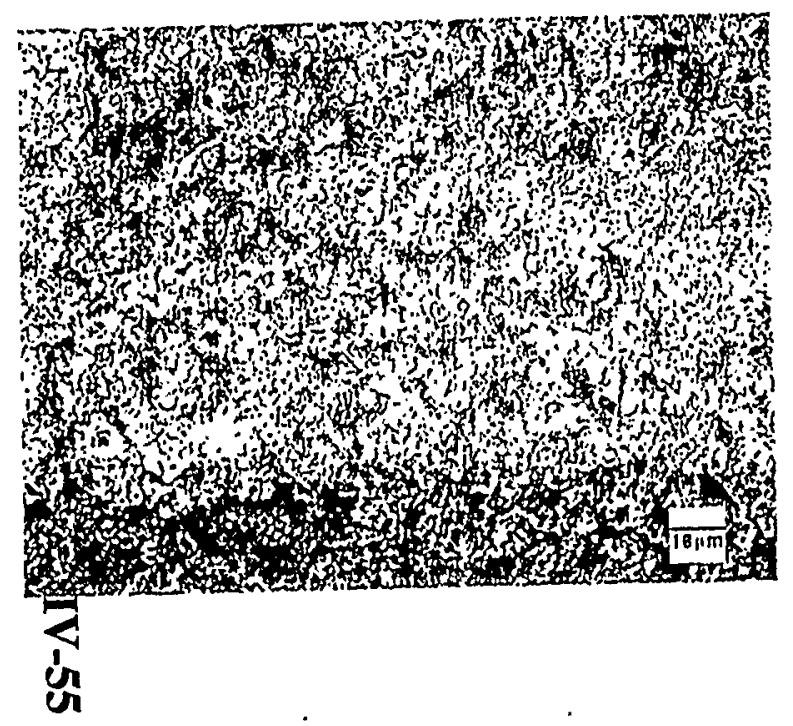

\section{Area 2 - Low-temperature exposure $\left(200^{\circ} \mathrm{C}\right)$}

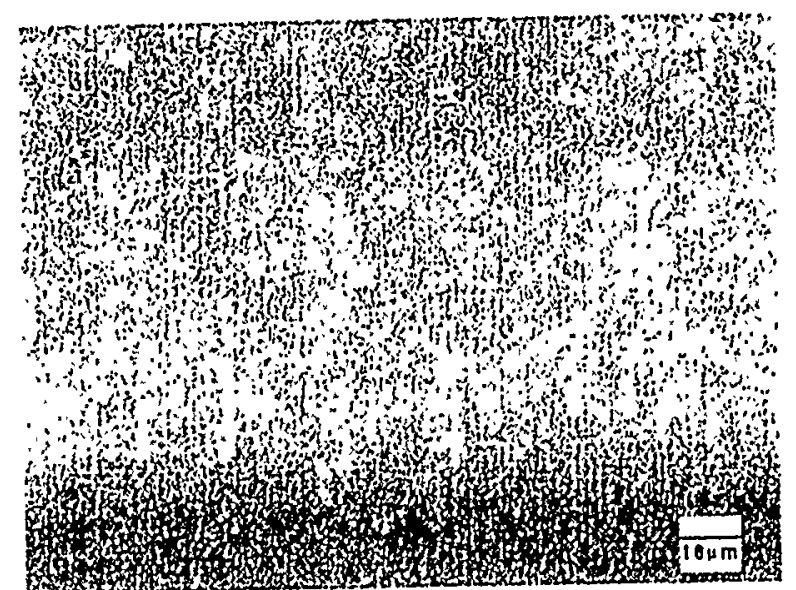

Area 3 - Surface hidden from plasma exposure

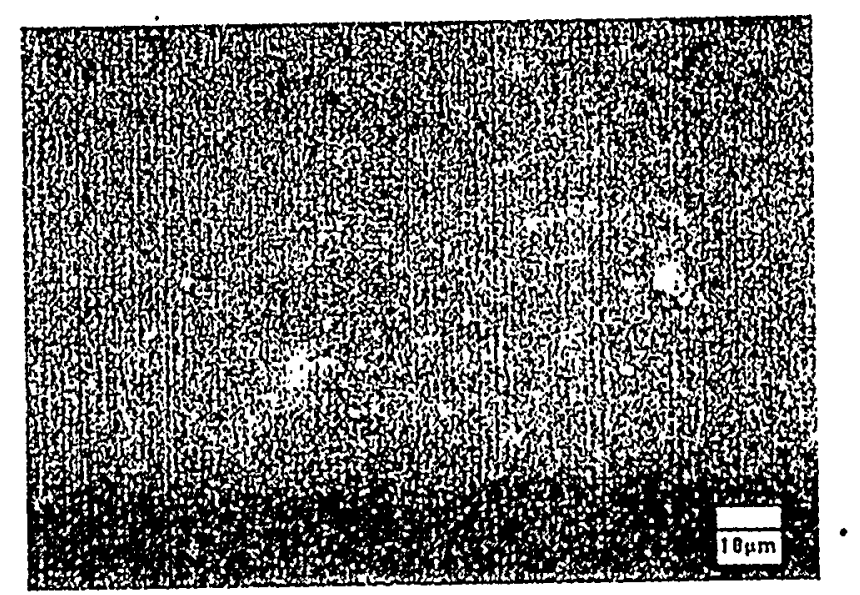

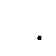




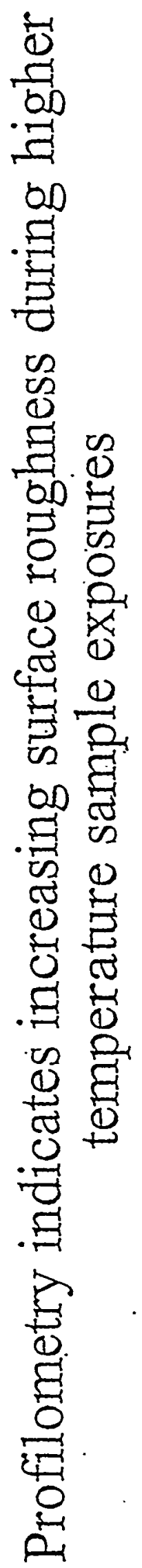

IV-56
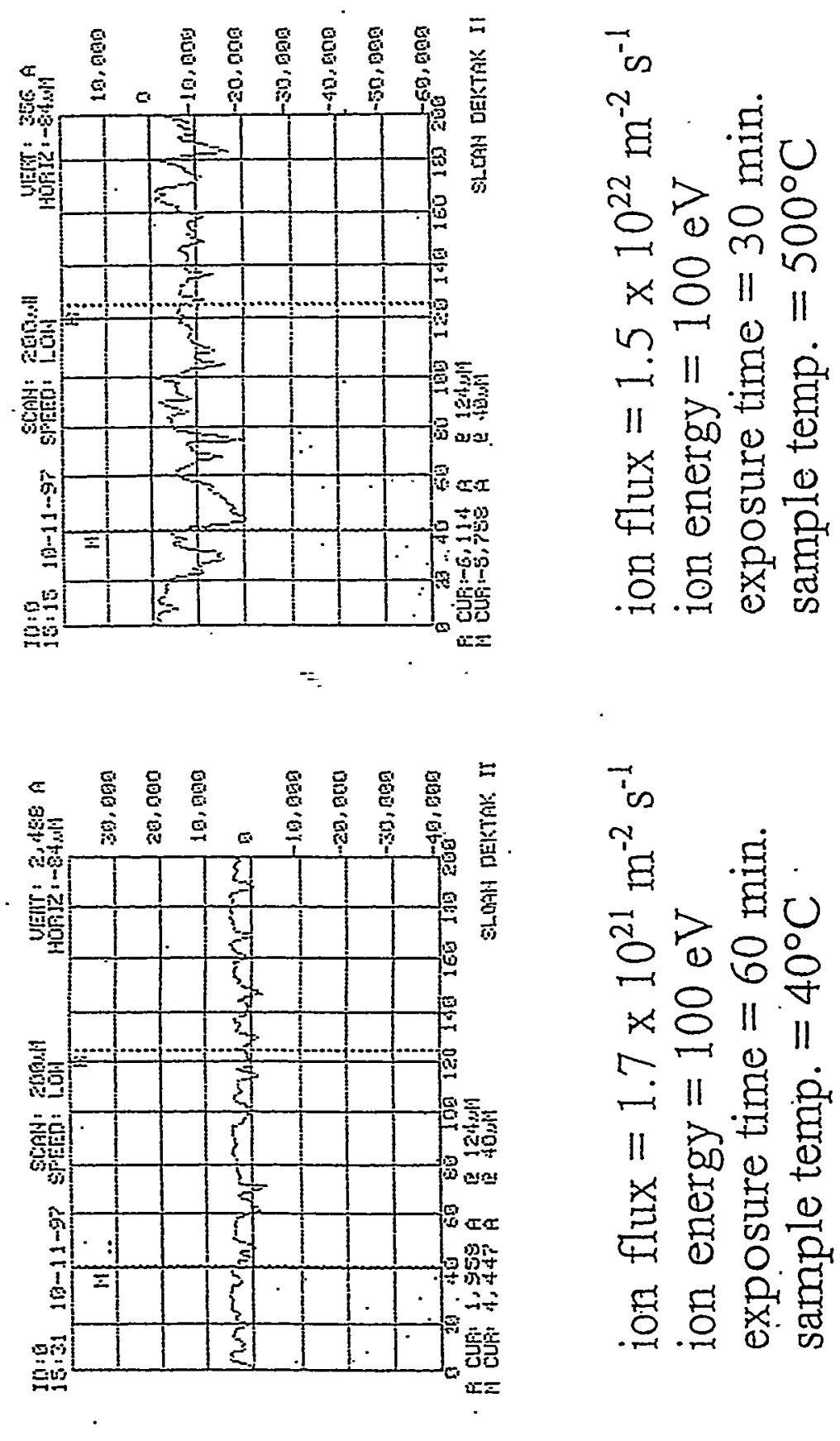

in

回, 焉

$\underset{0}{\sim}, 00$

स $\underbrace{}_{0} \| 1$

- $\stackrel{0}{0}$

आ

II 0000

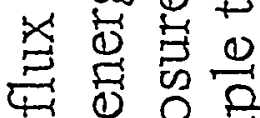

曽

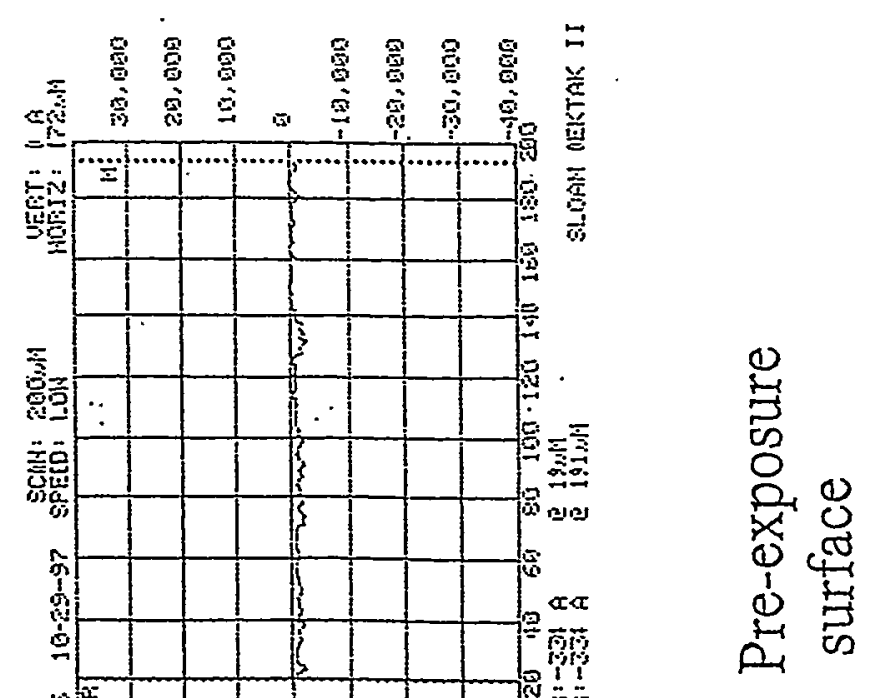


Surface damage increases withincreasing fluence during high temperature sample exposure to plasma.

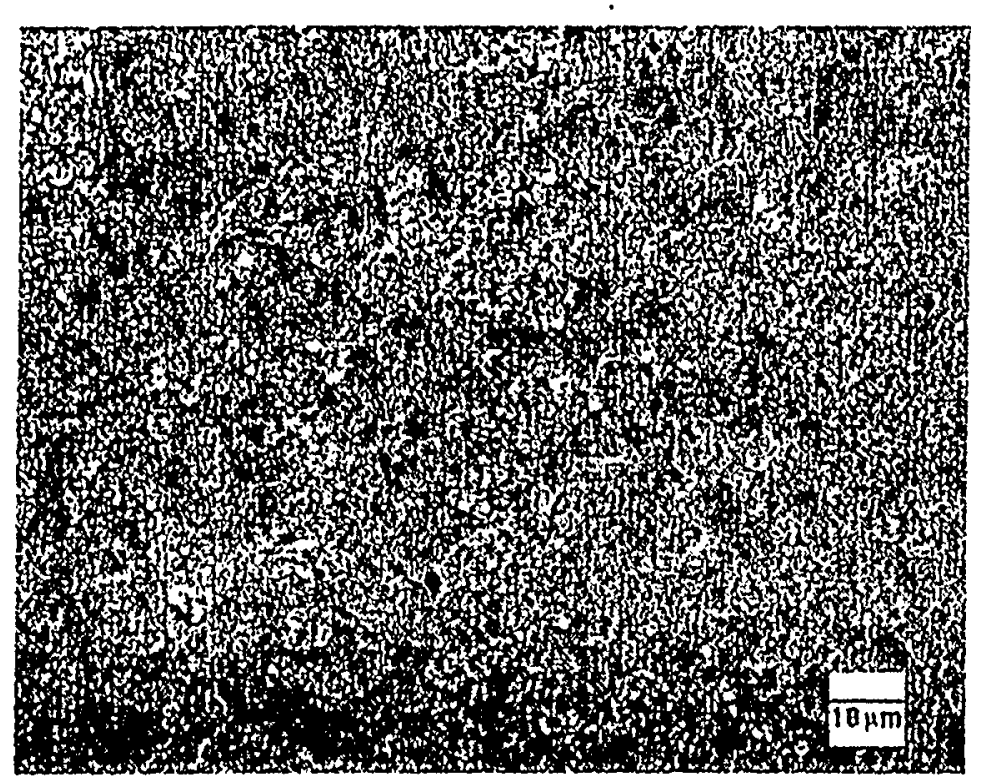

ion flux $=1.5 \times 10^{22} \mathrm{~m}^{-2} \mathrm{~s}^{-1}$ ion energy $=100 \mathrm{eV}$ exposure time $=30 \mathrm{~min}$.

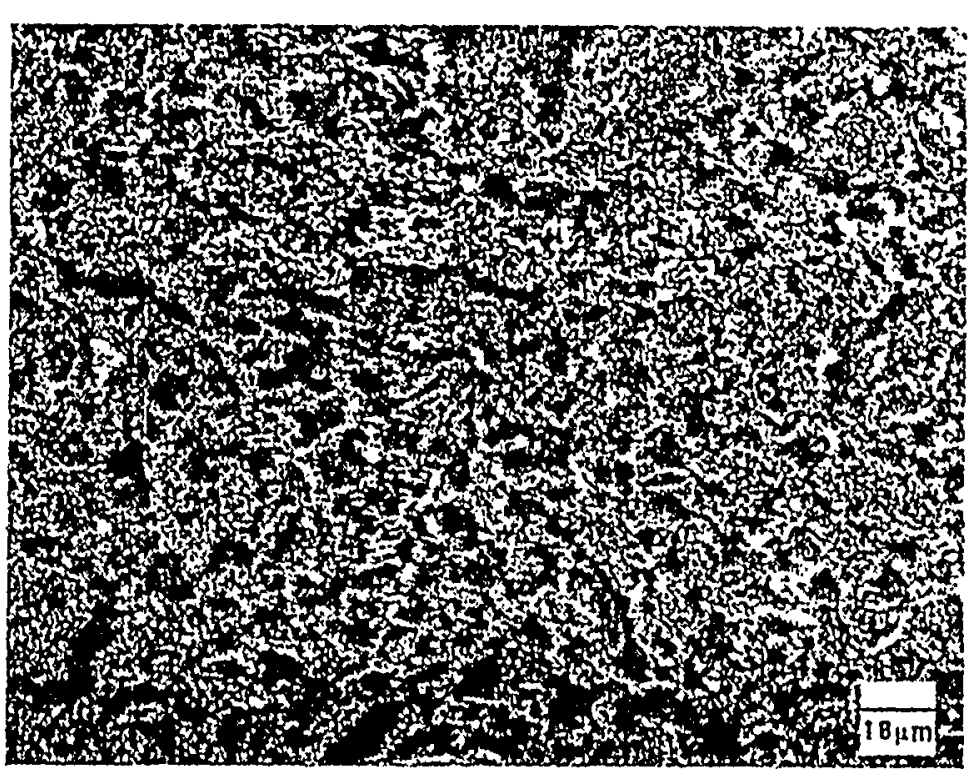

ion flux $=1.5 \times 10^{22} \mathrm{~m}^{-2} \mathrm{~s}^{-1}$ ion energy $=100 \mathrm{eV}$ exposure time $=6 \mathrm{hrs}$.

菅 

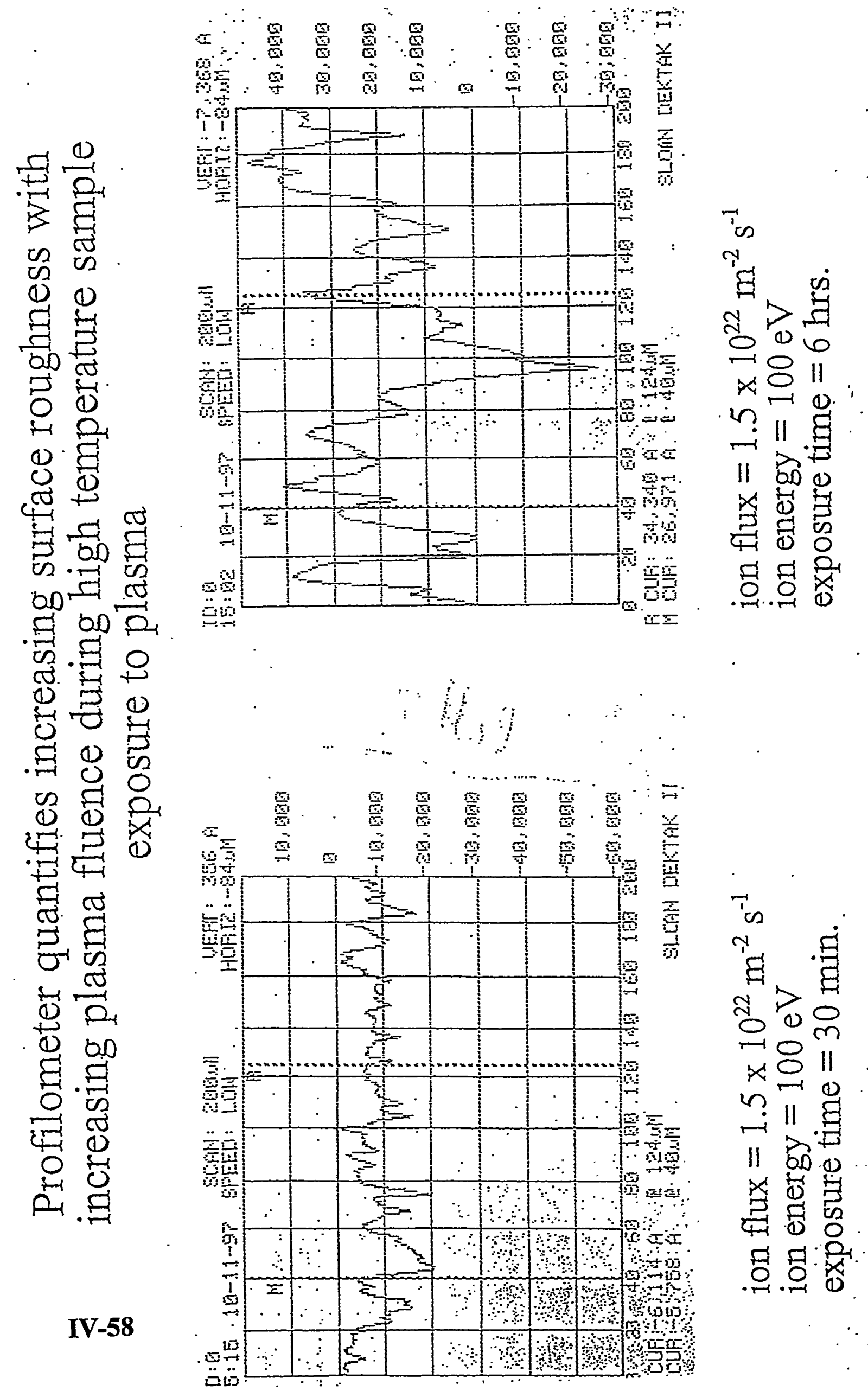

IV-58 


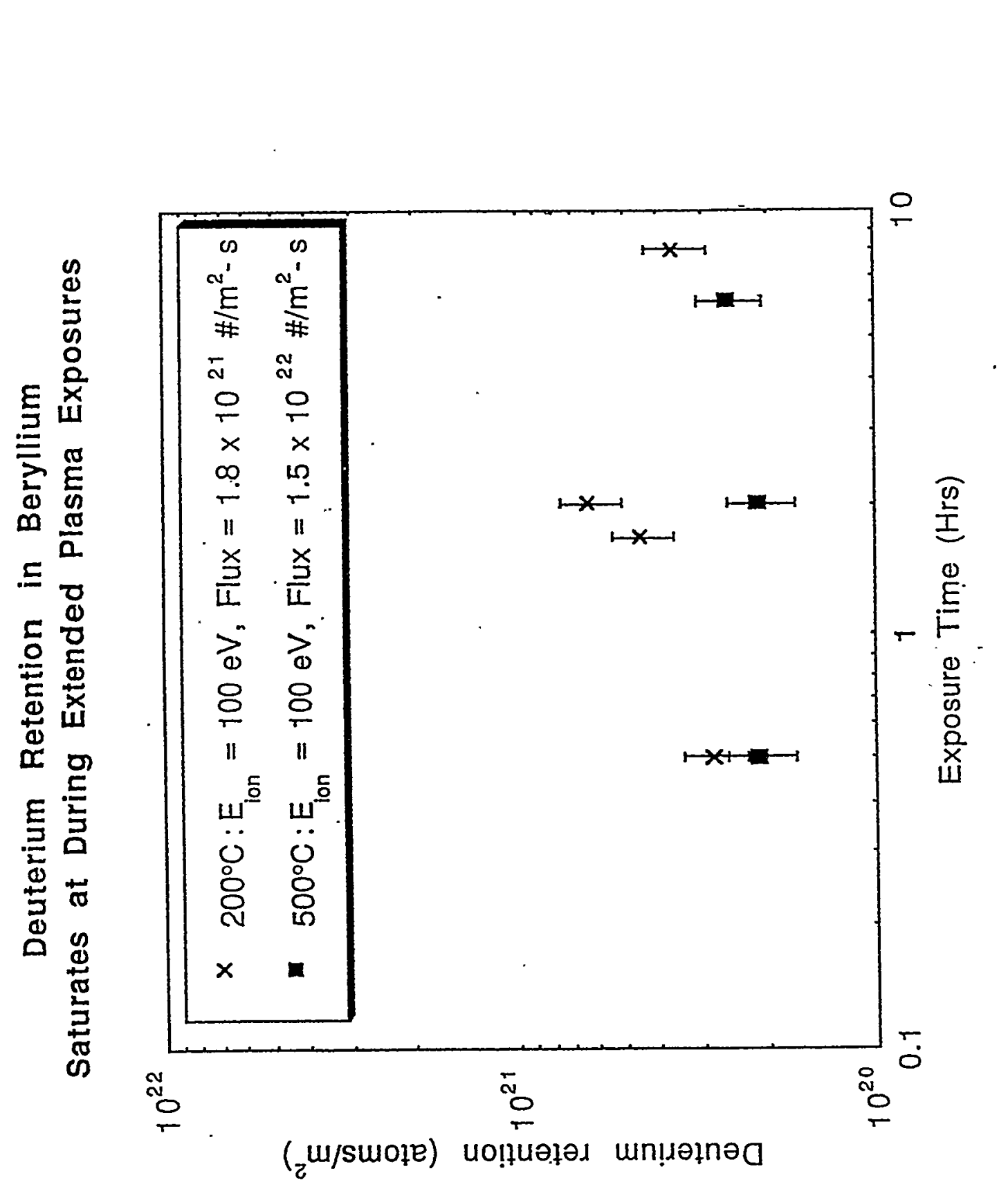




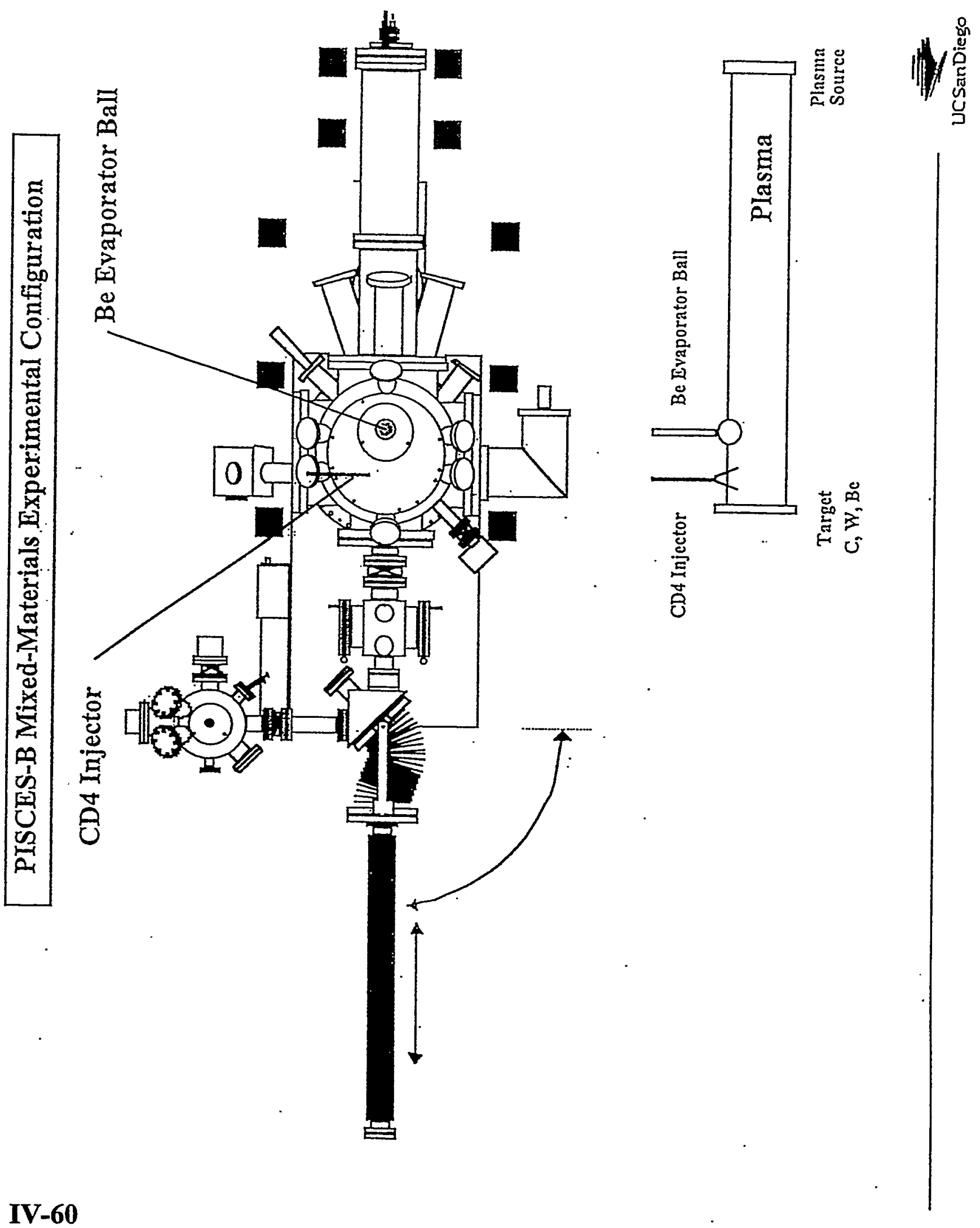




\section{嵪}

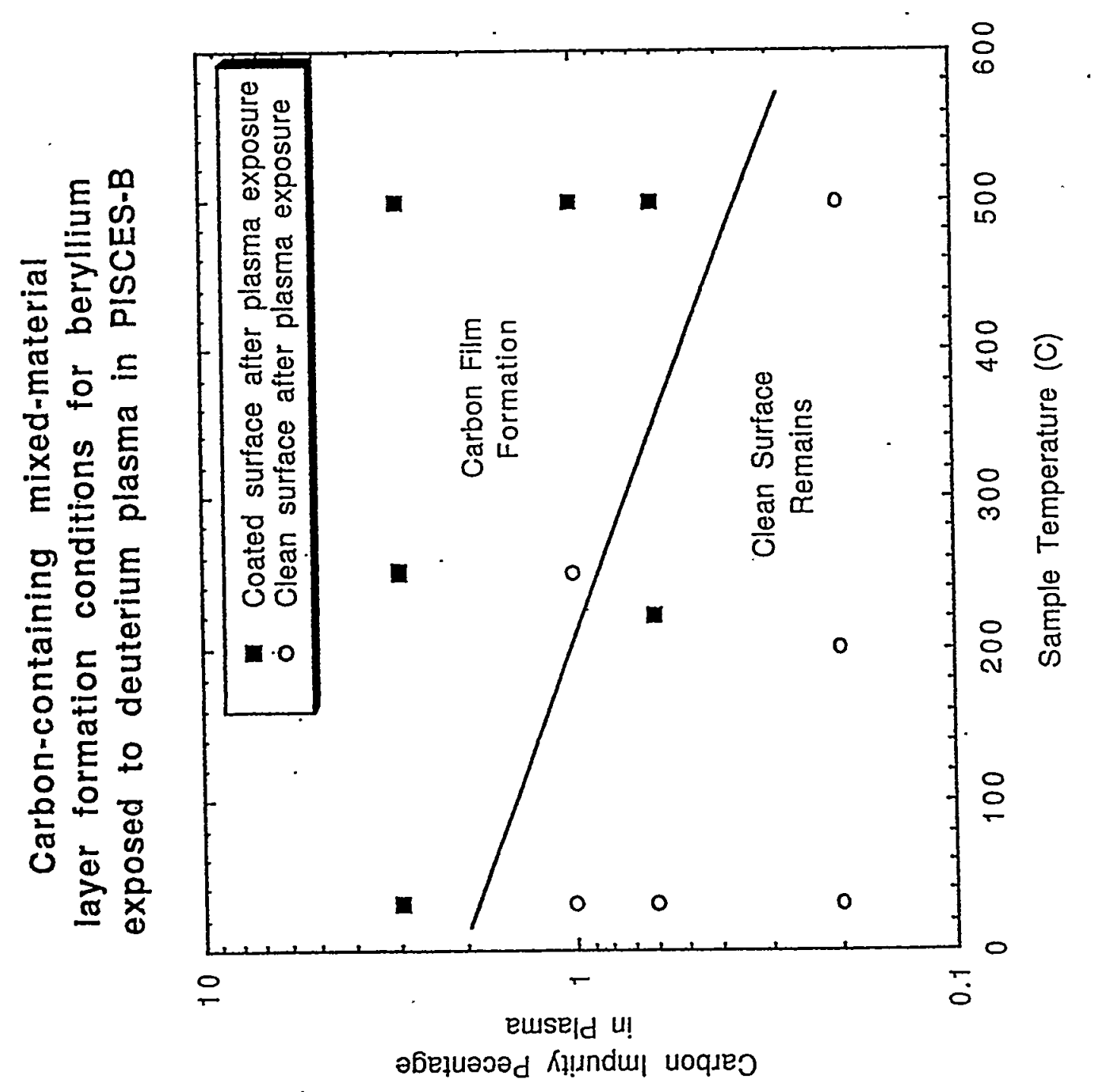

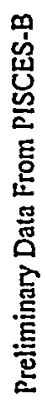




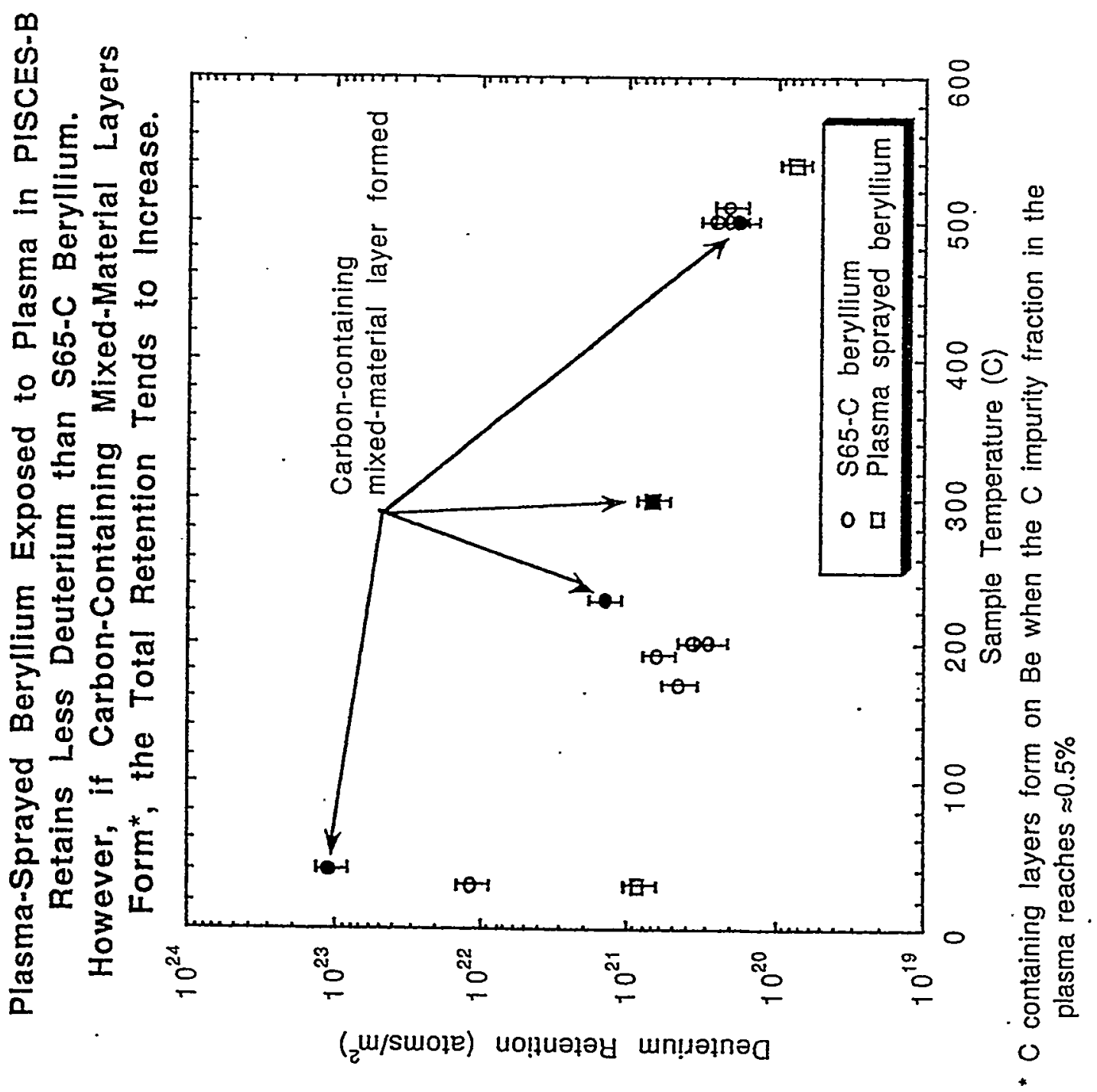




\section{Tungsten ( $1 \% \mathrm{LaO})$ exposed to $\mathrm{D}$ plasma}

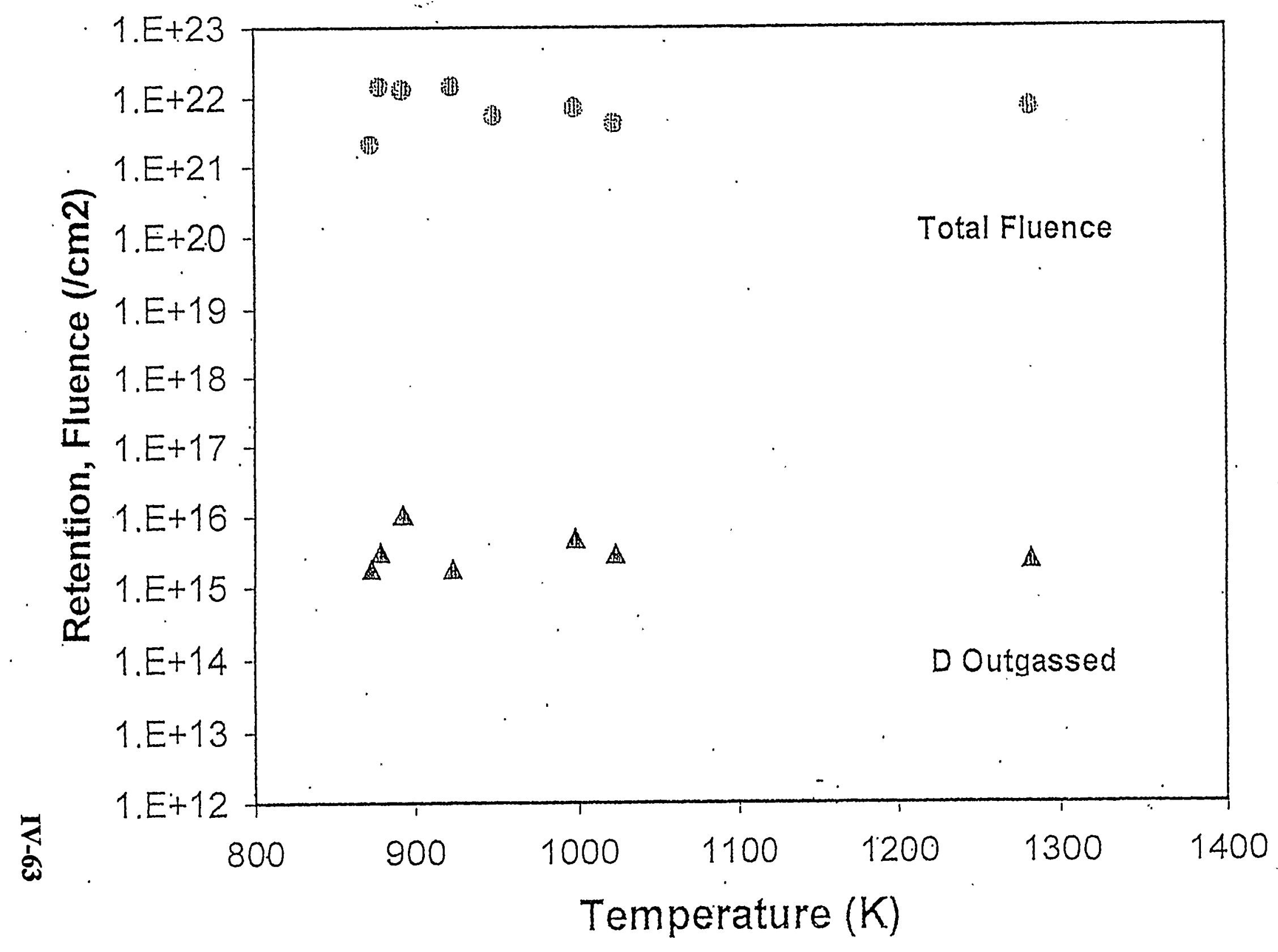




\section{Deuterium Retention in Carbon Coated W Samples}

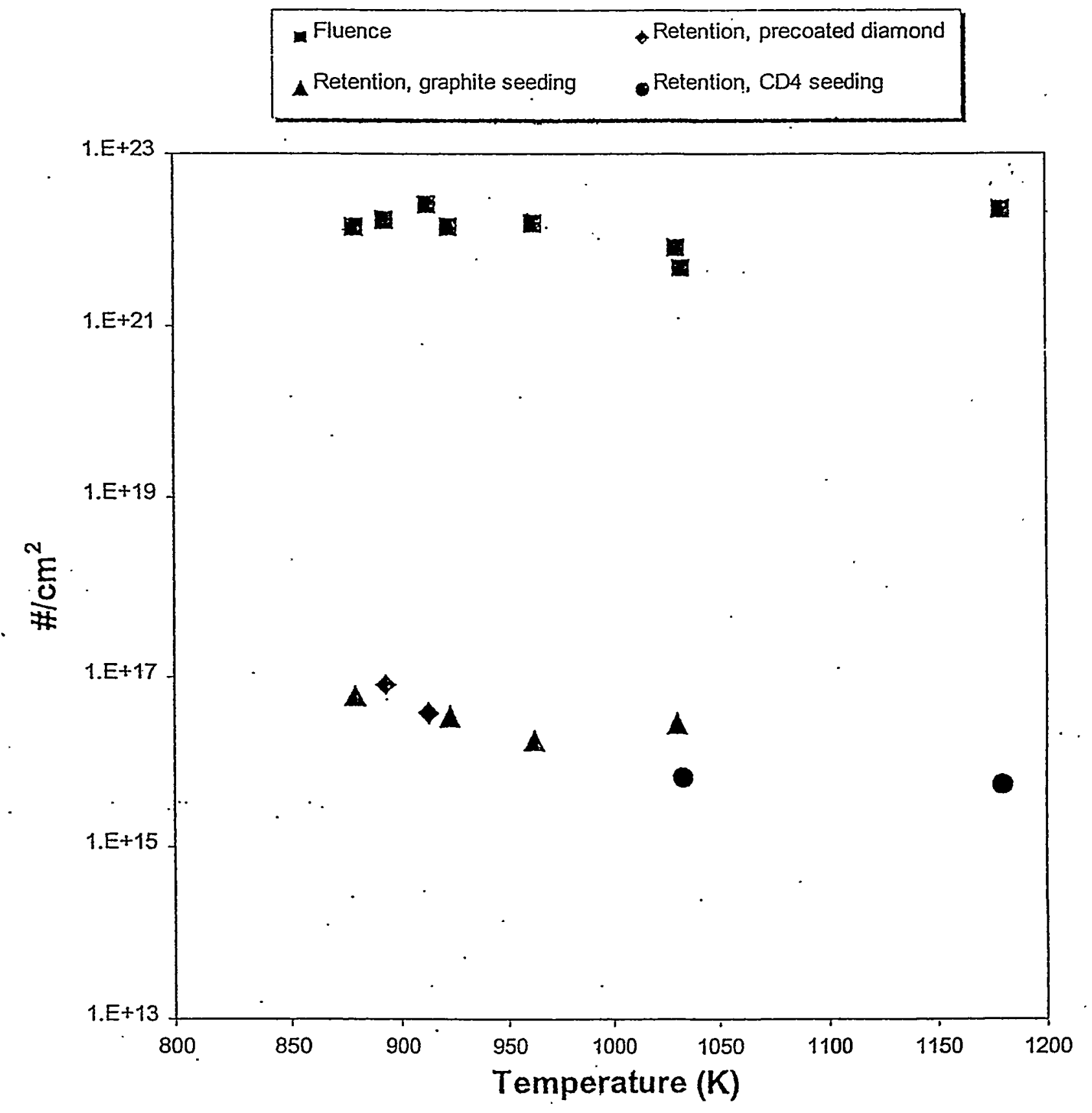




\section{Deuterium Retention in Tungsten}

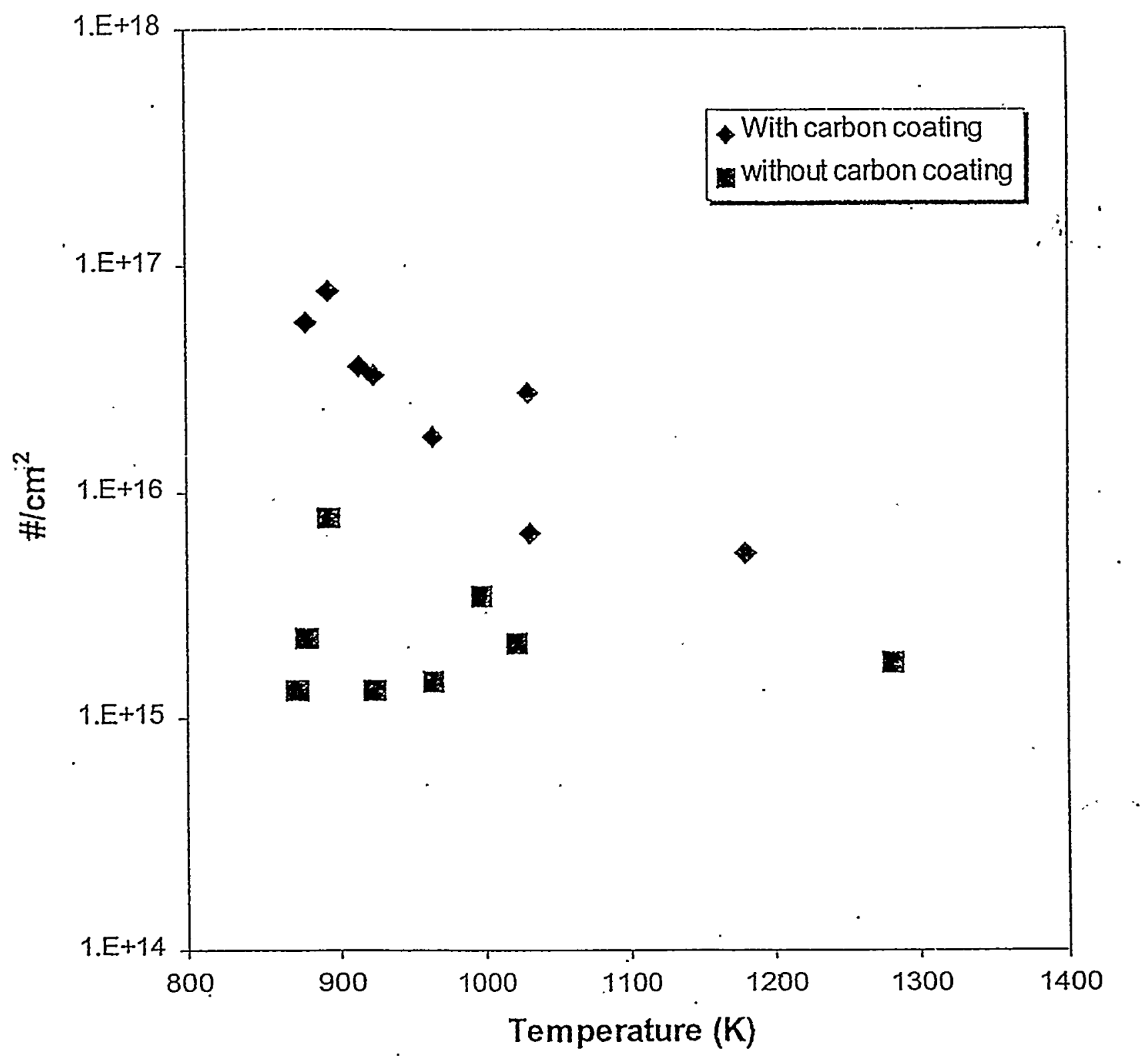




\section{Exposed Tungsten with carbon seeding}
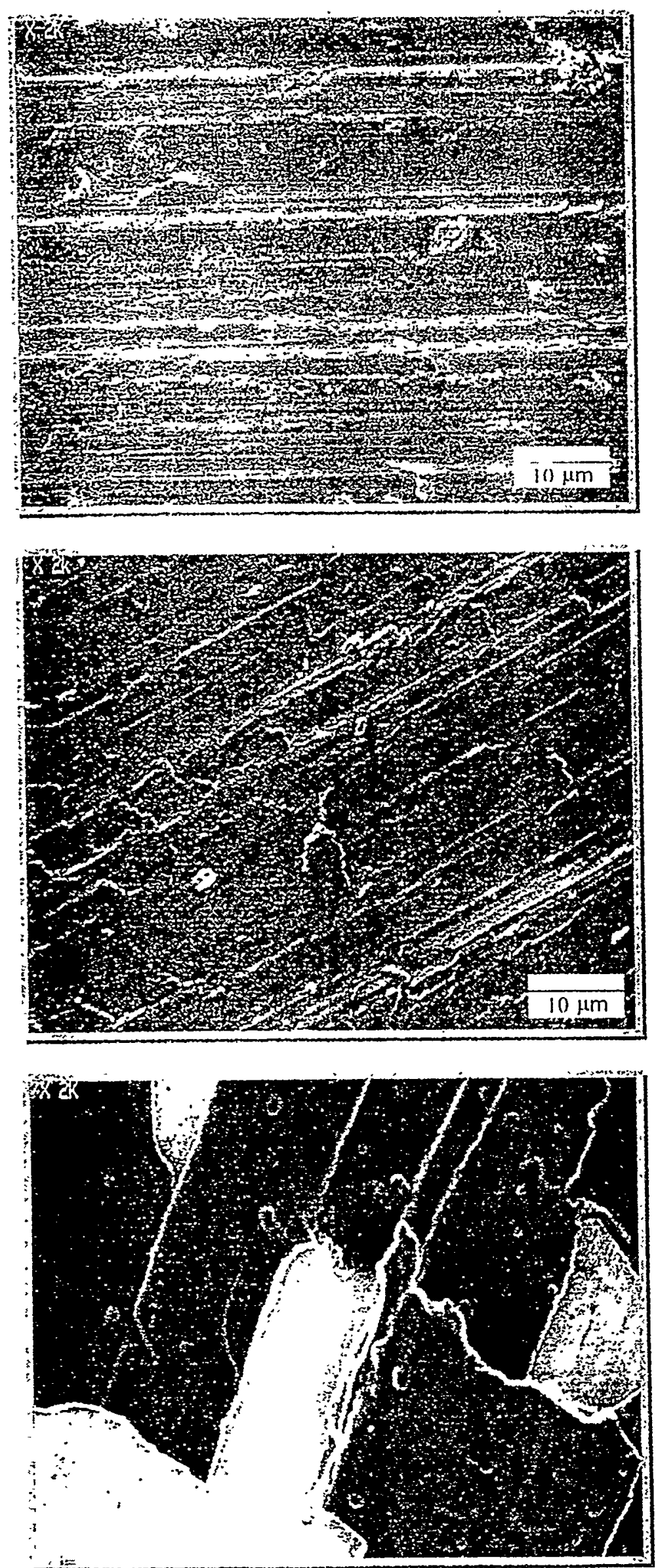

Pre-exposed Tungsten surface

Exposed tungsten surface with carbon seeding at $907 \mathrm{~K}$.

Exposed tungsten surface with carbon seeding at $1031 \mathrm{~K}$. 
Exposed Tungsten with $\mathrm{CD}_{4}$ seeding in Deuteriume asma
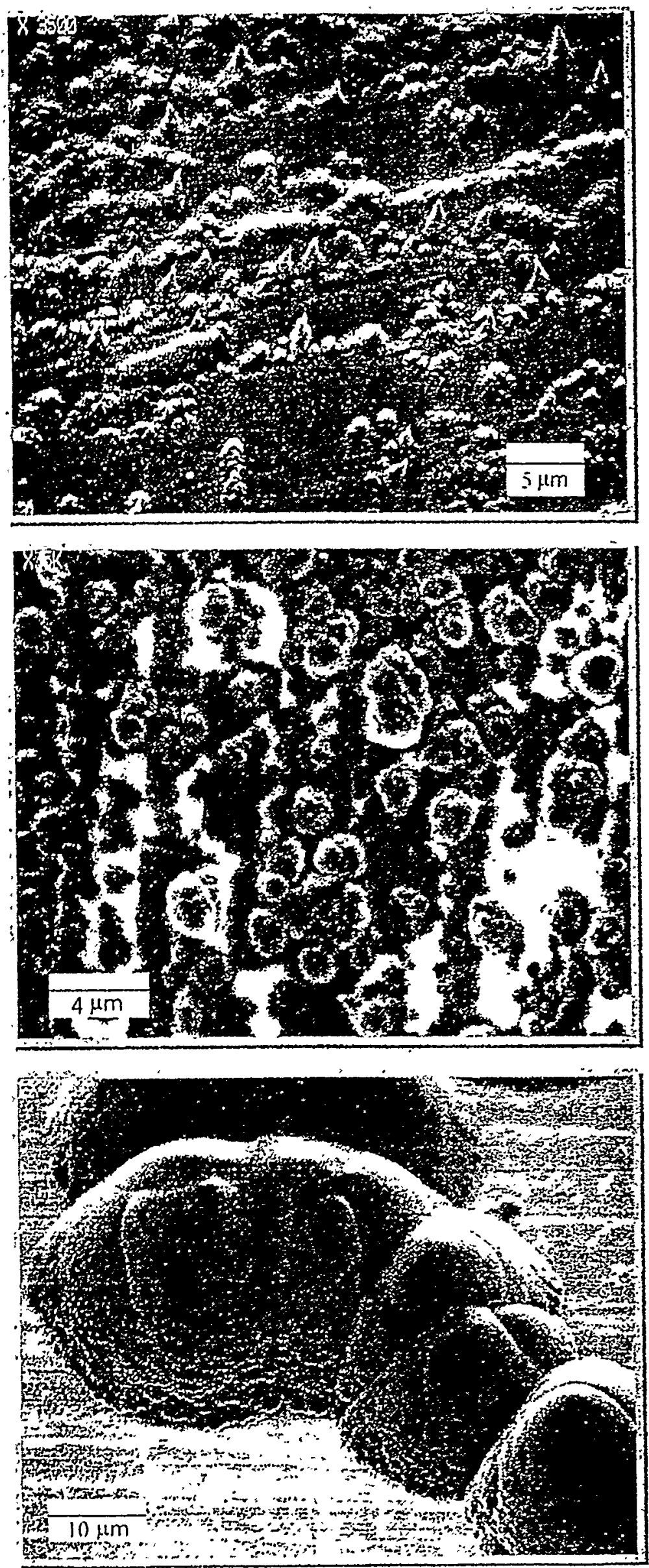

Sample temperature at $1033 \mathrm{~K}$ with $0.8 \% \mathrm{CD}_{4}$

Exposed tungsten at $1033 \mathrm{~K}$ with $1 \% \mathrm{CD}_{4}$

Exposed tungsten at $1180 \mathrm{~K}$ with $1.4 \% \mathrm{CD}_{4}$ 


\section{Conclusions \& Future Directions:}

- Net sputtering yield of 'clean' beryllium agrees with the expected sputtering yield of beryllium-oxide

- Near surface damage of beryllium results from $100 \mathrm{eV} D+$ plasma bombardment

- damage extends well beyond implantation depth

- surface temperature effects damage formation

- Deuterium retention in plasma-sprayed beryllium is less than press-sintered beryllium

- temperature dependent reduction

Impurity injection experiments are underway in PISCES-B to study mixedmaterial layers

- Determine formation conditions and growth rates for mixed-material layers on different substrate materials

- Carbon-containing layers can have a drastic impact on deuterium retention

- Investigate the role of metallic impurities incorporated in mixed-material layers 


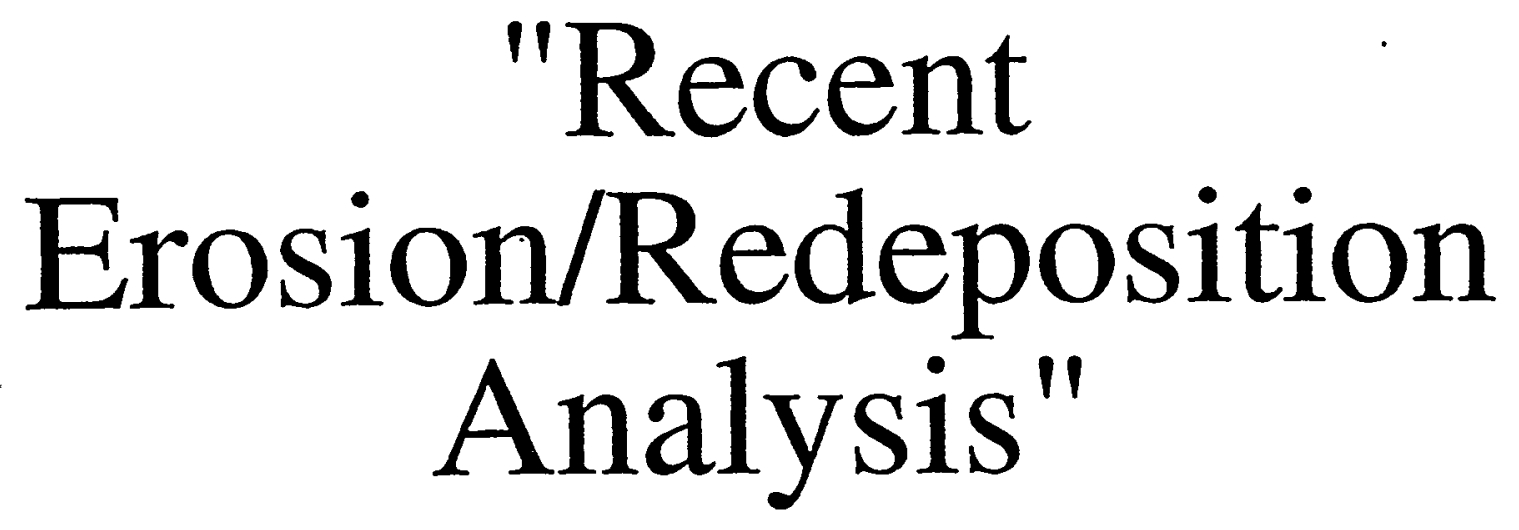

J. N. Brooks/D. K. Sze Argonne National Laboratory Argonne IL, USA

US-Japan Workshop on

High Heat Flux Components \& Plasma Surface Interactions for Next Fusion Devices, San Francisco, December 8-11, 1997 


\section{EROSION/REDEPOSITION ANALYSIS}

PERSONNEL: J.N. Brooks, A. Hassanein

COLLABORATIONS: D. Ruzic et al. (UIUC), F. Federici et al. (ITER/JCT), D Whyte et al. (GA), R. Bastasz, R. Causey et al. (SNL), Y. Hirooka et al (PISCES).

GOAL: use, develop, and validate models/codes to predict:

1) Sputtering-erosion-limited plasma facing component lifetime

2) Impurity transport and plasma contamination

3) Tritium co-deposition

4) Heat deposition and related sheath phenomena

\section{CURRENT ACTIVITIES:}

1) ITER Vertical Divertor and First Wall erosion/ codeposition analysis

2) DIII-D/DiMES modeling and code validation

3) ALPS (just starting), JET modeling (just starting)

4) Theory and code development; maintenance of ANL Integrated Erosion Code Center capability. 


\section{DIII-D/DiMES Code Validation; D79 Brooks/Whyte}

- DiMES 79 experiment - ELM'ing $\mathrm{H}$ mode attached plasma

- Carbon divertor analyzed with REDEP/WBC codes

- Code output compared with data:

1) predicted carbon net erosion rate compared with probe erosion/redeposition data.

2) predicted core plasma contamination compared with core measurement data.

3 ) predicted gross carbon erosion rate compared with photon data.

- Code/data comparison is good. 


\section{REDEP/WBC Code Matches the Features of Carbon Net Erosion at the DIII-D Divertor as Measured by DiMES}
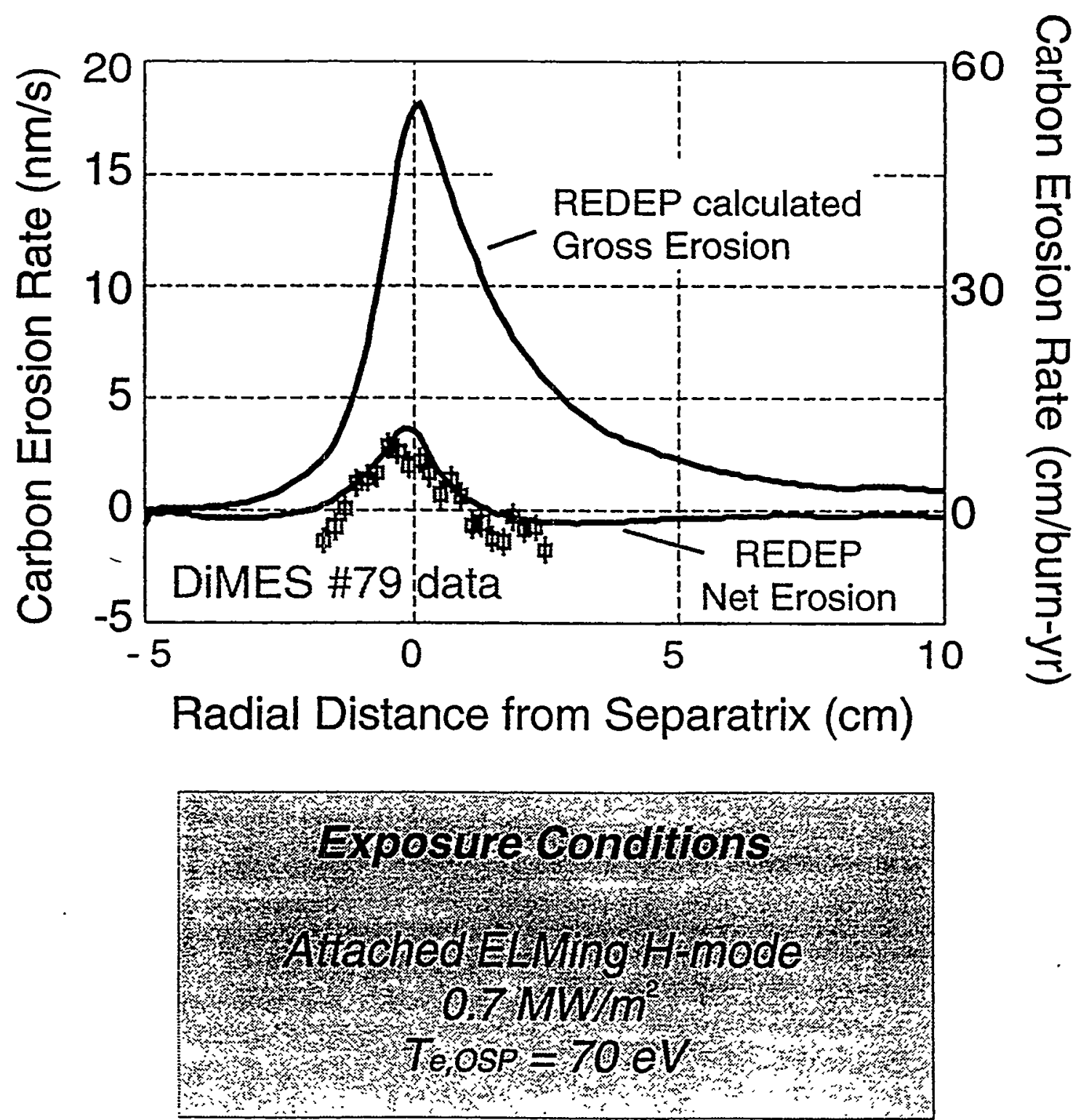

[i] Sandia National Laboratories
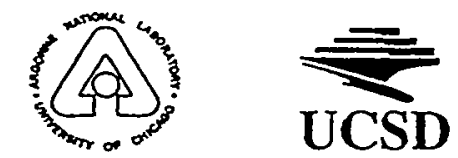


\section{Erosion/Redeposition Analysis of ITER}

- REDEP/WBC/DEGAS+/VFTRIM codes used to compute physical and chemical sputtering of carbon divertor plate and beryllium first wall for "semidetached" plasma solution (Kukushkin et al.)

- Tritium codeposition is computed using surface temperature dependent $\mathrm{H} / \mathrm{C}, \mathrm{H} / \mathrm{Be}$ trapping ratios.

- Methane-only chemical sputtering analyzed, due to lack of data/models for higher hydrocarbon atomic and molecular cross sections. (Future work will examine higher hydrocarbon transport).

- Dominating process for net chemical erosion and codeposition is formation of carbon atom from complex molecular transport, and subsequent non-local redeposition due to low electron temperature in semidetached plasma region. 
REDEP analysis, ITER carbon vertical target.

Semi-detached plasma.

Physical sputtering only.

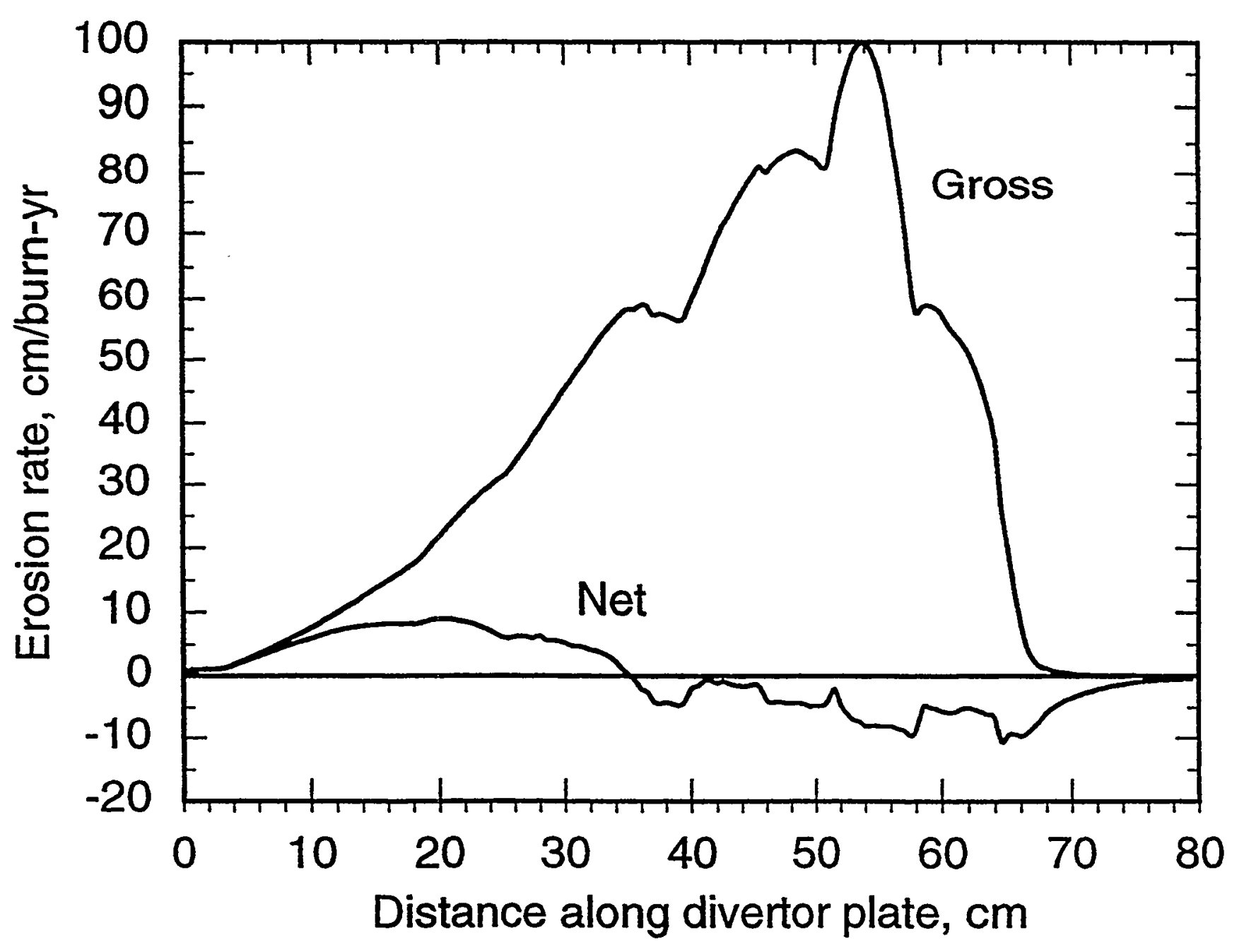


WBC analysis, ITER carbon vertical target. Semi-detached plasma.

Chemical sputtering only.

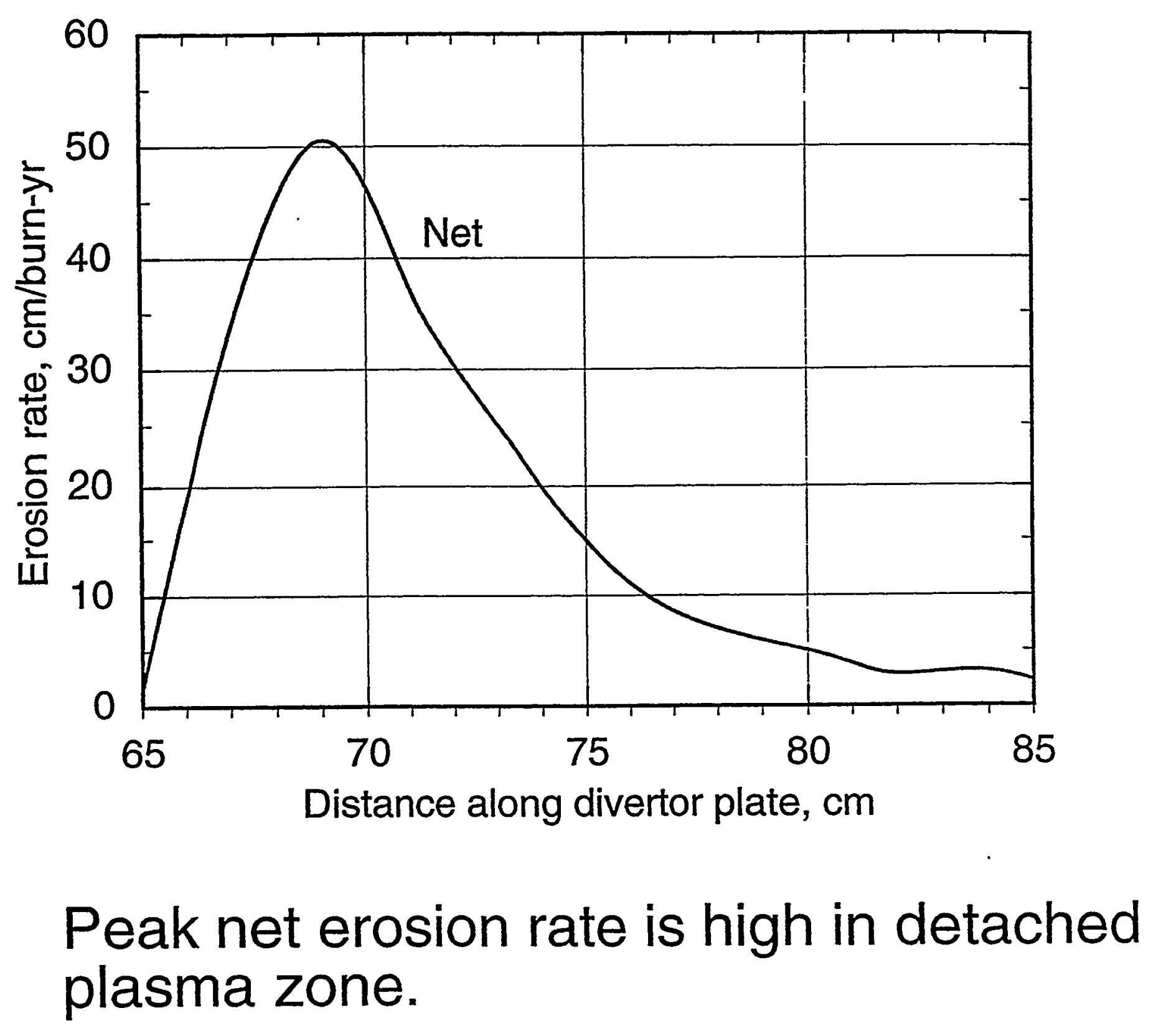




\section{ITER Erosion/Redeposition Analysis- Results for semi-detached edge plasma regime ("Case 98" , 1\% Neon). (Preliminary results- numerous models to be upgraded).}

- Peak net erosion rate, (pure) carbon coated divertor: 50 $\mathrm{cm} /$ burn-yr

- Average net erosion rate, beryllium coated first wall: $0.05 \mathrm{~cm} / \mathrm{burn}-\mathrm{yr}$

- Peak net erosion rate, beryllium, due to gas-puffing: very high-implies need for more spread out gas puffing

- Tritium codeposition rate in redeposited carbon: 10.1 $\mathrm{g} / 1000 \mathrm{~s}$ pulse

- Tritium codeposition rate in redeposited beryllium: $\sim 0.1$ $\mathrm{g} / 1000 \mathrm{~s}$

- Conclusions: Semi-detached regime is better than fully detached regime, net carbon erosion and tritium codeposition is probably acceptable for ITER low dutyfactor operation 


\section{Dependence of graphite erosion yield on irradiation flux close to actual edge plasma condition}

Y. Ueda, Y. Ohtsuka, M. Isobe, M. Nishikawa

Osaka University, Japan

(Presented by Y. Ueda)

December 8-11, 1997 at Warwick Regis Hotel

US-Japan workshop on High Heat Flux Components and Plasma Surface Interactions for Next Fusion Devices

\section{Outline}

1. Background of this work

2. Experimental setup

- high flux irradition test stand

- temperature control

3. Experimental results of RES of graphite

4. Discussion on $\mathrm{RES}$ in high flux regime

5. Summary and future plan 


\section{Background 1}

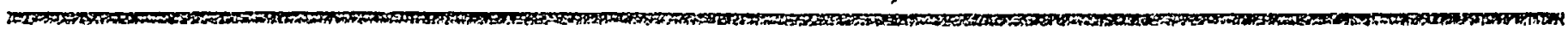

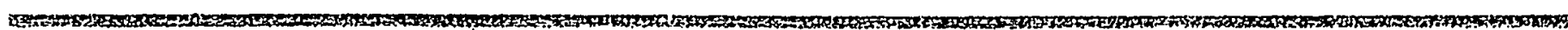

\section{Erosion Process Unique to Graphite}

- Radiation Enhanced Sublimation (RES)

Enhanced erosion of graphite over $1200 \mathrm{~K}$ by any

energetic particle impact

- Chemical Sputtering

Enhanced erosion of graphite around $800 \mathrm{~K}$ by

energetic hydrogen and oxygen impact

\section{Erosion of Graphite under High Heat Flux Condition in Tokamak Devices \\ - $\underline{\mathrm{RES}}$}

TEXTOR : not observed [1]

JET : not observed clearly [2]

TFTR : slightly observed over $1900 \mathrm{~K}$ [3]

- Chemical

JET : not observed clearly [4]

JT-60 : not observed clearly [5]

[1] V. Philipps et al., Nucl. Fusion 33 (1993) 953.

[2] R. Reichle et al., J. Nucl. Mater. 176\&177 (1990) 375.

[3] A. Ramsey et al., Nucl Fusion 31 (1991) 1811.

[4] K. H. Behringer, J. Nucl. Mater. 145-147 (1987) 574.

[5] H. Kubo et al., J. Nucl. Mater. 196-198 (1992) 71. 


\section{Background 2}

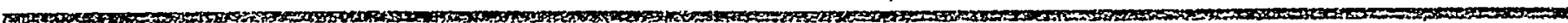

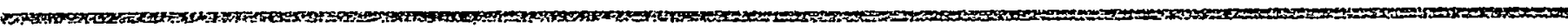

\section{Complex Situation for PFC}

- High (Heat - Particle) Flux

- Many Species of Ions

Proton, Deuteron, Triton

Helium ash

Low $\mathrm{Z}$ materials $(\mathrm{Be}, \mathrm{B}, \mathrm{C})$

High Z (W)

- Redeposition

- Energy Distribution

Maxwellian plasma ions

Fast Neutral

Ripple Loss Ion

- Angular Distribution

- High Fluence Irradiation 


\section{Aims of This Work}

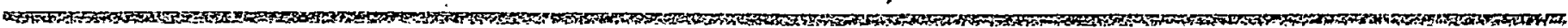

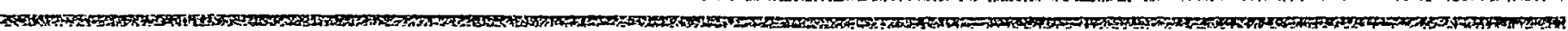

\section{Detailed Database of Graphite Erosion by}

High Flux Beam Irradiation

- Dependence on irradiation flux

- Dependence on angle of incidence

- Dependence on graphite types

(isotropic, pyrolytic etc.)

\section{Models for Graphite Erosion Applicable} to Actual PFM Conditions

- Lifetime estimation of graphite PFC

- Proposal of favorable edge plasma conditions 


\section{High Flux Irradiation Test Stand}

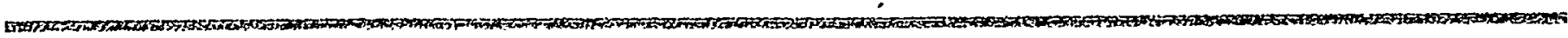

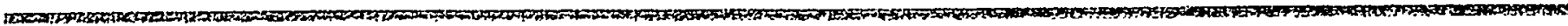

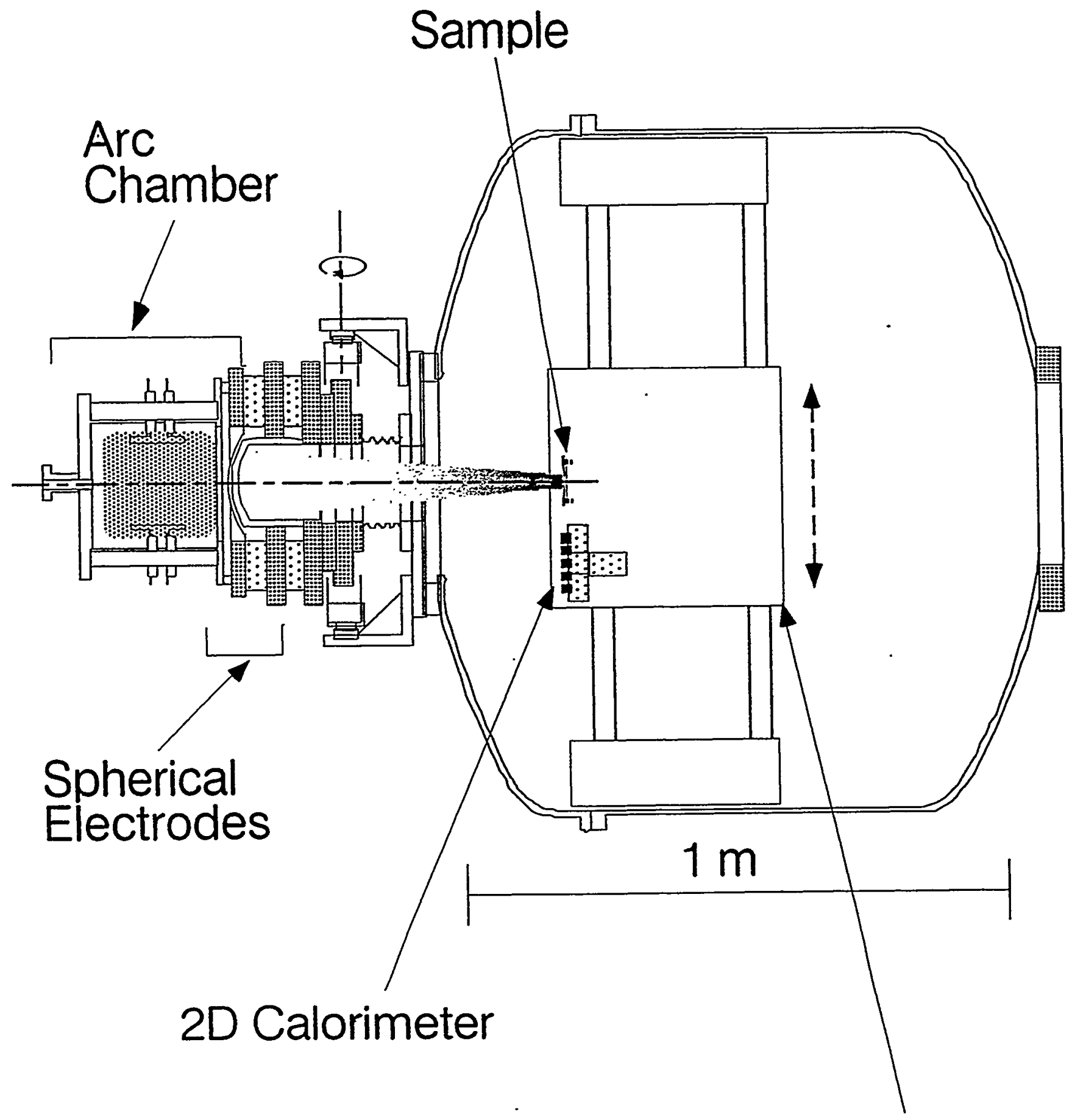

Sliding Base 


\section{Advantages of Our Device (compared with Plasma Simulator)}

Control of irradiation angle

- Angular Dependence

Multi-beam irradiation

- Multi-species Irradiation (D+C, D+He, and so on)

- Irradiation with Different Energy

Detailed diagnostics of sputtered particle

- Separation of Beam Source and Samples

- Direct Measurements of Sputtered Particles

\section{Disadvantages}

- Difficulty in producing very low energy $(\leq 100 \mathrm{eV})$ and high flux $\left(>10^{21} \mathrm{~m}^{-2} \mathrm{~s}^{-1}\right)$. 


\section{Beam Specification}

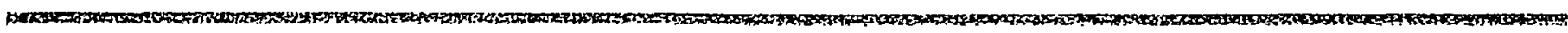

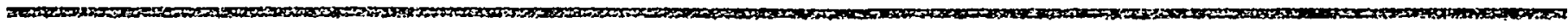

\section{Ion Source}

- Bucket source

\section{Arc discharge with thermionic cathodes}

- Electrodes

Spherical electrode

Effective diameter : $14 \mathrm{~cm}$

Radius of curvature : $50 \mathrm{~cm}$

- Gas : $\mathrm{D}_{2}: 5$ - 10 mTorr

$$
\text { Ar: } 5 \text { mTorr }
$$

\section{Beam Characteristics}

- Energy : $5 \mathrm{keV}$

- Power density : $3.5 \mathrm{MW} / \mathrm{m}^{2}$ (D)

$$
1.2 \mathrm{MW} / \mathrm{m}^{2} \text { (Ar) }
$$

- Flux : $1.0 \times 10^{22} \mathrm{D} / \mathrm{m}^{2} \mathrm{~s}$

$$
1.5 \times 10^{21} \mathrm{Ar} / \mathrm{m}^{2} \mathrm{~s}
$$

- Pulse length : $4 \mathrm{sec}$ 


\section{Sample Holder}

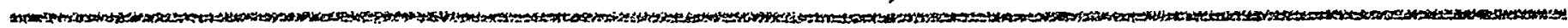

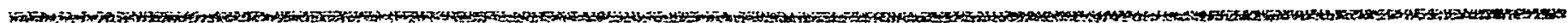

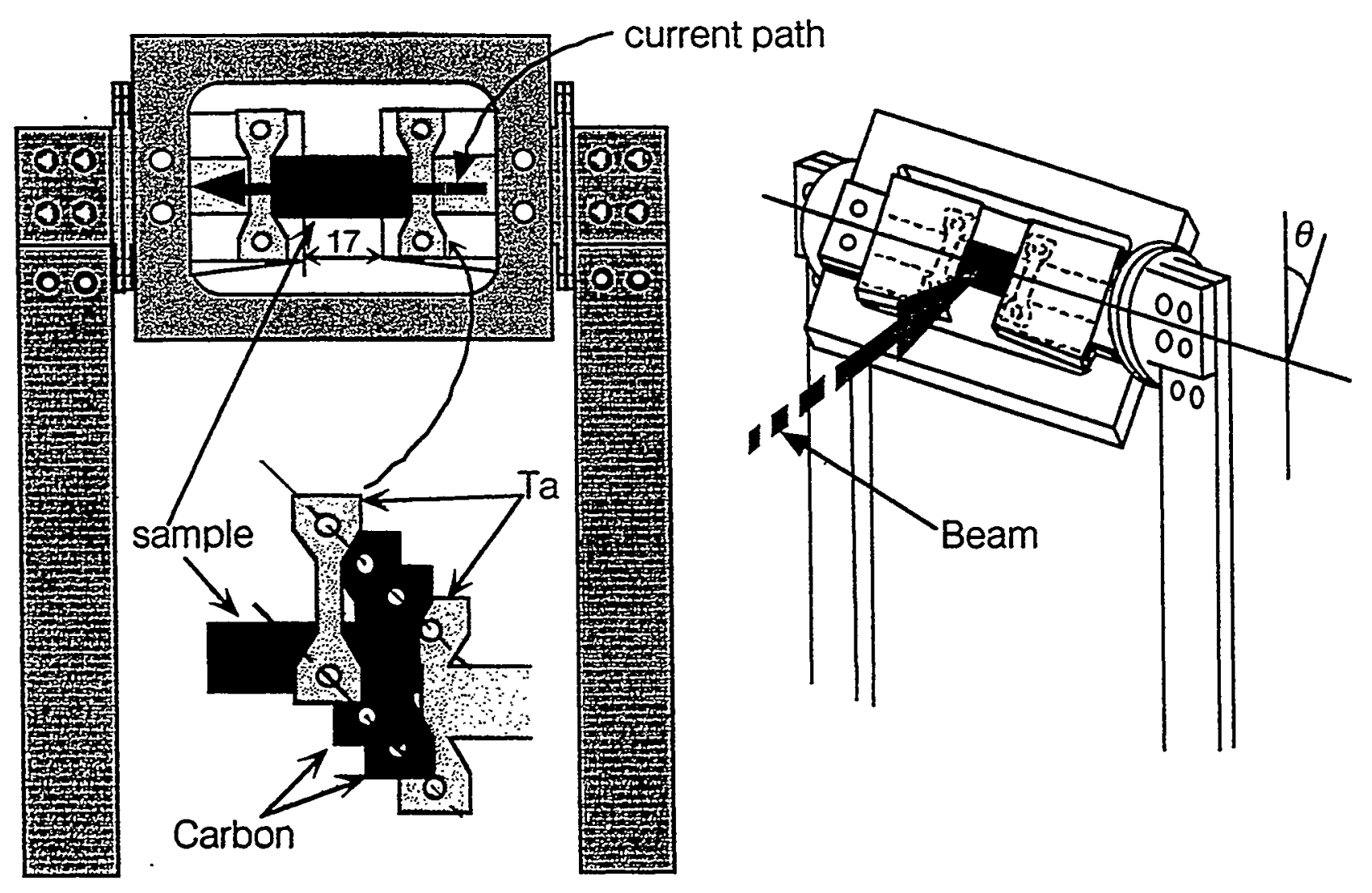

\section{Sample}

Isotropic graphite (IG-430, ISO-630 [Toyo Tanso])

Pyrolytic graphite [Union Carbide]

Doped Graphite (RG-Ti [NIIGrafit])

\section{Sample Size}

$$
(8 \sim 10) \times 40 \times(0.1 \sim 0.2)^{t} \mathrm{~mm}^{3}
$$

Irradiation Angle

IV-840 deg $\sim 75 \mathrm{deg}$ 


\section{Temperature Control}

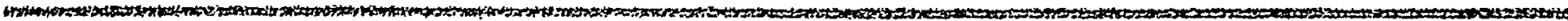

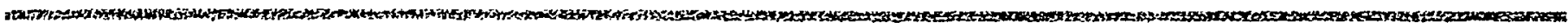

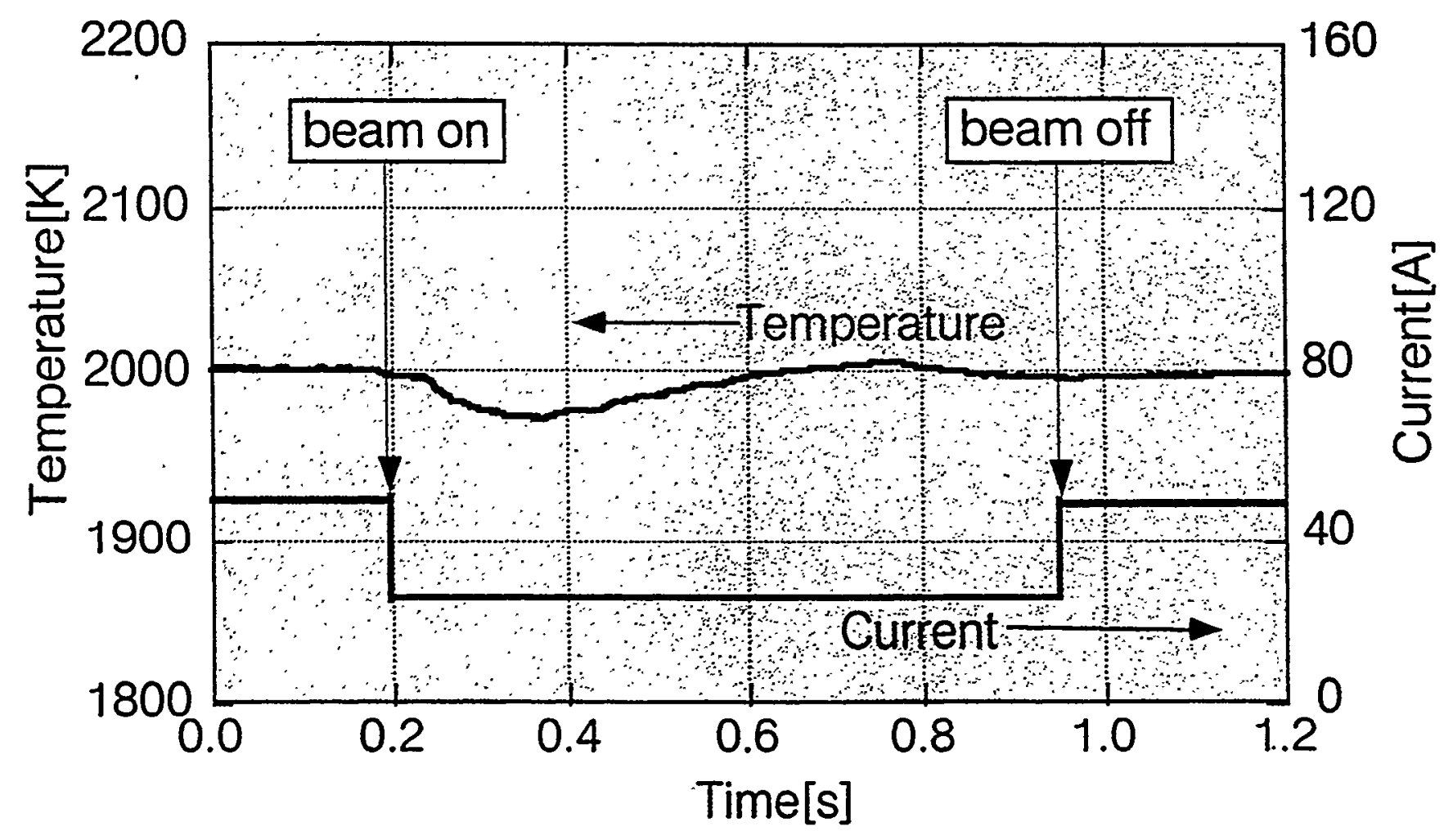

Beam Power : $0.96 \mathrm{MW} / \mathrm{m}^{2} 、 5 \mathrm{keV}$ Ar

Sample : Isotropic Graphite (ISO-630) $40 \times 10 \times 0.2 \mathrm{~mm}^{3}$

Surface temperature of samples was controlled by changing heating current during beam irradiation. 


\section{Temperature dependence}

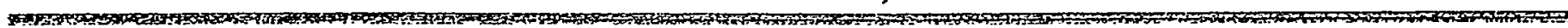

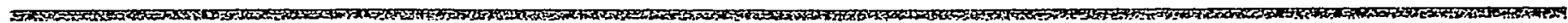

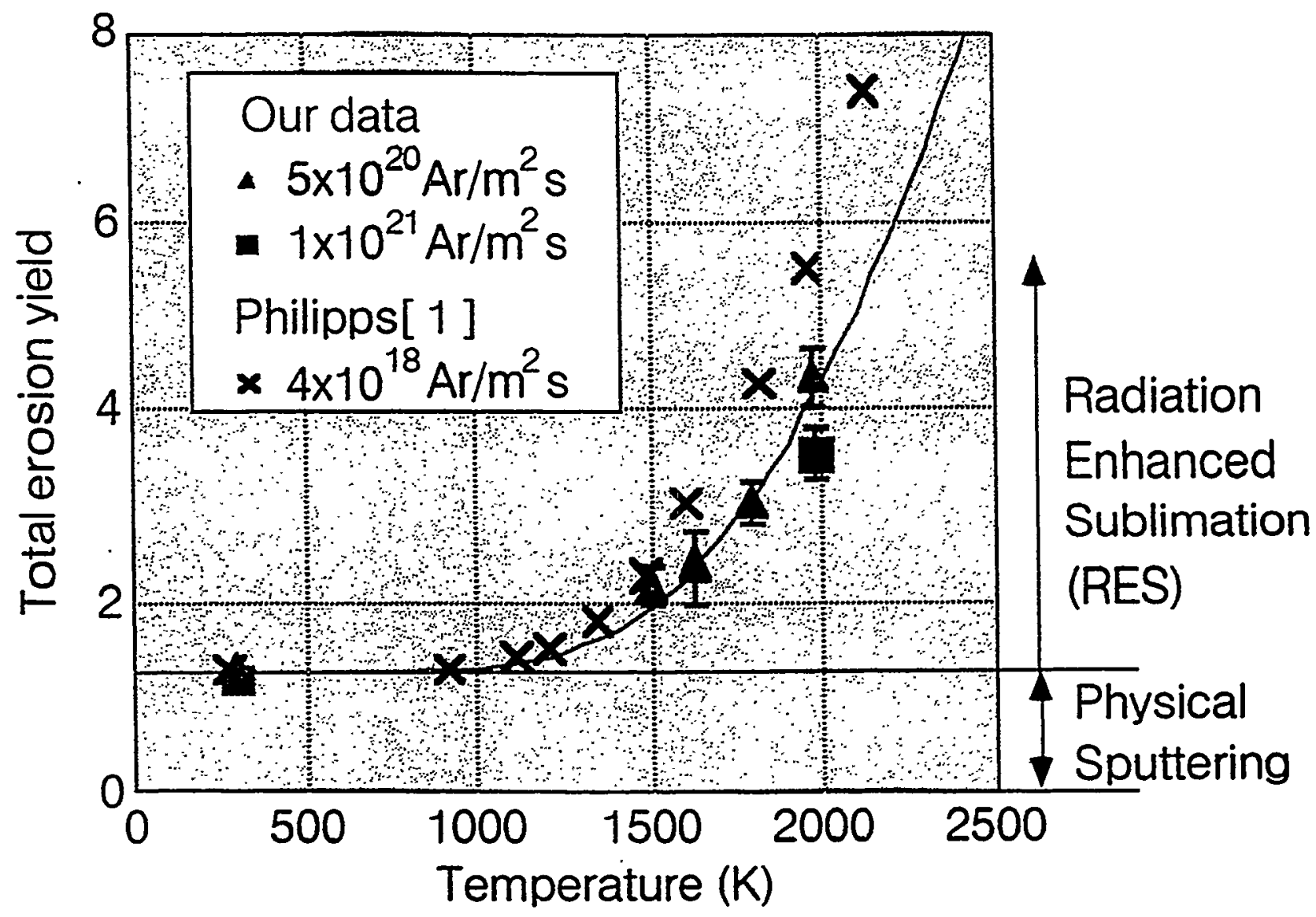

Beam : $5 \mathrm{keV} \mathrm{Ar}$

Sample : Isotropic Graphite (ISO-630) Irradiation Angle : $0 \mathrm{deg}$

[1] Philipps et al., J. Nucl. Mater. 155-157 (1988) 319. 


\section{Flux Dependence}

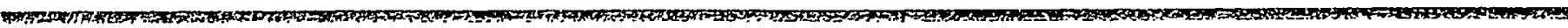

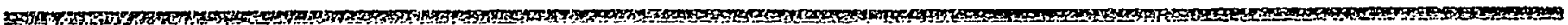

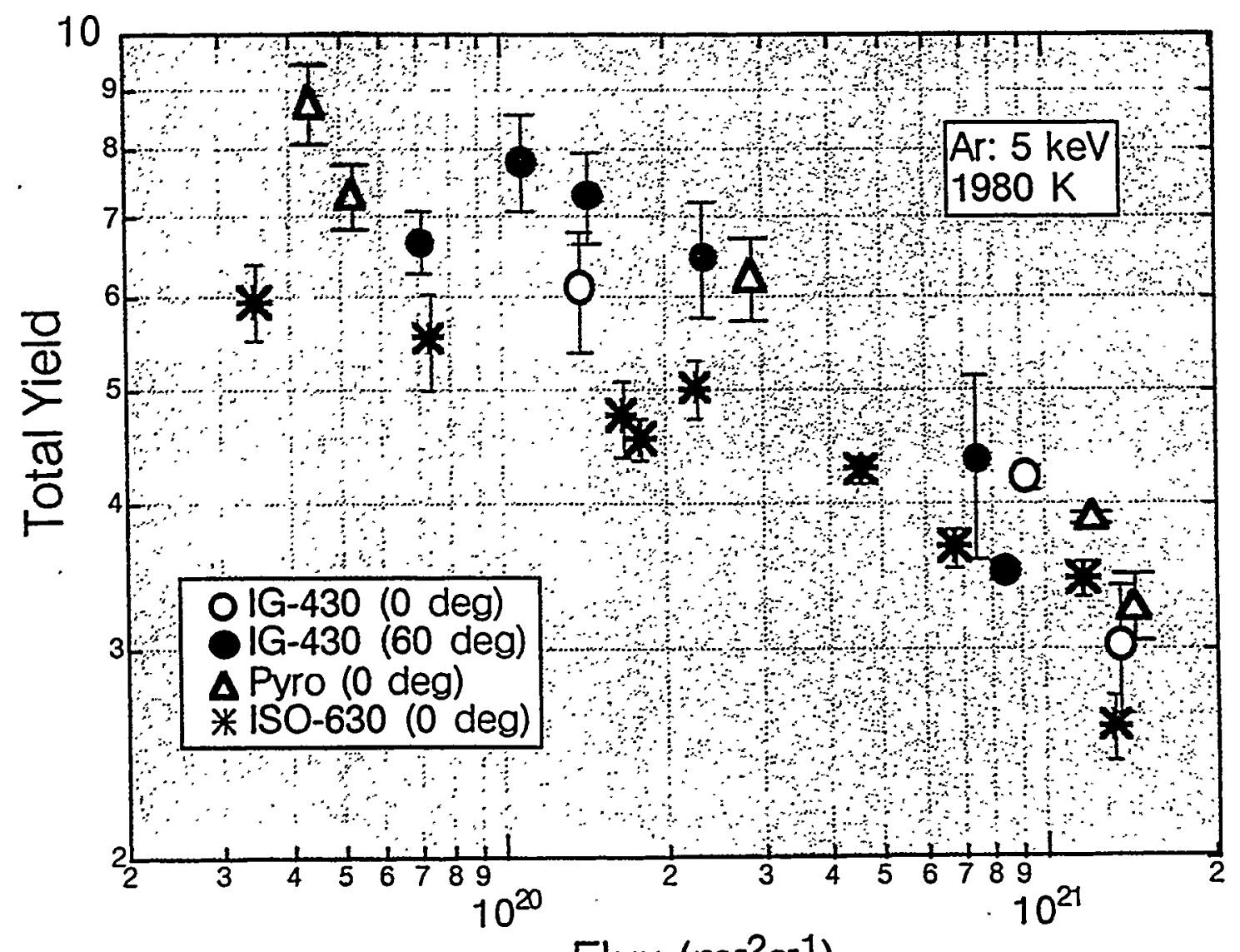

Flux $\left(m^{-2} s^{-1}\right)$

Flux dependence of total yield at $1980 \mathrm{~K}$

Total yield at $1980 \mathrm{~K}$ shows clear reduction with flux for IG-430 (0 deg and $60 \mathrm{deg}$ ) and ISO-630 (isotropic graphite), and pyrolytic graphite.

The dependence is similar for these materials. 


\section{Surface Morphology of RG-Ti}

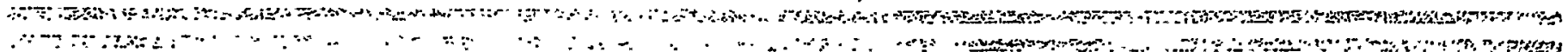

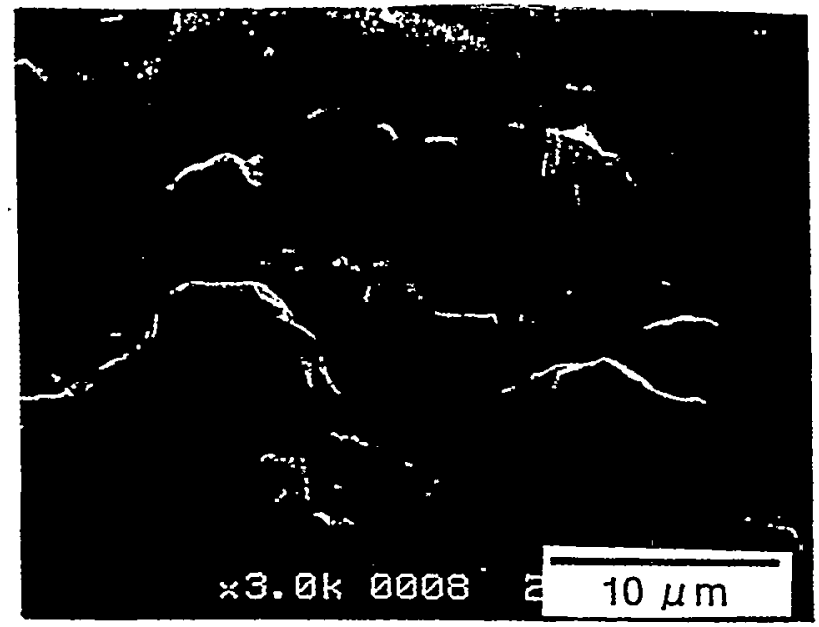

Low Flux

$1.2 \times 10^{20} \mathrm{Ar} / \mathrm{m}^{2} \mathrm{~s}$

$\left(1.9 \times 10^{23} \mathrm{Ar} / \mathrm{m}^{2}\right)$

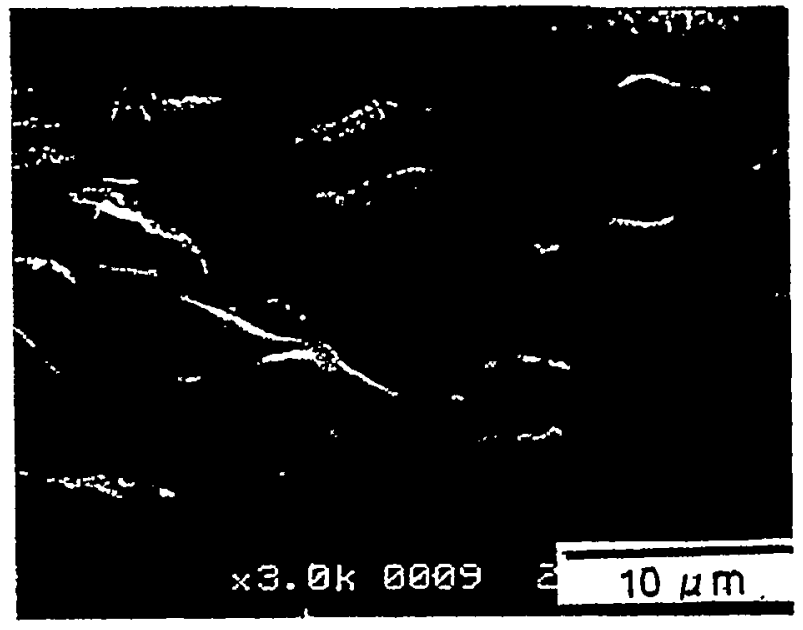

High Flux

$\underline{8.0} \times 10^{20} \mathrm{Ar} / \mathrm{m}^{2} \mathrm{~s}$

$\left(4.2 \times 10^{23} \mathrm{Ar} / \mathrm{m}^{2}\right)$

Samples : $\underline{\text { RG-Ti }}$ (1.7at\% Ti included)

Irradiation Beam : $5 \mathrm{keV} \mathrm{Ar}$

Temperature $: \underline{1780 \mathrm{~K}}$

TiC grains are eroded by physical sputtering, while graphite parts are eroded mainly by RES.

Erosion rate of graphite at elavated temperature is dependent on flux, which causes higher protuberances of $\mathrm{TiC}$ grains in the lower flux case. 


\section{Angular dependence}

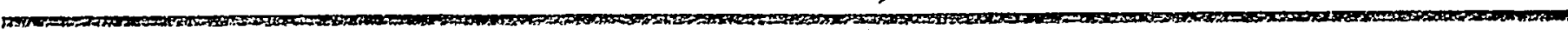

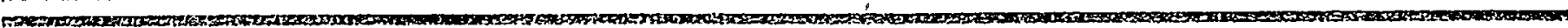

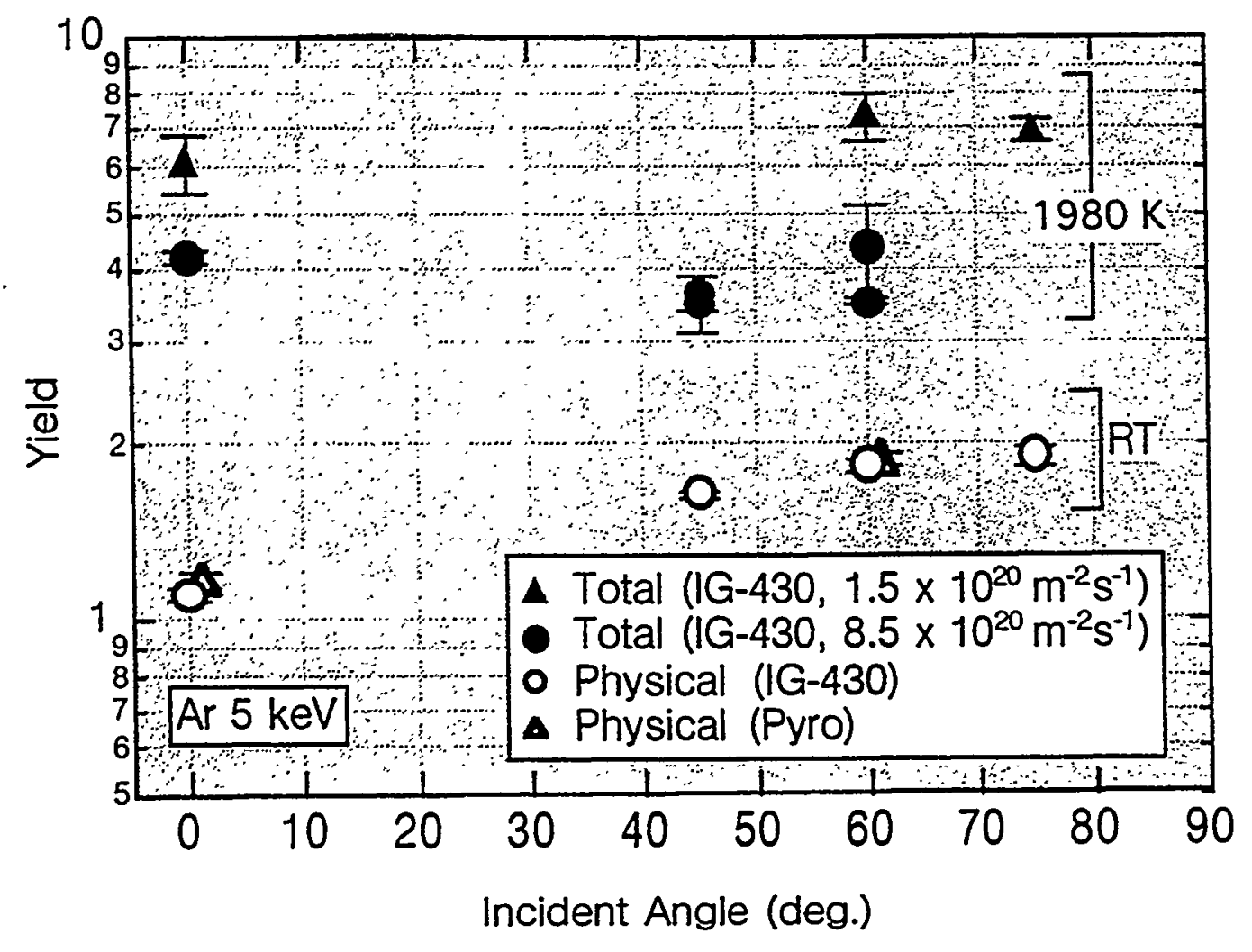

\section{Angular dependence of total yield for various condition}

\section{Physical sputtering (RT)}

- Increase with angle

- No difference between IG-430 and Pyro

RES + Physical (1980 K)

- No clear dependence on angle

- (clear flux dependence, 


\section{Illustration of Model}

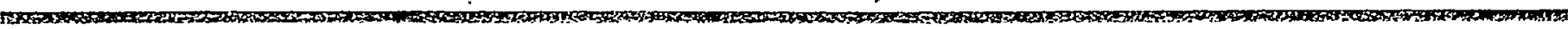

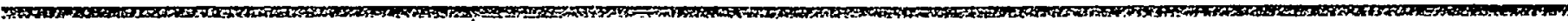

- Incident lon $\mathrm{O} C$ atom at lattice site

- C self-interstitial $\bigcirc$ vacancy

Stable defect

Generation of Frenkel pair

Surface

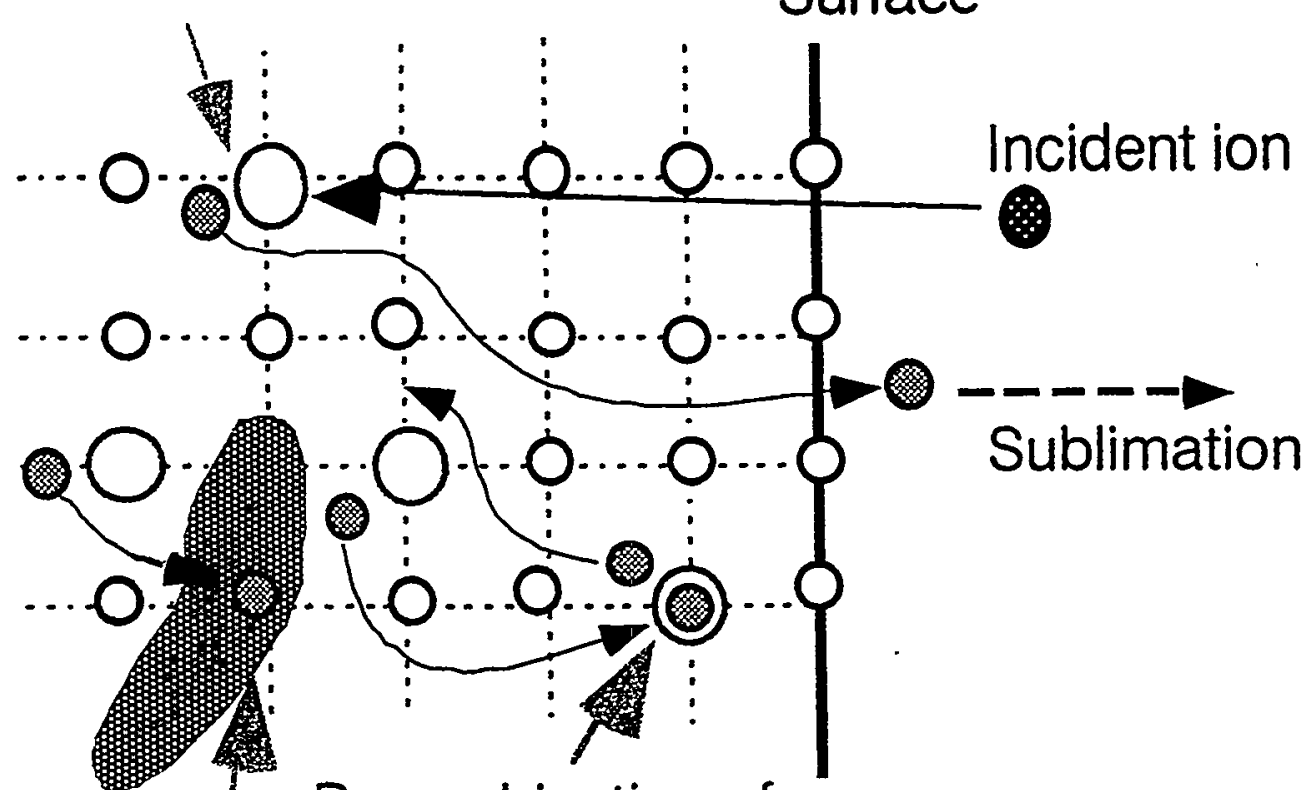

Recombination of interstitial and vacancy

Recombination of interstitial and stable defect 


\section{Model Calculation and Exp.}

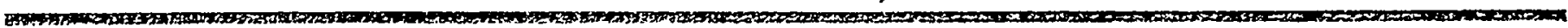

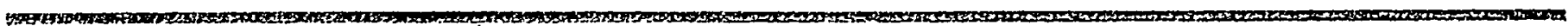

\section{Sinks for Interstitial}

- Low flux regime $\rightarrow$ Stable defects

RES Yield $\rightarrow$ Flux independent

- High flux regime $\rightarrow$ Vacancy

RES Yield $\rightarrow$ Flux dependent $\left(\sim \phi^{-0.25}\right)$

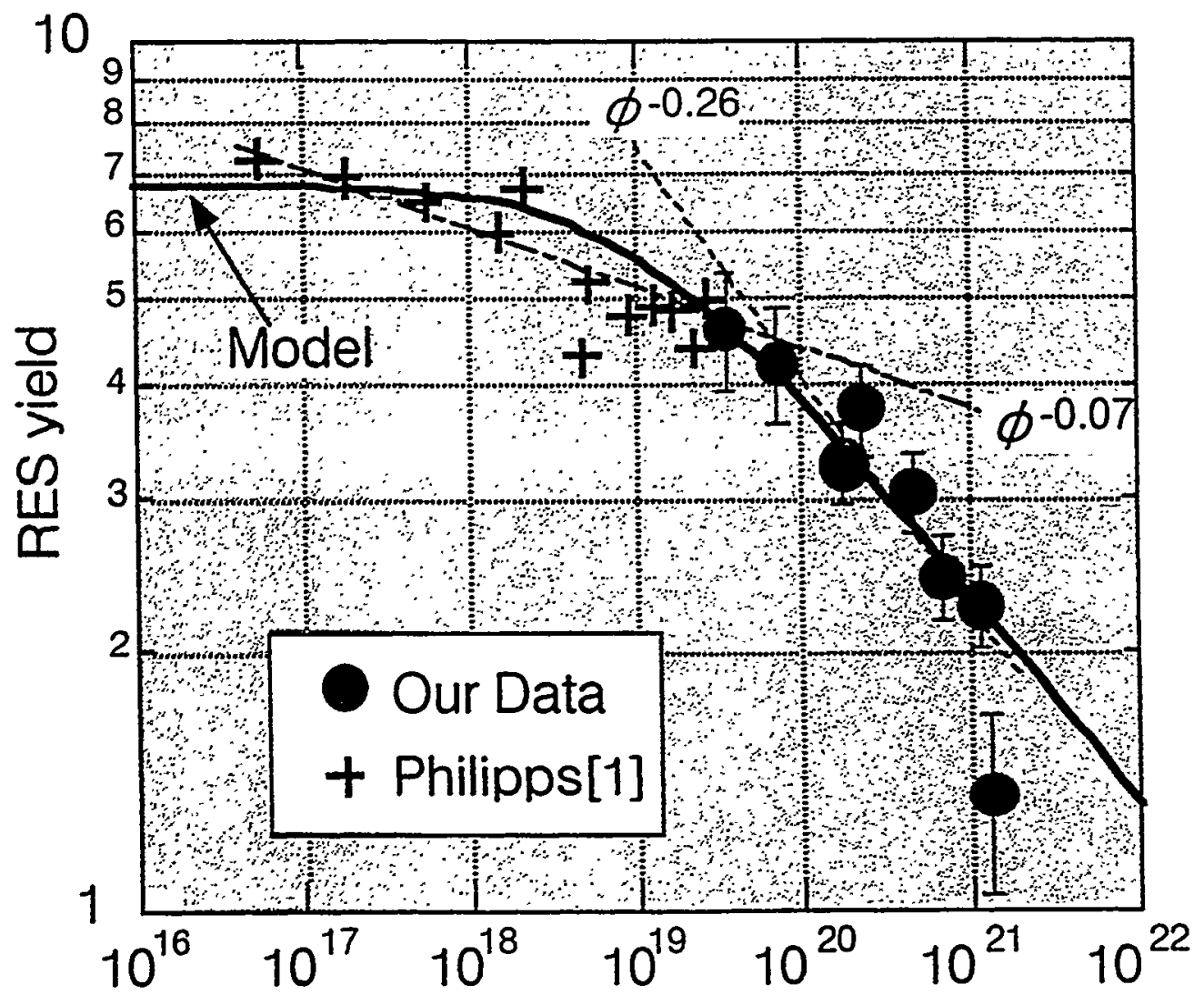

Flux $\left(\mathrm{Ar} / \mathrm{m}^{2} \mathrm{~s}\right)$

\section{Model assumption}

- Steady state

- Stable defect density : $5 \times 10^{-3} \mathrm{n}_{\mathrm{c}} \quad$ IV-91

$\mathrm{n}_{n}$ : carbon density 


\section{Erosion under PFM conditions}

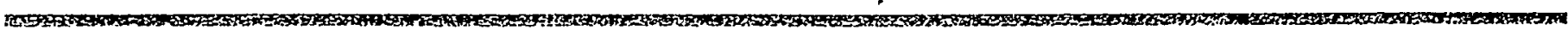

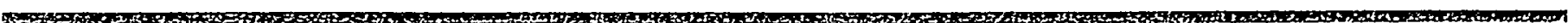

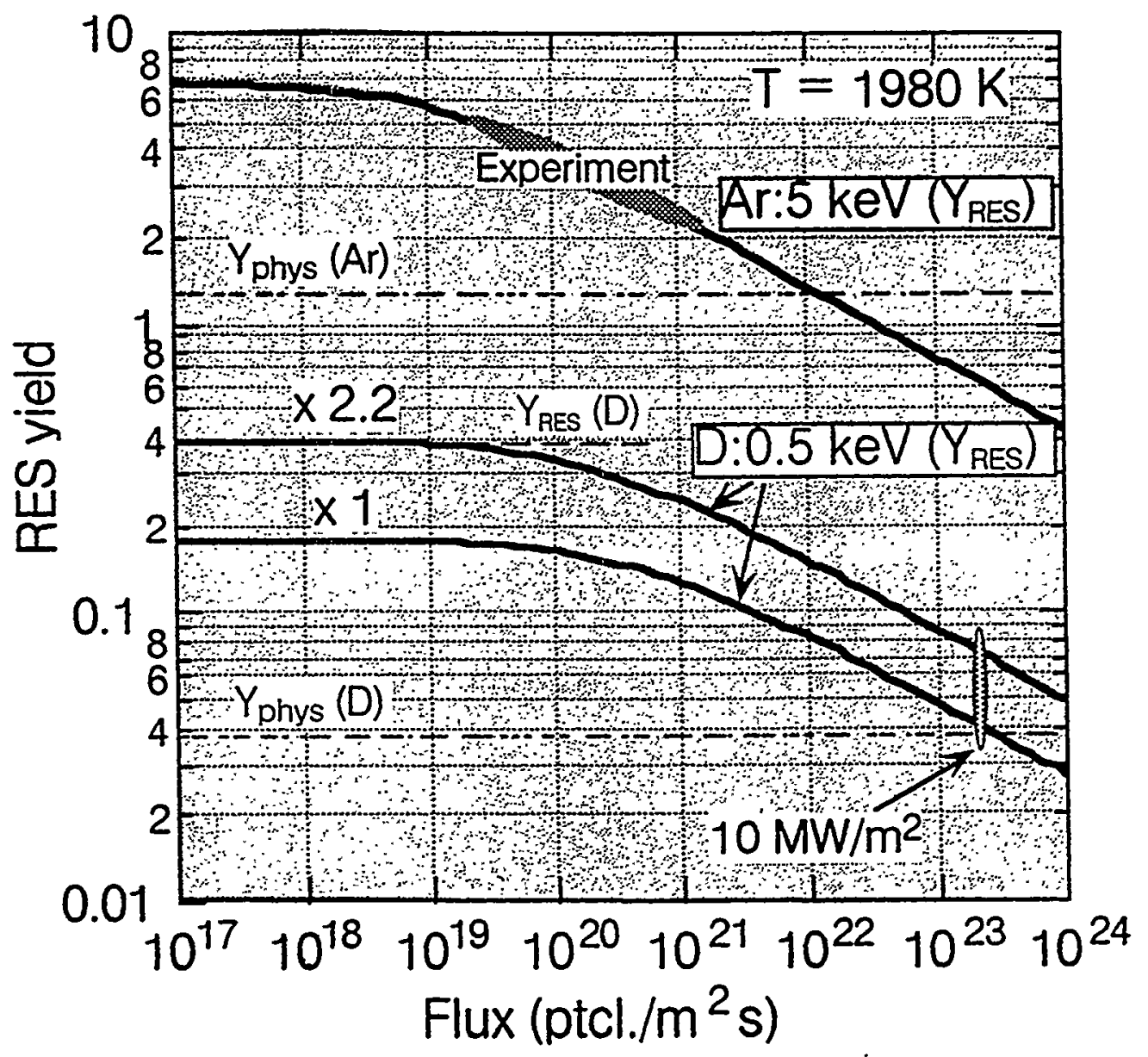

Under high heat flux edge plasma condition (Ion heat flux : $10 \mathrm{MW} / \mathrm{m}^{2}$ )

- RES yield is less than $1 / 5$ of previous low flux data

- Total erosion yield (RES+Phys.) is less than $1 / 4$ of previous low flux data $(T=1980 \mathrm{~K})$ 


\section{Particle Measurement}

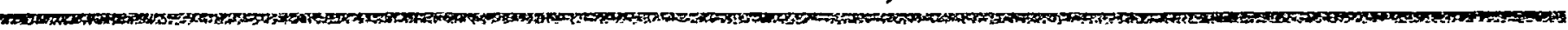

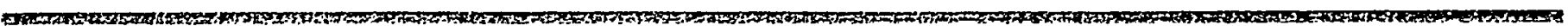

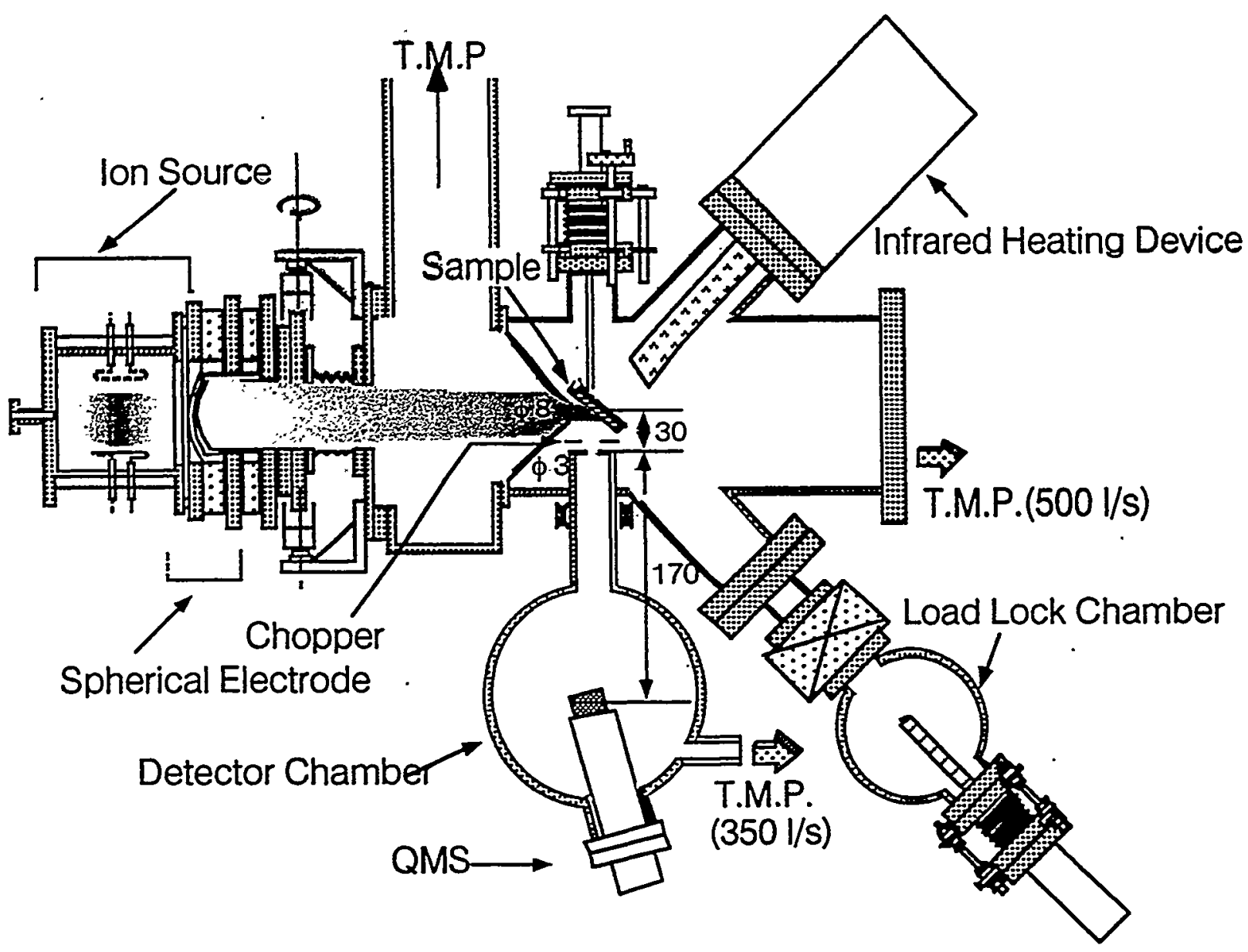

\section{Arrangement of Sputtered Particle Measurement}




\section{Summary and Future Plan}

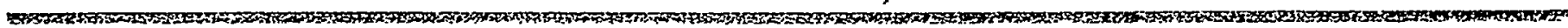

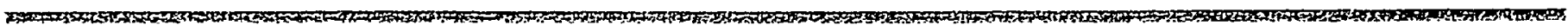

1. Erosion yield of radiation enhanced sublimation (RES) of various graphite (IG-430, ISO-630, PG) in high flux regime (5 $\mathrm{keV} \mathrm{Ar}, \sim 10^{17} \mathrm{Ar} / \mathrm{m}^{2} \mathrm{~s}$ ) significantly decreases with flux. Observation of surface structure of RG-Ti (Ti doped graphite) supports these results. No clear angular dependence of RES yield was observed.

2. The above result is consistent with RES model based on diffusion and annihilation of $\mathrm{C}$ selfinterstitial, in which dominant sinks are vacancies in high flux regime (strong flux dependence).

3. Under tokamak edge plasma condition $(0.5 \mathrm{keV} \mathrm{D}$, $10 \mathrm{MW} / \mathrm{m}^{2}$ ), RES yield can be reduced by more than a factor of 5 compared with that of low flux irradiation experiments.

\section{Future Plan}

- Direct measurements of sputtered particle

- New CW ion source for high fluenece and

IV-94 multi-beam irradiation 


\title{
DiMES Erosion and \\ Dust Experiments on DIII-D
}

\author{
presented by \\ D.G. Whyte, UCSD
}

at the US-Japan Workshop on High Heat Flux Components \& Plasma Surface Interactions for Next Fusion Devices

December 8-11, 1997

San Francisco

for the DiMES Team

R.B. Bastasz, W.R. Wampler,

Sandia National Laboratory

J.N. Brooks,

Argonne National Laboratory

C.P.C. Wong, W.P. West

General Atomics

and I. Opimach

Triniti Lab

[in] Sandia National Laboratories
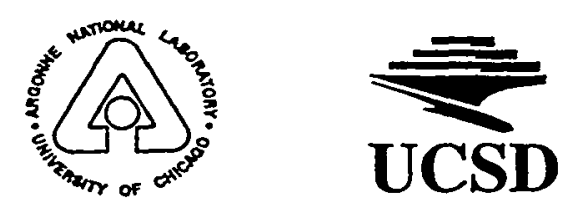


\section{Erosion Validation: Agreement}

1. Current understanding of erosion production and transport are sufficient to predict net erosion rates in attached regions of divertor plasmas for a single species PFC tokamak. This is validated by comparisons of models and experiment.

2. Short duration, dedicated erosion measurements accurately represent long term erosion rates.

3. Net erosion rate of the BIII-D carbon divertor, under attached conditions, will be $\sim 10$ 's cm/burn-yr and is peaked near the outer strike point. This is correctly predicted by REDEPMWB.

4. In DIII-D the hydrogenic inventory build-up rate is determined by the attached outer divertor's net erosion rate and subsequent redeposition at the detached inner leg. 


\section{Divertor Material Evaluation Studies (DiMES)}

\section{OBJECTIVES}

- Measure erosion rates and redeposition mechanisms under tokamak divertor conditions.

- Validate and improve erosion codes.

- Determine the implications for fusion power plant plasma facing components.

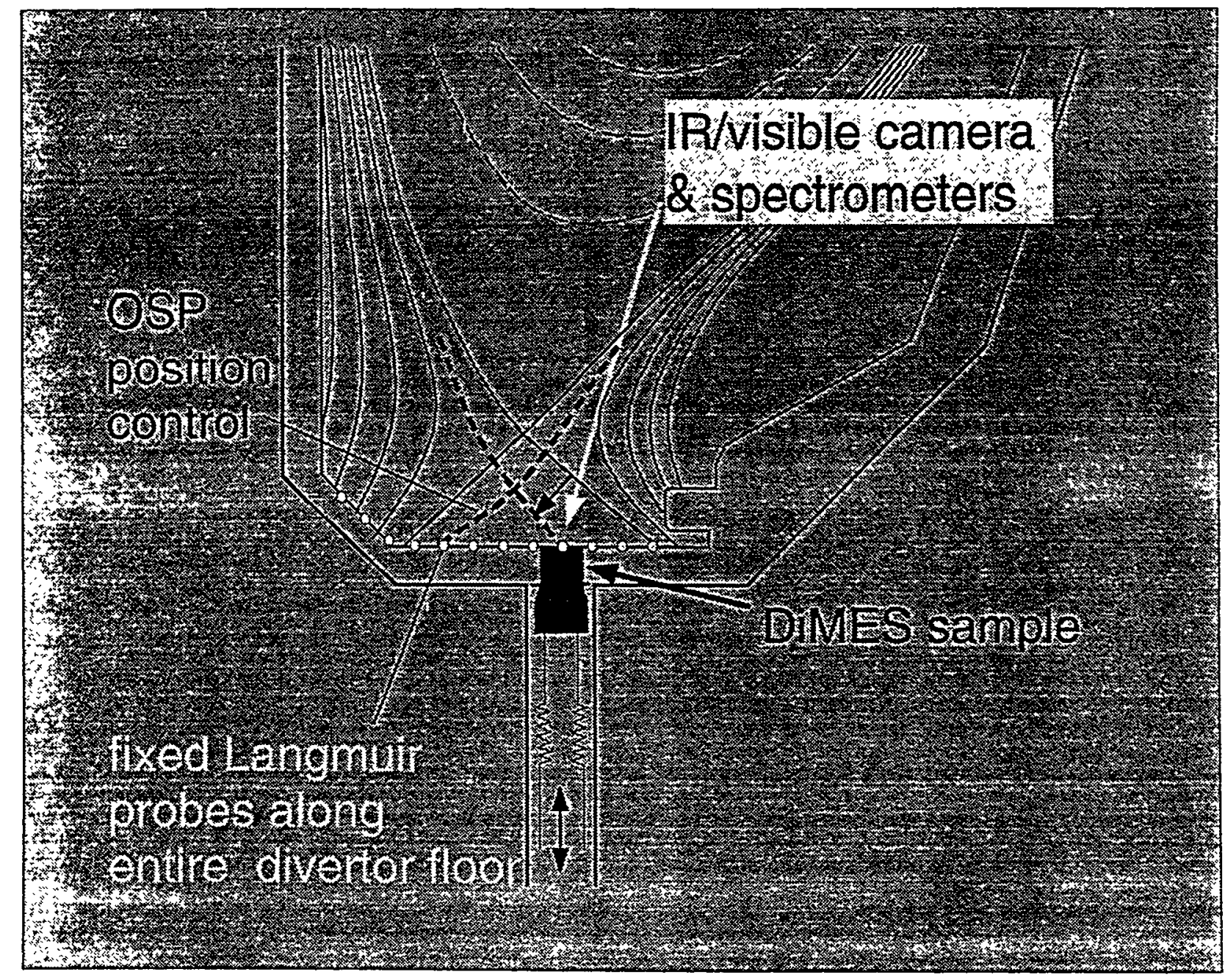

DiMES, DIII-D Divertor \& Diagnostics Provide Controlled, Characterized Exposure Conditions for Candidate Materials 


\section{Sample and Exposure Geometry}

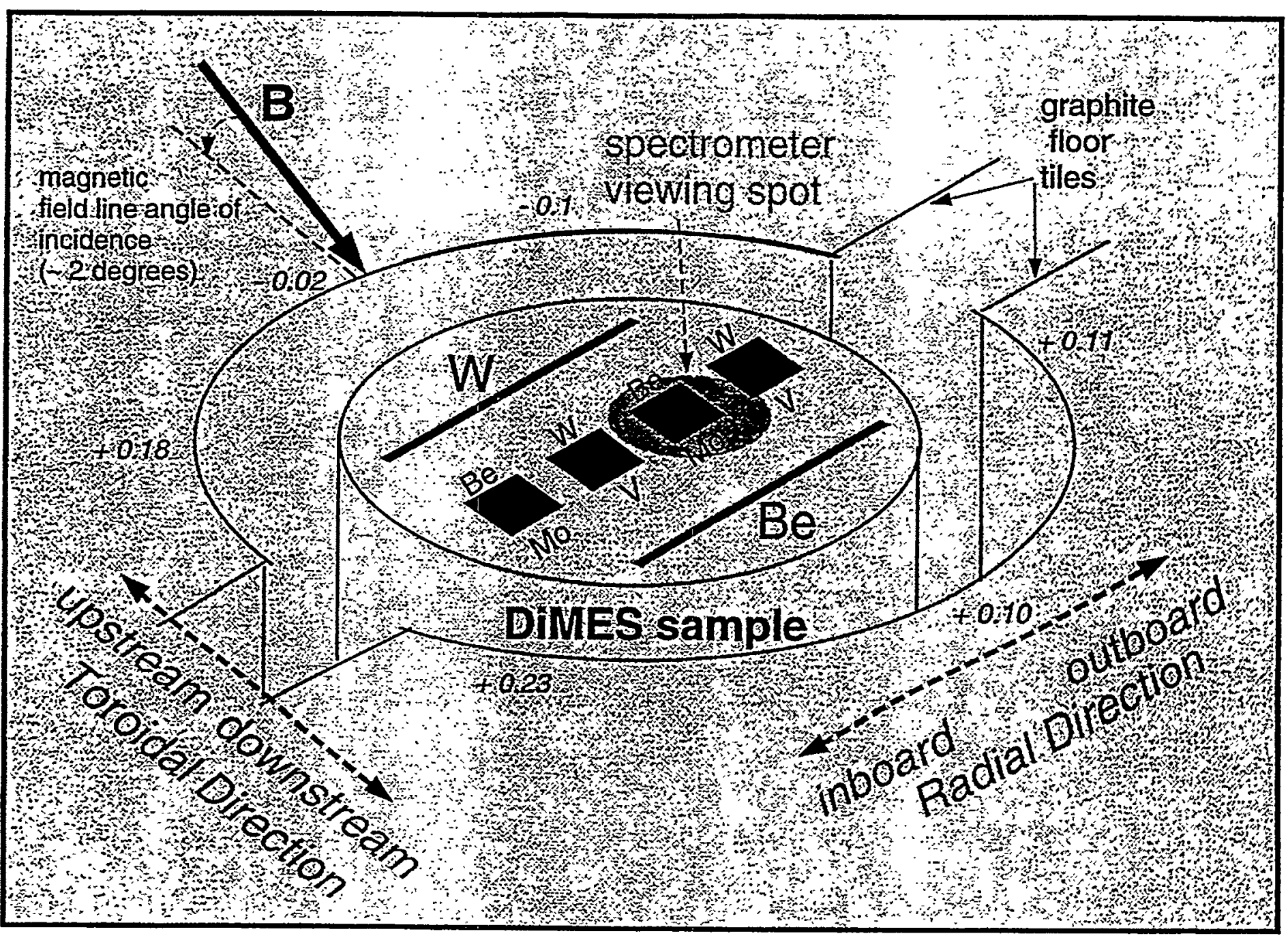

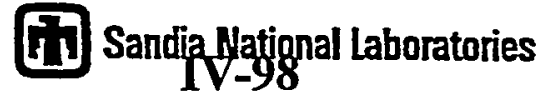
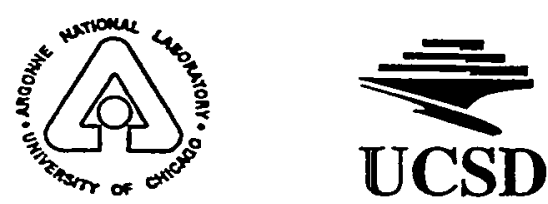


\section{DiMES Rooftop Alignment to Surrounding Tiles Allows for High Power Exposures with Uniform Heat Flux to DiMES and No Leading Edges}
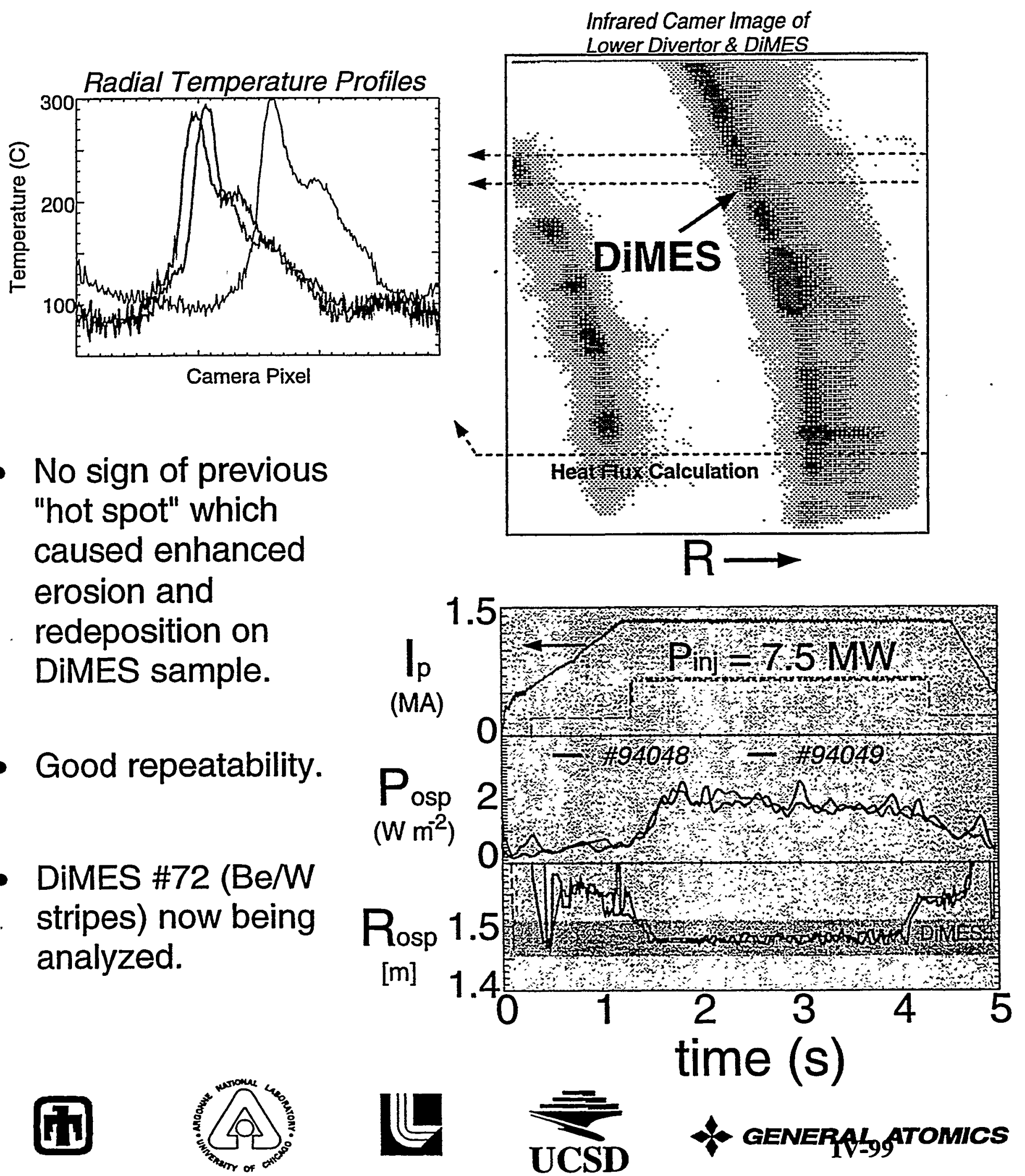


\section{DIII-D Diagnostics \& SNL Materials Evaluation Provide Complete Divertor Plasma Erosion Characterization to REDEP/WBC Erosion Models}

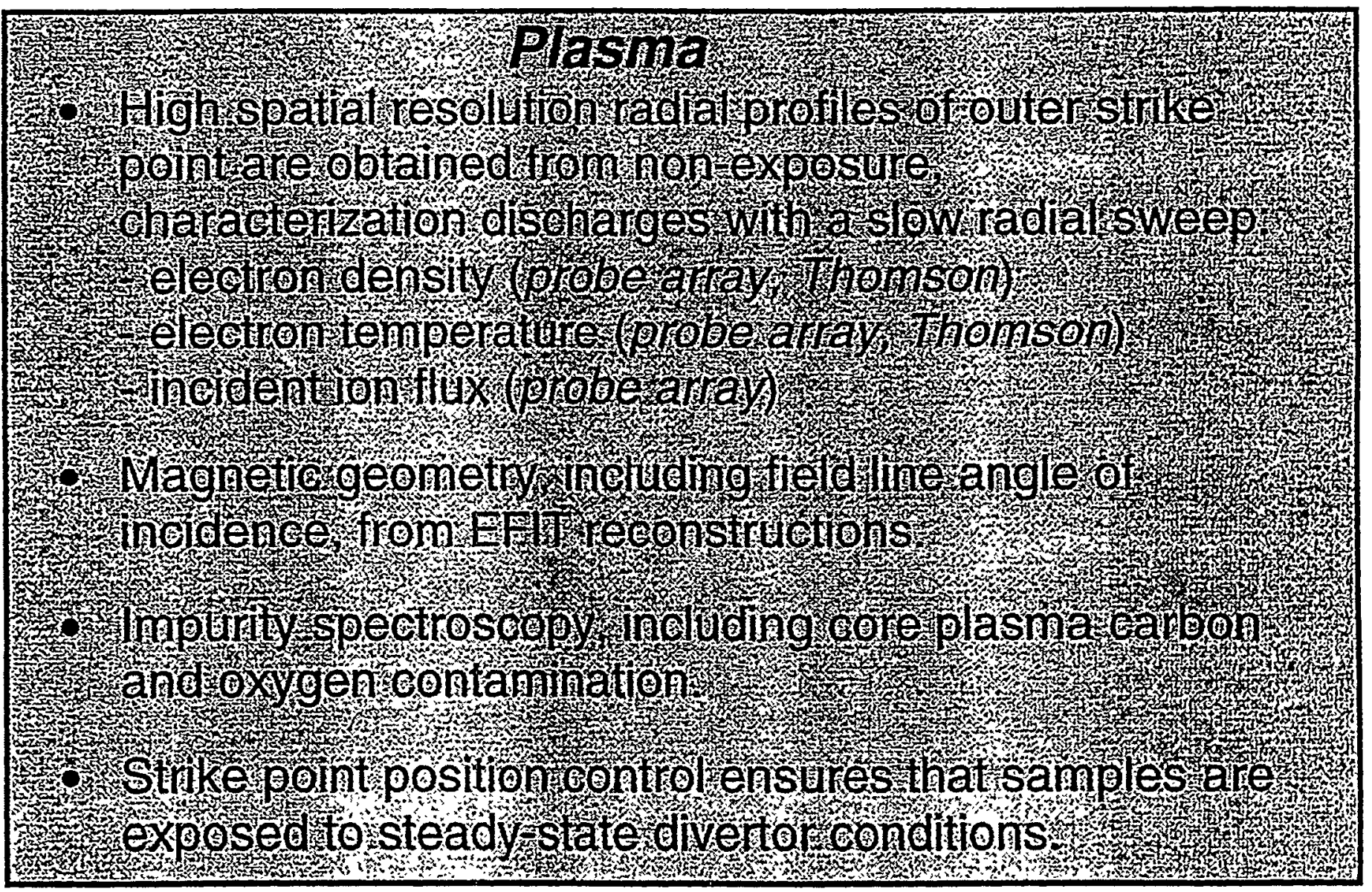

\section{Sample}

- ATJ graphite samples contain implanted Si depth marker RBS measures net carbon of Si measures. verosionlredepostion to $+10 \mathrm{~nm}$

- Pre and post-mortem sample analysis provides net carbonerosion and metallic redeposition patterns.

(6) Sandia Natigngl Laboratories
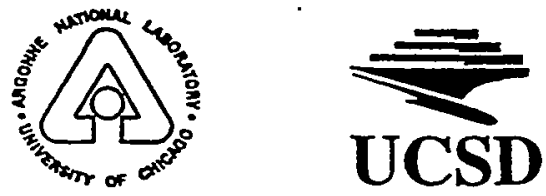


\section{Near-Surface Transport of Sputtered Material is Well Characterized by}

REDEP/WBC Code

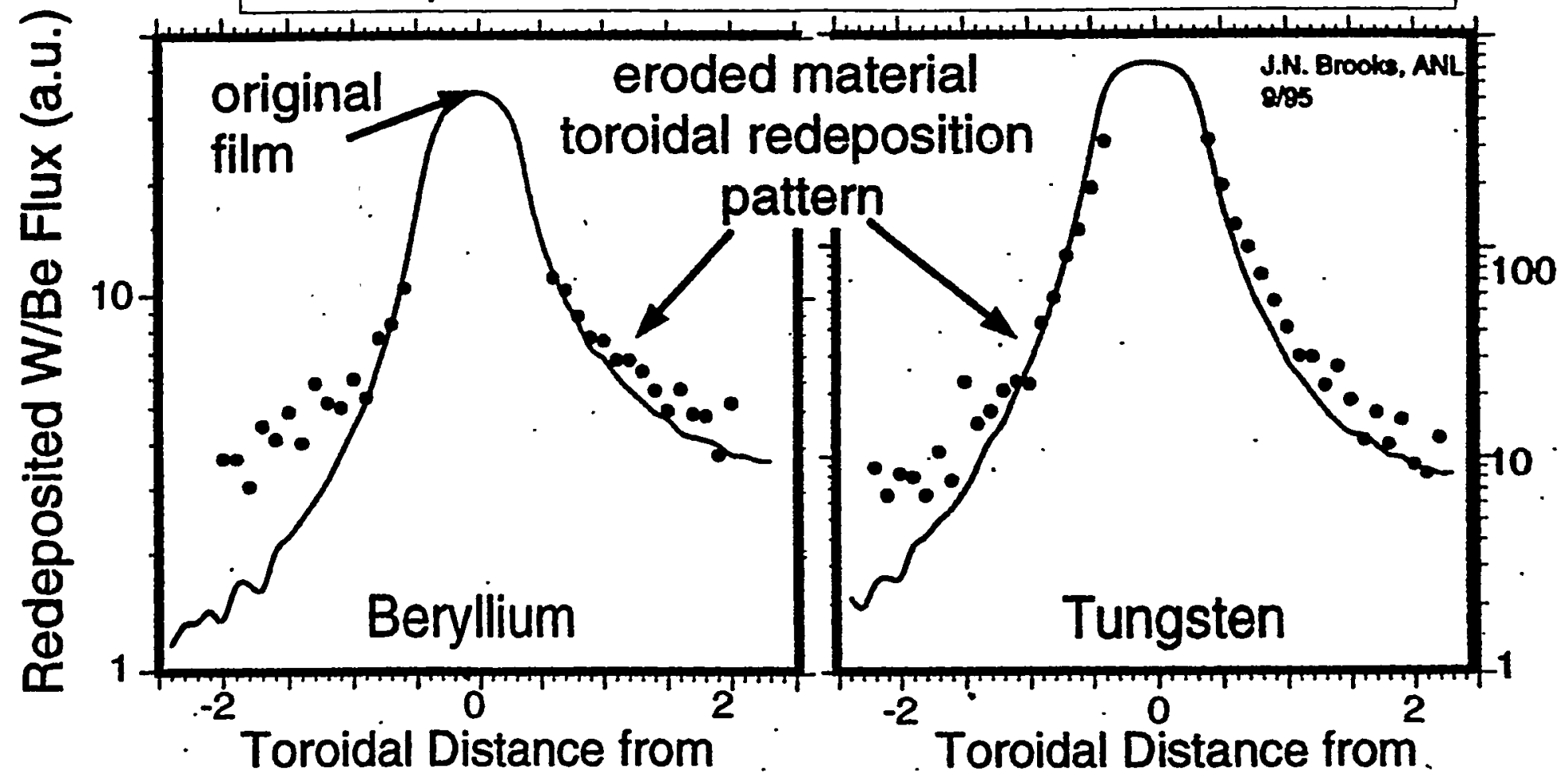

Film (cm)

Film (cm)

- WBC Monte Carlo impurity transport code computes the sputtering, in-plasma transport and redeposition of impurity atoms/ions. Code treats Lorentz motion and plasma-impurity collisions in detail and uses measured plasma and magnetic field parameters.

- Code/data comparison of Be II (467.3 nm) intensity predict an erosion rate of $2 \mathrm{~nm} / \mathrm{s}$, agreeing well with the surface analysis techniques.

(i) Sandia Mational laboratories

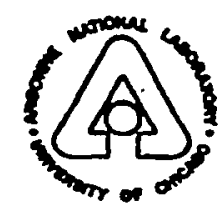
generataATPMICS 


\section{REDEP Code Agrees with Spectroscopic Measurements of Gross Carbon Flux. Experimental Redeposition Fraction $\approx \mathbf{8 8} \%$}

- CCD camera with CII filter and vertical view of lower divertor.

- Carbon outflux from CII emission $\times S / X B$ (ionization/photon ration from CR model).

- This method achieves best results when ionization length is short (i.e. near strike point).
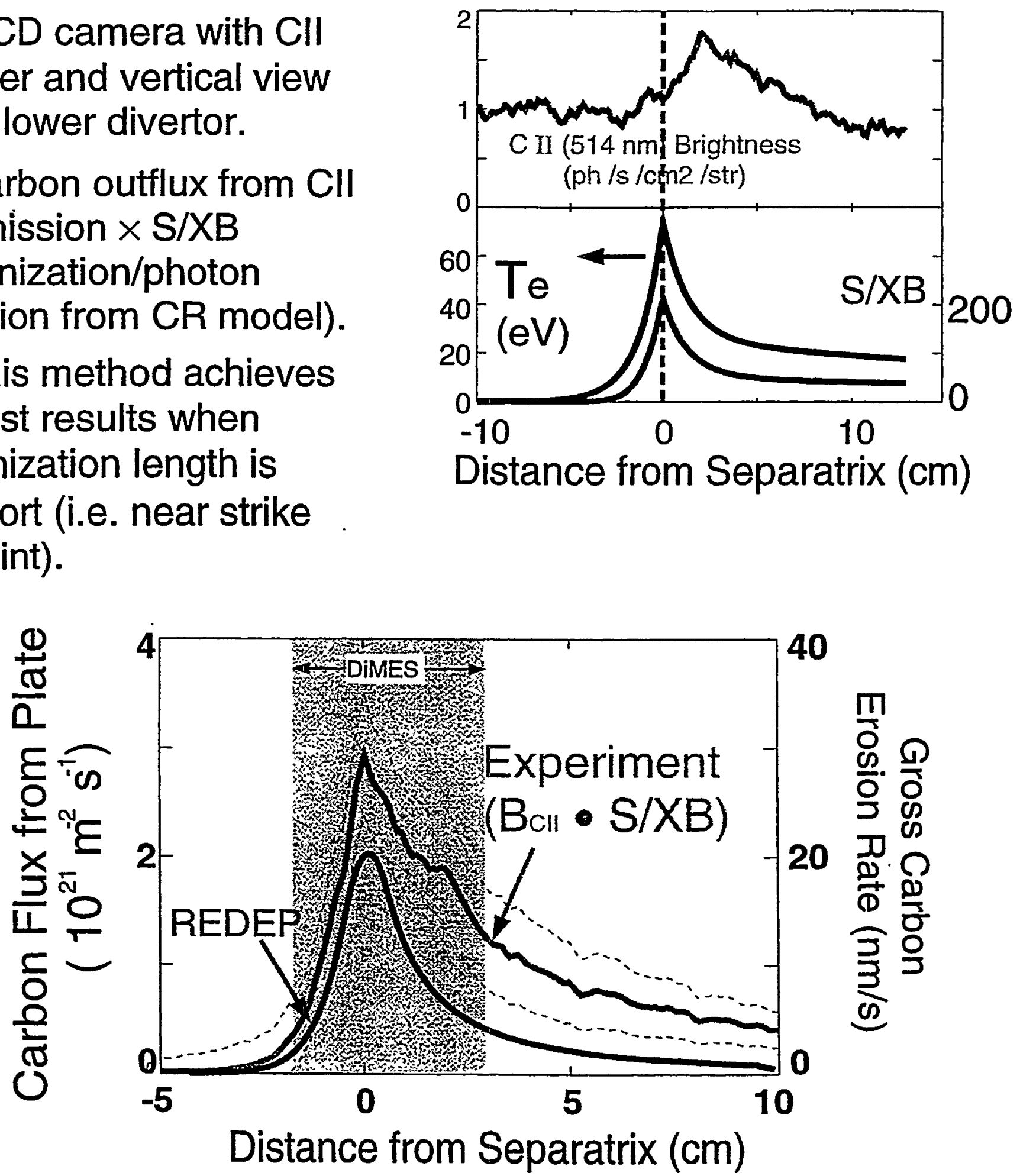

[7) Sandia National Laboratories
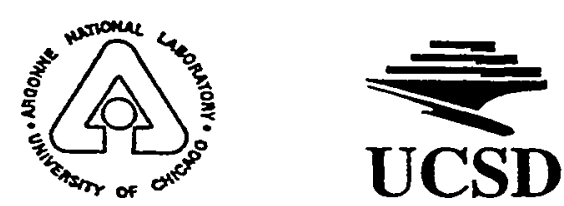


\section{REDEP/WBC Code Matches the Features of Carbon Net Erosion at the DIII-D Divertor as Measured by DiMES}
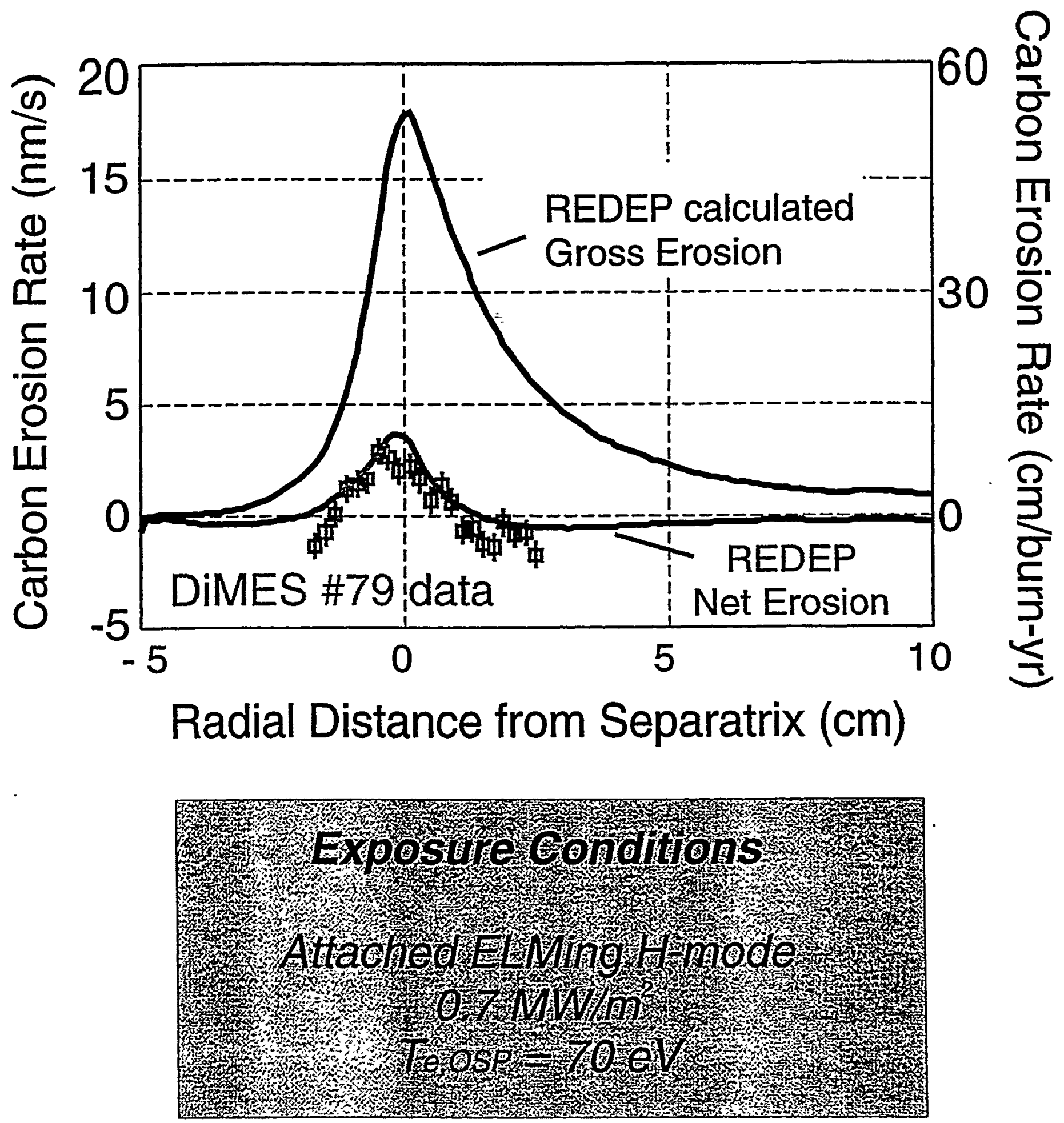

[17] Sandia National Laboratories
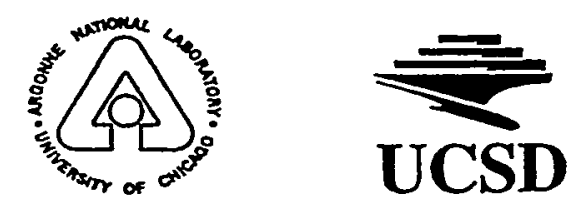

GENERAVIfJOMICS 


\section{Effective Carbon Sputtering Yield is $>10 \%$ for Attached Plasmas}

- Effective sputtering yield (Carbon outflux / ion influx) includes effects of carbon self-sputtering.

- Model and experiment agree near separatrix.
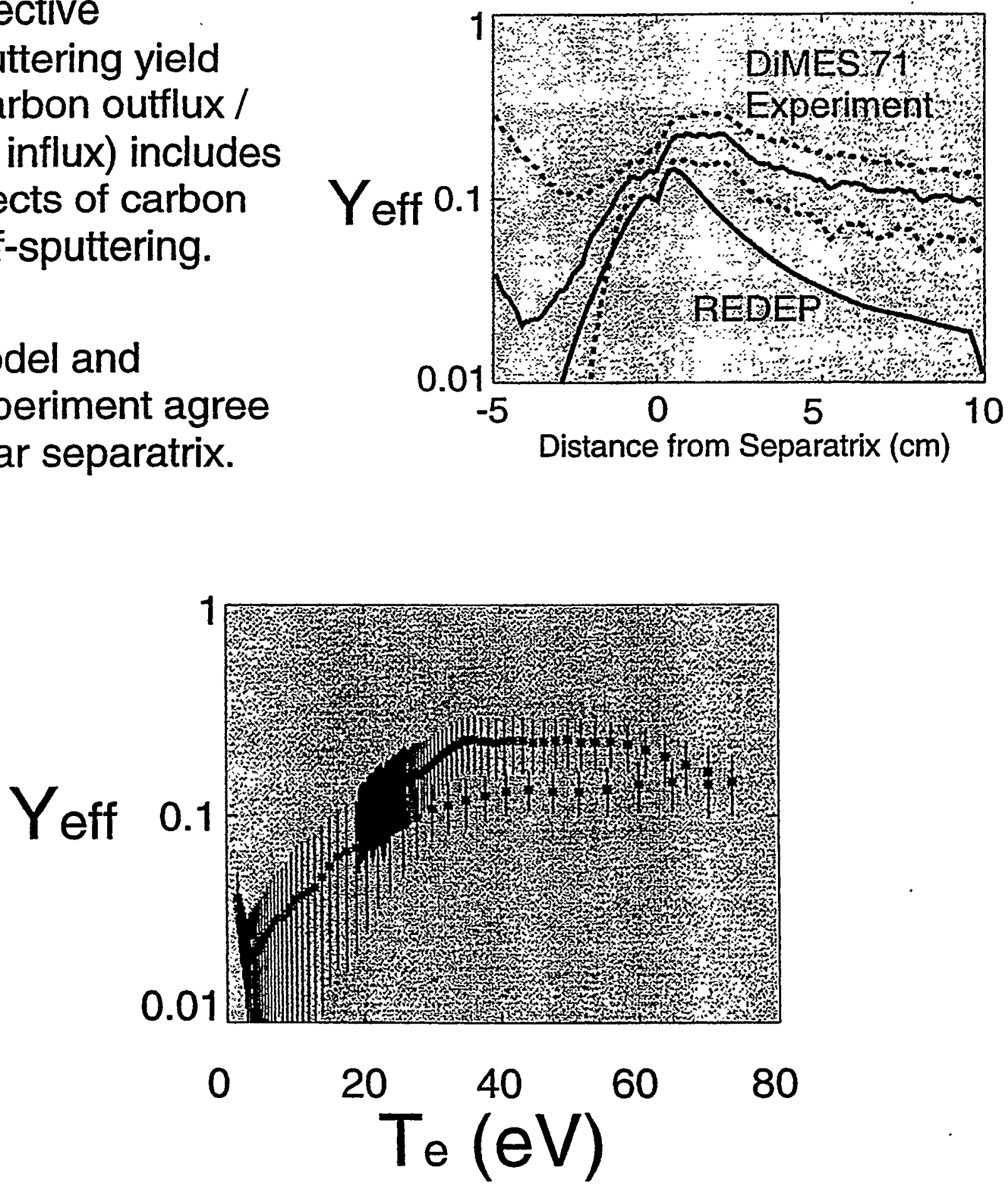

[i] Sandia National Laboratories
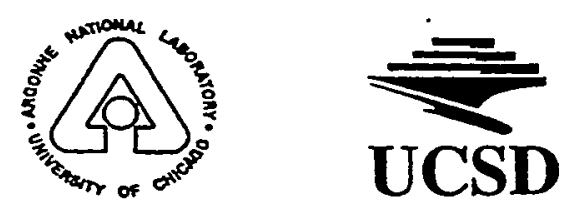


\section{The Peak Net Erosion Rate of the DIII-D Outer Divertor Graphite Plates Increases with Incident Heat Flux for Attached Plasmas}

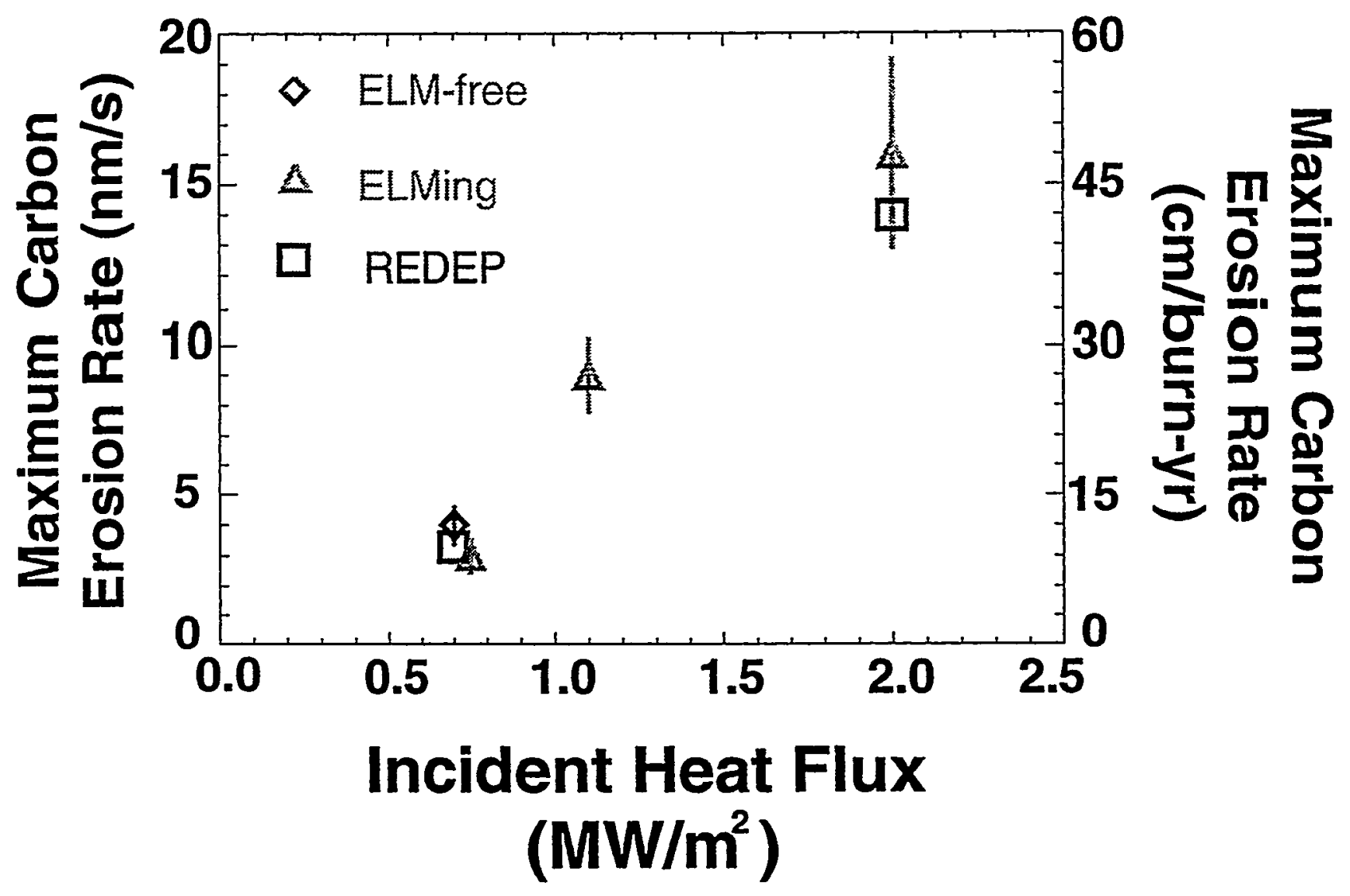

Simple models that neglect oblique inicidence and self-sputtering can underestimate the net erosion rate by a factor of 10 !

(ii) Sandia National Laboratories
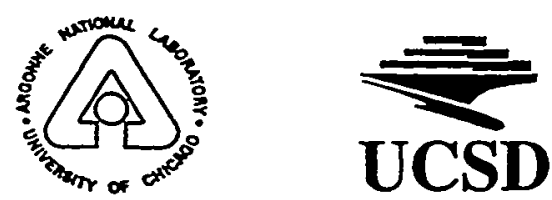


\section{Long term study of divertor tiles show that co- deposited inventory increases with erosion rate.}

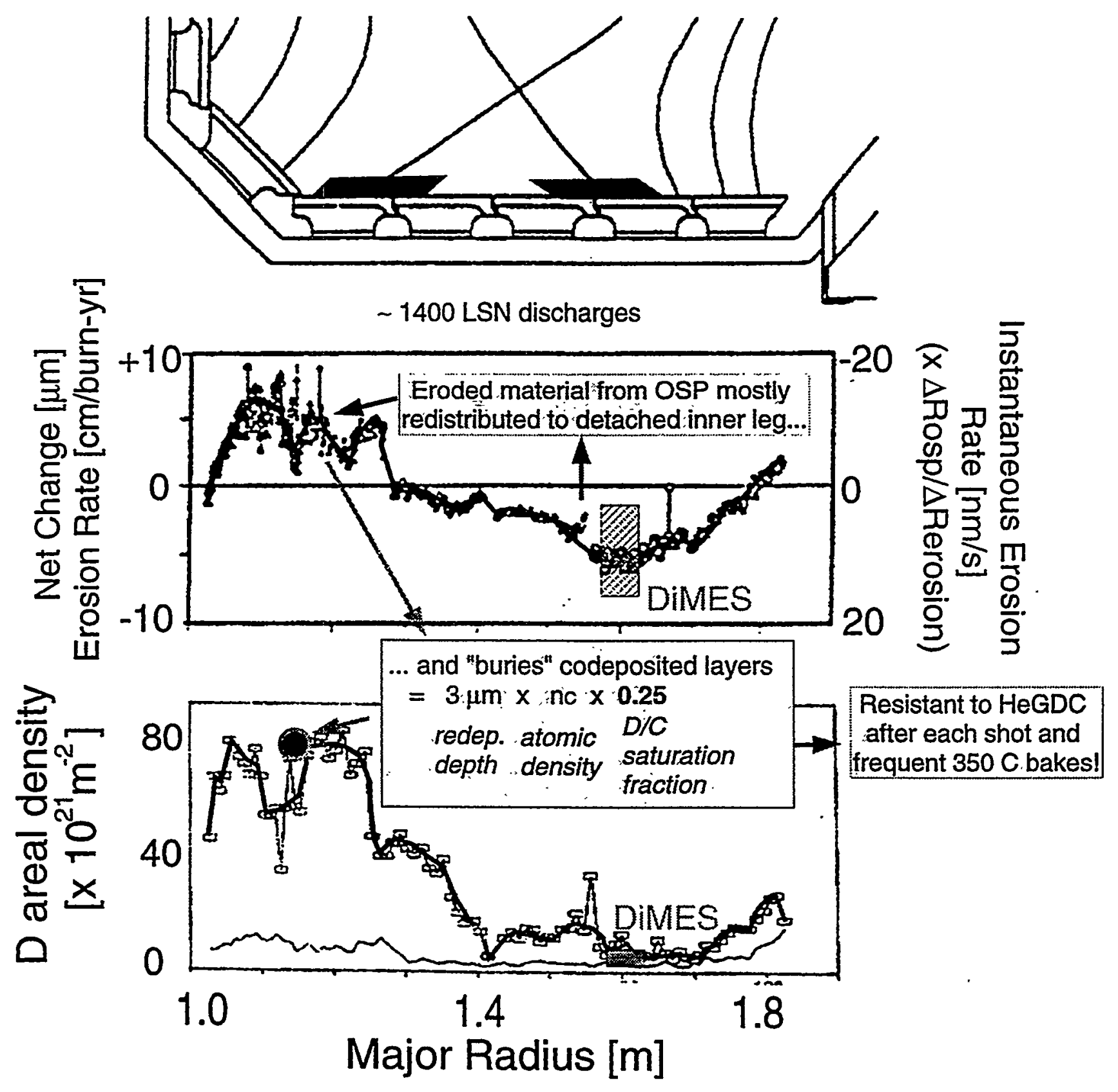

- The presence of large hydrogenic inventories in codeposited layers affects recycling and performance. This inventory is principally determined by the net erosion rates in the divertor. 
With Peak Heat Flux $<1 \mathrm{MW} / \mathrm{m}^{2}$, Outer Divertor Erosion Accounts for Observed Core Plasma Carbon Accumulation and Lack of Density Control in ELM-free H-mode

- Strike point is moved to DiMES probe (\#71) during ELM-free $\mathrm{H}$ mode.

- Net carbon erosion of $3.6 \mathrm{~nm} / \mathrm{s}$ and width $2 \mathrm{~cm}$, corresponds to $7 \times 10^{19}$ atoms/s net loss rate from outer divertor.

- Initial increase in electron density due to better particle confinement.

- After $2 \mathrm{~s}$ linear electron increase is accounted for by carbon accumulation and ionization, with the major source of carbon being outer strike point erosion.

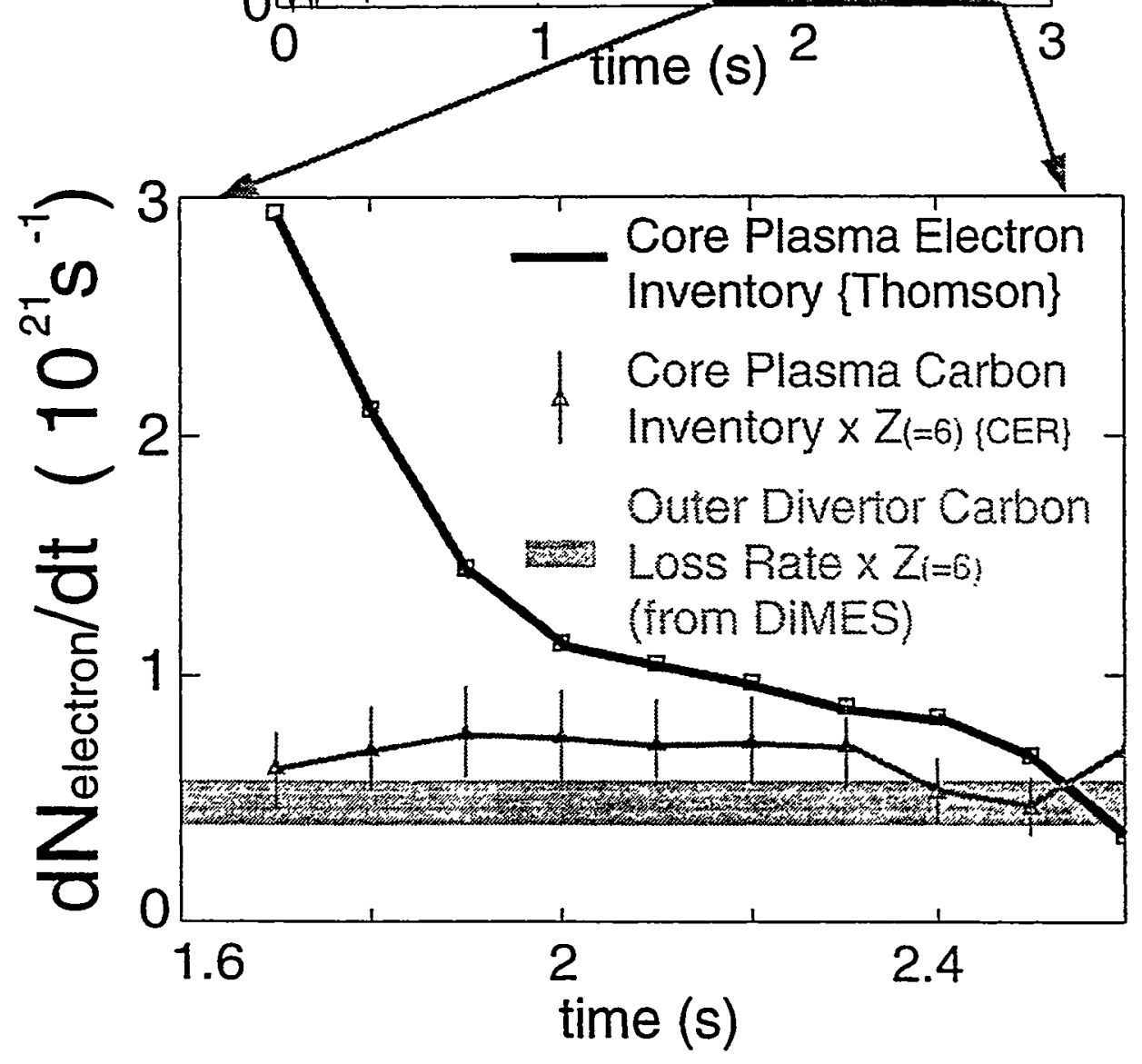

(ii) Sandia National Laboratories
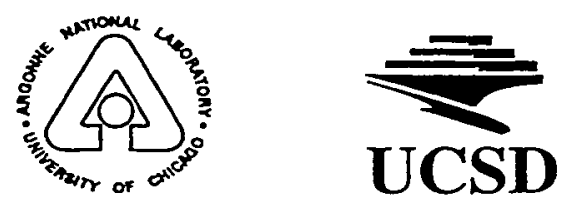


\section{Preliminary Results from DiMES Dust Production Experiments}

- Sample cap with $0.7 \mathrm{~mm}$ raised lip was exposed to $500 \mathrm{~ms}$ of 3 source ELMing H-mode plasma. Estimated parallel heat flux to lip $\sim 50 \mathrm{MW} / \mathrm{m}^{2}$.

- SEM shows 5-10 $\mu \mathrm{m}$ globules forming adjacent to lip in area of intense redeposition.

- Silicon dust collector wafer has a $0.5 \mu \mathrm{m}$ carbonaceous film and carbon dust particles of 1-10 $\mu \mathrm{m}$ size.

- Deuterium/Carbon fraction 0.1 for film on Si wafer. Note that film is not direclty exposed to ion flux.f

- Preliminary calculation shows that this results in: - Codeposited build-up rate of $0.2 \mathrm{~g}$ in 1,000 seconds for every square centimeter of misalignment (does not include dust formation).

- Dust formation: $10 \mathrm{mg}$ in 1,000 s/square $\mathrm{cm}$.

- Quantitative analysis of dust particles is ongoing. 


\section{DiMES Dust Collection Experiment}

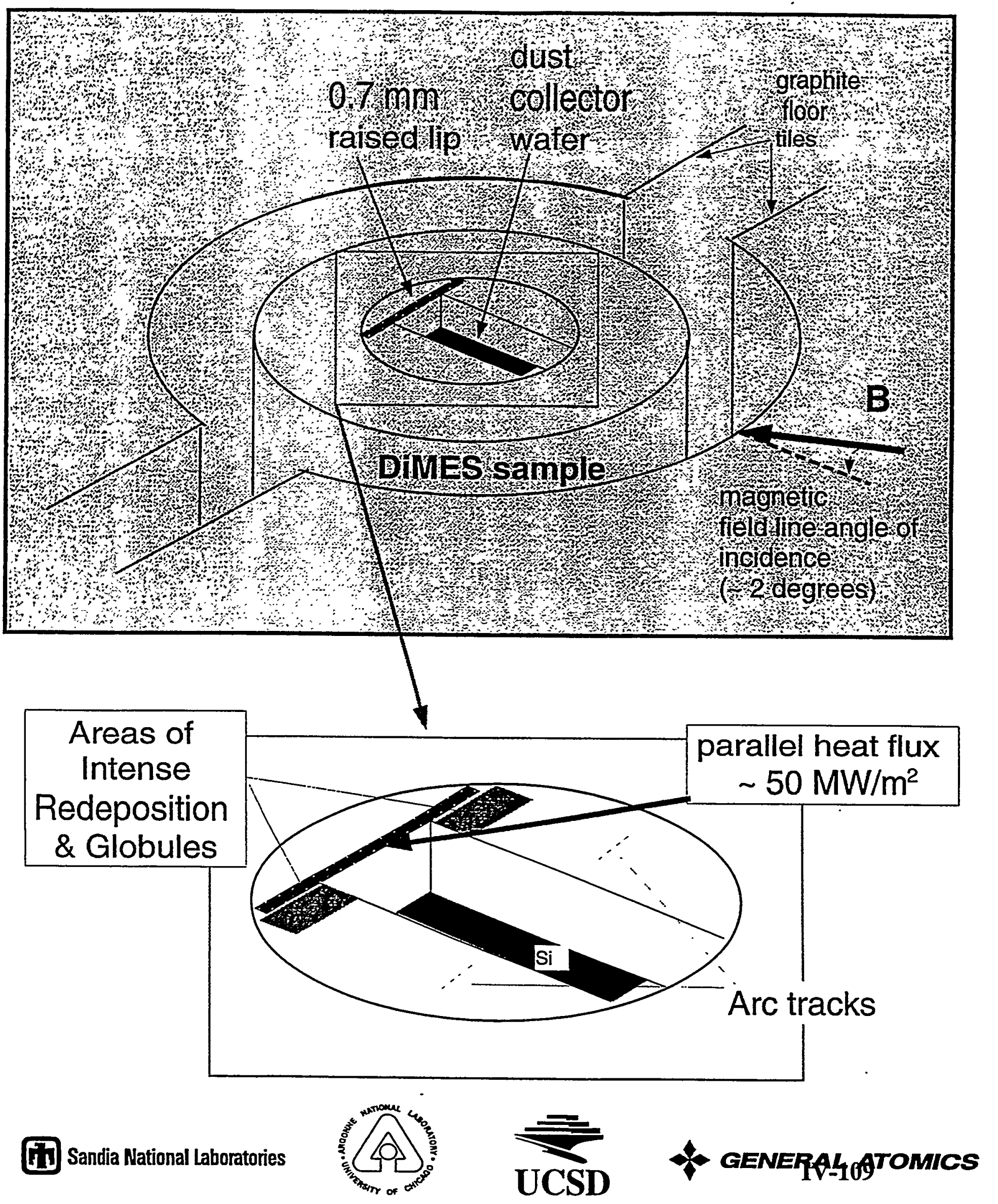



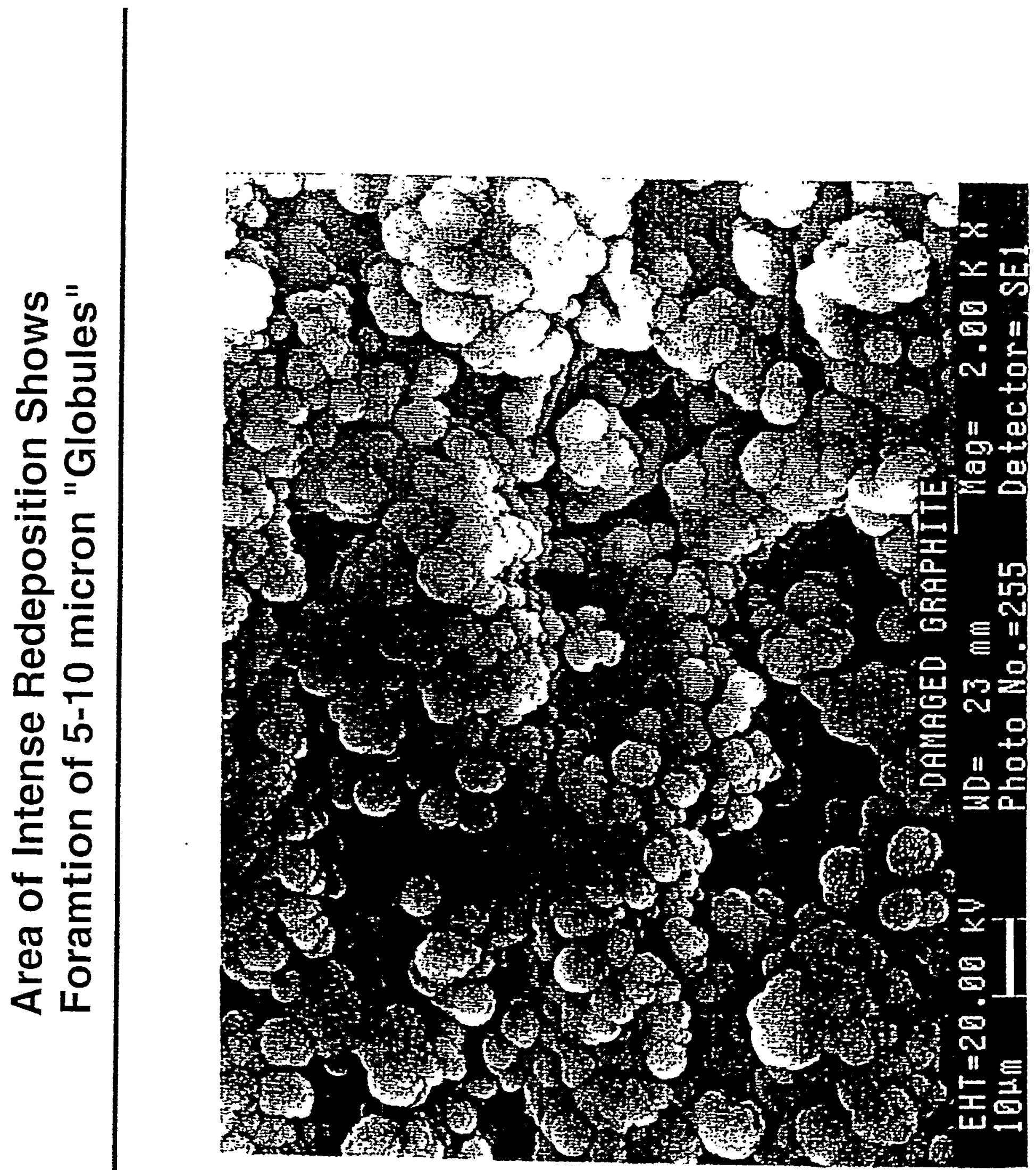
Si collection wafer has an adhered, uniform carbonaceous film (0.5 micron thickness) \& 5-10 micron carbon dust flakes

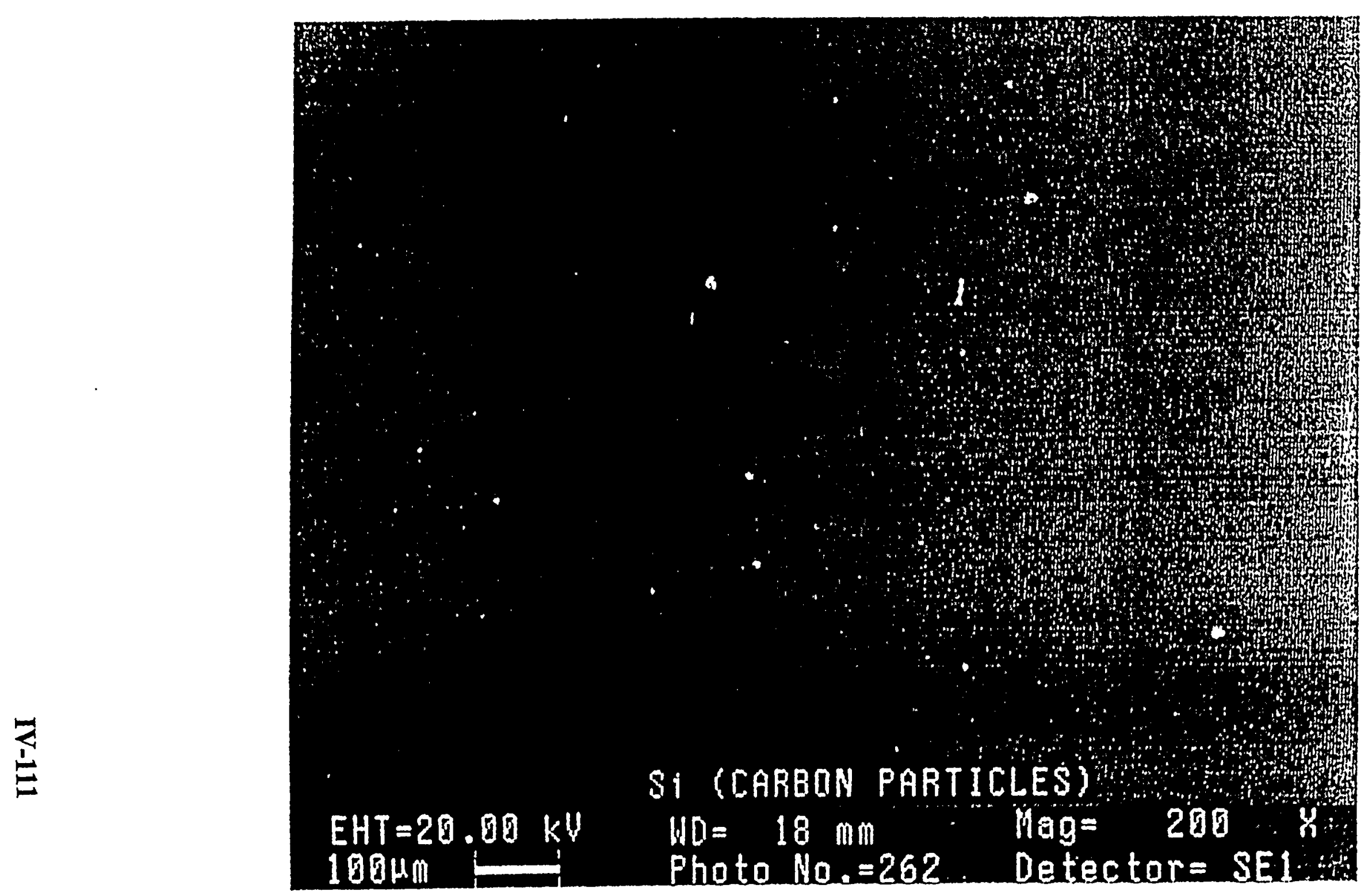


Reflected Neutral Particle Spectrum on MAI

S. Ohtsu, K. Kobayashi and S. Tanaka

Faculty of Engineering, University of Tokyo

US-JAPAN PMI/HHF Workshop

December 8-11th, San Francisco 


\section{Particle Reflection Process}

\section{Plamsa Material}

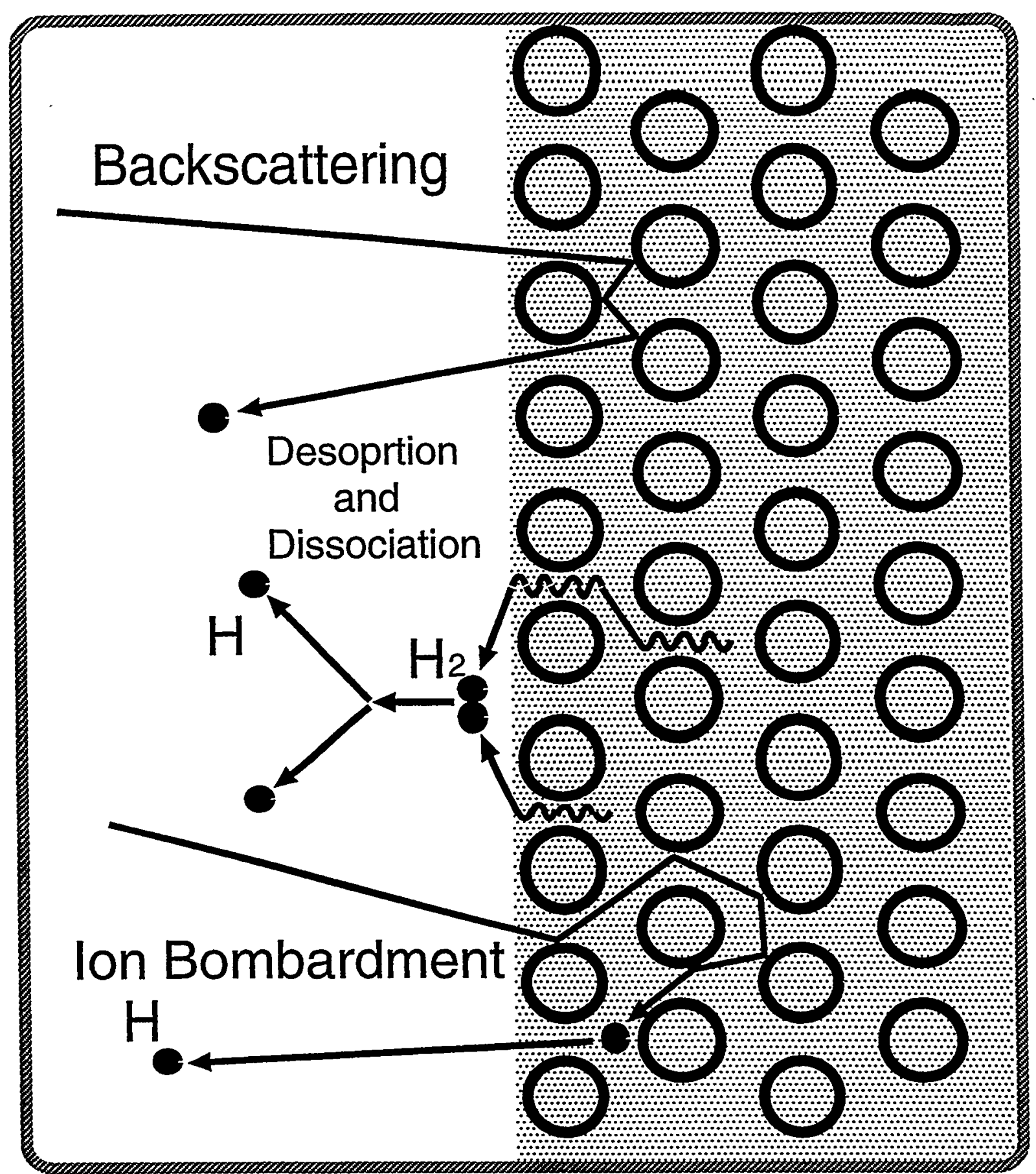

Particle reflection processes on the material serfar in the low energy region $(\sim 100 \mathrm{eV})$ should be investigated.

- Energy distribution

- Anglar distribution

- Excited states 


\section{Contents}

Spectroscopic measurements of the neutral particles near the solid target in linear steady plasma facility MAP

$\mathrm{H}$ alpha spectrum profiles at different incident angles to the target.

Time dependant mesurement of Halpho intensity profile

Monte-Carlo Simulation of neutral particle transports

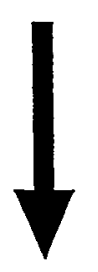

Investigation of the energy and angler distribution of backscattered neutral particles and their excited states. 
Linear Steady Plasma Facility

MAP(Material and Plasma)

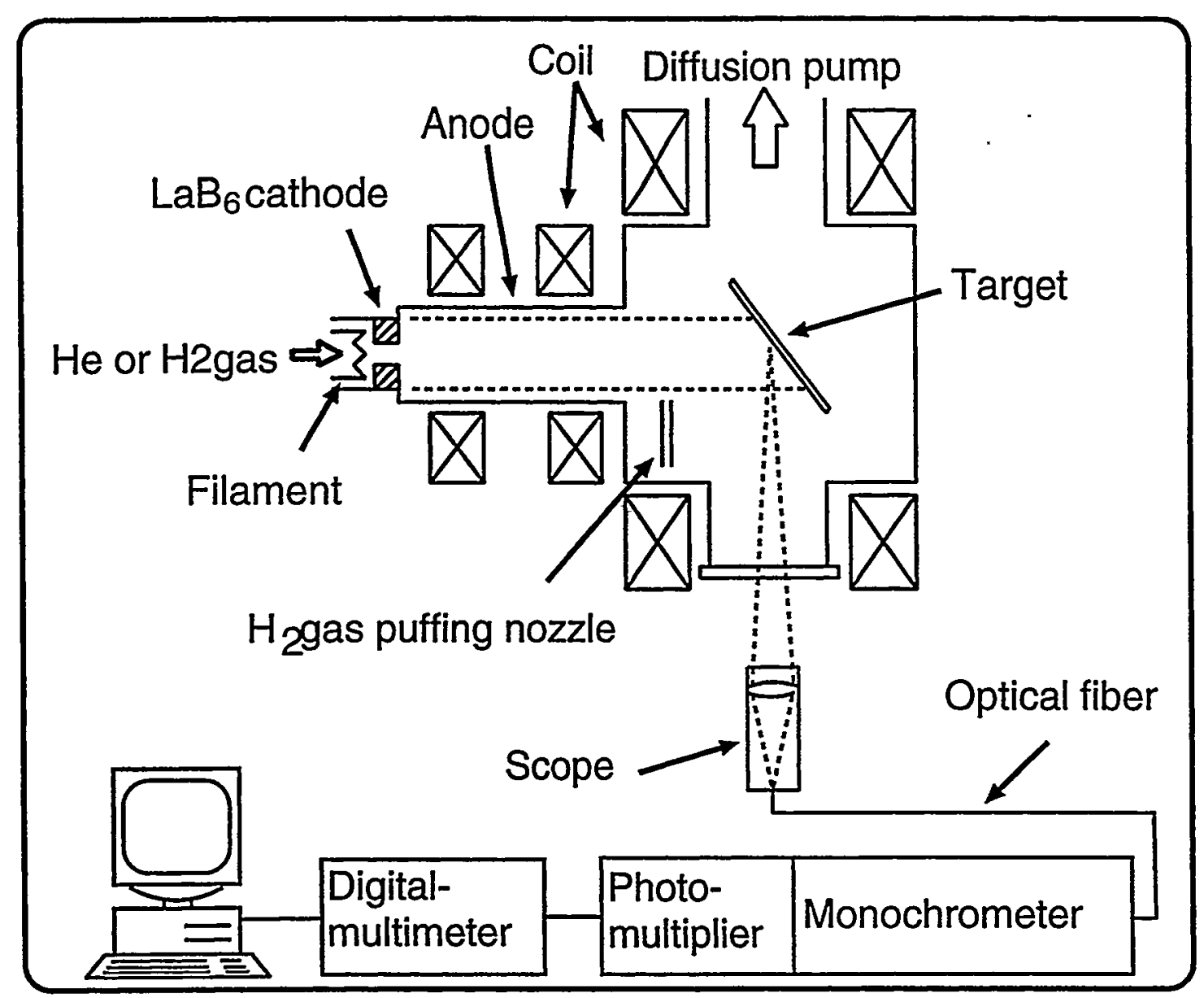

Experimental conditions

Temperature : $\sim 10 \mathrm{eV}$, Density :1.0X10E18 1/m^3.

Diameter of the plasma column : $3 \mathrm{~cm}$.

Plasma column length : $20 \mathrm{~cm}$.

- Target : Copper $(100 \mathrm{~mm} \times 100 \mathrm{~mm} \times 2 \mathrm{~mm})$.

- Magnetic field : 0.03T.

- Gas pressure : $5.0 \times 10^{15}$ Torr.

-Wave length resolution of the monochrometer $: 0.011 \mathrm{~nm}$.

- Target angle (Insident angle) : 0, $30,45 \underset{\text { IV-115 }}{60}$. 


\section{Spetrum Mesurement}

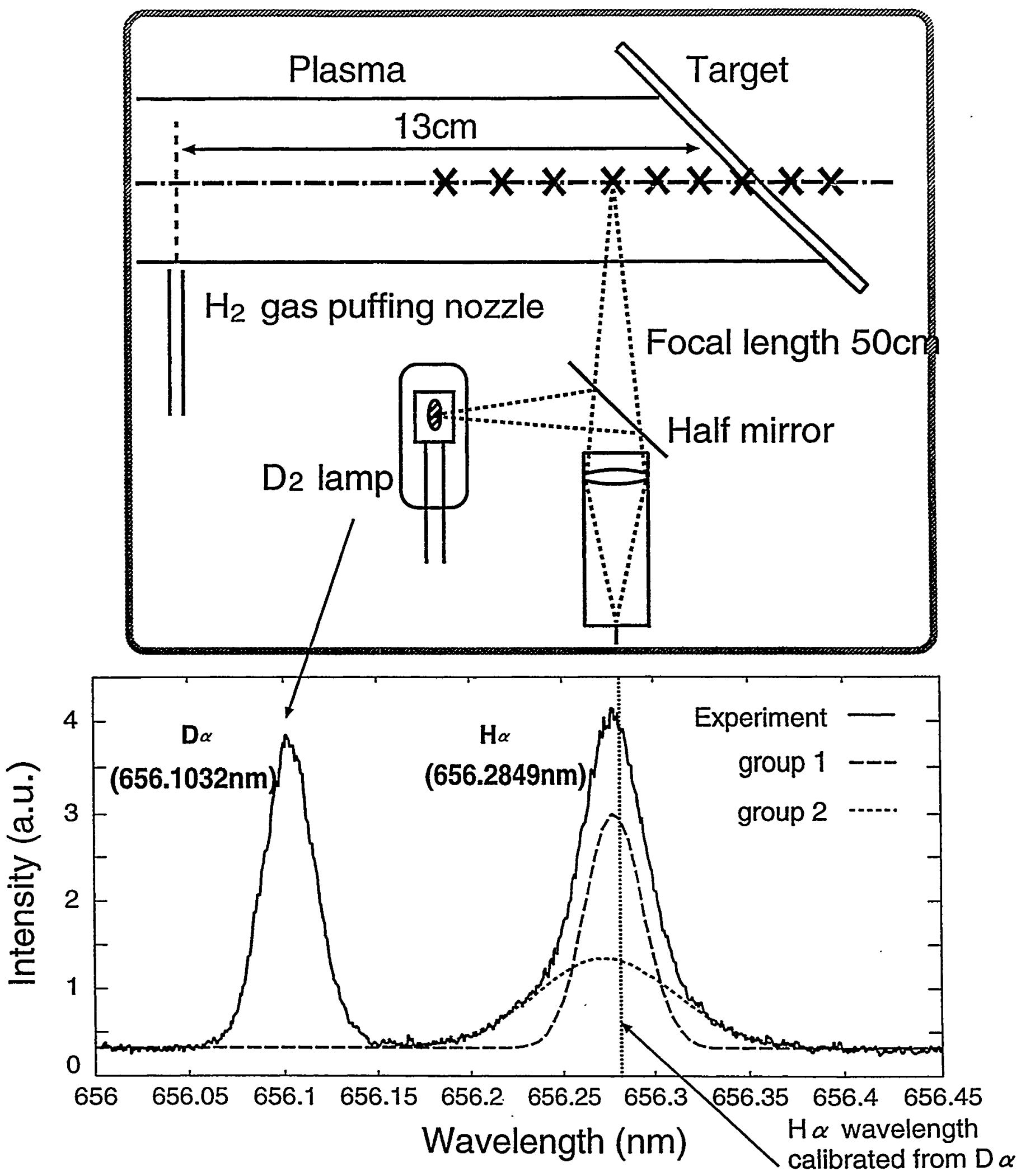

- $H \alpha$ spectrum is broader than $D \alpha$ spectrum.

Doppler-broadening

$\mathrm{H} \alpha$ spectrum at $3 \mathrm{~mm}$ from the target um into two gaussian curves

- $\mathrm{H} \alpha$ is not symmetric.

(wide at shorter wavelength)

IV-116

- The peak of the group 2 spectrum slightly shifts to lower wavenumber. 


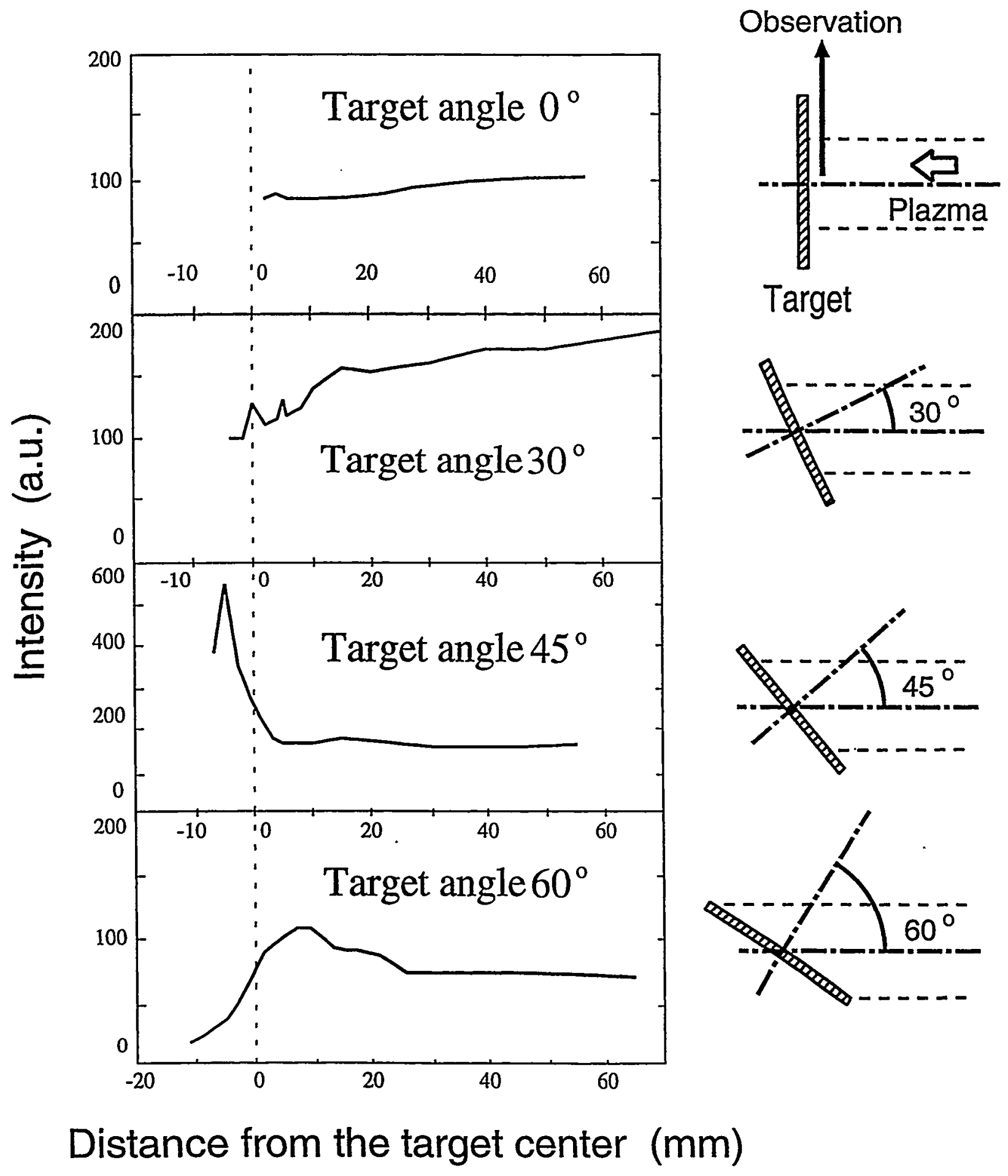

Axial distribution of the $\mathrm{H} \alpha$ intensity 


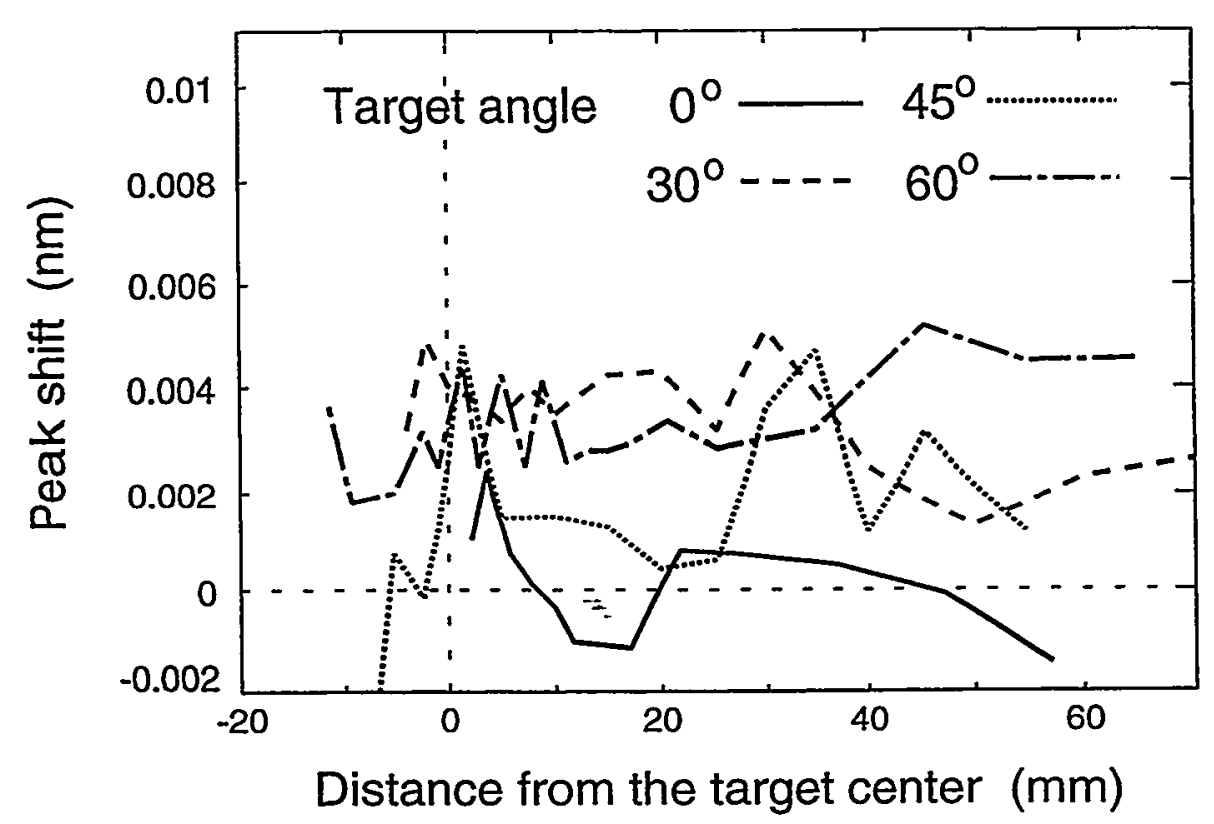

Peak shift to the shorter wavelength of the group 1 spectrum

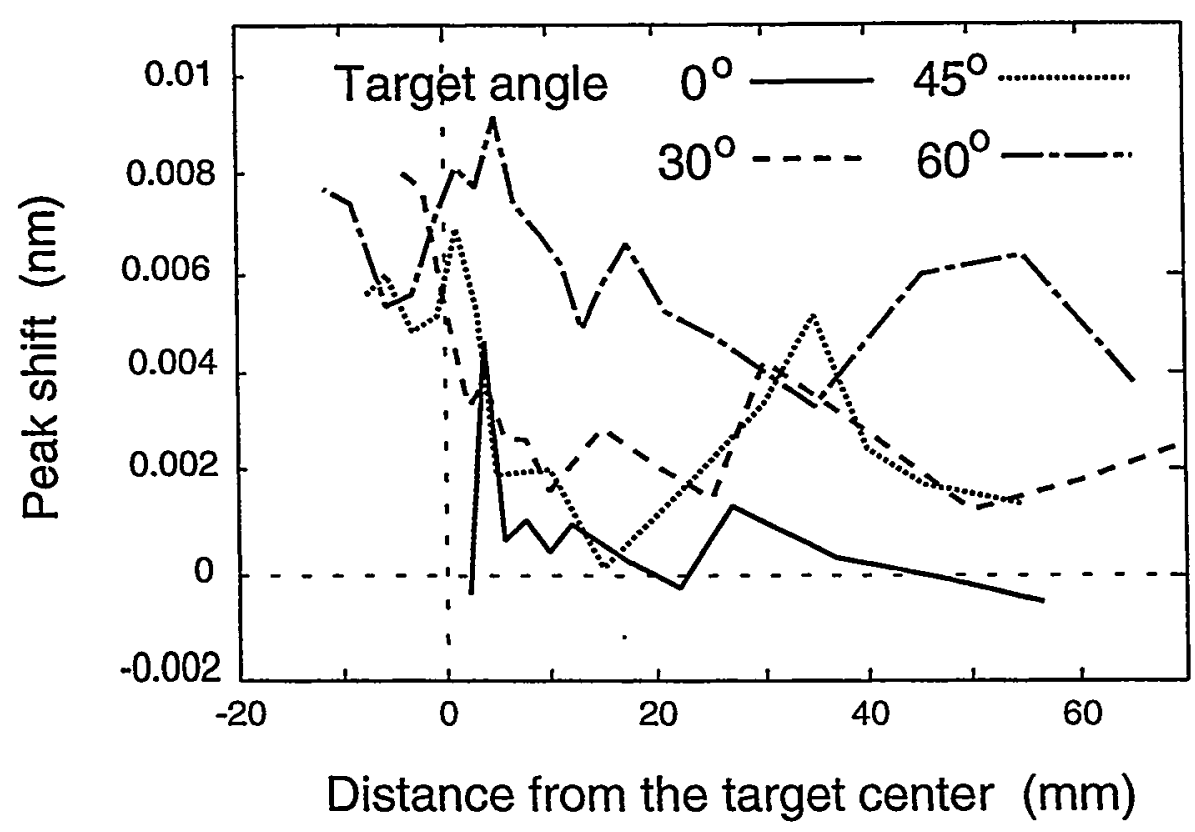

Peak shift to the shorter wavelength of the group 2 spectrum 

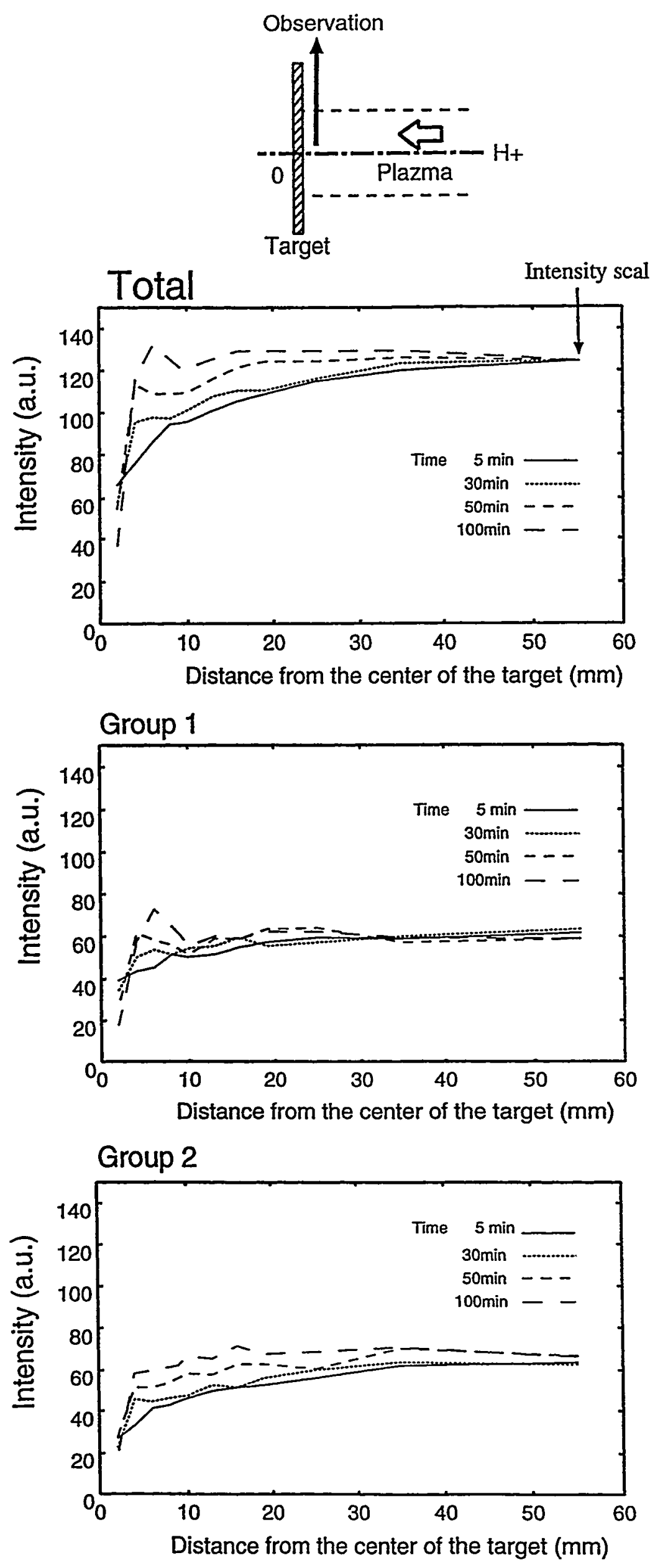


\section{- Calculation of the $\mathrm{H} \alpha$ spectrum}

We calculated $\mathrm{H} \alpha$ spectrum emitted from reflected hydrogen atoms by following two processes.

(1)Simulation of the reflecting process

by the TRIM code. $+$

(2)Simulation of the excitation process by

Monte Carlo method.

(1) Simulation of the reflecting process by the SRIM97 (aka TRIM)

code.

Incident angle (target angle) $: 0^{\circ} 、 30^{\circ} 、 45^{\circ} 、 60^{\circ}$ Incident particle : 10,000 hydrogen ions,

Incident energy : $30 \mathrm{eV}$,

- Nuclear stopping

$$
\theta=\pi-2 \int_{\mathbf{r}_{\min }}^{\infty} \frac{\mathrm{p}}{\sqrt{1-\frac{\mathbf{p}^{2}}{\mathbf{r}^{2}}-\frac{\mathrm{U}(\mathbf{r})}{\mathbf{E r}}}} \frac{\mathrm{dr}}{\mathbf{r}^{2}} \quad \mathrm{U}(\mathbf{R})=\frac{\mathrm{z}_{1} \mathrm{z}_{2} \mathrm{e}^{2}}{\mathrm{aR}} \mathbf{\Phi}(\mathbf{R})
$$

$\mathrm{p}$ : Impact parameter $\Phi(\mathrm{R})$ : universal-screening-function

- Electronic stopping

$$
\begin{gathered}
\Delta \mathrm{E}_{\mathrm{e}}=\mathrm{LNS}_{\mathrm{e}}(\mathrm{E}) \quad \mathrm{S}_{\mathrm{e}}(\mathrm{E})=\mathrm{S}_{\mathrm{L}}(\mathrm{E})=\mathrm{kE}^{\mathrm{p}} \\
\mathrm{k}=\mathrm{k}_{\mathrm{L}}=\frac{1.212 \mathrm{z}_{1}^{\frac{7}{6}} \mathrm{z}_{2}}{\left(\mathrm{z}_{1}^{\frac{2}{3}}+\mathrm{z}_{2}^{\frac{2}{3}}\right)^{\frac{3}{2}} \mathrm{M}_{1}^{\frac{1}{2}}}, \quad \mathrm{p}=\frac{1}{2}
\end{gathered}
$$

IV-120

$$
\Delta E_{e}: \text { Electronic energy loss }
$$


(2)Simulation of the excitation process by Monte Carlo method.

Target surface is di-
vided into rectanglu-
lar meshes and parti-
cles are supposed to
be reflected at the
center of each meshes.

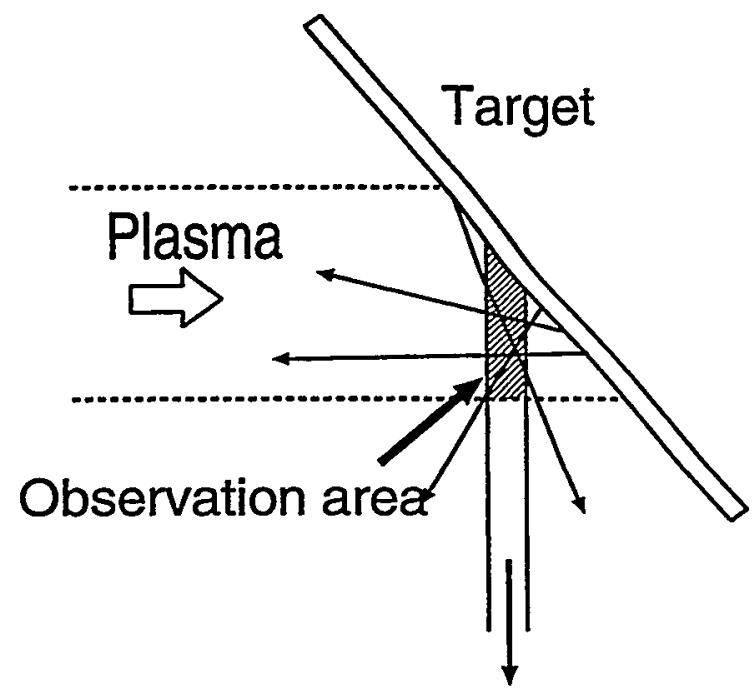

Observation directior

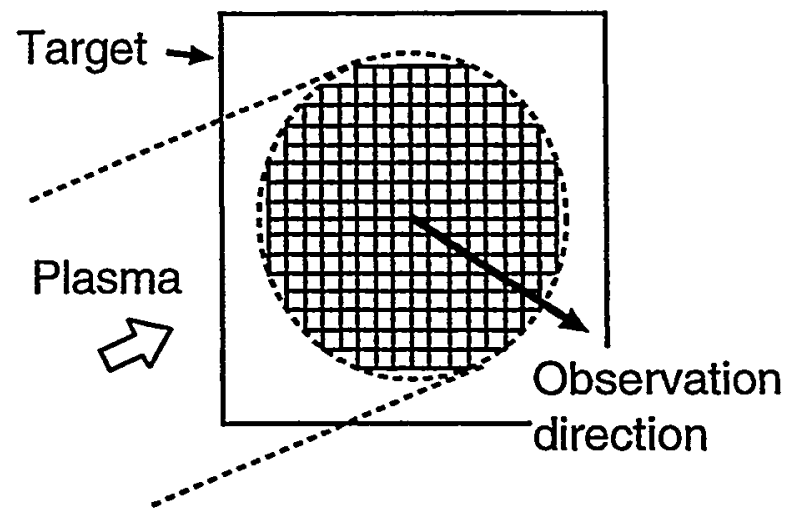

Then we calculate the coordinates of each particle until it emits $\mathrm{H} \alpha$ spectrum, and from the amount of the emission in the observation area, we obtain the intensity distribution of the $\mathrm{H} \alpha$ spectrum emitted by the reflected particles. 


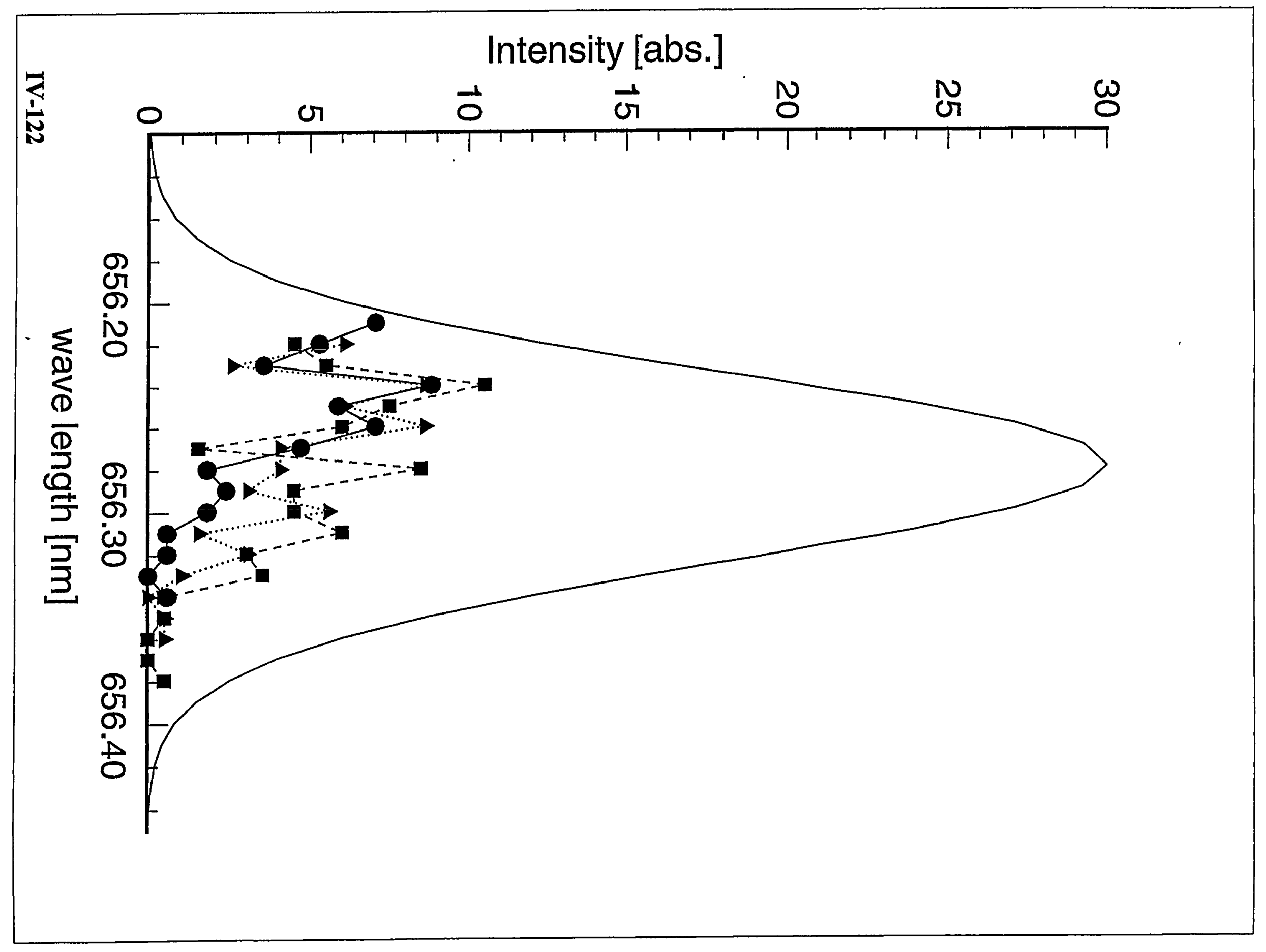




\section{Summary}

The $\mathrm{H} \alpha$ spectrum of the reflected hydrogen atoms were observed by spectroscopic analysis.

Spectrum of the backscattered particles were identified by the decomposition of the $\mathrm{H} \alpha$ spectrum. They were included in the high energy spectrum componet (group2).

After long time discharge, increase of $\mathrm{H} \alpha$ intensity in group1 (low energy group) was observed near the target. It is because low energy desorped particles are increased. 


\section{Session V: Plasma Studies}




\section{Ion Heating and Plasma Fluctuations in the UC San Diego PISCES Experiments}

S.C. Luckhardt, J. Cuthbertson*, R. Doerner, D. Whyte, and the UCSD PISCES Group

\section{US-Japan Workshop (97FT5-06)}

High Heat Flux Components \& Plasma Surface Interactions for Next Fusion Devices

December 8-11, 1997

San Francisco, CA

* Presently at San Diego Econometrics 


\section{PISCES and Collaboration Experimental Program Staff}

16 Scientists and Engineers, 4 Graduate Students

\section{Scientific and Research Personnel:}

J. Boedo (DIII-D and TEXTOR Collaborations)

Prof. R. W. Conn (Dean of engineering)

R. Doemer (PISCES-B Materials group leader)

D. Gray (DIII-D Collaborations)

A. Grossman (Plasma Materials Modeling)

Y. Hirooka (PISCES/TFTR Materials)

R. Lehmer (DIII-D Collaboration)

S. Luckhardt (Experimental Division, PISCES-A)

R. Moyer (DIII-D Collaborations)

R. Saraydarian (PISCES Materials, spectroscopy)

D. Sze (PISCES Materials surface science)

D. Whyte (joining in $2 / 97$, PISCES Materials, boundary)

\section{Engineering Staff:}

L. Chousal (Mechanical Engineer, Design, Beryllium Operations)

G. Gunner (Control Systems, Beryllium Operations)

P. Luong (Electronics, Vacuum Systems, Beryllium Ops.)

A. Viray (Safety Engineer, Beryllium Safety)
Graduate Students:

L. Blush!

Y. Duan

A. Liebscher

J. Zhang

Visiting Scientists:

Prof. H.Y. Chang (KAIST)

Dr. S. Zweben (PPPL)

Admin. Staff:

J. Hylton (Personnel, Payroll)

M. Garcia (Accounting)

T. Garcia (Clerical) 


\section{FACILITIES: UCSD Fusion Program Laboratories}

PISCES-B Mod Plasma Beryllium and Mixed Materials Interaction Facility

Surface Analysis Module Scanning Etching Ion Beam, Auger Electron Spectroscopy (AES), X-ray Photoelectron Spectroscopy (XPS), Thermal Desorption Spectroscopy (TDS).

PISCES-A Plasma Boundary Science Facility: Divertor and"Edge Plasma Simulator

PISCES Surface Science Laboratory (Scanning Electron Microscope, Back Scattered Electron Spectroscopy, Profilometer) 
UL SAN DIEGO PISCES $A+B$

"Flux Tube" Diverter Simulator

ION BEHAUIOR, MAIN H,D, He, ImPURITY $C, O$ IS ImPORTANT FOR

- enERGY SPECTRUM OF CX FLUX

- divertor un heat flux

$$
q_{11 i}=n v_{i}\left[\frac{5}{2} T_{i}+\frac{1}{2} m_{i} v_{n i}^{2}+I_{0}\right]
$$

- ION GYRO-RADIUS EFFEcTs: GYRO SHEATH.,Et

- mIXed materials efFEctS IMPURITY ION FLUXES, ENERGy RANCE, IMPURITY DENSITY,...

$\frac{\text { PAST YER }}{\text { PISCES }}$

PISCES CuR OR EXPerimenteR CampaiGn!

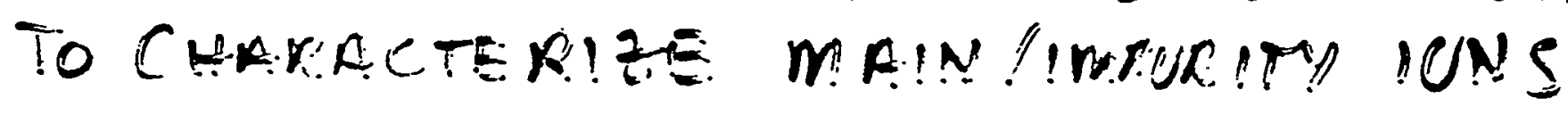

- main lon temperatures Til, $T_{+}$

- impurity lon density and temperature

- impurity DENSITY CONTROL: $C D_{4}$ PUFFING

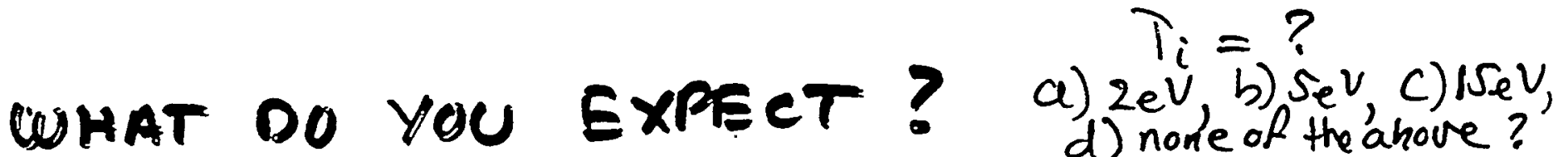

a) $2 \mathrm{eV}^{\mathrm{V}}$, b) $\left.\mathrm{se}^{v}, \mathrm{c}\right)(\mathrm{seV}$
d) none of the a hove?

? Dissociation Energies: 2 -3eV

? $T_{\dot{c}} \ll T_{e}$

$\vec{V}-6$ RESULTS $\sim 5$ - $20 \mathrm{eV}$ RANCE!! 

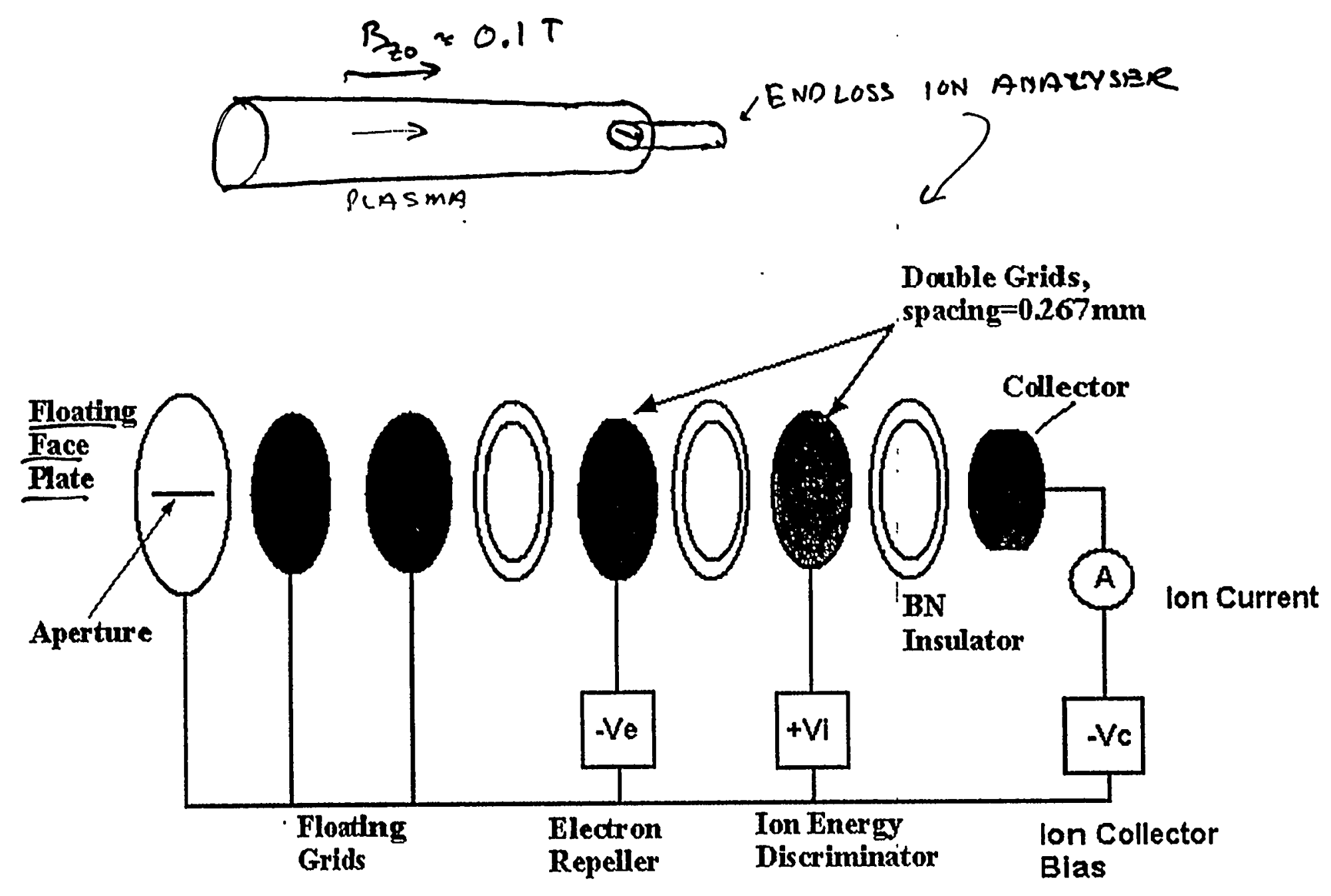
Best Fit ion Energy Spectrum PISCES $A$ \# 27045

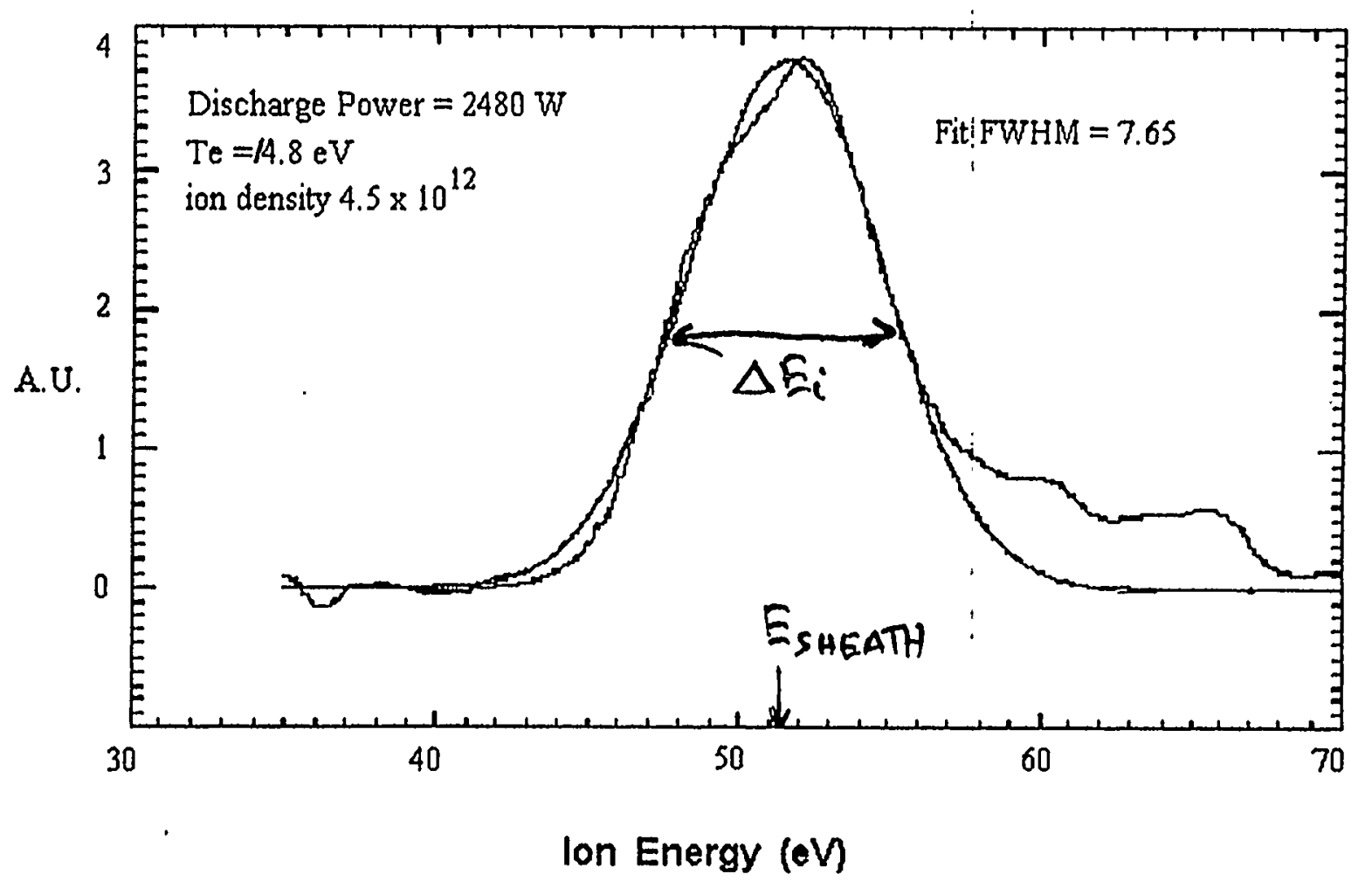

$$
\Delta E_{i \|}=7.6 \mathrm{cV}
$$$$
T_{e}=14.8 \mathrm{eV}
$$$$
n_{i}=4.5 \times 10^{18} \mathrm{~m}^{-3}
$$$$
c_{s}=\left(\frac{Z_{i} T_{e}+T_{i}}{m_{i}}\right)^{1 / 2}
$$

$V_{\text {SHEATH }}=2$

$\frac{T_{e}}{2} \ln \left[\frac{m_{i}}{2 m_{e}\left(1+T_{i} / T_{e}\right)}\right]$

Kinetic energy distribution of lons exiting the end of the PISCES-A plasma column as measured by the GEA. 


\section{FLOATING POTENTIAL AUTO-POWER SPECTRUM CHARACTERISTIC FREQUENCY RANGE $100 \mathrm{kHz}$ PISCES-A}

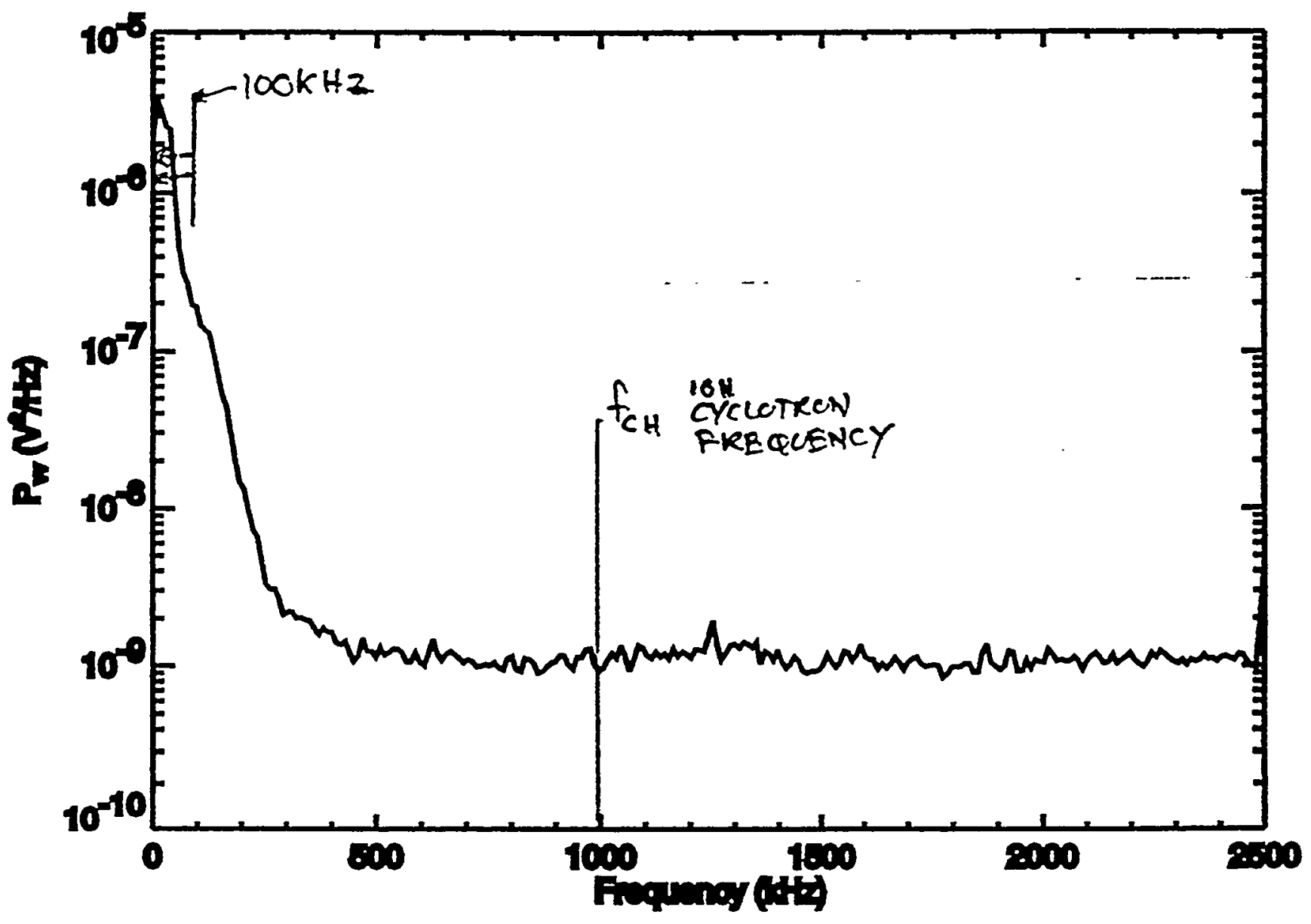



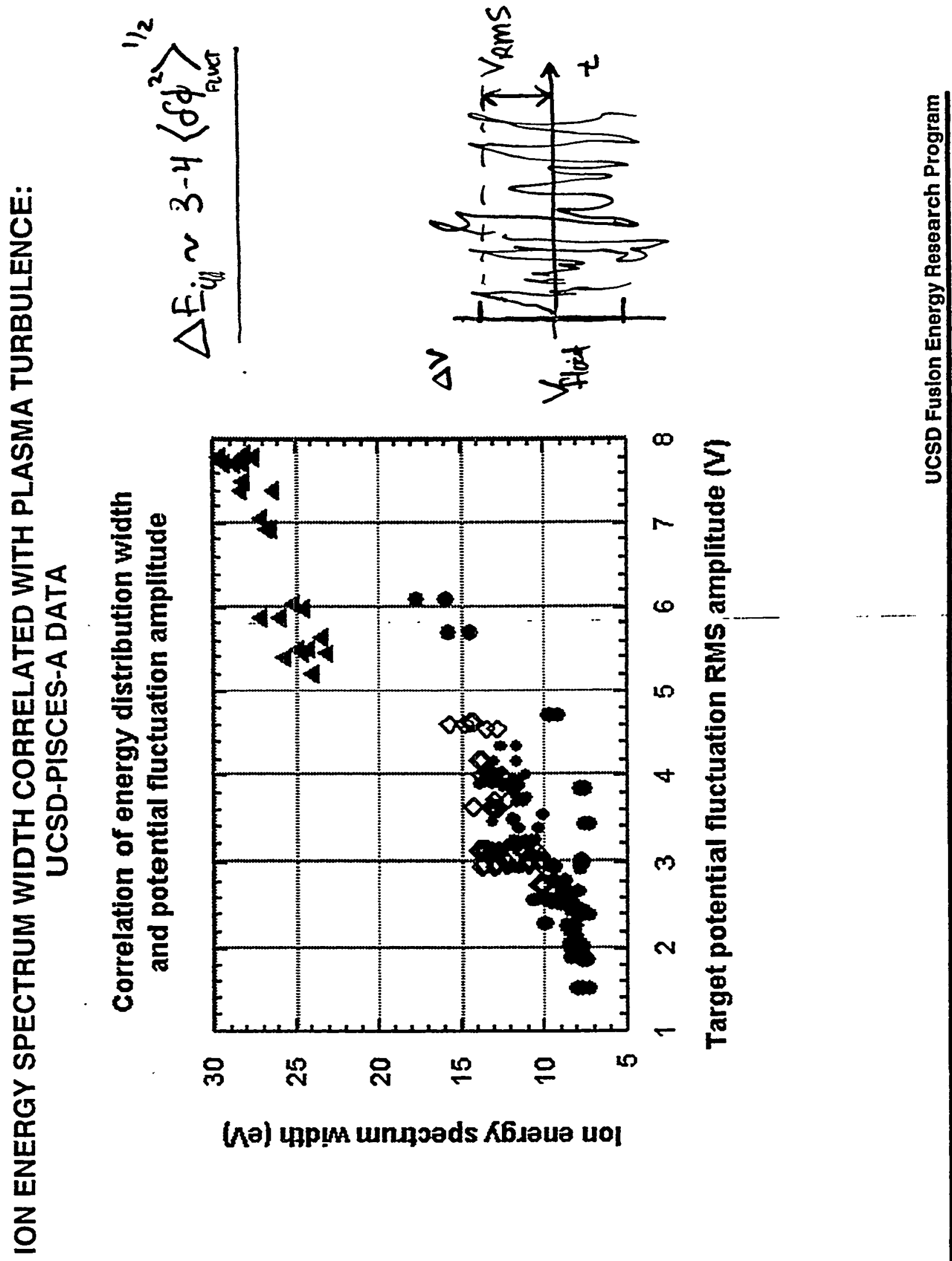

$N$

울

$\infty$

통

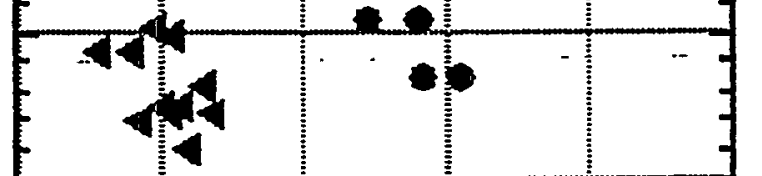

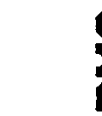

(1) 통

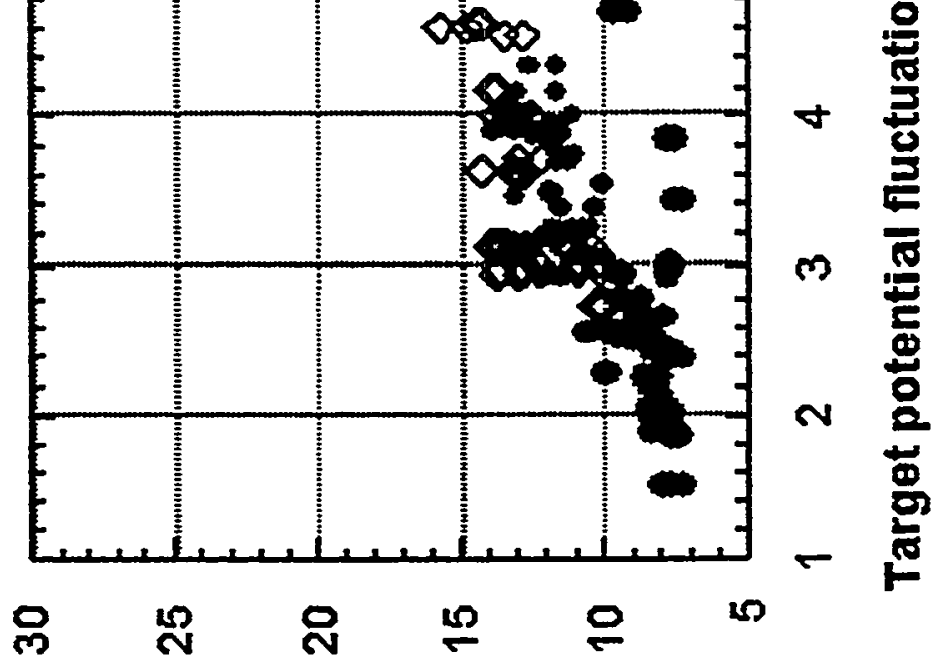

(na) цp!M unnoads K6」aua uol 


\section{PISCES-B Impurity Experiments}

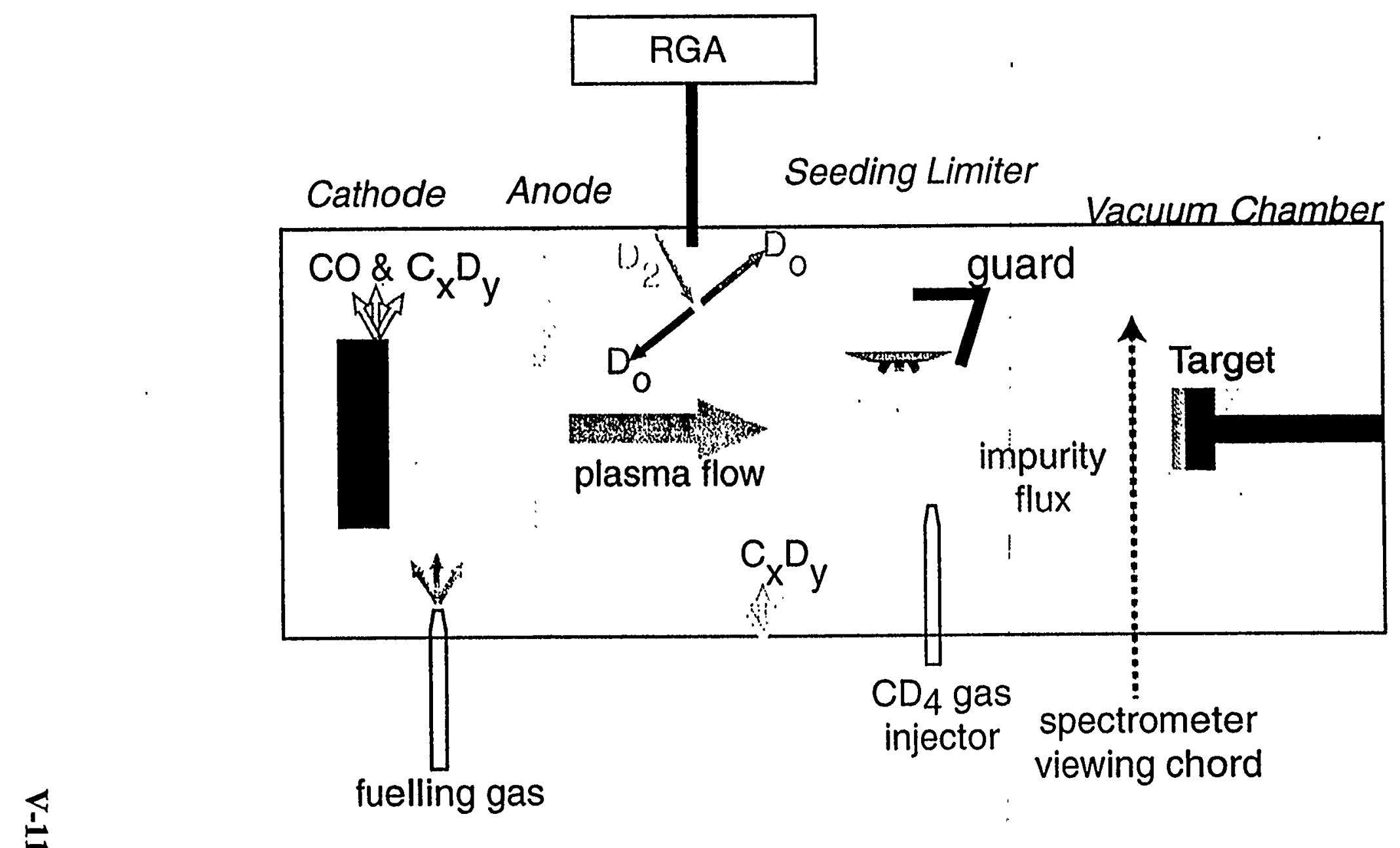




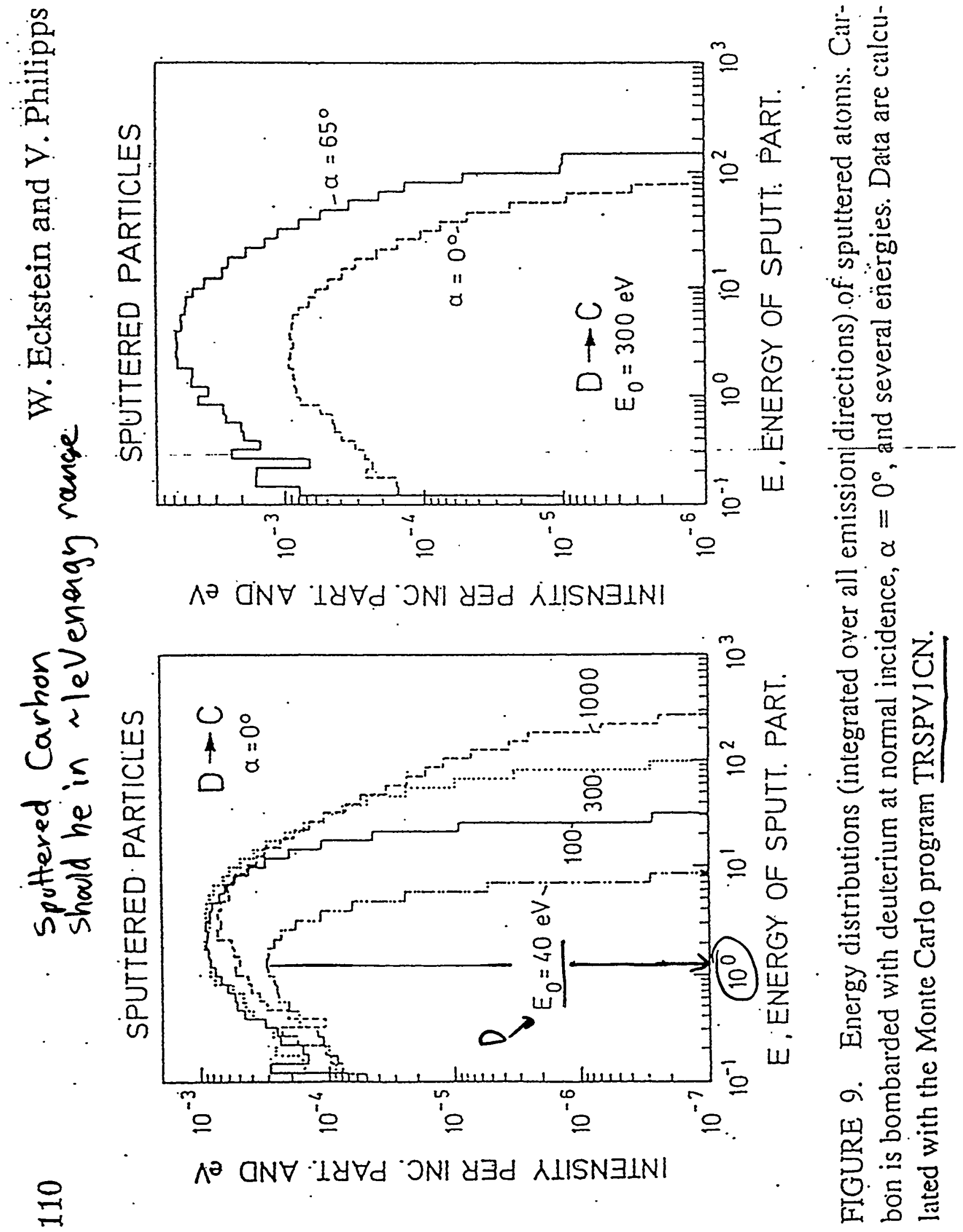



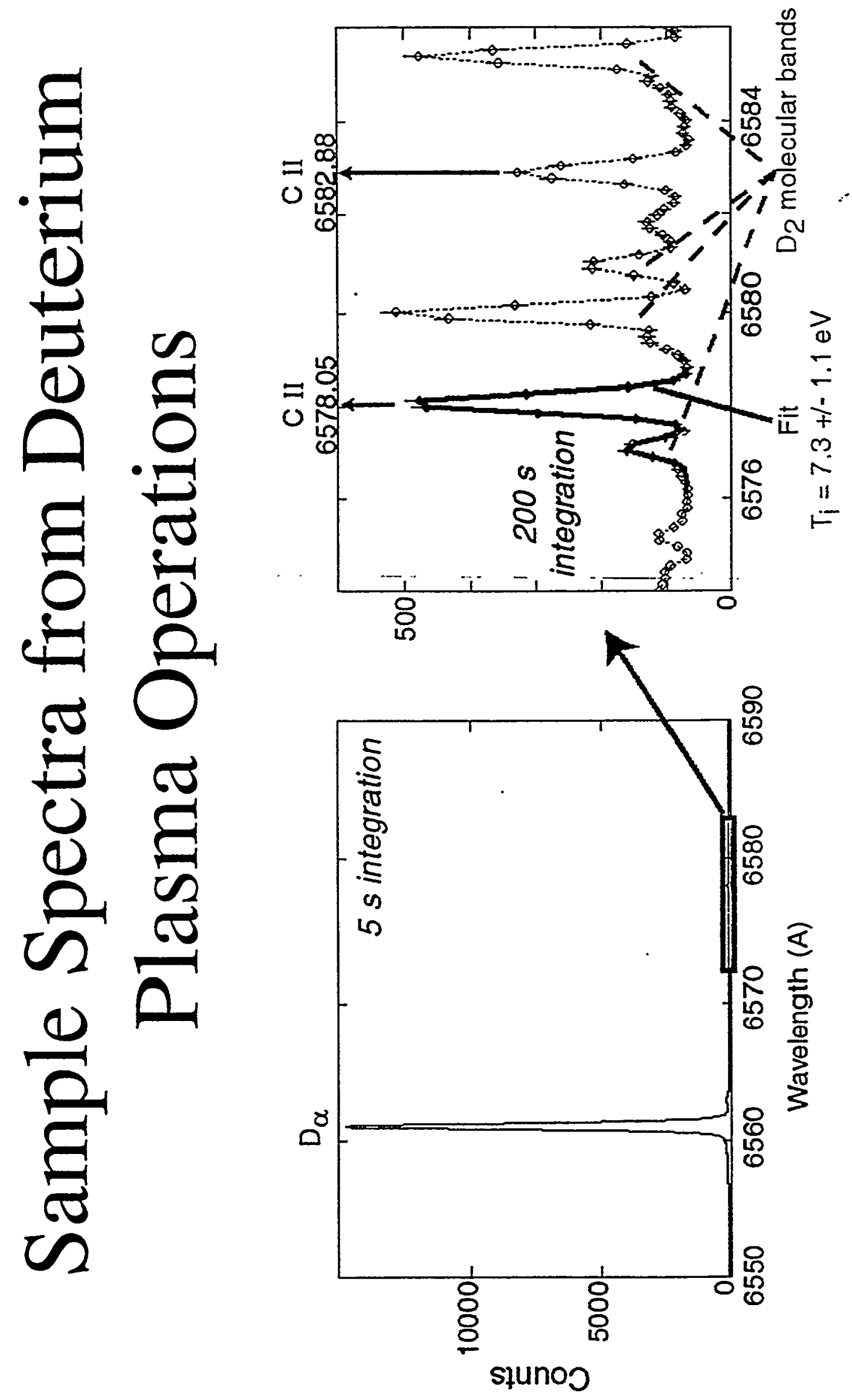


\section{Baseline Ion Temperatures}

Deuterium $T_{e}=40 \mathrm{eV} \quad \mathrm{n}_{\mathrm{e}}=0.7 \times 10^{12} \mathrm{~cm}^{-3}$

$\mathrm{T}_{\mathrm{i}}$, Carbon $=7.9 \mathrm{eV}$ (Doppler broadening)

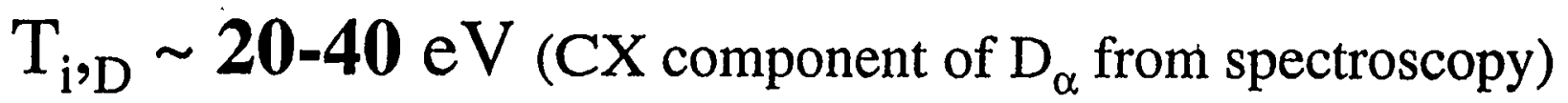

Helium $\mathrm{T}_{\mathrm{e}}=30 \mathrm{eV} \quad \mathrm{n}_{\mathrm{e}}=10^{12} \mathrm{~cm}^{-3}$

$\mathrm{T}_{\mathrm{i}}$, Carbon $=7-9 \mathrm{eV}$ (Doppler broadening)

$\mathrm{T}_{\mathrm{i}, \mathrm{He}} \sim \mathbf{1 0 - 1 5} \mathrm{eV}$ (Doppler broadening of He II)

$\mathrm{T}_{\mathrm{i}, \mathrm{He}} \sim \mathbf{1 0 - 2 0} \mathrm{eV}$ (Energy grid analyzer [Cuthbertson, et al.]) 


\section{Plasma Carbon Concentrations}

The CII doublet at 6578 and $6583 \AA\left(2 s^{2}\left({ }^{1} S\right) 3 p\right.$ $\left.2 s^{2} 2 p 3 s\right)$ can be measured simultaneously with $D_{\alpha}$ (6561 $\AA$ ) or He I (6678 $)$ ) to provide carbon ion concentrations.

$$
f_{C}=\frac{B_{C I I}}{B_{D_{\alpha}\left(H e I_{6678}\right)}} \frac{\langle\sigma v\rangle_{D_{\alpha}\left(H e I_{6678}\right)}}{\langle\sigma v\rangle_{C I I}} \frac{n_{D_{0}\left(H e_{0}\right)}}{n_{e}}
$$


"Baseline" Impurity Contamination (Spring 1997)

$$
\begin{gathered}
\text { Deuterium } \\
\mathrm{f}_{\text {Carbon }}=0.2 \% \\
\mathrm{P}_{\mathrm{Cx}} / \mathrm{P}_{\mathrm{D} 2}=0.3 \% \\
\mathrm{f}_{\mathrm{Oxygen}} \leq 0.1 \% \\
\mathrm{n}_{\mathrm{e}}=9 \mathrm{x} 10^{11} \mathrm{~cm}^{-3}, \mathrm{~T}_{\mathrm{e}}=40 \mathrm{eV}
\end{gathered}
$$

- Dominant source of carbon in hydrogenic plasmas appears to be hydrocarbon release from walls.

- Upper bound on oxygen content given from spectroscopy detection limit. 


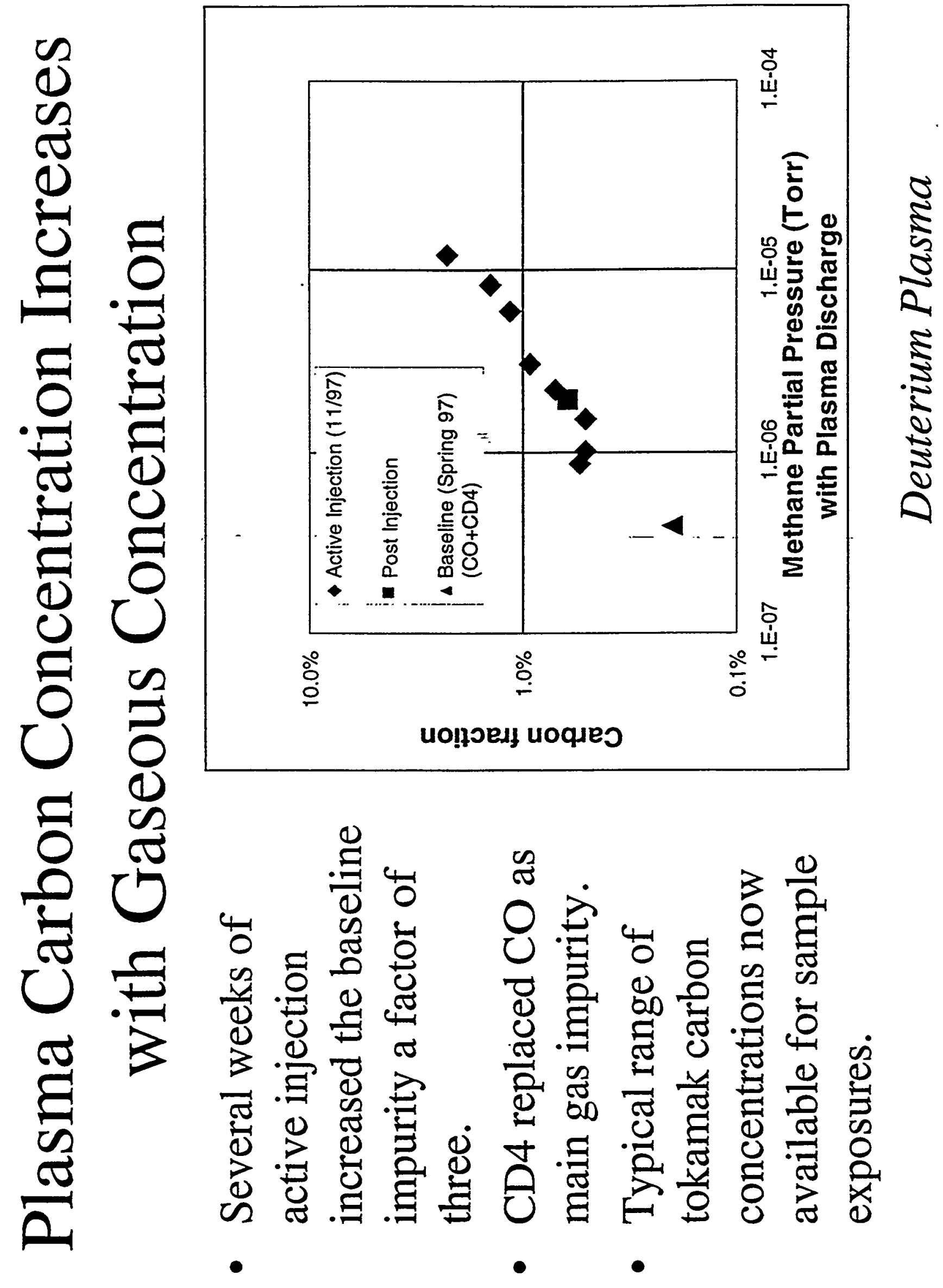




\section{DOLLOP : Improved Plasma}

Performance using a New Concept for Mitigating the Plasma-Wall Interaction in Fusion Devices

C.H. Skinner, D.K. Mansfield the TFTR Group

Motivation:

To Improve Performance by Modifying the Plasma - Wall Interaction with Minimal Perturbation to the Core 
Experimental Technique: Lithium Introduced onto Limiter by Injection of Pellets

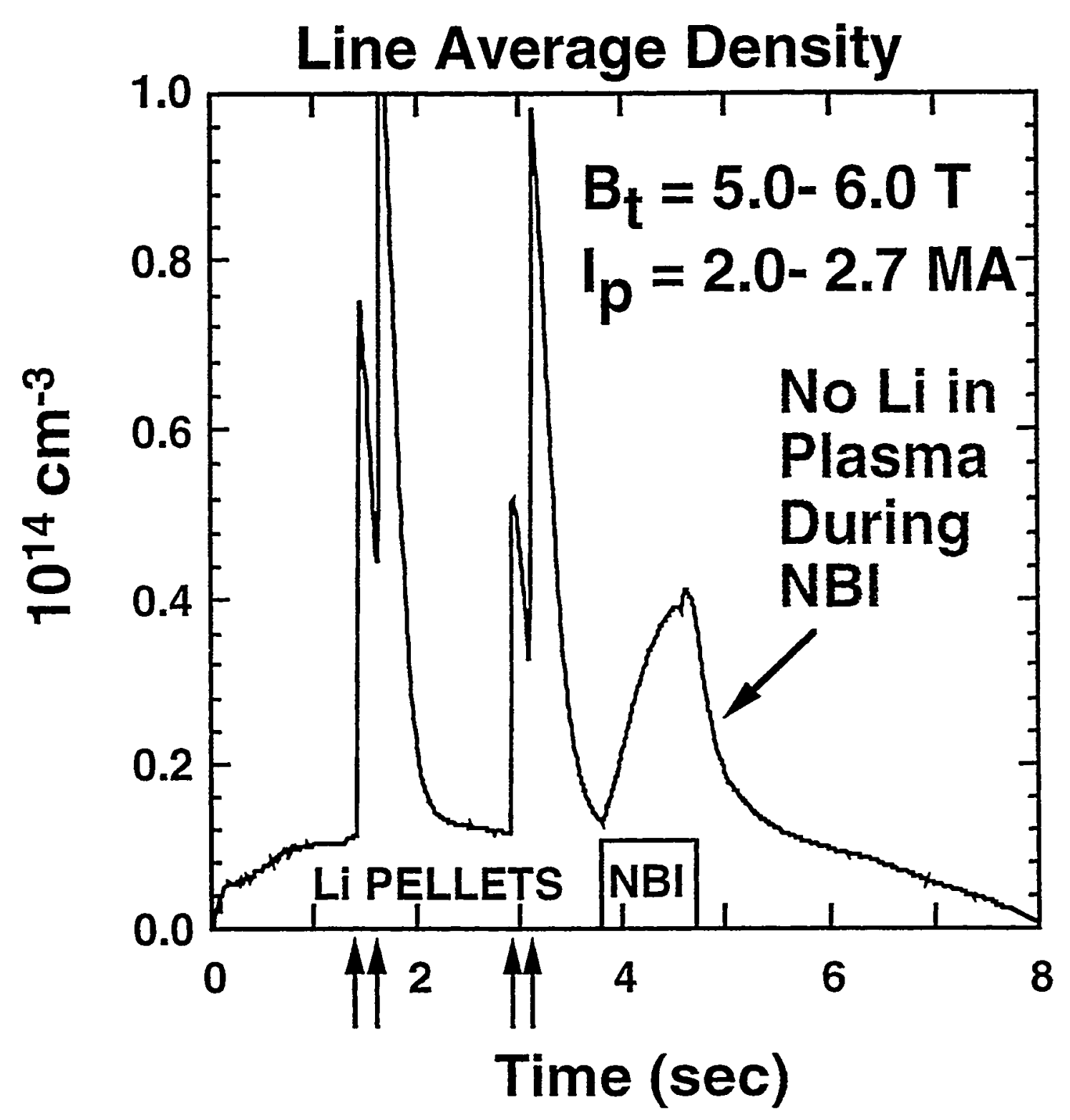

- Extended Clean-up Campaign Necessary

- Li Accumulated by Pre-conditioning

- Minimizing Limiter Contact is Helpful 


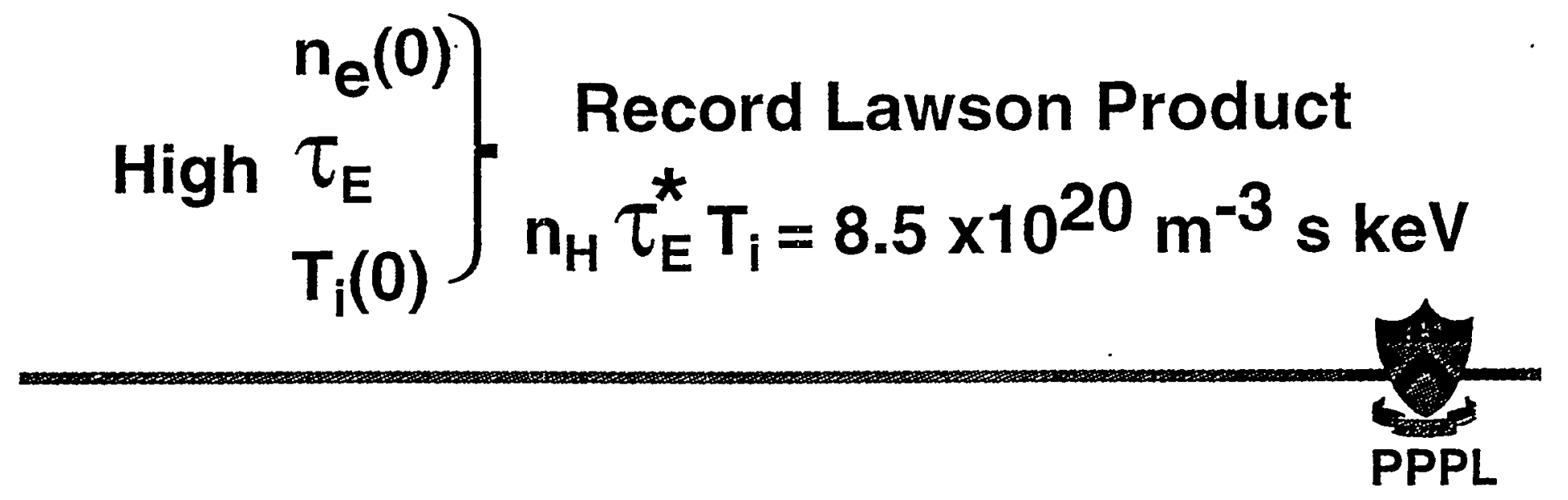

- Tritium-only Supershot 4 Pellets + Painting
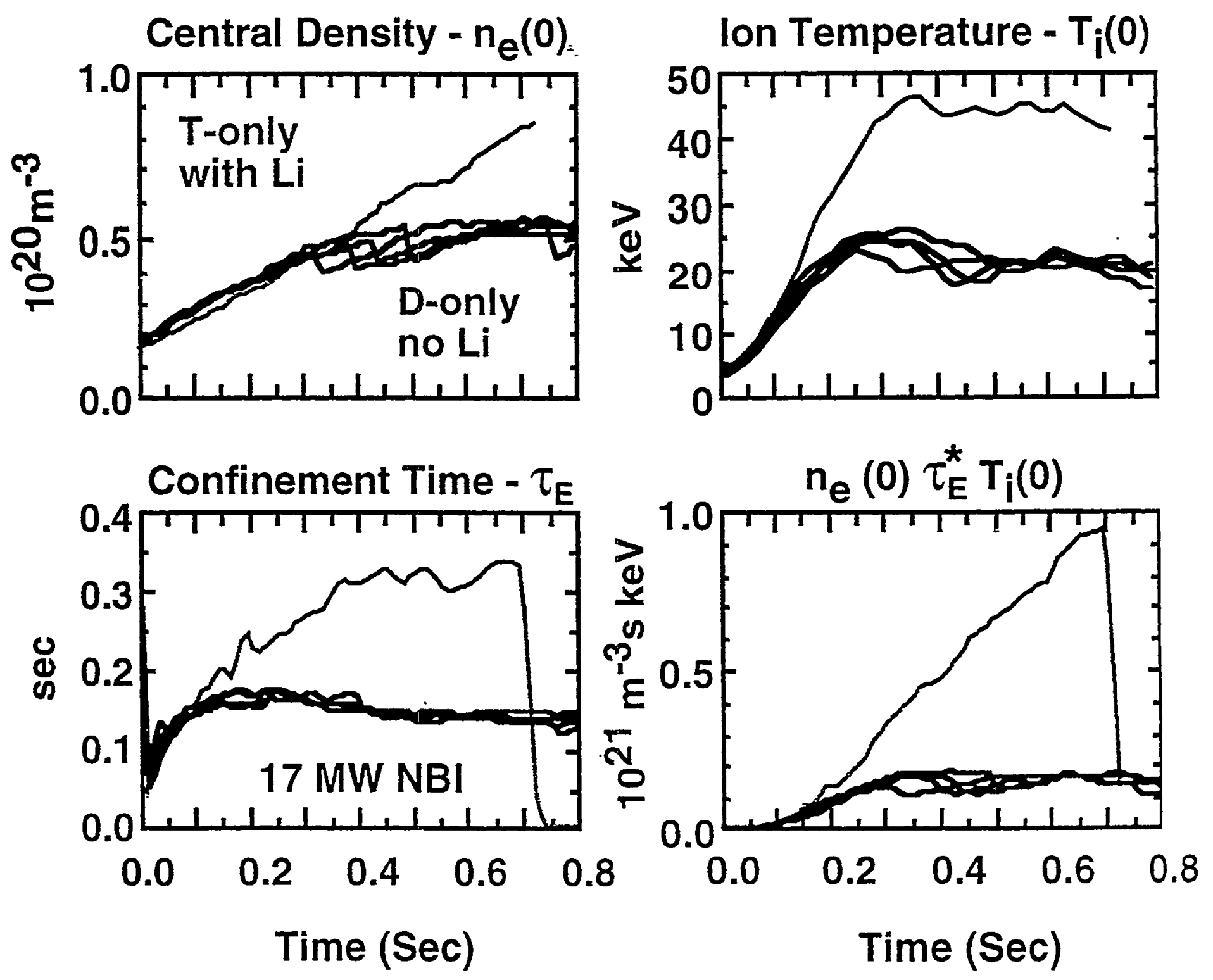


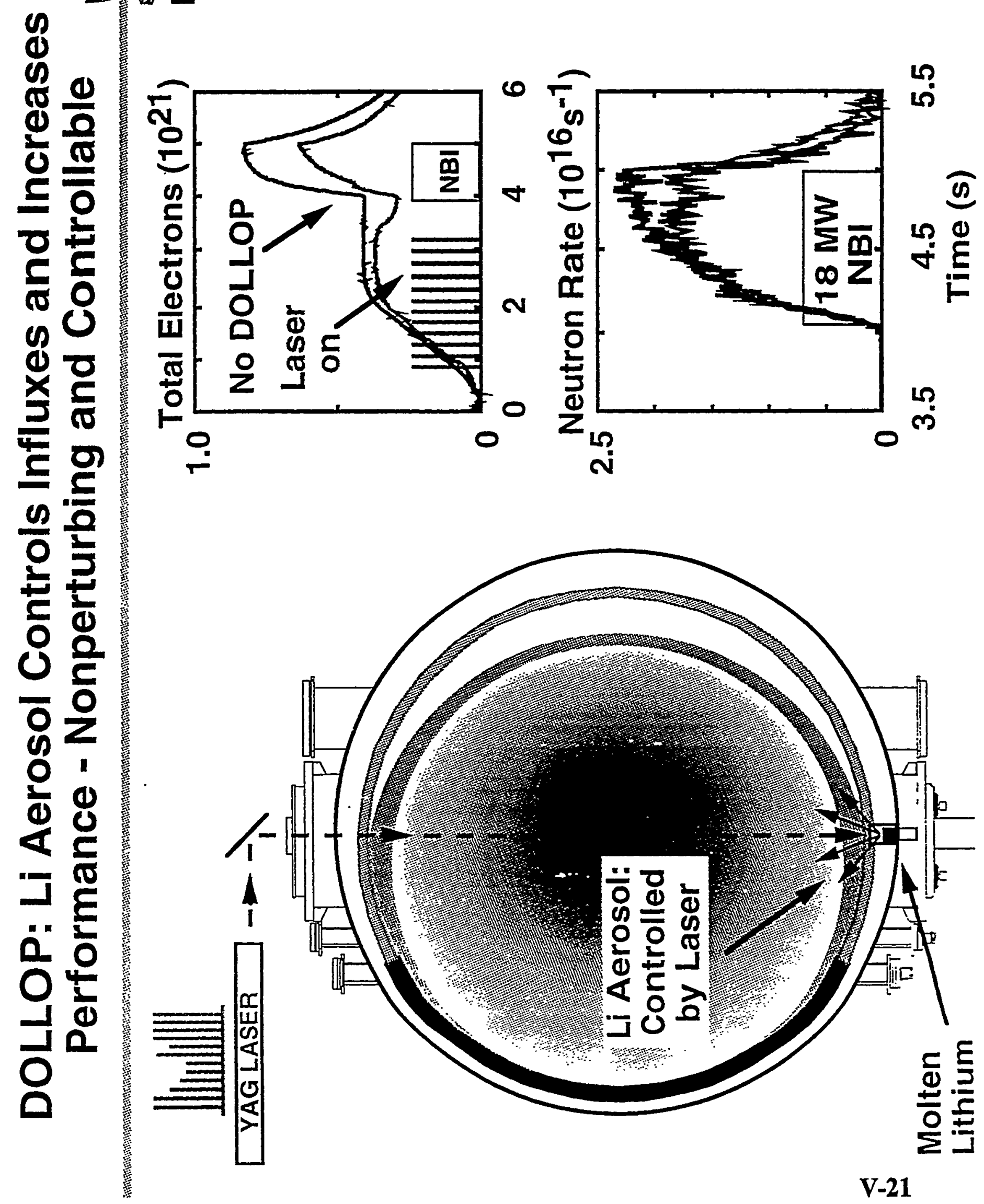




\section{DOLLOP : Initial Effects of Laser-induced Li Aerosol on Ohmic Discharges}

Total Electrons (1020)

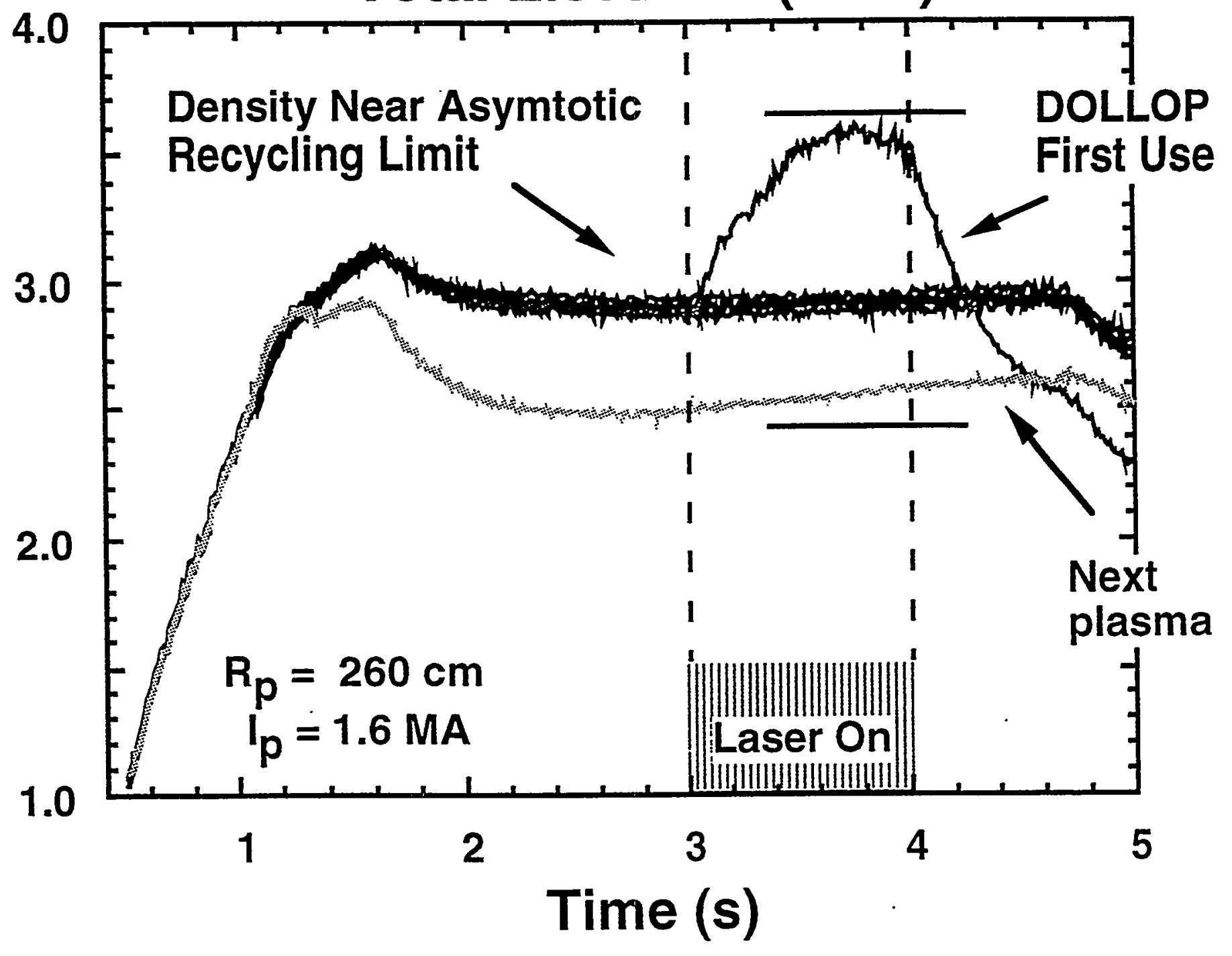

- Residual conditioning is clear

- Both a source and a sink of particles

- Several time constants at work 


\section{DOLLOP : Initial Effects of Laser-induced Li Aerosol on Ohmic Discharges}
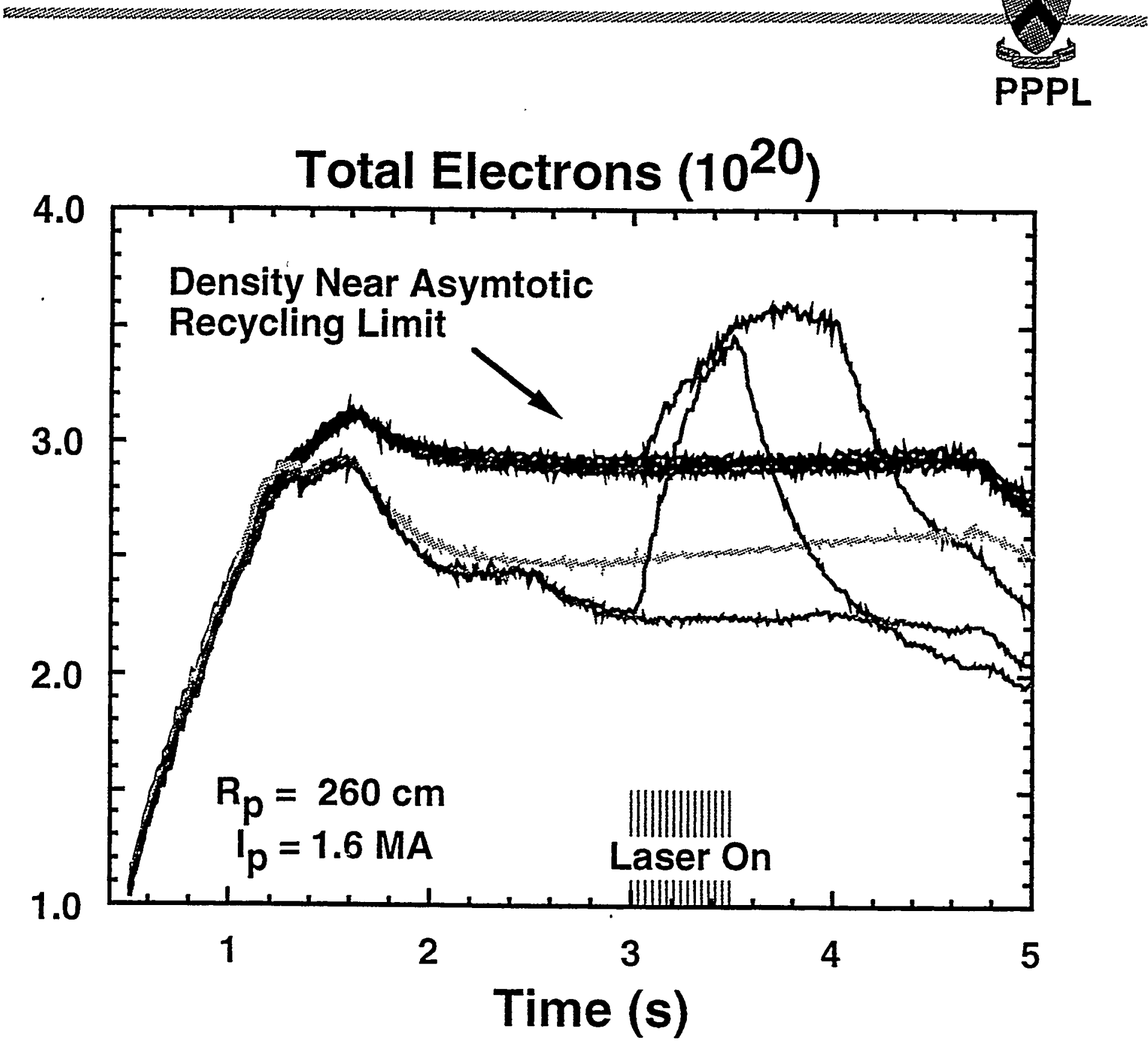

- Deposition controlled optically

- $5 \%$ of Li to Plasma - $95 \%$ to SOL

- Plasma reaction benign 


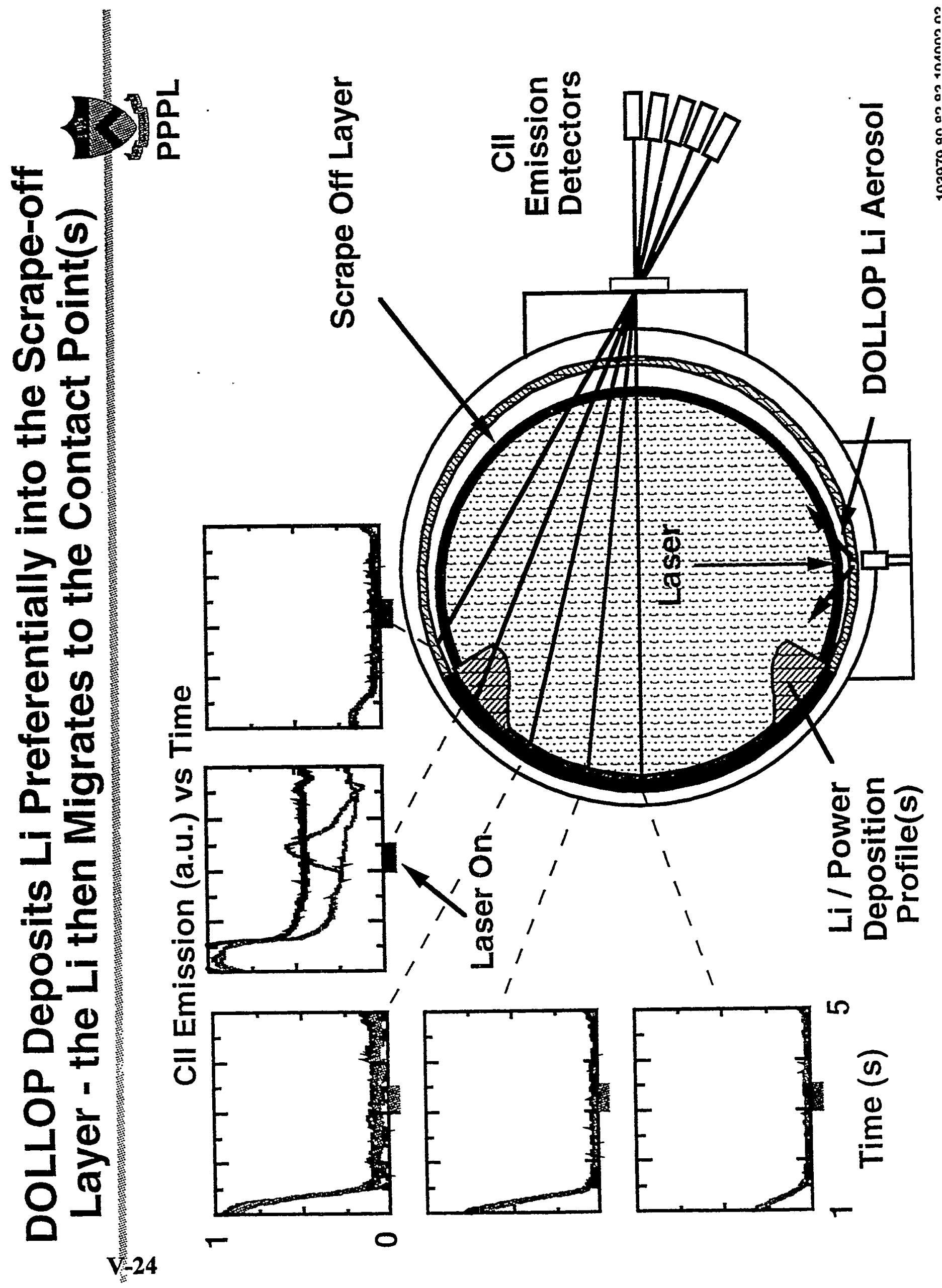




\section{DOLLOP Has Led to Enhanced and Sustained Performance with No Harmful Effects}

Confinement Time
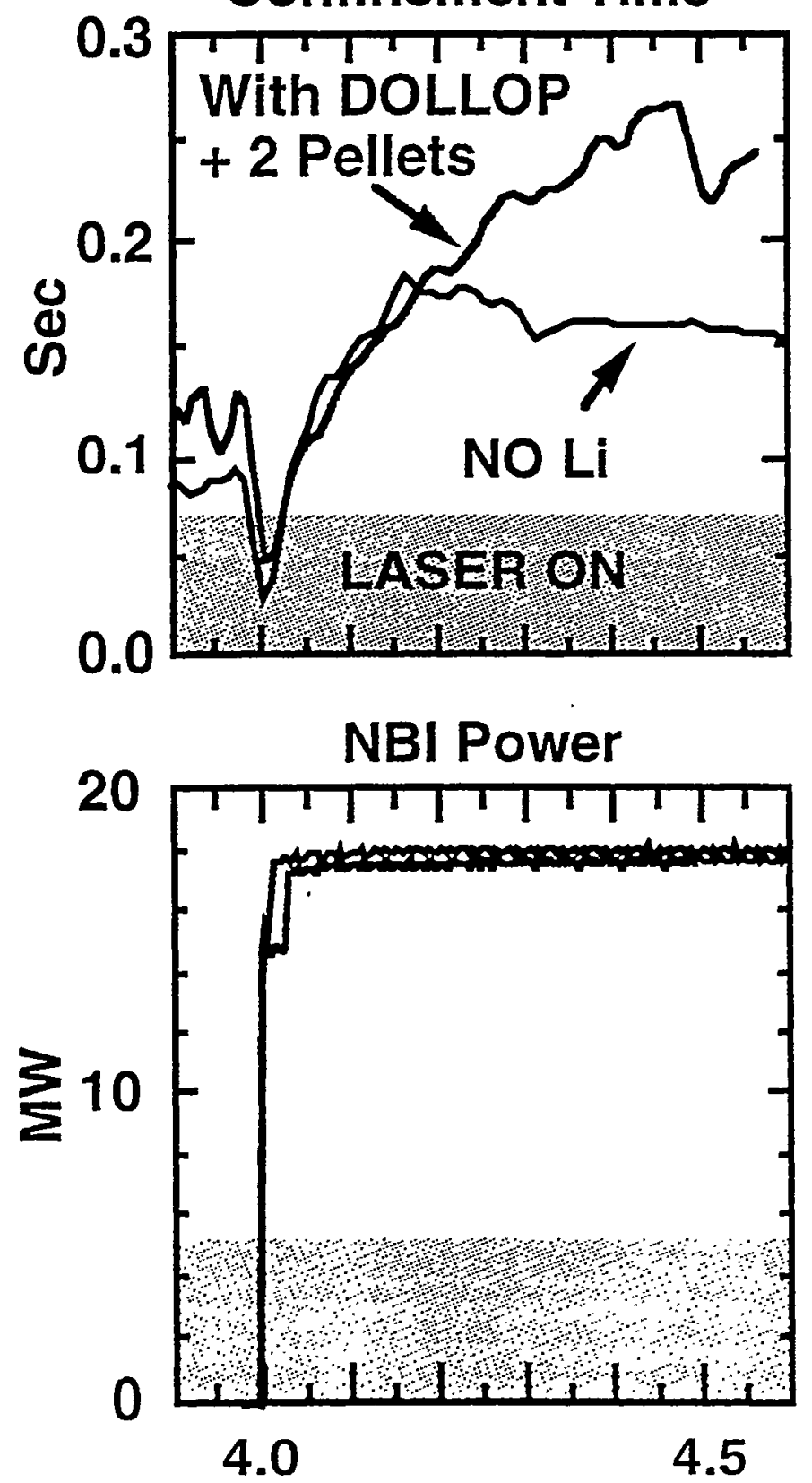

DD Neutrons
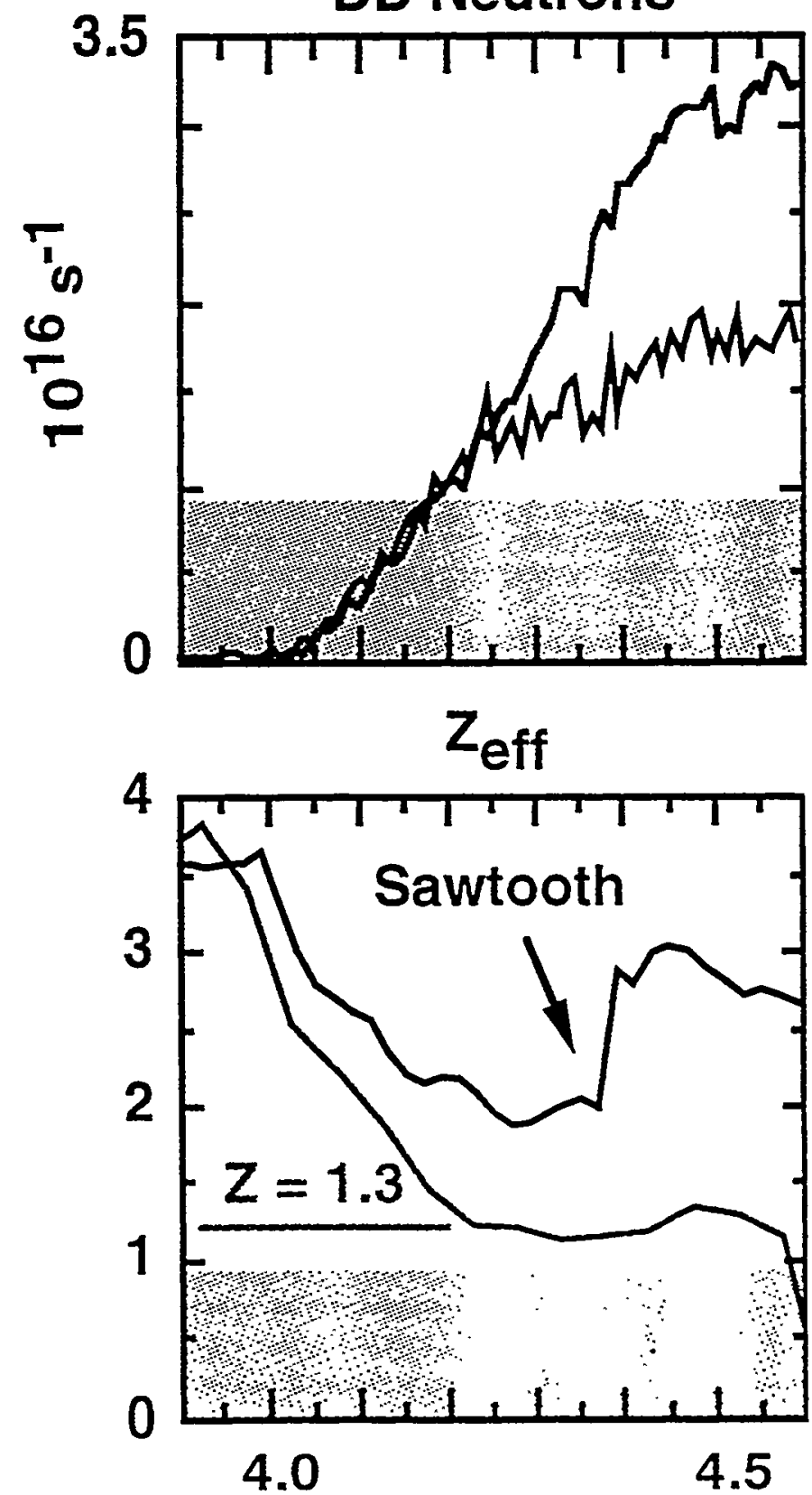

Time (s) 


\section{During its Initial Use, DOLLOP Has Raised the Plasma Internal Inductance}

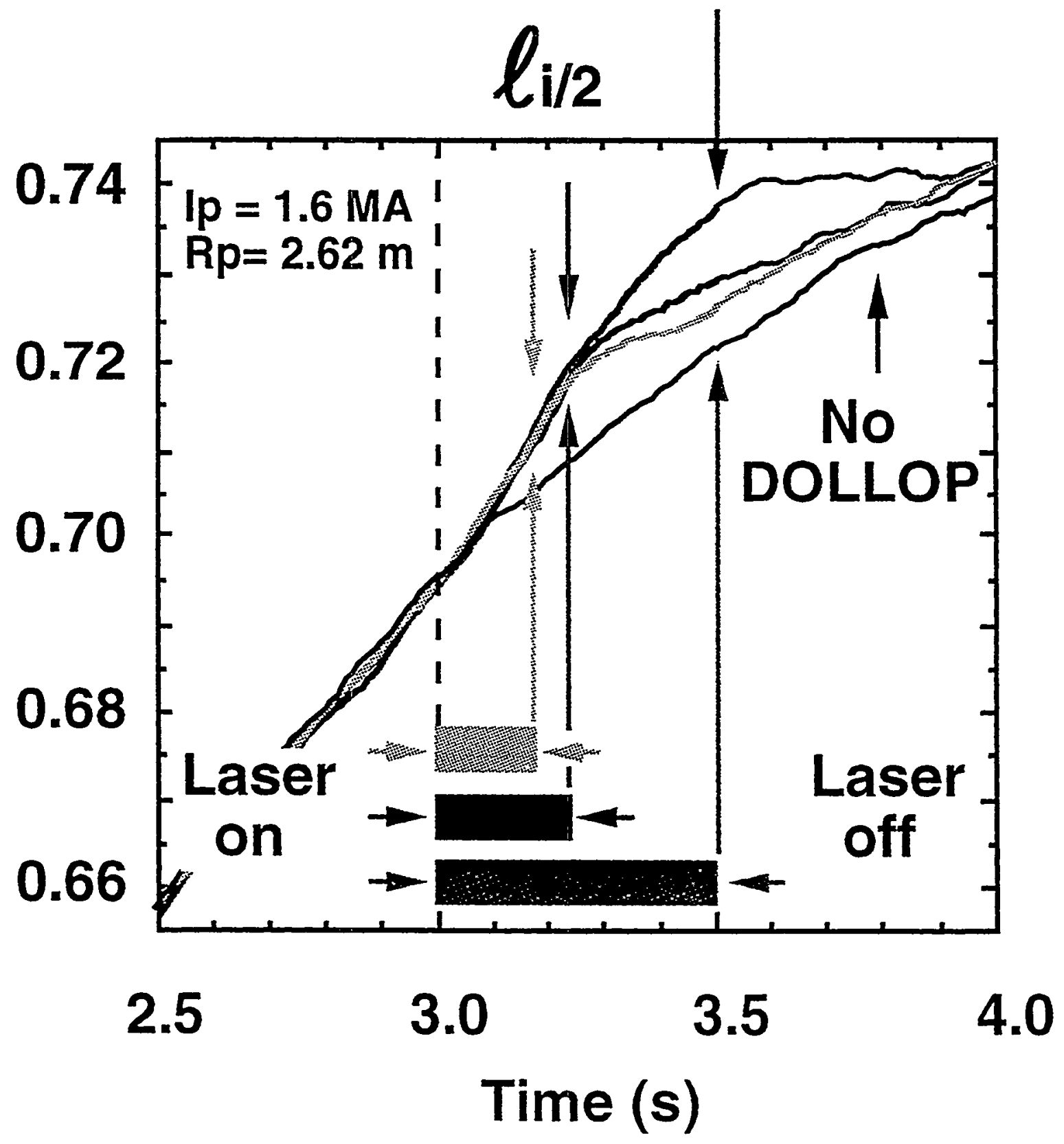

- Inductance Rises When the Laser is On - Optical Influence of Current Profile 
DOLLOP Causes a Prompt Improvement in Core Electron Energy Confinement
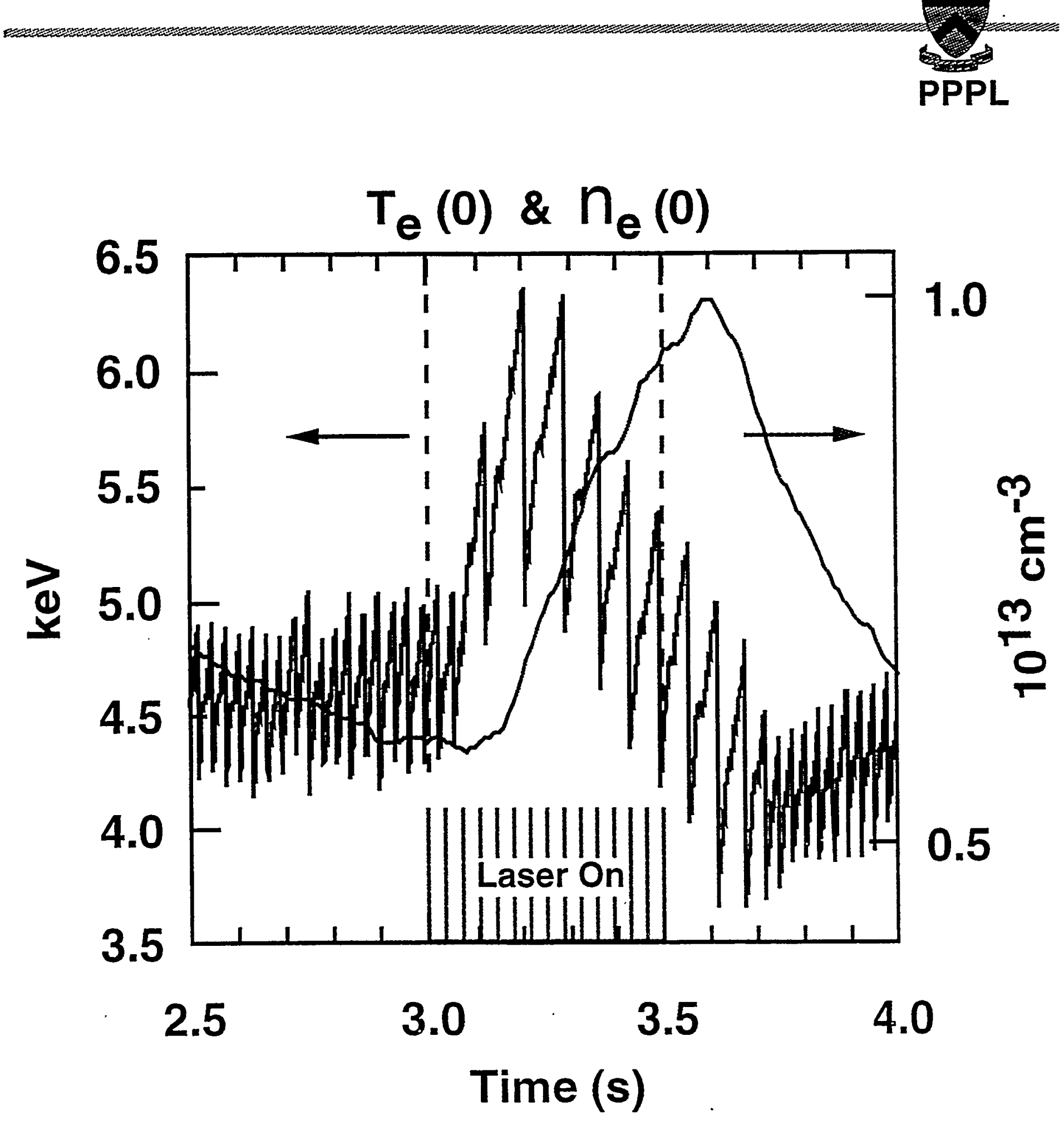

- Non-Local Paradigm for Electron Transport ? 


\section{High Speed Images of Li Aerosol}

R Maqueda. G Wurden; LANL.

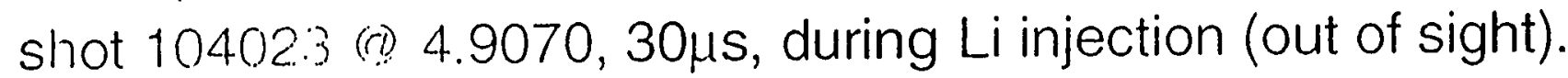
NB on @ $4 \therefore s$ unidirectional once NB on. visible light (no filter) videos on vel: http://wsx.lanl.gov/ricky/disrupt.htm

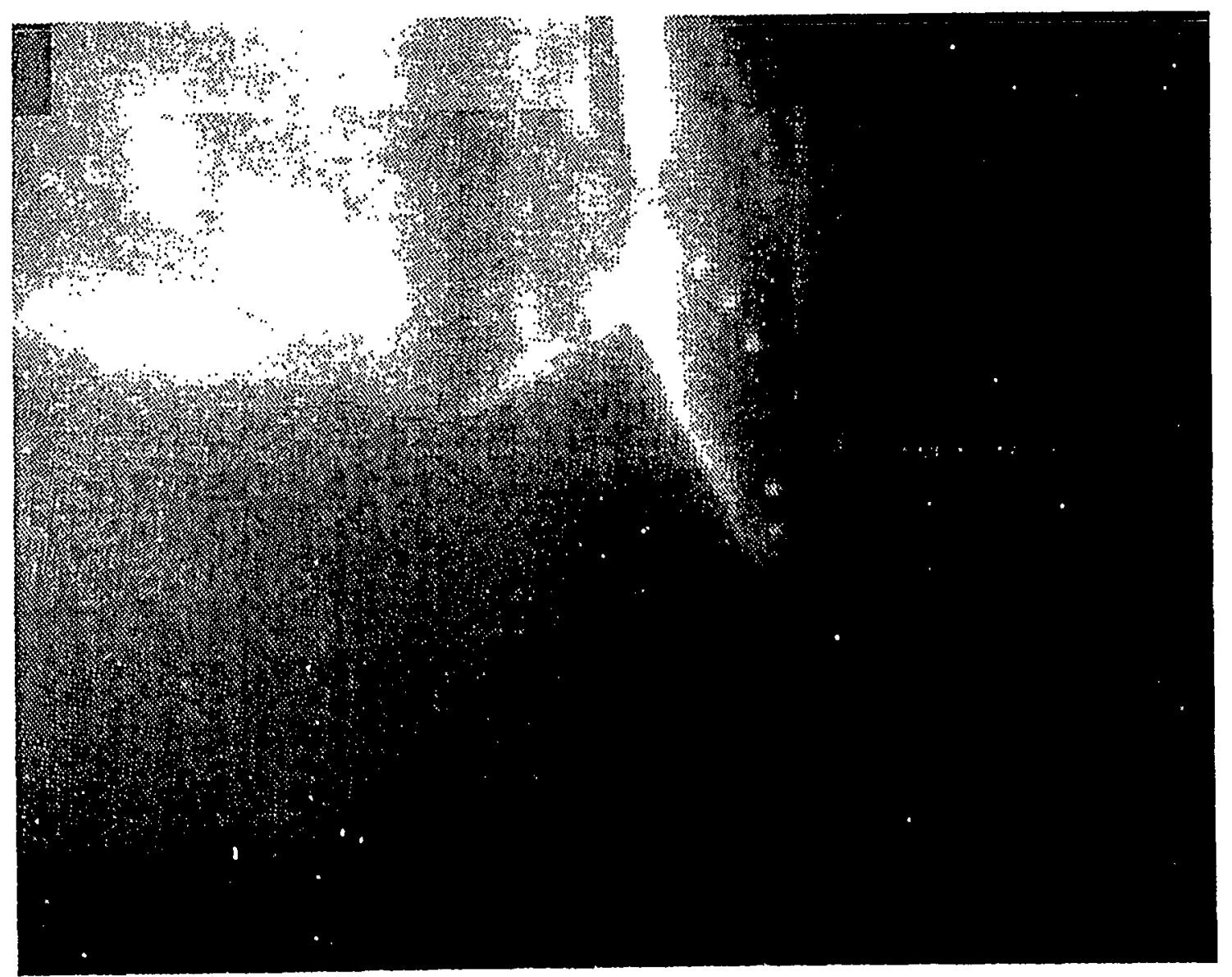




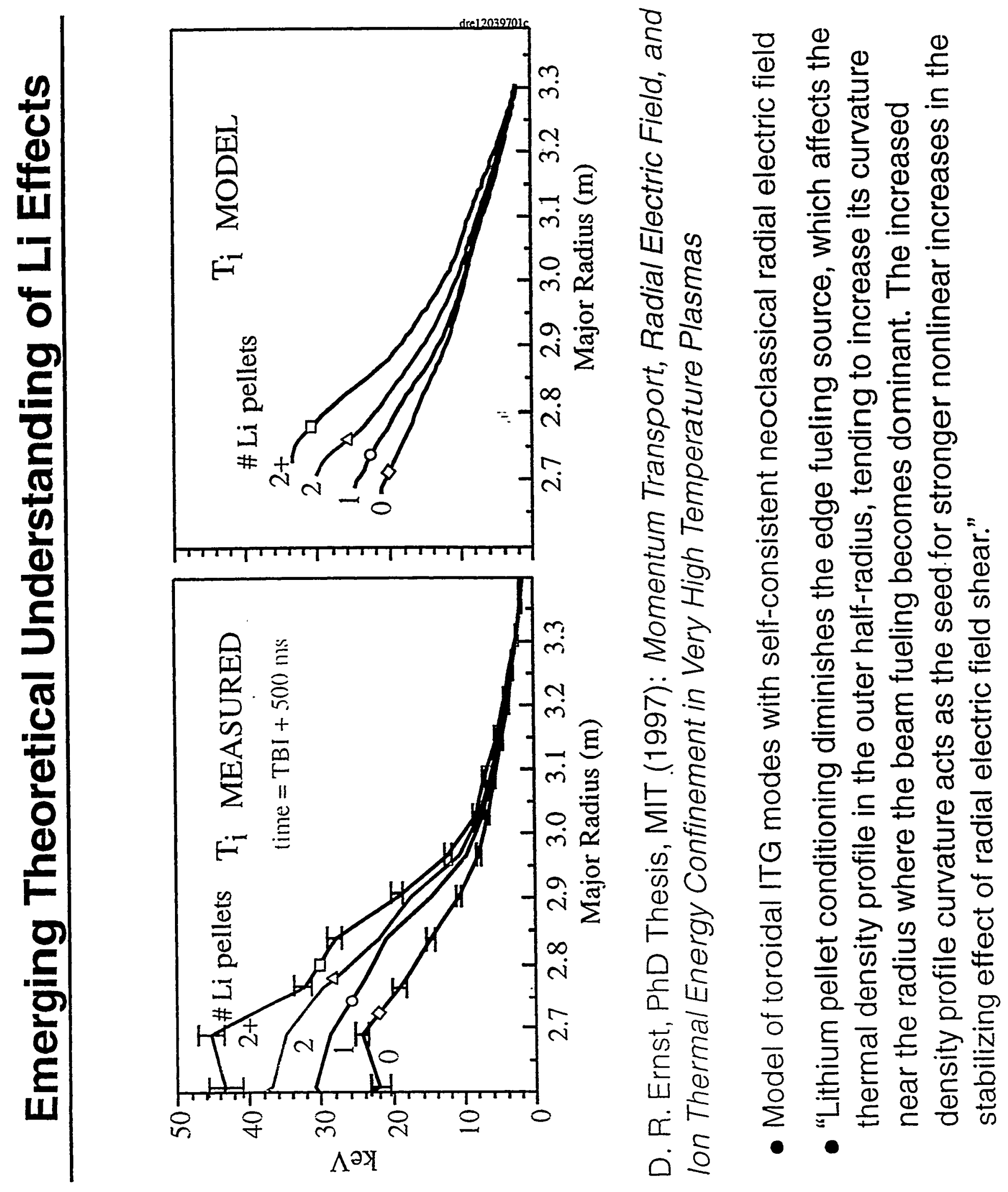




\section{LABORATORY STUDIES ON LIEFFECTS}

H Sugai, M Watanabe and H Toyoda; Nagoya University

Presented at PPPL October 1997

Basic Laboratory Studies reveal:

- abundant lithium chemistry on $\mathrm{H}, \mathrm{O}(\mathrm{C}$ ?)

- $\quad$ very strong gettering reaction

- $\quad$ saturation occurs in bulk (large diffusion constant)

- Once saturated, no gettering effect

- $\quad \mathrm{LiH}$ decomposed at $\mathrm{T}>400^{\circ} \mathrm{C}$

Many Li efiecis explained by Li Chemistry:

- Low $\mathrm{H}$ recycling due to $\mathrm{LiH}$ formation

Necessary conditions to get Li Effects:

- Low O impurities - to keep Li surface clean

- Low H retention in graphite

- Need sufficient $L i\left(N_{L i}>N_{D}\right)$ - free $L i$ atoms on walls key. 


\section{A Few Possibilities ...}

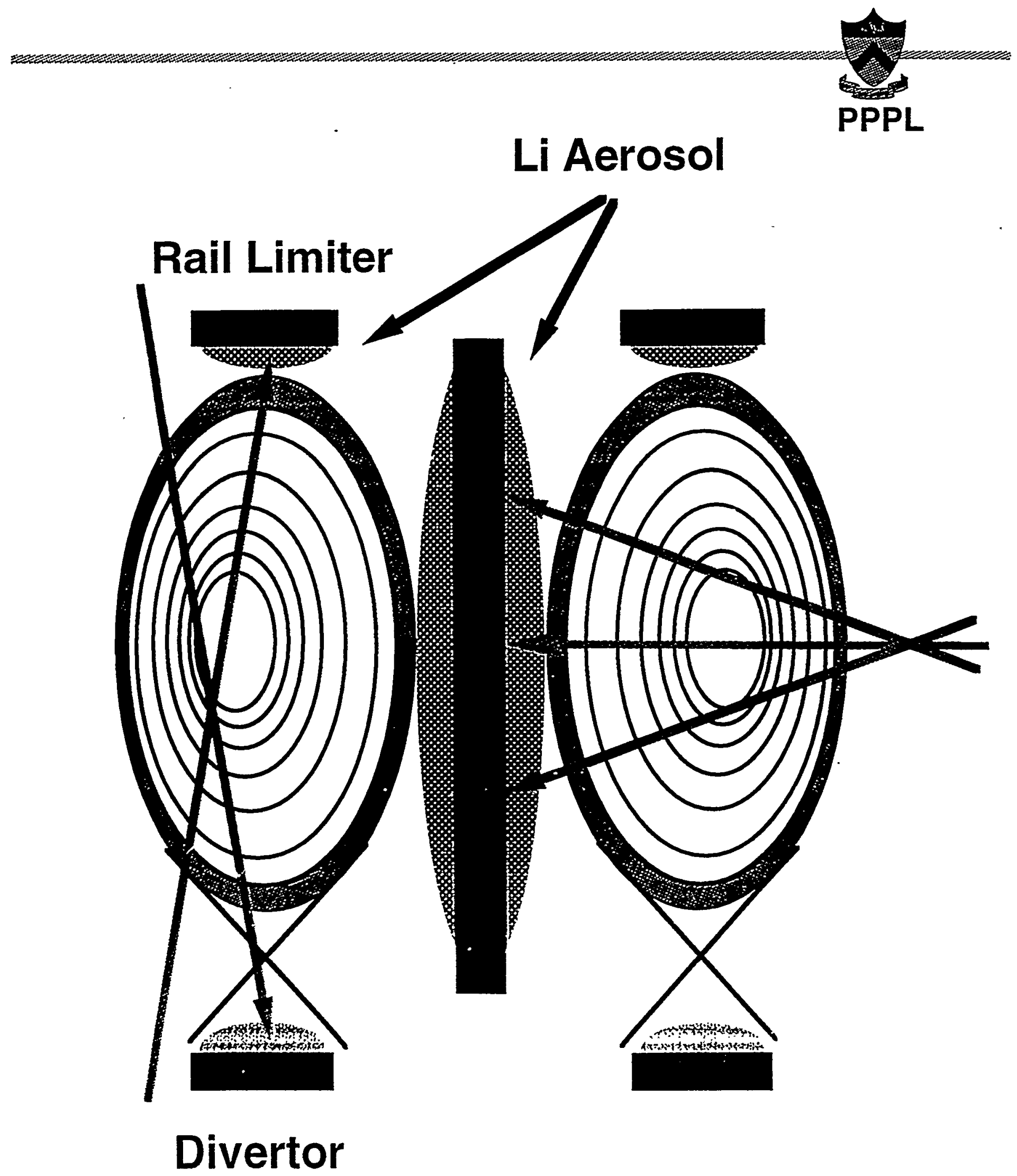




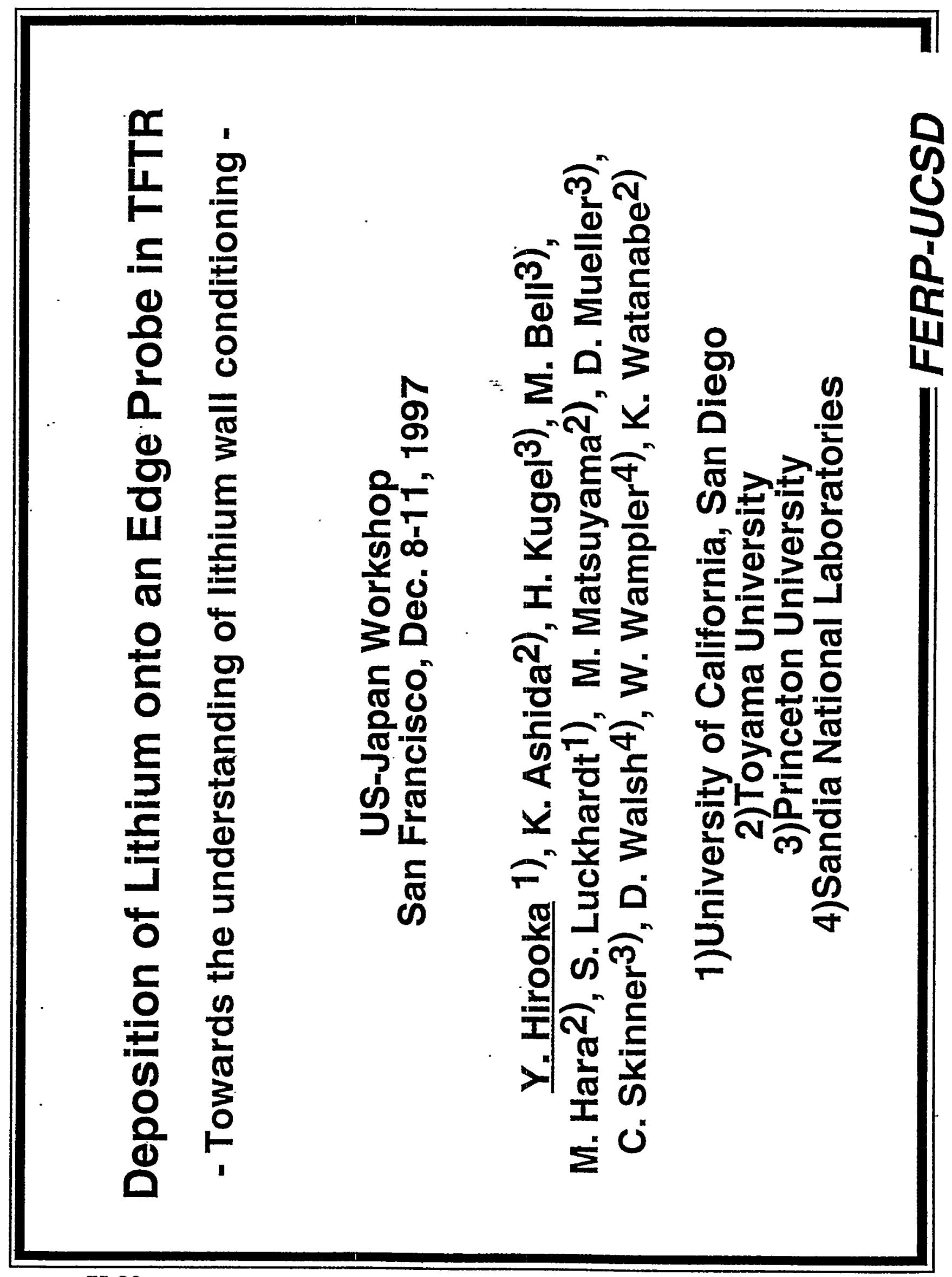




\section{Acknowledgement}

1. PPPL: TFTR Safety Staff

2. SNLS: EH\&S Staff: Brad Elkin

3. Toyama Univ.: Hydrogen Isotope Res. Center Staff

4. UCSD: Radiation Safety Staff: Sandy O'brien Ken Helm

5. PISCES-Lab. Staff: Leo Chousal

Alvin Viray

Daniel Sze 


\section{Table of Contents}

1. Motivation of the present work

2. Probe exposure experiments in TFTR

3. Post-exposure probe analysis

Tritium measurements

NRA-D, Li mapping

SIMS-depth profiling

XPS-surface chemistry

SEM-surface morphology

4. Summary 


\section{How does Li-coated wall interact with DT-plasma?}

1. Li coatings can trap both energetic and thermal $D, T, O, C, B$.

2. If eroded by DT, Li can be auto-redeposited (2ndary ions).

3. If coated with C, Li can thermally diffuse out to the surface.

4. Li migration leads to compounds formation (hydride, oxide, carbide, etc.), perhaps differently in the two directions.

5. Surface analysis can provide the key information.



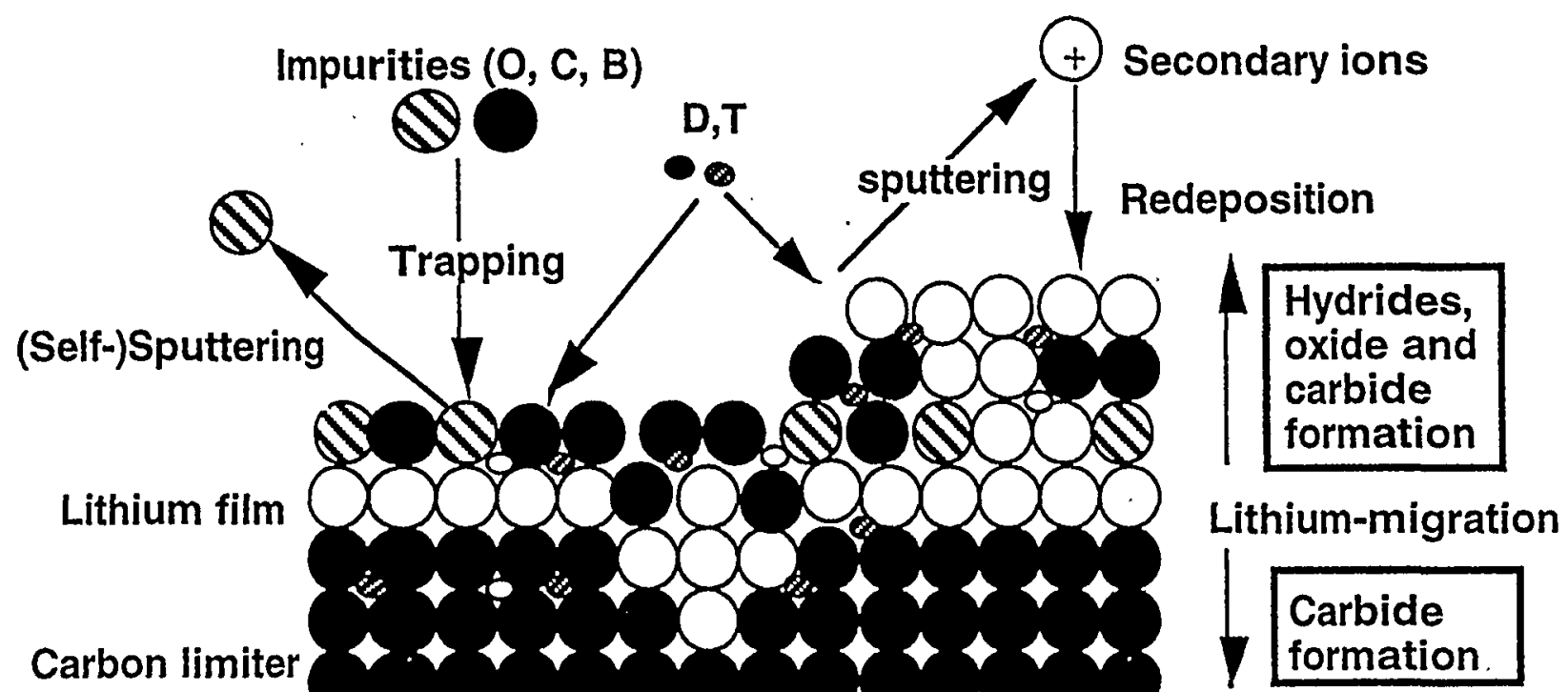




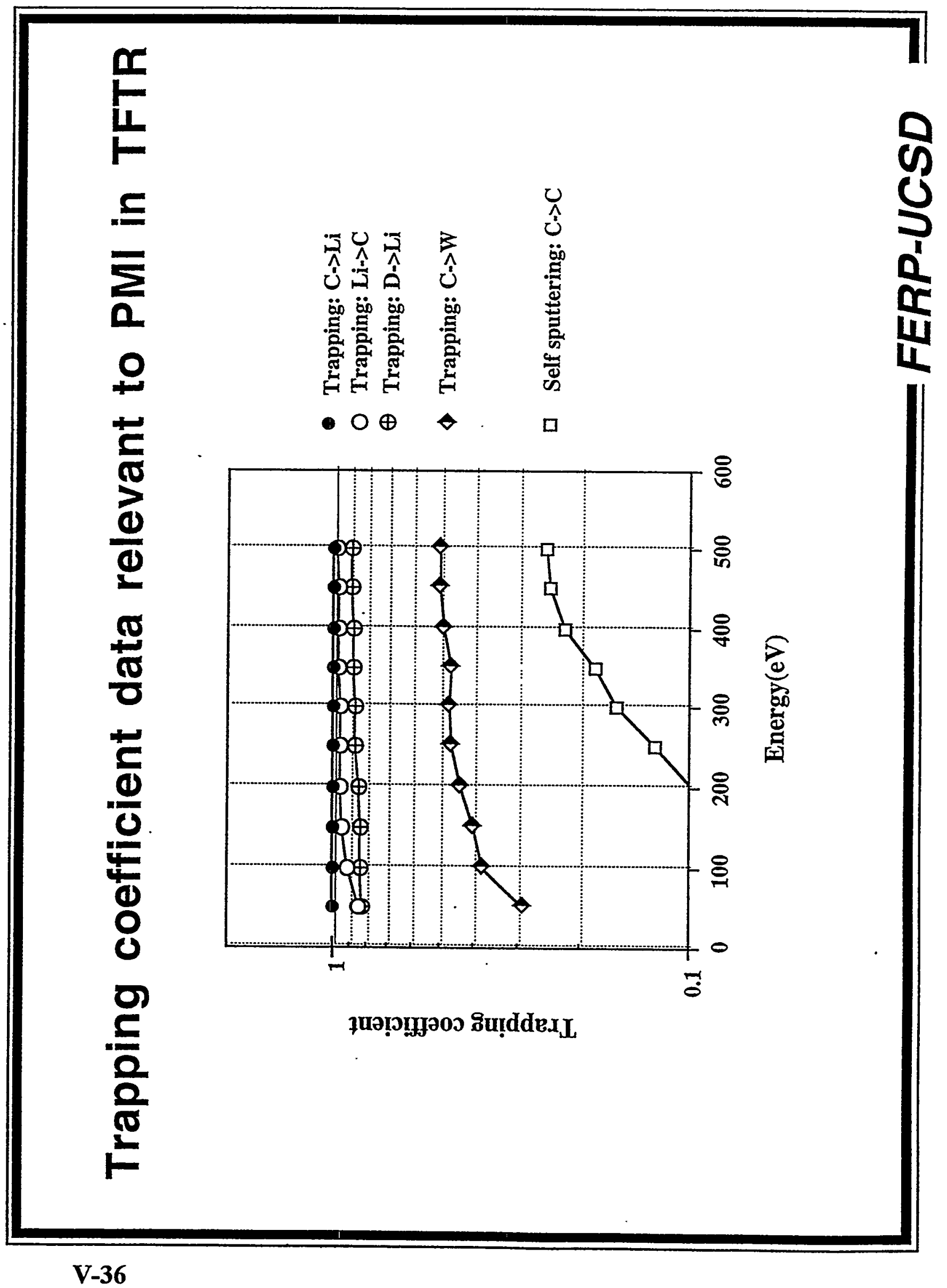




\section{TFTR deposition probe exposure conditions}

*Jan. 31st, 1997

*Exposed to $3 \times$ OH-DD-shots and one disrupted shot *Plasma current: $1.6 \mathrm{MA}$

*Major radius: $2.6 \mathrm{~m}$

*Probe position: $2.62 \mathrm{~m}$ (Bay-D)

*Probe material: 4D C-C composite (FMI)

*Li-pellet injection: $2,4,4$ pellets $\left(5 \times 10^{20}\right.$ atoms/pellet)

${ }^{*} \mathrm{Li}-6$ enriched pellets to $95 \%$ (5\%: $\mathrm{Li}-7$ )

ANT103067 01/31/97 12:39
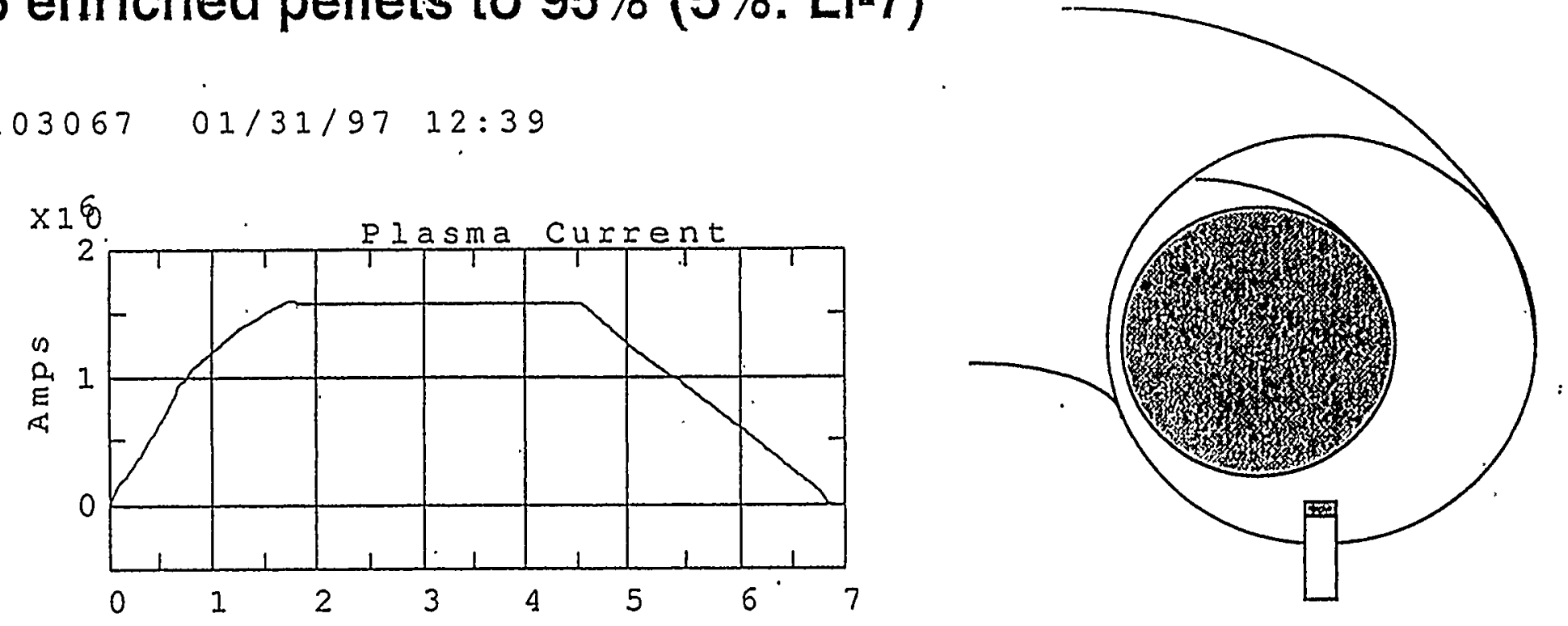
- $08 \cap 1 \forall J 0 \wedge 7 \exists \forall H$ HIW $ᄉ$

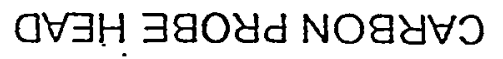
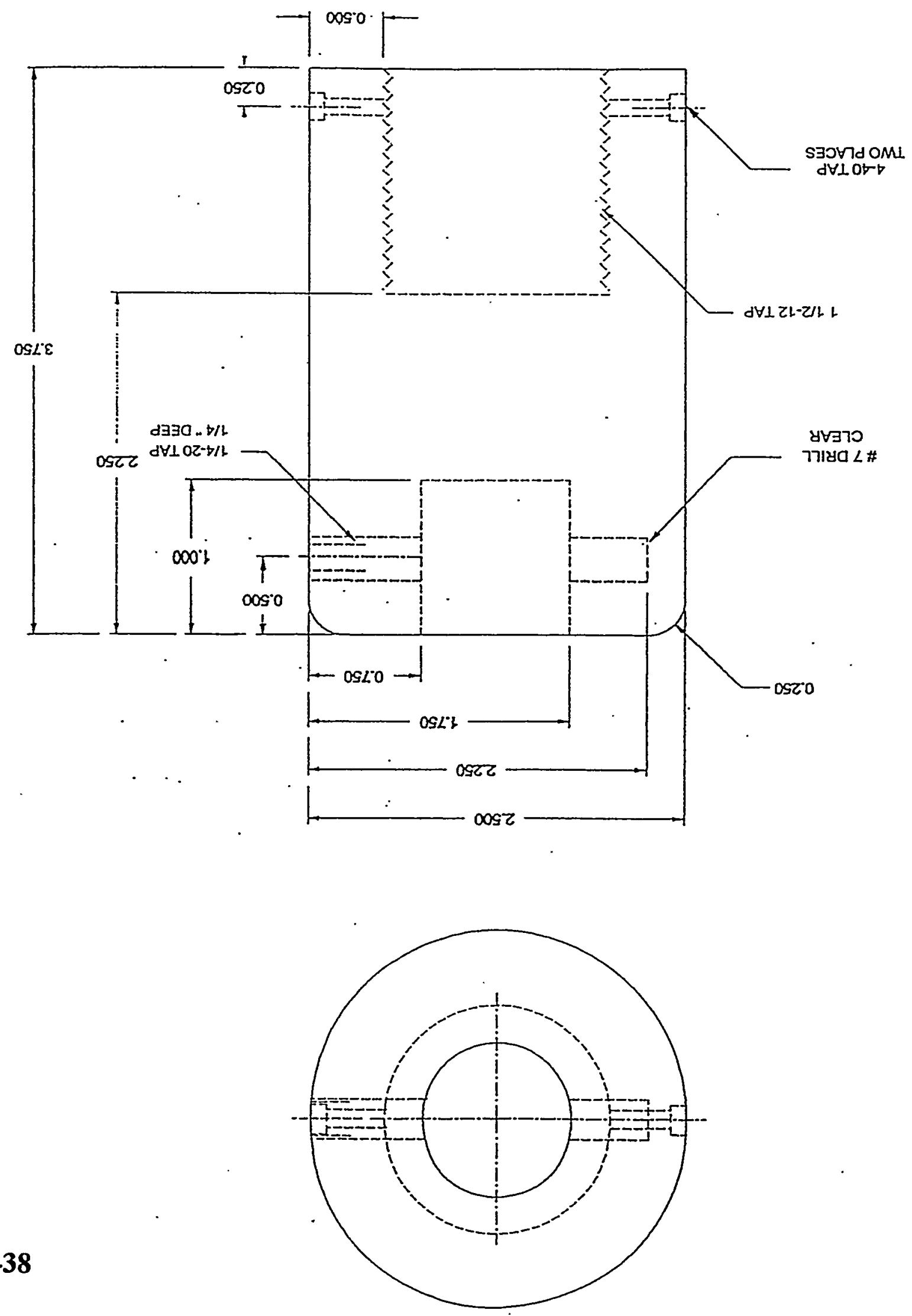


\section{D-mapping with ${ }^{3} \mathrm{He}(\mathrm{d}, \mathrm{p}) \alpha$ over the probe surface}

D-profile-front surface

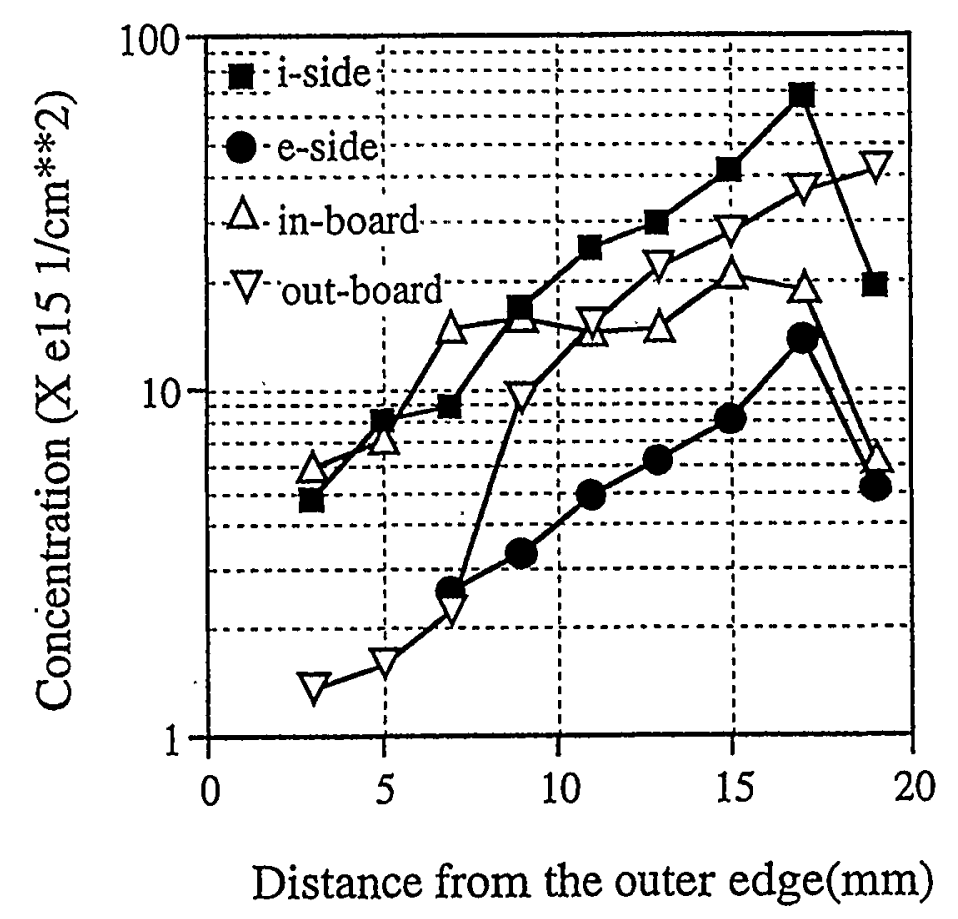

D-profile-sidewall

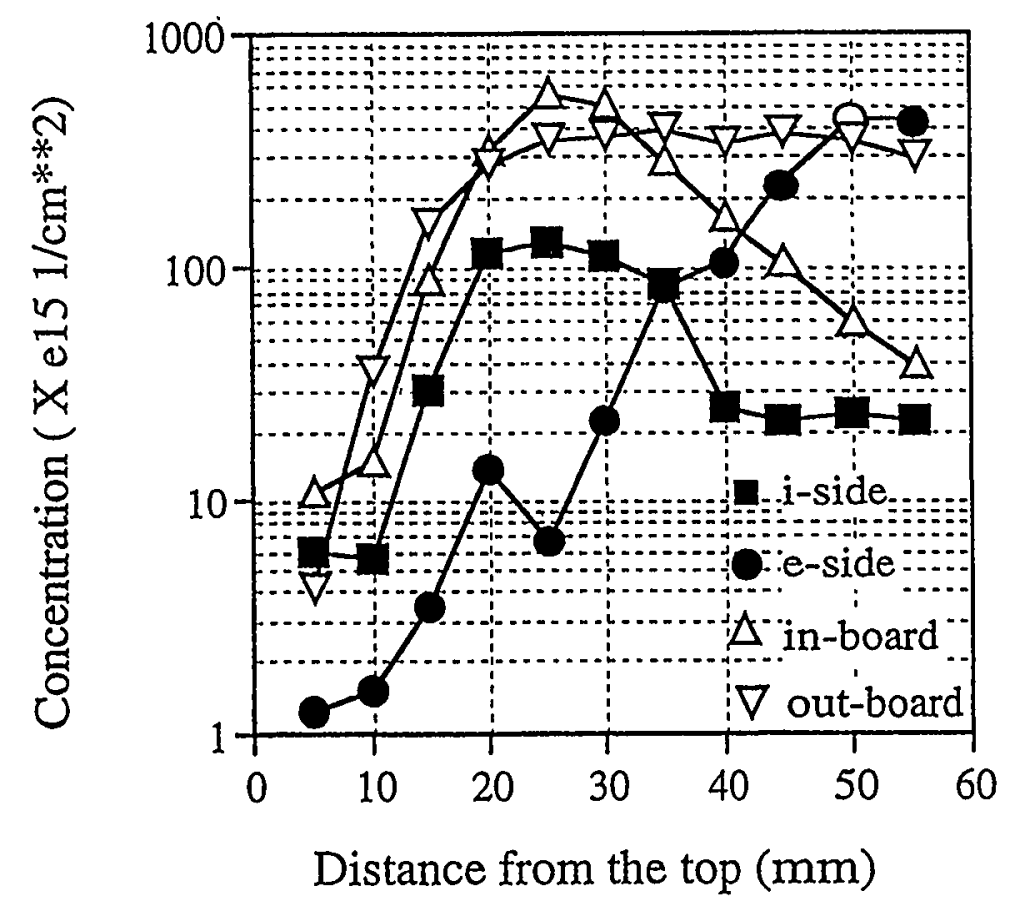




\section{Li-7 mapping with ${ }^{7} \mathrm{Li}(p, \alpha) \alpha$ over the probe surface - Recycling of Li-7 from previous shots -}

Li-profile front surface

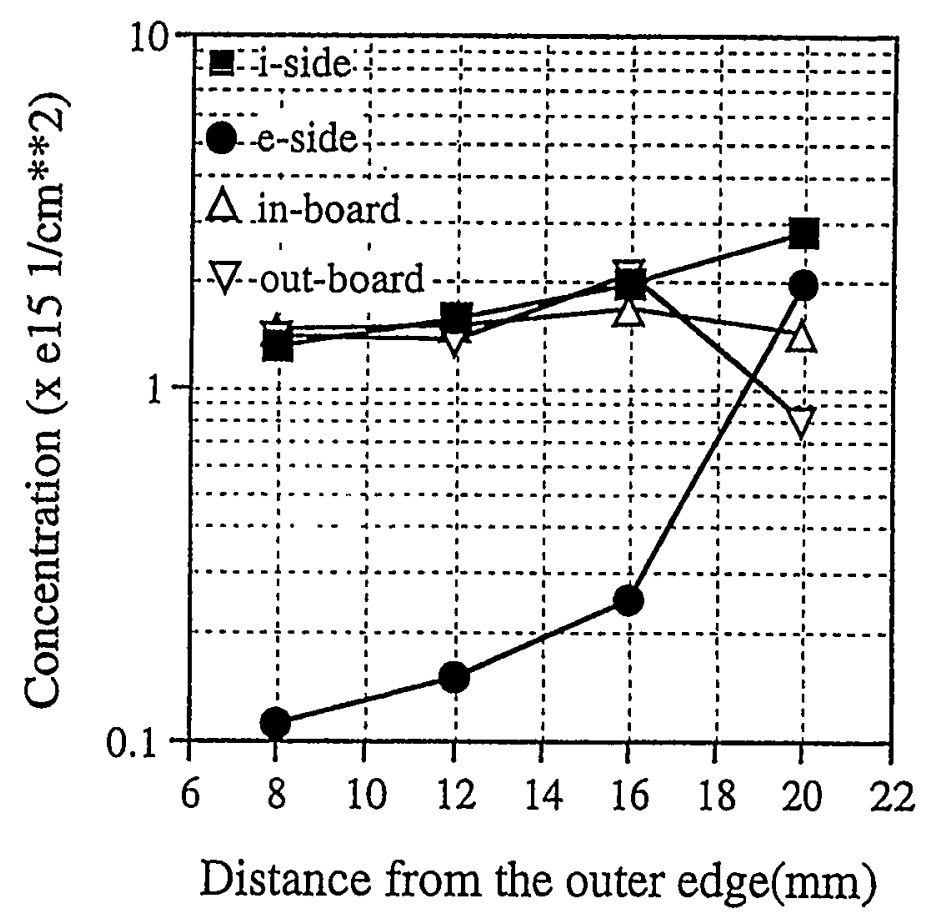

Li-profile sidewall

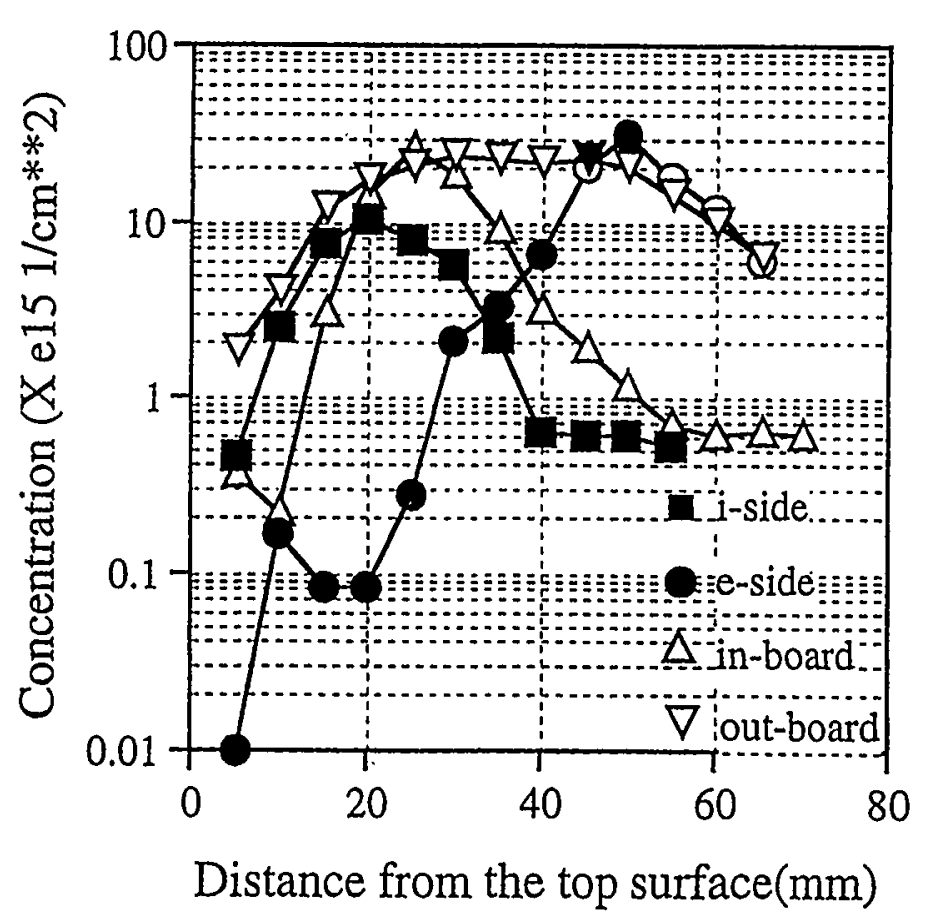

FERP-UCSD 


\section{Li-6 depth profiling with SIMS for the TFTR probe samples (4 directions on front surface)}

- Deposition of injected Li-6 -

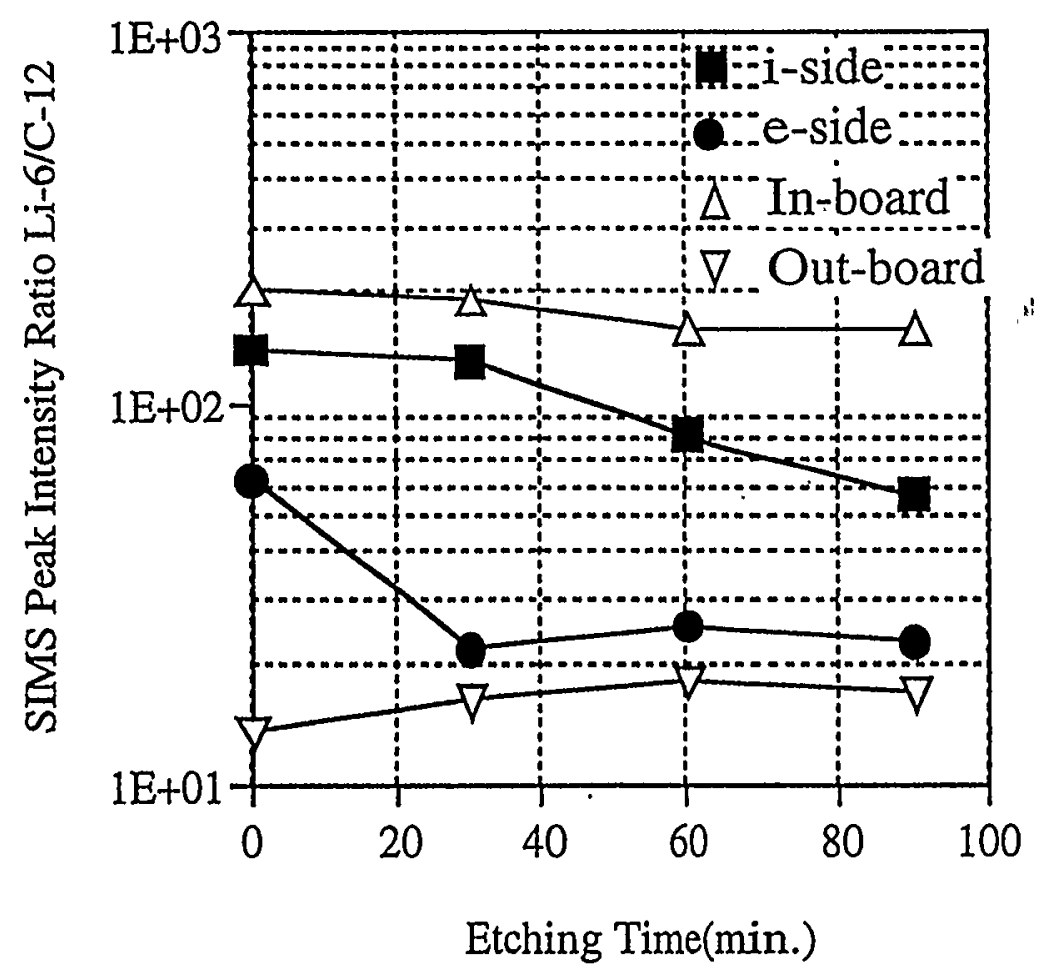




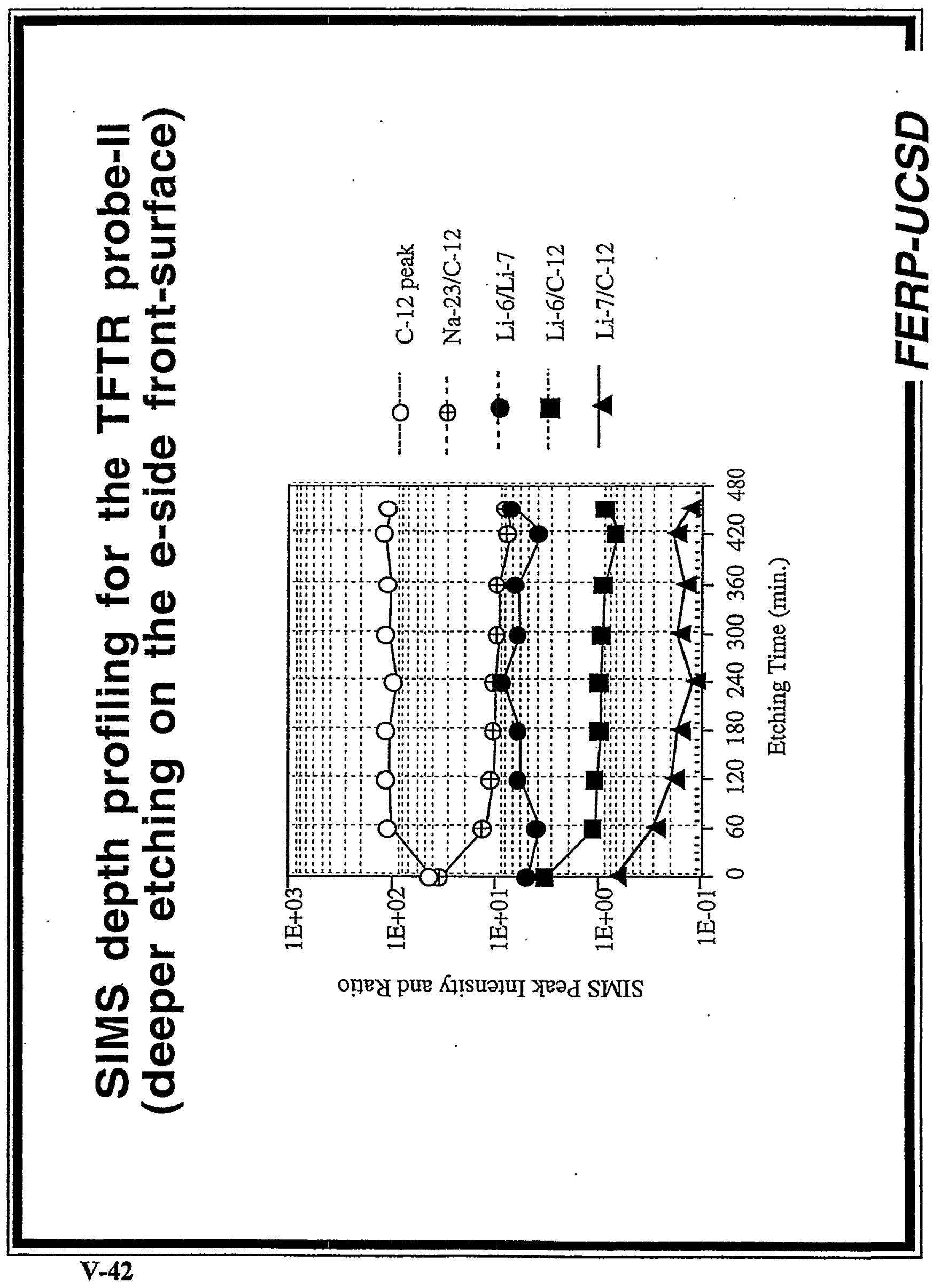




\section{Impurity contents in graphite materials}

\section{Impurity Contents(ppm) element FMI- Toyo Tanso \\ TFTR IG-43 IG-430U}

$\mathbf{L i}$

B

$\mathrm{Na}$

Al

Si

K

Ca

Ti

V

Cr

Fe

$\mathrm{Ni}$
2.9

17

$<10$

5-24

$<50$

$<50$

$<10$

1.7

10.9

0.3

10

$<0.5$
$<0.03$

3

$<0.5$

14

2

2

6

33

40

$<0.3$

26

4
$<0.001$

0.1

$<0.002$

$<0.001$

$<0.1$

$<0.03$

$<0.01$

$<0.001$

$<0.001$

$<0.004$

$<0.02$

$<0.001$

Remarks:

(1) IG-43 is a general purpose graphite and IG-430U is a ultrahigh purity graphite for special purposes (JT-60U and LHD).

(2) No significant difference as to other impurity elements. 


\section{Neg. SIMS data on $D$ and $O$ for the TFTR probe samples (4 directions front surface)}

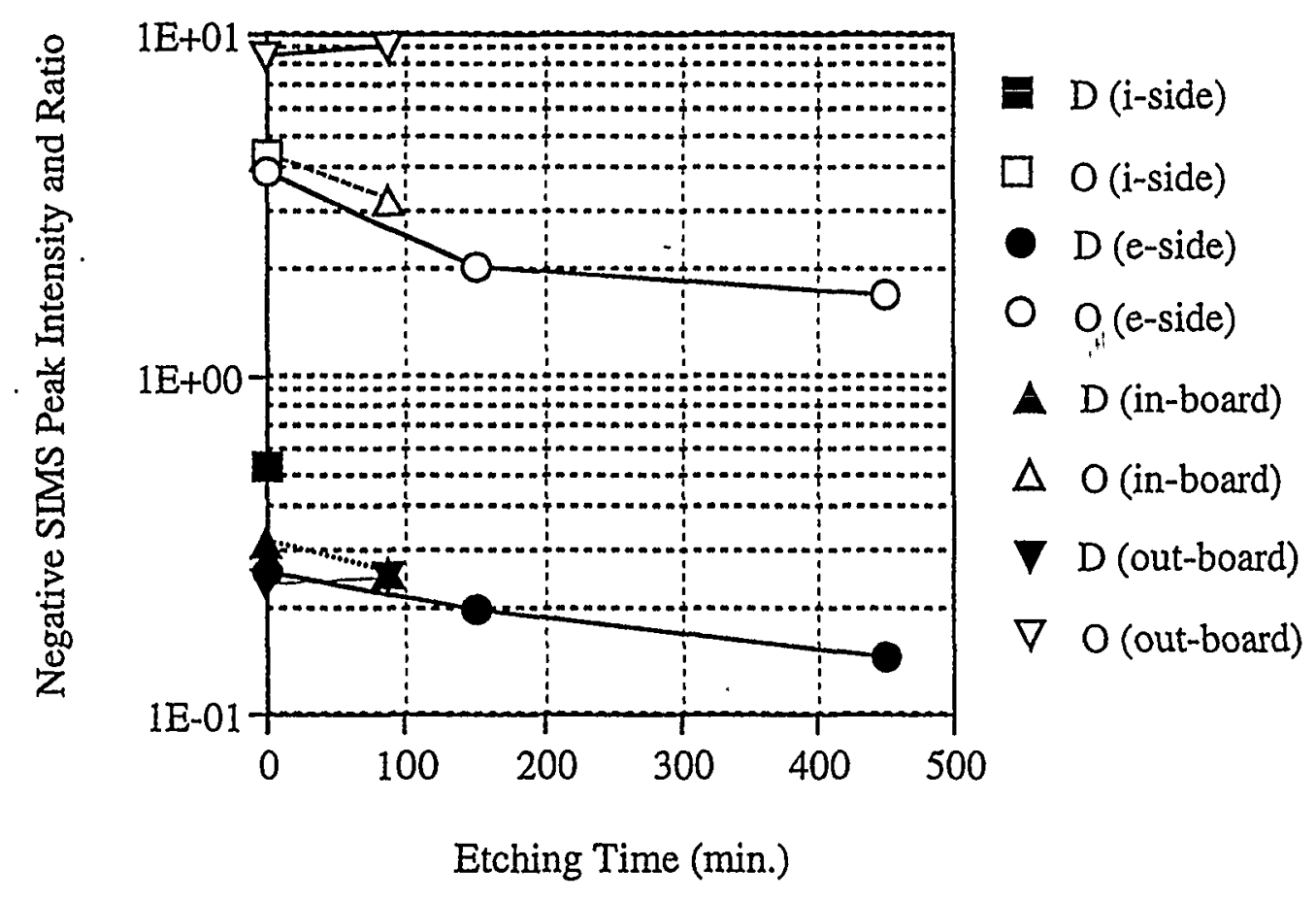




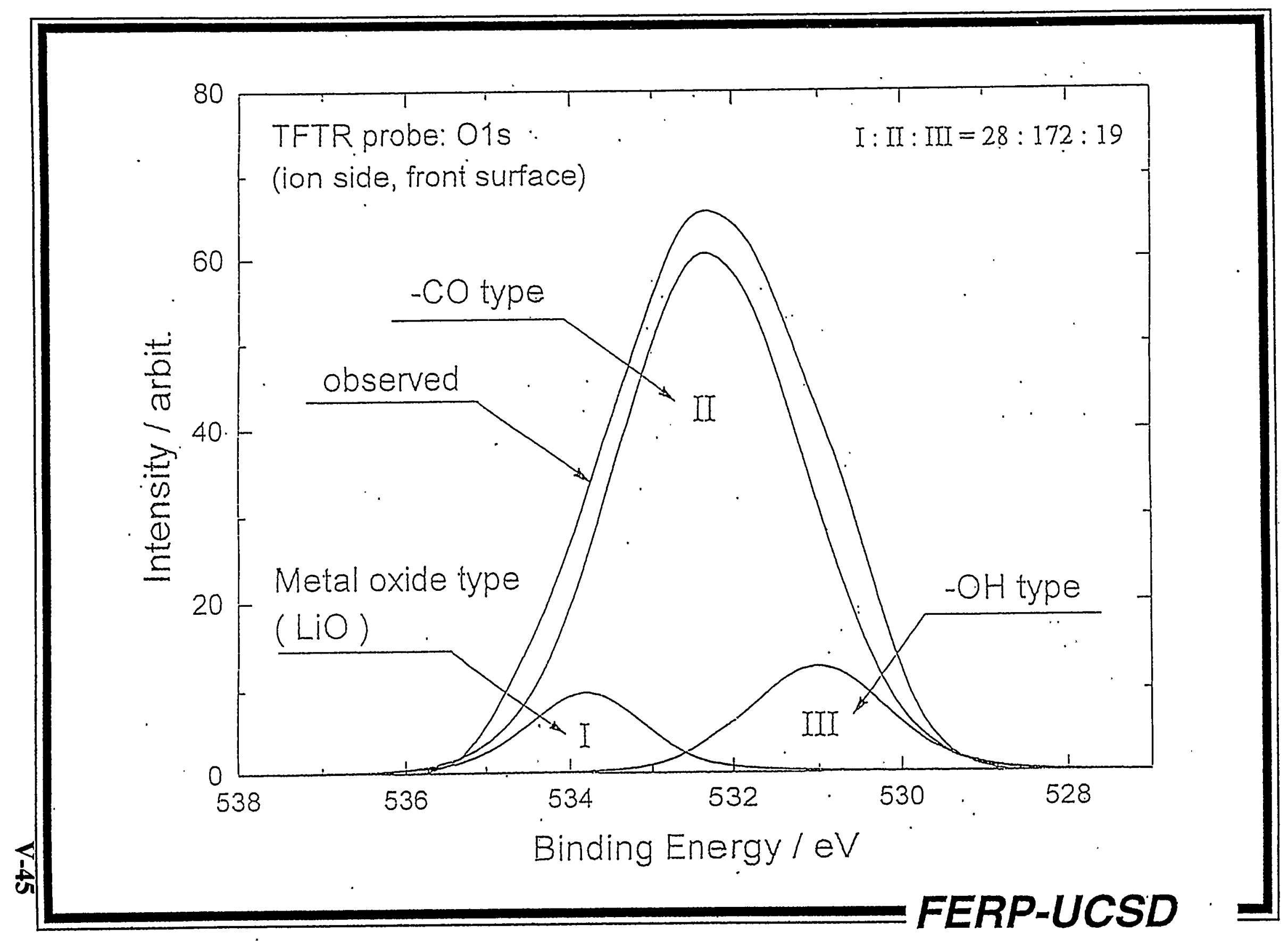




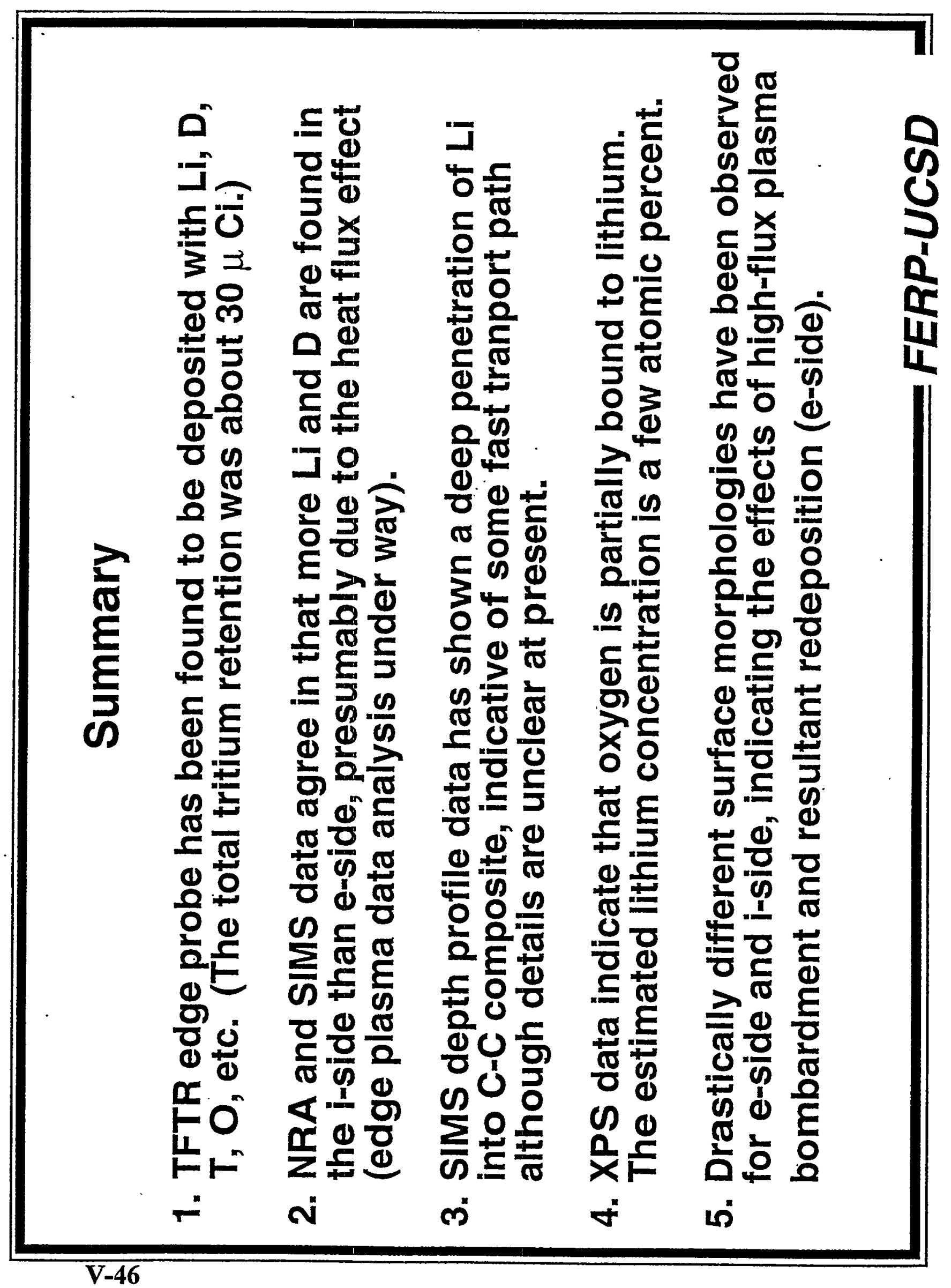


Session VI: Development Issues for Near Term PFC's

$\{$ Verbal Sessions\}

VI-1 
[page intentionally left blank] 
Solinated by Sapara 5 woup Issues for near term PFCs KSTAR (examples: LHD/WTX, NSTX, ITER)

(6)

Discussion topies:

i. Coupling with physics

2. Higher heat flux - louger pulse leupth

3. Use of $W, M_{0} \xi C$, Be (ITER)

4. S-c coil machine wall conditioning

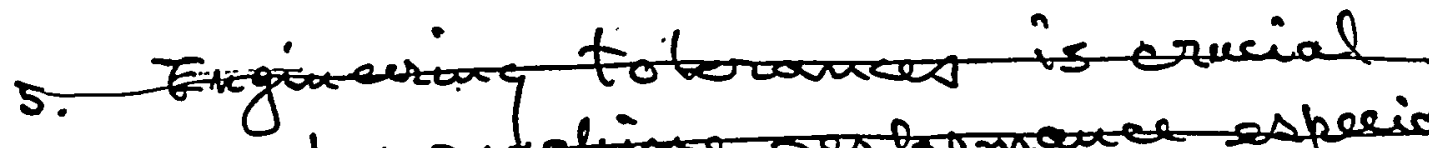

6. putial of ration

5 PFC Engineering design.

* $\mathrm{H}_{2} \mathrm{O}$ cooled

- eu-heat siuk - joints/inter face

- Mininum or zevo neutron vadiation daunge

- Low detty aycle

- Power comversion is not required 
Topic 1: Coupling with physics

$(\widehat{z}$

- Impacts on heat and particle flux spatial and temporal distributions

- Particles recycling, ash removal is pumping

- Radiative diverter

- Mantle / cove radiation

- ElMs

- Disruption

- Detached plasma

$\begin{array}{ccc}\text { LHD/WTX } & \text { NSTX } & \text { IER } \\ \checkmark & \vee & \checkmark \\ \checkmark & \checkmark & \checkmark \\ ? & ? & \checkmark \\ \text { No } & \checkmark & \checkmark \\ ? & ? & \checkmark\end{array}$

- stellarator has H-mode. ElMs not observed yet

- Closely coupled with edge condition

- Question on momentum balance on radiation diverter?

- Active pumping brings in new observations on recycling

- Coupling with fueling options - puffing/ pellet interaction with PS 1 inject:

- Modeling efforts med to be continued and coupled between physics 5 PS 1 effects

- More efforts on bunch marking between modeling and experiments, ar. erosion, ElMs, disruptions, imativial dam ape

- Technology \& PSI dedicated machines)?

VI-2 - There is a trend af paying attention to higher Z material. 
Topic 2.

(3)/5

Higher heat flux $5-25 \mathrm{mw} / \mathrm{m}^{2}$

Longer pulse length $5-20 \mathrm{~s}$ IIER (1000s) passively Actively eeg. HHD $5 \mathrm{~mW} / \mathrm{m}^{2}-10 \mathrm{~s}$ - cooled from passive to active

- A potential disconnect between the cooling of rear term PFC 5 advanced No signs That require active cooling $\vec{L} \hat{V}_{s} \mathrm{H}_{\mathrm{e}}$

- Cooling > IMV//me is not necessarily simple. High heat flux component design must also be assessedfor machines

- Stronger coupling required betevem physics $\xi$ engineering communities

- Should we focus ion ignition machine in the near term?

VI-3 
Topic 3

(4)

use of $W_{1} M_{0}, e$ $\xi B_{e}$ (TER)

- wi may be suitable locally. eng. high

- mixed material issues need to particle be addressed

being utilized metallic wall zacopes option is F - W-coated graphite being evaluated

- C-dust - Safety. Tritsin concern for D-topleration - move concerns for ITER.

coating - Li, B, si

VI -4 
Topic 4

Soc coil machine wall conditioning

- Vessel conditioning with is -on

- LHD wall limited to $\leqslant 100^{\circ} \mathrm{C}$

- Concern of baking in large machinesegg. machine deformation (ITER, HHD)

VI-5 
Topic 5 PEC Enginacing design

(6) $/ 1$

- Engineering tolerance is crucial for machine performance especially for initial operation

- For the next major device, we can run it to the serious problem of the inadequacy of engineering data in order to produce reliable components. (erg. I TE diverter tiles.)

VI -6 
Section VII: PFM Issues and Development

\section{VII-1}


[page intentionally left blank]

VII-2 
M.Shibui, Toshiba Corporation

\section{Design/R\&D Activities of Target Structure}

M.Shibui, M.Takahashi, K.Osemochi

Presented by M.Shibui

Toshiba Corporation

2-4, Suehiro, Tsurumi, Yokohama, 230 Japan

- $\mathrm{W} / \mathrm{Cu}$ gradient material

by composite VPS method

- Multi-layered cooling tube with tritium permeation resistant layer SUS316L/Cu/W/Cu trial structure 
M.Shibui, Toshiba Corporation

\section{Design issues on PFCs}

- Material issues on W

- High DBTT

- Very low ductility near RT

- Expensive curved surface by brazing

$\Rightarrow$ Reduction of residual thermal strain

$\Rightarrow$ Film rather than bulk material

- Safety issues

- (IA)SCC problem of coolant boundary

- Tritium permeation

$\Rightarrow$ Use of SUS316L as boundary

$\Rightarrow$ Use of $W$ as permeation barrier

Particles/Heat Flux
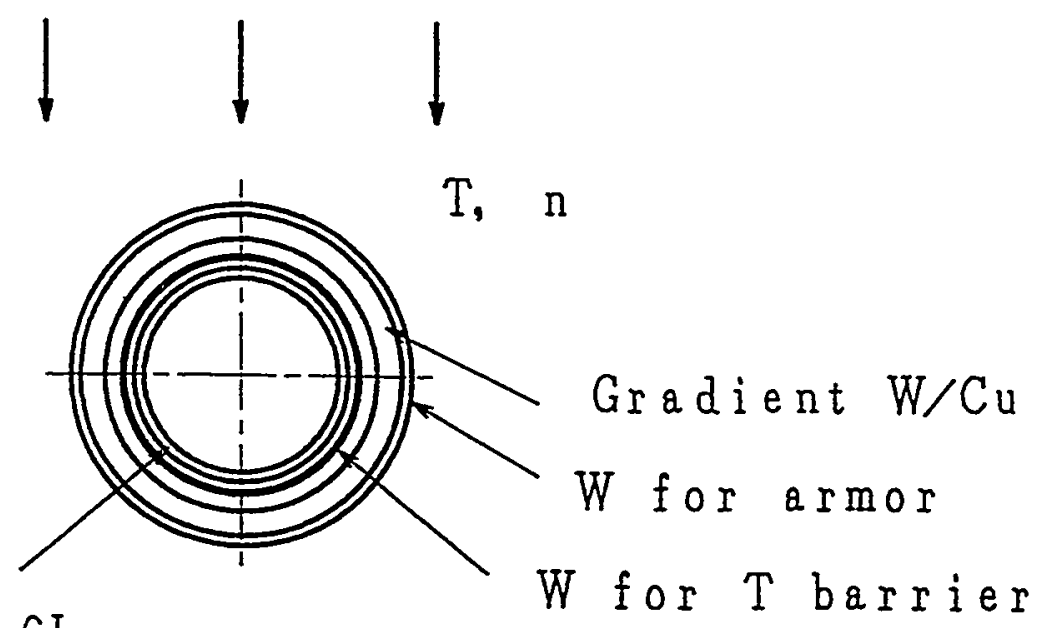

SUS $316 \mathrm{~L}$

for SCC resistance

VII-4 US/Japan Workshop, Warwick Regis Hotel, San Francisco,Dec.8-11,1997 
M.Shibui, Toshiba Corporation

W/Cu material by composite VPS method

- Advantages

- Composite gradient material

- Low cost

- Applicability to curved surface

- Potential in-situ repairability

- Analytical prediction

- R\&D of W/Cu composite VPS method

- Deposition rate

- Deposition efficiency of $\mathrm{Cu}$ powder

- Reduction of porosity in pure W layer

- Trial fabrication of $W$ target 

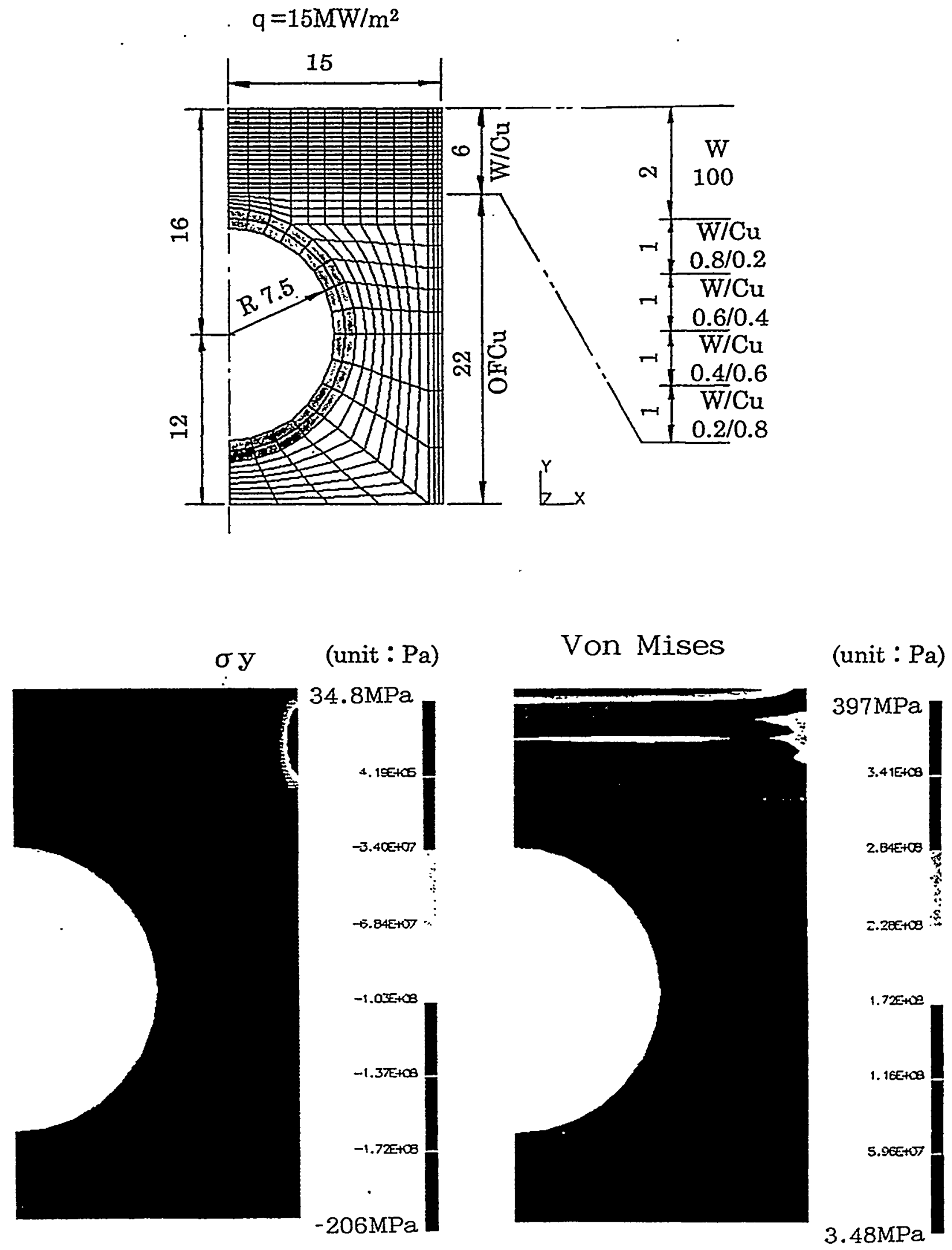


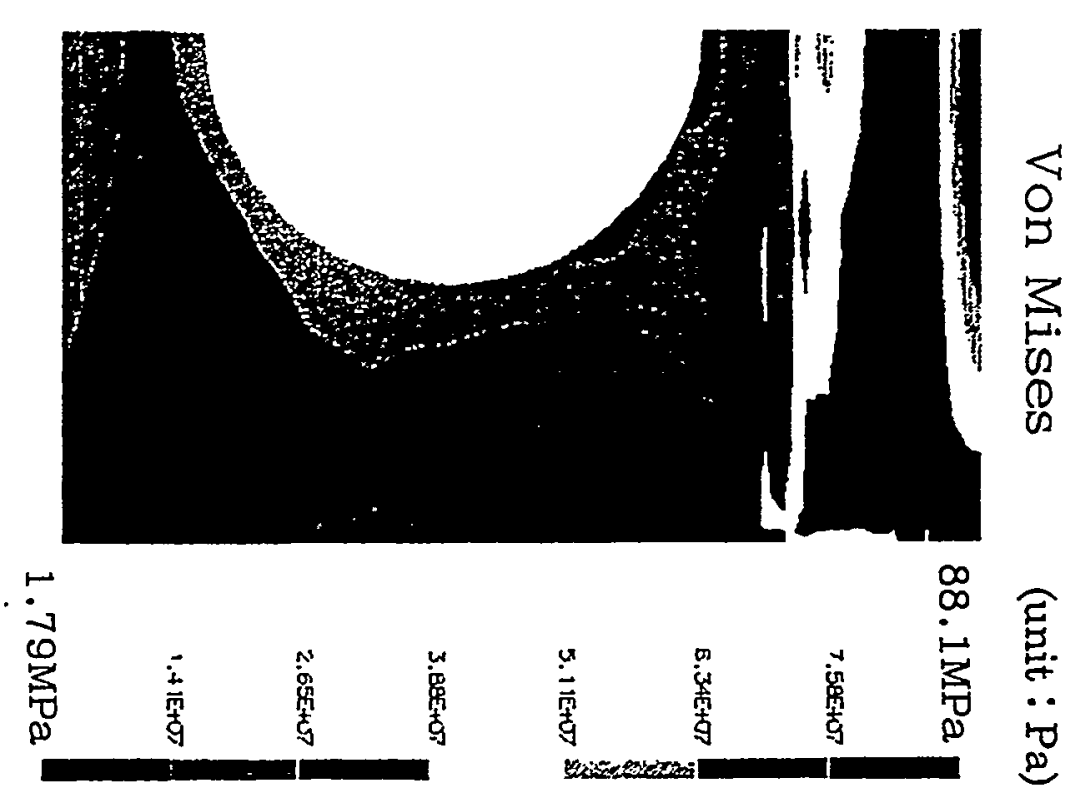

0
0
1
0
0
0
0
0
0
0
+1
0
0
0
0
0
0
0
3
5
0
0
1
0
0
0
0
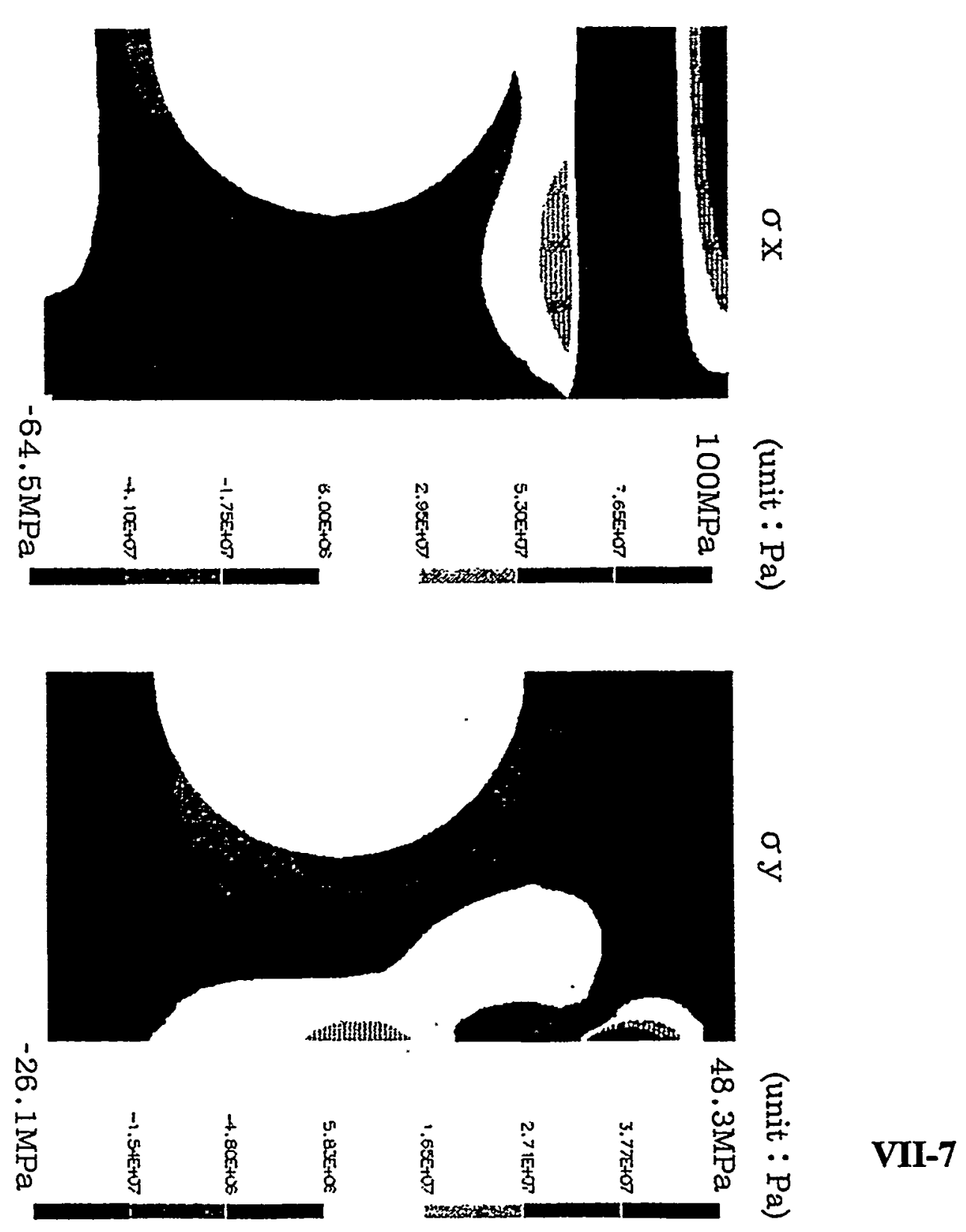

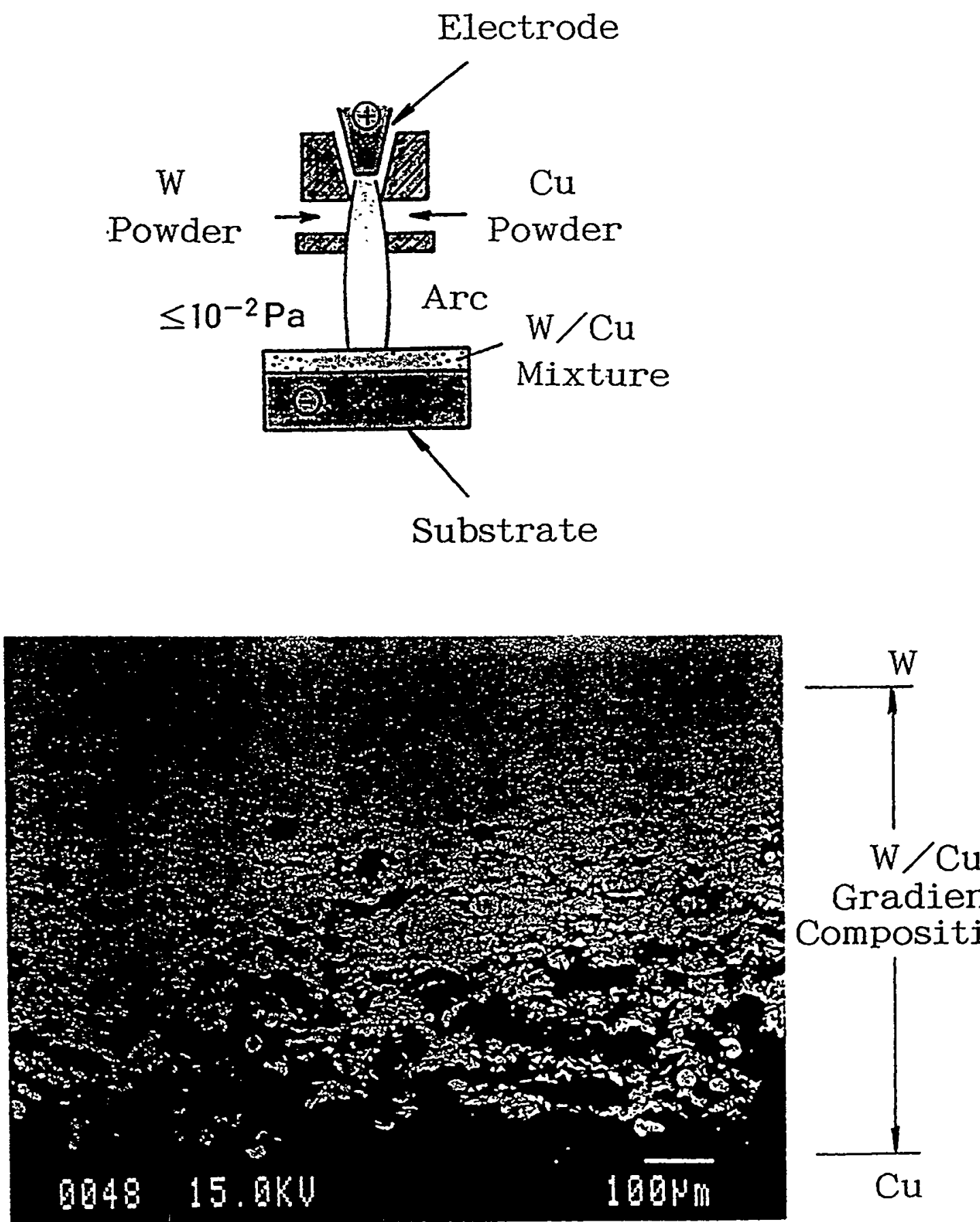

Gradient Compositios

VII-8 Composition gradient $\mathrm{W} / \mathrm{Cu}$ by VPS method 
VPS conditions for W/Cu composite

\begin{tabular}{|c|c|}
\hline Atmosphere & Ar, 137 Torr \\
\hline Power supply & $700 \mathrm{~A}-65 \mathrm{~V}$ \\
\hline Spray distance & $275 \mathrm{~mm}$ \\
\hline Spray rate & $400 \mathrm{~mm} / \mathrm{sec}$ \\
\hline Working gas & $\mathrm{Ar}: 25 \mathrm{~L} / \mathrm{min}$ \\
& $\mathrm{H}_{2}: 9 \mathrm{~L} / \mathrm{min}$ \\
\hline Surface preparation & Abrasive blasting \\
\hline
\end{tabular}




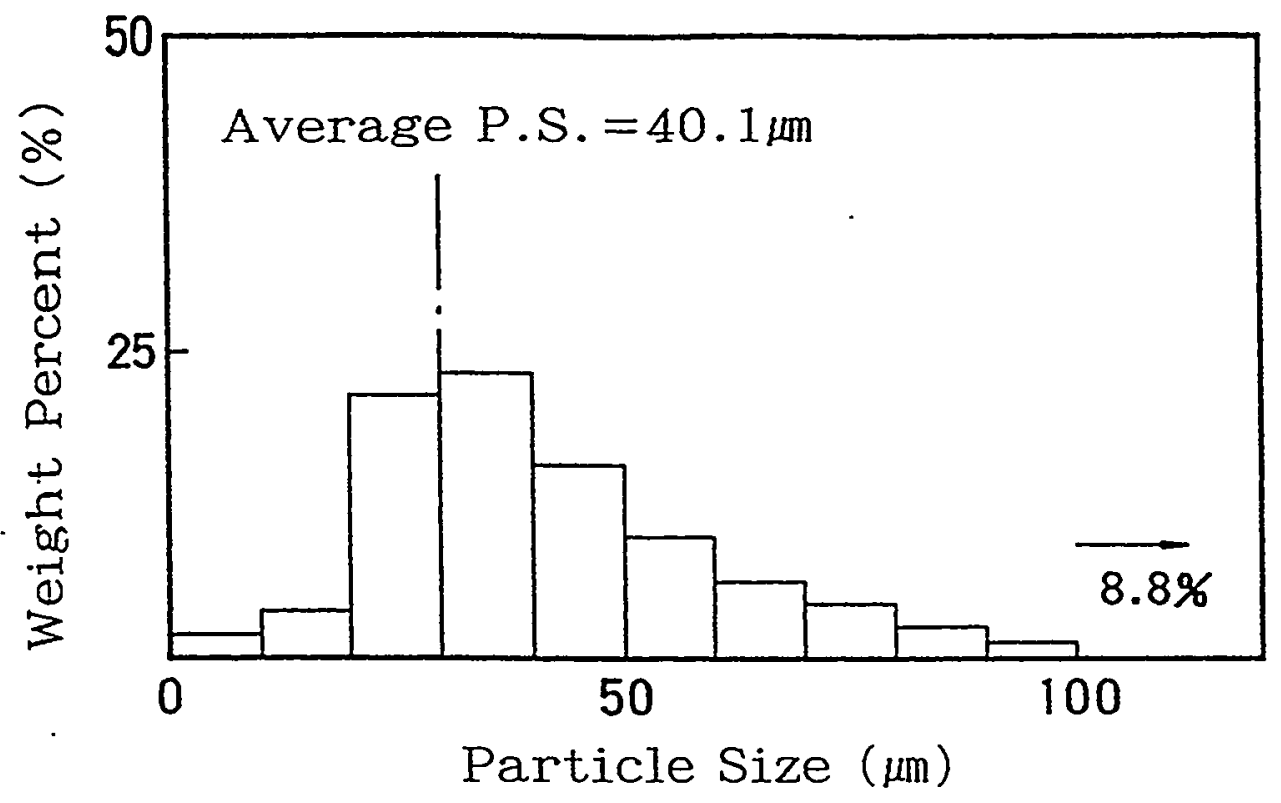

(a) W powder

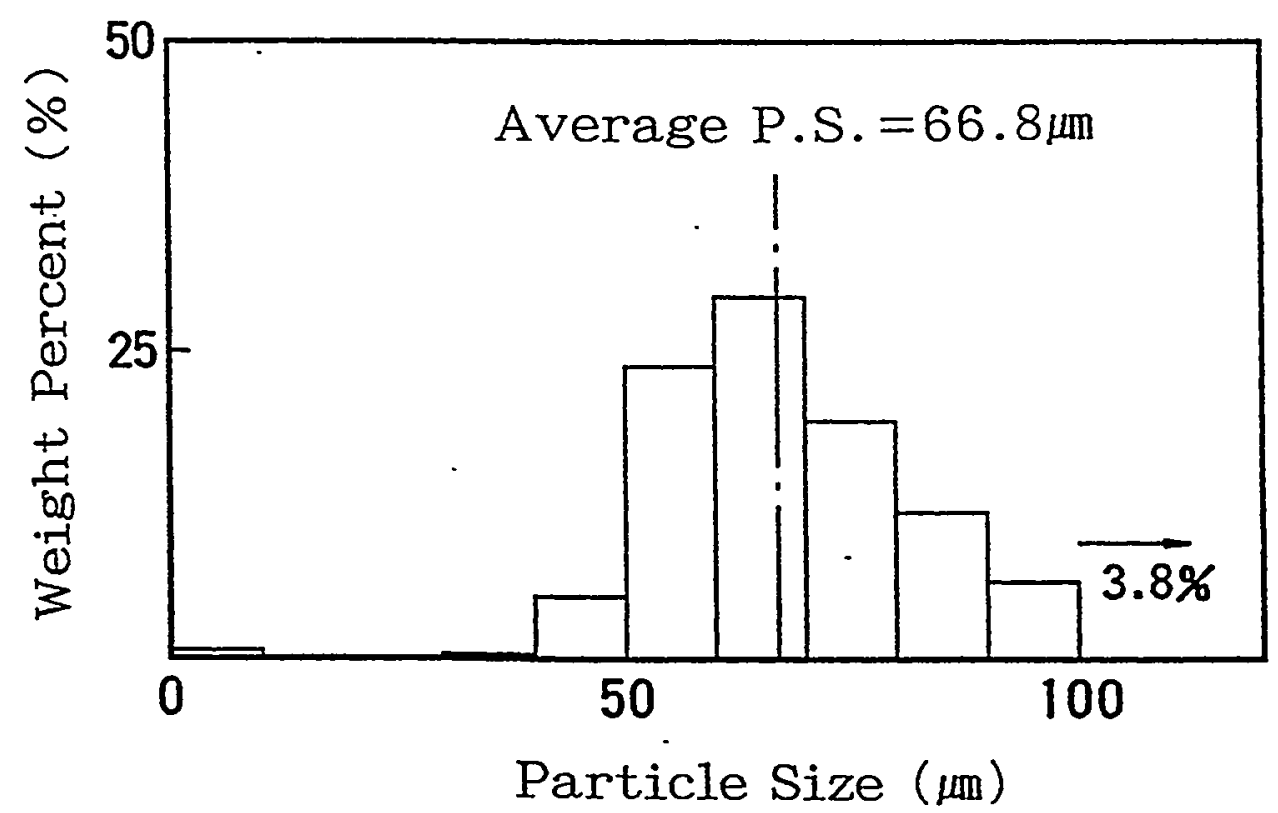

(b) $\mathrm{Cu}$ powder

$\mathrm{Cu}$ and $\mathrm{W}$ powder for composite spraying. 


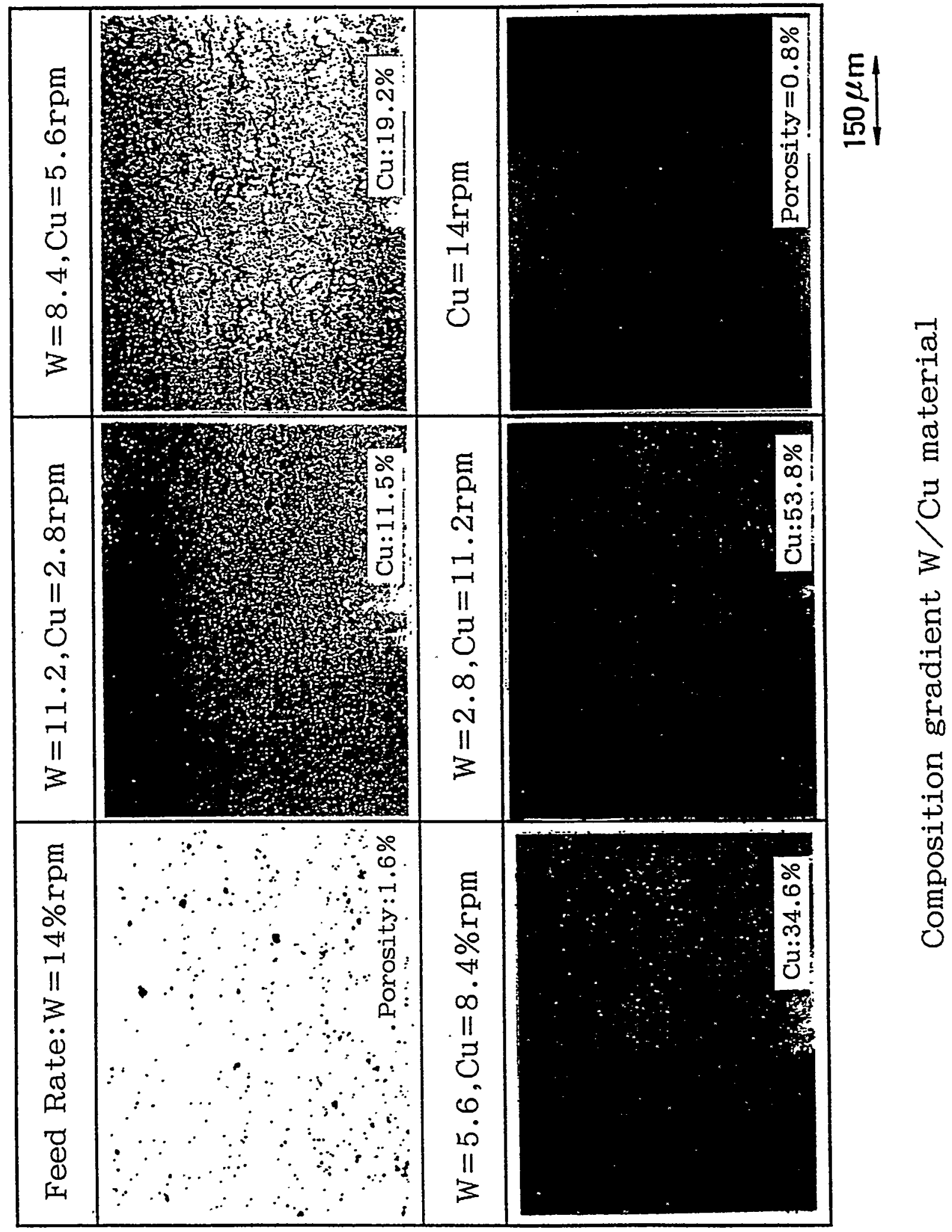




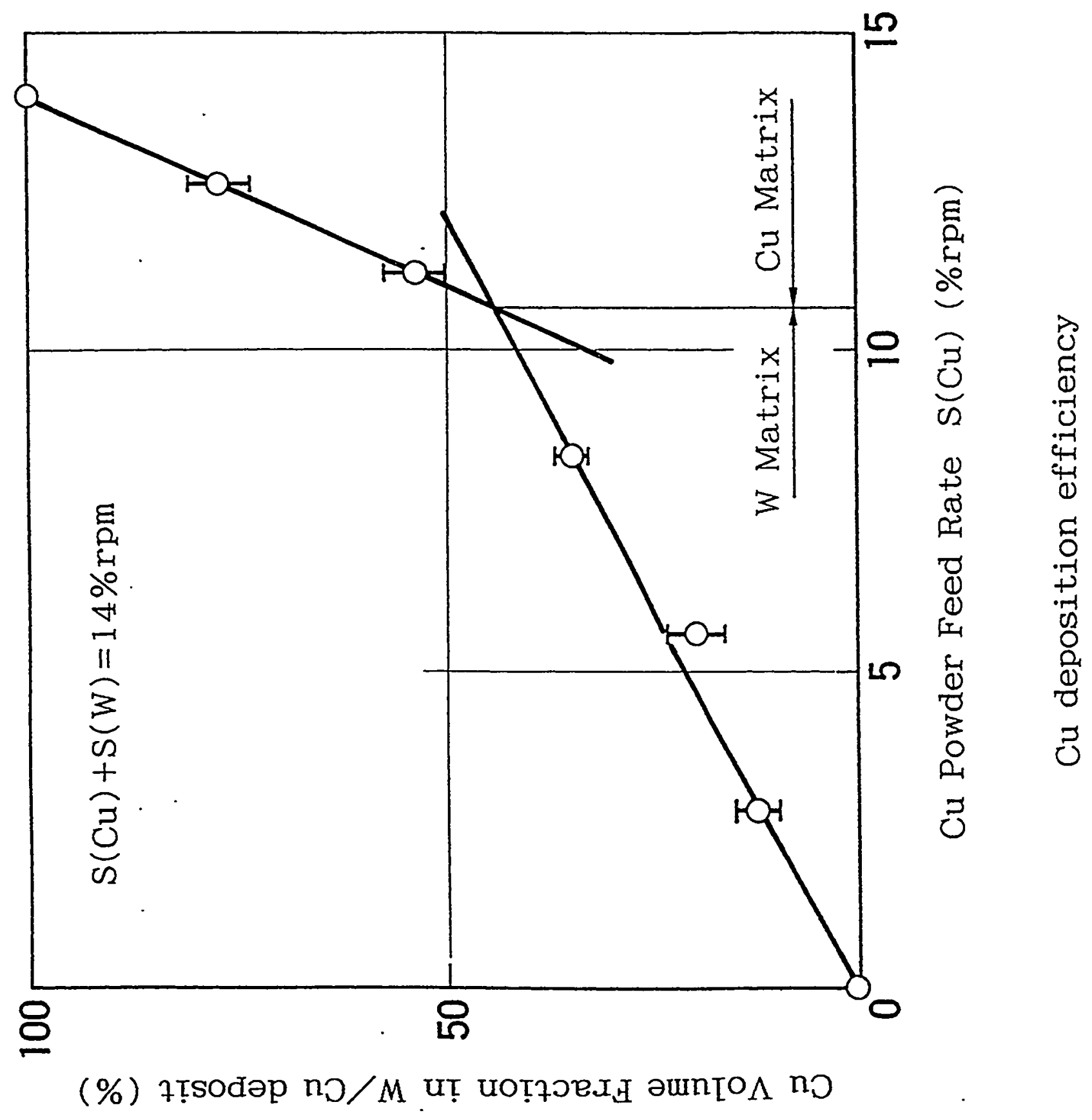

VII-12 


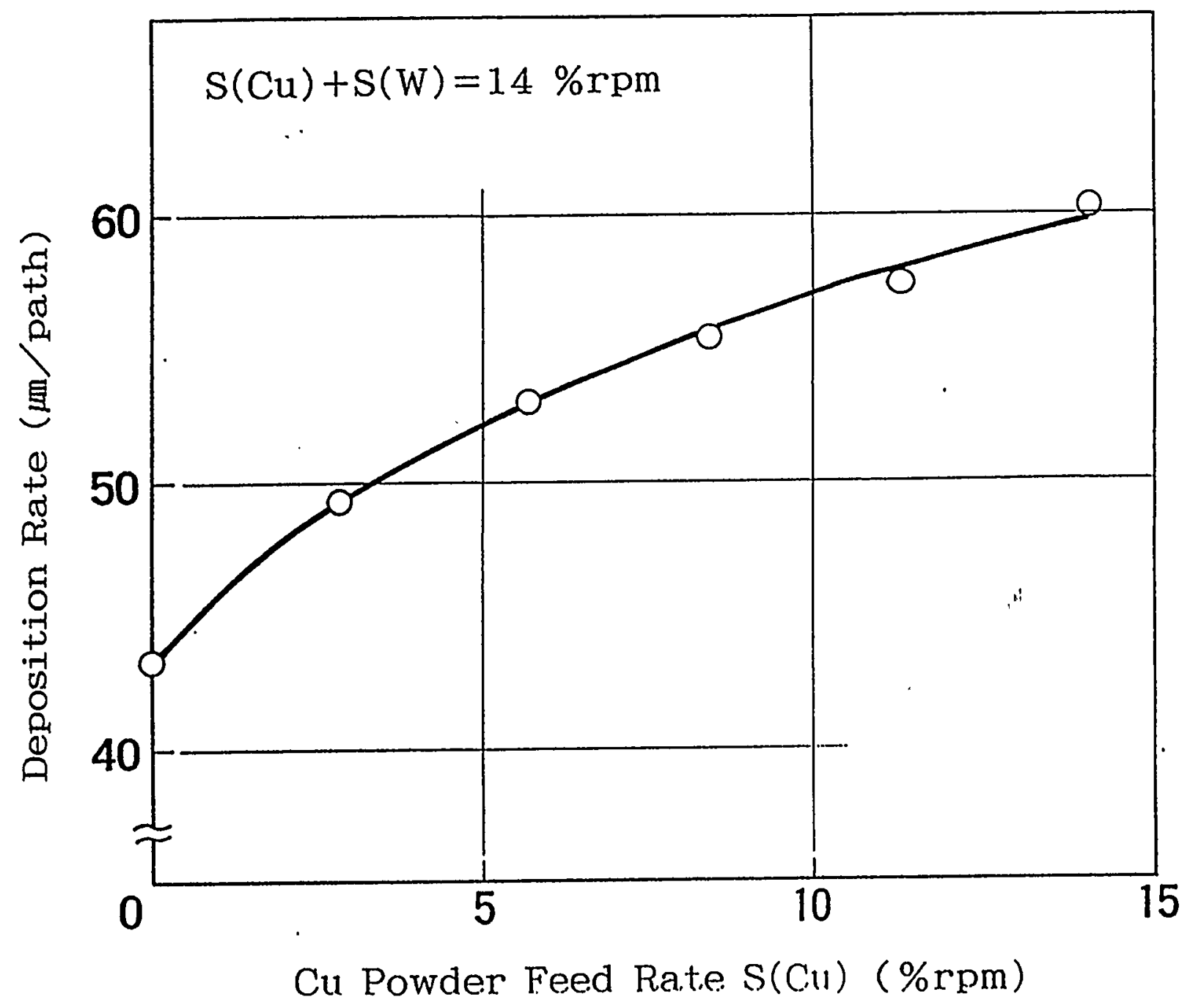

$\underset{\omega}{\mid}$

Deposition rate vs. $\mathrm{Cu}$ powder feed rate 


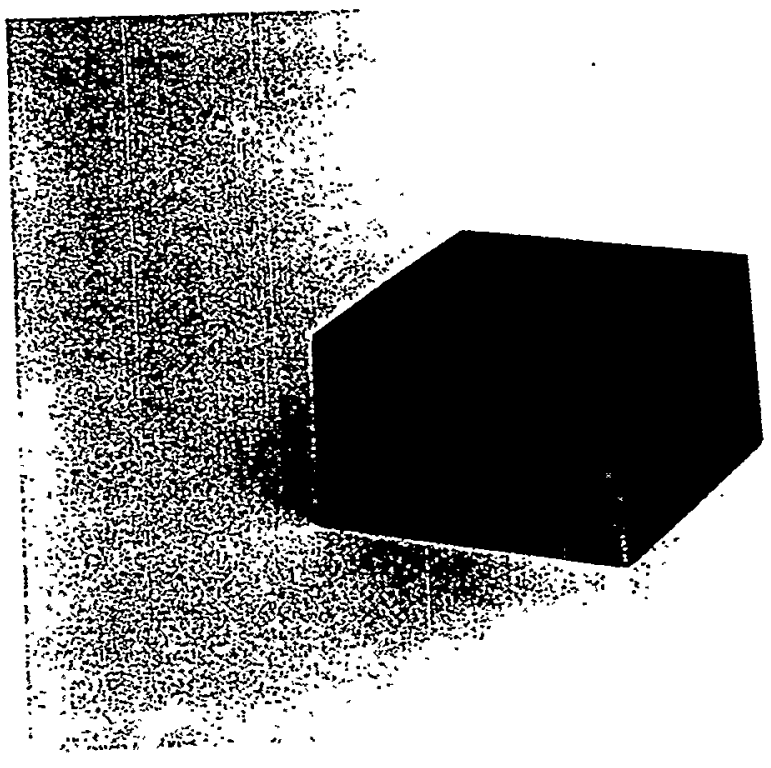

$\begin{array}{ll}\longmapsto & t(W)=2 \mathrm{~mm} \\ 20 \mathrm{~mm} & t(W / \mathrm{Cu}) \sim 3 \mathrm{~mm}\end{array}$

Trial fabrication of $\mathrm{W}$ target by composite VPS method 
M.Shibui, Toshiba Corporation

Development of multi-layered cooling tube

- Analytical prediction of $\mathrm{T}$ permeation

- 1-D analysis without armor

- Objective : Effective thickness of W layer

- Multi-layered structure

- SUS316L layer for SCC resistance

- Two $\mathrm{W}$ layers for $\mathrm{T}$ permeation resistance

- Cu layer for heat conduction.

- 0.2tSUS316L/Cu/0.03tW/Cu/0.03tW/OFCu 


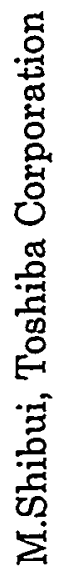

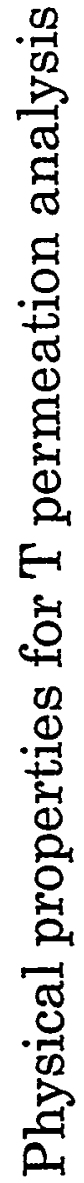

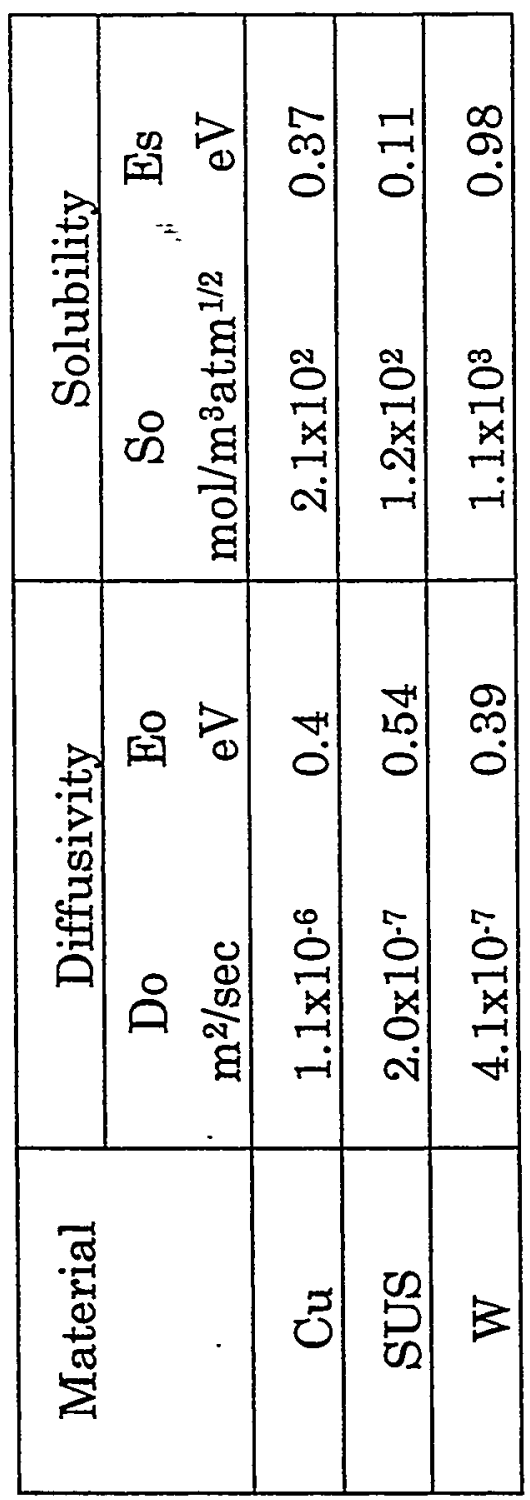

Oิ 


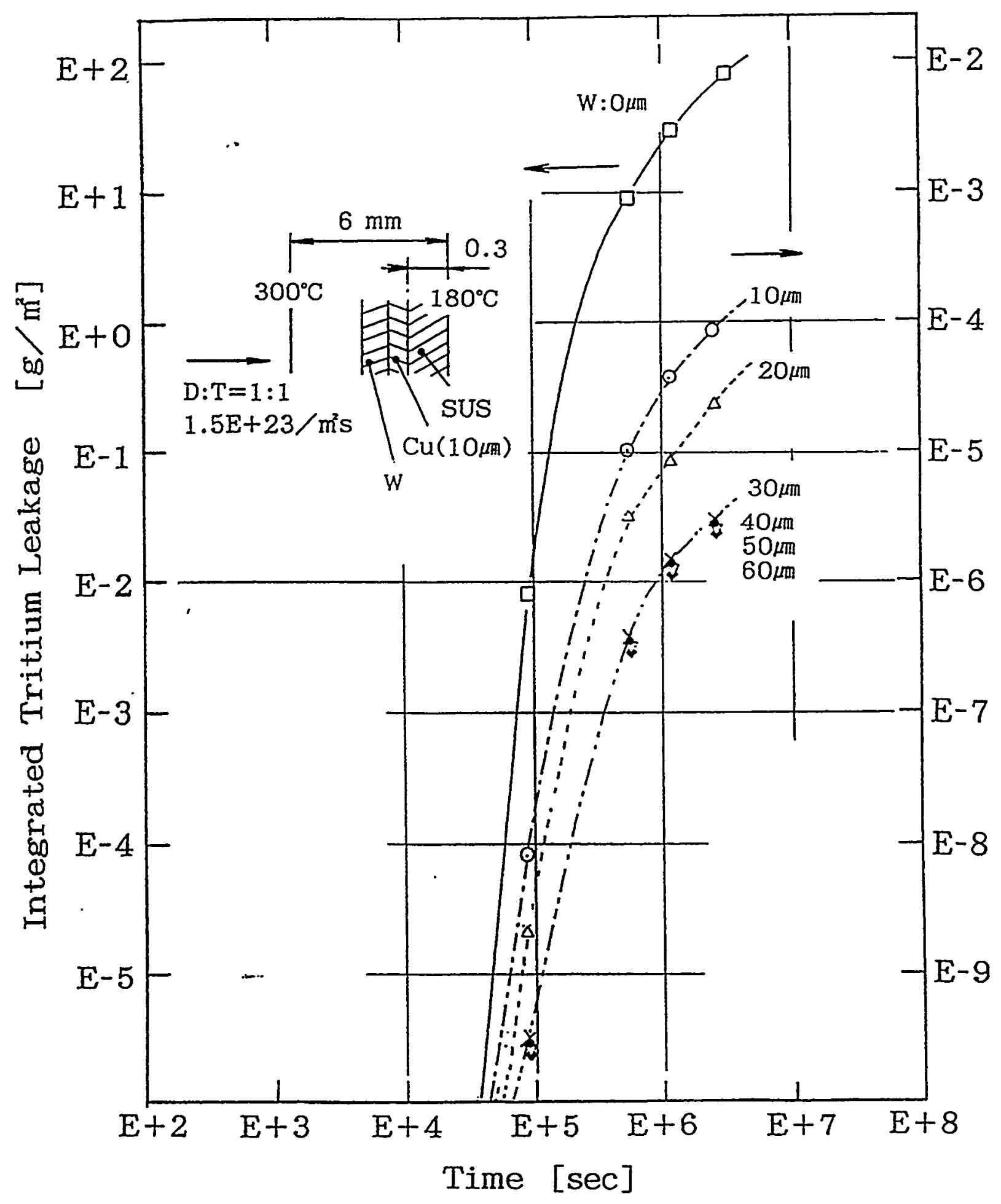

Intergated tritium leakage 


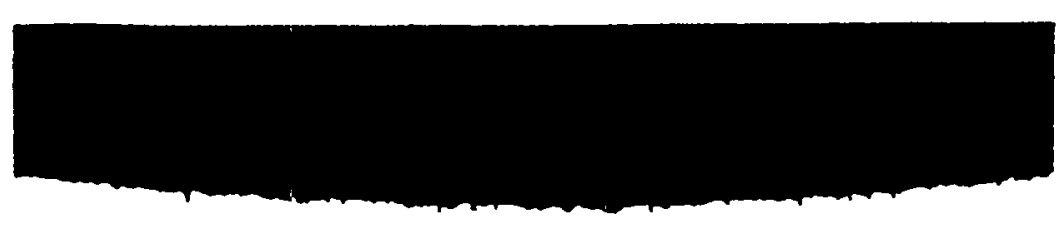

SUS316L

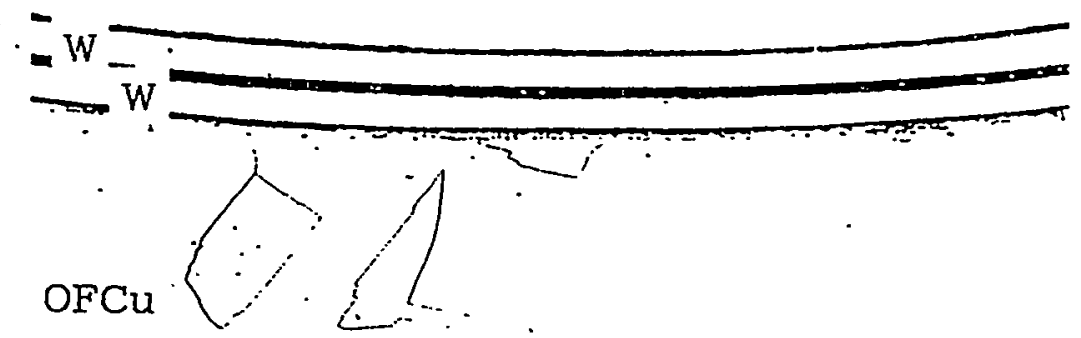

$0.1 \mathrm{~mm}$

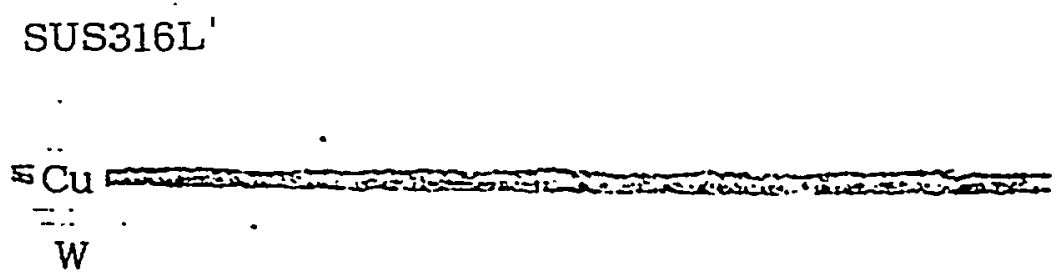

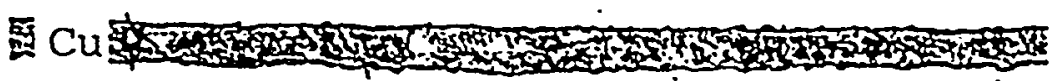

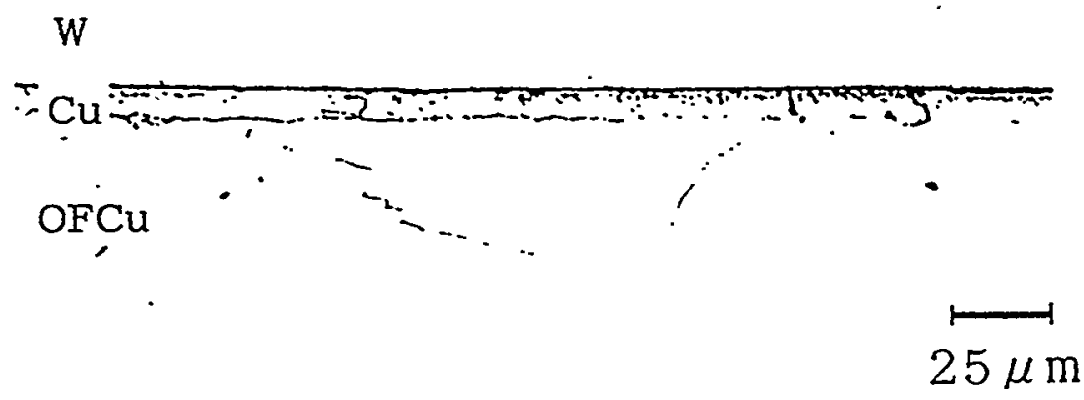

Optical micrographs of multi-layered cooling tube 
M.Shibui, Toshiba Corporation

\section{Concluding remarks}

1. Formation of W/Cu gradient material by the composite VPS method has been demonstrated :

@ with sufficiently small amount of porosity in pure $W$ layer,

@ with easy controllability of $\mathrm{Cu}$ volume fraction in the deposit.

2. Multi-layered cooling tube has been proposed :

(@) with SUS316L as SCC resistant layer and

@ W as T permeation resistant layer.

Its fabricability has also been demonstrated by using HIP. 


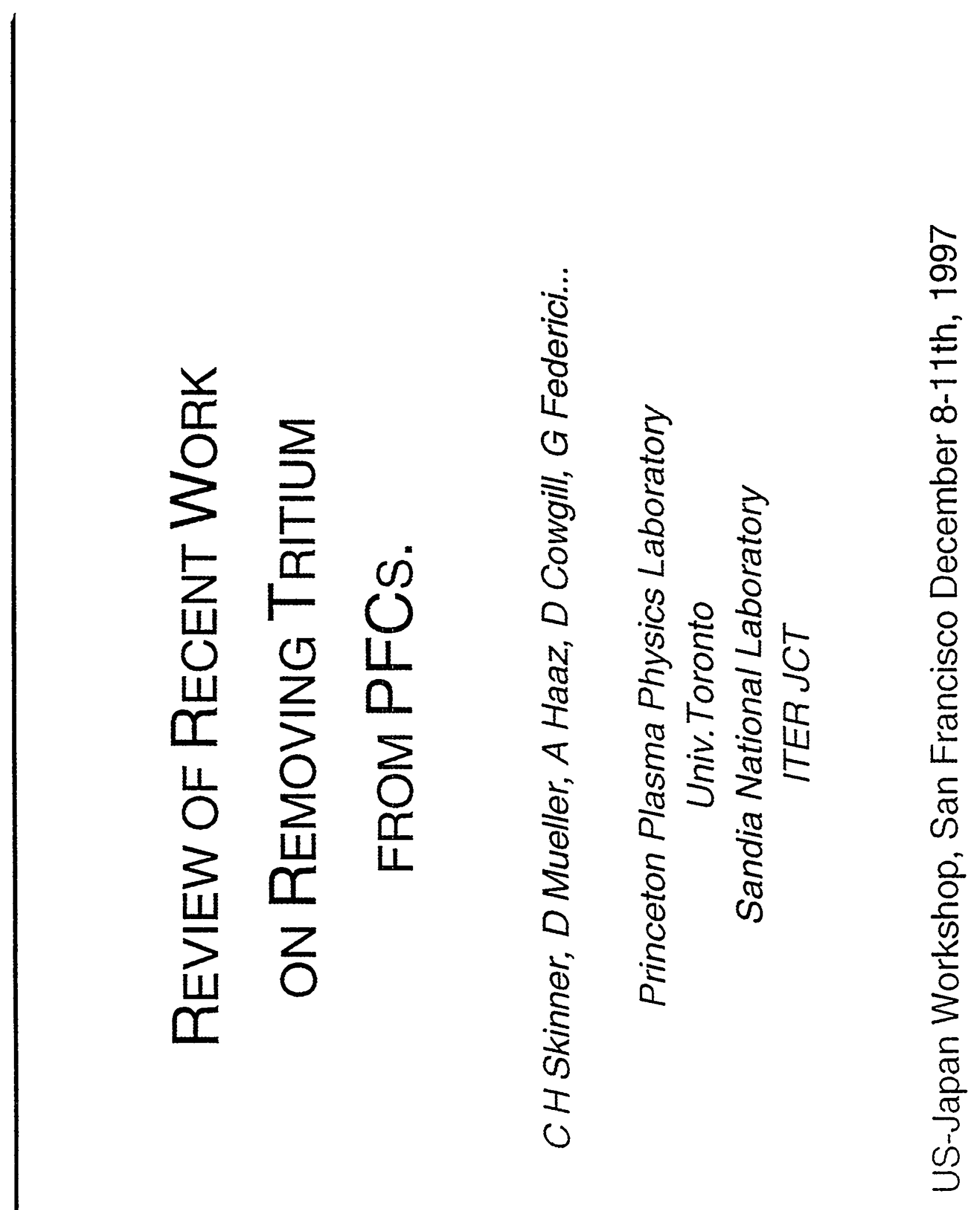




\section{TRITIUM REMOVAL}

\section{Motivation:}

- Tokamaks experience appreciable retention of tritium fuel.

- Tritium supply is limited

- In-vessel inventory is limited

- Tritium inventory control essential for fusion reactors. Steady state advantages of stellerators e.g. LHD would not result in practical fusion reactors without control of tritium inventory.

- Current retention levels are too high.

- Current removal methods are too slow and underdeveloped,

Development of highly efficient tritium removal techniques is essential for any DT fusion reactor. 


\section{TFTR Experience: how much tritium was retained and where?}

Summary of 3 run periods over 3.5 years in TFTR:

Total tritium injected (NBI+puff)

Total tritium retained in torus during run periods

Total tritium removed from torus in clean up months

\section{Deuterium Measurements:}

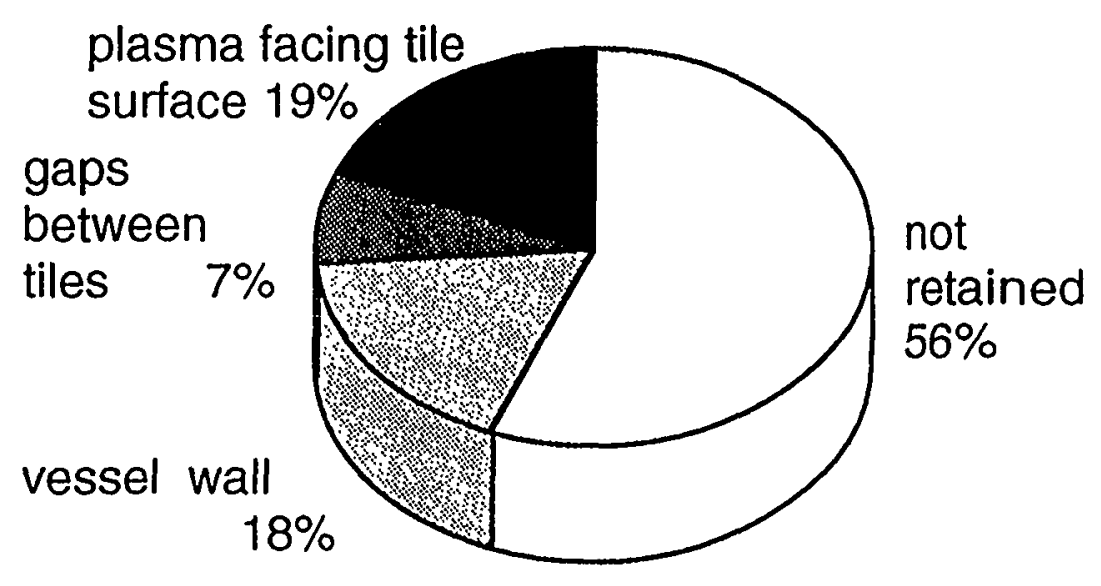

Tritium Analysis currently in progress:

- 10 Tritiated tiles, removed 9/97; to be shipped to Idaho State for nuclear elastic recoil analysis of $H, D$ and $T$ concentration vs. depth (range 15-40 $\mu$ ) Experiments planned at PPPL for tritium release by air baking.

- Dust vacuumed into filter housings and removed from diagnostic windows. Presently at INEL for particle size and BET analysis.

- Samples scraped from limiter surface at LANL for thermal outgassing of the tritium for total content and temperature of release. 


\section{ITER Fuel CyCLE}

\section{Fuel per 1,000 s pulse $(270 \mathrm{~g})=1 / 10$ th of annual supply !}

Tritium Retention experienced by:

TFTR -

JET DTE1

JT60 (exhaust)

JT-60 (tiles)

\$s

DIII-D (tiles)

CFTSIM - ITER dynamic fuel model

Assumes ITER retention $1-5 \%$ !

- lower \% than present tokamaks

Consistent with co-deposition rates

$\$$ predicted by Brooks.
CFTSIM - ITER dynamic fuel model

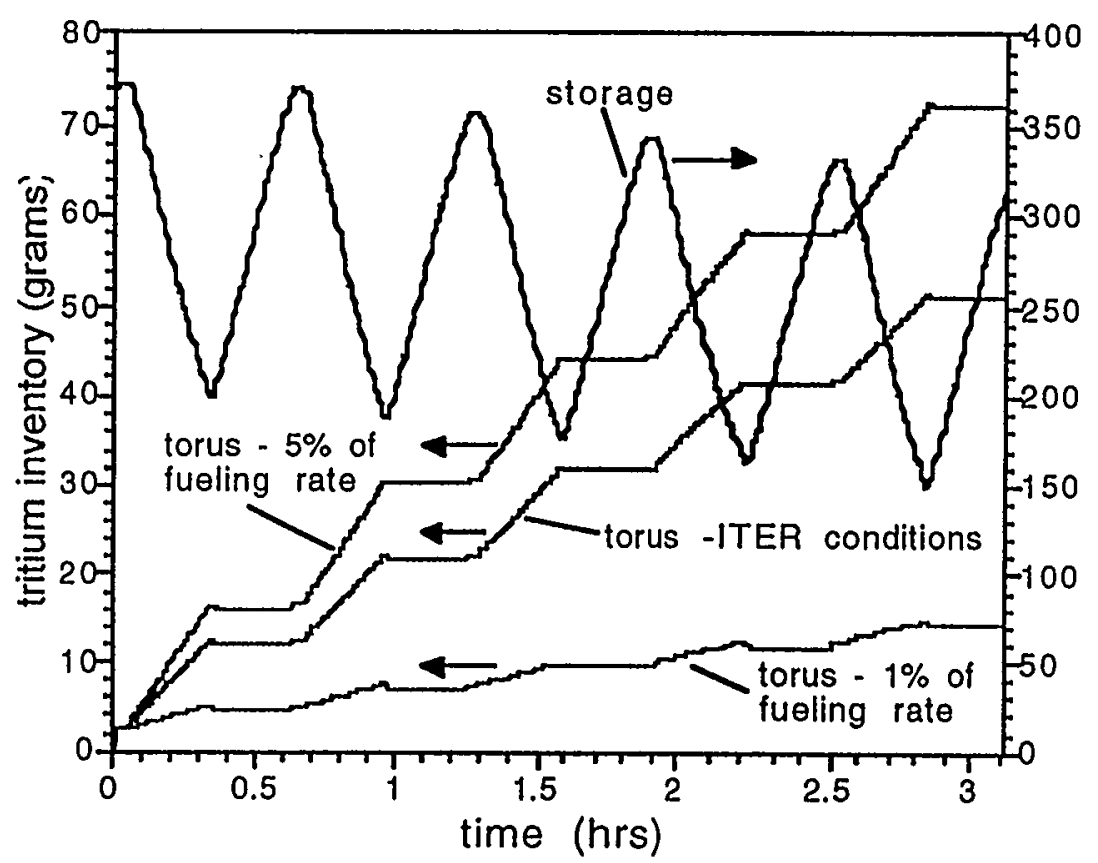

Fig. 3. ITER tritium inventories

Sugihara et al. EPS ‘97 Kuan et al. SOFE ‘97

Urgent need to develop ways to reduce retention and remove tritium ! 


\section{MODELING ITER T REMOVAL}

$\stackrel{20}{2}$

CFTSIM - ITER model applied

to tritium removal.

Kuan et al. SOFE' 97

Time available for removal:

$\approx 1 / 1,000$ of present tokamaks.

Desired removal rate in $\mathrm{nm} / \mathrm{s}$ range (or microns / hour).

Measured HeO glow removal rates:

CFTSIM - ITER dynamic fuel model

Laboratory

TFTR
$0.064 \mathrm{~nm} / \mathrm{s}$

$0.004 \mathrm{~nm} / \mathrm{s}$

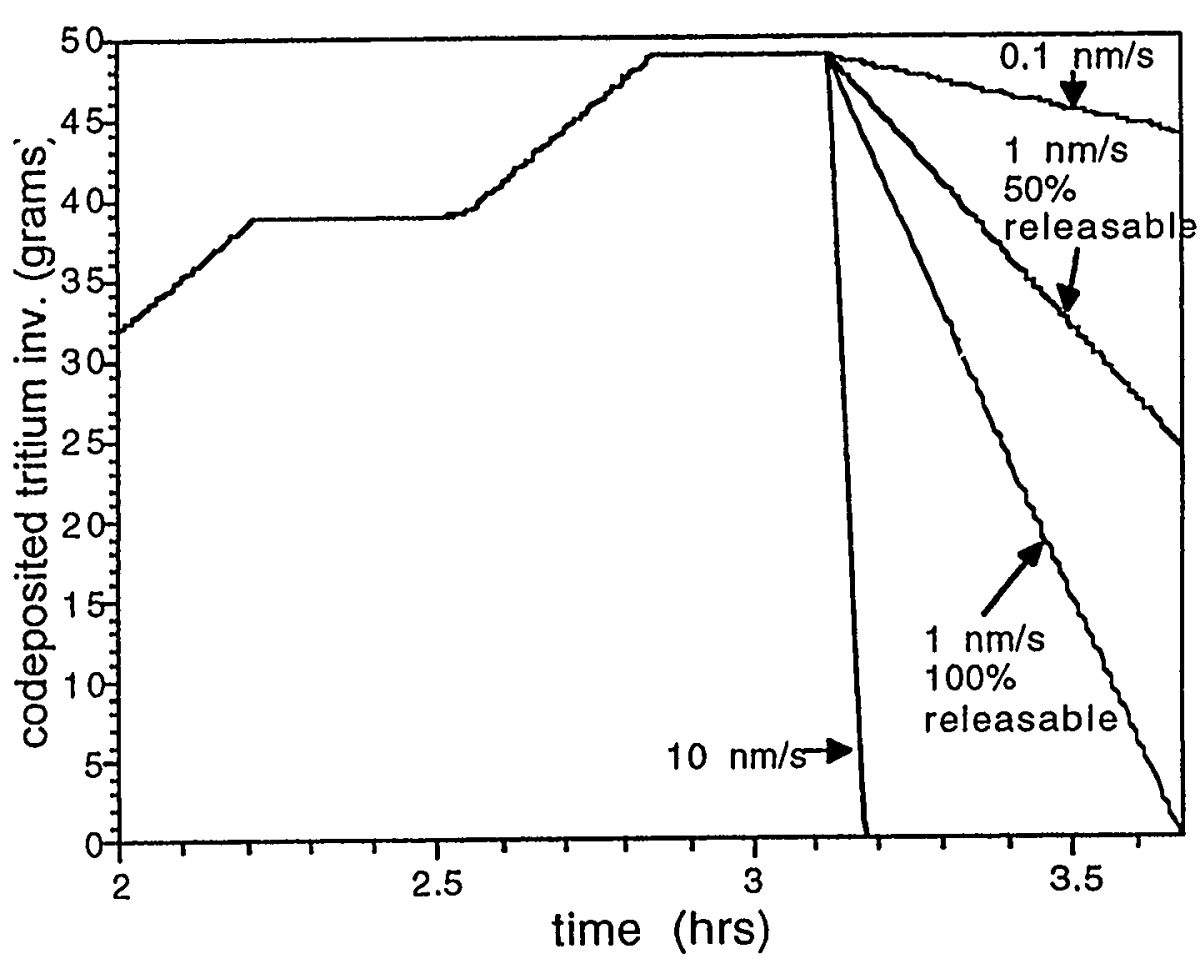

Fig. 4. Release of codeposited tritium 


\section{Comparison of tritium removal techniques, (1995)}

TFTR

He-GDC, outgas,

D soak $\}$ Ineffective

D-GDC Initial removal rate high (>170 Ci/hour), declining to $10 \mathrm{Ci} /$ hour.

Accesses only tritium on surfaces exposed to discharge.

HeO-GDC Rate $\approx 50 \mathrm{Ci} /$ hour - constant with time

room air 2,086 Ci removed, access to all surfaces

Disruptions Flash heating of limiter surface near midplane. - Release of recently retained tritium.

Pulse Heats limiter to $250^{\circ}$ C. $956 \mathrm{Ci}$ removed discharge over 23 hours.

cleaning

Boronization Little tritium released, most near surface in Irilium already removed.

Removal by $\mathrm{D}$ and $\mathrm{HeO}$ glow discharge (GDC)

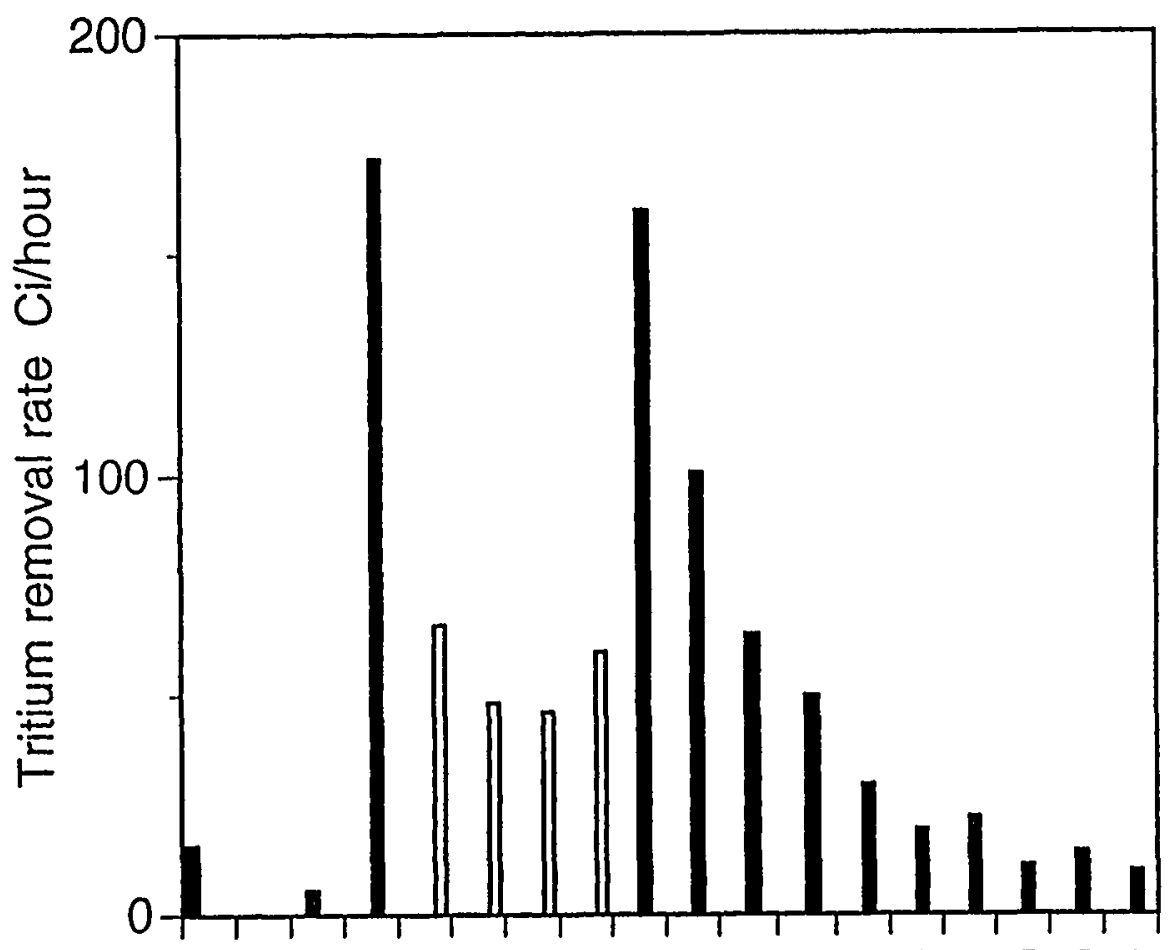

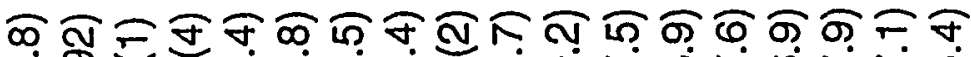
Q 0 西 0000000000000

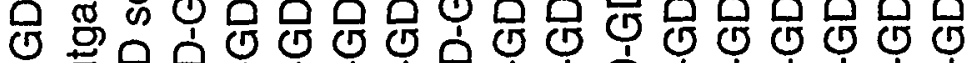

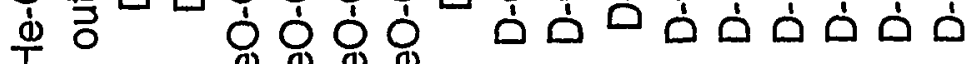
I

procedure (duration in hours)) 


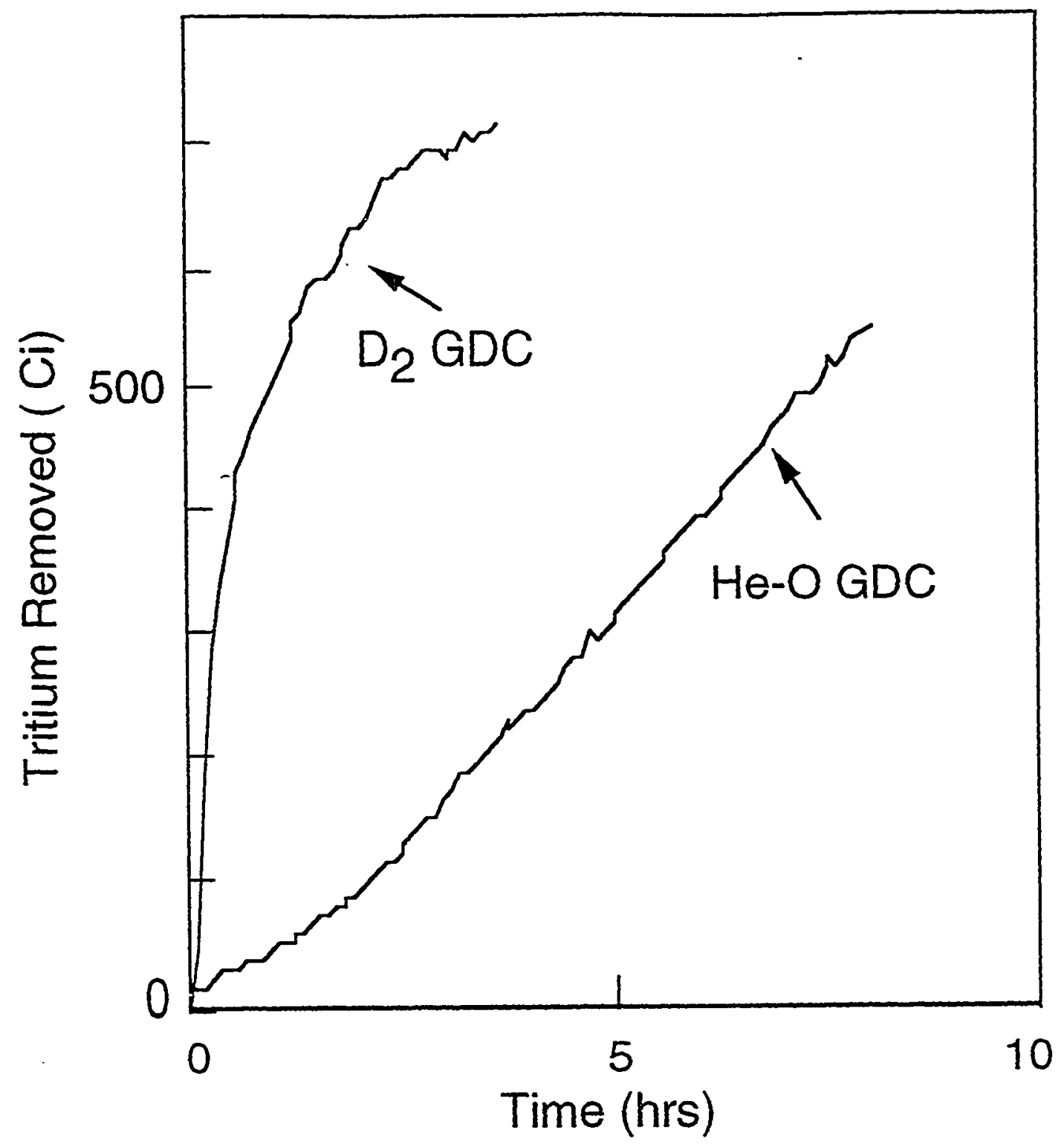




\section{Short term tritium retention high with strong tritium puffs}

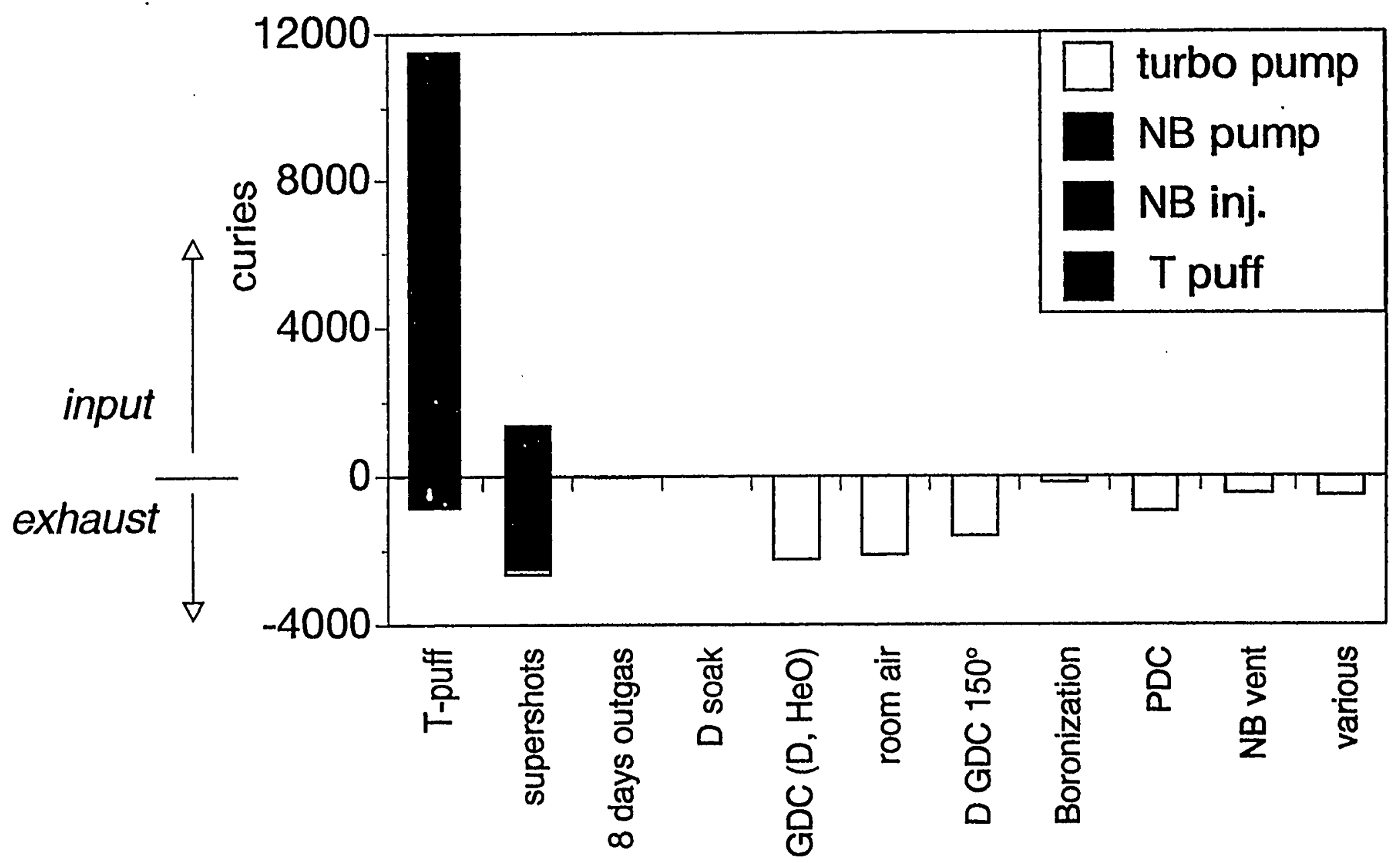

Tritium fraction maximized for L-mode study, (September 1995) Short term retention $>90 \%$

Tritium successfully removed by combination of glow discharge (D and $\mathrm{HeO}$ ), room air, and pulsed discharges. 
(sə!nก) Коричәли! ןәssәл-u!

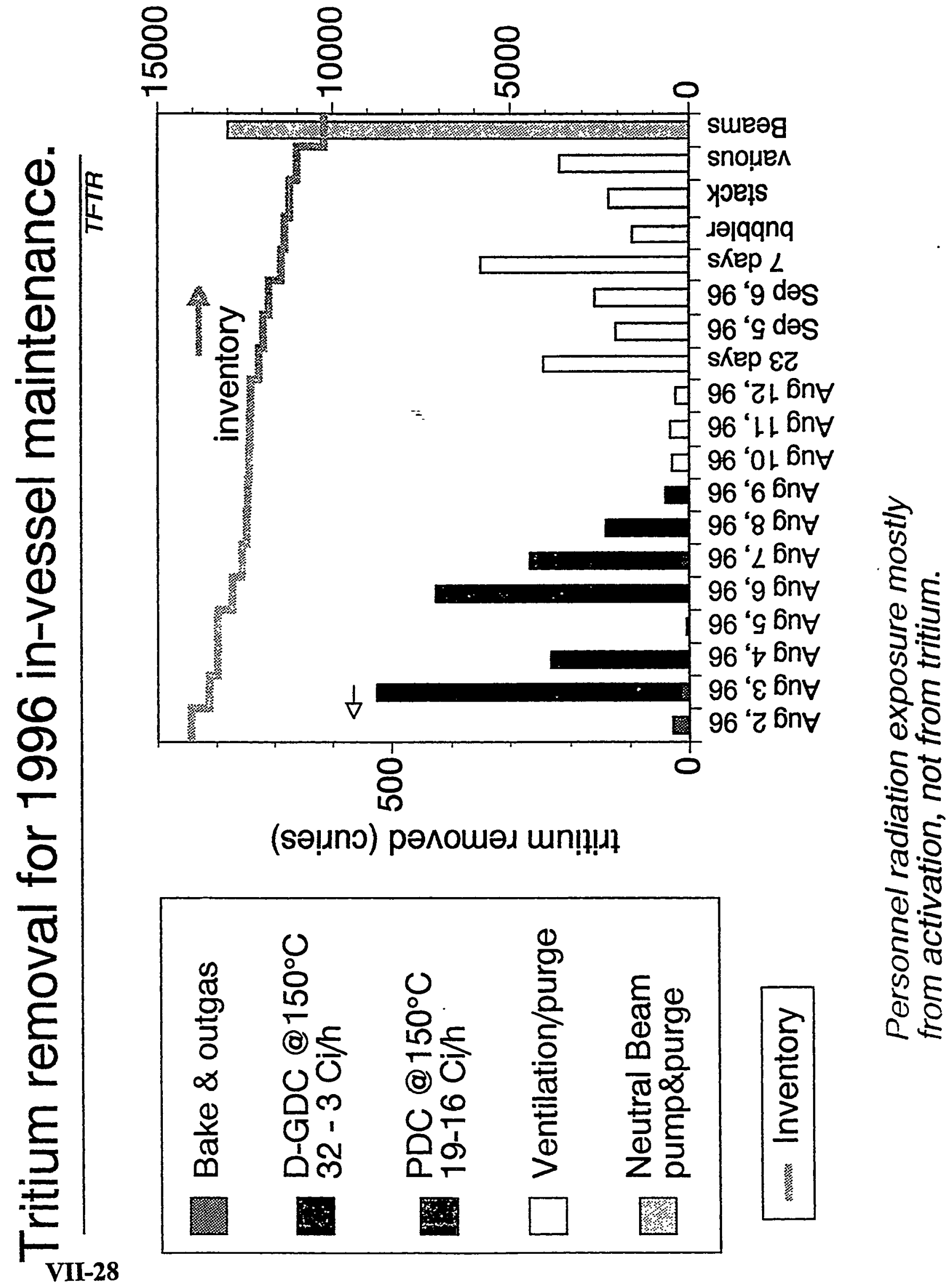




\section{Vacuum Vessel Tritium Recovery April 4 through May 8, 1997}

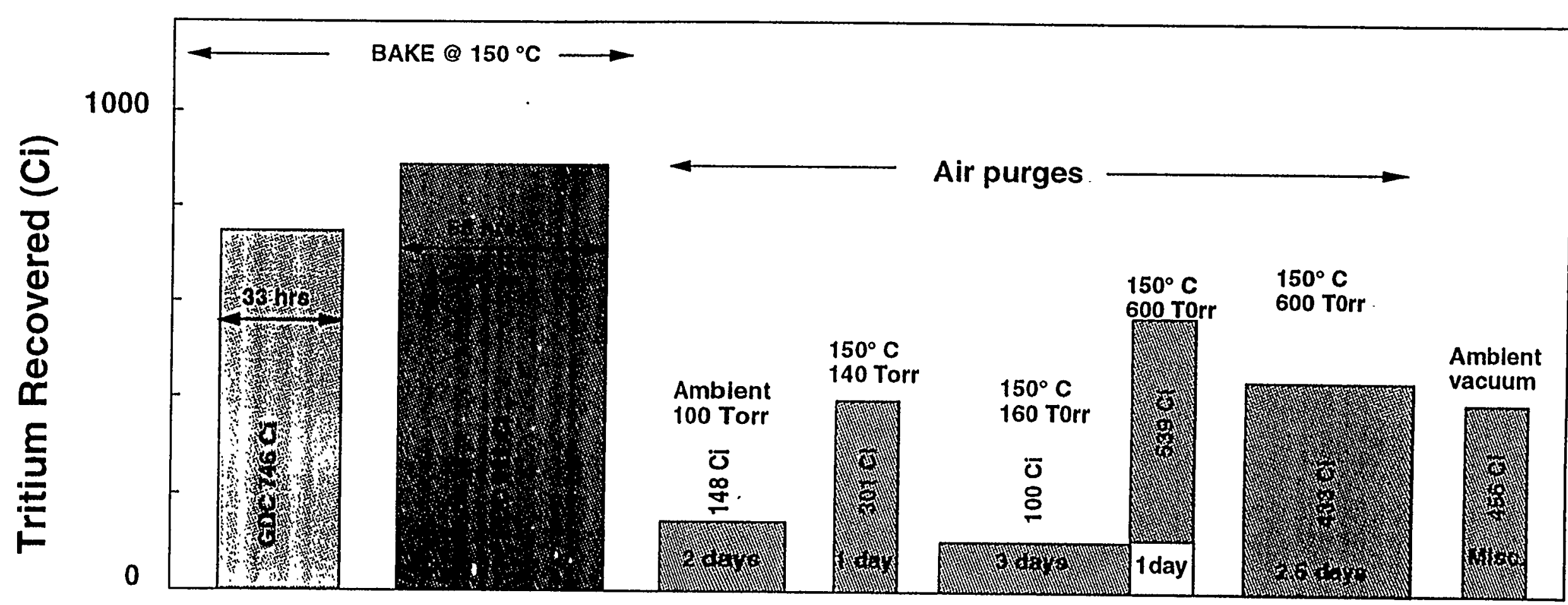

- During air purges, after about $1 / 2$ day following temperatue or pressure change, no change was observed in the tritium content of the air in the vacuum vessel.

Misc. refers to the sum of tritium removed between the various removal tactics. Mostly this is from outgassing of the vessel and various pumping appendages.

In addition $331 \mathrm{Ci}$ were recovered from the neutral beams during this period.

- In total $4279 \mathrm{Ci}$ were removed from the neutral beams up until August 14 mainly by purging them with air. 


\section{SUMMARY OF TFTR EXPERIENCE}

$\checkmark$ TFTR is the first tokamak with extensive tritium experience

$(\because=1,000$ DT discharges over 3.5 years).

TFTR high power phase is $\approx 1 \mathrm{~s}$, so total DT duration is $\approx 1,000 \mathrm{~s}$

$\approx 1$ ITER 1.000s pulse

In TFTR: of $5 \mathrm{~g}$ tritium fuel, $2.5 \mathrm{~g}$ was retained, and $1.7 \mathrm{~g}$ removed in 3 campaigns over $\approx 3$ months.

ITER Physics and Technology program requires a duty cycle $\approx 1-10 \%$ (compared to $:=10^{-4}$ for TFTR)

(future DT Reactor availability required is:

$\approx 50 \%)$

Much faster tritium removal required for ITER to fulfil its Physics and Technology goals. 


\section{Ten Removal Methods Considered for ITER.}

Adapted from Figure 3 in "Tritium Inventory in ITER PFC's, Predictions, Uncertainties, R\&D Status and Priority Needs"; International Symposium on Fusion Nuclear Technology, Tokyo April 6-11, 1997. Paper L164 G. Federici, et al.,

\begin{tabular}{|c|c|c|}
\hline $\begin{array}{l}\text { Identified } \\
\text { Options }\end{array}$ & Merits & Shortcomings \\
\hline $\begin{array}{l}\text { Glow } \\
\text { Discharge } \\
\text { Cleaning }\end{array}$ & $\begin{array}{l}\text { - well established tokamak practice } \\
\text { - does not require vent or opening of the } \\
\text { vacuum vessel }\end{array}$ & $\begin{array}{l}\text { - TF off, long shut-down } \\
\text { - limited access to shadowed areas } \\
\text { - HeO glow discharge requires active } \\
\text { conditioning to remove residual O. } \\
\text { - low removal efficiency for ITER (even with } \\
\text { O as a min. species) }\end{array}$ \\
\hline $\begin{array}{l}\text { D2 soaking } \\
\text { with heated } \\
\text { walls }\end{array}$ & - some tokamak experience & - low removal efficiency \\
\hline $\begin{array}{l}\text { Air/ } \mathrm{O}_{2} \\
\text { exposure with } \\
\text { hot walls. }\end{array}$ & $\begin{array}{l}\text { - expected good removal efficiency } \geq 50 \% \\
\text { and short cleaning time at wall temperature } \\
T * 250 " \mathrm{C}) \\
\text { - accessibility of non-line-of-sight and } \\
\text { shadowed regions, gaps, etc. } \\
\text { - mechanisms of removal are reasonably } \\
\text { well understood. } \\
\text { - may oxidise Be dust and may reduce its } \\
\text { chemical reactivity with steam in case of an } \\
\text { accident. }\end{array}$ & $\begin{array}{l}\text { - need venting; } \\
\text { - ratcheting effects could limit cleaning at } \\
240^{\circ} \mathrm{C} \text { (max temp. in ITER) } \\
\text { - wall conditioning is required for } \\
\text { decontamination of residual } \mathrm{O} \text { and } \mathrm{H}_{2} \mathrm{O} \text {. } \\
\text { - very limited tokamak practice and at too low } \\
\text { wall temperature (TFTR) } \\
\text { - removal of T from thick deposits and mixed } \\
\text { materials requires } \mathrm{R} \& \mathrm{D} \text {. }\end{array}$ \\
\hline
\end{tabular}

Recent Results...... 


\section{ERosion of Codeposited FILMS VIA OXYGEN EXPOSURE}

A.A. Haaz and J W Davis, University of Toronto; R Causey, Sandia; Jacob et al, Garching

Laboratory produced a-C:H and aC-D Films

$20 \mathrm{~nm}-2 \mu \mathrm{m}$ thick, at $200-350^{\circ} \mathrm{C}$ in low pressure $\mathrm{O}_{2}$ or air.

Erosion rates: $2 \mathrm{~nm} /$ hour $-50 \mathrm{~nm} /$ hour - too low.

Co-deposits from tokamaks:

- Causey et al [Sandia]

TFTR tile: $\sim 50 \mu \mathrm{m}$ thick $[\mathrm{H} / \mathrm{C} \sim 0.4]$ heated in air [760 Torr] at $350^{\circ} \mathrm{C}$ erosion $\sim 50 \mu \mathrm{m} / \mathrm{h} \quad$ [metals: $<0.2$ at $\%$; Mills et al]

- Jacob et al [Garching]

ASDEX-U tile: $~ 750 \mathrm{~nm}$ film [D/C 0.4] heated in air [760 Torr]

at $380^{\circ} \mathrm{C}$ erosion $\sim 0.3 \mu \mathrm{m} / \mathrm{h}$ [presence of $\mathrm{B}$ on the layer]

Erosion rates differ by two orders of magnitude!

The structure [and impurity type and content] of the codeposits from the two machines are different.

\section{NEW RESULTS}

[Haasz and Davis; US-HT/JCT Meeting on T inventory and Control in ITER PFCs [Pittsburgh, PA, 97 Nov16-17]

TFTR tile [POCO graphite] removed from machine in 1987, thickness:

$\sim 30 \mu \mathrm{m}[\mathrm{D} / \mathrm{C} \sim 0.35]$, effective density: $~ 320 \mathrm{~kg} / \mathrm{m} 3$

Exposure to oxygen, pressure: 16 Torr, temperature: $250^{\circ} \mathrm{C}, 300^{\circ} \mathrm{C}$, and $350^{\circ} \mathrm{C}$ 
348

B.E. Mills et al. / Characterization of del

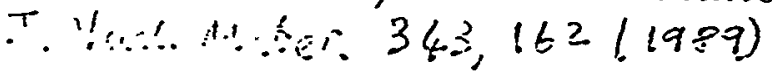

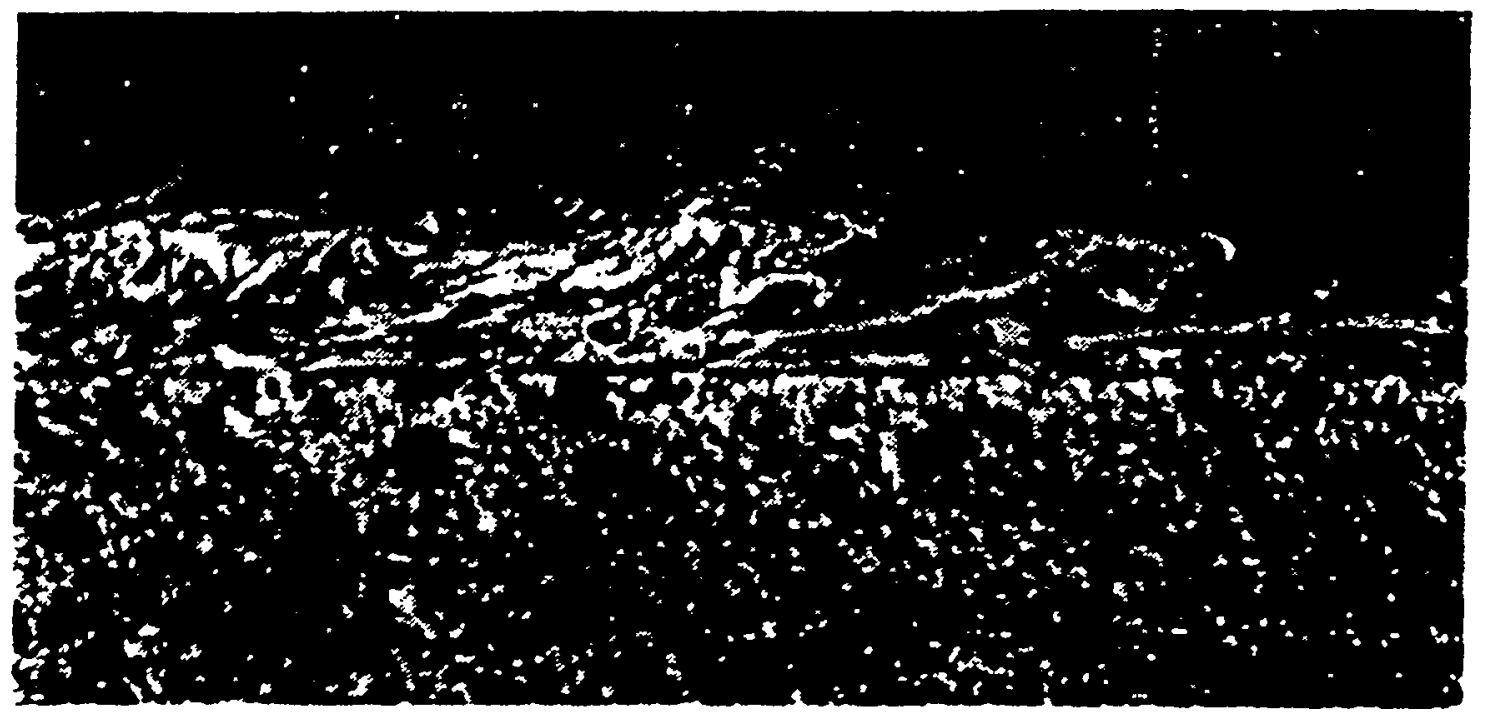

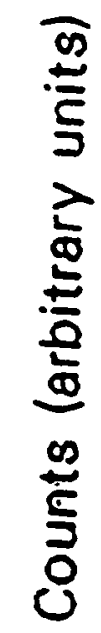

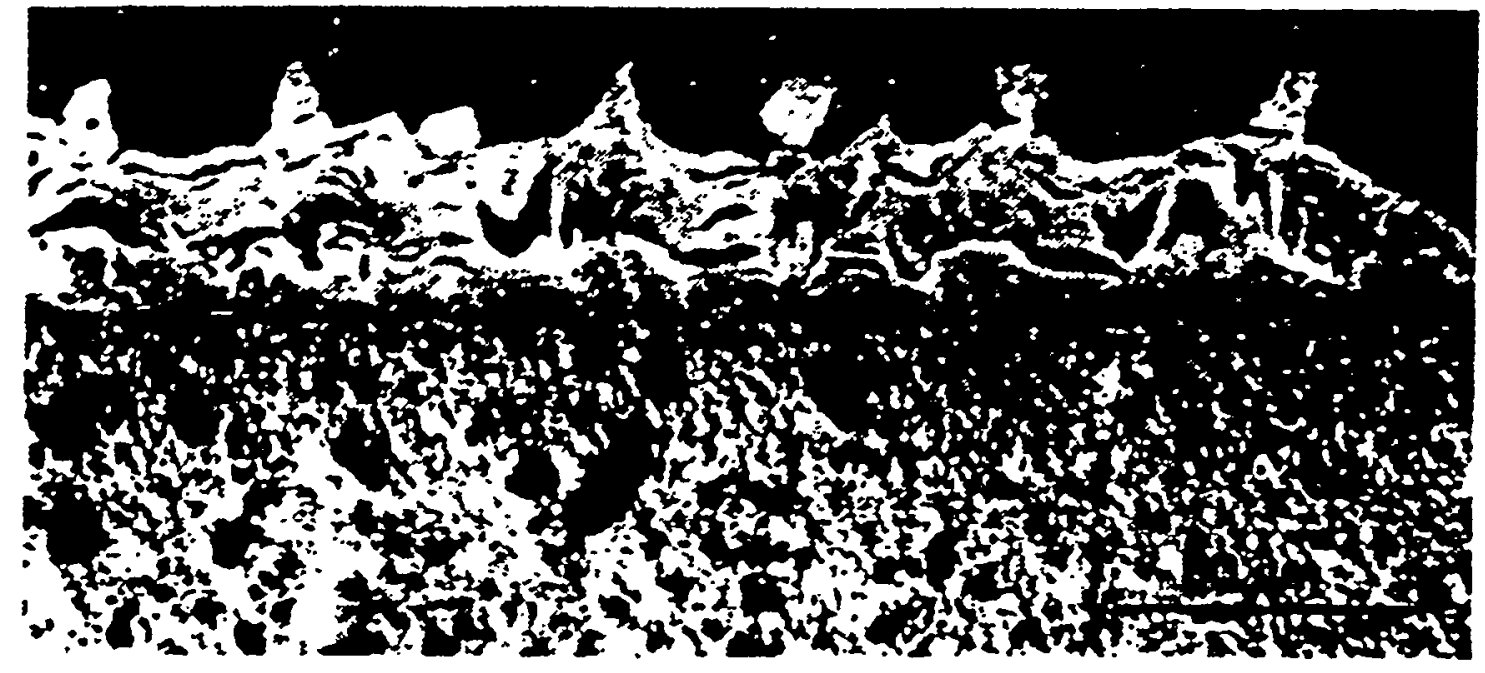

Fig. 5. Poloidal (top) and toroidal (bottom) cross-sections of the plasma-facing surface of a bumper limiter tile in the moderate deposition region, the surface of which is seen in figure $4 \mathrm{C}$.

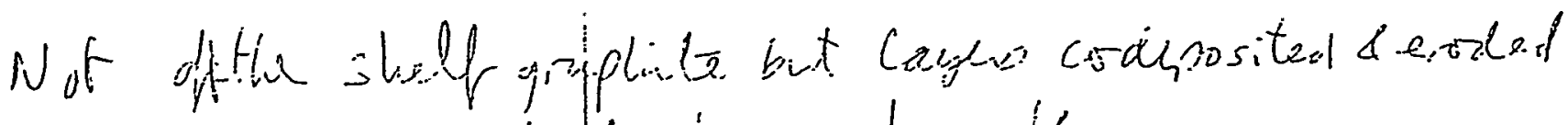

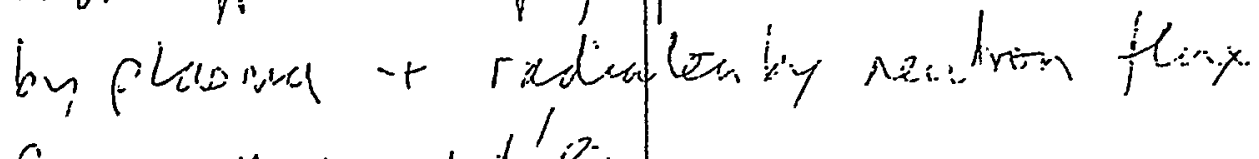

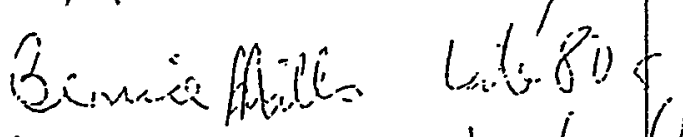

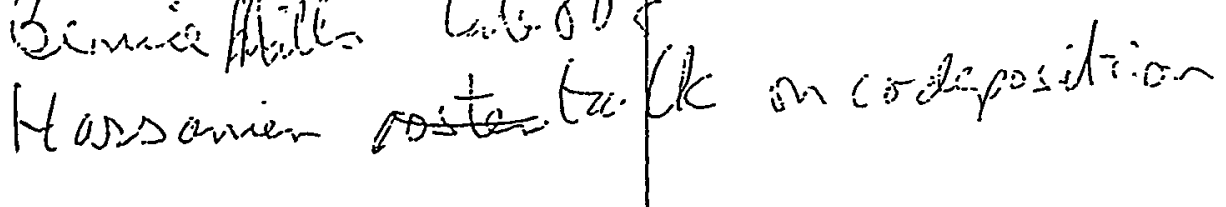

VII-33 


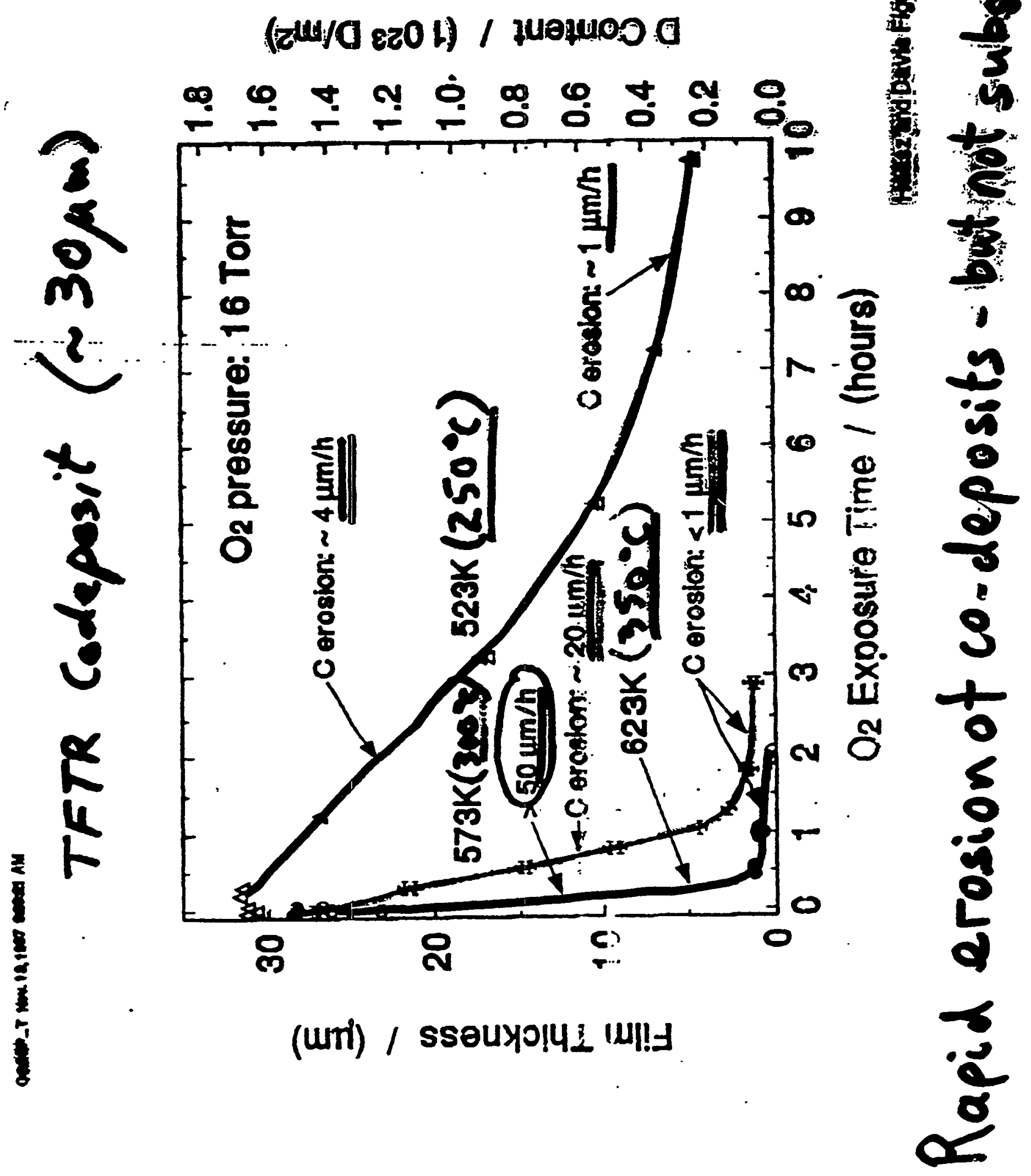




\begin{tabular}{|c|c|c|}
\hline $\begin{array}{l}\text { Identified } \\
\text { Options }\end{array}$ & Merits & Shortcomings \\
\hline $\begin{array}{l}\mathrm{Hot} \mathrm{Xe}_{2} \mathrm{O}_{2} \text { (or } \\
\mathrm{N}_{2} \mathrm{O} \text { ), with } \mathrm{Xe} \\
>>\mathrm{O}_{2} \text { (or } \mathrm{N}_{2} \mathrm{O} \\
\text { to minimise } \mathrm{O} \\
\text { contamination }\end{array}$ & $\begin{array}{l}\text { - enhanced impact-induced conditioning by } \\
\text { using Xe to increase momentum transfer. } \\
\text { - using } \mathrm{N} 2 \mathrm{O} \text {, rather than } \mathrm{O} 2 \text {, should } \\
\text { increase surface oxidation of codeposited } \\
\text { layers and reduce O-contamination. }\end{array}$ & - more study is needed. \\
\hline $\begin{array}{l}\text { Reaction with } \\
\text { gaseous } \\
\text { radicals (e.g., } \\
\text { O3) }^{2}\end{array}$ & $\begin{array}{l}\text { - efficient. } \\
\text { on. }\end{array}$ & $\begin{array}{l}\text { - short path length before decomposition } \\
\text { - conditioning may be needed afterward to } \\
\text { remove non-volatile products. }\end{array}$ \\
\hline $\begin{array}{l}\text { Isotope } \\
\text { exchange with } \\
\text { D plasmas. }\end{array}$ & $\begin{array}{l}\text { - no change in magnetisation needed } \\
\text { - no opening of the vacuum vessel } \\
\text { - no solid waste } \\
\text { - gaseous residue could be processed by } \\
\text { the existing fuel cleanup system } \\
\end{array}$ & $\begin{array}{l}\text { - some conditioning may be needed to re- } \\
\text { establish fuel recycling rates to the plasma } \\
\text { - R\&D needed to establish feasibility and } \\
\text { quantify process. }\end{array}$ \\
\hline $\begin{array}{l}\text { Abrasive } \\
\text { methods such } \\
\text { as } \mathrm{CO}_{2} \\
\text { blasting, LN2 } \\
\text { jets }\end{array}$ & $\begin{array}{l}\text { - high expected removal efficiency (short } \\
\text { cleaning time). } \\
\text { - may induce flaking and ease collection at } \\
\text { the bottom of the divertor through venting , } \\
\text { pump-out of debris. } \\
\text { - tokamak application: used for beryllium } \\
\text { cleaning in JET } \\
\text { - know-how available from other industrial } \\
\text { applications (e.g., paint removal/ cleaning } \\
\text { and decontamination of surfaces) } \\
\text { - some limited R\&D for ITER is in progress in } \\
\text { the US. }\end{array}$ & $\begin{array}{l}\text { - TF off, needs opening to allow access to } \\
\text { equipment; long shut-down, RH } \\
\text { intervention } \\
\text { - production of debris or residual waste; } \\
\text { ventilation required for debris removal, but } \\
\text { no abrasives in waste stream } \\
\text { - needs line-of-sight to surfaces to be } \\
\text { cleaned. } \\
\text { - no or limited access in gaps, limited access } \\
\text { in remote areas } \\
\text { requires some R\&D to extrapolate with } \\
\text { confidence to ITER }\end{array}$ \\
\hline
\end{tabular}




\begin{tabular}{|c|c|c|}
\hline $\begin{array}{l}\text { Identified } \\
\text { Options }\end{array}$ & Merits & Shortcomings \\
\hline $\begin{array}{l}\text { Collection of } \\
\text { flakes spalled } \\
\text { from the } \\
\text { plenum into the } \\
\text { bottom of the } \\
\text { divertor } \\
\text { cassette and } \\
\text { outgassing by } \\
\text { heating }\end{array}$ & $\begin{array}{l}\text { - in situ method } \\
\text { - tritium evolved can be simply handled by } \\
\text { the existing gas recycling system } \\
\text { - no interference with the pulse duty cycle } \\
\text { - minimal ancillary equipment required } \\
\text { - Jay be a major deposition site } \\
\text { JET is assessing technical feasibility }\end{array}$ & $\begin{array}{l}\text { - some modification to the cassette design } \\
\text { likely } \\
\text { experiments may suggest ways to } \\
\text { maximise spallation rate }\end{array}$ \\
\hline $\begin{array}{l}\text { ICRC (direct } \\
\text { ion resonance) }\end{array}$ & $\begin{array}{l}\text { - does not require vent or opening of the } \\
\text { vacuum vessel } \\
\text { - no conditioning requirements } \\
\text { some limited R\&D for ITER is in progress in } \\
\text { the US } \\
\text { - some tokamak conditioning experience } \\
\text { (TEXTOR, TORE Supra) }\end{array}$ & $\begin{array}{l}\text { - erosion is line-of-sight. Shadowed areas } \\
\text { are not eroded } \\
\text { - expected to be slow } \\
\text { requires active conditioning to remove } \\
\text { residual O. } \\
\text { - may be difficult to get long wavelength RF } \\
\text { into the divertor. } \\
\text { - requires significant R\&D. }\end{array}$ \\
\hline $\begin{array}{l}\text { Surface } \\
\text { heating to } \\
2000^{\circ} \mathrm{C} \text { with } \\
\text { continuous } \\
\text { wave } \mathrm{CO} 2 \\
\text { laser }\end{array}$ & $\begin{array}{l}\text { - no solid waste } \\
\text { gaseous residue could be processed by } \\
\text { the existing fuel cleanup system }\end{array}$ & $\begin{array}{l}\text { - R\&D needed to establish feasibility and } \\
\text { quantify process. }\end{array}$ \\
\hline
\end{tabular}




\section{Tritium Removal by Laser Heating}

- In vacuum, temp. $>1,000 \mathrm{~K}$ releases tritium over time scale of 30 minutes (Causey) but heating ITER vessel to $1000 \mathrm{~K}$ is impractical.

- Heating to $>\approx 2,000 \mathrm{~K}$ by nanosecond laser pulse releases surface tritium (Terrault).

- Transient surface heating by a scanning $\mathrm{CO}_{2}$ or $\mathrm{Nd}$ :Yag laser could release tritium in co-deposits without the severe engineering difficulties of bulk heating of the vessel.

- $A \approx 3 \mathrm{kw} / \mathrm{cm}^{2}$ flux with a exposure time of order $10 \mathrm{~ms}$ will heat a 50 micron co-deposited layer to 1,000-2,000 K.

- Substantial amounts of co-deposited tritium may be potentially removed by laser surface heating in an overnight cleanup.

- Improved wall conditioning may be a significant side benefit.

- Experimental validation is required. 


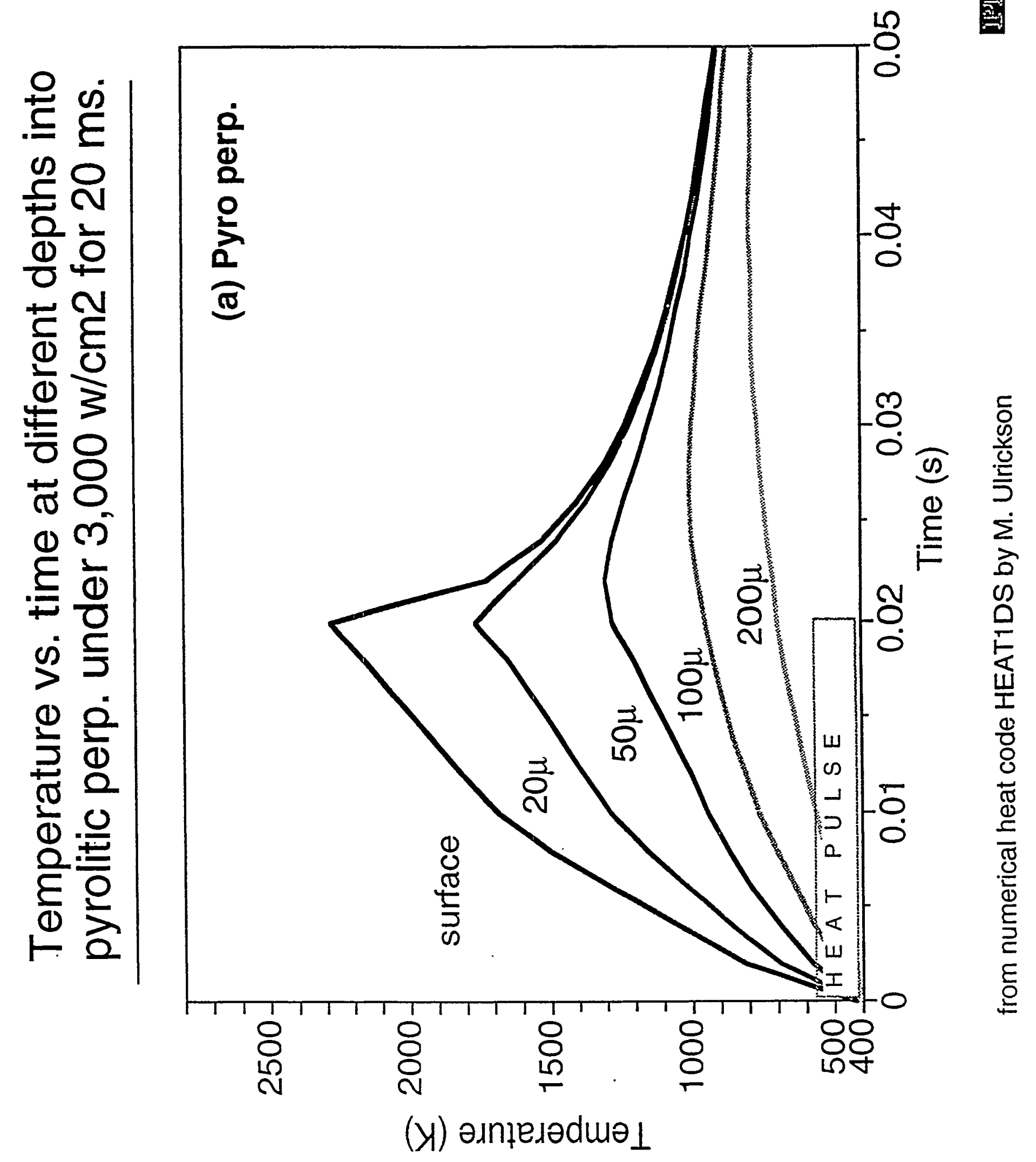




\section{FUTURE ?}

Need much lower retention than present tokamaks:

- More global physics understanding:

Need more in-situ, time dependent measurements e.g. DiMES, QCO's...

- relative contribution of startup, termination, long pulses, high power, attached/detached regimes, disruptions.... to compare to modeling.

- Need global models that combine PSI database, plasma codes, local co-deposition codes and tokamak geometry, - benchmarked with tokamak measurements.

Need much faster tritium removal than current techniques.

- Time available for removal is $\sim 1 / 1,000$ of present tokamaks.

- Global T removal from all surfaces would effectively double erosion rate and reduce divertor lifetime - need directed removal of co-deposits.

- Issues serious enough to warrant a dedicated tokamak program ?

- can one confidently model co-deposition during disruptions ?

- Need to justify research on basis of 'dual use' with relevance to advanced tokamaks (ST) and alternates (stellarators). 


\section{Chemical Compatibility of Carbon with Beryllium}

\section{Kan ASHIDA and Kuniaki WATANABE}

Hydrogen Isotope Res. Center

Toyama University

Gofuku 3190

Toyama 930

JAPAN 


\section{Background:}

Use of two or more components as Plasma Facing Materials caused the Formation of Mixed Plasma Facing Materials (MPFM) due to...

1. Erosion (sublimation, etc.)

2. Chemical Sputtering

3. Physical Sputtering 
II. Objectives:

To understand...

1. physical/chemical properties of MPFM

2. changes in the chemical states of MPFM due to solid state reactions

3. changes in the physical properties of MPFM due to the formation of new compound(s)

4. changes in the trap/release behavior of hydrogen isotopes in/from MPFM 


\section{Plausible Mixed Material systems:}

1. $(\mathrm{Li}+\mathrm{C}+\mathrm{Q})$

2. $(B e+C+Q)$

3. $(\mathrm{Mo}+\mathrm{C}+\mathrm{Q})$

4. $(W+C+Q)$

5. Other by-reactions with oxygen containing molecule(s) as $\mathrm{H}_{2} \mathrm{O}$ and $\mathrm{CO}$

$(Q=H, D$, and $T)$ 
IV. Data needs:

Toyama University

1. Phenomenological:

1-1. plausible chemical reaction(s) under various temp. and energy

$\longrightarrow$ XPS, AES, SIMS, RS, XRD

1-2. changes in trap/release behavior and distributions of fuel particles before and after reactions

$\longrightarrow$ TDS, SIMS, RBS 


\section{Change in the Be1s spectrum of [C/Be]}

sample with elevated temperatures

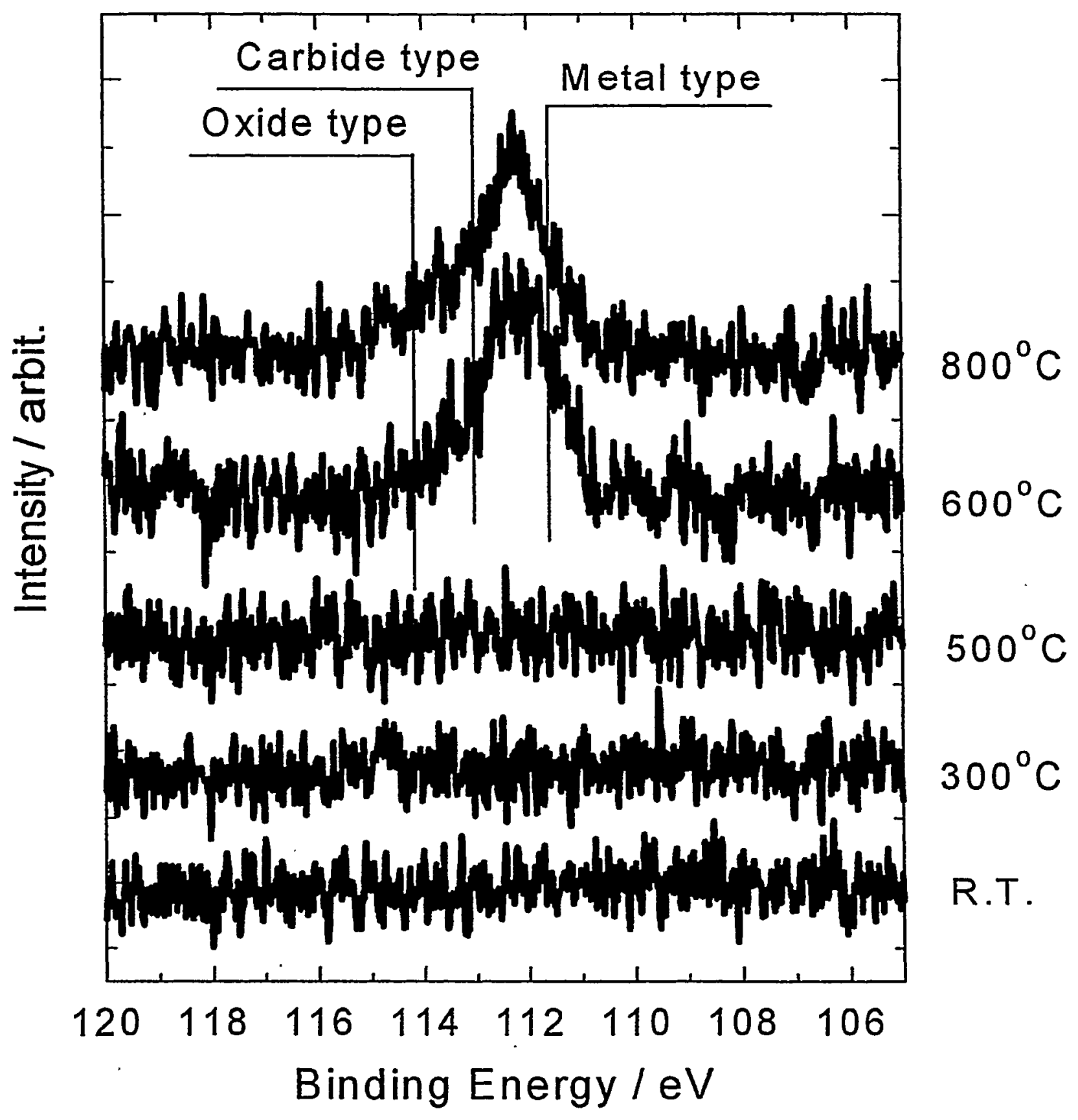


Change in the C1s spectrum of [C/Be] sample with elevated temperatures

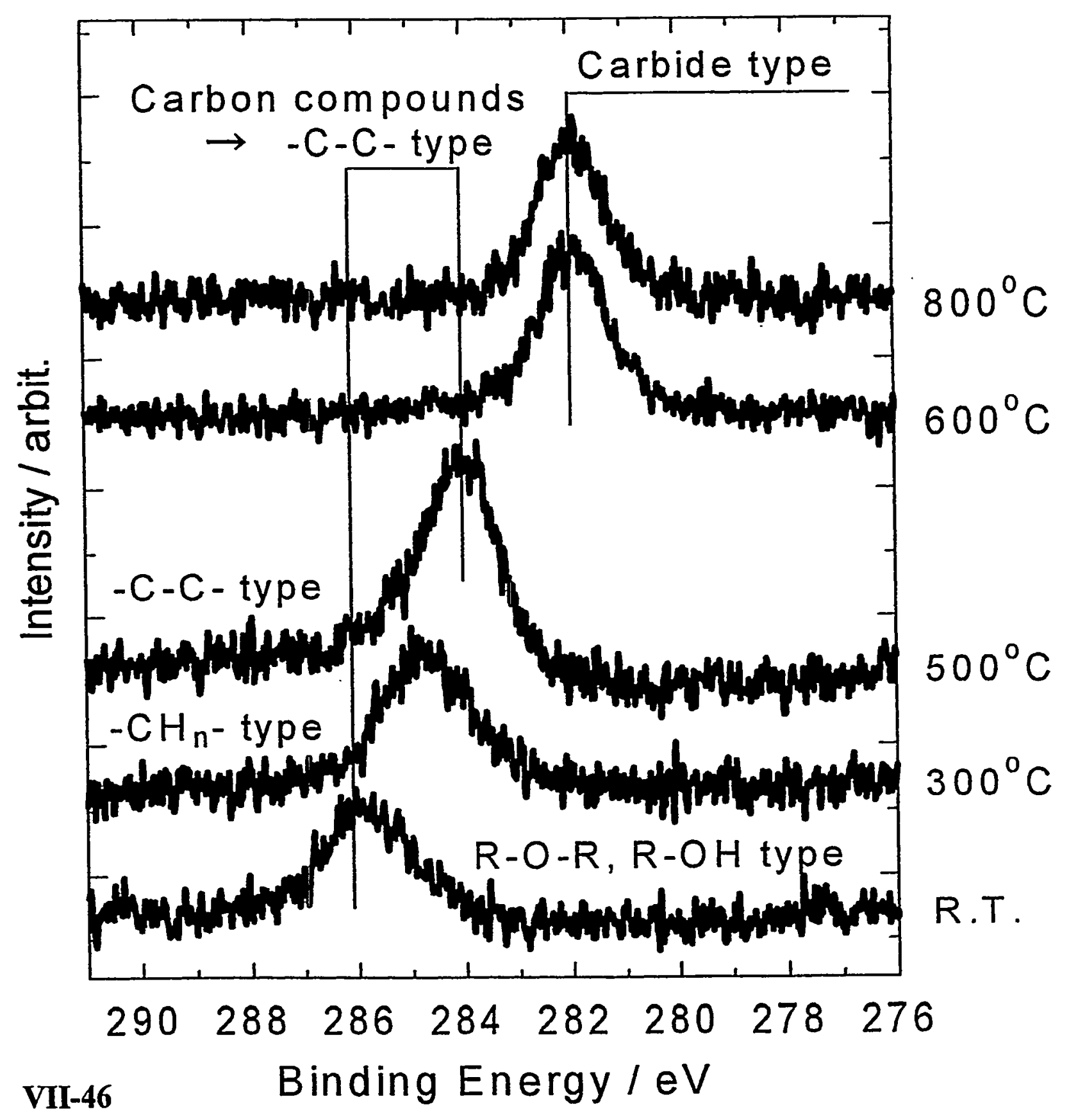




\section{Change in the 01s spectrum of [C/Be]}

\section{sample with elevated temperatures}

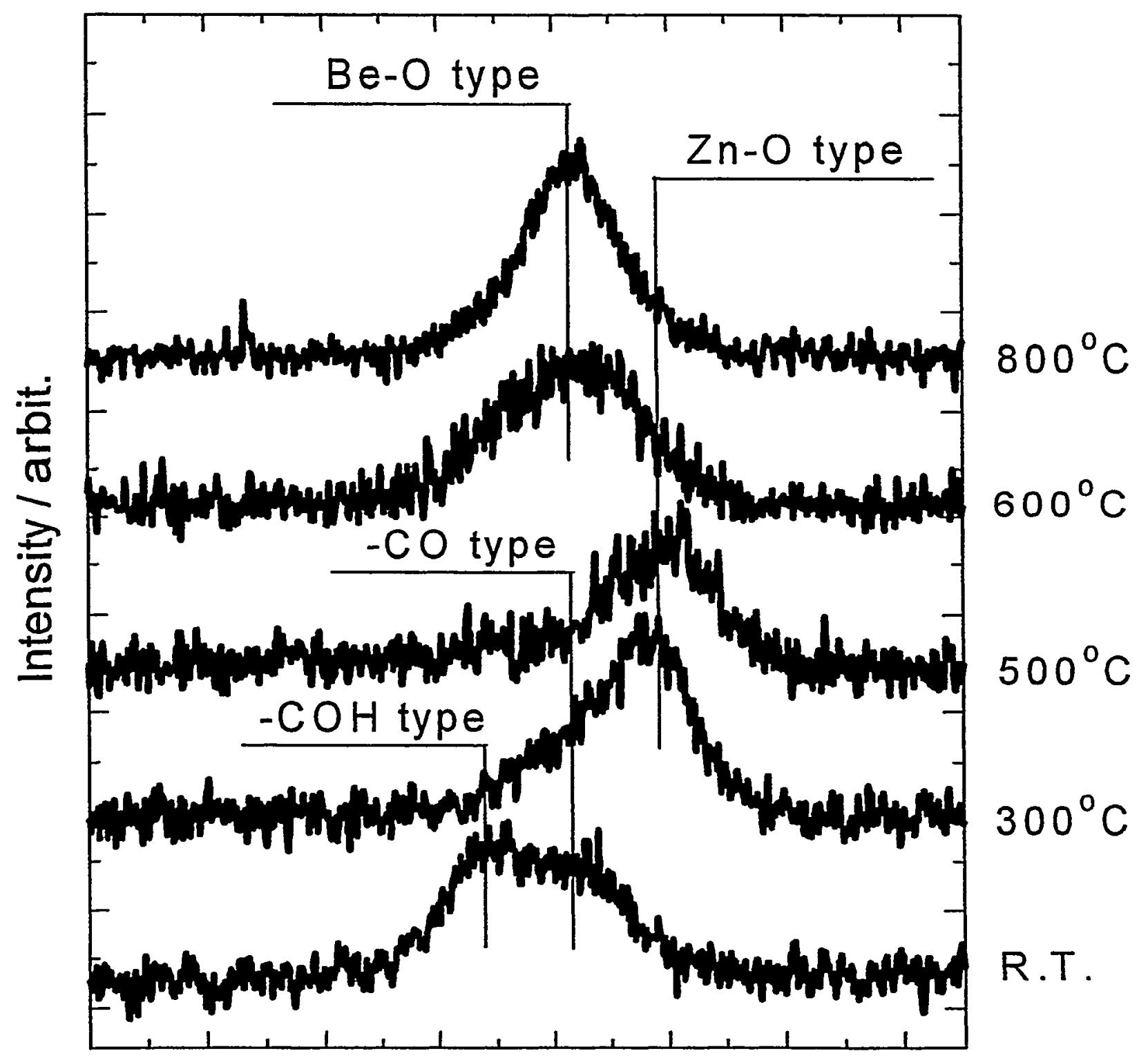

$\begin{array}{llllllll}540 & 538 & 536 & 534 & 532 & 530 & 528 & 526\end{array}$

Binding Energy / eV 


\section{Observed Be1s spectrum (solid line) and its three component peaks (dotted lines) of [C/Be] sample}

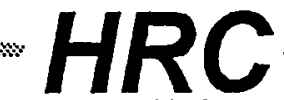

[oxide] : [carbide] : [metal] $=1: 5: 1([\mathrm{Be}]:[\mathrm{C}]:[\mathrm{O}]=65 \%: 23 \%: 10 \%)$ $[$ Be-oxide $]=65 \% \times(1 / 7)=9 \%$, $[$ Be-carbide $]=65 \% \times(5 / 7)=46 \%$ $[\mathrm{Be}]:[\mathrm{O}]=1: 1(\mathrm{BeO}),[\mathrm{Be}]:[\mathrm{C}]=2: 1\left(\mathrm{Be}_{2} \mathrm{C}\right)$

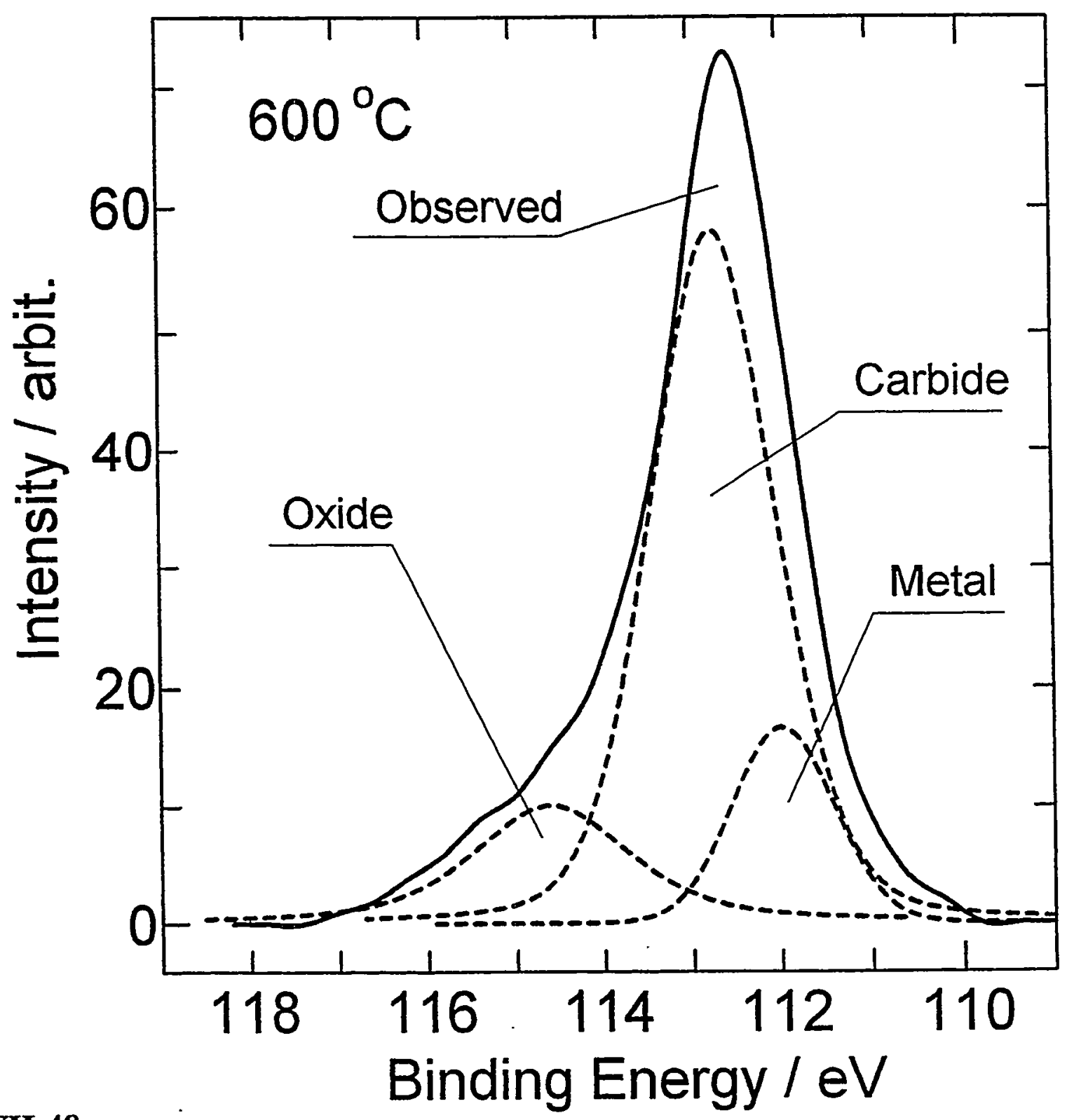


Positive SIMS spectrum of $[\mathrm{C} / \mathrm{Be}]$ sample with $\left(\mathrm{Ar}+\mathrm{D}_{2}\right)$ mixed gas as primary ion source

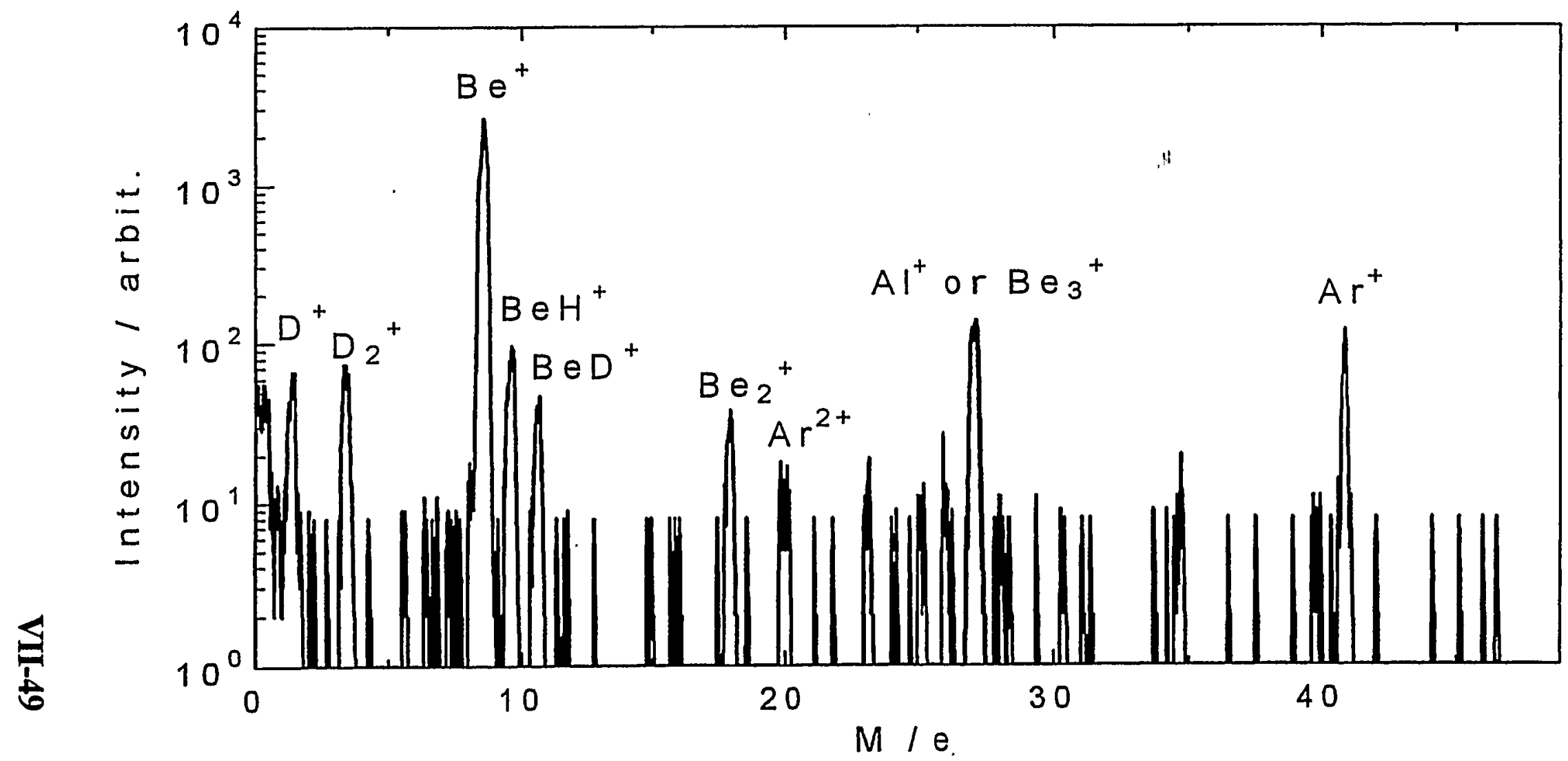




$$
\text { 矛 }
$$

Negative SIMS spectrum of [C/Be] sample with $\left(A r+D_{2}\right)$ mixed gas as primary ion source

$\underset{\text { toyama University }}{H R}$

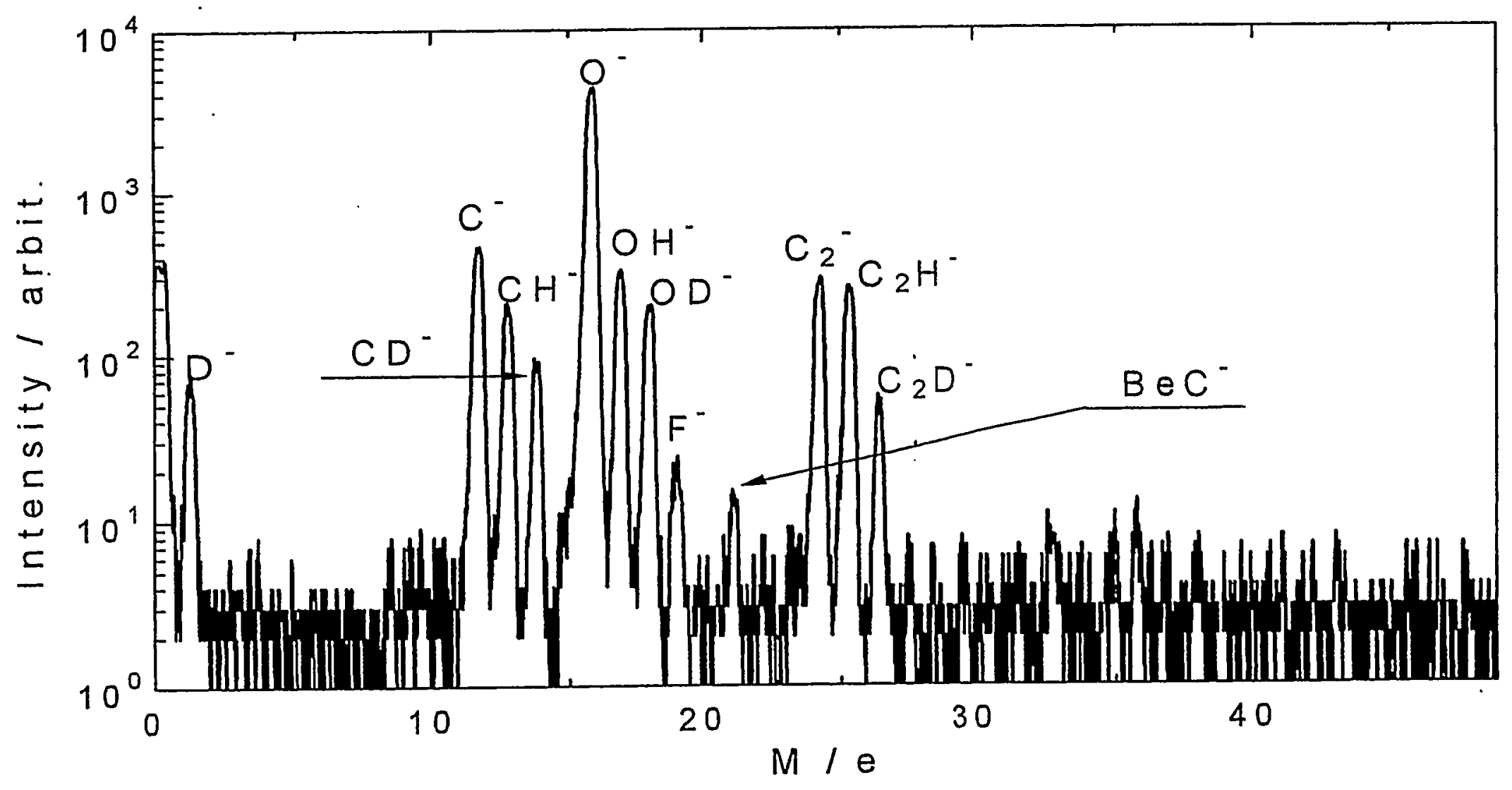




\section{Raman spectra for a hydrogen containing carbon deposits before and after vacuum heating at $800^{\circ} \mathrm{C}$ for $10 \mathrm{~min}$}

.

$H R C$

Toyama University

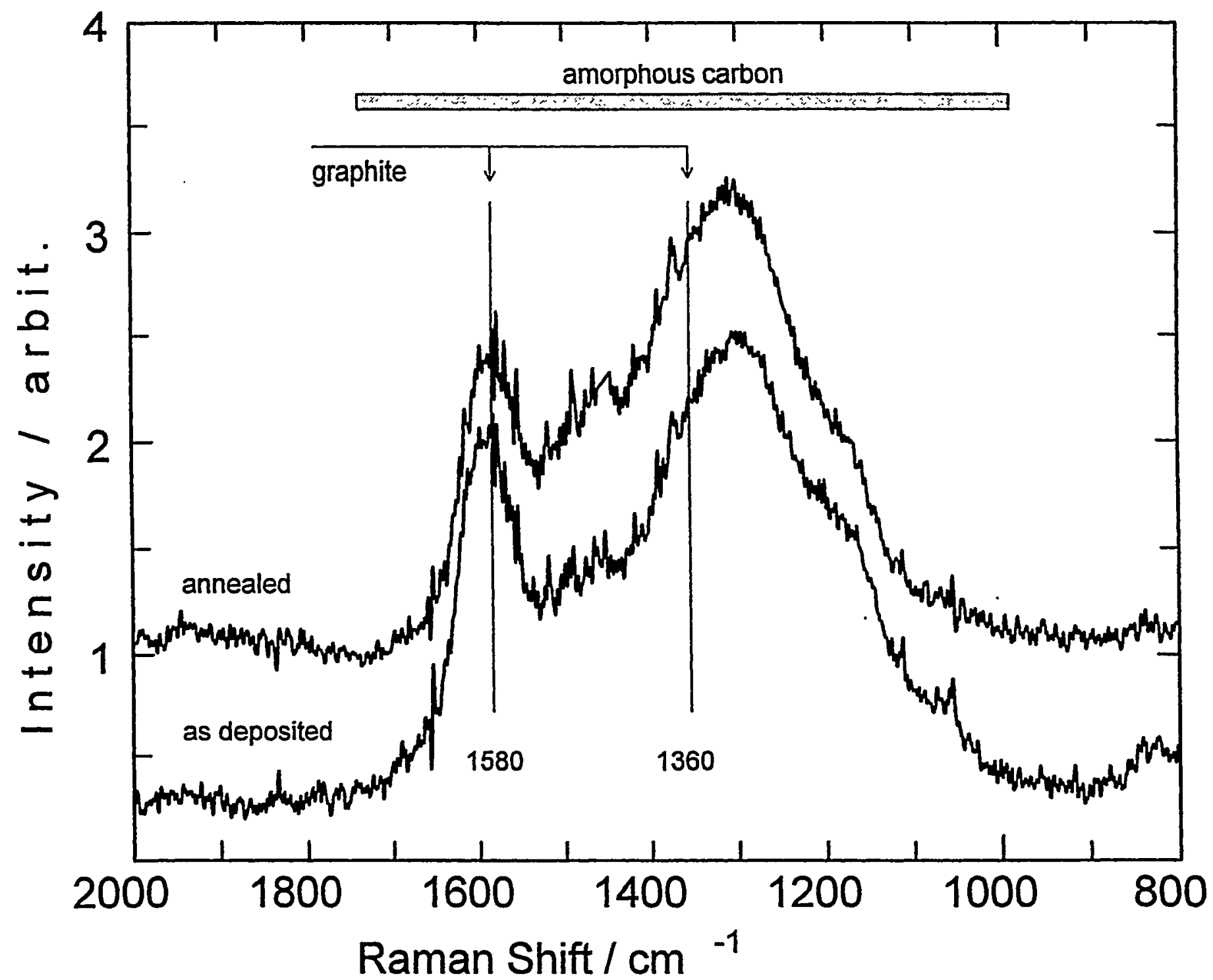




\section{TDS spectra of main desorption gases from Quartz plate covered with hydrogen containing carbon film}

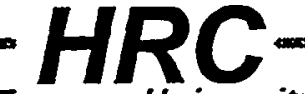

Toyama University

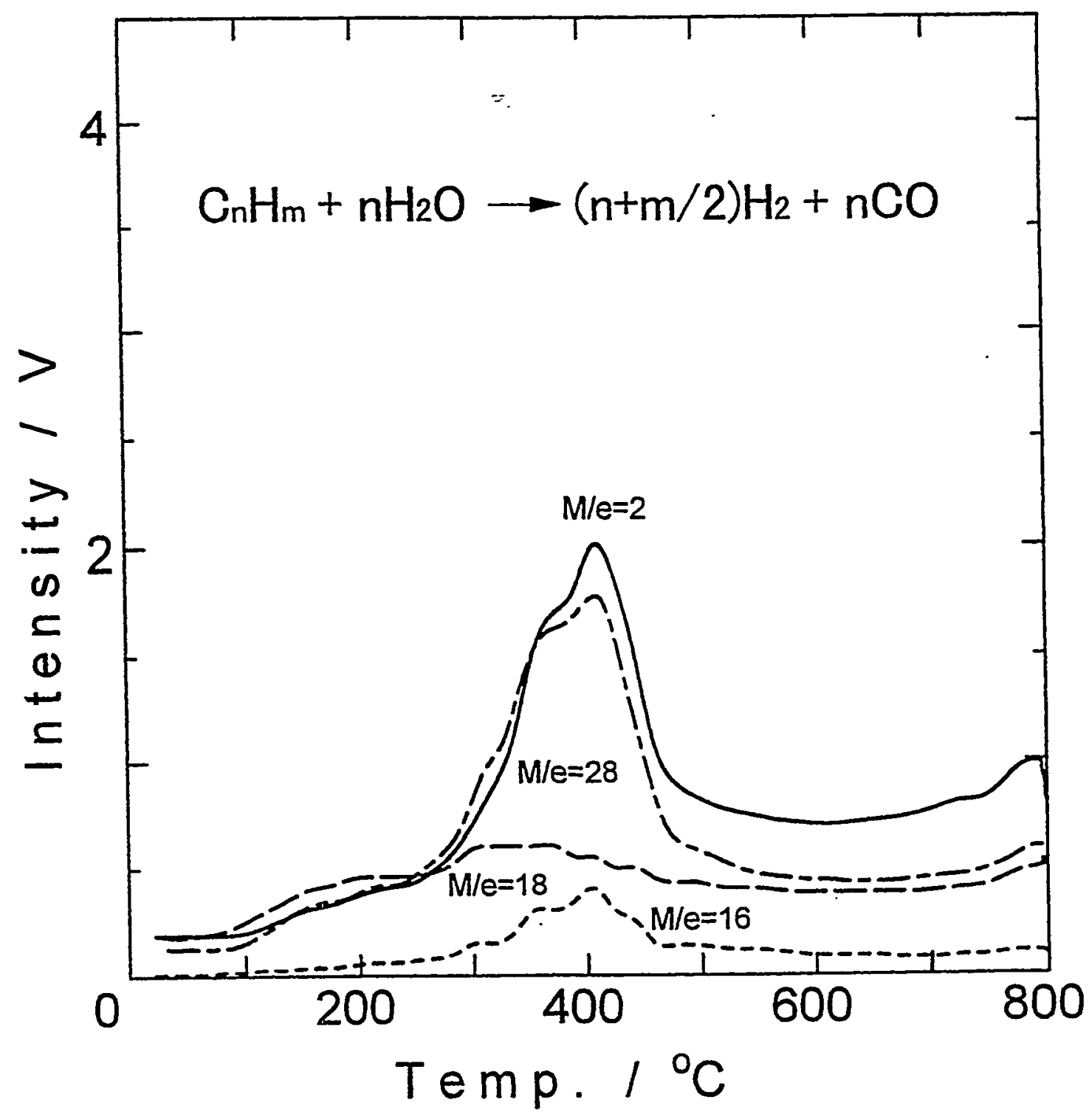

VII-52 


\section{TDS spectra of main desorption gases from Be plate covered with hydrogen containing carbon film}

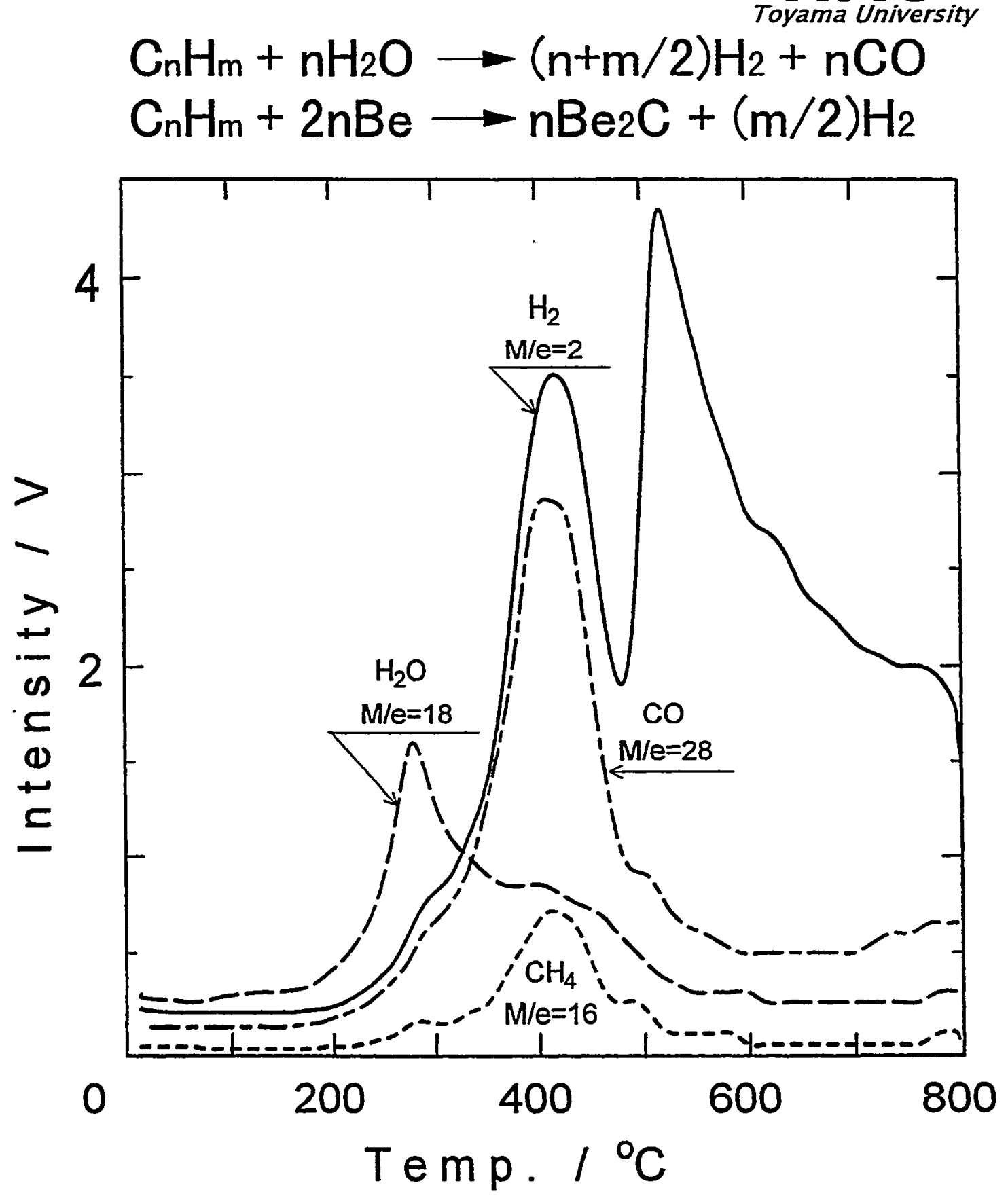




\section{Variation of XRD patterns for the $[\mathrm{C}(\mathrm{H}) / \mathrm{Be}]$ sample with vacuum heating for $10 \mathrm{~min}$ at given temperatures}

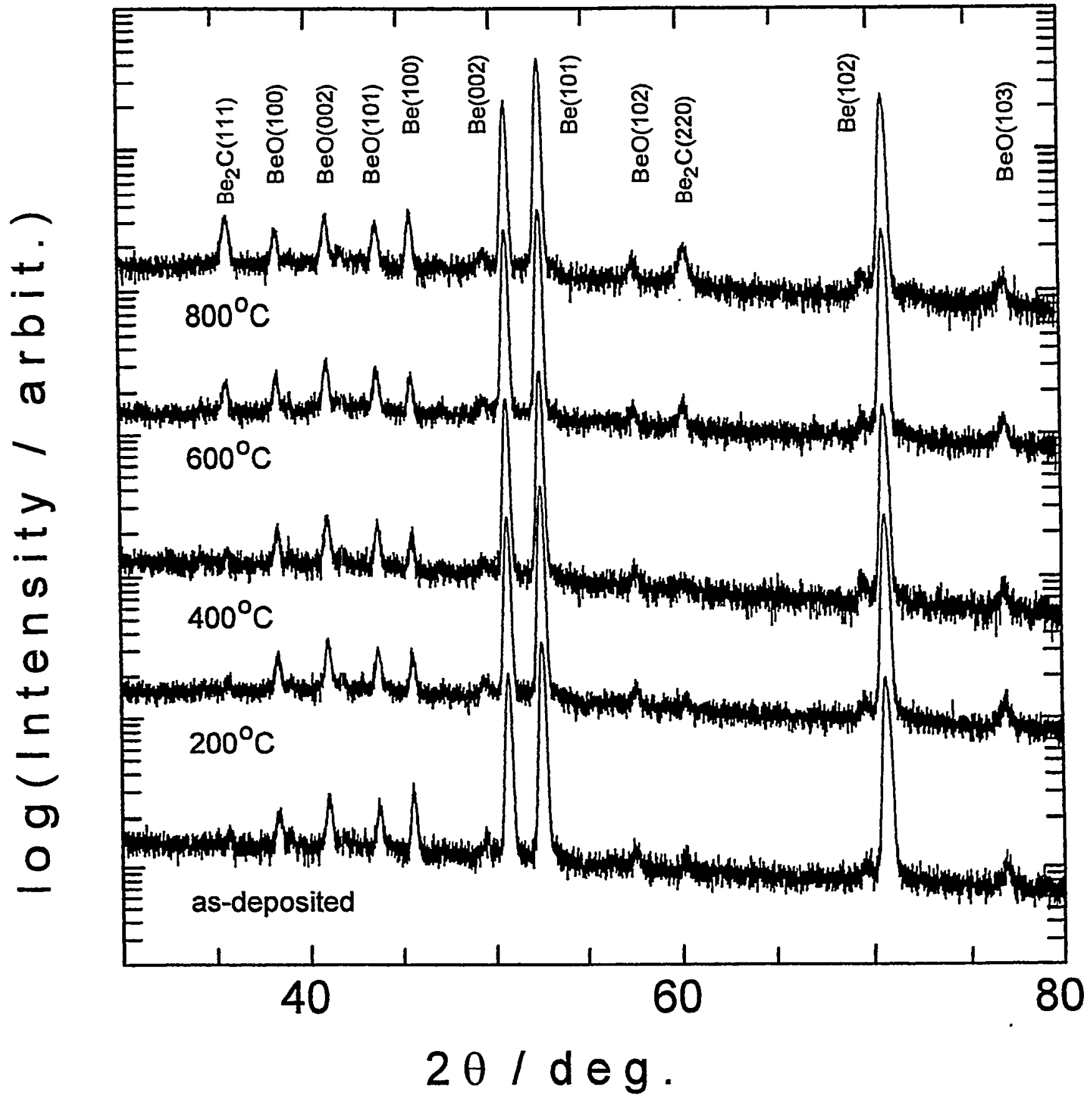




\section{Change in the relative intensities of Be2C(111) peak with vacuum heating at given temperatures}

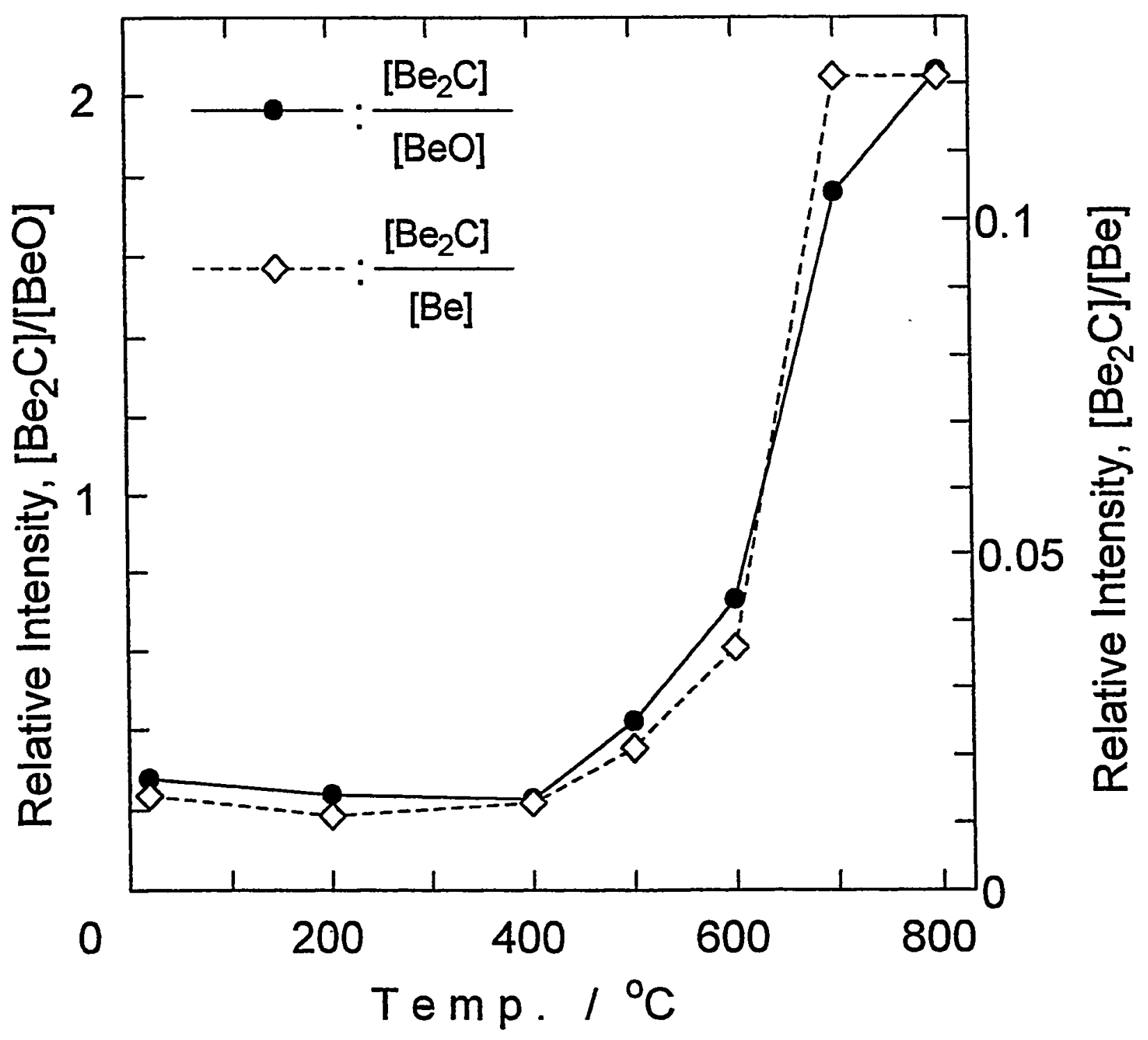




\section{Change in the XRD patterns for the $[C(H) / B e]$ sample with given heating time at $700^{\circ} \mathrm{C}$}

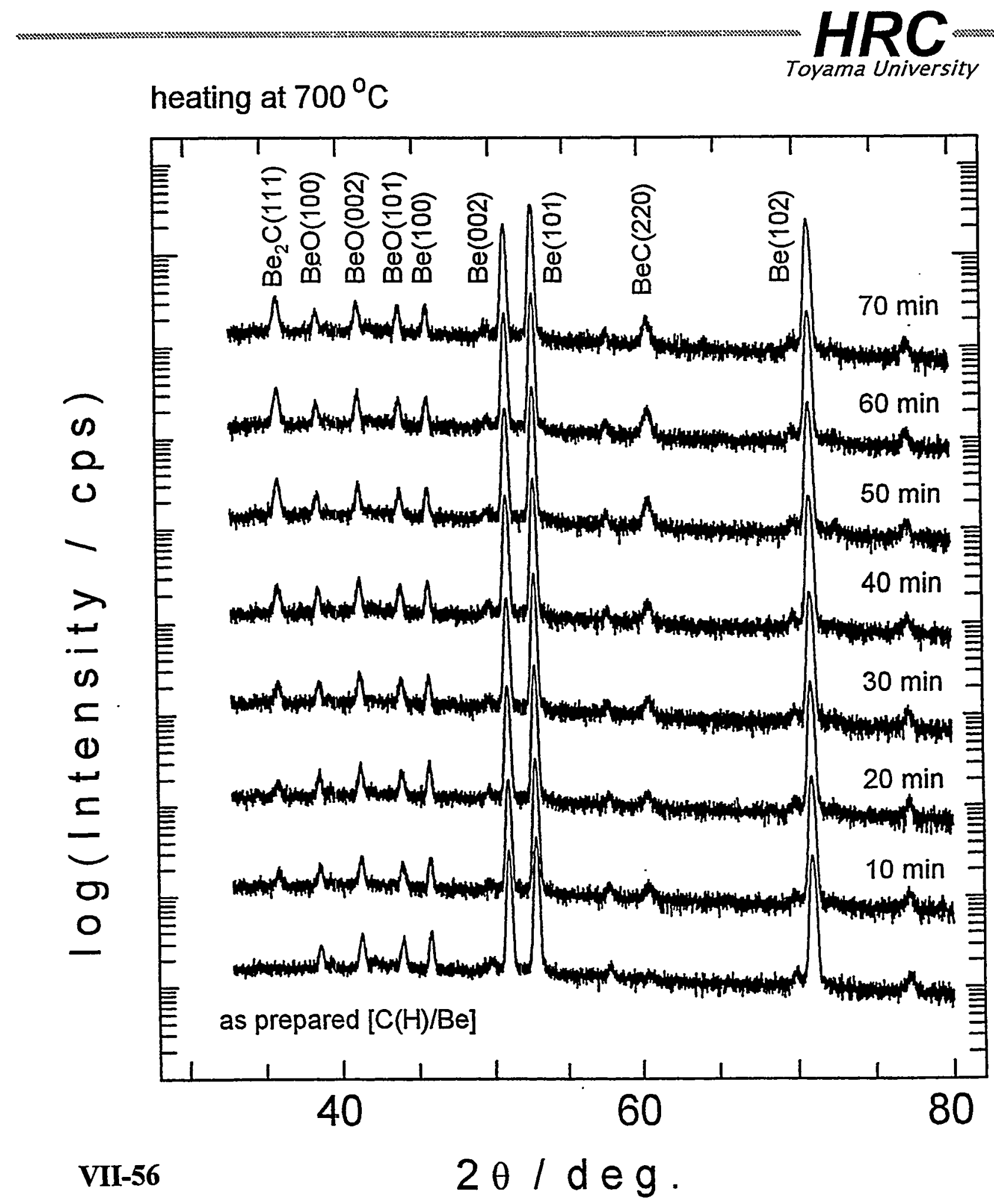




\section{Change in the relative intensities of $\mathrm{Be}_{2} \mathrm{C}(111)$ peak normalized by $\mathrm{Be}(101)$ with time}

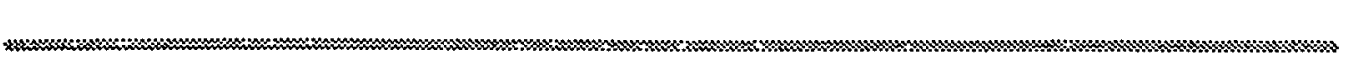

$H R C$

Toyama University

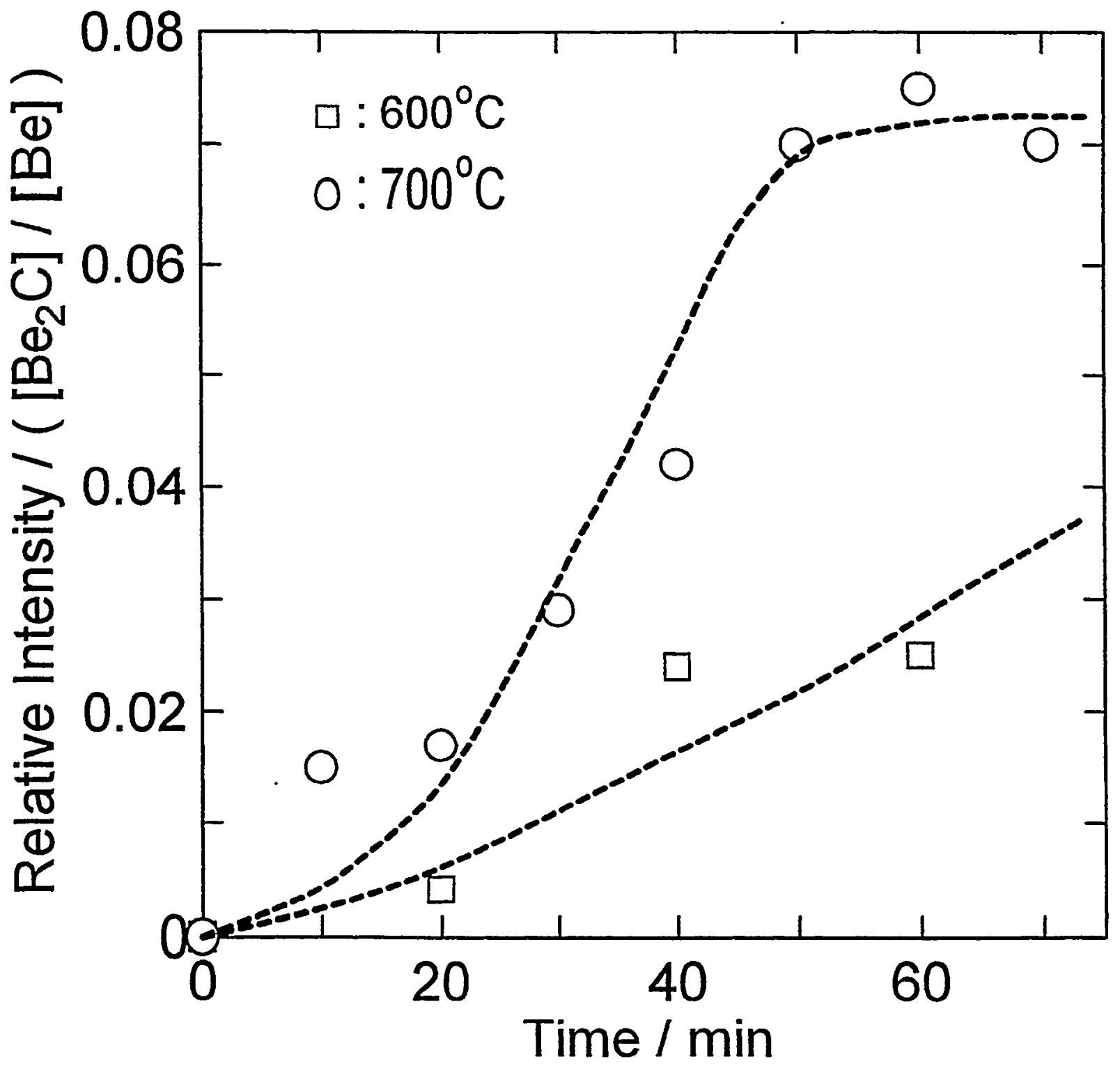




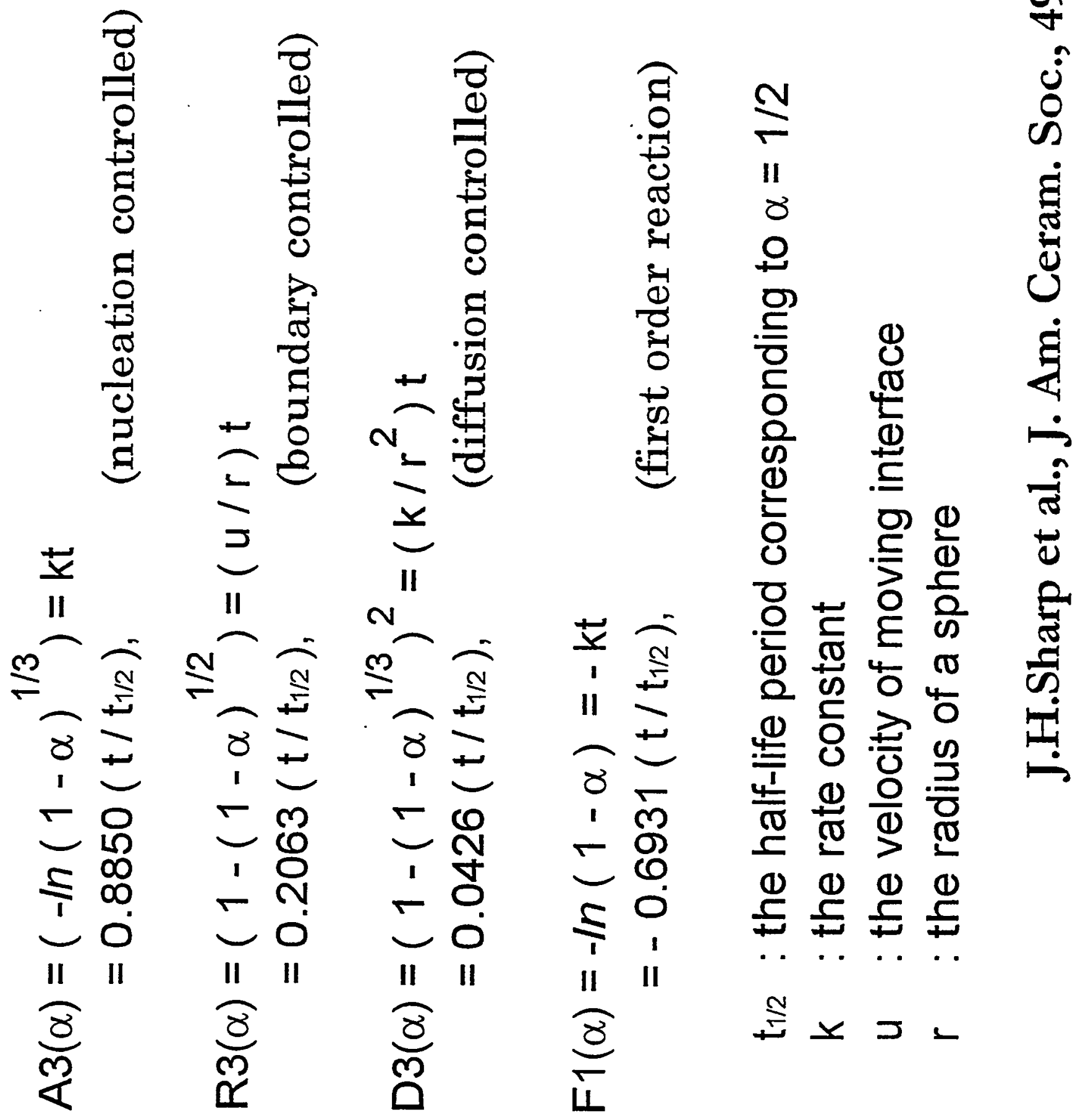


Plots of various crystal growth model against relative time scale with observed data

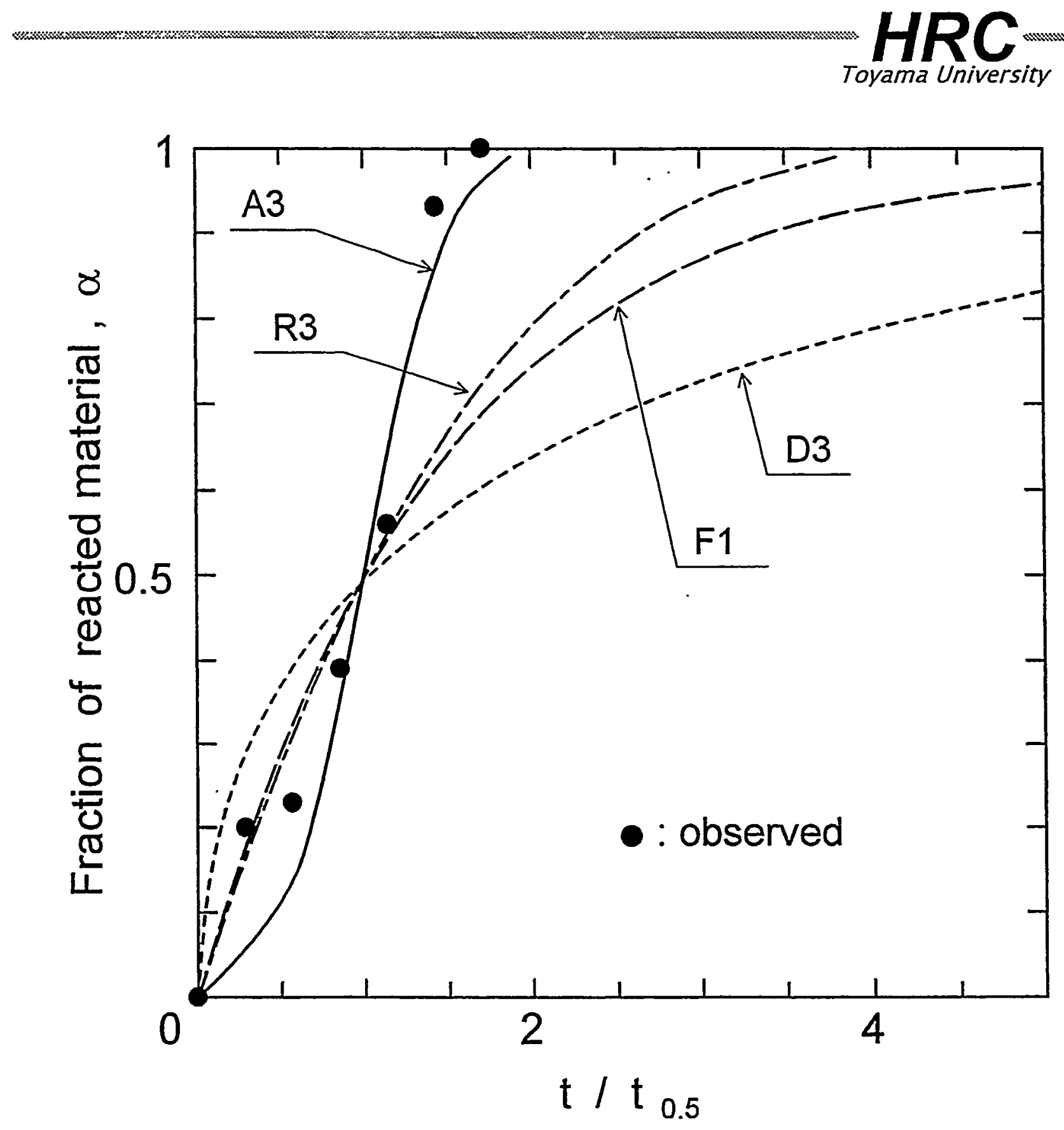

VII-59 


\section{Formation of $\mathrm{Be}_{2} \mathrm{C}$ in the $\mathrm{C}-\mathrm{Be}$ binary system by elevated temperature}

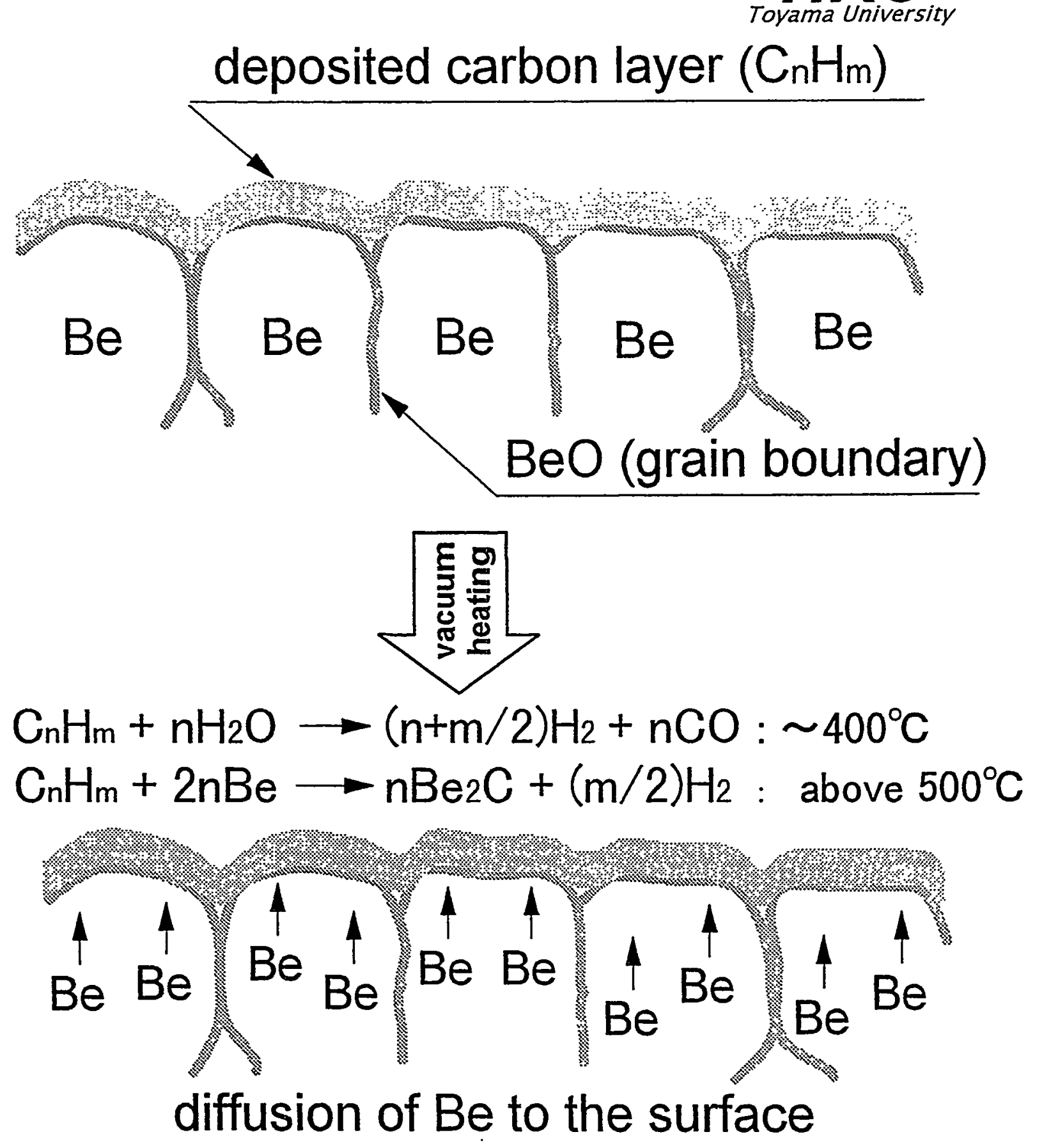

VII-60 


\section{Summary:}

- $\operatorname{HRy}_{\text {Toya University }}$

1. Formation of Be-oxide, $\mathrm{BeO}$, is unavoidable because Be has high affinity to oxygen and/or oxygen containing molecules such as $\mathrm{H}_{2} \mathrm{O}$.

2. Formation of $\mathrm{Be}$-carbide, $\mathrm{Be}_{2} \mathrm{C}$, takes place above $600^{\circ} \mathrm{C}$ for Be-C binary system.

3. Rate of $\mathrm{Be}_{2} \mathrm{C}$ formation reaction is limited by Random Nucleation mechanism.

4. Carbon atoms lose its ability to capture hydrogen (or deuterium) to release them to gas phase when it forms carbide.

5. Hydrogen isotope atoms are captured by $\mathrm{Be}$, $C$ and $O$ in the form of $B e-Q, C-Q$ and $O-Q$. $(Q=H$ and $D)$ 


\title{
Tritium Retention in Beryllium
}

\author{
Rion A. Causey \\ Sandia National Laboratories \\ Livermore, Ca 94550
}

US/Japan Workshop

San Francisco, Ca

December 8-10, 1997 


\section{Outline}

Beryllium Experiments

- High Flux Retention Measurements

- Low Flux Retention Measurements

- Codeposition Measurements

$=$

Tungsten Experiments

- Tungsten with $1 \%$ Lanthanum Oxide 
The Tritium Plasma Experiment (TPE) is a Unique Facility Devoted to Plasma-Materials Interaction Studies for the US DOE Magnetic Fusion Energy Program:

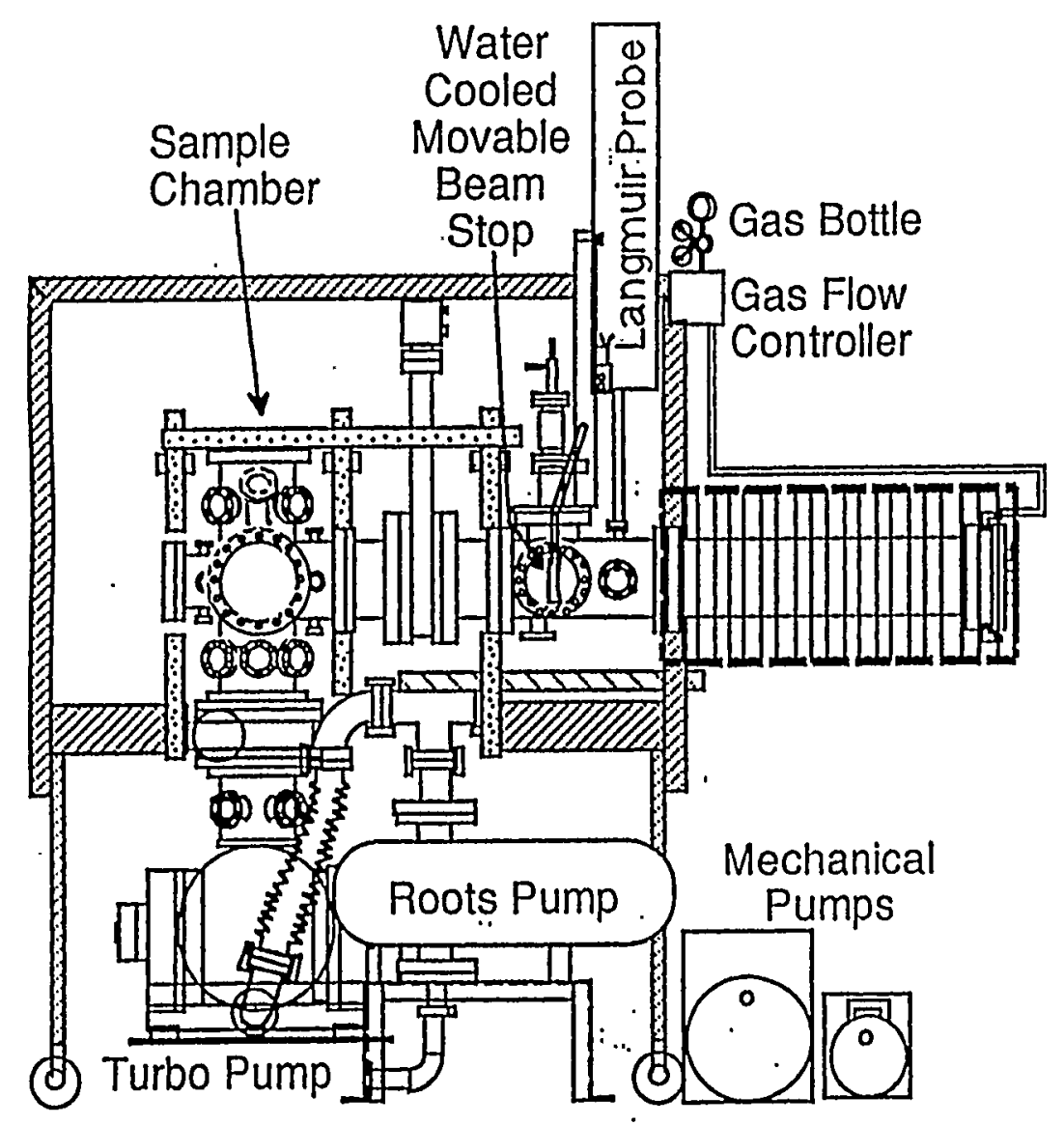

The Tritium Plasma Experiment is now located at the TSTA Facility in Los Alamos National Laboratory

This experimental facility is capable of delivering $1 \mathrm{~A} / \mathrm{sq} . \mathrm{cm}$ of $100 \mathrm{eV}$ tritons uniformly over a 2 inch diameter sample. The tritium inventory of this experiment is greater than 6 grams.

TPE is being used in experiments to determine the tritium migration parameters for materials (Be, $C$, and $W$ ) to be used in the ITER fusion reactor.

\section{Sandia National Laboratories}




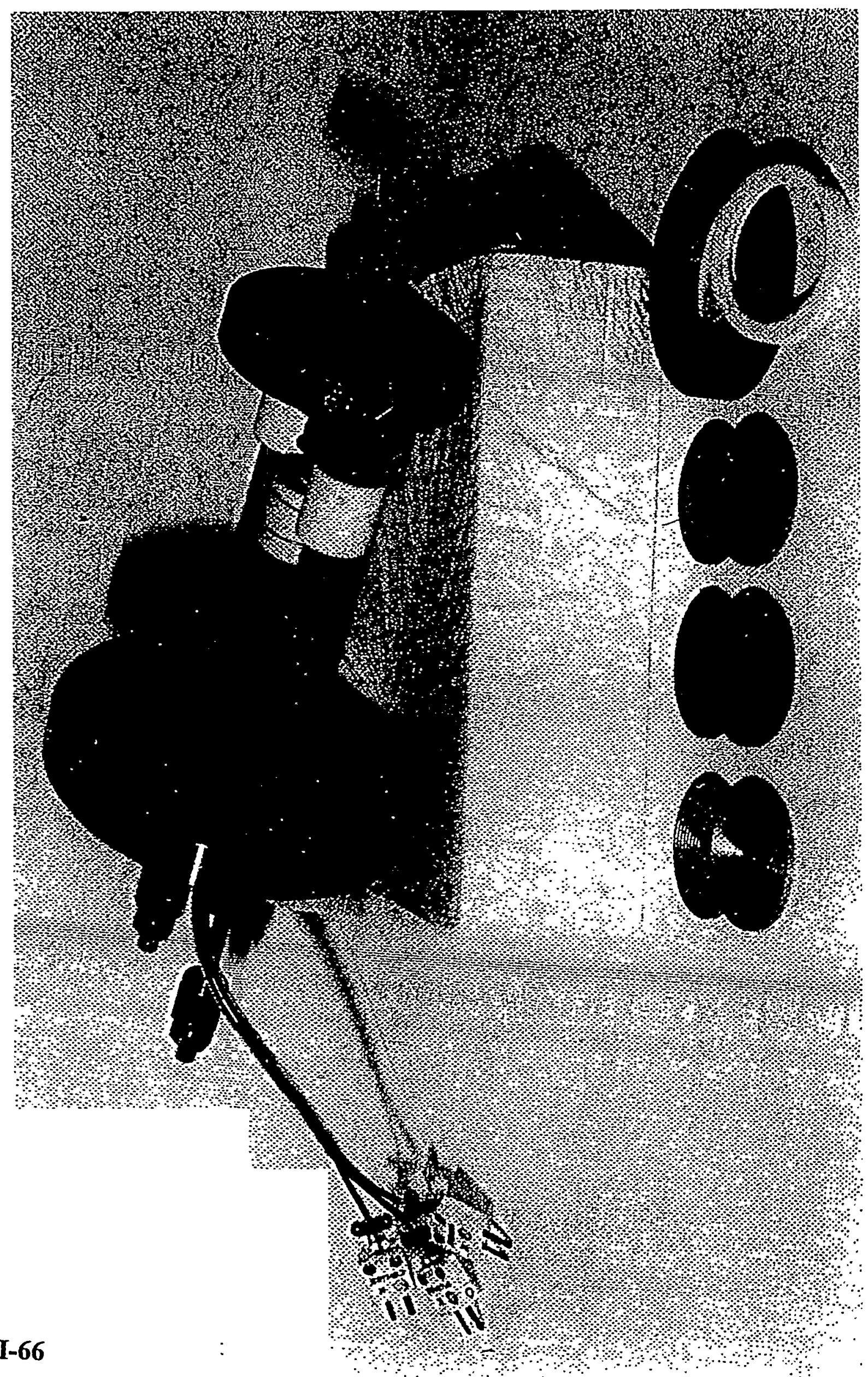




\section{Experimental Procedures}

\section{Material}

The beryllium used in this study was Brush Wellman S-65. It is $99.4 \% \mathrm{Be}$ and $0.6 \% \mathrm{BeO}$. It is hot pressed, and has $99.8 \%$ of theoretical density.

\section{Procedures .}

1. Sample was loaded into the TPE sample holder. Holder was installed and vacuum was established.

2. Plasma was started using pure deuterium. Once impurities generated by the initiation of the plasma were removed by the vacuum system, the bias was applied to the sample and the plasma intensity was increased until the desired sample temperature was obtained. Bias elevated the energy of the deuterons and tritons to $100 \mathrm{eV}$. Once the desired temperature was obtained, the tritium was added to the plasma gas. The plasma was maintained for one hour from the time the tritium was added.

3. After the plasma exposure, the sample was remove from TPE and transported to the outgassing system. Here the sample was linearly increased in temperature up to $800 \mathrm{C}$. During this time, gas consisting of $99 \%$ helium and $1 \%$ hydrogen was swept across the sample at a flow rate of $100 \mathrm{cc} / \mathrm{min}$. This gas was first sent through and ionization chamber. After exiting the ionization chamber, $10 \%$ oxygen was added before the gas was sent through a copper oxide bed. This converted the hydrogen and tritium to water for collection in the subsequent glycol bubblers. Liquid scintillation counting of the tritium in the water/glycol was used as a check on the data obtained by the ionization chamber. 


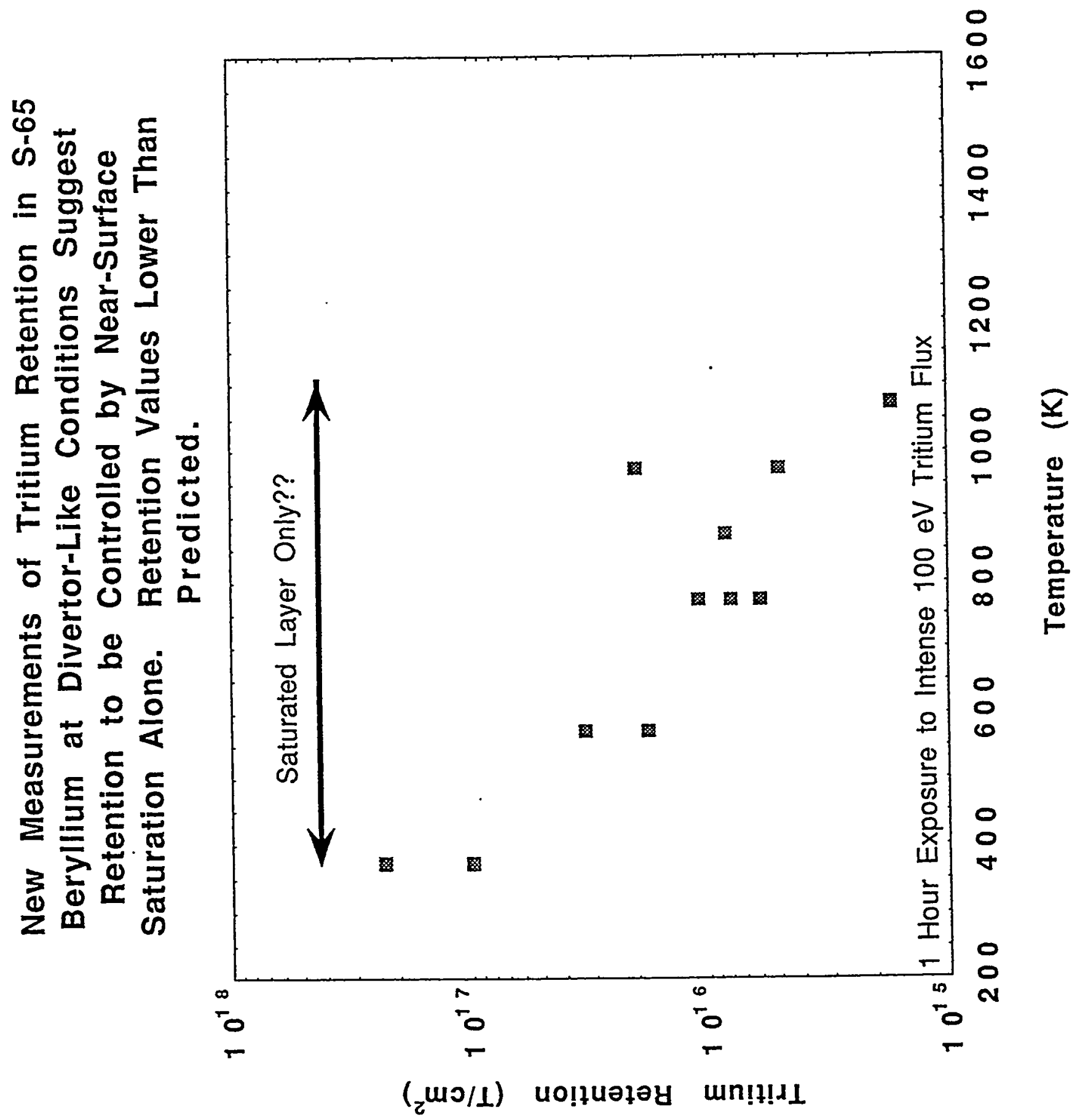


Comparison of TPE Beryllium Data to that Predicted by Assuming $\mathrm{C}=0$ at Boundary

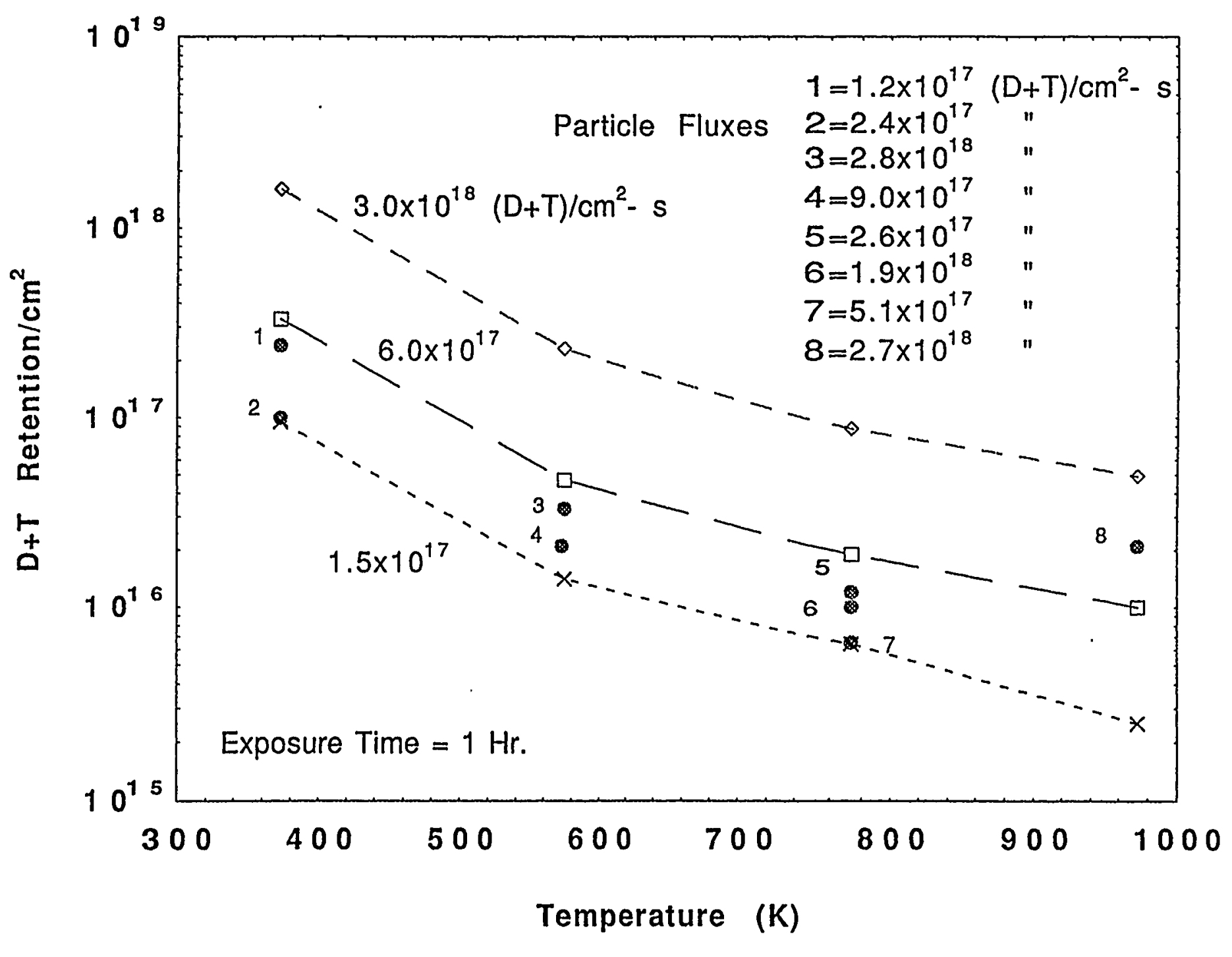


Earlier work by Chernikov et al. has shown hydrogen implantation into beryllium to open porosity in the implant zone. This would result in relatively rapid saturation in the hydrogen retention with longer or more intense exposure having no effect on the amount of hydrogen retained.

V.N. Chernikov, V.Kh. Alimov, A.V. Markin, and A.P. Zakharov, J. Nucl. Mater. 220222 (1996) 47.
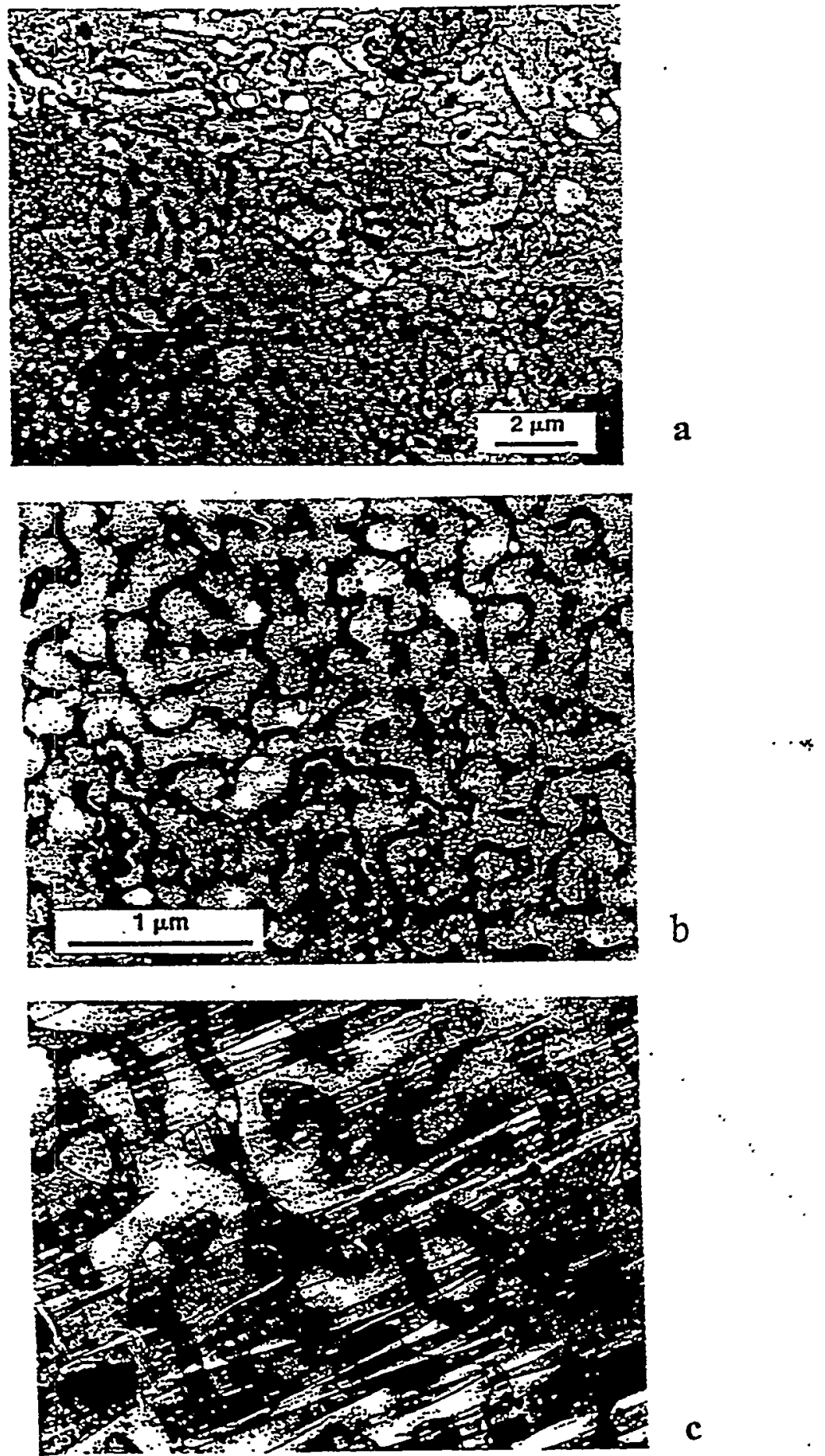

c

Figure 9. Deuterium bubbles and labyrinths of oblate interconnected channels after irradiation VII-70 $700 \mathrm{~K}$ with $10 \mathrm{keV} \mathrm{D}$ ions up to $2 \times 10^{21} \mathrm{D} / \mathrm{m}^{2}:$ a) viewing field covers some grains, low magnification; b) surface plane of the grain is close to $(0001)$; c) surface plane of the grain is nearly parallel to $c$ axis $\left(\mathrm{t}_{\zeta} \approx 450 \mathrm{~nm}\right)$. 


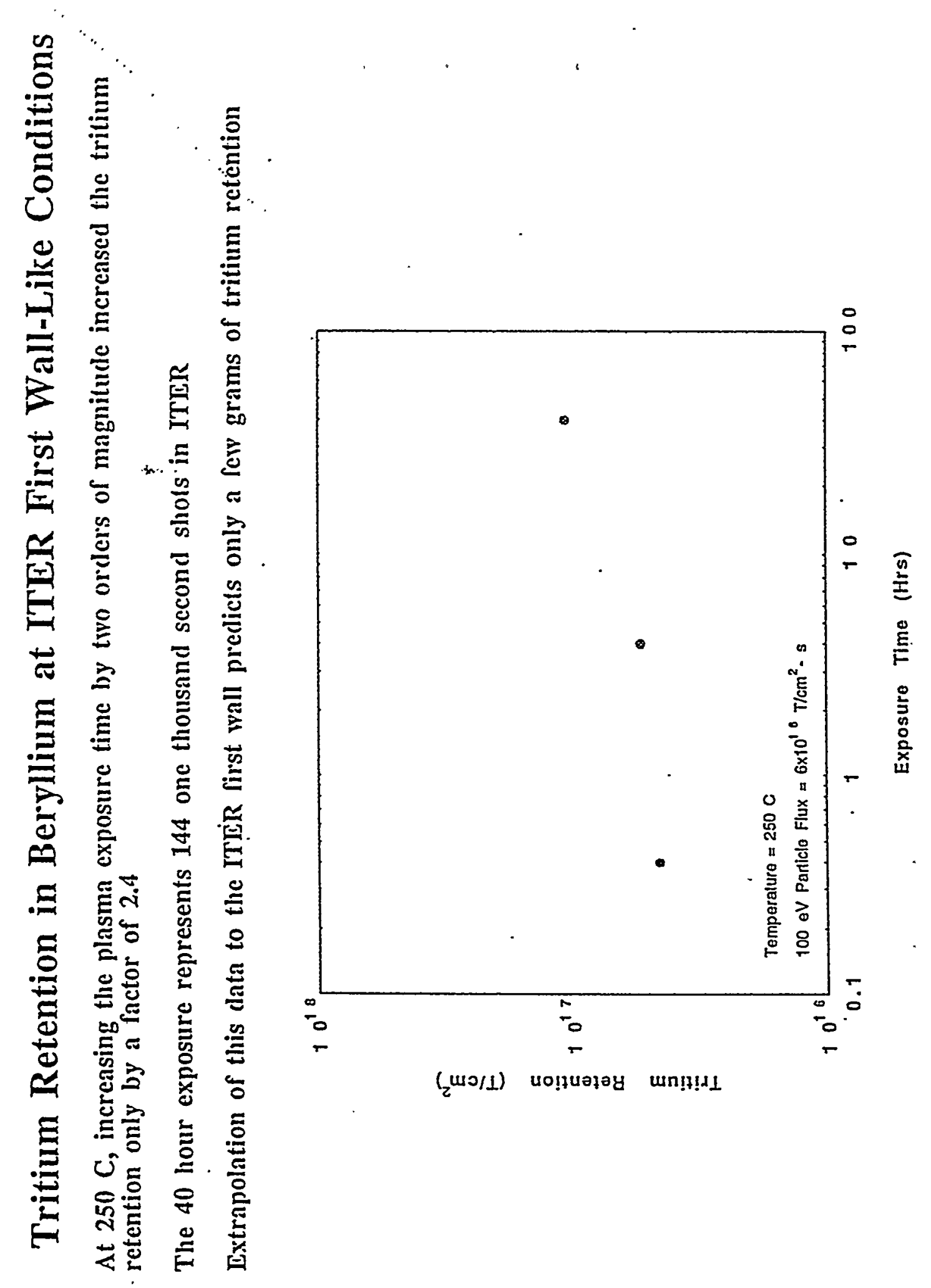

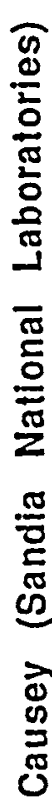




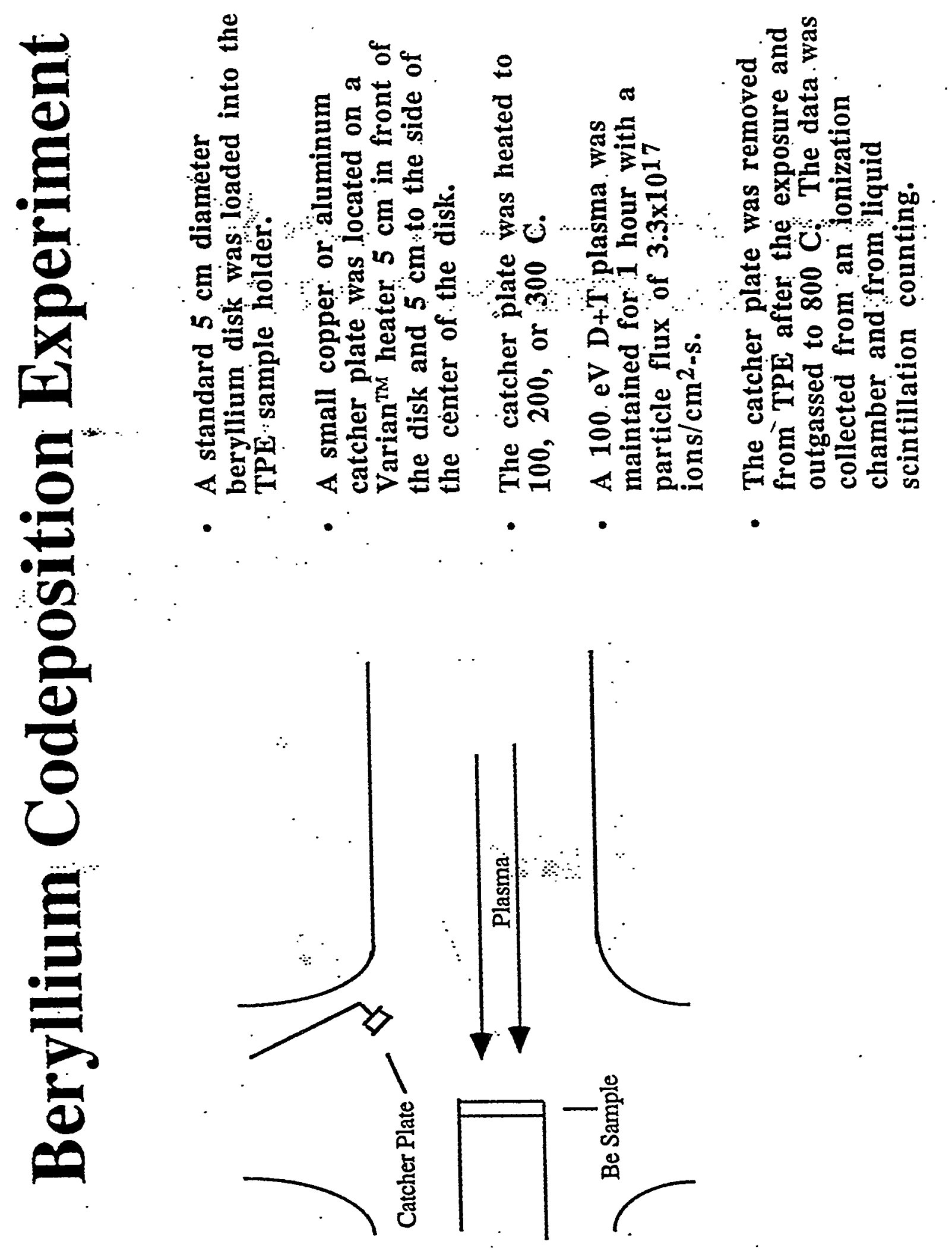




\section{Beryllium Codeposition Experiments}

- The beryllium/deuterium codeposition experiments have been repeated in the Tritium Plasma Experiment

- In these recent experiments, the amount of oxygen in the plasma was significantly reduced from that existing in the earlier experiments

- The samples were analyzed by Dave Walsh (SNL/NM) using accelerator techniques

\section{Sample Summary}

Sample Temp

$100 \mathrm{C}$

$200 \mathrm{C}$

$300 \mathrm{C}$

$150 \mathrm{C}$
Thickness

$1200 \AA$

$1200 \AA$

$1500 \AA$

$3200 \AA$
$\underline{\mathrm{Q} / \mathrm{Be}}$

0.125

0.125

0.06

0.03
$\underline{\mathrm{D} / \mathrm{Be}}$

0.15

$\mathbf{0 . 0 7}$

0.02

0.10

The carbon content of all samples was below $1.5 \%$ 


\section{Codeposition of Hydrogen Isotopes and Beryllium.}

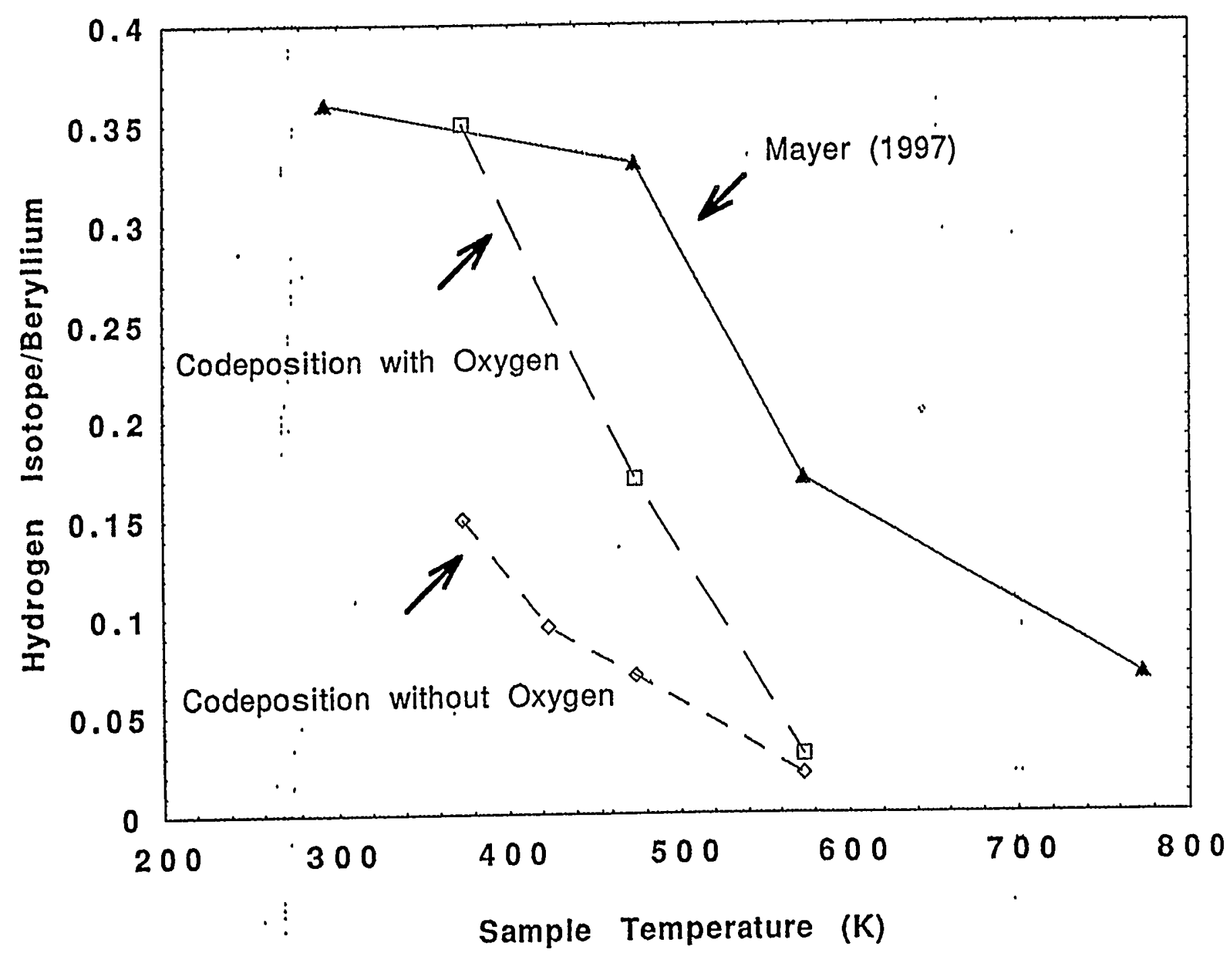



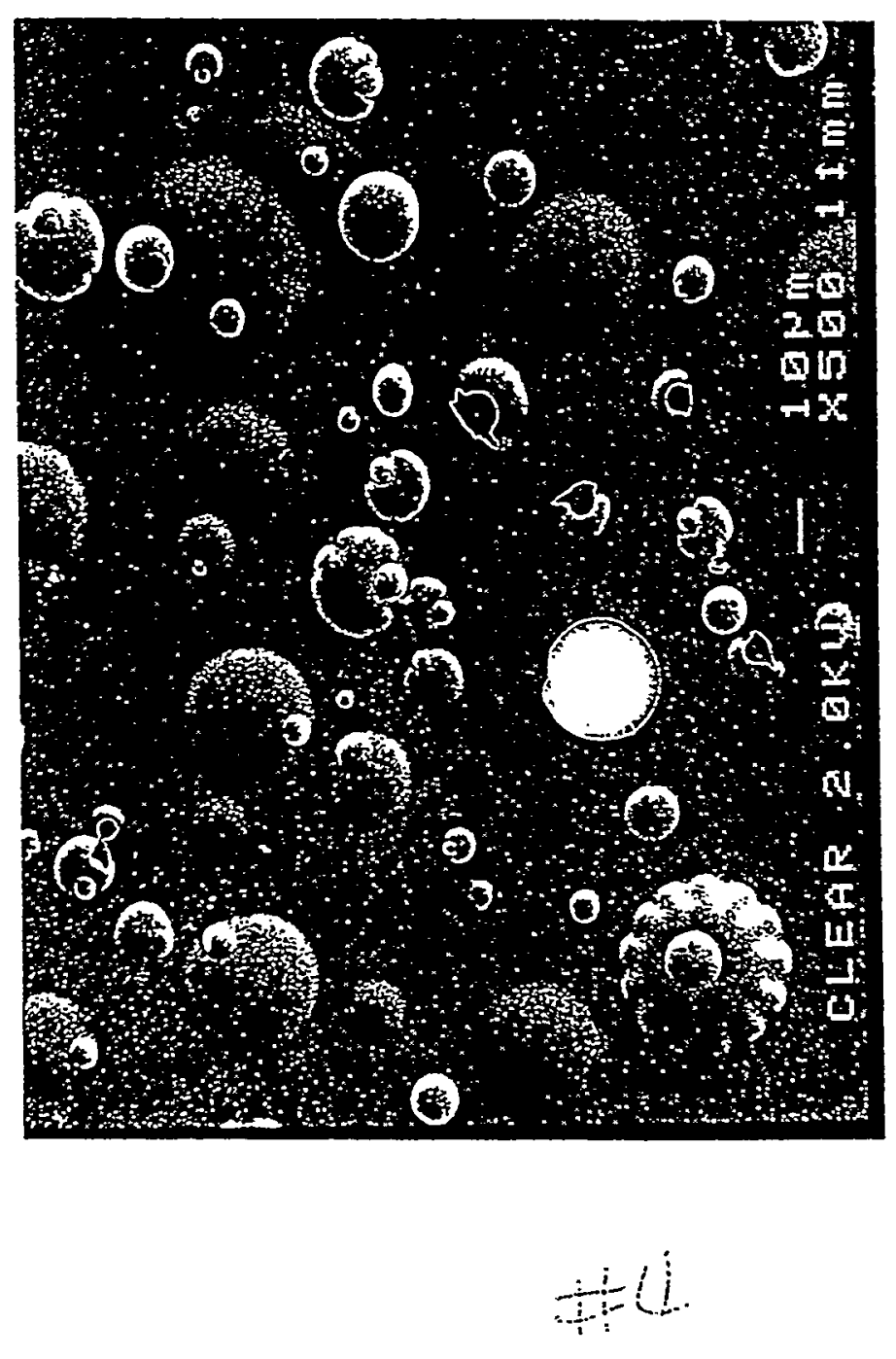

VII-75 


\section{Beryllium Results}

\section{Implications for ITER}

- If used as either a divertor or first wall material, the tritium retention of S-65 beryllium due to implantation should be small. The effects of neutron damage on the retention are unknown, but suspected to be minimal [work planned for next year].

- The codeposition of beryllium with tritium has been found to depend on the availability of oxygen. Even with oxygen present, the tritium inventory in sputtered beryllium will be below that of carbon (lower sputtering coefficient and lower dissociation temperature). 
Session VIII: First Wall Development

VIII-1 


\section{[page intentionally left blank]}

VIII-2 

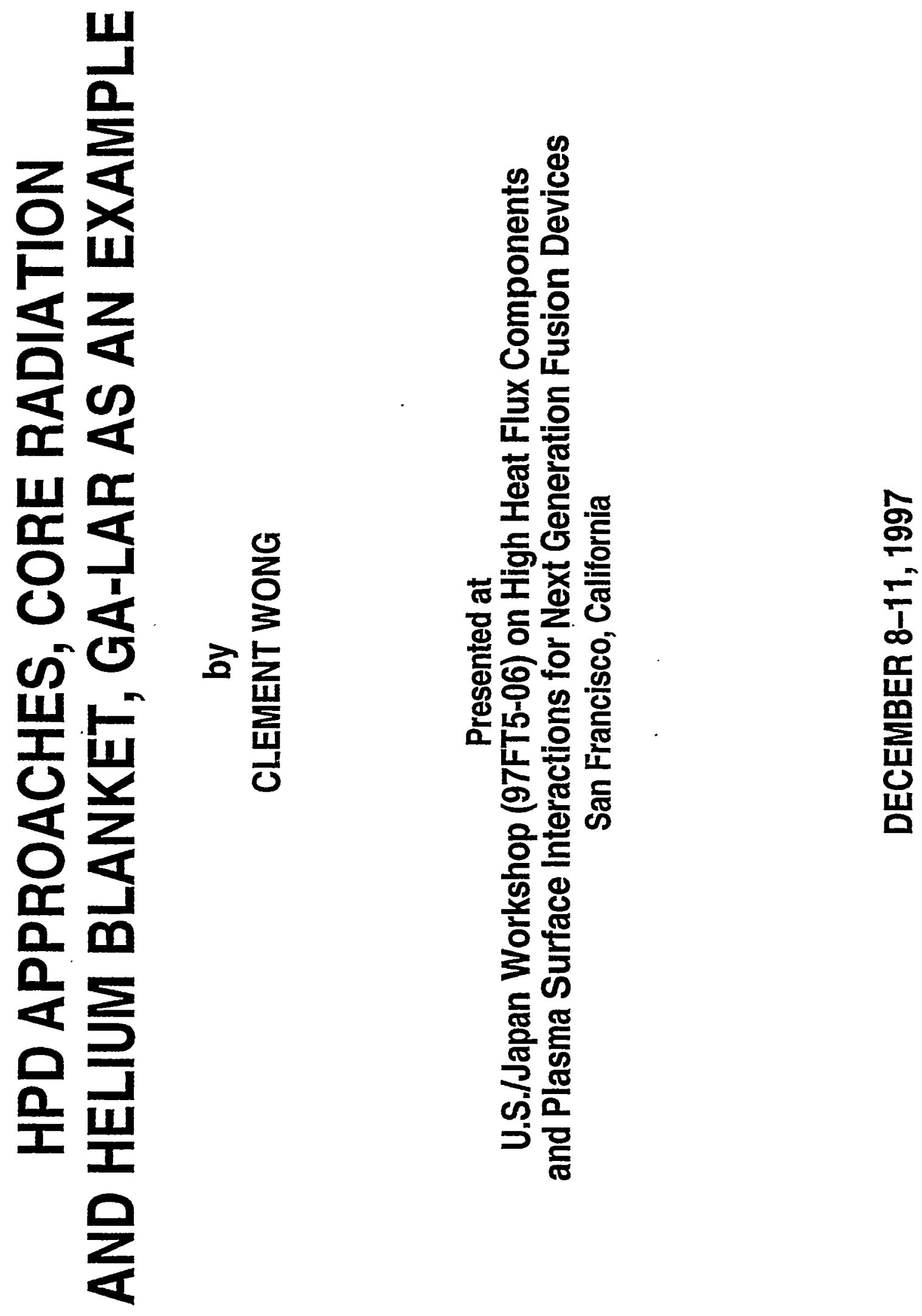


\section{WHY HPD? \\ (DESIGN AND ECONOMIC IMPLICATIONS)}

- A goal for economic fusion power is to compete with advanced energy sources*

- ARIES-RS ${ }^{\dagger}$ shows:

$$
\frac{\text { Reactor plant equipment cost }}{\text { Plant direct cost }}=\frac{\$ 1.4 \mathrm{~B}}{\$ 2.2 \mathrm{~B}}=0.64 \Rightarrow \mathrm{COE}=76 \mathrm{mill} / \mathrm{kWhr}
$$

- GA-LAR ${ }^{\ddagger}$ shows:

$$
\frac{\text { Reactor plant equipment cost }}{\text { Plant direct cost }}=\frac{\$ 1.1 \mathrm{~B}}{\$ 2.8 \mathrm{~B}}=0.4 \Rightarrow \mathrm{COE}=53 \mathrm{mill} / \mathrm{kWhr}
$$

$\Rightarrow$ Smaller and/or cheaper fusion power core

$\Rightarrow$ High power density design (plasma $q^{\prime \prime \prime}$ at $>5 \mathrm{MW} / \mathrm{m}^{3}$ )

- High heat flux and Tn FW/blanket design (e.g., Ave $\Gamma n \sim 8 \mathrm{MW} / \mathrm{m}^{2}$ )

${ }^{*}$ Coal and APWR: 50-60 mill/kWhr beyond the year 2000.

$\mathrm{t}_{\mathrm{Pe}}$ at $1 \mathrm{GW}$.

$\neq \mathrm{Pe}_{\mathrm{e}}$ at $2 \mathrm{GW}$. 


\section{CORE RADIATION LOGIC}

- Impurity in plasma core will radiate

- Minute fraction of impurity may be useful

- $\mathrm{f}_{\mathbf{z}} \Uparrow, \mathrm{Z}_{\mathrm{eff}} \Uparrow$, core radiation $\Uparrow, \varnothing_{\mathrm{fw}} \Uparrow, \varnothing_{\text {Div }} \Downarrow$

- Approach applicable to tokamak and LAR concepts

- Lower $\phi_{\text {Div }}$ can also means lower erosion rate

- Penalty: $f_{z} \Uparrow, n_{i} \Downarrow, Q_{\text {plant }} \Downarrow, Z_{\text {eff }} \Uparrow, C D$ - power $\Uparrow$ 


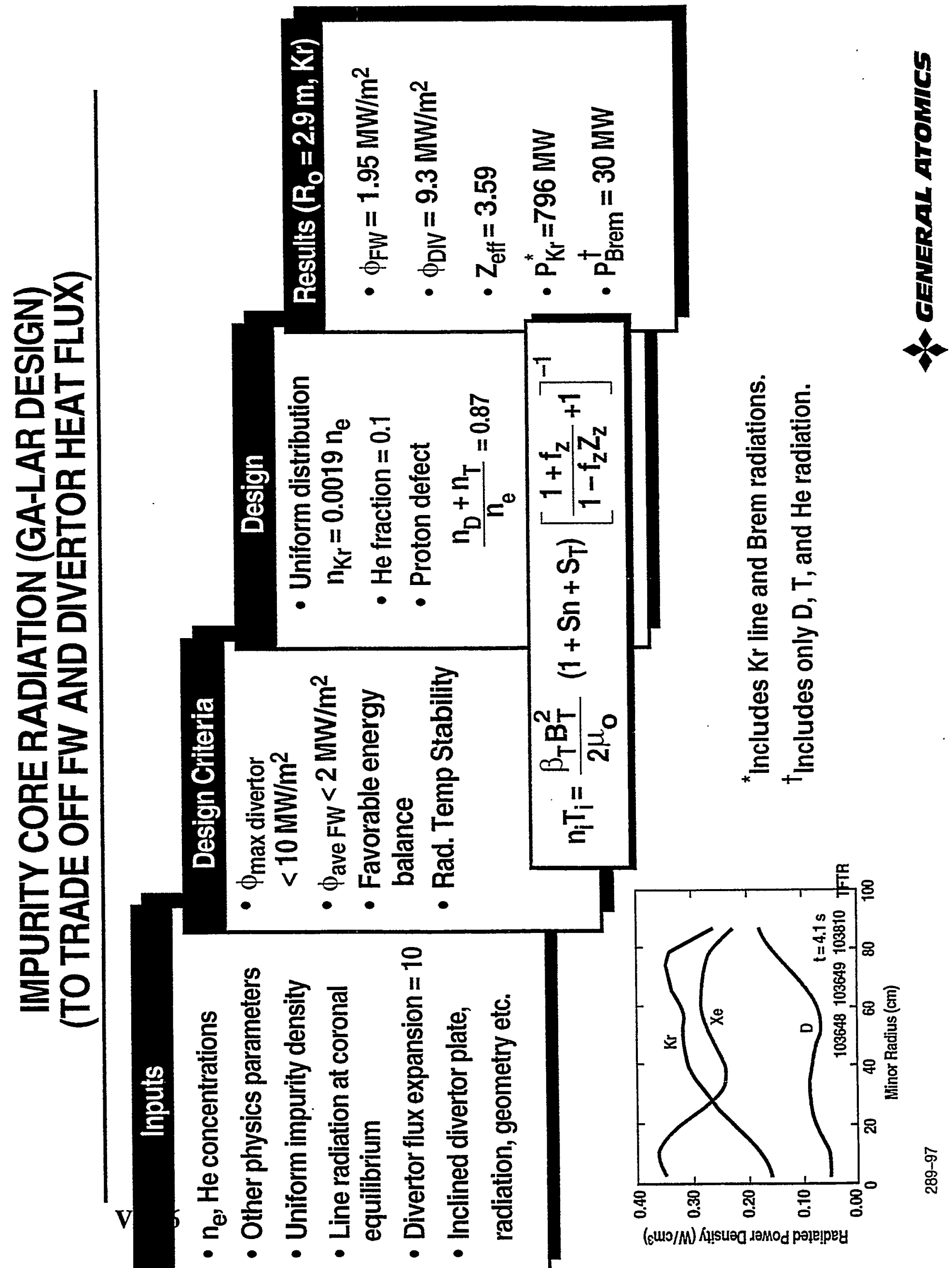




\section{OBSERVATIONS}

- $\mathrm{Kr}$ and $\mathrm{Xe}$ could be effective core radiation impurities

- $\phi$ div could be adjusted and made equal to $\phi \mathrm{fw}$

- TFTR showed supportive results

- Acceptable power balance is shown for GA-LAR design

- Core radiation approach applicable to tokamak, LAR and other confinement concepts

- Effects on transport and confinement not clear

- Temperature instability can be a concern

- Verification experiments are being proposed to be performed in present tokamaks 


\section{FW/BLANKET DESIGN}

- Functions

- Tritium production

- SC-coils and biological shielding

- Power conversion

- First wall heat flux removal

- Conventional approaches

\begin{tabular}{llll}
\hline Structural Material & Tritium Breeder & \multicolumn{1}{c}{ Coolant } & \multicolumn{1}{c}{ Comment } \\
\hline Ferritic steel & Solid & $\mathrm{H}_{2} \mathrm{O}$ & $\eta_{\mathrm{th}} \sim 33 \%$ \\
Ferritic steel & Solid & $\mathrm{He}$ & $\eta_{\mathrm{th}} \sim 33 \%$ \\
V-alloy & Li circulating & Li circulating & - MHD-insulator required for \\
& & & acceptable $\Delta \mathrm{P}\left(\eta_{\text {th }}=45 \%\right)$ \\
& & & 0 Safety concern \\
\hline
\end{tabular}

- Concept being evolved

\begin{tabular}{lccl}
\hline Structurai Material & Tritium Breeder & Coolant & \multicolumn{1}{c}{ Comment } \\
\hline $\begin{array}{l}\text { V-alloy + ferritic steel } \\
\text { (bi-metallic design) }\end{array}$ & Li or LiPb & He & $\eta_{\text {th }}=45 \%$ \\
& (stagnant) & & $\bullet$ High He pressure \\
& & & $\bullet$ Safety concern \\
& & & $\bullet$ Possible for HPD design \\
\hline
\end{tabular}




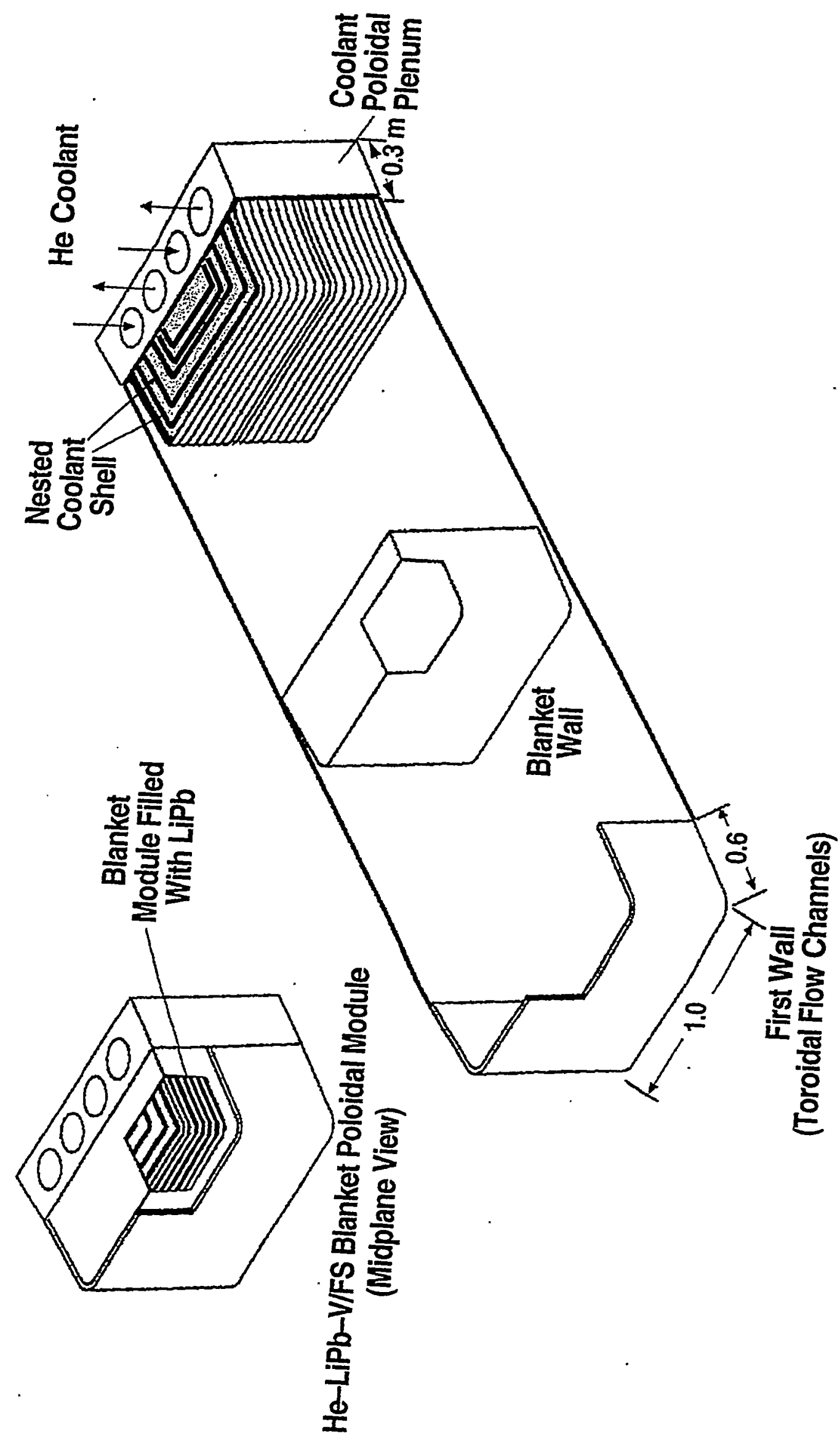




\section{FIRST-WALL BLANKET DESIGN $\left(R_{0}=2.9 \mathrm{~m}\right)$}

\section{Inputs}

$\cdot \Gamma_{\mathrm{n}}-$ Ave/max $=7.96 / 11.2 \mathrm{MW} / \mathrm{m}^{2}$

- $\phi_{\mathrm{FW}}$-Ave/max $=1.95 / 2.69 \mathrm{MW} / \mathrm{m}^{2}$

- Geometry

- Neutronics results

- He at $15 \mathrm{MPa}$

- $\mathrm{T}_{\text {in }}=250^{\circ} \mathrm{C}$

- $\mathrm{T}_{\text {out }}=650^{\circ} \mathrm{C}$

- He, Li Pb, V-alloy properties

- Nested shell geometry

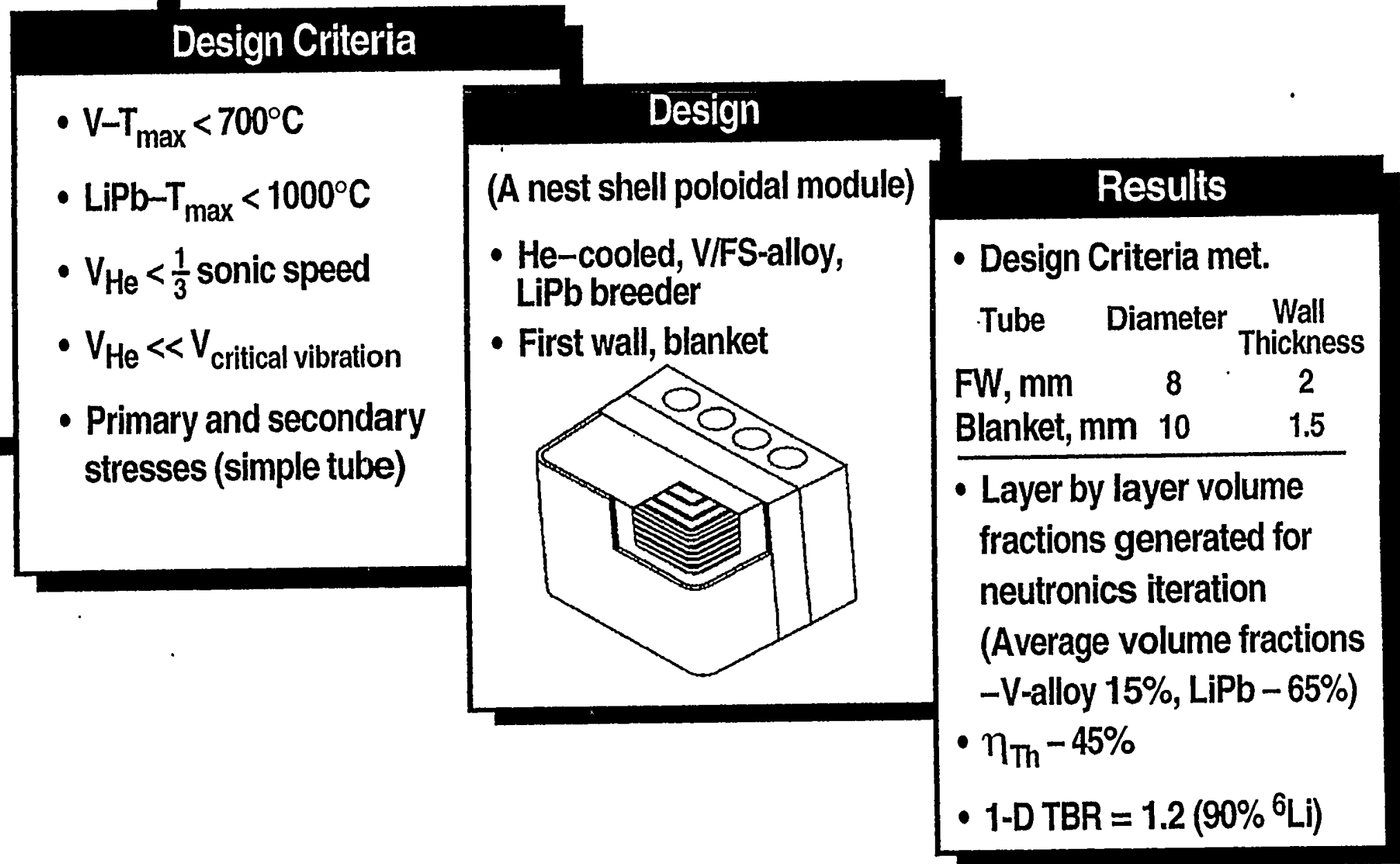

"Applicable to any high power density fusion system" 


\section{LOW ASPECT RATIO CONCEPT FOR A FUSION POWER PLANT}

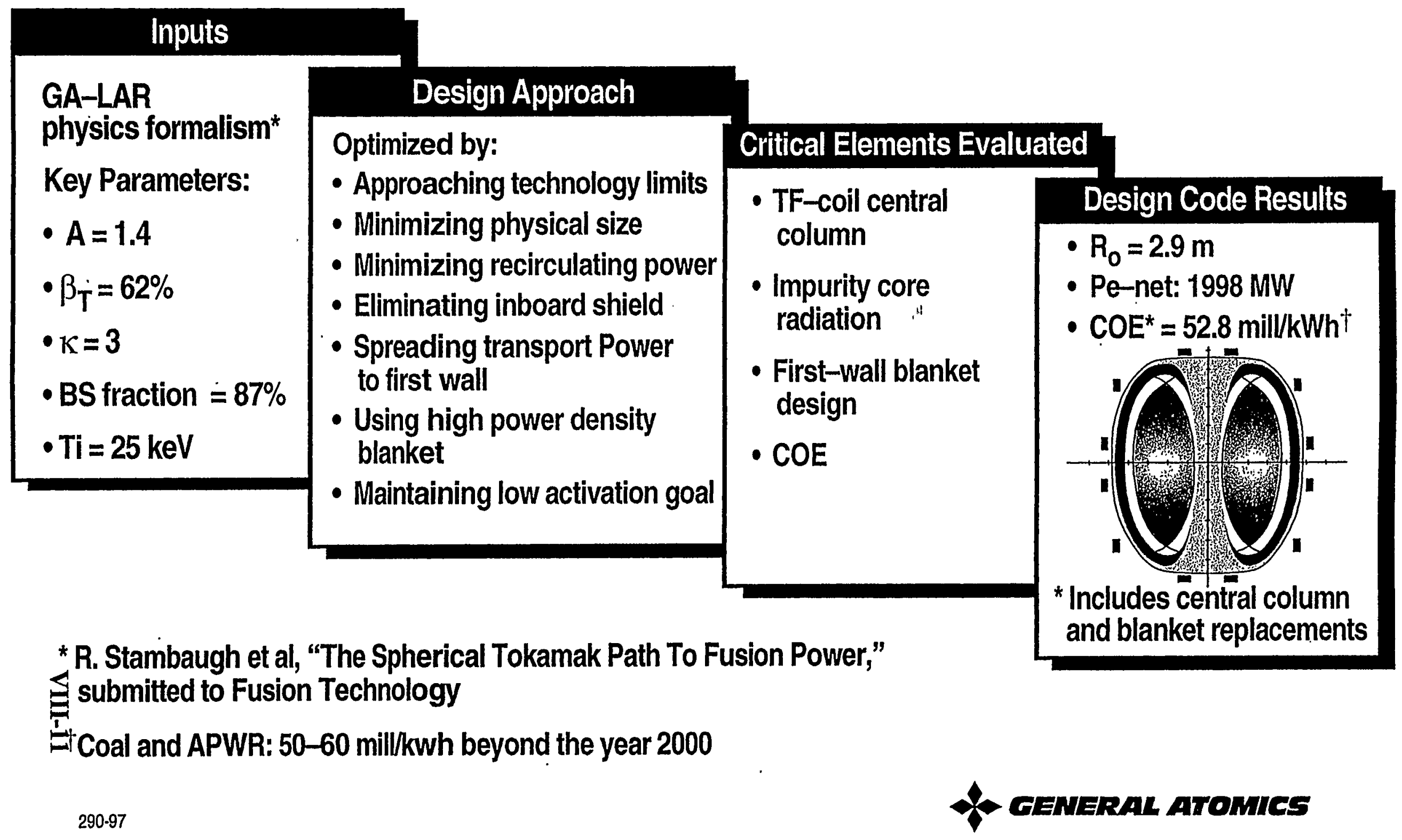




\section{PHYSICS AND ENGINEERING PARAMETERS OF A LAR 1998 MW(e) DESIGN}

Plasma aspect ratio, $\mathrm{A}$

Plasma vertical elongation

Minor plasma radius, a (m)

2.08

Major toroidal radius, $R_{0}(m)$

2.91

Plasma volume $\left(\mathrm{m}^{3}\right)$

741

First-wall surface area $\left(\mathrm{m}^{2}\right)$

493

Radial profile exponent for density, $s_{n}$

0.25

Radial profile exponent for temperature, $s_{T}$

0.25

Toroidal beta (\%)

62

Poloidal beta (\%)

On-axis toroidal field $(T)$

Plasma current, I (MA)

32.6

Plasma ion temperature (keV)

25

Plasma electron density, $n_{e}\left(10^{20} / \mathrm{m}^{3}\right)$

Plasma ion density $\left(10^{20} / \mathrm{m}^{3}\right)$

Kr fraction that of $n_{e}(\%)$

0.19

Effective plasma charge, $Z_{\text {eff }}$

3.6

Fusion power density (MW/m ${ }^{3}$ )

6.6

Fusion power (MW)

4909

Toroidal field coil summary

Number of TF coils

12

Mass of TF coil set (tonne)

TF-coil current per coil,(MA)

2.6

Central column average current density $\left(\mathrm{MA} / \mathrm{m}^{2}\right)$

TF coil resistive power consumption [MW(e)]

Engineering summary

Thermal conversion efficiency (\%) $\quad 45$

CD/heater [FWCD*] power (MW) .

Total useful thermal power (MW)

Gross electrical output power [MW(e)] 2625

Net electrical output power [MW(e)] 1998

Plant $Q$. $\quad 4.2$

14.06-MeV neutron load (MW/m $\left.{ }^{2}\right)$

Average LiPb blanket energy multiplication $\quad 1.4$

First wall heat flux (MW/ $\mathrm{m}^{2}$ ) 1.95

Divertor max. heat flux $\left(\mathrm{MW} / \mathrm{m}^{2}\right) \quad 9.3$

*Fast wave current drive 


\section{LAR FIRST WALL PARAMETERS}

Plasma aspect ratio, $\mathrm{A}$

Plasma vertical elongation

Minor plasma radius, a (m)

Major toroidal radius, $R_{0}(m)$

Plasma volume $\left(\mathrm{m}^{3}\right)$

First-wall surface area $\left(\mathrm{m}^{2}\right)$

Number of TF coil

Module midplane width $(\mathrm{m})$

Module length $(\mathrm{m})$

Fusion power density $\left(\mathrm{MW} / \mathrm{m}^{3}\right)$

Fusion power (MW)

$\Gamma \mathrm{n}$, ave/peak $\left(\mathrm{MW} / \mathrm{m}^{2}\right)$

$\varphi_{\text {fw }}$, ave/peak (MW/m ${ }^{2}$ )

Blanket energy multiplication

Helium pressure (MPa)

$\operatorname{Tin}\left({ }^{\circ} \mathrm{C}\right)$

First wall circular tube:

Inside diameter $(\mathrm{mm})$

Wall thickness $(\mathrm{mm})$
1.4

3.0

2.08

2.9

741

493

12

1.3

14.6

6.6

4909

$7.96 / 11.2$

$1.95 / 2.69$

1.4

15

250

8

2

\section{Inlet Middle $\underline{\text { Outlet }}$}

Tcoolant $\left({ }^{\circ} \mathrm{C}\right)$

Coolant velocity $(\mathrm{m} / \mathrm{s})$

Heat transfer coefficient $\left(\mathrm{W} / \mathrm{m}^{2} \mathrm{~K}\right)$

$T_{\max } \mathrm{V}$-alloy $\left({ }^{\circ} \mathrm{C}\right)$

250

138

280

310

Pressure drop first wall (MPa)

17780

146

154

591

17920

18060

Allowable primary stress (MPa)

0.5

Allowable secondary stress (MPa)

Primary stress (MPa)

621

650

Secondary stress (MPa) 


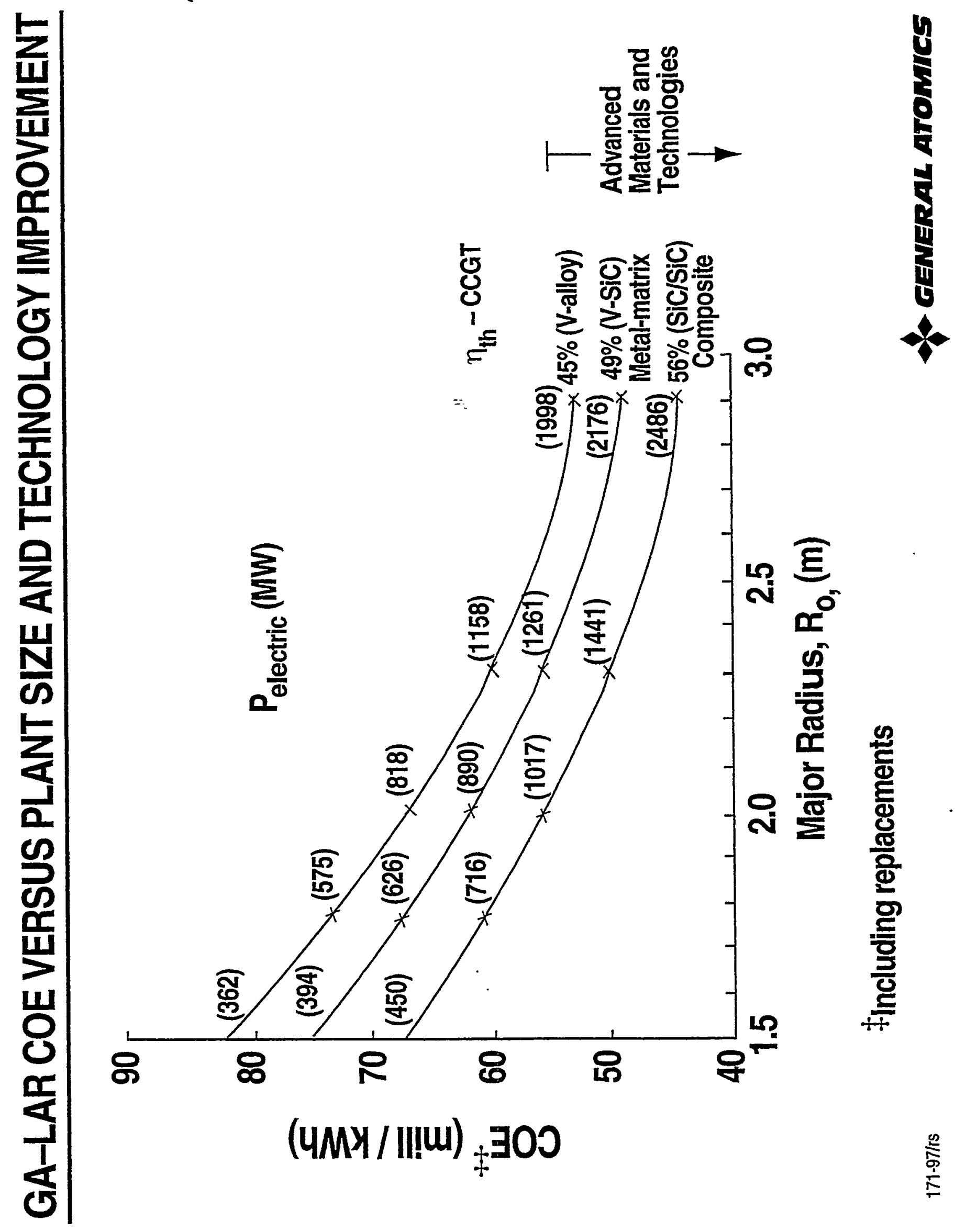




\section{CONCLUSIONS}

- HPD design approach is a key to reduce magnetic fusion COE

- Core radiation has the possibility of distributing transport power between first wall and divertor - further experimental verification is essential

- He-cooled, V/FS, LM breeder FW/blanket design can possibly handle $\Gamma \mathrm{n} \sim 8 \mathrm{MW} / \mathrm{m}^{2}$ - further technology developments are required

- GA-LAR shows the possibility of reaching a respectable and competitive COE of $53 \mathrm{mill} / \mathrm{kWhr}$ 


\section{SUMMARY OF ECONOMIC PARAMETERS}

Account \# Account Title

20.

21.

22.

22.1

22.1.1.

22.1.2.

22.1.3.

22.1.4.

22.1.5.

22.1.6.

22.1.7.

22.1.8.

22.1.9.

22.1.10

22.2.

23.

24.

25.

26.

90.

91.

92.

93.

94.

96.

97.

99.

Shield

Magnets

Owner's cost
Land and land rights

Structures and site facilities

Reactor plant equipment

Reactor equipment

FW/blanket/reflector

Supplemental-heating/CD systems

Primary structure and support

Reactor vacuum systems

Power supply, switching and energy storage Impurity control

Direct energy conversion system

ECRH breakdown system

Main heat transfer and transport systems

Turbine plant equipment

Electric plant equipment

Miscellaneous plant equipment

Special materials

Direct cost (not including contingency)

Construction services and equipment

Home office engineering and services

Field office engineering and services

Project contingency

Interest during construction (IDC)

Total cost $\left(\$ 10^{6}\right)$

Key design parameters:

Unit overnight cost [\$/kW(e)]

Capital return [mill/kW(e)h]

Plant availability

O\&M (1.68\%) [mill/kW(e)h]

Replace.[mill/kW(e)h]

Decommissioning [mill/kW(e)h]

Fuel [mill/kW(e)h]

$\mathrm{LSA}^{*}=2$ total COE [mill/kW(e)h]
\$M (1992)

10.438

557.5

1140

687.3

94.9

36

85.3

106.7

119.1

90

134.3

16.7

0.000

4.3

452.5

498.4

159.5

87.8

60.1

2503

330.4

137.7

165.2

508

677.3

755.3

5327
4572

39.4

0.75

9.16

3.7

0.5

0.03

52.8 at $\eta_{\text {th }}=45 \%$

49.3 at $\eta_{\text {th }}=49 \%$

44.4 at $\eta$ th $=56 \%$

*Level of safety assurance 


\title{
Concept of Flibe blanket in FFHR
}

\author{
Akio Sagara
}

O. Motojima, O. Mitarai 1), S. Imagawa, K. Watanabe, H. Yamanishi, T. Uda, H. Chikaraishi, A. Kohyama ${ }^{2}$, H. Matsui3), T. Muroga, N. Noda, T. Noda4), N. Ohyabu, T. Satow, A.A. Shishkin ${ }^{5)}$, Dai-Kai Sze6), A. Suzuki'7), S. Tanaka7), T. Terai7), S. Toda3), and FFHR Group

\section{National Institute for Fusion Science, Toki 509-52, Japan}

Collaboration works have made great progress in design studies for Force-Free Helical Reactor, FFHR, by standing on major advantages of current-less steady operation with no dangerous plasma disruptions. FFHR is a demo-relevant heliotron-type D-T fusion reactor $\left(\mathrm{Pf}=3 \mathrm{GW}, \mathrm{R}=20 \mathrm{~m}, a_{\mathrm{D}}=2 \mathrm{~m}, \mathrm{~B}_{0}=12 \mathrm{~T}, \mathrm{~B}_{\max }=16 \mathrm{~T},<\beta>=0.7 \%\right.$, enhancement factor $\mathrm{h}_{\mathrm{H}}=1.5$ for the energy confinement, and the neutron wall loading of $1.5 \mathrm{MW} / \mathrm{m}^{2}$.) based on the great amount of R\&D results obtained in the $L H D$ project. Aiming at power generation from 2025 by introducing innovative concepts available in a coming few decades, the design parameters at the first stage for concept definition have been investigated to make clear key issues required for power-plant engineering including materials development. Cost estimation and design optimization are planned at the next stage after the current Phase-I studies.

The main feature of FFHR is force-free-like configuration of helical coils, which gives three attractive merits : simplification of coils supporting structures by opening areas for maintenance works, widening of the coil-to-plasma clearance needed for the blanket and shield space, use of high magnetic fields allowing operation with a fairly low plasma beta, $\langle\beta\rangle$.

The other feature is the selection of molten-salt Flibe as a self-cooling tritium breeder from the main reason of safety : low tritium solubility, low reactivity with air and water, low pressure operation, and low MHD resistance which is compatible with our high magnetic field design.

- The 1-D blanket design with the forward layer of Be pebbles is optimized with the local TBR of 1.2 , saving the Be amount, and increasing the surface of Be reacting with corrosive $\mathrm{TF}$ molecules, where the nuclear heating in Flibe is as high as $60 \%$ of the total fusion output.

- The self-cooling Flibe of $40 \mathrm{~mol}^{\%} \mathrm{BeF}_{2}$ is operated at inlet/outlet temperatures of $450^{\circ} \mathrm{C} / 550^{\circ} \mathrm{C}$ with the pressure drop lower than $1 \mathrm{MPa}$ at the flow rate $7 \mathrm{~m}^{3} / \mathrm{s}$, where the double walled tube is reliable to sweep out the permeated tritium using He gas.

- The vacuum disengager is promising to recover more than $90 \%$ of tritium with the $T$ inventory less than $1 \mathrm{~g}$ in 400ton of Flibe in the loop.

- Nuclear properties such as radioactivity and transmutation at 450dpa are investigated on JLF$1, \mathrm{~V}$-alloy, $\mathrm{SiC}$ as well as materials compatibility with Flibe, aiming at replacement-free FFHR.

- Collaboration R\&D programs on Flibe chemistry and engineering have been set off by making a materials test device and an active flow loop.

In the course of FFHR design studies, many subjects have been pointed out as future works under the present encouraging positive results.

Reference : A. Sagara, et al, ISFNT-4 (1997) in press in Fusion Engrg. Design. 


\section{Concept of Flibe blanket in FFHR}

Akio_Sagara

O. Motojima, O. Mitarai1), S. Imagawa, K. Watanabe, H. Yamanishi, T. Uda,

H. Chikaraishi, A. Kohyama2), H. Matsui3), T. Muroga, N. Noda,

T. Noda4), N. Ohyabu, T. Satow, A.A. Shishkin5), Dai-Kai Sze6),

A. Suzuki7), S. Tanaka7), T. Terai7), S. Toda3), and FFHR Group

NationaLInstitute_for Fusion_Science,_Japan

1) Kyushu Tokai University, Japan

2) Kyoto University, Japan

3) Tohoku Univ., Japan

4) National Research Institute for Metals, Japan

5) NSC"Kharkov Phys. and Tech. Institute", Ukraine

6) Argonne National Laboratory, USA

7) University of Tokyo, Japan

US-Japan Workshop on

High Heat Flux Components \& Plasma Surface Interactions for Next Fusion Devices

Dec. 8 - 11, 1997

Warwick Regis Hotel, San Francisco

\section{Design studies of FFHR have been performed from 1993}

(1) as one of collaboration studies

(2) based on LHD under construction in NIFS

(3) to make clear key issues for D-T demo-plants

(4) to introduce innovative concepts

(5) by aiming at power generation from 2025.

Currentilas steady operation is the major advantage of 


\section{FFHR has two main features}

Reduction of the magnetic force between SC helical coils by giving three attractive merits:

- simplification of coils supporting structures which gives wide open areas for maintenance works,

- widening of the coil-to-plasma clearance needed for the blanket and shield space,

- use of high magnetic fields allowing operation with a fairly low beta, $\langle\beta\rangle$, requiring less-sever enhancement for $\tau_{E}$ :

Practical designs realizing these merits are actually required.
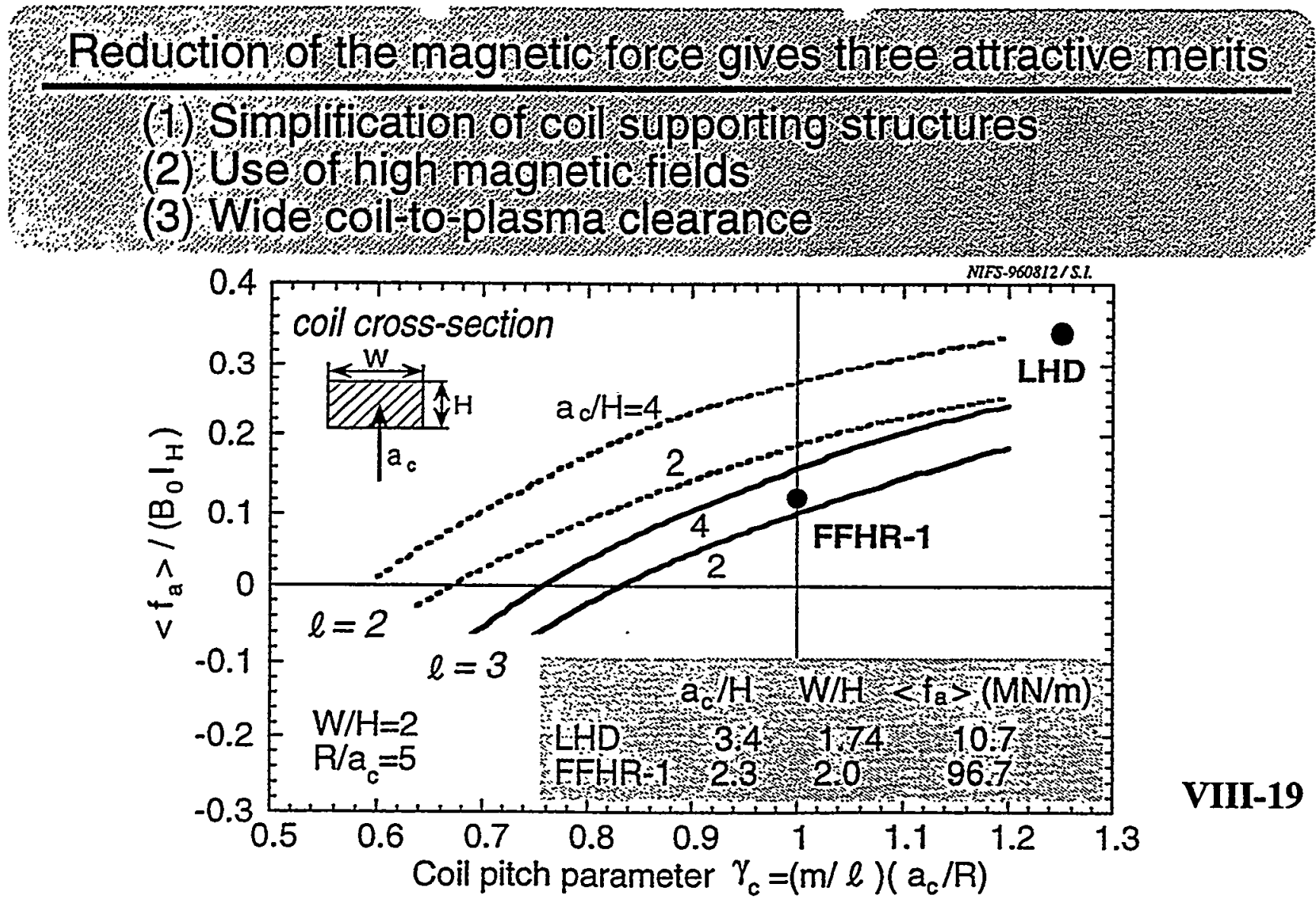


\section{Collaboration \& Publication Network}

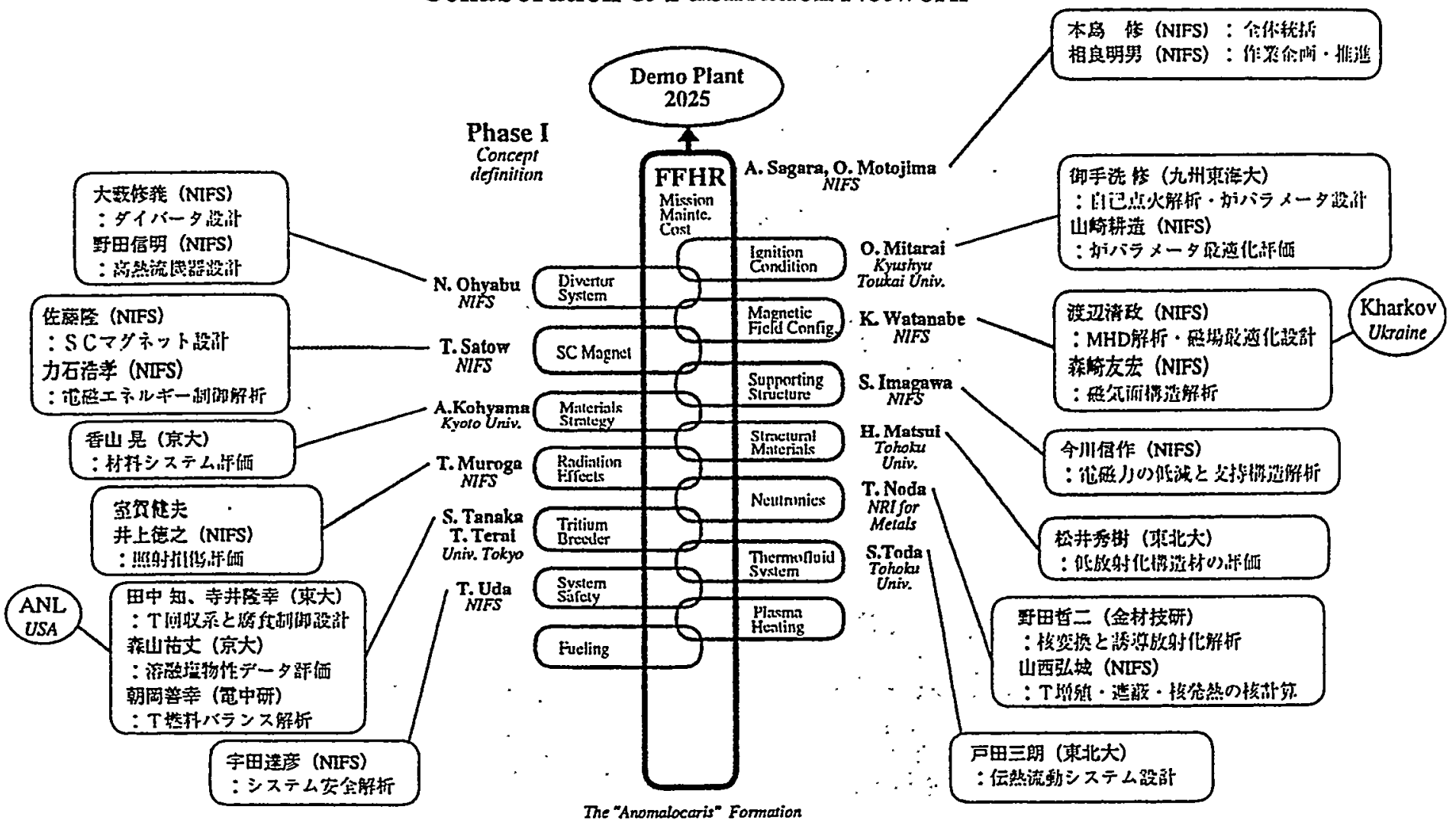

NIFS.970417.1.S.

\section{LHD-type Reactor Design - - FFHR - - .}

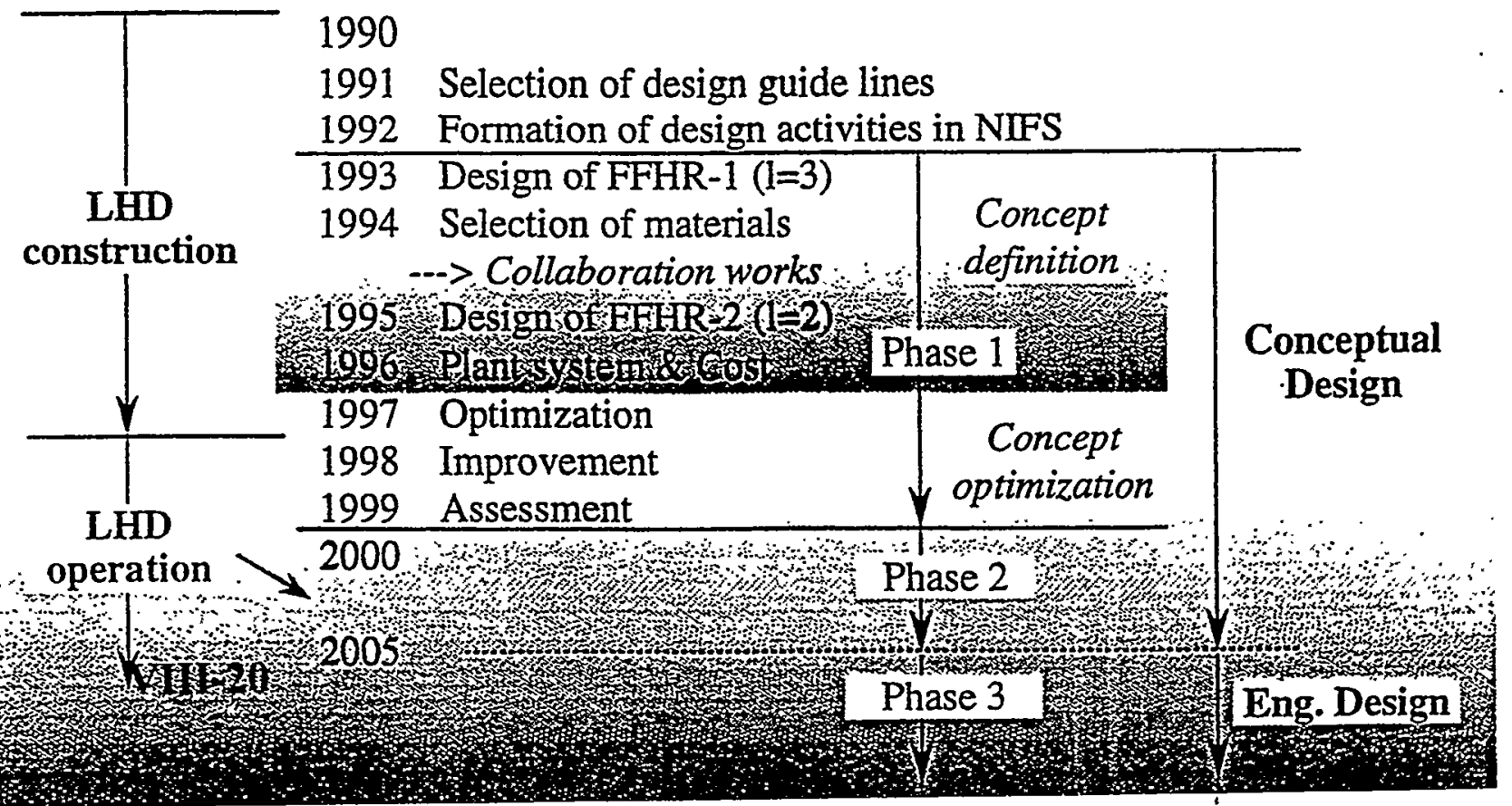




\section{FEM analyses have been done on cylindrical coils supporting structures}

- large maintenance holes

- under the merit of

force-free-like coils.

The max. stress is below

the yield stress of ss-316LN.

Structure designs of

the SC itself is the next work.

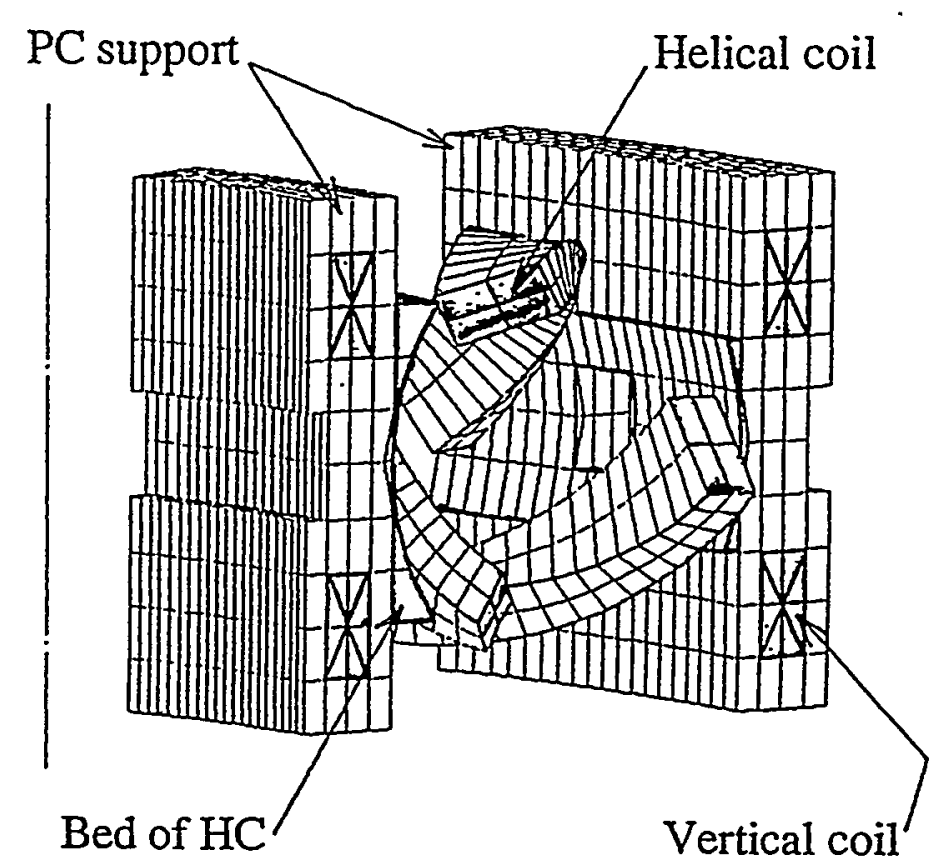

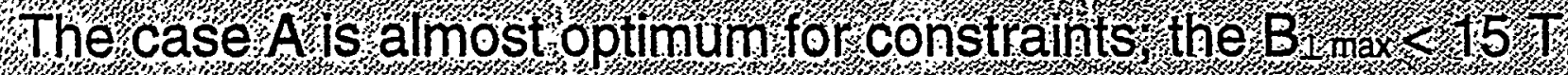

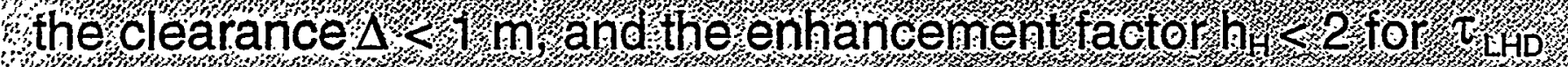

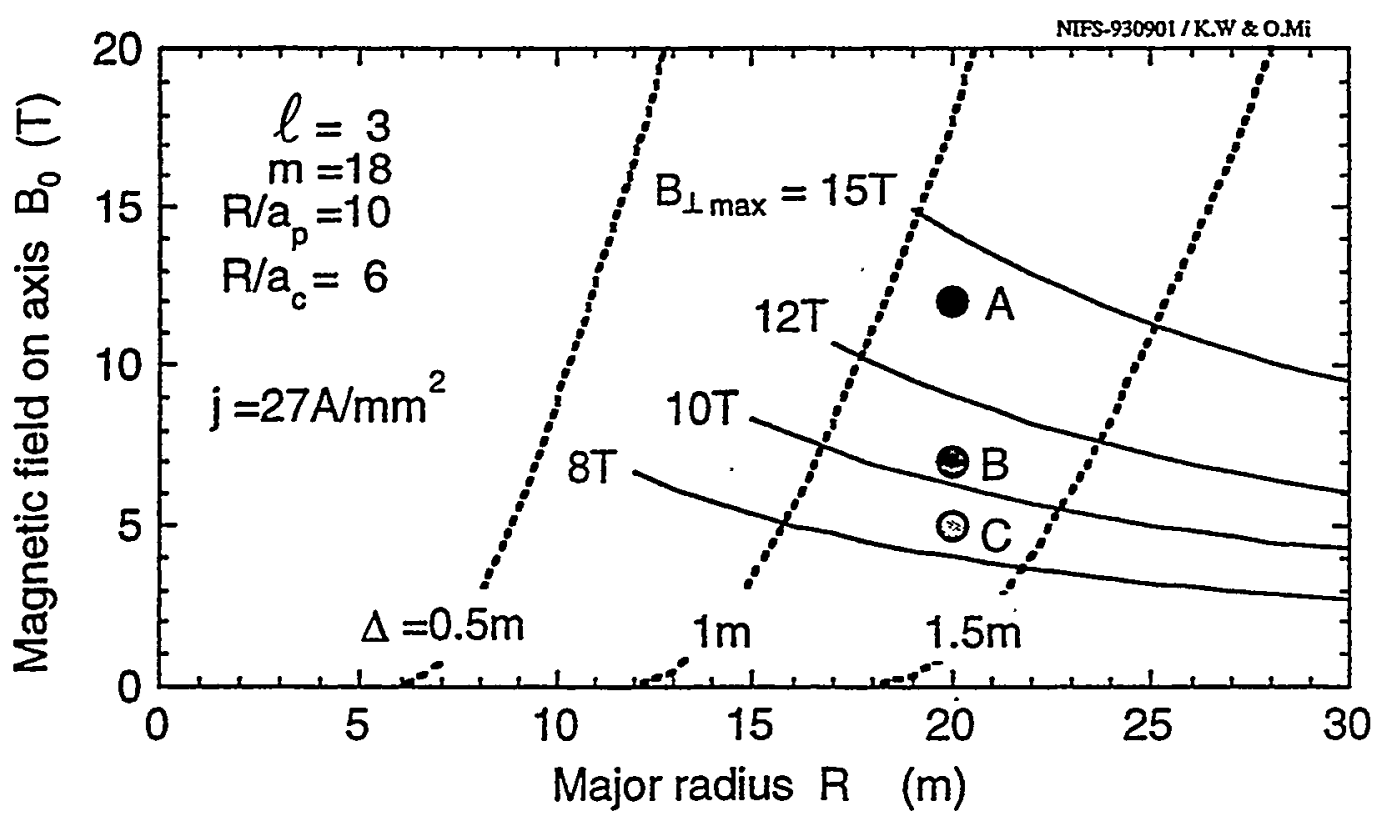

Ignition conditions for the $3 \mathrm{GW}$ fusion output case $A: T 1(0)=22 \mathrm{keV}, n(0)=2.0 \times 10^{20} \mathrm{~m}^{-3},\langle\beta\rangle=0.7 \%, h_{H}=1.5$ case $B: \pi(0)=24 \mathrm{keV}, n(0)=1.9 \times 10^{20} \mathrm{~m}^{-3},\left\langle\beta>=2.2 \%, h_{H}=2.25\right.$ 


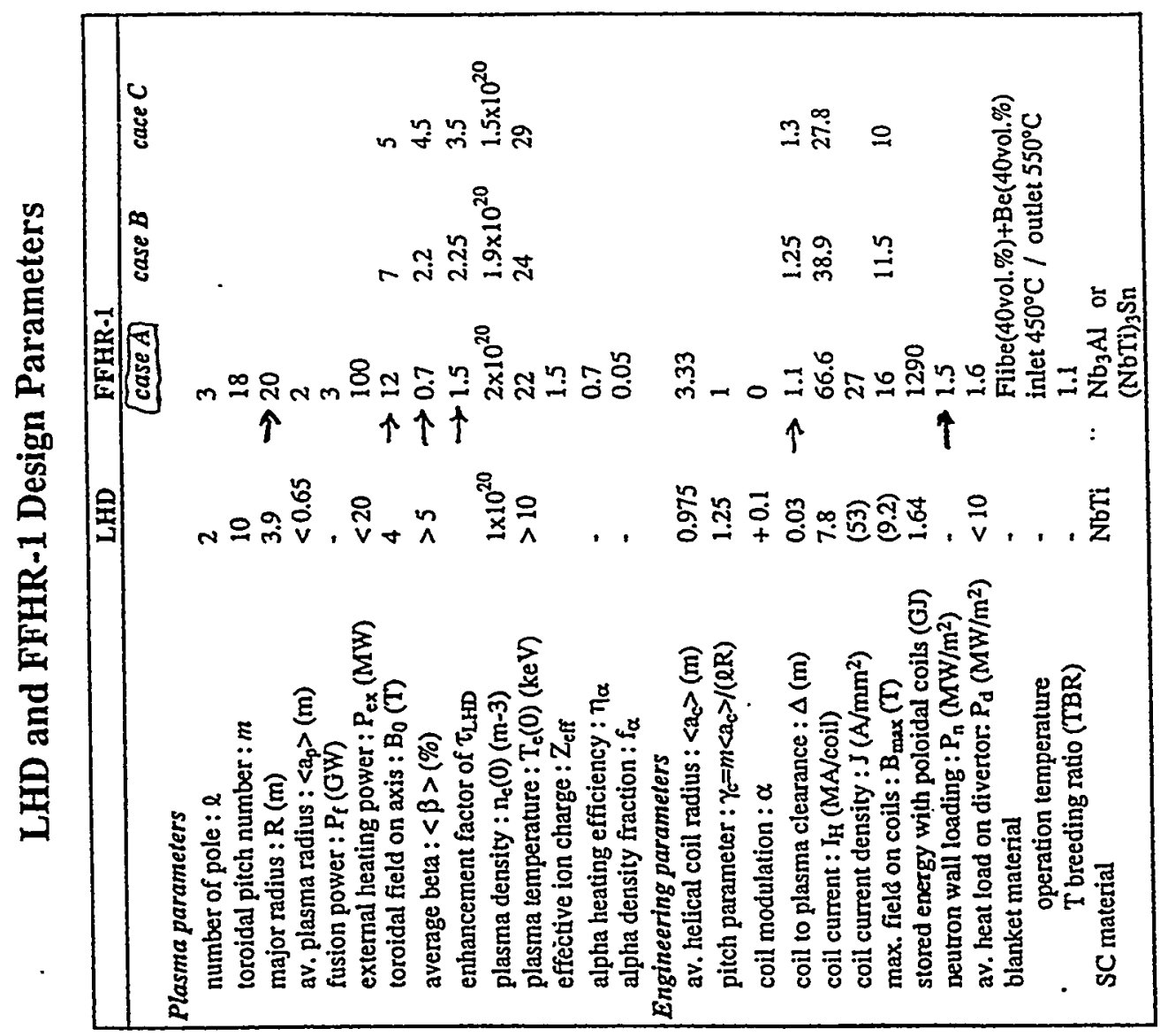

\section{Force-Free-like Helical Reactor F F HB} based on The LHD in National Institute for Fusion Science

$=20 \mathrm{~m}, a_{p}=2 \mathrm{~m}$

$=3, m=18, \gamma=1$

$0=I 2 T,\langle\beta\rangle=0.7 \%$

$f=3 \mathrm{GW}, \quad 1.5$ 乙L LA

${ }_{n}=1.5 \mathrm{MW} / \mathrm{m}^{2}$

ICC-SC coils of $b_{3} \mathrm{Al}$ or $(\mathrm{NbTi})_{3} \mathrm{Sn}$

riBe blanket ith ferritic-alloy lt. V-alloy, ODS)

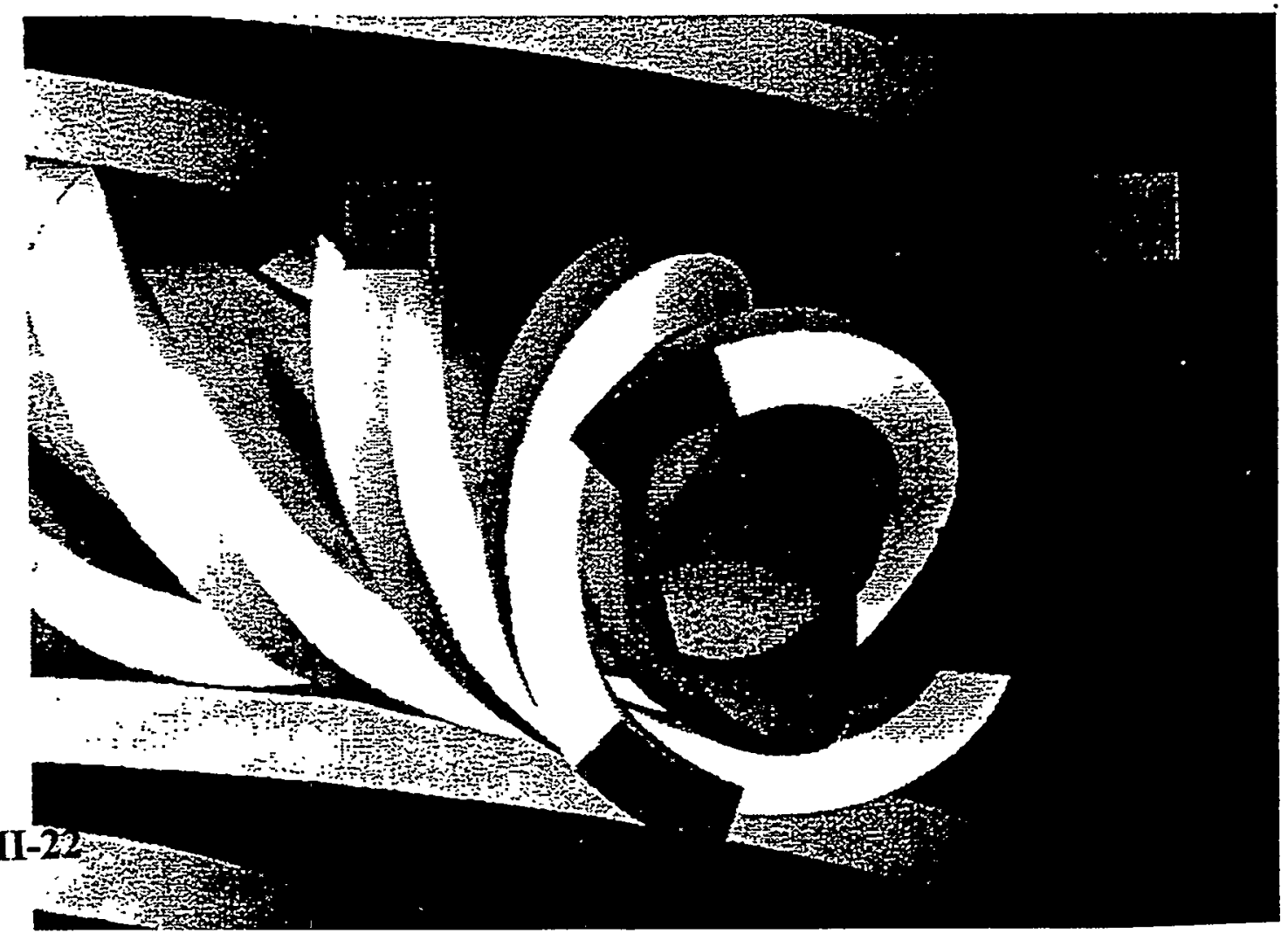


The Flibe of $40 \mathrm{~mol} \% \mathrm{BeF}_{2}$ is operated with $\triangle \mathrm{P}<1 \mathrm{MPa}$,

where the total pump power is only $0.8 \%$ of the fusion output $\mathrm{Pf}$.

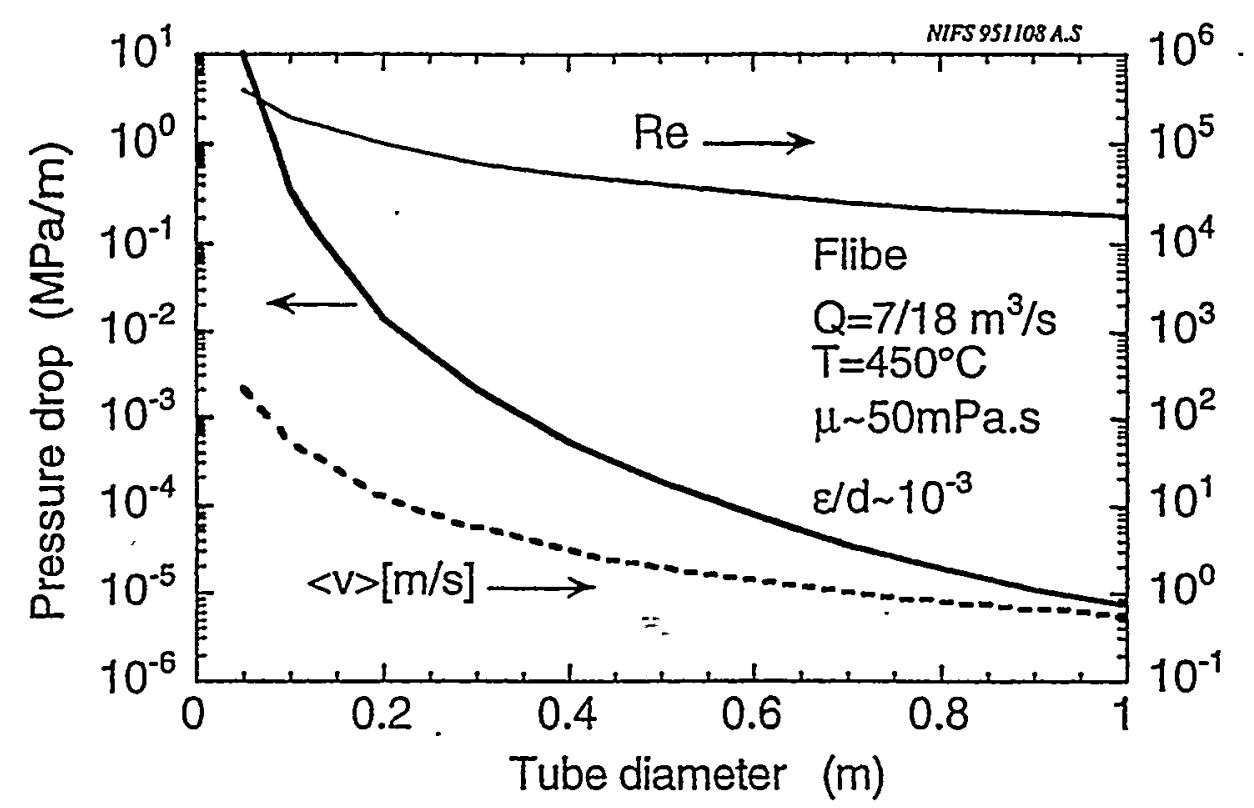

Flibe Blanket in FFHR

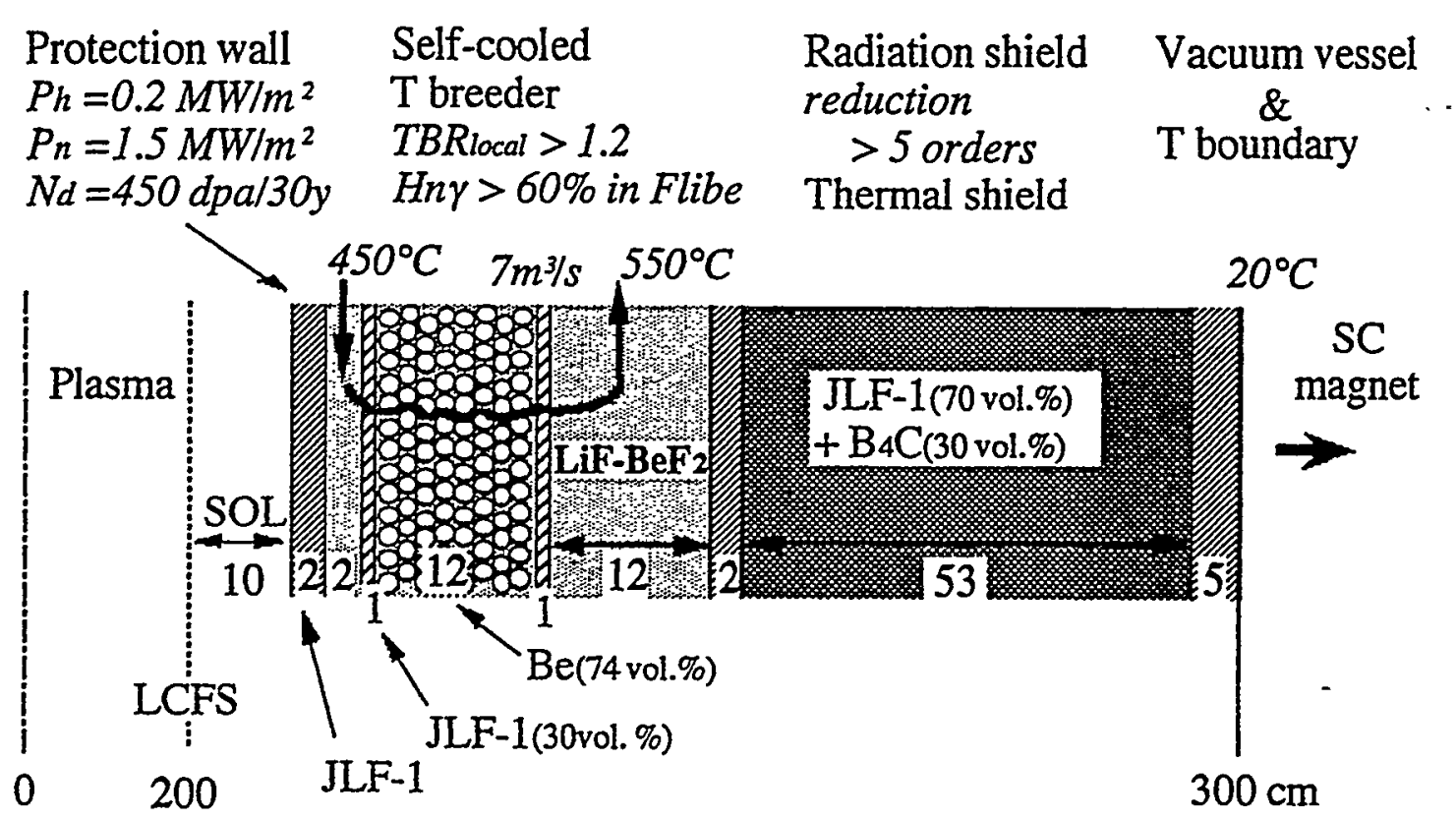

-Ferritic steel JLF-1 (Fe9Cr2W) was selected as the first candidate. - Vanadium alloy or ODS steel are second options. 
Selection of molten-salt Flibe as a self-cooling $\mathrm{T}$ breeder from the main reason of safety :

$\checkmark$ low tritium solubility ( 8 orders lower than liq.Li)

- low reactivity with air and water,

- low pressure operation(<1MPa),

- low MHD resistance $(\sim 1 \Omega \mathrm{cm})$ compatible with high $B$ field.

In order to fully take advantage of inherent safety with Flibe, it is still now required to improve the Flibe blanket concept and to clarify safety related issues.

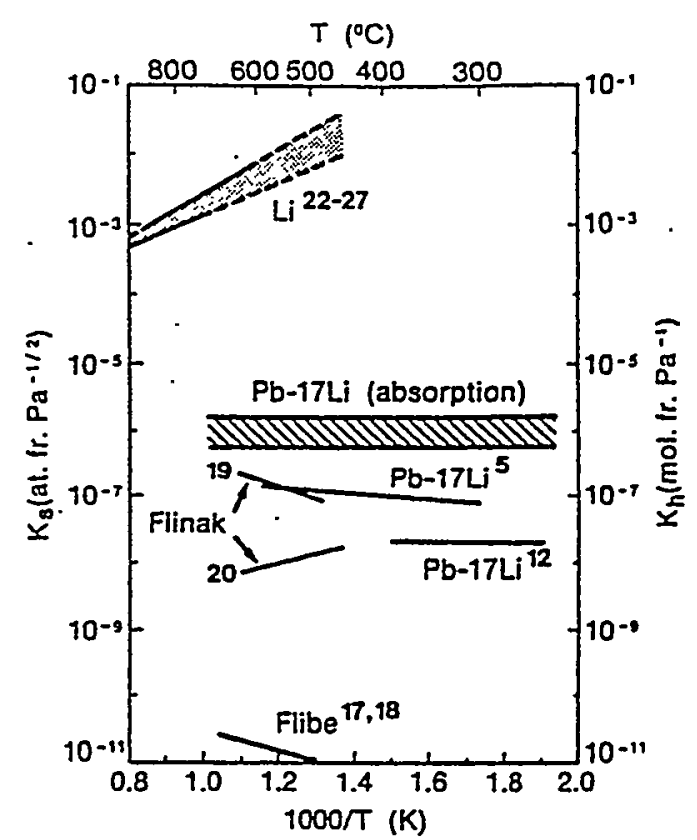

FIg. 1. Solubility constants for hydrogen isotopes in liquid breeders.

Caorlin et al. FUSION TECHNOLOGY

\section{The low solubility of $T$ in Flibe gives two advantages}

(1) $T_{2}$ recovery system is probably quite simple.

The vacuum disengager is promising

to recover $>90 \%$ of $T$ in Flibe,

where the double walled tube is reliable

to sweep out the permeated $\mathrm{T}$ with $\mathrm{He}$,

(2) In this case the T inventory is less than $1 \mathrm{~g}$

in $\mathbf{4 0 0}$ ton of Flibe in the loop.

vMgre specified data bases are desired on

rate-determining steps of $\dot{T}_{2}$ release, 
†D-T Demo Reactor $\quad\left(\mathrm{P}=3 \mathrm{GW}, \mathbf{R}=20 \mathrm{~m}, \mathrm{a}_{\mathrm{p}}=2 \mathrm{~m}, \mathrm{~B}_{0}=12 \mathrm{~T} \quad\right)$

-Current-less plasma (Steady operation, no disruption )

$\rightarrow$ Reduced wall loading $\left(1.5 \mathrm{MW} / \mathrm{m}^{2}, 30\right.$ years $\left.=450 \mathrm{dpa} \quad\right)$

\& Liquid Flibe blanket $\left(>450^{\circ} \mathrm{C}\right.$ for $\mathrm{T}_{\mathrm{m}},<550^{\circ} \mathrm{C}$ for JLF-1 )

If there is no need to replace in-vessel materials, FFHR can be operated with not only the high safety but also a high availability, resulting in reducing not only $\mathrm{COE}$ but also the total amount of radwaste.

The materials integrity at 450 dpa and compatibility with Flibe are the key issues to realize replacement-free FFHR.

\section{Induced Radioactivity}

The surface dose rate after $45 \mathrm{MWa} / \mathrm{m}^{2}$

$\downarrow$ JLF-1, V-alloy $<1 \mu$ Sv/h after 100 years cooling.

This level satisfies the shallow land disposal limits such as Class Climits of US 10CFR61 or the allowable hands-on dose rate of $10 \mu \mathrm{Sv} / \mathrm{h}$.

- Pure SiC satisfies the shallow land disposal limits.

- Mo and Nb must be lower than 10 ppm.
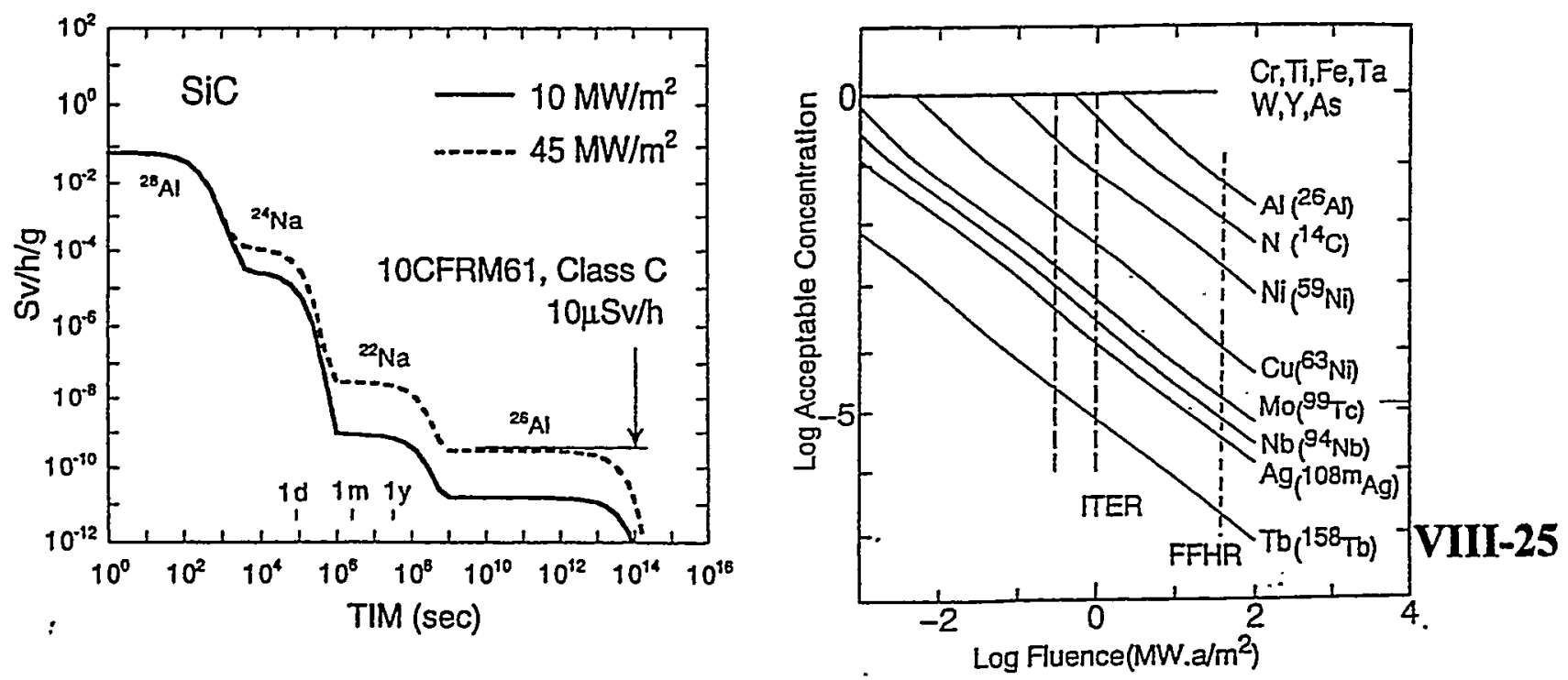
Flibeの運転温度
入口温度
: 融点と粘性の妥協点 $(40 \% \mathrm{BeF} 2)$ で $450^{\circ} \mathrm{C}$
出口温度
: JLF-1の照射下クリープより550、熱応力低減を検討
Flibe流量
: 玍出力3GW、 $\Delta T=100^{\circ} \mathrm{C}$ に対して $7 \mathrm{~m} 3 / \mathrm{s}$,
流動圧損
: $1 \mathrm{MPa}$ 以下（18流路、 $\phi 20 \mathrm{~cm} 、 50 \mathrm{~m} 、 5$ エルボー）
ポンプ動力：核融合出力の $1 \%$ 以下
初期充填 : ヒーターと加熱ガス併用を検討、回収含む手順が課題

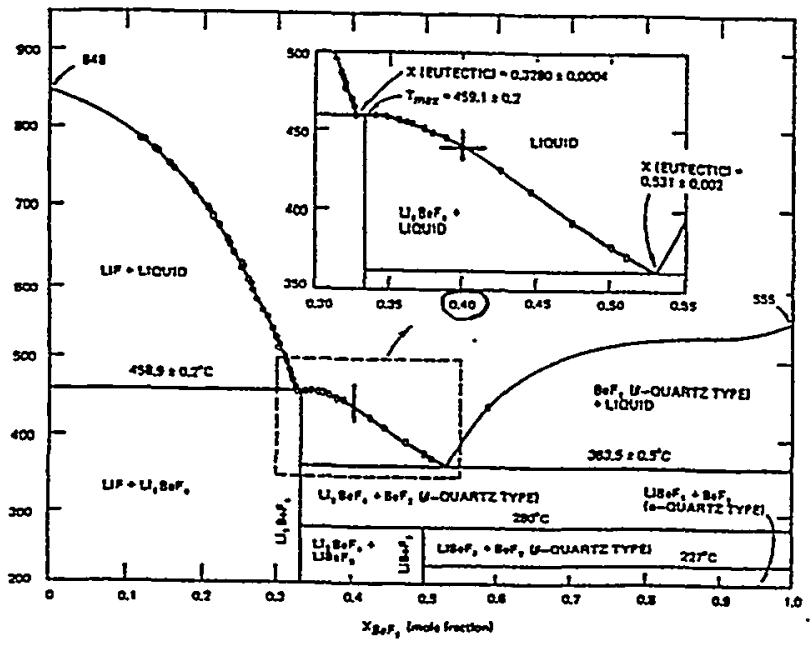

Ferritic Steels/ Helium Cooling

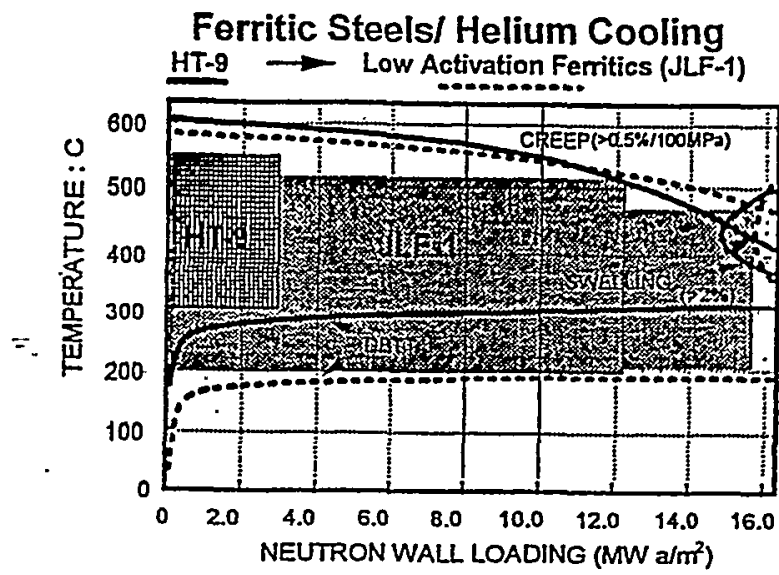

( after Kohyama);

\section{FFHR has many inherent and passive safety features}

\section{current-less plasma, \\ steady state operation, \\ $\checkmark$ use of Flibe, \\ - high-temp. \&T devices inside the torus area.}

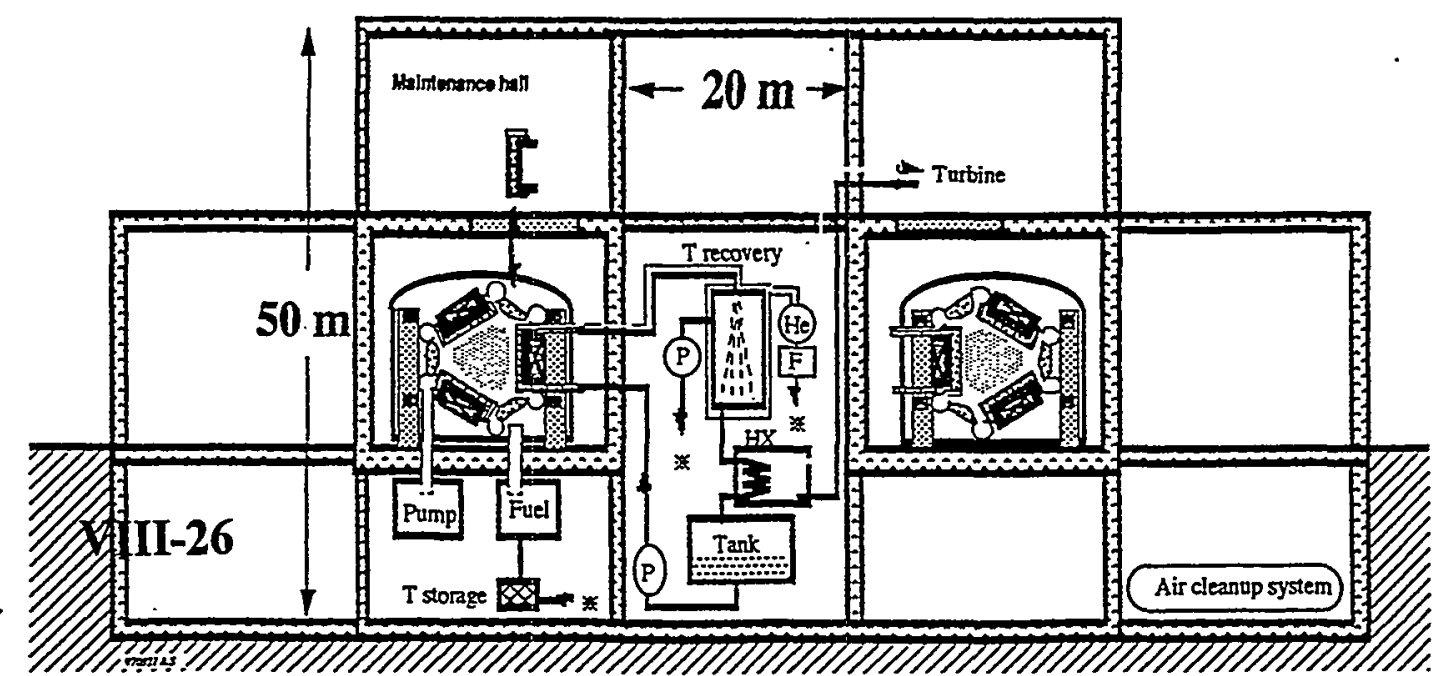




\section{Solid Transmutation Products after $45 \mathrm{MWa} / \mathrm{m}^{2}$}

- From W to almost $10 \% \mathrm{~W}, 20 \% \mathrm{Re}$ and $70 \%$ Os.

- Investigation JLF-1 after transmutation of $W$ are desired.

- From $\mathrm{V}$ to $\mathrm{Cr}$ is about $2 \mathrm{wt}$.\% under Flibe blanket.

$+\mathrm{V} 4 \mathrm{Cr} 4 \mathrm{Ti}$ has a sufficient margin to the DBTT shift.
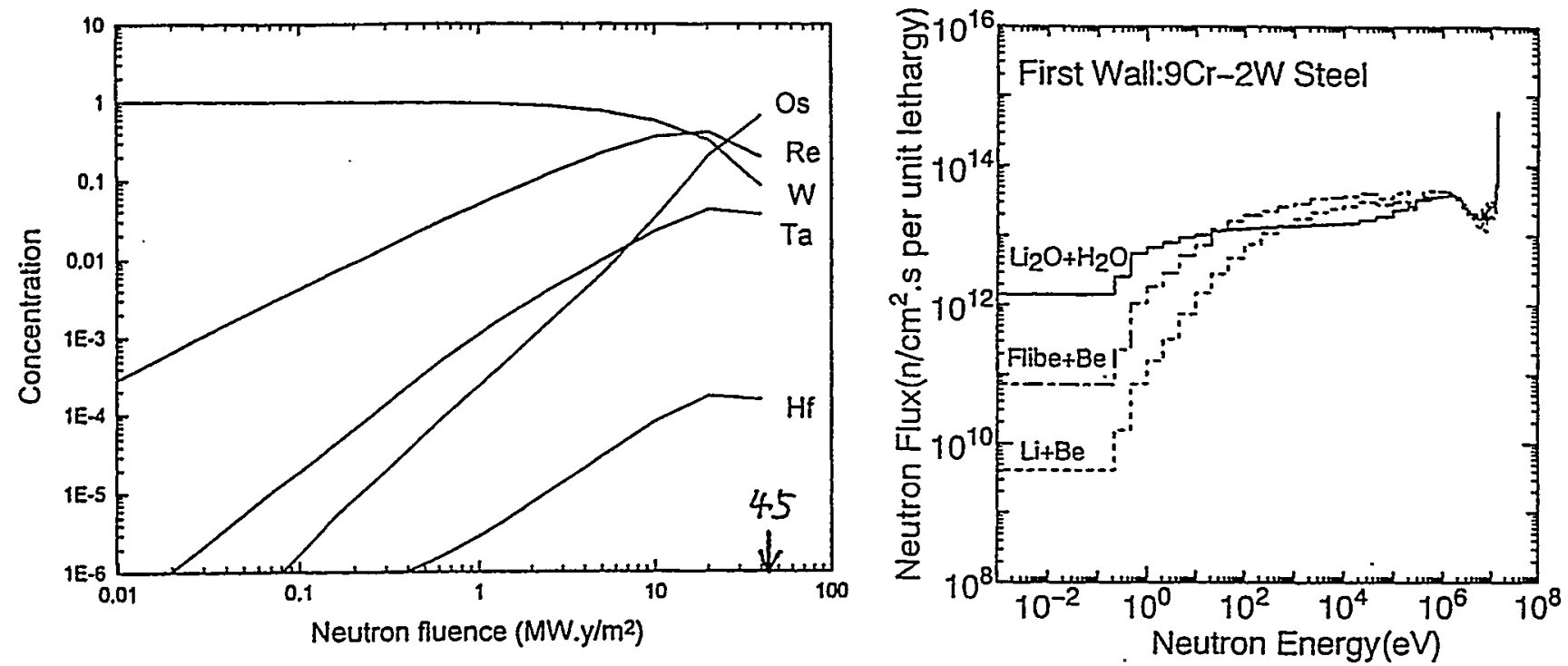

\section{Decay Heat $\mathbf{Q}_{d}$ \\ $\mathrm{dT} / \mathrm{dt}=\mathrm{Q}_{\mathrm{d}} / \mathrm{C}_{\mathrm{v}}$ under adiabatic condition}

- On W, Mo and Nb, dT/dt $<0.5^{\circ} \mathrm{C} / \mathrm{s}$ after 1 week cooling, this may be acceptable.

- On Ta, $\mathrm{dT} / \mathrm{dt}=15^{\circ} \mathrm{C} / \mathrm{s}$ for 100 days cooling is not acceptable.
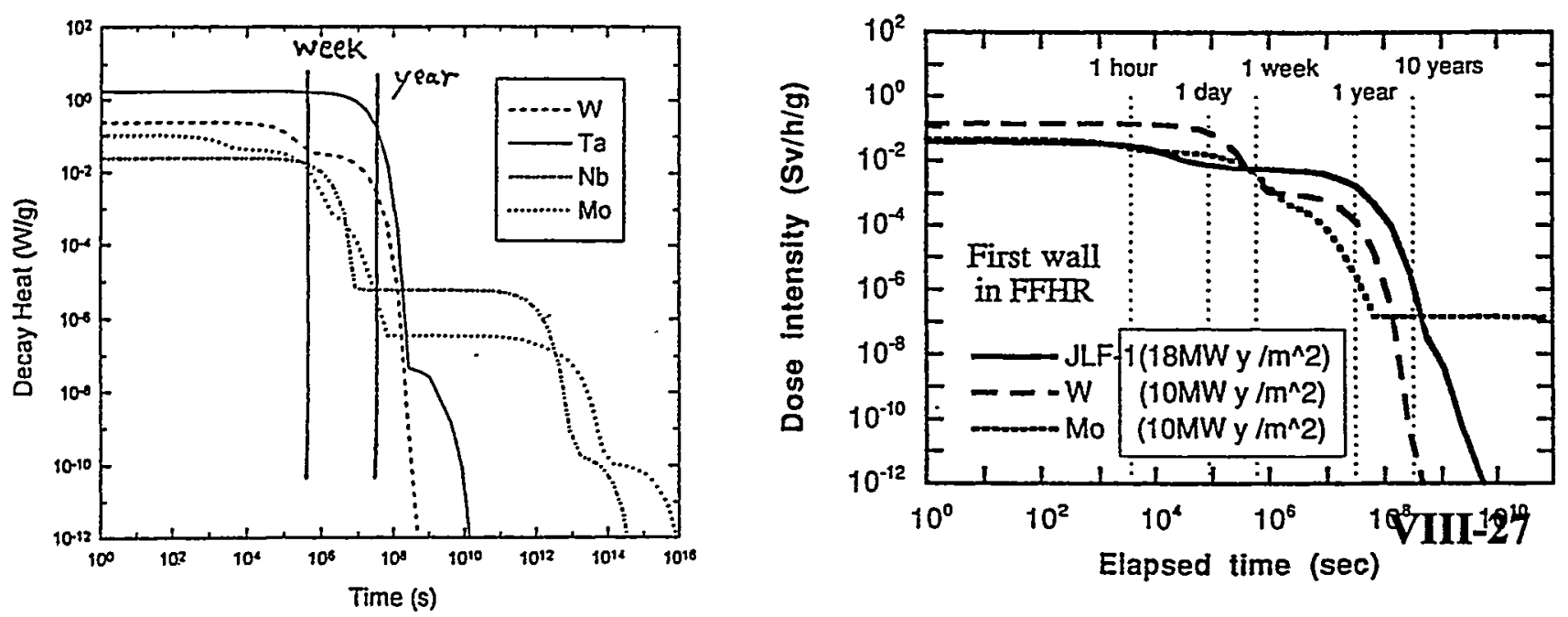


\section{Compatibility with Flibe}

- The neutron multiplier $\mathrm{Be}$ is used to reduce corrosive TF molecules. $\left(\mathrm{Be}+2 \mathrm{TF}-\rightarrow \mathrm{BeF}_{2}+\mathrm{T}_{2}\right)$

$-\mathrm{MoF}_{6}$ can be often used to form Mo layers on the coolant tube. $\left(\mathrm{MoF}_{6}+3 \mathrm{~T}_{2} \rightarrow \mathrm{6TF}+\mathrm{Mo}\right)$

- Data bases on chemical kinetics are strongly desired.

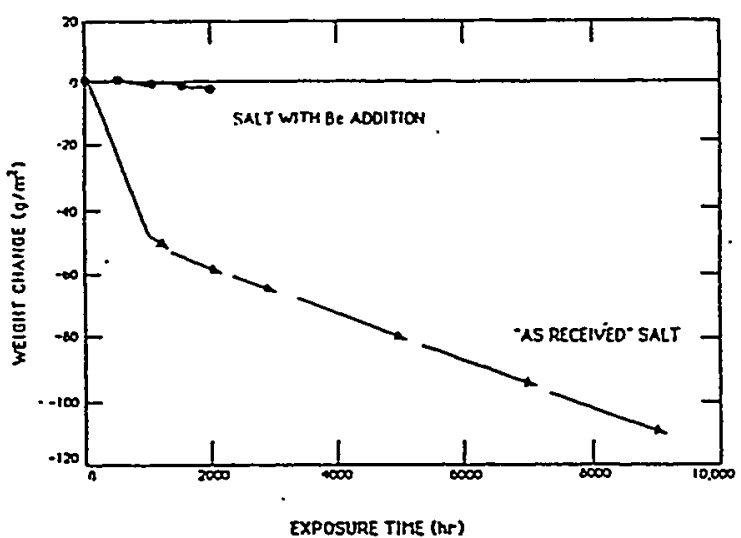

Fig. 2 Height change versus exposure time for type 316 stainless steel in LiF-BeF, sait at the maximum loop temperature of $650^{\circ} \mathrm{C}$.

by J.R. Keiser etal. (1979)

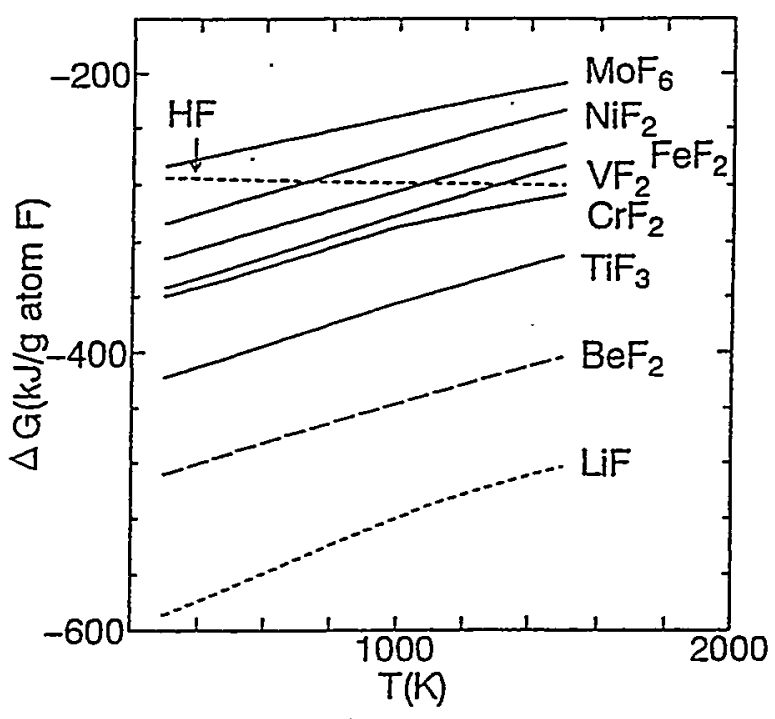

\section{R\&D's on Flibe chemistry and engineering have been set off}

\section{by making}

a materials test device (Univ. of Tokyo), an active flow loop (Tohoku Univ.).
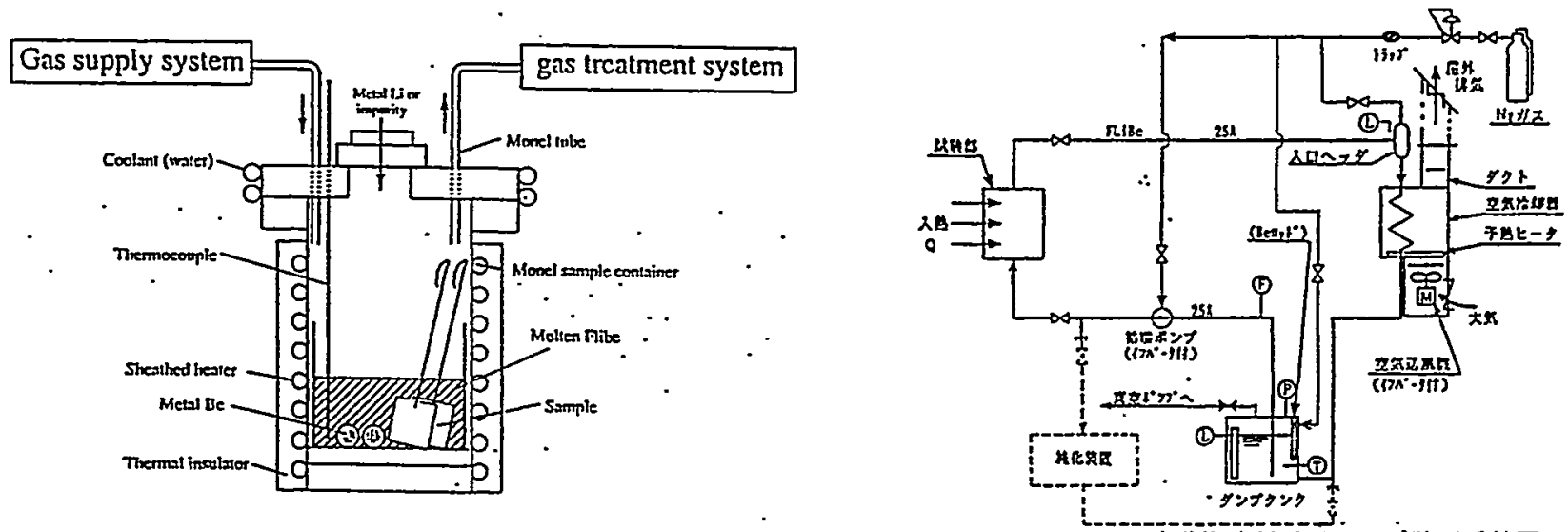

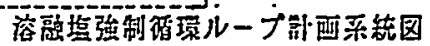




\section{The blanket units are replaced through maintenance ports by sliding along the continuous helical coils.}

(1) Since FLiBe is moved to a drain tank, each unit is below 5 ton.

(2) Radioactive wastes in each replacement are

-800 ton of JLF-1

$\rightarrow 160$ ton of Mo-TiC or 300 ton of W-TiC

which is-only $16 \mathrm{~m}^{3}$ in volume and can be managed-

- 350 ton of Be which is the mass of recycling use 200

as well as 400 ton of FLiBe

\section{Summary}

(1) Molten-salt Flibe is selected as a self-cooling tritium breeder from the main reason of safety.

(2) The 1-D blanket design with the forward layer of Be pebbles is optimized with TBRlocal $>1.2$, saving $\mathrm{Be}$ amount, and increasing the surface of $\mathrm{Be}$ reacting with corrosive TF, where the nuclear heating in Flibe $>60 \%$ of Pf.

(3) The self-cooling Flibe $\left(40 \mathrm{~mol}_{\%} \mathrm{BeF} 2\right)$ is operated at inlet/outlet of $450^{\circ} \mathrm{C} / 550^{\circ} \mathrm{C}$ with the $\triangle \mathrm{P}<1 \mathrm{MPa}$ at $7 \mathrm{~m} 3 / \mathrm{s}$, where the double walled tube is reliable to sweep out the permeated $T$ using He gas.

(4) The vacuum disengager is promising to recover more than $90 \%$ of tritium with the $T$ inventory $<1 \mathrm{~g}$ in 400 ton of Flibe in the loop.

(5) Nuclear properties such as radioactivity and transmutation at 450dpa are investigated on JLF-1, V-alloy, SiC as well as materials compatibility with Flibe, aiming at replacement-free FFHR.

(6) Collaboration R\&D programs on Flibe chemistry and engineering have been set off by making a materials test device and an active flow loop.III-29

(7) In FFHR design studies, many subjects have been pointed out as future works under the present encouraging positive results. 
Motivation, Scope, and Preliminary Approach for APEX

Neil B. Morley

Mohamed Abdou

Fusion Science and Technology, UCLA

US-Japan Workshop on High Heat Flux Components and Plasma Surface Interactions

San Francisco, USA

December 8-11, 1997 


\section{APEX}

\section{Ultimate Goal}

Significant contributions to making the (long-term) fusion energy system more competitive through exploring and developing more attractive concepts for Fusion Power Technology (FPT)

FPT: Region from the edge of the plasma to the inner surface of the magnets

\section{$\underline{\text { Near-Term Objective }}$}

Explore new (and possibly revolutionary) concepts that can provide the capability to efficiently extract heat from systems with high neutron and surface heat loads while satisfying all FPT functional requirements and maximizing reliability, maintainability, safety and environmental attractiveness 


\section{The Motivation for Conducting APEX Emerged from the New Vision for Fusion Restructured Program}

New Vision

- Take the long term view

- Emphasize science (including engineering sciences) as basis for innovation

- Key is Improving Fusion

$>$ Make the ultimate product more attractive

$>$ Have more effective R\&D pathways

\section{How to Improve Fusion}

1) Plasma Physics Innovation

2) Technology Innovation

- can make product more competitive

- can define the limits

$>$ provide boundary conditions to physics research

$>$ better evaluation of fusion's potential 


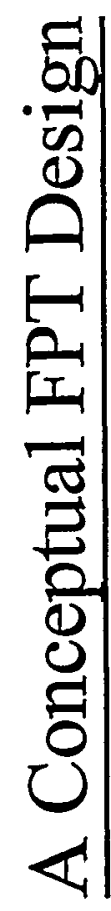
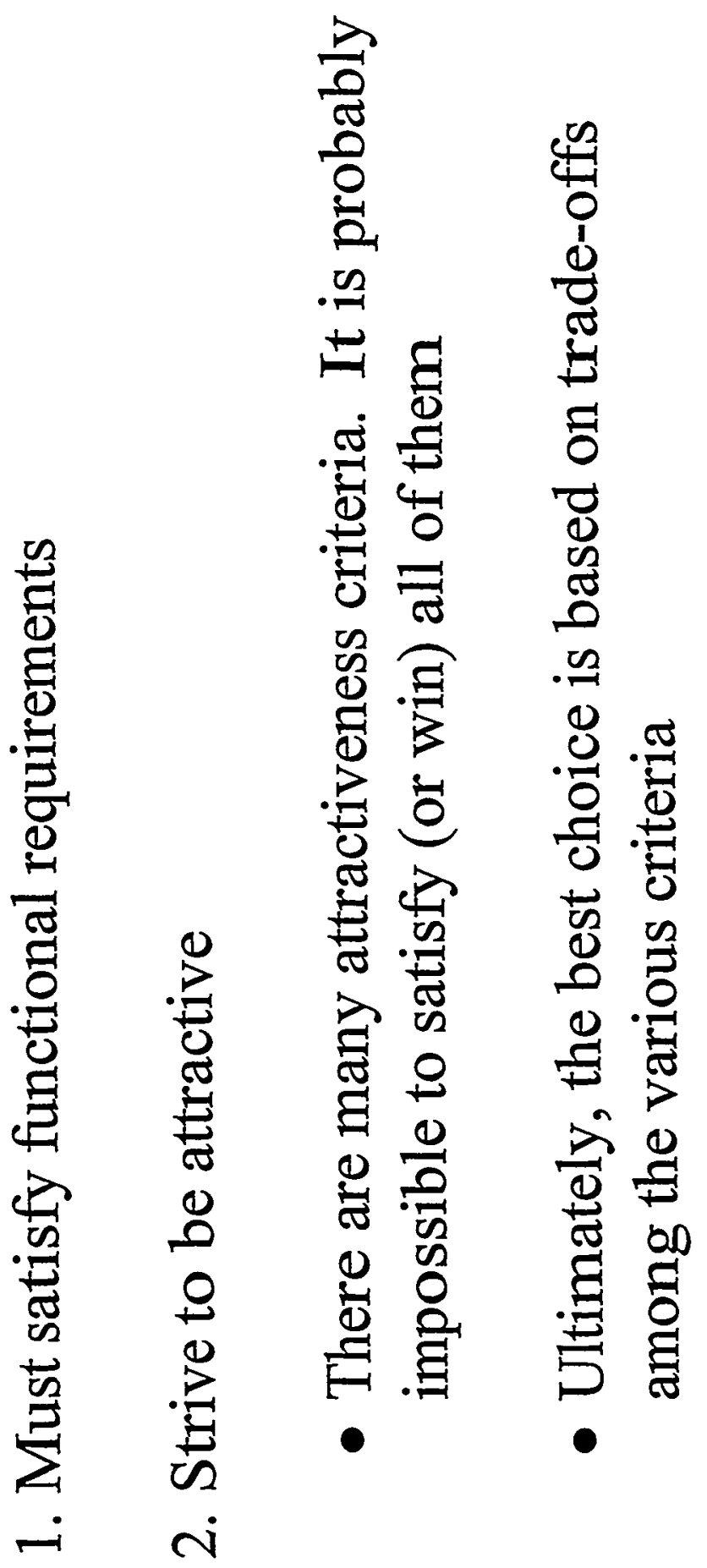

年

ए

$\ddot{0} ?$

$\sum_{1}^{\infty} \frac{p}{\infty}$

i 


\section{Functional Requirements of Fusion Power Technology}

1) provision of VACUUM environment

2) EXHAUST of plasma burn products

3) POWER EXTRACTION from plasma particles and radiation (surface heat loads)

4) POWER EXTRACTION from energy deposition of neutrons and secondary gamma rays

5) TRITIUM BREEDING at the rate required to satisfy tritium self sufficiency

6) TRITIUM EXTRACTION and processing

7) RADIATION PROTECTION 


\section{General Attractiveness Criteria for Fusion Energy System}

1. ECONOMICS

a) cost per unit thermal power

b) thermal conversion efficiency

c) mean time between failure (MTBF)

d) mean time to repair (MTTR)

e) lifetime

2. SAFETY

a) chemical reactivity

b) decay heat

c) tritium inventory

d) dose

e) etc.

3. ENVIRONMENTAL

a) waste disposal

b) routine releases (e.g. tritium)

c) material resources utilization

d) etc.

APEX (initial) focus: $\quad$ Economics

APEX (initial) DRIVER: Capability for High Neutron Wall Load and

Associated Surface Heat Flux 


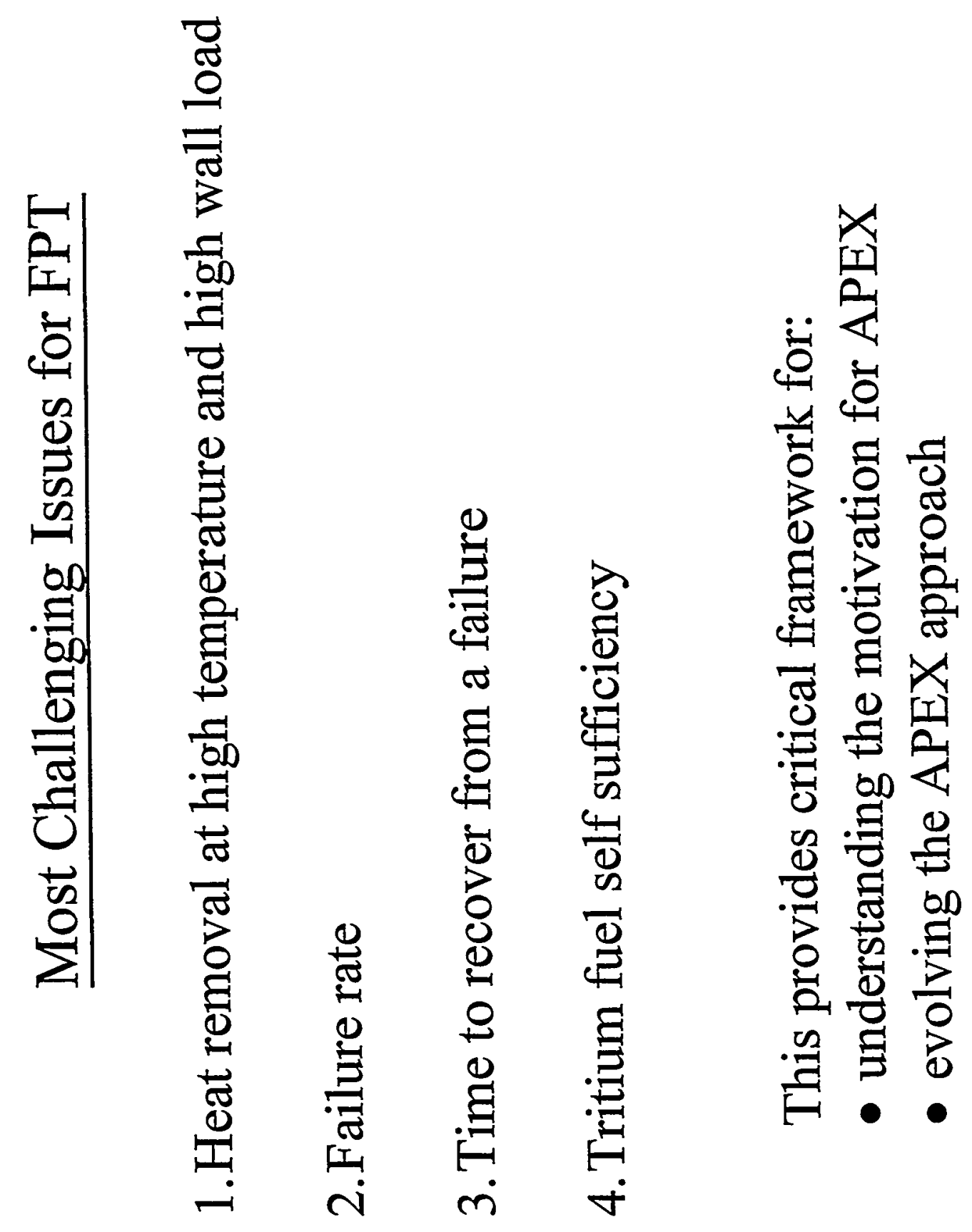


Current Design Concepts and Materials for First Wall / Blanket Do NOT Have the Capability to Meet the Fusion Challenge

\begin{tabular}{|c|c|c|}
\hline$\longdiv { \text { Concept } }$ & $\begin{array}{l}\text { Wall Load } \\
\text { Capability } \\
\text { MW/m } / \mathbf{m}^{2}\end{array}$ & Other Observations \\
\hline $\begin{array}{l}\text { Ferritic / He / Breeder } \\
\text { Ferritic / } \mathrm{H}_{2} \mathbf{O} \text { / Li Pb }\end{array}$ & 2 & $\begin{array}{l}\text { Magnetic material } \\
\text { Fracture toughness }\end{array}$ \\
\hline $\begin{array}{l}\text { Vanadium Alloy / } \\
\text { Lithium }\end{array}$ & 2.5 & $\begin{array}{l}\text { V works only with lithium } \\
\text { Is lithium acceptable? } \\
\text { Not feasible until a self healing } \\
\text { coating is found }\end{array}$ \\
\hline $\mathrm{SiC} / \mathrm{SiC} / \mathrm{He} /$ Breeder & 1.5 & $\begin{array}{l}\text { Serious feasibility issues } \\
\text { Do NOT know how to design } \\
\text { Poor thermal conductivity }\end{array}$ \\
\hline
\end{tabular}




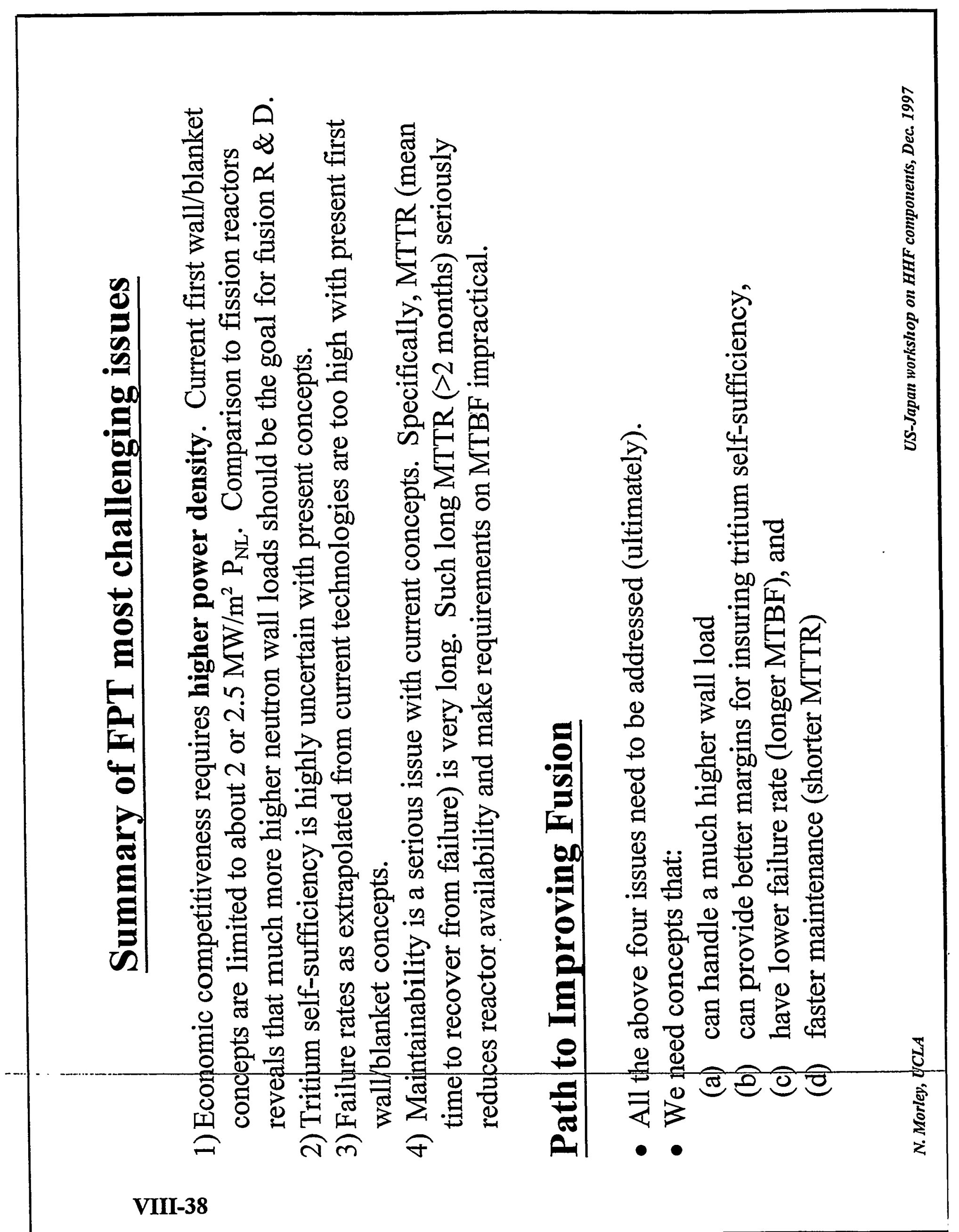




\section{APEX Focus}

- APEX is only the first leg along the path toward improving fusion

- APEX will focus specifically on simulating new design concepts for in-vessel components that are capable of handling high neutron wall loads and the associated surface heat flux

- Of course, we should keep an eye on maintainability, failure rate, and tritium self sufficiency plus many other criteria (low decay heat, low activation, etc.)

- However, we should not over-constrain the problem from the beginning. If we succeed in finding high power density concepts, we can work later on making them better for other issues.

\section{We invite comments on this}




\section{Proposed Goals for Neutron Wall Load and Surface Heat Flux at the First Wall}

1) Average Neutron Wall Load $P_{\mathrm{NL}, a v e}=5 \mathrm{MW} / \mathrm{m}^{2}$

Peaking Factor $=1.4$

Peak Neutron Wall Load $=7.0 \mathrm{MW} / \mathrm{m}^{2}$

$\underline{\text { Reasons }}$

- High enough to improve economics

- Not overly ambitious: we probably can find a concept or two that meet the goal

2) Surface Heat Flux

Radiate most of the $\alpha$-power to the first wall (reduce divertor problem)

- first wall surface area is more than ten times the divertor area

- this also allows useful (sensible) heat recovery for the $\propto$ - power

Suggested Peak Surface Heat Flux .85 x $0.25 \times 7 \sim 1.5 \mathrm{MW} / \mathrm{m}^{2}$

Design goals that must be met in a concept to be considered suitable for APEX Neutron Wall Load $=7$

Surface Heat Flux $=1.5$ 


\section{Participating Organizations}

University of California, Los Angeles (UCLA)

Professor Mohamed Abdou, Lead Investigator

Dr. Mahmoud Youssef, APEX Secretary (youssef@fusion.ucla.edu)

\section{Argonne National Labs (ANL) \\ General Atomics (GA)}

Idaho National Engineering \& Environmental Labs (INEEL)

Lawrence Livermore National Labs (LLNL)

Oak Ridge National Labs (ORNL)

Princeton Plasma Physics Lab (PPPL)

Rocketdyne

Sandia National Labs (SNL)

UC San Diego (UCSD)

University of Wisconsin (UWM) 


\section{APEX Project Groups}

(1) Design Conceptualization and Analysis

Chair: Mohamed Abdou, UCLA. Core of APEX project

(2) Mechanical Design and Availability Group

Chair: Brad Nelson, ORNL. This group will be responsible for assisting all design conceptualization groups in developing mechanical design and integration.

(3) Materials Group

Chair: Steve Zinkle, ORNL. Suggest materials for high power density applications and provide basic material properties for design.

(4) Power Conversion

Chair: D. Sze, ANL. Delineate operating temperature, materials, and technology requirements and issues. Also estimate efficiency as a function of blanket/first wall outlet coolant temperature.

(5) Physics Interface Group

Chair: Dale Meade, PPPL. Provide physics boundary conditions for FPT design (some issues may require interface with the physics community to get the best input.

(6) Safety Group

Chair: Kathy McCarthy, INEL

(7) Alternate Confinement Concepts

Chair: Dale Meade. Summarize the main configuration features and general range of parameters (wall load, surface heat flux, etc.) for alternate confinement concepts and contrast them to tokamaks.

(8) Expert Judgment and Selection Panel (TBD) 


\section{APEX Tasks}

Task 1: Delineate function requirements and develop evaluation Approach (criteria)
A. Special driver criteria (high wall load)
B. General Criteria (economics, safety, environmental)
C. $R \&$ D and potential success criteria

Task 2: Determine the key limiting factors on high power density

- understand the limits to learn how to extend them

Task 3: Explore concepts with high power density capability

- A primary task

- Primary sources of new concepts:

A) concepts previously proposed in literature

B) "Innovation through analogy" to other technologies (e.g. rocket engine)

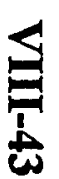

C) "Innovation through pursuit of engineering science logic" (building on what we learn from Task 2) 


\section{Task 4: Preliminary conceptual designs for new concepts}

- Approach:

- Concepts identified in Task 3 will be carefully analyzed and evaluated

- Initially, examine the scientific foundation of the concept

- If a concept has sound scientific basis, a preliminary conceptual design will be attempted to satisfy all functional requirements of FPT

- Only if such effort is successful for a concept, will we attempt to improve and optimize it using the evaluation criteria as a guide

- Please note that some concepts require new models and methods of analysis to predict behavior. This can be a major effort

- Initially, we will not constrain conceptualization too much. For example, low activation will not be an initial requirement.

- Output of this task

a) a set of preliminary conceptual designs for a number of promising concepts

b) preliminary evaluation of each concept

c) a set of key issues for each concept 
Task 5: Comparative evaluation and selection of most promising concepts

- The magnitude of this effort will strongly depend on the outcome of Task 4 , i.e. how many concepts (There may be none, or only one, or many)

- If there are several concepts, then the evaluation criteria developed in Task 1 will be utilized to select the most promising concepts that are worthy of further detailed studies

Task 6: Detailed analysis and evaluation of most promising concepts

- The most promising new concepts selected in Task 5 will be subjected to more comprehensive analysis and detailed evaluation

- Key issues will be identified and key R \& D items will be recommended

Task 7: Study conclusions and report 


\section{APEX: Relationships between Tasks and Groups}

\begin{tabular}{|c|c|c|c|c|c|c|}
\hline & \begin{tabular}{|l}
\multicolumn{1}{c|}{ Task 1 } \\
Functional \\
Requirements, \\
Scientific feasibility, \\
Evaluation \\
Approach
\end{tabular} & $\begin{array}{l}\quad \text { Task 2 } \\
\text { Key Limiting } \\
\text { Factors in } \\
\text { current } \\
\text { concepts }\end{array}$ & \begin{tabular}{l}
\multicolumn{1}{c}{ Task 3} \\
EXPLORE \\
concepts with High \\
Power Density \\
Capabilities
\end{tabular} & \begin{tabular}{|l|}
\multicolumn{1}{|c|}{ Task 4 } \\
Preliminary \\
Conceptual \\
Designs for \\
new concepts
\end{tabular} & \begin{tabular}{l}
\multicolumn{1}{c}{ Task 5} \\
Comparative \\
Evaluation \\
and Selection \\
of most \\
promising \\
concepts
\end{tabular} & $\begin{array}{l}\text { Task } 6 \\
\text { Detailed } \\
\text { Analysis \& } \\
\text { Evaluation of } \\
\text { most } \\
\text { promising } \\
\text { concepts }\end{array}$ \\
\hline $\begin{array}{l}\text { Group 1: } \\
\text { Design } \\
\text { Conceptualization \& } \\
\text { Analysis }\end{array}$ & & $\begin{array}{l}\text { (essentially } \\
\text { complete, no } \\
\text { further work } \\
\text { required) }\end{array}$ & $* * *$ & $* * *$ & X X & X X X \\
\hline $\begin{array}{l}\text { Group 2: } \\
\text { Mechanical Design } \\
\text { and Availability }\end{array}$ & $X$ & & $X X X$ & X X X & X X & X X \\
\hline $\begin{array}{l}\text { Group 3: } \\
\text { Materials }\end{array}$ & $\mathrm{X}$ & & $\begin{array}{l}\text { Material properties } \\
\text { and limits }\end{array}$ & \begin{tabular}{|l|} 
Material \\
properties and \\
limits \\
\end{tabular} & $\mathrm{XX}$ & $\mathrm{XX}$ \\
\hline $\begin{array}{l}\text { Group 4: } \\
\text { Power Conversion } \\
\text { System }\end{array}$ & & & $\begin{array}{l}\text { provide outlet } \\
\text { coolant temp. } \\
\text { requirements and } \\
\eta\left(\mathrm{T}_{\mathrm{in}}, \mathrm{T}_{\text {out }}\right)\end{array}$ & & & \\
\hline $\begin{array}{l}\text { Group 5: } \\
\text { Physics Interface }\end{array}$ & $\bar{X}$ & & $\begin{array}{l}\text { Physics boundary } \\
\text { conditions }\end{array}$ & & & 一 \\
\hline $\begin{array}{l}\text { Group 6: } \\
\text { Safety Environment }\end{array}$ & X X & & & & & \\
\hline $\begin{array}{l}\text { Group 7: } \\
\text { Alternate Confinement } \\
\text { Concepts }\end{array}$ & $X$ & & $\begin{array}{l}\text { Requirements for } \\
\text { alternate concepts- }\end{array}$ & & & \\
\hline $\begin{array}{l}\text { Group 8: } \\
\text { Judgement and } \\
\text { Selection Panel }\end{array}$ & $* * *$ & & & & & \\
\hline
\end{tabular}




\section{Current Work (Next Meeting, Jan. 1998)}

- Material database (FS, V, SiC, Nb-Zr, Ti-SiC, etc.)

- Continue creative evolution of new concepts (prize for best concept, any suggestions??)

- Solidify physics issues

1. Peaking factors

2. Physics radiation scenarios

3. Spectrum of radiated alpha power

4. Alternative confinement concept definition

- Concept Analysis

1. Convective liquid layers

2. Thick liquid walls

3. Porous walls

4. Flowing solid particulates 

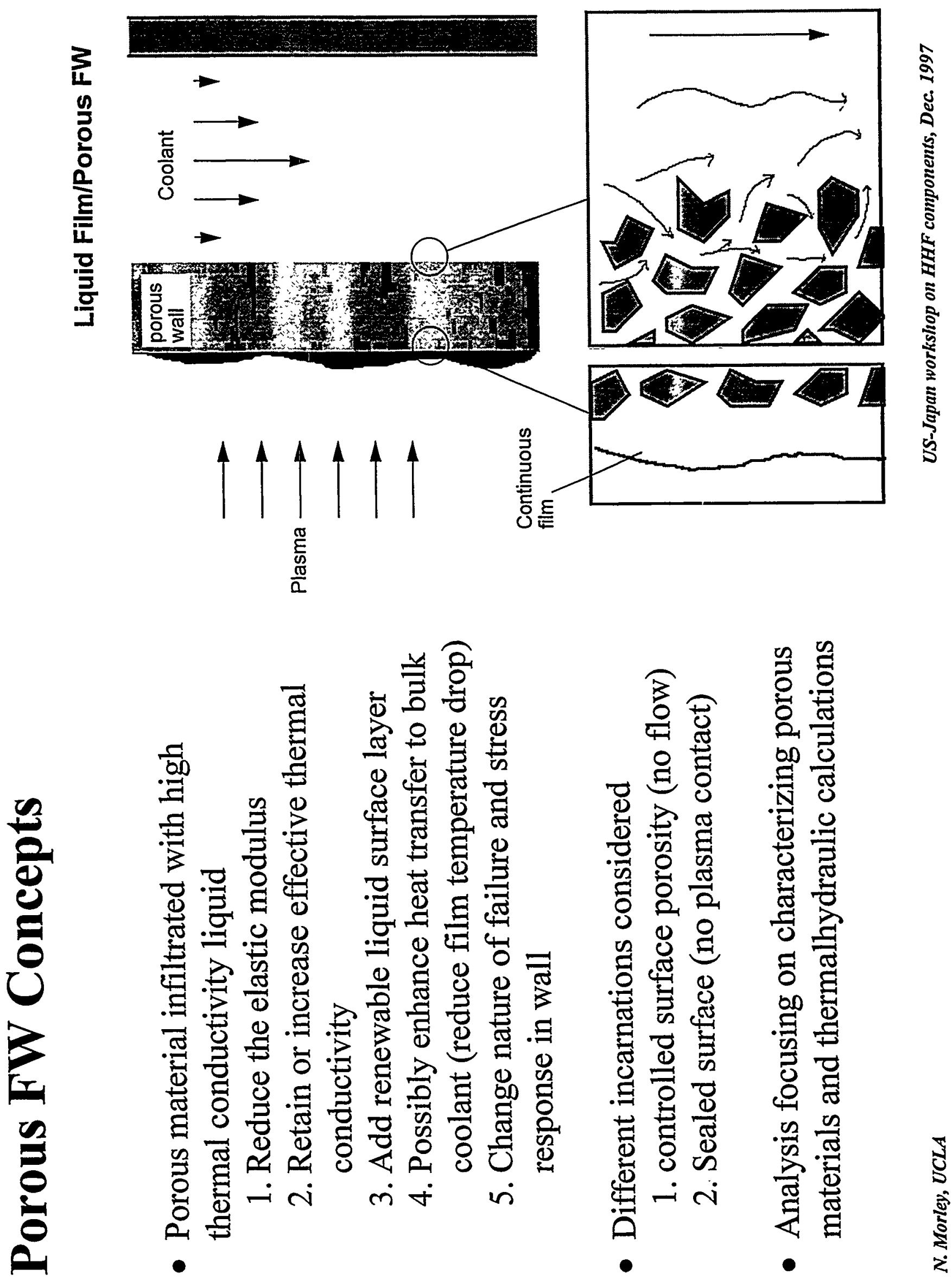


\section{Convective Liquid Layers}

- Fast flowing liquid layer:

1. Removes surface heat flux

2. Removes initial peak in neutron deposition

3. Contributes to breeder and/or neutron multiplication Change nature of failure and stress response in wall

4. Provides renewable surface

- Different incarnations considered

1. Recycle liquid to LM blanket

2. Thick layer (serves as FW/Blanket)

3. Multiple layers

4. EM adhesion

- Analysis focusing on photon penetration depth and thermalhydraulic and fluid mechanical calculations

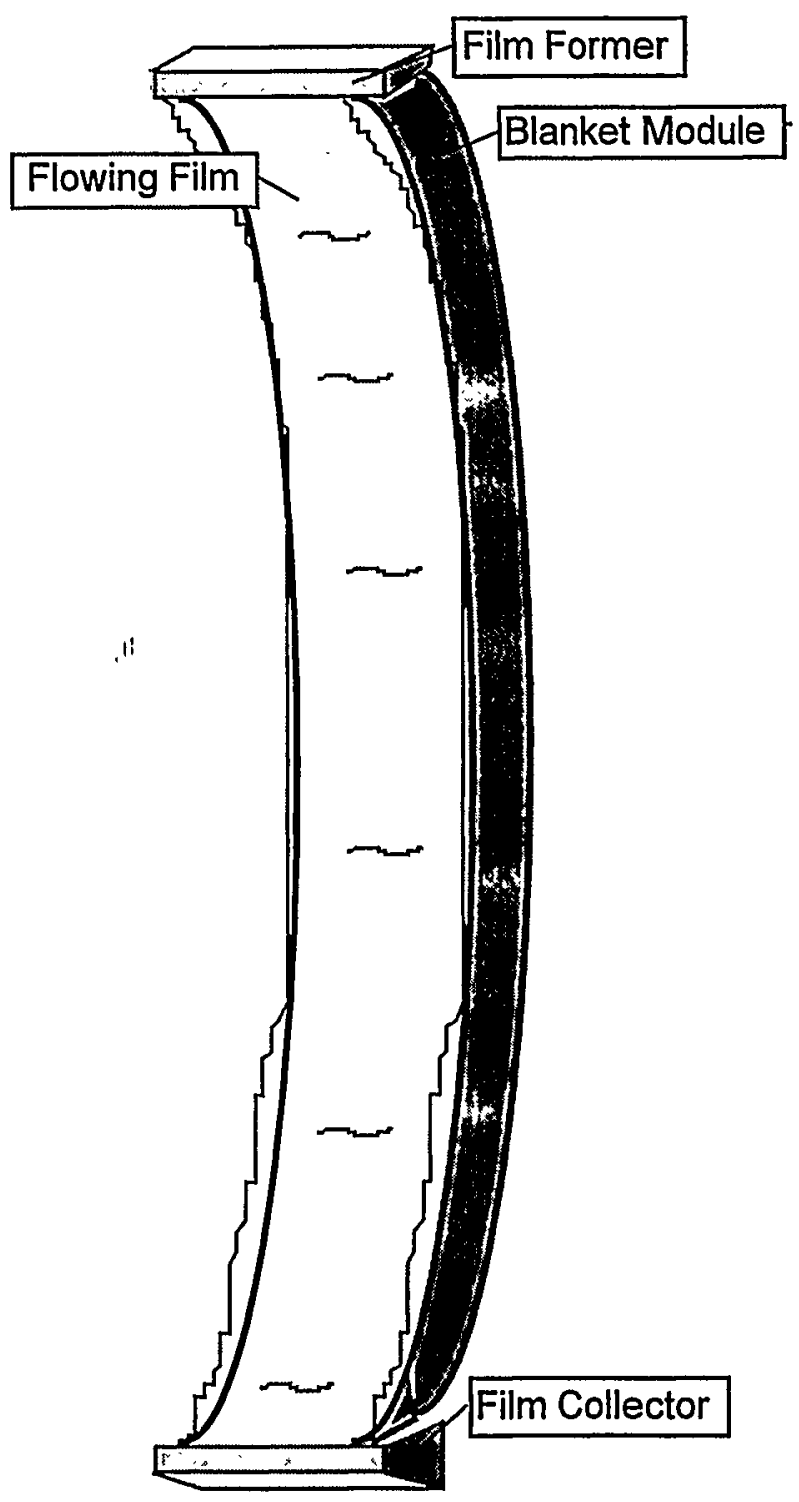




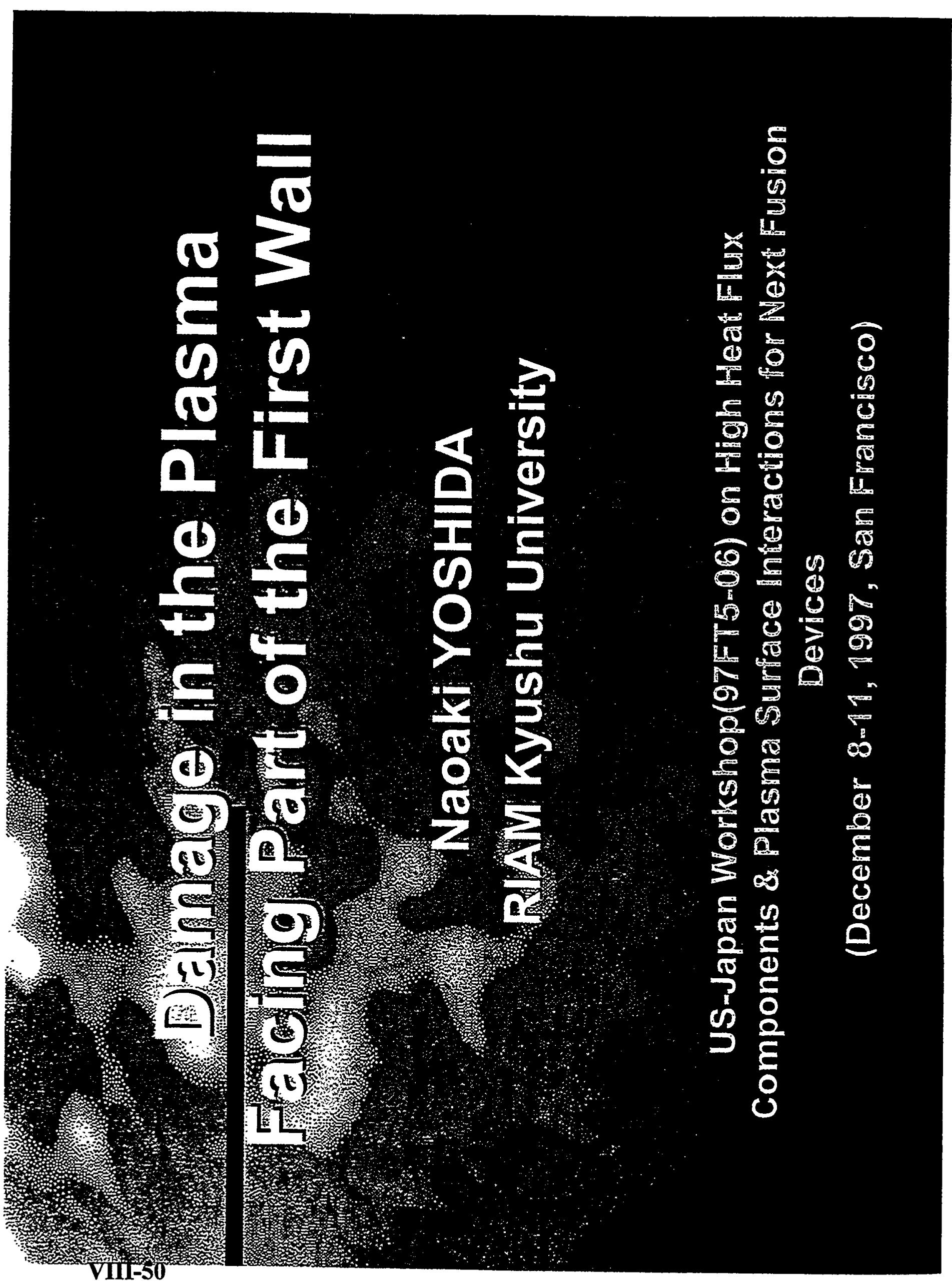




\section{Introduction}

What will happen in PFM by the bombardment of plasma particles, especially energetic particles?

surface damage, effect on bulk properties, etc.

- Damage by Tokamak Plasma

Results from the long pulse TRIAM-1M experiments

- In situ TEM Observation Experiments under Hydrogen Ion Irradiation

- In situ TEM Observation Experiments under Helium Ion Irradiation

- Temperature Variation Effects 


\section{Damage by Long Pulse Plasma in TRIAM-1M}

泉

T. Hirai et al. (P-1A-063)

$\left\langle\mathrm{n}_{\mathrm{e}}>\approx 2 \times 10^{18} / \mathrm{m}^{3}, \mathrm{~T}_{\mathrm{i}} \approx 500 \mathrm{eV}, \mathrm{t}_{\mathrm{j}}=78.4 \mathrm{~min} .(\mathrm{P} 1,5\right.$ shots $), \mathrm{t}_{\mathrm{j}}=72.1 \mathrm{~min} .(\mathrm{P} 2,1$ shot $)$

Tungsten

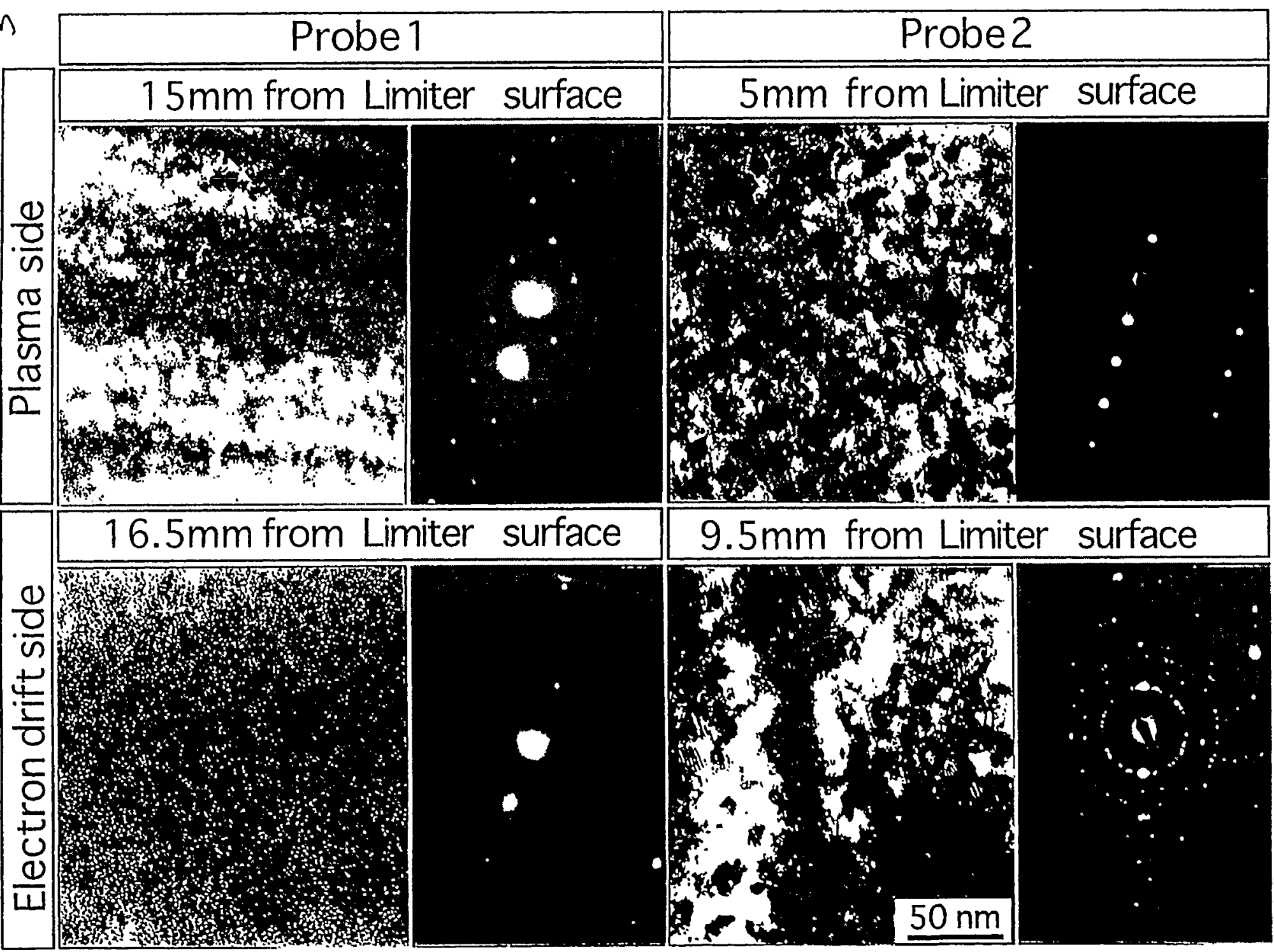




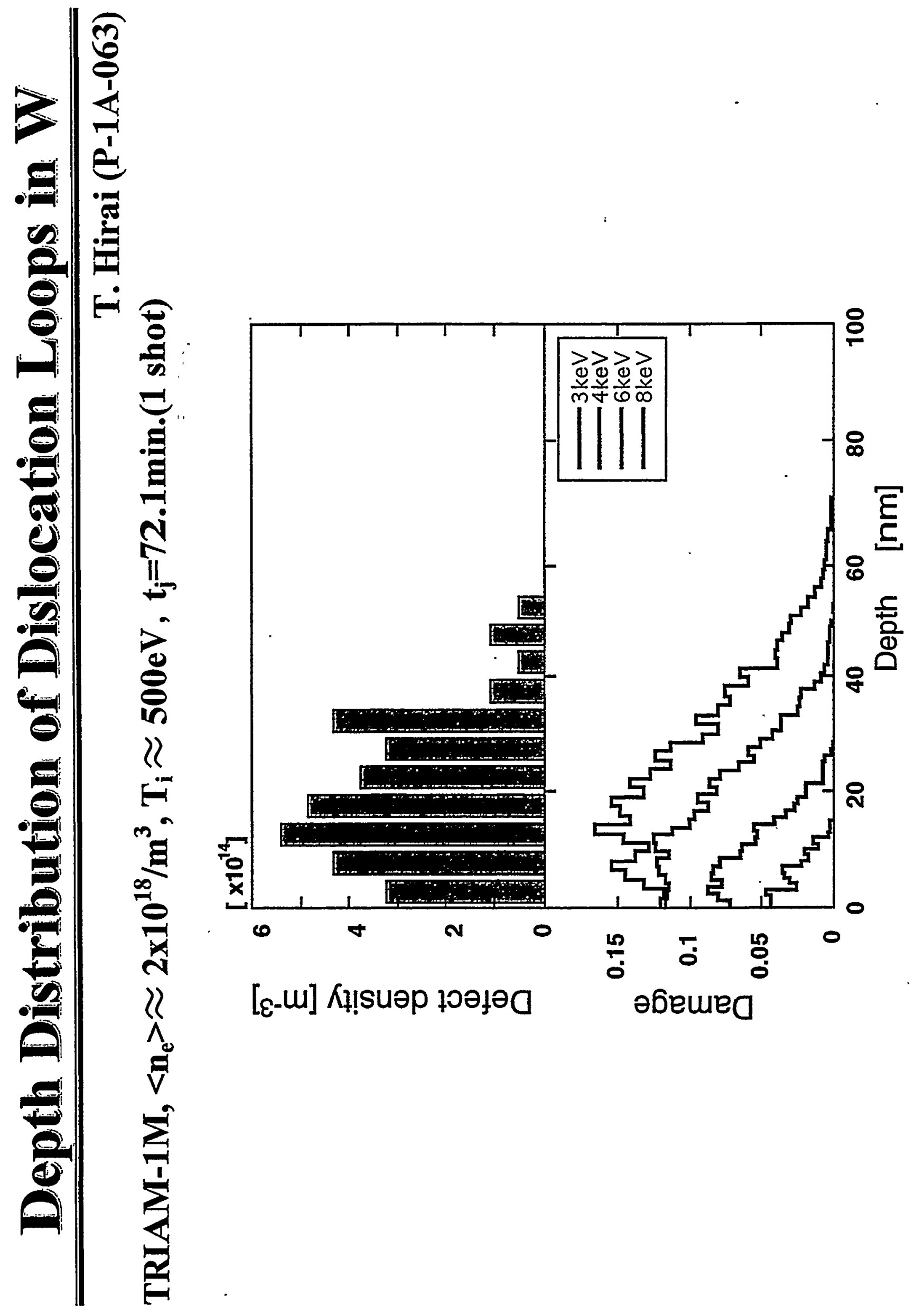




\section{Damage of Long Term W Specimen}

T. Hirai et al. (1997)

Pre-thinned TEM specimen: Exposed to TRIAM-1M LHCD discharges for a half year on the vacuum vessel wall

$\Rightarrow$ Embrittlement of bulk specimen $\cdots$ common phenomena of $\mathrm{H}$ irradiated $\mathrm{W}$

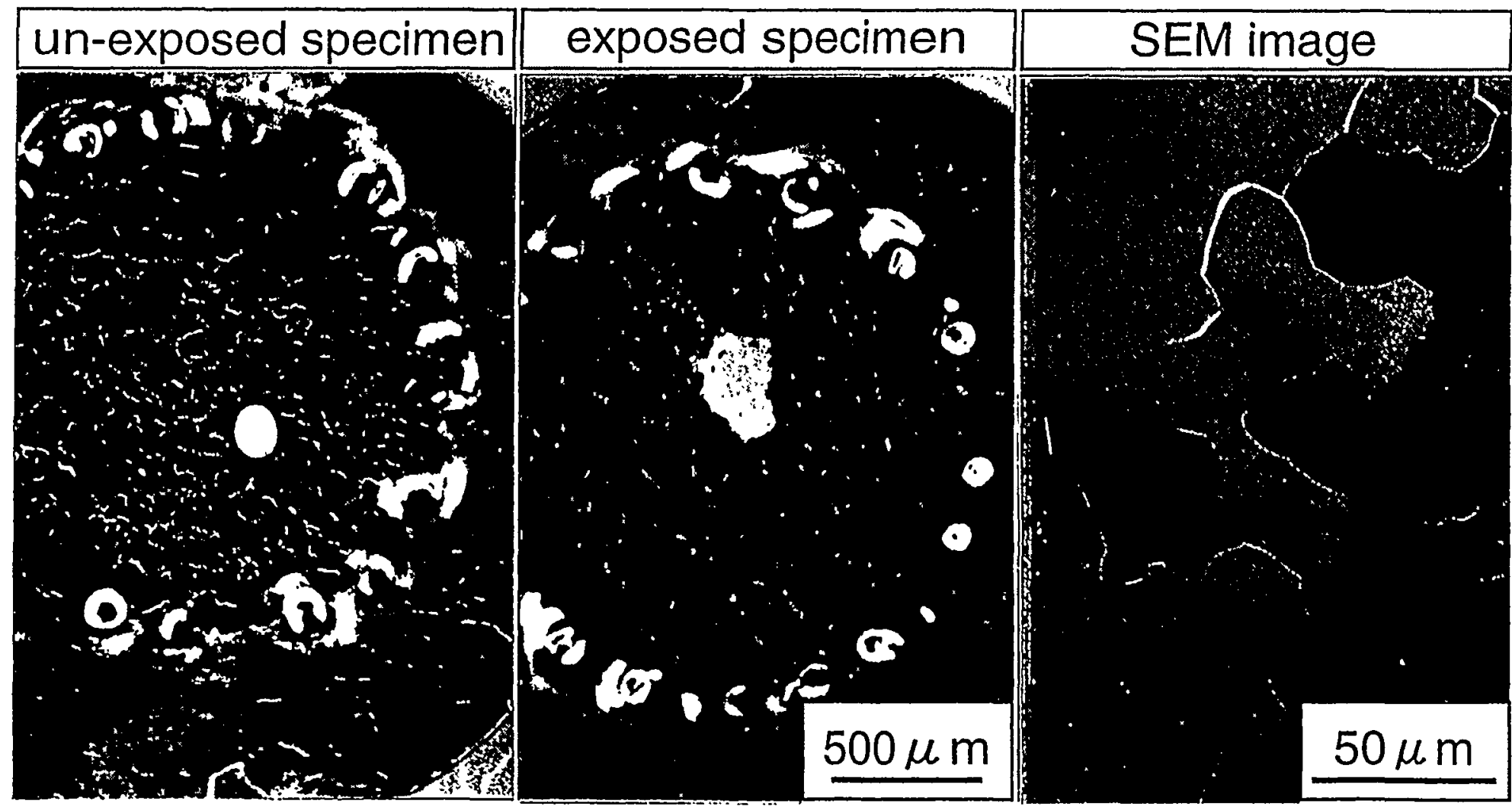




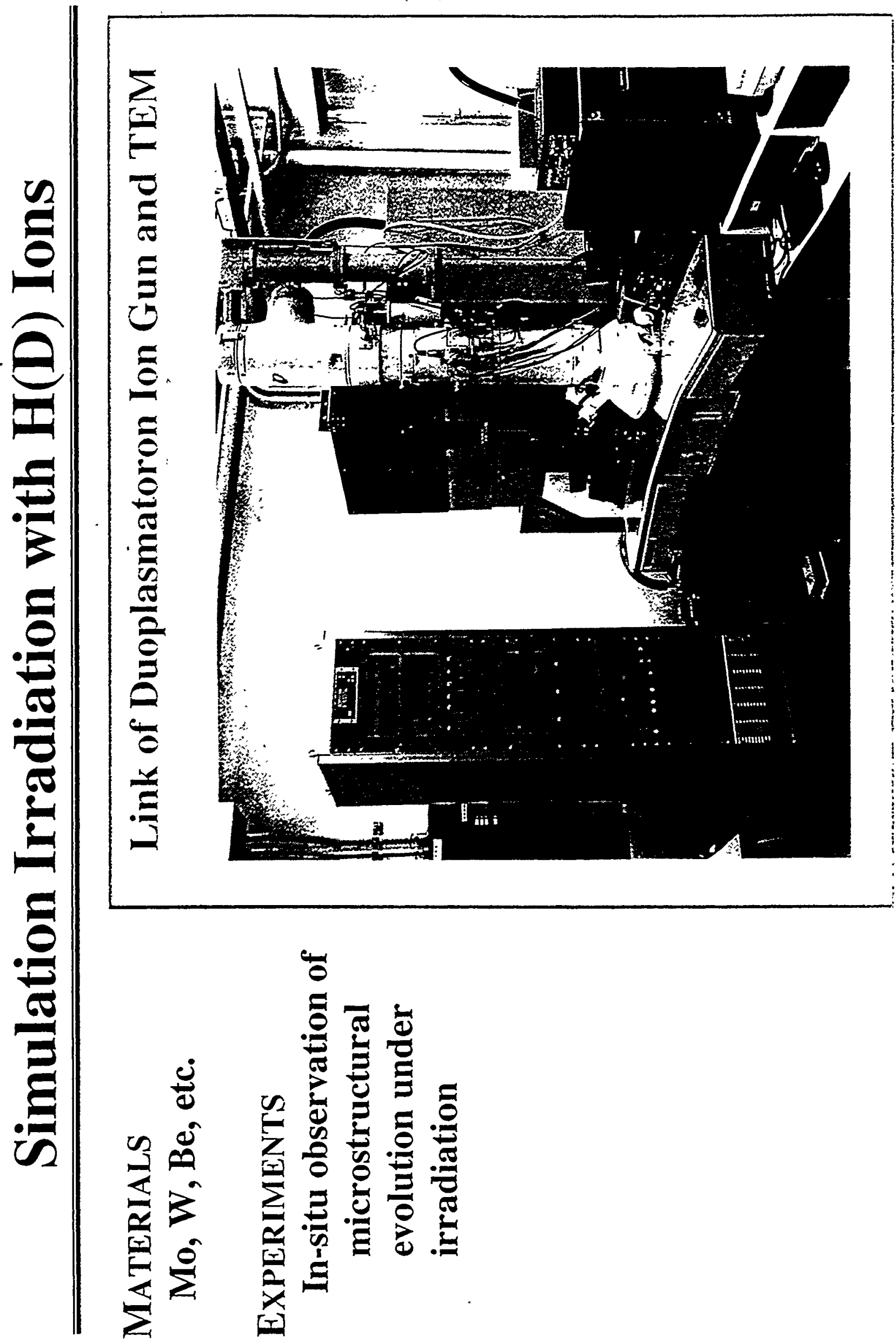




\section{Ion Energy Dependence of Damage in W and Mo by $\mathrm{H}^{+}$Irradiation at $300 \mathrm{~K}$}

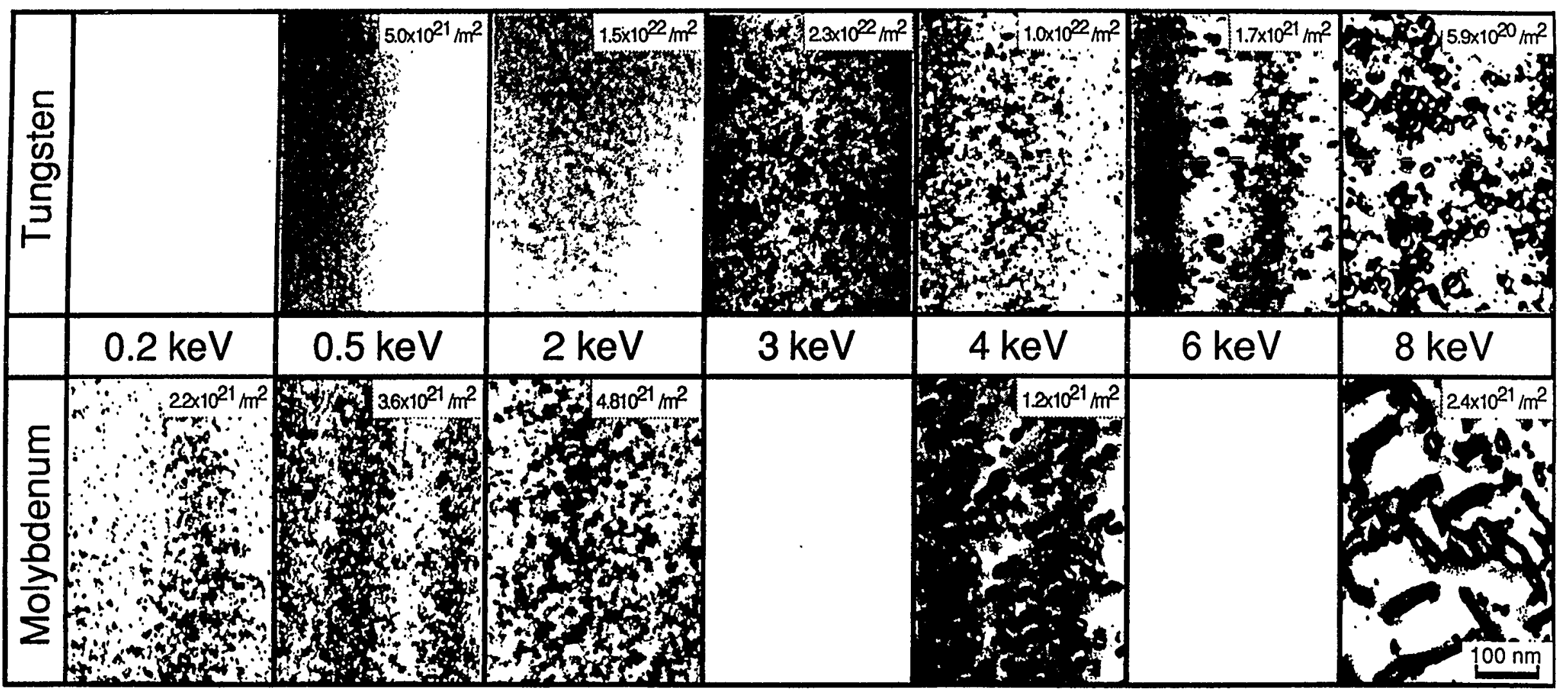




\section{Temp. Dependence of Damage of $\mathrm{W}_{\text {by }} \mathrm{H}^{+}$Irrad.}

8keV H ${ }^{+}, \mathrm{HP}-\mathrm{W}(99.995 \%), \mathrm{PM}-\mathrm{W}(99.95 \%)$

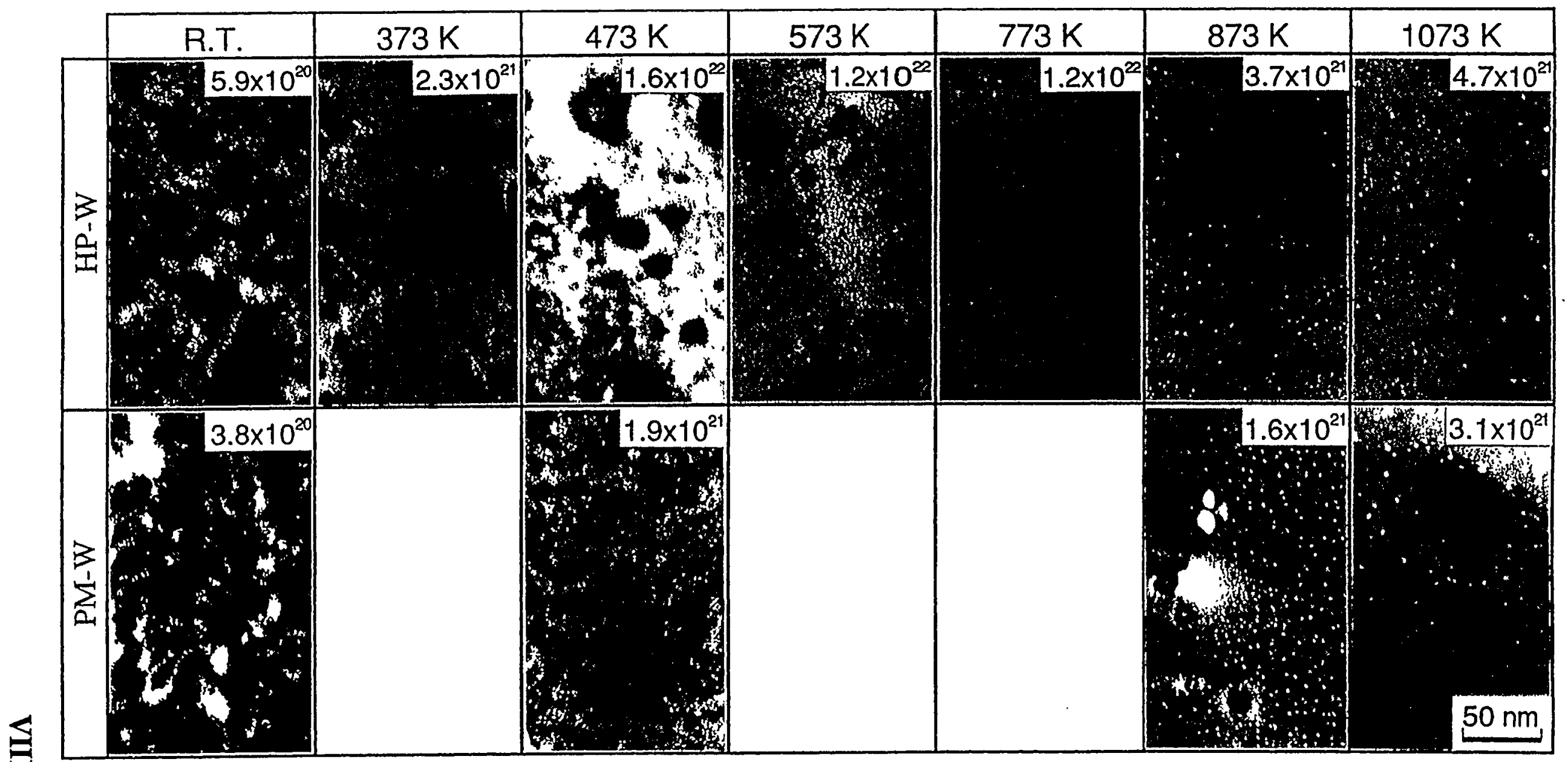




\section{Thermal Stability of Loops Formed by $\mathrm{H}^{+}$Irrad.}

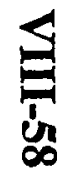

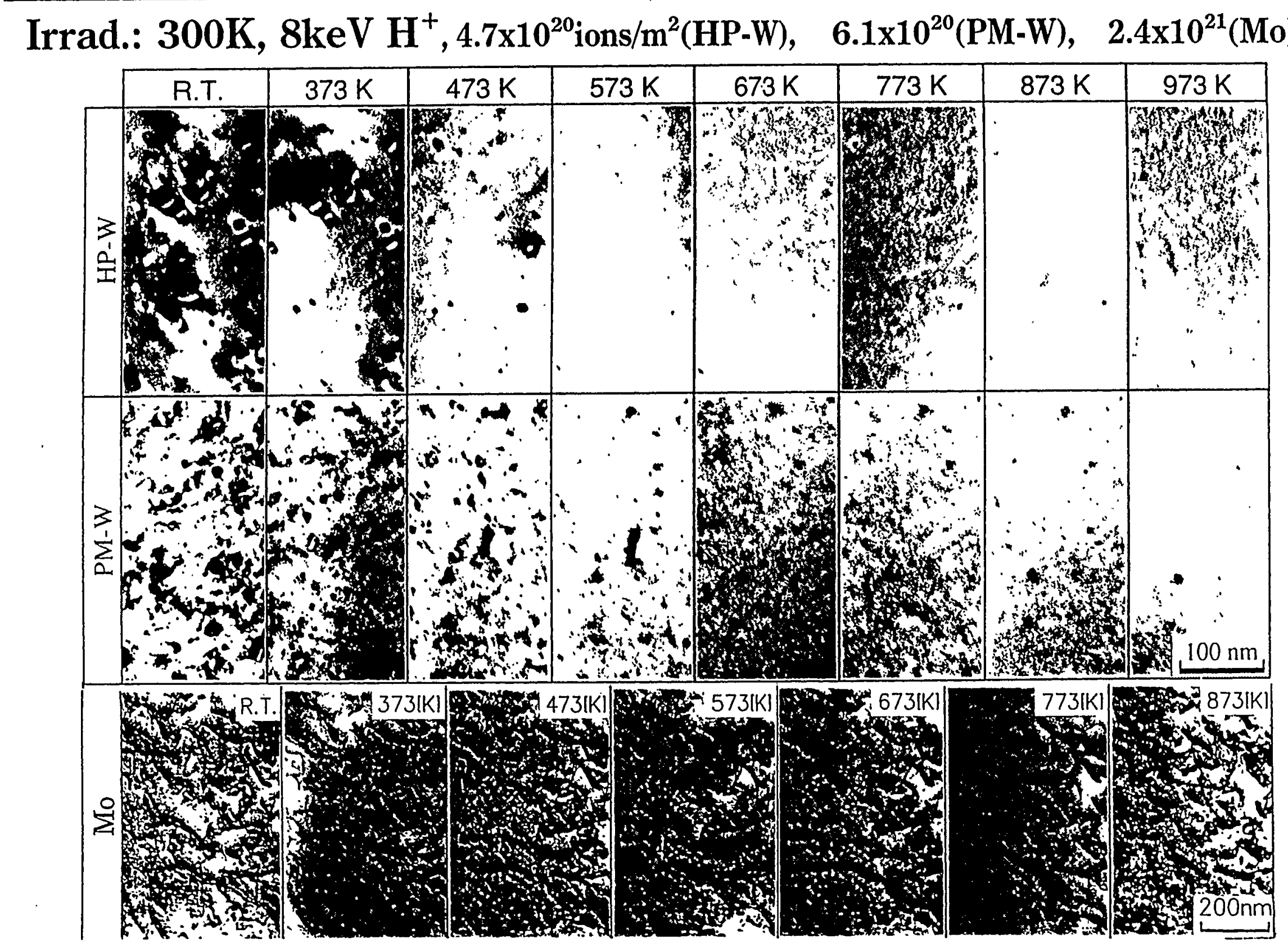




\section{Microstructure of D Ion Irradiated Be}

\section{$8 \mathrm{keV}-\mathrm{D}_{2}^{+}, 5 \times 10^{18}$ ions $/ \mathrm{m}^{2} \mathrm{~s}$}

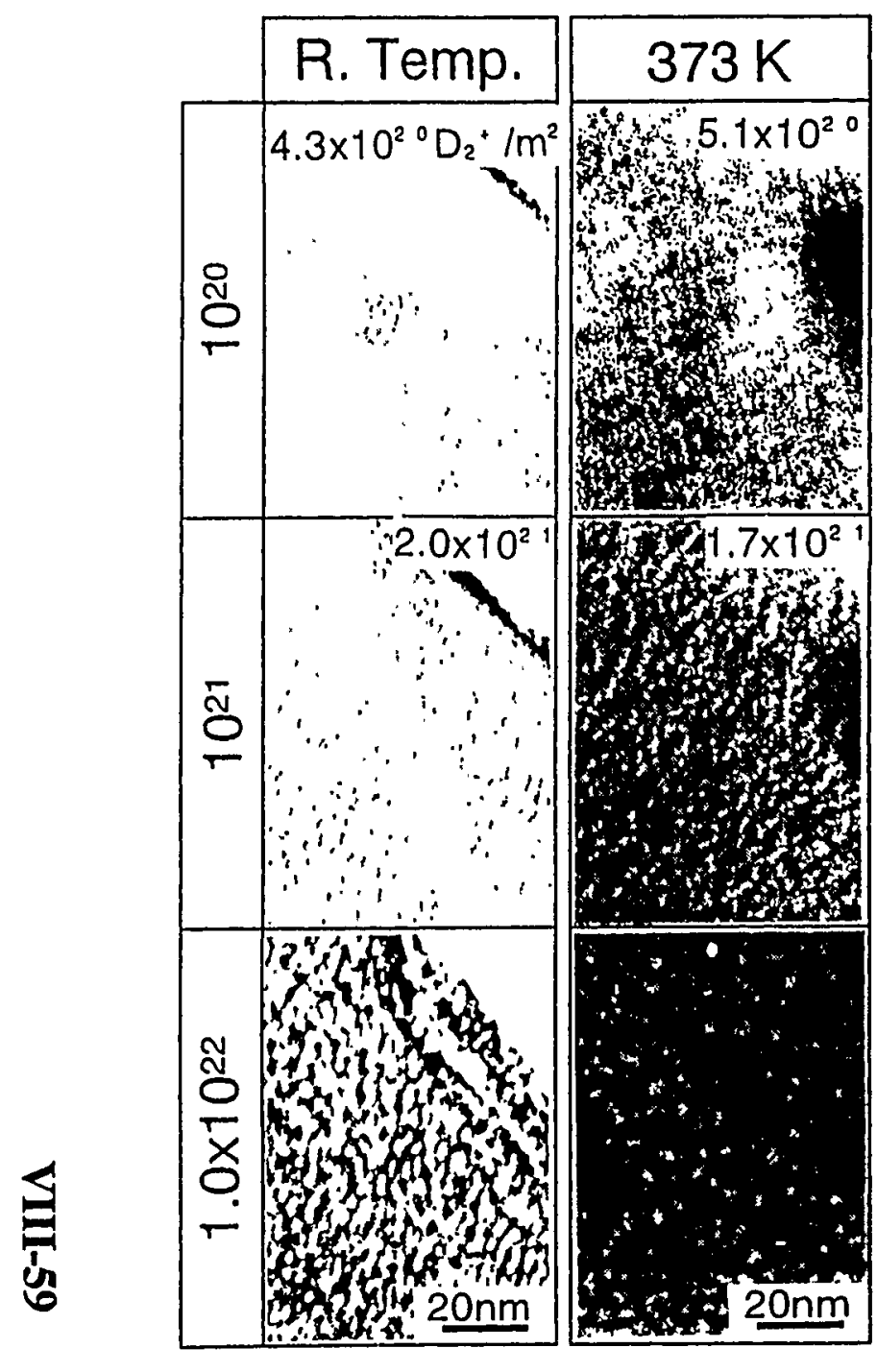

N. Yoshida et al.(1996

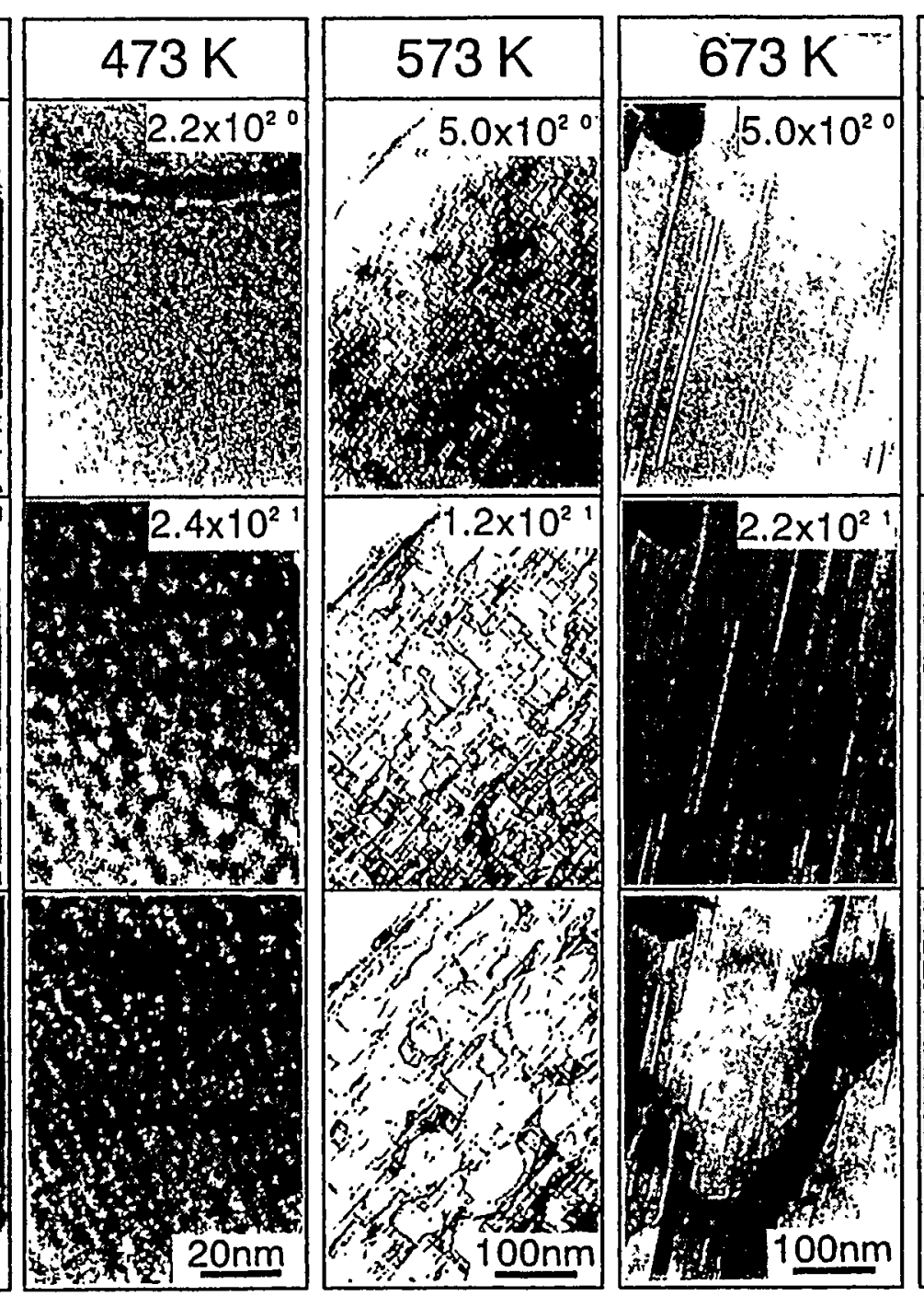

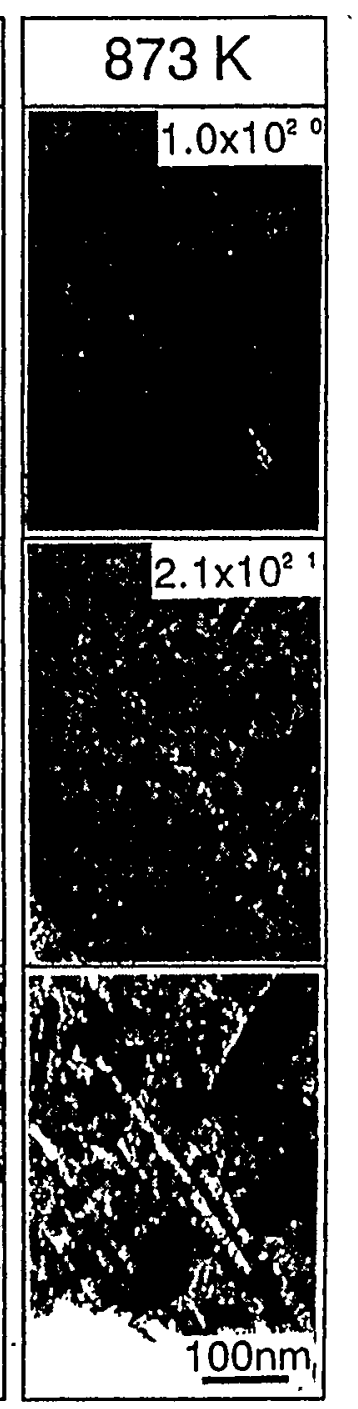




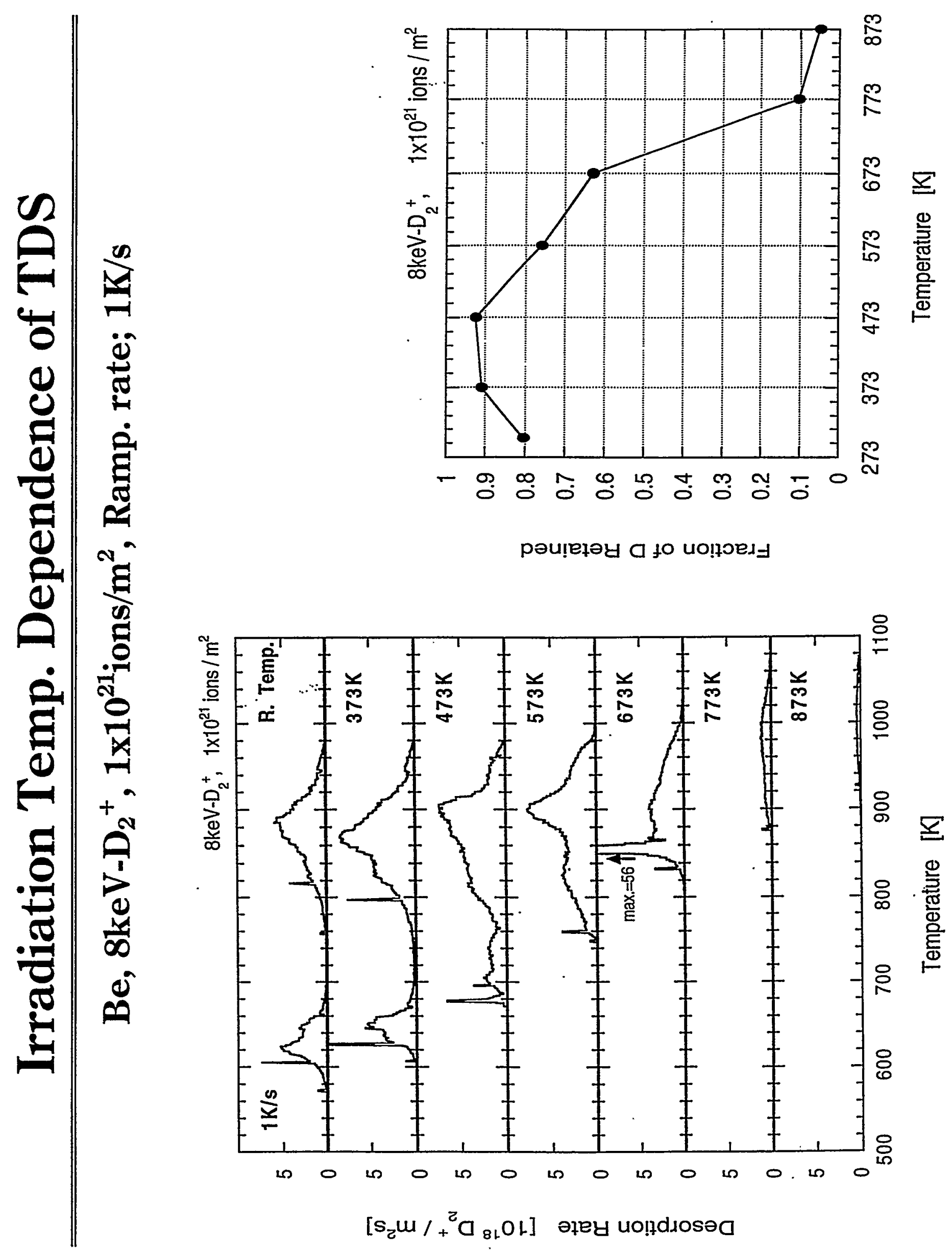




\section{Degradation Mechanism of PFM by Hydrogen}

(1) Elementary Phenomena

Direct knock-on damage of sub-surface region

$\square$

Dislocation network, bubbles" "hydrogen retention, hardening, etc.

W: weak effects at high temperature

Be: strong effects even up to $673 \mathrm{~K}$

(2) Secondary Phenomena

Bulk damage due to long range diffusion of $H$ and free defects

$\square$

Strong degradation of bulk materials (embrittlement, etc.)

The effects of hydrogen particle bombardments are not limited in the subsurface regions but spread into bulk. 


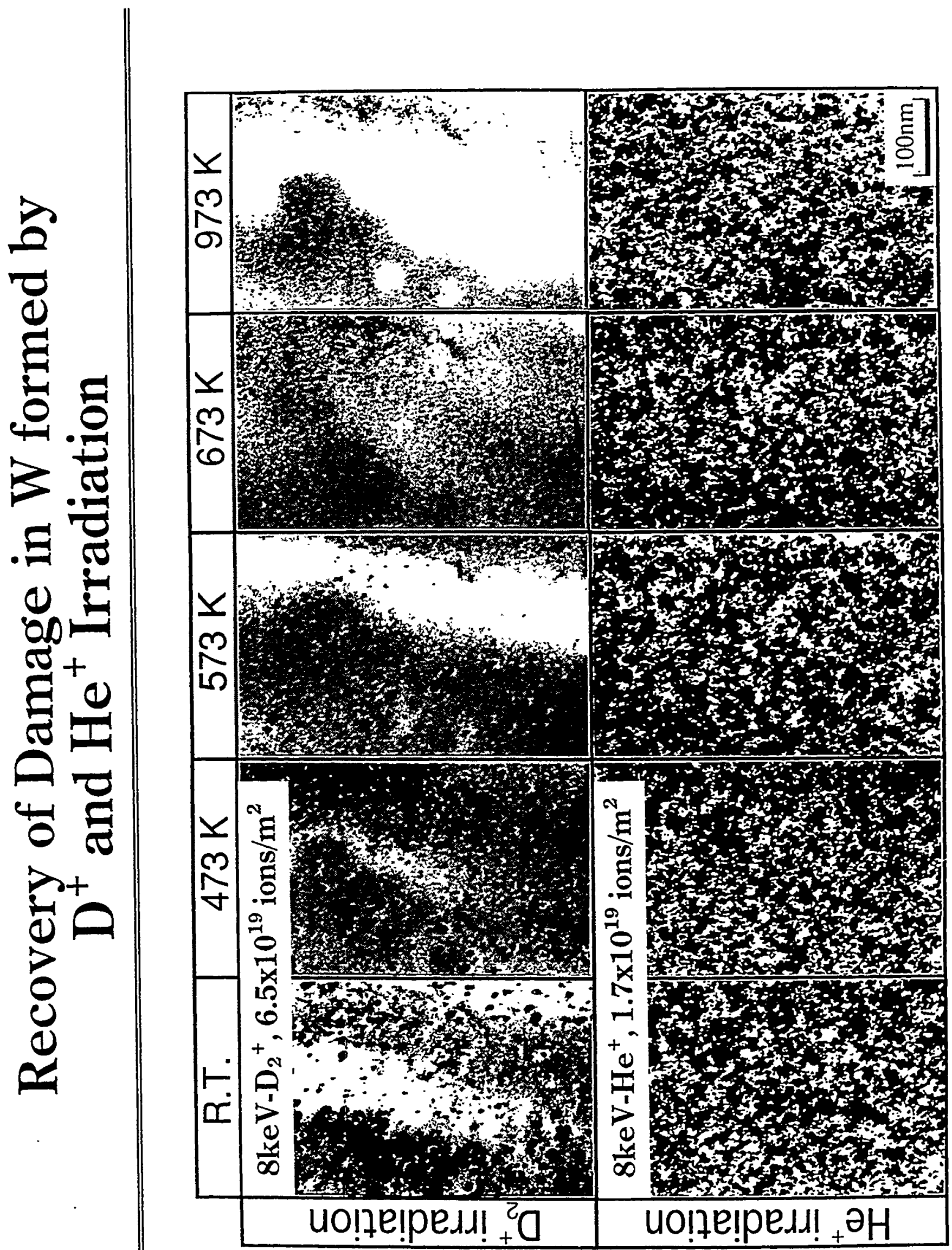




\section{Microstructure of He Ion Irradiated Mo}

N. Yoshida et al.(P1A-043)

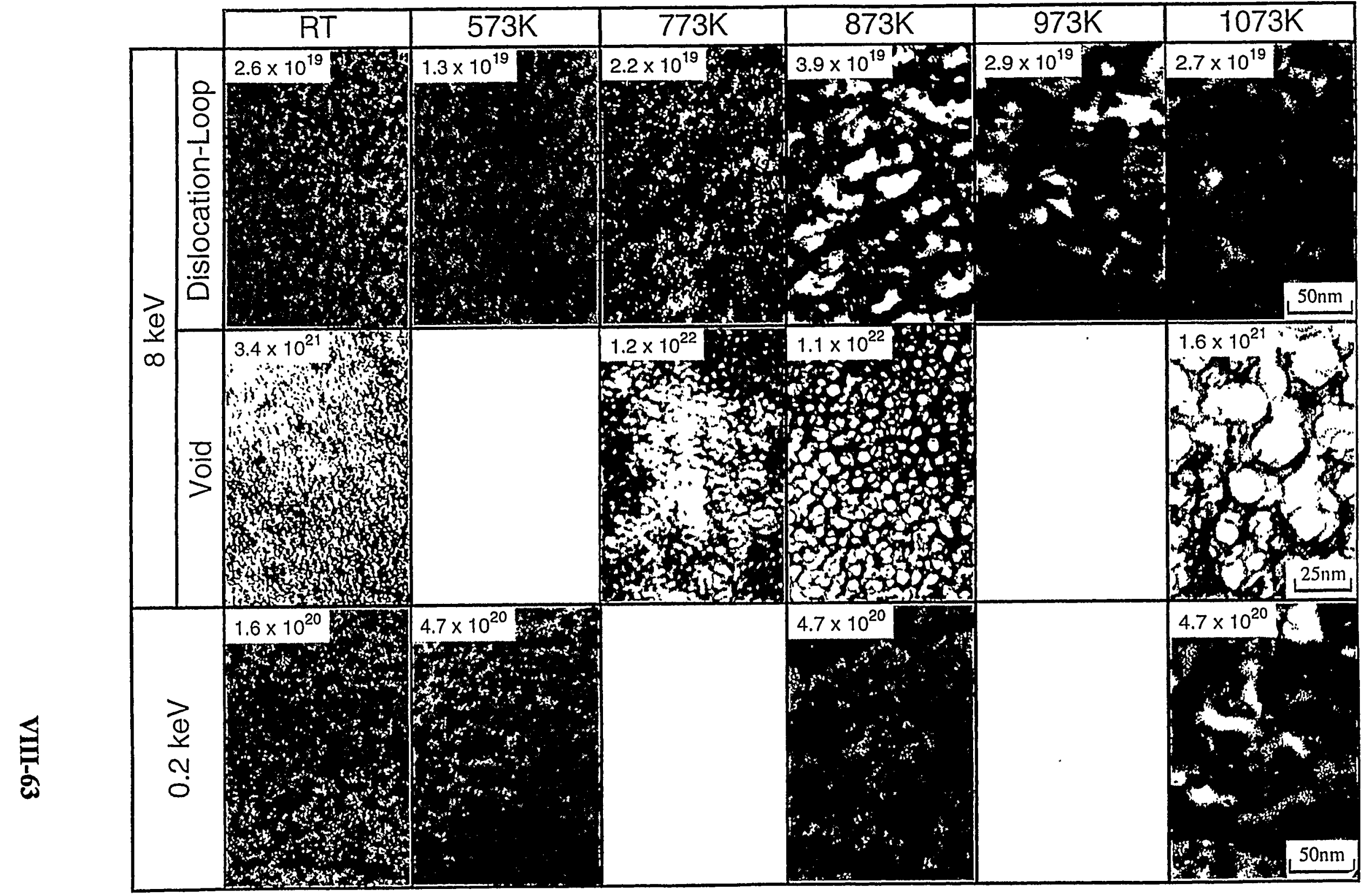




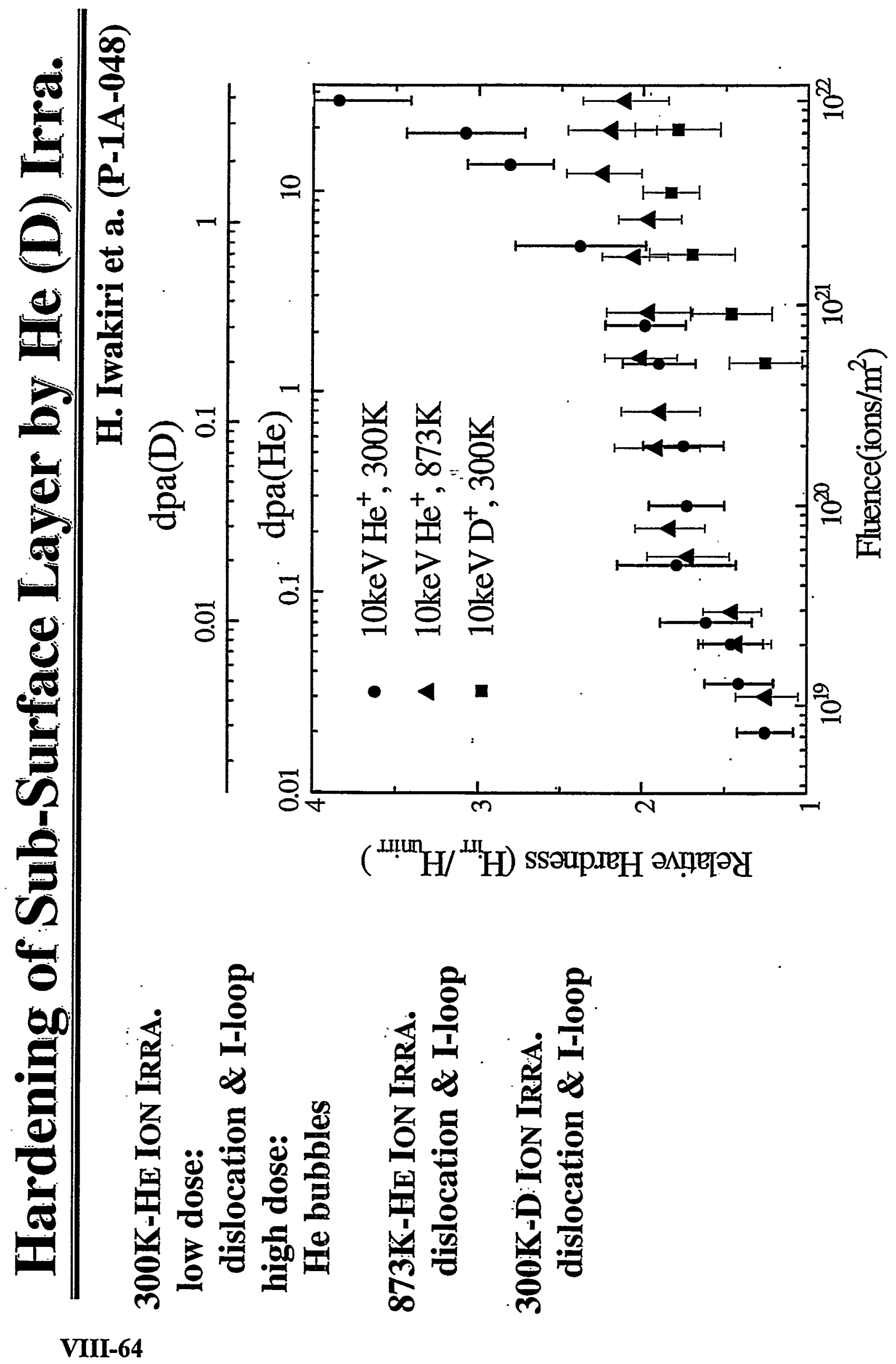




\section{Effects of He Irra. on Heat Loading Properties}

\section{Specimen:}

Powder Metallurgy W

He Ion Irradiation:

8keV-He Ions, $5 \times 10^{21}$ ions $/ \mathrm{m}^{2}$

Room Temp.

Heat Loading (Electron Beam): $20 \mathrm{MW} / \mathrm{m}^{2}$

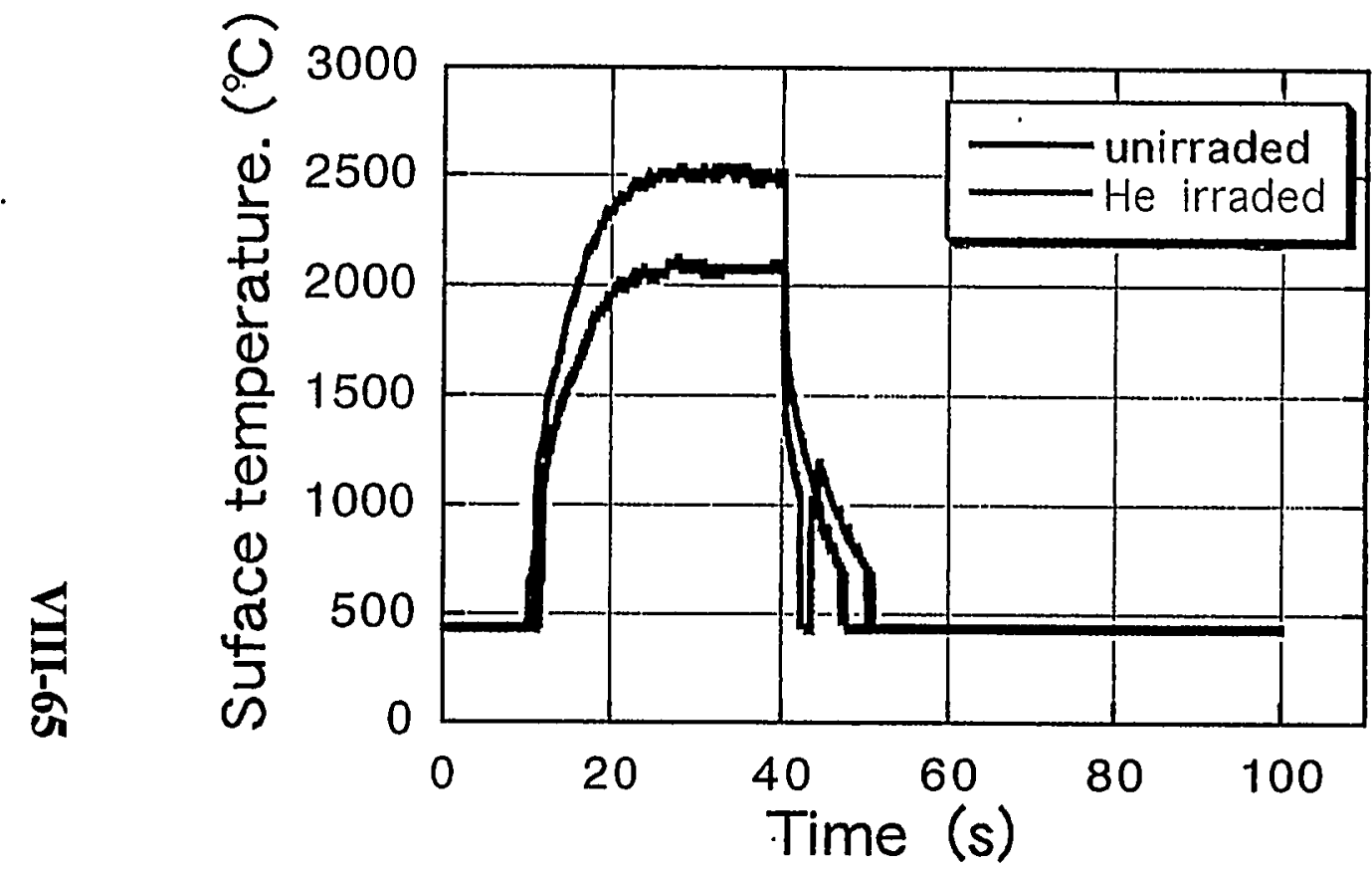

K. Makise et al.(1997)
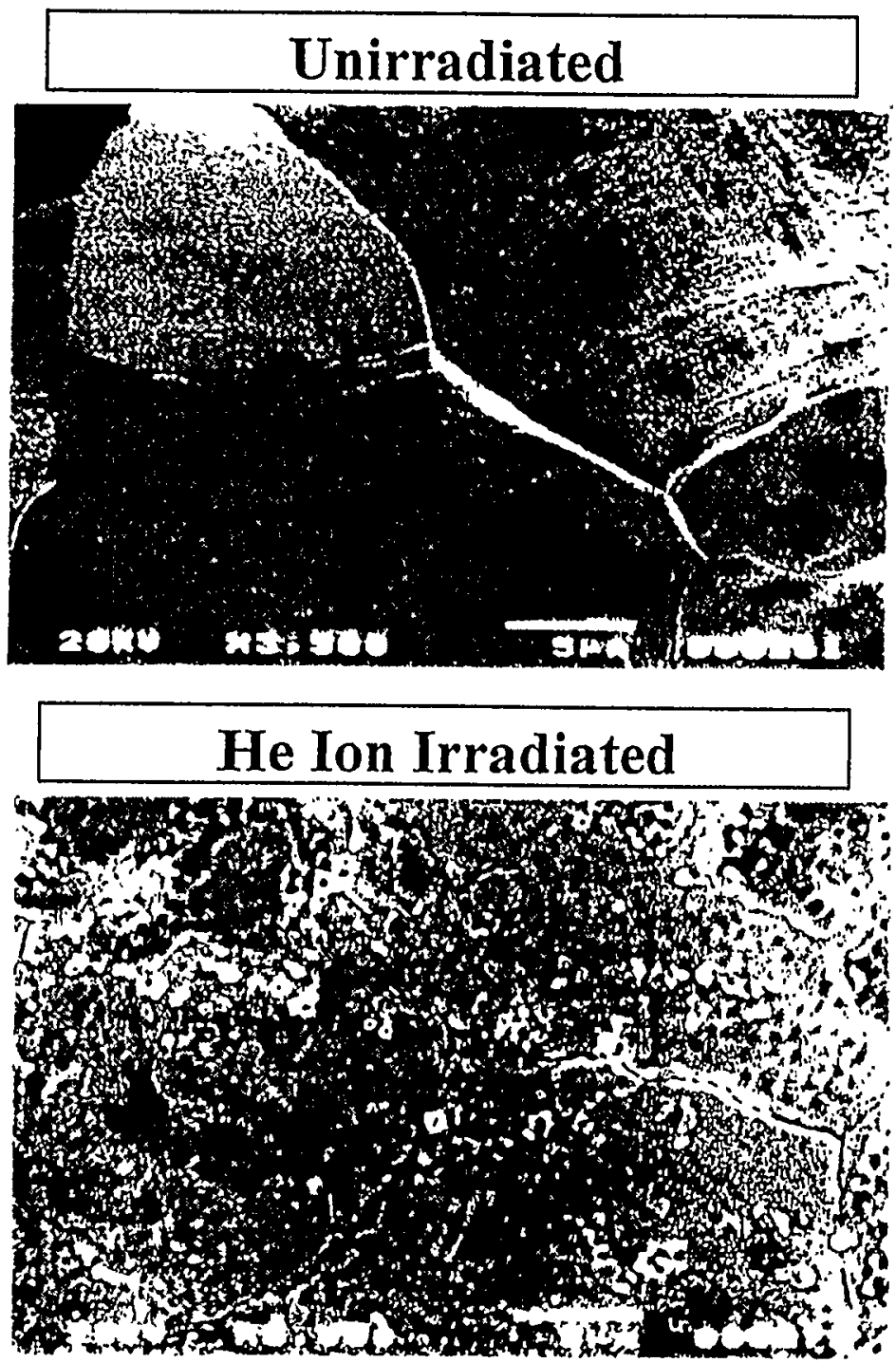


\section{Microstructure of He Ion Irradiated Be}

营

\section{$8 \mathrm{keV}-\mathrm{He}^{+}, 2 \times 10^{18}$ ions $/ \mathrm{m}^{2} \mathrm{~s}$}

T. Inoue et al.(1997)

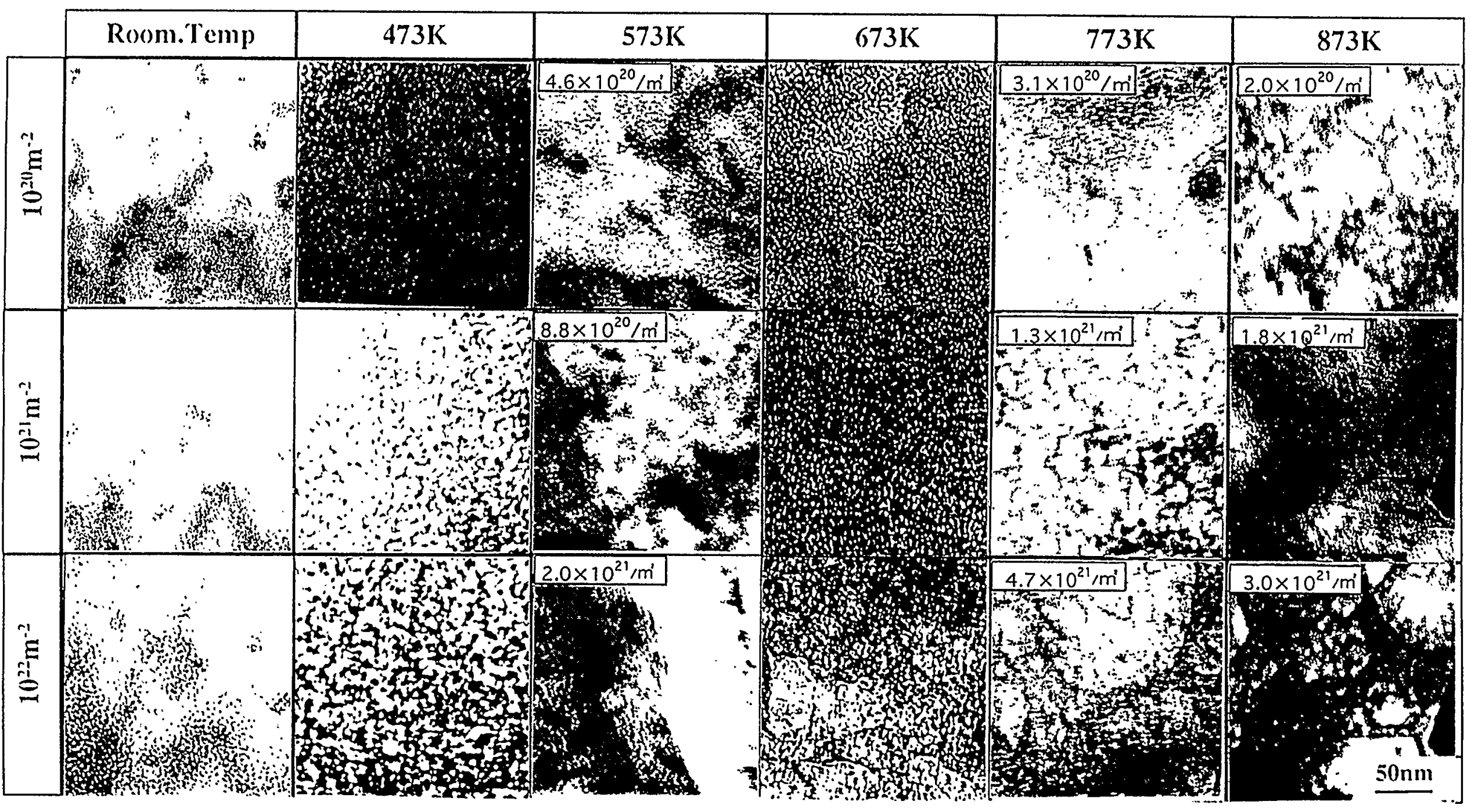




\section{Impacts of He Irra. on Material Properties}

Accumulation of defects is extremely high due to strong He-defect interaction.

- Accumulation of dislocation loop up to very high temp. (>1073K)

(high nucleation rate, strong stability "*)

- Active formation of He bubbles from low to high temp.

- Hardening, embrittlement of subsurface layer

- Embrittlement of grain boundary and matrix of bulk materials

- Reduction of thermal conductivity at subsurface layer

$\llbracket$

- Radiation embrittlement, Reduction of fatigue lifetime

- Increasing of tritium retention

紊

- Reduction of heat load resistance 


\section{Variation of Irradiation Environment --memperature Variation Effects--.-}

F

thermally activated process

(mobility of defects, binding force of defects, etc.)

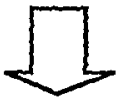

temperature is very essential for defect formation and damage accumulation

Irradiation Effect constant temp.irra. vs varying temperature irra.

Heating Effects slow heating vs fast heating 


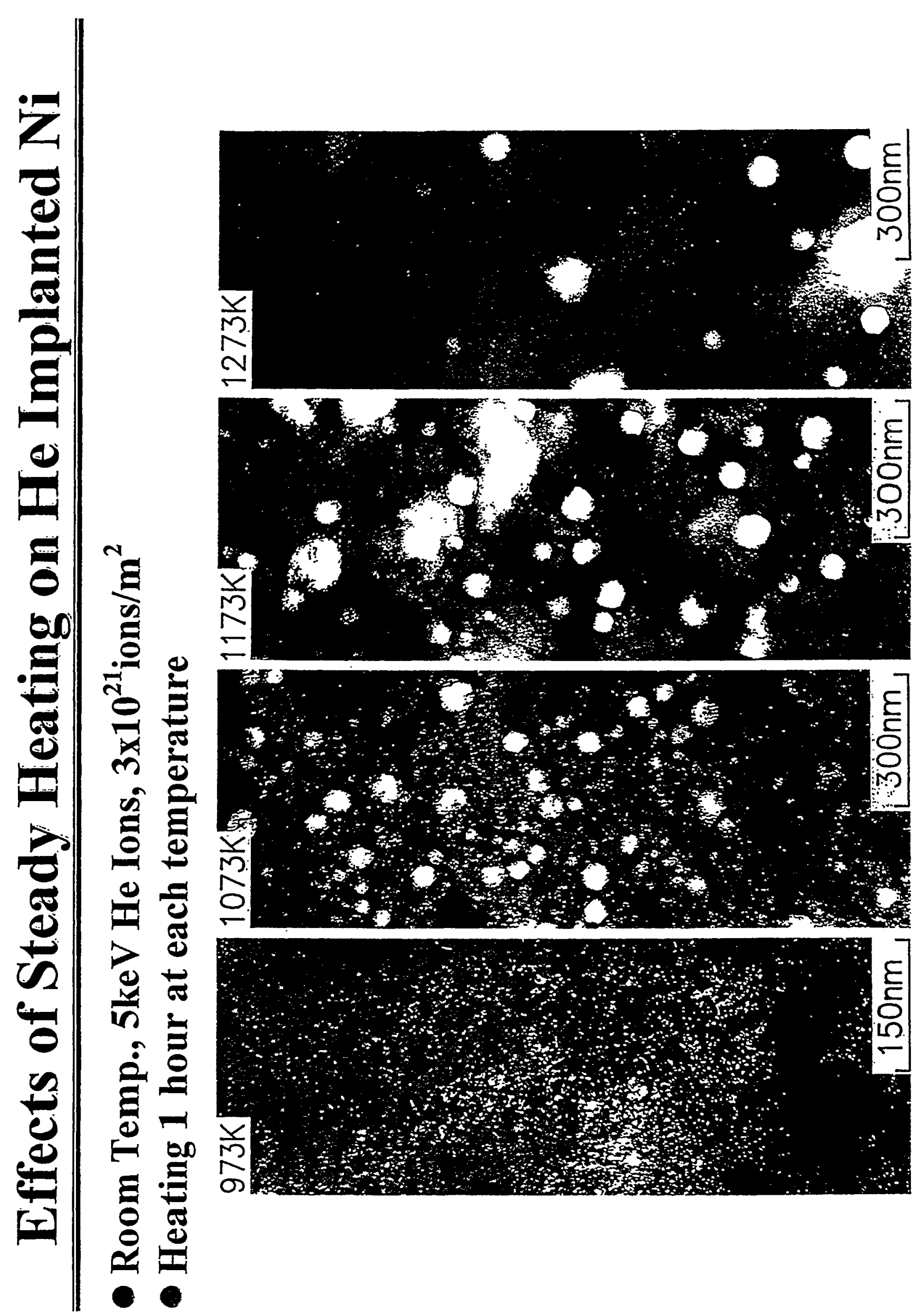




\section{Effects of Pulse Heating on He Implanted Ni}

- Room Temp., $5 \mathrm{keV}$ He Ions, $3 \times 10^{21}$ ions $/ \mathrm{m}^{2}$

- Ruby laser heating (1ms pulse, heating rate $\left.\sim 1000^{\circ} \mathrm{C} / \mathrm{ms}\right)$
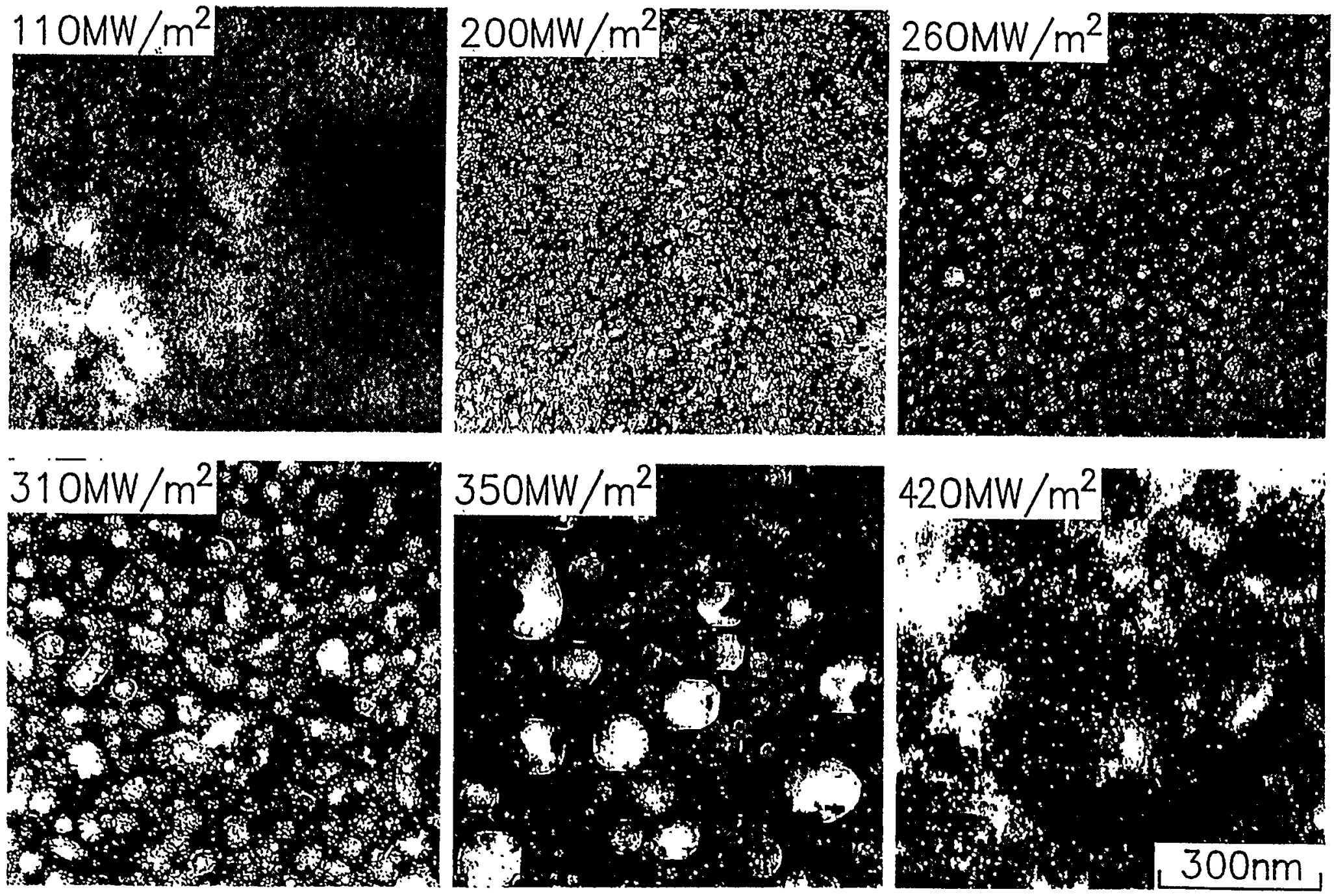


\section{Summary}

- Change exchanged neutrals cause heavy damage at first wall surface.

- Effect of implanted $H$ on bulk properties of PFM was demonstrated. This should be a next important issue.

- Energetic $H$ and He form sponge-like microstructure in Be at wide temperatures range.

$\Longrightarrow$ Tritium inventory, degradation of thermal and mechanical properties

- Helium irradiation enhances formation of defects such as I-loops and bubbles in Mo and $W$ at wide temperature range but rather weak effect of $\mathrm{H}$.

- Pulse heating changes microstructure very much; enhance bubble formation

- Irradiation effects under varying temperature condition and synergistic effects of plasma-neutron-heat is the next issue. 


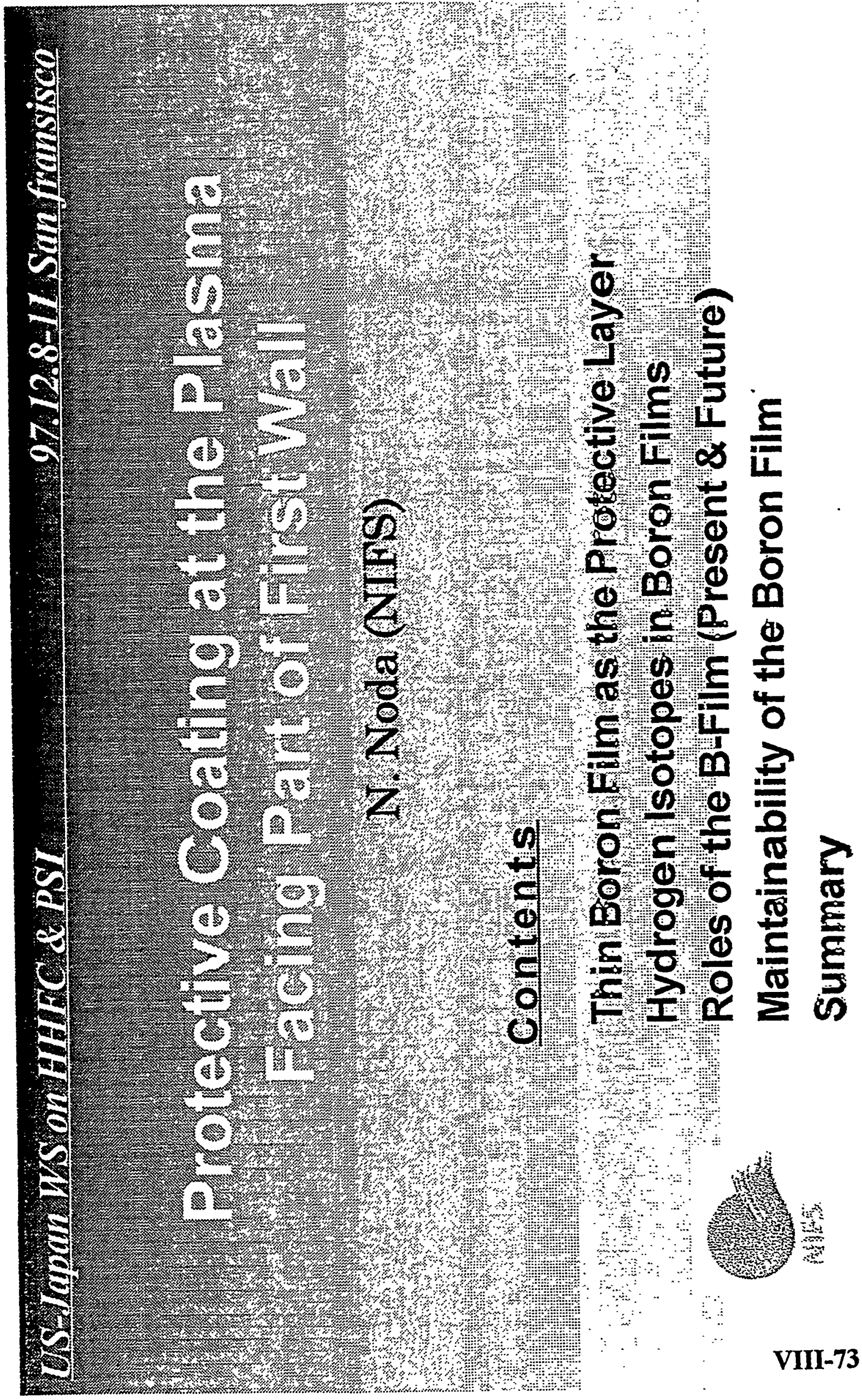




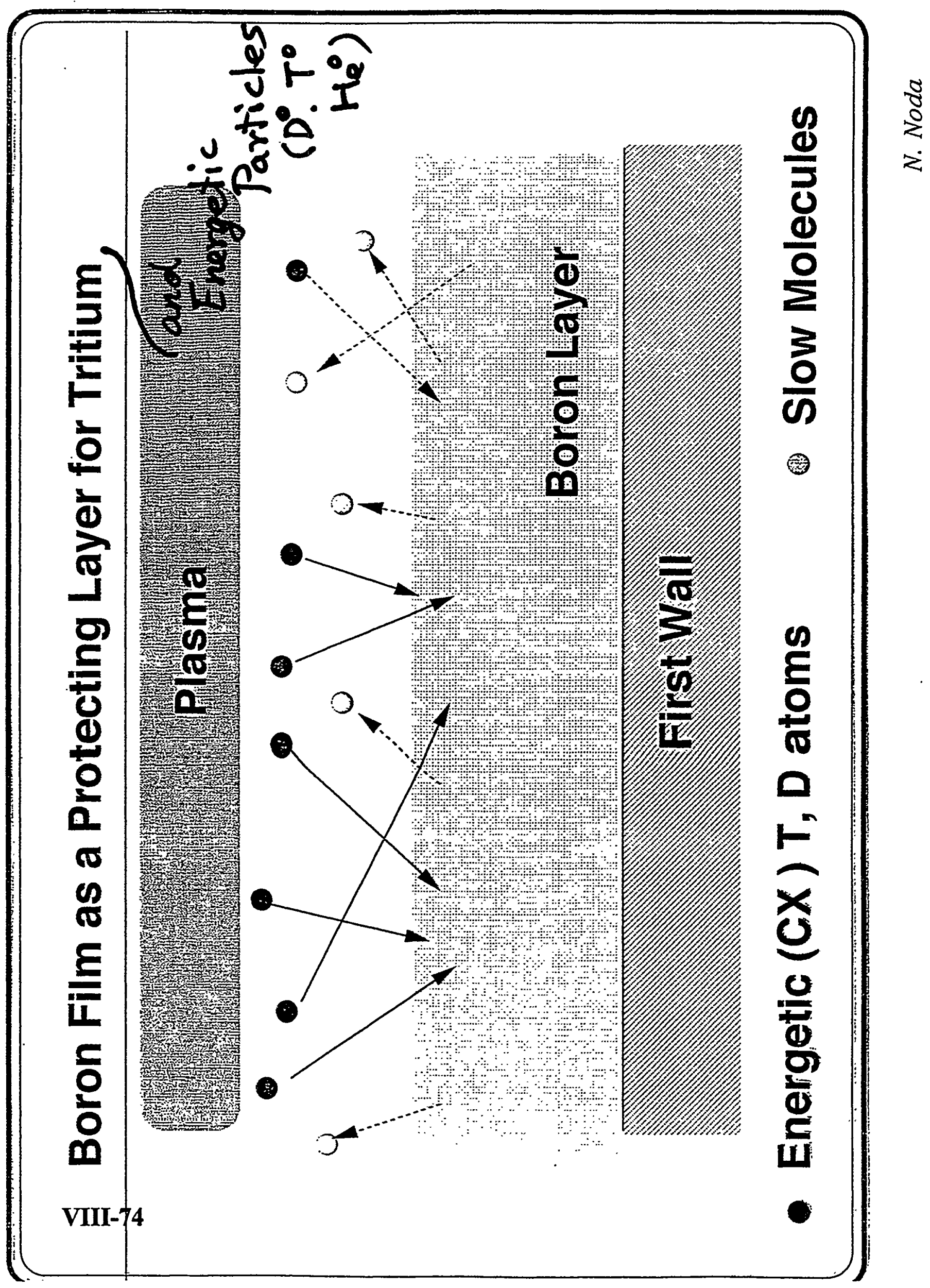




\section{What is the role of B-film? .}

Present Roles of reduction in

(1) oxygen (2) hydrogen? (3) wall materials?

Trelative to $\mathrm{C}$ walls

Future

Roles of (1), (2) must be taken over by divertor pumping

(3) will be effective

(4) T-free wall

(5) protection from energetic particle

$\frac{3}{3}$ 


\section{Thermal Desorption Experiment in SUT}

Hydrogen is removed from B-film below $400{ }^{\circ} \mathrm{C}$

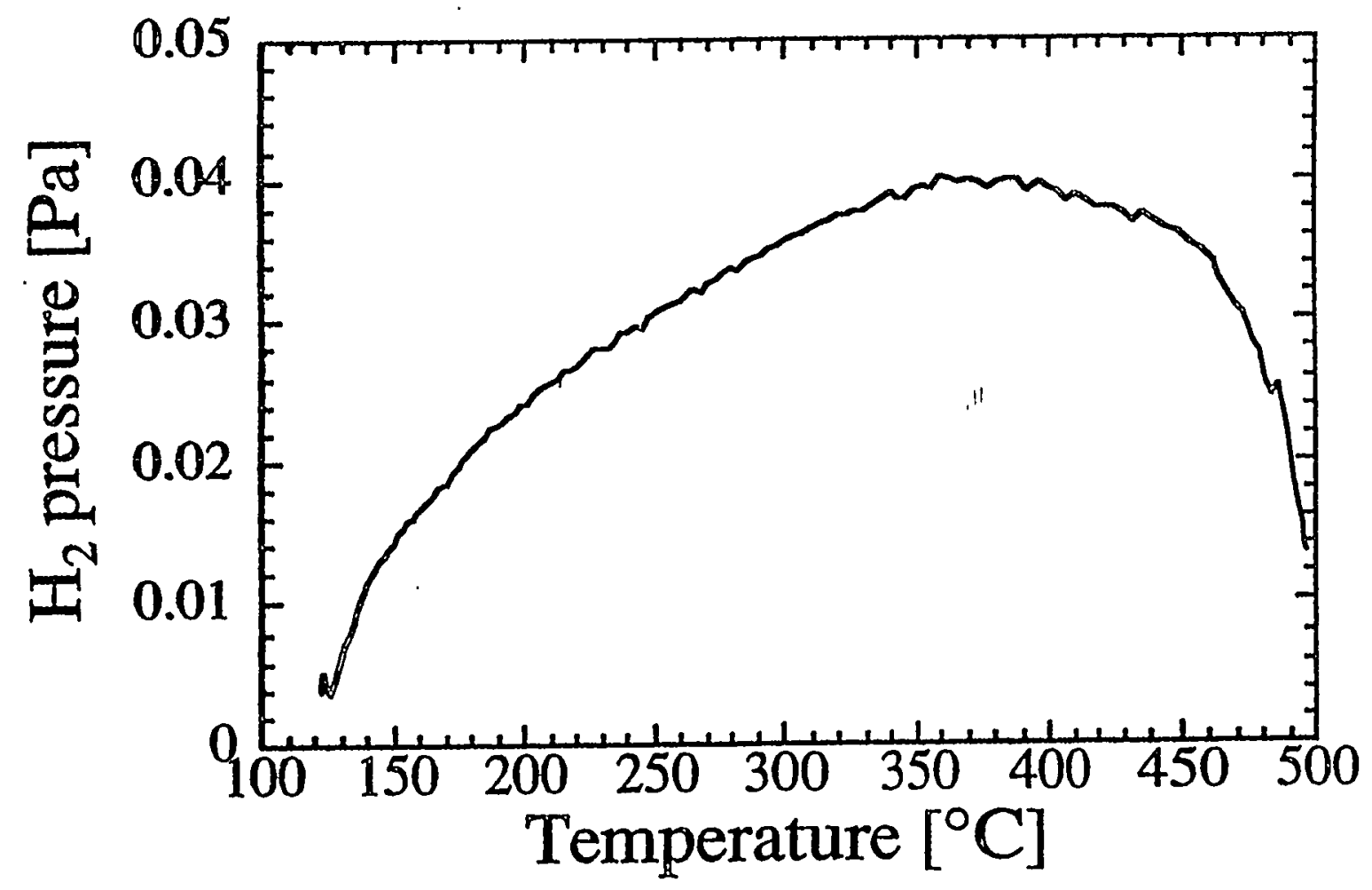

Wh gives us T-free first wall in future machines !! 


\section{Questions and problems}

(1) What role is really important in future?

(2) Detail behavior, quantitative information and mechanisms on hydrogen isotopes.

(3) Impact of impurity contamination $(O, C, W, \ldots)$

(4) What is the best material combination?

(5) What is equilibrium distribution?

(6) How the thin films can be maintained Is it possible to avoid gross immigration?

is it necessary to add boron during operation? If it is, is the dust formation tolerable? 


\section{Possible answers}

(1) A carbon film is lost due to methane formation because methane molecules reach pumping ducts and pumped away.

(2) It is not the case in a boron film. $\mathrm{B}-\mathrm{H}$ compound is fragile, easily broken by plasma impact, cannot reach pumping ducts.

$B$ atoms are redeposit on the first wall.

A boron films is expected to be kept long enough.

(3) Gross immigration is left as the major problem. If $\mathrm{B}$-addition is necessary, dust problem, too. p 
6L-IIIL. APRIJHAY 96 AFTER 95/96 CAMPAIGN (1090) SHOTS

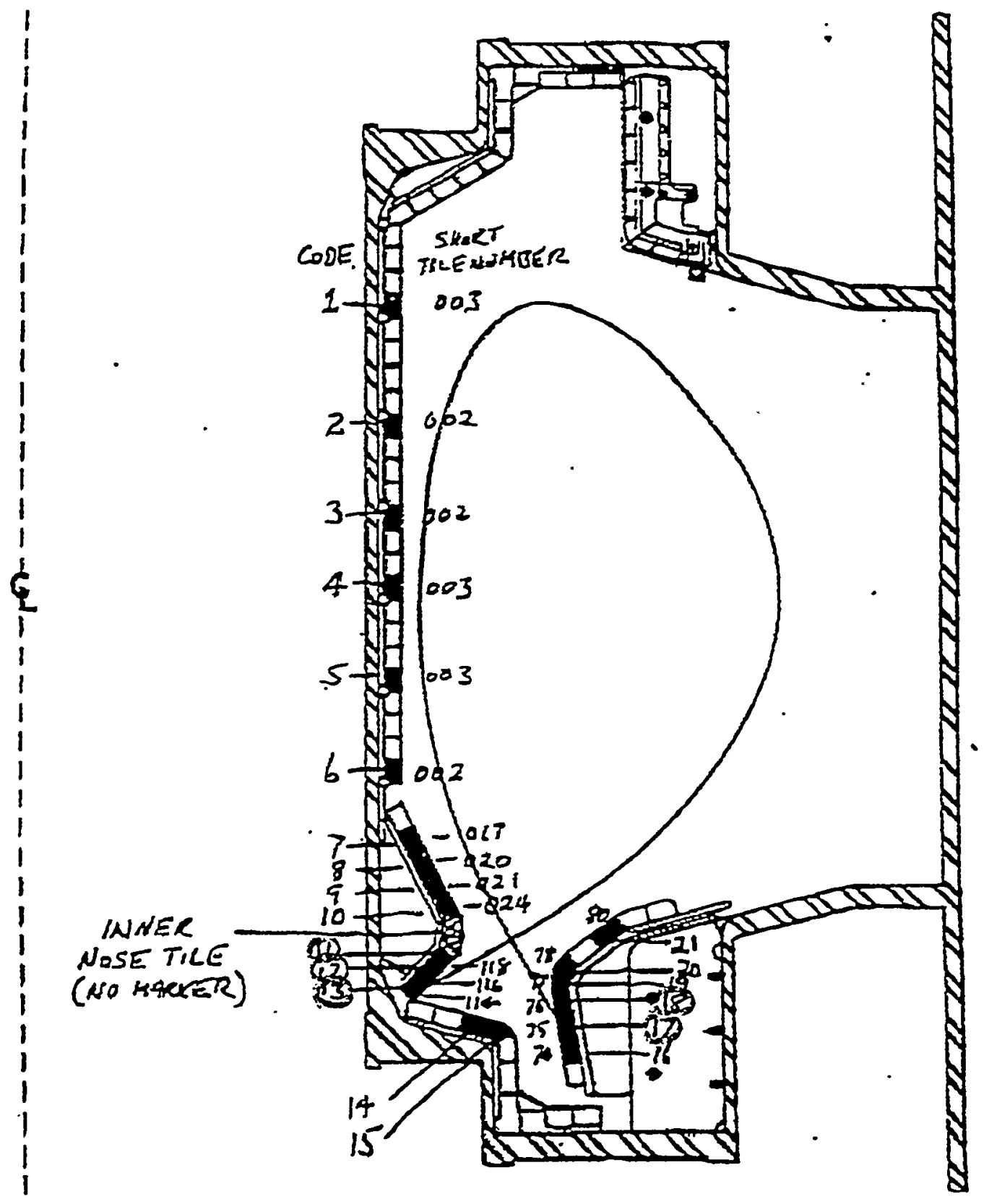

W. Wampler \& S. Pilciter

$$
\text { F:S } 3
$$

APR-28-199? 12:89

5058447775

90\%

P.84 


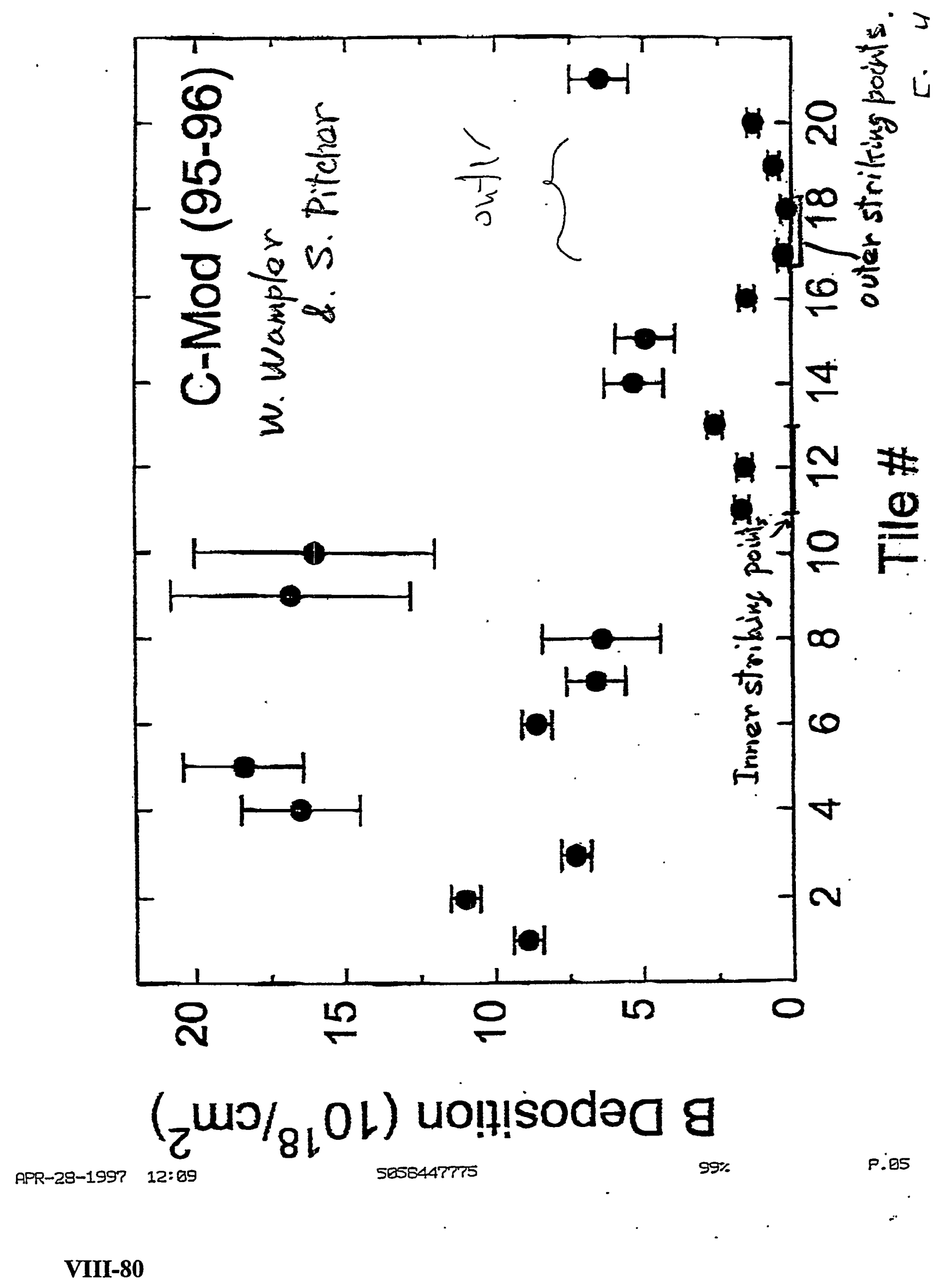




\section{Summary}

- A thin boron film is attractive as the protecting layer of the first wall

- It protects the surface against energetic particles such as CX neutrals of He, D, T

- Boron layer could be stable and resistant to erosion because of redeposition of $\mathrm{B}-\mathrm{H}$ compound during operation

- Gross immigration of boron atoms is one of the big problems to be investigated 


\section{Fabrication and High Heat Flux Testing of Plasma Sprayed Beryllium ITER First Wall Mock-Ups}

\section{R. G. Castro and K. E. Elliott}

Los Alamos National Laboratory Materials Science and Technology Division

Los Alamos, New Mexico 87545, USA

R. D. Watson and D. L. Youchison

Sandia National Laboratory

Fusion Technology Department

Albuquerque, New Mexico 87185, USA

\section{K. T. Slattery}

High Energy Systems

The Boeing Company

St. Louis, Missouri 63166, USA 


\section{U.S. Material and Joining Option Selections for ITER First-Wall/Shield Modules}

\begin{tabular}{|c|c|c|c|c|}
\hline $\begin{array}{l}\text { Limiler } \\
\text { Modules }\end{array}$ & $\begin{array}{l}\text { Heat Flux } \\
\left(M W / m^{2}\right)\end{array}$ & $\begin{array}{l}\text { Structural Alloy } \\
\text { (primary, Dackup) }\end{array}$ & $\begin{array}{c}\text { Armor/ } \\
\text { Segment Joint }\end{array}$ & Comment \\
\hline First Wall & 5.0 & $\begin{array}{l}\text { P: SS-DS Cu (AI-25, IGO) } \\
\left(950^{\circ} \mathrm{C} \text { HIP, slow }\right. \\
\text { Cool will degrade PH } \\
\text { properties). } \\
\text { B: CuNiBe (Hycon-3HP, } \\
\text { AT) (May consider } \\
\text { Alloy-3 due to lower } \\
\text { heat flux.) }\end{array}$ & $\begin{array}{l}\text { Be: EP CU-Cu DB at } 450^{\circ} \mathrm{C} \\
\text { Be: Al-Si HIP-Braze at } 600^{\circ} \mathrm{C} \\
\text { Be: Low Press Plasma Spray } \\
\text { (LANL process) }\end{array}$ & $\begin{array}{l}\text { Initial } 950^{\circ} \mathrm{C} \mathrm{HIP} \text { to form SS-Cu } \\
\text { structure. Then attach armor. } \\
\text { Could use EP to pre-join tiles into } \\
\text { matrix prior to canning. } \\
\text { Al-Si process adequate for FW heat } \\
\text { flux. Plasma spray Ti diffusion } \\
\text { barrier and Al-Si after } 950^{\circ} \mathrm{C} \mathrm{HIP} \mathrm{cycle.} \\
\text { See below. Properties need to be } \\
\text { verified for limiter heat fluxes. }\end{array}$ \\
\hline Shield Body & & Cast/HIP $(\overline{S S} 31 \overline{6} L-\mid \bar{G})$ & $\begin{array}{l}\text { Module size consistent with } \\
\text { single cast/HIP piece. }\end{array}$ & $\begin{array}{l}\text { Need mechanical properties, vacuum, } \\
\text { ferrite, irrad data. Also need to verify } \\
\text { core length limits. }\end{array}$ \\
\hline $\begin{array}{l}\text { Primary FW } \\
\text { Modules }\end{array}$ & $\begin{array}{l}\text { Heat Flux } \\
\left(M W / m^{2}\right)\end{array}$ & & $\begin{array}{l}\text { Armor/ } \\
\text { Segment Joint }\end{array}$ & Comment \\
\hline First Wall & 0.5 & $\begin{array}{l}\text { P: SS-DS Cu (Al-25, IGO) } \\
\text { B: CuNiBe (Hycon-3HP, } \\
\text { AT) }\end{array}$ & $\begin{array}{l}\text { Be: EP Cu-Cu DB at } 450^{\circ} \mathrm{C} \\
\text { Be: Al-Si HIP-Braze at } 600^{\circ} \mathrm{C}\end{array}$ & $\begin{array}{l}\text { Plasma spray process has achieved } \\
\text { properties sufficient for primary first } \\
\text { wall. Low-cost way to coat large areas. } \\
\text { Demonstrated } 80 \% \text { deposition rate } \\
\text { coats } 5 \mathrm{~mm} \text { over } 1 \mathrm{~m}^{2} \text { area in } 1 \text { hour. } \\
\text { See above. } \\
\text { See above. }\end{array}$ \\
\hline Shield Body & & Cast/HIP (SS316L-|G) & $\begin{array}{l}\text { Module size consistent with } \\
\text { single cast/HIP piece. }\end{array}$ & Same as for limiter module. \\
\hline \multicolumn{5}{|c|}{$\begin{array}{l}\text { Note: DS Cu selected where manufacturing cycle requires temperatures exceeding } 600^{\circ} \mathrm{C} \text { for extended periods. PH alloys preferred } \\
\text { elsewhere due to weldability and cost advantages. Armor joining options are listed in order of priority. }\end{array}$} \\
\hline
\end{tabular}




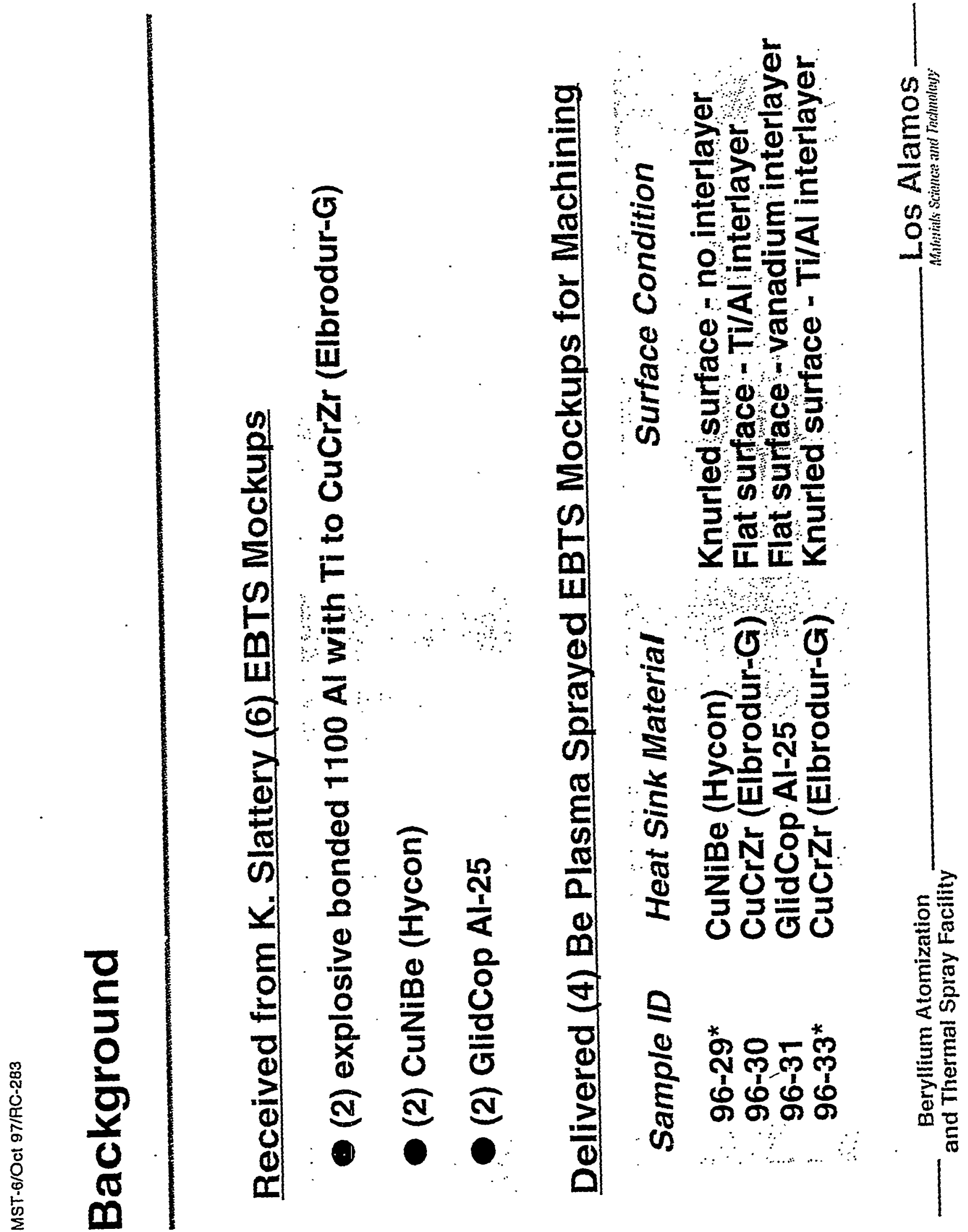




\section{Knurled Surfaces - EDM Machined}

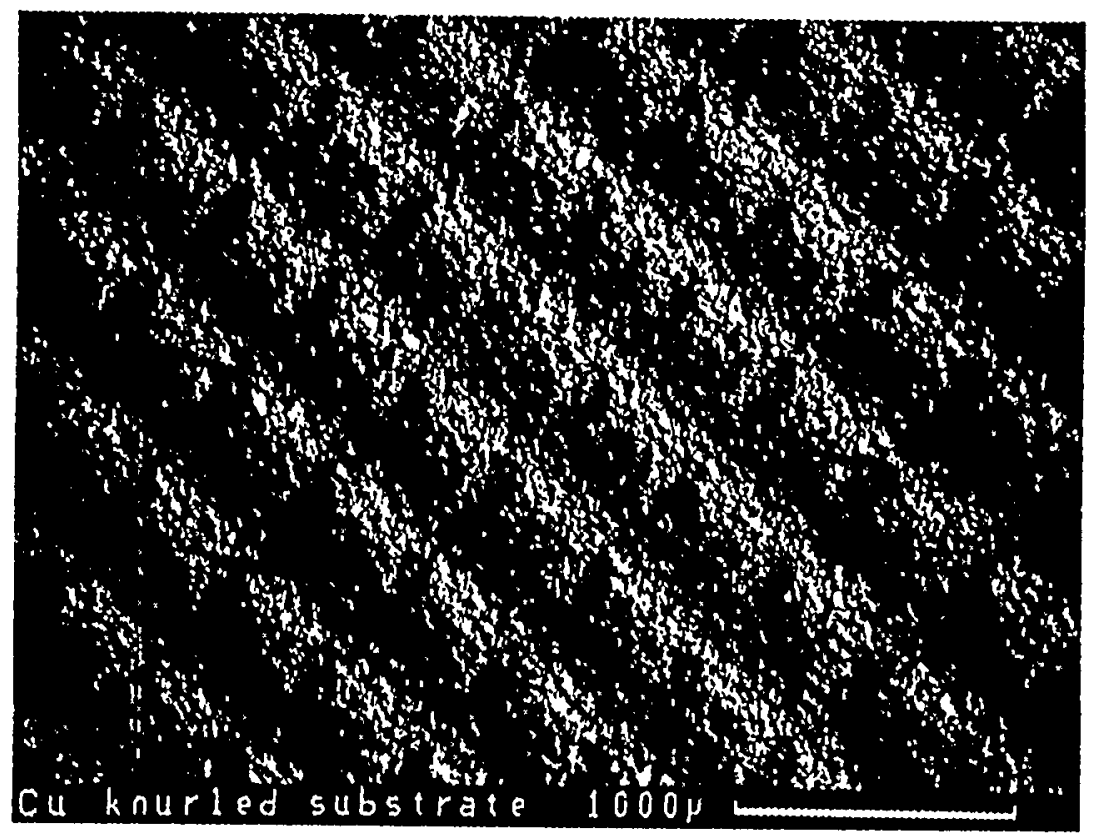

Copper

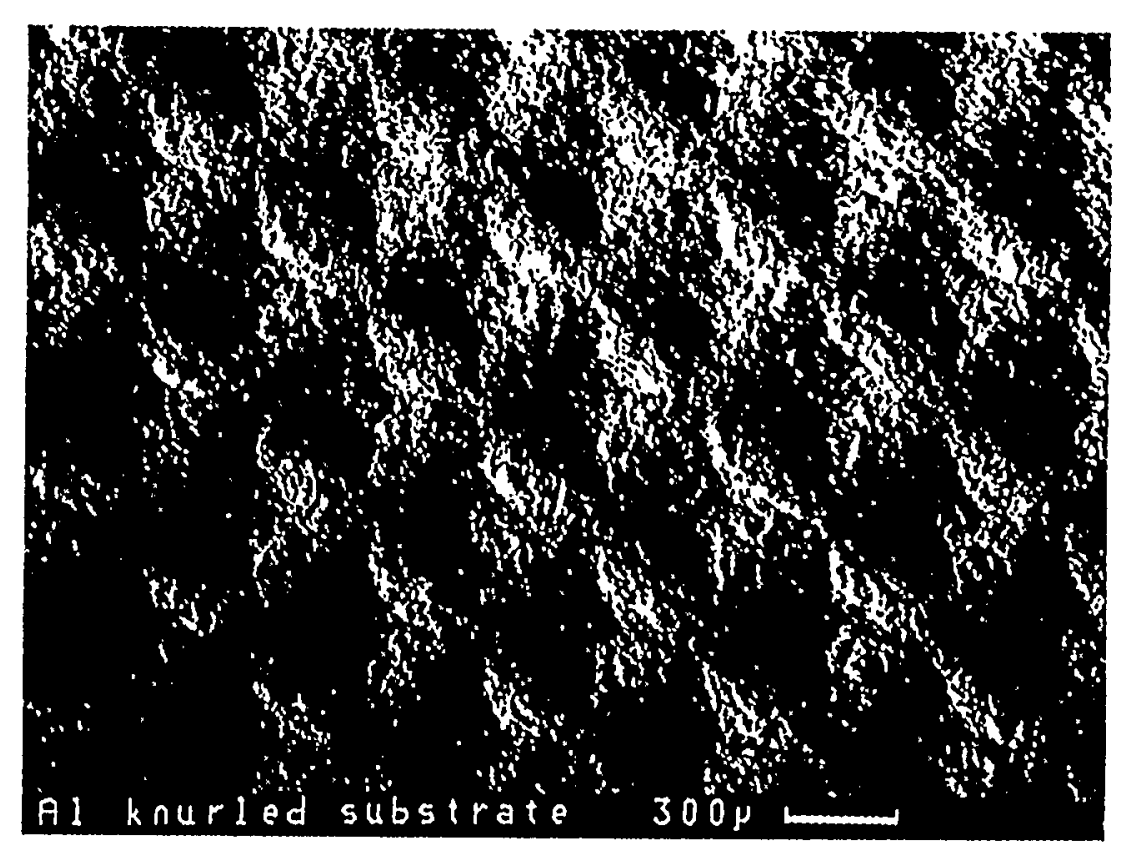

Aluminum

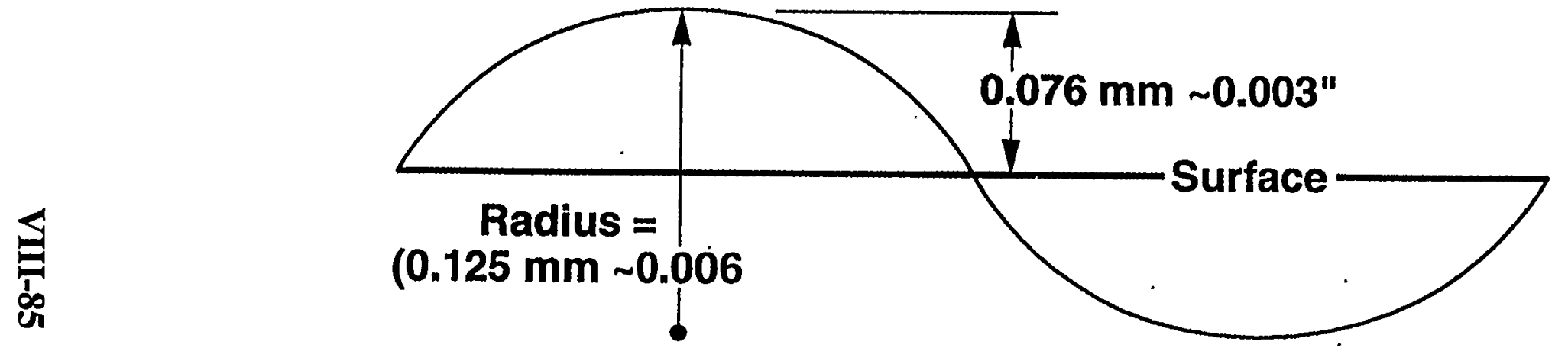

Beryllium Atomization and Thermal Spray Facility 


\section{Fabrication of EBTS Divertor Mock-Ups}

寻

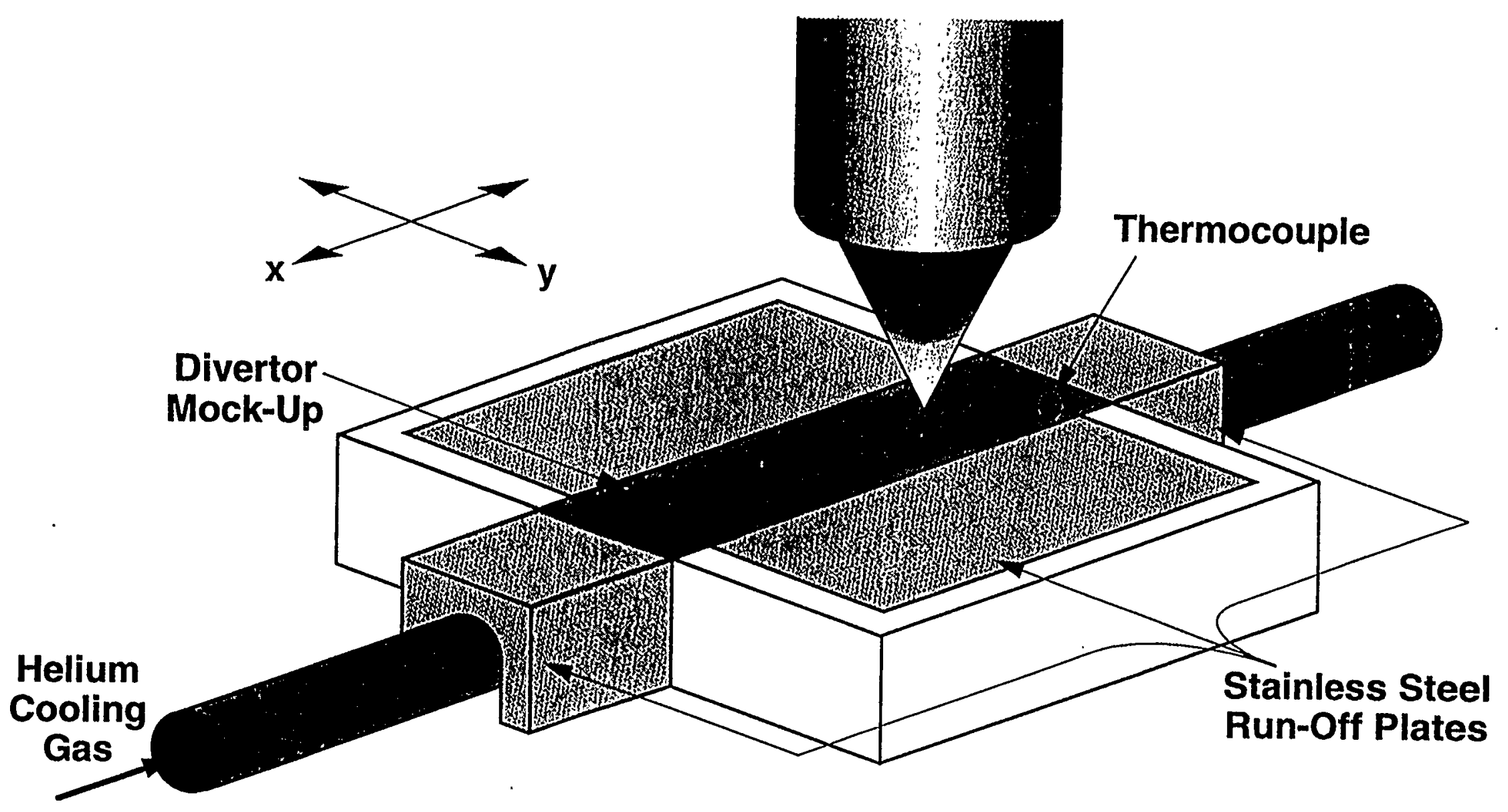




\section{Processing Conditions for Fabricating EBTS Mock-Ups}

\begin{tabular}{|c|c|c|c|}
\hline T/A Parameters & Settings & Torch Parameters & Settings \\
\hline Peak Amps (A) & 40 & Arc Gas (Ar) & $40 \mathrm{sIm}$ \\
\hline Background Amps & 40 & Secondary Gas $\left(\mathrm{H}_{2}\right)$ & $1 \mathrm{sim}$ \\
\hline Pulser (on/off) & (off) & Powder Gas (Ar) & $1 \mathrm{sim}$ \\
\hline Chamber Pressure & 40 torr & Feed Rate (lb/h) & $\sim 1$ \\
\hline Plasma Torch Current (A) & 400 & Current (A) & 400 \\
\hline Substrate Preheat Temp & $550-600^{\circ} \mathrm{C}$ & Volts & 35 \\
\hline \multirow[t]{2}{*}{ Distance $(\mathrm{cm})$} & $10 \mathrm{~cm}$ & Chamber Pressure & $400-450$ torr \\
\hline & & Substrate Temp & $550-600^{\circ} \mathrm{C}$ \\
\hline $\begin{array}{l}\text { Beryllium Alomization } \\
\text { and Therimal Spray Facility }\end{array}$ & & & Ala \\
\hline
\end{tabular}




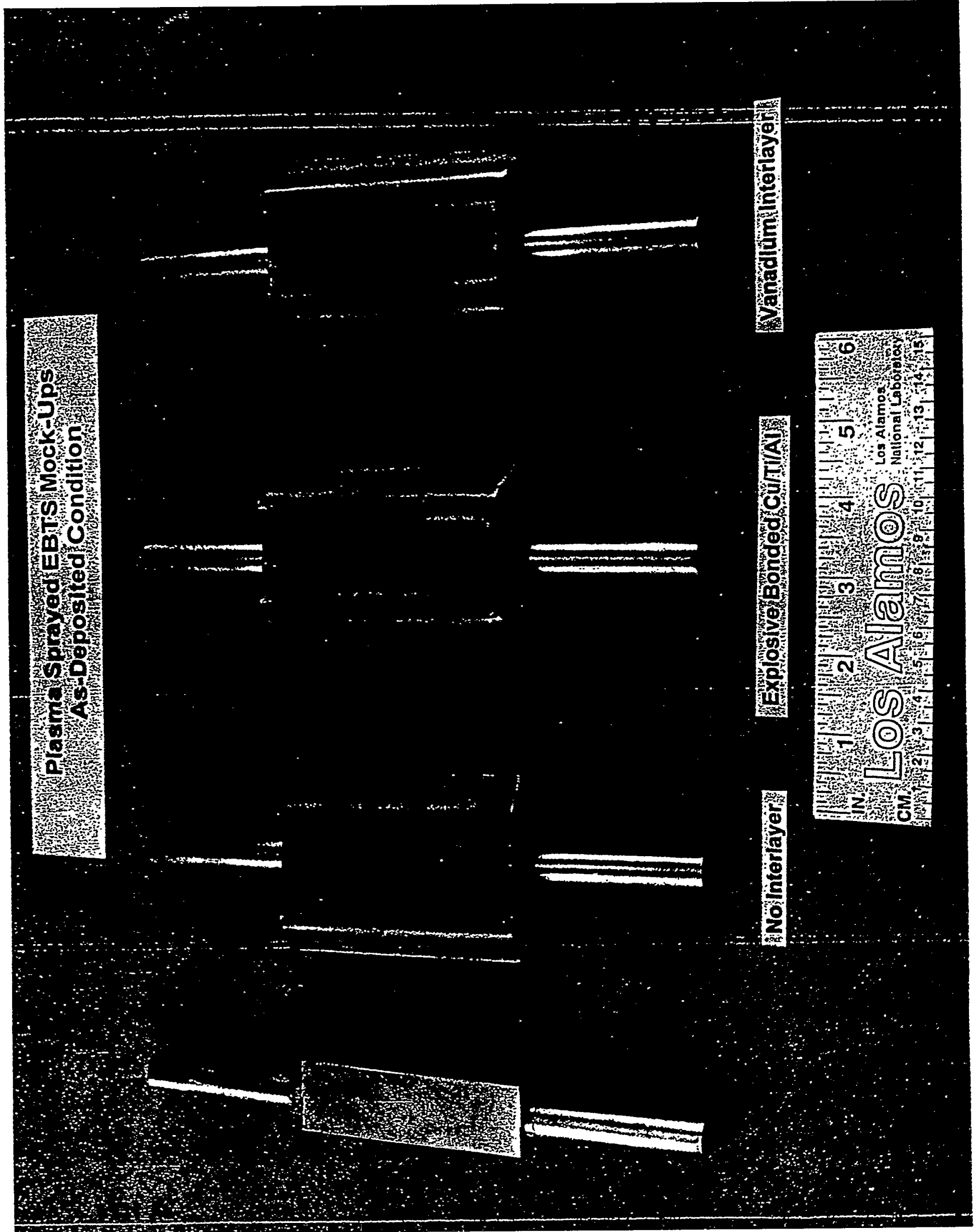




\section{Machining Beryllium Plasma Sprayed EBTS Mockups}

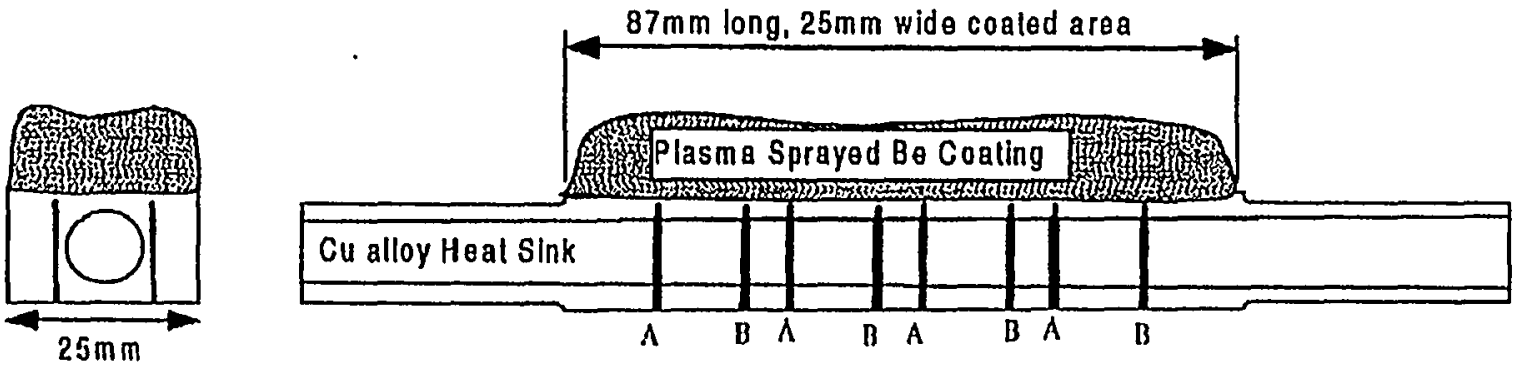

Armor and Heat Sink Diagram:

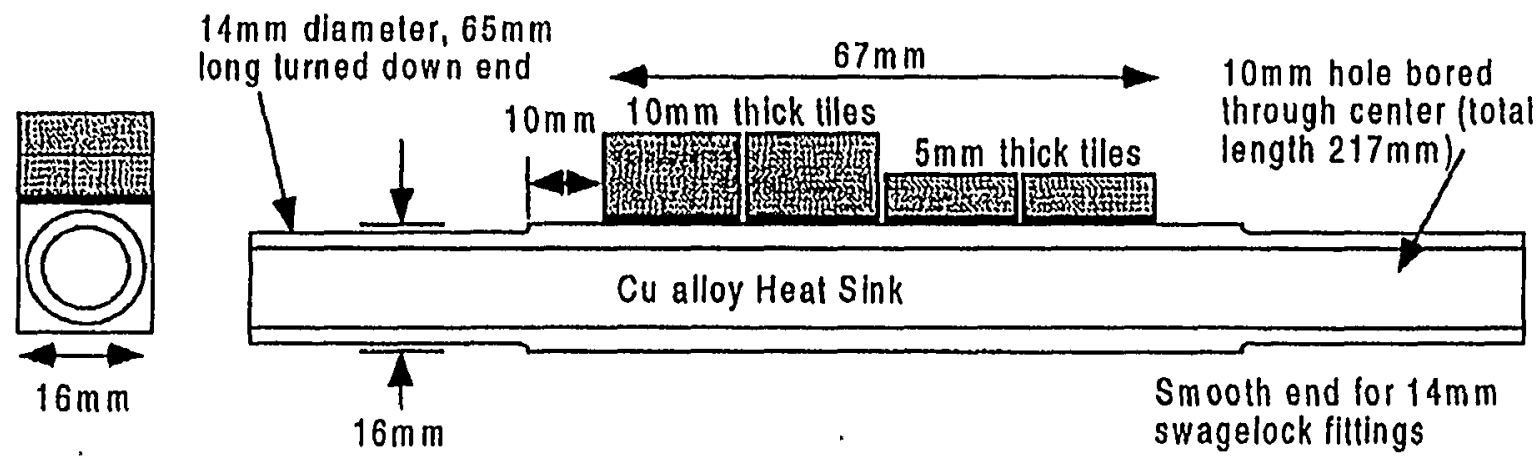

Armor Thermocouple_Hole_Dlagram:

holes centered

$1.5 \mathrm{~mm}$ from edge
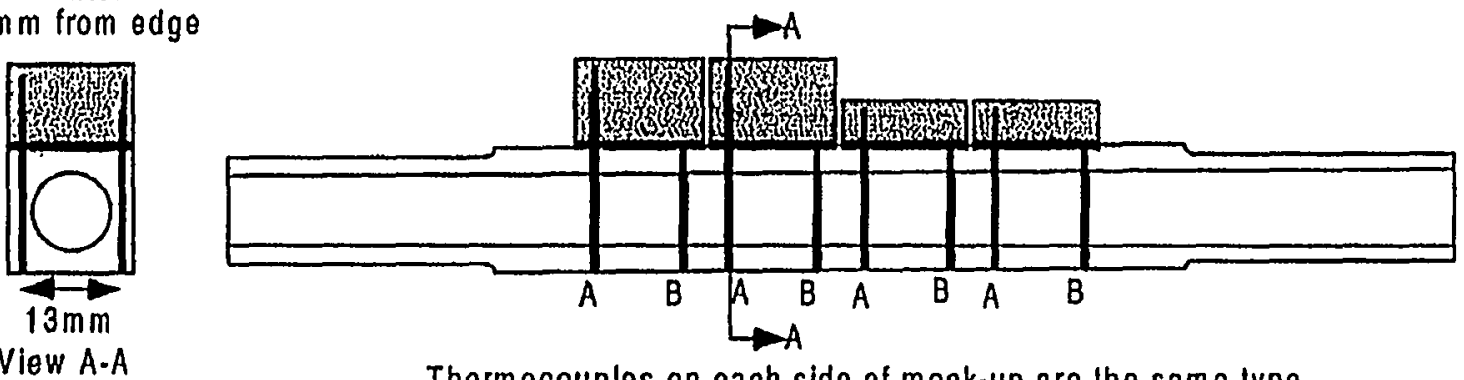

View A-A

Thermocouples on each side of mock-up are the same type 


\section{Machining of Subcastellations}

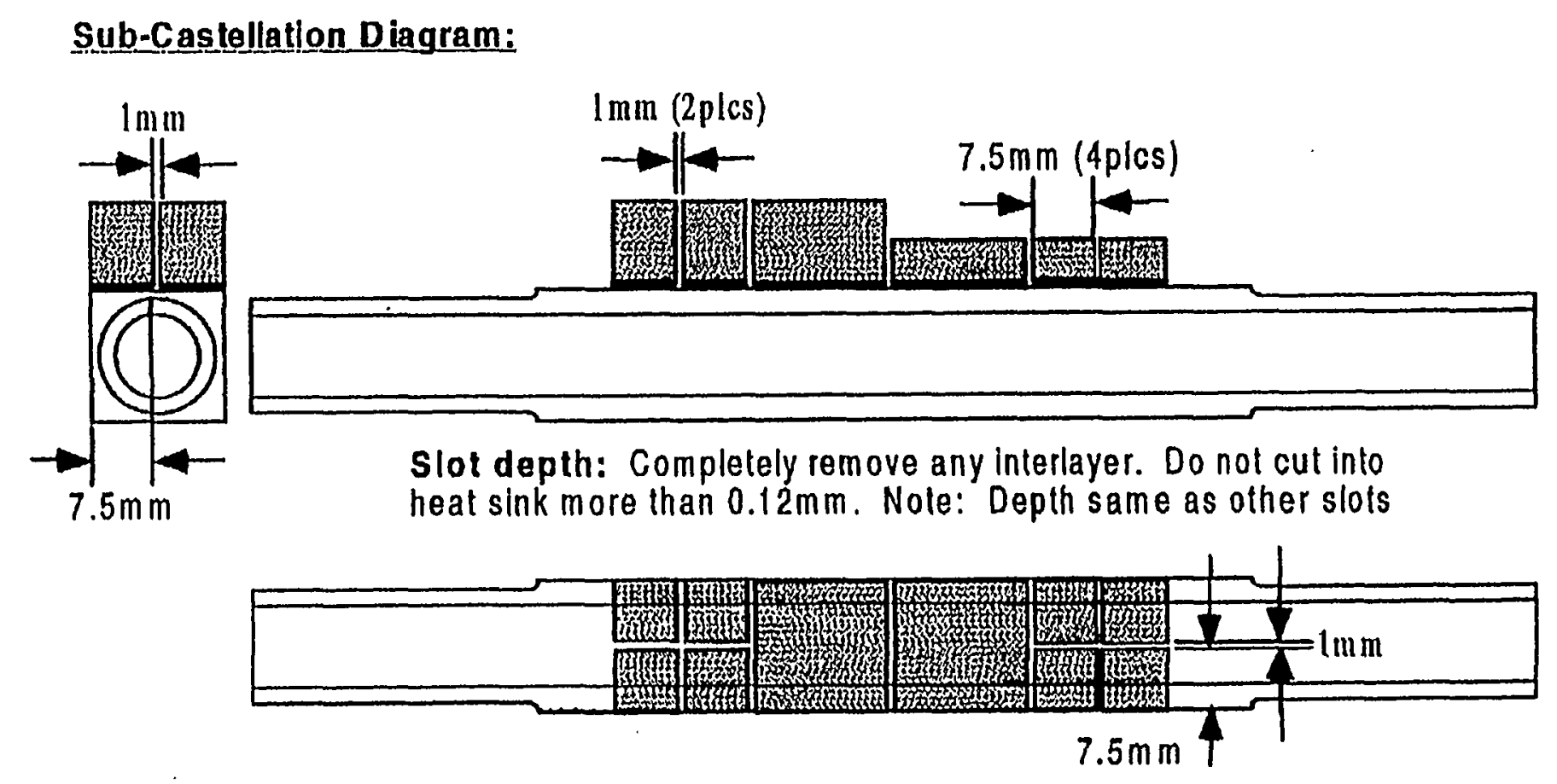




\section{Beryllium Plasma Sprayed EBTS Mock-Ups}

As-Machined Condition 


\section{Y7006 - 1 - S}

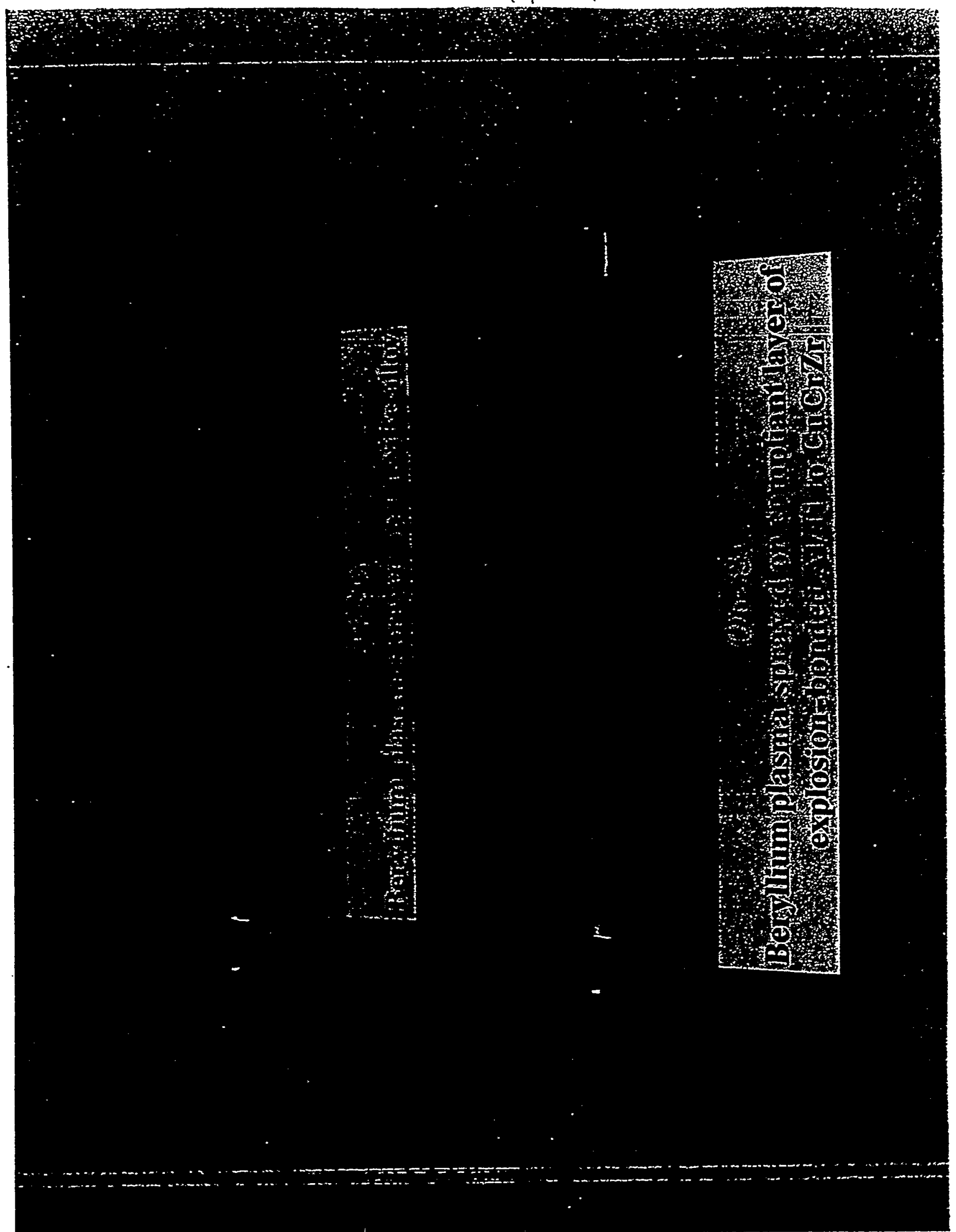

\section{VIII-92}




\section{Summary: High Heat Flux Test - Mockup 96-29}

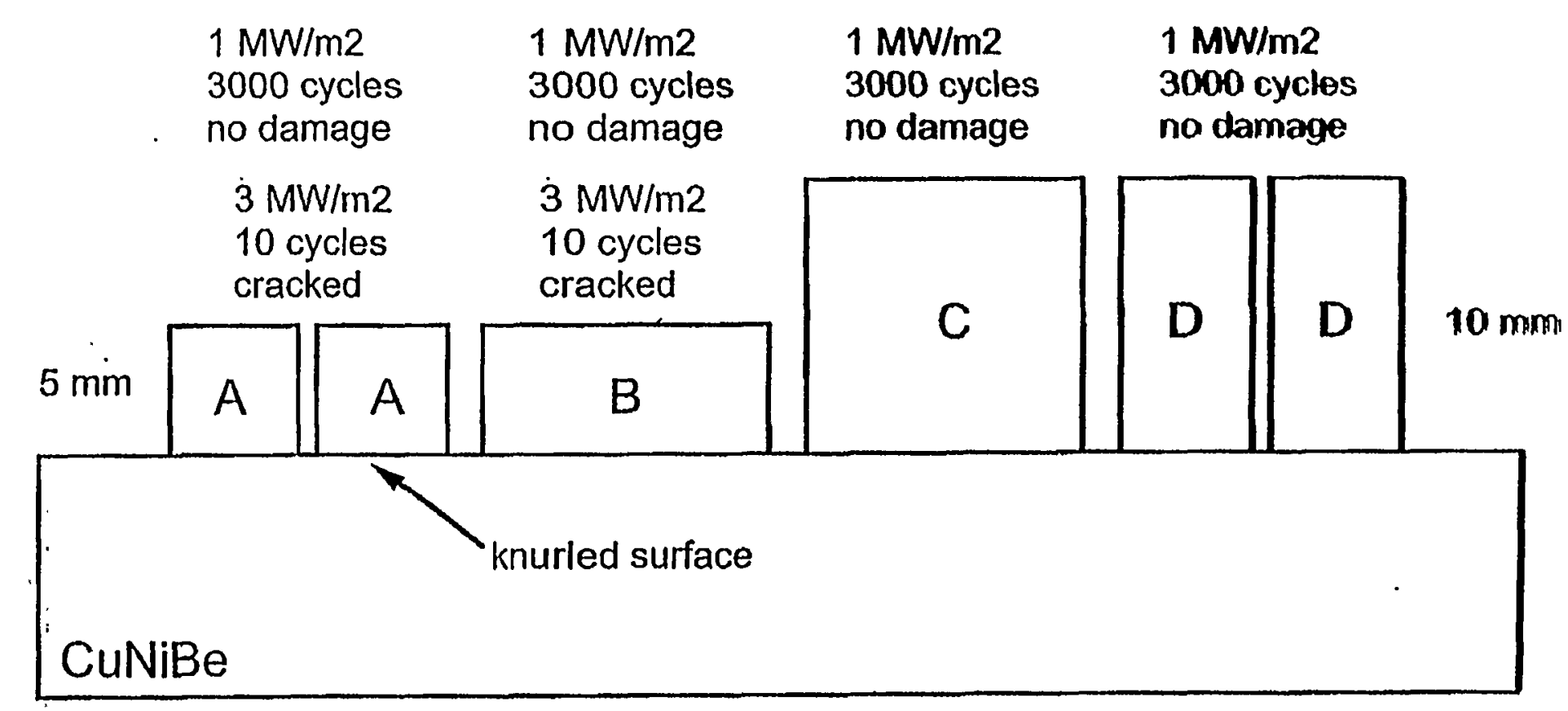




\section{Summary: High Heat Flux Test - Mockup 96-33}

$\sqrt{1}$

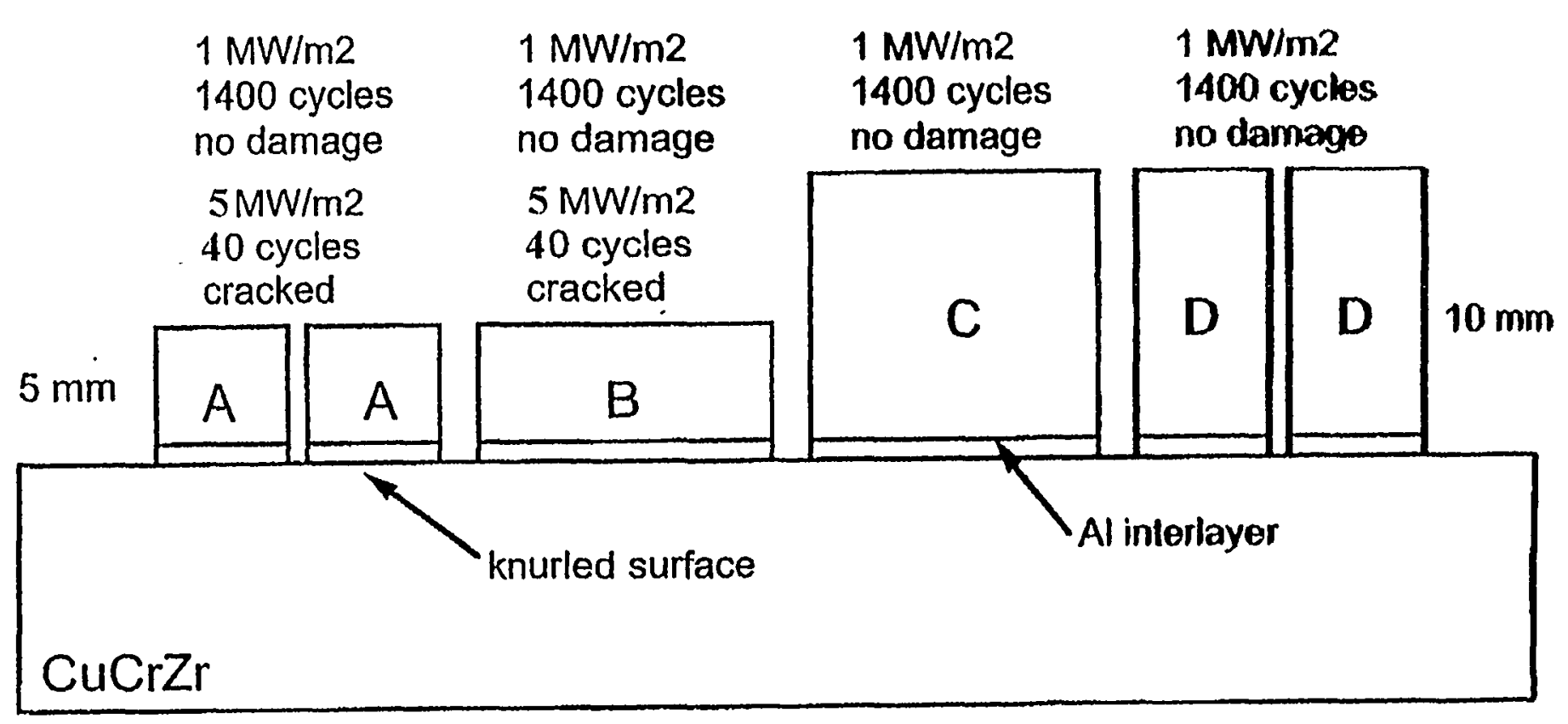




\section{EBTS Mockup 96-29 Be/Cu}

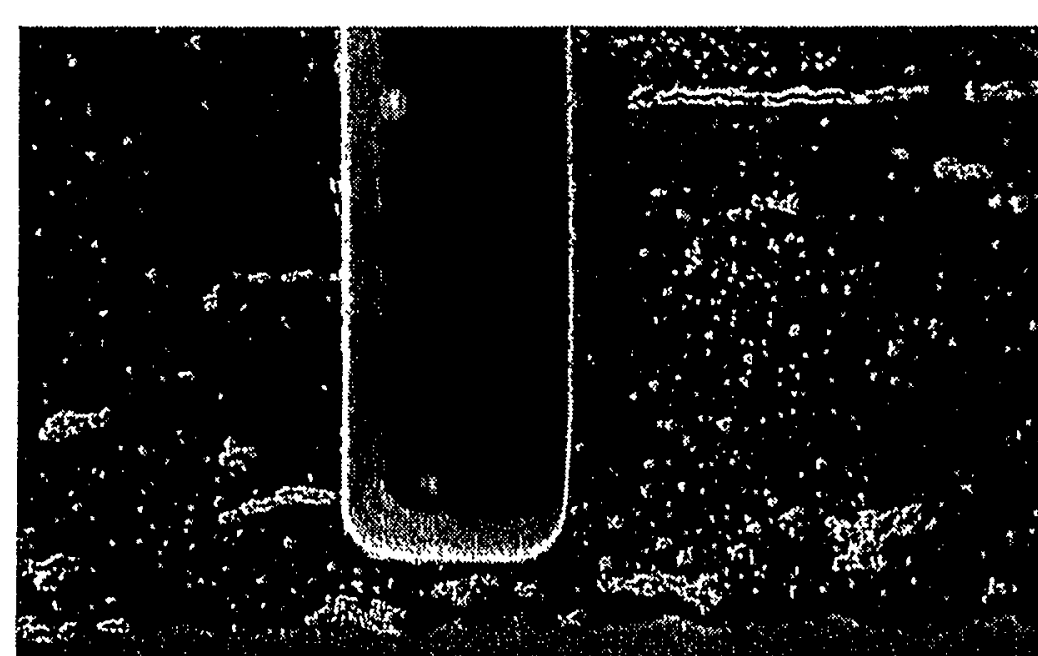

$96-29 \mathrm{Be} / \mathrm{Cu}$ $1000 \%$

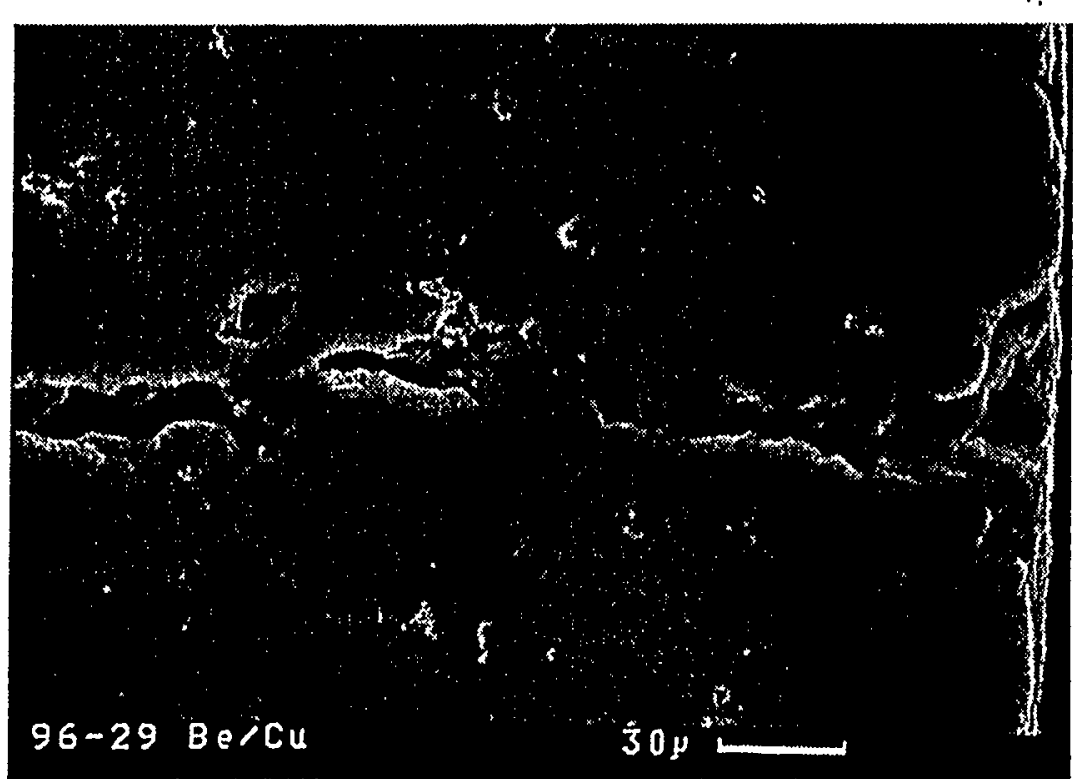

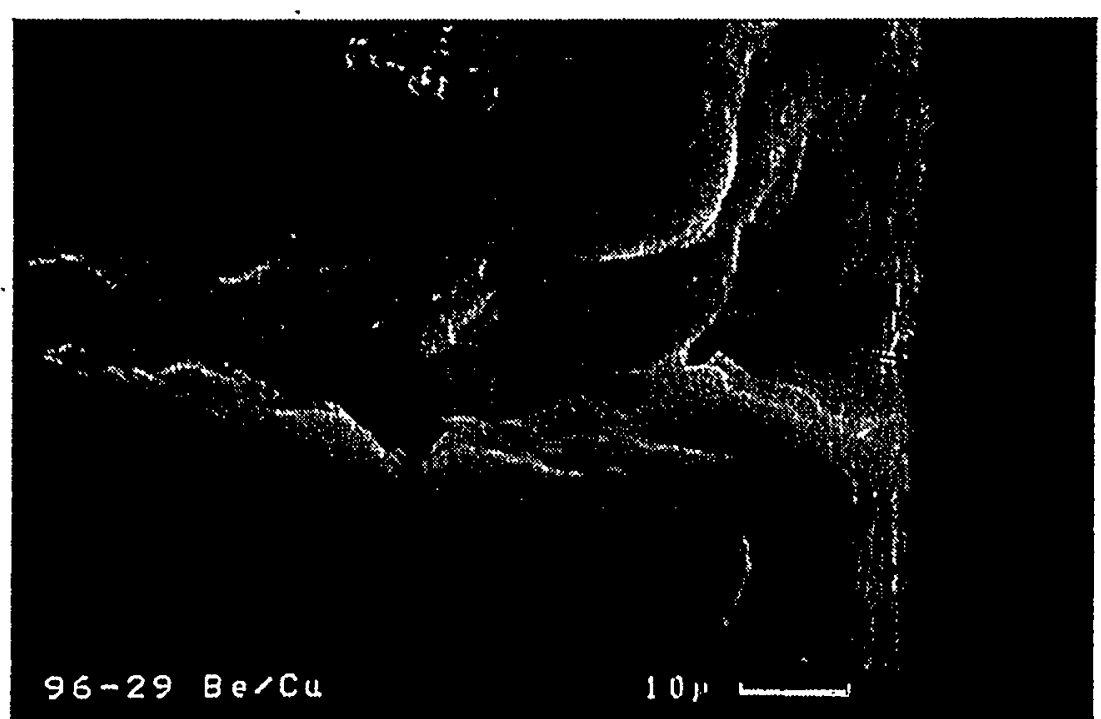

- Cracks may initiate at unmelted particles

- No evidence of cracking at root of castellation

- Cracks extend parallel to the $\mathrm{Be} / \mathrm{Cu}$ interface

- Cracks may be extending along unmelted particles 


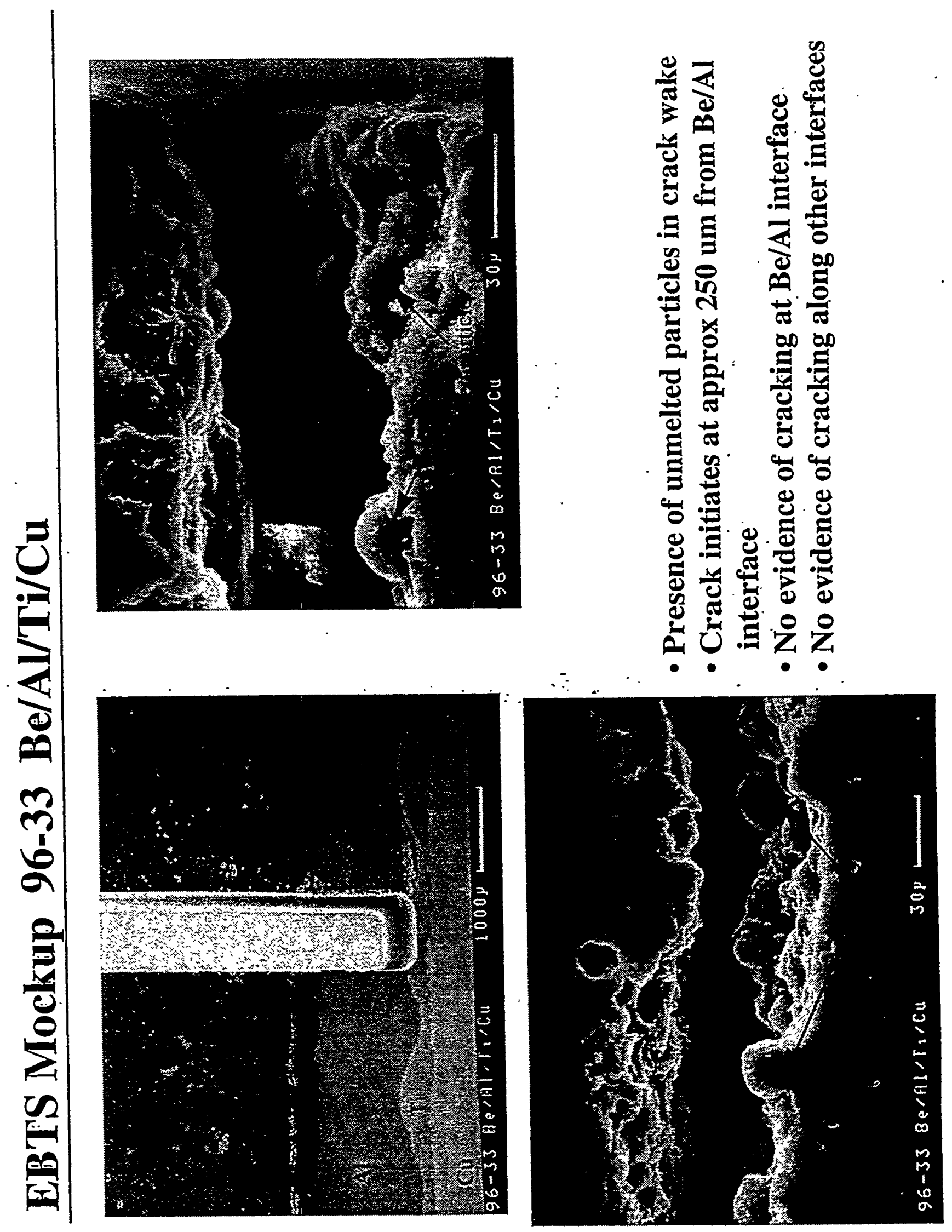




\section{DOE Newsnote 1/10/97}

Plasma Sprayed beryllium First Wall Mockup Survives 3000 HHF Cycles

A plasma-sprayed beryllium ITER first wall mockup fabricated by LANL has survived 3000 thermal fatigue cycles at $1 \mathrm{MW} / \mathrm{m}^{2}$ without damage during testing at the Plasma Materials Test Facility at Sandia National Laboratories. This heat flux is four times the expected average heat flux for ITER primary first wall modules $\left(0.25 \mathrm{MW} / \mathrm{m}^{2}\right)$, and is twice the peak design heat flux $\left(0.5 \mathrm{MW} / \mathrm{m}^{2}\right)$. These successful results demonstrate the potential for using plasma-sprayed beryllium as a method for both initial fabrication and for insitu repair of eroded beryllium armor-tiles in ITER. 


\title{
Development of PFC Armor Utilizing Vacuum Plasma Spray Processes
}

\author{
Scott O'Dell \\ and \\ Timothy McKechnie
}

Plasma Processes Inc. 


\section{Fabrication Technique for SBIR Phase I Mockup PW-3}
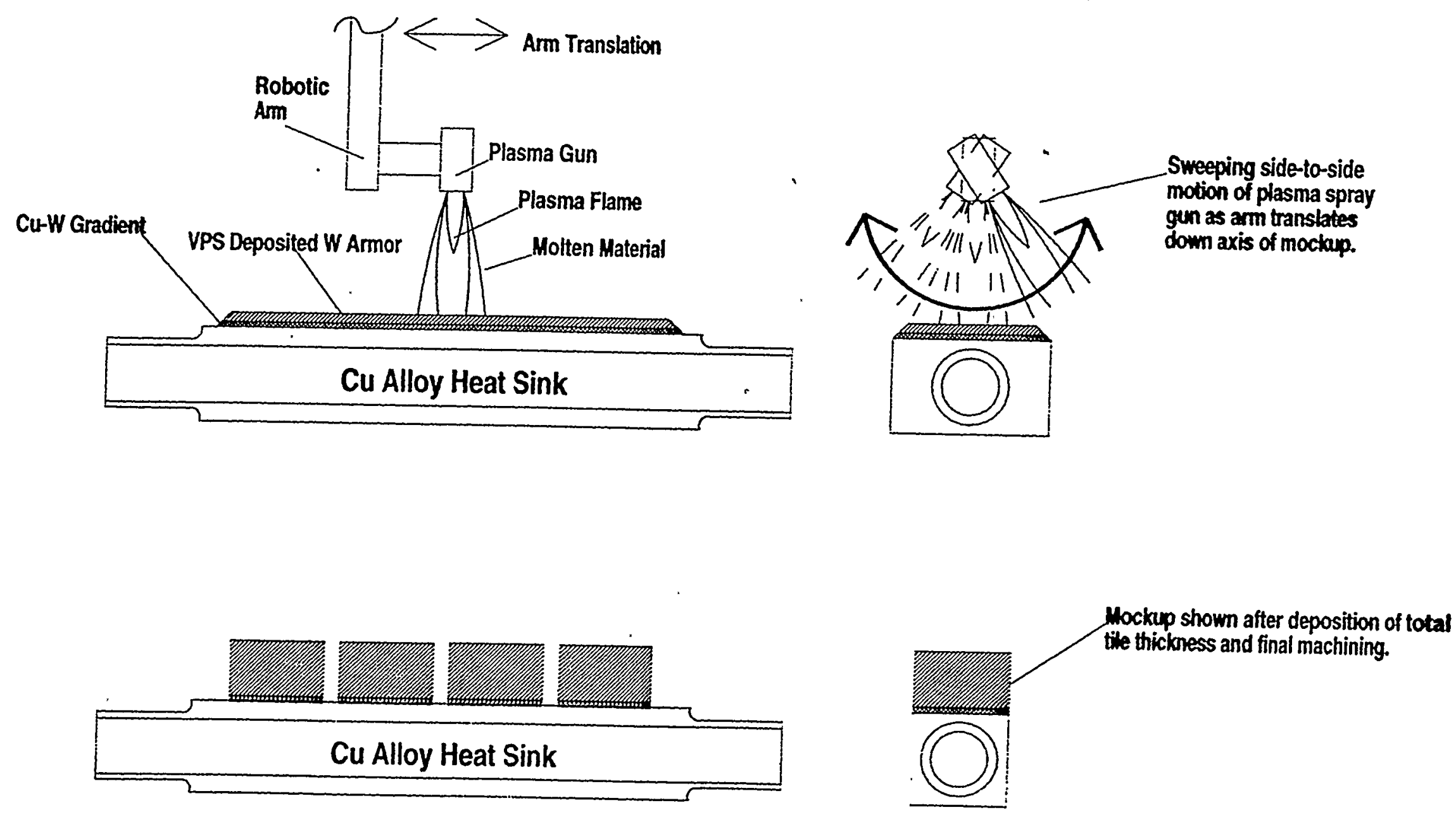


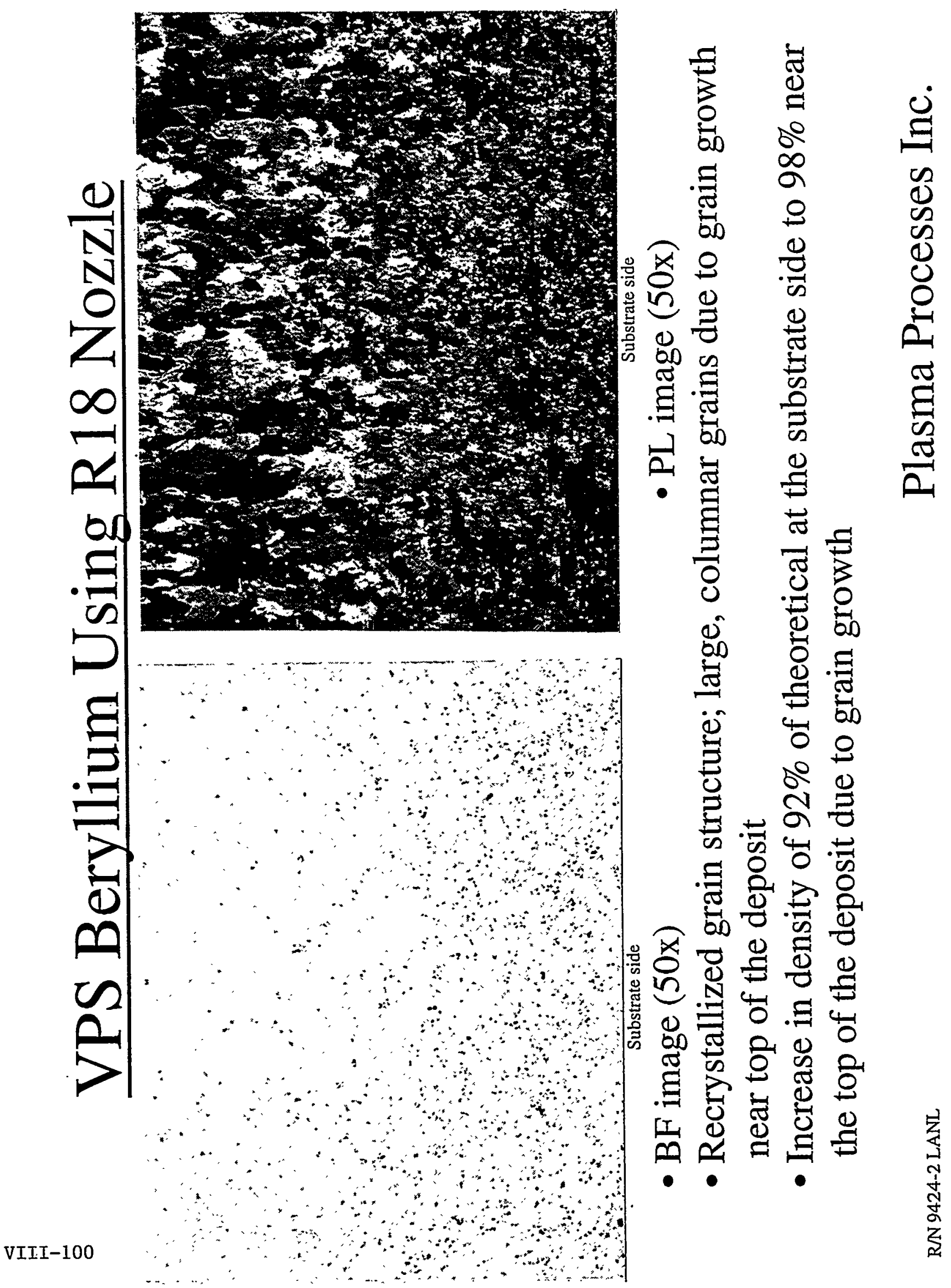




\section{Thermal Conductivity of VPS Be and VHP Be as a Function of Temperature}

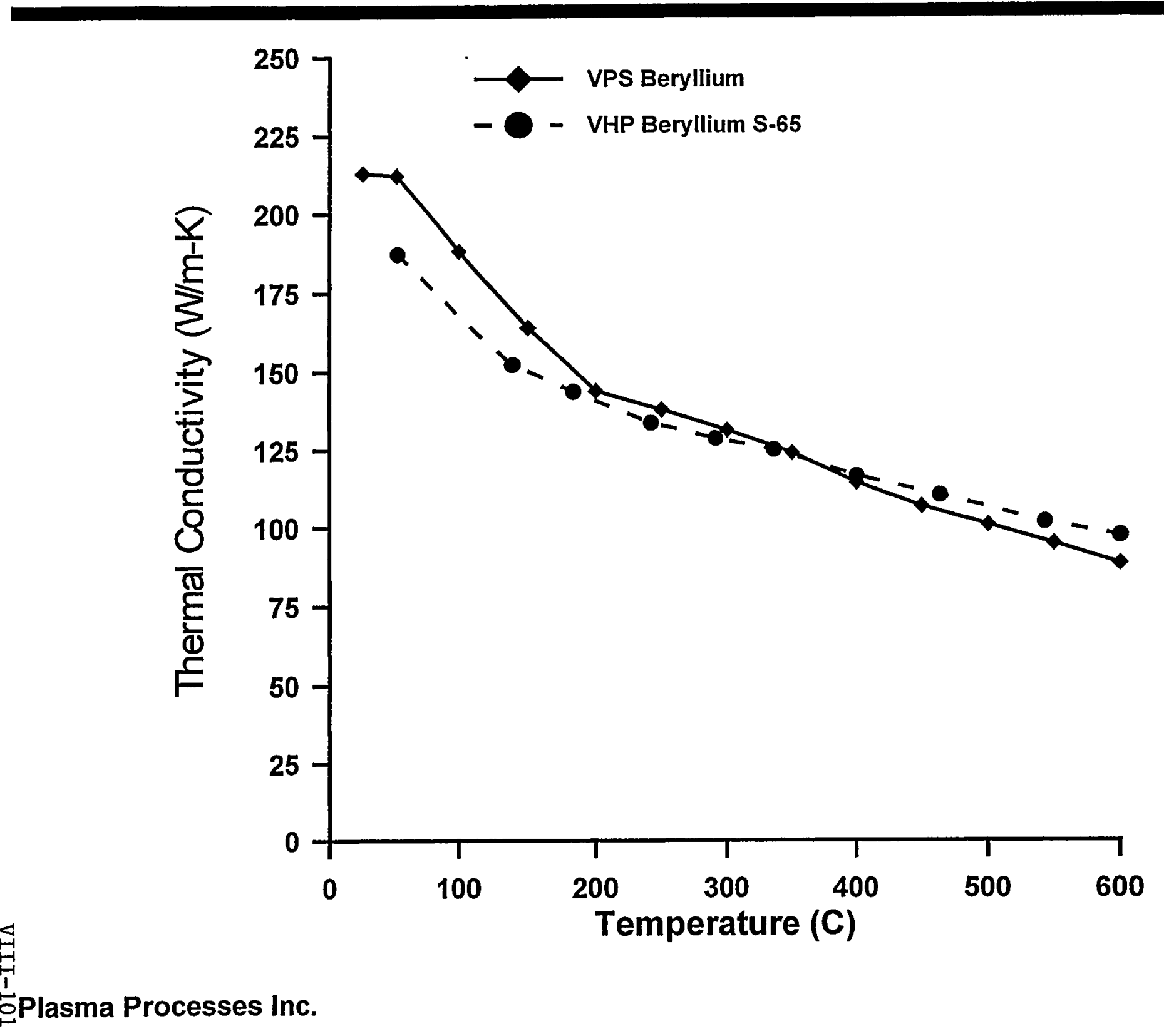




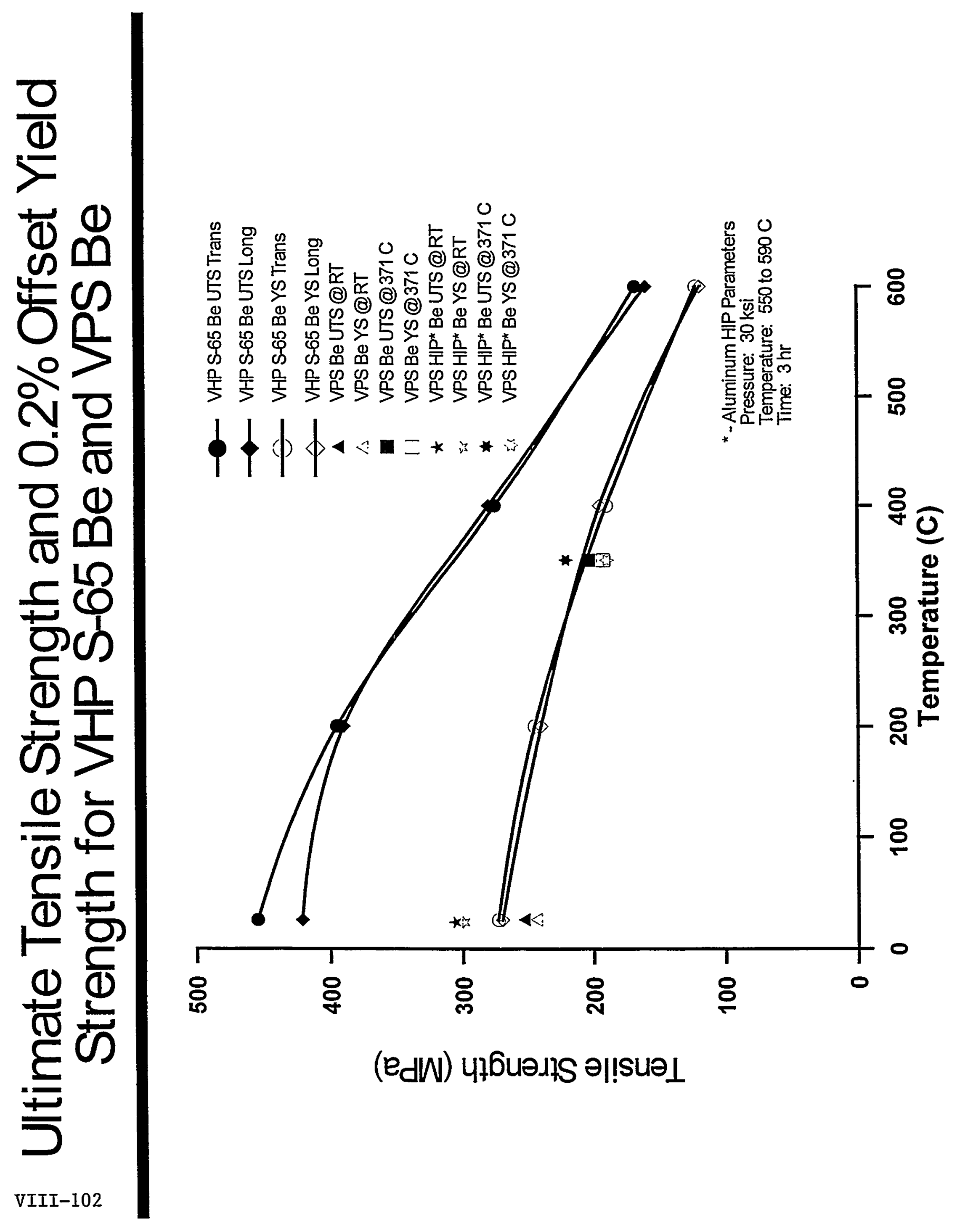

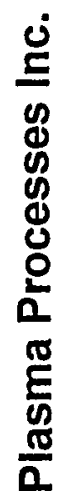




\section{Stress Analysis of Armor Joint}

- ABAQUS Finite Element Model (SNL)

- 2-D plane stress

- Elastic behavior

- Temp. dependent props.

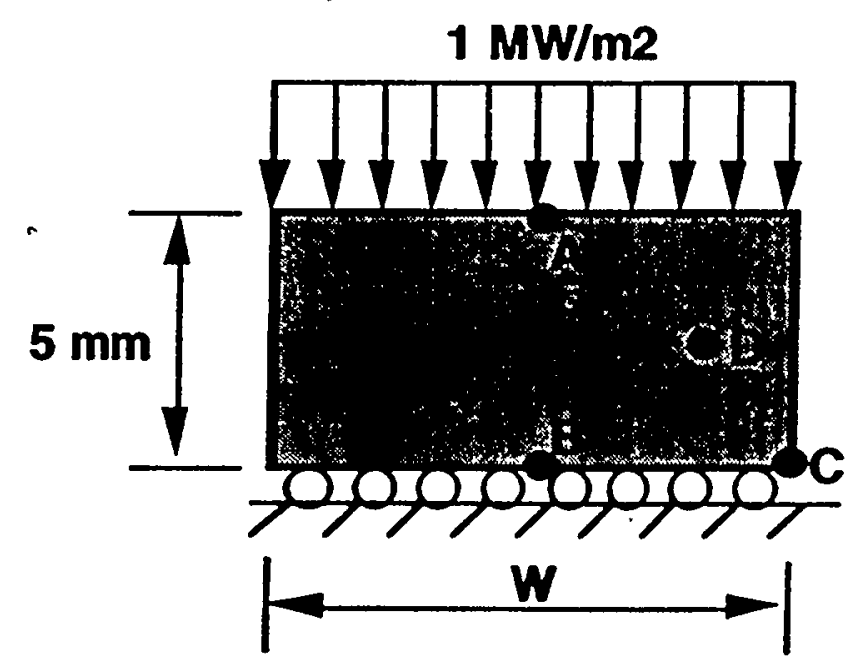

- 2000 elements (8- 


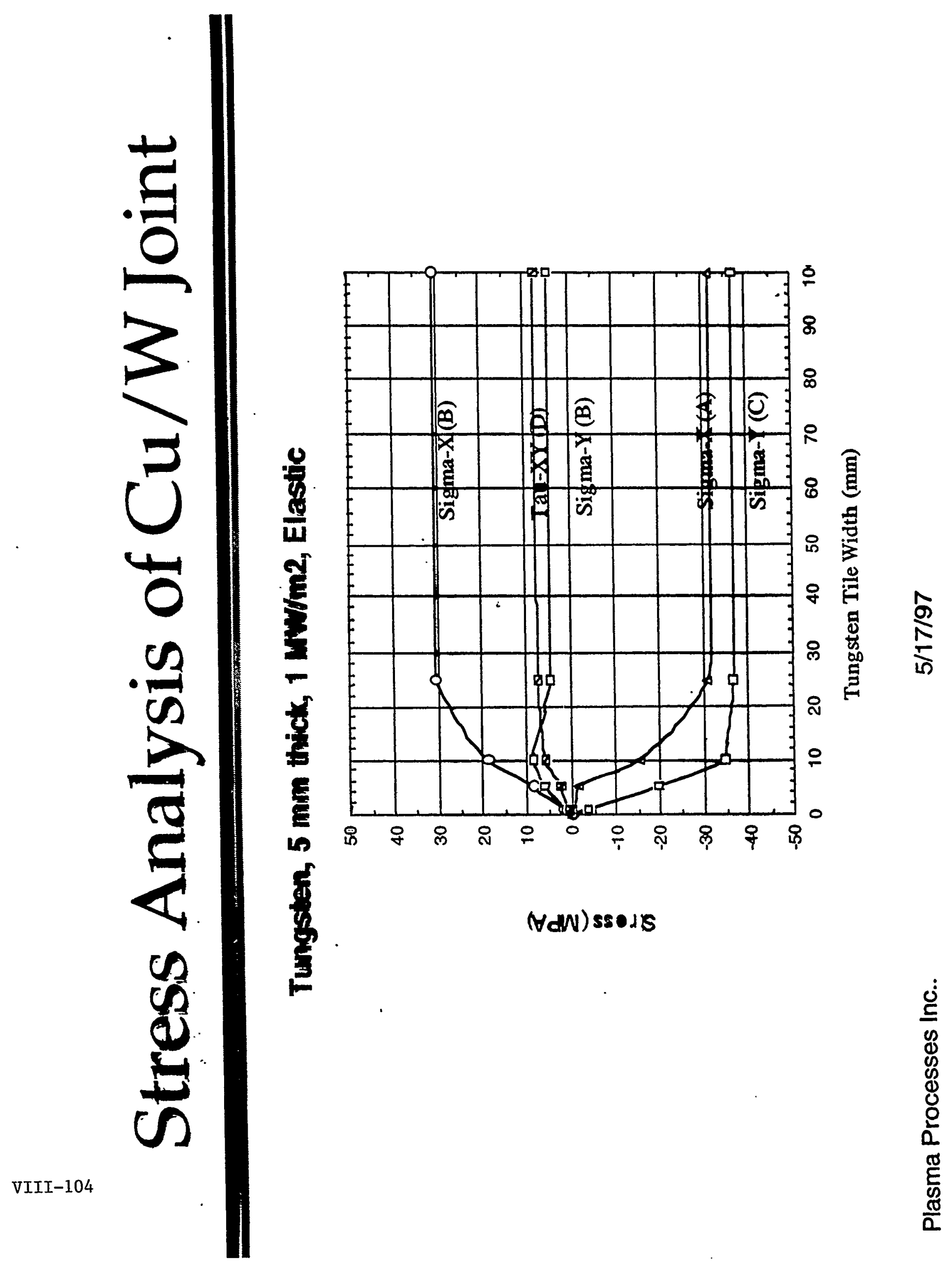




\section{Evolution of Plasma Facing Component Armor}
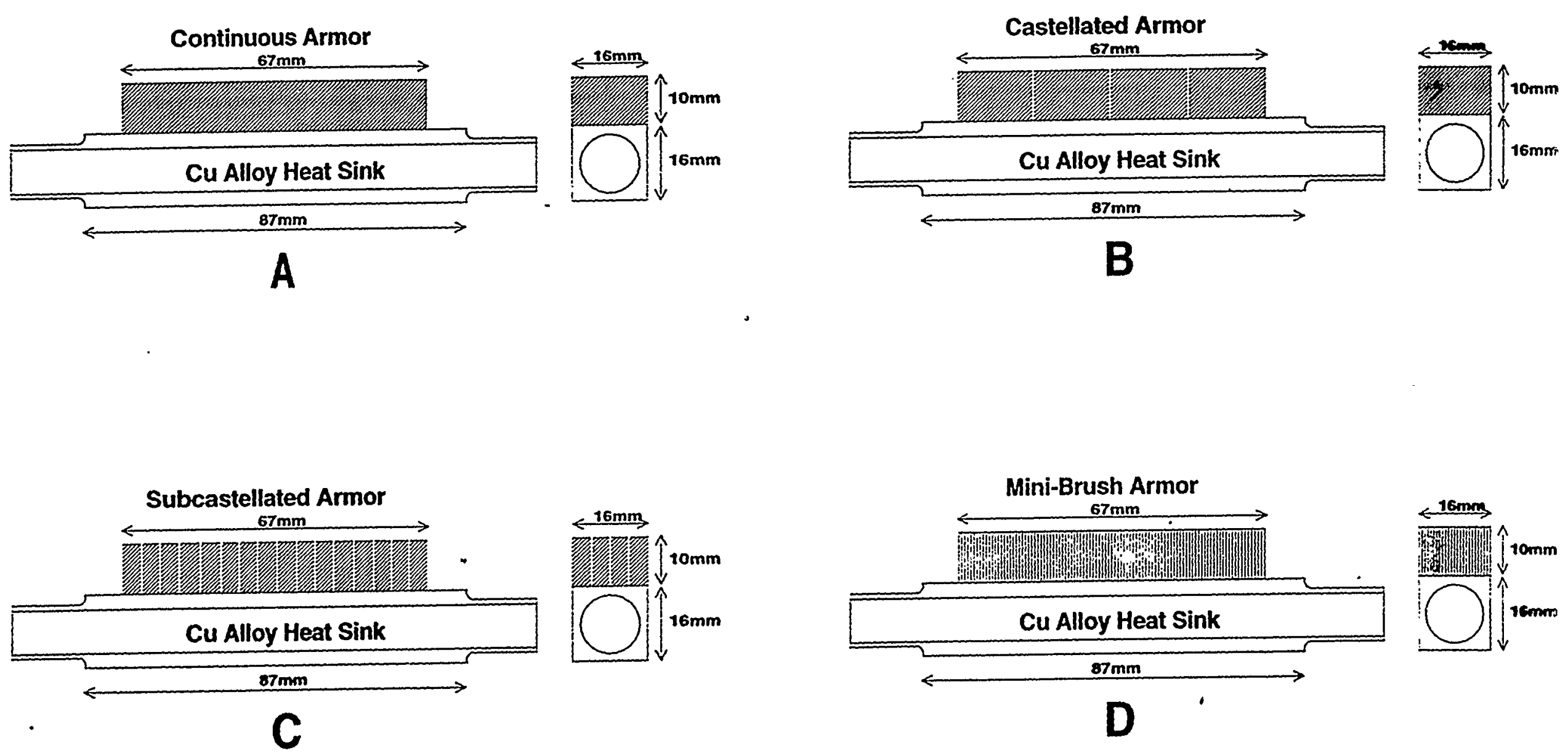

Figure 7 - The evolution of plasma facing component armor from the continuous covering to the mini-brush structure. 


\section{Vacuum Plasma Spray Brush Armor for Plasma Facing Components}

- Process is applicable to many different materials, i.e., W, Be, Carbon Composites

- Structure minimizes stress singularity of a flat bond line

- Grain orientation of the brush maximizes heat transfer to the copper alloy heat sink

- Small cross-sectional area reduces stress at interface (FEM)

\section{Brush Armor with End Points}

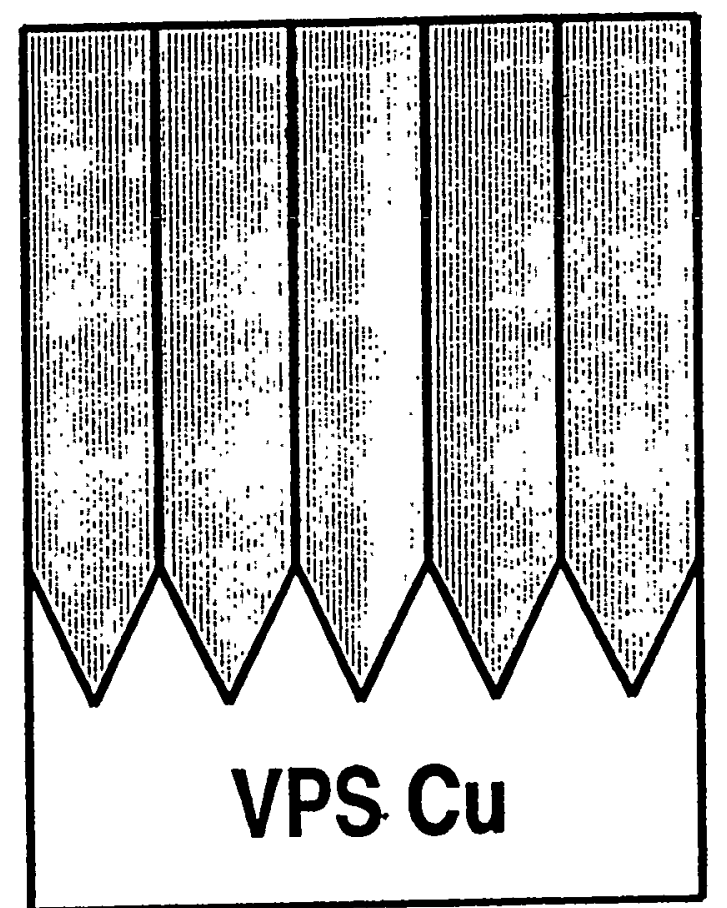

Plasma Processes Inc. 


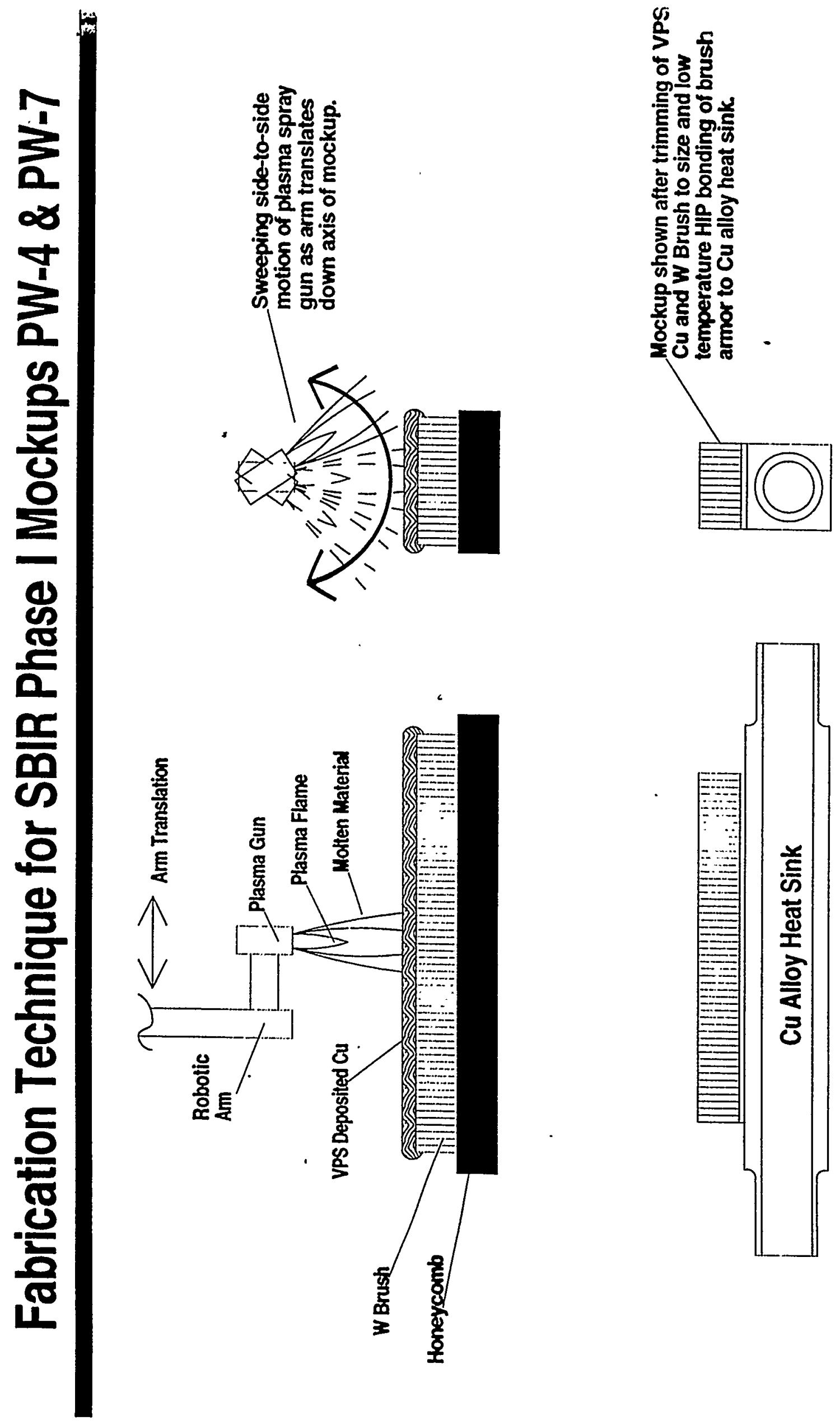



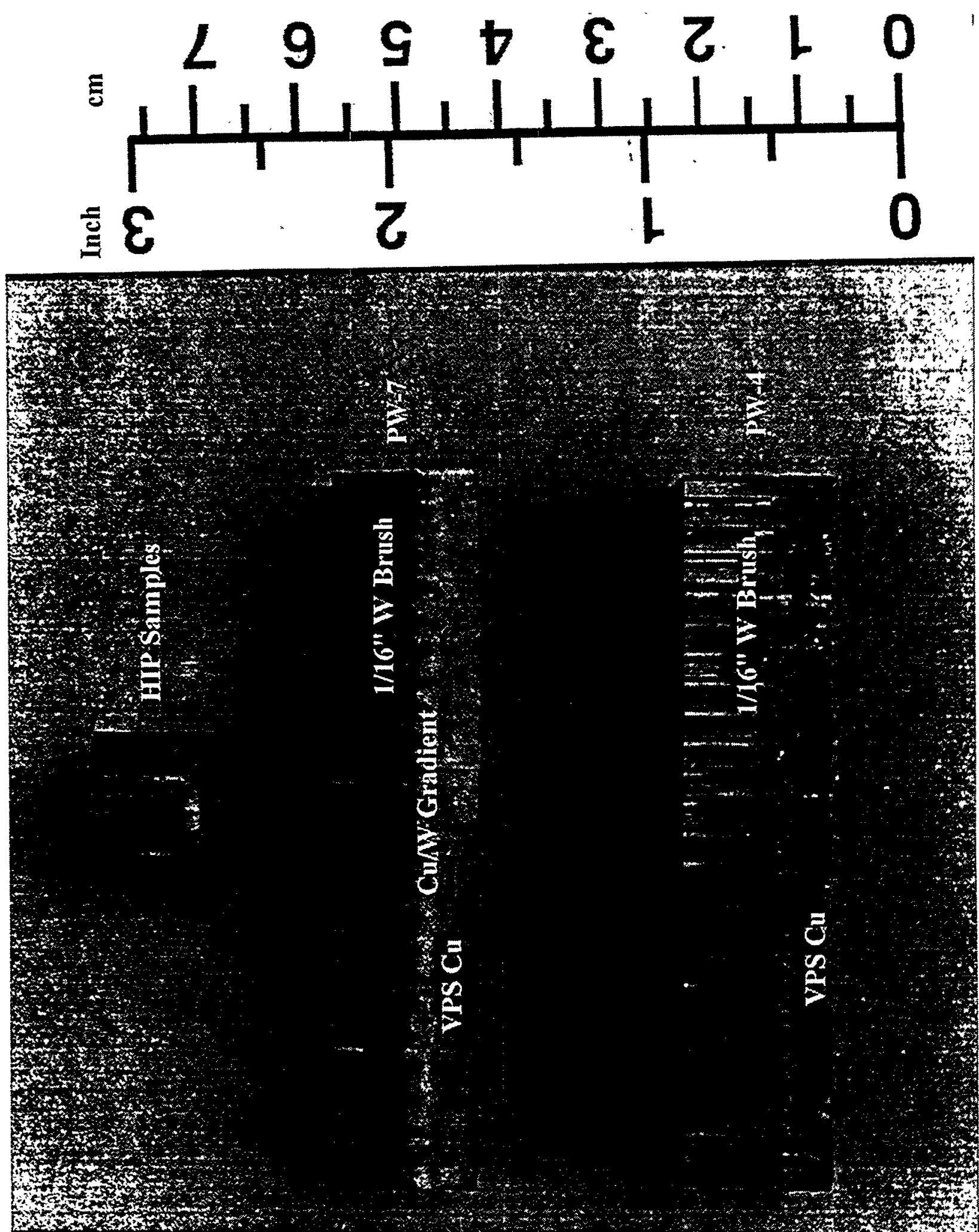


\section{Bonding Brush to Cu Alloy Heat Sink}

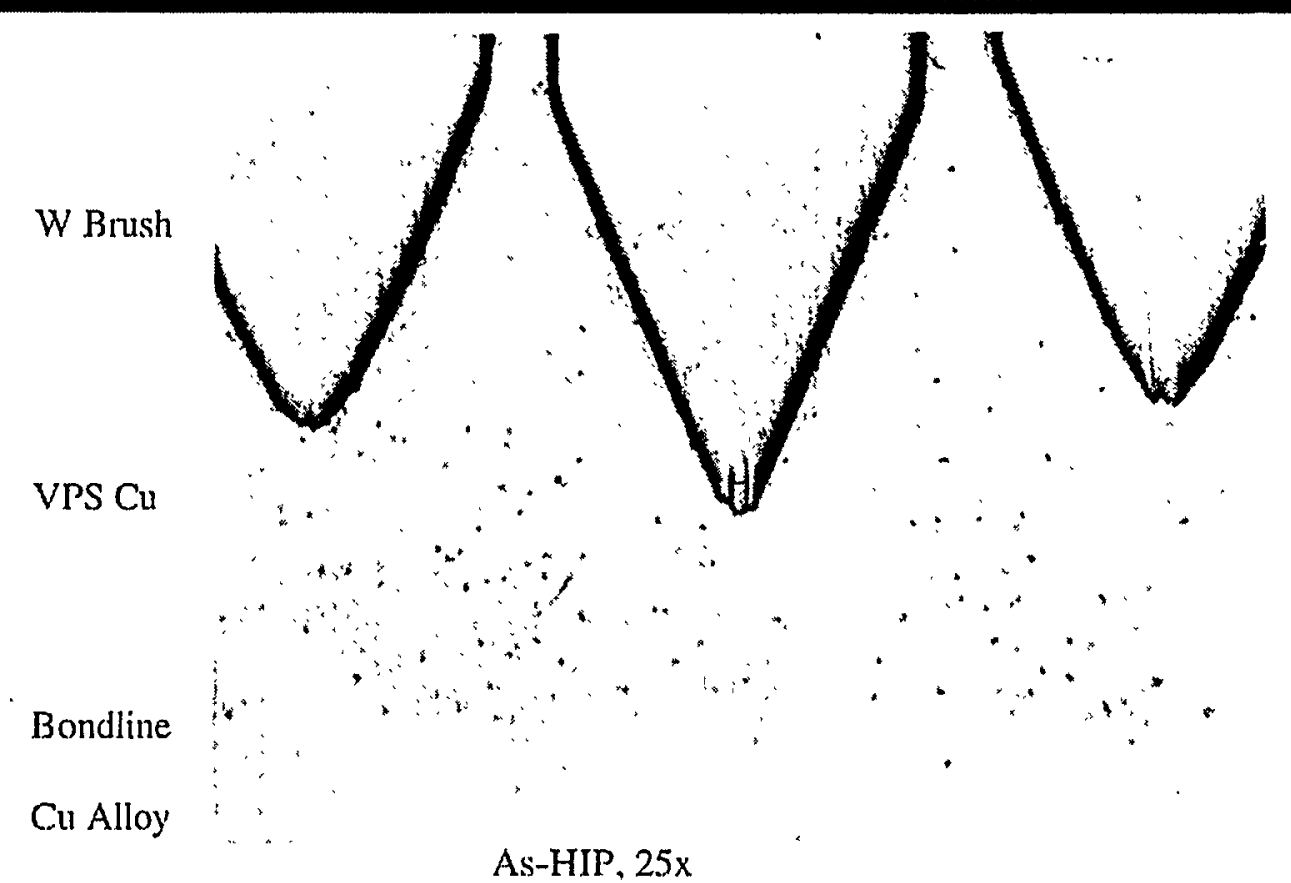

- VPS Cu and heat sink material cleaned and coated with PVD Ni

- Components placed in intimate contact in HIP can

- Joining accomplished through $450^{\circ} \mathrm{C}$ HIP diffusion bonding process

- Note penetration of W brush into VPS Cu ( 1 mm) for can \#107 (R/N V97-83A) 


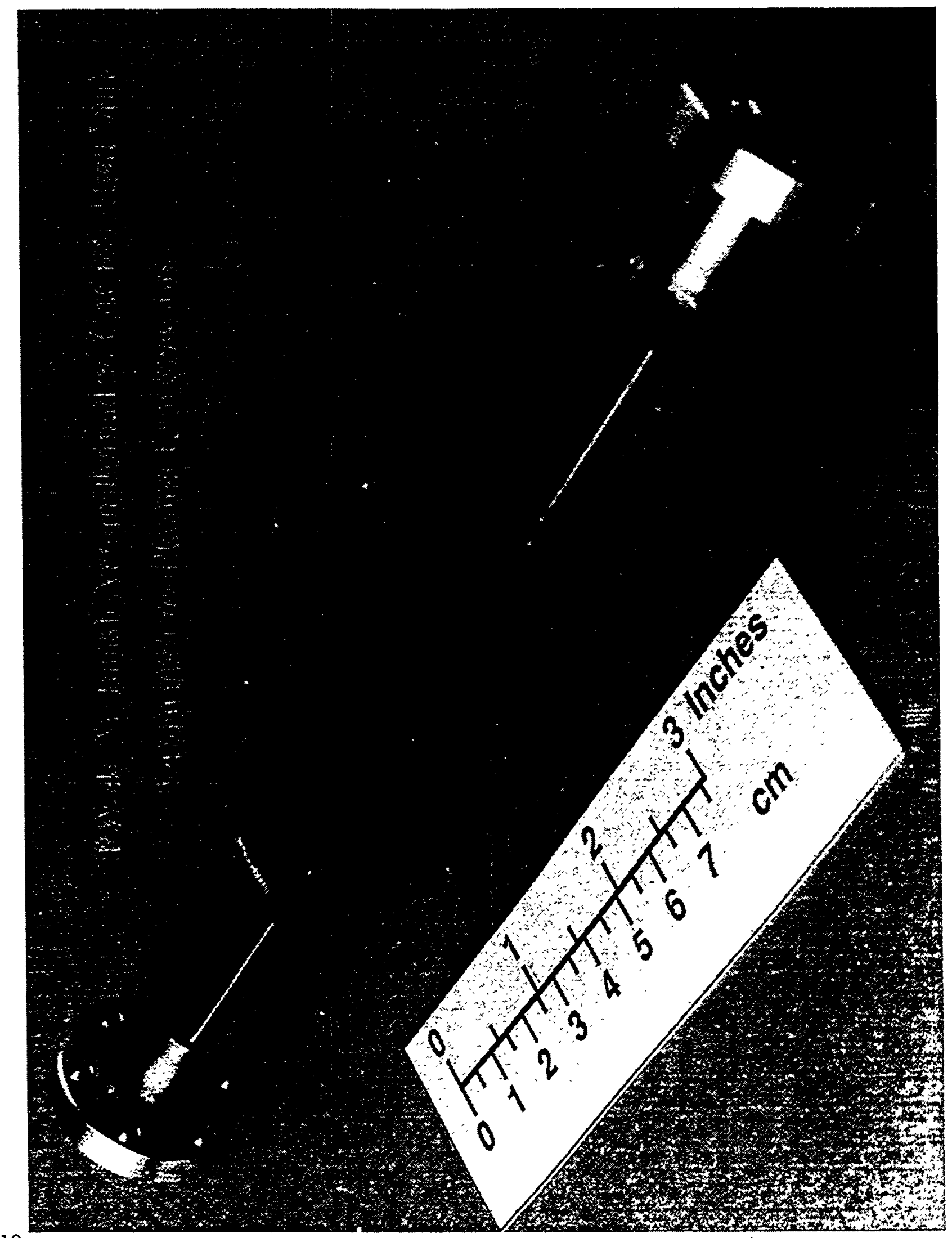

VIII-110 


\section{Optimization of Tip Design}

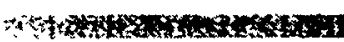

- Surface condition

- Coatings on tips (25-50 micron)

- Copper alloys

- Tip angles and depth of penetration in VPS Cu

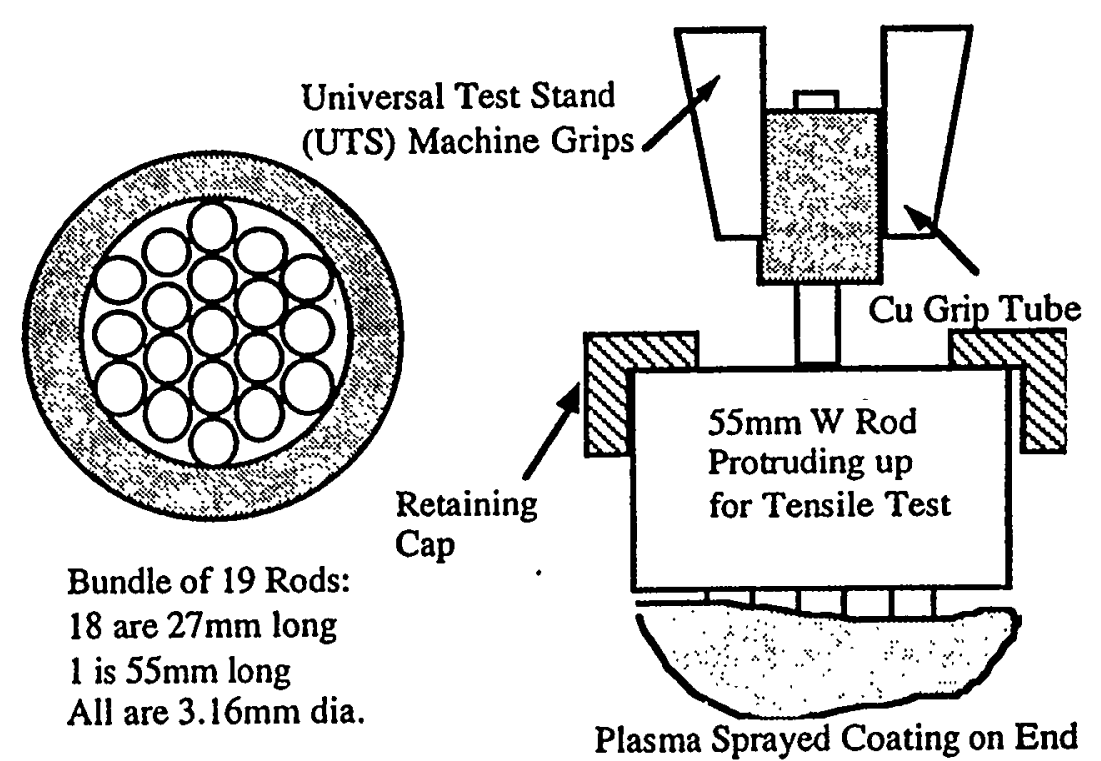

Plasma Processes Inc. 


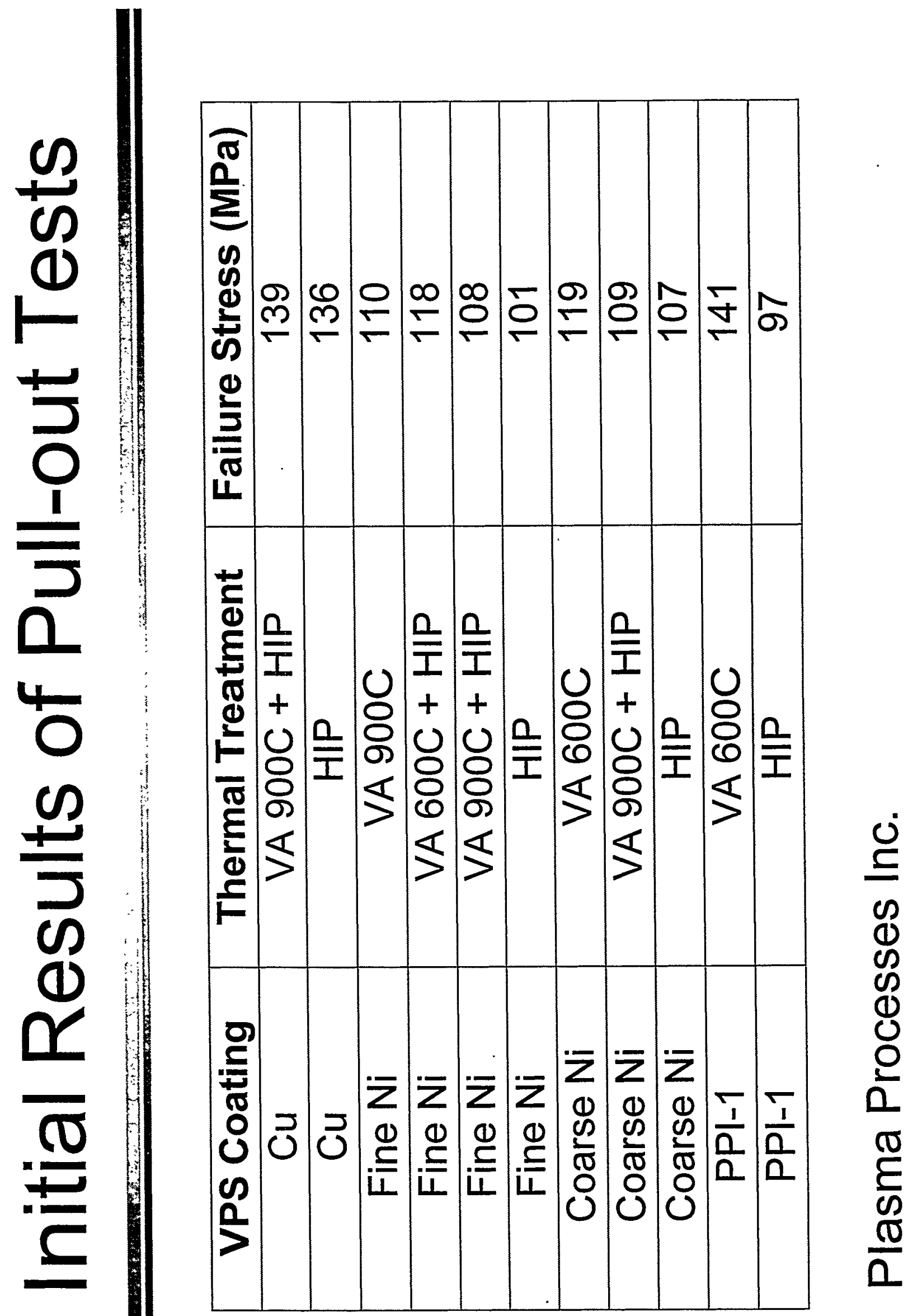




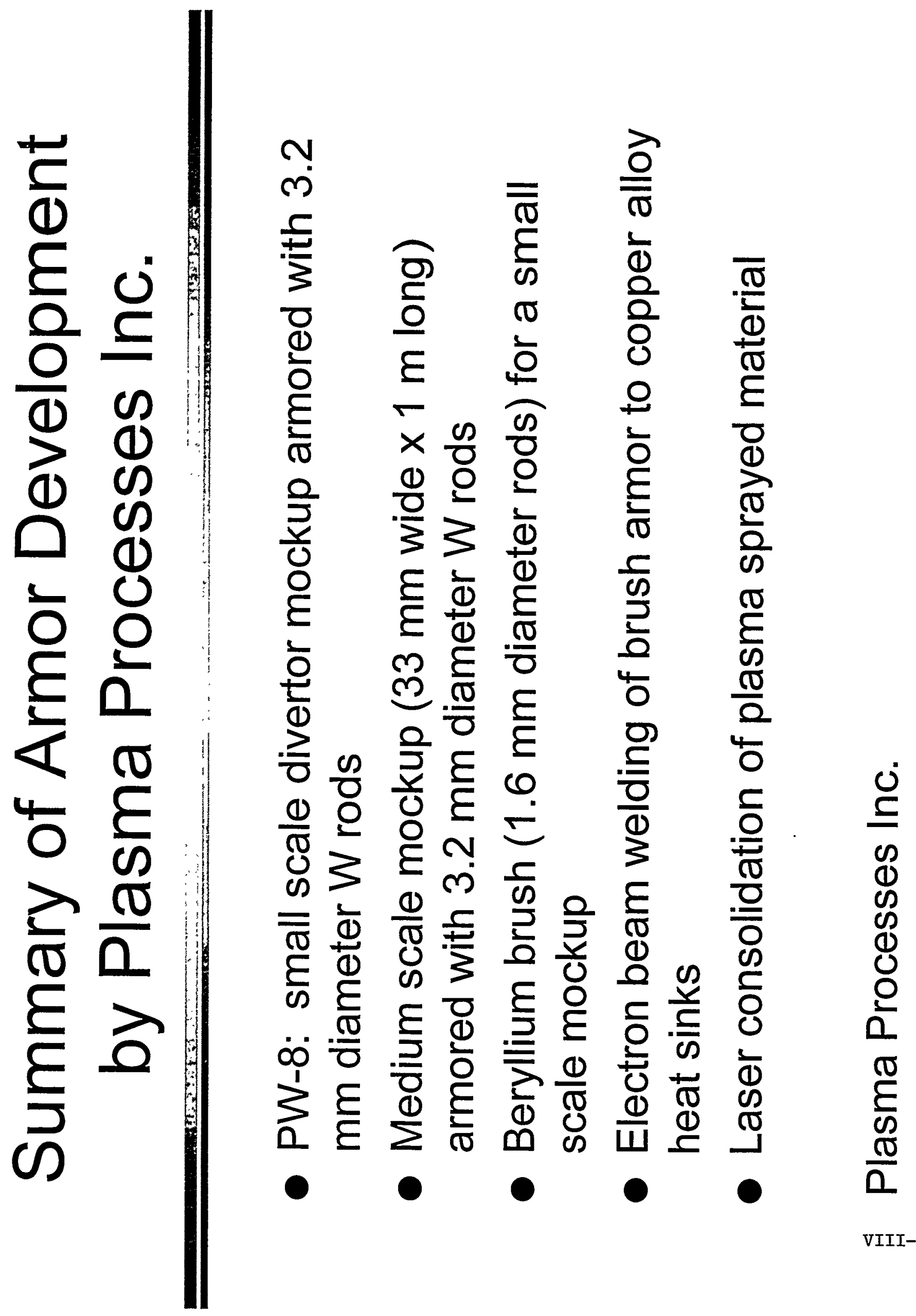


$\because$ 


\section{Section IX: PSI/PFM Issues of Collaboration}

IX-1 
[page intentionally left blank]

IX-2 
Subjects of collaborations

near term, US. JPN frame work

1. PSI studies, edge plasma physics in Wit to

SAL. UCSD, GA

proposing several programs.

$\therefore$ heat load distribution

- wall conditioning with mag. field

- boronization

- net erosion with long pulse ope.

- edge diagnostics

- identification of surface situation

$\mathrm{IX}-3$ 
2. Long term issues of PFC

- high z target plates

- protecting layers on last walls

- suet erosion studies:

- high heat flux components brazing. thermal hydrolics: materials, the gas cooling.

3. Series of WS on HHFE \&PSI

4. UNIV. in Japan Kyushr.U.

Nagoya:U.

- Osaka u. Hokkaido.U.

5. JAERI

Toyama 0

V. Tokyo 
7rरab 22

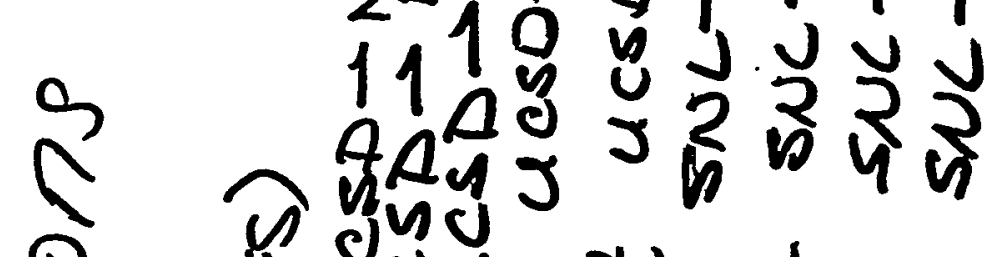<smiles>C=[As]</smiles>

o है

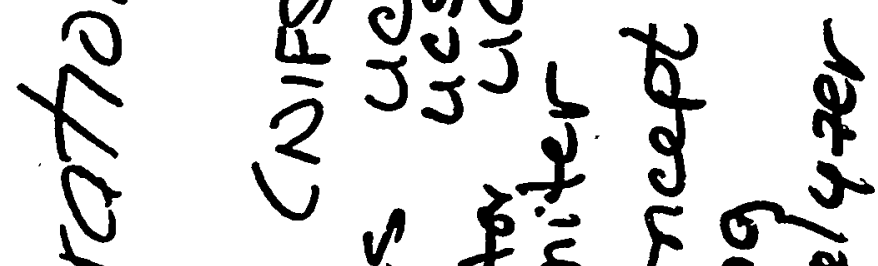

8

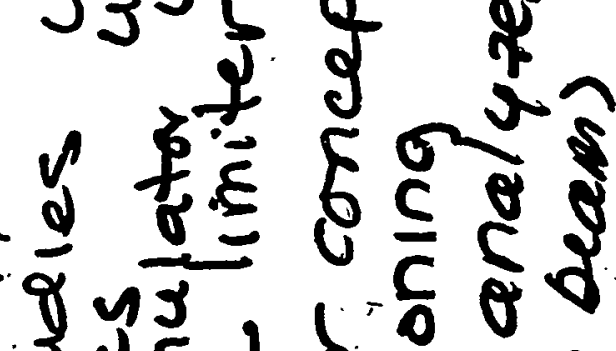

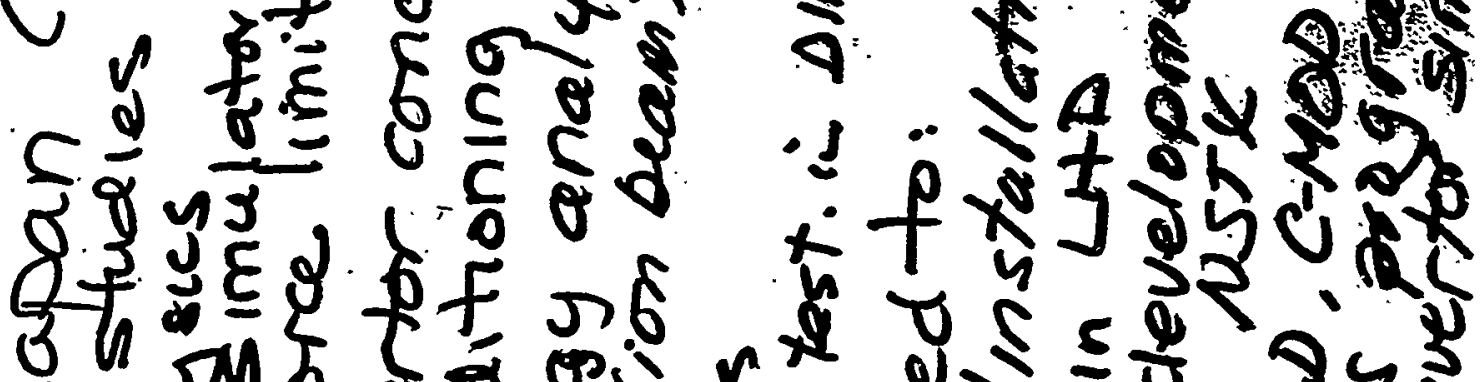

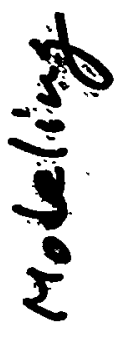

$\neq$

0 r

₹ \&

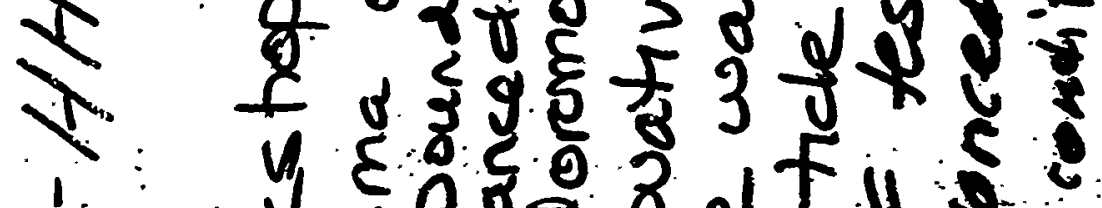

i

y y

पू t t - 


\section{Session X: Panel on Future PFC Concepts}


[page intentionally left blank] 


\title{
ALPS Summary
}

ALPS Working Group

\author{
Presented by Dai-Kai Sze \\ Argonne National Laboratory \\ Argonne, IL.
}

US-Japan Workshop on

High Heat Flux Components \& Plasma Surface Interactions for next Fusion Devices, San Francisco December 8-11, 1997 


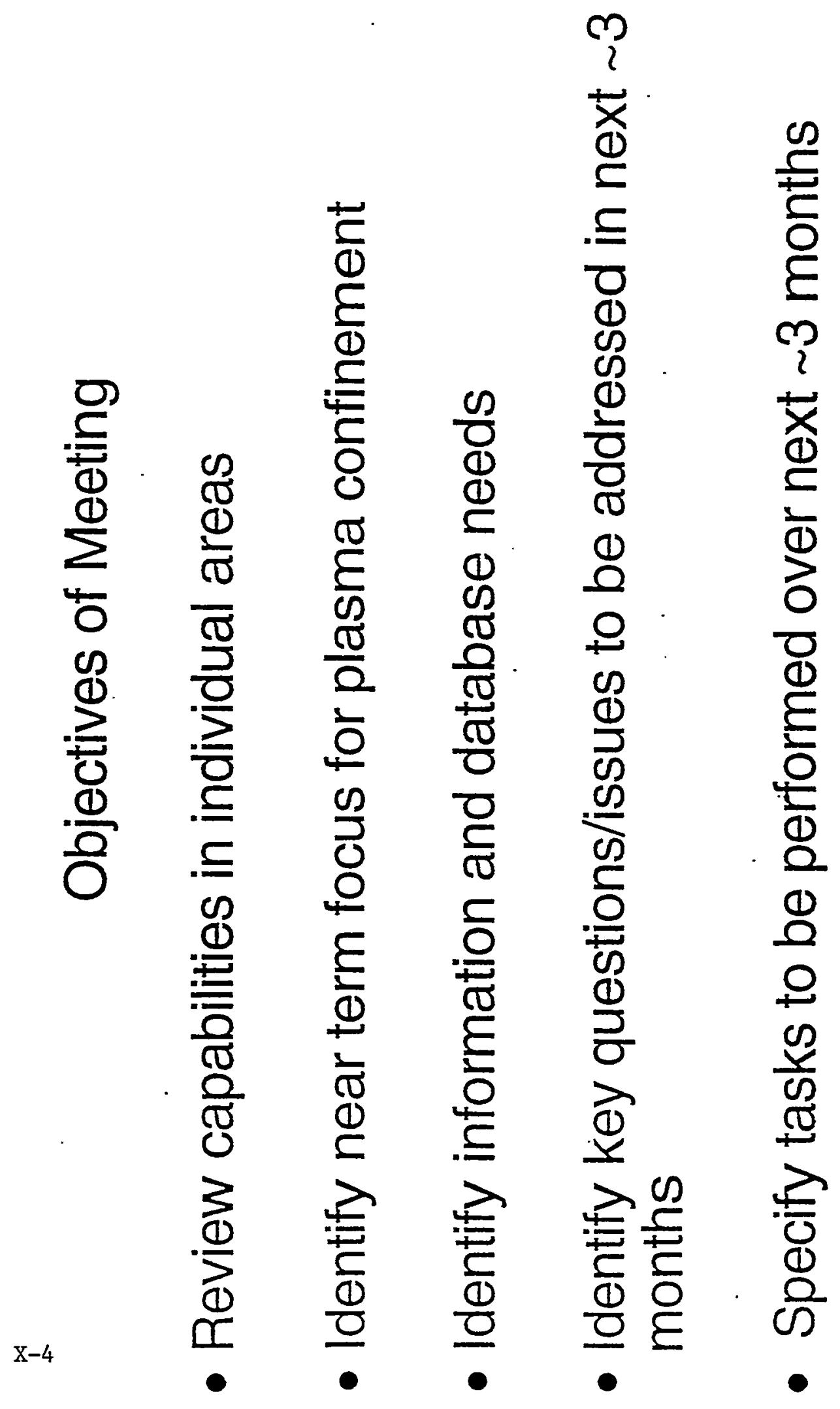




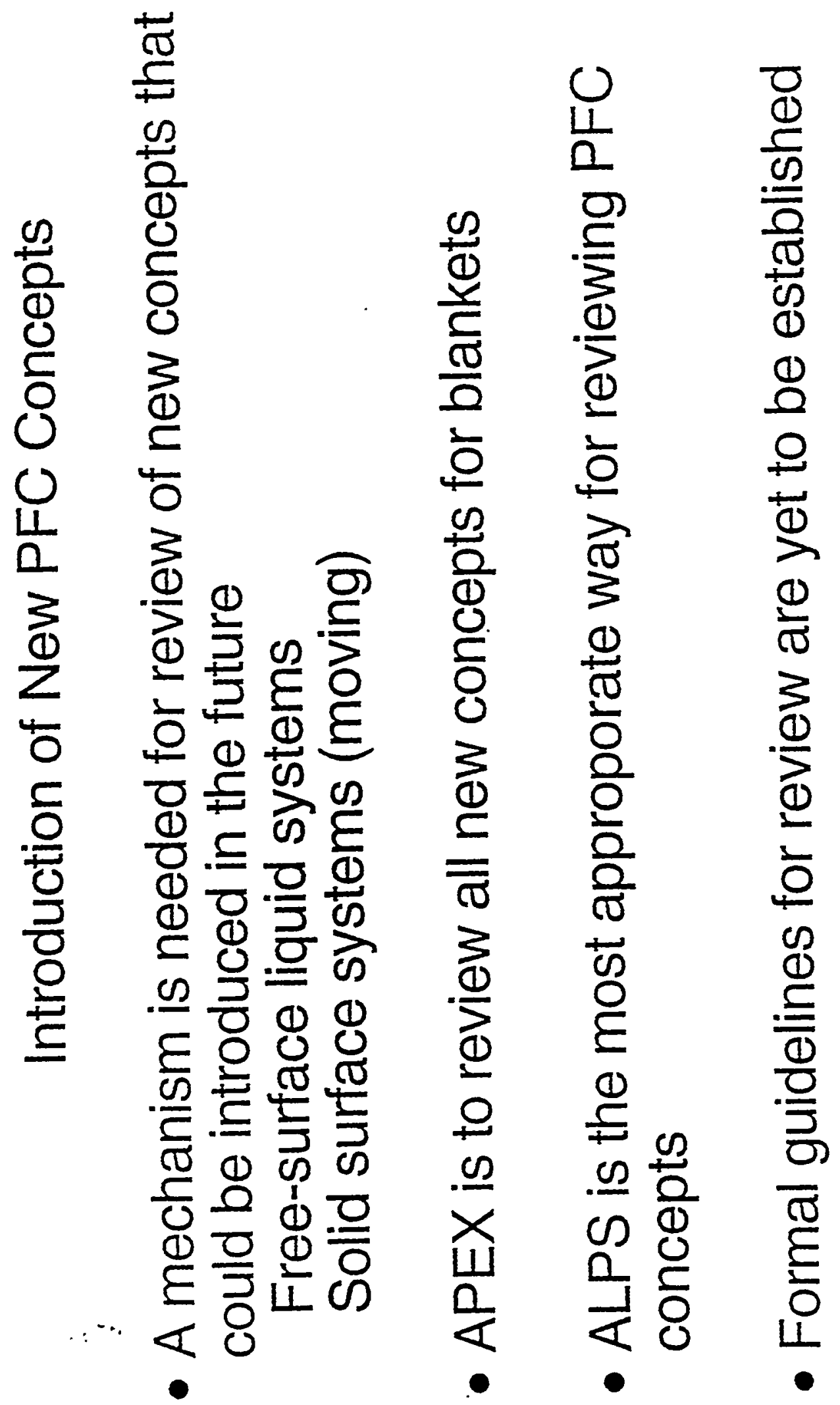




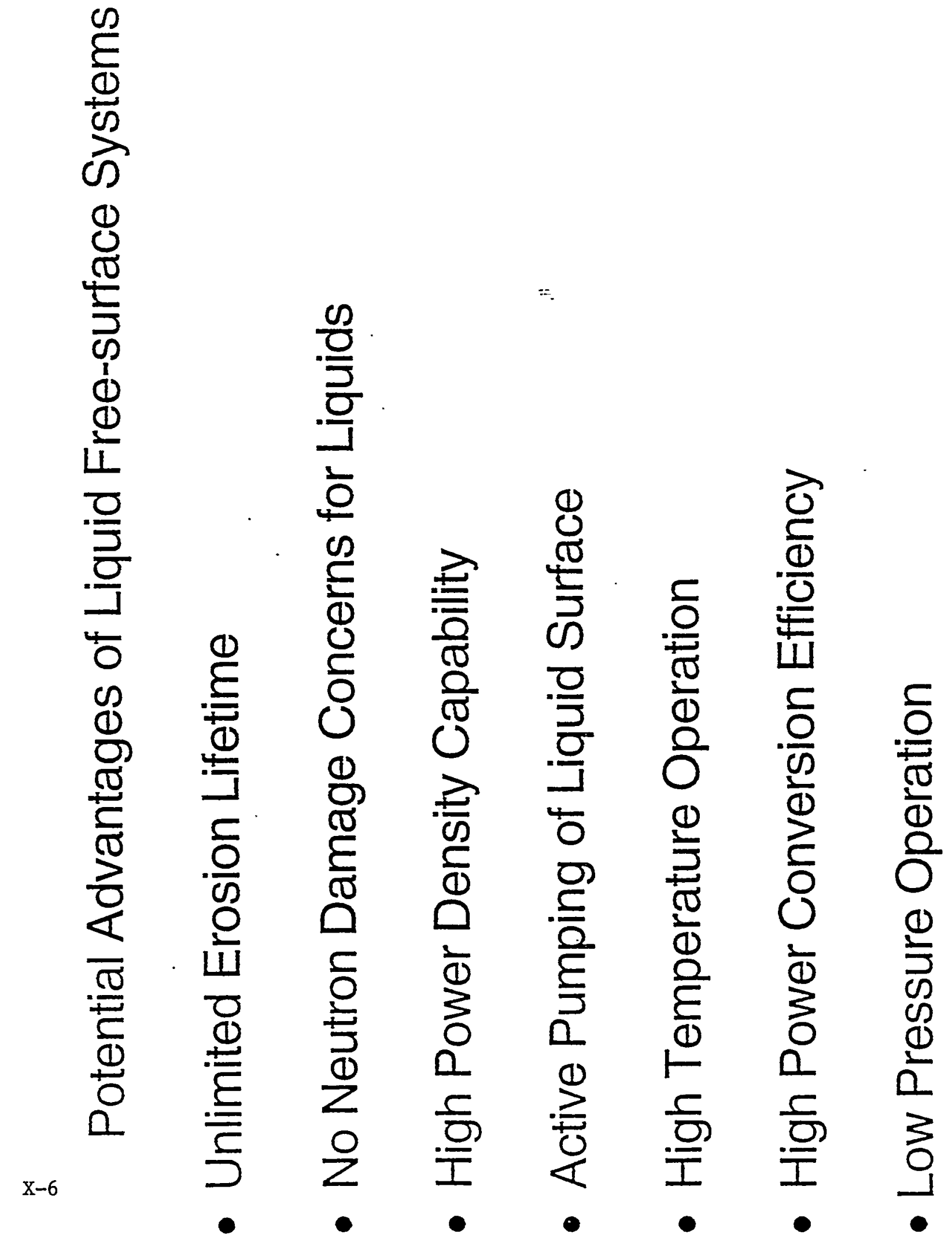




\section{Participating US Institutions}

- Argonne National Laboratory

- General Atomic

- Idaho National Environmental Engineering Laboratory

- Lawrence Livermore National Laboratory

- Oak Ridge National Laboratory

- Princeton Plasma Physics Laboratory

- Sandia Natioanal Laboratory

- University of California - Los Angeles

- University of California - San Diego

- University of Illinois

- University of Wisconsin 


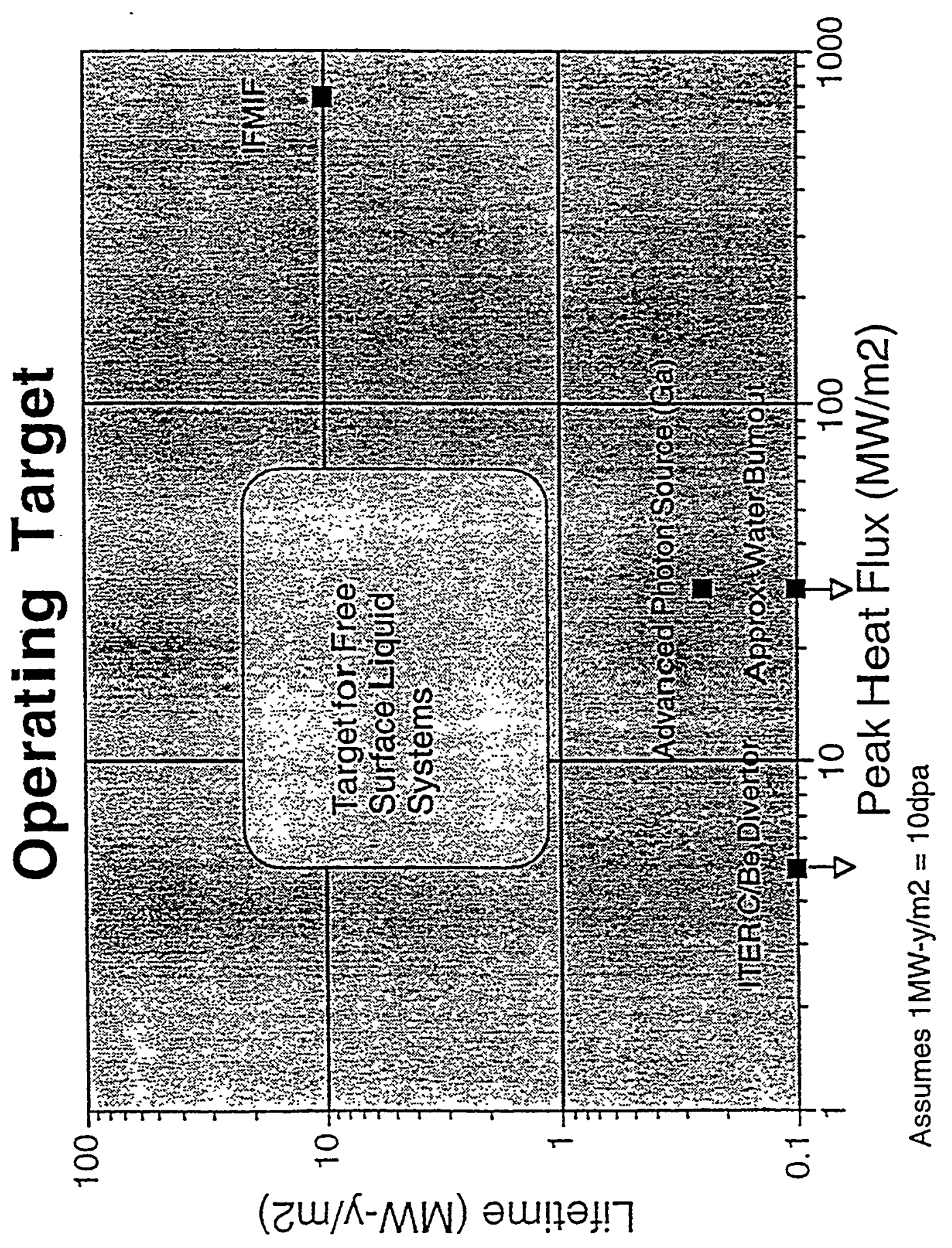

$x-8$ 


\section{Key Questions}

-What are the heat flux and power density limits for liquid freesurface systems?

-What are the maximum allowable evaporation rates for liquids that still insure stable plasma operation?

- How stable is the liquid surface during normal and off-normal conditions?

- How will the liquid free-surface systems alter the plasma edge conditions?

- How will the liquid free-surface systems affect other fusion systems? 


\section{Initial Assessment of Issues and R\&D for Liquid Plasma-Facing Components}

\begin{tabular}{|c|c|}
\hline Issue & R\&D Needs \\
\hline Sputtering and redeposition & $\begin{array}{l}\text { Assess sputtering yields along with sheath and } \\
\text { near-surface transport at liquid surfaces by } \\
\text { hydrogen, helium, and self-sputtering. Validate } \\
\text { models with plasma experiments. }\end{array}$ \\
\hline Species transport to plasma & $\begin{array}{l}\text { Measure } \mathrm{H}, \mathrm{He} \text { and self-sputtering rate vs. } \\
\text { energy for } \mathrm{Li}, \mathrm{Ga}, \mathrm{LiPb}, \mathrm{Sn} \text { and Flibe and other } \\
\text { canditate liquids. Model/measure edge plasma } \\
\text { transport from liquid surface. }\end{array}$ \\
\hline Plasma-liquid interface stability & $\begin{array}{l}\text { Modeling and data on plasma momentum flux } \\
\text { effects. Modeling and data on electric field and } \\
\text { current effects. }\end{array}$ \\
\hline Tritium (and $\mathrm{He}$ ) removal & $\begin{array}{l}\text { Measure tritium and deuterium uptake in TPE } \\
\text { and DiMES for candidate liquids, respectively. } \\
\text { Determine basic thermophysical properties. } \\
\text { Benchmark DIFFUSE with TPE, and DiMES } \\
\text { data. Define tritium extraction system, estimate } \\
\text { size and cost. Determine tritium inventory using } \\
\text { DIFFUSE }\end{array}$ \\
\hline Integrated plasma tests & $\begin{array}{l}\text { Multiple effect liquid surface / plasma interaction } \\
\text { tests in PISCES, DiMES, DIID tests. }\end{array}$ \\
\hline $\begin{array}{c}\text { Power density limits and heat } \\
\text { removal }\end{array}$ & $\begin{array}{l}\text { Calculate MHD external pressure drop. Define } \\
\text { maximum allowable temperature. Evaluate } \\
\text { thermal response to establish maximum q. } \\
\text { Produce benchmark heat transfer data }\end{array}$ \\
\hline $\begin{array}{l}\text { MHD Behavior of Liquid Metal } \\
\text { Free Surfaces }\end{array}$ & $\begin{array}{l}\text { Develop models of flows of free surfaces } \\
\text { including internal recirculation and turbulent } \\
\text { fluctuations. Provide benchmark data for intemal } \\
\text { flows. }\end{array}$ \\
\hline Insulator Coating Development & $\begin{array}{l}\text { Develop insulator coatings and test in-situ } \\
\text { resistivity. Determine irradiation effects on } \\
\text { coating resistivity. }\end{array}$ \\
\hline Radioactivity & $\begin{array}{l}\text { Define existing and goal impurity levels. Identify } \\
\text { chemical processes needed for impurity removal. } \\
\text { Identify missing cross section data data and dose } \\
\text { conversion factors.Investigate waste } \\
\text { management and safety characteristics of liquid } \\
\text { candidates and associated structure materials and } \\
\text { insulator coatings. }\end{array}$ \\
\hline Tritium Fuel Cycle & Develop models for overall fuel cycle \\
\hline $\begin{array}{l}\text { Material transport to vacuum } \\
\text { pump }\end{array}$ & $\begin{array}{l}\text { Plasma tests with liquid at high temperature. } \\
\text { Model the transport of liquid to the vacuum } \\
\text { pumping system }\end{array}$ \\
\hline
\end{tabular}




\section{Examples of liquid surface divertor systems}

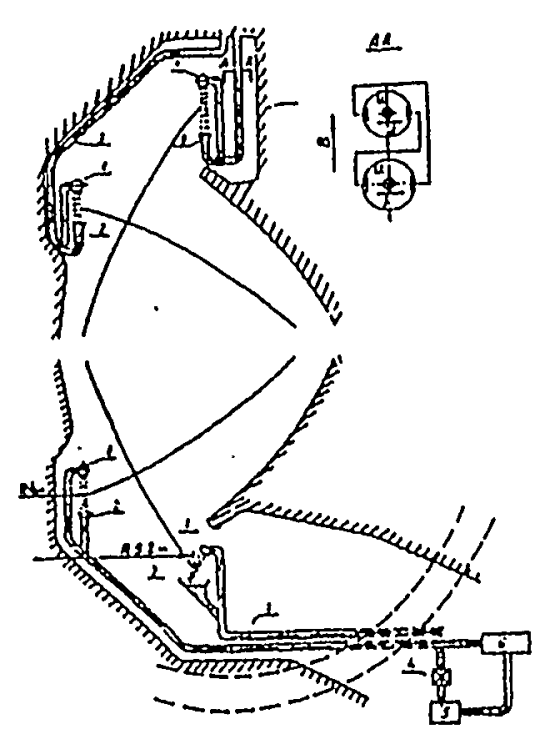

(a)

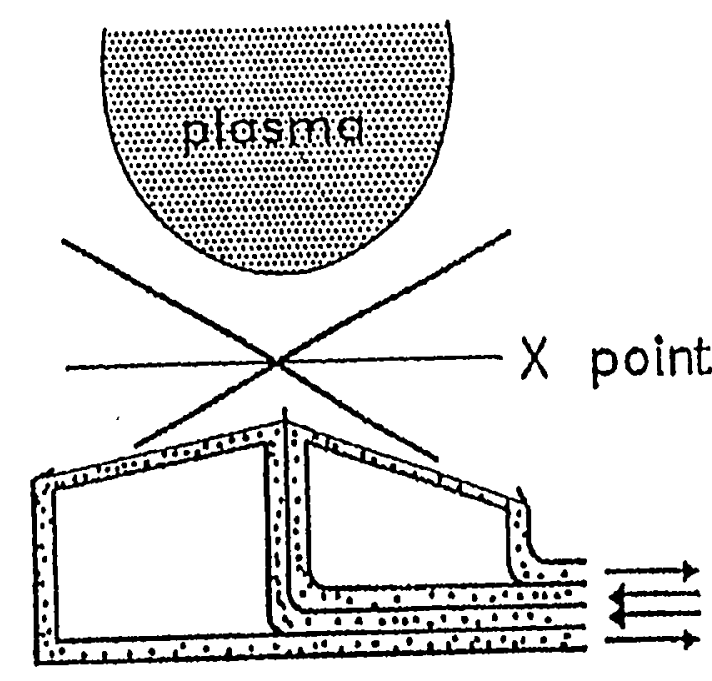

(b)

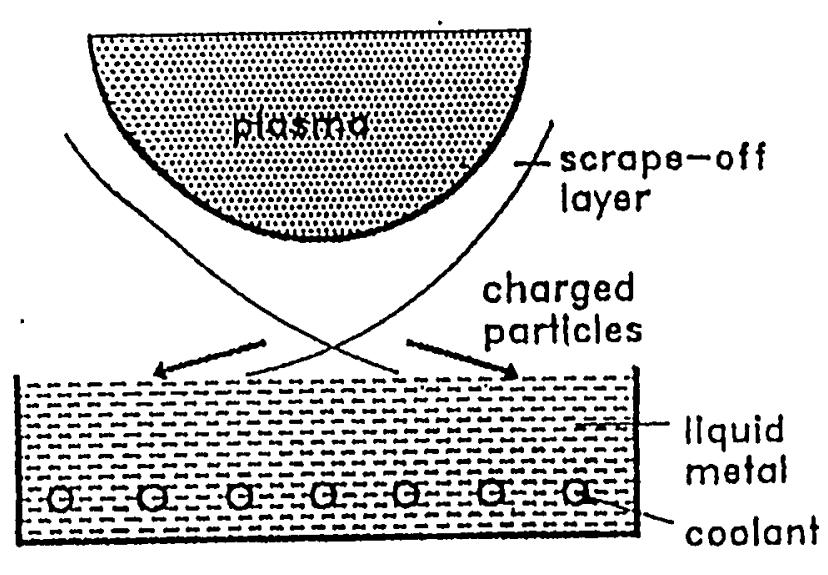

(c)

(a) Droplet double-null option, (b) Liquid flow over divertor plate option and (c) Stagnant pool with separate cooling

$\stackrel{\leftrightarrow}{5}$ 
Possible Materials, Configuration, and Confinement Options

- Liquid species

Li, Pb-17Li, Ga, Flibe, Sn, Flibe, Al, Al-Si

- Flow configuration

Fast film, droplets, water fall, stagnant film, pool, backside impinging jet

- Confinement

Tokamak, Advanced Tokamak, SphericalTorus, Field Reversed Configuration, Stellerator

- Near term objective is to reduce the number of options under consideration 


\section{Primary Objectives of the Evaluation Phase}

- Demonstrate that the advantages of free-surface liquid systems are real

One or more concepts are feasible and could operate as planned

- Provide sufficient confidence in their operation such that a significant follow-on activity can proceed

Installation of prototypes into plasma confinement devices. 


\section{There are Three, Closely Integrated Activities}

- Concept Evaluation

- PMI/Transport

- Engineering

- PMI/Transport and Engineering support the Evaluation

- Review and summary of the existing database

- Application of state-of-the-art models

- Providing key experimental data

- Developing improved codes. 


\section{Fig. 2-1 Evaluation Phase Schedule Free Surface Liquid Plasma Facing Systems}

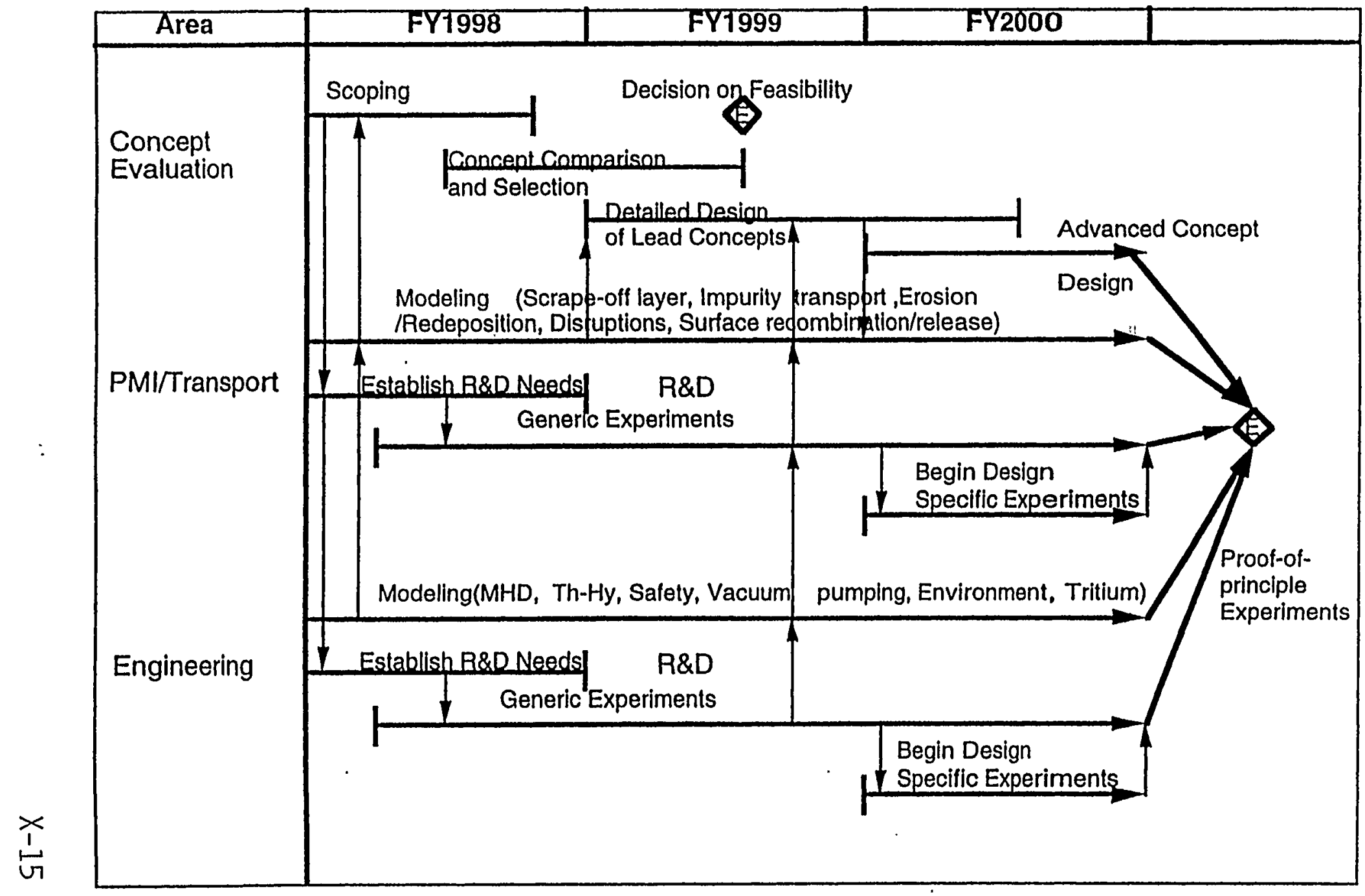




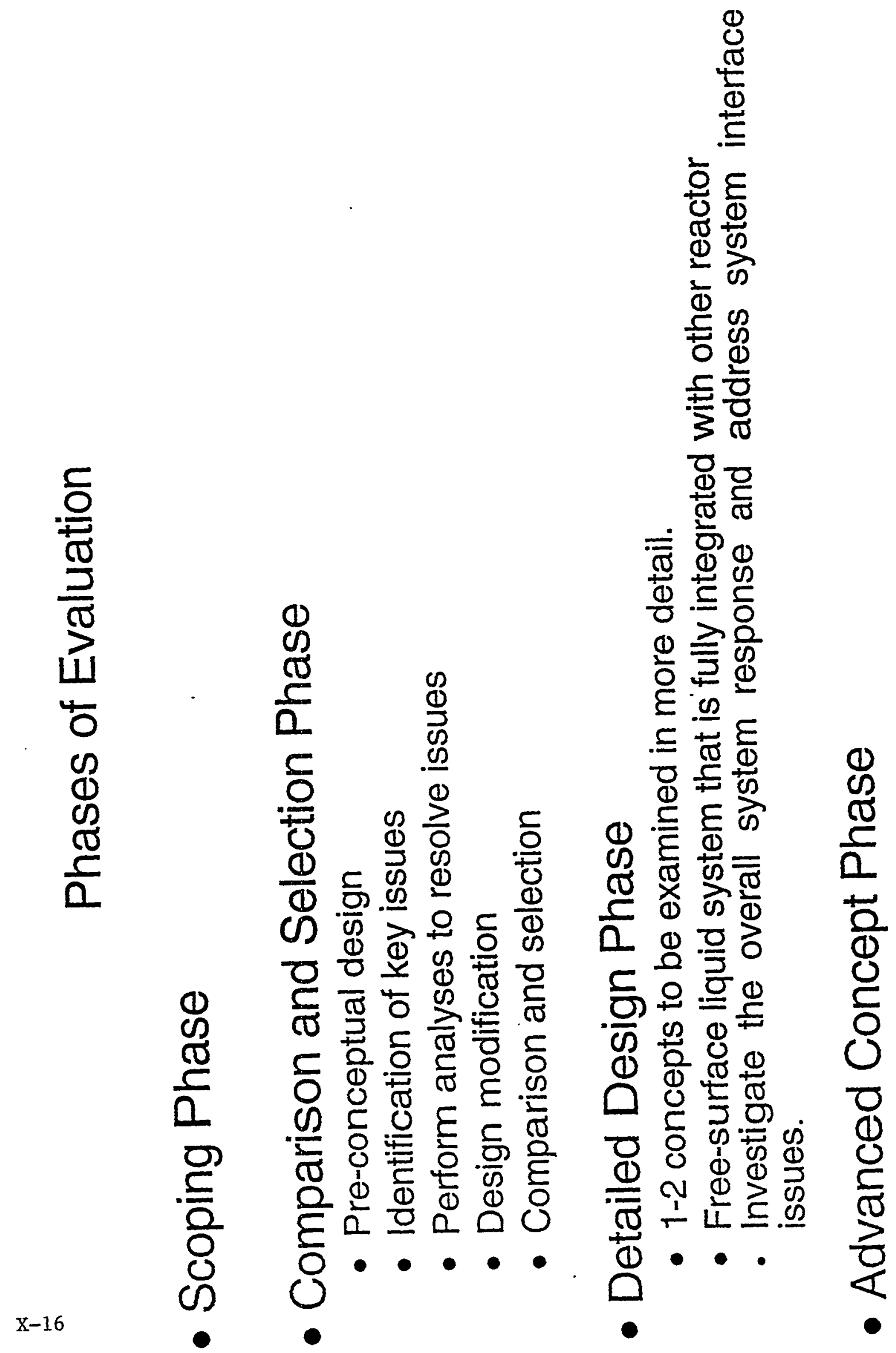




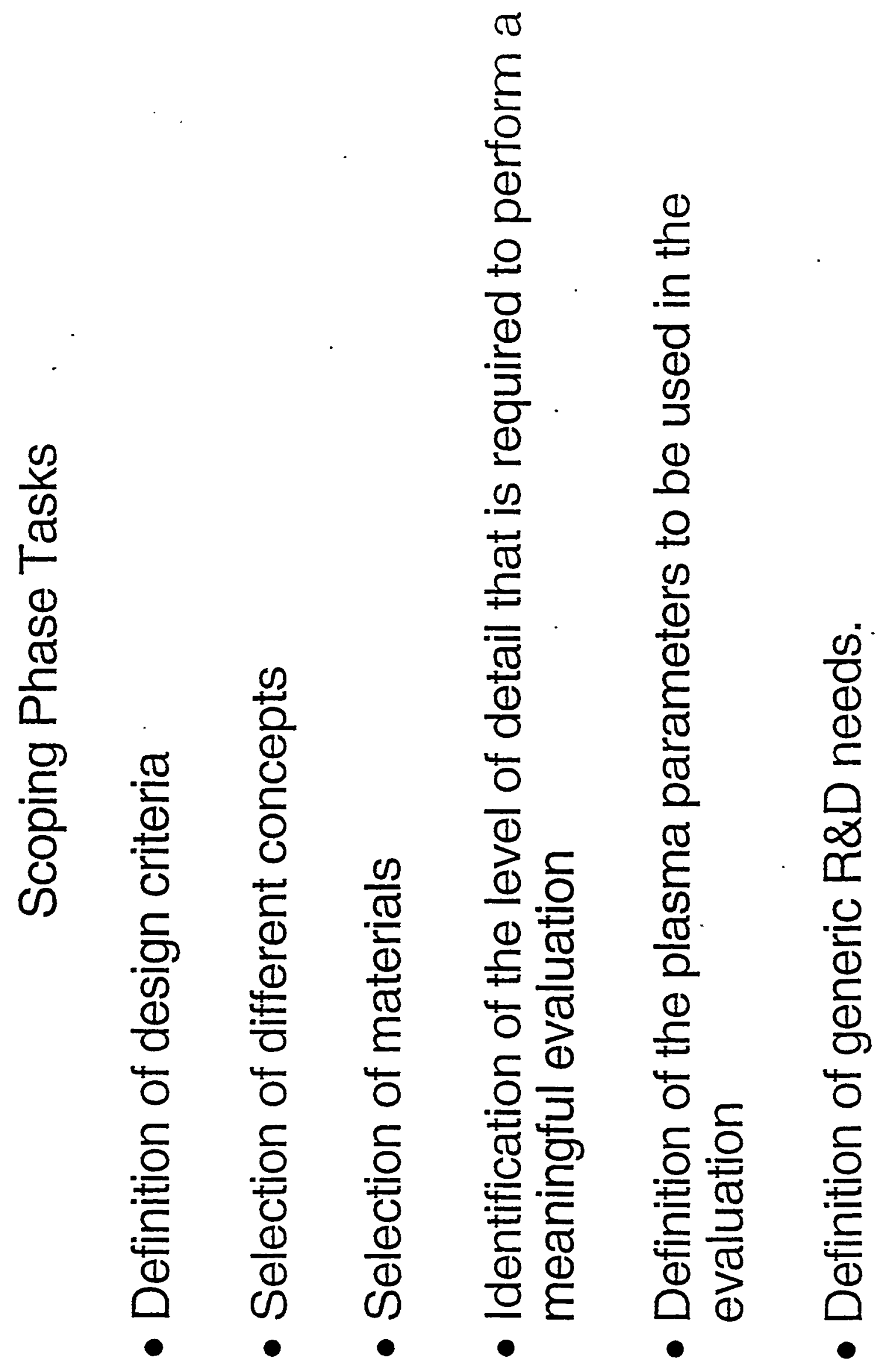




\section{Scoping Phase Tasks}

- Near Term Tasks for PMI/Transport

- Chamber surface configuration of conventional and alternate concepts

- Reference design physics parameters

- Allowable impurity fraction

- Impurity operation fraction Distribution of first wall and divertor heat flux

- Near Term Tasks for Engineering

- Preliminary liquid flow calculations

- Preliminary heat transfer calculations

- Preliminary estimates on liquid temperature limits 


\section{Performance Goals for Attractive Fusion Energy Systems}

\begin{tabular}{|c|c|c|}
\hline Attribute & Minimum Goal & $\begin{array}{l}\text { Grand } \\
\text { Challenge }\end{array}$ \\
\hline $\begin{array}{l}\text { Coolant Inlet/Outlet Temperature }\left({ }^{\circ} \mathrm{C} \text { ) }\right. \\
\text { (goal of } 45 \% \text { conversion efficiency) }\end{array}$ & $250 / 500$ & $250 / 1000$ \\
\hline Peak / Average Neutron Wall Load (MW/m²) & $6 / 3$ & $20 / 10$ \\
\hline Peak / Average Heat Flux (MW/m²) & $5 / 2$ & $50 / 20$ \\
\hline First Wall Fluence Lifetime (MW-y/m²) & 10 & 20 \\
\hline First Wall Erosion Lifetime $(y)$ & 2 & $\infty$ \\
\hline Time to Repair/Replace & $<1$ month & $<1$ week \\
\hline Average Cost of Core Materials $(\$ / \mathrm{kg})$ & 100 & $<50$ \\
\hline Waste Disposal Limit & $\begin{array}{c}\text { Class C } \\
\text { Major } \\
\text { Components }\end{array}$ & $\begin{array}{c}\text { Class C } \\
\text { All } \\
\text { Components }\end{array}$ \\
\hline Worst-Case Accident Dose at Site Boundary & $1 \mathrm{rem}$ & $0.1 \mathrm{rem}$ \\
\hline
\end{tabular}




\section{Set of Device Parameters for ARIES-RS}

Plasma major radius

Plasma minor radius

Divertor plate toroidal width

Divertor plate length

Magnetic field on axis

Plasma current

Neutron wall load normal to divertor

Total transport power

Divertor surface heating

Average divertor surface heat flux

Peak divertor surface heat flux

Heat exchanger outlet/inlet temperature

Plasma Zeff

Mid-plane SOL thickness

He exhaust rate

$\mathrm{H}$ exhaust rate

ne(a)
$5.5 \mathrm{~m}$

$1.4 \mathrm{~m}$

$1.5-2 \mathrm{~m}$

$\sim 1 \mathrm{~m}$

$8.0 \mathrm{~T}$

$11.32 \mathrm{MA}$

$\sim 1 \mathrm{MW} / \mathrm{m}^{2}$

$431 \mathrm{MW}$

$348 \mathrm{MW}$

$2 \mathrm{MW} / \mathrm{m}^{2}$

$6 \mathrm{MW} / \mathrm{m}^{2}$

$610 / 330{ }^{\circ} \mathrm{C}$

1.7

$1 \mathrm{~cm}$

$7.7 \times 1020 / \mathrm{s}$

1000 Torr-1/s

$0.6 \times 1020 / \mathrm{m}^{3}$ 


\section{PMI/Transport Proposed Tasks}

- Priority 1: (Needed in the first 3 to 6 months of the study)

- Chamber surface configuration of conventional and alternate concepts

- Reference design physics parameters

- Allowable impurity fraction

- Impurity operation fraction

(Defined as: The First wall and divertor having the same heat flux) 


\section{PMI/Transport Proposed Tasks (Cont.)}

- Priority 2: (Some results can be generated in the first year of the study and beyond)

Reference solid surface design:

- Definition of a solid surface reference divertor design

- PMI, basic and integrated data, for modeling and experiments:

- Sputtering yield of relevant liquid materials from hydrogen, He and selfsputtering

- Basic data on trapping and up-take of hydrogen and He

- Design limits modeling

- Erosion and redeposition integrated data

- Disruption simulations

- Review of TFTR results

- PMI integrated transient effects modeling

- Heated DiMES

Transport, basic and integrated, data for analysis, modeling and experiments:

- Transport of impurities at divertor, SOL, core...modeling

- Transport of impurities at divertor, SOL, core...experiments 


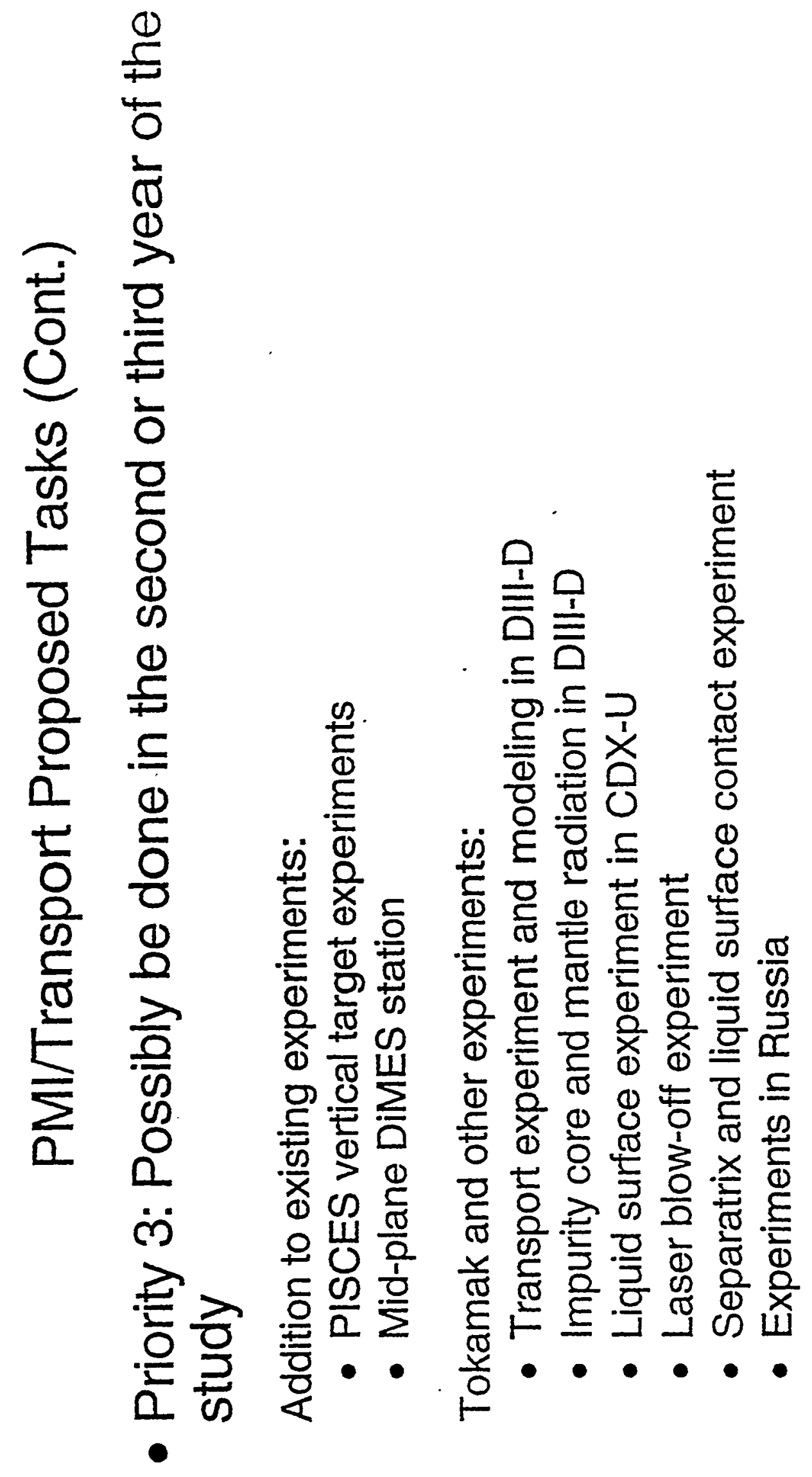




\section{Engineering Proposed Tasks}

Priority 1: Initial Assessement of Candidate Heat Removal Surfaces (Results needed in the first 6 months of the study)

- Preliminary liquid flow calculations

- Preliminary heat transfer calculations

- Preliminary estimates on liquid temperature limits

Priority 2: General System Analysis of Candidate Heat Removal Surfaces (Some results can be generated in the first year of the study and beyond)

Modeling

- Limits on material transport (plasma/pumps) (to refine the temperature limit on the liquid surface)

- Effect of transients and disruptions

- Waste management evaluation

- Continued heat transfer / liquid flow calculations 


\section{Engineering Proposed Tasks (Cont.)}

- Priority 2 (Cont.)

\section{Experiment}

- Properties of tritium in candidate liquids

- Activation cross sections and corresponding dose conversion factors (including impurities)

- Insulator coating development for liquid metals

- Priority 3: Detailed Engineering Performance Analysis of Promising Concepts (Possibly be done in the second or third year of the study) Modeling

- MHD flow behavior of free-surface liquid metals

- Heat transfer

- System response under off-normal conditions (scoping calculations)

Experiment

- MHD flow characteristics of free-surface liquid metals

- Thermalhydraulic/High Heat Flux tests in a high magnetic field 


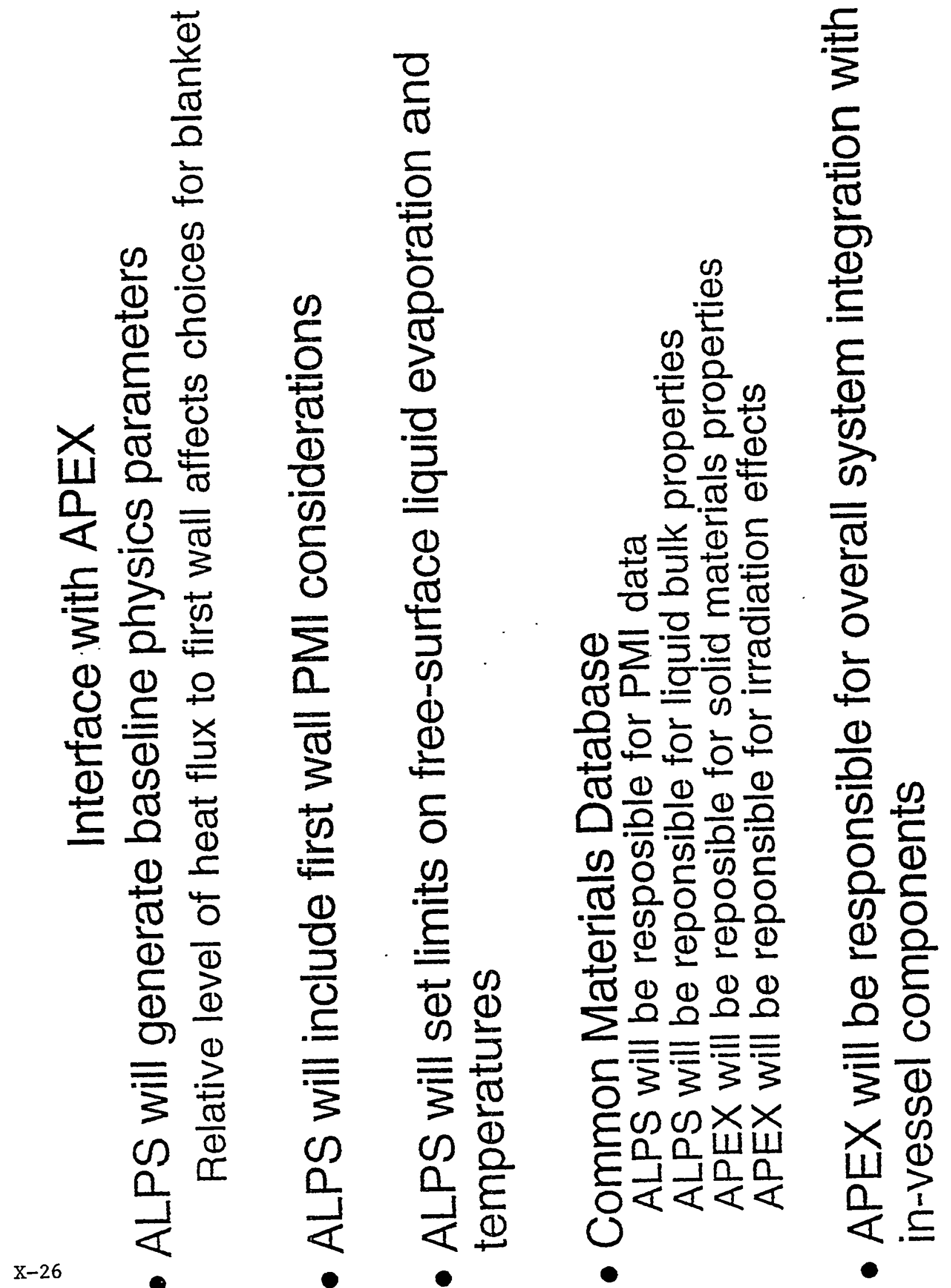




\section{Task Priorities Strawman}

Physics/Engineering Parameters

Focus on advanced tokamak (ARIES - RS)

Materials Database

Lists of needed data

Review of existing database

Needed R\&D

Establish the limitations of $\mathrm{Li}$ and $\mathrm{Ga}$ (Selection of materials)

Allowable evaporation

Core impurity limits

Edge transport and recycling

Design Selection Criteria (Selection of engineering concepts) 


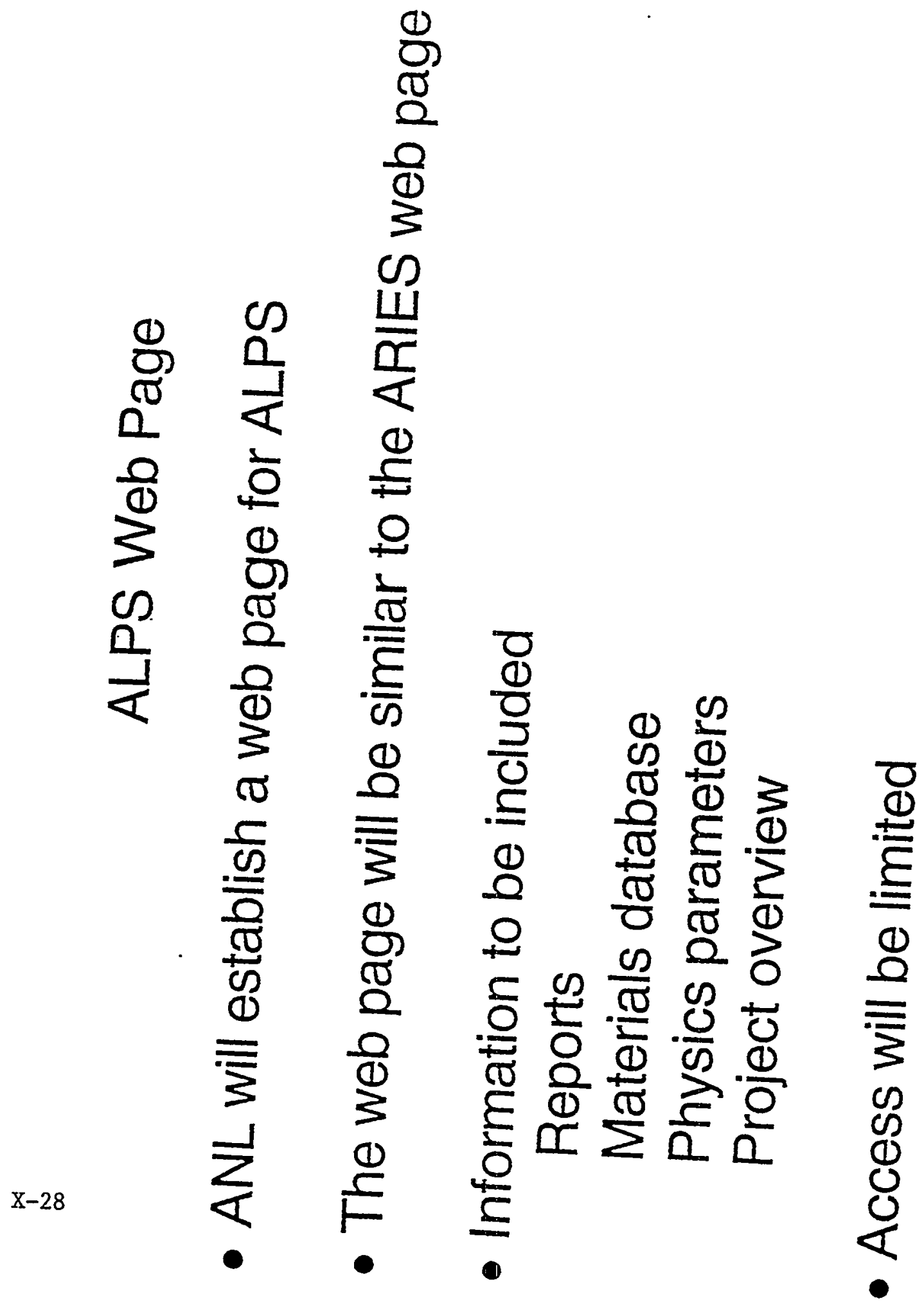




\section{Comments on Heat Transfer in Liquid Surface Plasma Facing Components}

Richard Nygren (Sandia)

This work represents preliminary ideas being discussed in two recently formed US programs, APS and APEX.

- basics concerns about what limits heat transfer

- specific issues regarding "waterfalls" and "droplet screens"

- general questions about how we measure heat transfer

- comment about global power loads on liquid surface PFCs 


\section{Liquid Surface High Heat Flux Technology}

ALPS basic issue: What limits heat removal in liquid surface PFCs?

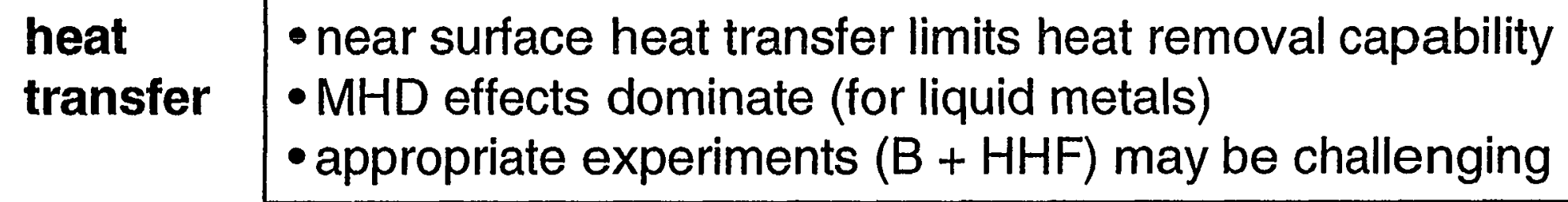

heat transfer

- near surface heat transfer limits heat removal capability

- MHD effects dominate (for liquid metals)

- appropriate experiments $(\mathrm{B}+\mathrm{HHF})$ may be challenging

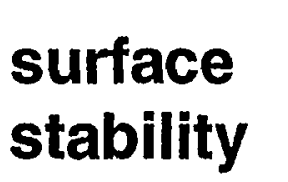

- sputtering erosion from liquid surfaces

- plasma wind effects

- transients (e.g., disruptions)

-problem of collection of "free flow" systems 


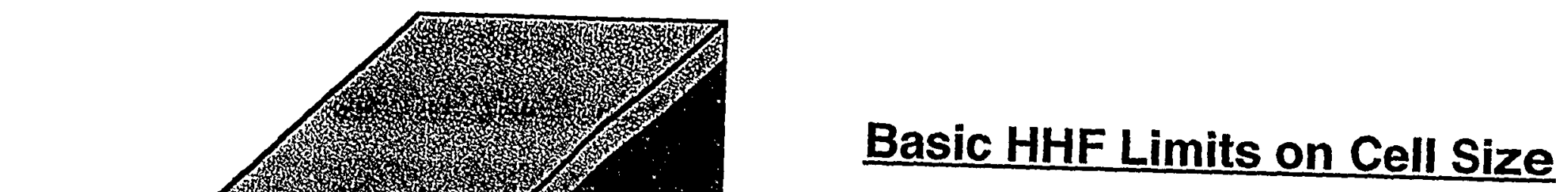

$50 \mathrm{MW} / \mathrm{m}^{2}$

$\Delta T=105^{\circ} \mathrm{C}, 0.1 \mathrm{~mm} \mathrm{Li}$

$\triangle T=285^{\circ} \mathrm{C}, 2 \mathrm{~mm}$ CuCrZr

$\Delta \mathrm{T}=500^{\circ} \mathrm{C}$ (too much), water,

$h=.1 \mathrm{MW} / \mathrm{m}^{2}-\mathrm{K}$,

need $h \sim 1$

heat pipes ( $q>100 \mathrm{MW} / \mathrm{m}^{2}$ )

distance for flowing $\mathrm{Li}$ to reach $500^{\circ} \mathrm{C}$ from $220^{\circ}$

$2.5 \mathrm{~mm}(t=0.0025)$ at $1 \mathrm{~m} / \mathrm{s}$ with $50 \mathrm{MW} / \mathrm{m}^{2}$

$24.5 \mathrm{~mm}$ at $10 \mathrm{~m} / \mathrm{s}$ with $50 \mathrm{MW} / \mathrm{m}^{2}$ 
希

T rise in Li with $50 \mathrm{MW} / \mathrm{m} 2$

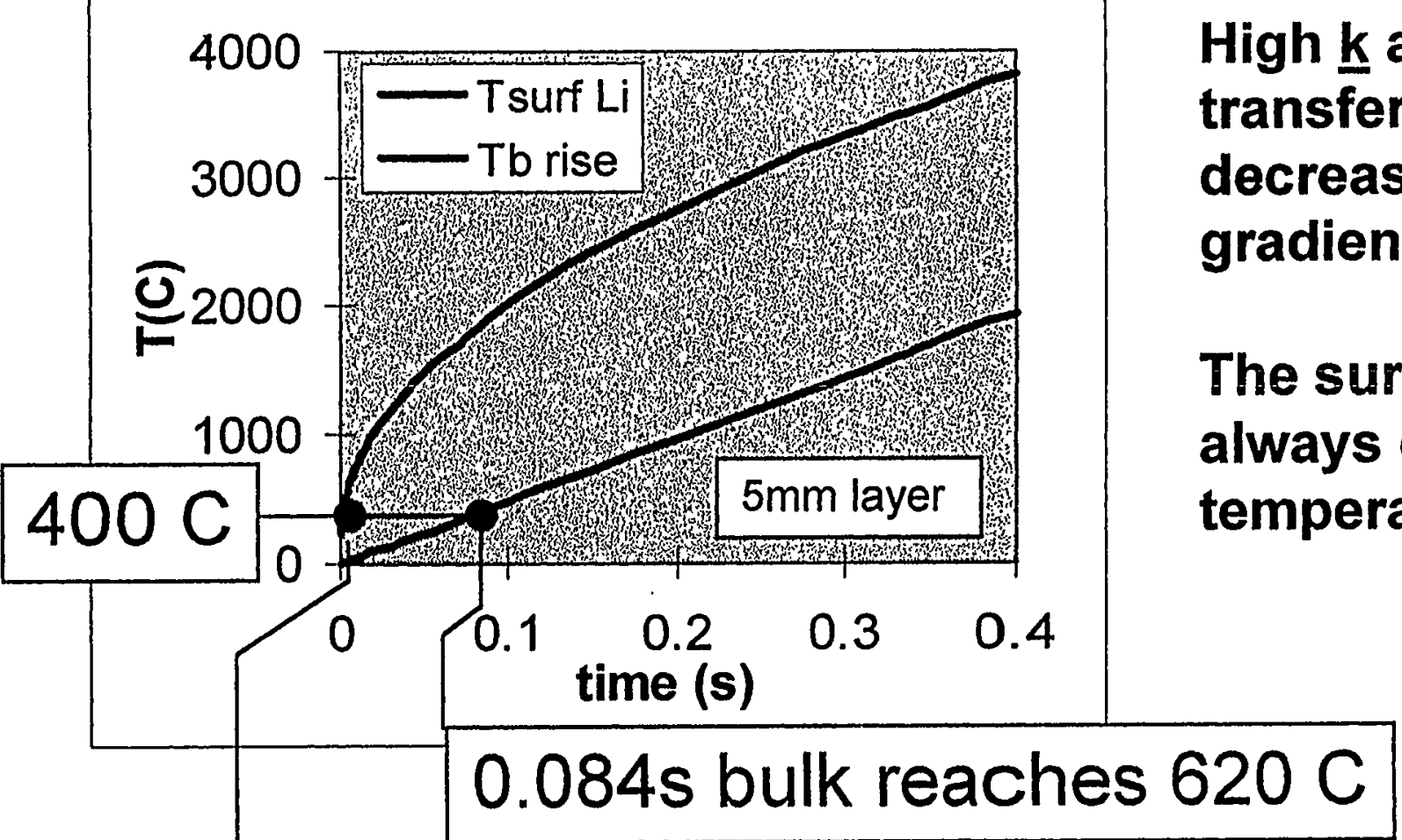

0.005 s surface reaches $620 \mathrm{C}$

$z(1 \mathrm{~m} / \mathrm{s})=5 \mathrm{~mm}$

$z(10 \mathrm{~m} / \mathrm{s})=50 \mathrm{~mm}$ $z(1 \mathrm{~m} / \mathrm{s})=84 \mathrm{~mm}$
$z(10 \mathrm{~m} / \mathrm{s})=0.84 \mathrm{~m}$ $-\cdot-$

High $k$ and enhanced heat transfer (turbulence) decrease the temperature gradient.

The surface temperature always exceeds the bulk temperature. 


\begin{tabular}{|l|}
\hline Heat Acceptance Limit \\
- laminar (MHD slug) flow has \\
high temperature gradient \\
- limit is probably evaporation \\
rate at the maximum surface \\
temperature \\
- total power is more important \\
than heat flux profile \\
- mixing is necessary for long \\
flow paths
\end{tabular}

出

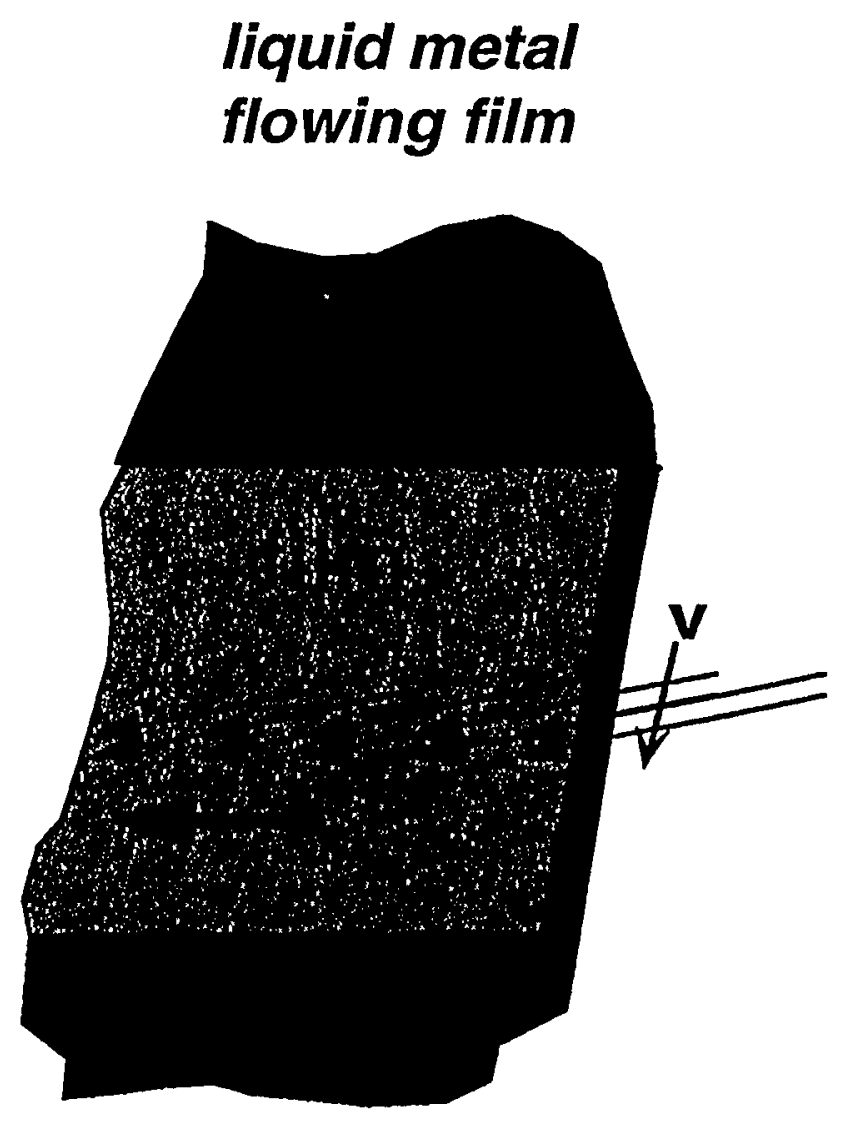




\section{Heat Source?}

- e-beam: $40 \mathrm{keV} \mathrm{e}-R_{\text {Larmor }}<1 \mathrm{~mm}$ in $1 T$ field, not feasible in $B$ field.

- laser: High power steady state laser needed, feasible, $\mathbf{\$} \mathbf{\text { . }}$

- neutral beam: Feasible, $\mathbf{\$ \$}$.

- sun: Feasible, SNLsolar facility (concentrator, $20 \mathrm{MW} / \mathrm{m}^{2}$ ). liquid metal

flowing film

\section{heat source???}

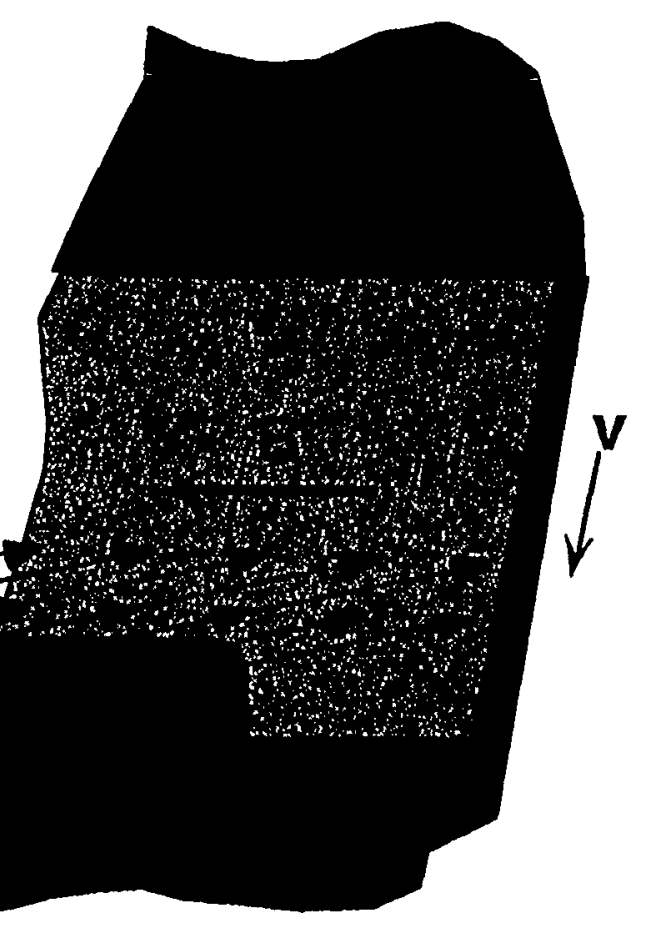




\section{liquid metal droplet curtain}

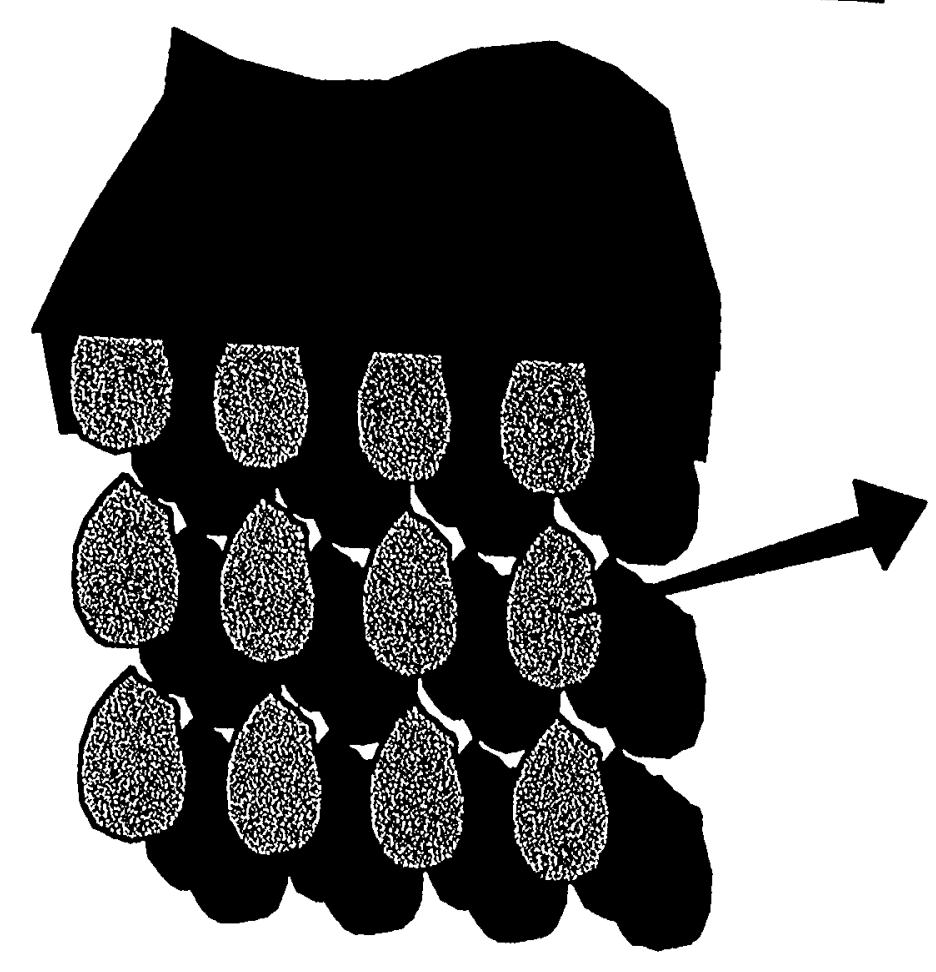

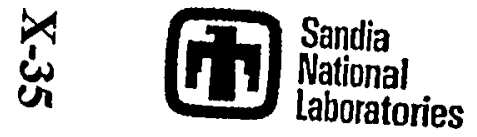

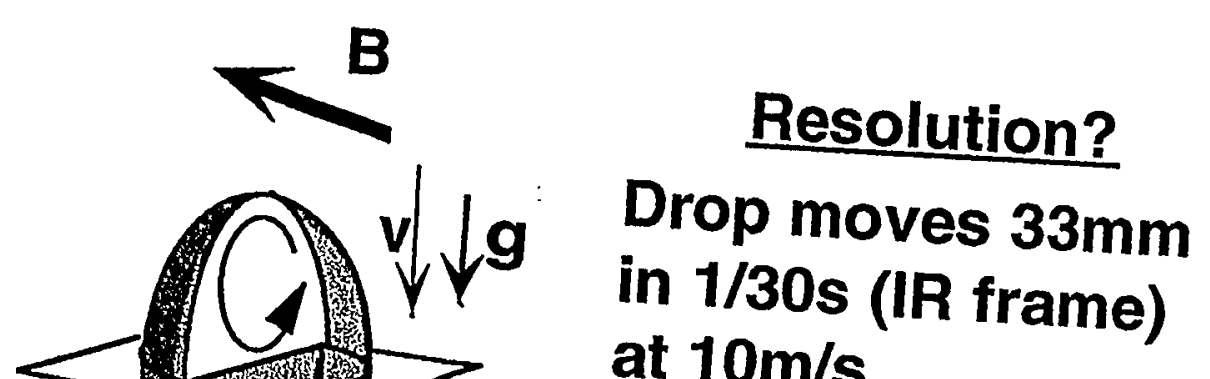
at $10 \mathrm{~m} / \mathrm{s}$. Emmisivity?

Pixel size?

rotation, shape, internal circulation

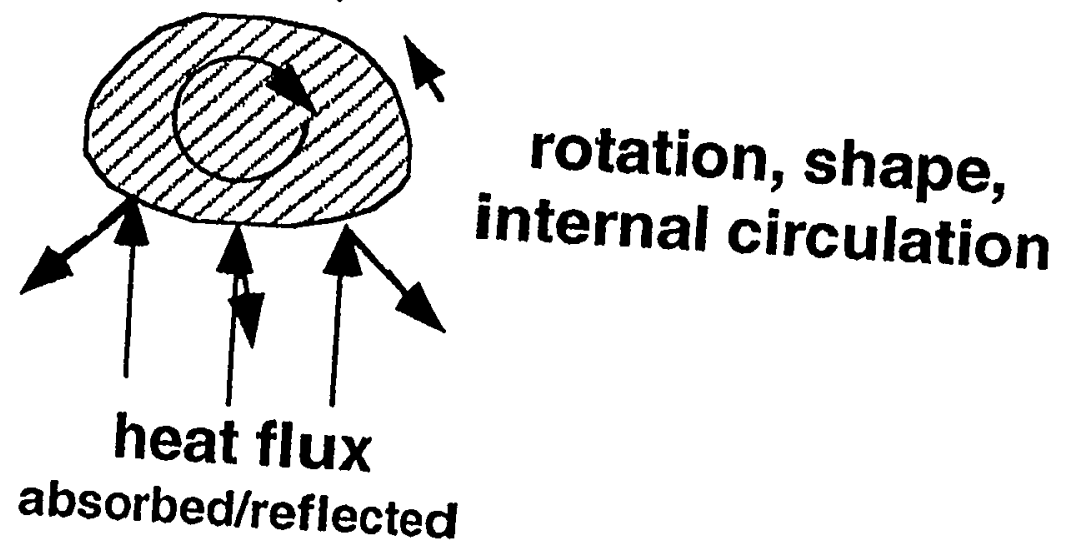




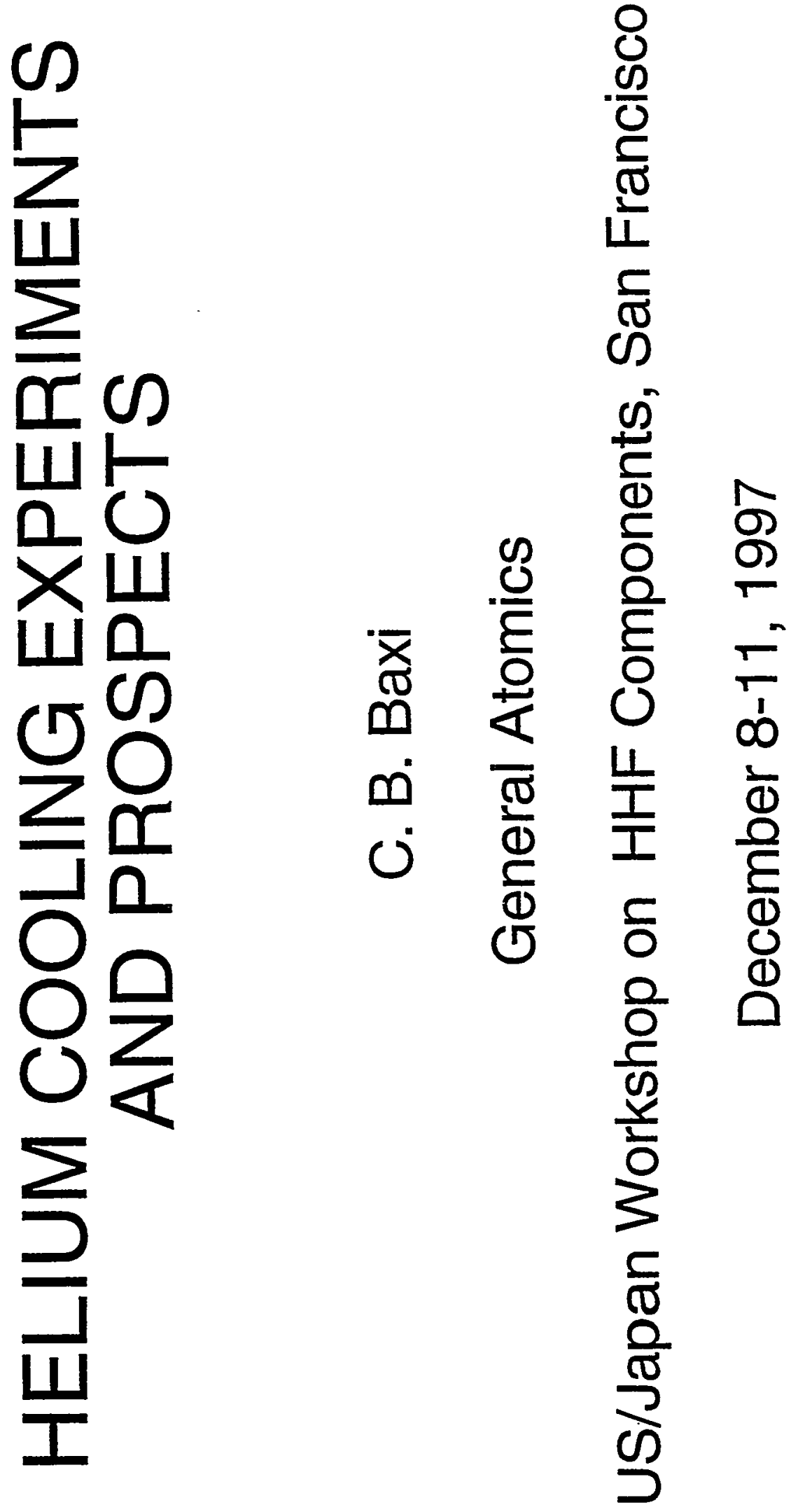




\section{ADVANTAGES OF HELIUM COOLING}

- Safety leaks not serious

- Helium characteristics

- Chemically inert

- Neutronically inert

- No phase changes

- Developed technology

- Heat transfer

- Purification (including tritium recovery)

- Maintenance advantages

- No activation

- No trace heating 


\section{SNLA HELIUM LOOP}

- Pressure $=4 \mathrm{MPa}$

- Flow $=23 \mathrm{~g} / \mathrm{sec}$

- Pressure drop $=0.5$ bar

- Helium inlet temperature $20^{\circ}$ to $45^{\circ} \mathrm{C}$

- Heat flux source electron beam

- Beam Power $30 \mathrm{~kW}$

- $57 \mathrm{~cm}$ diameter and $96 \mathrm{~cm}$ long vacuum chamber

- Maximum sample size $=25 \mathrm{~cm}$ long 


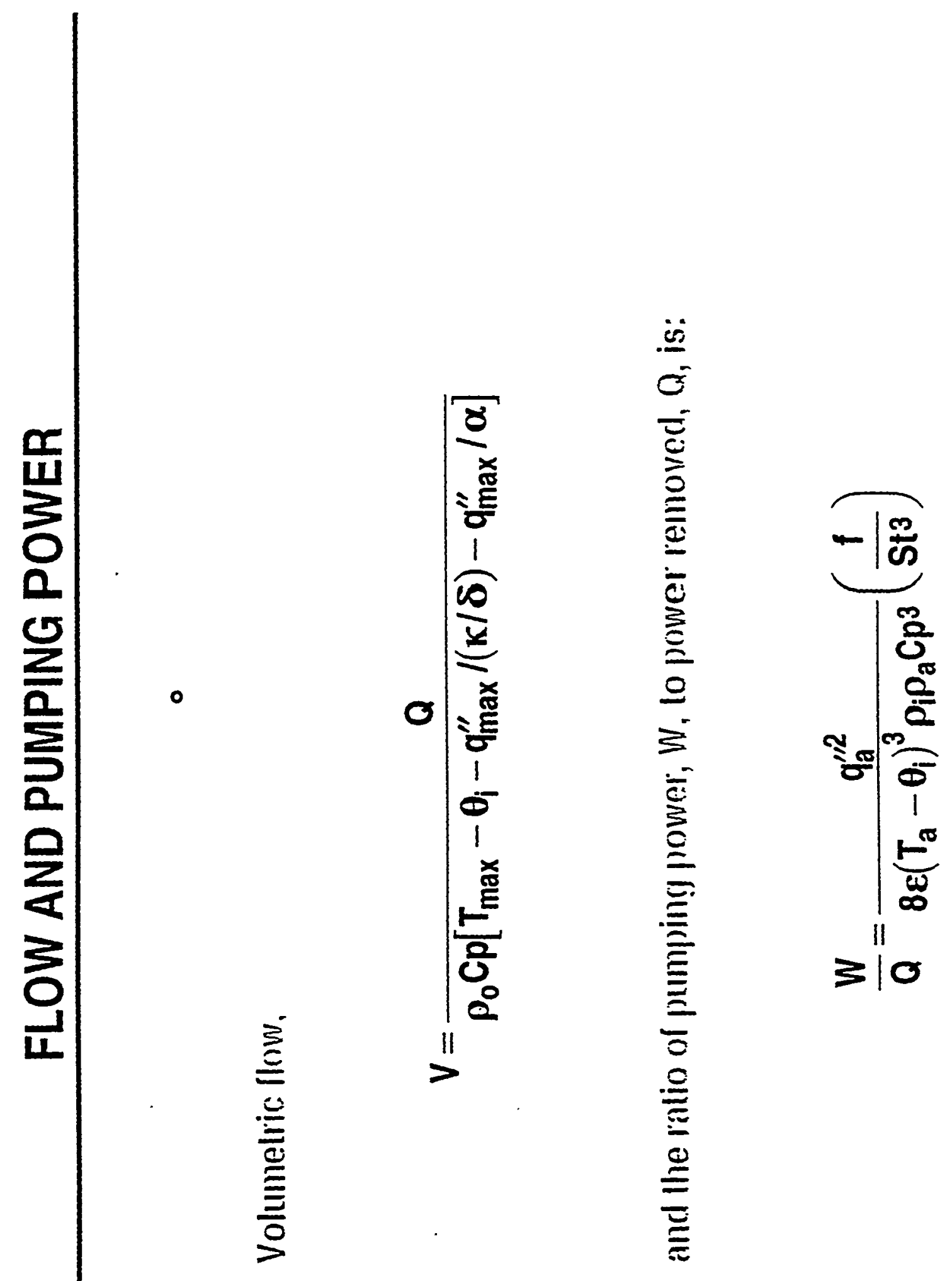




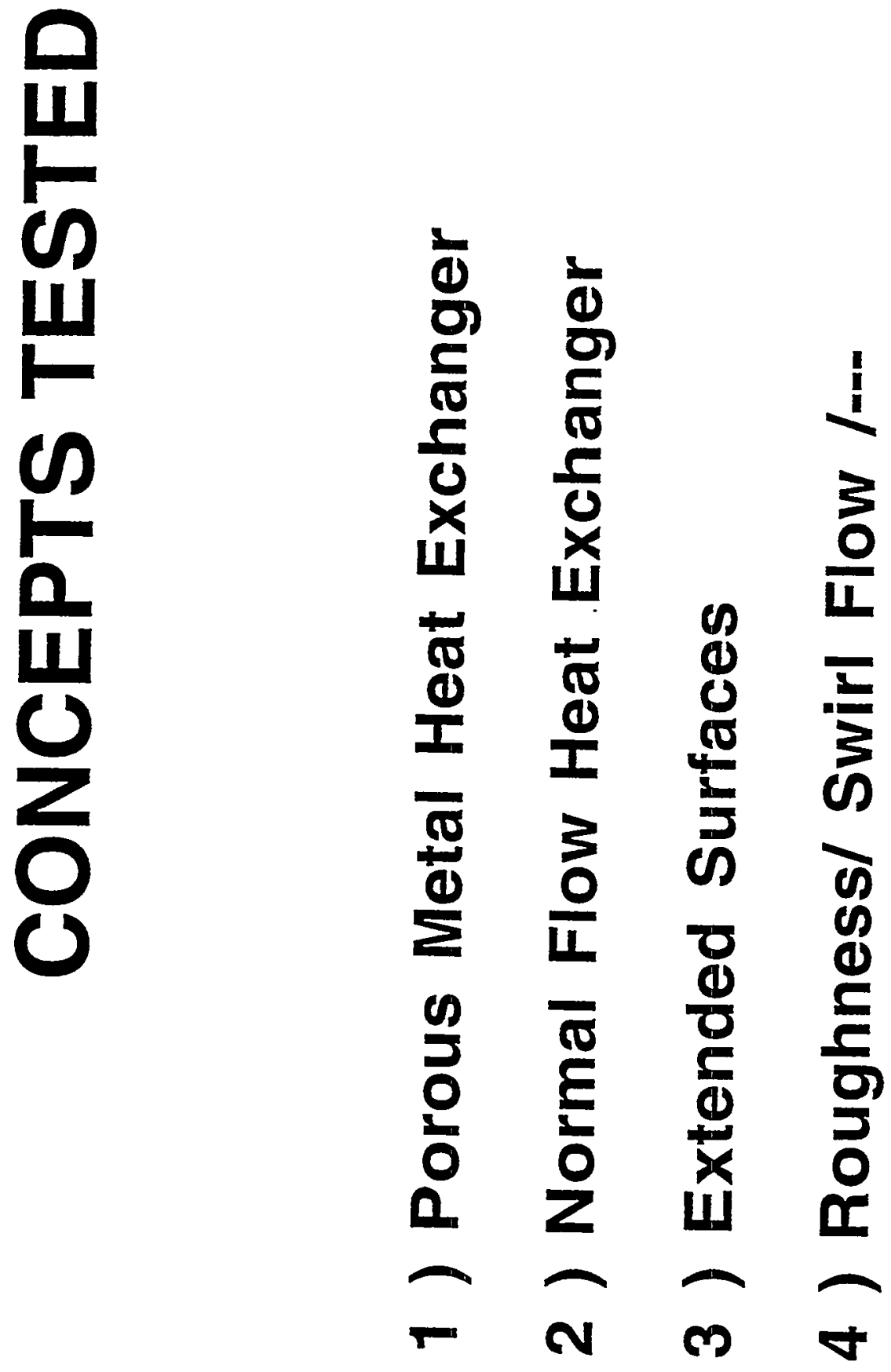


FINS:

PITCH $=1 \mathrm{~mm}$

THICKNESS $=0.4 \mathrm{~mm}$

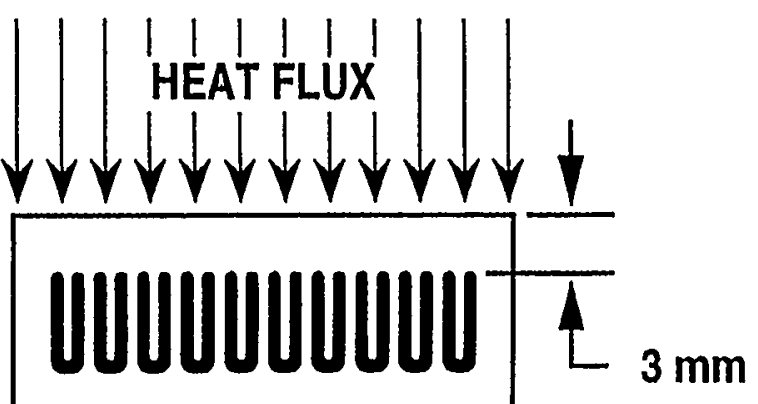

MATERIAL: Dispersion strengthened copper (GLIDCOP by SCM)

\section{RESULTS OF TESTS AT SNLA}

AT INLET PRESSURE $=4 \mathrm{MPa}$

\begin{tabular}{|l|c|c|c|}
\hline $\begin{array}{c}\text { Flow Rate } \\
(\mathrm{kg} / \mathrm{s})\end{array}$ & $\begin{array}{c}\text { Heat Flux } \\
\left(\mathrm{MW} / \mathrm{m}^{2}\right)\end{array}$ & $\begin{array}{c}\text { Peak Surface } \\
\text { Temperature } \\
\left({ }^{\circ} \mathrm{C}\right)\end{array}$ & $\begin{array}{c}\text { Pumping Power } \\
{[W(\% \text { of }} \\
\text { power removed })]\end{array}$ \\
\hline 0.022 & 10 & 380 & $157(0.8)$ \\
0.011 & 6 & 422 & $21(0.2)$ \\
0.0064 & 3 & 242 & $3.4(0.06)$ \\
\hline
\end{tabular}

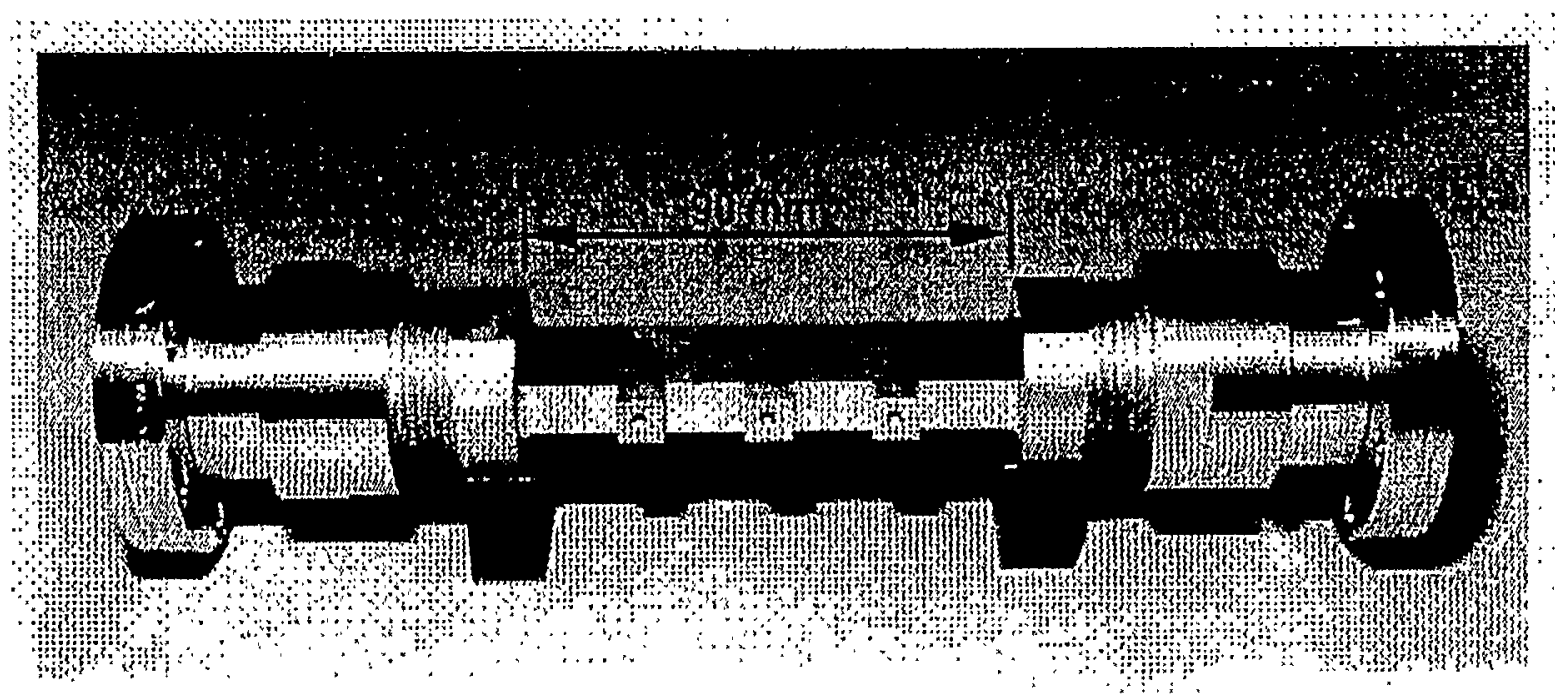

$\underset{⿱ 亠 䒑}{\$}$
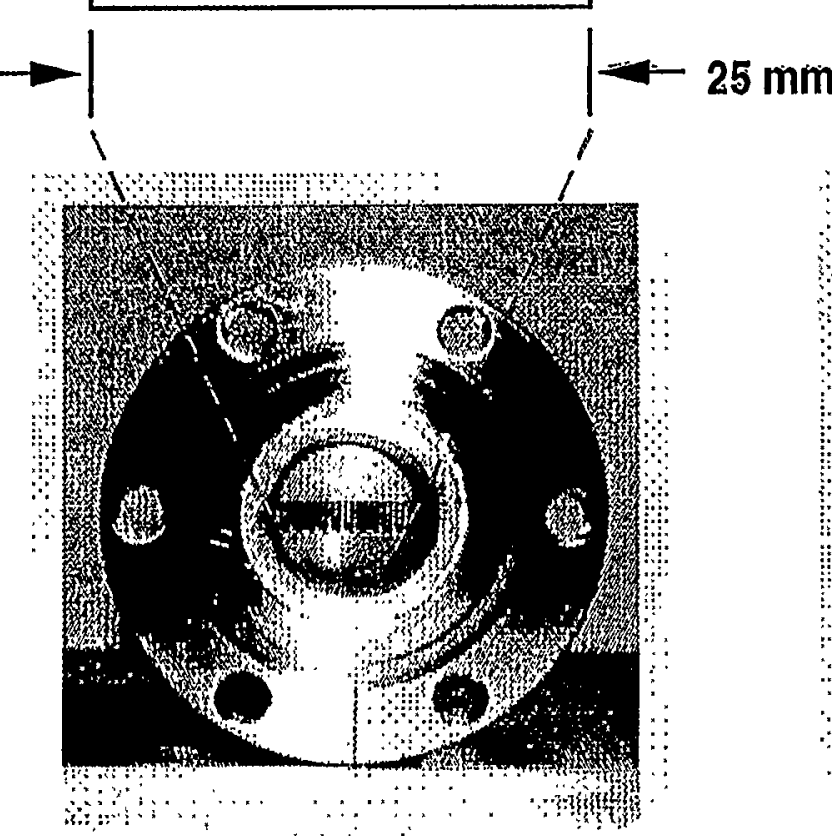

104-94 


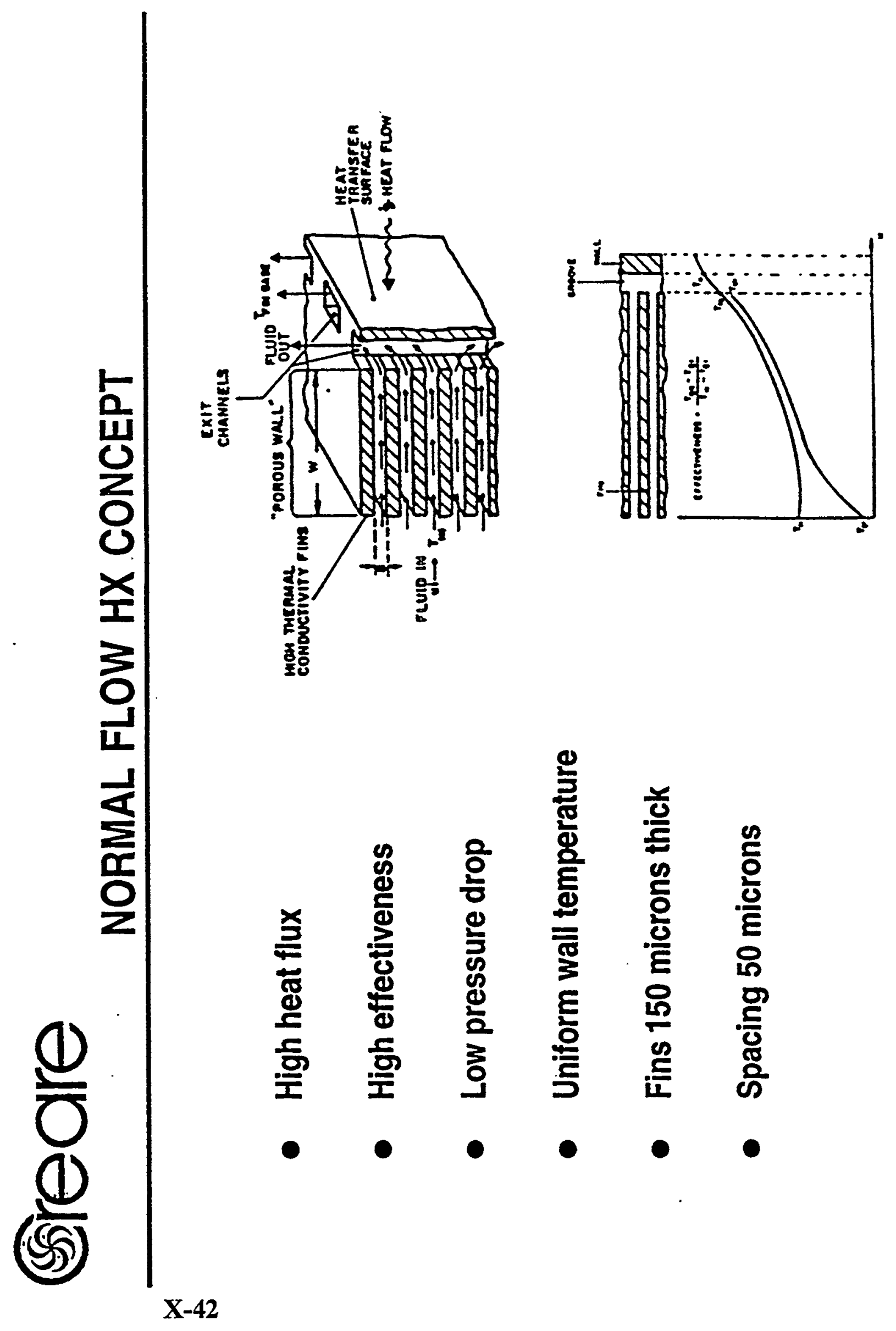




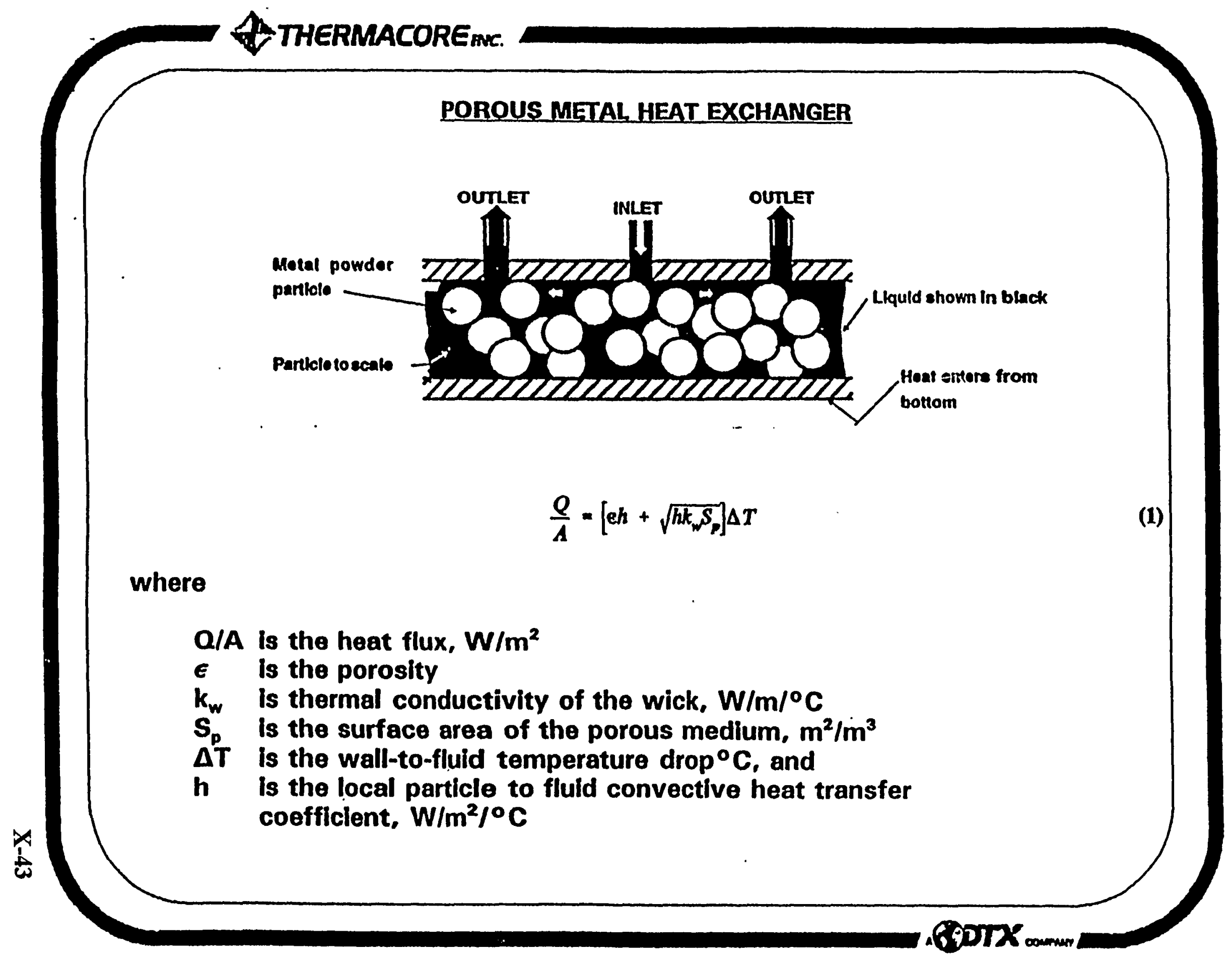




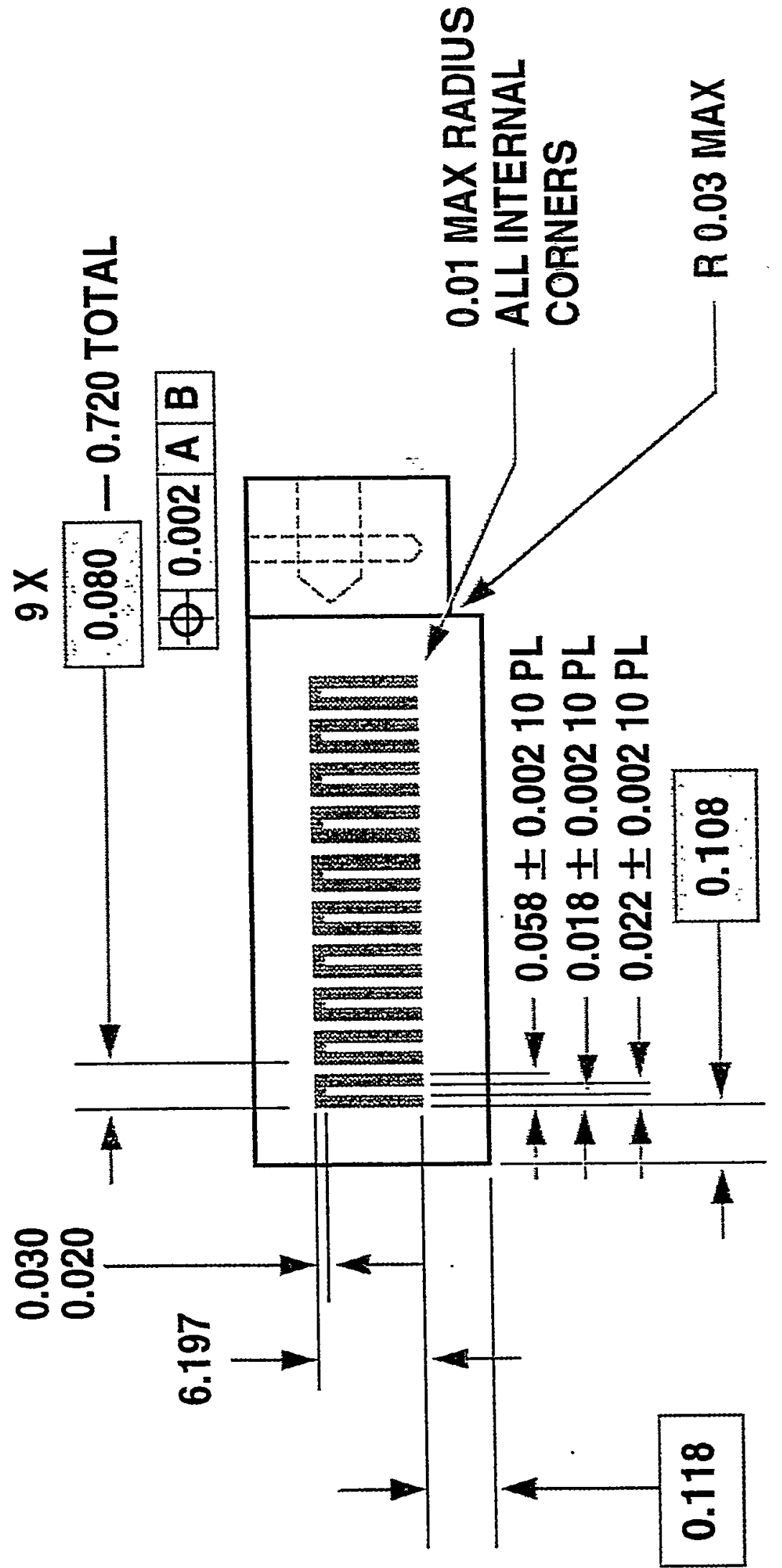

齐 


\section{HELIUM-COOLED VANADIUM MODULE}

Internal surface modification

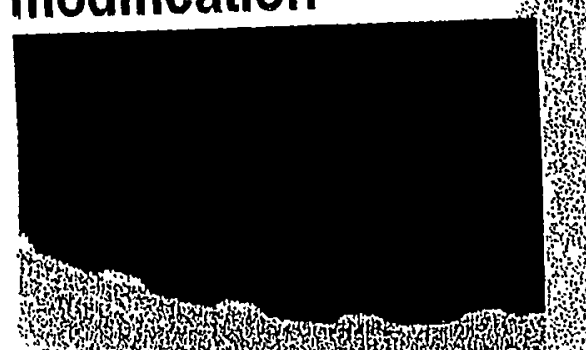

- Heat transfer enhancements provide a 3MW/m2 capability

The Vanadium tube has been tabicated by boring drawing and electrodis charge machining of the I.D,
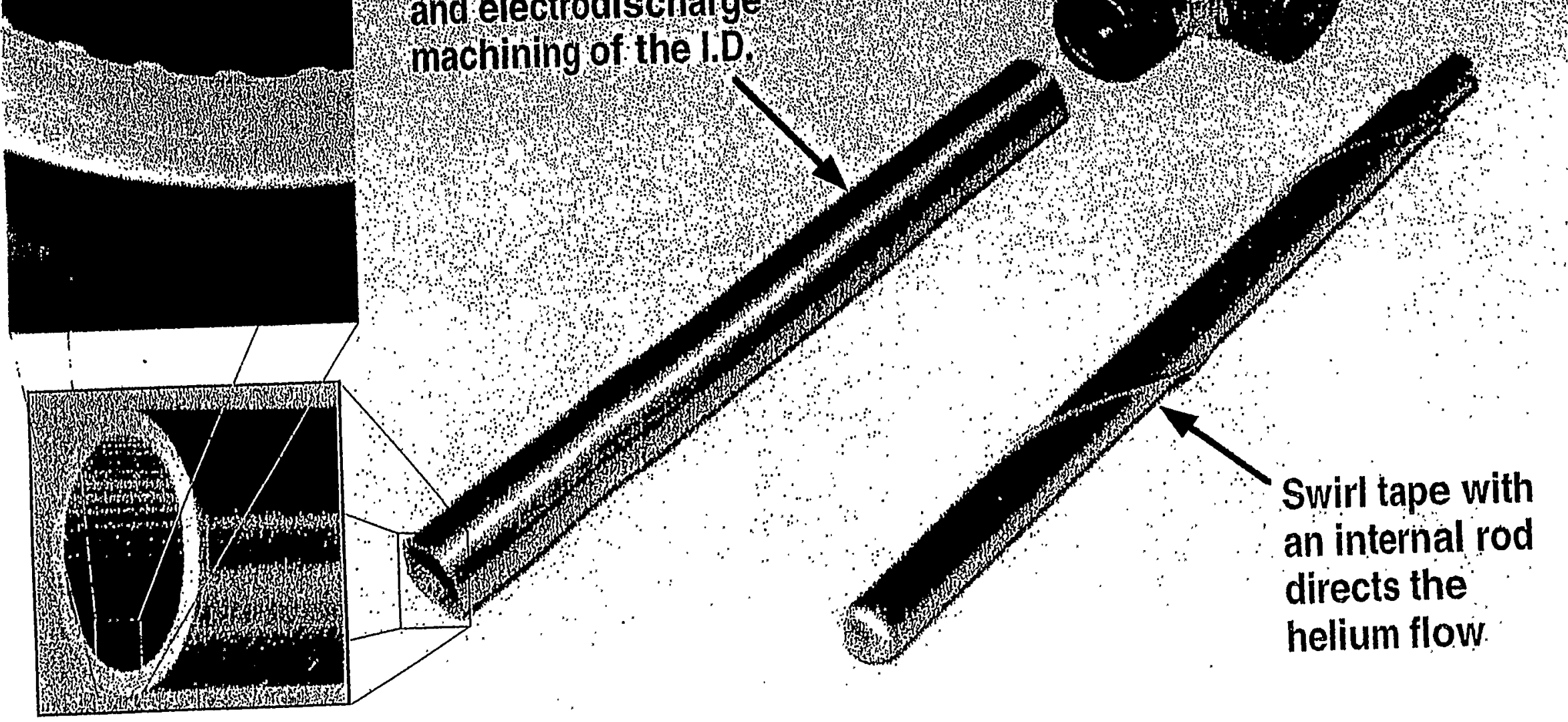

i 


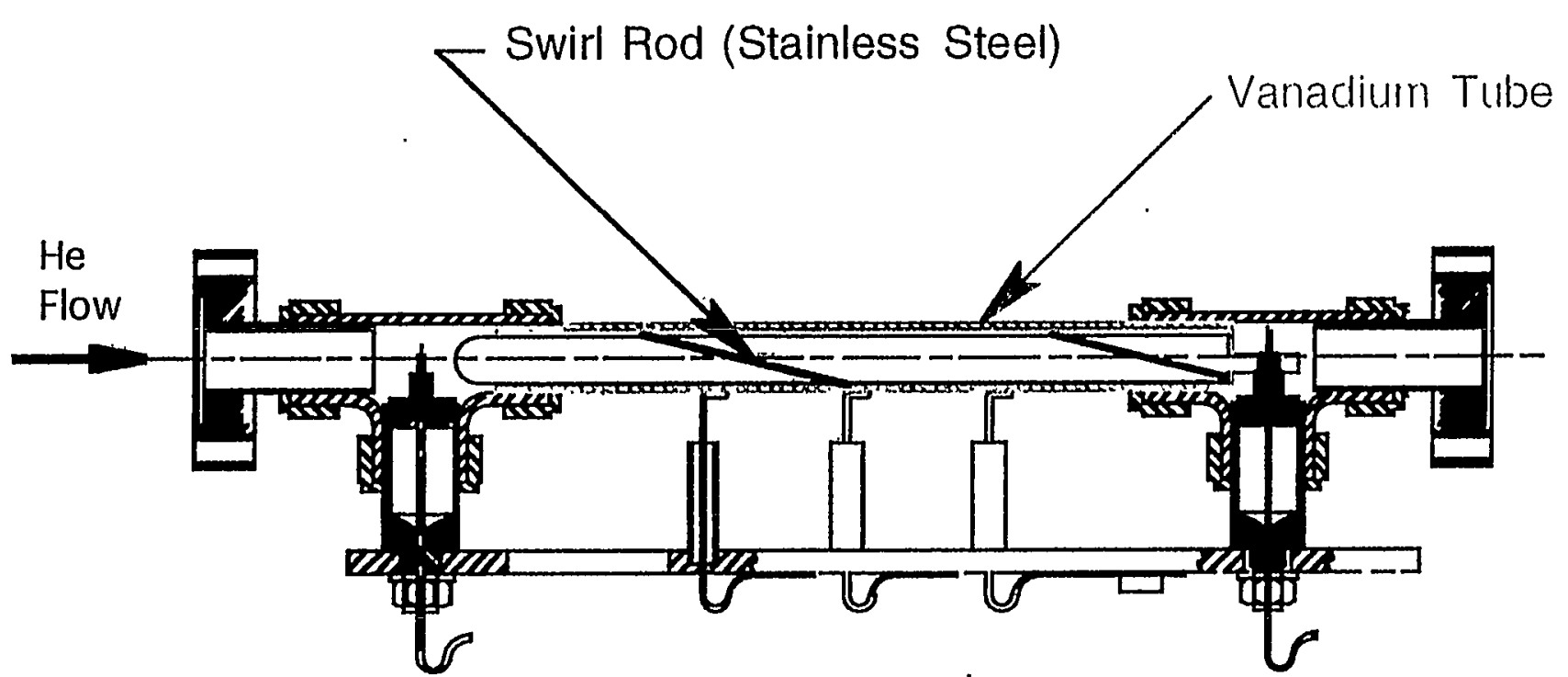




\section{GA DIVERTOR MODULE}

\section{RESULTS OF DECEMBER 7, 1994 TESTING AT SANDIA NATIONAL LABORATORY, ALBUQUERQUE}

\begin{tabular}{lll} 
Heat Flux & $\begin{array}{c}\text { Area } \\
\left(\mathrm{Cm}^{2}\right)\end{array}$ & Limited By \\
\hline $9 \mathrm{MW} / \mathrm{m}^{2}$ & 20.0 & Facility Limit \\
$18 \mathrm{MW} / \mathrm{m}^{2}$ & 5.8 & Surface Temperature ( $700 \mathrm{OC})$ \\
$34 \mathrm{MW} / \mathrm{m}^{2}$ & 2.0 & All Objectives Achieved
\end{tabular}




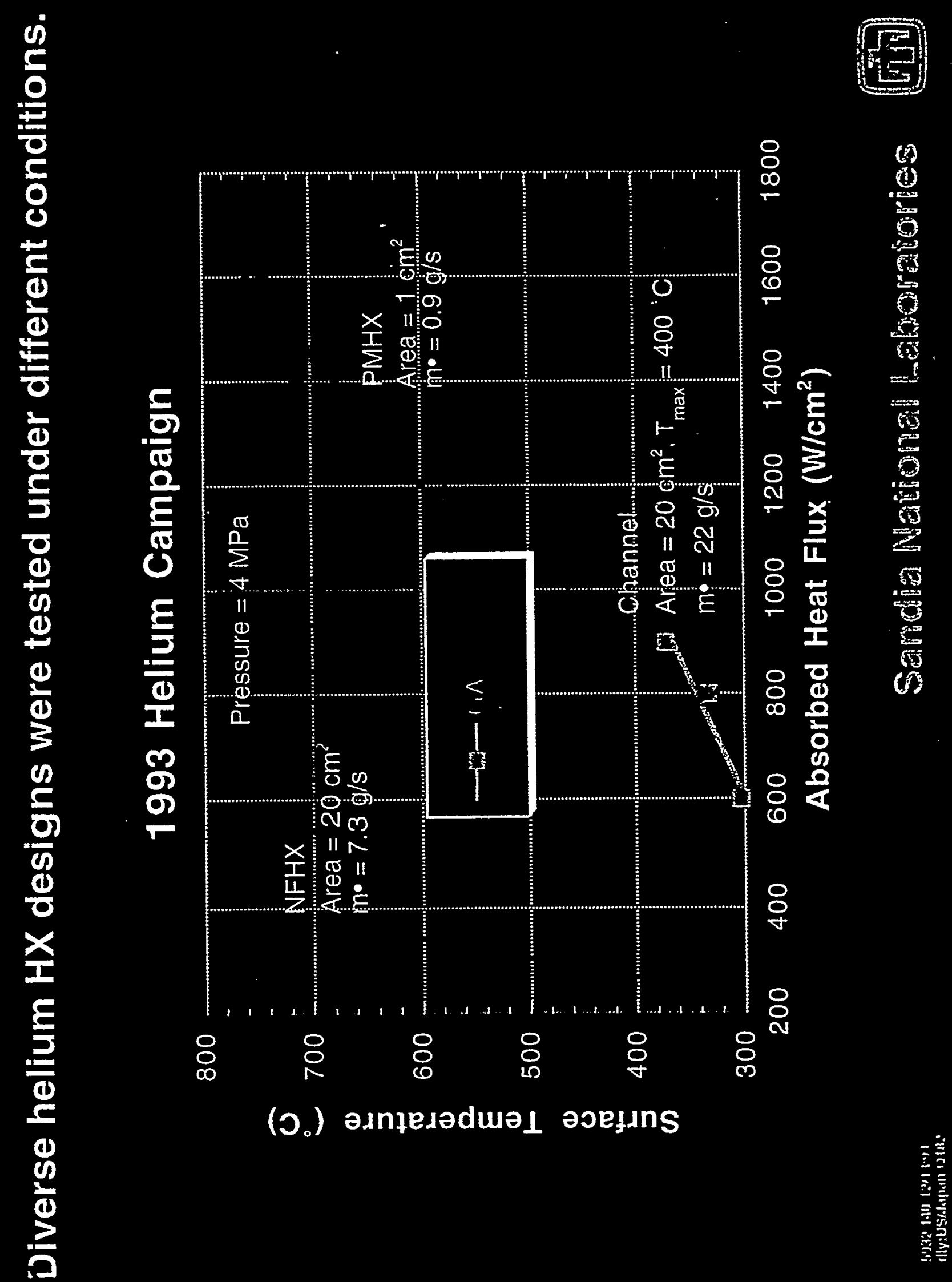



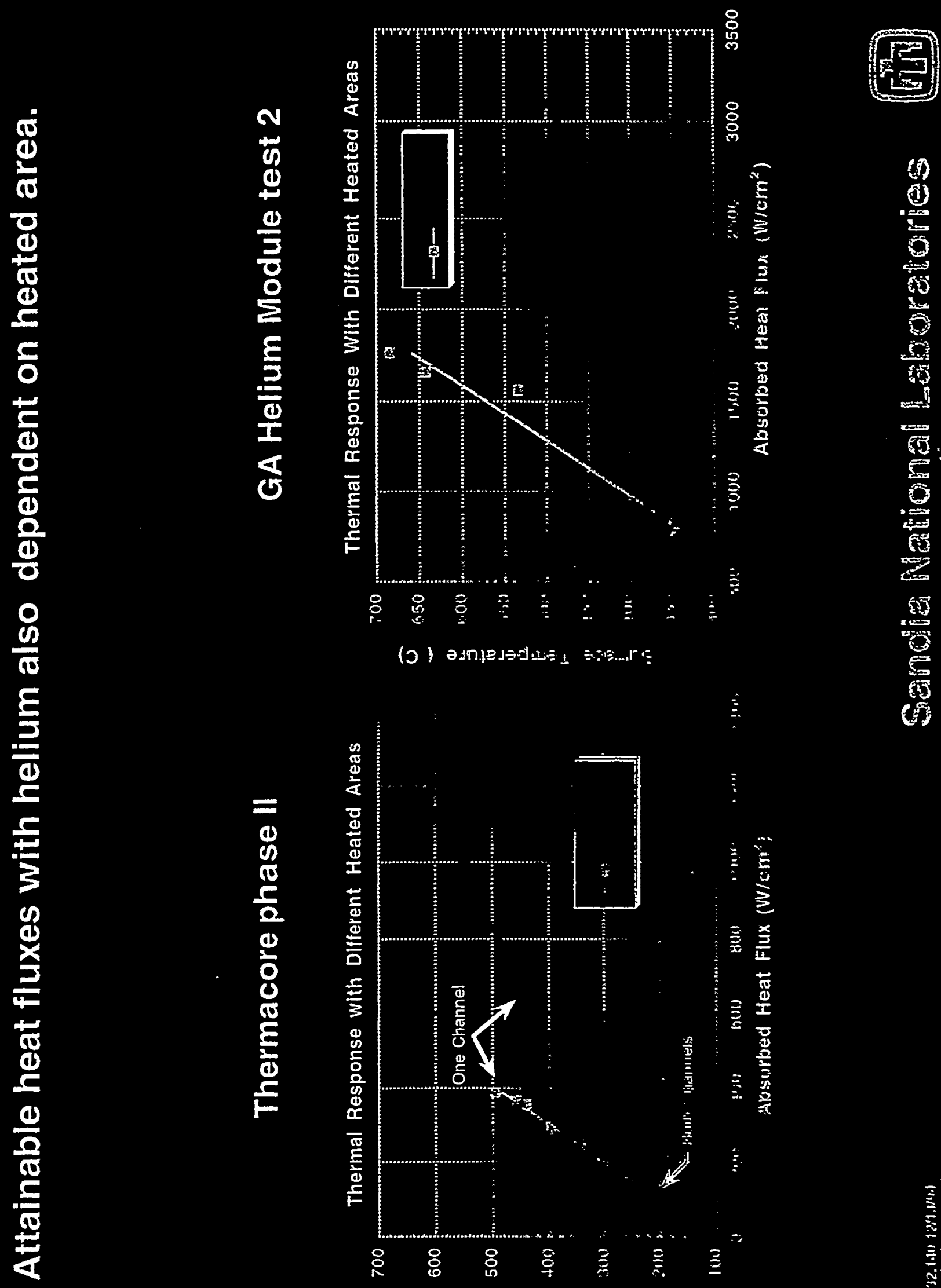

4

$\mathbf{N}$

$E$

6

a)

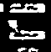

5

\%

$\infty$

9

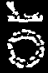

s.

8

50

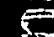

(5) asme

赵

6

0

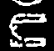

63

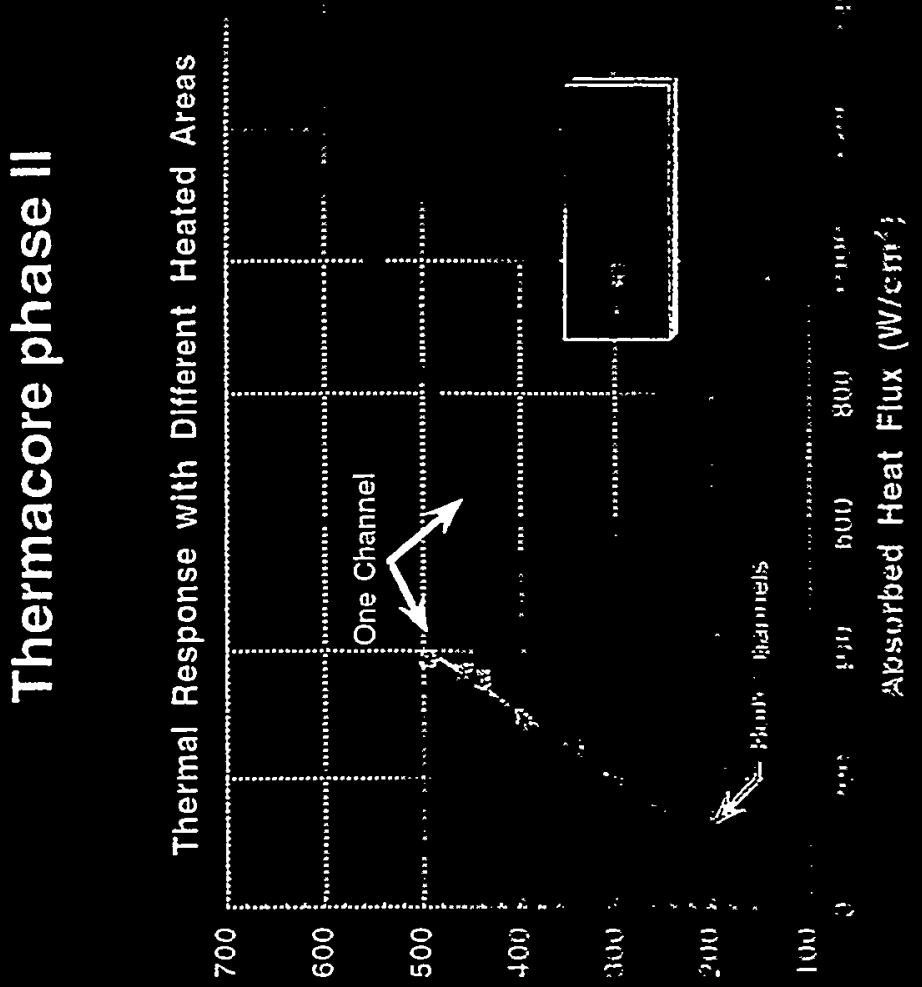

(0.) axnesaduə 1 әoejuns 


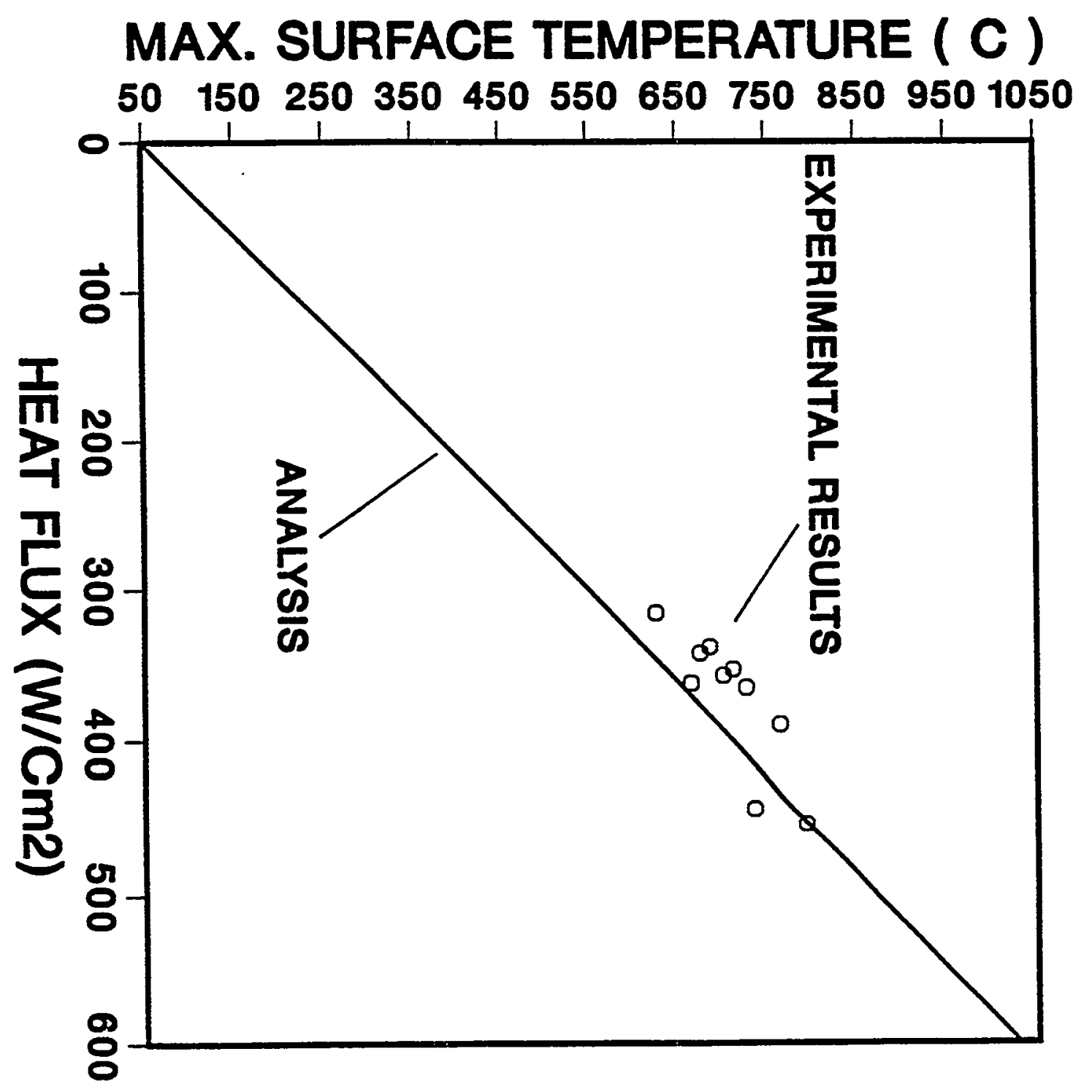




\section{FUTURE PLANS}

Helium cooled Faraday Shield Antenna to be tested on DIII-D within next few months 


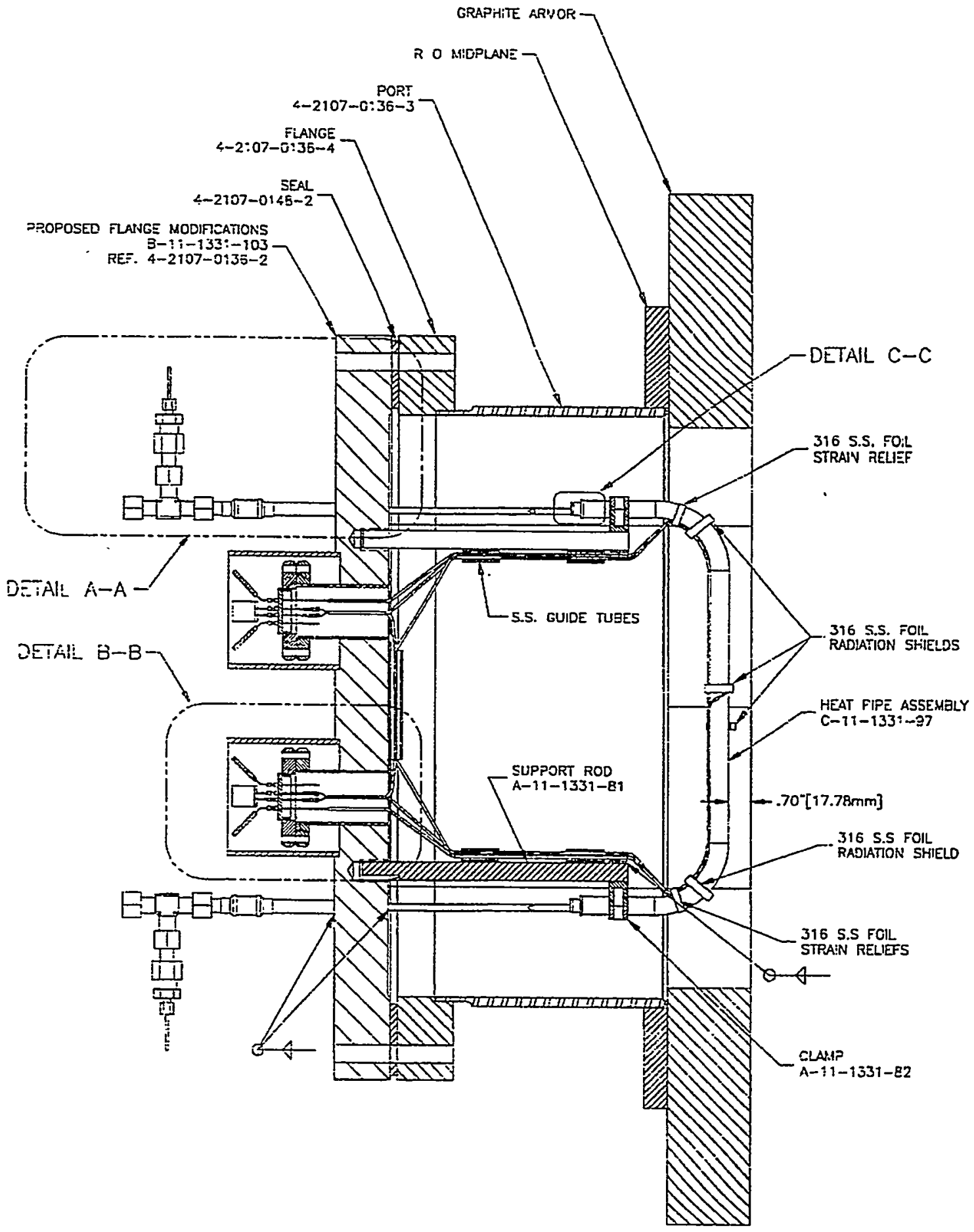

DII-D FARADAY SHIELD TEST ASSEMBLY - SWE VIEW. 


\section{CONCLUSION}

IT is feasible to remove a steady state heat flux in excess of $5 \mathrm{MW} / \mathrm{m}^{\wedge} 2$ with helium cooling at a moderate pressure ( $4 \mathrm{MPa}$ ) and modest pumping power $(5 \%)$. 


\title{
Advanced Plasma Facing Component by
}

- using Multi-layer Coated Pebbles

\section{(Comments on liquid/pebble divertor)}

\author{
M. Isobe, M. Nishikawa \\ Graduate School of Engineering, \\ Osaka University \\ (presented by Y. Ueda)
}

\section{Outline}

1. Basic concept of pebble drop divertor

2. Divertor pebble and performance of pebble drop divertor

A. Maximum heat load

B. Fuel pumping function

3. Conclusions 


\section{Basic concept of pebble drop divertor using multi-layer coated pebbles}

Small (about diameter of $1-2 \mathrm{~mm}$ ) special coated pebbles are used as divertor surface. This pebbles consists of ceramic microspherical kernel, coating layer for tritium permeation barrier and plasma-facing layer.

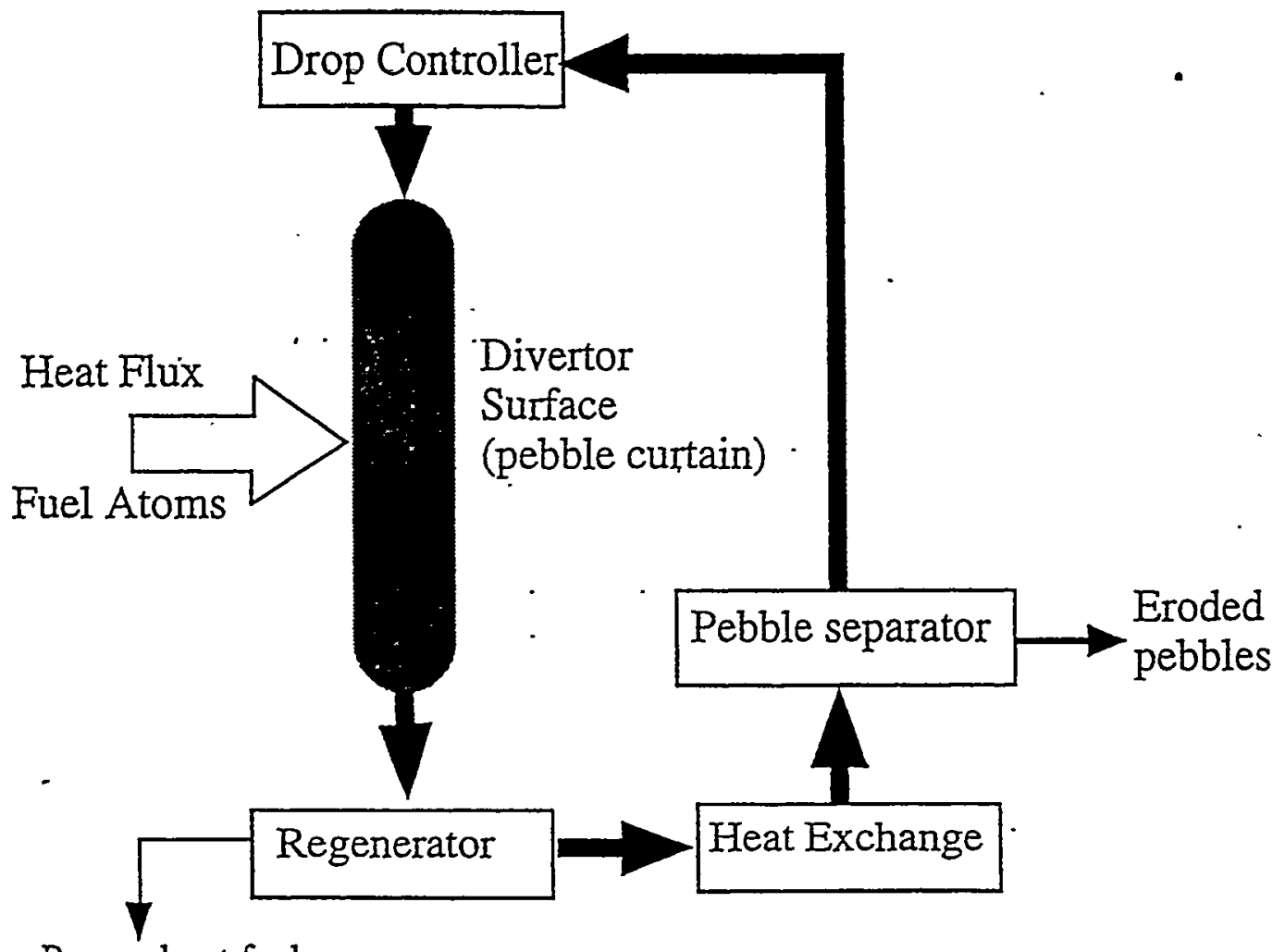

Pumped out fuel gas

Functional Scheme of pebble drop divertor 


\section{Multi-layer coated pebble}

\section{Tritium Permeation Barrier (TBL)}

Tritium permeation barrier layer prevents the diffusion of tritium from the PFL to the kernel and reduces the tritium retention in the bulk of pebble.

\section{Plasma Facing Layer} (PFL)

Plasma facing layer is optimized for the compatibility with core plasma. By using hydrogen gettering material, the pebbles have a function of fuel gas pumping.

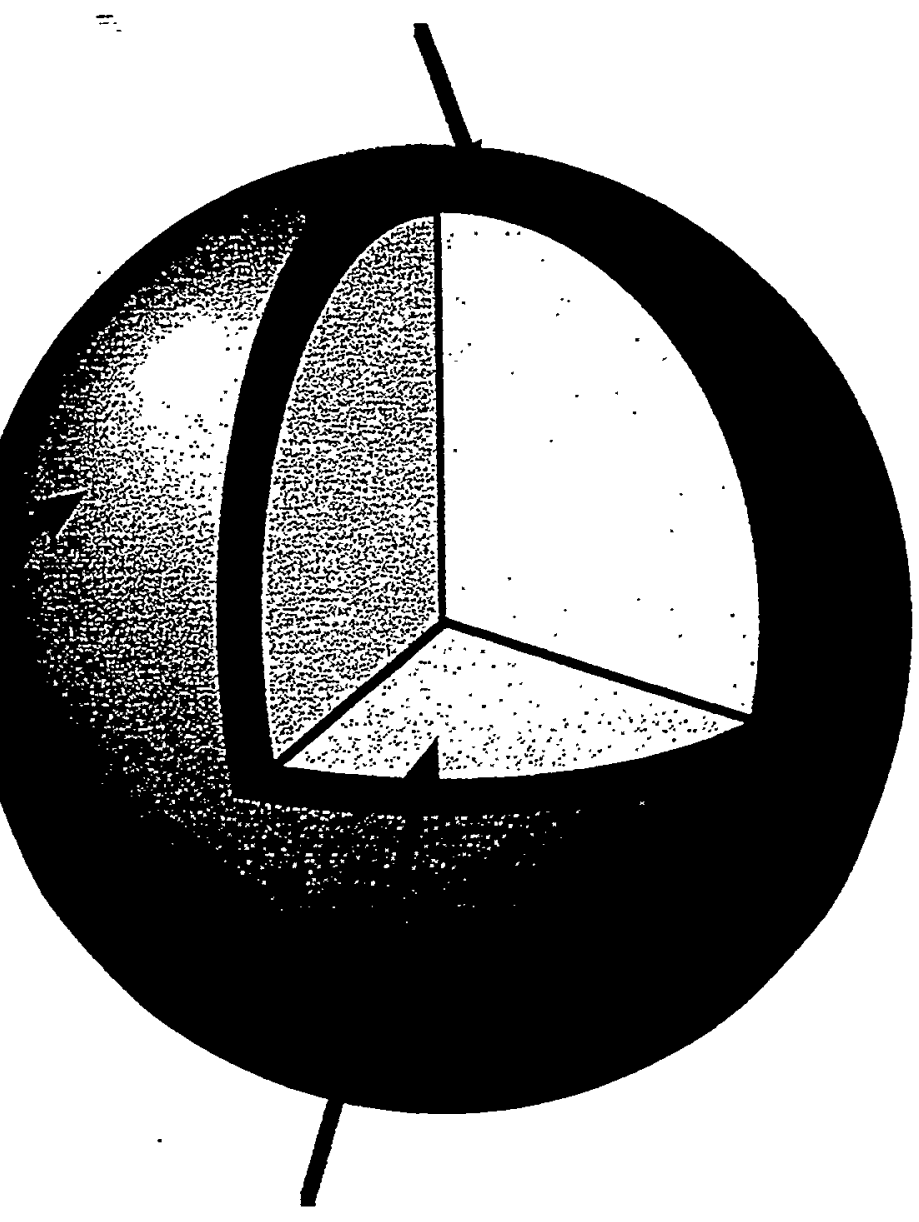

Kernel

A kernel sustains mechanical force and thermal stress. It also determines the heat capacity of a pebble. 


\section{Advantages of pebble divertor}

- Using insulator kernels, pebble circulation is not subject to MHD effects.

- Heat removal and fuel gas pumping can be realized simultaneously.

- Using tritium permeation barrier, the tritium retention of divertor pebbles can be significantly reduced. .

- Continuous replacement of eroded surface is possible.

- The fabrication technology of multi-layer coated pebble has been developed for High Temperature Gas-Cooled Fission Reactor's fuel particle. 


\section{Typical Stress Distribution in the Divertor Pebble}

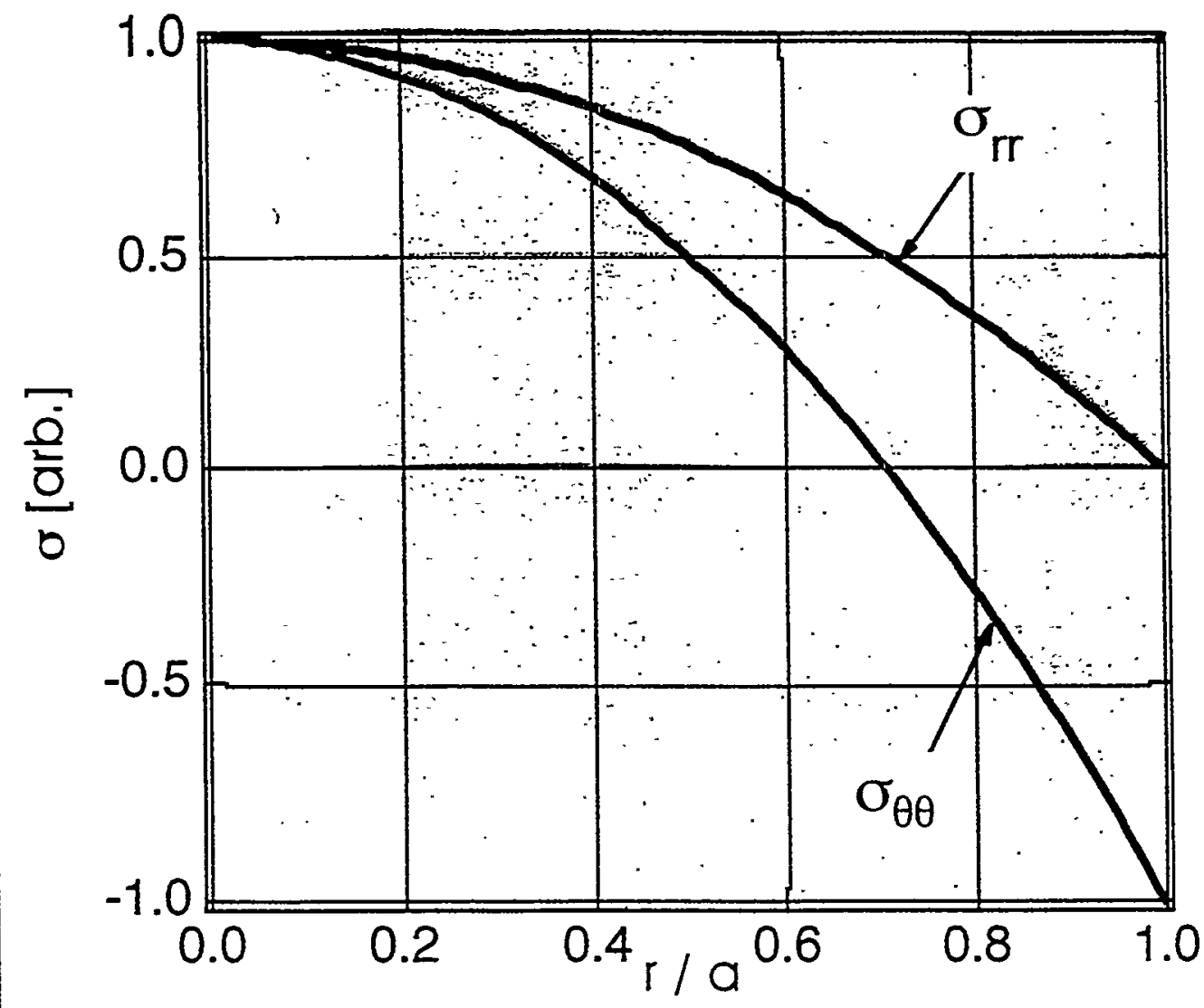

Tensile

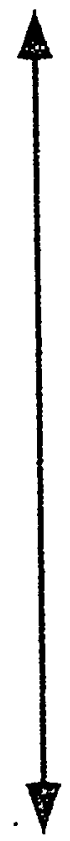

Compressive

- Radial stress shows maximum tensile force at the center of the pebble

- Angular stress shows maximum tensile force at the center of the pebble and maximum compressive force at the surface.

A pebble will be safe if surface compressive stress does not exceed the compressive strength of the kernel material. 


\section{Maximum Heat Load of Various Kernel Materials}

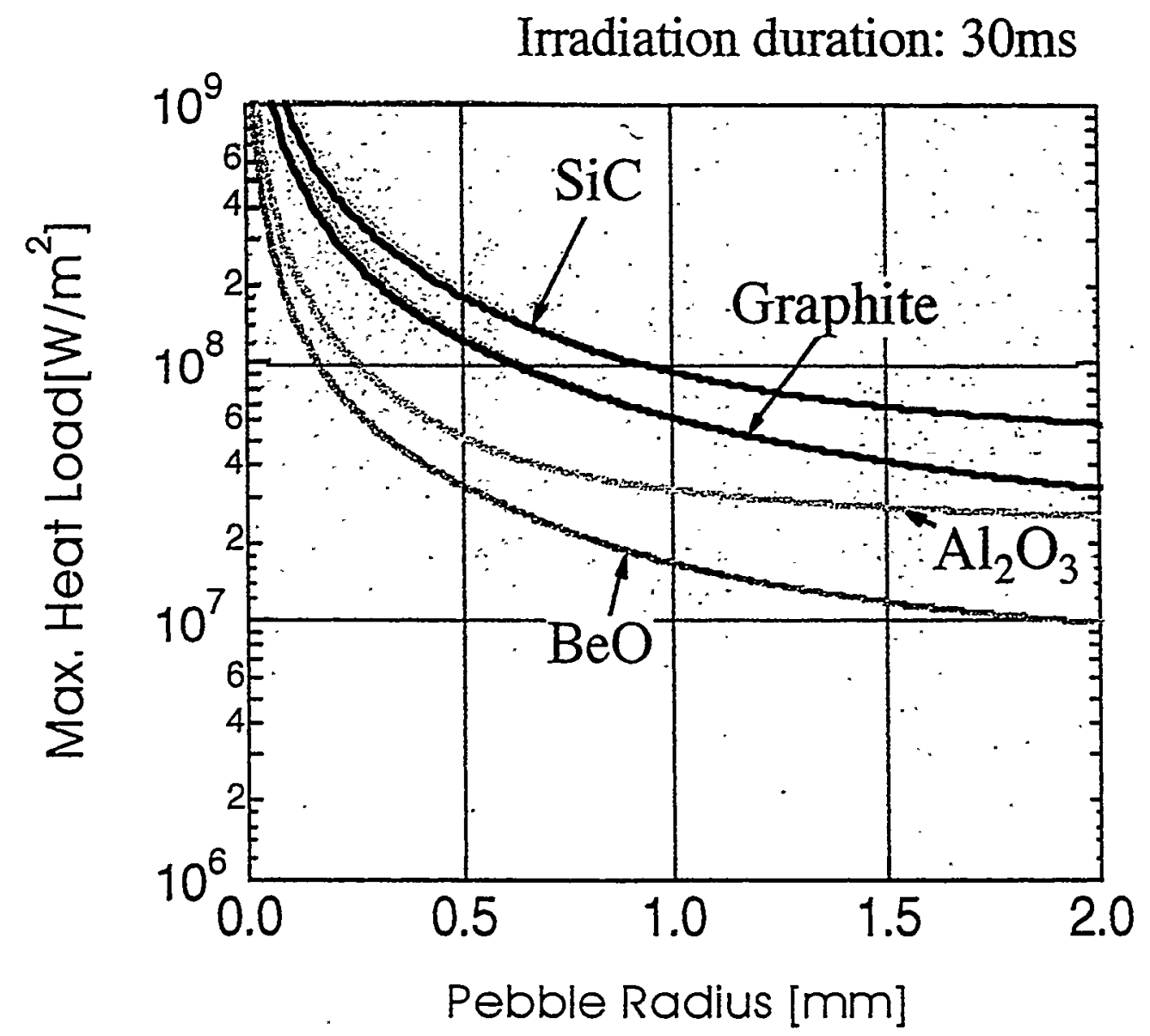

When the divertor pebble of $0.5-1 \mathrm{~mm}$ in radius is used, all candidate materials can be used in $15 \mathrm{MW} / \mathrm{m}^{2}$ of surface heat flux. 


\section{Surface Temperature of pebbles}

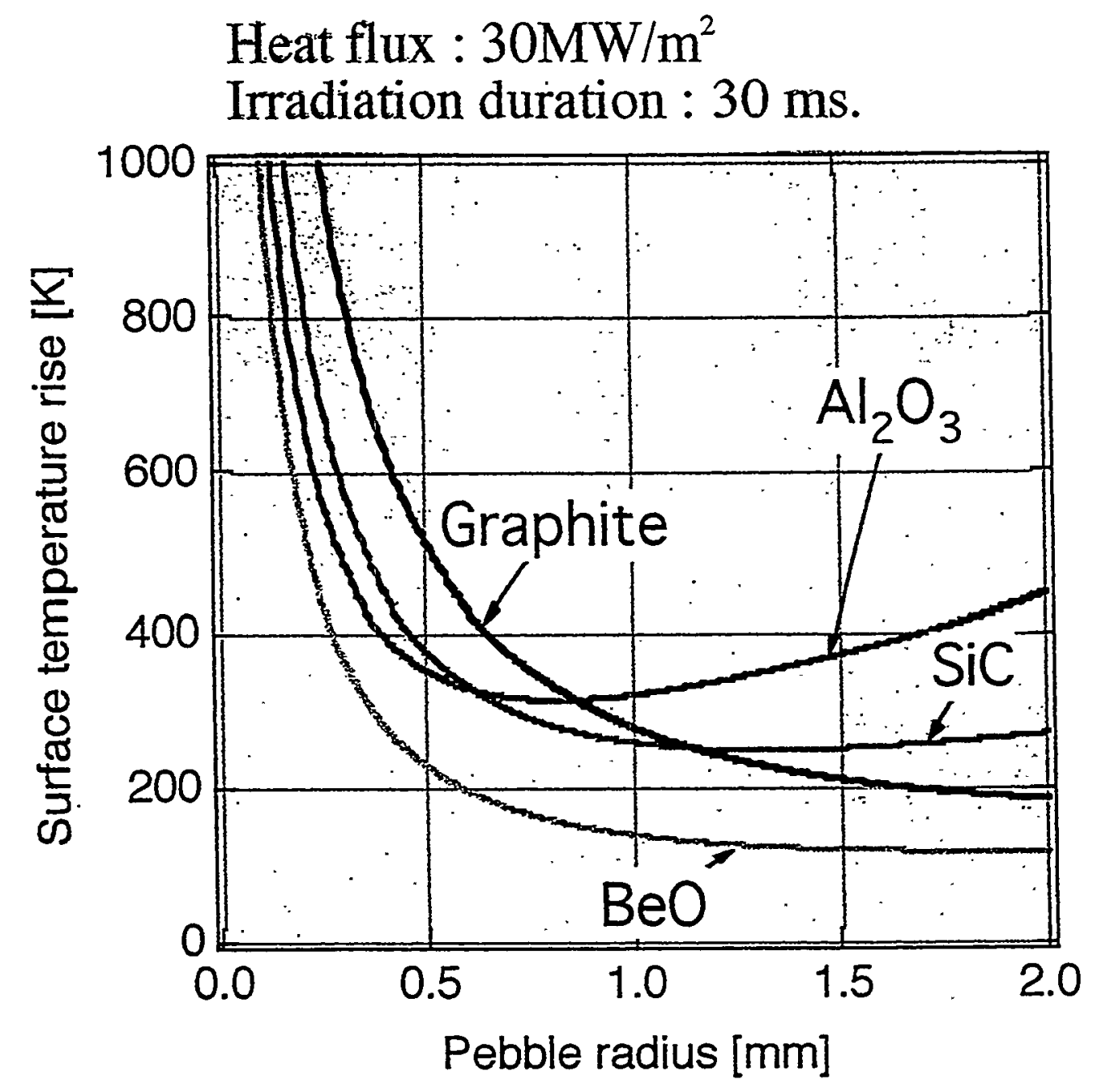




\title{
Fuel gas pumping performance
}

\section{Basic application of wall pumping}

Temperature rise $200 \mathrm{~K} \sim 300 \mathrm{~K}$ (dropping height $\sim 1 \mathrm{~m}$ )

\author{
Ref. A. Sagara, et.ai
}

"Design of Carbon Sheet Pump for LHD and

Demonstration of Hydrogen Pumping"

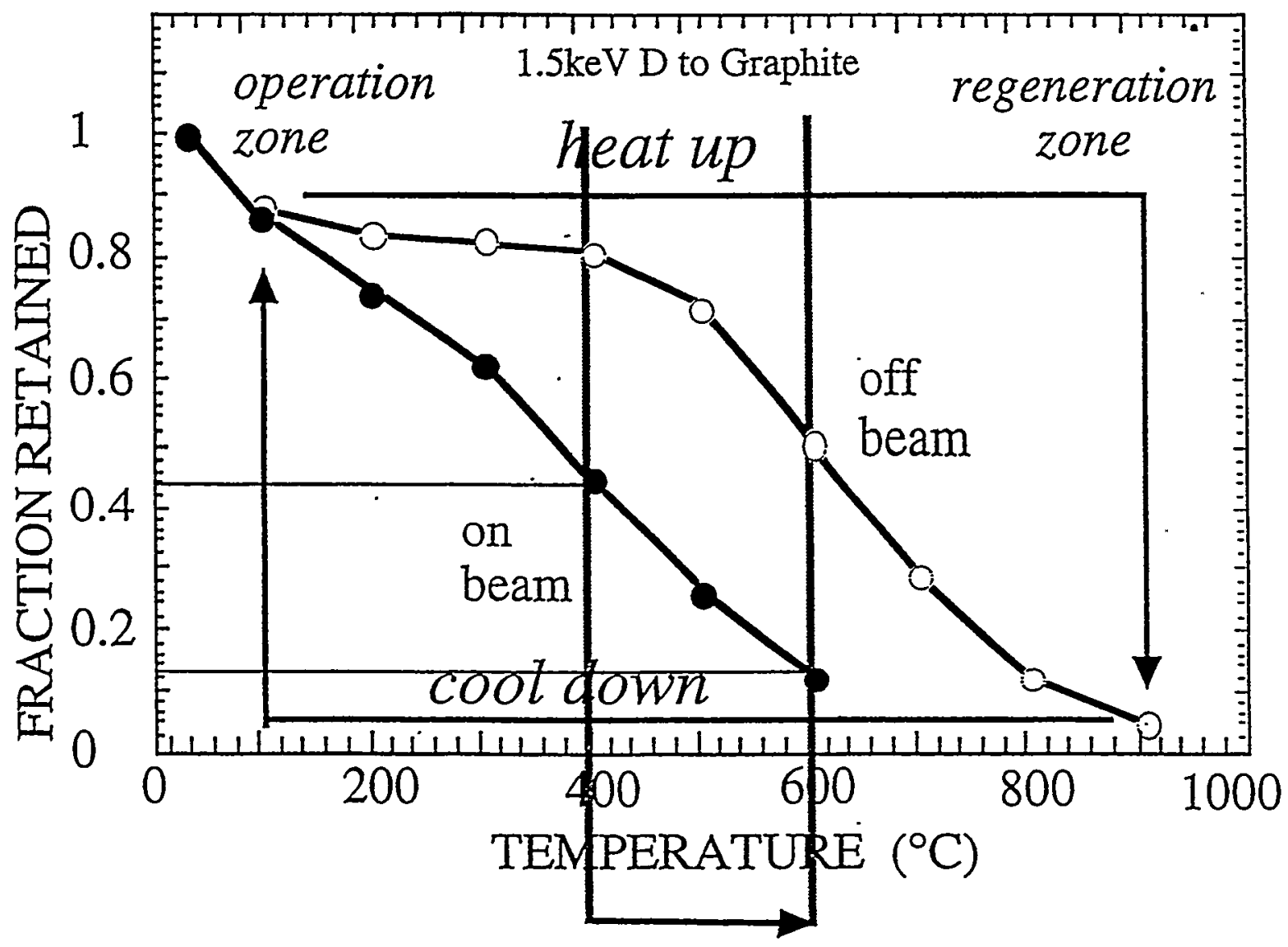

Supra-High Temperature Engineèring Lab.; 


\section{Calculated saturated concentration of hydrogen during irradiation}

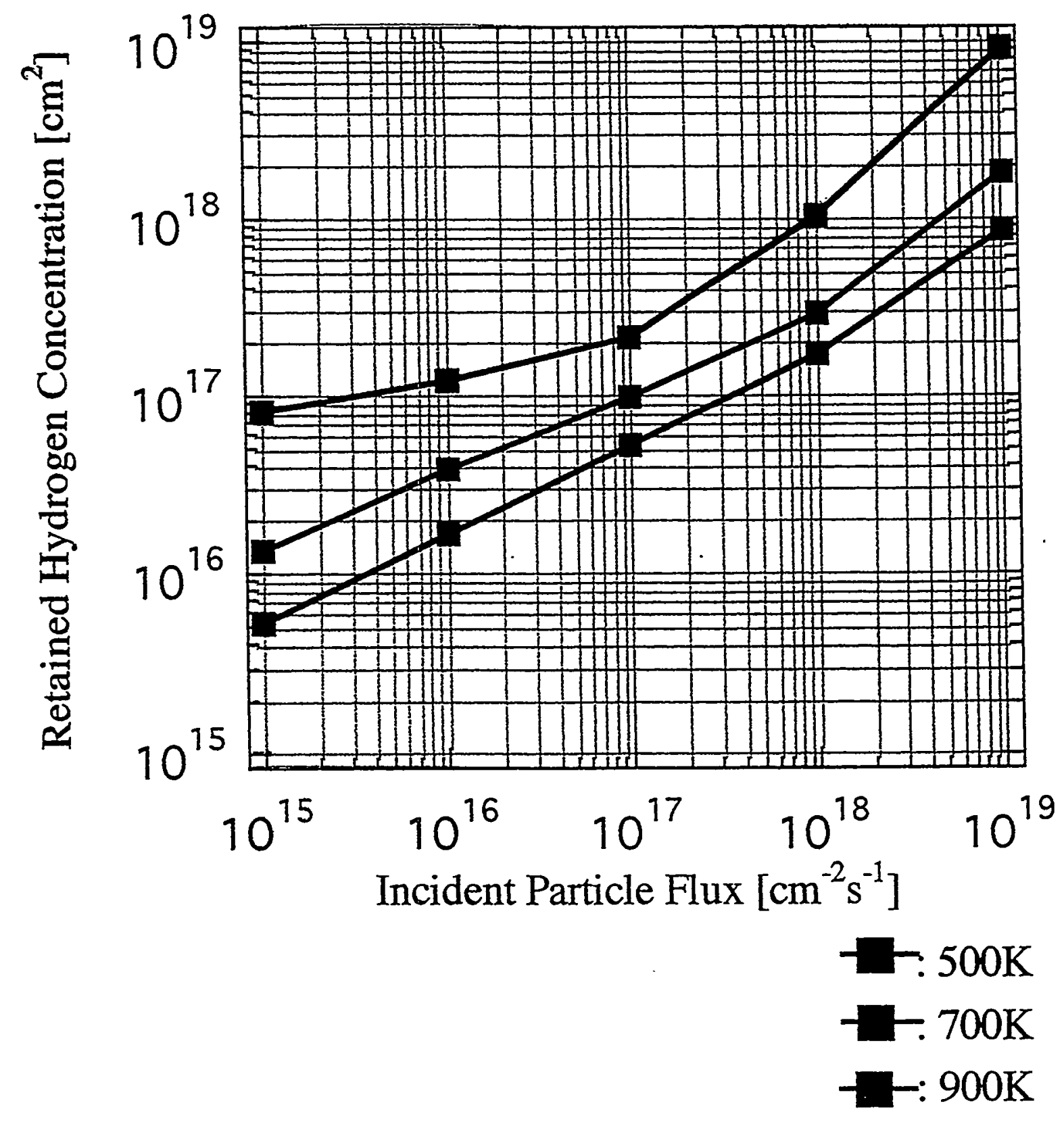




\section{Transient behavior of retained hydrogen}

r
Particle Flux: $5 \times 10^{22} \mathrm{~m}^{-2} \mathrm{~s}^{-1}$
Heat Flux: $17.5 \mathrm{MW} / \mathrm{m}^{2}$

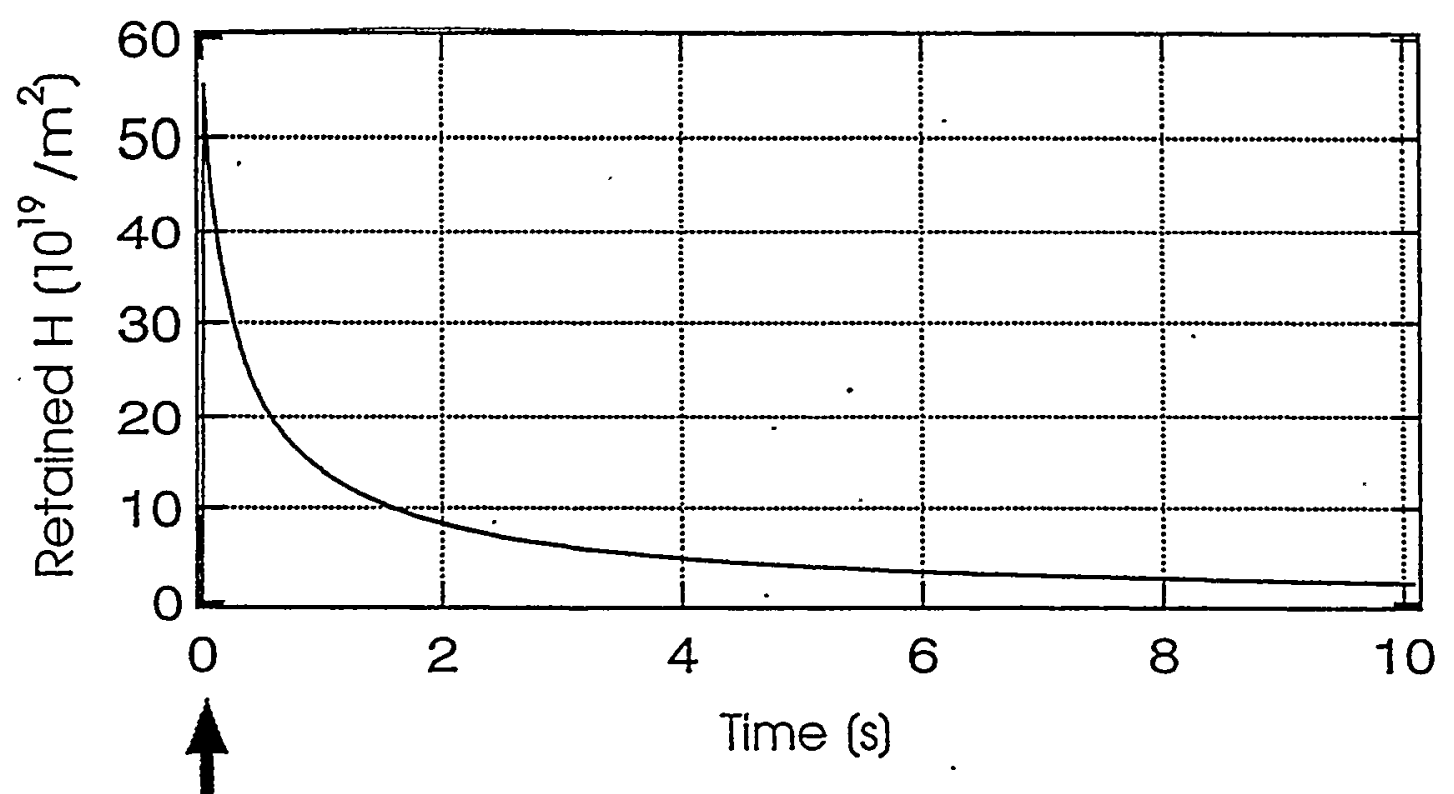

Pebble drop

Spontaneous release of retained hydrogen at high temperature operation 


\section{Estimation of pumping performance of the pebble drop divertor}

- The divertor surface area

Major radius of torus : $7 \mathrm{~m}$

Width of strike zone : $50 \mathrm{~mm}$ about $4.4 \mathrm{~m}^{2}$

- Retained hydrogen: $5.5 \times 10^{23} / \mathrm{m}^{2}$

- Pebble flow rate: $4.4 \mathrm{~m} / \mathrm{s}$ (drop from $1 \mathrm{~m}$ in height)

$\rightarrow \underline{2.1 \times 10^{23}}$ hydrogen atoms/s 


\section{Conclusions}

- The maximum heat load was determined by the induced thermal stress in the pebble. The divertor pebble 1-2 $\mathrm{mm}$ in diameter can be used in $15 \mathrm{MW} / \mathrm{m}^{2}$ heat load.

- The pumping performance of pebble drop divertor was numerically studied by calculating mass balance equations. It was found that dynamic retained hydrogen in the pebble increased with heat load (particle flux), and sufficient pumping could be achieved. 


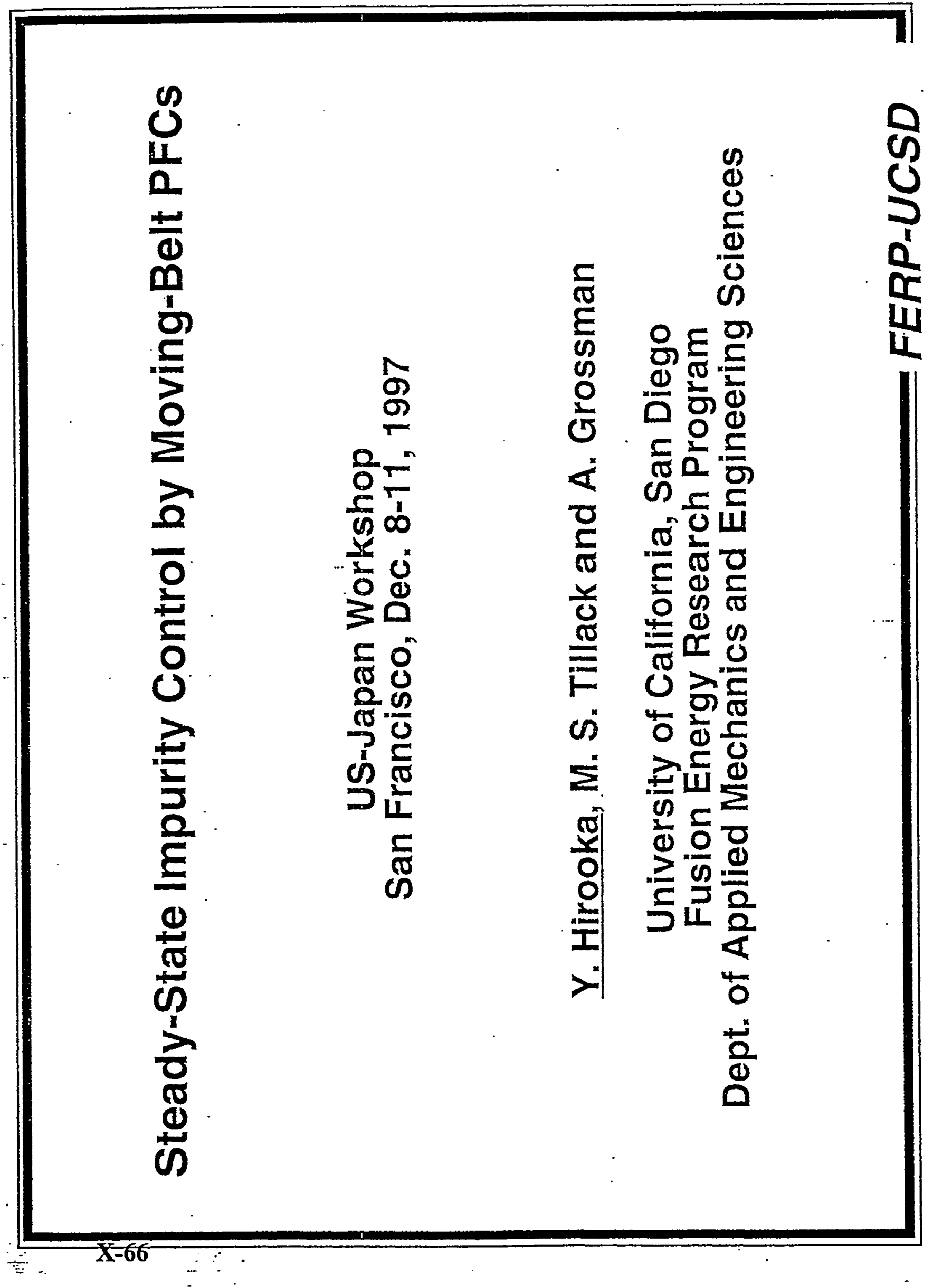




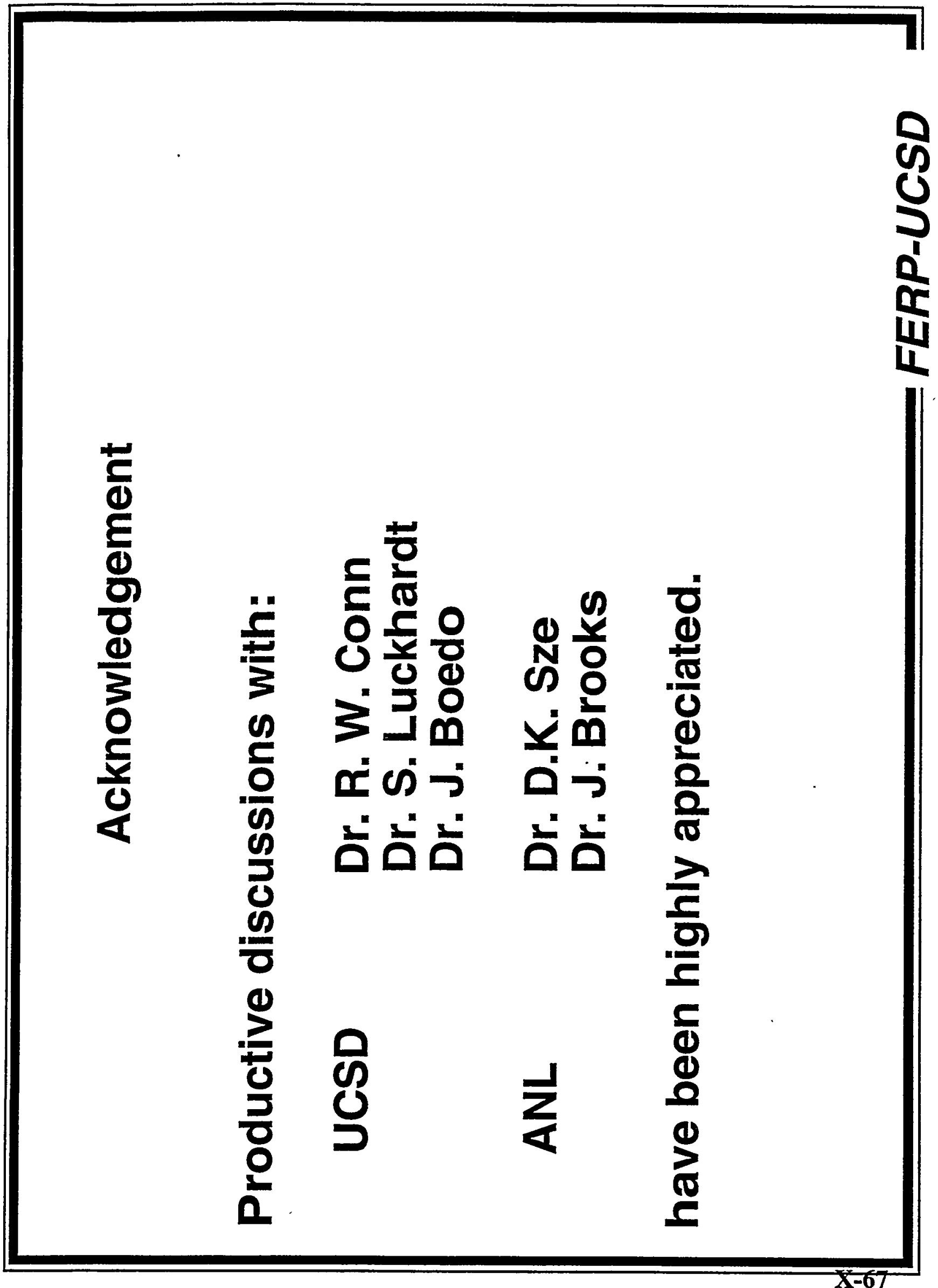




\section{Table of Contents}

1. Motivation of the present work

Technical issues on impurity control technologies

2. Review of innovative PFC concepts

Liquid lithium waterfall divertor

Rotating shell divertor, etc.

3. MB-PFC with ex-situ inline belt processing systems

Possible applications

4. Case study resutls

5. Summary. 


\section{Issues on the impurity control by PFC technolgies}

1. Wall conditioning-boronization, lithium injection, etc:

Effective but saturable, necessitating re-conditioning. Not desirable to steady-state reactors.

2. Tritium recovery necessary to meet the site regulation:

Codeposition leading to a continuous build-up of tritium. Periodic removal of codeposited materials

3. Core plasma contamination by eroded PFC materials:

Low-Z materials preferred but limited lifetime.

Can we have a long-lifetime low-Z PFC ??

4. Heat removal:

Thermal conductivity being the key, quality control for thousands of brazed tiles on heat sink becomes an issue. 
Comparison between conventional and moving-belt PFCs

ISSUE

Lifetime

Impurity

Heat removal

Tritium

MHD-effects

PMI-damages

Neutron effects
Stationary PFC

Limited (low-Z)

Periodic wall cond.

Conduction

Periodic removal

None

Periodic repair

Radioactivity (Mo, W) Bubble formation (Be)
Moving-belt PFC

Unlimited (w/gettering)

Continuous gettering

Radiation or contact

Continuous removal

Minimal (for SiC)

Continuous repair

Reduced radioactivity (for SiC) 


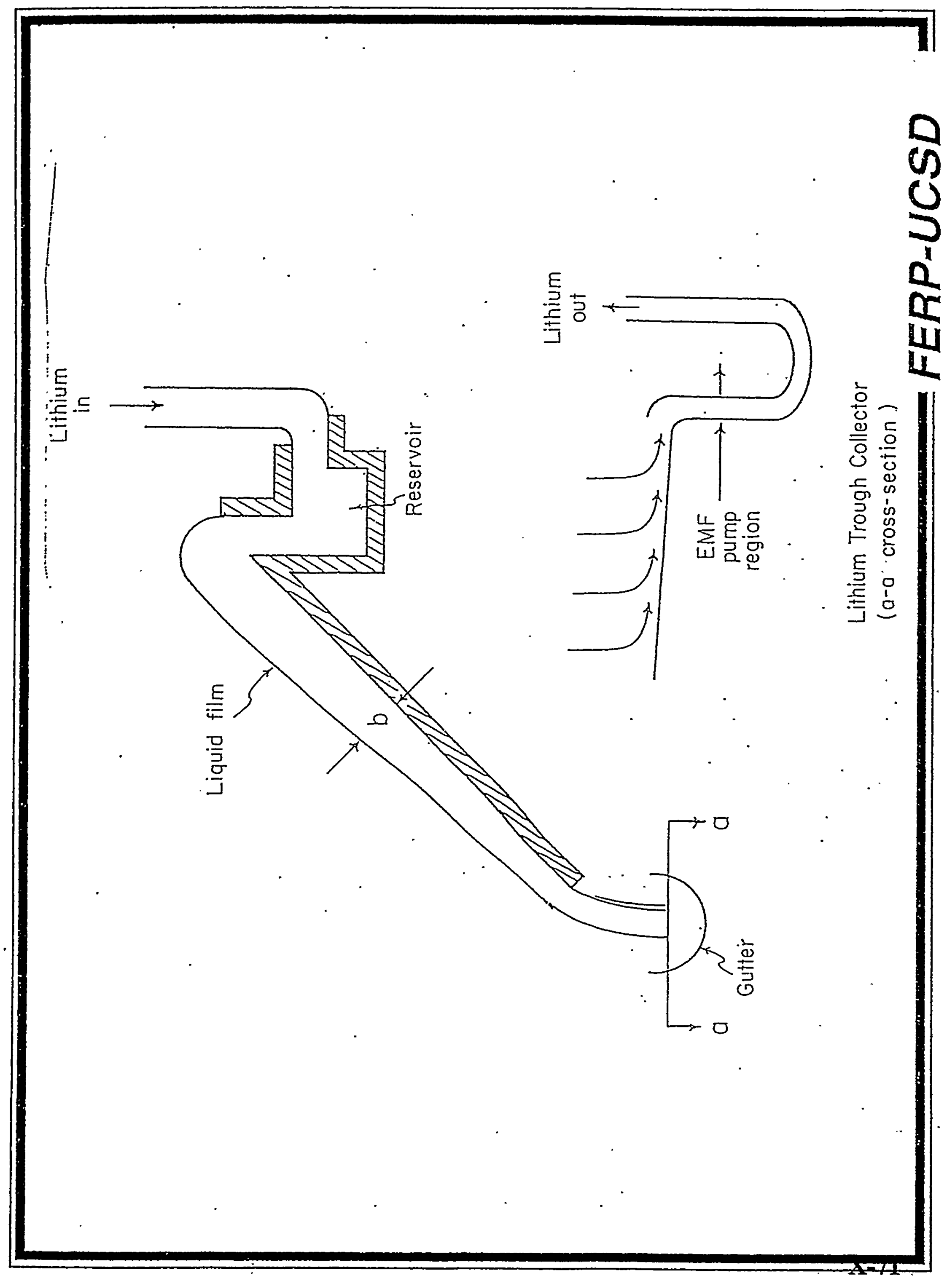




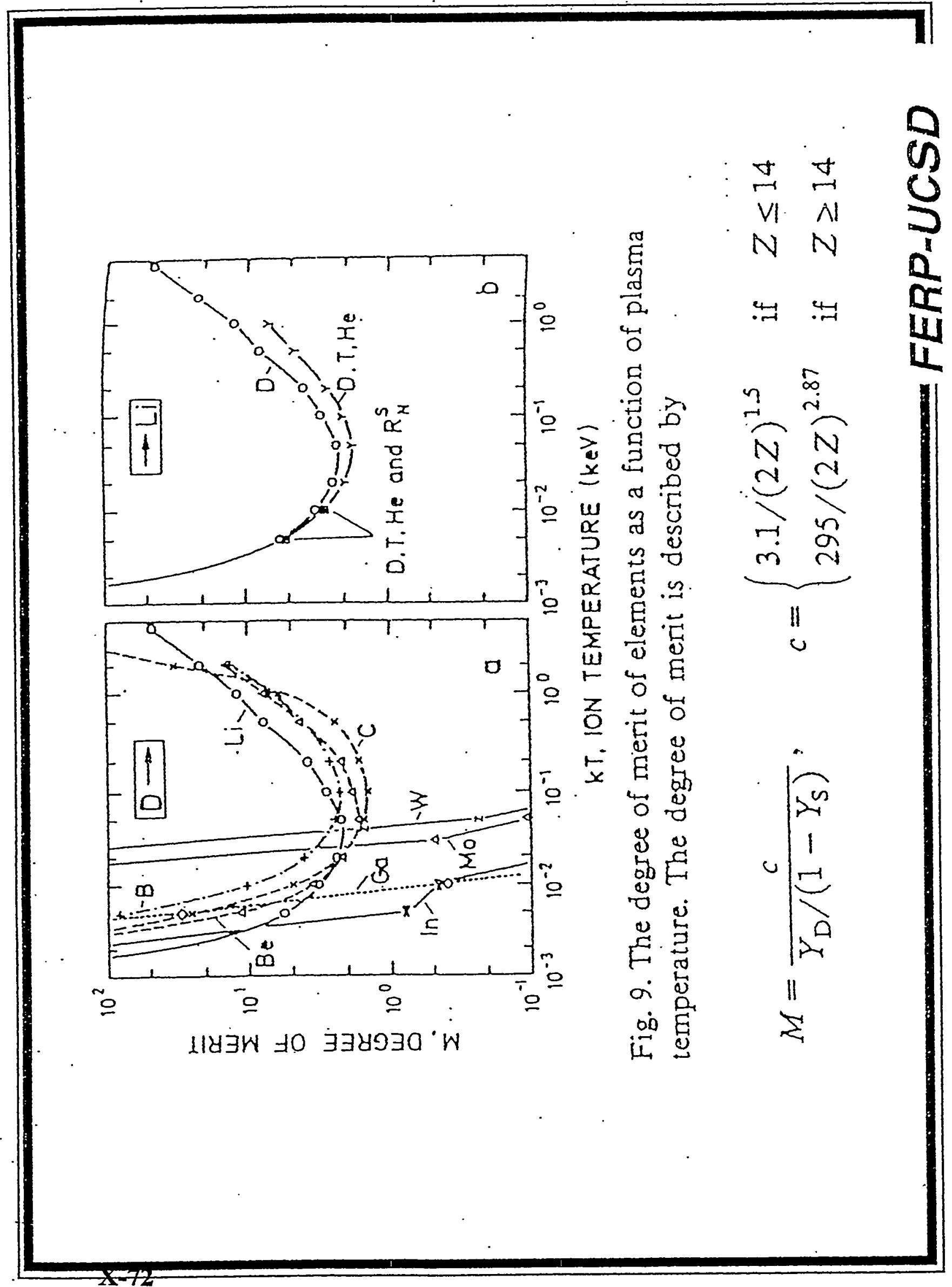




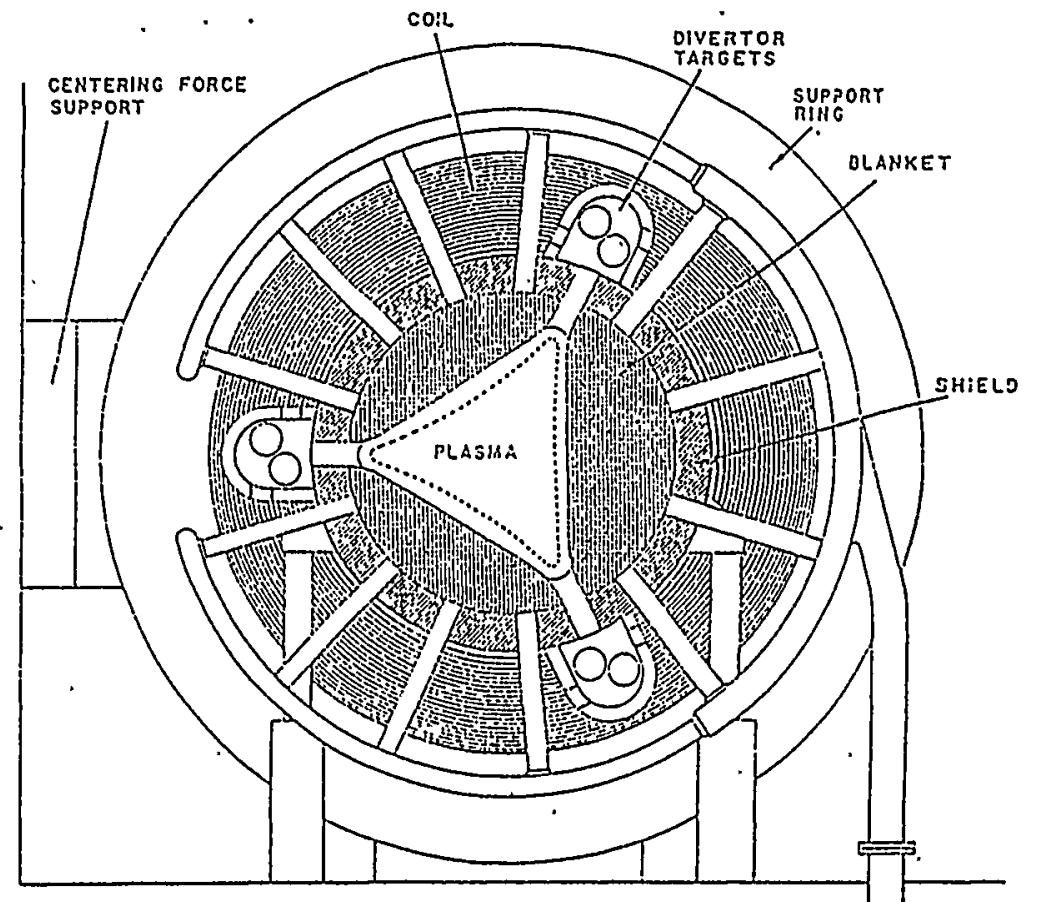

Figure yill.2-1 cross secticn of resetor showing location of dfvertor turgets.

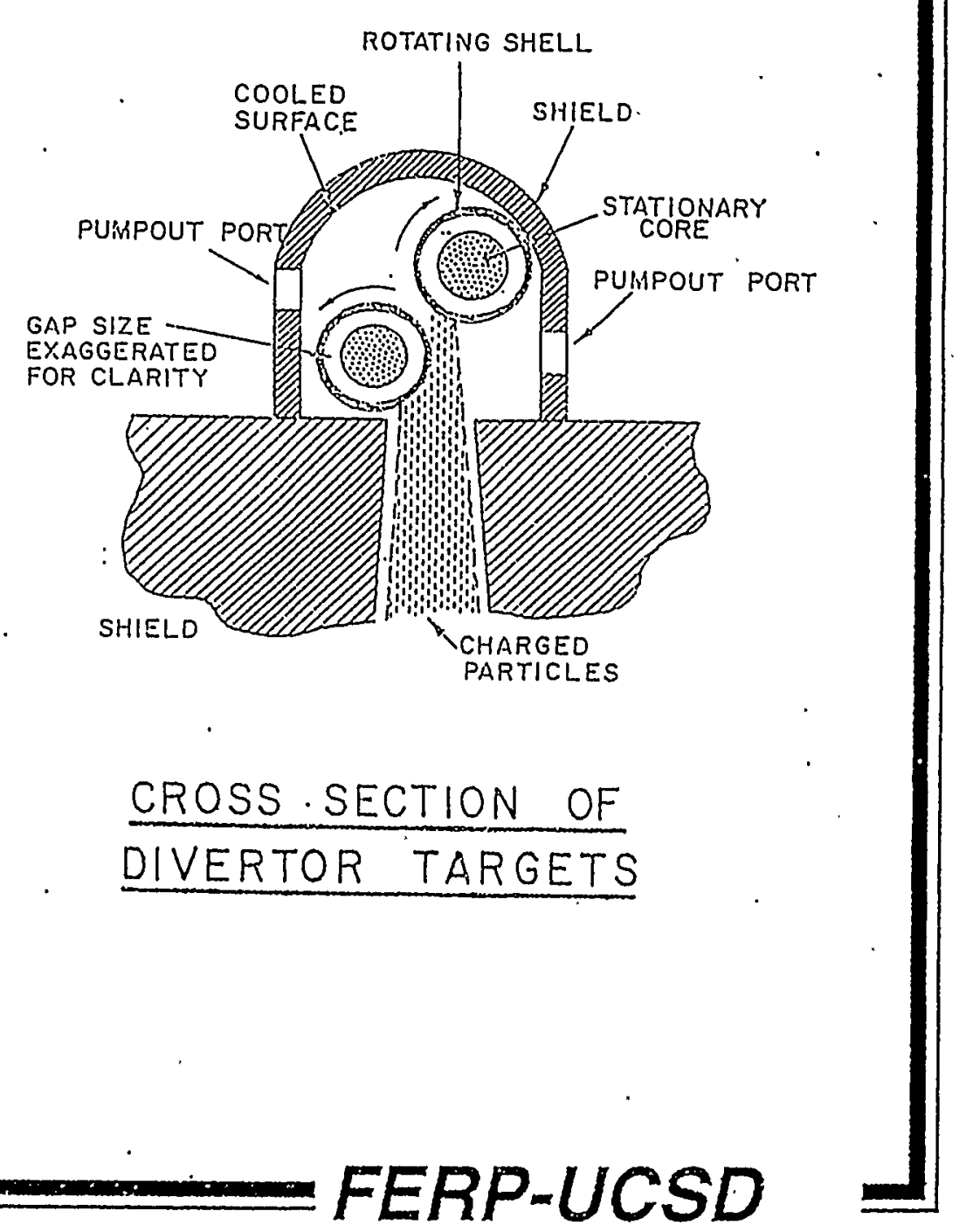




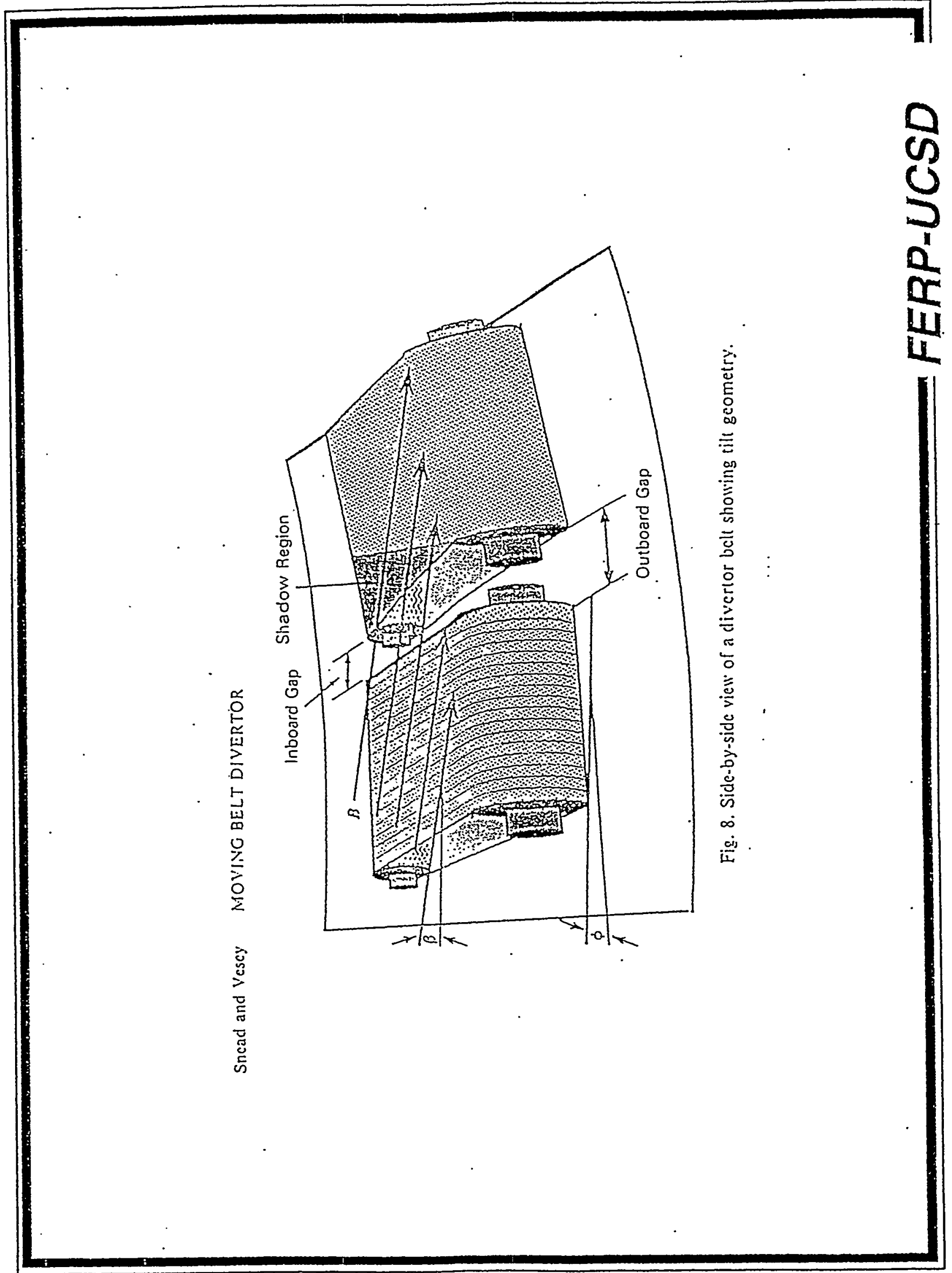




\section{Moving-belt plasma-facing components with ex-situ belt processing systems}

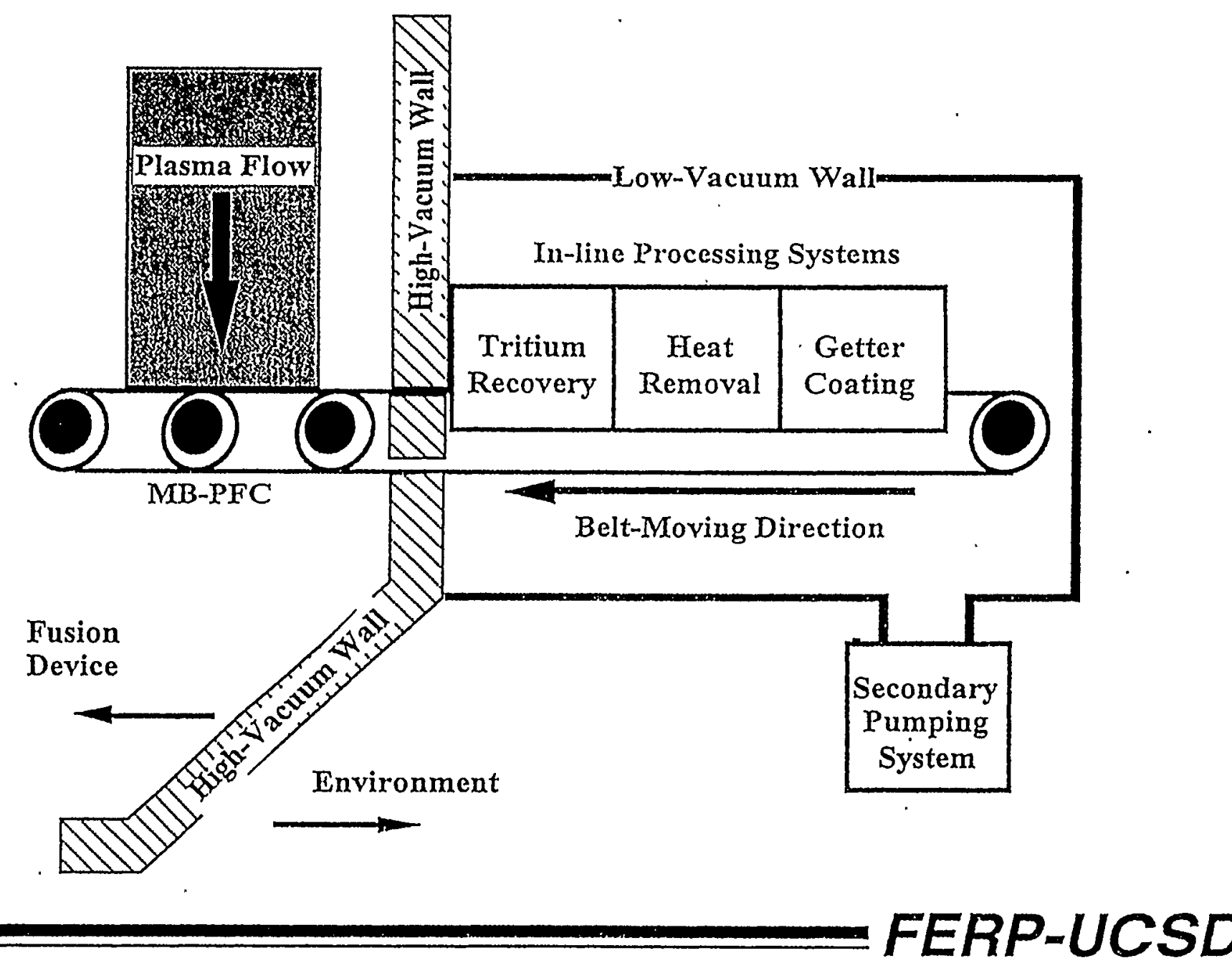


0
$\frac{0}{2}$
$\frac{0}{0}$
$\frac{5}{0}$
$\frac{0}{2}$

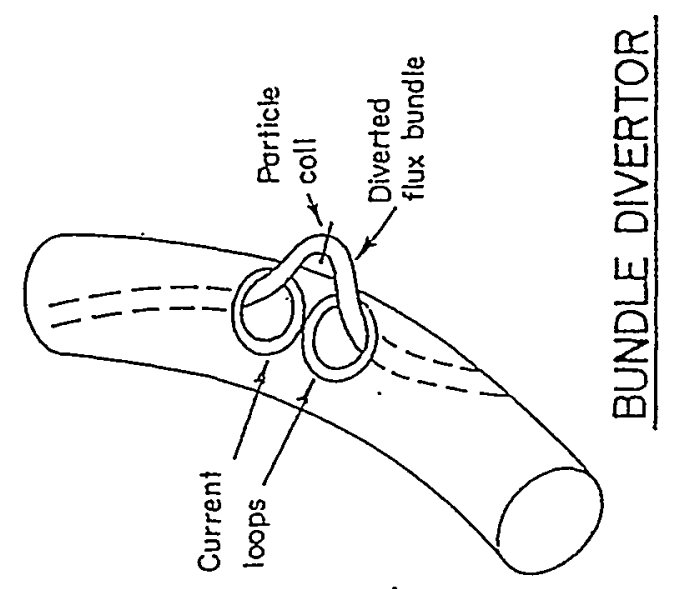

ธี

E

$\frac{0}{n}$

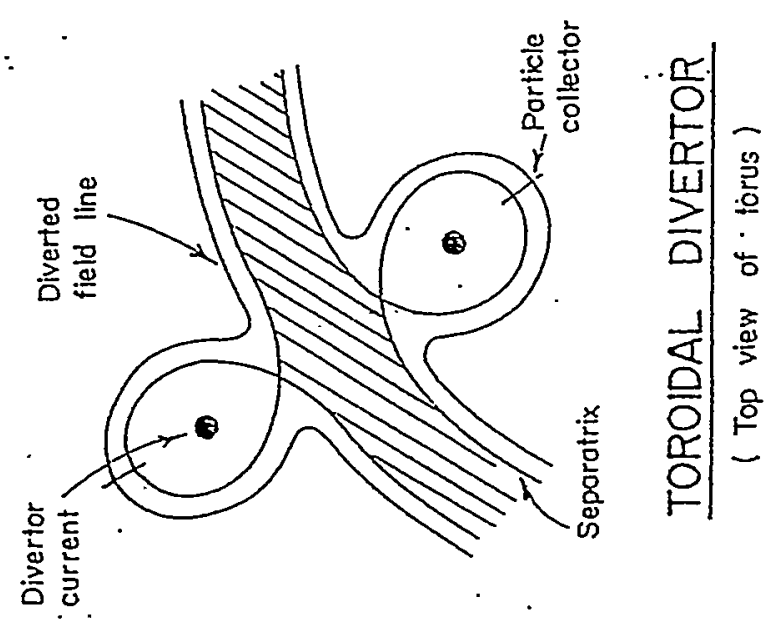

$\frac{1}{0}$
0
$\frac{2}{0}$
$\frac{0}{0}$
$\frac{0}{2}$
$\frac{2}{4}$

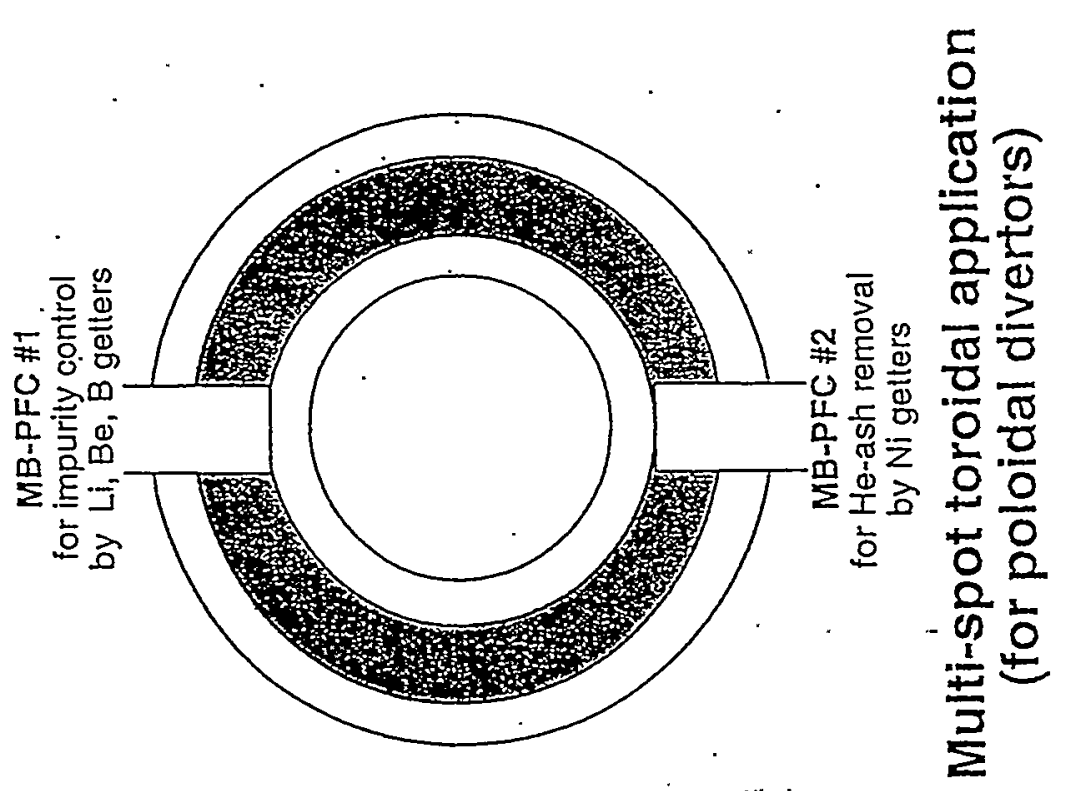


Table 1 MB-PFC system operating conditions and belt properties*.

Belt length, $L$

Belt width, W

Belt thickness, $t_{b}$

Belt density, $p$

Belt speed, $v_{b}$

Plașma interaction length, $1_{1}$

Tritium recovery section length, $l_{T}$

Getter-coating section length, $1_{2}$

Fuel plasma fluxes, $\Gamma_{\mathrm{D}}+\Gamma_{\mathrm{T}}$

Oxygen impurity flux, $\Gamma_{0}$

- Particle bombarding-energy; $E$

Redeposition probability, $\mathrm{P}_{\text {redcp }}$

Belt surface temperature

Surface emissivity, $\varepsilon$.

Getter deposition efficiency; $\vee \phi(\vartheta)$

Therrial conductivity, $\mathrm{k}$

Heat capacity; $\mathrm{C}_{\mathrm{p}}$ :

Thermal diffusivity, $\alpha$

Stefan-Boltzman constant

*Property data for carbon materials are listed here.
$20 \mathrm{~m}$

$\operatorname{lm}$

$1 \mathrm{~mm}$

$2.2 \mathrm{~g} / \mathrm{cm}^{3}$

$2 \mathrm{~m} / \mathrm{s}$

$1 \mathrm{~m}$

$2 \mathrm{~m}$

$2 \mathrm{~m}$

each $0.995 \mathrm{~A} / \mathrm{cm}^{2}$

$0.01 \mathrm{~A} \mathrm{~cm}^{2}$

$100 \mathrm{eV}$

$50 \%$

$1000^{\circ} \mathrm{C}$

0.8 .

. $50 \%$

$5 \mathrm{~W} / \mathrm{m}-\mathrm{K}$

$0.710 \mathrm{~J} / \mathrm{g}-\mathrm{K}$ $0.032 \mathrm{~cm} / \mathrm{s}$

$5.7 \times 10^{-12} / \mathrm{sW} / \mathrm{cm}^{2}-\mathrm{K}^{4}$ 


\section{Impurity control scenario}

(1) Erosion rate of a moving belt (Independent of moving speed)

$$
\Gamma_{\text {MBB-net }}=\Gamma_{\text {net }}\left(l_{1} / L\right): \text { "Diluted" erosion over the belt length }
$$

(2) Deposition rate of low-Z getter material (evaporation source*)

$$
\Gamma_{\text {MBB-depo. }}=\Gamma_{\text {evap. }}\left(I_{2} / \mathrm{L}\right) \vee \phi(\vartheta)
$$

*Plasma spray is possible but coverage uniformity over a moving-belt is a potential issue.

(3) Is "zero-erosion" condition possible at a practical eyaporation source temperature? -......'Yes!"” 


\section{Getter coating deposition rates for $\mathrm{Ll}, \mathrm{Be}, \mathrm{B}$}

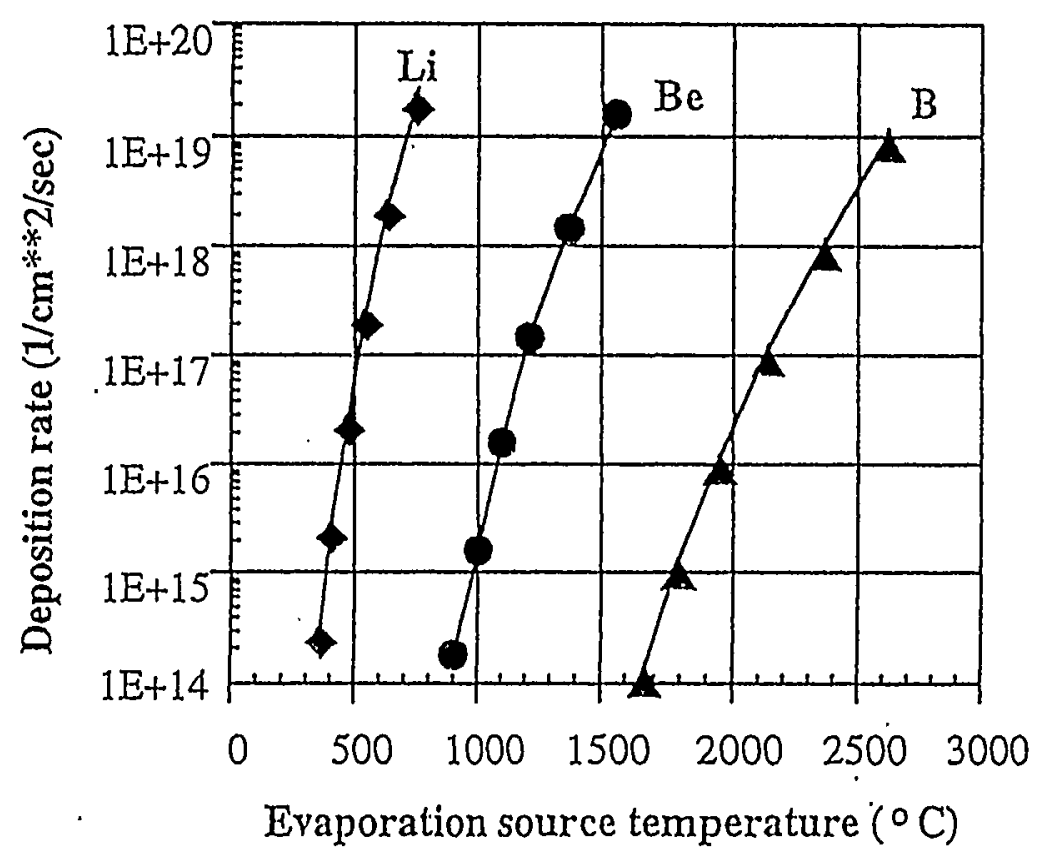

$$
\begin{gathered}
\Gamma_{\text {evap. }}=\frac{P_{\text {eq. }}}{\sqrt{2 \pi m k T_{s}}} \\
\Gamma_{\text {MB-depo. }}=\Gamma_{\text {.vvap. }}\left(I_{2} / I\right) \vee \phi(\vartheta)
\end{gathered}
$$




\section{Tritium recovery and in-belt inventory}

(1) TMAP + TRIM.SP code calculation

Numerical solution of diffusion equation with boundary conditions related to particle implantation in carbon.

(2) High-recovery efficiency: 99\%

High surface temperature after plasma exposure $\left(1000^{\circ} \mathrm{C}\right)$

(3) Tritium inventory: not an issue for MB-PFCs

Slow (parabolic) increase due to bulk diffusion Saturation not occur until $1.8 \times 10^{9}$ rotations (each $10 \mathrm{sec}$ ), i.e., 570 years ! 


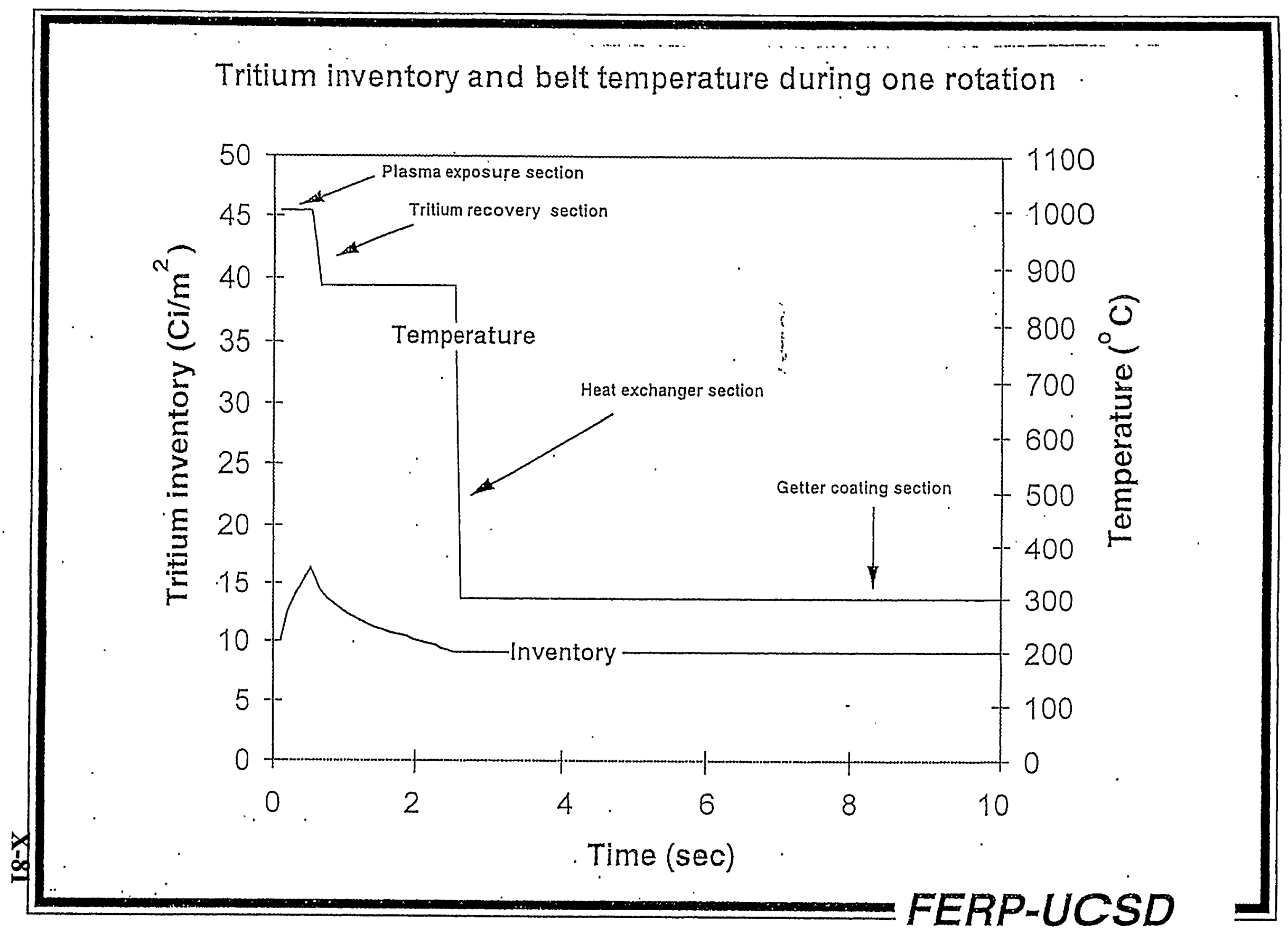




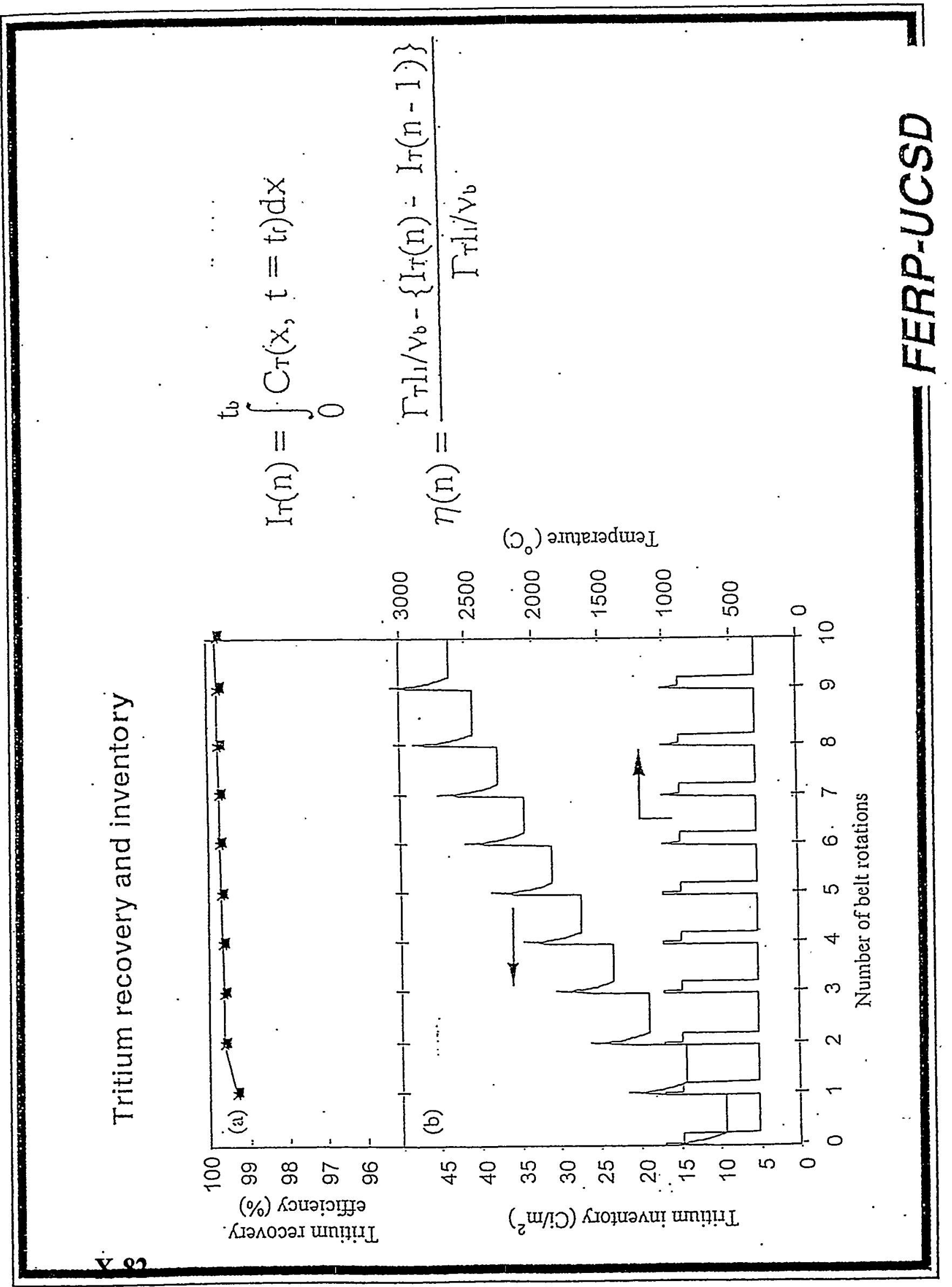




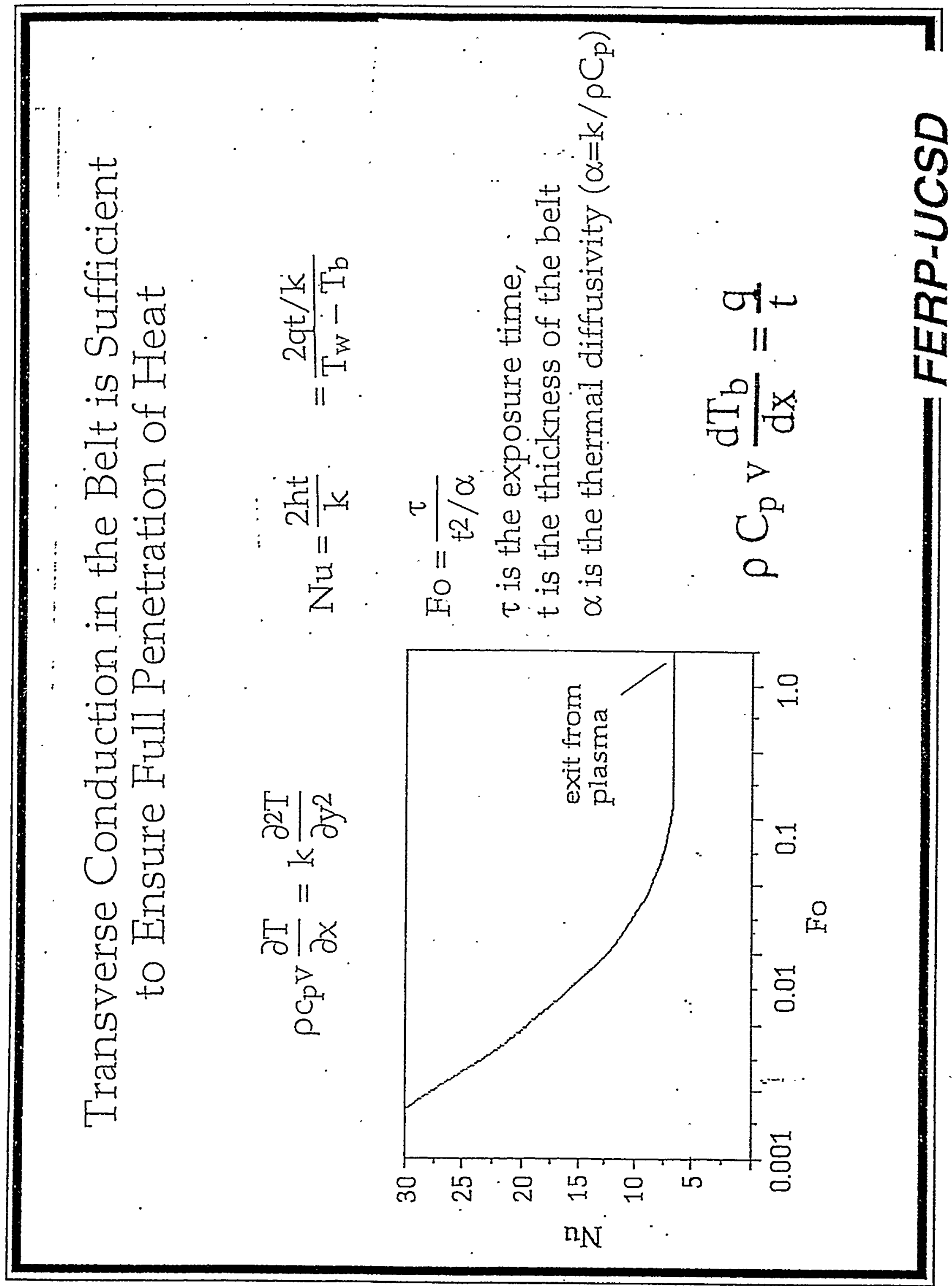




\section{Temperature profiles on a $\mathbf{C}-\mathbf{C}$ moving belt}

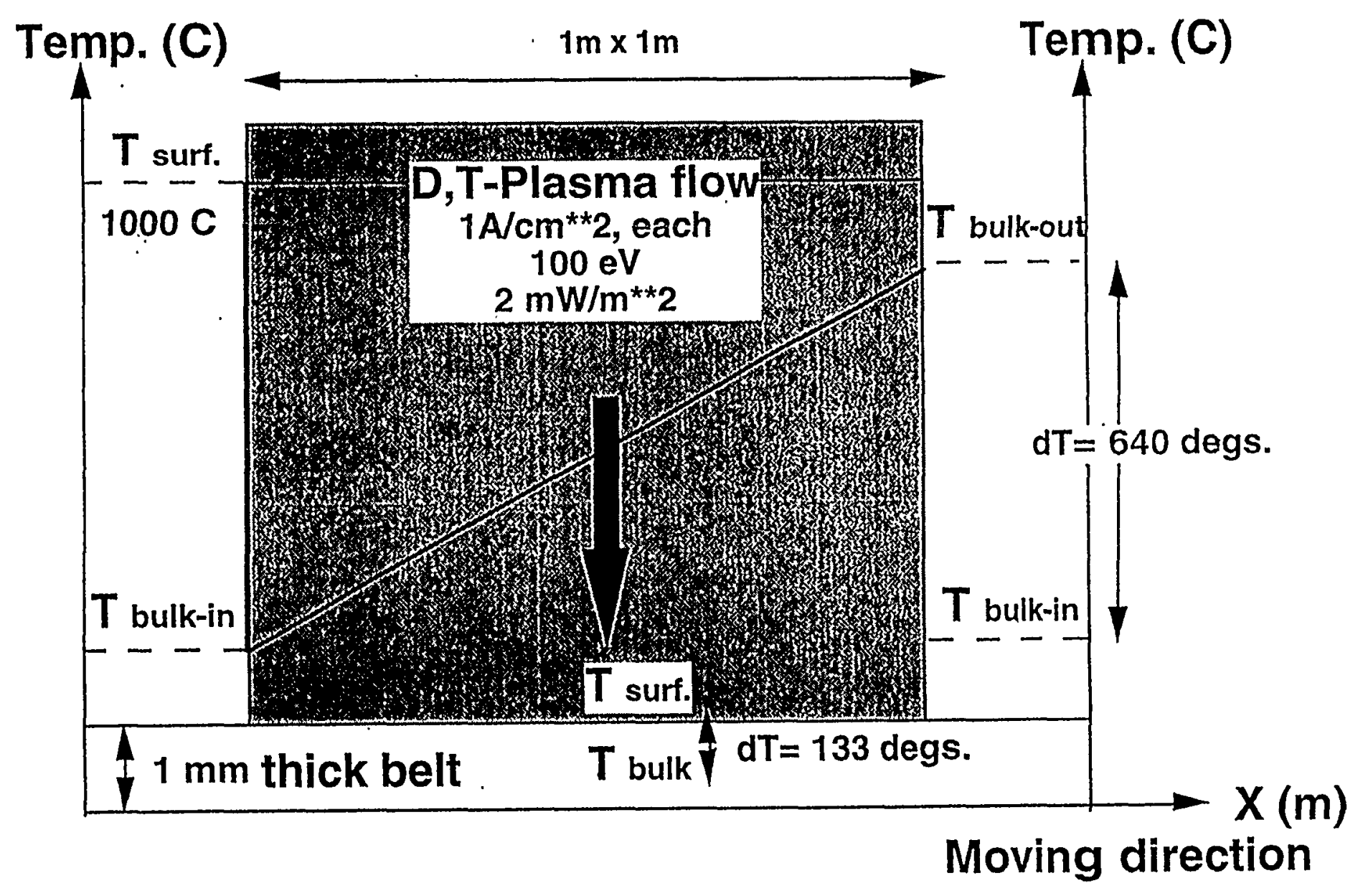




\section{Summary}

1. Moving-belt PFC with ex-situ belt-processing systems have been proposed for steady-state impurity control, heat removal, and tritium recovery.

2. To minimize the MHD effects and induced radioactivity, semimetallic and semi-conductor materials such as $\mathrm{C}-\mathrm{C}$ and $\mathrm{SiC}-\mathrm{SiC}$ fabrics are proposed as the belt materials.

3. In the case study assuming DT fluxes of $20 \mathrm{kA} / \mathrm{m}^{2}$ (at $100 \mathrm{eV}$ ), heat flux of $2 \mathrm{MW} / \mathrm{m}^{2}$, belt temp. of $1000^{\circ} \mathrm{C}$, belt-speed $2 \mathrm{~m} / \mathrm{s}$, a MB-PFC system has demonstrated the following possible:

(1) Unlimited lifetime with non-saturable impurity gettering,

(2) Effective heat removal by radiation or contact heat transfer

(3) Efficient tritium recovery for long-term operation.

4. Optimization and limitations of MB-PFCs will be investigated.

5. Currently, the application for LHD is under discussion. 


\section{Review of He Self-Pumping Concept}

\section{work by Jeff Brooks (ANL) and Richard Nygren (Sandia)}

in collaboration with:

- Sandia surface physics lab (Doyle, Wampler, Walsh)

- ANL chemistry lab (Krauss)

- IPP Garching surface physics lab

- PISCES group

- TEXTOR group

- MIT Alcator C-MOD group

Papers by Nygren et al and Brooks et al. (He implantation and TEXTOR experiments) 10th PSI (Monterey, 1992, JNM 196-198), 9th PSI (Bournemouth, 1990 JNM 176-177) 


\section{Summary of work on He self pumping}

- concept proposed in 1980s by ANL and Sandia

- He implantation work at low energies (<100 eV)

- TEXTOR He self pumping experiment

- experimental plan for Alcator C-MOD

- preliminary concepts for fusion reactors

\section{Goal for He self pumping effort}

- reestablish program

- perform follow on test (C-MOD?)

- further develop concepts for fusion reactors

- develop larger concept demonstration test

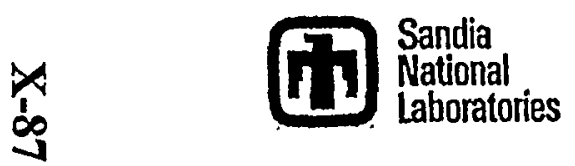




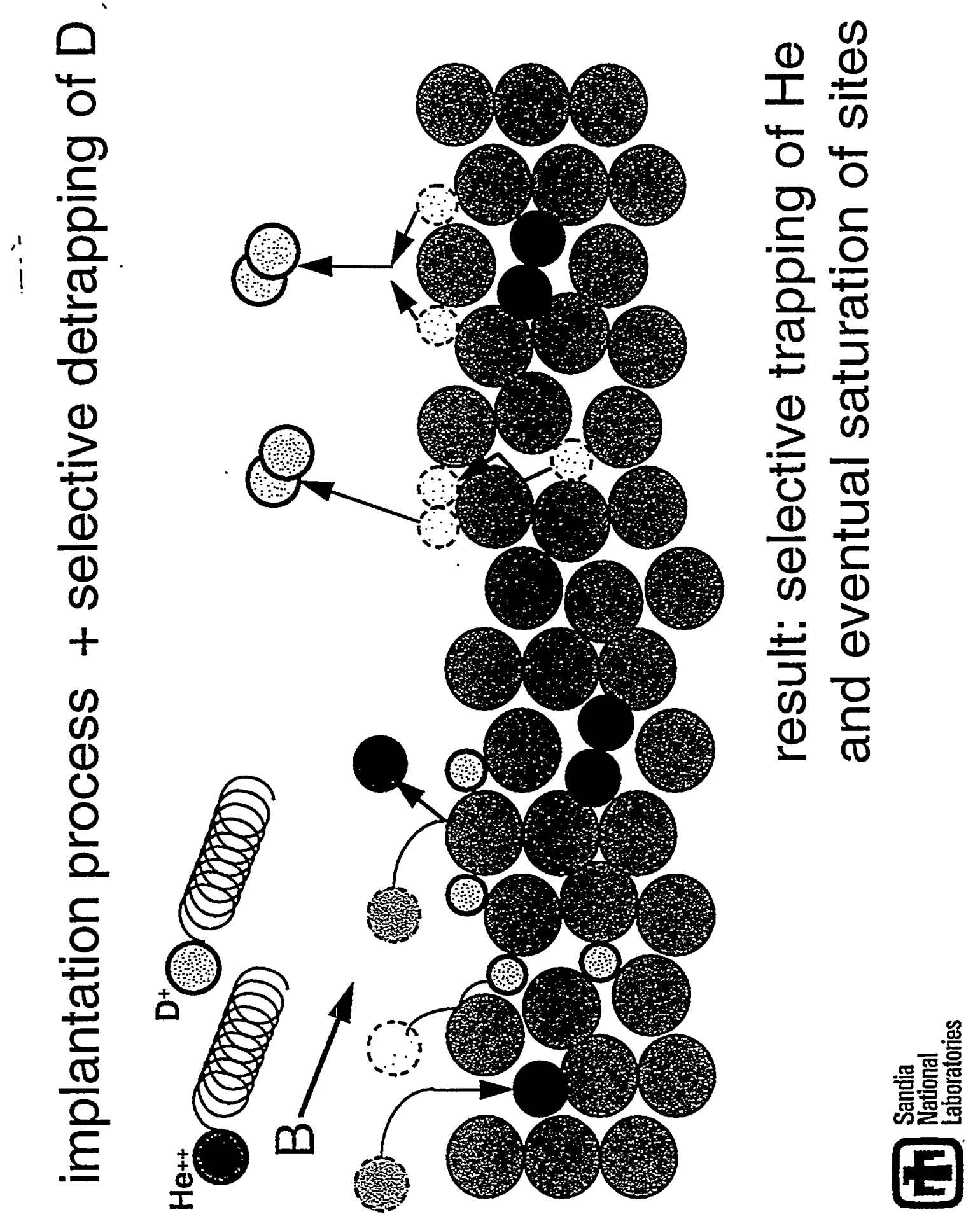




\section{Key requirements for materials}

- high probability of He trapping • high D solubility, diffusivity

- high He saturation level

- no hydride formation

- temperature window: $T_{\text {He release }}<T_{D \text { release }}<T_{\text {max operation }}$

- adequate heat removal (good k)

- adequate neutronics

- self sputtering coefficient less than unity

Candidates: $\mathrm{Ni}, \mathrm{V}, \mathrm{Fe}, \mathrm{Nb}, \mathrm{Mo}, \mathrm{Ta}$

We have used $\mathrm{Ni}$ in our experiments. 


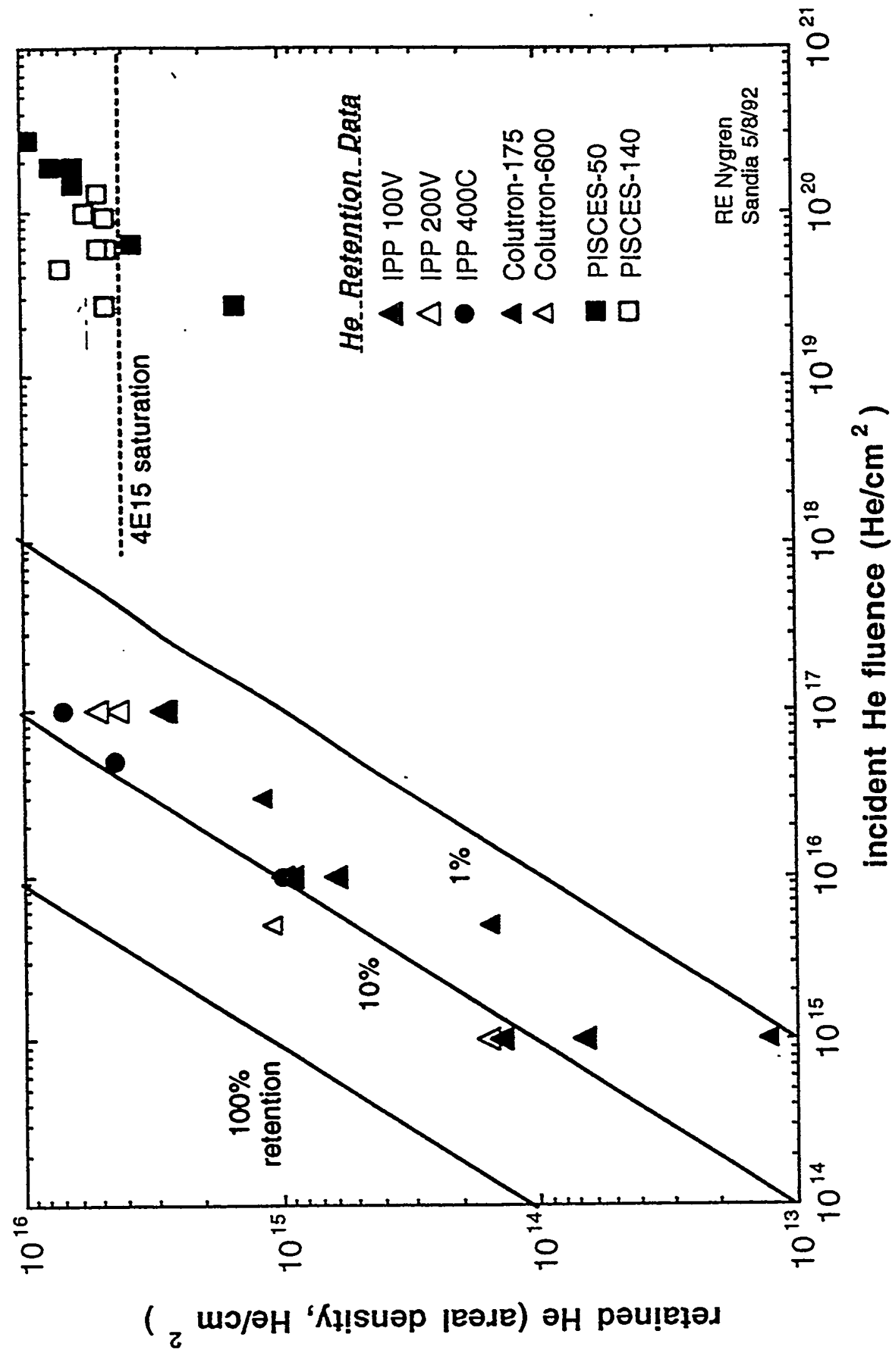




\section{TEXTOR experiment with ALT-I modular limiter}

Two Ni plates with embedded heaters, a Ni deposition system

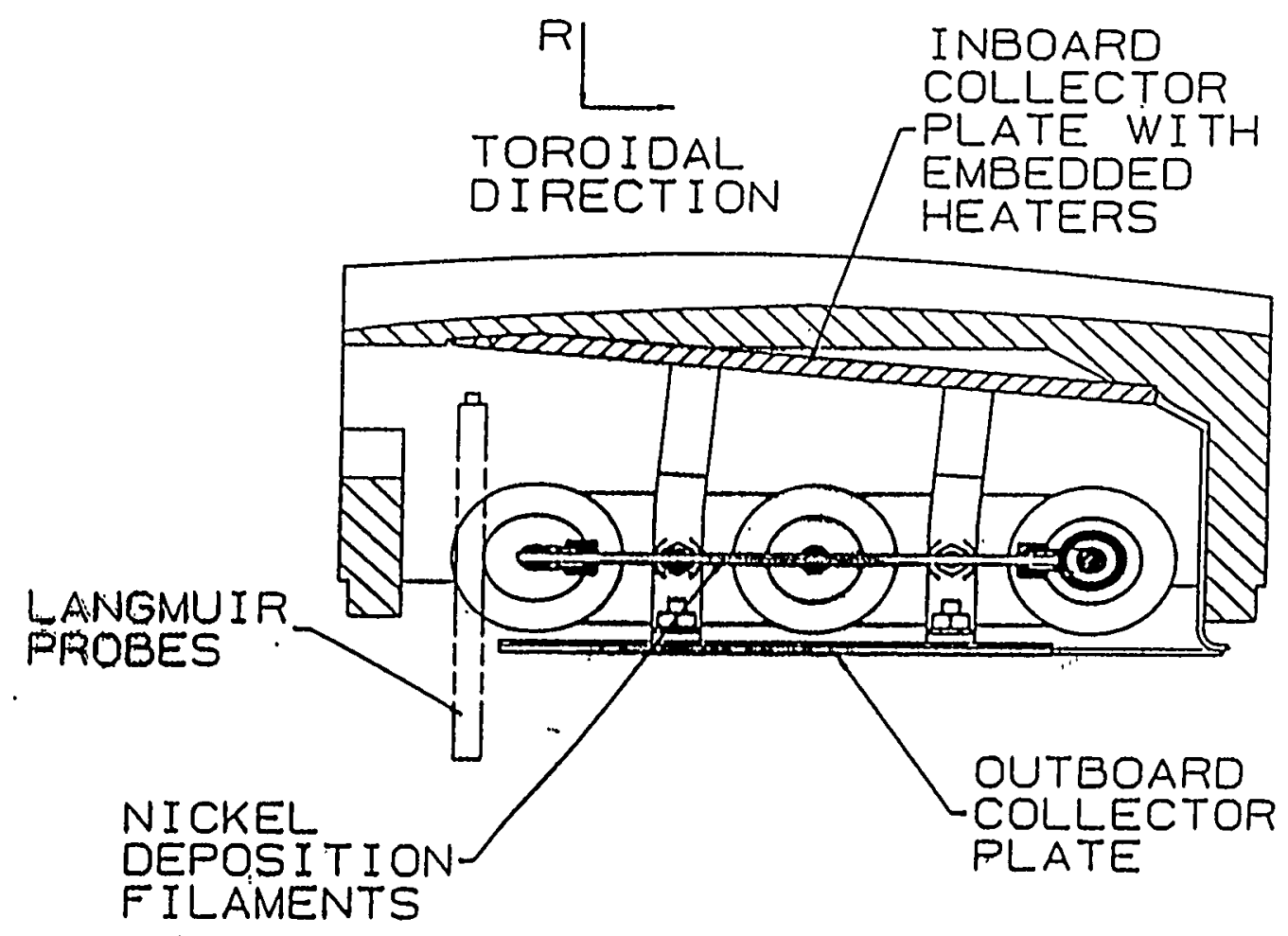

We operated the plate at $\sim 450^{\circ} \mathrm{C}$ and did observe He self pumping by comparing He in the plasma in shots with saturated $\mathrm{Ni}$ to $\mathrm{He}$ in shots with fresh $\mathrm{Ni}$. 


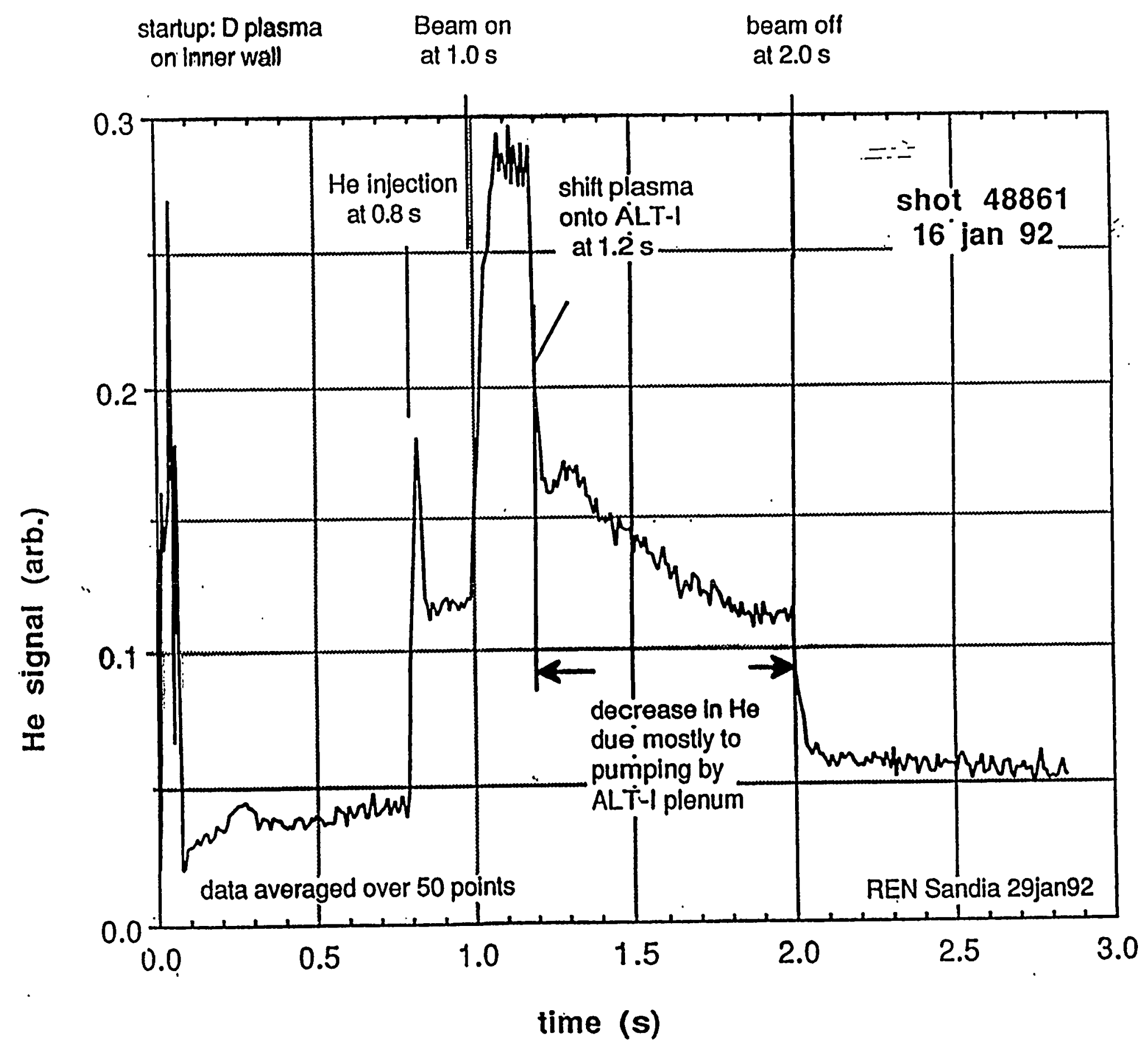




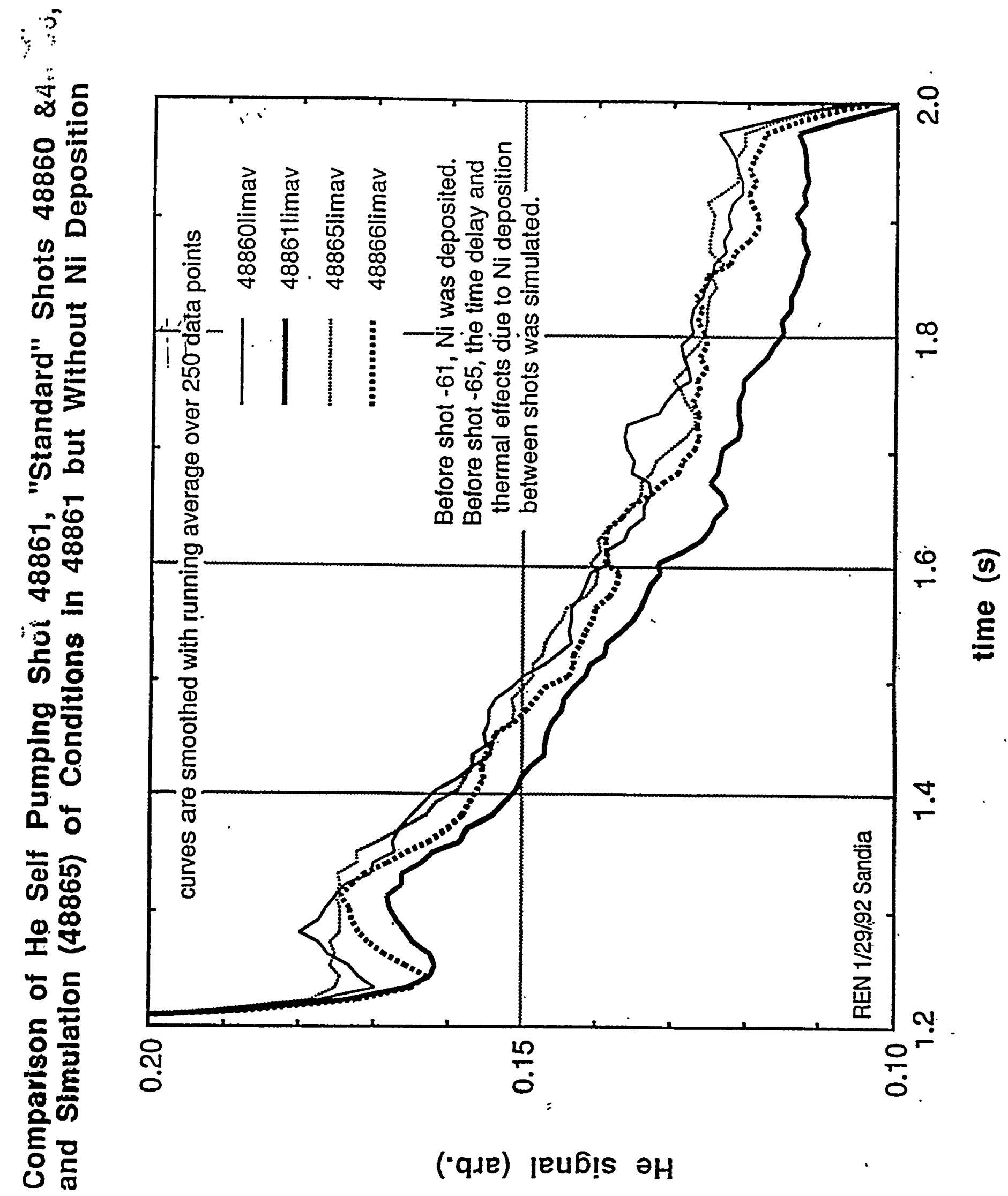




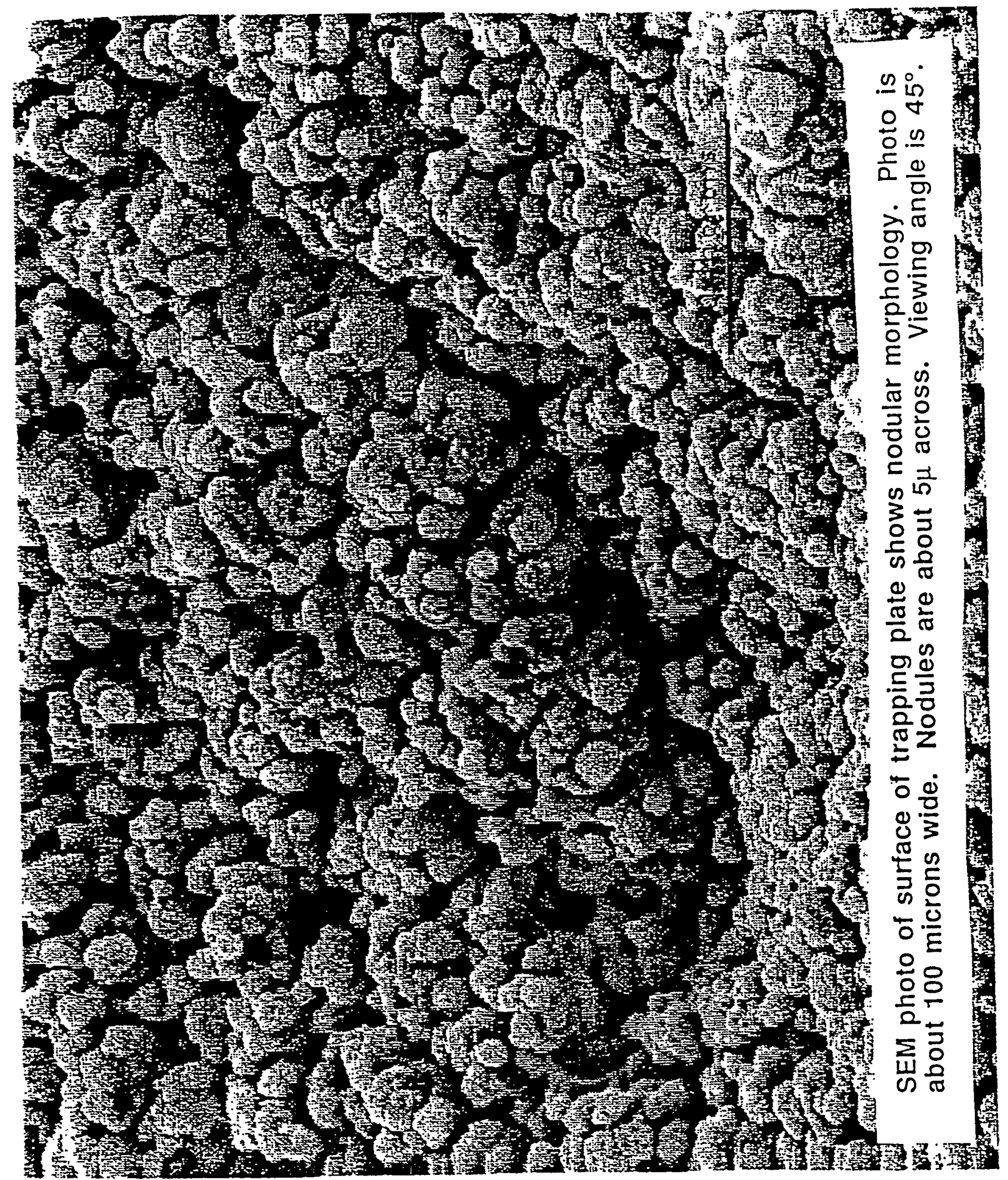




\section{TEXTOR experiment: post test analysis}

The two Ni plates were analyzed:

- Rutherford backscattering (W, Ni, O, C, B)

- elastic recoil detection (He)

- nuclear reaction analysis (D)

Both the inner plate (exposed to plasma) and the outer plate had significant (1-2x1016 He/cm $\left.{ }^{2}\right)$ and roughly amounts of He. The trapping by the outer plate implies that trapping of reflected neutral He occurred and was important.

The inner plate had very little $D$, but did have significant carbon deposition. (This might trap $D$ but not He.)

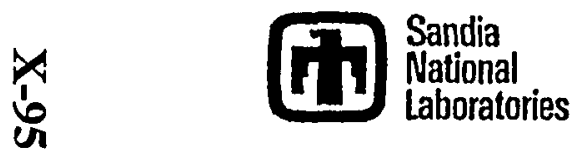


Concept development should explore:

- erosion as source of host material

- auxiliary deposition system

- biasing to increase implantation depths

- trapping located in a deposition region

- trapping located to exploit reflected neutral He

- realistic edge conditionsm including impurities

- assessment of D/T recycling along with He pumping

Materials development should include:

- samples that simulate a mix of impurities and host material

- materials directed toward specific designs,

e.g., system A for W walls, system B for Be walls 
A Strategy for Development of Helium Self Pumping

Richard Nygren, Sandia National Laboratories

Jeff Brooks, Argonne National Laboratory

from June 1992 plan

A novel concept for helium $(\mathrm{He})$ self pumping offers the potential to reduce and simplify vacuum pumping and tritium processing in fusion reactors. This year an ANL-Sandia-KFA team completed a successful proof-of-principle experiment of $\mathrm{He}$ self pumping on TEXTOR. After a brief explanation of the He self pumping concept, a proposed strategy for further development is outlined here.

To avoid diluting the plasma in a magnetic fusion reactor, He "ash" formed when deuerium (D) and tritium (T) combine, must be continually removed. Conventional vacuum pumping poses the problem that the $D$ and $T$ (fuel) would be pumped along with the He; this pumped fuel must then be recovered from the exhaust stream. With $\mathrm{He}$ "self pumping", there is no exhaust. He ions from the plasma are implanted and trapped in a host material at the plasma edge. Simultaneously implanted D and $T$ are mobile in the host material and diffuse out and recycle.

The TEXTOR experiment verified two important features of He self pumping. First, the host material, nickel at about $450^{\circ} \mathrm{C}$ trapped $\mathrm{He}$ and no D from a D-20\% $\mathrm{He}$ plasma. Second, even with a relatively low edge temperature of $25 \mathrm{eV}$ (at the throat of ALT-I) and only $0.08 \mathrm{~m}^{2}$ of host material, a significant fraction (5-10\%) of the $\mathrm{He}$ in the plasma was trapped. The amount of He pumped would have even been larger but carbon contamination of the nickel decreased its capacity to trap He.

The next step in developing this concept is to confirm He self pumping in a diverted plasma. In these experiments, the host material must be specifically chosen for the plasma conditions present. For example, different host materials would be chosen for a carbon machine than for a metal machine. Work in design and in materials development is also necessary to support the development of a well founded experiment.

Diverted Plasma: He self pumping involves ions accelerated through the sheath at surfaces where magnetic flux lines are incident at an oblique angle and important neutral interactions, including reflection and trapping of energetic neutral He. These features cannot now be well simulated outside a tokamak and an experiment with an ITER-like (diverted) plasma is needed.

Here are some guidelines specific to the configuration of a tokamak experiment.' (1) A prerequisite for tokamak experiments is a good understanding of the possible options for the applications of He self pumping in ITER. (2) The trapping surface must be in a deposition region at the plasma edge rather than a region of erosion. (3) The placement must permit adequate He pumping. (4) The configuration should exploit the trapping of reflected energetic neutral $\mathrm{He}$. (5) Plasma impurities should be representative of the end application. For example, if impurity seeding in the ITER divertor is anticipated, then it would be desirable to simulate this in the experiment. (6) The experiment will require sufficient edge diagnostics to measure the He and $\mathrm{H}$ or $\mathrm{D}$ content, $\mathrm{n}_{e}$ and $T_{e}$ at the plasma edge at a minimum. (8) There will be limitations on how tokamak experiments represent ITER applications. The aim of the experiment should be to maximize the He pumping and deal effectively with the plasma conditions (e.g., impurities) that will exist in the tokamak 
experiment itself. It can then be argued by analogy that effective solutions specific to ITER can be obtained. The placement of the trapping surface in a tokamak experiment will be a compromise based on the configurations of components, locations of diagnostics, etc. In principle, compromises in placement that decrease He pumping can be compensated by increasing the $\mathrm{He}$ in a $\mathrm{H}$-He plasma. However, the basic objective should be to develop an experiment in which particle fluxes, heat loads, impurities, etc. are as representative as possible of ITER applications. This may mean that several experiments, each specific to a certain application and range of conditions should be proposed and developed.

Materials: The primary application of interest is for the ITER Technology Phase, i.e., most probably for an all metal machine (Be or $W$ ). He self pumping might also be considered for a carbon machine, either for the Physics Phase or as the alternate for the Technology Phase. Metal versus carbon is an important distinction because the primary issue for carbon contaminated systems is probably degradation of the He trapping capacity due to carbon poisoning; whereas for all metal systems, the primary issue is probably adequate recycling of hydrogen. It is not necessary to argue for one instead of the other but simply to recognize that there may be differing sets of issues for these applications.

Here are some guidelines related to materials. (1) Applications for the Technology Phase of ITER should be tested in an all metal tokamak. (2) The impurity levels in the plasma should be representative of the end application in ITER. (3) The product of the area, integrated flux and the trapping rate (equal to the arrival rate of He times the pumping efficiency) must exceed the rate of He production. (4) The net rate of deposition of the host material must exceed the He trapping rate divided by the atomic fraction of $\mathrm{He}$ at saturation $\left(\mathrm{f}_{\mathrm{He}}\right)$. (5) In materials development supporting tokamak experiments (e.g., He trapping and $H$ recycling studies), simulations of plasma surface interactions should be representative of the surface impurities anticipated in the tokamak experiments.

Design : Several options for depositing the host material should be pursued. Criteria should be developed that are specific to each option for each ITER application.

Here are some guidelines related to design. (1) The trapping surface must be replenished at a rate that keeps up with the He production in the plasma. (2) If sputtering from an adjacent region is the source then the sputtering rate will have to be great enough to supply the desired deposition rate.

$$
\sum\left\{\mathrm{A}_{12}\left(1-\delta_{\mathrm{i}}\right) \Gamma_{\mathrm{i}-1} \mathrm{Y}_{\mathrm{i}-1}\left(\mathrm{E}_{1}\right)-\Gamma_{\mathrm{i}-2} \mathrm{Y}_{\mathrm{i}-2}\left(\mathrm{E}_{2}\right)\right\}>\frac{\Gamma_{\mathrm{He}-2} \mathrm{rHe}_{\mathrm{He}}}{\mathrm{f}_{\mathrm{He}}}
$$

$A_{12}$ is a ratio of area- 1 (erosion) to area-2 (deposition) and $\delta_{i}$ is the loss of sputtered particles between the source and the region of deposition. $\Gamma$ 's are the fluxes of 'various plasma constituents in area-1 and area-2. Y's are the sputtering yields which depend on energy $E$. At area-2, $\Gamma_{\mathrm{He}-2}, \mathrm{r}_{\mathrm{He}}$, and $\mathrm{f}_{\mathrm{He}}$ are respectively the $\mathrm{He}$ flux, $\mathrm{He}$ trapping efficiency and atomic fraction of trapped $\mathrm{He}$ at saturation. (3) For a host material different from the chamber and deposited from a filament, a batch process would probably be necessary in which the surface would be isolated during the deposition process. It is conceivable that this could be done mechanically and the outage for deposition would be rotated among several pumping stations to provide sufficient pumping. (4) If a "heat and dump" process were used to release trapped He from the host material, then some type. of isolation from the plasma would be needed. Again it is conceivable that either the surface itself could be moved mechanically or an interceding gate could be moved and rotation of active trapping stations would provide continuous pumping. 


\title{
Characterization of Liquid Metal Surfaces
}

\author{
R. Bastasz*, R. Causey, and K. Wilson \\ Sandia National Laboratories \\ Livermore, California \\ *(tel: 510 294-2013 email: bastasz@sandia.gov ) \\ topics
}

- Use of liquid metals as plasma-facing surfaces

- PMI effects at liquid metal surfaces

- Research needs

\section{Potential advantages of liquid systems for plasma facing components:}

1. Unlimited erosion lifetime.

2. No neutron damage concerns.

3. High power density capability.

4. Active pumping of DT and/or He.

5. High temperature operation at high efficiency.

6. Low pressure operation. 
Issue: How will a liquid divertor surface behave when bombarded by energetic particles?

Need: Fundamental data on ion-liquid interactions needed to properly model conditions that will be found in a fusion reactor.

Tasks: - Measure $H$ and D sputtering of liquid metals

- Measure liquid metal self-sputtering

- Examine surface composition and impurity effects

- Measure T uptake and release

\section{Lithium Self-Sputtering Yields:}

\begin{tabular}{|r|c|c|c|c|r|r|}
\hline \multicolumn{6}{|c|}{ TRIM Calculation Results } & ( for solid Li ) \\
\hline $\begin{array}{r}\text { Energy } \\
(\mathrm{eV})\end{array}$ & $00^{\circ}$ & $15^{\circ}$ & $30^{\circ}$ & $45^{\circ}$ & $60^{\circ}$ & $75^{\circ}$ \\
\hline 10 & $\approx 0$ & $\approx 0$ & $\approx 0$ & 0.01 & $\approx 0$ & 0 \\
\hline 20 & 0.01 & 0.02 & 0.04 & 0.14 & 0.16 & $\approx 0$ \\
\hline 50 & 0.07 & 0.10 & 0.18 & 0.44 & 0.72 & 0.10 \\
\hline 100 & 0.14 & 0.17 & 0.29 & 0.55 & 1.1 & 0.59 \\
\hline 200 & 0.20 & 0.21 & 0.36 & 0.65 & 1.4 & 1.6 \\
\hline 500 & 0.21 & 0.24 & 0.39 & 0.68 & 1.4 & 2.4 \\
\hline
\end{tabular}

preliminary calculations (1997 August) 


\section{Liquid metal surfaces appear to be stratified.}

density profile

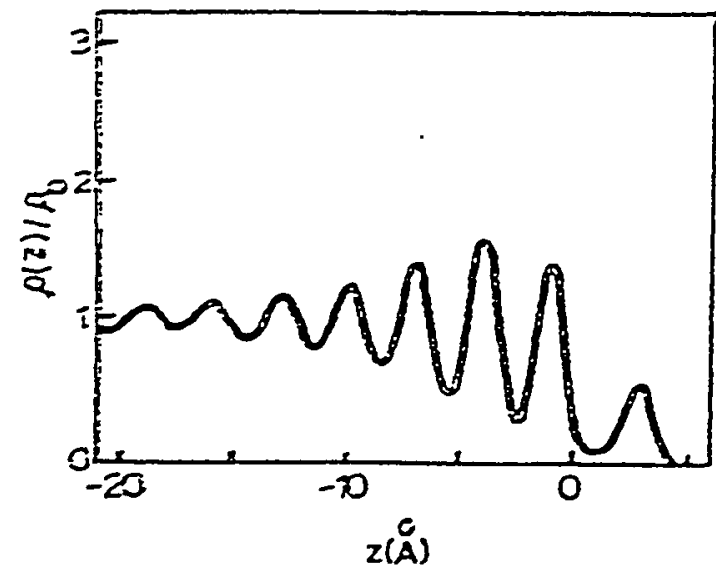

side view of liquid surface

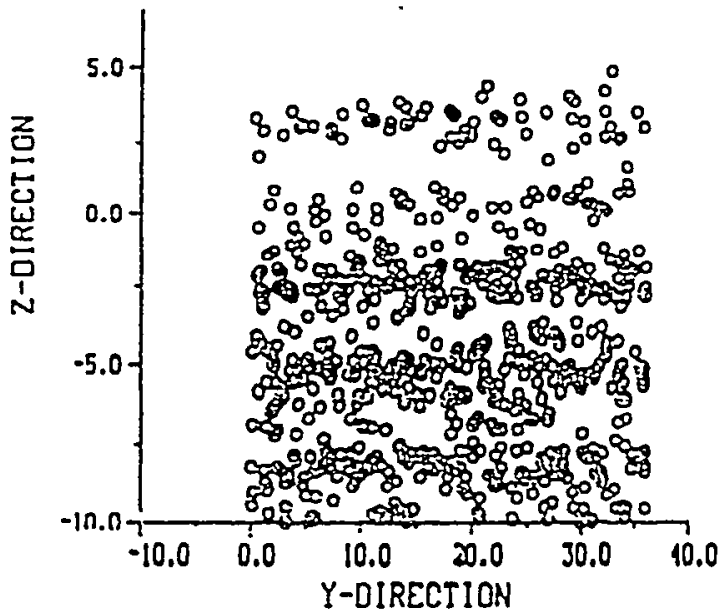

References: P. D'Evelyn and S. A. Rice, J. Chem. Phys. 78 (1983) 5081.

W. L. Morgan, J. Appl. Phys. 65 (1989) 1265.

\section{Self-sputtering simulations using a stratified liquid-metal model show an enhanced low-energy sputter yield.}

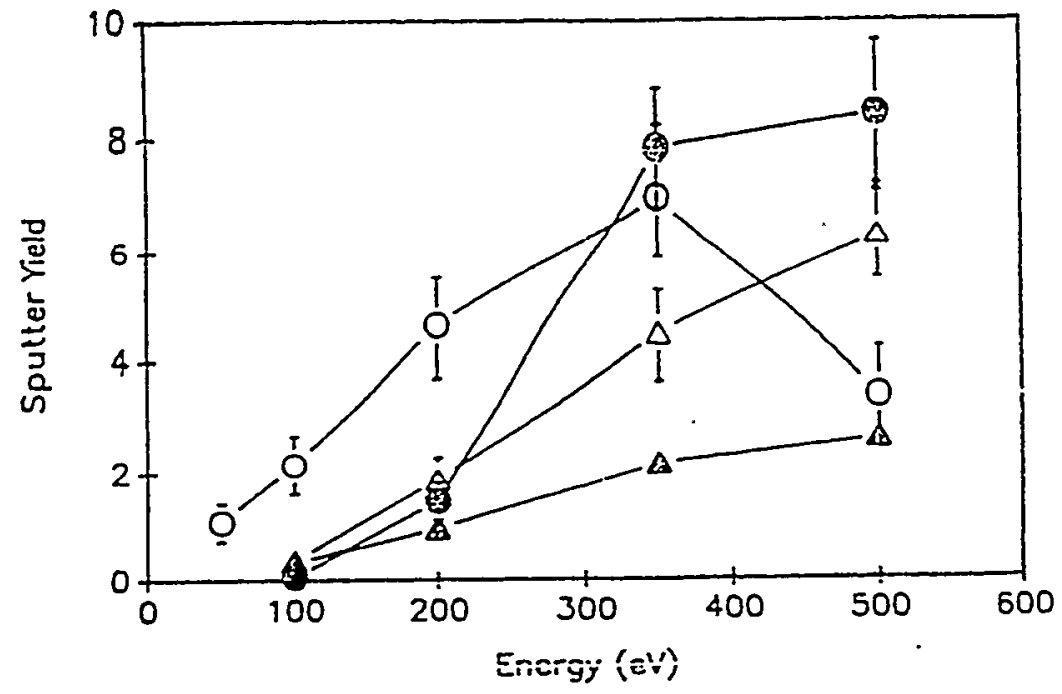

stratified liquid

$\triangle$ uniform liquid

solid

A TRIM

900 atom liquid metal model

Reference: W: L. Morgan, J. Appl. Phys. $\underline{65}$ (1989) 1265. 


\section{The composition of a liquid surface can much different than the composition of the bulk liquid.}

- The component with the lowest surface tension tends to segregate to the surface.

- For binary liquids, the Gibbsian segregation rule is

$$
\gamma_{A}+\frac{R T}{\sigma_{A}} \ln \left(\frac{1-x_{s}}{1-x_{b}}\right)=\gamma_{B}+\frac{R T}{\sigma_{B}} \ln \left(\frac{x_{s}}{x_{b}}\right)
$$

- Example: the surface of the Ga-In eutectic alloy.

$$
\begin{array}{ll}
\text { surface composition: } & \geq 94 \% \text { In } \\
\text { bulk composition: } & 16.5 \% \text { In }
\end{array}
$$

Reference: M. J. Regan, et al., Phys. Rev. B $\underline{55}$ (1997) 15874.

\section{Surface tension of the elements as liquids}

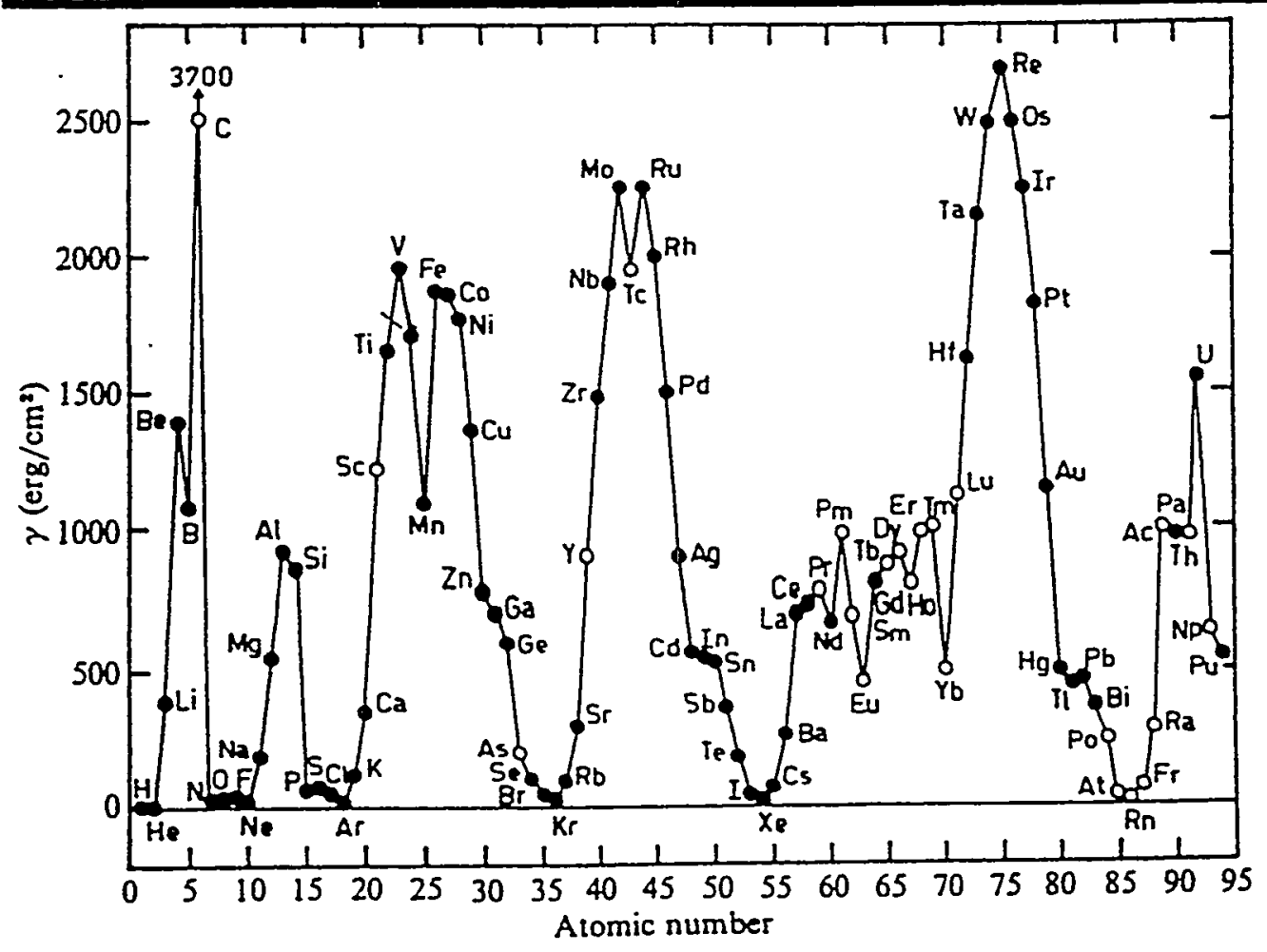

Reference: A. Zangwill, Physics at Surfaces 


\section{Low-Energy lon Beam Laboratory: The primary research tools are ARIES \& SIMS.}

- ARIES: Angle-Resolved lon Energy Spectrometer

$\rightarrow$ extremely surface-sensitive method for studying ion-surface interactions.

$\rightarrow$ measures energy and intensity of reflected and ejected ions from surfaces.

$\rightarrow$ provides fundamental data on sputtering, reflection, composition, and structure.

- SIMS: Secondary-Ion Mass Spectrometer

$\rightarrow$ sensitive detector of atoms and molecules on surfaces.

$\rightarrow$ mass analyzes ions sputtered from the surface (including $H, D, T$ ) and can measure depth profiles.

$\rightarrow$ useful for characterizing the distribution of impurities in the near-surface region.

\section{The ARIES instrument measures the energy and intensity of low-energy ions scattered or recoiled in the forward direction.}

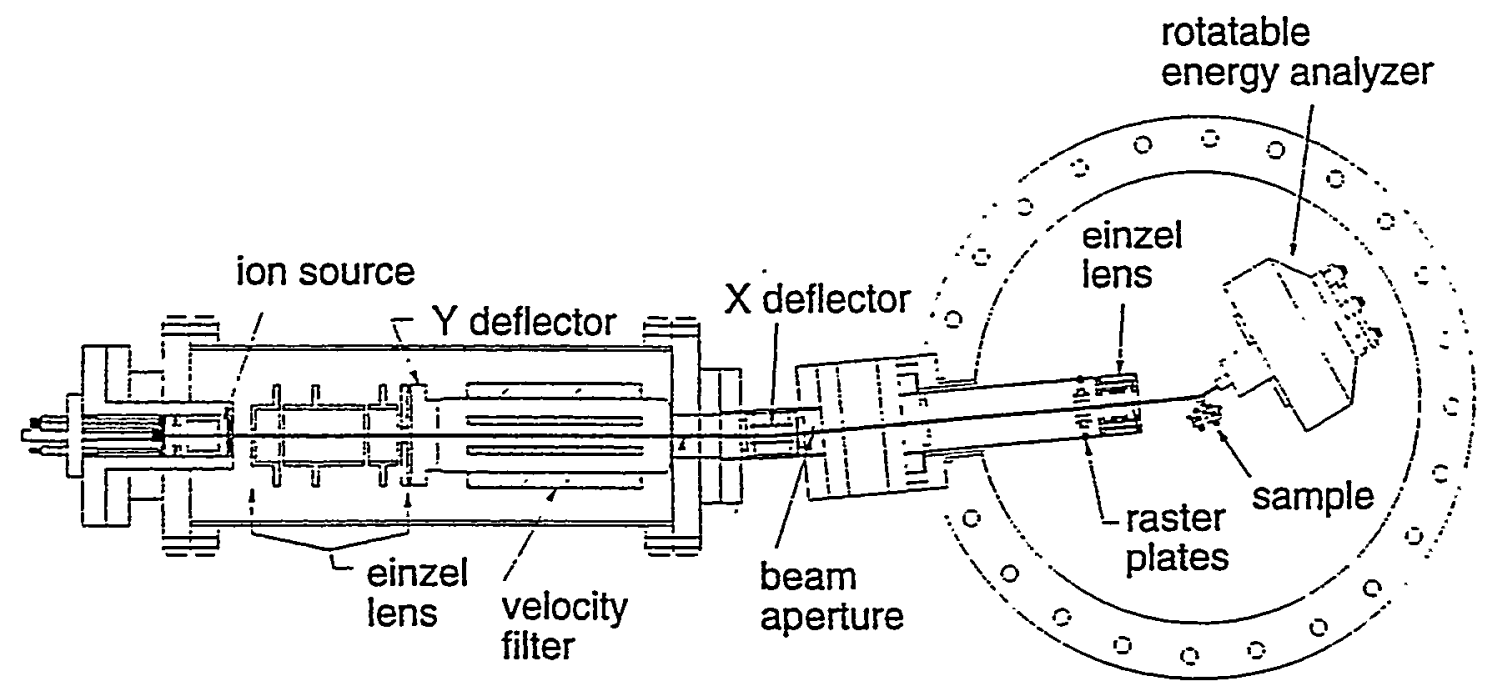

UHV analysis chamber 
The SIMS instrument contains a low-energy ion source, a quadrupole analyzer, and a heated sample stage (4 exchangable samples).

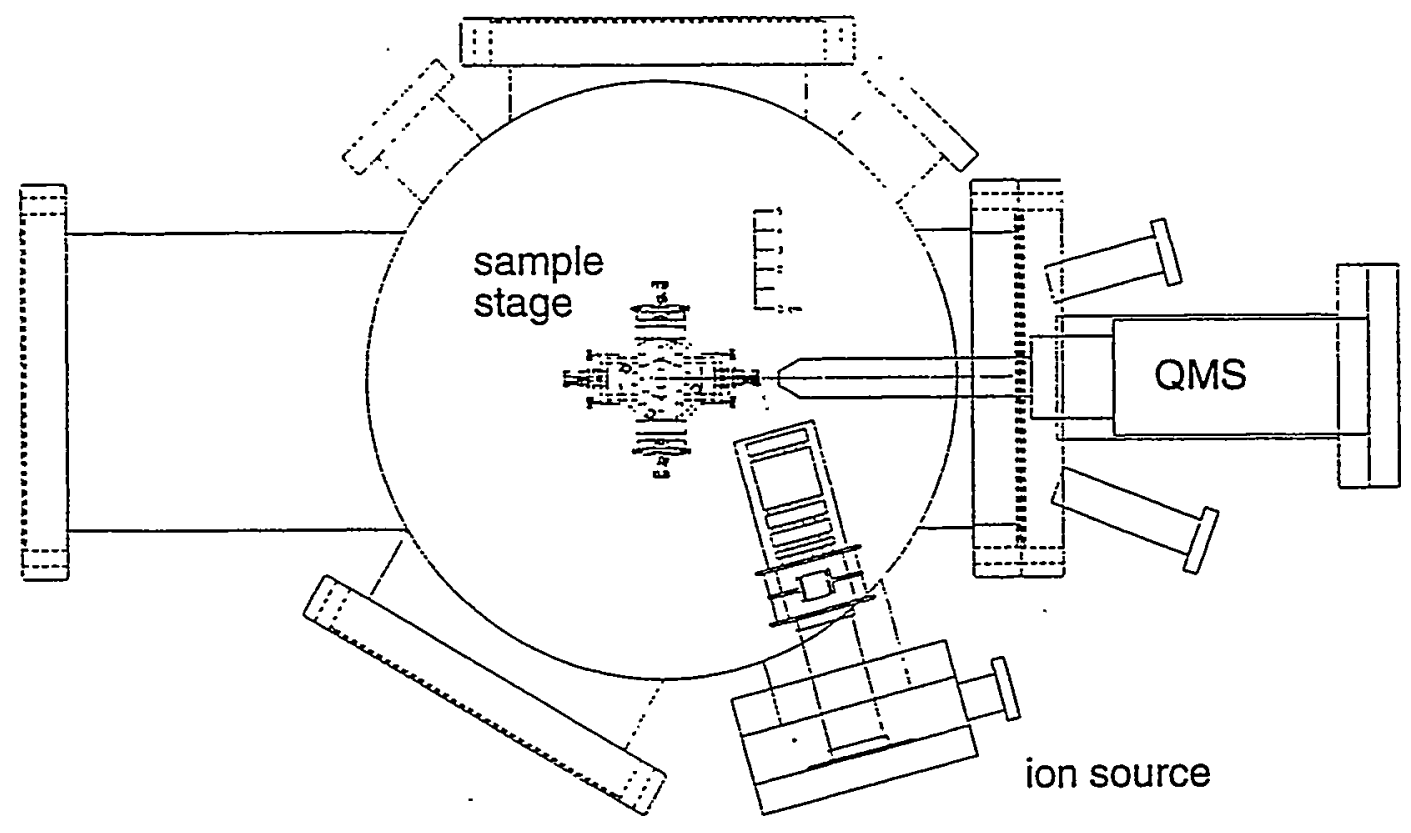

\section{Low-energy SIMS geometry is arranged to maximize depth resolution and sensitivity.}

sample stage 䢖

- Pl impact at $75^{\circ}$ reduces ion range and recoil mixing.

- QMS axis normal to sample surface for efficient SI collection.

QMS analyzer

for secondary ions

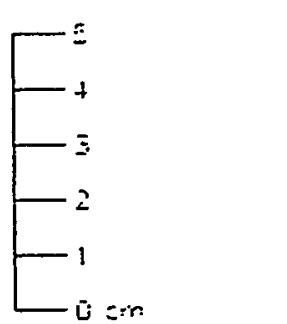

$10 \mathrm{eV}$ to $3 \mathrm{keV}$ primary ion source 
The Tritium Plasma Experiment (TPE) will play an important role in the ALPS experiments. Several of the experiments can only be completed with the use of Tritium

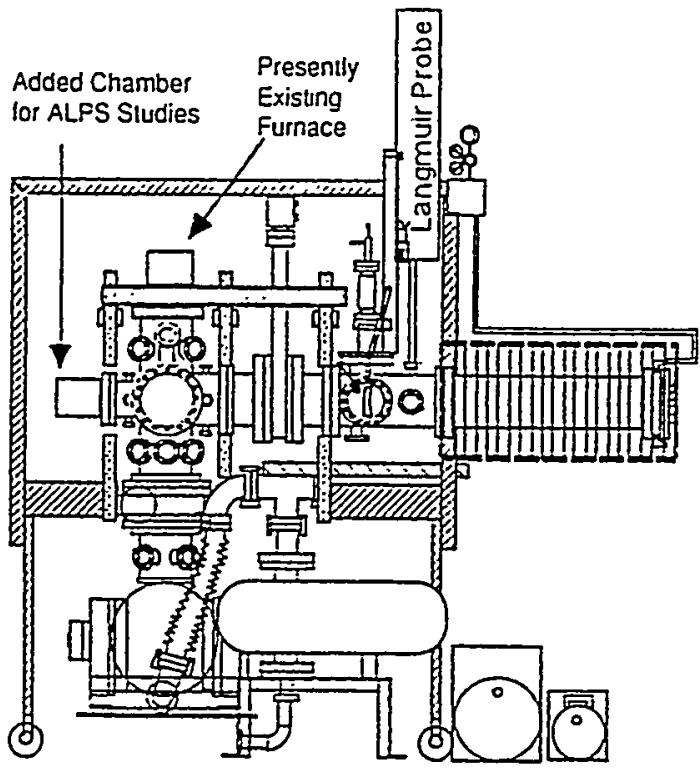

A high temperature furnace already exists
on the TPE experiment. It can be used in
studies of tritium solubility and diffusivity.

A new chamber can be bolted directly onto the end of TPE. This chamber can be used to house several different types of ALPS prototypes. The liquid metal can be directly measured for tritium uptake after plasma exposure. Heated catcher plates located in this special chamber can also be used to determine the codeposition rate of tritium with the sputtered liquid metal.

Sandia National Laboratories

\section{DiMES can be used to test ALPS concept.}

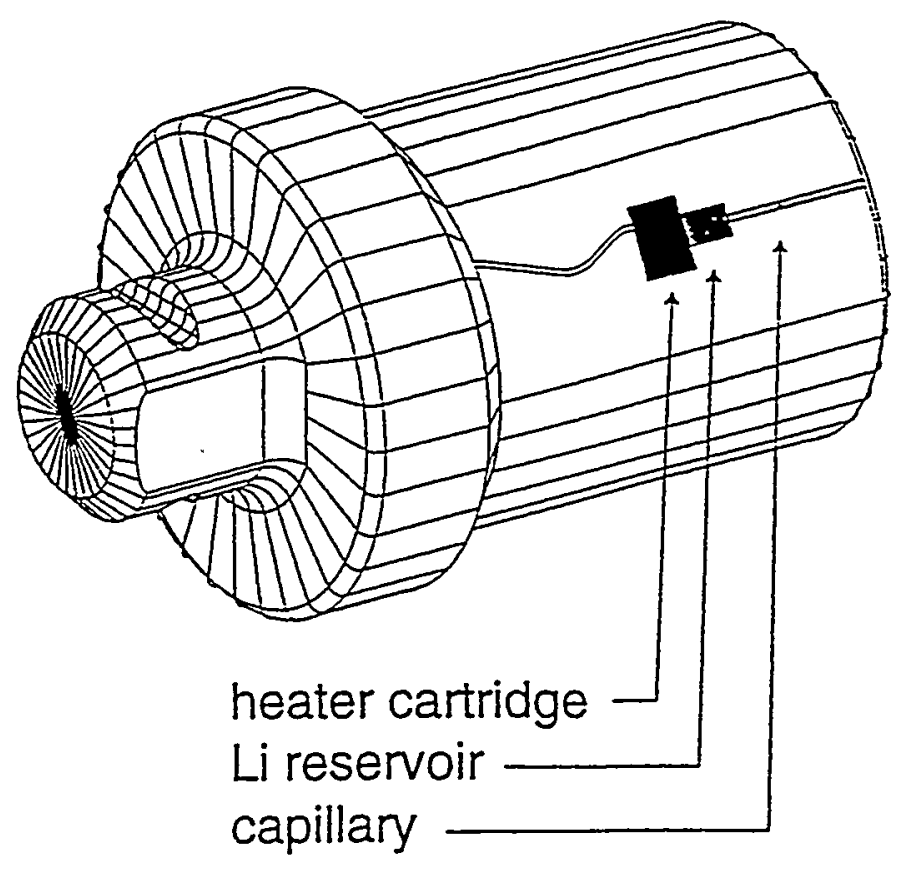

Concept of DiMES sample for ALPS test

Study:

- stability

- erosion

- transport 


\section{Summary:}

- Liquid metal surfaces can be:

- stratified

- compositionally altered

- transiently structured.

- Plasma-surface interactions at liquid metal surfaces may be fundamentally different than at solid surfaces.

- Accurate data are needed for:

- D, T, and He pumping, retention, and release

- D, T, and He sputtering yields

- Liquid metal self-sputtering yields

- Surface composition of liquid alloys and mixtures.

- A realistic model of plasma-surface interactions needs to be developed for liquids. 
Section XI: Long Range PFC Development and Collaborations 
[page intentionally left blank] 
(lof 3) +PMI

LONG RANGE PFC/PFS (gstems) tcollaboretions goals (1). $\times 10$ Inereage of heatfluy, pant, flex

(2). Self healing continvoesly venewa ble wall, off norm dl event handling.

(3). Optimization Tools for boundary/cone
interaction and control

Apponachs

(3) Develep integnated physics models

that couple PFS - EDGE - CORE
- (Nueleer) -

and

useter optimize system as auble

1),(2) Nouel PFS ideas.

in-site coatings, Li, B, ete coatings gas boundany

pehble beds - flews e.9. Osaku Vaiv. belts RF bounday

XI-3

blasing, currents 
2 of 3

LONG RANGE NEEDS

SCIEnCE Folus

$\rightarrow$ Intequated plaoma matenials models

$\rightarrow$ optimized boundany PMI/PFC syst CORE - EDGE - MATERTALS

$\rightarrow$ IN.SITU MATERIACS DIAGNCSTTCS

$\rightarrow$ Handling of off. normal events

FACIUTES

Divertor / Bountary simveators - Flux tube - Piseres-Upgrade

- Flux sheet long connection length island, chain, magneties effe. Dedicated Tokamak an Stellmator? XI-4 High Proven donsity, $50 \mathrm{~m}\left(\mathrm{~W} / \mathrm{m}^{2}\right.$ fn ACPS. 
3 of 3

Advanced/Novel / PFS/PMII concepts Convection squstems $\quad V_{\text {conv. }} \gg v_{\text {cond }}$.

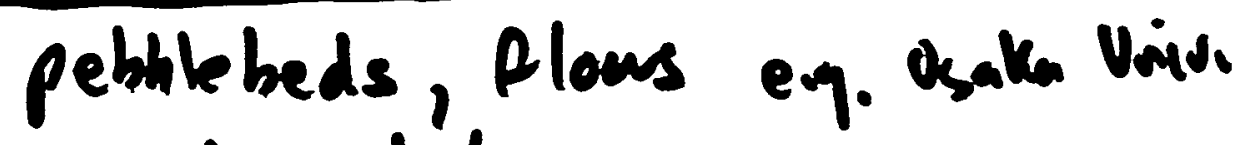
liquid metals

belts - m. B. Diventor Yittriker etale

boundany control systems

in-situ coatings li, $B$ ete. gas mantle, RI like RF boundony biasing, corrents fnomedge, walls.

interaction with new magnetic cafigs ST, ppstelloneton, FRC, ... ete.

$X I-5$ 
Session XII: Supplement Session

\section{XII-1}


[page intentionally left blank]

XII-2 


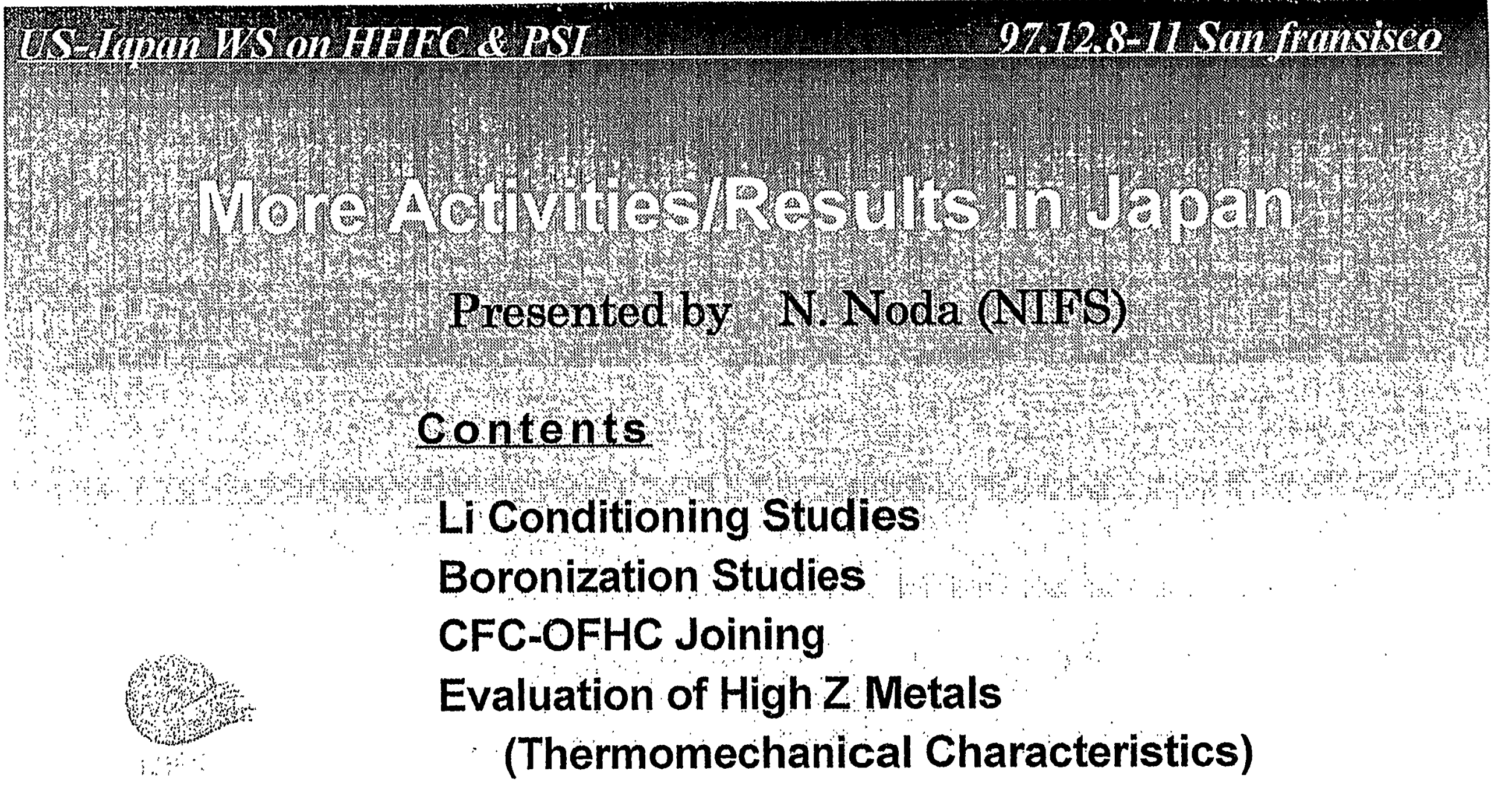

출 


\section{Simulation Experiments on Screening of Lithium by boundary plasma}

$\Rightarrow$ LF measurement of Li atom density profile -

$\mathrm{H}$. Sugai and $\mathrm{H}$. Toyoda

Nagoya University, Nagoya, Japan

Boundary Plasma

\section{e}

$\mathrm{Li} / 1, \frac{1}{2}, 0,1 \mathrm{Li}^{+}$

Lithum layer

Wall
Ionization threshold of $\mathrm{Li}$

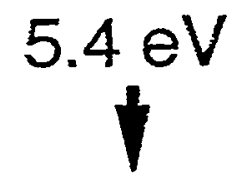

Easily screened by plasma $\downarrow$

Redeposition of $\mathrm{Li}$

\section{Laboratory Simulation Experiment}

- Lithium effusion from a small orifice

- RF helium plasma without magnetic field

$$
\mathrm{n}_{\mathrm{e}}=10^{11} \mathrm{~cm}^{-3}, \mathrm{~T}_{\mathrm{e}}=3 \mathrm{eV}, 35 \mathrm{mTorr} \mathrm{He}
$$

detection of lithium atom 
Schematic of experimental set up

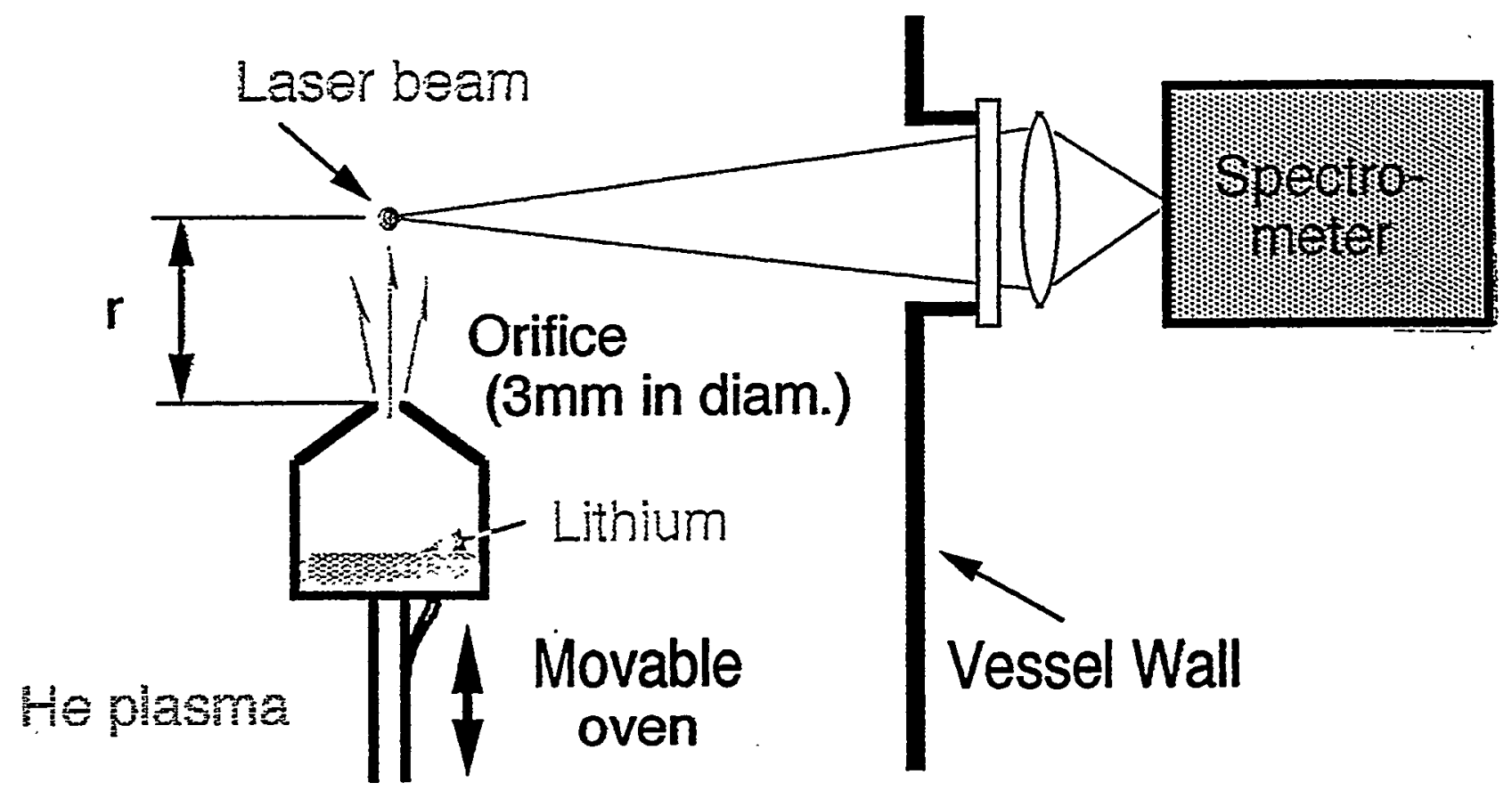

\section{Li atom density profile}

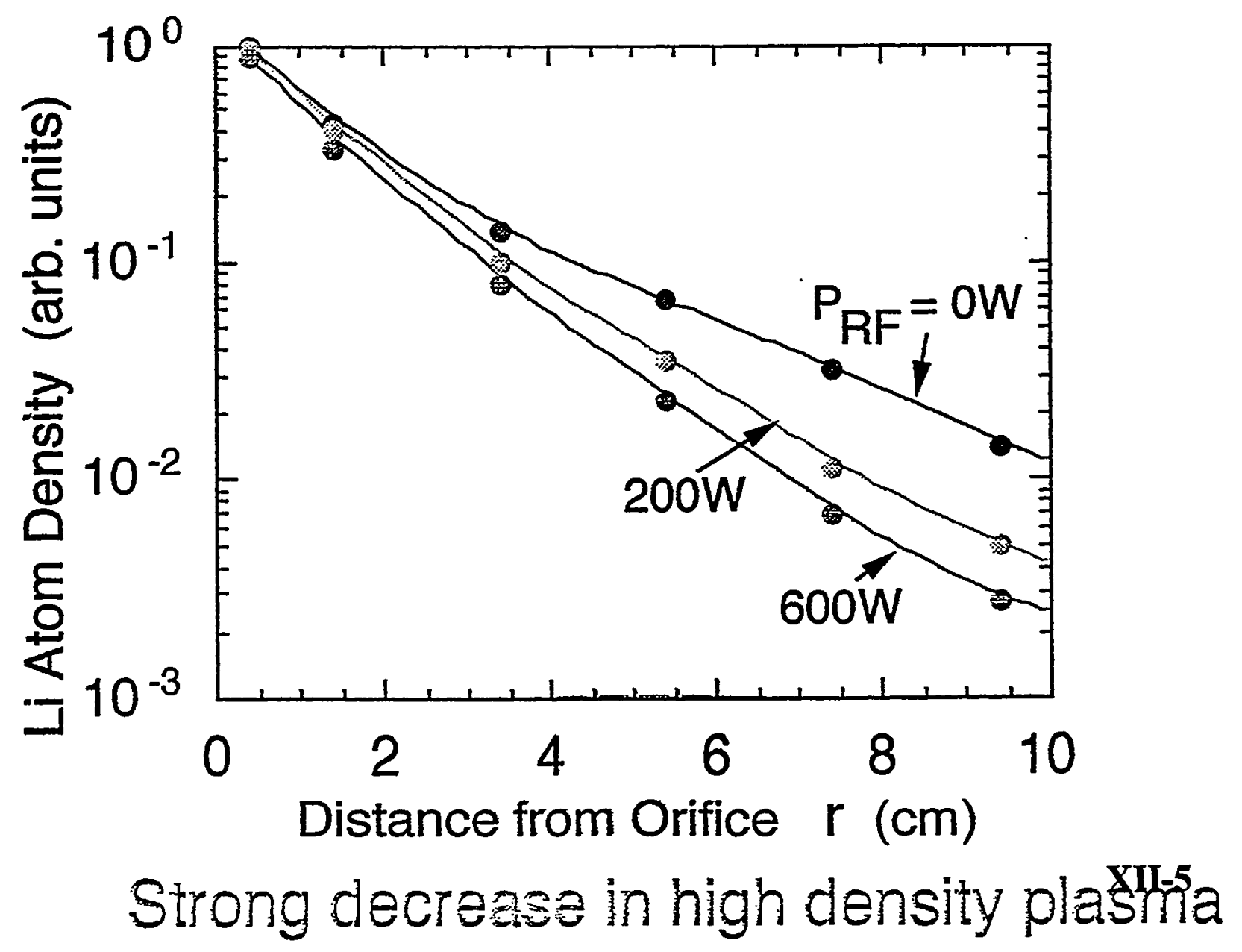



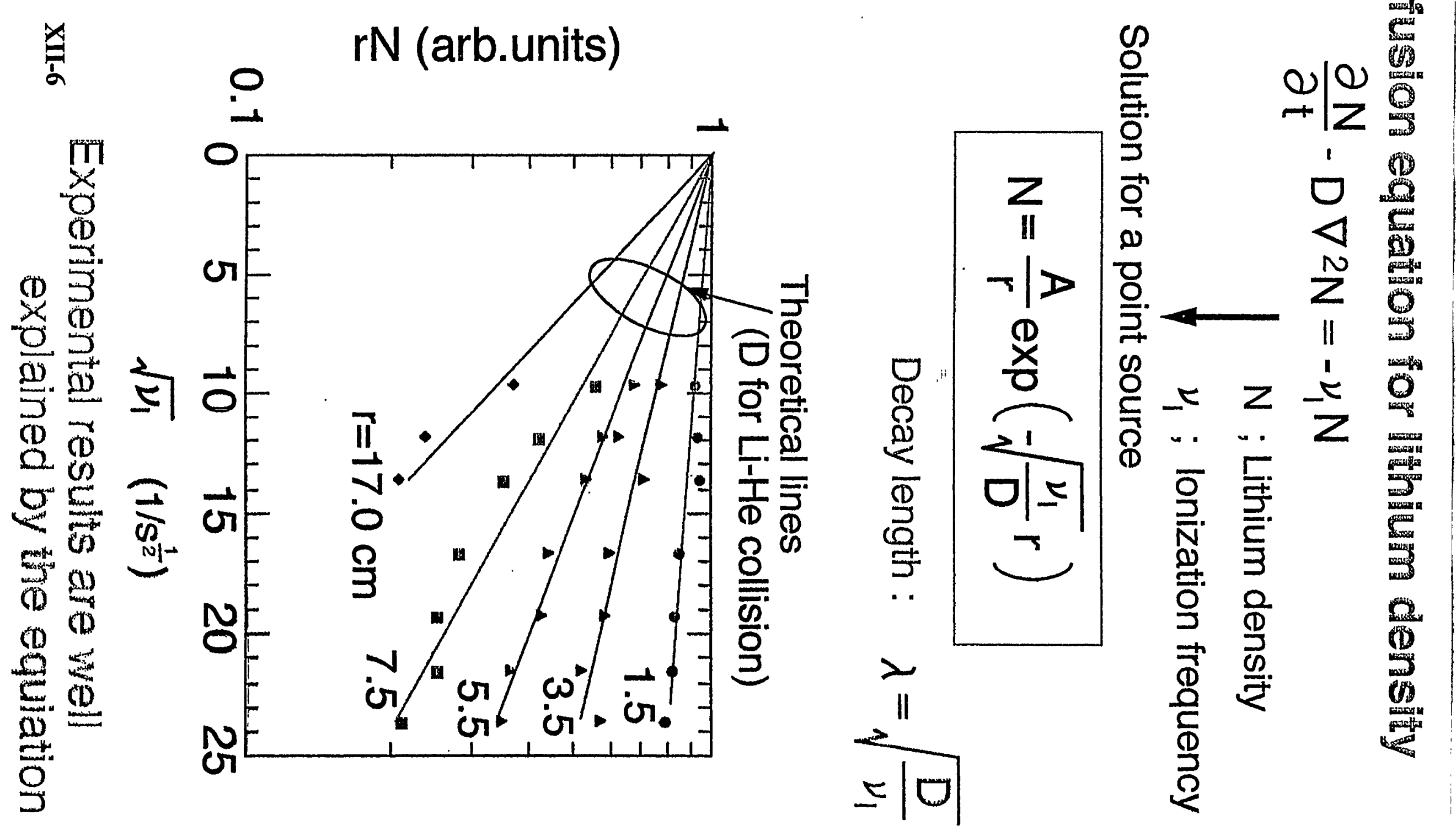


\title{
Hydrogen Absorption/Desorption by Oxygen Contaminated Boron film
}

\author{
H. Eiki (Hokkaido University) \\ K. Tsuzuki (National Inst. Fusion Science)
}

- Hydrogen absorption is reduced by $\mathrm{O}$ contamination

- Temperature for Max. desorption is not changed 


\section{Impact of Oxygen on H-Behavior in B-film}
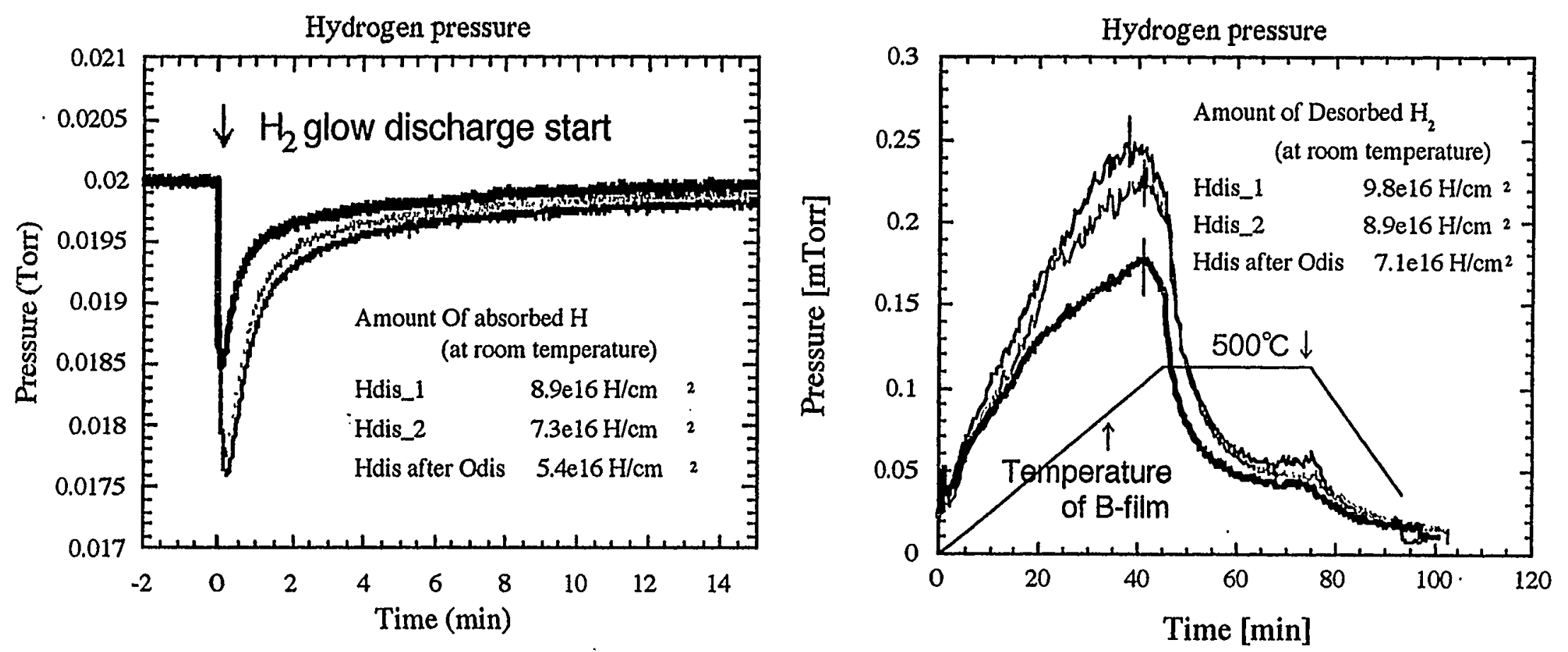

[Pure B film] [Pure B film]

[B film fully implanted by oxygen $(\mathrm{B} / \mathrm{O}=3 / 2)$ ]

$\rightarrow \mathrm{H}$ absorption is reduced

$\rightarrow$ peak temperature becomes higher ??

More precise investigation needed for O-contaminated B-films !!! 


\section{Joining of C/C Composite with \\ Oxygen-Free Copper by Titanium Foil}

Tatsuo OKU*, Yoshio IMAMURA*, Akira KURUMADA*, Kiyohiro KAWAMATA*, Nobuaki NODA**, Yusuke KUBOTA** and Osamu MOTOJIMA**

* Faculty of Engineering, Ibaraki University

$(4-12 \cdot 1$, Nakanarusawa, Hitachi, Ibaraki, 316, Japan)

** National Institute for Fusion Science

(322-6, Oroshi, Toki, Gifu, 509-52, Japan)

Purpose

- Development of a joining material for LHD divertor

幽

- Joining by titanium foil only 
Joining material: $\left(10 \times 10 \times 40 \mathrm{~mm}^{3}\right)$

- CX-2002U (Rayon carbon fiber felt C/C composite)

今. Oxygen-free copper

- Titanium foil (thickness : $50 \mu \mathrm{m}$, purity : 99.6\%)

Joining condition:

- Argon gas, no-pressure

- 10 minutes folding at $900 \sim 1000^{\circ} \mathrm{C}$

(heating rate $: 40^{\circ} \mathrm{C} / \mathrm{min}$, cooling rate $: 4^{\circ} \mathrm{C} / \mathrm{min}$.)

Tiestis:

3. Apeint bendung strength $\left(3 \times 4 \times 40 \mathrm{~mm}^{3}, 10 \mathrm{~mm}\right.$ and $30 \mathrm{~mm}$ spans)

: Continumisindentation test (Berkovich diamond indenter,

load: $49 \mathrm{~m}$, loading speed : $2.2 \mathrm{~m} \mathrm{~N} / \mathrm{s}$, holding time : $5 \mathrm{~s}$ )

Parameter $B$ : the slope at the maximum load point in load/depth versus depth curve for loading tests (proportional to hardness and strength)

Parameter D : the slope at the maximum load point in load/depth versus depth curve for unloading tests (proportional to Young's modulus)

- Observation of the microstructure by SEM 
Results:

- Success of joining above $900^{\circ} \mathrm{C}$ (eutectic alloy of $\mathrm{Ti}$ and $\mathrm{Cu}$, formation of $\mathrm{TiC}$ )

- Bending. strength was almost equal to that of $\mathrm{C} / \mathrm{C}$ composite. (fractured in $\mathrm{C} / \mathrm{C}$ composite)

- Joining area became widely with increase in joining temperature. (diffusion of $\mathrm{Ti}$ )

- Peaks of parameter $B$ and $D$ decreased with increase in joining temperature. (diffusion of $\mathrm{Ti}$ )

- Parameter B and D changed gradually at joining point. (good joining)

- Cracks were not observed in the joining area. (good joining) 

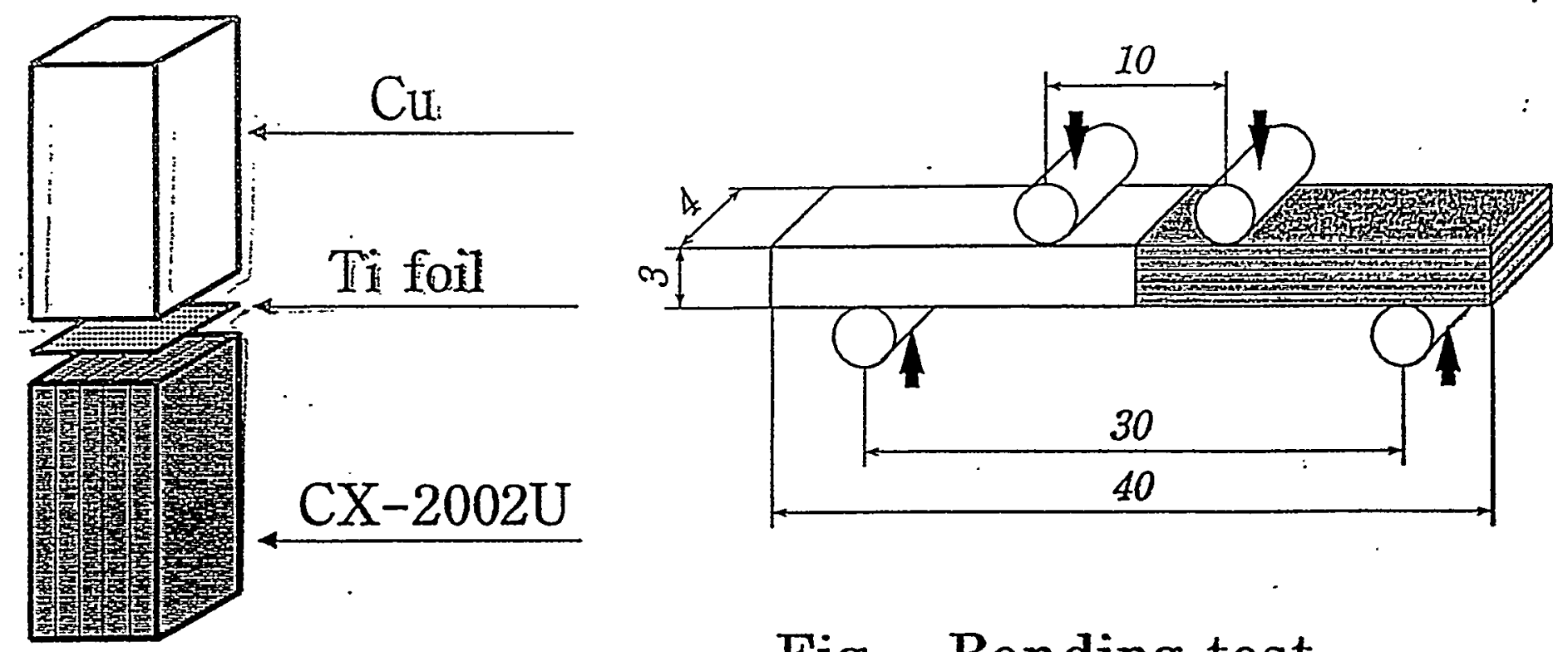

Fig. Bending test.

Fig. Test piece.

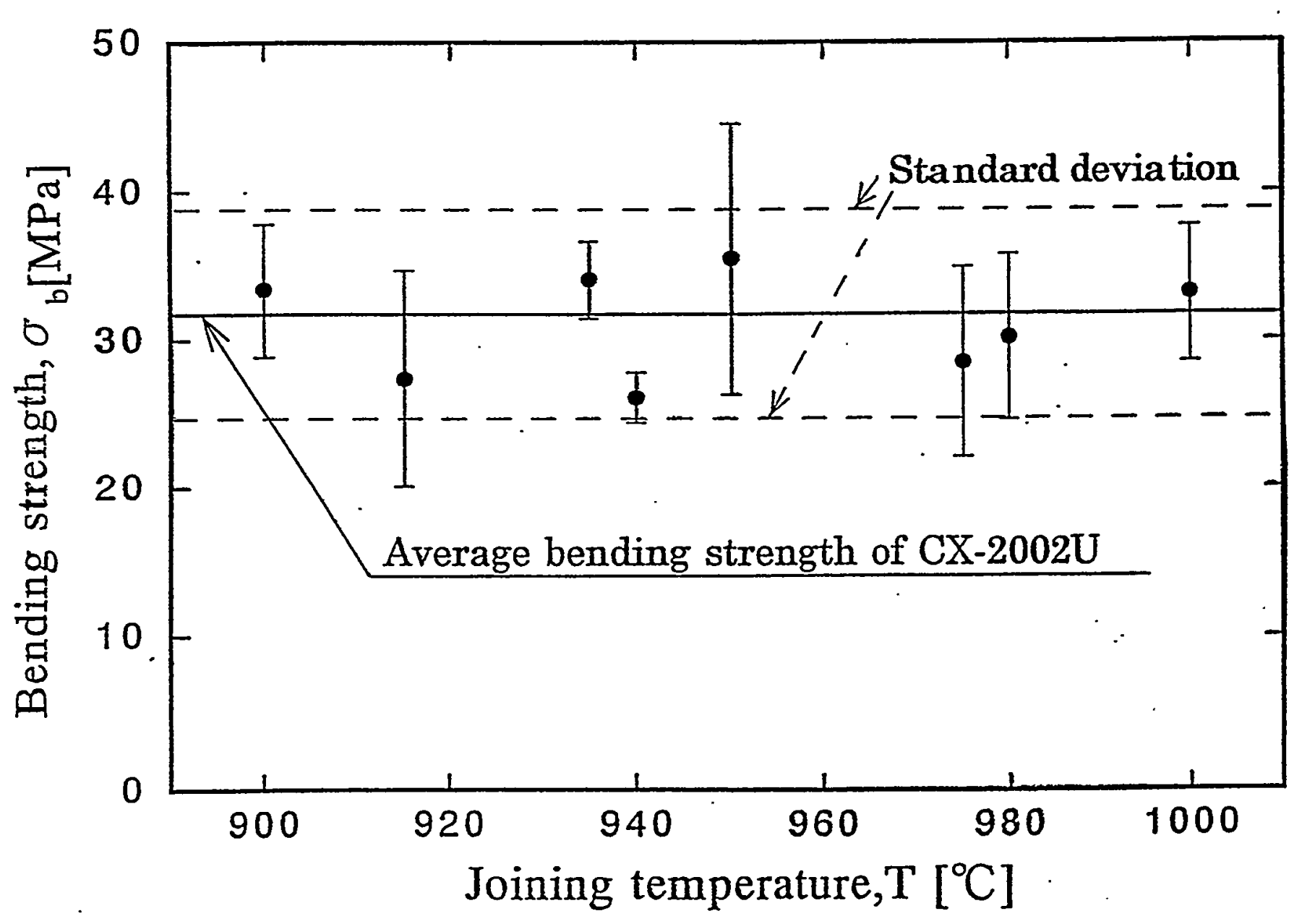

Fig. Relatiph 2 between bending strength, $\sigma_{b}$ and joining temperature, $T$. 


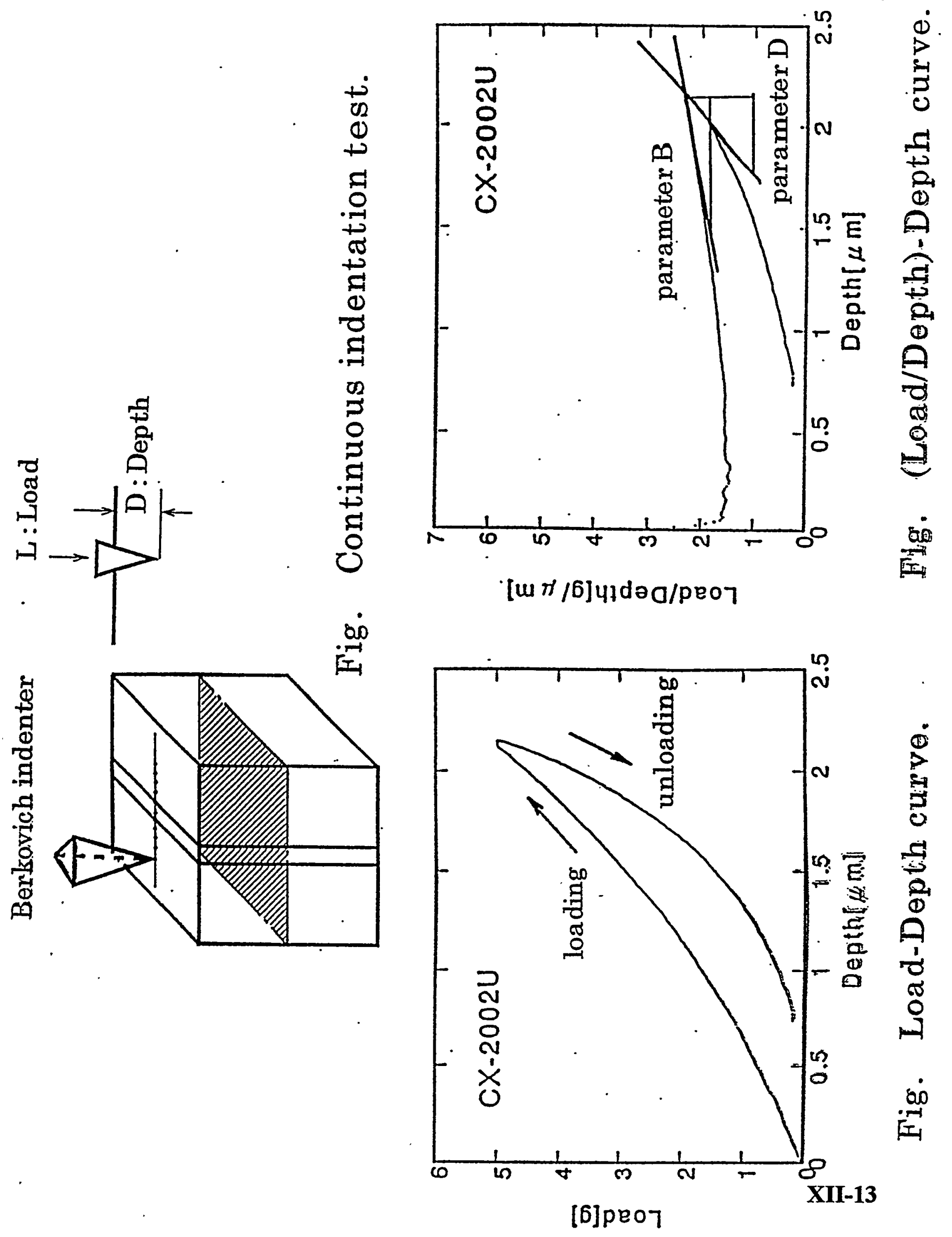




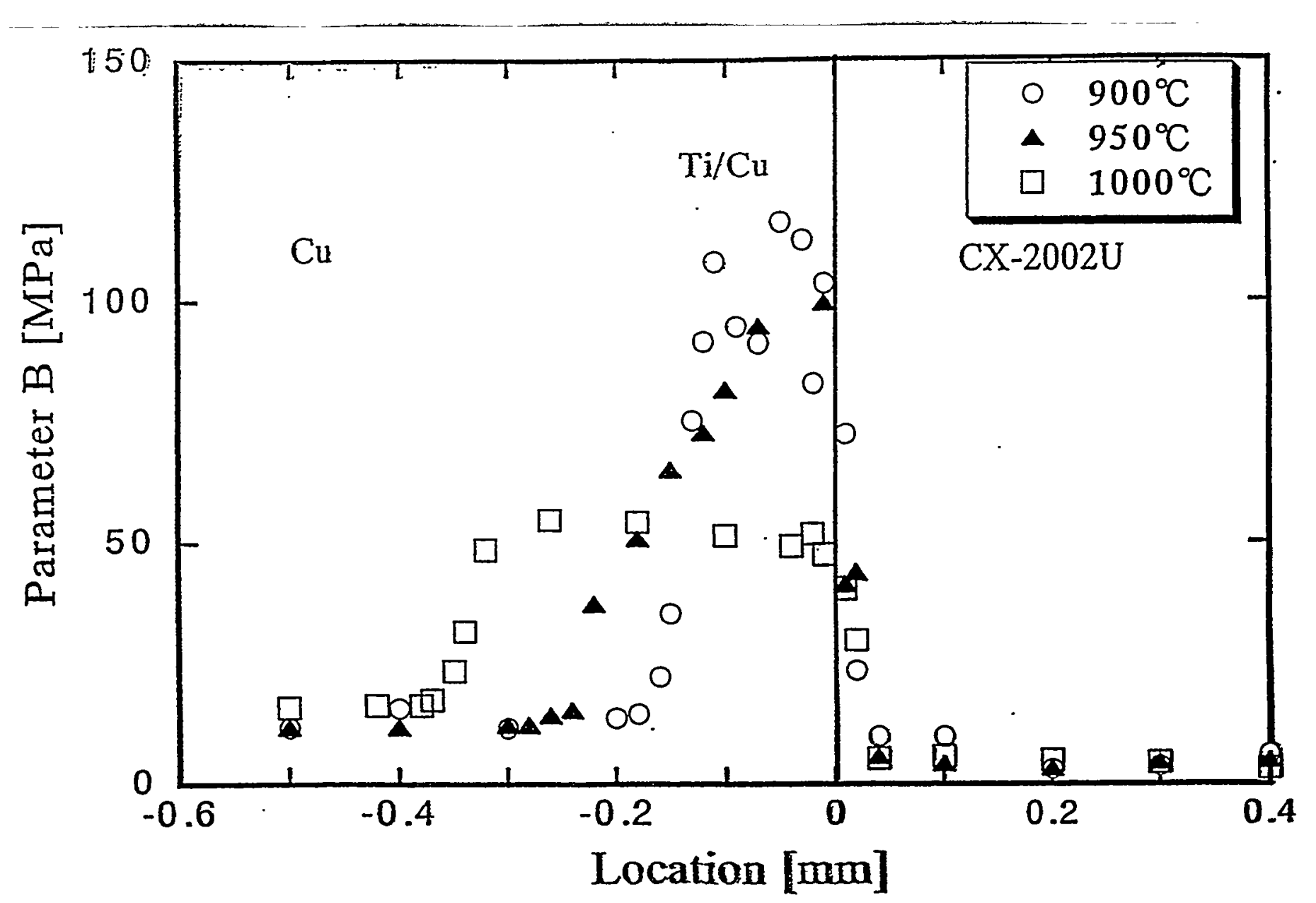

Fig. Distribution of parameter B.

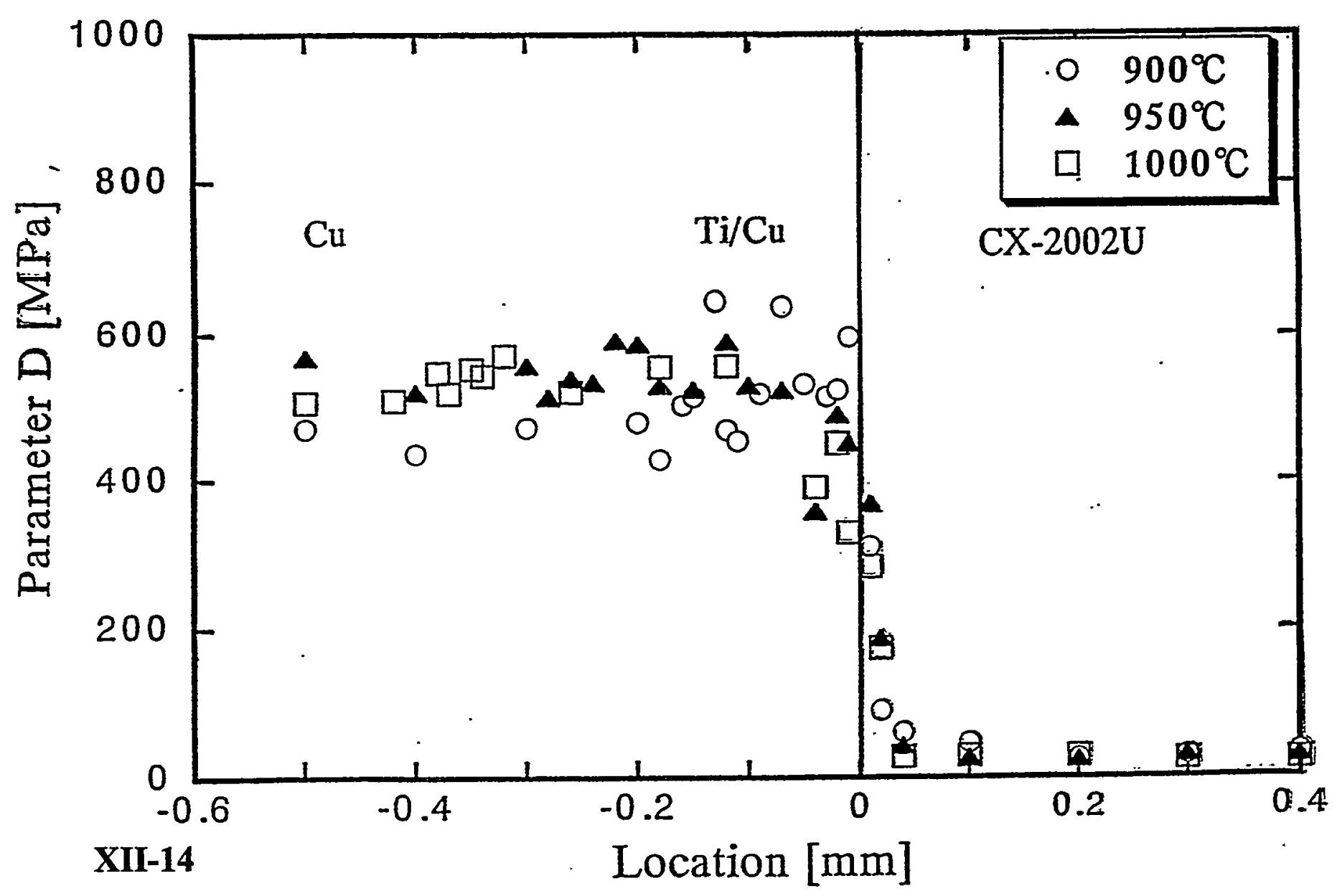

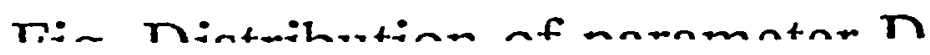



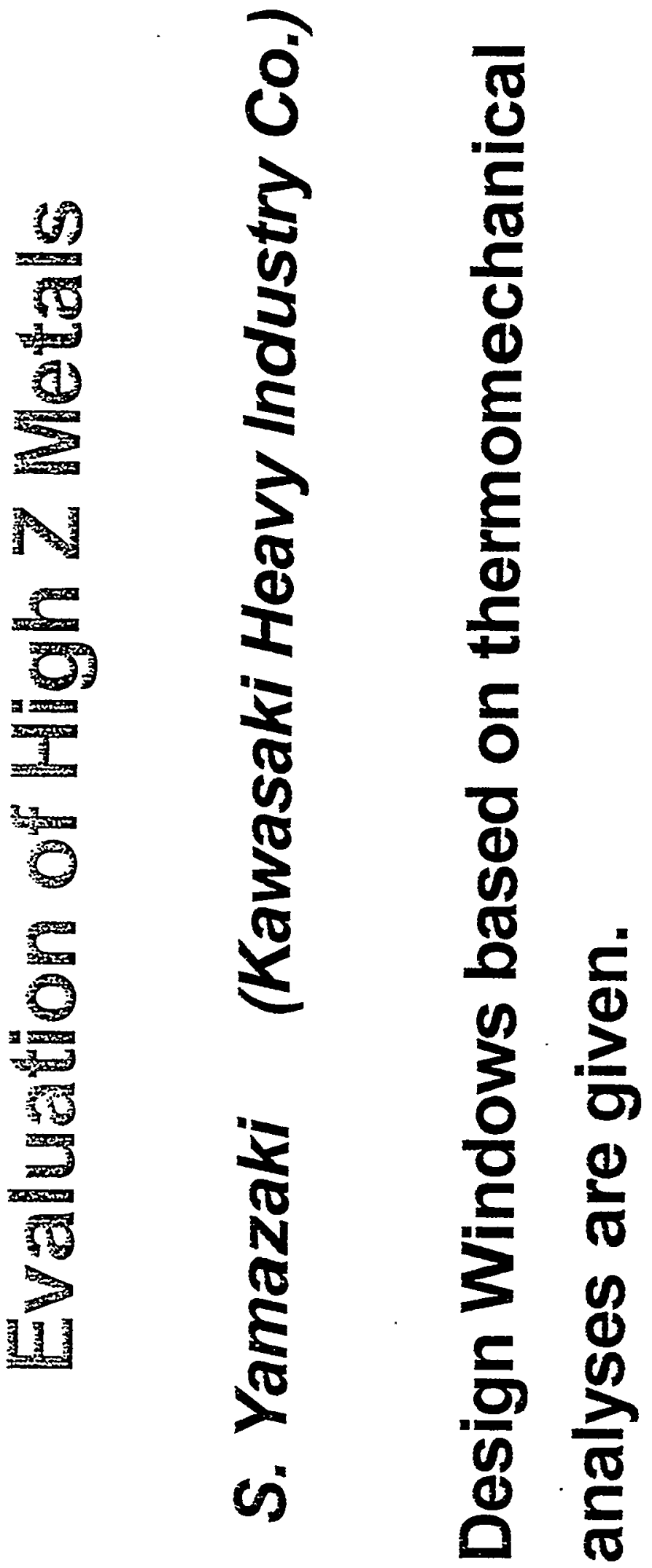


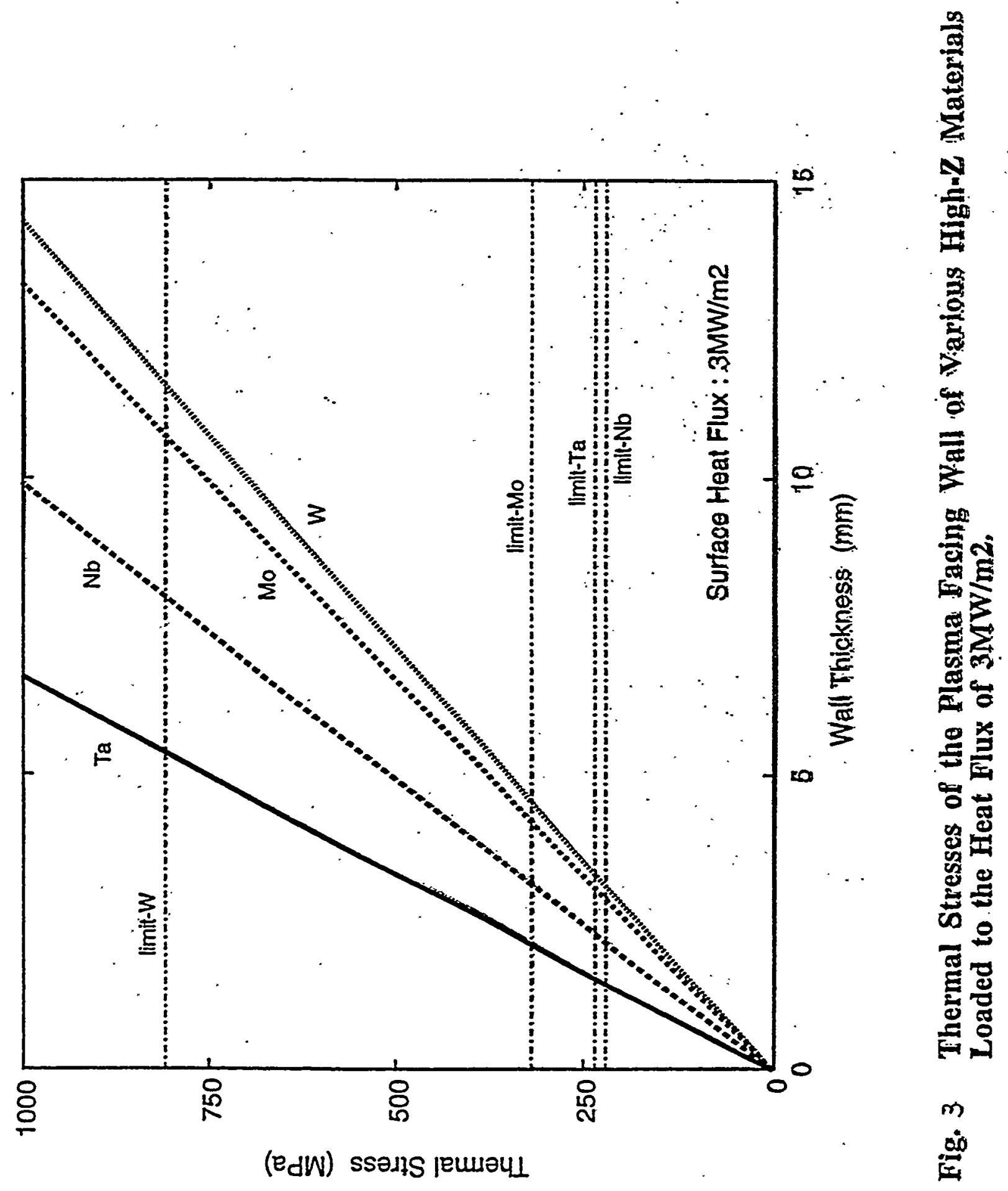

XII-17 


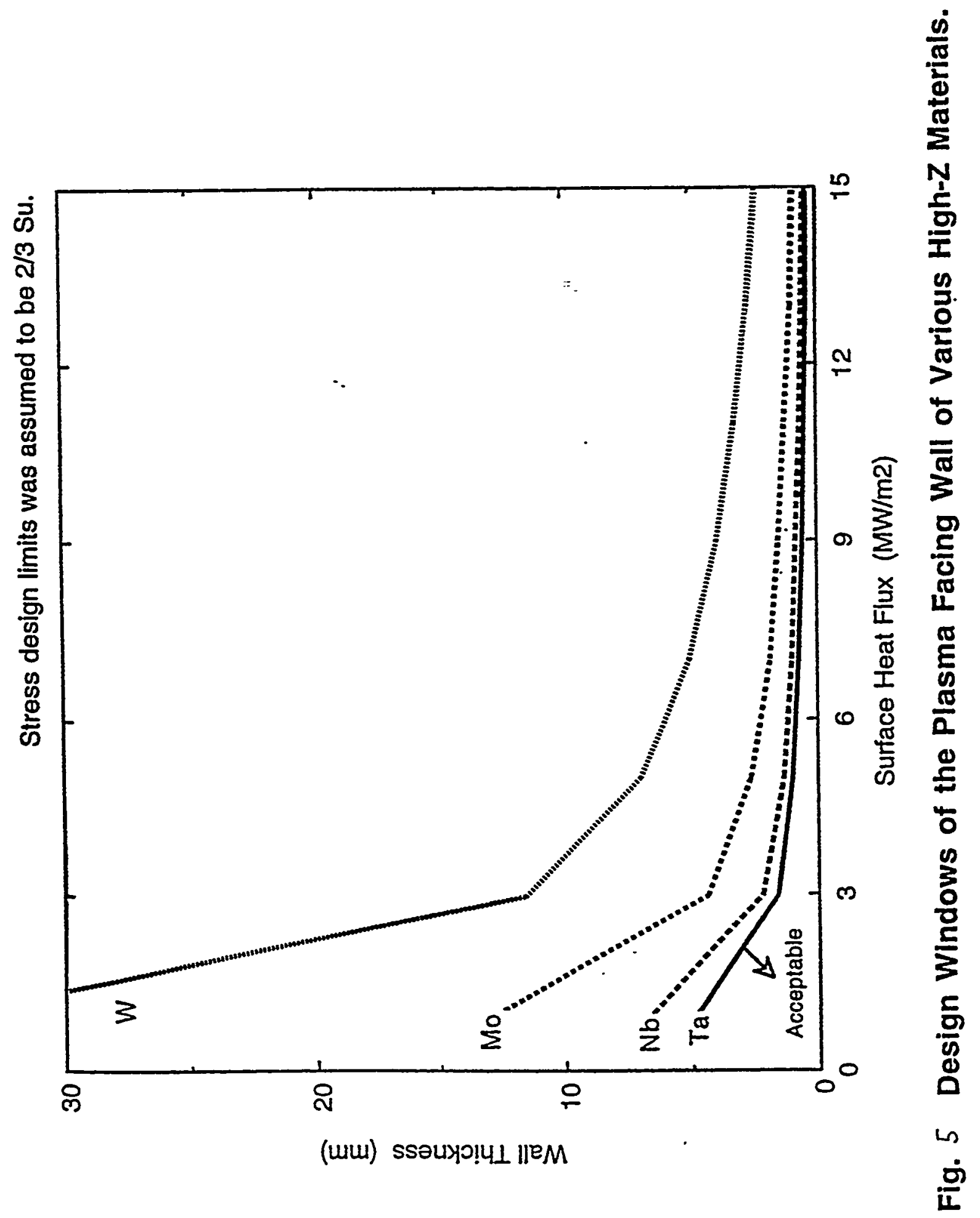

XII-18 
9

Threshold Energy (eV)

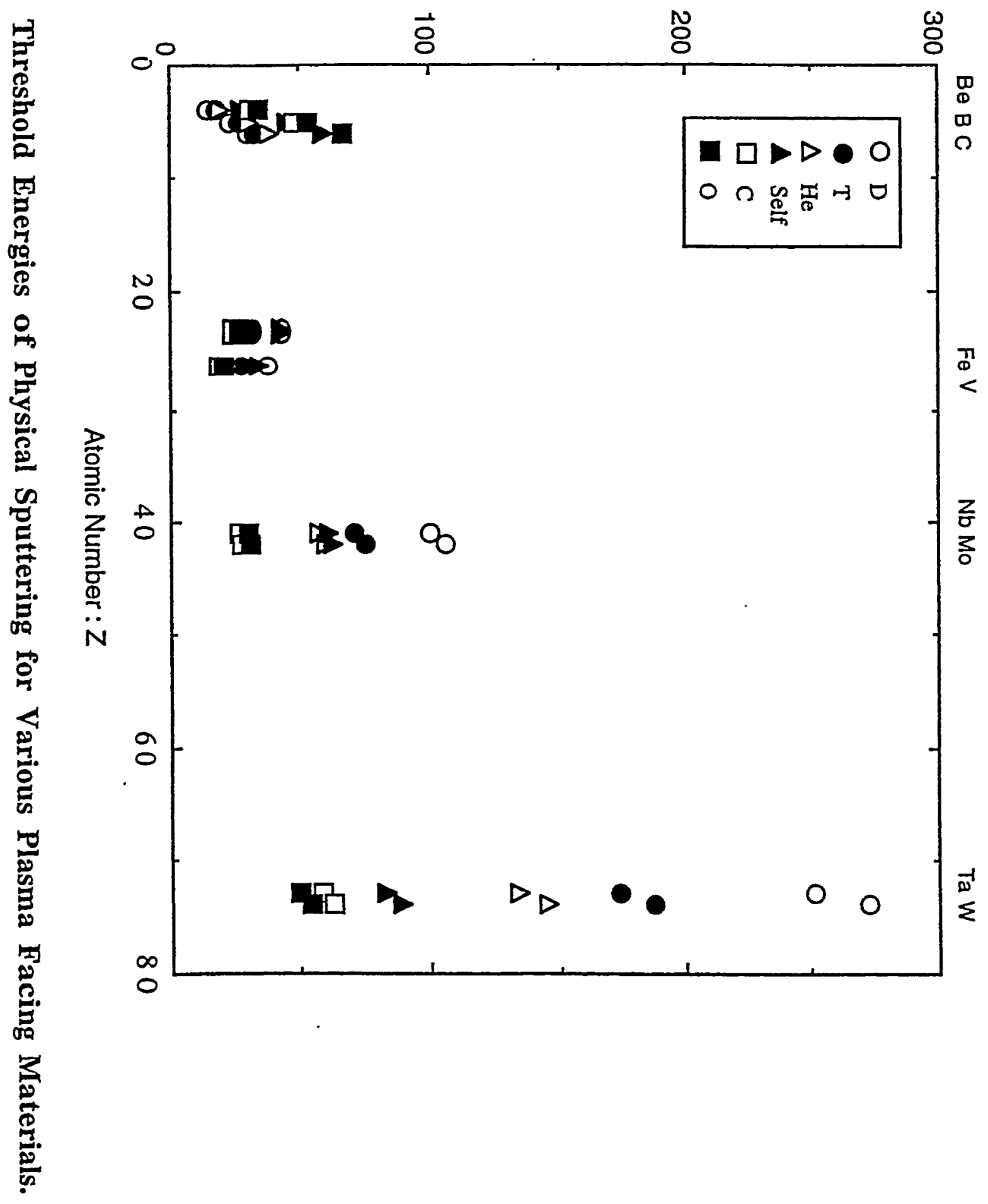




\section{CERTIFICATION OF SYSTEM SANITIZATION/CLEARING}

It is the responsibility of the person sending a computer system or component to Property Reapplication to ensure that all information is removed. In most cases, this consists of removing all information from the hard drive, if any, and any other disks being sent with the system.

If the computer (or disk) was ever used to process classified information, the discs must be degaussed. This is a process where a certified device uses an intense magnetic field to remove all information from the disk. Degaussing at SNL/NM can be performed in the Central Computing Facility in Building 880. Call Computer Operations at 844-5976 to schedule an appointment for degaussing..

If the computer (or disk) was used to process sensitive unclassified information (PRIVATE, UCAI, UCNI, OUO, etc.) the disk must be overwritten. A utility, such as Norton Utilities can be used to overwrite every location on the disk with a single character. Using delete, erase, and format commands does NOT meet the requirement, as these cormmands often. leave the file data on disk where it can be "undeleted". If the disk is inoperable and cannot be overwritten it must be degaussed.

Even for those computers that have processed only non-sensitive unclassified information, the information must be overwritten. Therefore, these systems are cleared in the same way as sensitive unclassified systems.

Property Reapplication will not pick-up the system or disk until the required sanitization has been performed and this form has been certified. Users at SNL/NM who require assistance in sanitizing a system can contact a CSR/CSU via CCHD @ 845-2243. A case number is required.

At SNL/CA, users should call Albert James at 294-2508.

For additional questions, contact the Computer Security Department.

Control Number (from Pick-Up Request Form):

Property Number.

Vendor:

Model No.:

Serial No.:

Check the highest sensitivity level for which this system (or disk) has been used. Then check the box indicating how the information has been removed.

\section{Classified}

$\square$ System contains no fixed drive.

All disk drives have been degaussed.

Sensitive Unclassified

$\square$ System contains no fixed drive.

$\square$ All disk drives have been degaussed.

$\square$ All disk drives have been wiped by Norton Utilities or equivalent.

Non-Sensitive Unclassified

$\square$ System contains no fixed drive.

$\square$ All disk drives have been degaussed.

$\square$ All disk drives have been wiped by Norton Utilities or equivalent.

1 certify that all information has been removed from this system (or disk) as described above. 


\title{
High heat flux testing of neutron irradiated divertor modules
}

\author{
R. Duwe, J. Linke, M. Rödig \\ Forschungszentrum Jülich, EURATOM Association, D-52425 Jülich, Germany \\ R. Nygren
}

Sandia National Laboratories Albuquerque, New Mexico 87185-1129, USA

US-Japan PMI / HHF Workshop

San Francisco, December 08 - 11, 1997 
Forschungszentrum Jülich

\section{Conclusions}

- Unirradiated CFC monoblock mock-ups have been tested at power densities up to $25 \mathrm{MWm}^{-2}$

- Neutron irradiation has been performed in the HFR Petten up to fluences of $0.3 \mathrm{dpa}$ at 320 and $770^{\circ} \mathrm{C}$.

- Irradiated ${ }^{C F C}$ modules show a significant increase in surface temperature during electron beam loading. This effect is less themial distintive for samples irradiated at $770^{\circ} \mathrm{C}$.

cycluig - Similar tests with neutron irradiated (see beryllium mock-ups are in progess. 


\title{
$\Rightarrow$ Draft
}

Testing of Actively Cooled High Heat Flux Mock-Ups

M. Rödig, R. Duwe, W. Kühnlein, J. Linke; M. Scheerer, I. Smid ${ }^{a}$. B. Wiechers

Forschungszentrum Jülich, EURATOM Association, D-52425 Jülich, Germany

a) Forschungszentrum Seibersdorf, A-2444 Seibersdorf, Austria

\begin{abstract}
Several un-irradiated CFC monoblock mock-ups have been loaded in thermal fatigue tests up to 1000 cycles at power densities $<25 \mathrm{MW} / \mathrm{m}^{2}$. No indication of failure was observed for these loading conditions. Two of the mock-ups were inspected by ultrasonic methods before thermal cycling. It could be proved that the voids found in the post-mortem metallography existed before and had no effect on the integrity of the mock-up.
\end{abstract}

For the first time, neutron-irradiated CFC monoblock mock-ups have been tested in the electron bear: facility JUDITH. These mock-ups had been irradiated before in the High Flux Reacto: at Petten up to $0.3 \mathrm{dpa}$ at 320 and $770^{\circ} \mathrm{C}$. All samples showed a significant increase of surface temperature, due to the irradiation induced decrease in thermal conductivity of the CFC materials.

\section{Keywords}

carbon and carbon materials (C01), divertor materials (D06), electron irradiation (E02), high heat flux materials (H03), joining (J01), neutron irradiation (N01)

\section{Introduction}

High heat flux components of ITER will be exposed to heat loads of up to $5 \mathrm{MW} / \mathrm{m}^{2}$ under normal and $20 \mathrm{MW} / \mathrm{m}^{2}$ under transient conditions. In order to remove these high heat loads, tiles of a plasma compatible armour material must be attached to a water-cooled heat sink. Candidates for plasma facing materials are beryllium, tungsten and carbon reinforced carbon materials (CFCs). Heat sink materials are copper alloys and, as a back-up, molybdenum alloys. Several joining processes have been developed for the attachment of the plasma facing materials to the heat sinks. in order to assess these bonds, high heat flux tests with actively cooled mock-ups have been carried out in the electron beam facility JUDITH. The results for beryllium - copper modules have been reported elsewhere [1, 2]; this paper deals with- -23 high heat flux performance of CFC monoblock modules. 
In former experiments, only un-irradiated mock-ups have been tested. But during the operation of ITER, the first wall and divertor components will be affected by $14 \mathrm{MeV}$ neutrons. In order to study the degradation of material properties under neutron irradiation, the irradiation experiment PARIDE has been performed in the High Flux Reactor (HFR) at Petten, The Netherlands. First mock-ups from this irradiation experiment have been tested under screening and thermal fatigue conditions in JUDITH.

\section{Experimental Details}

\subsection{Samples}

Fig. 1 shows the drawing of the CFC monoblocks. Three different CFC armour materials were used: Dunlop Concept 1, SEPcarb N31 and SEPcarb N112. Heat sink tubes were made from Glidcop Al25, CuCrZr and Mo5Re. All samples were produced by Plansee $A G$ by active metal casting $\left(A M C^{\odot}\right)$. After drilling, the CFC tiles were coated with liquid copper at $1250^{\circ} \mathrm{C}$; Ti additives were used as carbide formers. Then the tubes made from the two copper alloys were brazed in by means of pure titanium [3]. The Mo5Re tubes were joint in one step with the the AMC.

\subsection{Test Facility}

The electron beam facility JUDITH in general was described in [4]. It consists of an electron gun of $60 \mathrm{~kW}$ electric power and a number of powerful diagnostic devices. The heating of the mock-ups is performed by sweeping of a focussed electron beam $(\approx 1 \mathrm{~mm} \varnothing)$ over the sample surface at high frequencies up to $100 \mathrm{kHz}$. During the thermal heating tests, the heat sink tubes are water-cooled (water pressure: 40 bars, flow rate: $50 \mathrm{l} / \mathrm{min}$ ). A swirl is mounted inside the tube to avoid burn-out. The following diagnostics have been used in the tests reported in this paper:

- infra-red camera system (RT ... $\left.3000^{\circ} \mathrm{C}\right)$,

- one-color pyrometer $\left(200 \ldots 1100^{\circ} \mathrm{C}\right)$,

- two-color pyrometers $\left(550 \ldots 1600^{\circ} \mathrm{C}\right.$ and $\left.1000 \ldots 3500^{\circ} \mathrm{C}\right)$,

- video camera,

- thermo-couples,

- instrumented cooling loop (flow rate, in/outlet temperature).

For the investigation of neutron-irradiated samples, some modification to former testing procedures were required. These modifications concerned on one hand the sakjeztand on the other hand the testing facility. 
1

Due to the limited space in the neutron irradiation rig, the samples had to be miniaturized. Therefore the length of the cooling tubes exceeded the length of the CFC tiles only by $5 \mathrm{~mm}$ on each side of the module. This was not sufficient for commercial squeezing or flange connectors, and a special clamping mechanism was developed. For installing, the radioactive samples are placed on a small tray which is transported to the clamping mechanism by manipulator. When the sample is in the correct position, the water connectors are clamped to the sample. This is performed by a motor while the force is controlled by a load cell. Sealing is achieved by special sealing adapters machined from soft copper in combination with O-ring sealings and springs. The whole clamping system is attached to the door of the vacuum chamber of JUDITH. Once the sample has been installed, the door is closed and the sample is in the correct shooting position.

For better comparison, the samples in the pre-irradiation reference tests were designed identically to those of the post-irradiation experiments.

\subsection{Evaluation of Data}

The power absorbed by the mock-up during high heat flux loading $P_{\text {abs }}$ can be calculated directly from the increase of cooling water temperature. If the absorbed power is compared to the incident electrical power, an absorption coefficient of 80 to $85 \%$ is found for CFC monoblock modules.

Therefore the absurbed power can be measured rather exactly, but the definition of power density is more complicated. During the heat loading, the area covered by the electron beam is a little smaller than the total surface. If the power density is calculated, the value depends strongly on the assumed loading area (heated area or total surface area). For the assessment of the joints, a power density which refers to the total surface area $D_{t}$ is thought to be more suitable and the corresponding numbers are used in the following.

\subsection{Neutron Irradiation Experiment PARIDE}

The neutron irradiation experiment was performed in the High Flux Reactor in Petten. More than 600 samples of beryllium, CFC and tungsten alloys have been irradiated in this campaign. Nominal loading conditions were $0.5 \mathrm{dpa}$ at $350^{\circ} \mathrm{C}$ and $700^{\circ} \mathrm{C}$ respectively. The actual irradiation condition differed more or less from these nominal values according to the position of samples in the reactor. For the CFC monoblock mock-ups which are discussed in this paper, the following irradiation conditions must be assumed:

- $320^{\circ} \mathrm{C}, 0.34 \mathrm{dpa}$ ( according to $=0.33 \times 10^{25} \mathrm{~m}^{-2}, E>0.1 \mathrm{MeV}$ ), 49.6 full power days,

- $770^{\circ} \mathrm{C}, 0.35 \mathrm{dpa}$ ( according to $=0.37 \times 10^{25} \mathrm{~m}^{-2}$, E>0.1 MeV), 23.7 fulX 36 F5. days. 


\section{Results}

\subsection{Testing of Un-Irradiated Mock-Ups}

Three CFC monoblock mock-ups several times have been exposed to 1000 heating cycles (10 s heating, $10 \mathrm{~s}$ cooling) at different power densities. Aim of these tests was on one hand to study the heat removal efficiency of the different variants and on the other hand their performance under thermal cycling conditions as they are expected in the operation of ITER. The tested materials combinations and power densities $D_{1}$ were:

\section{- SEPcarb N31/ Glidcop: 7, $18 \mathrm{MW} / \mathrm{m}^{2}$}

- Dunlop Concept 1/ Glidcop: 7, 15, $19 \mathrm{MW} / \mathrm{m}^{2}$

- Dunlop Concept 1/ CuCrZr: 7, 15, 24 MW/m²

A more detailed description of the loading conditions is given in [5].

The surface temperature measured by means of the infra-red camera showed strong fluctuations. Normally (e.g. in the case of $\mathrm{Be} / \mathrm{Cu}$ mock-ups) this is an indication of a bad braze connection. But here strong fluctuations in the thermal conductivity of the CFC materials are responsible for this behaviour [6]. Such fluctuations lead to differences of the surface temperature of up to $200^{\circ} \mathrm{C}$. In spite of this non-uniformity, the thermal fatigue behaviour of the three mock-ups was excellent. Each of the modules was loaded several times up to 1000 heating cycles at different power densities, but no failure or degradation was observed. The distribution of surface temperatures measured by the infra-red camera stayed stable during all tests, this is an indication that no failure occurred during the tests.

In the post-mortem metallography of the first mock-up (SEPcarb N31/ Glidcop), small voids up to $1 \mathrm{~mm}$ approx. were observed in the braze layer. It was assumed that these voids were generated during the production process. In order to clarify this topic, the two other mock-ups were inspected by ultra-sonic methods before they were loaded with the last 1000 heating cycles at the highest power densities. Fig. 2 compares the result of this ultra-sonic inspection with the post-mortem metallography (mock-up Dunlop 'Concept $1 /$ Glidcop). This ultrasonic inspection is performed with a transducer inside the copper tube. The left picture shows the two dimensional map of the intensity of reflection. Areas with a high reflectance (red) are a sign for pores, voids or detachments. By comparison of these areas with the post-mortem metallography (right picture) it becomes clear that the voids in the braze at angular positions of $210^{\circ}$ and $310^{\circ}$ existed before the fatigue loadings. Nevertheless, they were stable during the thermal fatigue loading.

\subsection{Post-Irradiation Testing}

After-26utron-irradiation, most of the mock-ups were optically in a good condition. In the screening tests, one mock-up showed over-heating during loading by the 
electron beam (SEPcarb N31/ Glidcop $\mathrm{T}_{\text {ir }}=350^{\circ} \mathrm{C}$. But it cannot be proven that this fault was due to the neutron irradiation.

Only a limited number of mock-ups was pre-tested in screening experiments before they were irradiated in the fission reactor (mock-ups with Mo5Re heat sink tubes). The other irradiated mock-ups have to be compared with identical reference samples of the same materials combination (modules with $\mathrm{Cu}$ tubes).

\section{Mock-ups with Mo5Re tubes}

Identical monoblock mock-ups with Mo5Re heat sink tubes were compared in the electron beam facility at constant power densities before and after neutron-irradiation $\left(T_{\text {ir }}=770^{\circ} \mathrm{C}\right)$. Fig. 3 gives an example for such a comparison for a mock-up made from Dunlop Concept 1 . The distribution of surface temperatures measured by the infra-red camera did not change after neutron irradiation, but the surface temperature increased significantly. In fig. 4 for three mock-ups with different CFC armor the surface temperatures (measured by pyrometer) are plotted versus the absorbed power density. In all cases a significant increase of temperature after exposure to neutrons is observed. This is due to a decrease in thermal conductivity which was reported before for the CFC material SEPcarb N112 [7], and which is expected for the other CFC materials too [8]. The exact values of thermal conductivity will be available later from samples which had been included in the irradiation experiment PARIDE.

Dunlop Concept 1 which before irradiation had the best thermal conductivity of all three CFCs, was more influenced by the neutron irradiation than SEPcarb N31 and shows a higher increase of surface temperature than the latter. SEPcarb N112 shows the lowest thermal conductivity of the three CFCs before and after neutron irradiation.

\section{Mock-ups with copper iubes}

In a second test series, CFC monoblock mock-ups with copper heat sink tubes were loaded under screening conditions (steady state) from the top (12 mm CFC) and from the bottom (6 mm CFC) side. The tests were limited to surface temperatures below $2200^{\circ} \mathrm{C}$, according to power densities of 10 and $15 \mathrm{MW} / \mathrm{m}^{2}$ approximately. After screening, all samples were loaded by 100 heating cycles at power densities between 8 and $15 \mathrm{MW} / \mathrm{m}^{2}$ and one sample (Dunlop Concept $1 /$ Glidcop) up to 1000 cycles at $15 \mathrm{MW} / \mathrm{m}^{2}$. None of these samples showed failure or any instabilities.

Due to the better annealing effects of irradiation damages with increasing temperature, the decrease of thermal conductivity for the samples irradiated at $770{ }^{\circ} \mathrm{C}$ was found to be less distinctive. This is shown in fig. 5 for the materials combination Dunlop Concept 1/ Glidcop. 


\section{Summary}

No indication of failure was observed for CFC monoblock mock-ups loaded under thermal fatigue condition up to 1000 cycles at power densities $\leq 25 \mathrm{MW} / \mathrm{m}^{2}$. Two of the mock-ups were inspected by ultra-sonic methods before the last campaign of thermal cycling. It could be proved that the voids found in the post-mortem metallography existed before and had no effect on the integrity of the mock-up.

First neutron-irradiated CFC monoblocks have been tested in the electron beam facility JUDITH. These mock-ups had been irradiated in the High Flux Reactor in Petten up to $0.3 \mathrm{dpa}$ at 320 and $770^{\circ} \mathrm{C}$. All samples showed a significant increase of surface temperature, due to the decrease in thermal conductivity of the CFC materials. This effect is less distinctive for those samples irradiated at the higher temperature of $770^{\circ} \mathrm{C}$. During short thermal fatigue tests $(100$ cycles at 8 to 15 $\mathrm{MW} / \mathrm{m}^{2}$ ) no failure or instability occurred at any of the mock-ups.

\section{Acknowledgements}

The authors would like to acknowledge the help of F. Meuser in the preparation of the neutron-irradiation experiment. $\mathrm{H}$. Klöcker and $\mathrm{H}$. Münstermainn assisted in the electron beam experiments. In addition V. Gutzeit and $H$. Hoven assisted in the metallographic examination.

\section{References}

[1] M. Rödig, R. Duwe, J. Linke, A. Schuster, B. Wiechers, $3^{\text {rd }}$ IEA International Workshop on Beryllium Technology for Fusion, Mito City (Japan), Oct. 22-24, 1997

[2] M. Rödig, R. Duwe, J. Linke, A. Schuster, Fusion Engineering and Design, 37(1997), p. 317-334

[3] G. Vieider et al., $19^{\text {th }}$ Symposium on Fusion Technology (SOFT), Lisbon (Portugal), Sept. 16-20, 1996

[4] R. Duwe, W. Kühnlein, H. Münstermann, $18^{\text {th }}$ Symposium on Fusion Technology (SOFT), Karlsruhe (Germany), Oct. 22.-26, 1994

[5] M. Rödig et al, $4^{\text {th }}$ International Symposium on Nuclear Fusion Technology (ISFNT-4), Tokyo, April 06-11, 1997

[6] M. Rödig, R. Duwe, A. Gervash, J. Linke, A. Schuster, Physica Scripta Vol. T64, $60-66,1996$ 
[7] D. Moulinier: "Evolution des proprietes thermiques des materiaux composites carbone-carbone sous irradiation neutronique", Ph.D. thesis Conservatoire National des Arts et Metiers, Paris 1996

[8] V. Barabash et al., this conference

Figure captions

Fig. 1:Drawing of CFC monoblock mock-up

Fig. 2: Comparison of ultrasonic inspection (left) and post mortem metallography (right)

Fig. 3: Infra-red image of a monoblock mock-up made from Dunlop Concept 1 and Mo5Re, power density $D_{t}=2 \mathrm{MW} / \mathrm{m}^{2}$

Fig. 4: Surface temperature during electron beam loading before and after neutron irradiation for three CFC materials brazed to Mo5Re tubes.

Fig. 5: Surface temperature during electron beam loading for three CFC mockups (unirradiated, $\mathrm{T}_{\text {irr }}=350^{\circ} \mathrm{C}$ and $\mathrm{T}_{\text {ir }}=700^{\circ} \mathrm{C}$ )
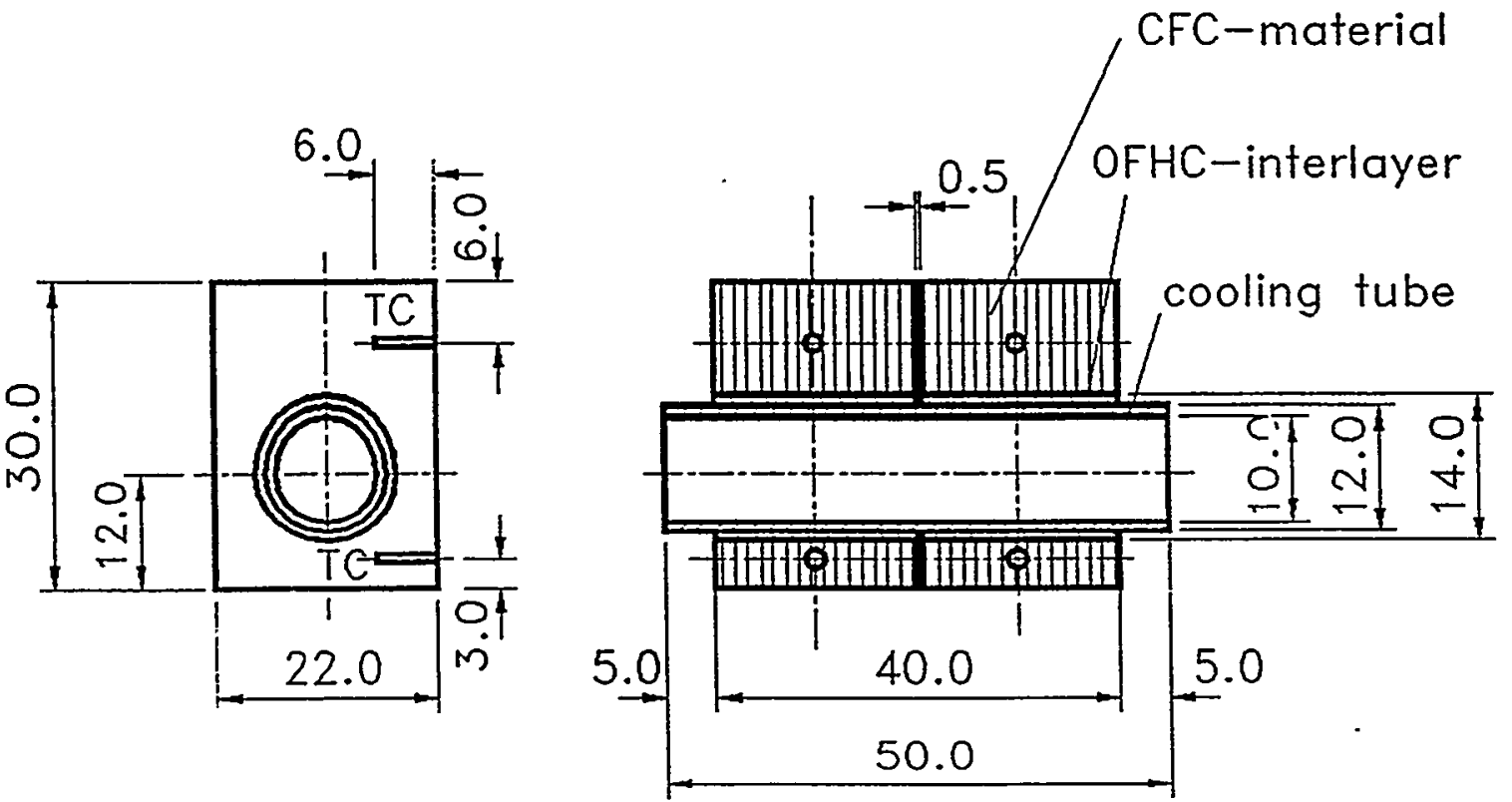

Fig. 1 

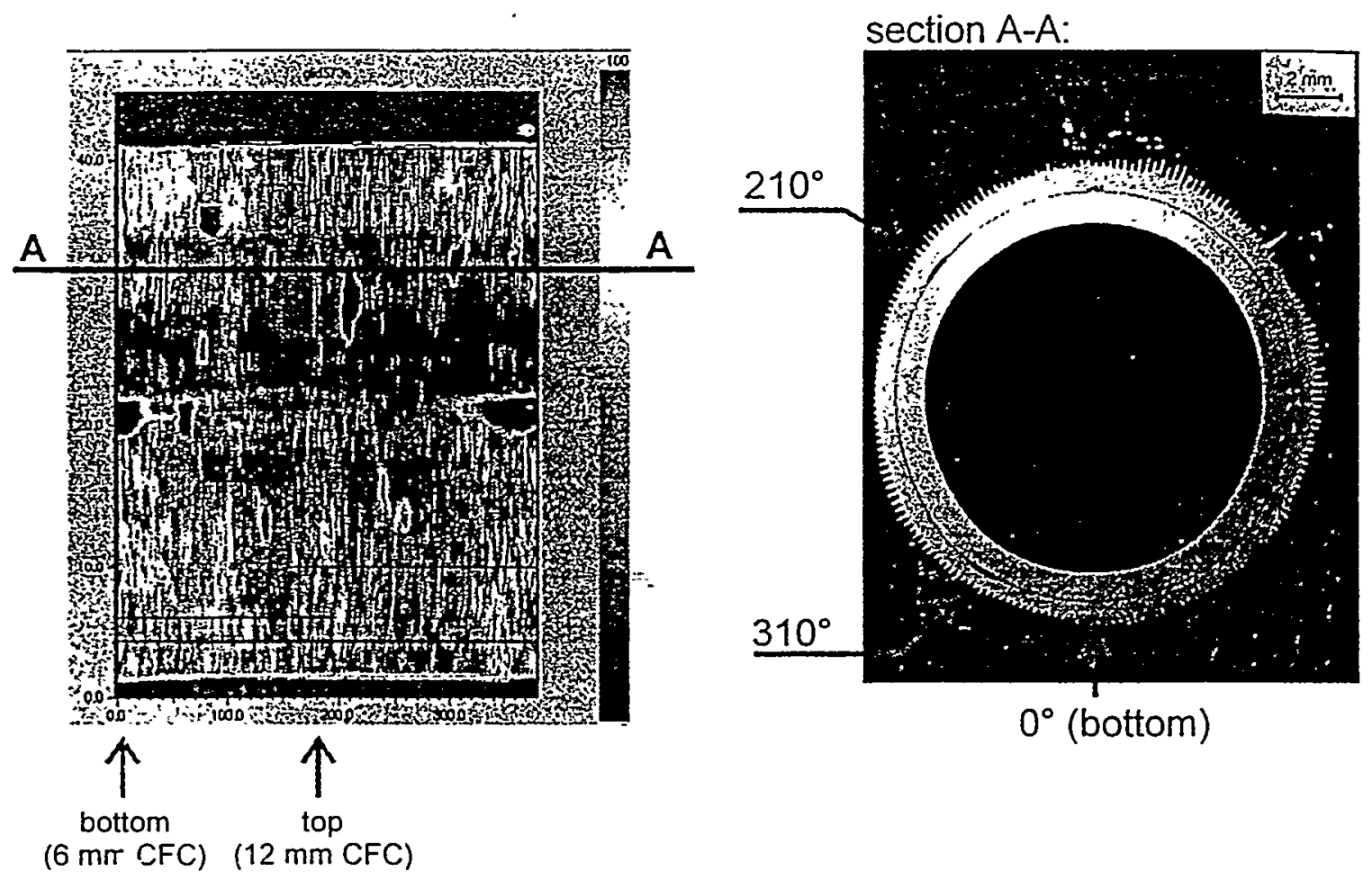

Fig. 2
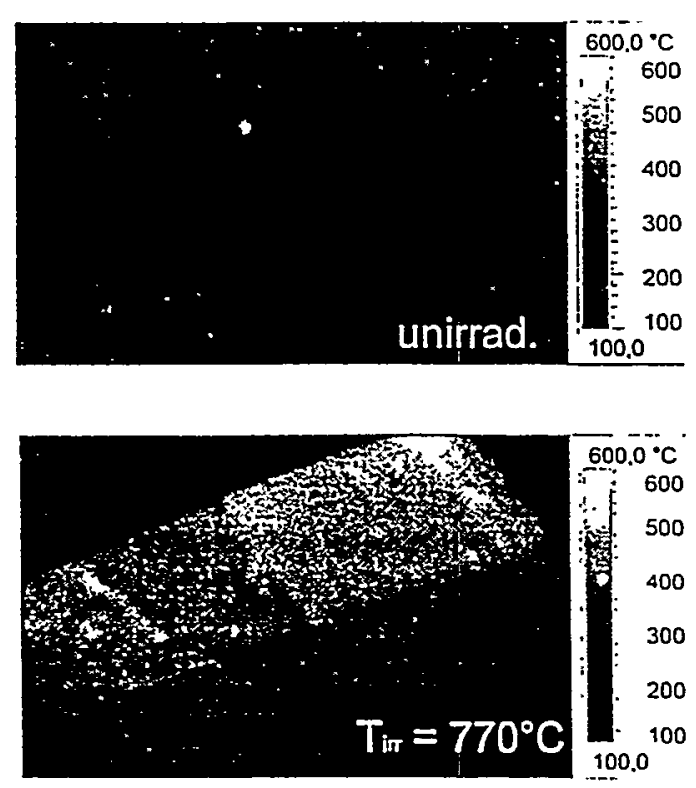

Fig. 3 


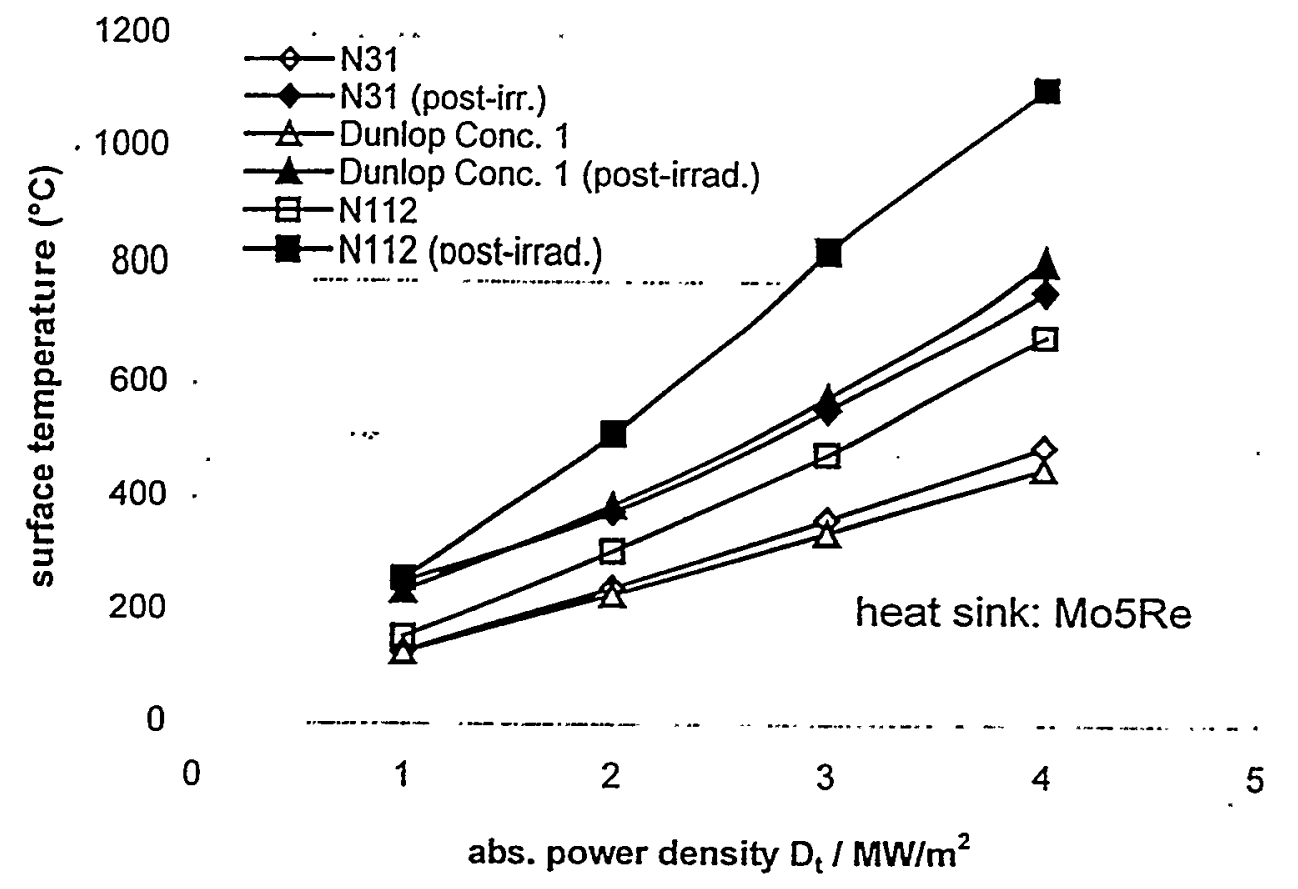

Fig. 4

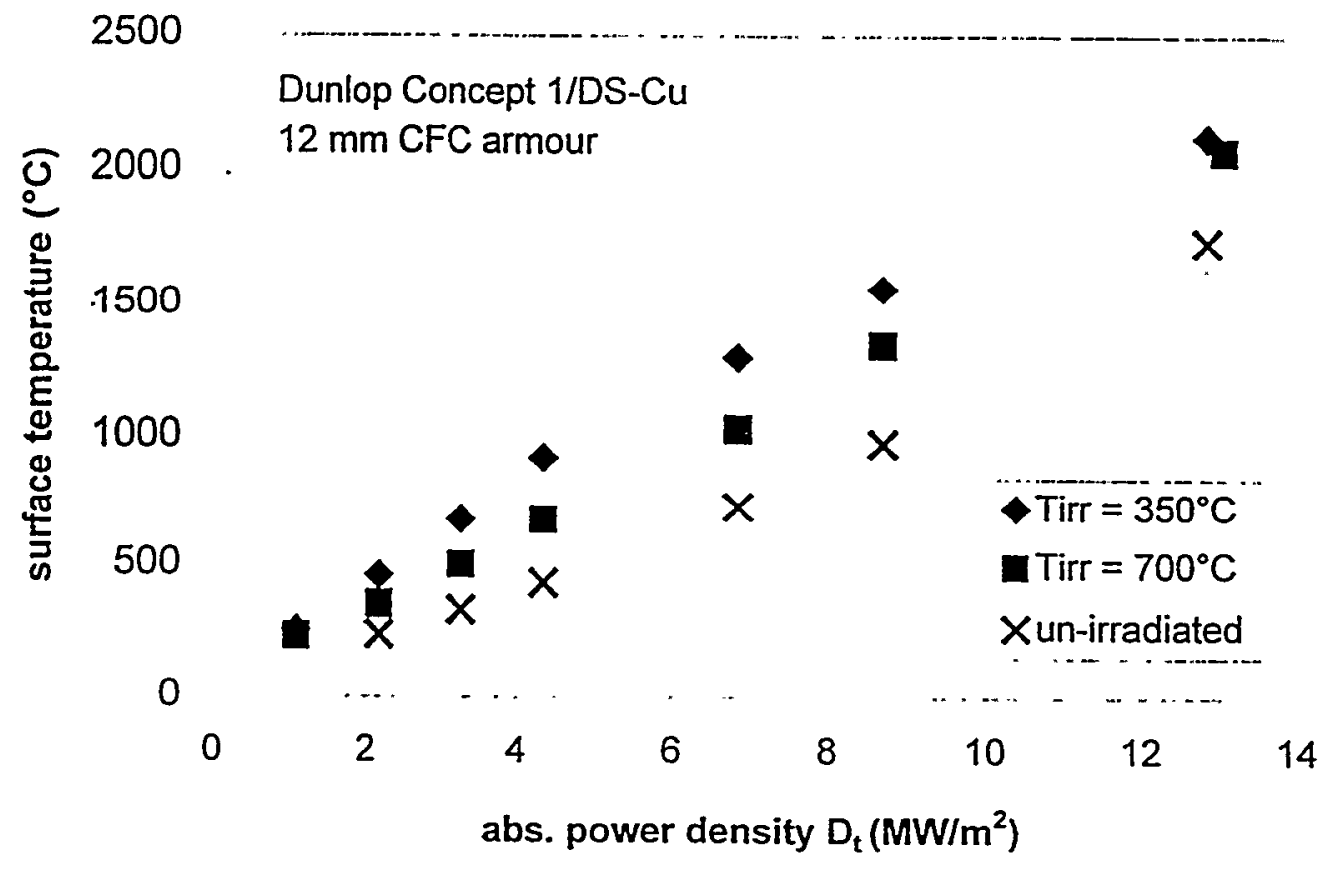

Fig. 5 


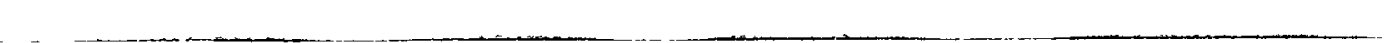


Summary Session

\{Verbal Discussions\}

XIII-1 
[page intentionally left blank]

XIII-2 


\section{Appendix A}

\section{Workshop Agenda}

A-1 

Agenda: US-Japan Workshop (97FT5-06 ) on

High Heat Flux Components \& Plasma Surface Interactions for Next Fusion Devices

December 8-11, 1997

12/1/1997

Warwick Regis Hotel

490 Geary St., San Francisco

800-827-3447, 415-928-7900, fax 415-441-8788

December 8 (Mon.)

9:00 Opening Remarks (20) R. Nygren (Sandia), S. Berk (DOE),

Session 1: activities in present and near term devices

N. Noda (NIFS), K. Wilson (Sandia)

9:20 Present status of LHD (30)

9:50 Divertor, first wall and PSI issues in LHD (30)

10:20 Status of NSTX and PSI issues (25)

10:45 coffee break

11:00 Design \& initial operation of W-shaped divertor in JT-60U (30)

11:30 Progress in DIII-D (25)

11:55 Highlights and plans for C-MOD (20)

12:15 announcements and lunch

Session II : PFC Development for near term devices

14:00 Utilization of high Z materials as PFCs (30)

14:30 Develoment of $W$ brush armor (20)

14:50 Development of high heat flux components at JAERI (30)

15:20 coffee break

15:40 Be-Cu Joining (20)

16:00 Problems and evaluation of plasma facing materials (30)

Special Session III : Historical Progress in PSI Studies

16:30 Small personal history on plasma surface interactions

17:00 adjourn until reception

O. Motojima \& S. Berk

O. Motojima (NIFS)

N. Noda (NIFS)

M. Peng (PPPL)

K. Masaki (JAERI)

C. Wong (GA)

MIT/Nygren (Sandia)

K. Nakamura \& C. Wong

T. Tanabe (Nagoya U.)

G. Wille (Boeing)

K. Nakamura (JAERI)

C. Cadden (Sandia)

N. Yoshida (Kyushu U.)

N. Noda \& K. Wilson

T. Yamashina (Hokkaido

U.)

19:00 Reception

The reception, hosted by Sandia National Laboratories and the US Department of Energy, will be held in the reception area at one end of the main dining room adjacent to the restaurant on the first floor of the Warwick Regis Hotel. 
US-Japan Workshop Agenda continued

December 9 (Tue.)

Session IV: wall conditioning, sputtering, erosion

8:30 Wall conditioning at the start up phase of LHD (30)

T. Tanabe \& Y. Hirooka

9:00 RF wall contioning (20)

9:20 Erosion/redeposition of high- $Z$ materials in a linear divertor simulator (30)

9:50 Erosion and impurity effects on PFC materials in PISCES-B (20)

10:10 Recent erosion/redeposition analysis (15)

A. Sagara (NIFS)

D. Cowgill (Sandia)

N. Ohno (Nagoya U.)

R. Doerner (UCSD)

Sze/Brooks (ANL)

10:25 coffee break

10:45 Dependence of graphite erosion yield on irradiation flux close to actual edge plasma condition (30)

11:15 DiMES experiments (20)

11:35 Reflected neutral particle spectra on MAP (30)

12:05 lunch

Session V: Plasma Studies

13:40 Effects of turbulent fluctuations on boundary ion temperatures in PISCES (20)

14:00 TFTR Experiments with Li $L$ (15)

14:15 Deposition of Li on a probe in TFTR (15)

Session VI: Development Issues for Near Term PFCs

14:30 Discussion, development issues for near term PFCs

15:30 coffee break

Session VII: PFM issues and development

15:45 W/Cu layers resistant to erosion and tritium permeation (30)

16:15 Review of recent work on removing tritium from PFCs (25)

Y. Ueda (Osaka U.)

D. Whyte (GA)

S. Ohtsu, K. Kobayashi, S. Tanaka (U. Tokyo)

S. Luckhardt

S. Luckhardt (UCSD)

C. Skinner (PPPL)

Y. Hirooka (UCSD)

A. Sagara \& C. Wong

A. Sagara \& C. Wong

N. Yoshida \& R. Causey

M. Shibui (Toshiba)

16:40 Chemical compatibility of $\mathrm{C}$ with $\mathrm{Be}(30)$

$\therefore$ Ashida \& K. Watanabe (Toyama U.)

17:10 Tritium retention in $\mathrm{Be}(20)$

R. Causey (Sandia)

17:30 Modeling of $\mathrm{H}$ isotope retention/release in PFC materials (15)

A. Grossman (UCSD)

$17: 45$ adjourn

Dinner arrangements on Tuesday and Wednesday evenings can be made during the day at the workshop if participants wish to dine together in groups for work or pleasure. San Francisco has many fine restaurants and a list of nearby restaurants is included with the workshop materials. 
US-Japan Workshop Agenda continued

December 10 (Wed.)

Session VIII: First Wall Development

M. Tillack \& N. Noda

8:30 HPD approaches, core radiation and He blanket, ST example (25)

C. Wong (GA)

8:55 Concept of FliBe blanket in FFHR (30)

9:25 APEX high fusion power density evaluation (20)

A. Sagara (NIFS)

N. Morley (UCLA)

9:45 Damage in the plasma facing part of the first wall (20)

10:05 Protective coating at the plasma facing part of first wall (20)

10:25 coffee break

10:35 Plasma spray coating development (20)

10:55 Recent progress at PTI in plasma spraying (15)

Session IX: PSI/PFM Issues and Collaboration

11:10 Discussion on PSI/PFM issues and collaborations

12:10 lunch

Session X : Panel on Future PFC Concepts

13:40 ALPS summary (20)

14:00 Heat removal issues with liquid metal PFCs (15)

14:15 Helium cooling experiments and prospect (15)

14:30 Comments on liquid/pebble divertor (15)

14:45 Novel concept for a moving belt PFC (15)

N. Yoshida (Kyushu U.)

N. Noda (NIFS)

15:00 He self pumping summary (15)

15:15 Characterization of liquid metal surfaces (15)

15:30 coffee break

Castro/Nygren (LANL)

S. Odell (Plasma Processes)

N. Noda \& R. Nygren

N. Noda \& R. Nygren

M. Tillack \& Y. Ueda

D. K. Sze (ANL)

R. Nygren (Sandia)

C. Baxi (GA)

Y. Ueda (Osaka U.)

Y. Hirooka (UCSD)

R. Nygren (Sandia)

R. Bastasz (Sandia)

\section{Session XI: Long Range PFC Development and Collaborations}

15:45 Group A Discussion: Liquid surface PFCs \& collaborations

15:45 Group B Discussion: Other PFCs \& collaborations

R. Nygren \& A. Sagara

17:15 adjourn

18:00 dinner groups per request of participants

December 11 (Thur.)

\section{Session XII : Supplement Session}

9:00 more activities / results in Japan (20)

N. Noda \& S. Luckhardt

9:20 Recent highlights from Judith (15)

9:35 Contributions from U. Toronto (15)

\section{Summary Session}

9:50 Remarks on the outlook for collaborations (20)

10:10 Summary/discussion: Liquid surface PFCs \& collab. (20)

10:30 coffee break

10:40 Summary/discussion: other PFCs \& collaborations (20)

11:00 Summary/discussion: Dev. issues for near term.PFCs (20)

11:20 Summary/discussion: PSI/PFM issues \& collaborations

K. Masaki \& D. K. Sze

N. Noda

KFA/R. Nygren

UT/R. Nygren

K. Wilson \& N. Yamashina Motojima/Noda, S. Berk

R. Nygren \& A. Sagara

N. Noda \& S. Luckhardt A. Sagara \& C. Wong N. Noda \& R. Nygren 
(20)

11:40 Closing remarks

N. Noda \& R. Nygren

11:50 adjourn

A-6 


\title{
Appendix B
}

\author{
List of \\ Participants \\ And \\ Addresses
}

B-1 



\section{List of Participants}

Dr. Kan ASHIDA

Phone: 81-764-45-6927

Fax: 81-764-45-6931

Bob BASTASZ

Phone: 510-294-2013

Fax: $\quad 510-294-3231$

Chandu BAXI

Phone: 619-455-3150

Fax: $619-455-2266$

Sam Berk

Phone: 301-903-4171

Fax: $\quad 301-903-1233$

Chuck CADDEN

Phone: 510-294-3650

Fax: $\quad 510-294-3410$

Rion CAUSEY

Phone: 510-294-3326

Fax: $\quad 510-294-3231$

Don COWGILL

Phone: 510-294-2146

Fax: $\quad 510-294-3231$
Toyama University

Hydrogen Isotope Research Center

Gofuku 3190, Toyama 930-8555

JAPAN

e-mail: ashida@hrc.toyama-u.ac.jp

Sandia National Laboratories, MS9162

P.O. Box 969

Livermore, CA 94551, USA

e-mail: bastasz@ca.sandia.gov

General Atomics

P.O. Box 85608

San Diego, CA 92186-5608

e-mail: Baxi@gav.gat.com

US Department of Energy

Office of Energy Research

ER-52, Germantown

Washington, DC 20585, USA

e-mail: sam.berk@mailgw.er.doe.gov

Sandia National Laboratories, MS9403

P.O. Box 969

Livermore, CA 94551, USA

e-mail: chcadde@sandia.gov

Sandia National Laboratories, MS9161

P.O. Box 969

Livermore, CA 94551, USA

e-mail: CAUSEY@sandia.gov

Sandia National Laboratories, MS9161

P.O. Box 969

Livermore, CA 94551, USA

e-mail: DFCOWGI@sandia.gov 
Russ DOERNER

Phone: 619-534-7830

Fax: $\quad 619-534-7716$

Arthur GROSSMAN

Phone: 619-534-9712

Fax: $\quad 619-534-7716$

Yoshi HIROOKA

Phone: 619-534-9720

Fax: $\quad 619-534-7716$

\section{Stan LUCKHARDT}

Phone: 619-534-9725

Fax: $\quad 619-534-7716$

Dr. Kei MASAKI

Teruo MATSUDA

Neil MORLEY

Phone: 310-206-1228

Fax: $\quad 310-825-2599$

Prof. Osamu MOTOJIMA

Phone: 81-572-58-2140

Fax: $81-572-58-2617$
University of California at San Diego 9500 Gilman Drive, Building 302

La Jolla, CA 92093-0035, USA

e-mail: rdoerner@fusion.ucsd.edu

University of California at San Diego

9500 Gilman Drive

La Jolla, CA 92093-0035, USA

e-mail: grossman@fusion.ucsd.edu

University of California at San Diego

9500 Gilman Drive, Building 302

La Jollà, CA 92093-0035, USA

e-mail: yhirooka@fusion.ucsd.edu

University of California, San Diego

9500 Gilman Drive

La Jolla, CA 92093, USA

e-mail: sluckhardt@fusion.ucsd.edu

Japan Atomic Energy Research Institute 801-1 Mukouyama

Naka-machi, Naka gun

Ibaraki-ken, JAPAN 311-01

e-mail: masakik@fusion.naka.jaeri.go.jp

Toyo Tanso America

University of California, Los Angeles

43-133 Engineering IV

405 Hilgard Avenue

Los Angèles, CA 90024-1597 USA

e-mail: morley@fusion.ucla.edu

National Institute for Fusion Science 322-6 Oroshi-cho, Toki 509-52, JAPAN e-mail: motojima@LHD.nifs.ac.jp 
Hiroo NAKAMURA

Dr. Kazuyuki NAKAMURA

Prof. Noda NOBUAKI

Phone: 81-572-58-2152

Fax: $81-572-58-2618$

Richard NYGREN

Phone: 505-845-3135

Fax: $\quad 505-845-3130$

Scott O'DELL

Phone: 205-851-7653

Fax: $205-859-4134$

Dr. Noriyasu OHNO

Phone: $81-52-789-3145$

Fax: 81-52-789-3944

Shigeki OHTSU

Phone: 81-3-3812-2111

$$
\text { Ext } 7010
$$

Fax: 81-3-3818-3455
Japan Atomic Energy Research Institute/ITER 801-1 Mukouyama

Naka-machi, Naka gun

Ibaraki-ken, JAPAN 311-01

e-mail: nakamuh@ipp..mpg.de

Japan Atomic Energy Research Institute

801-1 Mukouyama

Naka-machi, Naka gun

Ibaraki-ken, JAPAN 311-01

e-mail: nakamuk@naka.jaeri.go.jp

National Institute for Fusion Science

322-6 Oroshi-cho, Toki-shi 509-5292

JAPAN

e-mail: noda@LHD.nifs.ac.jp

Sandia National Laboratories, MS1129

Fusion Technology Department, 6428

Albuquerque, NM, 87185-1129, USA

e-mail: renygre@sandia.gov

Plasma Processes, Inc.

4914 D Moores Mill Road

Huntsville, AL 35811, USA

\section{Nagoya University}

Department of Energy Engineering and Science

Graduate School of Engineering

Nagoya, 464-8603, JAPAN

e-mail: ohno@nuee.nagoya-u.ac.jp

University of Tokyo

Dept. of Quantum Eng. \& Systems Science 7-3-1 Hongo Bunkyo-ku Tokyo, 113 JAPAN

e-mail: ohtsu@q.t.u-tokyo.ac.jp 
Martin PENG

Phone: 609-243-2305

Fax: $609-243-3315$

Dr. Akio SAGARA

Phone: 81-0572-58-2155

Fax: $\quad 81-0572-58-2618$

Dr. Masanoa SHIBUI

Phone: 045-510-5879

Fax: $\quad 045-500-1412$

Charles SKINNER

Phone: 609-243-2214

Fax: $609-243-2418$

Dai Kai SZE

Phone: $630-252-5180$

Fax: $630-252-5287$

Prof. Tetsuo TANABE

Mark TILLACK
Princeton Plasma Physics Laboratory

P. O. Box 451

Princeton, NJ 08543-0451

National Institute for Fusion Science

Research Operations Division, LHD Project

Shimoishi-cho-322-6, Toki-shi, 509-52

JAPAN

e-mail: sagara@LHD.nifs.ac.jp

Toshiba Corporation

Advanced Engineering Gr.

2-4, Suehiro, Tsurumi, Yokohama, 230-0045

JAPAN

e-mail: masanao.shibui@toshiba.co.jp

Princeton Plasma Physics Laboratory

Princeton University, P. O. Box 451

Princeton, NJ 08543, USA

e-mail: cskinner@rax.pppl.gov

Argonne National Laboratory

9700 South Cass Avenue, Bldg. 207

Argonne, IL 60439

e-mail: u1747@f.nersc.gov

Nagoya University

Department of Energy Engineering and Science

Nagoya, 464-8603, JAPAN

tanabe@cirse.magoya-u.ac.jp

University of California at San Diego

9500 Gilman Drive

La Jolla, CA 92093 USA 
Yoshio UEDA

Phone: $81-6-879-7236$

Fax: $81-6-879-7867$

Gerry WILLE

Ken WILSON

Phone: 510-294-2497

Fax: $\quad 510-294-3057$

Clement WONG

Phone: $619-454-4258$

Fax: $619-454-2266$

Prof. Toshiro YAMASHINA

Phone: 81-11-706-6659

Fax: $81-11-747-9366$

\section{Prof. Naoaki YOSHIDA}

Phone: $81-92-583-7716$

Fax: $81-92-583-7690$
Osaka University

Department of Electronic, Information Systems, and Energy Engineering

Graduate School of Engineering

2-1 Yamada-Oka, Suita, Osaka 565-0871

JAPAN

e-mail: yueda@ppl.eng.osaka-u.ac.jp

\section{Boeing Company}

MS 1067211

P.O. Box 516

St. Louis, MO 63166 USA

Sandia National Laboratories, MS9161

P.O. Box 969

Livermore, CA 94551-0969 USA

e-mail: klwilso@sandia.gov

General Atomics

P.O. Box 85608

San Diego, CA 92186-5608 USA

e-mail: won@gav.gat.com

Hokkaido University

Division of Quantum Energy Engineering

Sapporo, JAPAN 060

e-mail: yamasina@hune.hokudai.ac.jp

Research Institute for Applied Mechanics

Kyushu University

6-1 Kasugakoen, Kasuga, Fukuoka 816-8580

JAPAN

e-mail: yoshida@riam.kyushu-u.ac.jp 


\section{DISTRIBUTION}

$D-1$ 



\section{DISTRIBUTION}

1 Mohamed ABDOU

University of California, Los Angeles

Mechanical \& Aerospace Engineering

Department

44-114 Engineering IV

Box 951597

Los Angeles, CA 90095-1597, USA

1 Dr. Masato AKIBA

NBI Heating Laboratory, JAERI,

Naka-machi, Naka-gun, Kbaraki-ken, 311-0193, JAPAN

1 Dr. Kan ASHIDA

Toyama University

Hydrogen Isotope Research Center

Gofuku 3190, Toyama 930-8555, JAPAN

1 Charles BAKER

University of California, San Diego

9500 Gilman Drive

La Jolla, CA 92093, USA

1 Chandu BAXI

General Atomics

P.O. Box 85608

San Diego, CA 92186-5608, USA

1 Sam BERK

US Department of Energy

Office of Energy Research

ER-52, Germantown

Washington, DC 20585, USA

1 Jeffrey BROOKS

Argonne National Laboratory

Fusion Power Program

9700 S. Cass Avenue

Argonne, IL 60439, USA 
1 Robert CONN

University of California at San Diego

9500 Gilman Drive, Building 302

La Jolla, CA 92093-0035, USA

1 James DAVIS

University of Toronto

21 King's College Circle

Toronto, Ontario M5S 3J3, CANADA

1 Russ DOERNER

University of California at San Diego

9500 Gilman Drive, Building 302

La Jolla, CA 92093-0035, USA

1 Gianfranco FEDERICI

ITER Garching Joint Work Site

Max-Planck-Institut fur Plasmaphysik

Boltzmannstrasse 2

D-85748 Garching bei Munchen, GERMANY

1 Arthur GROSSMAN

University of California at San Diego

9500 Gilman Drive

La Jolla, CA 92093-0035, USA

1 Anthony HAAS

University of Toronto

21 King's College Circle

Toronto, Ontario M5S 3J3, CANADA

2 Ahmed HASSANEIN

Argonne National Laboratory

Fusion Power Program

9700 S. Cass Avenue

Argonne, IL 60439, USA 
1 Yoshi HIROOKA

National Institute for Fusion Science

322-6 Oroshi-cho, Toki 509-52, JAPAN

1 Prof. Satoshi ITOH

Advanced Fusion Research Center

Research Institute for Applied Mechanics

Kyushu University

87, Kasuga 816, JAPAN

1 Dr. Y. KUBOTA

National Institute for Fusion Science

322-6 Oroshi-cho, Toki-shi 509-5292;.JAPAN

1 Bruce LIPSCHULTZ

Massachusetts Institute of Technology

Plasma Science and Fusion Center

Room No. 17-103

77 Massachusetts Avenue

Cambridge, MA 02139, USA

1 Stan LUCKHARDT

University of California, San Diego

9500 Gilman Drive

La Jolla, CA 92093, USA

1 Dr. Kei MASAKI

Japan Atomic Energy Research Institute

801-1 Mukouyama

Naka-machi, Naka gun

Ibaraki-ken, 311-01 JAPAN

$1 \quad$ Dr. S. MASUZAKI

National Institute for Fusion Science

322-6 Oroshi-cho, Toki-shi 509-5292, JAPAN 
1 Richard MATTAS

Argonne National Laboratory

Fusion Power Program

9700 S. Cass Avenue

Argonne, IL 60439, USA

1 Dale MEADE

Princeton Plasma Physics Laboratory

P.O. Box 451

Princeton, NJ 08543-0451, USA

1 Peter K. MIODUSZEWSKI

Fusion Energy Division

Oak Ridge National Laboratory

P. O. Box 2009

Oak Ridge, TN 37831-8070, USA

1 Dr. T. MORISAKI

National Institute for Fusion Science

322-6 Oroshi-cho, Toki-shi 509-5292, JAPAN

1 Neil MORLEY

University of California, Los Angeles

43-133 Engineering IV

405 Hilgard Avenue

Los Angeles, CA 90024-1597, USA

1 Prof. Osamu MOTOJIMA

National Institute for Fusion Science

322-6 Oroshi-cho, Toki 509-52, JAPAN

1 Farouk NAJMABADI

University of California at San Diego

9500 Gilman Drive, Building 302

La Jolla, CA 92093-0035, USA 
1 Hiroo NAKAMURA

Japan Atomic Energy Research Institute/ITER

801-1 Mukouyama

Naka-machi, Naka gun

Ibaraki-ken, 311-01, JAPAN

1 Dr. Kazuyuki NAKAMURA

Japan Atomic Energy Research Institute

801-1 Mukouyama

Naka-machi, Naka gun

Ibaraki-ken, 311-01, JAPAN

1 Prof. M. NISHIKAWA

Osaka University

Department of Electronic, Information Systems, and

Energy Engineering

Graduate School of Engineering

2-1 Yamada-Oka, Suita, Osaka 565-0871, JAPAN

1 Prof. Nokuaki NODA

National Institute for Fusion Science

322-6 Oroshi-cho, Toki-shi 509-5292, JAPAN

1 Scott O'DELL

Plasma Processes, Inc.

4914 D Moores Mill Road

Huntsville, AL 35811, USA

1 Dr. Noriyasu OHNO

Nagoya University

Department of Energy Engineering and Science

Graduate School of Engineering

Nagoya, 464-8603, JAPAN

1 Shigeki OHTSU

University of Tokyo

Dept. of Quantum Eng. \& Systems Science

7-3-1 Hongo Bunkyo-ku Tokyo, 113, JAPAN 
1 Dr. N. OHYABU

National Institute for Fusion Science

322-6 Oroshi-cho, Toki-shi 509-5292, JAPAN

1 Martin PENG

Princeton Plasma Physics Laboratory

P.O. Box 451

Princeton, NJ 08543-0451, USA

1 Dave RUZIC

University of Ilinois

Fusion Studies Laboratory

100 Nuclear Engineering Laboratory =

103 South Goodwin Avenue

Urbana, IL 61801-2984, USA

1 Dr. Akio SAGARA

National Institute for Fusion Science

Research Operations Division, LHD Project

Shimoishi-cho-322-6, Toki-shi, 509-52, JAPAN

1 Michael J. SALTMARSH

Fusion Energy Division

Oak Ridge National Laboratory

P. O. Box 2009

Oak Ridge, TN 37831-8070, USA

1 Dr. Masanoa SHIBUI

Toshiba Corporation

Advanced Engineering Gr.

2-4, Suehiro, Tsurumi, Yokohama, 230-0045, JAPAN

1 A. SHIMIZU

Research Institute for Applied Mechanics

Kyushu University

6-1 Kasugakoen, Kasuga, Fukuoka 816-8580, JAPAN 
1 Charles SKINNER

Princeton Plasma Physics Laboratory

Princeton University

P. O. Box 451

Princeton, NJ 08543, USA

1 Ron STAMBAUGH

General Atomics

Fusion Group

P.O. Box 85608

San Diego, CA 92186-5608, USA

1 Dai Kai SZE

Argonne National Laboratory

9700 South Cass Avenue, Bldg. 207

Argonne, IL 60439, USA

1 Prof. S. TAKAMURA

Nagoya University

Department of Energy Engineering and Science

Graduate School of Engineering

Nagoya, 464-8603, JAPAN

1 Prof. Tetsuo TANABE

Nagoya University

Department of Energy Engineering and Science

Nagoya, 464-8603, JAPAN

1 Mark TILLACK

University of California at San Diego

9500 Gilman Drive

La Jolla, CA 92093, USA

$1 \quad$ Prof. K. TOKUNAGA

Research Institute for Applied Mechanics

Kyushu University

6-1 Kasugakoen, Kasuga, Fukuoka 816-8580, JAPAN 
1 Yoshio UEDA

Osaka University

Department of Electronic, Information Systems, and

Energy Engineering

Graduate School of Engineering

2-1 Yamada-Oka, Suita, Osaka 565-0871, JAPAN

1 Gerry WILLE

Boeing Company

MS 1067211

P.O. Box 516

St. Louis, MO 63166, USA

1 Clement WONG

General Atomics

P.O. Box 85608

San Diego, CA 92186-5608, USA

1 Prof. Toshiro YAMASHINA

Hokkaido University

Division of Quantum Energy Engineering

Sapporo, 060, JAPAN

1 Prof. Naoaki YOSHIDA

Research Institute for Applied Mechanics

Kyushu University

6-1 Kasugakoen, Kasuga, Fukuoka 816-8580, JAPAN

1 MS0736 N. R. Ortiz, 6400

2 MS1056 B. L. Doyle, 1111

W. R. Wampler, 1111

7 MS1129 M. A. Ulrickson, 6428 (1)

R. E. Nygren, 6428

D. L. Youchison, 6428 (1)

6428 File (3) 
$5 \quad$ MS9161 Ken Wilson, 8716

Rion Causey, 8716

Don Cowgill. 8716

Bob Bastasz, 8716

Dean Buchenauer, 8716

1 MS9403 Chuck Cadden, 8240

1 MS9018 Central Technical Files, 8940-2

2 MS0899 Technical Library, 4916

1 MS0619 Review \& Approval Desk, 12690 
